JEAN-BAPTISTE MAUDET

TERRES

DE TAUREAUX

LES JEUX TAURINS

DE L'EUROPE À L'AMÉRIQUE

PRÉFACE DE JEAN-ROBERT PITTE

$\mathrm{C} \Lambda \mathrm{S} \Lambda \mathrm{DE}$ VEL $\Lambda$ ZQVEZ 


\section{Terres de taureaux}

Les jeux taurins de l'Europe à l'Amérique

\section{Jean-Baptiste Maudet}

Éditeur : Casa de Velázquez

Année d'édition : 2010

Date de mise en ligne : 8 juillet 2019

Collection : Bibliothèque de la Casa de Velázquez

ISBN électronique : 9788490962459

\section{Sbooks}

http://books.openedition.org

Édition imprimée

ISBN : 9788496820371

Nombre de pages : 512

Référence électronique

MAUDET, Jean-Baptiste. Terres de taureaux : Les jeux taurins de l'Europe à l'Amérique. Nouvelle édition [en ligne]. Madrid : Casa de Velázquez, 2010 (généré le 02 février 2021). Disponible sur Internet : <http://books.openedition.org/cvz/7680>. ISBN : 9788490962459.

(c) Casa de Velázquez, 2010

Conditions d'utilisation :

http://www.openedition.org/6540 
Cet ouvrage est diffusé en accès ouvert dans le cadre du projet OpenEdition Books Select.

Ce programme de financement participatif, coordonné par OpenEdition en partenariat avec Knowledge Unlatched et le consortium Couperin, permet aux bibliothèques de contribuer à la libération de contenus provenant d'éditeurs majeurs dans le domaine des sciences humaines et sociales.

La liste des bibliothèques ayant contribué financièrement à la libération de cet ouvrage se trouve ici :

https://www.openedition.org/22515.

This book is published open access as part of the OpenEdition Books Select project.

This crowdfunding program is coordinated by OpenEdition in partnership with Knowledge Unlatched and the French library consortium Couperin. Thanks to the initiative, libraries can contribute to unlatch content from key publishers in the Humanities and Social Sciences.

Discover all the libraries that helped to make this book available open access: https: / $/$ www.openedition.org/22515?lang $=e n$.

\section{IIIJ Qopenedition couperinore}




BIBLIOTHÈQUE DE LA CASA DE VELÁZQUEZ 

BIBLIOTHĖQUE DE LA CASA DE VELÁZQUEZ

VOLUME 46

\section{JEAN-BAPTISTE MAUDET \\ TERRES DE TAUREAUX \\ LES JEUX TAURINS \\ DE L'EUROPE À L'AMÉRIQUE \\ PRÉFACE DE JEAN-ROBERT PITTE}


Directeur des publications: Jean-Pierre Étienvre.

Responsable du service des publications : Marie-Pierre Salès.

Secrétariat d'édition et mise en pages : Blanca Naranjo.

Traitement des illustrations: Carlos Sánchez.

Maquette de couverture : Manigua.

Traduction : Alistair Ross (anglais).

ISBN : 978-84-96820-37-1. ISSN : 0213-9758.

(c) Casa de Velázquez 2010 pour la présente édition

Casa de Velázquez, c/ de Paul Guinard, 3. Ciudad Universitaria 28040 Madrid EsPaña

Tél. : (34) 9145515 80. Fax : (34) 9154972 50. Site Internet : www.casadevelazquez.org

En application du Code de la propriété intellectuelle, il est interdit de reproduire intégralement ou partiellement, par photocopie ou tout autre moyen, le présent ouvrage sans autorisation du Centre français d'exploitation du droit de copie (20, rue des Grands-Augustins, 75006 Paris).

Cualquier forma de reproducción, distribución, comunicación pública o transformación de esta obra sólo puede ser realizada con la autorización de sus titulares, salvo excepción prevista por la ley. Diríjase a CEDRO (Centro Español de Derechos Reprográficos, www.cedro.org) si necesita fotocopiar o escanear algún fragmento de esta obra.

Le catalogue des publications de la Casa de Velázquez peut être consulté sur le site Internet de l'établissement ou expédié sur demande. 


\section{PRÉFACE}

Plongeons d'abord dans la nuit des temps taurins, quitte à prendre à rebroussepoil l'auteur de ce beau livre qui se méfie dans son préambule de toute linéarité historique. Pourtant, oui, nos ancêtres du Magdalénien étaient déjà saisis de crainte et d'admiration face à la majesté de l'aurochs mâle, au point de recouvrir de ses représentations les parois d'une salle de la grotte de Lascaux. Plus tard, une dizaine de millénaires avant notre ère, comme l'a naguère montré Jacques Cauvin, les civilisations mésolithiques du Proche-Orient commencèrent à rendre un culte à des figures de déesses-mères et de taureaux, principes de fécondité et de puissance de la vie. Dès lors, les hommes apprirent à dompter l'univers sauvage, tant végétal qu'animal. Ils comprirent qu'au lieu de se comporter en cueilleurs, chasseurs et pêcheurs, ils pouvaient maîtriser le processus de reproduction. De prédateurs fragiles, ils devinrent agriculteurs et éleveurs ingénieux, conscients de leur statut procréateur, comme des moyens d'assurer la pérennité de l'espèce humaine et son expansion sur terre. La révolution néolithique était en marche, fondée sur une révolution culturelle et non sur une réponse technique au changement du climat et à la nécessité démographique. C'est au moins de ces époques reculées que date la fascination de l'homme pour le taureau que l'on retrouve dans toutes les grandes civilisations méditerranéennes de l'Antiquité et aussi dans d'autres aires culturelles de la planète.

Bien des siècles plus tard, alors que le christianisme semblait avoir balayé les cultes païens, la péninsule Ibérique, puis le Midi de la France, réinventent le singulier rituel du combat d'un homme avec un taureau. Qu'il n'y ait aucune continuité entre les croyances anciennes et les jeux modernes et contemporains, que ceux-ci aient peu de lien avec la religion, comme Jean-Baptiste Maudet tient beaucoup (trop ?) à le souligner, n'enlève rien à la proximité des significations et des expressions. Il n'existe aucune parenté directe entre les ziggourats mésopotamiennes, les pyramides d'Égypte, celles des Mayas et celle de Pei au Louvre. Pourtant, les formes et les significations sont proches, car il existe un patrimoine mental commun à toutes les civilisations. C'est là l'énigme de la condition humaine, de son histoire et de sa géographie.

Pourquoi là ? Comment ces jeux taurins sont-ils devenus constitutifs d'une identité, d'une fierté, d'une aire culturelle? Comment se répartissent-ils dans 
l'espace du Sud-Ouest européen avec d'innombrables variantes, ici décrites par le menu, mêlant dans des configurations diverses le cheval au taureau ? Comment s'est diffusé le phénomène outre-Atlantique ? Pourquoi survit-il envers et contre tout, malgré les clameurs des bien-pensants de l'Europe et de l'Amérique du Nord ? C'est à toutes ces questions que répond Jean-Baptiste Maudet dans l'essai le plus complet qui soit sur ce phénomène envisagé sous l'angle de la géographie, c'est-à-dire de la localisation et de la répartition. Il faut être aficionado pour y consacrer des années de recherche, mais il faut aussi conjuguer sa passion avec des méthodes scientifiques éprouvées, avec une vraie culture géographique et avec le désir de donner à comprendre. Toutes les sciences de l'Homme ont été sollicitées pour concourir à dégager les explications que demande la très fine cartographie intégrée à cet essai et qui en est même la base documentaire la plus originale. Il est fait appel à l'anthropologie, à l'histoire, à l'archéologie, à la psychologie, à la sociologie, à l'économie, au droit, aux sciences politiques, à la linguistique, etc. C'est là le signe d'une maturité qui se traduit aussi par la limpidité d'expression et l'intégration discrète au développement de la pensée de l'appareil théorique nécessaire. Avant de devenir un livre, ce fut une thèse, au vrai sens du terme. La gymnastique des échelles est l'un des talents du géographe. Elle est ici pratiquée avec bonheur et l'on passe constamment des villages aux quartiers urbains, aux villes, aux régions, aux pays, aux portions de continents, aux solidarités transatlantiques selon les besoins de la démonstration.

Notre Europe est faite d'espaces culturels contigus et parfois emboîtés. La tauromachie dessine l'un d'entre eux, l'un des plus insolites qui soit dans un univers dominé par la technique. Elle prouve que l'humanité ne vit pas seulement de pain, dans une perspective purement matérialiste, et qu'elle a besoin de rêve. L'utile et le nécessaire ne se laissent pas si facilement enfermer dans des statistiques de production, de consommation et de disponibilités monétaires, ce qui est plutôt rassurant. Le sport, la poésie, la musique, le bon vin et l'amour sont biologiquement parlant inutiles; ils n'en sont pas moins nécessaires à la survie de l'espèce humaine sur terre. Dans cette partie latine de l'Europe et de son miroir déformant américain, les jeux taurins expriment l'affirmation exacerbée de la virilité dans un univers affectif dominé par les femmes. Ils disent aussi la supériorité de l'homme sur la bête et sur tout ce qu'il est convenu d'appeler "nature ", ce qui n'empêche pas une véritable admiration pour le taureau. Ce sont des constantes chez les riverains de la Méditerranée et, singulièrement, dans les sociétés de tradition catholique.

La tauromachie concerne l'affrontement de l'homme avec toutes sortes de bovins. Souvent ce sont des vaches, plus légères que les mâles, mais dotées néanmoins de cornes et se livrant à des charges et des ruades qui ne sont pas sans risque. Lorsqu'il s'agit de taureaux, c'est une autre histoire. Cet animal est le plus puissant du cortège animal des sociétés d'éleveurs. Il est domestiqué, mais jamais débarrassé de ses pulsions violentes. Il est indispensable à la multiplication des vaches, pourvoyeuses de lait et de veaux, mais un seul individu par troupeau suffit. L'idée même d'élevage de taureaux est une folie du point de vue du projet et des besoins de l'élevage. Le Sud-Ouest européen et une partie du 
continent américain l'ont codifié et développé afin d'offrir les plus beaux spécimens possibles à l'admiration des foules. Là où la mise à mort se pratique, la chance de survie de l'animal face à l'épée du torero est faible, mais elle n'est pas nulle et c'est ce risque de transgression, de renversement de la norme qui donne toute sa grandeur au rite, dans un monde qui remplace chaque jour un peu plus l'art de vivre et de gouverner par le frileux principe de précaution.

Lecteurs qui allez entrer dans la somme érudite, patiemment rassemblée et mise en forme élégante par Jean-Baptiste Maudet, quel que soit votre sentiment vis-à-vis de ces jeux qui dérangent parfois en dehors des régions où ils se déroulent, nul doute que vous en ressortirez plus riches, ayant mieux compris ce que représente la tauromachie pour ceux qui en ont besoin afin d'habiter pleinement leur territoire. Vous saurez aussi que la géographie a changé, qu'elle s'intéresse désormais à tout, porte un regard éclairant sur chaque réalité qui s'inscrit à la surface de la terre, y compris lorsqu'elle relève de l'immatériel et de la culture. L'amour des taureaux n'est pas la moins intrigante d'entre elles, lui qui enflamme les coeurs et qui exacerbe les sens jusqu'aux larmes qui se mêlent au sang de la bête et, parfois, de l'homme. Sans cette tension venue du tréfonds des cultures méditerranéennes, qu'elle possède ou non une fonction symbolique sacrificielle (laissons cette question pendante), l'Europe manquerait de ressort, de ce sentiment tragique de la vie hors duquel les peuples s'ennuient. Que des Allemands ou des Anglais assistent subjugués, tous les étés, aux fêtes et spectacles taurins d'Espagne, du Portugal ou de France, montre combien le Nord a besoin du Sud. Que le drapeau espagnol flotte sur bien des lieux tauromachiques d'Europe et d'Amérique montre que le souvenir des guerres européennes et coloniales a laissé enfin place à l'écoute et à l'estime. Que l'amour des taureaux contribue à la compréhension mutuelle est une belle et bonne chose.

Jean-Robert PITTE

Membre de l'Institut 



\section{REMERCIEMENTS}

S'intéresser aux jeux taurins à l'échelle de leur extension géographique a nécessité des kilomètres d'aventure humaine pour rencontrer les autres, ceux qui en savent plus que moi, ceux qui en parlent mieux que moi, éparpillés du Sud-Ouest européen à l'Amérique. Je les remercie pour ce voyage qui s'achève avec ce livre.

Ce livre commence dans le bureau de Jean-Robert Pitte, lorsque je décidai de partir en Espagne et qu'il me dissuada de travailler sur les objets géographiques qui n'existent pas, en l'occurrence : le magnifique fleuve qui ne traverse pas Madrid.

— À part ça, qu'est-ce qui vous intéresse en Espagne?

- Les taureaux.

- Alors travaillez sur les taureaux et entourez-vous.

Je ne m'imaginais pas alors à quel point j'allais m'intéresser à un sujet de géographie qui existait si peu et qui, de proche en proche, me fit depuis l'Espagne rejoindre l'Amérique, depuis la corrida, la grande famille des rodéos, et finalement traverser tant de lieux où s'affrontent des taureaux et des hommes. De la maitrise jusqu'au doctorat, Jean-Robert Pitte n'a cessé de passer mes recherches au crible du bon sens scientifique : poser d'abord des questions simples, donner toujours des réponses claires et surtout éviter les jargons d'écoliers pour être lu de tous.

M'entourer fut effectivement le meilleur des apprentissages qui me permet aujourd'hui de remercier ceux qui n'ont pas été surpris que je débarque chez eux, avec mon sac-à-dos et mes questions. Ne sachant pas alors qu'il deviendrait aussi un ami, la rencontre la plus décisive d'un point de vue scientifique fut celle de Frédéric Saumade, en 1999, dans son village du Cailar, en Camargue, tout étonné de savoir que l'on pouvait arriver en train jusque chez lui depuis la capitale. Un soutien de la première heure me fut également accordé par Pedro Romero de Solís, sans lequel je n'aurais jamais pu travailler à Séville et jamais compris jusqu'à quel point il était possible de marier la truculence d'un sourcil pointu et la curiosité scientifique. Parmi les soutiens de la première et de la dernière heure, je remercie également Araceli Guillaume-Alonso, pour ses conseils 
clairvoyants et ses lectures irremplaçables sur l'histoire de la corrida qui malheureusement fait encore l'objet d'étranges détournements. Je remercie également Luís Capucha et José María Sotomayor pour tout ce qu'ils m'ont apporté sur le Portugal et l'Espagne taurine, en m'ouvrant leur bibliothèque, leurs archives et finalement leur maison pour avoir le temps d'en apprendre le plus possible.

Je souhaite ensuite remercier les géographes qui m’ont guidé dans mes réflexions ou qui m'ont apporté des informations utiles à mon propos: Michel Favory, habité d'une docte passion pour la tauromachie, Marilys Dauga pour tout ce qu'elle sait sur la course landaise, Jean-Pierre Augustin, Guy Di Méo, André Humbert, Sophie Lignon, Claire Guiu et Jean-René Trochet qui ont toujours fait preuve d'attention à l'égard de mon travail chaque fois que je les ai sollicités. Plus largement, je tiens à remercier le laboratoire "Espace, Nature et Culture " ainsi que toute l'équipe du laboratoire "Société, Environnement, Territoire » avec qui je travaille depuis déjà quelques années.

Merci aussi à tous ceux qui m'ont encouragé et m'ont fait profiter de tout ce qui était en leur pouvoir pour m'éclairer : Annie Maïllis, Antoinette Molinié, Jean-François Jouneau, Jean-Michel Gouffrand, Jean-François Claverie, Bernard Traimond, François Zumbiehl, Francis Jauréguiberry, Dominique Fournier, Francis Wolff, Jean-Pierre Digard, Miguel Darrieumerlou, Jean-René Laval, José Carlos Arévalo, Bénédicte Langue, Jean-Marie, Odile, Marco, MarieAnne, Cyril, Mathieu, David, Sophie, Thomas, Matthieu, Torrente. J'exprime également toute ma reconnaissance à ceux qui m'ont accompagné pendant mes enquêtes, parfois pour de longues heures, parfois pour quelques instants qui n'ont pas moins d'importance dans le rythme curieux du terrain : Alberto de Jesús en Espagne, Nuno Carvalho au Portugal, Bartolomé Puiggros Plana, Pablo Gómez de Barbieri, Claudio Sillero, Fanni Muñoz et Juan Ossio au Pérou, Janan, Hernán Tapia, Santiago Yepes, David Alemán en Équateur, Javier Arriola au Mexique. Merci aussi à ceux que je n'ai pas pu rencontrer physiquement, mais avec qui j'ai pu échanger par courrier, parfois sur plusieurs années : Raúl Gordon Blasini pour le Venezuela, Julián Parra Díaz pour la Colombie, Luis Ruiz Quiroz pour le Mexique. J'aimerais également remercier des nouveaux venus dans un parcours qui se prolonge depuis 2008 en Californie avec Frédéric Saumade grâce à l'obtention d'un programme de l'Agence nationale de la recherche qui m’a déjà permis de préciser des données plus anciennes travaillées dans le cadre de mon doctorat : Buck Carothers et sa famille, Rick et Linda, Liliana et Manuel Souza, Marcos Franco, Olga Nájera-Ramírez de l'université de UC Santa Cruz, Alex Saragoza et Nathan Sayre de l'université de Berkeley ainsi que Paul Starrs de l'université du Nevada.

La Casa de Velázquez est un lieu dont on sort différent. Je tiens à remercier toutes les personnes qui la font exister et les directeurs de recherche Xavier Huetz de Lemps, Pierre Moret et Benoît Pellistrandi que j'ai connus à Madrid durant deux années qui n'offrent pas meilleur cadre de vie à l'approfondissement d'un travail : de la liberté dans des murs solides. Après plusieurs années hors de ce lieu, c'est encore la Casa de Velázquez et son actuel directeur JeanPierre Étienvre qui me font l'honneur de publier ce livre. Mes recherches voient 
ainsi le jour grâce au travail de Marie-Pierre Salès, de Blanca Naranjo et de Carlos Sánchez qui désormais connaissent peut-être cet ouvrage mieux que moi, tant il faut souligner l'art et le soin qu'ils ont apportés à en faire rien de moins précieux qu'un livre.

Enfin, merci à toutes les personnes qui se sont impliquées dans la réalisation concrète et parfois ingrate de ce travail, allant des relectures fragmentaires au décryptage de formats de fichiers insolites : Monique Morales, Julien Félix, Elvire, Virginie Boilé, Damien Martin, Aurélien, Hervé et Marie-Pierre Mazeau.

Bien sûr, merci à ma famille qui a eu la patience d'écouter les mêmes refrains si longtemps : mes parents, Nelly, Stéphane, Pascale, mes grands-parents qui n'ont vu qu'une partie du projet, ma grand-mère Amatxi qui m'a fait connaître les taureaux et ma Violaine, princesse de caste, qui pourrait apparaître à chaque étape de ces dix années de remerciements.

J.-B. M. 



\section{ABRÉVIATIONS}

ACOCHA Asociación Cofradía del Chagra (Pérou)

AFCE Association française des coureurs d'encierros (France)

AGL Asociación de Ganaderías de Lidia (Espagne)

ABQM Associação Brasileira de Criadores de Cavalo

Quarto de Milha (Brésil)

AEGRB Agrupación Española de Ganaderos de Reses Bravas (Espagne)

CAUE Conseil d'architecture, d'urbanisme et d'environnement (France)

CCNAT Comisión Consultiva Nacional de Asuntos Taurinos (Espagne)

COFM Comissão Organizadora das Festas da Moita (Portugal)

DF Distrito Federal - México DF (Mexique)

FARC Fuerzas Armadas Revolucionarias de Colombia (Colombie)

FEDECOLEO Federación de Coleo de Colombia (Colombie)

FEVECO Federación Venezolana de Coleo (Venezuela)

FITA Federación Internacional de Toreros Americanos

(Colombie, Mexique, Pérou, Équateur)

FFCC Fédération française de course camarguaise (France) 
FFCL Fédération française de course landaise (France)

GLU Ganaderos de Lidia Unidos (Espagne)

IGAC Inspecção Geral das Actividades Culturais (Portugal)

INEGI Instituto Nacional de Estadística, Geografía e Informática (Mexique)

MAPA Ministerio de Agricultura, Pesca y Alimentación (Espagne)

NATC National Association of Taurine Clubs of United States of America (États-Unis)

PBR Pro Bull Riders (États-Unis)

PRCA Professional Rodeo Cowboys Association (États-Unis)

SNTP Sindicato Nacional de Toreiros de Portugal (Portugal)

UCTL Unión de Criadores de Toros de Lidia (Espagne)

UNDETOC Unión de Toreros de Colombia (Colombie)

UVTF Union des villes taurines françaises (France) 


\section{INTRODUCTION}

\section{I. — UNE AIRE CULTURELLE DE LA TAUROMACHIE}

\section{LA SINGULIÈRE DIVERSITÉ DES JEUX TAURINS}

La diversité des jeux taurins est méconnue du grand public. La raison tient à l'emploi même du terme tauromachie. En effet, ce terme fonctionne le plus souvent comme une représentation culturelle et subjective du fait taurin, et non comme un terme permettant d'identifier des pratiques de même nature. Dans sa version la plus caricaturale, la tauromachie est synonyme de corrida, reléguant en arrière-plan la grande diversité des affrontements entre les hommes et les bovins qui sont pourtant tributaires d'une même analyse. Si l'on accepte de considérer que le taureau du mot tauromachie est un animal générique (les bovins auxquels les hommes se mesurent), alors la famille des pratiques tauromachiques s'agrandit considérablement. Comment justifier un tel regroupement ? La réponse est le fruit d'une observation factuelle : il n'existe guère d'autres espèces avec lesquelles les hommes se mesurent en des corps à corps violents au cours de spectacles publics d'une telle ampleur. La chasse et la pêche échappent à cette catégorie, en ce qu'elles ne sont pas des spectacles. Il existe des combats d'animaux, organisés par l'homme ou par les lois de la nature, mais l'homme n'y participe pas en personne : soit il en est l'organisateur, généralement avec des animaux de même espèce (taureaux, chevaux, béliers, chiens, coqs, poissons, scarabées, criquets), soit il est un simple observateur, derrière son poste de télévision, fasciné par le spectacle magnifié de la vie sauvage. Les numéros de cirque avec des animaux, en dépit des risques, s'apparentent plus à des exercices de dressage qu'à des combats réels. Enfin, l'équitation, si présente dans les pratiques tauromachiques, repose moins sur l'affrontement, même si cette dimension est présente, que sur l'entente harmonieuse de l'homme et de l'animal. Pour autant, les jeux taurins ne sont pas totalement étrangers à ces activités et participent d'un débat plus général, très souvent polémique, sur les relations complexes entre les hommes et les animaux dans notre monde contemporain. Préférer les termes de jeux taurins à celui de tauromachie a sim- 
plement pour objectif d'attirer l'attention sur la nécessité de ne pas isoler cette activité de l'ensemble des interactions homme-animal et d'élargir l'observation des faits taurins à des pratiques et des espaces géographiques qui généralement échappent à son analyse.

\section{TAUROMACHIES TRANSATLANTIQUES}

Sous cet angle, les terres de taureaux n'ont ni le même contour ni le même contenu que les terres de corridas et, malgré des travaux qui nous précèdent dans cette entreprise et sans lesquels une analyse d'une telle envergure territoriale n'aurait pas pu être menée à bien, elles n'ont jamais été observées dans leur totalité. Parmi ces travaux, citons d'emblée ceux de l'anthropologue Frédéric Saumade sur les tauromachies européennes qui ont incontestablement démontré la nécessité d'une prise en compte géographique élargie des phénomènes tauromachiques ${ }^{1}$. Plus récemment, ses analyses sur les jeux taurins au Mexique ainsi que celles de Dominique Fournier ont confirmé la nécessité d'une approche hispano-américaine de la question, permettant d'intégrer, au sein d'une problématique commune, les jeux taurins de la vieille Europe et du Nouveau Monde ${ }^{2}$. De l'autre côté de l'Atlantique certains travaux anglo-saxons, tels ceux de Richard Slatta, ont retracé l'histoire commune et comparée des rodéos américains, mais sans mettre à jour la matrice transocéanique de transformation des jeux taurins ${ }^{3}$. Certes, il existe quelques jeux taurins en dehors de notre espace d'étude, montrant qu'il n'y a pas lieu d'affirmer une quelconque essence euro-américaine de la culture taurine, mais les relations formelles, historiques et territoriales unissant les tauromachies transatlantiques apparaissent suffisamment solides pour les traiter comme un tout cohérent et en retracer les logiques de différenciation. Puisse notre travail permettre d'approfondir cette entreprise, d'élargir l'analyse à des terres et des pratiques insuffisamment mises en relief, et d'établir des liens fructueux entre géographie, histoire et anthropologie.

Ainsi considérée, l'aire culturelle de la tauromachie s'étend donc principalement du Sud-Ouest européen à l'Amérique et regroupe un large spectre de pratiques : depuis la grande famille des rodéos américains (rodéo et bull riding nord-américains, charreada et jaripeo mexicains, toros coleados colombiens et vénézuéliens, rodéo montubio équatorien, vaquejada et rodeio crioulo brésiliens, rodéo chilien...) jusqu'aux diverses " courses de taureaux » (corrida espagnole, corrida portugaise, course camarguaise, course landaise, course de recortadores). Ces pratiques exécutées par des spécialistes, plus ou moins institutionnalisées en des spectacles indépendants, ne doivent pas masquer

\footnotetext{
${ }^{1}$ F. SAUmade, Des sauvages en Occident; ID., Les tauromachies européennes.

${ }^{2}$ D. Fournier, « Du taureau considéré comme outil d'acculturation au Mexique »; ID., « Corrida, charreada et jaripeo »; F. SAUmade, « Du taureau au dindon. Domestication du métissage »; ID., « Animal de rente, animal de loisir »; ID., Maçatl.

${ }^{3}$ R. Statta, Cowboys of the Americas.
} 


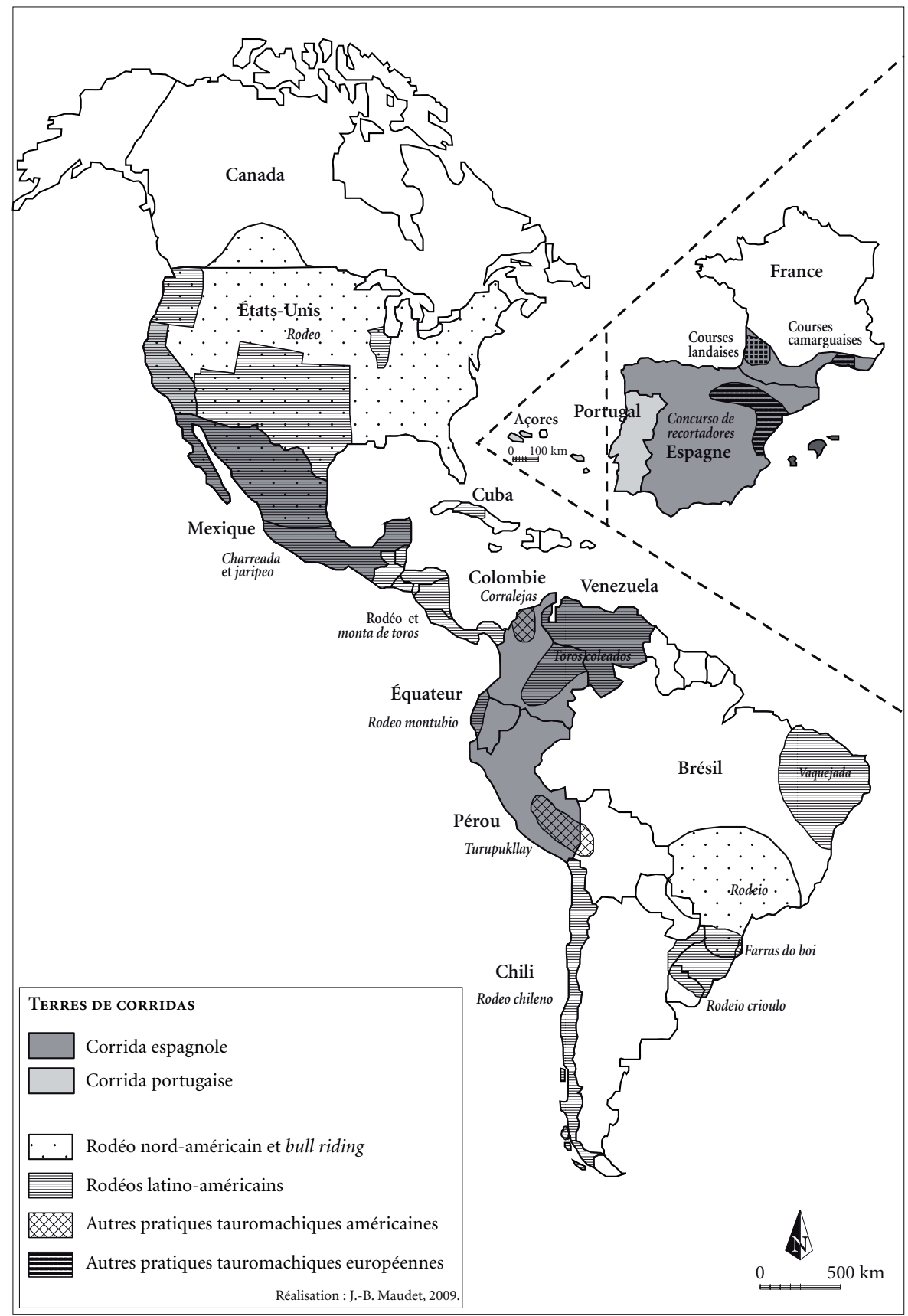

CARTE 1. - Les pratiques tauromachiques du Sud-Ouest européen à l'Amérique 
l'immense diversité des jeux taurins à participation collective : les encierros, dont celui de Pampelune, connu dans le monde entier, et divers lâchers de bovins en lieux ouverts ou clos. C'est sans doute l'une des grandes originalités de la tauromachie que cette dualité entre pratiques professionnelles et pratiques participatives. Tout se passe comme si, en terres taurines, la tauromachie était consommée sous ces deux espèces : le spectacle, celui de la représentation à mi-chemin entre l'art et le sport de haut niveau, et le jeu traditionnel collectif, pratiqué par des amateurs et des néophytes. À cette différenciation sociologique fondamentale, s'ajoute celle d'une inscription spatiale qui la recoupe en grande partie : une tauromachie d'arène, qui peut prendre des formes diverses, du moment qu'elle sépare la piste, des gradins, et les acteurs, des spectateurs; et une tauromachie de rue, aussi variée que le sont les contours matériels et idéels de l'espace public. En dépit de leur évidente opposition sociale et spatiale, ces deux genres tauromachiques entretiennent des relations complexes de parenté puisque la forme participative est perçue, tantôt, comme la matrice originelle de la forme professionnelle, une sorte d'antécédent élémentaire qui contiendrait en germe la professionnalisation du jeu, tantôt, à l'inverse, comme un avatar mineur du spectacle, une sorte de déclinaison pour les amateurs d'un jour. Cette relation tient ces deux genres tauromachiques dans une proximité qui renforce les processus d'identification entre les amateurs (ceux qui aiment ou qui pratiquent) et leurs héros. Il n'est alors pas étonnant de constater que les «fêtes taurines " associent le plus souvent les deux genres tauromachiques, aussi opposés que complémentaires, et participent à cet enracinement presque viscéral de la tauromachie sur ses territoires.

\section{Fête et tauromachie}

En cela, l'expression de «fête taurine " devient un objet d'observation plus complexe que ne le laisse croire l'association presque banale entre fête et tauromachie, si banale qu'on en oublie la nature de leur relation. Il y a là quelque chose de propre à la tauromachie et quelque chose de propre aux phénomènes festifs. Peu de tauromachie sans fête, peu de fêtes sans tauromachie : dans les régions taurines, tel pourrait être l'axiome des relations entre la fête et la tauromachie au point qu'elles finissent par être assimilées à un seul et même phénomène de société. Pour preuve, dans les régions taurines abondent les expressions visant à les associer ; mieux, les termes de feria et fiesta, sans qualificatif, suffisent souvent à désigner le loisir taurin inséparable d'une certaine effervescence collective. La tauromachie est-elle une fête ? Aujourd'hui tout spectacle qui possède les caractéristiques d'un grand rassemblement autour d'une passion commune semble prendre les allures d'une fête, au moins celle d'une fête officielle. Et tous les jeux traditionnels qui impliquent la participation collective de la foule semblent conserver nombre d'attributs distinctifs de la fête : le tumulte, le désordre, l'excès, au moins ceux associés à l'idée de faire la fête. Par ailleurs, les phénomènes festifs possèdent l'extraordinaire capacité d'amalgamer et d'intégrer les raisons les plus diverses au rassemblement des hommes, de sorte qu'il est souvent dif- 
ficile de distinguer l'objet même de ce qui est fêté au-delà des effets d'annonce propres aux calendriers officiels, civils ou religieux. En cela, la fête est, pour partie, intransitive. L'agglomération spontanée de la foule se révèle alors essentielle à la reconnaissance des phénomènes festifs. Or sur ce plan-là, quelles que soient les régions observées, les jeux taurins professionnels et participatifs sont de formidables catalyseurs de densité festive. Ces rassemblements créent des lieux de centralité tauromachique, au rayonnement plus ou moins vaste.

\section{L'ESPACE TAUROMACHIQUE}

De part et d'autre de l'Atlantique, notre étude recense une vingtaine de pays où il existe des pratiques qui mettent en scène un affrontement réel entre les hommes et les bovins. L'immensité océane sépare naturellement l'espace d'exercice de la tauromachie. L'ensemble européen paraît très compact et continu en comparaison d'un vaste ensemble américain, qui s'étend du Canada au Chili, marqué par de profondes discontinuités et par des jeux taurins qui forment, par endroit, des isolats au milieu d'immensités où la tauromachie n'est rien. Cependant, l'Atlantique ne constitue pas une barrière qui scinderait les pratiques en deux ensembles sans relation. Bien au contraire, cette discontinuité naturelle implique des continuités culturelles et de fonctionnement qui donnent forme à ce que l'on pourrait appeler "l'espace tauromachique ", quand bien même s'agirait-il d'une mosaïque complexe.

Qu'il n'y ait aucune équivoque sur l'utilisation de la notion d'espace tauromachique. Il s'agit avant tout d'une représentation construite par le géographe pour rendre compte de la spatialité d'un phénomène social. L'idée d'un espace tauromachique qui serait fragmenté à l'image d'une mosaïque n'est sans doute pas d'une grande originalité, tant cette métaphore s'adapte à une infinité d'étendues marquées par l'hétérogénéité et la discontinuité. Avancer l'idée d'un espace tauromachique au singulier, c'est considérer que suffisamment d'interactions relient entre eux les jeux taurins. Cette cohérence repose sur la coexistence territoriale de ces pratiques au sein d'un espace transatlantique qui hérite des relations culturelles entre la vieille Europe et le Nouveau Monde.

La configuration d'ensemble de l'espace tauromachique peut être appréhendée à travers deux approches complémentaires : d'une part l'étude de ses limites et de ses discontinuités, d'autre part l'étude des principales étapes qui ont présidé à la formation de cet espace. La première approche est attentive à caractériser la nature des frontières de l'espace tauromachique, la seconde approche s'intéresse aux logiques de diffusion spatiale et de transformation des jeux taurins. La première pose le problème des décalages entre les frontières historiques de leur diffusion, les frontières légales des jeux aujourd'hui autorisés ou interdits et les frontières réelles de leur extension. La seconde interroge les processus de formation des jeux taurins et les principales étapes de leurs évolutions. Bien que les chronologies diffèrent grandement d'un jeu à l'autre, le XIX ${ }^{\mathrm{e}}$ siècle apparaît comme une phase déterminante de la formation des spectacles tauromachiques modernes et de la stabilisation de leurs limites. 
Dans le Sud-Ouest européen, cette période remanie des héritages anciens à partir d'une diffusion accélérée de la corrida espagnole. En Amérique, cette période inaugure la transformation, dans le cadre d'États indépendants, des jeux taurins hérités de la période coloniale, qu'il s'agisse des fêtes taurines des élites urbaines ou bien des jeux populaires élaborés dans le cadre des élevages extensifs de bovins.

Les pratiques tauromachiques possèdent des caractéristiques spatiales et territoriales très différentes qui varient en fonction de nombreux critères : leur extension géographique, leur dynamique spatiale progressive, stabilisée ou régressive, leur degré d'institutionnalisation, leur caractère emblématique de la culture locale, régionale ou nationale. La corrida espagnole est la seule pratique véritablement transatlantique, même si la corrida portugaise, dans une bien moindre mesure, peut faire l'objet d'une analyse équivalente. Les corridas espagnoles sont pratiquées dans une quinzaine de pays. Dans sept d'entre eux, elles se déroulent dans le plus strict respect de sa forme canonique : l'Espagne, la France, le Mexique, le Venezuela, la Colombie, l'Équateur et le Pérou. Les règles du jeu et le déroulement d'ensemble du cérémonial sont ainsi identiques à Mexico, Caracas, Bogotá, Medellín, Cali, Quito, Lima, Madrid, Barcelone, Séville, Nîmes, Dax ou Bayonne. Quelques pays font exception à cette remarquable unité du rituel taurin, dont le Portugal où la corrida espagnole est privée de la pique et de la mise à mort publique du taureau. Au Portugal s'ajoute la Californie qui propose des corridas sans trace de sang, adaptées aux lois de protection animale en vigueur. Les petits pays d'Amérique centrale qui n'offrent que rarement des corridas le font parfois dans le respect de la règle espagnole.

En raison de leur extension transatlantique, les corridas sont à différencier des pratiques apparentées à la grande famille des rodéos, fondamentalement américaines si l'on excepte le cas Australien. En deçà des deux grandes catégories de spectacles transnationaux que sont les corridas et les rodéos, on observe de nombreuses pratiques locales, qui s'en réclament ou s'en distinguent, enracinées dans des régions de taille variable : les toros coleados des llanos entre la Colombie et le Venezuela, la vaquejada du Nordeste brésilien, les turupukllay de l'Altiplano andin, les fiestas en corralejas de la Costa Atlántica colombienne, le rodéo montubio du littoral équatorien, les farras do boi du littoral de Santa Catarina au Brésil, la course camarguaise et la course landaise en France, les capeias raianas à l'est de la Sierra da Estrella au Portugal et les touradas à corda sur l'île Terceira aux Açores. Parmi ces pratiques, certaines se sont affirmées comme un phénomène national, indiquant l'existence d'une dynamique spatiale progressive et d'une institutionnalisation volontaire dans le cadre d'un État-nation : le rodéo aux États-Unis, la charreada au Mexique, le rodeo chileno au Chili, les toros coleados au Venezuela et bien évidemment, dans le cas de l'Espagne et du Portugal, leur corrida respective, que l'on conteste ou non le bien-fondé de l'appellation «fête nationale » servant parfois à les désigner.

Parmi les plus conquérantes, figurent aujourd'hui les courses de recortadores en Espagne, qui ont quitté leur berceau originel de l'Èbre moyen, à la jonction 
de la Navarre, de l'Aragon et de La Rioja, pour s'étendre aux pays du Levant, puis à toute l'Espagne. En Amérique, il en va de même pour le bull riding étasunien qui progresse au Mexique, là où préexistent des jeux comparables (le jaripeo), et au Brésil, principalement dans la région de São Paulo, pour ce qui demeure l'un des exemples les plus spectaculaires de délocalisation récente. À l'inverse, il existe des pratiques qui menacent de disparaître. C'est le cas des turupukllay andins du Pérou, associant le taureau, le condor et l'homme, en raison d'une transformation du tissu rural traditionnel. C'est le cas également des farras do boi de l'État de Santa Catarina au Brésil qui sont contraintes d'évoluer pour échapper à leur récente interdiction, dans un contexte de développement touristique littoral et d'ouverture de la société locale.

Au-delà de la géographie particulière de chacune d'elles, l'intérêt est bien de comprendre les relations territoriales qu'elles entretiennent les unes avec les autres. Cette question passe par une attention particulière aux modalités de coexistence spatiale des jeux taurins. L'espace tauromachique se caractérise en effet par la coexistence de pratiques d'extension variable qui se recoupent et se chevauchent partiellement. Les unes sont cloisonnées par des frontières strictes, à l'image de la corrida, généralement autorisée ou interdite à l'échelle d'un pays entier. Les autres ne semblent avoir pour seules limites que l'extension apparemment non contrainte de leur exercice, à l'image des rodéos en Amérique. Les jeux taurins à participation collective, en raison de leur plus grande informalité, échappent en partie aux délimitations précises et résistent souvent aux tentatives de régionalisation. En revanche, tous possèdent dans leur histoire et dans leur fonctionnement des relations qui les unissent, ne les rendant totalement intelligibles que prises ensemble. Autrement dit, à l'échelle euro-américaine, l'espace tauromachique actuel, avec ses foyers de forte densité, ses lacunes et ses zones de chevauchement, résulte d'un long processus de genèse, de diffusion, de transformation, d'enracinement ou de rejet des jeux taurins. Les modalités de coexistence spatiale doivent alors être envisagées à plusieurs niveaux géographiques. Les pratiques tauromachiques coexistent à l'échelle transatlantique, aux échelles continentales, nationales, régionales, jusqu'à l'échelle de certaines villes, voire de certaines arènes, parfois même de certains spectacles. Le cloisonnement plus ou moins étanche des cultures taurines résulte, en partie seulement, des modalités de leur coexistence spatiale. Dans cette perspective, les relations entre sociétés et espaces tauromachiques sont analysées à partir des découpages nationaux qui demeurent ceux de la régulation institutionnelle des jeux. Pour pallier au mieux ce que le découpage par pays ne fait pas directement apparaître, les dynamiques transfrontalières occasionnées par les cultures taurines font l'objet d'une attention particulière. Les frontières entre les jeux et entre les pays s'avèrent être, en effet, de formidables supports de relations territoriales croisées.

En Europe, l'Espagne peut être considérée comme le cœur de la géographie taurine, flanquée de deux pays qui partagent avec elle certaines caractéristiques, mais qui possèdent aussi leur propre tradition. Au Portugal, la corrida à l'espagnole demeure minoritaire en comparaison de la corrida portugaise, considérée 
comme la forme tauromachique nationale même si, sur le plan de la répartition, ce qualificatif mérite d'être nuancé. Dans le Sud de la France, en des lieux définis par la loi, la corrida espagnole connaît un grand engouement et s'affirme comme pleinement assimilée au patrimoine culturel local. S'y ajoutent des pratiques tauromachiques d'origine autochtone : la course camarguaise dans le sud-est et la course landaise dans le sud-ouest. La qualification des pratiques tauromachiques comme " camarguaise » et « landaise » renvoie plus à une identité culturelle, ici régionale, qu’à une délimitation géographique précise.

En Amérique, il est possible de diviser les pays en trois groupes dont les continuités spatiales n'ont rien d'évident. Un premier groupe est constitué de pays possédant des jeux taurins qui coexistent largement avec la corrida : le Mexique, le Pérou, l'Équateur, le Venezuela, la Colombie. La Bolivie et certains des petits pays d'Amérique centrale tels que le Guatemala, le Nicaragua, le Costa Rica, Panamá, pourraient être rattachés à ce groupe, mais les corridas y sont rares et généralement adoptent des formes remaniées. Il serait également possible d'y inclure les États-Unis, une fois noté que les corridas ne sont présentes qu'en Californie sous une forme portugaise excluant toute effusion de sang. Dans ces pays, à l'exception notable du Pérou, la corrida coexiste avec des jeux de la famille des rodéos : charreada et jaripeo au Mexique, toros coleados en Colombie et au Venezuela, rodéo montubio en Équateur. Au Pérou, malgré l'existence de fortes traditions équestres, comme celle du caballo de paso, aucun spectacle professionnel de grande envergure apparenté au rodéo ne s'est développé. Tous ces pays possèdent un éventail très étendu de jeux taurins populaires pour lesquels la frontière entre ce qui relèverait de la tauromachie à l'espagnole et ce qui relèverait du rodéo se perd dans de multiples formes syncrétiques.

Un deuxième groupe est constitué de pays possédant des jeux taurins importants et bien institutionnalisés d'où la corrida est absente ou très exceptionnelle : le Canada, les États-Unis, le Chili et le Brésil. Dans ces pays, les jeux taurins relèvent principalement du rodéo qui en est la forme dominante. Le jeu le plus connu est le rodéo nord-américain, développé aux États-Unis et au Canada. Malgré l'éloignement, le Brésil se rattache à l'Amérique du Nord depuis que le bull riding professionnel s'y est implanté. Ce que les Brésiliens appellent le rodeio cohabite donc avec la vaquejada, version brésilienne de l'exercice du coleo, présent dans de nombreux pays. Cuba, l'Uruguay, le Paraguay et l'Argentine pourraient faire partie de cet ensemble avec néanmoins une pratique du rodéo moins affirmée à l'échelle nationale. L'Argentine, alors même qu'on aurait pu croire tous les ingrédients réunis pour voir s'y développer un rodéo de large audience, se distingue par le faible épanouissement des jeux taurins toutes catégories confondues. En marge de rodéos maintenus dans une relative atonie, seuls quelques jeux taurins isolés sont à remarquer : le toreo de la vincha dans le village de Casabindo situé dans la province septentrionale de Jujuy, et la coutume des yerras liée au marquage et à la castration du bétail dans les élevages de la Pampa, donnant lieu à des pratiques ludiques et festives. Quant au Chili, il possède une forme de rodéo unique en son genre, 
INTRODUCTION

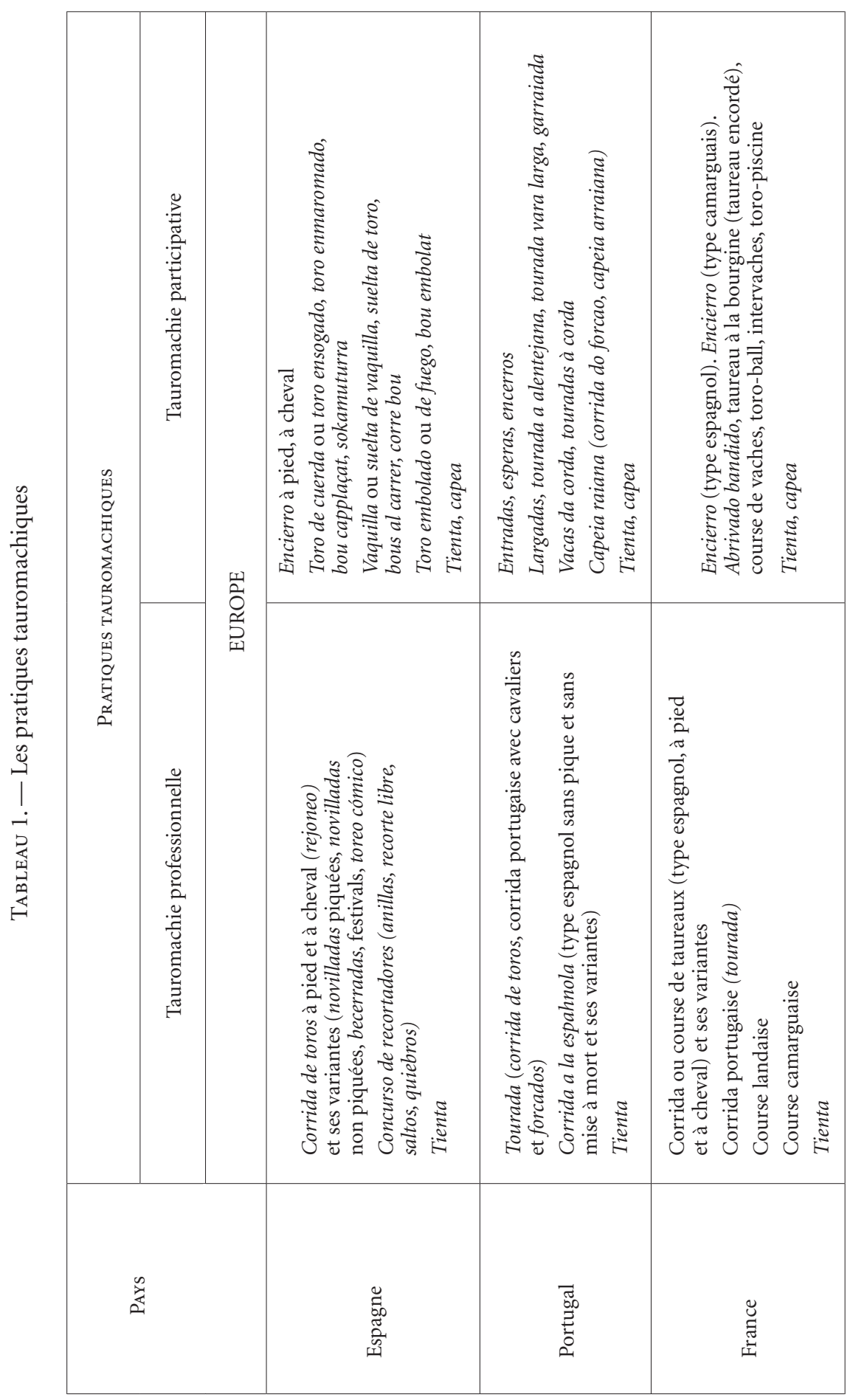


INTRODUCTION

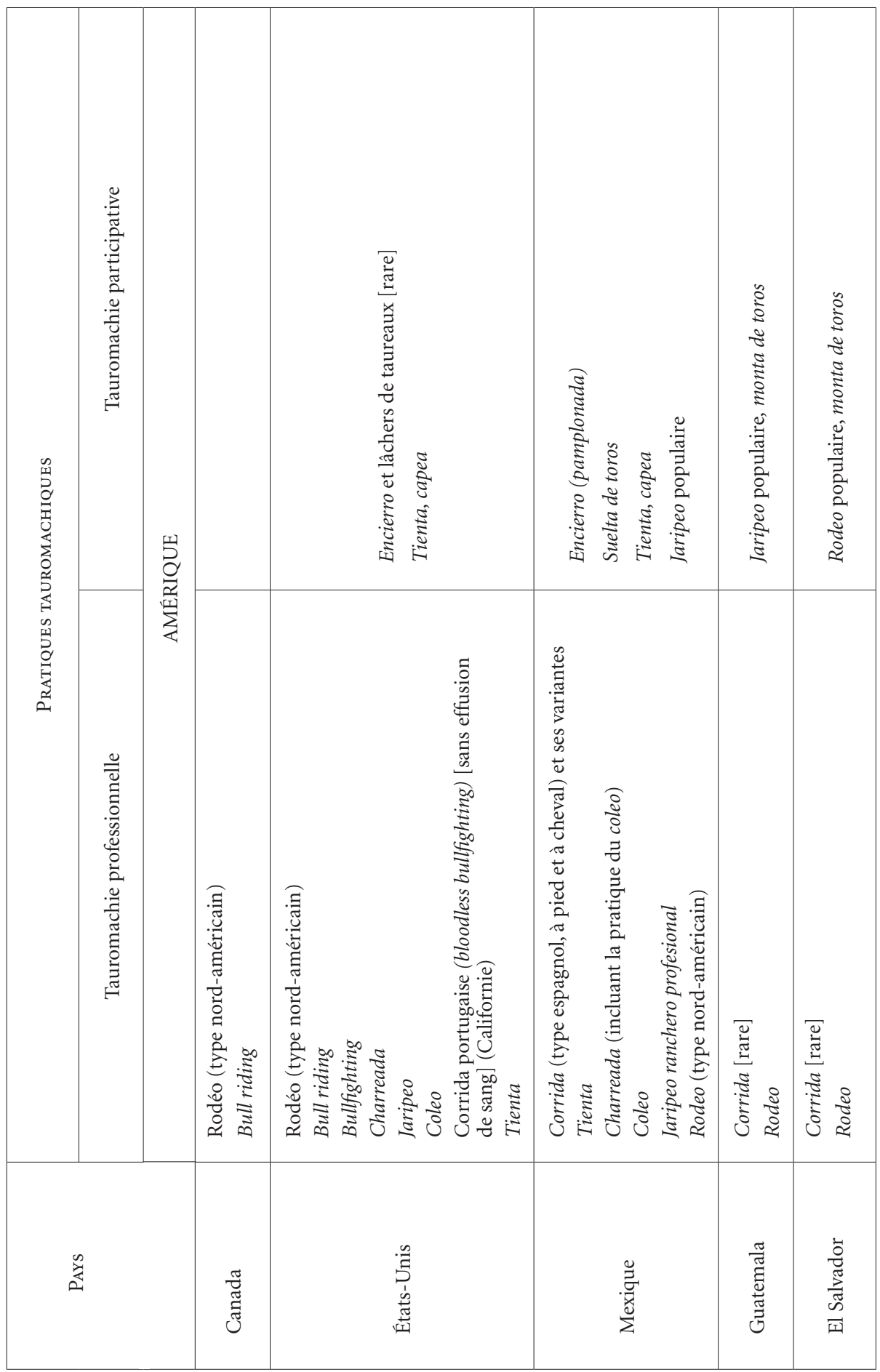


INTRODUCTION

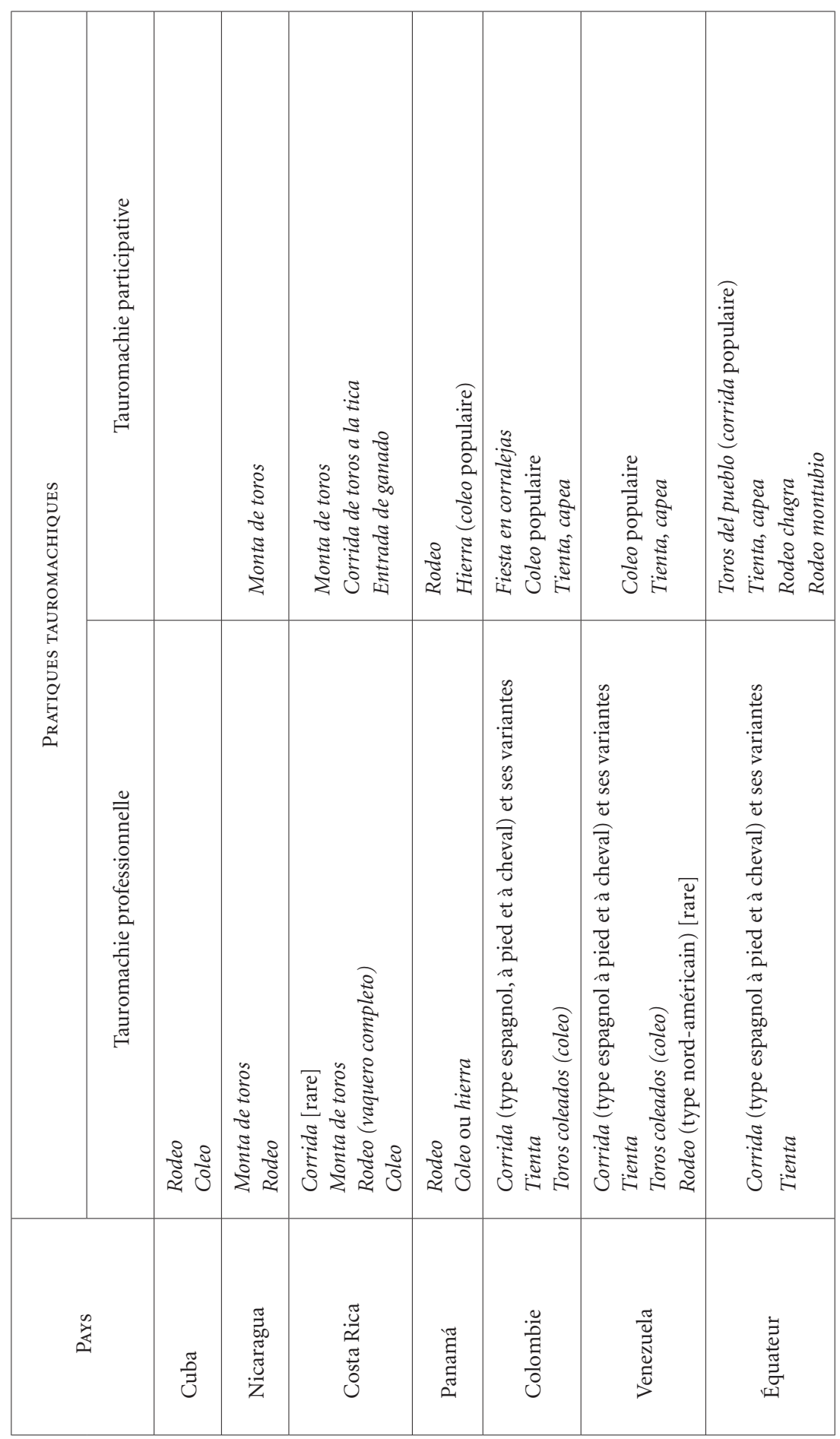


INTRODUCTION

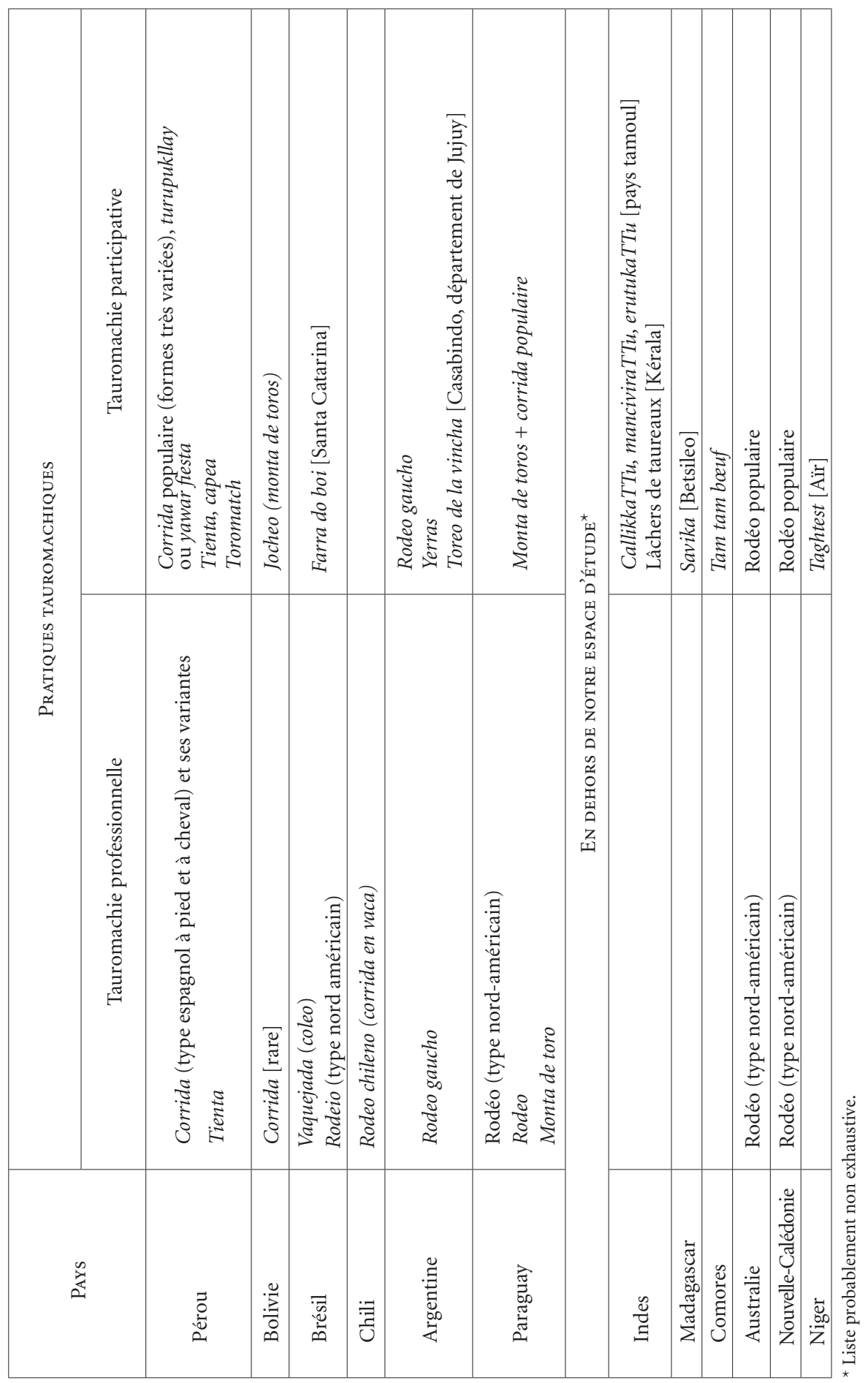


dont l'appellation officielle à caractère territorial témoigne d'un fort particularisme : le rodeo chileno.

Ce sont bien les jeux taurins apparentés au rodéo, en dépit des variantes nationales, régionales et locales, qui constituent le trait culturel commun de la tauromachie outre-Atlantique. Elle y consacre la trilogie formée par l'homme, le cheval et le taureau, possédant une valeur paradigmatique qui dépasse largement le seul cadre des jeux tauromachiques. En effet, en ces terres de centaures, cette trilogie taurino-équestre a toujours quelque chose à voir avec la construction des États-nations, faisant des rodéos un puissant vecteur d'identité territoriale.

\section{GÉOgRAPHie, SCIENCES HUMAINES ET TAUROMACHIE}

Vaste chantier que celui d'analyser la spatialité d'un fait culturel d'une telle ampleur géographique. Nous ne prétendons pas l'épuiser pour chacune des pratiques tauromachiques, ni cacher l'inégal approfondissement des recherches, selon les espaces et les pratiques observés. Les données recueillies et les enquêtes de terrain sont plus détaillées pour l'Europe que pour l'immense Amérique. Cette démarche procède tout autant de la réelle difficulté d'accéder uniformément à l'ensemble des sources d'informations propres à chaque pratique, que du choix méthodologique d'approfondir au mieux l'étude géographique de l'espace qui est au cœur de notre propos général : la péninsule Ibérique. Il y a quelques raisons à cela qui tiennent aux processus de diffusion spatiale des jeux taurins sur le temps long et aux modalités de leur enracinement, plaçant assurément la péninsule Ibérique au centre des territoires historiques et culturels de la tauromachie.

L'intérêt scientifique de cette approche n'est pas de satisfaire à un devoir d'inventaire des éléments matériels et immatériels de l'espace tauromachique. L'intérêt est plutôt d'ordre méthodologique et disciplinaire. Les rares travaux comparatifs sur les pratiques tauromachiques (ils n'en sont que d'autant plus précieux) ont principalement développé une approche qualitative des phénomènes comparés. Dans la plupart des cas, la dimension quantitative et spatiale n'a pas été suffisamment prise en compte. Cela ne garantit pas d'achever la compréhension des relations entre les hommes et leur territoire. Mais au fondement de notre discipline, n'est-il pas nécessaire de considérer la dimension quantitative et spatiale comme constitutive de la qualité même des objets comparés? Autrement dit, l'étude de la forme, de la signification sociale, anthropologique et territoriale des jeux taurins ne peut faire l'économie ni d'un recensement exhaustif, ni d'une localisation précise, ni d'une analyse de leur coexistence, car les relations quantitatives et spatiales impliquent des rapports idéologiques de force et de pouvoir déterminant leur fonctionnement.

Enfin, cette approche est à resituer dans le cadre d'un questionnement géographique de plus en plus attentif aux loisirs et aux phénomènes festifs de tous ordres, à leur dimension territoriale et, comme le laisse entendre Guy Di Méo, à une exigence à laquelle la géographie ne doit sans doute pas renoncer. Dans 
La géographie en fêtes, l'auteur rappelait que les rares géographes qui se sont intéressés à la fête, ont davantage insisté sur le rôle de celle-ci dans la construction des identités locales que sur son rôle dans celle des identités régionales ou nationales ${ }^{4}$. La place particulière des jeux taurins au sein de ce vaste champ de recherche a attiré, depuis peu, l'attention de quelques géographes français, mais pour la plupart dans une dimension strictement locale et sans la prise en compte de la superposition des cultures taurines ${ }^{5}$. Pour nous, l'enjeu n'est pas d'affirmer de façon systématique qu'il est du ressort de la géographie d'étudier la construction des identités aux échelles locales, régionales et nationales, comme s'il s'agissait d'épuiser par strate le champ de l'explication. Il n'est pas non plus question de répéter de façon dogmatique que l'analyse par échelle peut apporter aux explications en sciences humaines une spécificité propre à la géographie. L'objectif est d'évaluer dans quelle mesure l'identité locale prend sens en fonction d'un contexte spatial plus englobant et jusqu'à quel point les différents niveaux d'identification culturelle et territoriale fonctionnent ensemble. L'objectif est également de montrer que certains traits culturels, non pas isolés mais resitués dans l'histoire et la géographie complète de leur transformation, peuvent constituer ce que nous proposons d'appeler « un champ commun de distinction territoriale ». Voilà alors l'occasion, à partir d'un travail mené sur un espace géographique très étendu, d'observer sous un autre angle l'imbrication et la porosité des aires culturelles.

\section{II. — LA DIVERSITÉ TAUROMACHIQUE EN IMAGES}

Les photographies qui suivent présentent un large éventail de jeux taurins, plus ou moins connus, avec le souci, d'une part, de marquer une différenciation entre les tauromachies professionnelles et les tauromachies à participation collective, et d'autre part, de fournir des informations sur les modalités techniques des affrontements homme-bovin. Ces photographies, permettent également de compléter avantageusement les descriptions qui s'attachent à expliquer les règles du jeu de chacune des pratiques tauromachiques et les dispositifs matériels mis en ouvre pour leur déroulement. Enfin, mises ensemble, ces photographies confirment un air de famille qui justifie de considérer ces jeux comme un tout et de rapprocher ce que la géographie et les imaginaires éloignent. Corrida, rodéo : même combat.

\footnotetext{
${ }^{4}$ G. Di Méo (dir.), La géographie en fêtes, p. 1.

${ }^{5}$ C. Bernié-Boissard, "Nîmes en Feria : une ville sous la ville "; EAd., "Le public des courses de taureaux »; M. DAuga et J.-Y. Puyo, "La course landaise, sport-spectacle »; ID., "Les écarteurs landais entre mythe et réalité "; M. DAUGA, "L'âge d'or de la course landaise »; M. FAVory, «Les bestiaires et l'espace »; I. GARAT, "La tauromachie dans la vie bayonnaise "; R. Keerle, Sport et territoires ; ID., "La tauromachie camarguaise en quête d'un territoire».
} 


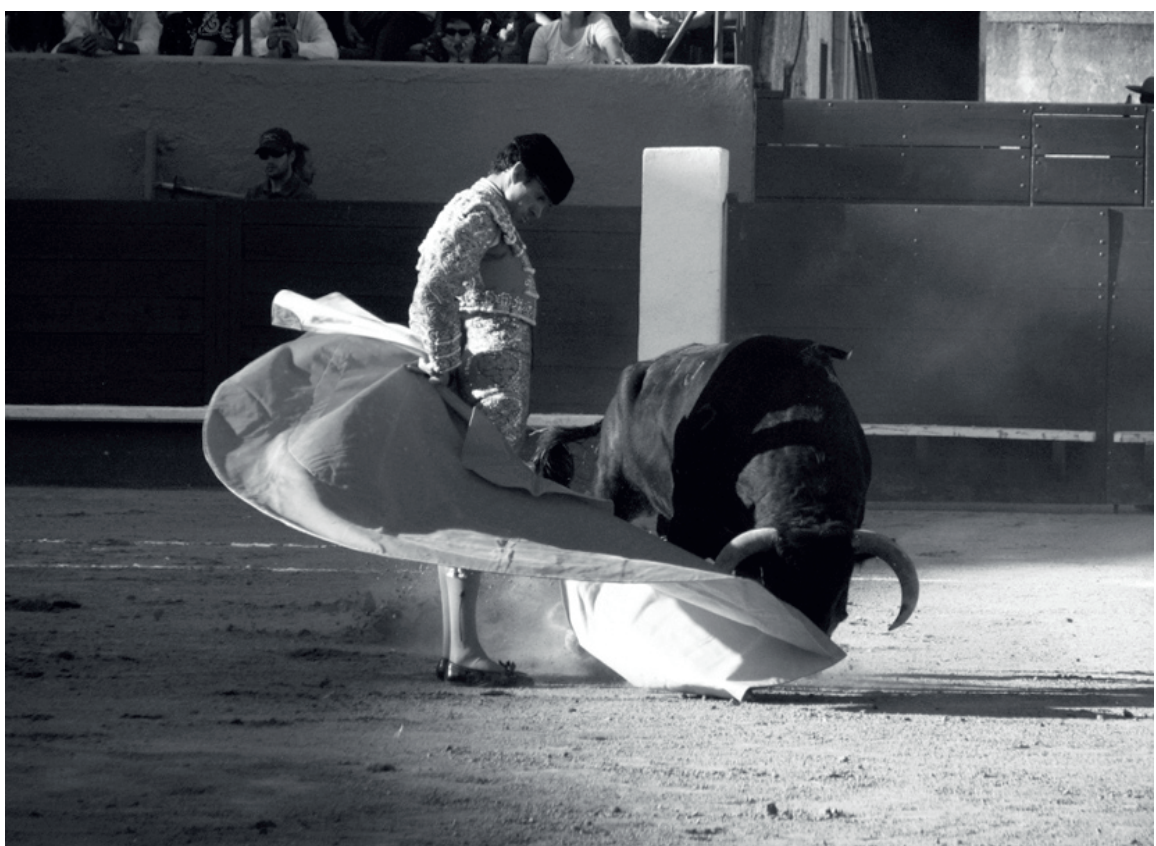

FIG. 1

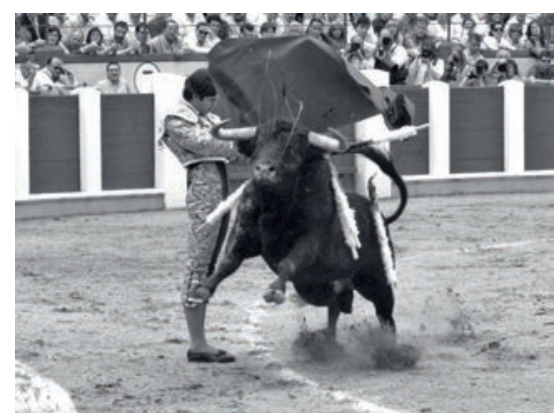

FIG. 2

FIg. 1 Corrida espagnole, Ávila, Espagne, 2007

(cliché : Guillermo Domínguez Torres)

Fig. 2 Corrida espagnole, Madrid, Espagne, 2007

(cliché : Guillermo Domínguez Torres)

FIg. 3 Corrida espagnole, picador, Espagne, 2007

(cliché : Guillermo Domínguez Torres)

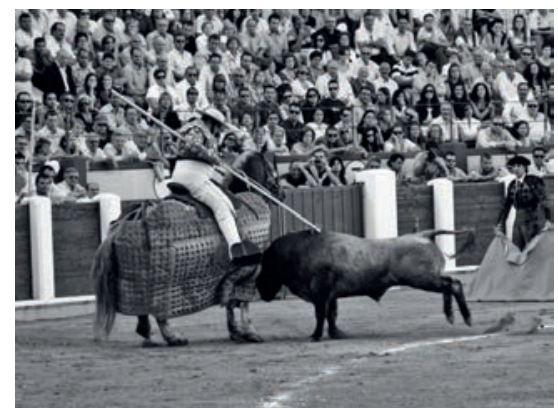

FIG. 3 


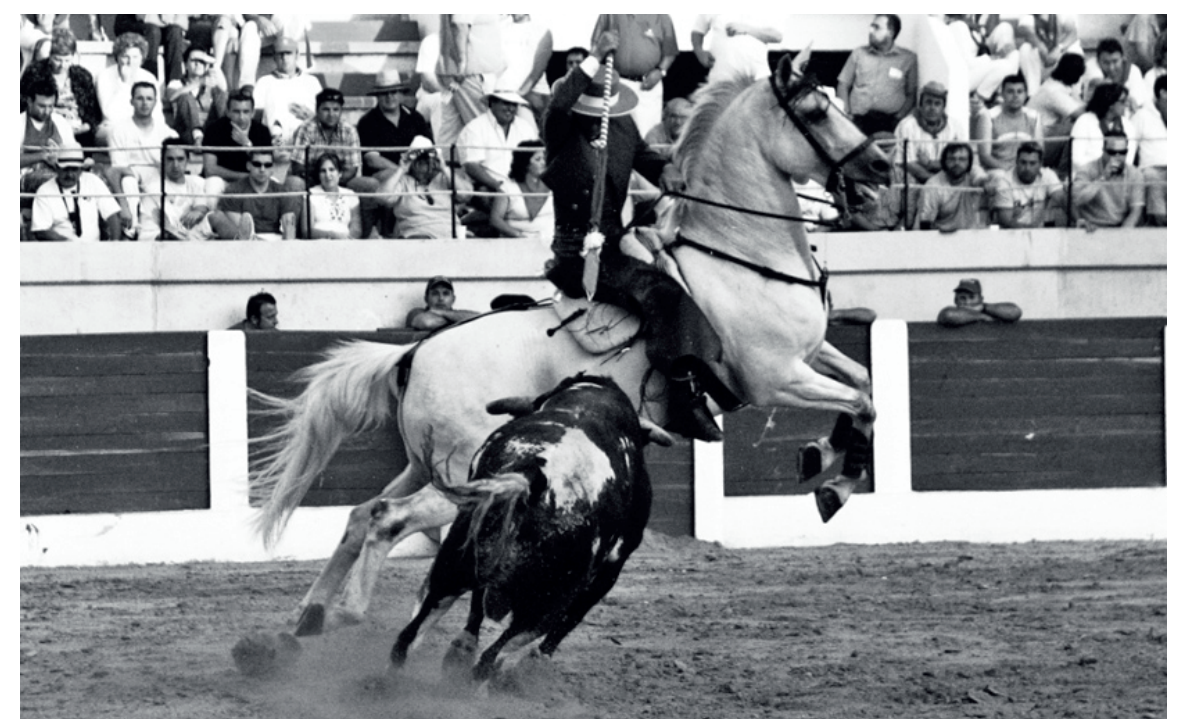

FIG. 4

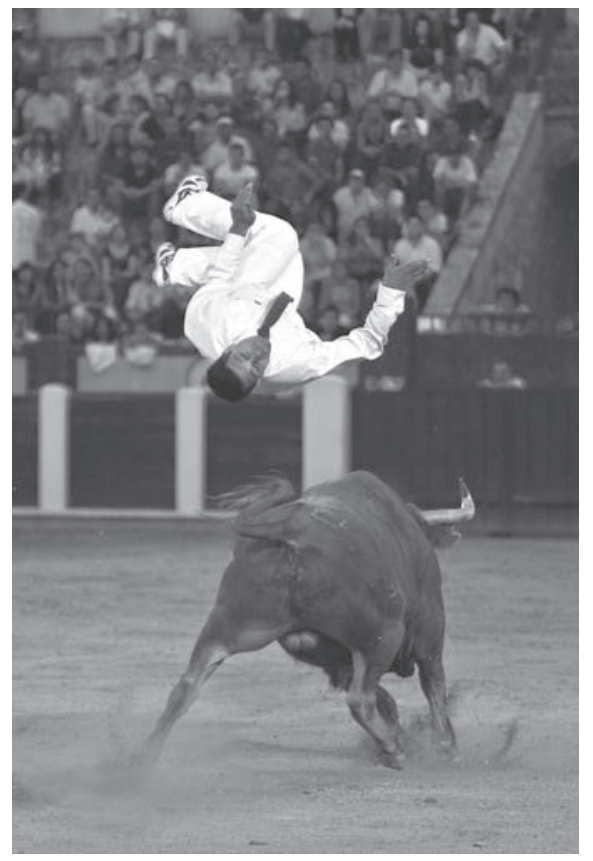

FIg. 4 Rejoneo, Espagne, 2007

(cliché : Guillermo Domínguez Torres)

FIG. 5 Concours de recortadores, salto, Espagne 2008 (cliché : Ángel Torres)

FIG. 5 


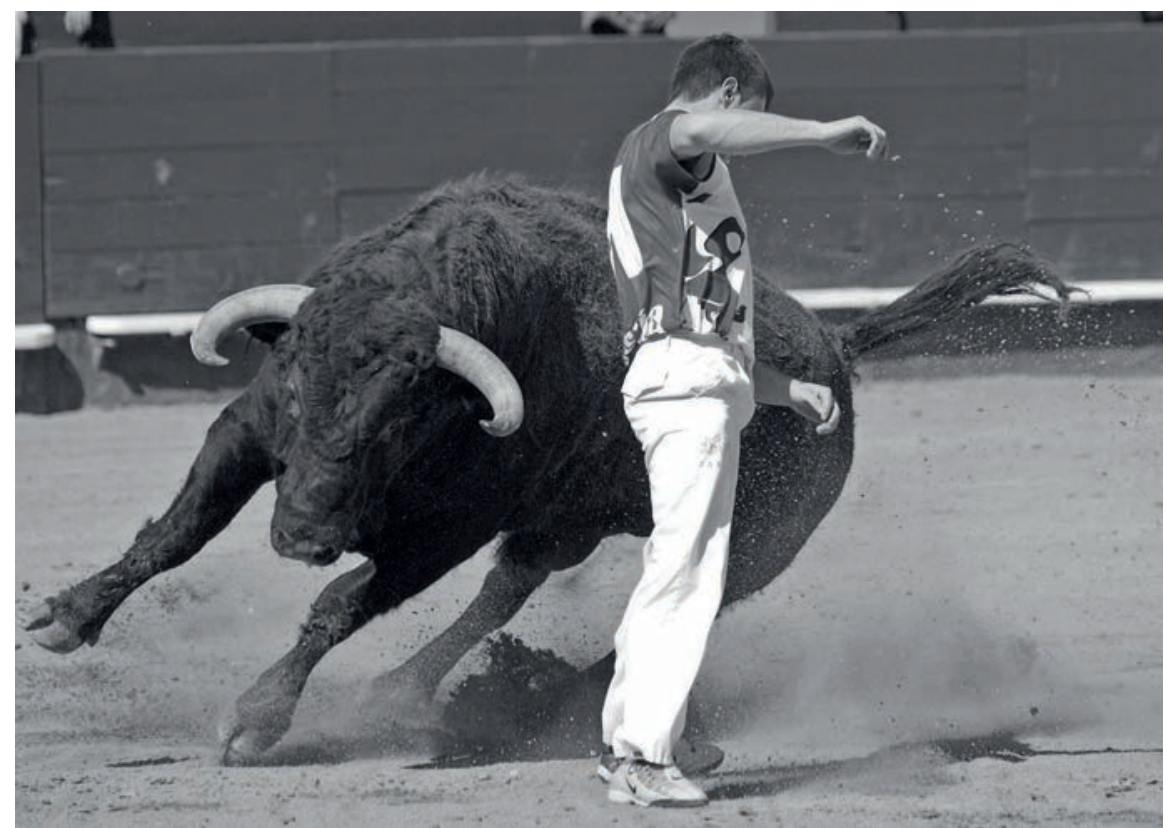

FIG. 6

FIg. 6 Concours de recortadores, Espagne, 2008 (cliché : Antonio González)

FIg. 7 Concours de recortadores, Espagne, 2008 (cliché : Ángel Torres)

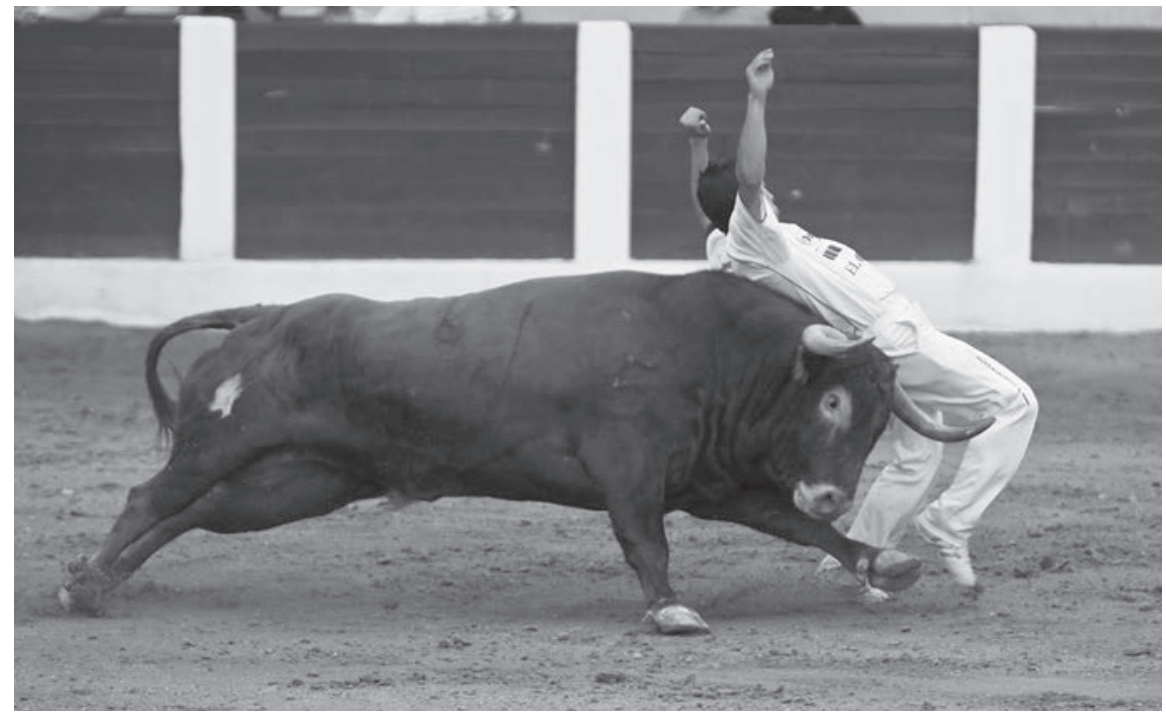

FIG. 7 


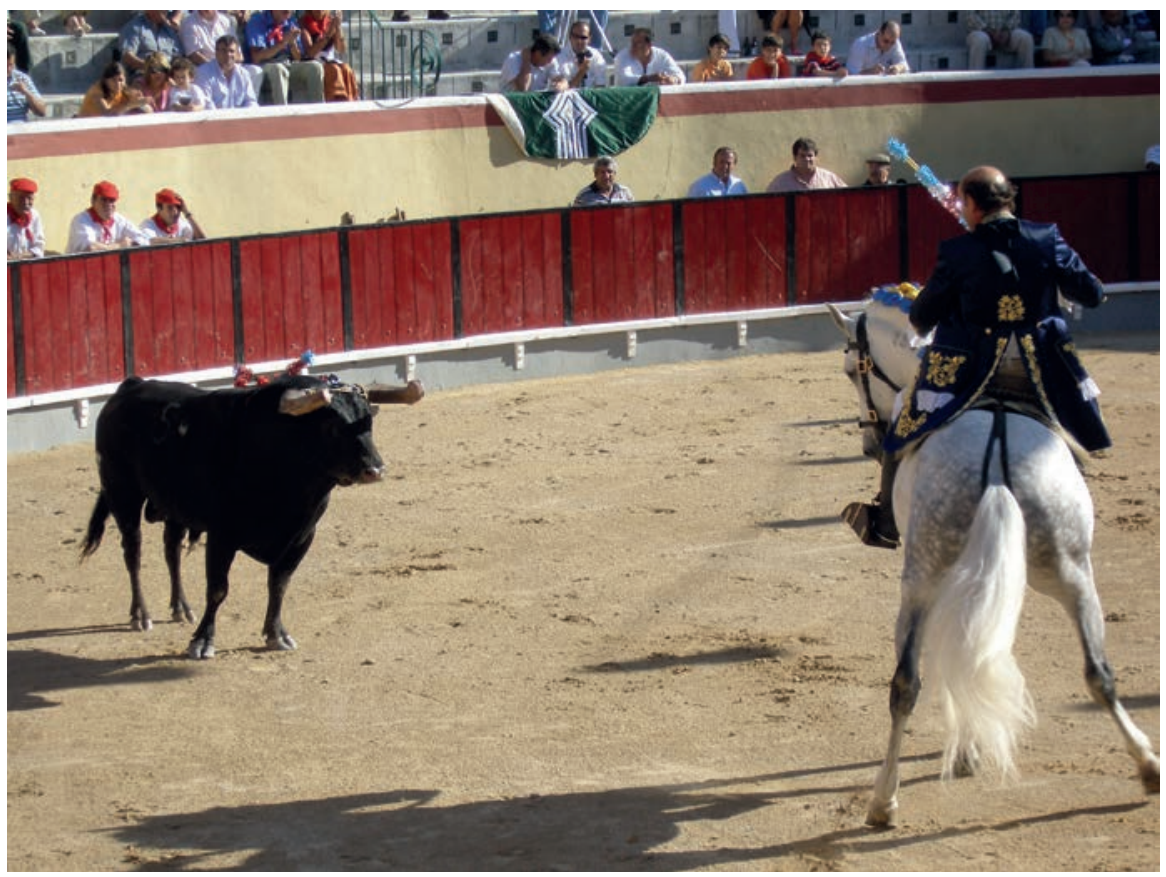

FIG. 8

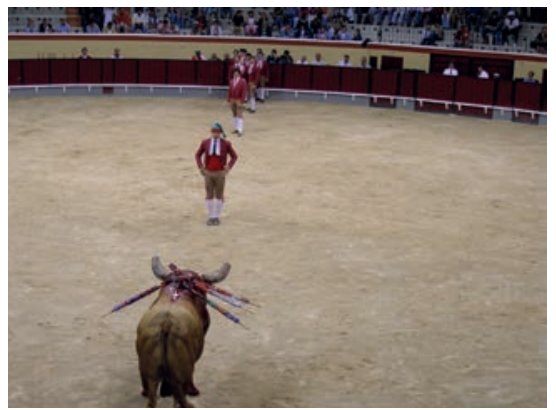

FIG. 9

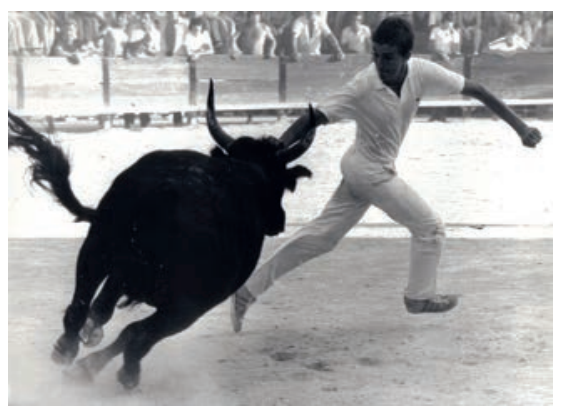

FIg. 8 Corrida portugaise, Vila

Franca de Xira, Portugal, 2005

(cliché : Jean-Baptiste Maudet)

FIG. 9 Corrida portugaise, Vila

Franca de Xira, Portugal, 2005

(cliché : Jean-Baptiste Maudet)

FIg. 10 Course camarguaise, France (cliché : Marc Leenhardt)

FIG. 10 


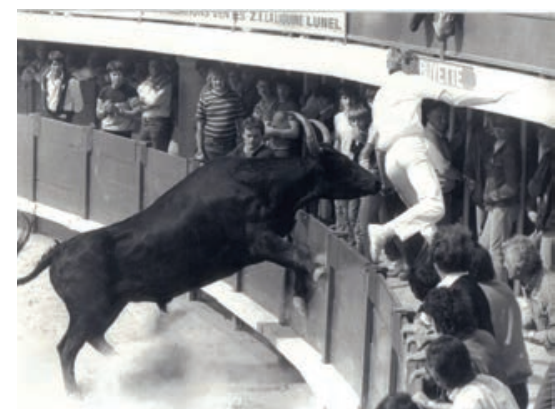

FIG. 11

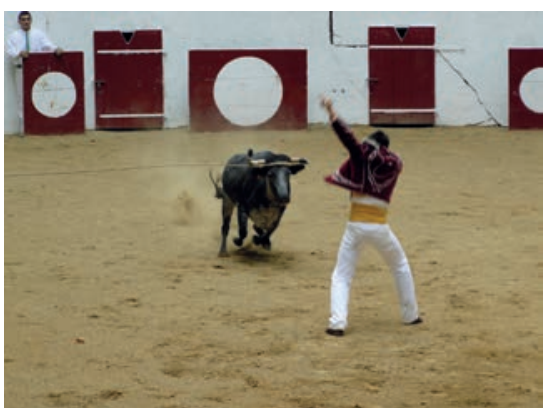

FIG. 12

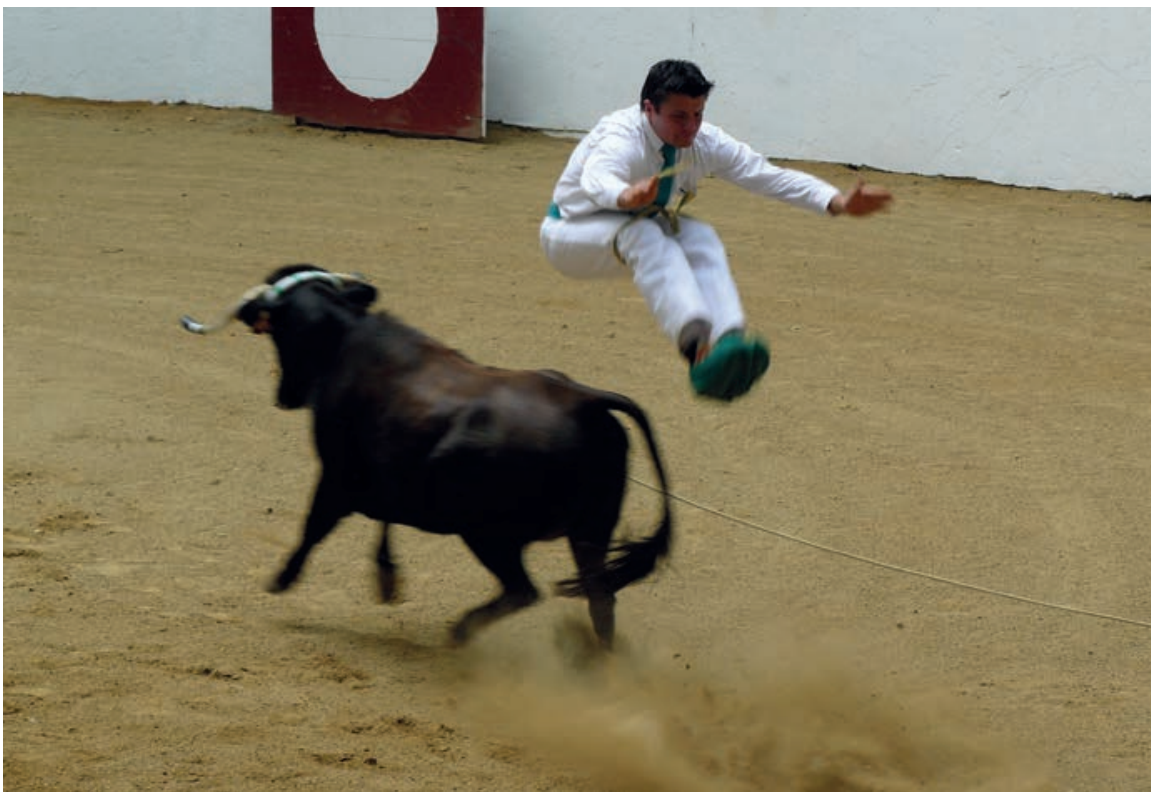

FIG. 13

FIG. 11 Course camarguaise, France

(cliché : Marc Leenhardt)

Fig. 12 Course landaise, Amou, France, 2008

(cliché : Guillaume Cingal)

Fig. 13 Course landaise, Amou, France, 2008

(cliché : Guillaume Cingal) 


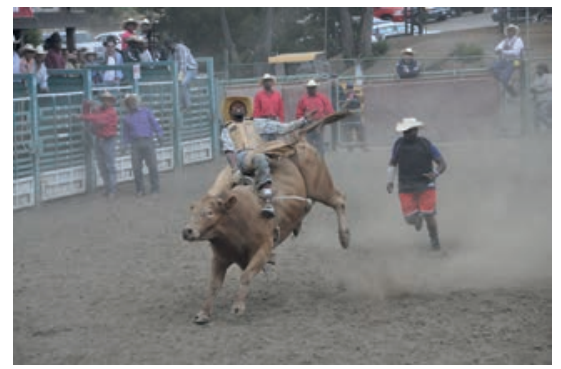

FIG. 14

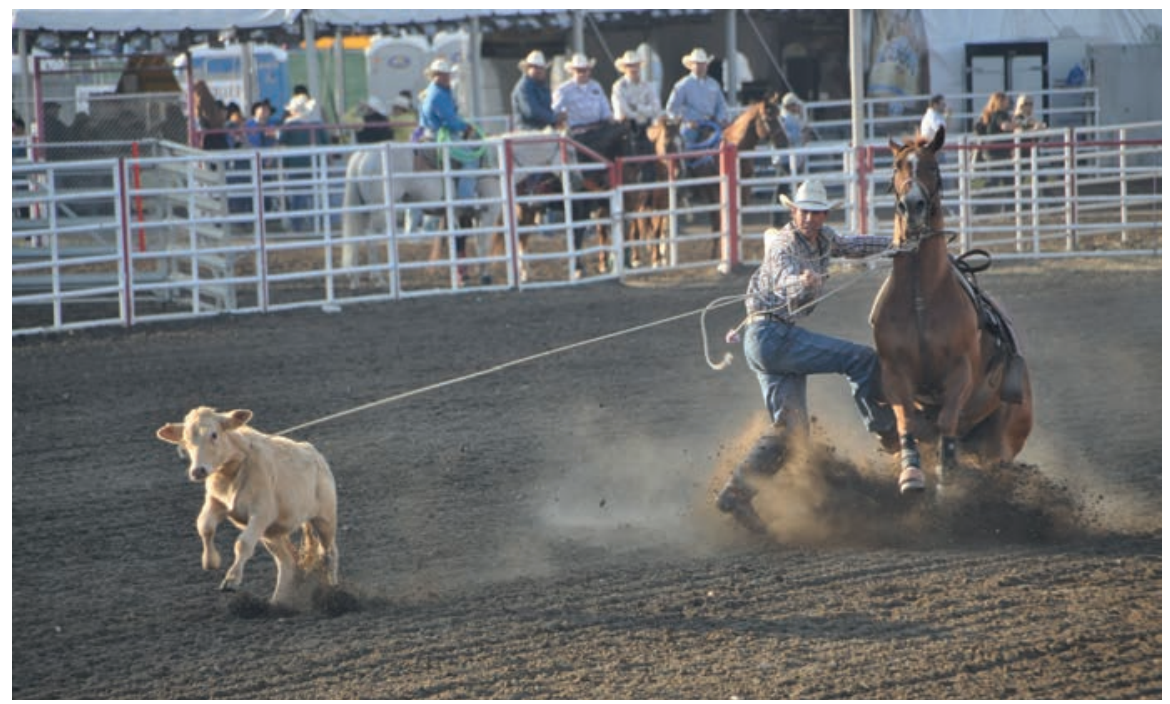

FIG. 15

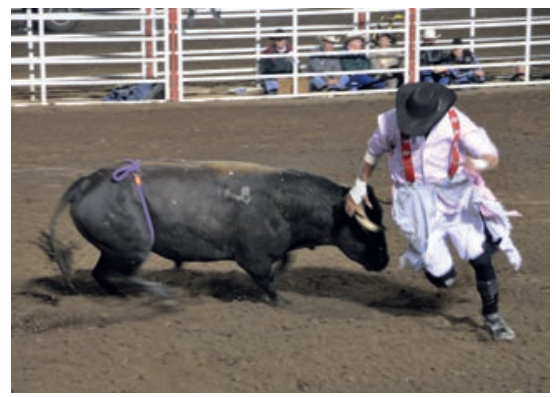

FIg. 14 Rodéo nord-américain, bull riding, Californie, États-Unis, 2009

(cliché : Jean-Baptiste Maudet)

FIg. 15 Rodéo nord-américain, calf roping, Californie, États-Unis, 2009

(cliché : Jean-Baptiste Maudet)

FIg. 16 Rodéo nord-américain, free style bullFighting, Californie, États-Unis, 2009 (cliché : Jean-Baptiste Maudet)

FIG. 16 


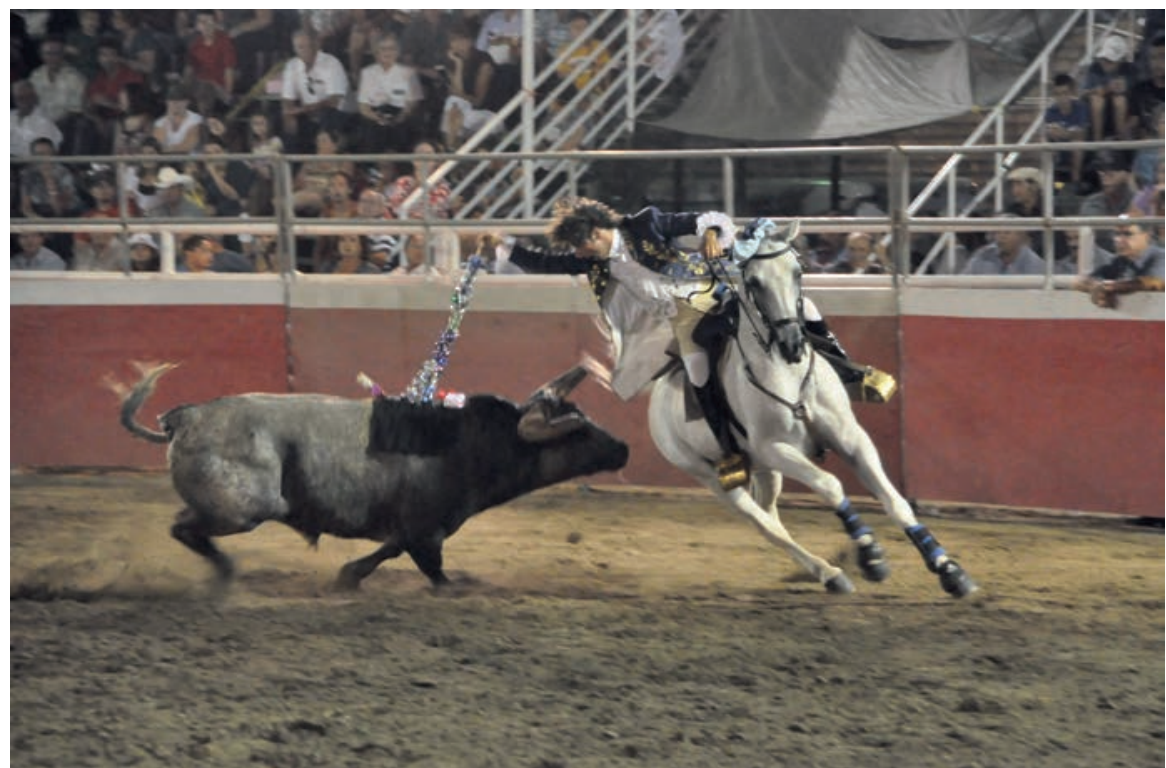

FIG. 17

FIG. 17 Bloodless bullFighting, Californie, États-Unis, 2009 (cliché : Jean-Baptiste Maudet)

FIg. 18 Jaripeo, Morelia, Mexique, 2007 (cliché : Frédéric Saumade)

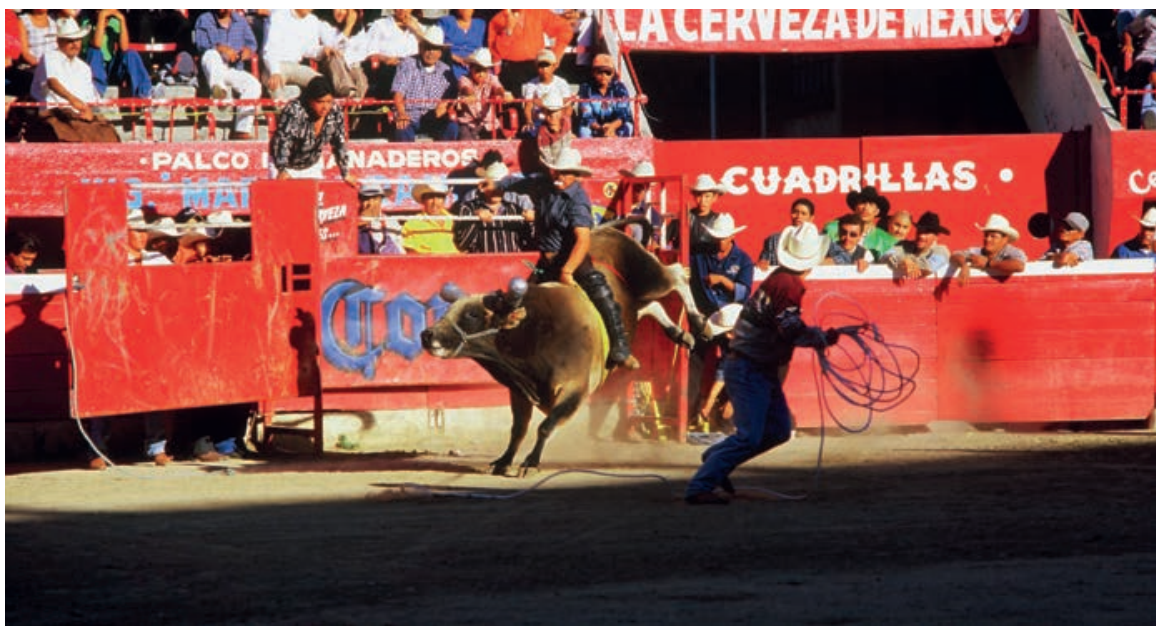

FIG. 18 


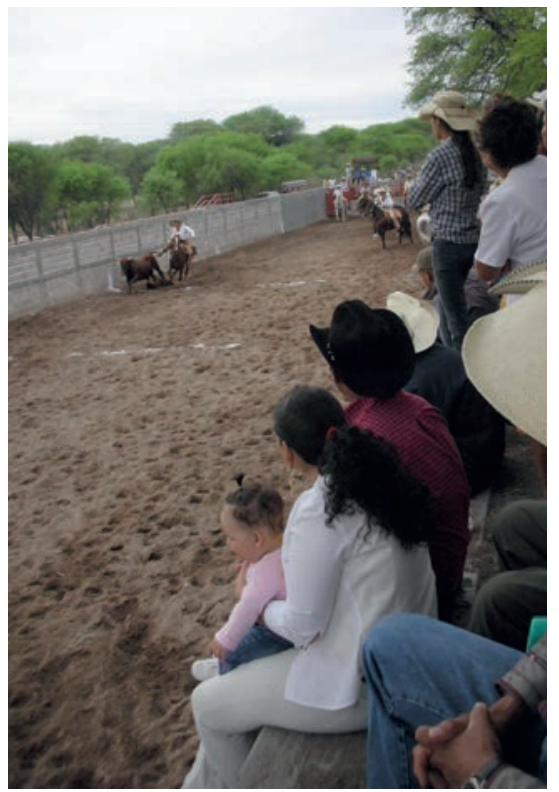

FIg. 19 Charreada rurale, coleada, Jalisco, Mexique, 2007 (cliché : Frédéric Saumade)

FIg. 20 Toros coleados, Venezuela, 2008 (cliché : Marcos Suárez)

FIG. 19

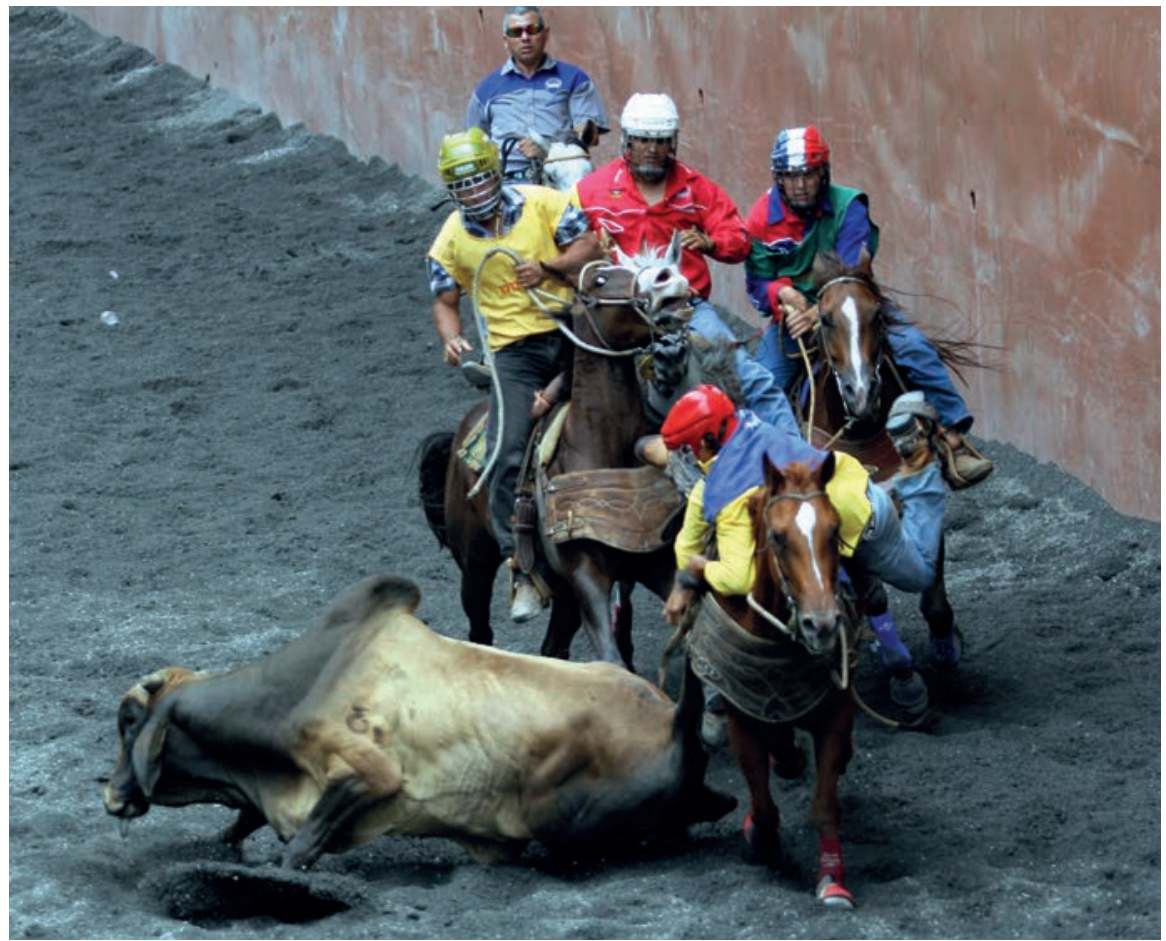

FIG. 20 
Fig. 21 Rodeo chileno, Chili (cliché : Patricia Moya Soulat)

Fig. 22 Suelta de vaquilla, Pampelune, Espagne (cliché : Baltasar García Salaberri)

Fig. 23 Encierro, Pampelune, Espagne

(cliché : Baltasar García Salaberri)

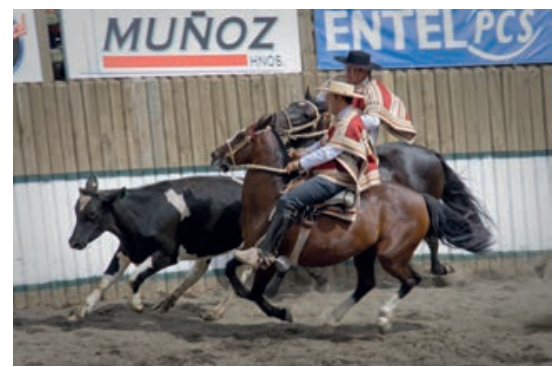

FIG. 21

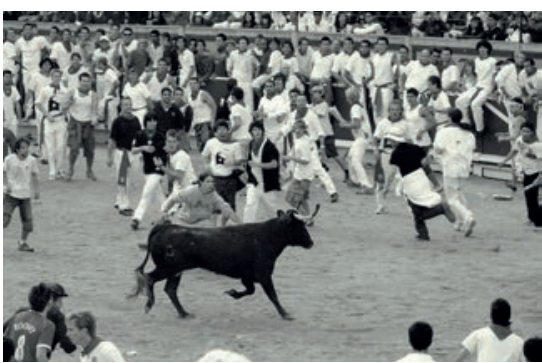

FIG. 22

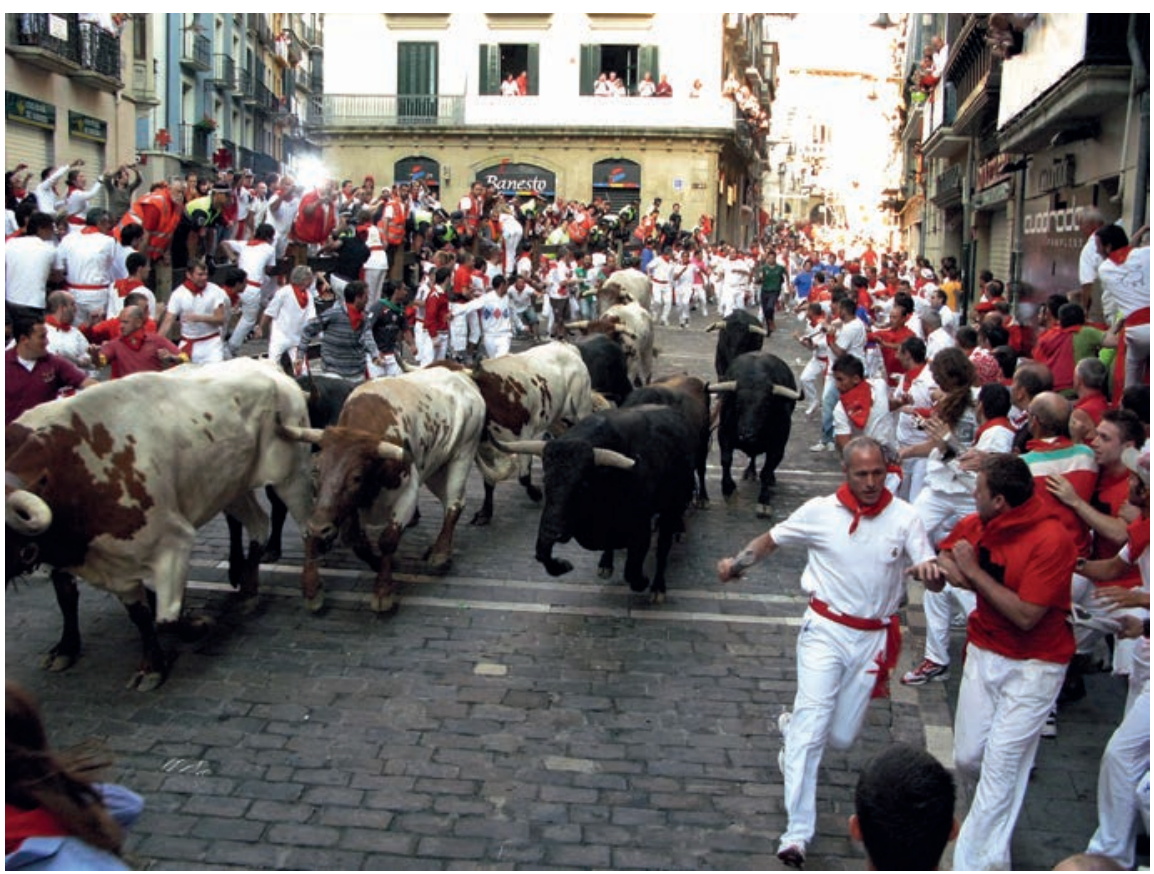

FIG. 23 


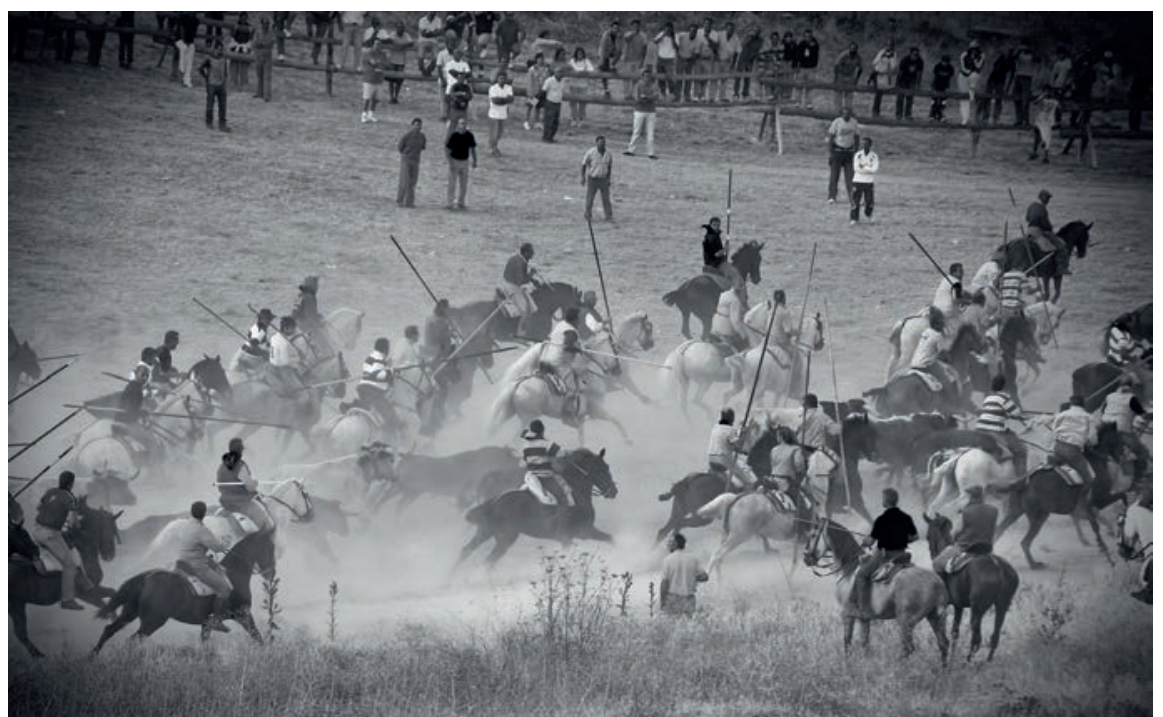

FIG. 24

FIg. 24 Encierro à cheval, Cuéllar, Espagne (cliché : Ángel Torres)

Fig. 25 Toro embolado, Communauté valencienne, Espagne

(cliché : Antonio González)

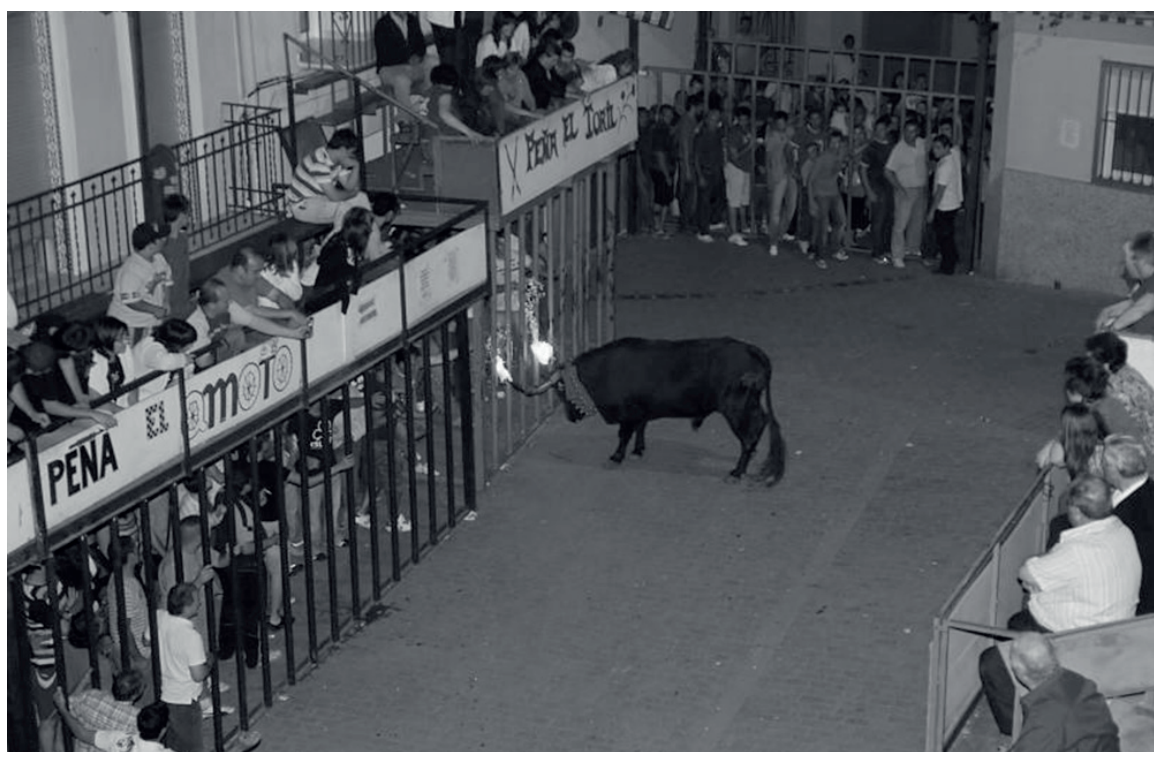

FIG. 25 
Fig. 26 Largada, Vila Franca de Xira, Portugal, 2005

(cliché : Jean-Baptiste Maudet)

FIg. 27 Tourada à corda, Riverdale, Californie, États-Unis, 2008 (cliché : Corey Ralston)

Fig. 28 Abrivade, Aigues Mortes, France (cliché : Marc Leenhardt)

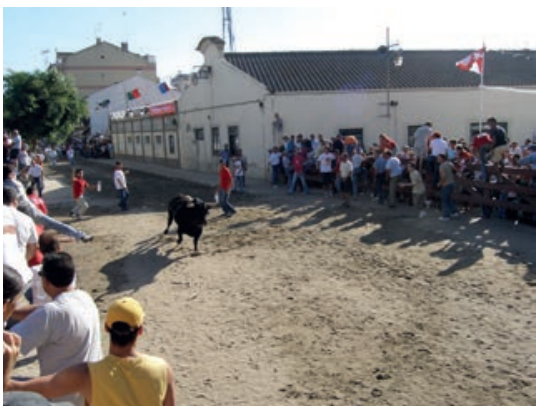

FIG. 26

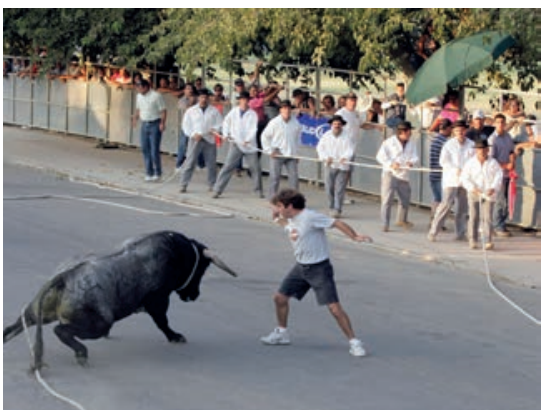

FIG. 27

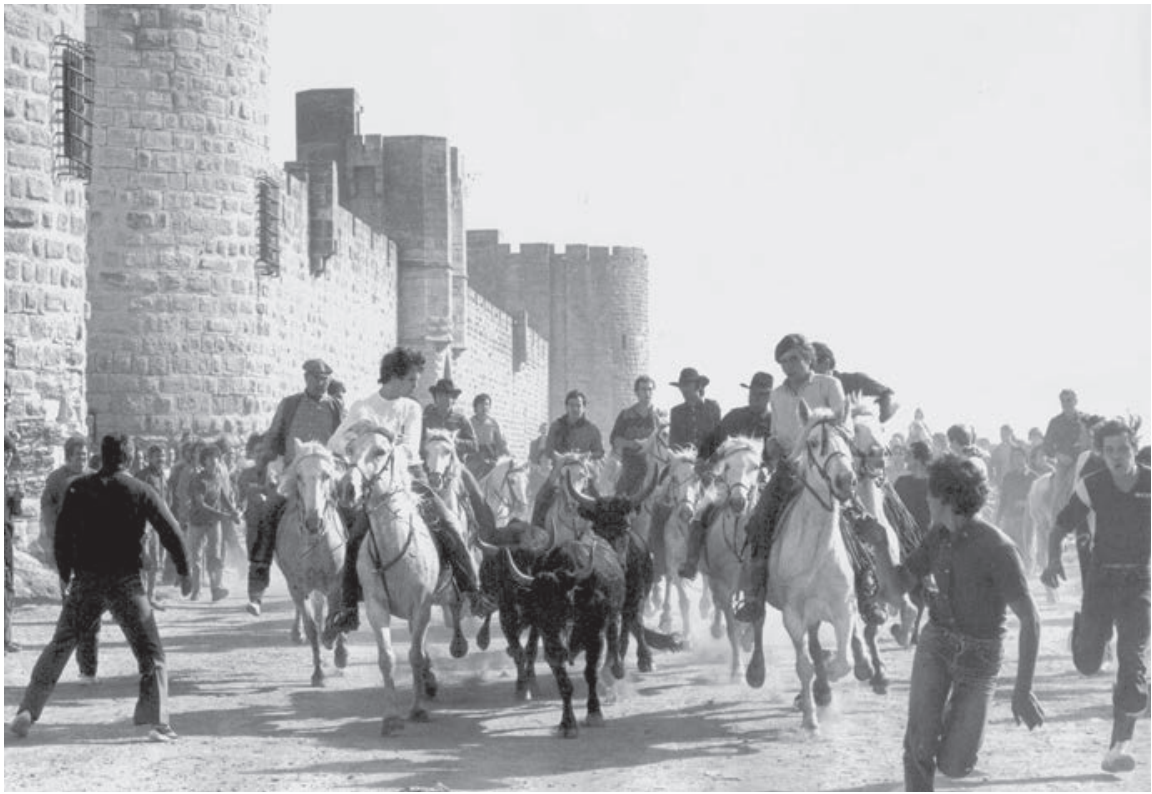

FIG. 28 


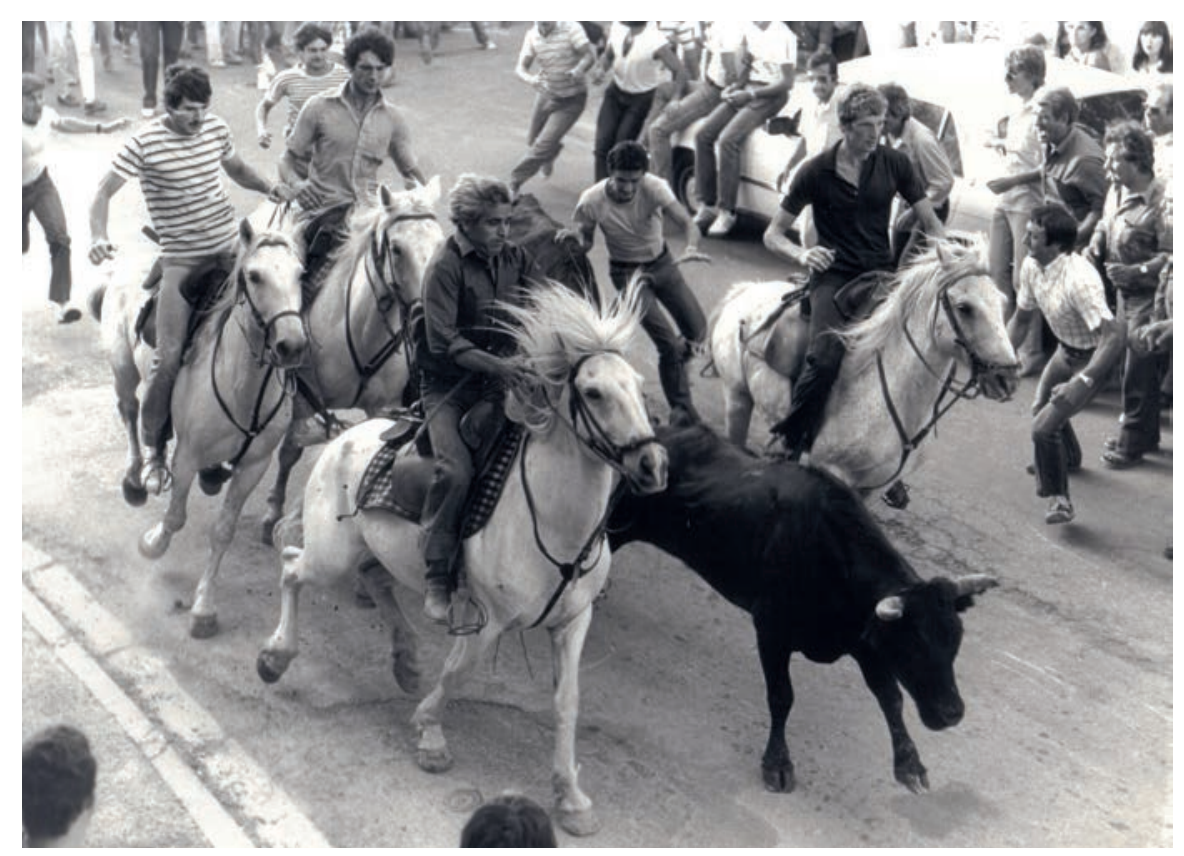

FIG. 29

FIG. 29 Bandide, Saint-Laurent d'Aigouze, France

(cliché : Marc Leenhardt)

FIg. 30 Capeia arraiana, Aldeia Velha, Portugal, 2008

(cliché : Paulo Sanches Fernandes)

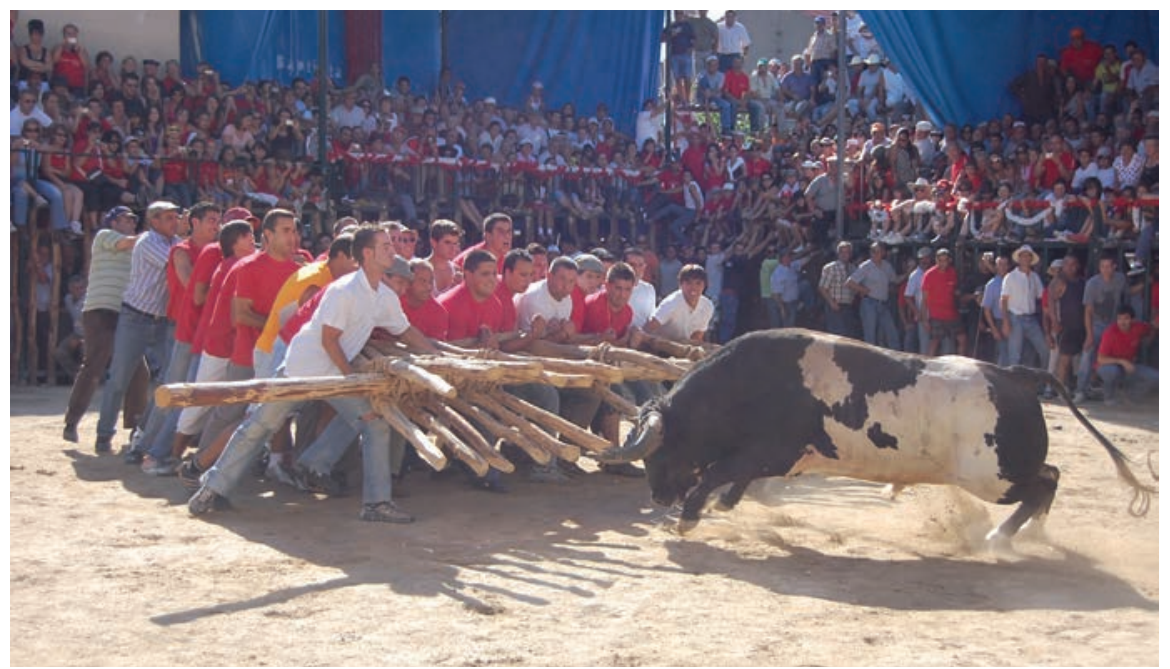

FIG. 30 
Fig. 31 Garraiada, Aldeia Velha, Portugal, 2008

(cliché : Paulo Sanches Fernandes)

Fig. 32 Monta de toros, El Salvador, 2007

(cliché : Miguel Ángel Servellón Guerrero)

FIG. 33 Fiestas en corralejas, Sincelejo, Colombie, 2008

(cliché : Cristiano Santiago

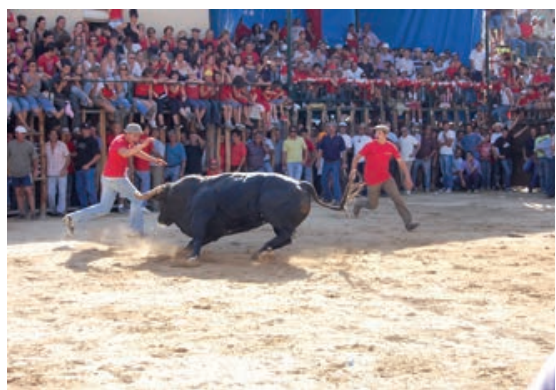

Gómez Zapata)

FIG. 31

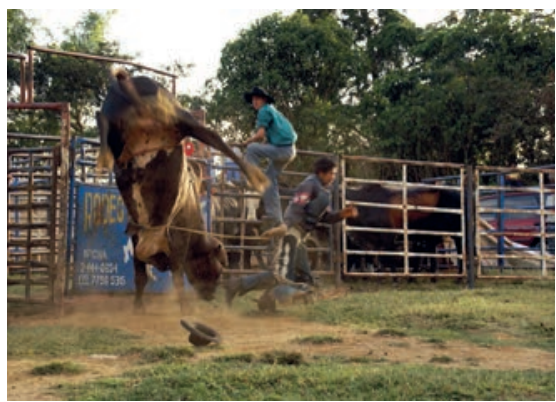

FIG. 32

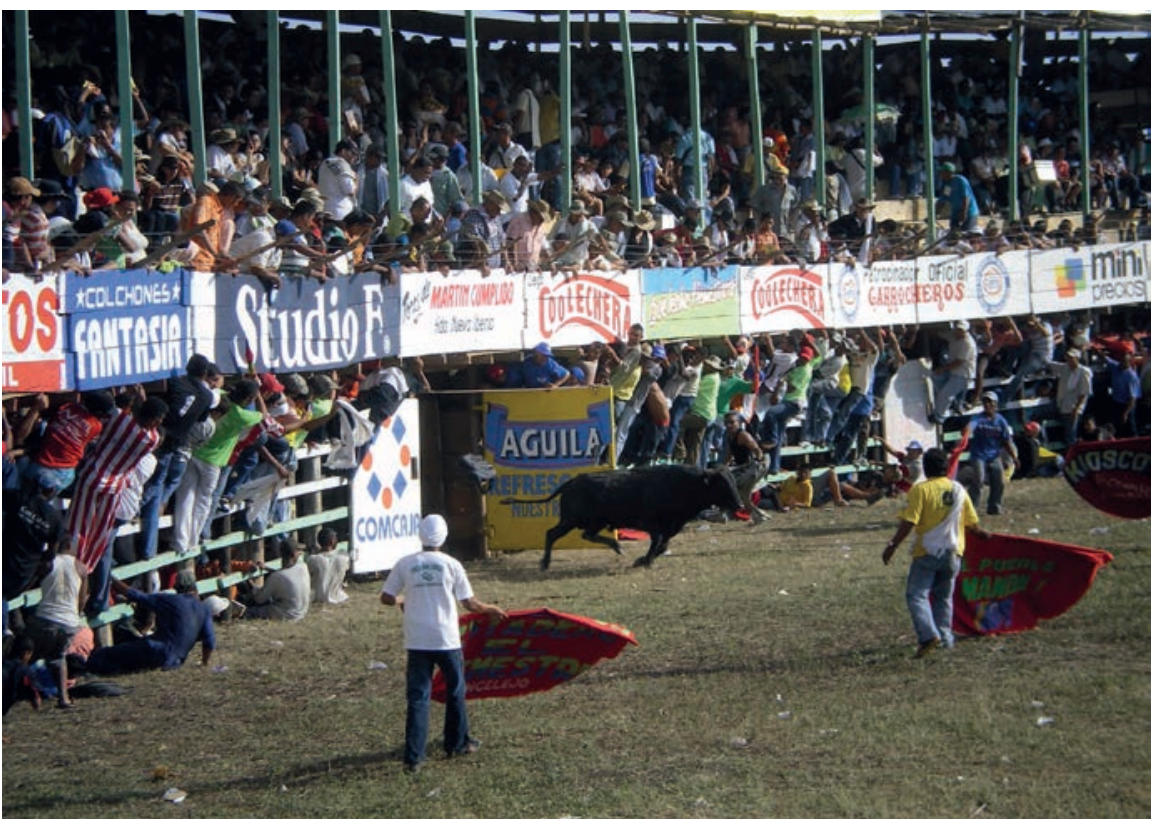

FIG. 33 


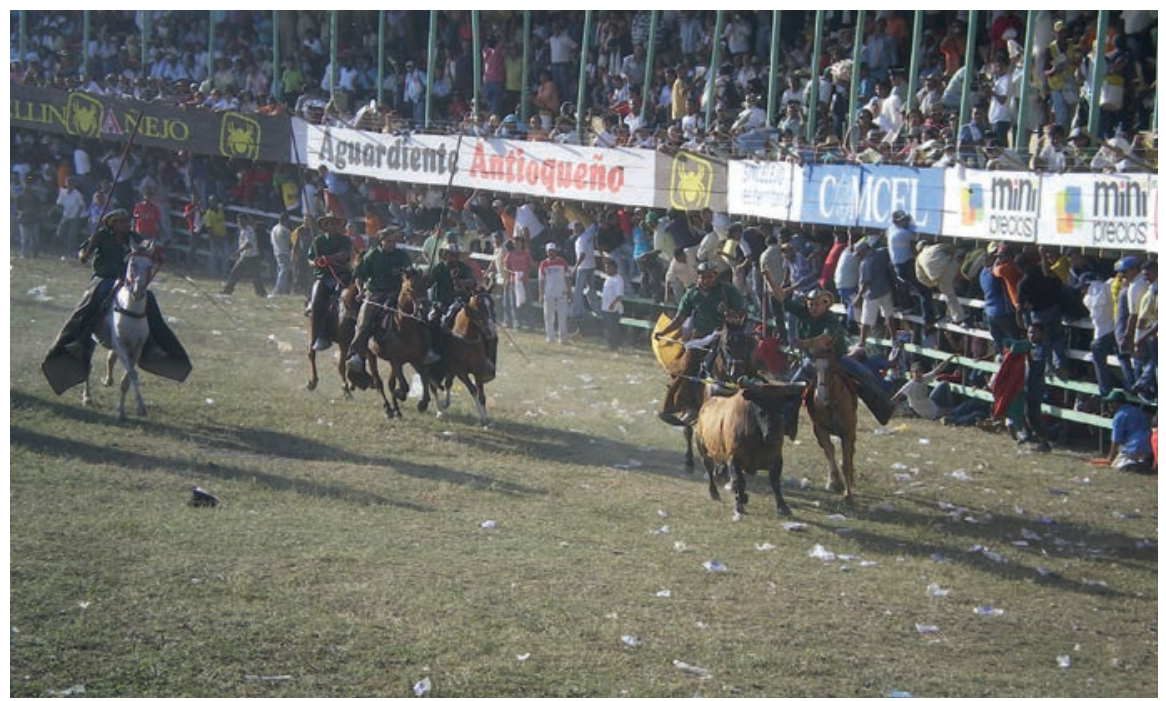

FIG. 34

Fig. 34 Fiestas en corralejas, Sincelejo, Colombie, 2008 (cliché : Cristiano Santiago Gómez Zapata)

Fig. 35 Turupukllay, Ccollurqui, Pérou, 2007 (cliché : Carlos Ojjo)

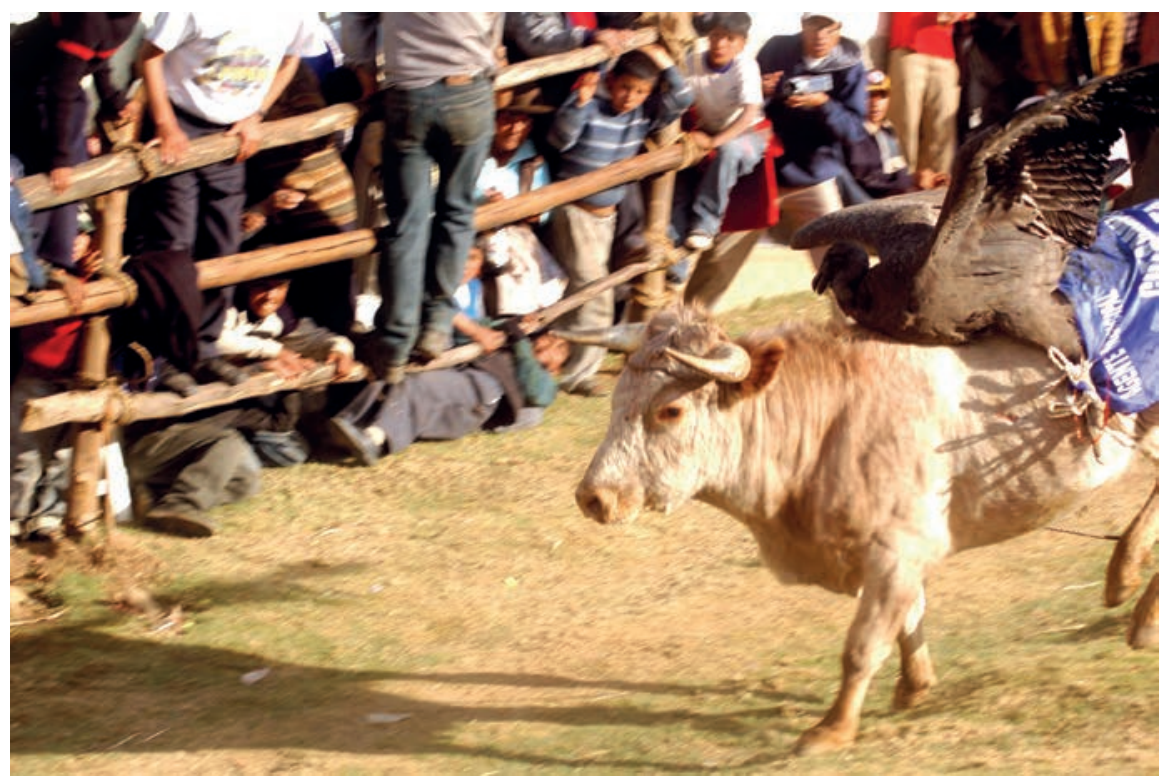

FIG. 35 


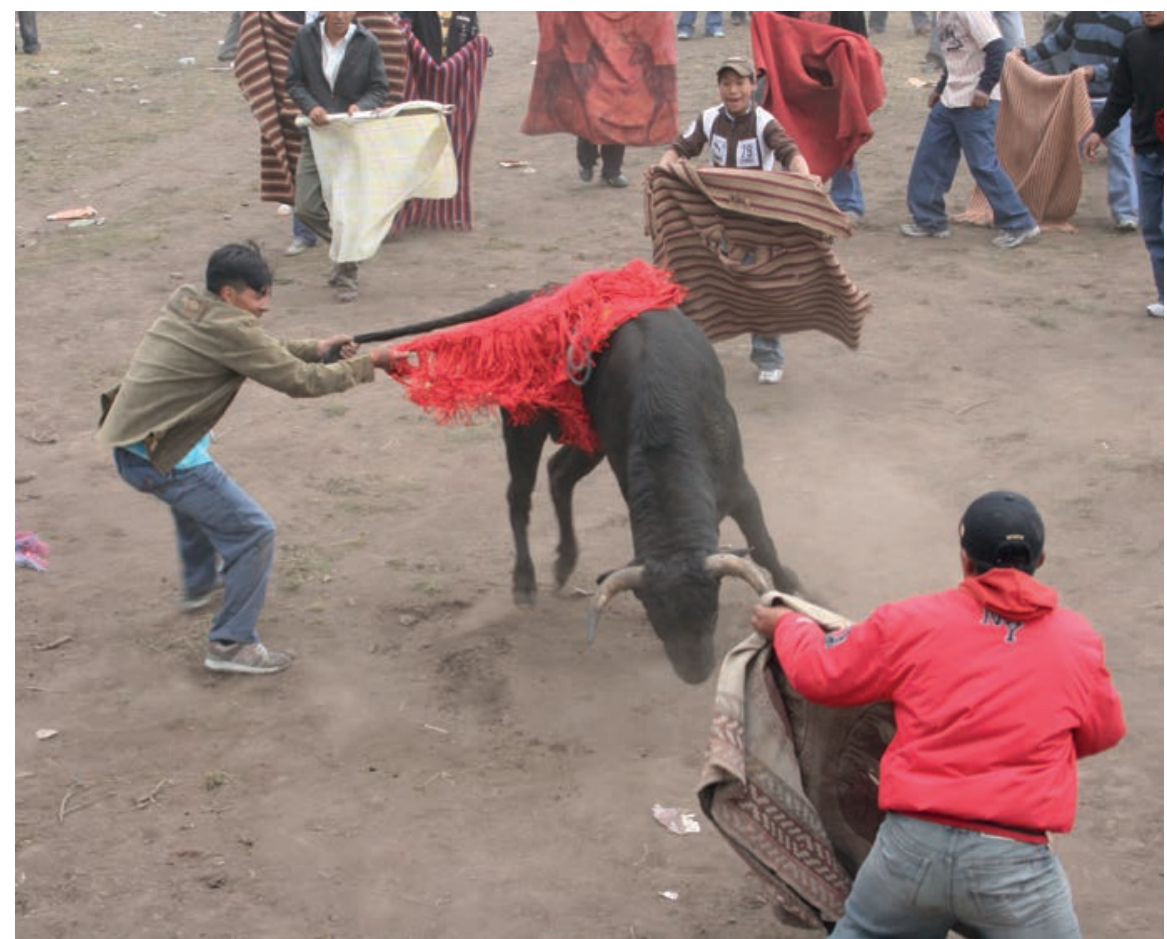

FIG. 36

Fig. 36 Toros de pueblo, Équateur, 2007 (cliché : Esteban Mendieta Jara) 

PREMIÈRE PARTIE

JEUX TAURINS D'EUROPE ET D'AMÉRIQUE 

Les représentations ethnocentriques et symboliques de la tauromachie n'ont pas contribué à rassembler la diversité des jeux taurins au sein d'une même définition. Les amalgames entre corrida et tauromachie, la domination d'une conception ritualiste du fait taurin, la quête obsédante de son enracinement historique ont masqué les principes élémentaires d'unité et de diversité des jeux taurins d'Europe et d'Amérique (chap. I). Rétablir ces principes permet des comparaisons transatlantiques touchant d'une part à la nature du fait taurin en tant que loisir et d'autre part à ses différentes modalités techniques et spatiales d'exécution (chap. II). Cela permet ensuite de mettre en évidence une forme de constante sociale des pratiques tauromachiques qui les place partout, et quelle que soit leur nature, sous le signe de la fête (chap. III). Il en résulte des liens assez originaux entre les sociétés « taurophiles » et leur territoire, qui naissent souvent d'une concurrence et d'une complémentarité entre des genres tauromachiques spatialement coexistants. 



\section{DÉFINITIONS ET REPRÉSENTATIONS DE LA TAUROMACHIE}

Ce que le mot tauromachie désigne oscille entre une acception restrictive du terme qui réduit son usage à la seule corrida et une conception extensive qui englobe un ensemble hétérogène d'interactions entre l'homme et le taureau. La première acception exclut, consciemment ou non, bon nombre de jeux taurins que l'on peut pourtant considérer comme appartenant à une même famille de pratiques. La seconde conception véhicule une représentation de la tauromachie aux contours flous, qui amalgame des activités sociales de natures fort différentes. À partir de ce constat, nous proposons une délimitation de la tauromachie fondée sur des critères matériels. À nos yeux, elle regroupe l'ensemble des pratiques qui mettent en scène un affrontement réel entre des hommes et des bovins.

La tauromachie a donné lieu à de nombreuses interprétations qui interrogent la nature profonde de cette pratique et sa fonction au sein des sociétés qui la produisent. Quelles que soient les interprétations sollicitées pour une meilleure compréhension de la tauromachie, nous pensons que l'autonomie de la sphère ludique est toujours suffisante pour l'inscrire pleinement dans le registre des divertissements. Autrement dit, nous étudions d'abord la tauromachie sous l'angle des jeux taurins, qui inscrit ces pratiques dans une géographie sociale et culturelle du loisir. Le loisir est à prendre au sens où l'entendent Norbert Elias et Eric Dunning selon lequel il constitue un champ de recherche à part entière et une catégorie positive hautement révélatrice du fonctionnement des sociétés humaines ${ }^{1}$. L'une des spécificités des jeux taurins en tant que divertissement est de se situer entre l'art, le sport et les jeux traditionnels, tant du point de vue formel que de leur organisation institutionnelle.

\footnotetext{
${ }^{1}$ N. Elias et E. Dunning, "Les loisirs dans le spectre du temps libre ", dans ID., Sport et civilisation, pp. 124-127. Les auteurs insistent sur la constitution du loisir comme catégorie positive qui dépasse la complémentarité travail - non travail, dans laquelle le loisir serait une oisiveté libératrice des seules tensions de l'activité. Sur ce point, les auteurs prolongent un raisonnement déjà ouvert par Jean Dumazedier lorsqu'il déclare que « le loisir n'est plus un problème mineur, sorte de "poste divers" sans importance placé à la fin de l'inventaire des grands problèmes, si l'on a encore de la place, du temps ou de l'argent pour s'occuper de lui ». J. Dumazedien, Vers une civilisation des loisirs?, p. 15.
} 


\section{I. — TAUROMACHIE ET CORRIDA}

\section{LA CORRIDA : UN ART TAUROMACHIQUE}

AU SOMMET DE TOUTES LES HIÉRARCHIES?

L'emploi du mot tauromachie comme synonyme de corrida est très courant. Cela s'explique d'abord par le simple fait que la corrida est la forme de jeu taurin la plus connue qui exerce une véritable hégémonie médiatique sur l'ensemble des pratiques tauromachiques. Cette position relègue en arrièreplan d'autres affrontements entre les hommes et les bovins, alors considérés comme des formes mineures (course landaise, course camarguaise, concours de recortadores, divers jeux taurins populaires), quand elles ne sont pas exclues des représentations tauromachiques (la grande famille des rodéos), dès lors qu'une distance formelle ou géographique trop importante les éloigne du modèle dominant, la corrida, et de son épicentre, l'Espagne. Tout se passe comme s'il existait des pratiques plus tauromachiques que d'autres, au sommet desquelles figurerait la corrida espagnole.

Cette conception du fait taurin peut également s'expliquer par un tout autre argument qui tient à la genèse même du mot tauromachie. En effet, le mot tauromachie apparait avec l'émergence de la corrida moderne en Espagne à partir du XviII ${ }^{\mathrm{e}}$ siècle. Le dictionnaire Le Robert situe le premier emploi français du mot tauromachie vers 1830. Mérimée utilise le mot en 1831, en francisant le terme espagnol tauromaquia, dans une lettre fictive adressée au directeur de La revue de Paris, écrite lors de son voyage d'Espagne. Pour cette raison, Mérimée est généralement considéré comme son introducteur dans la langue française. Le dictionnaire Le Robert propose deux définitions, celle de " course de taureaux ", qui est la traduction littérale de corrida de toros, et celle d'" art de combattre les taureaux dans l'arène ", qui reprend la définition du mot tauromachie inscrite dans le Diccionario de la Real Academia Española². L'historien des religions Ángel Álvarez de Miranda observe que le terme espagnol tauromaquia ne commence à être utilisé qu'au $\mathrm{XVII}^{\mathrm{e}}$ siècle par les écrivains baroques et s'est appliqué aux jeux tauromachiques de la noblesse ${ }^{3}$. Même si l'auteur ne donne aucune autre indication sur les écrivains en question, l'origine moderne du mot tauromachie est incontestable. Quelle que soit la date du premier emploi du mot tauromachie, il est admis que son usage se diffuse très largement à partir du traité datant de 1796, publié à Cadix sous le nom du matador Pepe-Illo : La Tauromaquia o arte de torear (La Tauromachie ou l'art de toréer) ${ }^{4}$. Ce traité rassemble les principales règles de l'époque pour combattre les taureaux en diverses circonstances, à pied ou à cheval, dans les champs ou en place publique. De ce contexte naît le double sens du mot tauromaquia qui désigne le traité de tauromachie lui-même et les

\footnotetext{
${ }^{2}$ Diccionario de la Real Academia Española : http://buscon.rae.es/draeI/ [réf. 5 février 2006].

${ }^{3}$ Á. Álvarez de Miranda, Le taureau. Rites et jeux, p. 78.

${ }^{4}$ Pepe-Illo, La Tauromaquia.
} 
règles qu'il expose ${ }^{5}$. Ce texte est reconnu comme celui jetant les bases de ce qui deviendra la corrida telle que nous l'observons aujourd'hui, du Sud-Ouest européen à l'Amérique latine. Cependant, la corrida moderne ne finit d'être codifiée, dans tous les aspects de son déroulement, qu'un demi-siècle plus tard par le torero Francisco Montes « Paquiro » qui publie en 1836 une Tauromachie complète ou art de toréer dans les plazas à pied comme à cheval". Le surnom de "Napoléon des toreros » qui lui est parfois attribué laisse apparaître toute son influence dans la codification moderne du spectacle. En Espagne, à partir de cette date, il n'y a plus de doute sur ce que la tauromachie désigne : il s'agit bien d'affronter le taureau en vue de sa mise à mort, en respectant des techniques adaptées et en exécutant des figures précises (les suertes) permettant à l'homme de ne pas être blessé7. Le mot tauromachie, à l'origine, est donc issu du milieu spécialisé qui l'a fait naître et il n'est pas employé de façon générique pour désigner l'ensemble des jeux où s'affrontent des hommes et des taureaux.

Dans les dictionnaires taurins récents, on aurait pu s'attendre à un élargissement explicite de la notion de tauromachie à d'autres pratiques proches, au moins pour celles qui ont acquis, à l'image de la corrida, une forme stable, des règles codifiées et une réelle institutionnalisation. Le dictionnaire encyclopédique de Claude Popelin, dont la première édition date de 1970, intitulé La Tauromachie, ne propose aucune définition du mot. Même si la corrida reste le sujet central du dictionnaire, l'ouvrage témoigne d'une certaine ouverture aux autres formes de jeux taurins. La préface s'en explique ainsi :

Ce dictionnaire de la tauromachie se propose d'offrir à l'aficionado, ou au simple curieux, l'information la plus étendue sur la corrida. [...] Une vue historique de la tradition tauromachique, partout où elle s'est manifestée (Espagne, Portugal, Provence et Landes, Vallespir, Andes et à un moindre degré, le Valais suisse ou l'Angleterre au temps de son bullbaiting), n'a pas davantage été négligée ${ }^{8}$.

L'énumération des lieux où « la tradition tauromachique s'est manifestée » apparaît très incomplète, mais elle a le mérite, sur le principe, de ne pas consi-

\footnotetext{
${ }^{5}$ Dans son propos au lecteur, Pepe-Illo déclare « qu'au point où est parvenu l'art de toréer, une œuvre se doit d'en exposer les règles et les techniques ». Il ajoute qu'il est le premier à parler du toreo et à présenter " [ses] pensées et idées Tauromatiques (sic) » fondées sur la "savante expérience, mère légitime de sa connaissance ». Pepe-Illo, La Tauromaquia, p. 43. L'emploi du mot « tauromatiques» (tauromaticás dans le texte) confirme sans doute l'usage encore instable du mot « tauromachie » et de l'adjectif « tauromachique».

${ }^{6}$ F. Montes, Tauromaquia completa.

${ }^{7}$ Le mot espagnol suerte signifie chance, et possède dans le vocabulaire taurin de nombreuses significations dont ici celles de figures, techniques ou actions exécutées pour toréer l'animal : passes de capes, piques, pose de banderilles, passes de muleta, mise à mort. Cette dernière est appelée «suerte suprême".

${ }^{8}$ C. Popelin, La Tauromachie, pp. 5-6. Le bullbaiting désigne les combats entre chiens et taureaux en vogue en Angleterre jusqu'au début du XIX ${ }^{e}$ siècle qui finissent par être interdits au milieu du siècle.
} 
dérer les termes tauromachie et corrida comme étant strictement synonymes. Le dictionnaire propose ainsi une entrée « course landaise » qualifiée de « forme tauromachique propre à l'Aquitaine » et une entrée "course à la cocarde » inscrivant ces jeux taurins dans le champ tauromachique. L'ouvrage collectif, intitulé La tauromachie, histoire et dictionnaire paru en 2003, ne possède aucune entrée tauromachie ${ }^{9}$. Il laisse à sa place le terme espagnol tauromaquia auquel est attribué le sens d' " art de combattre les toros ainsi que les règles et préceptes qui fondent cet art $»^{10}$. Les 1.800 entrées de la partie dictionnaire ne proposent aucune ouverture sur la diversité des jeux taurins, aucun article sur la course landaise, aucun article sur la course camarguaise, ni aucune allusion aux jeux taurins américains. En revanche y figurent des articles approfondis sur « cinéma et tauromachie ", " littérature et tauromachie ", " théâtre et tauromachie ". Faut-il y voir une lacune ? Probablement pas pour tous ceux qui font de l'art tauromachique le domaine réservé de la corrida. Le texte introductif affiche l'ambition de l'ouvrage : « donner à l'aficionado français, mais aussi au lecteur soucieux de s'instruire, un ensemble complet et accessible d'informations sur l'histoire, les techniques, la terminologie et les acteurs de l'art tauromachique, ainsi que sur la culture qui le fonde et qu'il perpétue ${ }^{11}$. Cette conception révèle un cloisonnement assez radical de l'orthodoxie tauromachique telle qu'elle est vécue, plus ou moins consciemment, par de nombreux amateurs de corrida. En témoignent par exemple, les propos d'André Viard, ancien matador et critique taurin reconnu :

Que notre Midi, avant d'épouser cette tauromachie qui lui viendra d'Espagne, ait développé des tauromachies affadies parce que la mort en est absente ? C'est peut-être vrai pour le jeu Gascon qui en pâtit, malgré ses feintes et ses voltes, empêtré dans ses ficelles et ses cornes emboulées, stigmates d'une grandeur tombée en décadence ${ }^{12}$.

Les écarteurs landais et la Fédération française de course landaise apprécieront que cela ne soit que "peut-être vrai ». Ils n'en continuent pas moins d'employer le terme de tauromachie pour désigner leur course, de même que les amateurs de course camarguaise emploient le terme de «tauromache » pour désigner les adeptes de la course à la cocarde. Derrière cette conception de la tauromachie se profile une opposition tenace entre la corrida, qui relèverait de

${ }^{9}$ R. BÉRARD (dir.), La Tauromachie. Histoire et dictionnaire.

${ }^{10}$ Ibid., p. 888.

${ }^{11}$ Ibid., p. x (numérotation en chiffre romain du propos introductif).

${ }^{12}$ A. Viard, Le Mythe du Taureau, p. 51. Nous aurons l'occasion de reparler de cet ouvrage tant il nous semble révélateur d'un véritable genre littéraire taurin à mi-chemin entre l'essai scientifique et la pensée symbolique qui participe pleinement d'une forme d'érudition très répandue parmi les aficionados. Pour ne pas isoler les propos d'André Viard d'un travail par ailleurs très éclairant, nous tenons à souligner la qualité des ouvrages que l'auteur et ancien torero a consacrés à l'analyse de la technique tauromachique, en particulier : A. VIARD, Comprendre la corrida. 
l'art et de la culture, et les autres formes d'affrontement entre les hommes et les bovins qui, au fond, ne relèveraient que du jeu et du folklore. On retrouve la marque d'une distinction classique entre culture savante et culture populaire, la première niant à la seconde ses lettres de noblesse. Cette distinction apparaît clairement dans de nombreux ouvrages sur la tauromachie parmi lesquels la célèbre encyclopédie, Los Toros, de José María de Cossío. Bien qu'attestant d'une connaissance approfondie de la diversité des pratiques tauromachiques, l'auteur considère les formes taurines " populaires » comme des épiphénomènes secondaires qui n'ont d'autre fonction que de rappeler les origines « anarchiques et désordonnées » de la corrida ${ }^{13}$. Les jeux taurins populaires et la corrida sont ici envisagés dans une continuité historique finaliste qui développe le passage progressif de l'un à l'autre, sans véritablement reconnaître aux premiers leur dimension parfaitement contemporaine. Dans un contexte actuel de croissance, de renaissance et de réinvention de nombreux jeux taurins populaires, cette dichotomie doit, plus que jamais, être soumise à un examen critique qui remet en cause nombre d'a priori relatifs à une conception évolutionniste des pratiques taurines.

\section{LA TAUROMACHIE}

\section{À L'ÉPREUVE DU RELATIVISME CULTUREL}

Selon les régions considérées, le mot « tauromachie » s'enrichit plus ou moins des spécificités de la culture taurine locale, comme le montrent les exemples landais et camarguais. Il en va de même en Amérique latine, même si le terme reste plutôt réservé à l'univers de la corrida. Dans les petits pays d'Amérique centrale (Guatemala, Nicaragua, Costa Rica, Panamá) où la corrida à l'espagnole ne constitue pas un spectacle régulier, la tauromachie désigne l'ensemble des jeux où s'affrontent l'homme et le taureau, que le taureau soit monté ou qu'il soit toréé, que le jeu appartienne, pour nous, à la famille des rodéos, ou à celle des courses de taureaux. À l'inverse au Mexique, le terme tauromachie ne regroupe pas l'ensemble des jeux où s'affrontent l'homme et le taureau. Ici, l'institut national de la statistique (INEGI) publie chaque année un rapport sur les activités culturelles du pays. Concernant notre objet, le rapport distingue la catégorie des « spectacles taurins» (corrida, charlotada, festival taurin, novillada) de la catégorie intitulée « charreada ou jaripeo $»^{14}$. Le glossaire donne comme équivalent à la notion de spectacle taurin celle d' " exhibition de tauromachie ", excluant ainsi la charreada et le jaripeo de l'activité tauromachique proprement dite ${ }^{15}$. La tauromachie apparaît ainsi comme une catégorie à géométrie variable qui pose un problème de géographie culturelle fondamentale à nos yeux : les relations entre la coexistence spatiale des pratiques

\footnotetext{
${ }^{13}$ J.-M. de Cossío, Los Toros, t. I, p. 704.

${ }^{14}$ Instituto Nacional de Estadística, Geografía e Informática, Estadísticas Culturales, p. 34 .

${ }^{15}$ Ibid., p. 61. Espectáculos taurinos: exhibición de tauromaquia.
} 
tauromachiques et le cloisonnement des cultures taurines ne sont pas partout identiques.

On retrouve les mêmes questions avec l'emploi de l'adjectif «taurin » et de l'expression los toros ( «les taureaux») qui s'emploient tantôt pour désigner toute forme de pratiques tauromachiques, tantôt seulement pour les jeux directement apparentés à la corrida. Les emplois varient en fonction de la culture taurine dominante des régions considérées, en fonction des relations entre les différentes pratiques taurines, en fonction des représentations qui les rapprochent ou les éloignent, et en fonction des cultures urbaines ou rurales à travers lesquelles ces représentations s'expriment. En Espagne, los toros désigne officiellement la corrida. Cette équivalence est précisée dans les dictionnaires où le mot toro, au pluriel, peut prendre le sens de "Fiesta o corrida de toros ${ }^{16}$. Nous reviendrons plus avant sur l'équivalence problématique entre fête taurine et corrida (voir chap. III). Mais, selon le contexte, los toros peuvent aussi désigner l'ensemble des pratiques taurines, et, plus généralement encore, les pratiques tauromachiques comme fait social, historique et culturel. Au Venezuela et en Colombie, dans les llanos, l'expression désigne le jeu des toros coleados. Au Mexique, dans le monde rural, l'expression los toros désigne fréquemment le jaripeo, terme qui lui-même, jusqu'à une époque récente est donné, dans de nombreux ouvrages de référence, comme un équivalent de charreada ${ }^{17}$. Dans tous les pays hispanophones, l'expression los toros aura tendance à désigner la corrida, marquant malgré tout un clivage assez prononcé entre la culture taurine urbaine, généralement acquise à l'univers du spectacle du haut de la hiérarchie, et une culture taurine rurale plus diversifiée.

Chez les amateurs de corrida du monde entier, los toros désigne bien les courses de taureaux, la plaçant ainsi au sommet de tous les jeux taurins. Les adeptes des autres formes de jeux ne partagent évidemment pas la même représentation hiérarchique des pratiques tauromachiques. Cependant, ces derniers ont souvent tendance à définir leur passion par rapport à la corrida considérée alors, plus ou moins consciemment, comme une forme dominante, indépendamment de l'intérêt qu'ils lui portent. On l'observe, par exemple, à travers la présentation de la course landaise par le site de la Fédération qui insiste sur le fait « qu'il n'y a pas ici de mise mort au cours de la course ${ }^{18}$. Cette précision démarque explicitement le jeu landais de la corrida sur la base d'un de ses éléments les plus caractéristiques : la mort du taureau. Cette attitude se retrouve peu ou prou dans l'immense majorité des pays qui possèdent des traditions taurines, y compris pour ceux, comme le Chili, qui ne pratiquent pas la corrida. En effet, les amateurs de rodeo chileno se défendent souvent des critiques énoncées par les associations de protection des animaux en soulignant qu'il n'y a ni sang ni mise à mort dans leur jeu, comme si le poids de la corrida dans les

${ }^{16}$ J. Casares, Diccionario ideológico de la lengua española, p. 823.

${ }^{17}$ D. Fournier, «Corrida, charreada et jaripeo », p. 187 ; F. SAumade, Maçatl, p. 80.

${ }^{18}$ Texte de présentation de la course landaise sur le site Internet de la Fédération française de course landaise : http://courselandaise.org/la-course.php [réf. du 10 juin 2007]. 
représentations était parvenu à imposer une image canonique de l'affrontement entre les hommes et les bovins. Mais le rodeo chileno, par la présence même de l'adjectif qui précise sa nationalité dans son appellation officielle, se démarque d'une autre forme tauromachique dominante : le rodéo étasunien. Tout se passe comme si le rodéo chilien, pour sa propre distinction, était pris entre deux pratiques tauromachiques hégémoniques : l'une issue d'un passé révolu : la corrida de l'Amérique espagnole, l'autre d'un présent triomphant : le rodéo de l'Amérique étasunienne.

La référence à la mise à mort constitue un point important dans la différenciation des pratiques et dans la différenciation des régions taurines. Il ne faudrait pas croire l'Espagne extérieure à ce débat sous prétexte que la corrida constitue la pratique dominante. Depuis trois ou quatre ans sont apparues, dans la presse spécialisée espagnole consacrée à la corrida, des publicités pour un spectacle intitulé El Rodeo en su salsa de Cuba qui s'affiche comme une forme tauromachique alternative. Le spectacle est annoncé comme " un échange de culture festive et de culture d'élevage entre l'Amérique et l'Espagne ». Le programme insiste évidemment sur le fait qu'il n'y a pas de mise à mort, afin d'attirer un large public. Le texte précise que « le spectacle ne comporte rien pouvant heurter la sensibilité du public ». En prenant le contre-pied de la corrida et pour inciter les entrepreneurs de spectacles à programmer " le rodéo à la sauce cubaine ", la publicité ajoute : "Modernise et rénove les spectacles de ta fête, pense à l'autre public ». D'une façon beaucoup moins anecdotique, on peut souligner que les spectacles taurins qui connaissent actuellement la plus forte croissance en Espagne sont les concours de recortadores dans lesquels les taureaux ne sont, ni tués, ni blessés. Cet aspect n'est évidemment pas le seul à expliquer l'engouement pour cette nouvelle forme de jeu tauromachique, mais l'absence de sang y participe certainement.

Retenons que le mot tauromachie ne désigne pas partout les mêmes réalités, ni ne reflète les mêmes clivages entre les pratiques. Son emploi est fortement attaché à la pratique de la corrida, même là où elle coexiste avec d'autres formes de jeux taurins. Quoi qu'il en soit, notre analyse ne peut se satisfaire d'une assimilation réductrice de la tauromachie à la seule corrida, qui témoigne d'une conception limitative des pratiques qui seraient proprement tauromachiques, ainsi que d'un fort hispano-centrisme des représentations culturelles du fait taurin.

\section{II. — TAUROMACHIE ET RITES TAURINS}

\section{LA TAUROMACHIE : « RITE ÉTERNEL »?}

À l'opposé d'une assimilation réductrice entre tauromachie et corrida, il existe une représentation extensive des faits taurins qui tend à considérer sur un même plan de nombreux rituels ayant pour principal protagoniste le taureau, quand bien même ils s'éloignent de toute dimension agonistique. En dépit de l'intérêt indiscutable que constitue une prise en compte élargie des relations 
homme-taureau, cette approche, par un défaut inverse, n'aide pas à définir la nature précise des pratiques tauromachiques.

Dans la littérature scientifique comme dans la littérature taurine, il existe peu de tentatives pour répertorier et éventuellement classer la totalité des jeux taurins. L'une des tentatives les plus explicites est celle de Jean-Pierre Fabaron dans un article de 1990, dont le titre laisse en outre apparaître une ambition géographique : «Répartition des jeux taurins dans le monde : essai de classification ${ }^{19}$. L'auteur ne propose aucune représentation cartographique des jeux taurins à travers le monde, mais développe plutôt une typologie des tauromachies à travers l'histoire, avec comme premier critère de différenciation celui de la mise à mort du bétail. Dans la catégorie des jeux avec mise à mort, l'auteur distingue les luttes du bovin contre un animal (lion, tigre, éléphant, blaireau, chien, ours) et les luttes contre l'homme (jeux du cirque, lanzadas entre chrétiens et musulmans, corrida navarraise, corrida espagnole, yawar fiesta), détaillant aussi les moyens techniques utilisés et l'éventuelle participation du cheval. Dans la catégorie des jeux taurins sans mise à mort, il répartit les pratiques en six soustypes : lutte face à un autre bovin, bovins tenus à la corde, bovins pris au lasso, bovins attelés, jeux taurins montés et bovins libres (non tenus à la corde). Le dernier sous-type est ensuite détaillé selon la gestuelle (course, sauts, taureaux renversés, provocation avec objets divers). Enfin l'auteur distingue un soustype sociologique appelé «toreo comique et mojiganga » où sont réunis des jeux burlesques. Cette démarche a le mérite d'avoir identifié et classé, selon des critères essentiellement formels, un nombre important de jeux taurins, même si le recensement comporte de nombreuses lacunes. En particulier, la typologie ne fournit aucun détail sur les différents jeux taurins apparentés à la famille des rodéos et passe sous silence la diversité des lâchers de bovins en Amérique latine. L'auteur reconnaît cependant lui-même les limites de son travail et précise : «Après ce tour d'horizon forcément incomplet et discutable (ce dont nous sommes conscients), nous souhaitons que cette ébauche de classification soit complétée ou remaniée, mais serve de point de départ à une investigation complémentaire $»^{20}$.

Comme principal élément de discussion, on peut s'interroger sur la pertinence d'adopter la mise à mort comme critère typologique transhistorique de premier ordre dans la différenciation des pratiques taurines à l'échelle mondiale. C'est sans doute se focaliser hâtivement sur l'aboutissement du spectacle (sa fin), sans s'interroger en première instance sur son ressort ludique (sa finalité) : sur ce qui fait véritablement jeu. On peut au contraire considérer que le travail à la muleta qui précède la mise à mort est aujourd'hui le cour du spectacle et ce pourquoi les aficionados vont préférer le style de tel ou tel torero. En outre, ce dernier peut triompher en dépit d'une mauvaise mise à mort. De même, le taureau peut être gracié sans que le spectacle perde de sa cohérence, ni que le

\footnotetext{
${ }^{19}$ J.-P. FABARON, "Répartition des jeux taurins dans le monde ».

${ }^{20}$ Ibid., p. 46. Les mots entre parenthèses sont ceux de Jean-Pierre Fabaron.
} 
succès du matador s'en trouve déprécié, bien au contraire. Le choix de l'auteur semble, une nouvelle fois, participer d'une fascination exercée par la mise à mort du taureau dans la corrida espagnole, à l'aune de laquelle, nous l'avons vu, les autres pratiques tauromachiques ont tendance à être appréhendées. C'est Frédéric Saumade, dans son travail de thèse doctorale, qui a sans doute le plus fermement démontré la nécessité de relativiser l'importance de la mise à mort dans la tauromachie. Selon l'auteur, cette fascination, portée sur le terrain de l'anthropologie, a eu des conséquences néfastes sur la compréhension générale des pratiques tauromachiques ${ }^{21}$. Sans entrer pour l'instant dans ce débat, cette fascination semble avoir empêché, dans la classification de Jean-Pierre Fabaron, leur correcte identification, ne serait-ce que pour parvenir à les recenser de façon plus précise et à mieux les différencier.

Plus récemment, l'exposition «Rites éternels : l’homme et le taureau dans le monde ", qui a été présentée au Musée des cultures taurines de Nîmes du 20 mai au 17 octobre 2004 proposait une autre approche des rituels taurins. Cette exposition présentait au grand public un large éventail d'interactions rituelles entre les hommes et les bovins, observées dans le monde entier à des époques différentes. Les rituels taurins sont ici classés suivant « les attitudes de l'homme face au taureau » quelles que soient les époques et quels que soient les espaces géographiques : « 1 - trouver son adversaire; 2 - valoriser l'animal ; 3 - affronter le fauve ; 4 - achever le combat ; 5 - passer à la postérité $»^{22}$. Ce choix muséographique est justifié par la volonté de susciter « l'étonnement» et de « donner des directions de recherches $»^{23}$. Reconnaissons à cette exposition une importante valeur iconographique et une réelle efficacité dans la présentation de la diversité des interactions entre l'homme et le taureau, généralement méconnue du grand public. L'exposition prend toutes les précautions oratoires pour mettre en garde le public contre " les interprétations abusives » d'une " mise en rapport des représentations antiques avec des images contemporaines $»^{24}$. Mais cet avertissement est sans doute trop visible pour ne pas comprendre qu'il s'agit d'une trop forte tentation à laquelle le public ne peut difficilement échapper par la mise en scène proposée. En effet, l'exposition fait volontiers de la tauromachie une geste universelle de l'homme coupée de tout contexte culturel précis. En témoigne, le glissement opéré à propos de la «Fresque du Toréador du Palais de Cnossos » dont le commentaire précise qu' « il est tentant de voir dans les acrobates du palais de Cnossos les illustres ancêtres des sauteurs landais et des recortadores espagnols $»^{25}$. Il y aurait quelque fausse naïveté de notre part à prendre cette invitation au pied de la lettre. Il s'agit bien sûr d'un rapprochement de la gestuelle. Cependant, cette mise en relation est assez dommageable dans la mesure où la thèse des origines égéennes de la tauromachie moderne

\footnotetext{
${ }^{21}$ F. SAumade, Des sauvages en Occident, p. 12.

${ }^{22}$ Musée des cultures taurines, Rites éternels, p. 9.

${ }^{23}$ Ibid., pp. 8-9.

${ }^{24}$ Ibid., p. 10.

${ }^{25}$ Ibid., p. 36.
} 
est un des lieux communs erronés de l'historiographie taurine qui continue de circuler en dépit d'études sérieuses qui l'invalident depuis longtemps. Álvarez de Miranda, qui meurt en 1957, cite en exemple l'œuvre de l'historien de la tauromachie Ortiz Cañavate qui, à partir des travaux d'Arthur Evans en Crète sur le palais de Cnossos, n'a pas eu de scrupule à supposer que les populations de l'archipel égéen pouvaient avoir apporté à la péninsule Ibérique le spectacle du combat contre des taureaux sauvages ${ }^{26}$. Il est par conséquent plus opportun de résister à cette tentation, même si la question des origines des jeux taurins reste en partie obscure et donne lieu à des interprétations contradictoires. En cela, on peut reprocher à cette démarche de reconstruire une «Civilisation du Taureau » universelle qui passe entièrement sous silence la question des ruptures et des continuités géo-historiques existant entre les différentes formes de rituels qui n'ont, en tous les cas, rien " d'éternels ${ }^{27}$. Elle n'opère, en outre, aucune véritable distinction conceptuelle qui permettrait de classer les pratiques par catégorie : pratiques d'élevages, pratiques cynégétiques, jeux, dévotions cultuelles, sacrifices... quand bien même s'agirait-il d'en interroger les limites. Il en résulte une appréhension floue de la tauromachie et de sa diversité.

\section{LES VERTIGES DE L'HISTOIRE TAURINE}

Cette approche révèle un aspect récurrent des représentations de la diversité tauromachique. La tauromachie est souvent pensée dans un cadre historique et symbolique vertigineux, mais dans un cadre géographique et matériel incomplet. Chez de nombreux aficionados, il existe une vulgate qui fait de la tauromachie (entendons, dans l'immense majorité des cas, de la corrida) la dépositaire de tout un ensemble de rituels taurins issus de contextes historiques, géographiques et culturels très hétérogènes. Elle recompose des informations tirées de supports variés (légendes, essais d'érudition, études scientifiques, fictions littéraires, œuvres d'art, déductions toutes personnelles) au service d'une reconstruction historique linéaire de la tauromachie et d'une vision essentialiste du fait taurin. Au plus fort de son expression, sont convoqués tout à la fois : les chasses préhistoriques, le bestiaire taurin de l'iconographie paléolithique et néolithique, un culte méditerranéen du taureau qui aurait migré sous diverses formes du croissant fertile vers la péninsule Ibérique. Dans cette conception de l'histoire taurine, certaines pratiques sont considérées comme des étapes fondamentales de la continuité des rituels taurins, tels que les jeux crétois dont témoignent les fresques du palais de Cnossos, les taurokhatapsies des plaines de Thessalie, la religion mithriaque et les venationes de la Rome Antique ${ }^{28}$.

Aussi ne doit-on pas être surpris de voir, dans de nombreux ouvrages sur la tauromachie, le Cid Campeador promu au rang de matador pionnier de l'his-

\footnotetext{
${ }^{26}$ Á. Álvarez de Miranda, Le taureau. Rites et jeux, pp. 34-35.

${ }^{27}$ Musée des Cultures taurines, Rites éternels, p. 6.

${ }^{28}$ Taurokhatapsie : terme francisé, du grec taurokhatapsia désignant des jeux taurins à cheval pratiqués en Thessalie vers le $\mathrm{v}^{\mathrm{e}}$ siècle av. J.-C.
} 
toire, lorsqu'on rapporte qu'il fut le premier à tuer un taureau d'un coup de lance du haut de son cheval, quand ce n'est pas Jules César en personne qui le précède. Dans le cas du Cid, Bartolomé Bennassar souligne que l'épisode, qui daterait de 1038 ou 1040, est mentionné pour la première fois dans les Quintillas de Fernández de Moratín et précise que selon l'historien Ramón Menéndez Pidal, il n'existe aucun document qui permette de l'accréditer ${ }^{29}$. Nombre d'ouvrages sur la tauromachie font référence à cet épisode, suivant en cela les propos de Francisco Montes "Paquiro », dans "son discours historico-apologétique des fêtes taurines ", qui atteste qu'il s'agit déjà d'une idée bien établie dans le premier tiers du XIX ${ }^{\mathrm{e}}$ siècle : " Tous conviennent que le célèbre chevalier Ruy, Rodrigo Díaz del Vivar, dit le Cid Campeador, fut le premier à avoir combattu les taureaux à coup de lance du haut de son cheval ${ }^{30}$. Pour ce qui est de Jules César, l'épisode est évoqué dans l'article "Tauromaquia » de la Enciclopedia Universal Ilustrada publiée dans le premier tiers du $\mathrm{xx}^{\mathrm{e}}$ siècle ${ }^{31}$.

Pour prendre un exemple plus récent, examinons une version érudite de l'histoire de la tauromachie, telle qu'elle est présentée dans l'œuvre de José María de Cossío déjà mentionnée ${ }^{32}$. Dans l'organisation même du discours, le premier chapitre de Los Toros, intitulé "La fête depuis ses origines à nos jours ", fournit une éloquente démonstration de reconstruction évolutionniste de l'histoire taurine. L'auteur ne peut être plus explicite dans sa démarche :

En poursuivant la comparaison fluviale, on finit par être convaincu que nos corridas de toros ont reçu comme affluents stimulants, des exemples ou des apports d'un ensemble complexe de traditions religieuses, de coutumes rurales et de prémices sportives jusqu'à se fixer dans la fête [taurine] qu'aujourd'hui nous connaissons ${ }^{33}$.

De façon très significative, le fleuve prend sa source dans le célèbre commentaire de Strabon comparant la forme de l'Espagne à une "peau de taureau ", pour finir sa course par les photographies de quatre matadors encore en activité au milieu des années 90 : Joselito, Ponce, Espartaco et Jesulín de Ubrique. Il ne s'agit pas ici d'expliquer la genèse et les évolutions des pratiques tauromachiques sur lesquelles nous reviendrons et pour lesquelles l'œuvre de Cossío demeure une source d'informations du plus grand intérêt. Contentons-nous d'observer l'existence de trois espaces de mise en relation historique des rituels taurins qui retracent le droit fil de l'évolution des pratiques pour de nombreux aficionados : la péninsule Ibérique, le bassin méditerranéen et un espace monde d'avant l'histoire. L'espace le mieux représenté est la péninsule Ibérique où se multiplient les

\footnotetext{
${ }^{29}$ B. BenNASSAR, Histoire de la tauromachie, p. 13.

${ }^{30}$ F. Montes, Tauromaquia completa, p. 19.

${ }^{31}$ Enciclopedia Universal Ilustrada, t. LIX, p. 912.

${ }^{32}$ Pour les commentaires qui suivent nous utilisons une réédition abrégée publiée en 1995 : J.-M. de Cossío, Los Toros.

${ }^{33}$ J.-M. de Cossío, Los Toros, t. I, p. 53.
} 
témoignages d'une ancestralité de la posture tauromachique qui s'appuie sur l'art pariétal (la Cueva Remigia de Castellón, les peintures rupestres de Cogull à Lerida), sur l'archéologie (taureaux de Guisando, taureaux de Osuna, Taureaux de Costig, le taureau de la stèle de Clunie) et sur la mythologie (les taureaux de Géryon, les travaux d'Hercule) $)^{34}$. Le deuxième espace de mise en relation des rituels taurins, plus vaste, est le bassin méditerranéen dans son ensemble (La Crète, les fresques du palais de Cnossos; le culte de Mithra ; les venationes des cirques romains $)^{35}$. Le troisième espace de référence, aux contours plus flous, s'inscrit dans un temps préhistorique où l'accent est mis sur l'homme chasseur de taureaux ${ }^{36}$. De la mise en scène d'une histoire fleuve de la tauromachie des origines à nos jours, prend forme l'idée que toutes les pratiques évoquées relèvent peu ou prou de la tauromachie. La multiplication des rituels taurins de toute nature et leur dispersion spatio-temporelle trouvent ainsi une cohérence au sein d'un espace-temps tauromachique réinventé. Cet espace est centré sur la péninsule Ibérique qui devient par la survivance de la corrida espagnole, le réceptacle de la culture classique du bassin méditerranéen et de la culture universelle de l'homme d'avant l'histoire. Les excès d'un tel positionnement, qui ne sont d'ailleurs que les conséquences d'une simplification outrancière de l'œuvre de Cossío utilisée à satiété par ses successeurs, ont été critiqués par les historiens et les anthropologues de métier. Pour autant, la question complexe des origines de la tauromachie reste un sujet de controverse que l'on ne saurait balayer sous prétexte que les lieux communs usent et abusent de ces amalgames. Tout ne doit pas être jeté avec l'eau du fleuve.

Quoi qu'il en soit, de telles références continuent de peupler l'imaginaire taurin de l'afición de part et d'autre de l'Atlantique. Elles sont autant d'arguments sur lesquels s'appuient la plupart des discours de légitimation de la tauromachie. Le paragraphe suivant que nous reproduisons dans son intégralité vaut toutes les démonstrations :

La question se pose alors : peut-on faute de preuve irréfutable, relier jeux et cultes de l'Antiquité à la corrida d'aujourd'hui, assimiler Joselito à Jason, Aparicio à Thésée, Curro Romero à Mithra, faire d'Ojeda, cet autre fils de Tartesse, un nouvel Hercule? Osons répondre oui ! Mais objectera-t-on, si tant est qu'une logique dans leur évolution ait présidé à leur métamorphose, par quel chaînon manquant rattacher l'une aux autres? L'homme, bien sûr! Et, au cœur de son essence, plus fort que les

\footnotetext{
${ }^{34}$ J.-M. de Cossío, Los Toros, t. I, pp. 21-22, 25, 33, 35, 38-40. On notera que l'auteur fait référence à la célèbre légende des taureaux de Géryon, dispersés sur les rives du Guadalquivir et cite le poète et éleveur Fernando Villalón pour noter qu'ils paissaient « exactement à l'endroit où continuent de ruminer tranquillement aujourd'hui les plus célèbres élevages de taureaux de combat andalous» (p. 25).

${ }^{35}$ Ibid., pp. 27-32, 42-48, 51-54. «La présence du taureau dans les cirques romains est l'impulsion d'une tradition à laquelle ne peuvent pas être étrangères les corridas espagnoles» (p. 53).

${ }^{36}$ Ibid., pp. 21 et 54-56.
} 
clivages de races, de lieux ou de cultures, son perpétuel besoin d'idéal, sa recherche pathétique du sacré, la force de ses passions et la sublimation de son instinct de puissance, innocente fierté primitive, lorsque l'on est fort, de se savoir admiré. Car, même si vouloir faire de la corrida moderne ou des tauromachies qui en sont proches (écart ou saut landais, rejoneo ou pega portugaise, recorte navarrais ou razet languedocien, saut de cheval à taureau ou attente au fer camarguais, acoso et derribo andalou...) l'héritière directe de ces pratiques antiques serait audacieux dans la mesure où rien ne permet d'affirmer qu'elles furent aussi pratiquées dans la péninsule Ibérique ou le midi français, celles-ci témoignent en revanche de la permanence, durant quarante siècles, d'une symbolique faisant du Taureau, image d'un éternel masculin sacralisé, le véritable étalon-or de bravoure originelle que l'homme, pour prouver sa valeur et affirmer sa propre identité, défie à l'infini ${ }^{37}$.

En l'état actuel des recherches, osons répondre non, même si, répétons-le, il serait déraisonnable d'affirmer de façon tout aussi catégorique qu'il n'existe aucun lien de parenté entre ces pratiques ${ }^{38}$. Il n'en demeure pas moins que ce type de raisonnement, dans sa méthode, relève bien d'une reconstruction qui remplace la "mémoire » par "l'histoire » au sens où Pierre Nora les oppose, considérant la première comme "inconsciente de ses déformations successives, vulnérables à toutes les utilisations et toutes les manipulations, susceptibles de longues latences et de soudaines revitalisations $»^{39}$.

Cette forme de reconstruction n'est pas réservée aux temps anciens et mythiques. Il est une façon de lier les faits entre eux qui relève incontestablement de l'histoire apologétique, ainsi que ne s'en cachait pas « Montes » dans son discours introductif à sa Tauromaquia. Ce type de discours contribue à déconnecter l'évolution de la tauromachie de toute histoire sociale pour faire d'elle un discours autonome. En effet, les héritages des anciens temps, une fois recentrés sur la péninsule Ibérique, ne resurgissent dans l'histoire qu'au moment de la Reconquête, lors des tournois et des fêtes royales qui célèbrent les grands événements de la couronne et de l'Église. Comme preuve évidente de la haute importance de la tauromachie, l'histoire officielle n'oublie jamais de mentionner les princes toreros, tels que Le Cid, Charles Quint ou encore Philippe IV. Une fois que la noblesse détient les rênes de l'art taurin, le récit s'oriente vers une description

\footnotetext{
${ }^{37}$ A. Viard, Le Mythe du Taureau, pp. 50-51. Joselito, Aparicio, Curro Romero et Paco Ojeda sont des matadors espagnols de très grand renom.

${ }^{38}$ Comme le dit Julio Caro Baroja : «Si dans une série de rites et de mythes, il existe un élément transmis, ce n'est pas lui qui permet de comprendre les autres, et nous ne devons pas en profiter pour mettre le Carnaval et le Carême dans la même marmite qu'Attis, Adonis, Isis et toute une série de dieux et d'êtres antiques ou médiévaux, pour extraire, finalement, le pur esprit de la végétation, un bouillon végétal sans mélange aucun, véritable, authentique et surtout primitif ». J. CAro Baroja, Le Carnaval, p. 21.

${ }^{39}$ P. Nora, Les lieux de mémoire, pp. 15-42.
} 
de plus en plus précise des techniques de combat à cheval et à pied. Comme le rappelle Bartolomé Bennassar, la thèse classique a ensuite longtemps soutenu que l'arrivée des Bourbons sur le trône d'Espagne fut responsable du recul de la tauromachie à cheval, pratiquée par la noblesse, laissant aux auxiliaires à pied, issus du peuple, le soin d'élaborer les fondements de la tauromachie moderne ${ }^{40}$. José María de Cossío déclare : «Le XviII ${ }^{\mathrm{e}}$ siècle assiste à la fin du toreo aristocratique à cheval devant l'invasion populaire, qui devait préparer le terrain dans tous les registres à la suprématie du peuple, qui a souvent été plutôt celle de la plèbe $»^{41}$. L'irruption du peuple dans l'histoire de la tauromachie laisse ensuite la place à la chronique des grands hommes qui lui ont donné sens. À la fin du $\mathrm{XVIII}^{\mathrm{e}}$ siècle, apparaissent les premiers codificateurs : Pedro Romero, Joaquín Rodríguez «Costillares » et José Delgado " Pepe-Illo ». Au xix e siècle, ce sont les toreros emblématiques de la consécration sociale du spectacle et de sa formalisation classique qui surgissent : Francisco Montes « Paquiro » le grand législateur, Francisco Arjona Herrera "Curro Cúchares », José Redondo y Domínguez «El Chiclanero ", Cayetano Sanz, considéré comme le premier torero de Madrid et Antonio Sánchez «El Tato ». À la fin du XIx $x^{e}$ siècle, s'affirment les toreros de l'âge d'or, Rafael Molina "Lagartijo ", Salvador Sánchez " Frascuelo ", Rafael Guerra Bejarano «Guerrita ». Au début du xx siècle, s'imposent les régénérateurs et les révolutionnaires de l'art taurin : Juan Belmonte dont la confrontation avec Joselito, dans les années 1910, est considérée comme un moment charnière de l'évolution du spectacle. Ensuite, la décadence annoncée de l'art taurin laisse néanmoins surgir ce que la langue taurine nomme des " monstres ", des "phénomènes » et autres innovateurs tels que Manolete dans les années 1940, Dominguín et Ordóñez dont la rivalité culmine en 1959, «El Cordobés » dans les années 1960, Paco Ojeda dans les années 1980 et José Tomás à partir du milieu des années 90. Ainsi s'enchaînent mythe fondateur, genèse, âge classique, révolution, âge d'or, décadence et renouveau : le récit de l'histoire taurine témoigne assurément d'une certaine façon d'écrire l'histoire.

Dans une approche constructiviste de la tauromachie où les représentations mentales participent de l'organisation objective du fait taurin, l'identification de ces espaces-temps emboîtés et d'une trame narrative qui enracine la tauromachie sur le temps long est pour le géographe d'un grand intérêt. Retenons que cette reconstruction historique, esquissée à grands pas, conçoit la tauromachie comme une sorte de pyramide dont le sommet serait la corrida, ici et maintenant, et dont la base serait les diverses formes de rituels taurins du passé, la notion de rite permettant souvent de faire l'économie d'une qualification précise des pratiques tauromachiques. Remarquons également que ce type de discours, exacerbé dans le cas de la corrida, existe pour d'autres jeux taurins, comme s'il s'agissait d'une trame narrative idéale des traditions taurines cherchant à faire valoir leur ancestralité. Ainsi, dans un ouvrage qui vient de fêter les cinquante

\footnotetext{
${ }^{40}$ On trouve un exemple de cette « thèse classique » dans l'œuvre de N. LuJÁN, Historia del toreo, pp. 9-28.

${ }^{41}$ J.-M. de Cossío, Los Toros, t. I, p. 84.
} 
ans de la Fédération française des courses landaises peut-on lire en ouverture : "Le premier drame taurin a lieu à Lascaux. [...] Sur la paroi des grottes, un homme meurt éventré par un bison. Un autre appelle la charge de l'aurochs, levant prophétiquement les bras au ciel, comme un écarteur de nos jours. Voilà la tauromachie paléolithique $»^{42}$. L'histoire tauromachique du Nouveau Monde suit généralement les mêmes sentiers. Tout commence en 1492 avec l'arrivée de Christophe Colomb et le débarquement des premiers taureaux et des premiers chevaux sur l'île d'Hispaniola. À peine sorti des caravelles changées en nouvelles arches de Noé, le bétail prend part aux premières corridas au cours desquelles combattent en personne les figures tutélaires de la Conquête. Aucune histoire de la tauromachie en Amérique latine n'oublie de mentionner Pizarro et Cortés tuant de leur lance de farouches taureaux, à l'image de Jules César et du Cid. Ce geste fondateur soude l'histoire taurine du Nouveau Monde à celle de la culture classique méditerranéenne et de la culture espagnole. Un reportage produit par la Télévision nationale du Pérou sur les traditions taurines dans ce pays s'ouvre, sans surprise, sur les " cavernes méditerranéennes, 17.000 ans avant J.-C. » et se poursuit sur l'idée que «le taureau produit chez l'homme péruvien la même fascination qu'en Crète ${ }^{43}$. On perçoit aisément la portée de telles reconstructions en termes d'enracinement territorial des pratiques et d'héritage culturel. Remarquons simplement pour l'instant la propension à étirer dans le temps ce que recouvre la tauromachie, à laquelle semble répondre une incapacité à rassembler les jeux taurins dans leur géographie présente. Avançons l'idée prématurée que cette attitude psychologique n'est pas étrangère à la façon dont fonctionne l'appropriation territoriale, prompte à invoquer l'histoire pour découper l'espace.

\section{III. — LES JEUX TAURINS}

\section{LA RECHERCHE D'UNE DÉFINITION MINIMALE}

Un homme affronte un taureau : tel pourrait être le plus petit dénominateur commun des pratiques tauromachiques si l'on accepte de fonder l'identification du fait taurin sur des critères débarrassés au mieux de ses représentations subjectives. Le mot taureau possède ici une valeur générique, puisque prennent part aux pratiques tauromachiques des bovins de toutes natures, des taureaux, des bœufs ou des vaches, de tous âges et de toutes races. Ce sont d'abord les cornes, la charge, la force, la course ou les ruades du taureau qui assurent la fonctionnalité du jeu taurin. Le taureau ne doit donc pas être envisagé dans sa dimension sexuée, mais à travers le rôle ambivalent de partenaire-adversaire que les jeux lui confèrent. Les pratiques tauromachiques relèvent alors de principes fonction-

\footnotetext{
${ }^{42}$ Fédération fRAnÇAise de COURSE landaise, Course landaise, p. 2.

${ }^{43}$ C. Sillero, Los toros en el Perú.
} 
nels communs : montrer son adresse, son courage et son style en se mesurant à l'animal. Sur ce point Bernard Traimond formule une hypothèse qu'il tient pour fondamentale dans son analyse des pratiques tauromachiques du vieux continent : "Aux taureaux, vaches et bœufs, les jeux taurins substituent un seul type de bovins [...] même si l'intégralité des uns et le sexe des autres tendent à les enfermer dans un statut symbolique précis ${ }^{44}$. Rappelons enfin que l'animal combattu, même lorsqu'il donne à voir une sauvagerie idéalisée, est toujours issu d'un élevage domestique.

Dans une conception extensive de la tauromachie, les combats opposant des taureaux entre eux pourraient faire partie des pratiques tauromachiques dès lors que ces affrontements sont mis en scène par l'homme et que les processus d'identification homme-animal sont en jeu. Mais ces pratiques constituent incontestablement une catégorie à part puisque l'homme ne combat pas en personne l'animal. Nous avons recensé de telles pratiques en des lieux qui ne se limitent en rien à l'aire culturelle latino-américaine et qui confirment qu'il s'agit d'une pratique quasiment universelle autant que peuvent l'être les sociétés où les bovins occupent une place importante.

- Chega de bois au Portugal dans les montagnes du Tras os Montes ;

- pelea de toros au Pérou dans la région d'Arequipa. Le terme de peleya de toros est également employé ;

- mayo au Kenya : une fois par mois à Khayega dans cette province de l'ouest du Kenya, à 500 kilomètres de Nairobi, les communautés Idakho et Isukha de l'ethnie Luhya engagent leur taureau champion dans la tradition du mayo (combat en langue locale) ${ }^{45}$;

— combats des reines d'Hérens dans le Valais Suisse ;

- combats de taureaux au Japon en plusieurs lieux périphériques, à Okinawa, à Uwajima (Shikoku), au nord de Niigata et Iwate, à Tokunoshima (sur l'île Amami) et sur l'île d'Oki ;

- combats de taureaux en Corée, spécialement à Chongdo dans la province de Kyongsang, au sud du pays, avec parfois des rencontres opposant taureaux japonais et taureaux coréens ;

- combats de taureaux en Chine chez les peuples Dong, à Liping, Rongjiang, Congjiang et Jinping ;

— combats de buffles à Haiphong et dans le Nord-Est du Viet-Nam ;

— combats (dhirio) de taureaux brahmas à Goa en Inde. Ces combats sont plus ou moins clandestins;

${ }^{44}$ B. Traimond, Les fêtes du Taureau, p. 18.

${ }^{45}$ Dépêche de L'Agence France Presse du 24 mai 2005. 
- combats de taureaux en Turquie, en Iran et dans le sultanat d'Oman

(dans la région de Dhafar et d'Al-Musanaa).

Il y a là matière à une investigation plus approfondie qui pourrait déterminer, parmi ces combats de taureaux, la part revenant à des foyers endogènes, si tant est que cette notion ait un sens dans un contexte élargi d'échanges et d'emprunts, et celle revenant aux processus de diffusion spatiale des jeux taurins de la péninsule Ibérique. En particulier, il semblerait que l'origine portugaise des combats entre taureaux soit établie pour Goa, elle est revendiquée par certains Omani et elle m'a été soutenue comme une évidence à propos du Japon et de la Corée, par un éleveur de taureaux portugais... qui ne connaît pas le doute. Quoi qu'il en soit, il est possible de rapprocher ces jeux d'autres types de combats organisés entre animaux de même espèce : combats de coqs, combats de chiens, combats de serpents, etc.

En revanche, les courses d'attelages et autres épreuves de force, mettant en scène des bovins sans qu'il y ait de combat ni de contact entre les protagonistes, nous semblent être à la limite de la définition, tant ces jeux paraissent éloignés de la logique agonistique qui préside au fonctionnement de la tauromachie. Ils témoignent, dans tous les cas, d'une médiation différente dans l'expression des rapports de force liés à l'assujettissement de la nature à travers la mise en scène de bovins. De même, les sacrifices de bovins qui ne sont précédés d'aucune mise en scène d'affrontement réel entre l'homme et l'animal possèdent une logique différente de celle qui commande le jeu tauromachique. Cette logique ne lui est cependant pas étrangère dans certains exemples qui attestent d'un lien étroit entre jeux taurins et pratiques sacrificielles. Aux vues de cette analyse, la tauromachie peut être définie comme l'ensemble des jeux qui mettent en scène un affrontement réel entre l'homme et le taureau.

\section{LES DIVERGENCES D'INTERPRÉTATION DE LA TAUROMACHIE}

Il existe de nombreuses interprétations de la tauromachie, de ses origines, de sa fonction sociale, de sa nature profonde. Parmi elles, les relations entre tauromachie et pratiques sacrificielles ont fait couler beaucoup d'encre. Cette question fait suffisamment débat en sciences humaines parmi les chercheurs qui s'intéressent à la tauromachie, pour qu'il paraisse nécessaire, dès à présent, d'en préciser les termes. Le débat oppose les tenants d'une interprétation sacrificielle de la tauromachie, aux tenants d'une interprétation profane des pratiques tauromachiques. La première interprétation, soutenue par des intellectuels et des chercheurs de renommée internationale tels que Michel Leiris et Julian Pitt-Rivers, a longtemps fait figure d'école dominante pour l'analyse des rituels taurins ${ }^{46}$. Ce courant fonde principalement son argumentation sur

\footnotetext{
${ }^{46}$ M. Leiris, Miroir de la tauromachie; J. Pitt-Rivers, « Le sacrifice du taureau ». Pour une vue d'ensemble des travaux de Julian Pitt-Rivers sur la tauromachie voir P. Romero de Solís (éd.), Antropología de la tauromaquia.
} 
une lecture de la célèbre théorie du sacrifice développée par Henri Hubert et Marcel Mauss ${ }^{47}$. Pedro Romero de Solís figure de nos jours parmi les auteurs qui fournissent les analyses les plus approfondies en ce sens ${ }^{48}$. Dans le détail, au sein de cette famille d'interprétation, il existe plusieurs positions attentives à la dimension sacrificielle des rituels taurins dont les conceptions peuvent varier, entre ceux qui considèrent la tauromachie comme un véritable culte du taureau et ceux qui interprètent cette activité comme la transformation de rites propitiatoires d'origine sacrée. Les travaux de Frédéric Saumade sur ce thème prennent le contre-pied de cette lecture par une démarche comparative désengagée des problématiques de l'anthropologie religieuse. Dans le cadre de sa thèse doctorale, il relativise l'invariant anthropologique que constituerait le sacrifice taurin à partir d'une comparaison des cultures tauromachiques andalouses et camarguaises $^{49}$. Il poursuit cette réflexion par une ouverture sur Les tauromachies européennes et par un discours plus radical à l'endroit de la théorie sacrificielle ${ }^{50}$. Selon l'auteur, cette théorie "n'est autre chose que la projection intellectuelle d'un archétype ethnologique sur une réalité rendue ainsi plus suggestive à défaut d'avoir été éclairée de façon convaincante » et de conclure en affirmant que « la tauromachie apparaît en fait comme la résultante ritualisée de deux moments constitutifs de l'exploitation des bovins : la conduite et l'abattage $»^{51}$. Son approche récente des pratiques tauromachiques au Mexique prolonge outre-Atlantique son argumentation qu'il met ici au service d'une relecture des processus d'acculturation et de métissage ${ }^{52}$.

Pour autant, ces interprétations divergentes sont-elles aussi irréconciliables que la controverse scientifique se plait à l'exprimer ? Deux précisions permettent de relativiser leur caractère apparemment antinomique. D'une part, la définition préliminaire de la tauromachie au sein de ces interprétations n'est pas strictement identique. Frédéric Saumade accorde au mot tauromachie un sens volontairement restreint aux spectacles modernes d'arènes, codifiés et institués, pour en montrer toute la spécificité alors que la plupart des auteurs englobent un panel de pratiques plus large où les affrontements de l'homme et du taureau sortent du cadre de la seule représentation spectaculaire. Il en résulte, assez logiquement, des différences d'interprétations. D'autre part, pour certaines pratiques, à commencer par la corrida, la validité de la dimension sacrificielle de la tauromachie

\footnotetext{
${ }^{47}$ H. Hubert et M. Mauss, «Essai sur la nature et la fonction du sacrifice».

${ }^{48}$ P. Romero DE Solís (coord.), Sacrificio y tauromaquia en España y América. Mentionnons également un article qui répond aux positions de ce que l'auteur qualifie « d'école anti-sacrificielle française » dans ID., "La dimensión sacrificial de la tauromaquia ».

${ }^{49}$ F. Saumade, Des sauvages en Occident, pp. 11-12.

${ }^{50}$ ID., Les tauromachies européennes.

${ }^{51}$ Ibid., pp. 10 et 14 . Voir également sur ce point F. Saumade et E. Desvaux, « Relativiser le sacrifice ou le quadrant tauromachique ». Dans cet article les auteurs posent les fondements d'une analyse structurale des rituels taurins qui met en valeur les correspondances et les oppositions des codes sexuels, ludiques, eschatologiques et sociologiques.

${ }^{52}$ F. Saumade, Maçatl.
} 
(ou son déni) ne tient souvent qu’à la définition préalable accordée à la notion même de sacrifice. Plus précisément, les différents auteurs engagés dans le débat n'ont pas tous la même lecture du système sacrificiel élaboré par Marcel Mauss. Pour Frédéric Saumade, l'interprétation de la tauromachie comme réminiscence d'un culte du taureau, qui serait justifiée sur la base du système sacrificiel élaboré par Mauss, achoppe sur l'impossibilité pour le taureau d'être à la fois la victime sacrificielle et la divinité destinataire du sacrifice. L'auteur ajoute en outre « qu'il n'y a nulle trace, ni implicite, ni explicite, d'un être transcendant à qui serait dédié le bétail immolé $»^{53}$. Pour les tenants de l'interprétation sacrificielle, le modèle demeure éclairant pour comprendre les rites taurins, au-delà des phénomènes de substitution, d'abstraction ou d'indéfinition du destinataire. Dominique Fournier se demande si dans le monde contemporain, l'existence d'un destinataire identifié est vraiment indispensable pour que se constitue un rite sacrificiel ${ }^{54}$. Antoinette Molinié envisage que le destinataire puisse être une entité abstraite (le pouvoir génésique) et souligne qu'il n'y a pas d'incompatibilité à confondre victime et destinataire comme le montre par exemple la dimension sacrificielle de la messe dans le christianisme ${ }^{55}$. Enfin, Pedro Romero de Solís considère que les logiques de substitution animal-homme-divinité sont essentielles à la compréhension de la dimension sacrificielle des rituels taurins dans le monde méditerranéen, comme en témoigne l'épisode du sacrifice d'Isaac et la figure de l'agneau pascal ${ }^{56}$. L'auteur ajoute que le système sacrificiel serait incomplet sans l'ingestion collective des animaux sacrifiés faisant ici référence à l'institution du banquet sacrificiel analysé par Sigmund Freud dans son ouvrage Totem et Tabou. Il dégage ainsi une structure originelle ternaire des fêtes du taureau en Méditerranée, composée de la conduite de l'animal vers le sacrifice, du sacrifice lui-même et de la cérémonie du banquet. Le caractère protéiforme des pratiques tauromachiques et la difficulté à les identifier comme appartenant à une même activité s'expliquerait alors par le fait que les éléments de cette structure ternaire sont aujourd'hui, ou bien masqués, ou bien disjoints, ou bien partiellement désactivés. Quoi qu'il en soit, et telles sont les vertus du débat scientifique, il n'est plus possible de se cantonner à un seul point de vue. L'affirmation que faisait encore Julian Pitt-Rivers en 1983, dans un article intitulé "Le sacrifice du taureau », par ailleurs d'un apport décisif, semble aujourd'hui difficile à soutenir : «Qu'il s'agisse [la tauromachie] d'un sacrifice paraît à l'anthropologue trop évident pour qu'il se sente obligé de le justifier $»^{57}$.

\footnotetext{
${ }^{53}$ F. SAUmade, Les tauromachies européennes, p. 10.

${ }^{54}$ D. Fournier, « Les spectacles tauromachiques naissent aussi de l'histoire ». Ce texte est une note critique de l'ouvrage de Frédéric Saumade sur les tauromachies européennes à laquelle l'auteur a répondu : F. SAUMADE, «L'obsession sacrificielle».

${ }^{55}$ A. Molinié, «El toro y el cóndor en lidia ».

${ }^{56}$ P. Romero de Solís, « La dimensión sacrificial de la tauromaquia » ; ID., « Corpus Christi y tauromaquia ».

${ }^{57}$ J. PitT-Rivers, « Le sacrifice du taureau », p. 283. En réalité, l'auteur n’a cessé de creuser cette question, de justifier son approche et de proposer des démonstrations éclairant sa conviction.
} 
Notre observation de la diversité des pratiques tauromachiques nous invite, dans cette perspective, à nous méfier des systèmes explicatifs totalisants, d'un parti comme de l'autre, qui visent à l'élaboration d'une théorie générale valable pour l'ensemble des rituels taurins. Nous pensons qu'il existe des pratiques taurines où la dimension sacrificielle semble tout à fait défendable, que l'on $\mathrm{y}$ adhère ou non, en particulier pour celles où le sang coule et pour celles où l'animal est mis à mort. Les divergences sur ce point reposent, à nos yeux, sur des questions de définition préliminaire du rite sacrificiel dont la critique plus fouillée dépasse notre objet. Mais il existe, aussi sûrement, des pratiques pour lesquelles une lecture sacrificielle du jeu taurin s'avère totalement inopérante, en particulier pour celles où l'animal ne subit aucune transformation et où il n'est ni blessé, ni tué. Quoi qu'il en soit, il nous semble toujours possible d'affirmer une autonomie de la sphère ludique suffisante pour inscrire pleinement les pratiques tauromachiques dans le registre des divertissements. Sans vouloir réduire la complexité des faits, la tauromachie est ainsi appréhendée d'abord à travers les éléments matériels de ses manifestations contemporaines. Dans cette perspective, la notion de jeu taurin pourrait même remplacer avantageusement celle de tauromachie, tant cette dernière semble véhiculer de représentations idéologiques contradictoires. Le jeu est pris dans la pluralité de ses significations si fermement analysées par Roger Caillois ${ }^{58}$. Pour appréhender les pratiques tauromachiques, utiliser la notion de jeu, telle qu'elle est développée chez Caillois, possède l'intérêt de ne les enfermer ni dans une totalité hermétiquement profane, ni de présupposer chez elles une quelconque sacralité. Les caractéristiques attribuées au jeu par l'auteur trouvent une application pertinente pour notre propos visant à ne pas trancher de façon globale sur le caractère profane ou sacré de la ritualité tauromachique :

Le jeu est une activité : libre (à laquelle le joueur ne saurait être obligé sans que le jeu perde aussitôt sa nature de divertissement attirant et joyeux), séparée (circonscrite dans les limites d'espace et de temps précises et fixées à l'avance), incertaine (dont le déroulement ne saurait être déterminé ni dans le résultat acquis préalablement, une certaine latitude dans la nécessité d'inventer étant obligatoirement laissée à l'initiative du joueur), réglée (soumise à des conventions qui suspendent les lois ordinaires et qui instaurent momentanément une législation nouvelle, qui seule compte), fictive (accompagnée d'une conscience spécifique de réalité seconde ou de franche irréalité par rapport à la vie courante $)^{59}$.

C'est une des différences entre l'approche de Caillois et celle de Huizinga. La relecture que propose Caillois de l'œuvre de Huizinga, de laquelle toutes les études sur le jeu sont malgré tout tributaires, attire l'attention sur une « fragilité

\footnotetext{
${ }^{58} \mathrm{R}$. CAIllois, Les jeux et les hommes.

${ }^{59}$ Ibid., pp. 42-43.
} 
de la thèse la plus audacieuse de l'ouvrage : l'identification du ludique et $\mathrm{du}$ sacré $»^{60}$. Selon Huizinga, jeu et sacré ont en commun :

Un espace clos et délimité, séparé du monde et de la vie. Dans cette enceinte, durant un temps donné, on exécute des gestes réglés, symboliques qui figurent ou actualisent des réalités mystérieuses au cours de cérémonies où, comme dans le jeu, concourent à la fois les vertus contraires de l'exubérance et de la réglementation, de l'extase et de la prudence, du délire enthousiaste et de la précision minutieuse. On est enfin transporté hors de l'existence ordinaire ${ }^{61}$.

Que cette description s'appliquerait à merveille à une tauromachie qui confine au sacré ! Pourtant nous préférons adopter le positionnement théorique de Roger Caillois qui admet des connexions possibles entre ludique et sacré, mais qui précise qu'il « ne croit pas que les formes des jeux et des cultes, parce qu'elles prennent un soin égal de se séparer du cours ordinaire de l'existence, occupent pour autant par rapport à celle-ci une situation équivalente, ni qu'elles recouvrent pour cette raison un contenu identique $»^{62}$.

\section{LA TAUROMACHIE ENTRE L'ART,}

LE SPORT ET LES JEUX TRADITIONNELS

Ce positionnement nous permet de préciser la notion de jeu tauromachique. La tauromachie est-elle un sport ? Est-elle un art ? Est-elle un jeu traditionnel ? Il faut renoncer à une réponse simple et univoque. Tout d'abord parce que les pratiques tauromachiques sont éminemment protéiformes, ce qui empêche, répétons-le, toute forme de réponse globale. Ensuite, parce que ces trois notions ont alimenté des découpages multiples.

La majorité des recherches sur le sport ne prêtent guère attention à la tauromachie, pas plus que les recherches sur la tauromachie ne relient leur analyse à une réflexion sur le sport. Pourtant les spécialistes des cultures sportives sont les premiers à reconnaître l'extraordinaire hétérogénéité de leur objet d'étude, mais la tauromachie leur paraît le plus souvent trop archaïque, folklorique, ou traditionnelle pour prétendre à la modernité et à l'universalité sportive. De même, les études sur la tauromachie n'ont pas davantage suivi la piste d'un rapprochement entre les deux pratiques. Et ceux qui le font préfèrent généralement les opposer en affirmant que la tauromachie n'a rien à voir avec le sport, puisque là où le premier est un combat réel, le second est un combat mimétique, et là où le premier est une expression artistique, le second n'est au mieux qu'engagement physique et habileté technique. Si l'on dépasse les arguments d'ordre

\footnotetext{
${ }^{60}$ R. Caillois, L’homme et le sacré, p. 207.

${ }^{61}$ Ibid., p. 208.

${ }^{62}$ Ibid., p. 211.
} 
apologétique, ceux de la modernité contre l'archaïsme et ceux de l'artiste contre l'athlète, de nombreux points communs existent entre les deux pratiques. La plupart des définitions du sport moderne s'accordent au moins sur quatre composantes minimales qui, une fois réunies, le distinguent fortement des jeux traditionnels : un ensemble de situations motrices, un système de règles, un enjeu lié à la compétition et un caractère institutionnel ${ }^{63}$.

L'art pose des problèmes de définition non moins complexes. Les oppositions classiques entre l'artiste et l'artisan, entre les beaux-arts et les arts techniques, entre poeisis et technê suffisent à le rappeler. L'art est à la fois un procédé, une technique et une production esthétique qui les transcende ${ }^{64}$. Quant à la notion de jeu traditionnel, Norbert Elias et Eric Dunning en font une catégorie opposée à celle du sport moderne. Ces auteurs soutiennent une lecture " discontinuiste " des relations entre le jeu traditionnel et le sport, dont la rupture s'inscrit dans une phase "d'accélération du processus de civilisation des mœurs " nommé en la matière, processus de sportization ${ }^{65}$. Les données qui constituent le sport moderne dans sa spécificité sont alors : " l'abaissement du degré de la violence permise dans la mise en jeu des corps, l'existence de règles écrites et uniformes codifiant les pratiques, l'autonomisation du jeu (et du spectacle du jeu) par rapport aux affrontements guerriers ou rituels $»^{66}$.

$\mathrm{Du}$ sport, les pratiques tauromachiques possèdent incontestablement les attributs d'une activité physique. Les tauromachies participatives, par leur caractère plus informel, moins codifié, par leurs limites plus imprécises dans le temps et dans l'espace, par une plus faible différenciation des rôles, par la plus faible distinction entre les joueurs et les spectateurs, semblent assez bien correspondre aux spécificités des jeux traditionnels. Par opposition, on serait tenté de classer les tauromachies professionnelles dans la catégorie des sports modernes, si la question des risques et de la violence ne venait pas immédiatement rendre les clivages plus complexes. En effet, il convient de souligner à cet endroit que les tauromachies relèvent d'un processus de "sportization » sinon inachevé, tout au moins atypique, en comparaison des sports modernes caractérisés par « l'abaissement du degré de la violence permise dans la mise en jeu des corps ». De plus, la présence en piste d'un animal adversaire contribue à exclure la tauromachie d'une conception du champ sportif fortement marqué par le principe d'équité. Selon ce principe, la dimension sportive des jeux taurins est contestable si on la cherche dans l'affrontement entre l'homme et la bête, puisque par définition les adversaires n'ont pas les mêmes armes ni le même rôle. L'issue normative de ce qui est en jeu n'est pas soit la victoire de l'un, soit la victoire de l'autre, mais

\footnotetext{
${ }^{63}$ J.-P. Augustin, Sport, géographie et aménagement, pp. 11-12.

${ }^{64}$ Rappelons sur le sujet le bon mot de Paul Valéry « On dit de la médecine qu’elle est un Art; on le dit aussi bien de la Vénerie, de l'Équitation, de la conduite de la vie ou d'un raisonnement. Il y a art de marcher, un art de respirer; il y a même un art de se taire ". Cité dans A. Rer, Dictionnaire culturel en langue française, p. 525.

${ }^{65}$ N. Elias et É. Dunning, Sport et civilisation, p. 29.

${ }^{66}$ R. Chartier, dans N. Elias et É. DunNing, Sport et civilisation, p. 13.
} 
la manière dont l'homme parvient à triompher de l'animal dans le respect des règles imposées. Sous cet angle, dans la dramaturgie qu'elle engage, la tauromachie se rapprocherait davantage des sports où l'homme affronte les forces de la nature.

Un autre point de vue est de considérer que le sport porte en lui des propriétés contradictoires, car aujourd'hui beaucoup de sports dits « extrêmes » valorisent précisément une exposition toujours plus forte et violente des corps. Dans cette perspective, de nombreux jeux taurins s'inscrivent dans une indéniable modernité sportive liée à la prise de risque et au désir de repousser les limites de ce que l'homme peut affronter. Comment analyser autrement le succès actuel des concours de recortadores en Espagne où les participants affrontent couramment des taureaux aux cornes ni protégées ni épointées, dont ils provoquent la charge, debout, immobiles face à l'animal ou de plus en plus souvent à genoux et de dos afin d'accroître le danger? Comment expliquer autrement le renouveau dans la course landaise des sauts pratiqués non plus sur des vaches emboulées et encordées, mais sur des taureaux sans corde, aux cornes élimées sous l'unique pression des compagnies d'assurances qui, dans le cas contraire, refuseraient tout contrat ? De même, comment comprendre l'autonomisation et la croissance actuelle du bull riding considéré dans le rodéo nord-américain comme l'épreuve la plus dangereuse ? Comment expliquer ces transformations autrement que par une adéquation de la tauromachie à certaines évolutions sportives aujourd'hui à l'œuvre? Cela montre, s'il était besoin de le prouver, que le sport forme lui-même un ensemble de pratiques très hétérogènes que la classification d'Elias et de Dunning ne suffit bien évidemment pas à circonscrire.

Du sport, certaines pratiques tauromachiques professionnelles possèdent aussi la dimension institutionnelle et organisationnelle. En France, c'est le cas de la course landaise et de la course camarguaise dont les activités sont organisées dans le cadre de fédérations sportives. Les instances fédérales ont été respectivement agréées en 1973 et 1975 par le ministère de la Jeunesse et des Sports, accordant à ces pratiques une mission éducative. En Espagne, les personnes impliquées dans l'organisation des concours de recortadores se disent très favorables à une institutionnalisation sur le modèle fédéral français. OutreAtlantique, de nombreuses pratiques de la famille du rodéo se sont également ralliées à une institutionnalisation sur le modèle sportif. Au Mexique, les charreadas sont réglementées par l'Association nationale des Charros depuis 1921 et sont reconnues «sport national » sous la tutelle de la Fédérération mexicaine de Charrería depuis 1933. Aux États-Unis, la première organisation professionnelle de rodéo est créée en 1936, sous le nom de Cowboys Turtle Association qui deviendra la puissante PRCA (Professional Rodeo Cowboys Association) dont dépend une grande partie du système compétitif à l'échelle nationale. Au Venezuela, la Fédération vénézuelienne de Coleo (la FEVECO), qui regroupe toutes les associations du pays, est créée en 1959. En 1969, elle est inscrite à l'Institut national du Sport. Victor Manuel Sarmiento, coleador et actuel président de la Fédération souligne que « le coleo est organisé comme un 
sport en catégories, championnats et éliminatoires. L'entraînement physique et l'adresse exigés par le coleo lui confère sa valeur compétitive et récréative ${ }^{67}$. En 1981, le gouvernement (le Congrès national), fait officiellement du coleo « un sport criollo et traditionnel ${ }^{68}$. En Colombie, la Fédération colombienne de Coleo (la FEDECOLEO) est créée en 1994. Elle met en place un règlement et un championnat national au début de l'année 1999. Le coleo est officiellement reconnu en tant que sport en 1998 par le Comité olympique colombien qui entérine ainsi son caractère autochtone ${ }^{69}$. Au Brésil, depuis 1998, la vaquejada est officiellement considérée par le gouvernement fédéral comme « une pratique sportive et un spectacle populaire, dans de nombreuses régions du pays, dont l'importance économique, touristique et culturelle est croissante $»^{70}$. En 2001, une loi confirme le statut de sportif professionnel aux vaqueiros ainsi qu'aux peaes qui participent aux rodeios. Enfin, ces pratiques possèdent également un découpage territorial des compétitions relevant du modèle sportif. Les saisons sont généralement structurées en compétitions locales, régionales ou nationales, reliées entre elles par un système comptable de notation qui permet de classer la performance des protagonistes dans les différentes catégories et aux différents échelons. L'évolution de nombreuses pratiques tauromachiques semble donc faire entrer le jeu taurin dans la modernité par l'intermédiaire d'une institutionnalisation sur le modèle sportif. La notion hybride de «sport traditionnel », que ne renierait aucune des pratiques mentionnées, situe ainsi les jeux taurins dans un entre-deux qui nuance, à beaucoup d'égards, les clivages entre sports modernes et jeux traditionnels. Quoi qu'il en soit, l'idée d'un sport traditionnel contraste avec celle d'une hypermodernité véhiculée aujourd'hui par de nombreux sports internationaux.

En revanche, les corridas, dans leurs versions portugaise ou espagnole, semblent échapper en grande partie aux logiques institutionnelles et compétitives du modèle sportif. Il s'agit pourtant bien de pratiques fortement institutionnalisées, mais selon des modalités qui les distinguent assez nettement du champ sportif. Dans tous les pays où elles sont pratiquées, les corridas obéissent à des réglementations qui définissent strictement les conditions de leur déroulement. Cependant, il n'existe aucune entité organisatrice à qui incomberait la régulation de l'ensemble des manifestations, ni aucun championnat orchestré sur une quelconque base territoriale unifiée. Les corridas sont soit organisées en féria lorsqu'une arène propose un ou deux spectacles quotidiens coïncidant avec les fêtes de la cité, soit organisées en saison lorsqu'une arène propose une pro-

${ }^{67}$ El Nacional, « La fiesta de nuestro llano », 13 octobre 2002 : http://www.el-nacional.com/ revistas/todoendomingo/todo158/reportaje2.htm [réf. 5 février 2006].

${ }^{68}$ FEVECO (Fédération vénézuélienne de Coleo). Le mot criollo qui signifie littéralement créole, désigne ici ce qui est autochtone.

${ }^{69}$ Les normes du Comité international olympique autorisent les pays affiliés à accepter de nouvelles disciplines au sein des comités nationaux s'ils démontrent le caractère autochtone de la pratique.

${ }^{70}$ Projet de loi n ${ }^{\circ} 249$, Congresso Nacional. 
grammation de spectacles répartis sur l'année comme le ferait un théâtre. De même, en dépit des trophées éventuellement accordés à l'issue de la course, il n'existe aucune comptabilité permettant de classer officiellement les protagonistes du jeu taurin, pas plus qu'il n'existe de moyens de déclarer vainqueur tel ou tel torero sur des critères chiffrés et objectifs. On observe d'ailleurs que le champ lexical du sport est sensiblement réduit dans l'univers de la corrida. Il est même souvent dénigré comme si l'effort physique faisait mauvais ménage avec la dimension artistique à laquelle la corrida aspire. En témoigne la présence fréquente en piste de toreros dépassant l'âge de quarante ans, nullement attendus pour leurs qualités athlétiques. En témoigne également le recours au lexique de l'inspiration et de la composition chorégraphique pour analyser le travail des toreros, au-delà des considérations d'ordre technique. L'évaluation de la performance des protagonistes semble ici plus qu'ailleurs subordonnée aux critères subjectifs de l'interprétation. L'ensemble de ces éléments rapproche volontiers les corridas du modèle de la représentation artistique. Qu'il n'y ait aucune équivoque, il ne s'agit pas d'établir une hiérarchie de valeurs entre ce qui relèverait de l'art et ce qui relèverait du sport, mais de montrer que l'ensemble des jeux taurins n'a pas choisi la même voie de modernisation spectaculaire. Ajoutons que pour la plupart des jeux taurins, les spectateurs sont généralement plus attentifs au style des protagonistes qu'aux décisions comptables du jury ou de la présidence, de sorte que les catégories sont en réalité perméables. Comme le souligne Bernard Traimond à propos de la course landaise et de la course camarguaise : " ces jeux se trouvent entre deux dynamiques contradictoires, l'une qui leur fait adopter les formes et les valeurs du sport, l'autre qui les refuse ${ }^{71}$. La corrida les a sans doute davantage refusées que ne l'ont fait les autres formes de jeux taurins professionnalisés.

\section{IV. - LA TAUROMACHIE}

\section{DANS LE CADRE DES RELATIONS HOMME-ANIMAL}

\section{LE PARADIGME DE LA « COURSE »}

Au-delà de son emploi comme synonyme de corrida, la notion de « course de taureau » possède une valeur paradigmatique d'un grand intérêt pour interroger les logiques formelles des jeux taurins. Cette notion permet de réfléchir à l'unité et la diversité des jeux taurins en établissant des comparaisons géographiques, historiques et culturelles. Cela tient d'abord à la polysémie du verbe courir. Historiquement, Álvarez de Miranda souligne l'importance de l'expression « courir les taureaux ", d'un usage fréquent au moins depuis le XIII ${ }^{\mathrm{e}}$ siècle, et la considère comme un argument en faveur de l'origine populaire de la corrida ${ }^{72}$. L'expres-

${ }^{71}$ B. Traimond, Les fêtes du Taureau, p. 104.

72 Á. Álvarez de Miranda, Le taureau. Rites et jeux, p. 79. 
sion est donc bien antérieure à la notion moderne de tauromachie. En France, le verbe courir, appliqué aux bovins, apparaitt sur l'un des plus anciens documents d'archives évoquant des jeux taurins, datant de 1282, retrouvé par Claude Pelletier en 1979. Ce document établit qu'à cette époque existe à Bayonne, comme dans toute l'Aquitaine, la coutume de « lâcher taureaux, vaches et bœufs dans la rue pour les faire courir $»^{73}$. Sous l'Ancien Régime, le verbe courir est employé transitivement avec le sens de "poursuivre à la course » et s'appliquait le plus souvent à un animal. Cet emploi existait également dans le domaine cynégétique et s'est maintenu de nos jours dans le vocabulaire de la chasse à courre dont font l'objet le cerf, le chevreuil, le sanglier, le renard ou le lièvre. Les scènes décrites par Argote de Molina dans son Discurso sobre la montería montrent que les taureaux ont également été chassés comme des gibiers de grande vénerie ${ }^{74}$. Des nobles à cheval pourchassent les taureaux, secondés par une meute de chiens et tenant à la main une arme nommée demi-lune leur permettant de sectionner les tendons des proies. Argote de Molina précise que cette pratique avait aussi cours en Amérique pour chasser les taureaux dispersés par le marronnage ${ }^{75}$.

À l'origine, le verbe courir a donc une acception très large puisqu'il est l'objet d'un double emploi « dans lequel il faut voir à la fois l'action de galoper pour les bêtes, celle de s'esquiver pour les hommes, mais aussi celle de poursuivre, c'est-à-dire de courser l'animal ${ }^{76}$. Il faut donc comprendre l'expression « courir les taureaux » dans toute sa richesse sémantique et voir en elle une posture élémentaire de l'affrontement entre l'homme et l'animal qui s'est orientée, dans le cadre d'une histoire longue, vers des principes ludiques différents selon les jeux considérés et les contextes culturels qui leur donnent forme et signification.

Le verbe courir est encore à l'honneur dans la dénomination de nombreuses pratiques tauromachiques actuelles. En Europe, on le retrouve dans la corrida de toros (corrida de touros en portugais), dans la course landaise, la course camarguaise, la course de vaches, le corre bou des pays catalans, et il est au cœur du vocabulaire de l'encierro. En français, nous ne disposons que d'un seul mot pour parler des courses de taureaux et des courses de vitesse, là où les langues espagnole et portugaise utilisent le terme carrera et carreira pour ces dernières. Pour la plupart des jeux codifiés un verbe d'action précise les modalités d'affrontement propre à chaque type de course : torear pour la corrida, "écarter » pour la course landaise, « raseter » pour la course camarguaise, recortar pour les concours de recortadores, et pegar pour désigner le travail des forcados lors des corridas portugaises. Dans les jeux taurins à participation collective, le verbe

\footnotetext{
${ }^{73}$ M. LAFORCAde, La tauromachie dans le Sud-Ouest de la France, p. 36.

${ }^{74}$ G. Argote de Molina, Discurso sobre la montería, cité par F. Flores Arroyuelo, Correr los toros en España, p. 77.

${ }^{75}$ F. Flores Arroyuelo, Correr los toros en España, p. 77. Le taureau cimarrón, marron en français, est le taureau issu de l'introduction du bétail en Amérique latine par les Espagnols et les Portugais, dont beaucoup d'exemplaires se sont échappés dans la nature, ont proliféré et sont retournés à l'état sauvage. Ce phénomène porte le nom de marronnage.

${ }^{76}$ C. Martel, « Du vocabulaire des "gens de bouvine” à celui de la course landaise », p. 13.
} 
courir est couramment employé pour désigner l'action de ceux qui affrontent le taureau qu'elles qu'en soient les modalités.

L'action de courir, au sens de courser l'animal, trouve également une certaine pertinence pour décrire le fonctionnement de nombreux jeux taurins d'Amérique appartenant à la grande et complexe famille des rodéos. En Europe, le rodéo est surtout connu à travers le modèle de l'Ouest américain dont l'imagerie ne retient généralement que l'usage du lasso et la figure du cow-boy monté sur des taureaux ou des chevaux furieux. Derrière ce cliché, qui a la force des représentations diffusées par la puissance médiatique étasunienne, se profilent des pratiques panaméricaines beaucoup plus diversifiées. À elle seule, l'histoire du mot rodéo retrace un itinéraire géographique très instructif pour notre propos général. Citons l'article du Dictionnaire historique de la langue française:

Rodéo ou rodeo n. m. est emprunté (1923) à l'anglo-américain rodeo (1914), nom d'un jeu équestre consistant à maîtriser un cheval ou un bœuf non domestiqué, et lui-même emprunté (1834) à l'hispanoaméricain rodeo " encerclement du bétail, emplacement circulaire où l'on marque le bétail » (XvIII ${ }^{\mathrm{e}}$ siècle). Ce dernier est dérivé de l'espagnol rodear « tourner, encercler » [...]. Le mot, essentiellement employé pour désigner le jeu équestre pratiqué dans l'ouest des États-Unis et, par extension, le lieu où se déroule cette fête, se dit aussi, au figuré ( $\mathrm{XIX}^{\mathrm{e}}$ siècle), comme corrida, d'une course agitée, d'une vive agitation collective et spécialement d'une poursuite en voiture ${ }^{77}$.

Remarquons pour l'instant que le terme de rodéo a voyagé de l'Amérique espagnole à l'Amérique anglo-saxonne. On notera également, non sans quelque satisfaction, l'analogie qui est établie entre le rodéo et la corrida à propos de leur sens figuré, attaché à l'idée d'agitation collective. Il est également opportun de rapprocher le sens premier de l'hispano-américanisme rodear (encercler) du mot espagnol encerrar (enfermer) d'où vient le terme d'encierro. Les termes rodear et encerrar partagent l'action de courir les bêtes pour les conduire et les rassembler dans un lieu clos. La Enciclopedia Universal Ilustrada précise qu'à l'origine le rodeo désigne l'action de rassembler le bétail (équins et bovins) afin d'opérer différentes tâches : dresser les chevaux, rattraper une bête égarée, soumettre un spécimen récalcitrant, trier les populations, marquer les jeunes bestiaux, conduire les bêtes sur les pâturages ou vers les lieux de vente et d'abat$\operatorname{tage}^{78}$. Le mot rodeo désigne également le lieu circulaire où l'on réunit le grand bétail. De ce contexte utilitaire proviennent différentes formes de rodéos pratiqués de l'Amérique du Nord au Cône Sud, plus précisément du Canada à la Patagonie chilienne, dont les techniques attestent d'un principe général commun consistant à courser les bovins et à les maîtriser.

\footnotetext{
77 «Rodéo» dans A. Rey, Dictionnaire historique de la langue française, p. 3270.

78 «Rodeo» dans Enciclopedia Universal Ilustrada, t. LI, p. 1185.
} 
Les formes prises par le rodéo se partagent en trois modalités d'affrontement principales : l'une fondée sur l'utilisation du lasso ; une autre fondée sur la course-poursuite dont la forme la plus répandue utilise la technique du coleo consistant à rattraper le taureau au galop et le saisir par la queue afin de le faire chuter ; une dernière fondée sur la monte d'animaux non domestiqués, qu'il s'agisse de chevaux ou de taureaux. La course-poursuite finalisée par le coleo ou la coleada est l'une des modalités techniques les plus répandues (au Mexique, à Cuba, au Panamá, au Venezuela, en Colombie, au Brésil). Le rodéo chilien constitue un cas particulier puisqu'il n'y a ni coleada, ni lasso, ni monte de bétail non domestiqué. Ici est pratiquée une seule technique consistant à poursuivre le taureau à cheval afin de le plaquer contre les barrières de la piste. Il est intéressant de constater que le rodéo chilien porte également le nom de corrida en vaca. L'expression courir «en vaca » est un emploi archaïque qui signifie « courir à deux cavaliers » et ne fait aucunement référence à la vache (la vaca). Avec le temps cette expression a été déformée et le rodéo chilien est fréquemment appelé corrida de vacas (course de vaches), y compris par ses adeptes. Il ne faut pas exclure qu'il s'agisse là d'une influence de l'expression corrida de toros dans l'évolution sémantique des termes servant à désigner le rodéo chilien. Tout se passe comme si les appellations de jeu chilien manifestaient une double opposition : la corrida de vacas s'opposant à la corrida de toros d'origine espagnole, le rodeo chileno s'opposant au rodéo dominé par le modèle étasunien. Sans anticiper sur l'analyse des transformations des jeux taurins, notons qu'il s'agit là d'un bon indice du relais opéré par les hégémonies culturelles espagnoles, puis étasuniennes sur le continent américain, participant à la codification des pratiques tauromachiques.

Si l'on peut considérer qu'il y a bien une poursuite dans les modalités du rodéo qui utilisent le lasso, en revanche la monte du bétail pour résister à ses ruades semble échapper au paradigme de la course ${ }^{79}$. Pour autant, la monte n'est pas totalement dissociable de l'ensemble des exercices permettant de maîtriser les bovins, même si elle connaît un développement indépendant dans le cadre de jeux taurins spécifiques. D'un point de vue technique et utilitaire, la monte du taureau a constitué dans le passé un moyen de réduire les forces de spécimens récalcitrants pour empêcher leur fuite et les ramener ensuite au troupeau. Au Costa Rica, Jorge González « Cañero » raconte qu'à l'époque où il n'y avait pas encore d'enclos pour rassembler et trier les animaux, les vaqueros couraient en cercle autour du bétail et si un taureau s'échappait, il était attrapé au lasso, puis monté afin de réduire ses ardeurs ${ }^{80}$. En outre, si pour nous la course et la monte semblent éloignées, notons que dans certains pays tels que le Costa Rica et le Nicaragua, l'expression corrida de toros est couramment utilisée pour désigner

\footnotetext{
${ }^{79}$ Dominique Fournier et Frédéric Saumade font de la monte des taureaux une inversion de la corrida et de la monte équestre. D. Fournier, «Corrida, charreada et jaripeo », p. 192. F. SAUMAdE, «Animal de rente, animal de loisir ».

${ }^{80}$ V. Randall Corella, «El Mundo tras las espuelas », La Nación, 2004 : www.nacion.com/ dominical/2004/enero/04/dominical0.html [réf. 5 février 2006].
} 
des jeux où les taureaux sont montés. Au Costa Rica, lors des corridas de toros a la tica, un taureau est lâché au milieu de la foule, les participants devant dans un premier temps faire courir l'animal pour ensuite le monter lorsque celui-ci se laisse suffisamment approcher ${ }^{81}$. En Bolivie amazonienne, cette pratique, associant un lâcher de taureau suivi de sa monte, porte le nom de jocheo de toros. Dans les Andes péruviennes des années 1960, Augusto Goicochea Luna rapporte qu'il n'est pas rare que les mêmes taureaux soient courus, toréés, puis montés ${ }^{82}$. Enfin, nous avons rencontré de nombreux cas où l'expression de corrida de toros est utilisée pour désigner n'importe quelle forme de jeu taurin, y compris dans des contextes culturels où la distinction est habituellement de mise pour les différencier. En témoigne, l'emploi générique de la notion de corrida dans le règlement des «fiestas charro-taurinas » de la ville de Comala au Mexique ${ }^{83}$. En effet, le règlement parle de corrida de toros alors qu'il est explicitement question d'exercices de la charrería, deux formes de jeux taurins différents et habituellement différenciés. Le cas n'est pas isolé et témoigne, une nouvelle fois, d'une imbrication des pratiques tauromachiques beaucoup plus complexe qu'une vision ségrégative prompte à cloisonner des formes qui en réalité participent parfois d'une expression culturelle commune.

\section{Pratiques tauromachiques, Violence et Domestication}

Il est possible de voir dans le parcours du champ vers les arènes, en vue d'une domination de l'homme sur l'animal, l'existence d'un itinéraire matériel et idéel, de la nature vers la culture. Ce parcours peut également être replacé dans une histoire de la domestication et de ses transformations ludiques. La domestication est ici prise au sens défini par Jean-Pierre Digard qui y voit un fait culturel total, "non un état de l'animal, mais une action de l'homme, non un processus historique achevé, daté et localisé, ayant conduit à un état, acquis une fois pour toutes, de certaines espèces animales, [...] mais une action continue, qui doit être renouvelée et entretenue jour après jour, sous peine de dédomestication ${ }^{84}$. En quoi les tauromachies témoignent-elles d'une relation homme-animal particulière au regard du processus historique général de domestication ?

On doit à Jean-Pierre Digard d'avoir rendue intelligible, à partir du concept de "système domesticatoire », la complexité d'une grande partie des relations que l'homme établit avec les animaux. Dans son ouvrage intitulé L'Homme et les animaux domestiques, il considère que chaque société investit dans ses relations avec les animaux un ensemble particulier de techniques (élevage, dressage, etc.), de représentations et de croyances relatives aux animaux, voire de sentiments envers eux (attachement, respect, compassion, mépris, répulsion,

\footnotetext{
${ }^{81}$ L'expression a la tica signifie ce qui est le propre du Costa Rica.

${ }^{82}$ A. Goicochea Luna, Tauromaquia andina, pp. 52-53.

${ }^{83}$ Comala est une ville d'environ 20.000 habitants, située dans l'État de Colima.

${ }^{84}$ J.-P. Digard, Les Français et leurs animaux, p. 13.
} 
peur...), autant de facteurs qui ne sont compréhensibles que pris ensemble ${ }^{85}$. En France, l'auteur montre que l'engouement pour les animaux de compagnie à partir des années 50 est allé de pair avec un effacement des animaux de rente, en particulier le bétail et la volaille. À cela s'ajoute une fascination pour la faune sauvage qui partage avec les animaux de compagnie le fait qu'ils ne font l'objet d'aucune exploitation directement utilitaire. Enfin, l'auteur établit une dernière catégorie : celle des " animaux intermédiaires ». Ces animaux, tout en n'appartenant à aucun des groupes précédents, empruntent des attributs de plusieurs groupes à la fois. Le cheval est l'animal exemplaire de cette catégorie. Il quitte peu à peu à partir de 1950 la sphère utilitaire pour entrer dans celle des loisirs, tout en ne pouvant pas rejoindre la catégorie des animaux de compagnie, ne serait-ce qu'en raison de sa taille ${ }^{86}$. L'auteur classe également le taureau de corrida dans cette catégorie. En effet, il relève à la fois de l'animal domestique puisqu'il est issu d'un élevage très spécialisé, et de l'animal sauvage, puisque tout est fait pour que le taureau évolue en liberté et pour qu'il ait le moins de contact possible avec l'homme. "Autrement dit, le travail de domestication s'est ici effectué à contre-courant, dans le sens d'une sorte d'ensauvagement orienté ${ }^{87}$. Remarquons qu'il n'en va pas de même pour tous les bovins participant aux pratiques tauromachiques. Dans le cas de nombreux rodéos (charreada, toros coleados, vaquejada, rodéo chilien), les bovins utilisés ne sont autres que les animaux de rente détournés momentanément de leur filière. Seuls les exercices de monte des taureaux, exécutés dans un cadre professionnel, font l'objet d'une sélection individuelle des animaux combattus (bull riding du rodéo nord-américain, jaripeo ranchero professionnel mexicain, rodeio brésilien). Dans le cas des spectacles où les animaux font carrière durant plusieurs années, tels que les bull riding, les jaripeos professionnels, les courses camarguaises et landaises, les bovins apprennent le métier au fur et à mesure de leur sortie. Dans ce cas, il n'est pas impossible d'y voir une certaine forme de dressage empirique, même s'il s'effectue dans le sens d'une accentuation des qualités combatives. Fort de cette distinction catégorielle du règne animal, l'auteur propose d'inclure la tauromachie dans un ensemble plus vaste de pratiques où l'homme et l'animal s'opposent :

Les pratiques agonistiques avec des taureaux, au même titre que les courses de chevaux, le rodéo américain ou les numéros de fauves dans les cirques, malgré leurs évidentes différences, sont justiciables du même type d'interprétation que toutes les pratiques agonistiques qui associent ou opposent, d'une manière ou d'une autre, l'homme et l'animal. Car, après tout, entre l'action d'enfourner un cheval sur une piste et celle d'affronter un taureau dans une arène ou un lion dans une cage, il y a des différences

\footnotetext{
${ }^{85}$ ID., L'Homme et les animaux domestiques; ID., « Les Européens du Sud-Ouest ».

${ }^{86}$ ID., Les Français et leurs animaux, p. 51.

${ }^{87}$ ID., « Les Européens du Sud-Ouest ».
} 
de degré dans le risque et dans la performance, et des différences dans la manière, mais pas fondamentalement de différence dans la finalité de l'action : il s'agit dans tous les cas d'avoir le dessus sur l'animal ${ }^{88}$.

Nous partageons avec l'auteur l'idée que ces pratiques relèvent d'une attitude fondamentale qui est celle d'une domination de l'homme sur l'animal. Cependant, il nous semble que les jeux taurins relèvent d'une catégorie à part en ce qu'ils mettent en scène un affrontement réel. Si l'équitation marque dans son cheminement historique une forme de domination de l'homme sur l'animal, elle exalte aussi des valeurs d'entente harmonieuse qui sont à l'opposé d'une finalité combative. L'équitation ne désigne pas de vainqueur ni de vaincu entre l'homme et l'animal, ce qui en revanche est le propre du combat. Dans le cirque, le dompteur ne se risquerait à aucun numéro avec un animal qu'il n'a pas longuement dressé dans les limites permises par l'espèce. Dans ce cas, bien que le danger soit réel, l'affrontement relèverait plutôt d'un numéro de fiction répété. Or dans la majorité des jeux tauromachiques, l'homme ne se mesure pas à l'animal de façon métaphorique, mais dans un corps à corps qui valorise l'habileté et la prise de risque des hommes face au danger. Les blessures fréquentes reçues par les toreros, les raseteurs, les écarteurs, les forcados, les monteurs de bull riding et de jaripeo professionnel, les recortadores mais aussi les coleadores et les vaqueiros ainsi que les participants des divers lâchers de taureaux, rappellent la réalité de cet affrontement et son haut degré de violence. Les jeux tauromachiques constituent bien, de nos jours, un cas à part dans le registre des divertissements.

Historiquement, il n'en a pas toujours été ainsi. Éric Baratay nous rappelle que les combats entre animaux, organisés par l'homme, les affrontements entre l'homme et l'animal, ainsi que les jeux au cours desquels des animaux sont violentés traversent notre histoire jusqu'à une époque charnière qu'il situe entre le $\mathrm{XVIII}^{\mathrm{e}}$ et le début du XIX ${ }^{\mathrm{e}}$ siècle, à partir de laquelle s'engage un reflux progressif des spectacles violents auxquels participent des animaux ${ }^{89}$. Pour ce qui est des jeux taurins, ils concernent encore jusqu'au milieu du $\mathrm{XIX}^{\mathrm{e}}$ siècle une grande partie de l'Europe. Il existait jusqu'au Xviı ${ }^{\mathrm{e}}$ siècle des formes d'encierros en Angleterre à Stamford (Lincolnshire), à Tutbury (Staffordshire) et à Birmingham $^{90}$. L'Angleterre finit par interdire ces pratiques en 1840, au moment où les sports modernes commencent à émerger. En France, il est fréquent que les bouchers fassent courir les bêtes harcelées par des chiens, pratique qui donne lieu occasionnellement à des combats organisés. Tel est le cas à Paris, de 1788 à 1850 à « la barrière des combats » (actuelle place du Colonel Fabien) ou à Bordeaux, dans les quartiers de la Bastide ainsi qu'à Talence dans « l'auberge de Plume la

\footnotetext{
${ }^{88}$ Ibid.

${ }^{89}$ É. Baratay, Et l'homme créa l'animal, pp. 269-275.

${ }^{90}$ Cité par B. Bennassar dans Histoire de la tauromachie, p. 12. L'auteur fait référence à E. et J.-P. FAbAron, dans Toros $n^{\text {os }}$ 1267-1268, 22 décembre 1985. Également cité par B. TrAimOND dans Les fêtes $d u$ Taureau, p. 10, faisant référence à K. ThомAs, Dans le jardin de la nature, p. 244.
} 
poule $»^{91}$. De même, à Vitteaux, en Côte d'Or, avaient lieu tous les ans « la course et le combat de taureaux » qui n'ont disparu qu'au milieu du xvinI ${ }^{\mathrm{e}}$ siècle $^{92}$. Enfin, en Italie, on court les taureaux dans les Maremmes toscans et Buffalo Bill dans sa tournée européenne de 1905 pour présenter son Wild West Show fut mis au défi par les buterri de les dompter ${ }^{93}$. L'évolution européenne vers une raréfaction des spectacles violents qui mettent en scène des anima ux s'inscrit dans une transformation sociétale plus profonde des relations homme-animal selon laquelle l'exploitation des animaux est progressivement occultée au plus grand nombre. L’intermédiaire technique des abattoirs et des élevages industriels, éloignés des lieux de vies, qui désincarnent et dépersonnalisent la relation entre l'exploitation de l'animal et la pratique alimentaire en est l'un des témoignages les plus éloquents ${ }^{94}$. Dans le cas français, Éric Baratay considère que :

La dissimulation et l'exil [des abattoirs] sont le fruit de changements de sensibilité concernant l'hygiène, de plus en plus affirmée, la violence, de plus en plus réprouvée, la mort, repoussée, et l'animal dont la souffrance publique est de moins en moins tolérée. Ils sont contemporains du nettoyage grandissant des hommes et des rues, du déplacement des lieux d'exécution ou d'équarrissage et des cimetières, de la répression des brutalités entre les hommes et envers les bêtes : interdiction des combats d'animaux en 1833, des mauvais traitements envers les bêtes domestiques en $1850^{95}$.

L'industrialisation des sociétés et la transformation radicale des modes de vie qui en a découlé posent alors de nombreuses questions sur les relations homme-nature. La dénonciation des nuisances et des dangers imputables aux avatars mondialisés de la Révolution industrielle occupe désormais une place importante dans les débats des sociétés contemporaines. Dans son sillage, la distinction entre l'homme et l'animal s'efface souvent au profit d'un bon sentiment généralisé à l'égard des bêtes. Cette évolution n'est pas étrangère à l'hégémonie de la culture urbaine sur les sociétés rurales traditionnelles, riches de relations complexes et diversifiées entre les hommes et les animaux. Cette transformation rapide explique en grande partie le fait que les jeux taurins apparaissent pour beaucoup totalement anachroniques, quand ils ne sont pas considérés comme

\footnotetext{
${ }^{91}$ P. Dupuy, Toros, ${ }^{\text {os }} 1667-1668,20$ décembre 2001, p. 25.

92 J.-P. FABARON, Toros n ${ }^{\circ}$ 1661, 30 août 2001, p. 32, faisant référence à J. GARCIN, « La petite histoire taurine de la ville d'Autan ».

${ }^{93}$ Les buterri de la Maremme sont, à beaucoup d'égards, l'équivalent toscan des gardians de Camargue ou des campinos de l'Alentejo. Ils continuent de pratiquer leur métier notamment dans l'élevage de 4.000 ha (L’Azienda Regionale Agricola di Alberese) intégré au Parc naturel régional de la Maremme. La maremmana est une vache qui pèse entre 900 et $1.300 \mathrm{~kg}$. Le trident camarguais et le pampilho portugais sont ici remplacés par l'ucino, une branche de noisetier d'un mètre cinquante dont l'extrémité est fourchue.

${ }^{94} \mathrm{~N}$. Vialles, Le sang et la chair.

${ }^{95}$ É. Baratay, Et l'homme créa l'animal, p. 107.
} 
des pratiques barbares, au premier rang desquelles figure la corrida. Cette évolution sociologique est bien le fruit d'une dissimulation de la violence envers les animaux, et en particulier de ses formes publiques, mais sans doute pas d'une réduction fondamentale de celle-ci. Norbert Elias, dans sa réflexion sur la « civilisation des mœurs " parle bien d'un contrôle et d'une euphémisation de la violence liée à l'émergence des États modernes, mais pas de sa disparition. C'est alors la dimension publique de la violence dans les pratiques tauromachiques qui pourrait bien être problématique, plus que la violence elle-même. Les pratiques tauromachiques exhiberaient aux yeux de tous ce qu'il ne faudrait pas voir : la domination de l'homme sur la nature pour assouvir des besoins et des désirs, matériels et immatériels, comme principe ancien de nos civilisations traversant notre histoire jusqu'à nos jours.

Cette domination a toujours été accompagnée d'un travail complexe de classification des éléments naturels répartis dans des registres multiples et variables selon les sociétés. Or on constate que la montée des mouvements de « libération animale », du courant philosophique antispéciste ou des théologies animales anglo-saxonnes, est aujourd'hui peu soucieuse de questionner les catégories culturelles qui fondent notre rapport au règne animal ${ }^{96}$. En dépit des évolutions, des différences de civilisations et du statut changeant ou intermédiaire de certaines espèces, ces catégories sont toujours cloisonnées par des limites rendues relativement imperméables par la culture : les animaux sauvages, les animaux nuisibles, les animaux domestiques, les animaux familiers, les animaux de loisirs, etc. Ces catégories vident de son sens l'angélisme selon lequel ne pas faire de mal aux animaux serait un principe universel et bon. Il est alors tentant d'accepter le résumé sibyllin du philosophe Francis Wolff qui s'accorde à de nombreuses recherches anthropologiques : "L'animal, l'animal en général, n'existe pas. Ce qui existe ce sont des espèces de vivants. Ce qui existe ce sont des virus, des moustiques, des chiens... des taureaux et des hommes ${ }^{97}$. Conformément à une éthique profondément humaniste, à l'exception de l'homme, les autres espèces ont une place particulière dans des sociétés particulières, mais en aucun cas un statut universel qui les assigne en tout lieu à un même sort. Ou bien, comme le répète un aficionado de la Peña Ostrea d'Andernos-les-Bains, dans une version rétive au système philosophique et pour laquelle il faudrait considérablement nuancer la valeur de l'analogie: "Quand je mange une huître, je mange une huître », et d'expliquer par là qu'il ne s'adonne pas à la torture d'un être vivant par le citron et la mastication.

Certes, à l'échelle européenne, les rapports au règne animal et les catégories qui les structurent ne sont pas tout à fait identiques entre les pays méditerranéens et les pays anglo-saxons, ni entre pays ayant connu différents rythmes d'industrialisation et d'évolution des sociétés rurales. Cependant, aucun raccourci ne permet de trouver des découpages culturels qui suffiraient à expliquer

\footnotetext{
${ }^{96}$ Pour une approche philosophique des mouvements de « libération animale » voir L. Ferry, Le nouvel ordre écologique, chap. II « La "libération animale" ou le droit des bêtes ", pp. 57-86.

${ }^{97}$ F. WolfF, «Qui est le taureau ?», p. 33 ; ID., Philosophie de la corrida.
} 
pourquoi les uns aiment les jeux taurins et les autres les condamnent. Plus modestement, les éléments qui reviennent le plus souvent dans les témoignages personnels que nous avons pu recueillir, en Europe et en Amérique, pour expliquer la passion des jeux taurins, demeurent la tradition familiale et le fait d'avoir grandi dans une région tauromachique ou une région d'élevage. Pour d'autres, sans lien avec un héritage culturel local ou familial, la passion naît d'une admiration du courage des hommes, d'une fascination pour le taureau ou le cheval et d'une sensibilité à la dimension sportive ou artistique du spectacle qui s'accompagne d'une compréhension éthique globale des relations homme-animal. Cela étant, quelle que soit la rationalité de la justification des éthiques antagonistes, il semble, le plus souvent, qu'elles soient postérieures à la fascination du goût ou du dégoût, comme si en quelque sorte l'argument venait après la sensibilité.

En revanche, le raccourci selon lequel les positions anti-taurines seraient au-dessus de tout soupçon au nom d'une éthique prétendument universelle (mais qui ne l'est pas), relève d'une mystification. Sur ce point l'anthropologue Sergio Dalla Bernardina attire notre attention sur les manifestations pétries de bons sentiments visant à protéger les animaux, à grand renfort d'images sensationnalistes :

Tout aussi ambiguë, me paraît-il, est la compassion apparemment sans reproche qui inspire les performances que l'on vient d'évoquer : replacées dans le cadre spectaculaire, mondain et médiatique dans lequel elles se produisent, ces manipulations irrespectueuses du sang des animaux semblent correspondre moins à des appels humanitaires en faveur des visons et autres carnivores poilus, qu'à des exploitations symboliques, foncièrement narcissiques, jouant sur les vertus "charmeuses » de l'hémoglobine ${ }^{98}$.

La manipulation démocratique de nombreux mouvements anti-taurins qui considèrent, à grand renfort de sondages, que toute pratique n'obtenant pas un assentiment majoritaire devrait être interdite, nous semble être encore plus pernicieuse qu'une vision manichéenne et universalisante des relations hommeanimal. Cet argument est fréquemment utilisé dans de nombreux pays par les détracteurs des jeux taurins constatant qu'ils n'intéressent qu'une fraction minoritaire de la population et, de ce fait, devraient être logiquement supprimés. Rappelons, quelle que soit l'opinion de chacun sur les jeux taurins, que la démocratie ne se réduit pas au mode d'élection défini par le principe de l'opinion majoritaire, elle est aussi la garantie des libertés fondamentales d'activité et d'expression.

${ }^{98}$ S. Dalla-Bernardina, L'éloquence des bêtes, p. 132. 


\section{UNITÉ ET DIVERSITÉ DES PRATIQUES TAUROMACHIQUES}

Les planches photographiques présentées en introduction (fig. 1-36, pp. 15-29) donnent à voir des pratiques tauromachiques très diverses. Il existe assurément plusieurs façons de les classer selon les propriétés formelles que l'on souhaite mettre en valeur. Le tableau 1 (pp. 9-12) de présentation des pratiques tauromachiques par pays complète ce que la synthèse cartographique ne permet pas de faire apparaître en détail. Ce tableau met également en exergue un élément de différenciation fondamental des jeux taurins en tant qu'activité de divertissement. Dans le champ des loisirs, la tauromachie relève de deux réalités sociologiques distinctes, celle du spectacle professionnel et celle du jeu à participation collective. Cette distinction mérite d'être analysée en profondeur pour rendre compte de la nature des différents jeux taurins et des différents acteurs y prenant part.

Un second niveau d'analyse appréhende les différentes modalités d'affrontement entre les hommes et les bovins en fonction des règles du jeu et des lieux de mise en scène. Les règles du jeu varient en fonction du nombre de protagonistes en lice, de l'utilisation ou non du cheval, du type d'animal combattu, de l'utilisation ou non d'artefacts tenus par l'homme (cape, poncho, carton déplié, parapluie ouvert, journal roulé, banderilles, piques, épée, lasso) ou d'artefacts ajoutés au taureau (armatures métalliques supportant des boules de feu, cordes pour conduire l'animal ou pour faciliter sa monte, récompenses qu'il s'agit de décrocher : cocarde, colcha, enjalma, vincha). La tauromachie investit des types de lieux très différents, depuis des édifices spécialisés (les arènes de corrida, de course landaise, de course camarguaise, les enceintes de rodéo : manga des toros coleados, lienzo ou rancho de la charreada, medialuna du rodéo chilien, parque de vaquejada) jusqu'aux lieux les plus divers de la vie et des activités quotidiennes, les rues, les places publiques, les enclos, les champs, les terrains vagues, les chemins de campagne, etc. On observe, sur l'ensemble des pratiques, une corrélation forte entre le degré de professionnalisation de la tauromachie et la spécialisation des lieux de mise en scène. La portée d'une telle opposition est aisément perceptible entre d'un côté les jeux d'arènes (la tauromachie professionnelle pratiquée au sein d'équipements spécialisés) et de l'autre les jeux de 
rue (la tauromachie participative pratiquée au sein des espaces publics). Cependant la distinction entre jeux d'arènes et jeux de rue n'est pas aussi radicale qu'il n'y paraît. La première raison tient au fait que la tauromachie participative s'accommode en réalité de tout type d'espace et a fortiori des arènes construites pour la tauromachie professionnelle, dès lors qu'elle est autorisée à y pénétrer. La seconde tient à la nécessité, commune à la plupart des divertissements taurins participatifs, de circonscrire une aire de jeu pour des raisons évidentes de sécurité. Il faut comprendre l'expression de jeux de rue au sens où l'on parle parfois de sport de rue ou de théâtre de rue pour désigner des pratiques qui sortent des cadres conventionnels des spectacles professionnels. La rue doit ainsi être considérée dans sa dimension sociologique, tout autant que dans sa dimension spatiale, rassemblant ainsi des espaces hétéroclites servant occasionnellement de lieu de mise en scène tauromachique. À l'inverse, on peut considérer que l'arène constitue une structure élémentaire de l'affrontement entre l'homme et le taureau, quelle que soit sa forme et quelle que soit sa matérialité. On retrouve une idée proche chez le critique taurin et écrivain Jacques Durand qui s'interroge sur la formation des arènes et la logique qui leur donne forme :

Un taureau galope au milieu de la foule puis s'immobilise. Un cercle de coureurs se forme spontanément à une certaine distance autour de lui. On peut considérer cet espace brut comme la plaza de toros primitive, une sorte de degré zéro de l'architecture taurine qui aboutira plus tard à l'auto-invention d'un édifice spécialisé ${ }^{1}$.

\section{I. - TAUROMACHIE PROFESSIONNELLE \\ - TAUROMACHIE PARTICIPATIVE}

\section{UNE OPPOSITION FONDAMENTALE}

DANS LE REGISTRE DES DIVERTISSEMENTS

En tant qu'activité, la mise en scène réelle de l'affrontement entre l'homme et le taureau fait partie de la catégorie des divertissements sous la forme de deux réalités distinctes : le spectacle professionnel et la pratique participative. Dans les spectacles de la tauromachie professionnelle, il existe une frontière scénique stricte et une différence de statut entre les acteurs du jeu taurin et les spectateurs. Généralement, la configuration spatiale de l'arène ou de toute autre enceinte spécialisée dans le jeu taurin matérialise physiquement cette séparation. Les professionnels sont en piste, les spectateurs sont sur les gradins, séparés par des barrières. Les règles du jeu sont codifiées et définissent le rôle de chacun des protagonistes. Dans la tauromachie à participation collective, acteurs et spectateurs du jeu taurin sont plus souvent confondus et chacun peut librement

\footnotetext{
${ }^{1}$ J. Durand, Humbles et phénomènes, p. 112.
} 
éprouver son courage, son adresse ou sa témérité face au risque que constitue toujours, à quelque degré que ce soit, cette rencontre entre l'homme et l'animal. À la différence des spectacles taurins régis par un cérémonial fixe et des règles prescriptives, les jeux taurins à participation collective se caractérisent par une plus grande informalité et des normes plus lâches. Cette opposition fondamentale ne résiste pas totalement à l'analyse de certains cas intermédiaires.

Le terme de spectacle possède une ambivalence qui n'aide pas à la distinction des types de divertissements tauromachiques puisqu'il est couramment employé non seulement pour désigner ce qui relève d'une représentation (théâtrale, musicale, ici tauromachique) mais aussi pour désigner « un ensemble de choses ou de faits qui s'offre au regard, capable de provoquer des réactions $»^{2}$. On retrouve cette ambivalence dans le règlement taurin espagnol dont la classification fait apparaître plusieurs types de "spectacles tauromachiques ». Le règlement différencie huit catégories de "spectacles et festivités taurines » (espectáculos y festejos taurinos) : corridas de toros; novilladas con picadores; novilladas sin picadores; rejoneo ; becerradas ; festivales ; toreo cómico ; espectáculos o festejos populares ${ }^{3}$. Les sept premières relèvent bien d'un spectacle au sens d'une représentation payante où interviennent des spécialistes, la dernière prend la forme d'un divertissement ouvert à tous, le plus souvent gratuit, ici qualifié de «populaire » pour bien insister sur sa dimension collective et participative. Les spectacles tauromachiques «populaires » sont parfois qualifiés de « traditionnels », entérinant l'idée que la forme professionnelle du jeu constituerait une tauromachie " savante » et " moderne » en rupture, sur un plan sociologique et historique, avec la tauromachie participative.

Ensuite, on pourrait être surpris de voir qualifiés de professionnels certains spectacles tauromachiques exécutés par des amateurs. Ici, le professionnel n'est pas tant celui qui perçoit un salaire pour un travail que celui qui maîtrise une technique spécifique sur laquelle repose le spectacle ou la représentation. Par exemple, la charreada et le rodeo chilien sont des sports équestres revendiqués comme non professionnels. Il n'en demeure pas moins que les cavaliers mexicains (les charros) et les cavaliers chiliens (les huasos) possèdent une technique acquise par un entraînement assidu et que les meilleurs d'entre eux participent aux championnats et compétitions de haut niveau ${ }^{4}$. De même, les forcados de la corrida portugaise sont fiers de constituer une corporation d'amateurs qui n'engage le plus souvent aucune autre contrepartie financière que les frais de transport, le gîte et le couvert. Pour autant, ils participent aux spectacles pro-

\footnotetext{
2 «Spectacle» dans A. Rey, Dictionnaire culturel en langue française, pp. 960-963.

${ }^{3}$ Règlement taurin espagnol, article 25 « espectáculos y festejos taurinos » (spectacles et festivités taurines). Dans le langage taurin les termes espectáculo et festejos sont le plus souvent utilisés sans distinction alors même que le Diccionario de la Real Academia Española n'accorde au mot festejos aucune équivalence du mot spectacle. Nous reviendrons sur ce point apparemment anodin qui est d'un intérêt certain pour l'analyse de la dimension festive de la tauromachie.

${ }^{4}$ Pendant longtemps le rugby relevait d'une logique équivalente avec une éthique de l'amateurisme qui n'empêche en rien l'existence de compétitions professionnelles de haut niveau.
} 
fessionnels des corridas portugaises. Nous considérons donc les acteurs des pratiques tauromachiques comme des professionnels de l'arène en tant que spécialistes du jeu taurin.

Ces considérations nous incitent à identifier une autre sous-catégorie de divertissements tauromachiques : ceux qui relèveraient du sport de loisir. Ce type de divertissement est assez bien représenté en Amérique latine dans le cadre d'une équitation taurine pratiquée en club ou dans des associations sportives et culturelles. Pour un même jeu taurin on observe alors une pratique professionnelle intégrée à un système de compétitions de haut niveau et une pratique non professionnelle de loisir. C'est le cas de la charreada au Mexique, du rodéo chilien, des toros coleados au Venezuela et en Colombie, de la vaquejada au Brésil et de certains exercices du rodéo nord-américain. La tauromachie comme sport de loisir pourrait également désigner l'activité de nombreux passionnés, parfois organisés en associations, qui se déplacent, au rythme des calendriers des fêtes populaires, pour participer à tel ou tel lâcher de taureaux dès que leur emploi du temps le leur permet. Pour les plus fervents, la tauromachie s'inscrit dans une activité ludique et physique régulière. De même, comment classer autrement les jeux taurins populaires auxquels participent des pratiquants occasionnels qui s'entraînent plus ou moins régulièrement pour entrer en piste lors de quelques fêtes de villages. Il en est ainsi des concours de capeia raiana au Portugal, de roscadero en Aragon, des emboladores en Espagne. Certes il ne s'agit pas de jeux professionnels, mais ils possèdent des règles établies et la présence en piste d'équipes de spécialistes en représentation, distinctes des spectateurs momentanément exclus du jeu. Comme le soulignait déjà Norbert Elias au milieu des années 1980, «le sport de loisir [...] attire moins l'attention en tant qu'institution sociale que les sports professionnels de haut niveau $»^{5}$. C'est aussi une institution sociale intermédiaire plus difficile à saisir, tant le sport accepte luimême de définitions. Il en va de même pour les jeux pratiqués dans les élevages de taureaux à mi-chemin entre le spectacle privé et les travaux des champs, qu'il s'agisse des tientas où viennent s'entraîner les toreros ou bien de certains rodéos pratiqués dans les haciendas à l'initiative du propriétaire terrien. Une fois de plus, des catégories trop rigides ne résistent pas à la complexité des pratiques.

On peut alors distinguer plusieurs catégories d'acteurs affrontant le taureau :

- les professionnels, protagonistes d'un spectacle tauromachique intégré à la société de consommation qui peut être hautement rémunérateur : matadores de toros (à pied et à cheval) de la tauromachie espagnole, bull riders du rodéo nord-américain ;

- les spécialistes rémunérés d'un spectacle dont le degré de professionnalisation est moindre (raseteurs de la course camarguaise, écarteurs de la course landaise, recortadores, coleadores, vaqueiro de la vaquejada brésilienne, manteros des corralejas, jinetes du jaripeo ranchero professionnel;

\footnotetext{
${ }^{5}$ N Elias et É. Dunning, Sport et civilisation, p. 82.
} 
— les spécialistes se réclamant d'un sport amateur (cavaliers charros ou cavaliers huasos);

- les employés travaillant dans les élevages de taureaux prenant part occasionnellement à des jeux taurins : les campinos portugais, les gardians camarguais, les garrocheros de la Costa Atlántica colombienne, les cordiers des Açores ;

— les non spécialistes : tous les participants anonymes, occasionnels ou réguliers, prenant part aux festejos populares et aux divertissements taurins qui leur sont réservés. Il s'agit d'un groupe très hétérogène qui intègre en réalité de véritables spécialistes amateurs et des néophytes affrontant le taureau pour la première fois. Cette catégorie compte en effet des personnes qui par leur expérience de la tauromachie participative ont du métier. Dans le monde de la corrida, on appelle les plus expérimentés des aficionados prácticos (dont la traduction libre pour rendre l'expression significative pourrait être des " amateurs pratiquants »). Il s'agit généralement d'aficionados qui possèdent leurs entrées dans le mundillo ou font partie d'une association taurine ( "peña ») et qui ont acquis une expérience avec du petit bétail, soit en tienta chez des éleveurs, soit en capea qu'ils organisent eux-mêmes. Certains vont jusqu'à organiser des spectacles dans des arènes de première importance, comme c'est le cas par exemple dans les arènes de Acho à Lima, au grand regret de certains aficionados qui le vivent comme une profanation du temple. Certains coureurs d'encierros très expérimentés, appelés les divinos (les divins), figurent également en position intermédiaire puisqu'ils sont considérés comme des professionnels de l'encierro, connus et reconnus par les autres coureurs.

\section{QUAND LA TAUROMACHIE MÉLANGE LES GENRES}

La multiplication des catégories et la diversité des acteurs du jeu tauromachique nous invitent à reconsidérer la frontière entre tauromachie professionnelle et tauromachie participative. Cette dichotomie fondamentale au sein des jeux taurins ne doit pas occulter les relations complexes qui les unissent. En effet, il existe des relations de parenté équivoque entretenues entre le spectacle professionnel et le jeu populaire. En introduction de notre travail, nous avons souligné que la forme participative était perçue tantôt comme la matrice originelle de la forme professionnelle, une sorte d'antécédent élémentaire qui contiendrait en germe la professionnalisation du jeu, tantôt, à l'inverse, comme un avatar du spectacle, une sorte de déclinaison pour les amateurs d'un jour. Le mélange des genres de divertissements n'est d'ailleurs pas rare au cours d'une même manifestation. Deux cas de figure sont possibles. Le premier se présente lorsqu'un même spectacle prévoit une partie professionnelle et une partie participative. Le règlement taurin espagnol depuis 1982 prévoit une réglementation précise pour cette pratique officiellement nommée suelta de reses para fomento y recreo de la afición (lâcher de bovins pour la promotion et le divertissement de l'afición). 
Cette pratique est incluse dans la catégorie des spectacles taurins populaires au même titre que les encierros et que le toreo de vaquillas en plazas públicas, correspondant à la tauromachie de divers types de bovins sur la voie publique ${ }^{6}$. Les lâchers de bovins pour la promotion et le divertissement de l'afición se déroulent dans les arènes à la suite du spectacle professionnel pour que les volontaires s'essayent à l'art de toréer. Cette pratique requiert réglementairement la présence d'un professionnel et exige que les bovins aient les cornes protégées (emboulées) et qu'ils n'excèdent pas l'âge de deux ans. On retrouve cette association de la tauromachie professionnelle et de la tauromachie participative dans l'univers de la course landaise, lors des courses dites « mixtes », par opposition aux courses dites «formelles ». Les courses mixtes se composent d'une partie « sérieuse », réalisée par les professionnels de l'arène et d'une partie qui offre la possibilité au public de participer à différents types de jeux avec des vachettes, dans une ambiance de rire et de détente. Cette association s'observe également dans l'État du Yucatán au Mexique où une quinzaine de taureaux peuvent être affrontés successivement à la suite d'une corrida ou d'une novillada sérieuse. La plupart du temps, seul le premier taureau de caste est combattu à l'espagnole, de façon conventionnelle, par une cuadrilla locale, puis mis à mort. La viande servira à préparer le chocolomo, un plat typique de la région du Yucatán. Les autres taureaux généralement de demi-caste sont livrés à la foule pour être courus et toréés sans mise à mort.

Le second cas de figure se présente lorsque les acteurs spécialistes et amateurs se mélangent au cours d'une même séquence de jeu. La pratique est de mise dans de nombreux villages andins d'Équateur et du Pérou où la performance des professionnels de faible renom peut être interrompue par un habitant du cru qui présume d'un certain savoir-faire, posté dans l'arène avec ses émules qui l'encouragent à intervenir si une occasion se présente. En outre, le dernier taureau fait généralement l'objet d'un jeu à participation collective où les volontaires descendent en plus grand nombre dans l'arène pour se mesurer au taureau comme bon leur semble. C'est également le cas lors des corralejas de la Costa Atlántica colombienne où, à la foule anonyme qui court les taureaux, se mêlent des semi-professionnels, des manteros, des toreadores et des garrocherros engagés par les organisateurs de spectacles. Les garrocheros, qui sont des bouviers à cheval des élevages voisins, excitent l'animal en le défiant à l'aide de la garrocha (longue pique), rendant l'animal encore plus agressif. Parmi les hommes à pied on compte des amateurs d'un jour et des semi-professionnels manteros et toreadores pour qui les corralejas représentent une source de revenus pendant la période des fêtes. Les corralejas se situent ainsi à la charnière du spectacle professionnel et du jeu collectif, et offrent une image de ce que la tauromachie a été en Espagne dans un stade de professionnalisation peu avancé. On l'aura compris, il n'est pas rare que la tauromachie professionnelle et la tauromachie participative

\footnotetext{
${ }^{6}$ La traduction littérale de toreo de vaquillas en plazas públicas (tauromachie de vachettes sur les places publiques) entraînerait une approximation sur ce que la pratique désigne réellement, à savoir des pratiques très diverses, qui mettent en scène des bovins de taille, d'âge et de sexe différents, dans des lieux également très hétérogènes.
} 
coexistent au sein d'un même spectacle, même si historiquement la professionnalisation des jeux taurins a plutôt contribué à les séparer. Comme on le verra plus avant, en se situant non plus au niveau du spectacle, mais au niveau de l'ensemble des programmes festifs des localités taurophiles, on constate que ces deux types de pratiques sont régulièrement à l'affiche, comme si la fête se devait de consommer la tauromachie sous ses deux espèces.

Deux figures opposées continuent de symboliser cette proximité dans l'univers de la corrida. D'un côté, la figure de l'espontáneo, simple spectateur qui surgit illégalement des gradins pour descendre en piste toréer avant que la police ne se saisisse de lui. Cette pratique est partout en régression, mais elle demeure plus vivace en Amérique latine où les frontières entre spectacles professionnels et spectacles à participation collective sont souvent plus poreuses. De l'autre, la figure des matadors professionnels dont certains se mêlent à la foule anonyme pour courir l'encierro. Citons, pour la première, l'exemple d' «El Cordobés » qui pour sortir de l'anonymat faisait l'espontáneo comme l'ont fait beaucoup d'autres individus n'ayant pas connu la même fortune ${ }^{7}$. Citons, pour la seconde, l'exemple d'Antonio Ordóñez qui avait l'habitude de courir, le matin, l'encierro de Pampelune au milieu de la foule anonyme, avant de se produire dans les arènes l'après-midi. L'espontáneo anonyme qui devient célèbre et le matador illustre plongé dans l'anonymat reflète une certaine porosité entre la tauromachie professionnelle et la tauromachie participative attestant l'idée que ces pratiques ne sont pas hermétiquement cloisonnées. Cette dualité constitutive donne à la tauromachie une certaine originalité qui la distingue de beaucoup de divertissements où le monde des professionnels et celui des amateurs sont davantage séparés. Elle contribue aussi à les tenir dans une proximité qui, nous le pensons, renforce les phénomènes d'identification entre les aficionados et leur héros, et participe pleinement à un enracinement en profondeur des pratiques sur leur territoire.

\section{II. — LES TAUREAUX DES JEUX TAURINS}

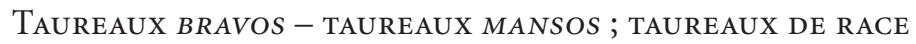 \\ - TAUREAUX CROISÉS}

Malgré l'intérêt d'accorder une valeur paradigmatique à la notion polysémique de " course de taureaux » pour juger de l'unité et de la diversité des jeux taurins, il est néanmoins nécessaire de distinguer plusieurs types d'animaux combattus. On observe une différence importante entre les jeux taurins où le bétail charge l'homme, ceux où le bétail doit éjecter l'homme monté sur son dos et ceux où l'homme doit maîtriser un bétail qui prend la fuite. Remarquons néanmoins que l'ordonnancement de ces distinctions perd de sa lisibilité au fur

\footnotetext{
${ }^{7}$ Les photos de Lucien Clergue ont immortalisé une scène célèbre ayant eu lieu à Madrid.
} 
et à mesure que les jeux gagnent en improvisation et sortent des cadres strictement codifiés, exploitant alors la réversibilité du comportement animal, de la fuite à la charge, de la charge à la fuite, de la capacité d'éjecter l'homme à celle de le charger au sol. Nombre de tauromachies populaires semblent d'ailleurs utiliser cette indistinction entre rodéo et course de taureaux comme un ressort ludique, révélant de façon élémentaire, mais très significative, les relations d'hybridations entre les jeux.

Observer les races bovines associées aux jeux taurins est d'un grand intérêt pour affiner la compréhension de la diversité tauromachique. Les jeux taurins pratiqués dans le Sud-Ouest européen reposent sur la présence en piste d'un bovin sélectionné et reproduit pour le combat, qu'il s'agisse des corridas espagnoles et portugaises, des courses landaises, des courses camarguaises, des concours de recortadores ou des divers jeux taurins à participations collectives. Cette sélection est le fruit d'élevages spécialisés. Il existe autour des races de taureaux de combat une prolifération de catégories et de ramifications. Pour simplifier, il est possible de différencier deux races de taureaux, scientifiquement identifiées, intervenant dans les jeux taurins. Les premiers, les plus répandus, sont les toros de lidia (littéralement taureaux de combat), originaires de la péninsule Ibérique, également appelés toros bravos (taureaux « braves ») appartenant à la race bos taurus de variété ibericus ${ }^{8}$. Il existe des élevages de taureaux bravos dans tous les pays où l'on pratique la corrida. Les seconds sont les taureaux de race Camargue qui font figure d'exception dans la géographie des taureaux de combats élevés pour charger l'homme. Le taureau de combat ibérique est le fruit d'un élevage spécialisé qui démarre au début du XviII siècle en Espagne, même si dès le XVII ${ }^{e}$ siècle, il est possible d'attester d'un travail de sélection de bovins en vue de jeux taurins. Au sein de cette race, il est coutume de distinguer ce que le vocabulaire taurin nomme des " castes ». Les spécialistes retiennent généralement quatre grandes castes historiques de taureaux de combat qui s'individualisent entre le milieu et la fin du XVIII ${ }^{\mathrm{e}}$ siècle :

- la caste Cabrera, du nom de l'éleveur d'Utrera en Basse Andalousie, Luis Antonio Cabrera qui réunit en 1740 du bétail des Pères Augustins de Carmona, ainsi que des bêtes appartenant à d'autres communautés religieuses ;

- la caste Jijona descendant de l'éleveur Juan Jijón Salcedo dont les bêtes vivaient autour de Madrid, dans les plaines de Ciudad Real, les escarpements des Monts de Tolède et les vallons qui entourent Colmenar Viejo ;

- la caste Vazqueña du propriétaire d'Utrera dans la province de Séville, Gregorio Vázquez ;

\footnotetext{
${ }^{8}$ Pour avoir en tête un ordre de grandeur significatif, il existe en Espagne 28 races autochtones de bovins dont 20 sont en voie d'extinction. Le taureau de combat bos taurus variété ibericus ne fait pas partie des espèces menacées. S. OrTUÑo PÉreZ, « Ecología y economía de las explotaciones de ganado de lidia ", p. 166.
} 
- la casta Vistahermosa du nom de Pedro Luis Ulloa comte de Vistahermosa à Dos Hermanas (Séville). Cette dernière caste est, à tous égards, la plus importante puisqu'elle représenterait l'origine de plus de $90 \%$ des taureaux de caste actuels .

Une cinquième caste est souvent prise en considération. Elle ne prend pas le nom de son propriétaire, mais de son origine géographique : la casta navarra. Elle serait plus ancienne que les autres du point de vue de la sélection empirique pour les jeux taurins (Pampelune fin du XIV siècle). Beaucoup la considéraient comme éteinte jusqu'à la voir resurgir à partir de la fin des années 1970, chez des éleveurs navarrais qui affirment que la race n'a jamais totalement disparu.

À propos de ces " castes fondatrices », inlassablement invoquées par une afición très friande de généalogie, Antonio Luis López Martínez, historien de métier et spécialiste des élevages de taureaux de combat, souligne que l'établissement des filiations historiques des races ne date que du dernier tiers du $\mathrm{XIX}^{\mathrm{e}}$ siècle. Il précise que cette généalogie repose sur des renseignements donnés par les éleveurs eux-mêmes. Et l'auteur d'ajouter que " nous ne pouvons ignorer les intérêts des éleveurs à démontrer une origine déterminée pour leur élevage qui confère à leurs taureaux un meilleur pedigree afin d'en exiger un prix supérieur ${ }^{10}$. Selon le contexte, l'expression de " taureaux de caste » peut être synonyme de taureaux de combat. Mais dire qu'un taureau a de la caste, c'est aussi insister sur un comportement individuel et contingent qui témoigne, sur le moment, d'une grande combativité.

Comme échelon intermédiaire, entre les castes fondatrices et le comportement individuel des taureaux, figurent les encastes, désignant des ramifications secondes et croisées entre elles, qui se détachent à partir d'une même caste historique, en l'occurrence principalement la caste Vistahermosa. Ainsi parle-t-on d'encaste Saltillo, Murube, Santa Coloma, Parladé, etc. Les taureaux varient ensuite en fonction du fer de l'élevage et de l'éleveur lui-même qui en hérite et modèle son cheptel. À l'image d'un vigneron qui travaille sa vigne et son terroir, les éleveurs cherchent à donner à leurs taureaux des caractéristiques en fonction des croisements de sang et des choix de sélection : physionomie, forme des cornes, couleur de robe et surtout comportement dans l'arène ${ }^{11}$. Lorsque le taureau entre en piste, il n'a jamais combattu et a grandi le plus possible à l'écart des hommes. Le comportement du taureau en piste est jugé à l'aune de deux

\footnotetext{
${ }^{9}$ Les chiffres qui rappellent la domination écrasante de la caste Vistahermosa comme origine de toutes les ganaderias varient d'un ouvrage à l'autre, de $90 \%$ à $98 \%$. Sigfredo Ortuño Pérez propose le chiffre de $90 \%$. S. Ortuño Pérez, « Ecología y economía de las explotaciones de ganado de lidia ", p. 167.

${ }^{10}$ A. L. López Martínez, Ganaderías de lidia y ganaderos, p. 17.

${ }^{11}$ Les ouvrages traitant de l'histoire et des caractéristiques des élevages de taureaux de combats sont très nombreux. Sur l'histoire des élevages, la référence irremplaçable, en dépit de son discours iconoclaste qui rompt avec une certaine mythologie du taureau de combat, est l'ouvrage d'A. L. López Martínez, Ganaderías de lidia y ganaderos. Sur les caractéristiques des castes, des encastes et des élevages, on pourra consulter J.-L. CAstanet, Campo Bravo.
} 
critères essentiels pour la tenue du combat : la «bravoure » et la «noblesse ». Ces termes possèdent un sens particulier dans le langage taurin. Étymologiquement, le terme de bravo désigne l'animal d'un naturel sauvage, par opposition à l'animal domestique, qualifié de manso, sa " mansuétude " faisant de lui un adversaire impropre au combat. Dans le champ tauromachique, la bravoure est synonyme de combativité. La noblesse qualifie la façon dont s'exprime cette agressivité qui dans l'idéal se doit d'être franche et sans détour, composée de charges longues, données tête basse. Pour beaucoup d'aficionados, la véritable bravoure implique nécessairement les qualités de noblesse et par conséquent ces deux notions ne sauraient être contradictoires ${ }^{12}$.

Le règlement taurin espagnol précise que toute bête participant à un spectacle taurin doit être inscrite dans le Registre des élevages de taureaux de combat sous tutelle du ministère de la Justice et de l'Intérieur ${ }^{13}$. Pour être inscrits dans ce registre, les animaux de l'élevage doivent être enregistrés par le Livre généalogique de la race bovine de combat dépendant du ministère de l'Agriculture, de la Pêche et de l'Alimentation. En Espagne, tous les taureaux combattus doivent figurer sur le Livre du ministère assurant la provenance de la matière première du jeu. Les élevages sont affiliés à différentes associations professionnelles spécialisées dans l'élevage de taureaux de race brave. L'Espagne en compte quatre : Unión de Criadores de Toros de Lidia (UCTL), Asociación de Ganaderías de Lidia (AGL), Agrupación Española de Ganaderos de Reses Bravas (AEGRB), Ganaderos de Lidia Unidos (GLU) ${ }^{14}$. La plus ancienne est l'UCTL créée en 1905 dans laquelle figurent les élevages espagnols les plus prestigieux ainsi que certains élevages portugais et français. Au Portugal et en France, il existe de même qu'en Espagne des livres généalogiques de races bovines de combat qui enregistrent les animaux destinés aux jeux taurins ainsi que des associations professionnelles regroupant les élevages : au Portugal, l'Asociação Portuguesa de Criadores de Toiros de Lide, en France, l'Association française des éleveurs de taureaux de race brave. Le système est le même dans les pays d'Amérique latine. Au Pérou, il existe deux associations professionnelles d'éleveurs : l'Asociación de Criadores de Ganado de Lidia del Perú et le Comité Nacional de Criadores de Ganado de Lidia. Au Venezuela, l'enregistrement des taureaux de race brave passe par l'intermédiaire d'une association unique l'Asociación Venezolana de Criadores de Toros de Lidia. En Colombie, cette fonction revient principalement à l'Asociación de Criadores de Toros de Lidia de Colombia, mais il en existe d'autres. En Équateur, il s'agit de l'Asociación de criadores de ganado de lidia del Ecuador.

\footnotetext{
${ }^{12}$ C'est l'avis autorisé de José Carlos Arévalo, directeur de la revue taurine 6 Toros 6, qui s'est souvent exprimé sur la dialectique complexe entre bravoure et noblesse.

${ }^{13}$ Règlement des spectacles taurins, titre II : Des registres des professionnels taurins et des élevages de taureaux de combats, chap. II : Registres des entreprises d'élevage de bêtes de combats, article 10 et 11 .

${ }^{14}$ En 1999, on comptait 1.139 élevages affiliés aux associations professionnelles de taureaux de combats répartis de la façon suivante : 448 dans l'AGL, 334 dans l'UCTL, 230 dans l'AEGRB, 127 dans la GLU.
} 
Dans le registre taurin, l'appellation générique de « taureaux de caste » les différencie des taureaux dits de « demi-caste » (media casta), qui sont des animaux de moindre qualité. Les taureaux de demi-caste, qui peuvent localement porter des noms différents (toro morucho dans la région de Salamanque), sont moins chers que les taureaux de caste et leur pedigree, sur le papier, ne garantit pas les qualités éprouvées chez leurs congénères de race pure. Il s'agit souvent d’un bétail plus rustique au comportement désordonné qui ne permet pas l'exécution d'une tauromachie artistique, fondée sur la connaissance du comportement animal et sa nécessaire anticipation acquise par l'expérience. Ces animaux sont très souvent utilisés lors des jeux taurins à participation collective sauf lorsque sont choisis, à dessein, des taureaux de combat d'élevages réputés, comme on l'observe de plus en plus souvent en Estrémadure et surtout lors des bous al carrer des pays valenciens.

Le taureau de race Camargue possède un comportement et un morphotype différent de celui du taureau brave espagnol. D'un point de vue morphologique, le taureau Camargue est plus petit et plus fin que son homologue espagnol. Il dépasse rarement 1,30 mètre de haut et son poids varie de 300 à $450 \mathrm{~kg}$. Il possède des cornes verticales et son pelage est toujours uniformément noir. D’un point de vue comportemental, Frédéric Saumade a montré que le type idéal du bon taureau " cocardier " était opposé à celui de taureau brave espagnol. Les deux partagent une combativité d'exception, mais le " cocardier » doit faire preuve de ruse et de vice pour dérouter les hommes, qualités qui chez le taureau espagnol passeraient pour un manque avéré de noblesse et finalement une défaillance de sa bravoure. Les vaches de la course landaise sont, quant à elles, achetées principalement en Espagne et au Portugal, marginalement en Camargue, par des ganaderos ou ganadères qui traditionnellement s'investissent peu dans la reproduction du bétail, même si l'on note aujourd'hui une évolution allant dans ce sens.

En Amérique latine, les élevages de taureaux braves issus des encastes d'origine espagnole sont réservés aux spectacles du haut de la hiérarchie taurine (corridas et novilladas). Dans les villages, et pour tous les spectacles à participation collective, sont généralement toréés des taureaux dits criollos (créoles) ou des taureaux dits de media casta (demi-caste). Les taureaux criollos regroupent les animaux considérés comme étant de races autochtones. Ces animaux proviennent du bétail introduit par les Espagnols immédiatement après la découverte du Nouveau Monde. Cette introduction, comme nous aurons l'occasion de le préciser, fut d'ailleurs en partie responsable de la progression rapide de la colonisation. Les taureaux de demi-caste peuvent ici désigner tous les taureaux qui ne sont pas exclusivement de race brave, qu'ils soient criollos ou qu'ils aient été croisés avec des taureaux criollos ou avec des taureaux d'autres races comme les zébus. Ces animaux sont le plus souvent issus d'élevages non spécialisés pour le combat, mais dont les propriétaires, dans les régions taurines, se plaisent à constituer un petit cheptel d'exemplaires agressifs, susceptibles de participer aux différents divertissements tauromachiques locaux. C'est le cas par exemple de certains élevages des départements colombiens de Sucre et Córdoba, destinés à 
l'alimentation, constitués à partir d'une race créole ou croisée avec la race zébu, dont les exemplaires les plus farouches participent aux fiestas en corralejas ${ }^{15}$. On remarquera que le comportement des taureaux lors des corralejas peut alterner entre la fuite et les charges mortelles, entre le taureau manso et le taureau bravo. Il n'est pas rare, lorsque les hommes à cheval poursuivent l'animal qui tente de leur échapper, qu'il se retourne soudainement pour les charger ou pour foncer vers des hommes à pied qui se trouvent à proximité. Il en va de même pour les courses de taureaux des villages équatoriens où les animaux sont choisis parmi les exemplaires descendus des pâturages d'altitude par les chagras, les paysans de retour de leur rodéo. Malgré le choix circonspect des organisateurs de spectacles, il est fréquent que les exemplaires donnent un jeu très décevant pour la course de taureau, mais cet aléa fait lui-même partie du spectacle. Sans aller chercher d'autres exemples dans le registre des tauromachies participatives, il arrive parfois dans des corridas formelles qu'un taureau de combat issu d'un élevage réputé ne se prête pas au jeu et ne charge absolument pas. Il sera alors changé pour un autre animal, sous les sifflets du public adressés à l'éleveur si l'opération se répète et si le nouvel animal en piste ne se montre guère plus combatif. Ne perdons pas de vue que la sélection du bétail de combat n'est pas une science parfaitement exacte. Cela permet de rappeler une évidence constitutive de l'espèce bovine : tous les bovins se servent de leurs cornes ou bien sont susceptibles de s'en servir dans une situation d'attaque ou de défense.

Les animaux qui participent aux jeux de la famille des rodéos américains répondent à des critères différents. Dans le cas du Mexique, les jeux taurins utilisent des taureaux mansos, c'est-à-dire des bœufs de labour, d'attelage ou d'embouche, promis à l'abattoir, détournés du circuit de la production de viande. C'est également le cas de l'ensemble des jeux où il est question de courser l'animal : toros coleados du Venezuela et de la Colombie, vaquejada brésilienne et rodéo chilien. Les jeux de monte posent un problème quelque peu différent. Dans le cas du Mexique, Frédéric Saumade observe que la monte du taureau, "diminuée » et " dérisoire » dans la charreada, se voit exaltée et poussée à l'extrême dans le cas du jaripeo professionnel, comme si la monte du jaripeo venait inverser les codes de la charreada ${ }^{16}$. Pour les jeux taurins professionnels où la monte constitue le cour du spectacle comme dans le bull riding étasunien et le jaripeo professionnel, les taureaux sont bien évidemment sélectionnés pour leur combativité, impliquant un travail de reproduction approprié aux exigences du combat. Parmi les taureaux de jaripeo professionnel réputés, citons ceux de la Selección Michoacana, l'une des entreprises organisatrices de spectacles leader au Mexique, créée en 1979 et qui compte aujourd'hui 70 toros de reparo et plus de 200 vaches destinées à la reproduction. Aux États-Unis, citons les taureaux produits par Chad Herrington, Nevada Berger, Page, Casper Vaca, Growney Brothers qui ont acquis une solide réputation. De façon plus

\footnotetext{
${ }^{15}$ Cette race dite raza Criolla Costeña con Cuernos serait issue de la variété du taureau de Galice.

${ }^{16}$ F. SAumade, Maçatl, pp. 31-32.
} 
étonnante en raison des caractéristiques du jeu, la Fédération de rodéo chilien dispose de son propre cheptel d'environ 1.000 têtes, sur un terrain de 500 ha (El fondo El Rodeo), réservé pour l'organisation du championnat national. Ce bétail est élevé et sélectionné pour répondre aux normes de gabarit imposé par le règlement marquant les prémices d'une spécialisation.

\section{LES SOCIÉTÉS 《 TAUROPHILES 》}

En dépit de ce qui pour les détracteurs des jeux tauromachiques fait figure de paradoxe majuscule, les amateurs de tauromachies aiment les taureaux, ils aiment les observer dans les champs, ils aiment les voir combattre et ils aiment les défier. Dans les tauromachies où le taureau se doit d'être combattant, certains ont acquis individuellement une grande célébrité faisant d'eux de véritables héros dont les exploits restent gravés. On le comprend assez bien dans le cas des animaux qui font carrière tels que les taureaux camarguais, les vaches landaises, les taureaux de monte (bull riding et jaripeo) et certains taureaux de corralejas. Cela se produit également pour les taureaux de corridas combattus et tués dans l'arène, dont l'heure de gloire est pourtant plus brève, mais non moins intense aux yeux du public, en particulier lorsque la dépouille de l'animal est récompensée d'un tour d'honneur sous les acclamations de la foule ou lorsqu'il est exceptionnellement gracié pour l'excellence de son comportement. En revanche, les taureaux des jeux taurino-équestres fondés sur la coursepoursuite restent dans l'anonymat, alors que c'est le cheval qui fait l'objet de toutes les fascinations.

Le phénomène est bien connu dans le cas de la course camarguaise où certains cocardiers sont statufiés tel "Le Clairon » de la manade Granon, situé à l'entrée de la ville de Beaucaire qui fut, jusqu'à la fin des années 1930, une grande vedette de la course, tel « Pascalet » de la manade Rebuffat à Lunel, ou encore « le Sanglier » de la manade Granon, inhumé au Cailar en 1933, une stèle rappelant sa mémoire. Pour la course landaise, les coursayres se souviennent de «Challengita » de la ganaderia Maigret, qui remporta la corne d'or à Nogaro de 1961 à 1967. Elle donna son nom au trophée Challengita qui depuis 1992 vient couronner pour les vaches le classement de la saison. Les coursayres se rappellent aussi de Joselita, dans les années 1970, morte dans l'arène, et de Fédérale, dans les années 1990, qui remporta la corne d'or ${ }^{17}$. Le monde des corralejas possède aussi ses célébrités, tel le redoutable taureau criollo «El Barraquete » du département de Sucre dans les années 1970, ou encore « El Chivo Mono », tristement connu pour avoir tué sept personnes lors d'une fiesta en corralejas de Planeta $\mathrm{Rica}^{18}$. Dans le bull riding nord-américain, les taureaux de légende sont ceux sur le dos desquels les monteurs ne parviennent quasiment jamais à rester les huit secondes réglementaires pour considérer la monte valide : Tornado, Oscar, Red

\footnotetext{
${ }^{17}$ Challengita, Joselita, et Fédérale ont été achetées à des éleveurs de la province de Salamanque.

${ }^{18}$ A. Lopera, Colombia, tierra de toros, p. 148. L'auteur cite de larges extraits du livre référence de Juan Santana Vega, El mundo de las corralejas.
} 


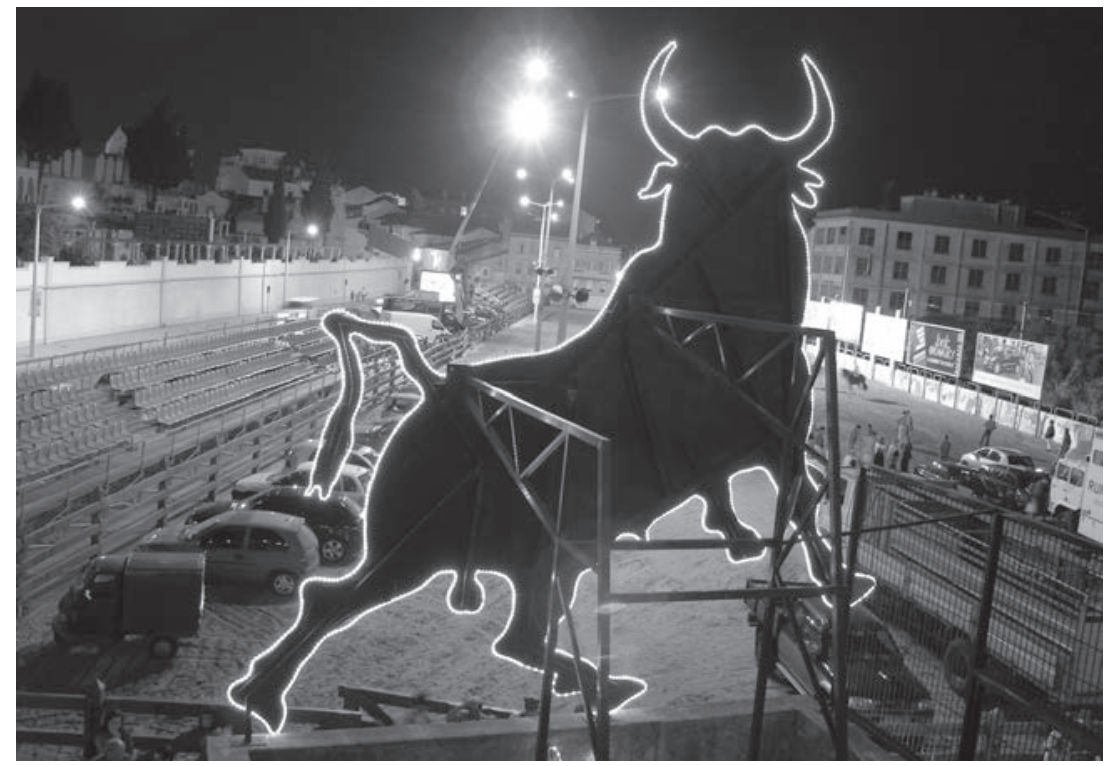

Fig. 37. - Taureau des arènes de Vila Franca de Xira (Portugal). Le taureau illuminé domine la ville pendant les fêtes du Colete Encarnado

(Vila Franca de Xira, 2005, cliché : Jean-Baptiste Maudet).

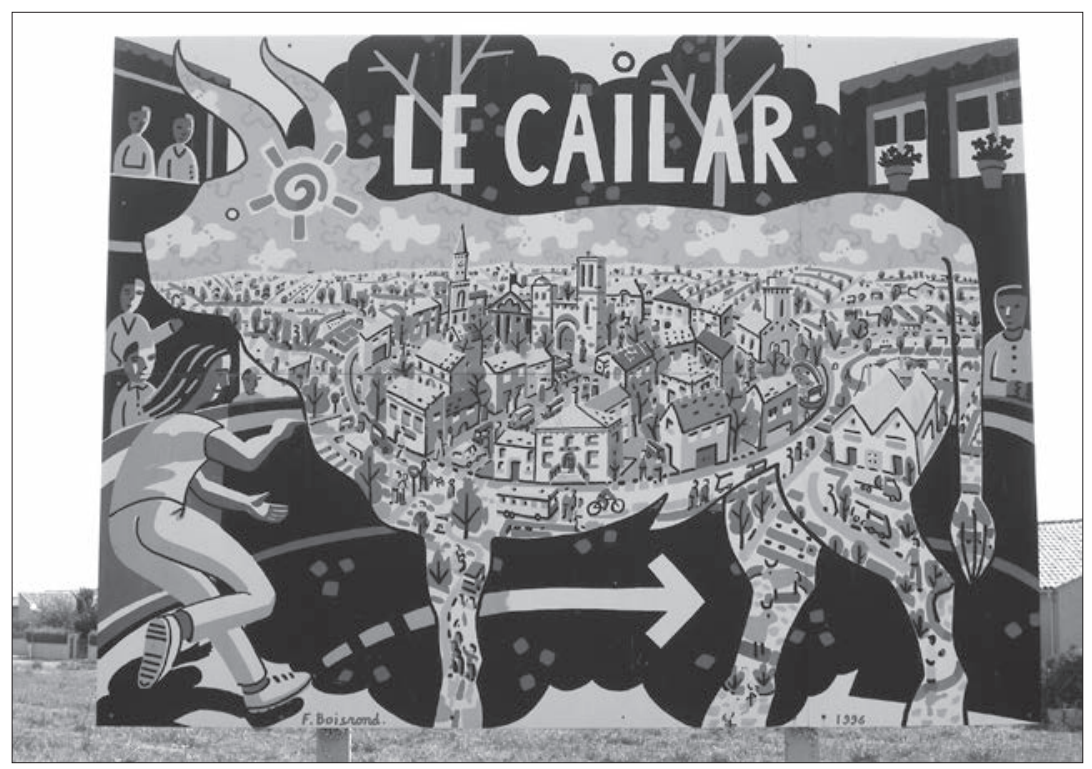

Fig. 38. - Taureau à l'entrée du Cailar (France). À l'image de la ferveur taurine, le village ne se voit pas autrement que dans le taureau

(Le Cailar, 2004, cliché : Jean-Baptiste Maudet). 


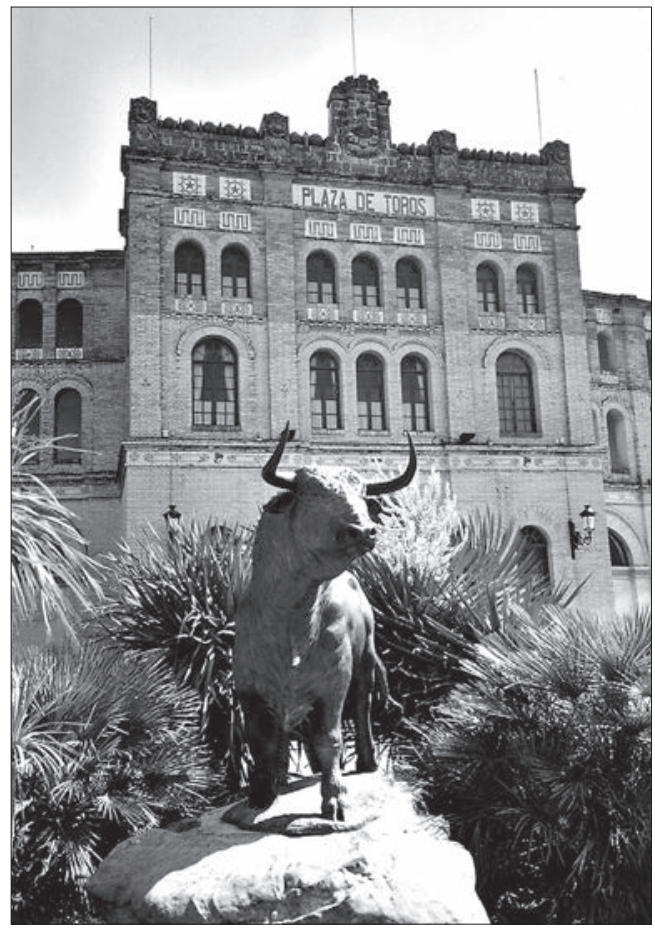

Fig. 39. - Taureau à l'entrée des arènes du Puerto de Santa María (Espagne).

Statue d'un toro bravo, si nombreux en cette province de Cadix

(Le Puerto de Santa María, 2003, cliché : Jean-Baptiste Maudet).

Rock, invaincu de 1984 à 1987 sur 309 tentatives, Bodacious dans les années 1990 avec seulement huit tentatives de monte validées sur l'ensemble de sa carrière, Dillinger et Little Yellow Jacket au début des années 2000, aujourd'hui Voodoo Child encore invaincu. Dans le monde du jaripeo ranchero profesional, certains toros de reparo sont connus dans tout le pays à l'image de "Vinagrillo " de l'entreprise Selección Michoacana. Au Nicaragua, dans la province de Chontales, les années 1990 ont consacré des taureaux de monte inégalables aux dires des habitants de Juigalpa : «El Calentura » et «El Mariposa ${ }^{19}$. Certains élevages locaux ont ainsi acquis une grande notoriété : les taureaux de don Abelino Martínez à Cuapa, ceux de La Quinta Lidia, de don Fernando González, ceux de Concepción González, à San Pedro de Lóvago, ceux de don Julio Miranda, Ramón Laguna, Juan Vinagra, à Juigalpa. Les taureaux célèbres du milieu des années 2000 se nomment «El Medio luto » (le demi-deuil), « El Policía » (le policier), «El Periodista » (le journaliste), «El Vendanal » (le coup de vent) et «El Saca Pedos» (le péteur). La personnification des surnoms confirme que la bête à défier est, dans une certaine mesure, un égal de l'homme.

${ }^{19}$ El Nuevo Diario, 9 avril 2005. Informations tirées de l'article intitulé « La pasión taurina » : http://elnuevodiario.com.ni/2005/04/09/turismo/24816 [réf. 5 février 2006]. 
Dans la culture taurine propre à la corrida, le taureau de combat fait également l'objet d'une forte " héroïsation ", soit qu'il ait fait montre d'une combativité d'exception, soit qu'il ait contribué au triomphe retentissant d'un matador. Certains ont laissé une empreinte profonde sur « la planète des taureaux », tel «Atrevido », passé à la postérité sous le nom du «taureau blanc d'Osborne ", qui permet au matador Antoñete de réaliser à Madrid en 1965 ce que d'aucuns considèrent comme l'une des grandes faenas de sa carrière ${ }^{20}$. Au Pérou, le taureau le plus célèbre reste "Arabi Pacha ", taureau reproducteur de plus de dix ans, "qui en savait davantage qu'un professeur de Salamanque ", tué à Lima le 24 mai 1885 par le plus célèbre des matadors péruvien Ángel Valdez, dont on aura l'occasion de reparler ${ }^{21}$. Certains taureaux obtiennent la grâce autorisée par l'article 82 du règlement taurin espagnol afin d'être utilisés comme géniteurs pour continuer à perfectionner la sélection des taureaux de combat, tel « Belador» de l'éleveur Victorino Martín gracié en 1982 lors de la corrida de la Presse à Madrid ${ }^{22}$. Enfin certains sont restés tristement célèbres pour avoir tué des grands noms de la tauromachie. Limitons-nous à trois sur une liste très longue : «Barbudo », qui tua Pepe-Illo à Madrid le 11 mai 1801; «Bailador» qui tua le torero Joselito à Talavera de la Reina le 16 mai 1920 ; « Islero » de la ganaderia de Miura qui tua Manolete, le 28 août 1947 à Linares ${ }^{23}$. Les taureaux de combat ont suscité une littérature prolifique qui ne laisse aucun doute quant à la fascination qu'ils exercent auprès des aficionados du monde entier. Cette " héroïsation » du taureau de combat est ostensible en terres de corridas, il n'est que d'observer partout les têtes de taureaux empaillées chez les éleveurs, chez les matadors, dans les arènes, dans les musées taurins, dans de nombreux bars et restaurants. En Espagne, les éléments les plus visibles de cette fascination sont peut-être les immenses taureaux des publicités Osborne qui ont fini par être classés au patrimoine national, et repris en autocollant à l'arrière des voitures de nombreux aficionados qui affichent ainsi leur passion pour ce que la tauromachie a de plus essentiel : le taureau. Les taureaux Osborne constituent aujourd'hui un élément du patrimoine culturel espagnol et un marqueur paysager qui symbolise l'Espagne de manière très efficace. Ils sont aussi utilisés périodiquement comme de grands tableaux noirs pour y écrire des revendications liées à la culture taurine, soit pour la condamner, soit pour la glorifier.

\footnotetext{
${ }^{20} \mathrm{La}$ « planète des taureaux » est une expression devenue courante dans le langage taurin, rendue célèbre par le journaliste Antonio Díaz Cañabate. Elle indique que la tauromachie forme un monde à part, presque autarcique, avec sa langue, ses héros, ses mythes, son histoire... et assurément sa géographie. La faena (travail, tache) dans le langage taurin désigne les exercices à la muleta à travers lesquels s'expriment les qualités techniques et artistiques du matador.

${ }^{21}$ H. López Martínez, Plaza de Acho, p. 126.

${ }^{22}$ Le taureau gracié sort vivant de l'arène. Ses plaies sont soignées et il retourne généralement dans son élevage d'origine ou bien il est vendu à un autre éleveur comme reproducteur.

${ }^{23}$ Notons qu'un groupe antitaurin porte le nom provocateur de «Islero Fan Club », traduisant, un amour pour cet animal totalement extérieur à celui des aficionados, qui ne s'inscrit jamais dans une opposition contradictoire entre l'amour des hommes et l'amour des bêtes.
} 


\section{III. - JEUX D’ARÈNES}

L'usage du mot arena, dans le contexte taurin, existe en espagnol, mais il est beaucoup plus rare que le terme de plaza de toros. Luis Nieto Manjón en donne une définition simple : « lieu fermé et disposé pour courir ou combattre les taureaux $»^{24}$. La langue espagnole n'est pas avare de termes pour désigner la piste du jeu taurin, enrichie par une tradition journalistique ancienne qui a contribué à une diversification du langage spécialisé. José María de Cossío recense treize termes dont les connotations peuvent varier en fonction de la nature exacte du signifié. Le ruedo et le redondel font référence au terrain circulaire délimité par les barrières. Ajoutons, parmi les emplois courants, le terme de coso qui est utilisé dans la presse spécialisée comme synonyme de plaza de toros.

En Amérique latine, le terme coso est également employé pour désigner la piste d'autres pratiques tauromachiques que la corrida, tels que les mangas de coleo ou les lienzos charros. On remarque, avec intérêt, le double sens du mot coso en espagnol. Le Diccionario de la Real Academia Española le définit d'une part comme " une place, site ou tout autre lieu circonscrit, où sont courus et combattus des taureaux et où sont célébrées d'autres fêtes publiques ", mais également comme une " rue principale » d'un espace urbanisé. Le terme convient ainsi pour qualifier les espaces de jeu centrés, tels que les arènes de corrida et les espaces de jeu longitudinaux, tels que ceux des mangas de coleo et des lienzos charros. Le mot manga signifie une manche de vêtement, un couloir ou un passage faisant le lien entre les champs et les enclos et désigne, dans le contexte taurin, la piste longitudinale du coleo. Le mot lienzo signifie clôture. Le lienzo charro se compose en réalité de deux parties : l'une longitudinale qui prend la forme d'un large couloir, le lienzo et qui donne le nom à l'ensemble de l'édifice spécialisé. Cette partie est utilisée pour les coleadas et débouche sur un espace circulaire, le ruedo, utilisé pour les autres épreuves de la charreada.

La plupart des pistes de la tauromachie professionnelle peuvent être classées en fonction de ces deux formes, les unes longitudinales, les autres centrées, qu'elles soient plus ou moins rondes, plus ou moins quadrangulaires, régulières ou irrégulières. Les parques de vaquajeda entrent dans la catégorie des pistes longitudinales avec la particularité qu'elles s'évasent dans le sens de la course. Les arènes de corridas espagnoles, portugaises et des courses camarguaises entrent dans la seconde catégorie, même si à l'intérieur de la piste les «terrains » du combat ne s'organisent pas de la même façon. Dans les corridas espagnoles et portugaises le centre de l'arène constitue le terrain le plus important ou tout au moins celui de l'affrontement idéal pour juger de la domination de l'homme sur l'animal. Dans la course camarguaise, c'est la périphérie de la piste qui constitue le terrain privilégié du combat, celle où le taureau se réfugie pour répondre aux assauts des raseteurs. C'est pourquoi, les amateurs de course camarguaise considèrent qu'une bonne piste possède des

\footnotetext{
${ }^{24}$ L. Nieto Manjón, Términos taurinos, p. 347.
} 
coins dans lesquels le taureau aime à se défendre contre ses assaillants. Dans les arènes du rodéo chilien, c'est également la périphérie de la piste qui constitue le « centre» du terrain de jeu, puisque les cavaliers doivent plaquer les taureaux contre les barrières. D'autres cavaliers sont d'ailleurs situés au centre de la piste, en position de spectateurs privilégiés. La course landaise constitue un cas intermédiaire puisque l'écarteur est immobile au milieu de la piste, mais la vache, lancée à pleine vitesse, en exploite toute la longueur. Si l'on précise que la vache est retenue par une corde, on comprend mieux la logique axiale $\mathrm{du}$ jeu et pourquoi certaines arènes de course landaise ne sont constituées que de loges contenant les vaches, surmontées d'une tribune, au-devant desquelles s'allonge une piste sans aucune barrière pour clore l'enceinte. Quant aux pistes du rodéo nord-américain ou du jaripeo professionnel, leur forme ne semble pas tributaire d'une règle du jeu qui les détermine. Les arènes, leur forme et leur taille ne sont donc pas totalement indépendantes des règles du jeu, sans pour autant y être entièrement assujetties. Pour preuve, certaines arènes reçoivent des jeux différents. C'est par exemple le cas pour de nombreuses arènes du Sud-Ouest français où se déroulent des corridas et des courses landaises. Il en est de même pour de nombreuses arènes du Sud-Est où se déroulent des corridas et des courses camarguaises. Pour nombre d'entre elles, en particulier celles du Sud-Ouest, il est souvent inutile de tenter de discerner s'il s'agit d'une arène de course landaise ou de course espagnole, puisque certaines procèdent d'une architecture hybride adaptée à la spécificité de deux pratiques. Ainsi existe-il des logiques incontestables de spécialisation des lieux de mise en scène taurine pouvant se traduire par des incompatibilités entre certains jeux et certaines pistes, sans exclure pour autant une polyvalence des arènes, lorsque les principes ludiques de tel ou tel jeu le permettent.

Ce point a son importance pour étudier les modalités de coexistence spatiale des pratiques tauromachiques. Dans le Sud-Ouest et le Sud-Est français, certaines arènes accueillent des corridas, et respectivement des courses landaises et camarguaises, alors que d'autres ne sont adaptées qu'aux formes tauromachiques régionales ne pouvant accueillir le spectacle d'origine espagnole. Soulignons enfin que le terme même de plaza de toros (place des taureaux exprime une rencontre entre le monde urbain et le monde rural. En tant que place urbaine, beaucoup d'arènes espagnoles reprennent les motifs architecturaux caractéristiques des plazas mayores de la Renaissance, tels que les balcons et les colonnades. En tant que lieu d'enfermement du taureau, les arènes reprennent les barrières et les couloirs des enclos de l'élevage bovin. En tant qu'édifices spécialisés dans le spectacle, les arènes font nécessairement resurgir le précédent historique des cirques romains. De nombreux livres leur sont consacrés témoignant de leur valeur architecturale et patrimoniale ${ }^{25}$.

\footnotetext{
${ }^{25}$ Parmi ceux que nous avons utilisés : P. AlCÁzar, Plazas de toros de Colombia ; CAUE des Landes, Arènes de la course landaise et de la corrida; F. Garibay Anaya et M. Luna Parra, México se viste de luces; M. Luna Parra et L. Ruiz Quiroz, Análisis y registro de las plazas de toros de México ; F. López Izquierdo, Plazas Mayores y de toros.
} 


\section{LES JeUX D'ARÈnes du Sud-Ouest européEN}

Dans la corrida espagnole à pied, le succès du matador dépend principalement du maniement artistique de la muleta lui permettant de maîtriser et de conduire les charges du taureau, ainsi que de l'exécution orthodoxe de la mise à mort d'un coup d'épée porté à proximité du cœur. Dans le détail, la corrida met en présence dans l'arène des taureaux de plus de quatre ans et des équipes de toreros (cuadrillas), composées chacune d'un chef, le matador de toros, également appelé maestro (maître), de trois banderilleros, deux picadors montés sur des chevaux protégés par un caparaçon et un valet d'épée qui n'intervient pas directement dans le combat. La corrida est découpée en trois phases appelées tercios (tiers) : le tercio de piques, le tercio de banderilles et le tercio de mort, conclu par la mise à mort du taureau. Aujourd'hui, le cœur du spectacle réside dans la capacité du matador, avec son style et sa technique, à enchaîner, durant le dernier tercio, les passes de muleta en fonction des caractéristiques de l'animal pour parvenir à le dominer. Une corrida formelle (celle qui constitue la norme et la pratique la plus courante) met à l'affiche trois matadors combattant deux taureaux chacun. Le modèle canonique de l'arène de corrida est celui d'une piste circulaire délimitée par une barrière comprenant des chicanes (burladeros), permettant de se protéger et d'accéder facilement à la piste.

Dans la corrida espagnole à cheval, ou corrida de rejoneo, le cavalier montre la maîtrise de sa monture face aux charges du taureau, en plantant sur son dos des rejones, en passant au plus près des cornes, en guidant la course du taureau lancé à sa poursuite, puis en concluant l'affrontement par la mise à mort de l'animal. Pour chacune des phases du combat (la sortie du taureau, la pose des banderilles et la mise à mort), le cavalier possède une monture appropriée au comportement du taureau et à l'exercice (suerte) à réaliser. Afin de diminuer les risques de blessures du cheval, la pointe des cornes des taureaux est limée conformément au règlement. Généralement, six taureaux par course sont combattus par trois rejoneadores, accompagnés de subalternes à pied dont le rôle reste mineur. De même que la corrida à pied, la corrida de rejoneo est pratiquée en Amérique latine.

Lors d'une tourada formelle de six taureaux, ou corrida portugaise, chaque animal est combattu selon deux modalités bien distinctes, d'abord celle des cavaliers (en général trois par course), ensuite celle de forcados (de une à trois équipes par course). Les cavaliers interviennent individuellement. Ils sont accompagnés de subalternes appelés banderilleros qui en dépit de leur nom ne servent qu'à placer ponctuellement le taureau, soit pour faciliter les manœuvres du cavalier si l'animal ne charge pas, soit pour attirer son attention pendant le changement de monture. Le taureau a les cornes gainées dans un étui pour diminuer les risques de blessures des chevaux et des hommes. Lors de la première phase du combat, le cavalier plante des farpas, équivalent des rejones, puis les banderilles dans un déroulement et selon des modalités d'affrontement très comparables à ceux décrits pour la corrida à cheval espagnole. La performance du cavalier ne se termine pas par la mise à mort du taureau, mais par l'intervention des 
forcados qui réalisent la pega. Les forcados se rangent en file indienne dans l'axe de l'animal et le premier d'entre eux, le chef de file, appelle le taureau pour l'affronter au corps à corps. Pour exécuter la pega, il lui faut parvenir à amortir la charge de l'animal en reculant, se glisser entre les cornes pour prendre son cou à bras-le-corps et rester accroché jusqu'à ce que ses partenaires le rejoignent, les uns après les autres, afin d'immobiliser le taureau. À la différence des corridas espagnoles, l'animal n'est pas tué en piste mais dans les coulisses à l'abri du public, conformément à la loi.

La course landaise est une tauromachie pédestre fondée sur l'esquive de l'animal lancé à pleine vitesse. Les hommes en piste, appelés génériquement écarteurs ou toreros, pratiquent sur une vache aux cornes emboulées des écarts et des sauts. Dans le détail, il existe plusieurs types d'écarts (écart sur la feinte ou écart sur le saut) et différents sauts (saut de l'ange, saut périlleux, saut périlleux vrillé ou saut pieds joints, attachés ou dans le béret). L'écart demeure la technique classique, la plus appréciée par les puristes de la course landaise et les sauts furent pendant longtemps considérés comme des amusements secondaires. L'écarteur placé au centre de la piste cite la vache de face pour provoquer sa charge et au moment où elle utilise ses cornes pour atteindre l'homme, celui-ci l'esquive d'un écart faisant passer la tête de l'animal au creux de ses reins cambrés. La charge de la coursière est en partie maîtrisée par une corde attachée à la base des cornes dont le maniement par le " cordier ", au moment attendu de l'impact, entre en considération pour juger de la qualité des écarts. Le sauteur, dans la même position de départ que l'écarteur, saute au dessus l'animal. Dans la course landaise, la cuadrilla (une douzaine d'écarteurs) est attachée à l'année à une même ganaderia (un troupeau de vaches). Le bétail n'est pas mis à mort et participe ainsi tout au long de sa carrière à différentes courses.

La course camarguaise oppose au taureau cocardier appelé biòu des équipes de raseteurs qui doivent s'emparer des attributs primés fixés sur le frontal de l'animal (une cocarde, un gland et deux ficelles) à l'aide d'un crochet métallique à quatre branches. Pour effectuer le raset, le «tourneur » place le taureau afin de permettre au raseteur de démarrer sa course sous un angle favorable et de croiser la trajectoire du taureau lancé à sa poursuite. La course du raseteur doit se finir en ligne droite en direction de la barrière délimitant la piste derrière laquelle il saute pour se protéger. Le bon cocardier doit poursuivre son adversaire jusqu'aux planches, contre lesquelles il vient buter pour tenter d'encorner l'homme. C'est ce qui est appelé le " coup de barrière ", très apprécié par les afeciounados et aussitôt salué par les premières notes de l'ouverture de Carmen. Chaque taureau reste en piste un quart d'heure avant d'être remplacé. La course camarguaise est également désignée par l'expression de « course libre » datant du $\mathrm{XIX}^{\mathrm{e}}$ siècle, pour différencier le jeu en cours de codification de celui où le taureau restait encordé.

Les courses de recortadores, également appelées concours ou exhibitions de recortadores, regroupent des modalités d'affrontement dont les règles du jeu peuvent varier d'une course à l'autre. On distingue malgré tout plusieurs spécialités dont la codification est établie : les concours d'anneaux (concurso de 
anillas), les concours de recortes libres, les concours de sauts. Selon les concours, on affronte des taureaux ou des vaches dont les caractéristiques comportementales impliquent des techniques adaptées. Les concours d'anneaux se pratiquent le plus souvent sur des vaches, alors que les concours de recortes libres et de sauts ont tendance à se pratiquer de plus en plus sur des taureaux aux cornes intactes. Les protagonistes ont des compétences spécialisées de la même façon que dans les courses landaises, les sauteurs n'écartent pas, et les écarteurs ne sautent pas. L'épreuve des anneaux se pratique par équipe de deux et consiste à enfiler en trois minutes des anneaux d'environ huit centimètres de diamètre sur les cornes de l'animal. La façon d'approcher la vache à demi-retournée ressemble à la technique utilisée par les raseteurs de la course camarguaise où il est nécessaire d'approcher au plus près le frontal de l'animal. Les concours de sauts et de recortes libres se pratiquent individuellement. Le recorte se fait en citant l'animal de face et consiste à décrire un arc de cercle en courant pour rejoindre la course de l'animal, puis, à l'instant de l'impact attendu, à esquiver la charge en cambrant le dos. Cette suerte est assez proche de l'écart de la course landaise. Le recorte peut aussi consister à faire un quiebro, c'est-à-dire à entraîner l'animal dans une direction, puis repartir au dernier moment dans la direction opposée, une fois que le taureau, emporté par sa vitesse, tout en le frôlant, ne peut plus atteindre l'homme. Le quiebro peut s'exécuter de face, de dos ou à genou. Il s'agit de tromper le taureau au plus près des cornes, en donnant la plus grande impression de facilité et de relâchement. Les sauts se réalisent de façon semblable à ceux de la course landaise et constituent désormais une épreuve à part entière. Notons dès à présent que les concours de recortadores constituent une forme tauromachique récente qui n'apparaît qu'à la fin des années 1970 et qui connaît actuellement un succès grandissant dans toute l'Espagne.

Enfin, il faudrait ajouter à ces jeux d'arènes des pratiques issues de l'élevage à mi-chemin entre la sphère ludique et la sphère utilitaire : les tientas. Les tientas servent à tester le bétail pour sa sélection et théoriquement n'entrent pas dans la catégorie des spectacles à proprement parler. Les tientas ont généralement lieu dans les arènes privées de l'élevage ou à " champ ouvert ». C'est lors des tientas à « champ ouvert » que l'on pratique à cheval la technique de l'acoso y derribo pour tester la bravoure de l'animal. La technique consiste à faire chuter l'animal dans sa course avec une garrocha (longue pique) jusqu'à ce qu'il décide d'attaquer la monture plutôt que de fuir, comme s'il finissait par comprendre qu'il s'agit là de la meilleure défense. Les tientas en arènes représentent une opportunité pour de jeunes toreros de faire leurs armes avec l'autorisation de l'éleveur. Elles permettent aussi à des toreros confirmés de s'entraîner pendant la saison creuse pour le plus grand plaisir des aficionados introduits auprès de l'éleveur. Il n'est pas rare que ce dernier se saisisse alors de l'occasion pour recevoir à sa table des amis, des journalistes, des voisins, des membres d'une « peña » taurine et quelques pique-assiettes en tout genre profitant de la prodigalité du seigneur. Certains éleveurs en font une source de revenus complémentaires. En lien direct avec des associations taurines ou des agences de tourisme, ils proposent des journées composées d'une visite de l'élevage, d'un déjeuner dans la propriété et 
d'une capea dans les arènes privées où chacun peut approcher de près la sensation de se trouver en face d'une bête qui charge.

\section{LES RODÉOS DE L'AMÉRIQUE}

La force de l'iconographie nord-américaine donne du rodéo l'image d'un jeu consistant principalement à monter des chevaux sauvages, s'agissant là de l'épreuve la plus ancienne désormais supplantée par la monte des taureaux sauvages considérée comme l'épreuve la plus spectaculaire. Comme nous l'avons évoqué précédemment, il existe bien d'autres modalités de jeu appartenant à cette grande famille panaméricaine.

Dans les vaquejadas au Brésil, l'ouverture des portes pour le bétail est précédée d'un cri «boi saído éboi corrido » que l'on pourrait traduire par « un taureau sorti est un taureau couru ", indiquant le départ du jeu. Ici, les cavaliers courent par équipe de deux (un puxador et un vaqueiro de esteiro ou bate-esteira), à la poursuite d'un bœuf ou d'une vache (boi). L'un des cavaliers (le bate-esteira) veille à faire courir l'animal en ligne droite, le rabat vers son co-équipier et lui transmet la queue du bovin. Celui-ci (le puxador) l'attrape par la queue et le déstabilise pour le faire chuter dans sa course, les quatre pattes en l'air, entre deux marques au sol. Le jeu se déroule sur une piste qui mesure environ 160 mètres. Les cent premiers mètres sont réservés à la poursuite et la prise de vitesse, les dix mètres suivants correspondent aux marquages à l'intérieur desquels il faut faire chuter l'animal, les cinquante mètres restants permettant la décélération du cheval. La piste s'évase progressivement en largeur, de 15 à 20 mètres de large au départ, à 25 à 45 mètres à l'arrivée, formant ainsi une sorte de trapèze allongé. On peut observer que la forme de la piste est la même que celle délimitée par les barrières des mangas (manches) ou embudos (entonnoirs), dressées dans le dernier tronçon des encierros pour mener les troupeaux depuis l'extérieur de la ville jusqu'à l'enclos où ils sont enfermés. La grande différence réside dans le parcours effectué par les bêtes : dans un cas elles courent dans le sens d'un évasement de la piste et dans l'autre, dans le sens d'un rétrécissement.

Les toros coleados au Venezuela et en Colombie reposent sur le même principe. Le cavalier doit rattraper le taureau au galop et le saisir par la queue afin de le faire chuter dans sa course. Le jeu est également désigné sous le nom de coleo, substantif du verbe colear, la cola signifiant la queue. Au Venezuela, le coleo met en compétition quatre coleadores en concurrence à la poursuite d'un taureau sur une piste longitudinale appelée manga de coleo. La piste mesure au minimum 200 mètres de long et entre 10 et 15 mètres de large. La piste est divisée en segments : "zone de préparation » où la coleada ne peut pas être effectuée, " première zone » et " deuxième zone " où la coleada est autorisée, puis «zone morte » où la coleada est à nouveau interdite. Au signal du juge arbitre, le taureau est lâché en piste. Il est pourchassé par les quatre cavaliers. Lorsque l'un d'entre eux parvient à attraper la queue de l'animal, les trois autres doivent se reculer pour le laisser correctement exécuter la coleada. Une 
fois qu'elle est réalisée, le cavalier s'écarte de l'animal et réintègre le peloton des coleadores pour que le jeu recommence et ainsi de suite. Chaque coleada permet de marquer des points selon la zone dans laquelle chute l'animal et selon la façon dont il chute. Les chutes les mieux notées sont les plus spectaculaires. La campana (la cloche) où l'animal se retrouve les quatre pattes en l'air est mieux notée que le costado où l'animal se couche simplement sur le flanc. Lors d'un coleo formel, chaque sortie de l'animal dure cinq minutes et les coleadores concourent deux fois chacun. Des centaines de concurrents interviennent et l'ensemble des épreuves se déroule sur deux jours. En Colombie, les pistes et les notations sont équivalentes à celles du Venezuela, mais les coleadores affrontent le taureau individuellement, chacun leur tour. Il existe de nombreuses façons de colear, à une main, à deux mains, en sautant de cheval ou en passant la queue par-dessous la jambe (colear a arción bolera). Cette dernière technique est caractéristique de la coleada pratiquée à Cuba, au Costa Rica et au Mexique dans le cadre des épreuves de la charreada. Au Panamá, le coleo porte généralement le nom de hierra. L'appellation panaméenne du coleo qui vient du terme hierro (fer de marquage) relie directement le rodéo ludique à l'activité de l'élevage. En Argentine, la hierra, généralement écrite yerra, prend le sens de ferrade, au cours de laquelle, à l'origine, les gauchos rassemblaient le bétail, puis attrapaient les exemplaires un à un au lasso, qu'il fallait ensuite marquer au fer ou qu'il fallait castrer ${ }^{26}$.

La charreada, à la différence des jeux précédents, regroupe un ensemble d'épreuves qui n'incluent pas toutes la présence en piste du taureau ${ }^{27}$. Les épreuves reposent sur le maniement du lasso (la reata), le dressage des chevaux et la maîtrise des bovins. Une charreada est composée de dix suertes différentes : la cala de caballo, les piales en el lienzo, les colas, la jineteada de toros, la terna en el ruedo, la jineteada de yegua, les manganas de a pie, les manganas de a caballo et le paso de la muerte. La cala de caballo consiste à faire galoper le cheval le plus vite possible et le faire s'arrêter d'un seul coup dans une zone déterminée. Les piales consistent pour le cavalier à piéger les pattes arrière d'une jument au galop, en attachant son lasso au pommeau de la selle et en le disposant au sol afin de former un piège (le pial). Les colas correspondent à la coleada décrite dans ses variantes vénézuélienne, colombienne et brésilienne. Ici, le cavalier poursuit seul le bovin et doit le faire chuter dès qu'il a pris assez de vitesse pour être déséquilibré dans sa course, mais avant qu'il ne parcoure une distance de 60 mètres. La jineteada de toro consiste à monter à cru sur le dos d'un taureau jusqu'à ce que l'homme soit désarçonné ou que le taureau cesse de ruer pour qu'il puisse descendre de l'animal. Lors de la terna en el ruedo, trois cavaliers charros doivent maîtriser un taurillon à l'aide d'un lasso, en moins de six minutes. L'un d'entre eux doit attraper la tête, les autres posent le lasso au sol pour piéger les pattes de l'animal et le faire chuter. La jineteada de yegua est

\footnotetext{
${ }^{26} \mathrm{H}$. Armaignac, Viaje por las pampas argentinas.

${ }^{27}$ Pour une description approfondie des techniques de la charreada, F. SAumade, Maçatl, et A. C. Ramírez Barreto, El juego del valor.
} 
la monte d'une jument sauvage. Lors de la mangana de a pie, il s'agit pour le charro à pied de manier le lasso selon des codes esthétiques déterminés (florear la reata), puis d'attraper une jument qui court autour de la piste, pressée par des cavaliers. Cette épreuve se pratique également à cheval (la mangana de a caballo). Le paso de la muerte consiste pour le cavalier à sauter de son cheval sur le dos d'une jument sauvage pour parvenir à arrêter sa course. Chaque épreuve est notée par un jury en fonction de critères techniques très précis. Lors de la compétition dite du charro complet (charro completo), un même cavalier reprend l'ensemble des épreuves. Enfin, il existe pour les femmes qui participent à la charreada, nommées les escaramuzas, des exercices équestres sans taureaux.

Le rodéo nord-américain a beaucoup de points communs avec les exercices mexicains. De même que la charreada, ce rodéo rassemble de nombreuses épreuves, une dizaine au total dont trois mettent en scène un affrontement direct entre l'homme et le bovin. Le calf roping consiste à poursuivre un veau à cheval, à le capturer au lasso, puis à l'immobiliser au sol en lui nouant trois pattes. Le team roping est la même épreuve exécutée à deux cavaliers avec un bétail plus âgé. Le steer wrestling, autrefois connu sous le nom de bulldogging (de " doggie » : bouvillon), consiste à rattraper le bovin à cheval et à le saisir par les cornes en pleine course avant de se jeter à terre pour le retourner et l'immobiliser. Enfin, le bull riding consiste à monter à cru un taureau sanglé par une corde, permettant au monteur de s'accrocher à une main, afin de tenter de rester sur l'animal pendant huit secondes. Les bull riders affrontent des taureaux qui peuvent avoisiner une tonne. Le bull riding connait aujourd'hui un développement croissant en tant que spectacle indépendant des autres épreuves du rodéo nordaméricain. Les bull riders sont aidés par des auxiliaires appelés cowboys protector ou rodeo clowns qui protègent le monteur au moment où l'homme descend du taureau, chute accidentellement ou reste accroché à la bête par la corde lui servant à résister aux ruades. Les rodeo clowns attirent alors l'attention de l'animal pour l'éloigner de l'homme à terre. Récemment est apparue une épreuve qui leur est spécifiquement réservée, le free style bullfighting souvent appelé simplement bullfighting, soit le même nom utilisé en Amérique du Nord pour désigner la corrida espagnole. Nous reviendrons sur cette évolution importante du rodéo nord-américain.

Au Mexique, le jaripeo ranchero professionnel est un jeu très proche du bull riding. Il s'agit, comme ce dernier, d'un spectacle à part entière fondé sur la seule monte du taureau ${ }^{28}$. Le monteur appelé jinete (cavalier) s'accroche également à une corde ceinturant la poitrine de l'animal, mais porte des éperons à crochets permettant de mieux résister aux ruades de leur adversaire qui redoublent d'intensité sous la blessure. Ici, à l'inverse du bull riding, il n'y a pas de limite de temps pour la monte. Pendant longtemps, jusque dans les années 1940, les

\footnotetext{
${ }^{28}$ Pour une analyse ethnographique approfondie du jaripeo ranchero professionnel au Mexique, F. SAumade, Maçatl.
} 
termes de charreada et de jaripeo sont donnés comme équivalents ${ }^{29}$. Dans certains États mexicains, comme celui de Jalisco, il arrive que le terme de jaripeo soit encore utilisé pour désigner la charreada. Le plus souvent, il désigne simplement le jeu prisé dans les zones rurales, consistant à monter à cru les bovins lors des fêtes de village.

Le rodéo chilien se distingue de toutes les autres formes de jeu en présentant une modalité d'affrontement qui n'existe nulle part ailleurs. Le rodéo chilien est pratiqué dans une arène circulaire de 20 à 25 mètres de rayon appelée medialuna (demie-lune) divisée en deux parties inégales par une barrière en arc de cercle. La plus grande partie forme la cancha, la piste principale, et la plus petite forme l'apiñadero. Les deux parties communiquent par des portes permettant les allers et venues des cavaliers et des bovins. Une équipe de deux cavaliers (la collera) prend le taureau en tenaille et le conduit au galop autour de la piste. Le cavalier qui se situe le plus au centre de la piste, lorsqu'il franchit la ligne dite de " posture ", doit faire en sorte que le poitrail de son cheval reste au contact du bovin, obligeant sa monture à galoper de côté. Lorsqu'il pénètre dans la zone réservée à cet effet, le cavalier doit coincer l'animal contre une sorte de butoir rembourré, la quincha. La course et le blocage du taureau (la atajada) sont notés par un jury selon des critères qui tiennent compte de la position du cheval, du suivi de la course et des zones d'impacts portés sur le taureau. Les cavaliers doivent effectuer quatre courses, deux au contact des pattes avant avec deux atajadas, et deux au contact des pattes arrière avec une atajada et un retour à l'apiñadero. Les championnats de rodéo chilien comprennent également des épreuves strictement équestres, les movimientos a la rienda (mouvement les rênes en main) qui comptent dix épreuves distinctes visant à mesurer la virtuosité du cheval et la qualité du dressage.

\section{IV. - JEUX DE RUE}

À la différence des spectacles taurins régis par un cérémonial fixe, des règles $\mathrm{du}$ jeu normatives et une frontière stricte entre acteurs et spectateurs, les jeux taurins populaires se caractérisent par une plus grande informalité et une frontière plus floue entre les acteurs et les spectateurs du jeu. Ces jeux prennent généralement la forme de lâchers de bovins avec lesquels la foule joue de façon élémentaire : faire charger le taureau, le faire courir, le tromper, le fuir, l'esquiver, le poursuivre, le toucher, l'attraper, le monter, l'immobiliser. Des cris, de la poussière, une même effervescence collective donnent un air de famille à ces jeux d'un bout à l'autre de la géographie taurine : qu'il s'agisse d'une course de vaches sur la place Saint-André à Bayonne, d'un encierro au pied des remparts d'Aigues-Mortes, d'une espera sur l'avenue Alves Redol à Vila Franca de Xira (Ribatejo), d'un bous al carrer sur la place San Vicente de Vall de Uxó (Cas-

\footnotetext{
${ }^{29} \mathrm{Ibid}$., p. 80. L'auteur souligne que tous les ouvrages classiques relatifs aux charros, tels ceux d'Álvarez del Villar ou de Rincón Gallardo font l’amalgame entre les deux termes, pendant longtemps indifférenciés.
} 
tellón), d'un toro de pueblo dans le village de Pintag dans la sierra équatorienne de Pinchincha, d'une fiesta en corralejas à Sabanalarga sur la Costa Atlántica colombienne ou d'un jocheo de toros à San Ignacio de Moxos dans le département amazonien de Beni en Bolivie. Dans le détail, on observe des variations géographiques dans les gestuelles privilégiées pour affronter l'animal, variations qui ne sont pas sans rapport avec les aires d'extension des spectacles de la tauromachie professionnelle. On observe également, au sein même des jeux à participation collective, des degrés variables de codification entre ceux aux techniques bien établies et ceux laissant une plus grande part aux improvisations de toutes sortes. L'analyse hésite souvent entre deux hypothèses, l'une selon laquelle les modalités d'affrontement les plus élémentaires constitueraient un fond de pratiques tauromachiques commun de part et d'autre de l'océan, l'autre selon laquelle la diversité géographique des jeux taurins offrirait des spécificités régionales bien plus remarquables. Autrement dit, le geste taurin serait contenu entre une posture universelle liée aux caractéristiques de l'homme et du taureau s'affrontant, d'un côté, et des styles locaux qui précisent ces gestes selon des codes culturels particuliers, de l'autre. En outre, il n'est pas rare que des pratiques très proches sur le plan fonctionnel portent des noms différents en fonction des langues et des emplois régionaux. À l'inverse, un même nom peut désigner, selon les lieux, des pratiques différentes, ce qui n'aide pas à l'identification de ces jeux taurins éminemment protéiformes.

Il n'est sans doute pas possible de séparer totalement les gestuelles adoptées pour affronter l'animal, des caractéristiques architecturales des lieux de mise en scène du jeu taurin. Cette relation est beaucoup moins forte pour les jeux à participation collective que pour les spectacles d'arènes où la configuration de l'aire de jeu participe de la règle et conditionne la gestuelle. Pour les tauromachies participatives, il en va jusqu'à la délimitation même de l'aire de jeu qui semble parfois accessoire. Cependant, les réglementations, pour des raisons évidentes de sécurité, obligent dans la majorité des cas les organisateurs à délimiter la piste taurine par de solides barrières. C'est le cas des cadalfas employées pour les bous al carrer de la région de Valence, ces barrières métalliques verticales, permettant un passage relativement aisé pour les hommes qui souhaitent circuler entre l'intérieur et l'extérieur de la piste, tout en empêchant le taureau de s'en échapper. Ces barrières sont souvent surmontées de tribunes comprenant plusieurs rangées de spectateurs. L'évolution des jeux taurins est allée globalement vers une plus stricte fermeture des espaces de jeu, là où des tonneaux, des charrettes et quelques planches suffisaient autrefois à bâtir l'arène. En dépit des évolutions techniques, tout se passe comme si la tauromachie populaire installait l'arène dans la rue, avec toutes les conséquences symboliques et matérielles que cela implique.

Il est possible de différencier deux types de jeu caractéristiques de la tauromachie participative, d'une part les encierros qui valorisent la notion de distance à parcourir au contact des animaux réunis en troupeau, et d'autre part, les autres lâchers de bovins qui valorisent le face-à-face répété des hommes et de l'animal, généralement seul en piste, évoluant dans un espace plus réduit. Il existe ensuite 
des formes hybrides tenant des deux modalités, et des variantes selon les moyens utilisés pour limiter le rayon d'action de l'animal. L'utilisation de cordes joue, à cet égard, un rôle important dans de nombreuses pratiques.

\section{ENCIERROS...}

Lors des encierros, le jeu consiste à courir des bovins le long d'un parcours sur plusieurs centaines de mètres. Le règlement taurin espagnol donne à l'encierro une définition précise : « la conduite, à pied, sur la voie publique, du bétail à combattre le jour prévu pour un spectacle taurin autorisé par le règlement, depuis le lieu du lâcher jusqu'aux arènes, accompagné au minimum de trois bœufs domestiques $»^{30}$. Dans le langage de la corrida, le mot encierro désigne également le lot de six taureaux destiné à la corrida, lot qu'il est nécessaire "d'enfermer » dans les dépendances de l'arène avant que la course ne commence. Le nom du jeu fait ainsi référence au parcours traditionnel des bêtes, accompagnées vers un lieu clos depuis les champs où elles sont élevées. Le sens du mot encierro témoigne, à lui seul, d'une articulation fondamentale entre la rue et l'arène, entre le champ et la place, entre la campagne et la ville, entre la tauromachie participative et la tauromachie professionnelle.

L'utilisation du mot encierro pour désigner cette pratique apparaît au XIX ${ }^{\mathrm{e}}$ siècle et remplace progressivement celui d'entrée (entrada), sous-entendu entrée dans la ville, que l'on utilise encore au Portugal ${ }^{31}$. Aujourd'hui, l'encierro constitue bien un jeu taurin autonome, que les bêtes soient ou non combattues ensuite, avec ses codes, sa technique et son langage. Pour les « encierristes ", néologisme d'usage fréquent qui désigne les coureurs d'encierros, il existe une véritable technique de course dont la sophistication n'a rien à envier à celles valorisées dans les spectacles professionnels. Pour les encierristes chevronnés, la rencontre idéale avec le taureau se compose de trois phases principales : " entrer, connecter, sortir ». « Entrer» consiste à chercher à se situer dans le flot de l'encierro par une bonne prise en compte de l'ensemble des paramètres dynamiques. «Connecter » permet de se faire voir par le taureau afin de prendre le rythme de sa course, en demeurant le plus longtemps possible à portée de cornes. "Sortir » consiste à rompre le lien qui unit l'homme et le taureau, sans provoquer de mouvement brutal risquant de détourner l'animal de sa course, ce qui constitue l'un des risques majeurs de l'encierro pour tous ceux qui demeurent en arrière ou à côté de l'animal' ${ }^{32}$. Il est bien évident que pour les coureurs d'un jour ces paramètres n'existent pas. Il s'agit de courir et de ne pas se faire prendre, de courir si possible devant les taureaux, et dans le cas contraire derrière, pour les approcher ou pouvoir raconter qu'on les a senti passer. Le plus « professionnel » des encierros courus à pied, au sens où

\footnotetext{
${ }^{30}$ P. Plasencia, La fiesta de los toros, p. 114.

${ }^{31}$ La commission de fêtes de Pampelune estime que le mot encierro a commencé à être utilisé dans ce sens autour de l'année 1856.

${ }^{32}$ Entretien personnel avec le coureur d'encierros et auteur E. De Marichalar, Le souffle dans le dos, chap. IV « L'art de courir les taureaux », pp. 137-162.
} 
de nombreux spécialistes s'y retrouvent, est celui de Pampelune. Il sert partout de référence au point qu'il est possible de voir en lui le modèle idéal de l'encierro navarrais, modèle qui est le plus imité à l'échelle transatlantique.

Il existe des encierros dans la plupart des pays possédant des traditions tauromachiques, certains sont pratiqués à pied, d'autres incluent la présence de chevaux guidant le troupeau. Le parcours de l'encierro peut être entièrement urbain, entièrement rural ou mixte lorsque les taureaux sont encore conduits des champs ou des espaces périurbains jusqu'au cœur de la cité. Le modèle navarrais à pied, que le règlement reconnaît d'ailleurs comme la forme canonique de l'encierro, est celui copié dans le Sud-Ouest de la France lorsque le jeu est à l'affiche. Rappelons néanmoins que la pratique est fort ancienne. Dans le Sud-Ouest, à la différence des encierros espagnols où sont courus les taureaux de la corrida, les participants courent généralement des vaches dont les cornes sont obligatoirement protégées. En Espagne, il existe aussi des encierros de vaches, comme le très réputé encierro de Pilón de Falces en Navarre, qui possède la particularité d'être couru en moyenne montagne dans une zone très accidentée. Ici les cornes ne sont pas protégées et le danger est tout aussi grand que s'il s'agissait de taureaux. Aucun coureur ne ferait preuve de sexisme en la circonstance. Au Mexique, en référence au modèle navarrais, les encierros sont très souvent désignés sous le nom de pamplonadas, sans qu'une réponse précise puisse être apportée sur les modalités exactes de transmission du terme d'un continent à l'autre. Au Mexique, par dérivation de ce premier emprunt, la pamplonada peut également porter le nom de la ville où elle se déroule : la sanmiguelada à San Miguel de Allende, la huamantlada à Huamantla, la xiqueñada à Xico, la Ixtencada à Ixtenco, la santanada à Querétaro organisée par la paroisse du quartier de Santa Ana. Même si la pratique est moins présente qu'au Mexique, il est à noter que le terme de pamplonada est également utilisé au Pérou pour désigner certains encierros urbains. Il est employé à Trujillo pour parler du lâcher de taureaux qui se déroule chaque année le 19 mars, le jour de la San José, patron de la cité, ainsi qu'à Surco dans la région urbaine de Lima. Cet emploi manifeste un lien explicite entre les jeux taurins populaires espagnols et ses avatars latino-américains.

Au Portugal, il existe des encerros à pied, comparables aux encierros espagnols, portant généralement le nom d'entradas (entrées). Le terme d'entrada correspond assez bien à l'irruption du troupeau entrant dans la cité, conduit par les coureurs ou par les campinos au grand galop, qui encadrent le bétail jusqu'aux arènes à l'aide de leur lance appelée pampilho. Ce que l'on appelle abrivado en Camargue est très proche de l'« entrée » portugaise à cheval. Les gardians, trident en main, conduisent les taureaux généralement réunis en troupeau de quatre. Leur tâche consiste à les guider jusqu'aux arènes et à empêcher que les hommes à pied (les attrapaires) ne fassent échapper des bêtes. La bandido relève du même principe que l'abrivado, mais se déroule après la course camarguaise, reconduisant, par un parcours inverse, les taureaux aux élevages ${ }^{33}$. Partout où

\footnotetext{
${ }^{33}$ C. Martel et J.-N. Pelen (éd.), L’homme et le taureau en Provence et en Languedoc.
} 
les zones périurbaines et l'éloignement des zones d'élevages ne permettent pas de réaliser le parcours des champs jusqu'aux arènes, les taureaux sont conduits en camion jusqu'à l'entrée de la ville. Il existe depuis 2004, une « charte des spectacles de rue » approuvée par la Fédération française de course camarguaise, qui stipule que « les taureaux sortiront cornes protégées ou sciées » et précise les conditions requises pour le déroulement de ces manifestations, en particulier celle de fournir des licences en règle pour les manadiers et les cavaliers ${ }^{34}$.

Parmi ces manifestations certaines se déroulent dans des conditions singulières à l'image des abrivado de plage à Palavas, aux Saintes-Maries-de-la-Mer ou au Grau du Roi. Les Saintes-Maries-de-la-Mer organisent le 11 novembre un festival d'abrivado qui regroupe plus de 200 gardians et mille chevaux venus de toute la région. Dans le même esprit, Lunel organise une abrivado de 100 taureaux. Il est à noter que les gardians pratiquent également d'autres formes de jeux dont certains n'incluent pas la présence du taureau, tels que le jeu de l'aiguillette, le jeu des oranges, le jeu de l'épervier ou du bouquet et le tournoi des écharpes. Ce sont des jeux d'adresse qui mettent en valeur les qualités équestres des gardians. En cela, ces jeux se rapprochent de certains exercices des campinos portugais, de certaines épreuves du rodéo nord-américain et de celles de la charreada mexicaine ou du rodéo chilien qui, de même, ne sont pas tous fondés sur la présence en piste du taureau. On remarquera, avec curiosité, l'existence du jeu gardian du saut de cheval à cheval et du saut de cheval à taureau qui formellement se rapproche de certaines épreuves appartenant à la culture du rodéo. Les campinos portugais, en plus d'exercices non taurins, possèdent des jeux incluant des taureaux, autres que les encierros : les picarías. Le jeu consiste à courser un bovin, seul ou à deux, avec la vara de campinar ou pampilho pour orienter sa course. Il existe donc des analogies entre les jeux taurino-équestres de part et d'autre de l'Atlantique, les uns inclus dans le cadre spectaculaire des rodéos, les autres cantonnés au monde des éleveurs de taureaux dans les pays où les traditions d'élevages demeurent fortes. Enfin, toujours attachées à la culture des éleveurs de taureaux camarguais, les ferrades consistant à marquer les veaux, font office de pratiques ludiques auxquelles assistent de nombreux spectateurs lorsqu'elles se déroulent dans les arènes à titre de spectacle ou lorsque les manadiers reçoivent dans leur propriété. De même que les yerras de la Pampa argentine, les tientas des élevages espagnols et portugais, de même les ferrades constituent des moments de convivialité et de reconnaissance sociale.

Ce que l'on nomme encierro en Camargue est différent de son homonyme espagnol, puisque l'encierro camarguais est un lâcher de bovins sur une place délimitée par des barrières. Cependant, l'encierro de type navarrais existe, il est alors qualifié alors d' «encierro à l'espagnole ». Le premier aurait eu lieu à SaintLaurent-d'Aigouze en Petite Camargue en $1985^{35}$. En Espagne, l'utilisation du cheval lors des encierros est minoritaire, mais ne constitue pas une pratique rare

\footnotetext{
${ }^{34}$ Document disponible sur le site Internet de la Fédération française de course camarguaise : www.ffcc.info/IMG/_article_pdf/article_198.pdf [réf : le 10 décembre 2006].

${ }^{35}$ P. Dupuy, Toros en Camargue, p. 37.
} 
comme le montre, en autres, ceux de la province de Guadalajara pratiqués à travers champ. Bien que le règlement l'interdise, des voitures, généralement des $4 \times 4$, sont parfois de la partie, dans un détournement le plus total de la loi et de la tradition. Il existe des encierros à cheval dans d'autres régions d'Espagne parmi lesquels sont célèbres ceux de Ciudad Rodrigo (Salamanque), de Cuéllar (Segovia), de Arévalo et de Segorbe (Castellón).

Si l'on accepte de situer l'encierro à la frontière entre la pratique ludique et la pratique utilitaire consistant à assurer le transfert des bêtes des champs vers l'espace de jeu, on observe que beaucoup d'autres pratiques s'en rapprochent, même lorsqu'elles n'ont pas atteint le même degré d'autonomie vis-à-vis du fondement utilitaire. Dans les Andes péruviennes, Augusto Goicochea Luna raconte dans les années 1960 comment les villageois attendaient avec impatience l'arrivée du troupeau descendu de la puna par les bouviers ${ }^{36}$. Un cavalier en avance prévenait de leur arrivée imminente. Les habitants se cachaient, montaient sur les toits des maisons et sur les branches des arbres. Les plus courageux ou les plus saouls tentaient de se mêler au convoi qui fonçait vers le toril pour enfermer les bêtes. Cette scène n'est pas très éloignée de ce que l'on observe encore en Équateur, sous le nom de rodéo chagra. Le terme de chagra désigne un habitant de la sierra équatorienne et reste attaché au monde paysan particulièrement rustique de l'Altiplano ${ }^{37}$. Le rodéo chagra consiste pour les éleveurs de bétail à partir dans la montagne pour rassembler les bêtes laissées sur les alpages du páramo et à redescendre aux enclos les bêtes devant être castrées, marquées ou vendues. À leur arrivée, se crée un attroupement pour tenter d'accompagner un instant les bêtes. Certains taureaux sont ensuite vendus à des organisateurs de spectacles pour une corrida ou un toro de pueblo.

\section{... ET AUTRES LÂCHERS DE BOVINS}

Les autres lâchers de bovins, dont le principe ludique ne repose pas sur une distance à parcourir, rassemblent une très grande diversité de jeux taurins. Les lâchers de bovins les rassemblerait tous s'il n'était question de mettre en évidence la spécificité des encierros, constituant un jeu charnière entre la rue et l'arène, qui éclaire l'évolution historique des pratiques tauromachiques et leur progressive individualisation au sein de la sphère ludique aux dépens de la sphère strictement utilitaire. Cependant, il arrive que la distinction entre encierro et lâcher de bovins en lieu clos perde de sa pertinence. À Jerez au Mexique, la pamplonada se déroule le long d'un parcours où les taureaux courent en boucle autour d'un vaste pâté de maisons. Cette configuration de l'encierro manifeste un stade avancé d'autonomie du jeu taurin, découplé de la fonction utilitaire puisqu'il n'est ni question de guider les taureaux du champ aux arènes, ni même question d'en reconstruire la symbolique. Une fois que les

\footnotetext{
${ }^{36}$ A. Goicochea Luna, Tauromaquia andina, pp. 26-27.

${ }^{37}$ Le mot chagra viendrait de chacra qui signifie terre où l'on cultive le maïs.
} 
taureaux ont perdu de leur mobilité, le troupeau se désagrège, les animaux se dispersent et répondent aux sollicitations des participants qui les toréent et les montent, comme s'il s'agissait d'un lâcher individuel en lieu clos. Au Portugal, les entradas ou esperas sont généralement suivies de largadas, où les taureaux sont relâchés individuellement sur les lieux mêmes du parcours de l'encierro. À Vila Franca de Xira, le taureau se déplace alors d'un bout à l'autre du parcours, depuis les arènes Palha Blanco jusqu'à l'enclos situé au bout de la rue Joaquin Pedro Menteiro, attiré par les uns, retenu pas les autres, au milieu d'un public qui ne se préoccupe de l'animal que lorsque celui-ci approche, annoncé par des cris et des mouvements de foule. De même, à Moita, les largadas se tiennent le long de l'avenue du Dr. Teófilo Braga. Ici c'est le parcours de l'encierro qui sert d'arène. Les lâchers de bovins varient donc en fonction de la taille des espaces barricadés et de la configuration spatiale des lieux réservés à la tauromachie à participation collective.

Ces lâchers portent des noms différents. En Espagne, les termes les plus couramment utilisés sont sueltas (lâchers), vaquillas, sueltas de vaquillas, toros a la calle, capeas, auxquels il faut ajouter les multiples désignations dans les langues basque, catalane (corre bou) et valencienne (bous al carrer). Localement on utilise d'autres expressions, telle que le toro de aguardiente, souvent utilisée en Andalousie pour désigner un lâcher de taureaux qui se déroule à l'aube, à l'heure où les paysans avaient l'habitude de prendre un verre d'eau-de-vie avant de commencer une longue journée de travail. Dans le détail les lâchers varient en fonction du type d'animal et, dans certains cas, en fonction des gestuelles utilisées pour l'affronter. Dans la région de Valence, qui fait partie des zones où l'engouement est très fort pour les tauromachies à participation collective, les jeux taurins proposent de plus en plus souvent d'affronter des taureaux de quatre ans, aux cornes non protégées et non épointées, issus d'élevages réputés, identiques à ceux présentés en corrida formelle. Cette pratique qui tend à s'affirmer dans les pays valenciens contraste avec toutes celles où les bêtes ont réglementairement les cornes épointées. Nous verrons qu'en la matière les législations régionales diffèrent grandement.

Au Portugal, les lâchers de bovins prennent le nom de largada, touradas a alentejana, de garraiadas ou encore de tourada à vara larga dans certaines localités du district de Portalegre. Les taureaux ont les cornes protégées ou épointées. Au Sud du Portugal, certains lâchers, qui pourtant ne répondent pas au fonctionnement de l'encierro navarrais, prennent le nom de pamplonas. Cet emploi confirme le rayonnement du modèle navarrais de l'encierro dont on a déjà montré les prolongements américains. Au Portugal, il n'est pas rare que les lâchers de bovins populaires ne se terminent par une pega, consistant à immobiliser le taureau à plusieurs à la manière des forcados, sans que cela ne représente pour autant l'objectif ultime du jeu.

En France, il existe aussi plusieurs sortes de lâchers de bovins. Dans le SudOuest, on parle généralement de courses de vaches. Le site officiel des fêtes de Bayonne précise : "Les courses de vaches qui s'y déroulent sont à Bayonne ce que l'encierro est à Pampelune : une tradition, un sport, avec ses règles et ses 
précautions $»^{38}$. Et les organisateurs de les détailler : « Sur le sable, les règles sont bien présentes et gare à ceux qui ne les respectent pas, car les vaches, elles, ne s'y trompent pas. Ne pas taper ces demoiselles, ne pas se jeter sur elles, ni leur tirer la queue mais les faire courir et tourner en bourrique fait partie des us et coutumes des courses à Saint-André $»^{39}$. Elles se rapprochent en cela des courses " mixtes » à la landaise. Il existe également dans la région les manifestations appelées « intervaches » ou « toroballs » qui reposent sur une participation libre. On observera le détournement homonymique cocasse du mot toroballs, qui est semblable au nom désignant les pratiques sacrificielles de l'Antiquité, les tauroboles, pour ici désigner l'organisation d'un match de foot dans l'arène, auquel participe une vachette. En Camargue, comme nous l'avons précisé, les lâchers de bovins en place fermée portent le nom d'encierros. On remarque, non sans parallèle avec les lâchers portugais où l'on pratique la pega, que la technique du raset est ici couramment employée. Là encore, on observe qu'il ne s'agit pas d'une modalité d'affrontement exclusive d'autres techniques puisqu'il est aussi question de donner quelques passes au taureau dès que l'animal le permet. Les " courses de tau » proposent le même type de jeu, mais avec de jeunes taureaux non castrés, testés par les manadiers pour apprécier leur comportement en piste, montrant que pour les éleveurs les jeux à participation collective ne sont pas déconnectés de la pratique professionnelle ${ }^{40}$. Enfin, ajoutons à ces jeux la pratique des «toro-piscines » qui s'épanouit à partir des années 1970, notamment grâce à la popularité que leur donnent les jeux télévisés d'Intervilles. Ils connaissent dans les stations balnéaires du littoral landais et du littoral languedocien un très fort succès en période estivale.

Ces lâchers de bovins sont très courants en Amérique latine où s'ajoutent à la ferveur populaire une gamme d'improvisations et un répertoire gestuel encore plus diversifiés. Aux lâchers de bovins courus (toros del pueblo dans les Andes équatoriennes, corridas populaires dans les Andes péruviennes, toros a la tica au Costa Rica, corralejas dans le Nord de la Colombie, farras do boi sur le littoral de Santa Catalina au Brésil) s'ajoutent les jeux de monte, qu'ils soient indépendants (jaripeo populaire au Mexique, monta de toros à Panamá, Cuba, au Nicaragua) ou pratiqués ensemble (jocheo en Bolivie, corrida de toros au Guatemala, rodeo au Paraguay). Au Pérou, les Andes font figure de véritable conservatoire de jeux taurins où les genres et les techniques se mélangent avec une grande liberté. Le yawar fiesta ou turupukllay répond à cette logique où se combinent des éléments rituels, au premier regard, très étonnants. Les turupukllay mettent en scène un double affrontement : le taureau face au condor fixé sur son dos et les hommes face à cette bête hybride qui rappelle la figure fantasmagorique du taureau-ailé. Toujours au Pérou, parmi les lâchers plus élémentaires, beaucoup ajoutent à une

\footnotetext{
${ }^{38}$ Disponible sur le site Internet : www.fetes.bayonne.fr/index.php?id=103 [réf. du 10 décembre 2006].

${ }^{39}$ Disponible sur le site Internet : $w w w . f e t e s . b a y o n n e . f r / i n d e x . p h p ? i d=103$ [réf. du 10 décembre 2006].

${ }^{40}$ Le tau est un jeune taureau non castré que l'on réserve également pour les « courses de nuit ».
} 


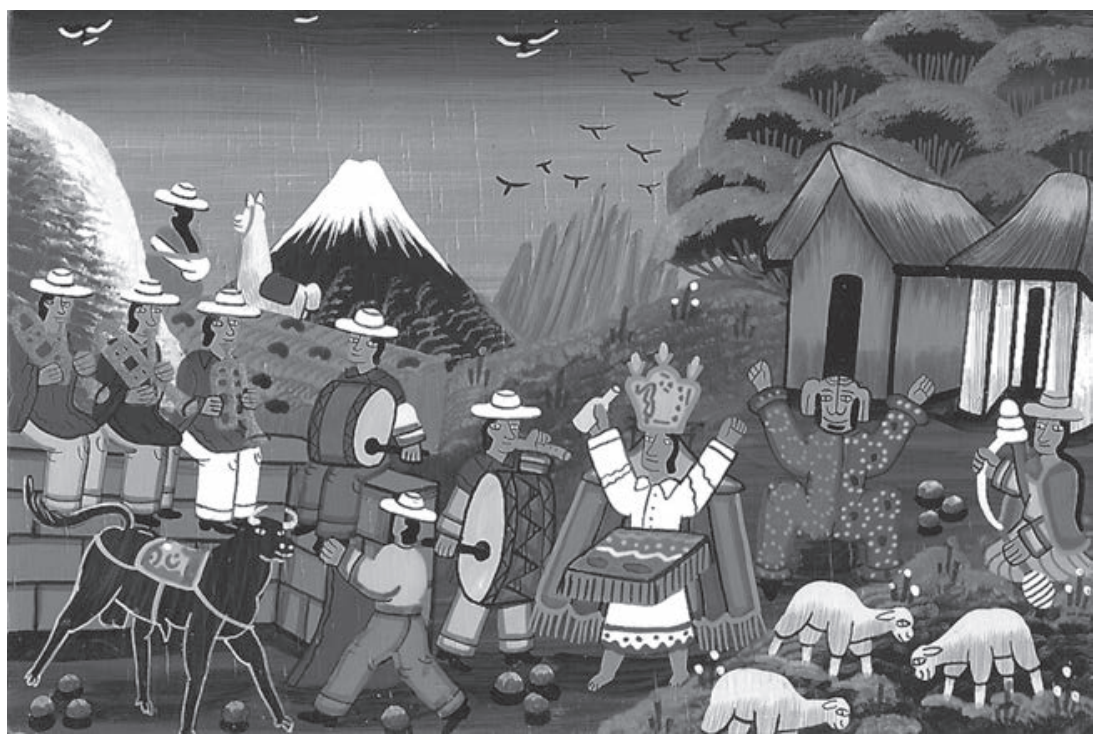

Fig. 40. - Taureau de la fête du Corpus Christi (Andes équatoriennes) sur lequel est fixé la colcha, à moins qu'il ne soit question de le monter. Couvercle d'une boite peinte (collection de l'auteur, peinture sur bois, cliché : Jean-Baptiste Maudet).

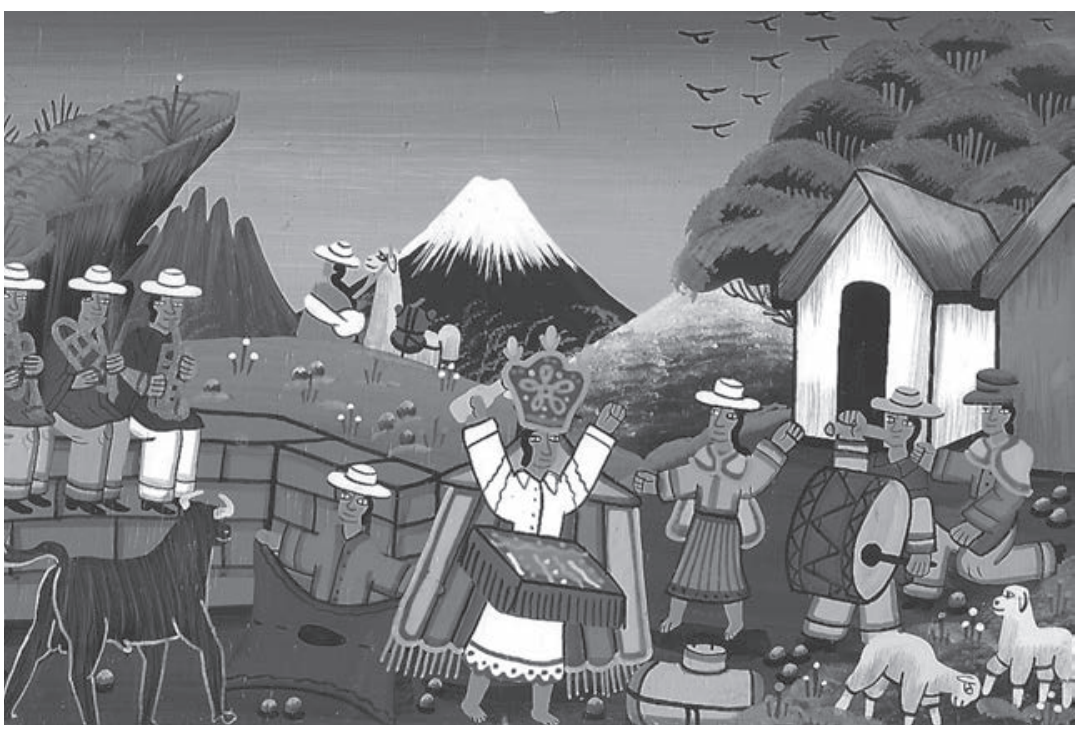

FIg. 41. - Taureau dans la fête du Corpus Christi (Andes équatoriennes), toréé à l'aide d'un poncho dans une arène élémentaire. Couvercle d'une boite peinte. (collection de l'auteur, peinture sur bois, cliché : Jean-Baptiste Maudet). 
tauromachie faite d'esquives et de passes, le défi de décrocher des récompenses fixées sur l'animal : la enjalma et la moña. La enjalma est un tissu richement brodé fixé sur le dos de l'animal à l'aide de petites cordelettes attachées sous son ventre. La moña est également une riche étoffe, fixée entre les cornes du taureau, qu'il est encore plus difficile de décrocher. Un peintre équatorien, spécialisé dans l'art naïf qui fait une large place aux thèmes taurins, nous a confirmé que cette pratique existait aussi dans son pays, en particulier lors des fêtes du Corpus Christi. L'étoffe fixée sur le dos du taureau s'appelle ici la colcha (couverture, toile). [Voir fig. 36 et 40, pp. 29 et 101].

Ces jeux taurins populaires font figure de grands rassemblements, au point que le nombre de participants peut dépasser le nombre de spectateurs des jeux d'arènes professionnels les plus courus. Dans le cas des corralejas du Nord de la Colombie, les arènes construites pour l'occasion sont beaucoup plus étendues que celles des autres jeux d'arènes. La corraleja vient du mot espagnol corral qui signifie cour et enclos. Le mot corraleja désigne à la fois le jeu et l'enceinte du jeu. En cela, il est à rapprocher du rodeo et de l'encierro pour lesquels les termes désignant le jeu sont directement inspirés de l'action de rassembler et d'enfermer les bêtes. La construction de cette grande arène en bois rappelle toutes celles qui autrefois dans les villes et les villages du Sud-Ouest européen étaient ainsi montées et démontées à chaque période de fête. La corraleja la plus connue est celle construite à l'occasion des fêtes de Sincelejo. Elle mesure plus de 350 mètres de diamètre soit environ six fois la taille maximum autorisée par le règlement des spectacles taurins espagnols ${ }^{41}$. Ses gradins bâtis sur deux ou trois étages peuvent contenir entre 5.000 et 10.000 personnes, et sa piste, souvent en herbe, peut accueillir des milliers de participants. D'une certaine façon, l'herbe de cette proto-arène aux grandes dimensions rappelant encore les champs s'oppose au sable imposé par les règlements des plazas de toros urbaines, qui au contraire éloigne le dispositif d'accueil des taureaux des espaces ruraux dont ils sont issus. Les autres corralejas n'ont pas les dimensions exceptionnelles de celle de Sincelejo, mais témoignent d'une même caractéristique commune à beaucoup de tauromachies à participation collective : le renversement fréquent des proportions entre la piste et les gradins. Chacune dans leur style, académique et populaire, les arènes de Mexico et celles de Sincelejo sont les deux plus grandes arènes du monde : la première pour la contenance de ses gradins, la seconde pour les dimensions de sa piste. Â beaucoup d'égards, les corralejas représentent un stade intermédiaire de jeu, volontairement bloqué dans son évolution sous une forme semi-professionnelle et ruralo-urbaine.

La pratique la plus représentative de la tauromachie de rue, dans ce qu'elle a de plus anarchique et chaotique, est probablement celle des farras do boi, dans l'État brésilien de Santa Catarina. Cette pratique semble être passée totalement inaperçue de ceux qui s'intéressent à la diversité des jeux tauromachiques. Il s'agit ici de poursuivre un animal « lâché dans la nature », de jouer avec lui, de

\footnotetext{
${ }^{41}$ Règlement des spectacles taurins : titre III. article 18, « La piste des arènes permanentes ne doit pas avoir un diamètre supérieur à 60 mètres ni inférieur à 45 mètres ».
} 
le harceler jusqu'à épuisement et dans certains cas jusqu'à sa mort pour ensuite répartir la viande entre les participants. Cette pratique est officiellement interdite, mais continue de se dérouler dans une semi-clandestinité, l'intervention des forces de l'ordre pour l'empêcher n'étant pas toujours efficace.

Généralement, lorsque tombent les barrières de la tauromachie participative, les cordes attachées aux taureaux se tendent pour limiter le rayon d'action de l'animal. L'encordement des bovins à la base des cornes pour manipuler le bétail est une pratique ancienne $\mathrm{e}^{42}$. Frédéric Saumade considère que cette technique est « la forme la plus archaïque du jeu taurin de rue et, hormis les Landes, la plus éloignée du moderne jeu d'arène $»^{43}$. Les jeux taurins populaires fondés sur l'utilisation d'un taureau encordé possèdent des noms différents : toros enmaromados, toros ensogados, toros de sogas, toros de cuerda en espagnol, bou de corda et bou ensogat en valencien, bou capllaçat en catalan, sokamuturra en basque. La corde permet de limiter le rayon d'action de l'animal et constitue un ressort ludique en limitant partiellement ses déplacements dans l'espace. Contrairement aux pratiques où les taureaux libres de cordes nécessitent une fermeture de l'aire de jeu, les taureaux encordés se passent généralement de tels aménagements. Pour des raisons qui tiennent aux traitements subis par l'animal, cette pratique est interdite dans certaines régions, comme Madrid et la Navarre, alors que beaucoup d'autres régions leur accordent une légalité d'exception justifiée par l'ancienneté de la tradition. C'est le cas en Andalousie où, à ce jour, douze villages ont obtenu une dérogation les autorisant à pratiquer le taureau à la corde : Villalba del Alcor dans la province de Huelva, Benamahoma, Benaocaz, Grazalema, San Roque et Villaluenga del Rosario dans la province de Cádiz, Gaucín dans la province de Málaga, Carcabuey dans la province de Cordoue, Arroyo del Ojanco et Beas de Segura dans la province de Jaén et Ohanes dans la province d'Almería. Certaines fêtes où se déroulent des «taureaux encordés » ont acquis une grande renommée, due à un effort de valorisation des traditions locales. Tel est le cas du toro de cuerda de Grazalema, petit village de la sierra de Grazalema (Cádiz), de celui de Gaucín (Málaga), du toro ensogado de Beas de Segura (Jaén), du toro enmaromado de Benavente (Zamora), du toro ensogado de Teruel, du toro de sogas de Rubielos de Mora (Teruel) en l'honneur duquel a été érigé un monument, et enfin du bou capllaçat d'Amposta (Tarragona) ${ }^{44}$.

Cette pratique qui pourrait apparaître sur le continent comme une exception minoritaire parmi les lâchers de taureaux est la norme sur l'île Terceira des Açores. Les touradas à corda connaissent en effet un très fort succès sur cette île et constituent la forme établie de la tauromachie populaire ${ }^{45}$. Le taureau

\footnotetext{
${ }^{42}$ S. Rodríguez Becerra, « Poder y fiestas populares con toros en Andalucía », p. 77. Selon l'auteur la mention la plus ancienne daterait de 1328.

${ }^{43}$ F. SAUmade, Maçatl, p. 19.

${ }^{44}$ À Rubielos de Mora, le monument du taureau à la corde est l'œuvre de l'artiste local, José Gonsalvo.

${ }^{45}$ V. Nemésio, Mau tempo no canal.
} 
encordé doit rester dans une limite spatiale déterminée par un marquage au sol à l'intérieur duquel il est couru par la foule. Les cordiers qui souvent travaillent dans les élevages de taureaux sont responsables des dégâts qui surviendraient à l'extérieur de l'aire de jeu. On retrouve cette pratique dans quelques cités californiennes de la San Joaquin Valley en lien avec la communauté des Açores qui y réside en nombre. Les taureaux à la corde existaient également en Camargue. Ici, le dernier bastion d'une pratique aujourd'hui interdite demeure celui de la commune d'Eyragues. Baptisé d'un nouveau nom, l'« encierro à l'eyraguaise » se déroule dans un espace délimité par des barrières conformément à l'évolution générale des jeux pratiqués sur la voie publique.

Les taureaux embolados des pays valenciens, ainsi que les bous capllaçats $e$ embolats du Sud de la Catalogne offrent encore une autre façon d'intégrer la corde comme ressort ludique du jeu taurin. Les termes mêmes de taureaux emboulés méritent un examen précis. L'expression est utilisée au Portugal pour qualifier les cornes du taureau de combat qui sont aujourd'hui épointées et gainées dans un étui de cuir et sur l'extrémité desquelles on fixait autrefois des boules, afin de réduire les risques. L'expression est également utilisée dans la course landaise pour parler des cornes " tamponnées » qui, peu avant 1890, recevaient des boules en fer ou en plomb, puis en bois. En Espagne, les taureaux emboulés de la sorte pour réduire les risques de blessures existent, mais ils sont assez rares. La technique de l'épointage des cornes (l'afeitado) est préférée lorsqu'il s'agit de réduire réglementairement le danger. En Espagne, un toro embolado désigne plus souvent un " taureau de feu » (toro de fuego), animal sur lequel est fixée une armature métallique, imitant grossièrement la forme des cornes, surmontée de boules inflammables. Cette technique a remplacé l'application sur les cornes elles-mêmes de substance inflammable. Dans les pays du Levant espagnol, l'action d'embouler le taureau constitue aujourd'hui une pratique ludique autonome qui fait l'objet de concours chronométrés, soit dans les arènes, soit sur la place publique. Le taureau sort en piste encordé, puis il est guidé grâce à la corde contre une poutre verticale, où un groupe d'emboladores fixe la structure sur le la tête du taureau, enflamme les torches chargées de matériel pyrotechnique, puis libère l'animal en coupant la corde, tout cela en un minimum de temps. Le taureau est ensuite couru dans la rue ou dans l'arène, généralement de nuit. Au même titre que pour les taureaux encordés, certaines provinces ont interdit cette pratique ou l'ont limitée à quelques exceptions bénéficiant de mesures dérogatoires (toro de júbilo de Medinaceli dans la province de Soria). Ailleurs, en France, en Espagne, au Portugal et en Amérique latine, les toros de fuego ne sont que des armatures chargées de pétards et de fusées, prises en charge par un ou deux individus qui reproduisent la furie d'un véritable taureau lâché au milieu de la foule.

Enfin, ajoutons un certain nombre de jeux, aux modalités d'affrontement uniques en leur genre, que nous pourrions qualifier de hors normes si ces normes n'avaient pas déjà été suffisamment malmenées par la dimension protéiforme des tauromachies collectives. En Espagne, deux d'entre elles attirent chaque année l'attention des médias et font l'objet de violentes polémiques, 
opposants contempteurs et thuriféraires : le toro de la Vega à Tordesillas et les lâchers de taureaux de Coria. Le toro de la Vega est une sorte de chasse à courre populaire : un taureau est lâché du centre de la cité vers les champs dans un parcours inverse à celui de l'encierro. Une fois que l'animal a franchi la limite de la ville, la chasse est ouverte par des hommes à pied et des cavaliers qui doivent l'abattre à la lance. À Coria, lors des lâchers de taureaux sur la place publique, les participants lancent sur le taureau de grosses fléchettes ${ }^{46}$.

Comment hiérarchiser les critères de différenciation des pratiques tauromachiques ? La différence entre les jeux faisant l'objet d'une représentation spectaculaire qui incombe à des spécialistes et les jeux à participation collective, nous paraît être de la première importance. C'est ce critère qui permet le mieux de poser les bases d'une lecture des jeux taurins interrogeant les liens entre les hommes et leur territoire. Ces deux grandes modalités de loisir taurin ont une inscription spatiale toute différente qui localement détermine une partie des relations complexes unissant à travers la fête, territoire et tauromachie.

\footnotetext{
${ }^{46}$ Cette pratique apparaît dans des miniatures du XIII ${ }^{e}$ siècle et, selon Ángel Álvarez de Miranda, les banderilles plantées par les toreros pourraient être les avatars modernes de ces petits harpons lancés par la foule. Á. Álvarez de Miranda, Le taureau. Rites et jeux, pp. 94-96.
} 



\section{LA TAUROMACHIE SOUS LE SIGNE DE LA FÊTE}

Reprenons l'axiome des relations entre la fête et la tauromachie dans les régions de tradition taurine tel qu'énoncé en l'introduction de notre travail : peu de tauromachie sans fête, peu de fêtes sans tauromachie. Dans ces régions, il est rare en effet que les jeux taurins ne soient pas parties prenantes des réjouissances festives des calendriers civils ou religieux. Partout la fête et la tauromachie semblent indissociablement liées au point qu'elles finissent par être assimilées à un seul et même phénomène de société : les fêtes taurines. Derrière cette évidence en trompe-l'œil, se cachent des réalités sociales et géographiques très hétérogènes. Cette hétérogénéité tient d'abord à la nature même des phénomènes festifs qui exigent d'être qualifiés pour être significatifs. Les recherches sur les phénomènes festifs montrent qu'il s'agit d'un objet complexe. De quelle fête parle-t-on ? Parle-t-on de ce qui est fêté, de l'objet de la fête ou parle-t-on de faire la fête ? Parle-t-on du jour de fête officiel, déclaré, institué, orchestré ? Parle-t-on de festoyer dans la démesure, l'exubérance et la spontanéité ? Cette hétérogénéité tient aussi, comme toujours, à la diversité des pratiques tauromachiques qui ne s'inscrivent pas toutes dans le même rapport à la fête et montrent qu'il existe des formes multiples de l'effervescence festive. La différence entre les spectacles de la tauromachie professionnelle et les jeux de la tauromachie à participation collective prend ici tout son sens.

En sciences humaines, les fêtes ont très souvent été reliées au sacré. En effet, la fête est, soit considérée comme un événement qui prend sa source dans le sacré, soit comme un temps social qui, par sa " ritualité », reconstruit une forme de sacralisation. Jean Duvignaud y reconnaît l'influence de l'École française de sociologie selon laquelle « la fête est une manifestation du mana et correspond à la catégorie du sacré, laquelle s'oppose aux moiteurs et aux incertitudes de la vie profane $»^{1}$. Nous pensons que l'indissociabilité de la fête et de la tauromachie, qui relève d'une construction sociale et sémantique, a en partie contribué à renforcer une lecture religieuse des ritualités taurines. Autrement dit, le regard porté sur la tauromachie, n'est pas le même lorsqu'on en parle comme une fête,

\footnotetext{
${ }^{1}$ J. Duvignaud, Fêtes et civilisations, p. 53.
} 
un spectacle, un jeu ou un sport. Si la dimension rituelle des phénomènes festifs est indiscutable, admettons comme point de départ qu'il existe des rites profanes et que la notion de rite est valable dans une acception qui déborde largement le champ religieux. Comme le souligne Claude Rivière : « Entre l'habitude classique de restreindre le rite au domaine du sacré et la tentation de dire rituels tous les comportements routiniers, le spectre du repérage et de l'explication du rite demeure vaste. Les définitions doivent être précisées et nuancées, celle de M. Mauss (acte traditionnel efficace qui renvoie à des choses appelées sacrées) comme celle de J. Beattie (acte social symbolique) $»^{2}$.

\section{I. - FÊTES ET TAUROMACHIES : UNE RELATION À INTERROGER}

\section{LA TAUROMACHIE ENTRE FÊTE ET SPECTACLE}

Toutes les pratiques tauromachiques semblent s'inscrire dans une culture de la fête très affirmée. En Espagne, dans l'univers de la corrida, le mot fiesta suffit à désigner la tauromachie. L'histoire de la tauromachie présentée par José María de Cossío dans son ouvrage Los Toros s'intitule : « la Fiesta depuis ses origines jusqu'à nos jours $»^{3}$. Cette association est reprise par un large champ sémantique où les frontières entre fête et jeu taurin se dissipent : fiesta taurina, festejos taurinos, fiesta brava, fiesta de los toros ou tout simplement fiesta. Mieux, en Espagne, l'appellation controversée de fiesta nacional, dans un pays où le concept de nation apparaît comme éminemment problématique, est encore couramment employée pour évoquer les corridas et pour ce qui relèverait d'une relation identitaire particulièrement intense entre les Espagnols et la tauromachie. Notons d'emblée que cet emploi relève aujourd'hui davantage d'une formule héritée de l'histoire que d'un label officiel à caractère idéologique. Il n'en demeure pas moins qu'une relation forte unit la fête et la tauromachie, dans les mots et dans les faits. Le Diccionario de la Real Academia Española donne comme définition à l'expression "corrida de toros": " une fête qui consiste à combattre un certain nombre de taureaux sur une place fermée $»^{4}$. D'un point de vue strictement définitionnel, il est symptomatique que le terme de jeu ou de spectacle ne lui ait pas été préféré. De même, la loi taurine du 4 avril 1991 sur les compétences administratives en matière de spectacles taurins parle, dès la première phrase, du régime juridique de la Fiesta de Toros et entend instaurer les meilleures conditions pour garantir « la pureté de la fête $~_{5}$. Il apparaît clairement que la fête taurine n'est pas un spectacle comme les autres et a fait l'objet d'une certaine codification d'État.

\footnotetext{
${ }^{2}$ C. RIVIÈRE, Les rites profanes, p. 10.

${ }^{3}$ J.-M. de Cossío, Los Toros, t. I, p. 17.

4 «Corrida de toros ", dans Diccionario de la Real Academia Española. Disponible sur le site Internet : www.http://buscon.rae.es/draeI.

${ }^{5}$ Loi 10/1991 sur le règlement des spectacles taurins. Exposé des motifs, article 1 et 2.
} 
En Espagne, le temps long de la tauromachie aurait-il fini par amalgamer les distinctions conceptuelles entre rite, fête, cérémonie officielle, spectacle et jeu ? Comment expliquer que la fiesta (taurine) tende à les réunir dans un fait social qui semble ne pas tenir compte de ces distinctions conceptuelles. On sait qu'à partir du XI ${ }^{\mathrm{e}}$ siècle, la noblesse espagnole prend l'habitude de donner des courses de taureaux en place publique pour célébrer les naissances, les baptêmes, les mariages princiers ou la canonisation d'un saint. Les autorités locales des villas financent également des corridas votives pour que les saints intercèdent en leur faveur à l'occasion d'une épidémie ${ }^{6}$. La Renaissance entretient à merveille une ambiguïté baroque où la fête est un spectacle et le spectacle une fête. Certains défunts ordonnent sur leur testament que l'on donne une course de taureaux au bénéfice de leur âme $e^{7}$. Le peuple possède aussi ses propres jeux où l'on court les taureaux à l'occasion des noces ${ }^{8}$. Les marchés médiévaux avaient déjà rapproché les activités commerciales, les foires, les fêtes municipales et le divertissement des foules. Au XIX ${ }^{\mathrm{e}}$ siècle, les spectacles taurins se greffent aux foires commerciales, les ferias. Les foires aux bestiaux des régions d'élevage ne sont-elles pas alors des occasions idéales, comme la célèbre feria de Séville officialisée en 1846 ? Quoi qu'il en soit de ce processus sur lequel les historiens n'ont pas encore fait toute la lumière, le divertissement taurin, parmi les événements programmés de la fête officielle et parmi les distractions de la foire commerciale, apparaît bien comme le grand moment d'effervescence communautaire au point qu'il accapare et cristallise les attributs de la fête : le temps et le lieu d'exception, le rassemblement, le tumulte et l'enthousiasme. Les fêtes taurines résulteraient-elles d'une fusion lente du rite, de la fête, de la foire, du spectacle et du jeu taurin, dans le moule de la représentation où s'amalgament le fond, le prétexte et la forme?

On aurait pu croire cet effet réservé à l'Espagne et aux corridas. En réalité, cet amalgame se retrouve dans l'immense majorité des pratiques tauromachiques et bien au-delà des frontières espagnoles, comme si la tauromachie était prédisposée en terres taurines à occuper une place à part dans les phénomènes festifs, aussi sûrement que le font universellement la musique, la danse, la gastronomie et l'ivresse des sens. En Amérique latine, l'univers de la corrida s'inscrit aussi dans une égale intimité avec la fête faisant des courses taurines plus qu'un simple spectacle. L'exemple du Pérou est très significatif. Dans ce pays, l'article 5 de la loi taurine précise que « l'État pourra adopter des mesures destinées à promouvoir et protéger les activités auxquelles se réfère la présente loi, pour garantir le maintien de la tradition et de la culture de la fiesta de los toros ${ }^{9}$. Dans le texte introduc-

\footnotetext{
${ }^{6}$ A. García-Baquero González et alii, Sevilla y la fiesta de los toros, p. 17.

${ }^{7}$ Ibid., p. 18.

${ }^{8}$ Á. Álvarez de Miranda, Le taureau. Rites et jeux, pp. 89-96.

${ }^{9}$ Loi taurine du 27 juillet 2001, article 5 (mesures de promotion) alinéa a. L'auteur de cette loi, Marcial Ayaipoma Alvarado, était alors président du Congrès de la République péruvienne. Il est également éleveur de taureaux de combat (les élevages « La Huaca » et « Lomas de Villa »). Il fut pendant longtemps l'organisateur des spectacles des arènes de Lima. Il est président de la " peña » taurine « Perú » et Membre actif et honoraire du Cercle des journalistes taurins du Pérou.
} 
tif exposant les motifs de cette loi, il est mentionné que « les corridas de toros ou festejos taurinos, sont parties prenantes de la tradition culturelle du Pérou, puisque des corridas sont célébrées depuis la fondation de Lima, ville capitale en 1536, spectacles qui sont considérés comme Fiesta Nacional dans le pays ${ }^{10}$. L'occurrence de l'expression fiesta nacional pour désigner dans le contexte péruvien un spectacle que chacun sait d'origine espagnole donne la mesure des logiques complexes d'acculturation liées aux activités tauromachiques dans les pays latino-américains.

Cette relation entre la fête et les taureaux dépasse le cadre de la culture liée aux corridas. Dans le Nord de la Colombie, les fêtes taurines populaires s'épanouissent sous le nom de fiestas en corralejas. La corraleja désigne l'enceinte du jeu tauromachique invariablement associée à la fête qui s'y déroule. Les fiestas en corralejas sont très proches d'une ambivalence absolue entre la fête et le spectacle qui rendrait les deux notions parfaitement inséparables. L'enceinte est ouverte toute la journée aux initiatives du public au milieu des taureaux lâchés successivement. Le soir venu, c'est dans la corraleja que se tient le bal et que se poursuit la fête. La fiesta en corralejas désigne donc d'abord le jeu taurin, mais aussi l'ensemble de la séquence festive qui s'organise autour de lui.

Au Brésil, dans l'État de Santa Catarina, l'expression de farra do boi a remplacé, depuis les années 1970, les autres noms qui désignaient les divers jeux taurins, en particulier le boi-no-campo (le taureau dans le champ), boi-na-varra (taureau à la corde). Cette transformation sémantique qui rehausse la notion de fête (farra) peut être interprétée comme la nécessité de considérer les traditions festives locales et les jeux taurins comme un tout face aux tentatives de les faire disparaître ou d'épurer les fêtes traditionnelles des éléments problématiques aux yeux du regard extérieur. Il en va de même dans la famille des rodéos latino-américains. Au Mexique, les termes charreada et fiesta charra sont employés presque indifféremment avec l'idée que l'une ne va jamais sans l'autre. Au Chili, il n'y a pas de fiesta huasa sans rodeo, pas plus qu'il n'existe de rodeo chileno sans fiesta. Au Venezuela et en Colombie, les expressions fiesta del coleo et toros coleados sont interchangeables, le jeu tauromachique constituant la clé de voûte de toute la fiesta llanera, la fête rurale des llanos. L'élection d'une reine de la fête, les combats de coqs et le bal, dansé au rythme ininterrompu du joropo, cette musique locale interprétée à la guitare et à la harpe qui accompagne aussi les concours de joutes oratoires rimées, viennent toujours compléter les toros coleados. Ces jeux taurins apparaissent bien comme la raison d'être de la fête et du rassemblement, son noyau dur, sa substance active, son facteur coagulant qui rassemble les différents ingrédients de la fête.

D'un point de vue lexical, la relation d'indissociabilité entre fête et jeux tauromachiques n'est pas facilement transposable d'une langue à l'autre, même lorsqu'apparaît une logique similaire. Cette relation est moins immédiatement appréciable en anglais, la terminologie anglo-saxonne faisant généralement

\footnotetext{
${ }^{10}$ Exposé des motifs du projet de loi 0077.
} 
fusionner la fête et le spectacle dans l'univers démonstratif du show. Le rodéo nord-américain y trouve son mode d'expression rutilant : musique country, mode vestimentaire attachée au Far West (chapeau, jambières en cuir, bottes, chemise à manches longues boutonnées, Stetson). Le rodéo apparaît bien, à la fois, comme le sport, le spectacle, la fête et le show du cow-boy.

La langue française est en apparence moins marquée par cette assimilation quasi systématique, mais la réalité sociologique est bien la même. Dans le cas de la corrida, un exemple très concret et riche d'enseignement en donne la mesure : celui de l'annulation de la corrida de la féria des Vendanges de Nîmes en raison des événements du 11 septembre 2001, survenus le mardi précédent à New York. Rappelons d'abord que le mot féria est la transposition directe du mot espagnol feria qui signifie foire et qui désigne dans le contexte taurin, un ensemble de courses de taureaux programmé à l'occasion d'une fête. Cette acception du mot féria est valable en péninsule Ibérique, en France et en Amérique latine. Précisons qu'en France, la féria est par définition taurine, n'étant jamais utilisée pour désigner la foire commerciale. Suivons sur cette affaire les propos rapportés par le chroniqueur Manolillo dans la revue Toros ${ }^{11}$. Après deux jours de palabres, la décision de l'annulation de la corrida et de toutes les festivités du vendredi est finalement prise par les élus locaux et les organisateurs de la féria des Vendanges :

Le trio fête-féria-corrida est en place, avec comme conséquence que les trois forment un ensemble indistinct dont il ressort aujourd'hui que la corrida est assimilée à une fête. [...] La regrettable association fériafête-corrida a finalement pris le dessus. Il ne pouvait être question de laisser se dérouler une corrida-fête un jour de deuil national. Ce fut donc l'annulation de tout ${ }^{12}$.

En aurait-il été de même avec une forme de divertissement moins associée à la notion de fête ? Si l'on peut comprendre la déception ponctuelle de l'aficionado, il n'est pourtant pas certain que cette association soit regrettable, ni pour les aficionados, ni pour la société locale, puisque dans les régions taurines, fête et tauromachie contribuent chacune à la vitalité de l'autre. Puisqu'elles ne peuvent être tenues pour équivalentes, il est nécessaire d'interroger la nature des liens qui les unissent. L'analyse des calendriers taurins offre un éclairage instructif.

\section{CALENDRIERS TAURINS ET CALENDRIERS FESTIFS}

S’il fallait retenir une idée forte au sujet des calendriers taurins, nous insisterions sur la remarquable coïncidence de la tauromachie avec la célébration des fêtes patronales locales. Il n'en demeure pas moins qu'il existe une incontestable autonomie des jeux taurins qui imposent leur propre rendez-vous en

${ }^{11}$ Toros, $\mathrm{n}^{\circ} 1662,27$ septembre 2001, p. 1 .

${ }^{12}$ Ibid., p. 1. 
marge des calendriers officiels des festivités traditionnelles. Dans l'ensemble, tout se passe comme si la majorité des jeux taurins se coulait dans le temps festif préexistant, tout en sécrétant et fixant des temps forts d'une temporalité propre. Pour reprendre une observation de Joffre Dumazedier " Les fêtes traditionnelles [...] fixent un cadre rituel aux loisirs modernes, mais en même temps les loisirs modernes leur donnent un sens et un rôle nouveaux $»^{13}$. De ce point de vue la coïncidence patente entre le loisir taurin et les fêtes patronales ne doit pas conduire à penser mécaniquement que la tauromachie a quelque chose à voir avec la religion. Il serait plus prudent de considérer comme point de départ que les fêtes traditionnelles, qu'elles soient d'origine civile ou religieuse, offrent des moments d'autocélébration de la communauté au sein desquels la tauromachie occupe, en terres taurines, une place de choix. Cette communauté peut être locale, comme dans le cas des fêtes patronales de nombreux villages et villes, ou bien régionale, voire nationale, dès lors qu'à travers la fête, toujours localisée, s'expriment aussi ces niveaux de représentation. Guy Di Méo distingue trois branches principales du modèle festif : fêtes calendaires, fêtes patronales et fêtes commémoratives auxquelles se greffent d'autres rameaux, dont les foires, les festivals et les fêtes privées ${ }^{14}$. L'importance quantitative des fêtes locales sur l'ensemble des événements festifs d'un territoire donné confère aux fêtes patronales une place prépondérante. Étant donné qu'en Occident, le cycle calendaire fut très tôt absorbé par le calendrier chrétien, fêtes patronales et fêtes calendaires ont souvent eu tendance à se recouper. C'est ainsi que l'on peut repérer la trace des fêtes calendaires païennes, largement fondées sur les cycles agricoles et les rythmes saisonniers, à travers un calendrier chrétien qui leur est en partie surimposé. On observe un phénomène analogue en Amérique latine où le calendrier festif chrétien ou civil s'est, dans certains cas, surimposé à des fêtes préhispaniques, aztèques, mayas ou incas, ce qui a pu faciliter, par endroits, les progrès d'une évangélisation syncrétique. En Amérique, les fêtes locales correspondent souvent à la commémoration de la fondation de la ville, généralement placée sous le signe d'un saint du temps de la colonisation espagnole, ensuite converti en figure patronale. Les articulations des jeux taurins à la fête offrent ainsi une palette de situations très diverses depuis le simple spectacle qui parait dénué de toute relation avec le reste des activités festives jusqu'au loisir omniprésent qui semble structurer l'espace-temps de la fête. Prenons l'exemple du calendrier taurin espagnol qui offre un large éventail de situations.

En Espagne, la plupart des férias taurines et des « jours de taureaux » coïncide avec la célébration du saint patron de la cité. Citons les exemples les plus importants à l'échelle du territoire. La San José, le 19 mars, coïncide avec l'une des férias taurines de Valence, également célébrée par des courses de taureaux à Aranjuez, Elda, Ontur, Utiel. La San Juan, le 24 juin, coïncide avec les férias taurines de Badajoz, Soria et Alicante, également célébrée par des courses à Albacete,

${ }^{13}$ J. Dumazedier, Vers une civilisation des loisirs ?, p. 64.

${ }^{14}$ G. Di Méo (dir.), La géographie en fêtes, pp. 7-8. 
Éibar, León, Tarragone. La San Jaime ou Santiago, saint Jacques, saint patron de l'Espagne, le 25 juillet, coïncide avec la féria de Santander à laquelle s'ajoutent de nombreuses corridas, à Almadén, Ávila, La Línea de la Concepción, Puerto de Santa María, Tudela, Valence. La liste n'est pas exhaustive. Enfin, la date la plus féconde en «jours de taureaux » est l'Assomption de la Vierge, le 15 août où sont régulièrement données des courses à Alfaro, Barcelone, Burgo de Osma, Cenicientos, Calatayud, Gijón, Málaga, Puerto de Santa María, Séville, Tafalla.

D'autres férias correspondent à des dates religieuses mobiles qui dépendent du jour de Pâques : le mardi gras ou le carnaval, le dimanche des Rameaux, le jeudi de l'Ascension, le dimanche de Pentecôte, le jeudi de la Fête-Dieu (Corpus Christi). Le cycle festif de Pâques est sans doute celui qui met le mieux en valeur un lien substantiel qui aurait survécu entre le calendrier taurin et le calendrier religieux. En 325, le concile de Nicée fixa la fête en mémoire du Christ au dimanche de la résurrection tombant après la pleine lune qui a lieu soit le jour de l'équinoxe de printemps (21 mars), soit aussitôt après cette date, Pâques oscillant ainsi entre le 22 mars et le 25 avril. On remarquera que le mot " carnaval ", emprunté à l'italien carnevalo est composé de carne (viande, chair) et de levare (lever, ôter). Le premier sens du mot a pu être " entrée en carême », puis " veille de l'entrée en carême ", temps caractérisé par des fêtes, des ripailles et des divertissements en tout genre. Cette relation autour de la fête carnavalesque qui établit un lien fort entre la fête, la subversion de la norme et la consommation exceptionnelle de viande, offre un ancrage solide pour enraciner les jeux taurins dans une ancienne ritualité festive. Parmi les férias et les jours de taureaux modernes qui suivent le calendrier du cycle festif de Pâques, citons le cas de Séville (la corrida du dimanche de résurrection et la féria taurine de Pâques), la féria de Castellón au moment du Carnaval, les férias de Tolède et de Grenade pour le Corpus Christi (Fête-Dieu).

Certaines dates taurines, en rupture avec le calendrier religieux, correspondent à des fêtes civiles commémoratives, tel que le jour de l'Andalousie à Séville, la très attendue corrida du Dos de Mayo à Madrid, ou encore celles du 12 octobre qui célèbrent el día de la Hispanidad (autrefois nommé día de la Raza) correspondant à la date anniversaire de la découverte de l'Amérique ${ }^{15}$. D'autres événements taurins s'affranchissent entièrement de la chronologie festive des calendriers civico-religieux. Ce sont, tout d'abord, les courses proposées dans le cadre des arènes dites de temporada (de saison). Ces arènes élaborent, en marge de leur féria, une programmation hebdomadaire de spectacles comme le font les théâtres et les opéras. Seules Madrid et Séville peuvent s'inscrire véritablement dans cette catégorie même si, dans deux styles différents et géographiquement très éloignés, les arènes de Barcelone et du Puerto de Santa María (Cadix) proposent une programmation de ce type. À Barcelone, il existe officiellement une

\footnotetext{
${ }^{15}$ La Raza, qu'il serait maladroit de traduire par race sans complément d'information, est une notion complexe, utilisée à des fins idéologiques jusque dans les années 1940. Elle désigne sous un même ensemble une communauté « hispanique » constituée de l'Espagne, du Portugal et des pays latino-américains, définie par des critères historiques, linguistiques et culturels.
} 
saison taurine qui se réduit en réalité à 6 ou 7 dates, soit beaucoup plus que de nombreuses férias, mais beaucoup moins qu'une véritable saison de courses de taureaux comme celle de Madrid et de Séville. Au Puerto de Santa María, le cycle des courses est annoncé sous l'appellation de féria, mais elles sont réparties en courses hebdomadaires sur les mois d'été. Ajoutons à cette catégorie la tradition des courses de solidarité, de bienfaisance, offertes pour financer un projet, indemniser des victimes d'une catastrophe, rendre hommage à un torero qui se retire ou renflouer les caisses d'une gloire désargentée. Les plus célèbres de ces manifestations sont, à Madrid, la corrida de la Presse, au bénéfice des journalistes, et la corrida de la Bienfaisance, au bénéfice de diverses œuvres de la Communauté. Les corridas de bienfaisance perpétuent la tradition des spectacles et des jeux qui n'étaient autorisés qu'à condition qu'ils contribuent à une œuvre caritative ou au financement d'un projet pour la cité. Sont à mettre dans cette catégorie ce que la nomenclature taurine nomme " festival », à savoir une course mineure, sans habit de lumière, avec des taureaux aux cornes réglementairement épointées, au cours duquel les professionnels ne sont pas rémunérés. Enfin, certains rendez-vous pourraient être rapprochés du modèle sportif, composé de plusieurs rencontres qui cherchent à créer une émulation au-delà des représentations isolées et indépendantes les unes des autres. C'est le cas pour des courses réservées aux jeunes toreros : le concours de novilladas d'Arnedo qui décerne au meilleur espoir le trophée du zapato de oro (la chaussure d'or), les concours de novilladas composés de phases éliminatoires pouvant avoir lieu dans différentes arènes. Cette évolution récente montre que l'univers de la corrida n'est pas totalement hermétique aux évolutions organisationnelles de type sportif, même si ce modèle est constamment mis à distance pour asseoir la dimension artistique de la tauromachie.

\section{II. - TAUROMACHIES FESTIVES \\ ET FESTIVITÉS TAURINES}

\section{La TAUROMACHIE,}

CAUSE OU CONSÉQUENCE DE LA FÊTE

À travers cette diversité de situations, il n'est pas toujours facile de savoir si la tauromachie est cause ou conséquence de la fête, tant les logiques festives les imbriquent jusqu'à faire disparaître l'objet même de ce qui est fêté, au profit de ses manifestations plurielles. On touche ici sans doute à l'un des aspects inhérents à l'étude de tout phénomène festif.

$\mathrm{Au}$ Portugal, pays où le temps festif est de même qu'en Espagne, fortement marqué par la tradition catholique, on retrouve la même place dominante des fêtes patronales qui semblent fixer le rythme de la saison tauromachique. Mais, là encore, les articulations entre la fête religieuse, la fête civile et les loisirs taurins possèdent de multiples facettes. Les fêtes taurines du Ribatejo nous enseignent que cette articulation est propre à chaque lieu. À Moita do Ribatejo, la féria tau- 




Fig. 42. - Bouée décorative, Vierge du Bon Voyage au club nautique de Moita. Le responsable du club nous explique avec pédagogie quels sont les piliers de l'identité locale : ici le fleuve et la Vierge du Bon Voyage...

(Moita, 2005, cliché : Jean-Baptiste Maudet).

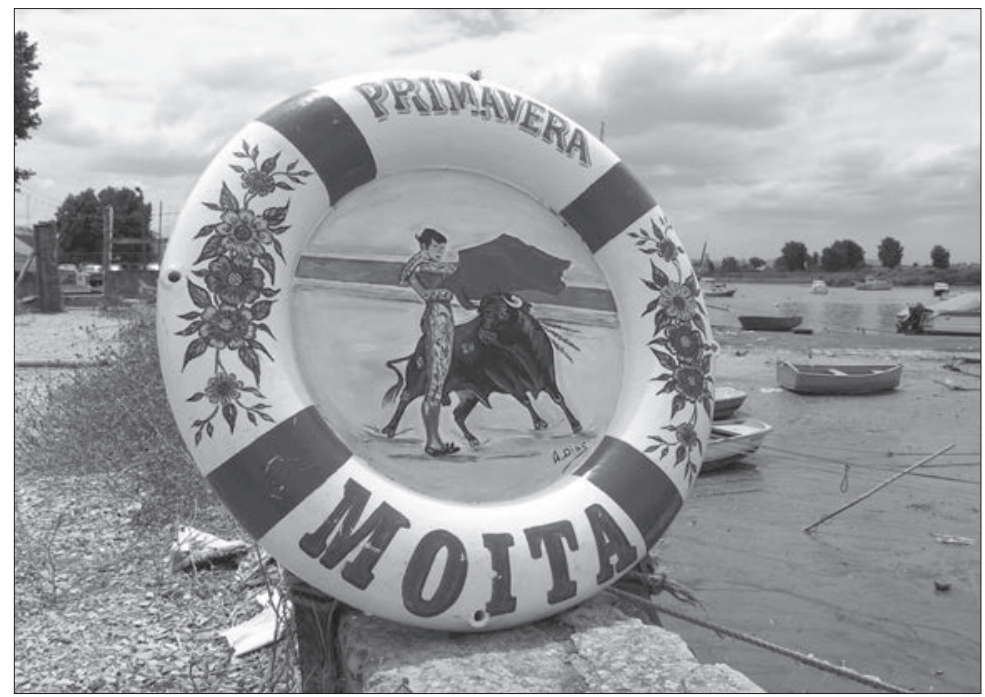

Fig. 43. - Bouée décorative, corrida de toros... là : le fleuve et la tauromachie, plus précisément la corrida à pied (Moita, 2005, cliché : Jean-Baptiste Maudet). 
rine est l'une des principales activités venant célébrer les fêtes en l'honneur de Nossa Senhora da Boa Viagem. Il s'agit d'une des férias taurines les plus importantes du Portugal, qui présente un cycle de 5 à 6 spectacles consécutifs. En interrogeant les habitants de Moita sur l'objet principal de la fête, beaucoup ont exprimé l'idée que le fleuve, les taureaux et la Vierge du Bon Voyage en étaient les trois composantes inséparables. Un responsable du club nautique de Moita, pour me confirmer ces dires, a décroché une bouée d'ornement fixée au mur du restaurant. Un côté de la bouée représente la Vierge du Bon Voyage, l'autre une scène tauromachique et de commenter : « cette bouée représente ce qu'est la fête pour nous : le Tage et ses bateaux, notre Vierge qui nous protège et les courses de taureaux » (voir fig. 42 et 43, p. 115).

Derrière l'apparente complémentarité harmonieuse d'une fête tricéphale, certains dénoncent au contraire la tension des intérêts divergents. Le directeur de la revue taurine portugaise Tauromaquia, Rui Patrocino, dans le numéro de septembre 2004, paru juste avant les fêtes de Moita, critique le manque de collaboration entre les principaux décideurs des festivités ${ }^{16}$. D'une part, l'Église paroissiale possède une commission responsable des célébrations religieuses qui n'est pas intégrée à la Commission organisatrice des fêtes de Moita (COFM). D'autre part, l'entreprise organisatrice de la féria taurine programme ses spectacles en concurrence avec la COFM. En effet, sur les programmes de la fête de 2004, on observe que les corridas sont données en nocturne, à 22 heures, en même temps que les concerts, les bals et autres podiums musicaux. Et l'auteur de conclure : «Tout se passe comme si chacun organisait sa fête de son côté » ${ }^{17}$. Pour l'anthropologue Vitor Pereira Mendes qui s'exprime dans l'éditorial du même numéro, il s'agit d'une dénaturation de la fête, car les courses de taureaux, pour remplir leur fonction, doivent remplacer le travail : « En ce que la fête est fête, elle doit refuser le sens pratique et utilitariste de la vie $»^{18}$. L'exemple de Moita montre à quel point les vues peuvent diverger au sujet de la complémentarité des activités festives et de leur concurrence qui, selon les groupes d'intérêt, peuvent manifester, pour les uns, une diversité de l'offre festive et pour les autres, un dysfonctionnement de la programmation et l'essence même de la fête.

Les fêtes du Colete Encarnado de Vila Franca de Xira offre un exemple différent. Le colete encarnado est le nom du gilet rouge porté par les campinos et place immédiatement la grande fête annuelle de la cité sous le signe de l'élevage des taureaux de combat. La fête a d'ailleurs été lancée en 1932 par les édiles locaux, à la tête desquels se trouvait l'éleveur José Van-Zeller Pereira Palha, souhaitant récompenser les pompiers volontaires de Vila Franca. Le programme des fêtes tourne aujourd'hui autour de la figure centrale du campino auquel il est rendu hommage. Pendant les trois jours de fête se multiplient les activités taurines et équestres: les esperas, les largadas, la garraiada dans les arènes de Palha Blanco,

\footnotetext{
${ }^{16}$ Tauromaquia, septembre 2004, $\mathrm{n}^{\circ} 6$.

${ }^{17}$ Ibid., p. 5.

${ }^{18}$ Ibid., p. 3.
} 
les compétitions où s'illustre le savoir-faire des bouviers sur l'esplanade qui prolonge les arènes, et une à deux courses de taureaux ${ }^{19}$. À cela s'ajoutent des activités festives telles que des concerts de fado et de flamenco, des spectacles de danse et des sardinades en plusieurs points de la ville. La référence religieuse reste discrète et se limite généralement à une messe en soirée, célébrée à l'église Matriz de Vila Franca. Ici la fête a bien lieu en l'honneur des campinos et ne s'articule à aucune fête d'origine religieuse. Les fêtes du Colete Encarnado établissent en lien fort et visible entre les travailleurs du bétail brave et l'activité tauromachique.

Le cas des fêtes d'Alcochete nous permet en quelque sorte de boucler le tour d'horizon de la diversité des festivités taurines du Ribatejo. Les grandes fêtes annuelles d'Alcochete sont les festas do barrete verde e das salinas qui ont lieu la deuxième semaine d'août. Par cette appellation, la fête rend hommage à deux éléments constitutifs de l'identité d'Alcochete à mi-chemin entre l'activité tauromachique et l'activité économique. Le barrete verde est le bonnet vert porté par les forcados et les campinos ${ }^{20}$. Les salinas sont les salines qui pendant longtemps ont constitué la principale industrie de la cité, en relation avec le port de Lisbonne et le salage des morues. Les festas do barrete verde e das salinas initialement intitulées Festas do barrete verde ont été créées au début des années 1940 par un journaliste d'Alcochete, José André dos Santos. Cette fête venait remplacer celle de Nossa Senhora da Vida (Notre-Dame de la Vie) que les habitants d'Alcochete font remonter au XVII ${ }^{\mathrm{e}}$ siècle. Notons que les corridas furent introduites dans les festivités en 1930. On observe ainsi qu'il existe des phénomènes de relais et de substitution dans les programmations et les appellations des fêtes, qui font varier la place de la tauromachie dans le système festif. Dans le cas d'Alcochete, on est passé d'une fête dont l'objet officiel était l'hommage à Notre Dame de la Vie à la célébration de trois figures emblématiques de la terre, de "notre terre » comme se plaisent à le dire les habitants de la cité : le travailleur du bétail, le salinier et le forcado. Le lien avec le terroir est immédiat dans le cas des campinos et des saliniers auquel s'agrège la figure populaire du forcado.

Assister à ces événements nous enseigne qu'il existe toujours une part d'intransitivité de la fête qui, par un jeu d'accumulation d'activités, de mise en scène, d'occultation, d'exhibition ou de médiation de ce qui est fêté, rend toutes les fêtes possibles. L'objet premier de la fête, lorsqu'il est possible de l'identifier au-delà des effets d'annonce, peut être central pour les uns, marginal pour les autres, absent pour les troisièmes, sans que ne soit jamais remise en cause la loi selon laquelle la fête engendre et attire la fête. Guy Di Méo rappelle en ce sens les analyses de François-André Isambert qui notait que les modes d'expression de la fête, par leur consistance propre « deviennent souvent opaques, au point de voiler aux yeux des spectateurs, voire du participant, leur signification, c'est-àdire l'objet qu'ils célèbrent $»^{21}$.

\footnotetext{
${ }^{19}$ Nous nous référons ici au programme des fêtes de 2005 auxquelles nous avons participé.

${ }^{20}$ Lors des encierros de Pampelune, le barrete verde est un signe de reconnaissance des Portugais qui les courent, pour la plus grande joie des aficionados de ce pays qui suivent les retransmissions télévisées.

${ }^{21}$ G. Di Méo (dir.), La géographie en fêtes, p. 3.
} 
Lors du IV congrès mondial des villes taurines qui s'est tenu en septembre 2001 à Vila Franca de Xira au Portugal, Jocelyne Pezet, conseillère régionale du Languedoc-Rousillon et conseillère municipale de Nîmes, évaluait à près d'un million de visiteurs le nombre de personnes attirées par la féria de Nîmes, soit environ sept fois la population résidente de la ville. Ce fut également l'occasion de révéler le résultat d'un sondage qui permet de rendre compte des raisons pour lesquelles les participants se rendent à la féria de Nîmes. À la question "pourquoi venez-vous à la féria ? ", 16,8 \% répondent pour les corridas et les manifestations taurines, $8,9 \%$ pour les chevaux et le folklore, $49,5 \%$ pour l'ambiance des fêtes, 24,8\% pour d'autres raisons. Bien qu'il semble difficile de pouvoir segmenter sans recoupement les raisons de la participation, cette enquête confirme le rôle clé de l'activité tauromachique dans le succès de la féria, sans qu'elle soit pour autant la première motivation des participants. Il est intéressant de constater qu'une notion aussi difficile à définir que l'ambiance, invoquée à près de $50 \%$, soit finalement le principal facteur d'agglomération de la population, alors que cet élément échappe en partie aux projections des programmateurs. Que fête la féria de Nîmes ? La Pentecôte, la tauromachie ou Nîmes elle-même ? La Pentecôte fixe la date plus qu'elle n'est célébrée. La tauromachie donne le thème officiel et détermine une partie de la viabilité financière. Le tissu local des aficionados nîmois s'investit dans la fête à travers les clubs taurins, les «bodegas », les associations. Les habitants participent à la fête, certains la fuient. Les populations alentour, les touristes, les visiteurs convergent vers Nîmes et participent à la féria. Les uns ne reconnaissent plus « leur " féria, les autres se demandent si la tauromachie est vraiment indispensable à une si belle fête. En dépit de la forte affluence à la féria de Nîmes, la tauromachie reste perçue par le plus grand nombre comme la raison d'être de la fête. Cette perception n'est pas démentie par le poids économique de la tauromachie au sein de la Féria. Le Bureau d'information et de prévention économique estime que les dépenses cumulées de 100.000 spectateurs taurins lors de la féria s'élèvent à 13 millions d'euros et Jocelyne Pezet d'ajouter « la féria est une des rares manifestations de cette envergure qui coûte à la collectivité moins de $1 \%$ du flux économique obtenu ». En 1996, d'après le Conseil économique du Languedoc-Roussillon, les corridas de la féria de Nîmes auraient permis de dégager un excédent budgétaire de 2,7 millions de francs qui « parviennent presque à couvrir les frais engagés par la ville de Nîmes pour l'ensemble des festivités qui se sont déroulées sur cinq journées et qui ont attiré 260.000 visiteurs par jour »22. Quant à l'origine géographique des spectateurs de corridas à Nîmes, une enquête menée en 2003 et 2004 a montré que sur 10 spectateurs, 6 sont originaires du Languedoc-Roussilon, 2 viennent de régions plus septentrionales et 2 ont des origines espagnoles, confirmant ici le caractère localisé de la culture taurine ${ }^{23}$.

\footnotetext{
${ }^{22}$ Conseil Économique et Social du Languedoc-Roussillon, Le rôle et l'impact du taureau, p. 45.

${ }^{23}$ C. Bernié-Boissard, « Le public des courses de taureaux », p. 27. Voir également ID. (dir.), Tauromachies et identités locales.
} 
On comprend mieux l'engouement suscité par les fêtes taurines auprès de nombreuses municipalités en matière de développement local. Mieux, le spectacle peut être déficitaire, il reste bien souvent avantageux au vu des effets d'entraînement exercés sur l'ensemble des activités économiques. L'un des porte-paroles de la Fédération française de la course camarguaise nous a confirmé qu'avec un coût d'organisation moyen de 5.000 euros par course, chaque spectacle était déficitaire de 2.000 euros. Rapporté aux 900 courses camarguaises de l'année 2004, le déficit s'élève à 1.800.000 euros, entièrement comblé, par les activités annexes des clubs taurins, buvettes, sandwicherie, tombolas, etc. Un processus analogue se produit avec le fonctionnement économique de la course landaise.

Dans cette imbrication des faits, la tauromachie professionnelle et la tauromachie à participation collective s'inscrivent chacune dans une conception différente de la fête, ou plutôt manifestent une façon différente de faire la fête. Elles impliquent aussi un rapport au territoire différent. Prenons le cas des corridas formelles à l'espagnole pour lesquelles les codes sont quasiment identiques en tout lieu, à quelques nuances près. Il s'agit dans la majorité des cas d'un spectacle de la fin d'après-midi, sauf en Équateur où les corridas commencent à midi. L'heure venue, les amateurs se pressent vers les arènes, beaucoup se sont habillés spécialement pour l'occasion, certains préparent digestifs et cigares à offrir à leur voisin, d'autres ont emporté de quoi boire et manger. L'ouverture du spectacle est très cérémoniale. Le spectacle commence toujours à l'heure, les alguaciles qui représentent l'autorité en piste reçoivent les clefs permettant de faire sortir les taureaux, les toreros défilent en habit de lumière au rythme des pasodobles et au son des applaudissements de la foule, ils saluent la présidence, les drapeaux aux couleurs de l'Espagne, parfois de la région, de la province ou de la ville flottent au-dessus des arènes. Le déroulement du spectacle est pris dans une logique festive contradictoire, comme tout divertissement réglé reposant sur l'exaltation d'une passion collective.

En effet, le spectacle est régi par des normes, des droits et des devoirs assignés aux spectateurs et aux professionnels qui, en théorie, sont à l'opposé du désordre, de la licence ou de la subversion que l'on identifie comme des caractéristiques constitutives de la fête. Mais, dans le même temps, ces normes déterminent les conditions d'existence du rassemblement, de l'effervescence collective, voire de la transgression. Quoi qu'il en soit, les grands triomphes des matadors ont la saveur des grands moments de fête pour ceux qui les vivent. Le matador est acclamé. Il reçoit les trophées accordés par la présidence à la demande d'une foule en liesse : une oreille, deux oreilles ou deux oreilles et la queue. La musique accompagne le tour de piste du matador. Il reçoit sur son passage les félicitations du public, des fleurs et des cadeaux de toutes natures, fréquemment liés aux produits du terroir, des jambons, du vin, un pain géant offert par le boulanger qui profite de l'occasion pour se faire de la publicité. On lui jette également des châles, mantilles, chapeaux, casquettes et parfois même d'autres présents plus inavouables lancés comme autant de liens personnels tissés entre la foule et le héros du jour. De ce qu'il reçoit de la foule, certains accessoires sont portés 
par le matador d'autant plus volontiers qu'ils symbolisent le lieu du triomphe, généralement des foulards aux couleurs de la cité et de la fête ou des chapeaux traditionnels : chapeau des charros mexicains, panama originaire d'Équateur, plus rarement barrete verde des campinos et forcados portugais, foulard rouge à Bayonne et à Dax, foulard bleu à Bilbao, foulard à carreau noir et rouge à Saragosse... À la fin du spectacle, le torero triomphateur sort par la grande porte, il est porté par la foule, autrefois jusqu'à son hôtel, aujourd'hui jusqu'à sa voiture où l'on se précipite à nouveau pour espérer le toucher, parfois lui arracher un morceau de son habit de lumière. L'après-corrida peut se poursuivre tard dans les bars alentour, servant souvent de siège aux associations d'aficionados qui commentent et rejouent inlassablement les gestes d'une émotion fugitive. La course de taureau tient de la fête, si l'on accorde une telle reconnaissance aux manifestations collectives structurées par un spectacle qui crée des temps de rassemblement, de décharges émotionnelles et de forte convivialité.

La corrida ajoute un fait essentiel, le public intervient réglementairement dans les décisions prises par la présidence pour accorder les trophées. La première oreille est accordée par la présidence à la demande du public, quand bien même sa propre conviction serait différente. Les trophées supplémentaires sont à la discrétion de la présidence. Mal lui en prendrait de contredire un avis largement majoritaire qui serait immédiatement suivi d'une bronca retentissante. La bronca n'a pas d'équivalent français et semble avoir disparu des spectacles plus policés. Voici la définition qu'en donne Claude Popelin :

[Dispute] Nom donné aux colères extrêmement bruyantes qui dressent le public contre un torero déficient, un taureau inapte, une décision contestée du président ou un détail d'organisation négligé. Pas toujours intégralement justifiée, cette expression de la vox populi est l'arme majeure des spectateurs pour se faire écouter dans l'arène $e^{24}$.

Cette colère du public peut encore se solder aujourd'hui par l'intervention de la force publique pour assurer la sortie d'un torero ou la retraite de la présidence. Elle est assurément une emprise démocratique sur le spectacle qui en fait un acte politique avec d'autant plus d'acuité que le président est toujours un membre des pouvoirs publics. En Espagne, rappelons que la course demeure sous le contrôle du ministère de l'Intérieur. C'est une différence importante avec les autres spectacles taurins où les juges ne prennent pas leur décision en fonction du public, relégué dans une position plus passive à laquelle ne s'est finalement jamais tout à fait résolu le public des corridas.

La tauromachie participative pousse ce principe à son comble ou plutôt n'a peut-être jamais perdu son fondement : la participation collective du peuple à un moment exceptionnel d'euphorie et de divertissement. Les jeux taurins populaires mettent en scène une fête que l'on rattache plus facilement aux représentations habituelles des événements à caractère festif. À la place du spectacle

\footnotetext{
${ }^{24}$ C. Popelin, La Tauromachie, p. 50.
} 
ordonné, de la séparation entre acteurs et spectateurs, des temps généralement respectés d'acclamation et de silence, s'exprime ici une plus grande spontanéité individuelle et collective qui confine au désordre. Les jeux taurins populaires signifient d'autant mieux l'exceptionnalité du temps et du lieu de la fête qu'ils transfigurent les espaces de la vie quotidienne, comme lorsqu'ils occupent les rues et les places publiques de la cité, aménagées et décorées pour l'occasion. La coïncidence du jeu taurin et de la ville en fête offre un décorum qui place immédiatement la scène dans une atmosphère festive : des drapeaux aux fenêtres, des fanions suspendus le long des rues, des odeurs de grillades, d'anticuchos au Pérou, de sardines au Portugal, le son des fanfares, le bruit des pétards. À Vila Franca de Xira, le lâcher de taureau dans l'espace public dure toute la matinée, les participants traversent la rue, les mains prises par les victuailles et les verres de bière, certains se prennent en photo tant que le taureau n'y est pas, d'autres s'approchent encore d'un balcon ou d'une balustrade où ils ont repéré celui ou celle à qui plaire. Au passage du taureau, ils se jetteront sur l'élu, feignant un danger imminent pour une première prise de contact. Les lieux quotidiens sont investis et transfigurés par la fête taurine dont les valeurs semblent à l'opposé de la fête réglée par une mise en spectacle rationnelle. Elle donne à voir une improvisation ludique permanente, même si les jeux taurins populaires obéissent en réalité, pour la plupart, à une réglementation précise, tout au moins en Europe : la taille des barrières, la largeur du parcours, la longueur de la corde...

En Amérique latine, le niveau de violence peut être beaucoup plus élevé pour les hommes et pour les bêtes, et les normes réglementaires pour le déroulement des jeux taurins populaires beaucoup plus aléatoires, quand elles existent. Le dicton parcourt les Andes depuis les corralejas du Nord de la Colombie jusqu'au turupukllay de l'Altiplano péruvien : "une fête réussie est une fête où le sang coule », de préférence celui de l'homme, ressuscitant fantasmes ou réalités de la pratique sacrificielle.

\section{TROIS EXEMPLES D'IMBRICATION :}

Madrid, Pampelune, SÉville

Trois exemples concrets nous permettent de montrer les relations complexes d'imbrication entre festivités taurines et tauromachies festives en insistant sur leur rapport à l'espace. Ils permettent aussi de présenter les trois fêtes taurines les plus imitées à travers le monde et dont les codes servent de références à de nombreuses férias. En outre, Madrid et Séville sont des centres de commandement du monde des corridas tant d'un point de vue économique que d'un point de vue iconographique. En cela, avoir à l'esprit ces exemples dans le détail de leur organisation permet une analyse plus fine des espaces taurins, du SudOuest européen à l'Amérique latine.

a) Madrid : la San Isidro et la sanisidro

Les principales fêtes de la ville de Madrid sont celles données en l'honneur du saint patron Isidore, autour du 15 mai. Isidore était un laboureur de Madrid 


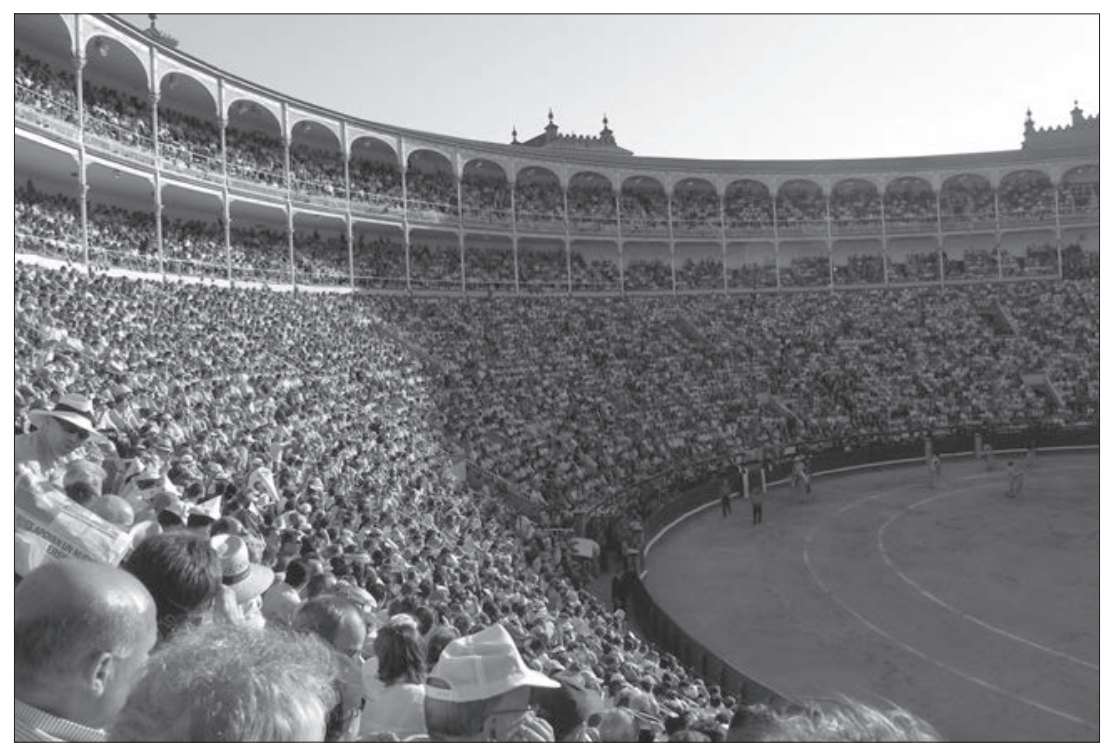

FIG. 44. - Plaza de toros de Madrid. Les arènes de Las Ventas sont combles pour la San Isidro (Madrid, 2005, cliché : Jean-Baptiste Maudet).

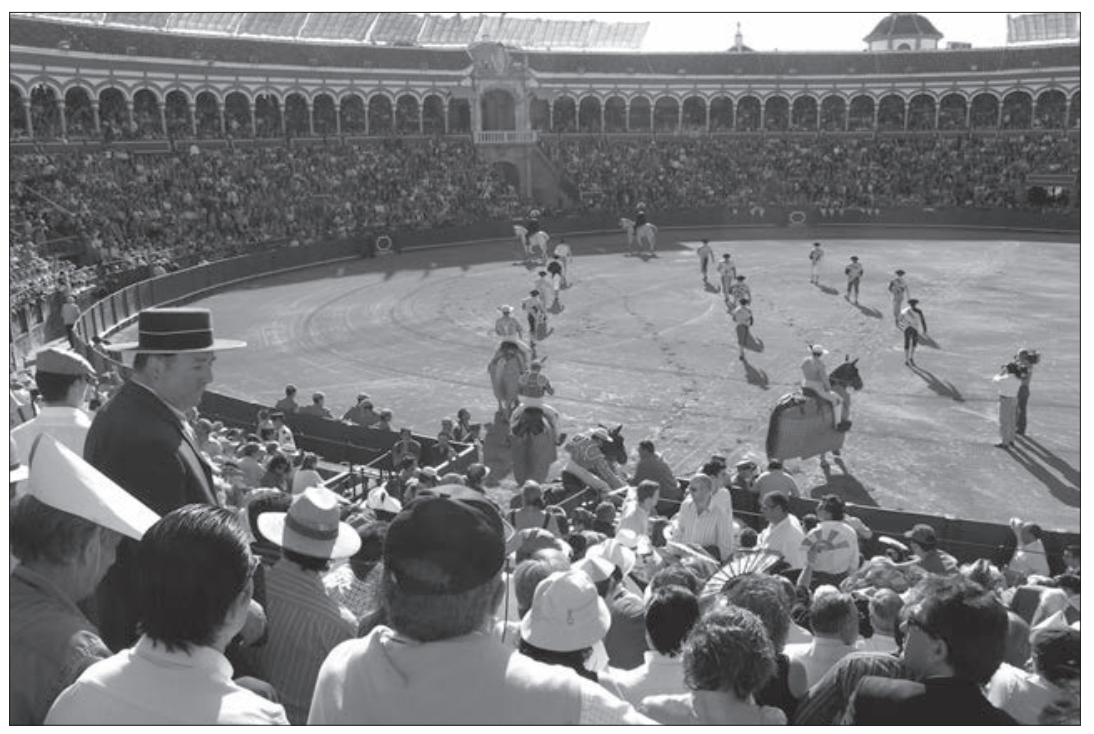

Fig. 45. — Plaza de toros de Séville. Les arènes de La Maestranza de Séville pendant la Féria. Public hétérogène des places au soleil comme en témoignent les couvre-chefs (Séville, 2004, cl iché : Jean-Baptiste Maudet). 


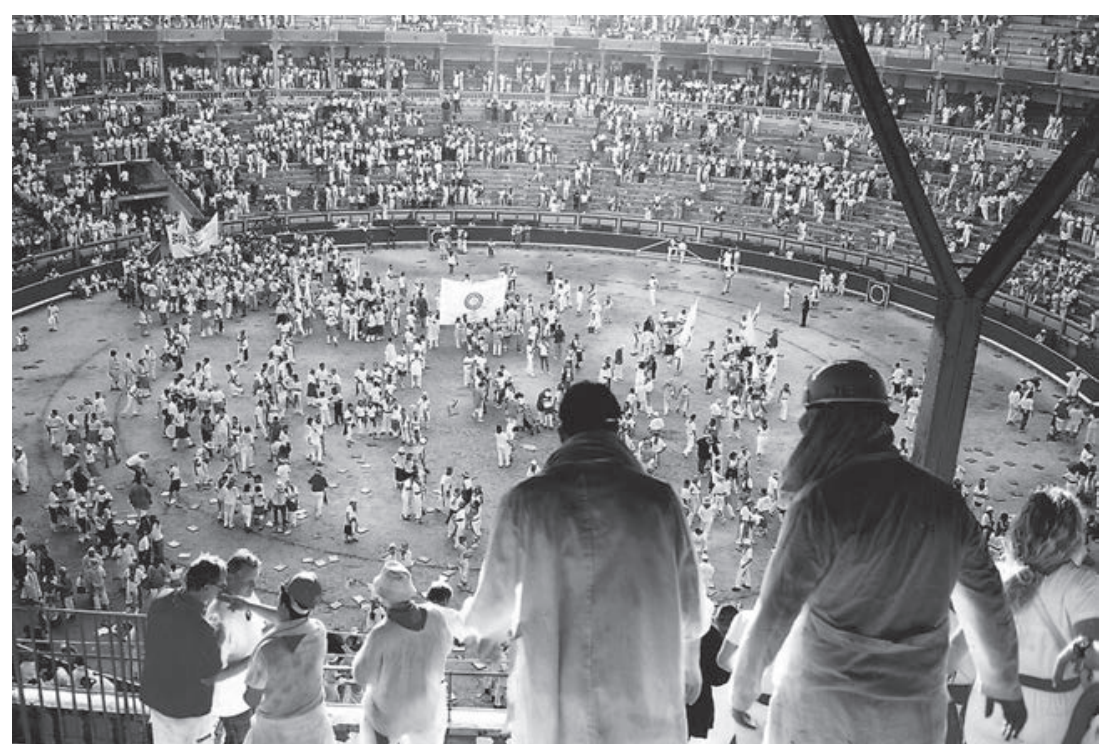

Fig. 46. - Plaza de toros de Pampelune. Les arènes de Pampelune se vident par la piste au rythme des «bandas » pour la San Fermín

(Pampelune, 2003, cliché : Jean-Baptiste Maudet).

né en 1080 et canonisé en 1622 pour avoir, parmi beaucoup d'autres miracles dont certains posthumes, fait jaillir une source dont l'eau guérit tous les maux. À l'endroit où la source jaillit, au sud-ouest de la ville, fut construit l'ermitage de San Isidro, peint par Goya en 1787 et dont le tableau se trouve aujourd'hui au Prado. Le jour du saint ne fut définitivement fixé au 15 mai qu'en 1669. Les actes religieux liés à la célébration du saint patron démarrent le dimanche qui précède le 15 mai. Tout commence par la bénédiction de la source miraculeuse attenante à l'ermitage où sont exposées les reliques du saint. Le matin du 15 mai a lieu une messe célébrée par le cardinal de Madrid, suivie d'une procession qui traverse le centre historique de la ville. La dernière cérémonie religieuse qui clôt les fêtes de la San Isidro se tient le dimanche suivant dans l'ermitage de San Isidro, ponctuée par le chant Regina coli adressé à la vierge. Lors de ces fêtes qui durent une semaine sont organisés différents divertissements : des concerts publics en plein air, du théâtre de rue, un festival de théâtre (Distrito Quijote), un festival de musique (Titirimundi), des défilés de géants (gigantes y cabezudos), des expositions, une fête foraine et... une féria taurine. Les dépliants promotionnels des fêtes de la San Isidro proposés par la mairie insistent moins sur la présence de la féria taurine que ne le font ceux des agences de voyages et des offices du tourisme, visant une clientèle internationale pour qui les corridas sont présentées comme l'événement à ne pas manquer, puisqu'elles constituent une spécificité des fêtes espagnoles en général et des fêtes de San Isidro en particulier. 
La féria taurine est appelée la San Isidro parfois orthographiée en un seul mot sanisidro et sans majuscule, comme s'il s'agissait d'un nom commun découplé du nom propre dont elle tire pourtant son appellation. À la différence des fêtes de la San Isidro, la sanisidro dure plus de trois semaines du début du mois de mai au début du mois de juin. Avec un spectacle par jour, c'est la féria taurine la plus longue et la plus importante du monde. Si l'on y ajoute la courte féria de la Communauté de Madrid fin avril et la Feria del Aniversario, crée en 2006 à l'occasion du $75^{\mathrm{e}}$ anniversaire des arènes, se déroulant début juin, les aficionados peuvent assister à Madrid à plus d'un mois de corridas quasiment consécutives. La féria taurine madrilène fut créée en 1947 par Livinio Stuyck, alors imprésario des arènes. Elle a d'abord été appelée "feria de Madrid » et réunissait sur cinq jours, à partir du 15 mai, les corridas normalement programmées sur tout le mois. Depuis le début du $\mathrm{xx}^{\mathrm{e}}$ siècle, une corrida avait lieu le jour de la San Isidro. Dans les années 1960, le nombre de courses données pour la San Isidro oscille entre 10 et 15. Le nombre de courses dépasse la vingtaine au début des années 1970. La sanisidro réunit généralement tous les plus grands toreros du moment, sauf lorsque les exigences de certains d'entre eux en termes économiques ou en termes de choix des élevages s'avèrent incompatibles avec la volonté ou le budget de la direction des arènes. Pour la sanisidro, tous les éleveurs conservent leurs meilleurs lots de taureaux ou plutôt les plus respectables en termes de poids, de musculature et de cornes, bien que sur ce terrain les ferias de Bilbao et de Pampelune la surpassent. Il est d'usage assez courant de comparer la sanisidro à la grande bourse des valeurs taurines, qui chaque année fait varier la cote des professionnels, leurs prétentions salariales et leur nombre de contrats pour la saison en cours. Pour les arènes, le 15 mai est une date presque comme les autres, même si nous avons pu remarquer que ce jour promet généralement une affiche de qualité. Le 15 mai 2005, les arènes ont cherché à programmer une corrida en cohérence avec la dimension locale de la fête en mettant à l'affiche trois matadors de Madrid : El Juli, Matías Tejela et César Jiménez qui confirmait alors son alternative ${ }^{25}$. L'expérience n'a pas été renouvelée en 2006, ni en 2007.

Si la tauromachie est une fête, et que la sanisidro représente la féria la plus importante au monde, il est surprenant de constater qu'il s'agit aussi d'une des férias les moins festives à l'intérieur des arènes, comme à l'extérieur. Dans les arènes, aucune ambiance de fête pour des spectateurs réputés sérieux et intransigeants, qui préfèrent volontiers jouer le rôle d'un tribunal de l'orthodoxie taurine, plutôt que de se laisser aller à la moindre complaisance. Cette tradition de rigueur et de sévérité est ancienne. Comme le rappelle Jacques

\footnotetext{
${ }^{25}$ Dans le langage taurin, « confirmer son alternative » signifie venir toréer à Madrid pour la première fois après avoir acquis le statut de matador de toros. L'alternative est un rite qui marque le passage du rang de novillero au rang de matador de toros, ce qui implique d'affronter des taureaux qui ont plus de quatre ans. Dans le détail, «El Juli » et Matias Tejela sont nés à Madrid alors que César Jiménez est originaire de Fuenlabrada, ville de la Communauté de Madrid. Les trois matadors sont passés par l'école taurine de Madrid.
} 
Durand, lorsqu'en 1973, le matador Palomo Linares coupe la deuxième queue de l'histoire des arènes de Las Ventas, le lendemain, sous l'effet du scandale provoqué par une récompense fortement contestée, une partie du public portait un brassard en signe de deuil ${ }^{26}$. Certains secteurs de l'arène poussent cette exigence à l'intransigeance, voire à l'intégrisme comme hier la andanada 8 et comme aujourd'hui le très redouté tendido 7. Cette partie de l'arène correspond aux premières places du soleil qui passent à l'ombre quand le soleil décline. Elles surplombent le terrain où a lieu la pique. Les spectateurs du tendido 7 ne font aucune concession à la présentation et à la qualité des taureaux, pas plus qu'ils n'acceptent le moindre manquement au règlement taurin. Cette intransigeance affichée est considérée, tantôt comme une juste rigueur, tantôt comme une attitude irrespectueuse et contestable, voire comme la manifestation d'une ignorance crasse ou délibérément provocatrice. Quoi qu'il en soit le tendido 7 aime jouer un rôle important et ses occupants ne se voient pas comme de simples spectateurs. La réputation de Madrid n'est pas seulement fondée sur les caractéristiques de son public. Un détail lourd de sens : aucune musique n'est jouée pendant que le matador torée contrairement à toutes les autres arènes du monde où lorsque l'homme parvient à lier les passes de façon satisfaisante, l'orchestre se met à enchaîner les pasodobles. L'orchestre ne joue qu'au début de la corrida lors du paseo, lors des intermèdes entre chaque taureau et à la fin du spectacle. Ce détail est à l'image d'un intérieur très sobre : des gradins en béton, des piliers en acier basque, le drapeau de l'Espagne et de la Communauté autonome qui flotte au-dessus des arènes, une girouette en forme de taureau qui surmonte l'horloge, un balcon royal généralement vide. Les seuls éléments qui tranchent avec la sobriété du décorum festif sont les vendeurs de boissons agréés par les arènes et les victuailles apportées par les spectateurs qui généralement nourrissent aussi la cousine d'un cousin qu'ils ne connaissent pas. C'est sans doute dans les parties hautes de l'arène, tout au moins dans les andanadas et les gradas du soleil que les spectateurs sont le plus à la fête. Les lieux résonnent et la hauteur vous met à distance du spectacle lorsqu'il est ennuyeux. C'est aussi là que l'on trouve le public le plus bigarré où se côtoient des abonnés de longue date, un public populaire, des étudiants étrangers et des touristes qui découvrent à leur manière cet étrange spectacle.

Immédiatement à l'extérieur des arènes, seuls quelques stands proposent des boissons fraîches non alcoolisées, des graines en tous genres, des chapeaux pour se protéger d'un mois de mai souvent caniculaire, des éventails, des souvenirs et autres verroteries taurines qui vont de l'autocollant à l'effigie du taureau de combat, à la paire de banderilles en plastique, en passant par les affiches de corridas où votre nom peut être inscrit au pochoir. Au-delà, s'ouvrent les bouches de métro de la station Las Ventas où s'engouffre une grande partie des spectateurs qui ne commenteront la corrida avec leur voisin que le temps d'un trajet. D'autres attendront que les transports en commun se désengorgent dans les bars de la calle de Alcalá et de ses rues perpendiculaires. Là, certains

\footnotetext{
${ }^{26}$ Libération, 16 mai 2001 ou J. DuRAnd, Chroniques taurines, p. 130.
} 
aficionados évoquent avec passion le spectacle : les mains tendues pointent vers le bas pour reproduire les gestes des toreros, les index se dressent, parallèles, pour reproduire les cornes, les verres et les tapas se déplacent sur les tables pour rejouer la position exacte des protagonistes, certains toréent les voitures qui roulent au pas à l'approche des trottoirs bondés. Pour une petite minorité, la nuit se poursuit tard dans les quelques adresses taurines que les arènes ont fixées. Pour autant l'ambiance festive qui serait imputable aux courses de taureaux s'essouffle dans l'ensemble assez vite. C'est pourquoi en 2003, les propriétaires des bars El Albero, La Tienta, et El Rincón de Jerez qui ne possèdent pas de terrasses sont furieux des mesures prises pour vérifier que l'on ne puisse consommer d'alcool sur la voie publique. Les voitures de la police passent et exigent qu'aucune boisson ne soit consommée dehors, au moment même où les bars débordent sur la rue, avant que le reflux ne s'amorce lorsque le métro parvient à drainer sans encombre les passagers. Il faut dire que les arènes de Las Ventas ne sont pas situées dans les quartiers du centre-ville où se concentrent les animations. Le début de la construction des arènes date de 1929. Encore en construction, elles sont inaugurées une première fois en 1931, puis une seconde fois en 1934. Leur édification visait à remplacer les arènes de la Puerta de Alcalá, plus centrales, mais insuffisamment grandes pour répondre à la demande. Le projet de les agrandir en rognant sur la taille de la piste et en surélevant la structure d'un étage avait bien été envisagé, mais c'est finalement celui d'une nouvelle arène qui l'emporta. Les arènes sont alors construites tout au bout de la calle Alcalá qui prend son origine à la Puerta del Sol où se situe le kilomètre zéro de la ville et du pays. L'urbanisation galopante de Madrid a rapidement inséré les arènes dans le tissu urbain, mais elles demeurent à l'est de la ville, très excentrées par rapport au cœur historique et situées en bordure de la M 30, une autoroute urbaine qui contourne la partie centrale de l'agglomération.

Mais la distance au centre de la ville et la localisation des arènes n'expliquent pas tout pour comprendre l'absence de fête autour d'elles. Il faut aussi remarquer la faiblesse des liens et des références culturelles communes entre les spectacles taurins et les autres supports des fêtes de la San Isidro. Rien dans les festivités programmées pour la San Isidro ne tisse de relation entre l'univers de la tauromachie et les autres formes d'activités ou de divertissements. La plupart des aficionados qui ne sont pas de Madrid, n'ont d'ailleurs généralement aucune connaissance des autres aspects de la fête urbaine, certains ignorent même que la San Isidro puisse être autre chose qu'une féria taurine. La fiesta taurina relève ici d'une industrie culturelle dont la coïncidence avec la célébration du saint patron apparaît comme un prétexte lointain. En tous les cas, il n'existe nul signe extérieur marquant une quelconque continuité entre les courses de taureaux et les autres manifestations liées aux autres aspects de la fête urbaine, qu'il s'agisse des divertissements ou des actes religieux. Ce qui relie la fête et la tauromachie semble bien être, dans ce cas, une logique d'agglomération circonstancielle d'activités juxtaposées au sein d'une grande ville, où il est difficile de mesurer si le succès des unes profite au succès des autres. 


\section{b) Pampelune : San Fermín et sanfermines}

À l'inverse de la San Isidro, il est des exemples où la fête urbaine semble surgir de la tauromachie elle-même. C'est le cas à Pampelune où la célébration de la San Fermín et l'omniprésence des taureaux ont tendance à fusionner, ou plutôt, à ne plus tout à fait pouvoir être séparées par la rigueur analytique, sans rater l'essentiel. Les fêtes de San Fermín mieux connues sous le nom de fêtes de Pampelune ont acquis une renommée internationale aussi forte que le Carnaval de Venise ou celui de Rio. Ernest Hemingway est pour beaucoup dans la réputation et le rayonnement de la San Fermin en publiant, en 1926, le roman Le soleil se lève aussi, intitulé Fiesta dans le monde hispanique. Pampelune d'ailleurs le sait et le montre. La ville a rendu hommage à l'écrivain en édifiant un buste près des arènes, inauguré lors de la San Fermin de 1968, en l'honneur de celui qui fit connaître la féria. Sur ce buste est gravé « À Ernest Hemingway, prix Nobel de littérature, ami de ce peuple et admirateur de ses fêtes, qui sut décrire et faire connaître la ville de Pampelune. San Fermín. 1968 ». Sa vénération rivalise avec celle du saint patron. De nombreuses plaques, dans le vieux centre, mentionnent la fréquentation des lieux par Hemingway. Pendant la San Fermín, est organisé chaque année un concours de sosies pour élire le meilleur Ernest Hemingway. Certaines enseignes signalent avec un humour qui n'aurait sans doute pas déplu à l'écrivain lui-même : "Hemingway n'est jamais venu dans ce bar ${ }^{27}$. Les brochures consacrées aux fêtes de Pampelune de l'office du tourisme de la Navarre, reprises sur leur site Internet, présentent successivement l'histoire de la fête, celle du saint et celle de l'écrivain.

Qui est le rival de celui que l'on a adopté ici sous le nom de Don Ernesto ? Firmin est le fils d'un des sénateurs de la Pampelune romaine du III $^{\mathrm{e}}$ siècle, Firmus, converti au christianisme. Firminus (Fermin) répondit à la vocation d'évangélisateur et fut ordonné prêtre. Il revint à Pampelune une fois nommé évêque. La tradition fait de lui le premier évêque de Pampelune qui fut décapité à Amiens, lors d'une pérégrination évangélisatrice menée loin des terres navarraises. La liturgie fixa le jour du martyre le 25 septembre et le jour de la saint Firmin le 10 octobre à la date de son entrée dans la ville. À Pampelune, les actes religieux en l'honneur de San Fermín ne sont pas attestés avant le XII siècle. Contrairement aux apparences, San Fermín n'est pas le saint patron de la ville, mais de la Navarre, rang qu'il partage avec saint François Xavier. Les premières sources qui évoquent l'existence de courses de taureaux à Pampelune datent du $\mathrm{XIV}^{\mathrm{e}}$ siècle. Jusqu'en 1590, la San Fermín était fêtée à la date du 10 octobre, mais cette année-là, la fête fut noyée sous un déluge. Le changement de date pour fêter la San Fermín eut lieu l'année suivante en 1591, quand les édiles eurent l'idée de faire coïncider la célébration du Saint Patron navarrais avec la foire au bétail, fixée généralement entre la Saint-Jean et la mi-juillet. Le 7 juillet, qui tombait cette année-là un dimanche, fut décrété officiellement par l'évêque de Pampelune, Bernardo Rojas, jour de la San Fermin. Beaucoup d'exégètes locaux

\footnotetext{
${ }^{27}$ Cette formule apparaît également sur une enseigne d'un restaurant de la Plaza Mayor à Madrid.
} 
font remonter la naissance des Sanfermines à cette date. La première San Fermín dans sa nouvelle version estivale dura deux jours et semble avoir rassemblé les principales composantes de la fête-féria : actes religieux, foire commerciale, divertissements en tous genres (théâtre, danse, musique) et courses de taureaux. $\mathrm{Au}$ début $\mathrm{du} \mathrm{xx}^{\mathrm{e}}$ siècle, la féria taurine comptait six corridas et constituait déjà une longue féria pour l'époque.

Aujourd'hui, l'élément le plus connu des fêtes de Pampelune est l'encierro, couru chaque matin à 8 heures précises, du 7 au 14 juillet. Au-delà de l'encierro, les manifestations taurines semblent rythmer le temps et structurer l'espace de la fête urbaine, sans pour cela éclipser la célébration du saint patron. La fête débute en réalité la veille avec au douzième coup de midi l'embrasement du chupinazo depuis le balcon de l'hôtel de ville sur la Plaza Consistorial. Le chupinazo est une fusée pyrotechnique mise à feu par un membre du conseil municipal, désigné par le maire en fonction de la majorité électorale. Cette habitude date du début des années 1940. La foule est réunie sur la place de la mairie et attend le départ de la fusée qui tonne comme un coup de canon pour annoncer le démarrage des festivités. Alors, tous en cour, les pamplonicas poussent le cri traditionnel « ;Viva San Fermín, Gora San Fermin! » et attachent à leur cou, le fameux foulard rouge qui complète la tenue blanche ${ }^{28}$. En matière de costume, ce que la tradition voit comme immémorial débute en 1931, lorsque le peintre navarrais Juan Marquina, membre de la « peña » La Veleta, s'habille en blanc, avec une ceinture rouge et un foulard rouge, pour rappeler le martyre du saint et sa décapitation ${ }^{29}$. En quelques années, les blouses noires des membres des autres « peñas » sont rangées au placard, à Pampelune, puis dans toute la Navarre, ainsi que, plus tardivement, dans de nombreuses villes du Sud-Ouest français.

À en croire les événements du chupinazo de 2006, dans le rituel millimétré des fêtes de Pampelune, les mots ont leur importance. En effet, cette année-là, le conseiller municipal Javier Eskudi, chargé de lancer le chupinazo, s'est refusé à prononcer depuis le balcon le traditionnel cri d'ouverture, remplacé pour la circonstance par « ;Gora Fermindonorean Jaiak, Vivan las fiestas de San Fermín! ». Ce n'est en aucun cas le discours prononcé d'abord en basque qui créa la polémique, mais le fait d'avoir remplacé «Vive San Fermín! » par «Vivent les fêtes de la San Fermín! », au point que le maire de Pampelune, Yolanda Barcina, s'empara du micro pour rétablir la formule officielle comme l'exige la tradition. Javier Eskudi justifia sa position dans un entretien en expliquant qu'en tant qu'agnostique, il se refusait à crier «Vive San Fermín! » et ajouta que l'événement était mondialement connu en tant que fêtes de la San Fermín. Cette polémique pose très explicitement le problème de l'identification de ce qui est fêté, lors des fêtes de Pampelune et, plus généralement comme nous l'avons suggéré, lors de toutes fêtes.

Dès le 6 juillet, la fête explose au rythme des nombreuses "bandas » et du groupe musical de la municipalité, La Pamplonesa, qui arpentent les rues sans

\footnotetext{
${ }^{28}$ Gora San Fermin est la traduction en basque de Viva San Fermín.

${ }^{29}$ J. Durand, Chroniques taurines, p. 179.
} 
discontinuer. En plus de la fête spontanée s'égrène, toute la semaine, une longue liste d'actes officiels. Le programme des fêtes de 2006 en recense 302, parmi lesquels les défilés de géants, la fête foraine, les feux d'artifice, les concerts, divers spectacles de rues, les cérémonies religieuses et les événements taurins. Les cérémonies religieuses commencent le 6 à 20 heures par les vêpres en l'honneur de San Fermín, dans l'église de San Lorenzo qui abrite la chapelle du saint. Le 7 juillet ont lieu la procession et la messe solennelle. Les Pamplonais ont introduit, non sans humour, dans les litanies à San Fermín des préoccupations païennes et prosaïques dont certaines intéressent de près l'univers tauromachique :

pour que les bonnes coutumes soient observées pendant les fêtes, nous te prions, écoute-nous ;

pour que par «bonnes coutumes » on entende ce que l'on doit comprendre, nous te prions, écoute-nous ;

pour qu'il n'y ait pas d'espontáneos, nous te prions, écoute-nous ;

pour que nous nous divertissions un peu dans les corridas de cette féria, nous te prions, écoute-nous ;

pour que les prix ne soient pas abusifs, nous te prions, écoute-nous ;

pour qu'il y ait une paire d'heures pour le repos, nous te prions, écoute-nous ;

pour que les tourments s'éloignent, nous te prions, écoute-nous ;

pour que les parents, $s^{\prime}$ ils doivent venir, nous préviennent ${ }^{30} \ldots$

Ces litanies constituent un premier lien carnavalesque entre la dimension religieuse et la dimension tauromachique de la fête. La foule n'a aucun mal, en cette première nuit de fête, à atteindre le petit jour pour assister ou participer à l'encierro. La veille, a lieu chaque soir vers 23 heures, l'encierrillo, qui se déroule sans coureur, permettant de transférer les bêtes d'un premier enclos (los corrales del Gas) vers l'enclos du départ de l'encierro (los corralillos de Santo Domingo). L'encierro démarre lorsque l'église de San Saturnino sonne les coups de 8 heures. Alors, la première fusée est lancée pour annoncer l'ouverture de l'enclos où sont gardés les taureaux de combat. Une deuxième fusée annonce que tous les taureaux sont sortis. Quelques minutes avant que l'encierro ne commence, les coureurs réunis devant l'image pieuse de saint Firmin située dans une niche au bas de la Cuesta de Santo Domingo demandent, le quotidien du jour roulé

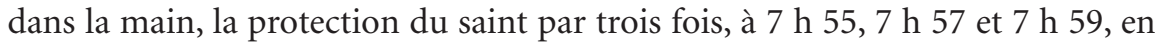
chantant :

\section{A San Fermín pedimos, \\ Por ser nuestro patrón, \\ Nos guíe en el encierro, \\ Dándonos su bendición ${ }^{31}$.}

\footnotetext{
${ }^{30}$ Cité par P. Dupuy, Guide des férias, p. 194.

31 Traduction : À San Fermín nous demandons / pour être notre saint patron / qu’il nous guide pendant l'encierro / en nous offrant sa bénédiction.
} 
Voici un deuxième point de contact entre la fête taurine et la fête religieuse, l'humour le cédant ici à la gravité et la concentration d'avant la course. Les hommes et les taureaux parcourent l'itinéraire menant aux arènes en un temps qui varie en moyenne de 2 à 4 minutes si aucun incident majeur ne vient séparer les bêtes, toujours plus agressives lorsqu'elles sont isolées. Une troisième fusée annonce à la ville que les taureaux sont entrés dans l'arène et une quatrième qu'ils sont enfermés dans les dépendances en attendant l'heure de la corrida. Commence alors une vaquilla dans les arènes remplies par les centaines de coureurs attardés et par les spectateurs qui se décident à descendre une fois les taureaux à l'abri. Plusieurs vaches aguerries au combat, aux cornes gainées ou épointées, se succèdent et n'ont pas de mal à bousculer les néophytes inattentifs et les soûlards téméraires ${ }^{32}$. Après l'encierro, la ville retombe dans un calme relatif et une ambiance plus familiale où ceux qui ne sont pas encore couchés croisent ceux qui viennent de se lever. La fête reprend du volume et du rythme dès la mi-journée. Beaucoup se projettent déjà vers le temps de la corrida. La foule enfle progressivement sur la Plaza del Castillo (la place centrale) ainsi que dans les rues alentour à mesure que s'ouvre l'appétit et que se fait sentir la soif.

Les corridas commencent à $18 \mathrm{~h} 30$, mais elles créent un temps et un lieu d'effervescence qui déborde largement le spectacle lui-même, de même que les quelque 2 ou 3 minutes de l'encierro structurent l'espace-temps de la fête. Une bonne heure avant le début de la corrida, les " peñas " pénètrent dans l'arène par la même porte qui a vu passer les coureurs. Les "peñas » sont des associations festives créées pour et par la San Fermín. Il existe officiellement 16 « peñas " pamplonaises regroupées au sein d'une fédération. Pendant les fêtes convergent d'autres associations et d'autres formations musicales : «bandas » du Sud-Ouest, parfois «peñas» du Sud-Est et fanfares venues d'ailleurs. La première " peña », La Única, a été créée en 1903. Les " peñas » défilent en piste en jouant de la musique, agitent des banderoles humoristiques sur des thèmes d'actualité locale, nationale et internationale. Ils gagnent progressivement leur place dans les gradins situés au soleil ainsi que dans les parties les plus hautes et les moins chères de l'arène. Dans cette zone, l'ambiance festive ne fera que monter en puissance tout au long de la corrida quels que soient les événements qui se déroulent en piste. Les boissons coulent à flot au sens propre et au sens figuré : vin, sangria, bière, alcools forts et divers mélanges qui combinent presque à l'infini les possibilités. À partir du troisième taureau, circulent également les mets les plus divers préparés pour être consommés dans les arènes,

\footnotetext{
${ }^{32}$ Pour avoir participé à de nombreuses San Fermin, il convient de relativiser les nombreuses descriptions qui insistent assez lourdement sur le fait que les étrangers seraient comme prédestinés à être bousculés par les bovins, soit parce qu'ils ont trop bu, soit parce qu'ils n'y connaissent rien. Ce lieu commun se voit également répété pour les encierros où les risques sont beaucoup plus élevés. Cette simplification ne se vérifie d'aucune façon dans les faits. Au cours du $\mathrm{xx}^{\mathrm{e}}$ siècle, sur les 13 personnes mortes au cours des encierros de Pampelune, seuls un Américain et un Mexicain ne sont pas Espagnols et 9 sont Navarrais. Cet écart entre les faits et les représentations ouvre une piste intéressante pour qualifier les processus identificatoires mis en jeu par les fêtes locales qui sont à la fois nécessairement ouvertes aux personnes venues d'ailleurs, tout en les stigmatisant.
} 
plateaux de charcuterie, croquettes, tortillas, empanadas, bocadillos ainsi que des plats en sauce, ragoûts, boulettes de viande, morues, tripes, oreilles, mangés à grand renfort de pain et distribués tous azimuts. Les « peñas » chantent et se répondent au son ininterrompu des grosses caisses. Le contraste est fort avec les spectateurs plus conventionnels situés à l'ombre. Pas d'orchestre spontané, l'atmosphère y est plus retenue, les tenues vestimentaires plus soignées, sans que l'ambiance pour autant ne soit guindée.

Sur ce point, les descriptions des arènes de Pampelune souffrent souvent de deux approximations : l'une concernant le public assis à l'ombre, l'autre concernant le public installé au soleil. Souvent l'existence même d'un public à l'ombre est quelque peu occulté par l'impact visuel et sonore des « peñas » faisant alors des arènes un cratère rugissant uniformément embrasé par la fête. Et lorsque le public à l'ombre est pris en compte, les hommes en complet et les femmes en robe sont souvent cantonnés dans un rôle exagérément sérieux, comme s’il était besoin d'accentuer un contraste déjà si fort. De même dans une accusation excessive des contrastes, la plupart des descriptions du public du soleil tendent à considérer qu'il s'intéresse à toute autre chose qu'à la prestation en piste des hommes et des taureaux. Là encore, il conviendrait de nuancer le propos. On préfère affirmer que le public du soleil se désintéresse lorsqu'il ne se passe rien d'exaltant (ce qui en tauromachie n'est pas rare), jusqu'à tourner le dos à la piste en signe de protestation lorsque la prestation est particulièrement ennuyeuse (ce qui est paradoxalement la manifestation d'un intérêt certain). Ce public est également rattrapé en permanence par la fête dans les gradins, qui constitue un intérêt en soi, mais il sait aussi faire preuve d'une ferveur immense lorsque le torero réussit à capter son attention. Le nom du torero El Juli n'a sans doute jamais été hurlé aussi fort qu’à Pampelune lorsqu'il triomphe : le cri "ijulijuli! », avec une forte accentuation de la jota, accompagné à la grosse caisse, en est même devenu un chant rituel pour accueillir le torero, acclamé par les uns, sifflé par les autres. Lorsque la corrida prend fin, les « peñas » envahissent à nouveau la piste et ressortent lentement, une à une de l'arène, au rythme des fanfares suivies par une partie du public qui suit le cortège sonore dans toute la ville. Les plus endurants atteindront l'heure de l'encierro.

À Pampelune, la féria taurine rebaptisée, en 1959 la feria del toro fait corps avec la ville et les fêtes de la San Fermín. Même si les éléments qui la composent conservent leur raison d'être et leur autonomie, des relations nombreuses se tissent entre les différents objets de la fête, entre les lieux et les pratiques.

c) Séville : la Féria et la féria taurine

Séville offre un cas encore différent d'imbrication complexe entre la féria taurine et la grande fête de la ville, la Feria, généralement écrite avec une majuscule. Les deux férias ne doivent pas être confondues. Bien que la féria taurine soit située, de même qu'à Pampelune, au cœur du dispositif festif, les relations entre fête et tauromachie se traduisent très différemment. Soulignons en premier lieu que Séville possède plusieurs fêtes d'importance, appelées Fiestas Mayores (Grandes Fêtes) dont la Semana Santa (Semaine sainte) et la feria de abril (féria 
d'avril), également appelée fiestas de primavera (fêtes de printemps). Toutes deux sont des fêtes qui attirent de nombreux visiteurs nationaux et internationaux $^{33}$. La première est une fête fondamentalement religieuse qui commémore la passion et la mort du Christ. La seconde est une fête qui éclate en de multiples activités complémentaires, dont la tauromachie est une des composantes importantes. La féria d'avril commence le troisième mardi postérieur à la Semaine sainte et dure jusqu'au dimanche.

On considère généralement que la première féria d'avril est celle de 1847 qui a duré trois jours, du 19 au 21. Dès sa première édition a eu lieu une course de taureaux à laquelle n'a pris part aucun grand torero de l'époque ${ }^{34}$. La première féria d'avril naquit d'une initiative mercantile qui visait à promouvoir une grande foire au bétail, afin de relancer l'activité commerciale d'une région en crise en ce milieu du $\mathrm{XIX}^{\mathrm{e}}$ siècle, dont les principales richesses étaient celles de l'agriculture et de l'élevage. À l'origine de cette initiative, on trouve deux représentants de la bourgeoisie locale émergente, José María Ybarra et Narciso Bonaplata, l'un basque et l'autre catalan, qui proposèrent le projet aux autorités municipales. L'idée fut appréciée en haut lieu et la féria autorisée par décret royal, signé de la main de la reine Isabelle II. Ce décret renouait ainsi avec une autorisation d'Alfonse X le Sage, de 1254, qui avait accordé la tenue de deux foires à Séville, l'une au printemps et l'autre à l'automne. La foire au bétail du milieu du xix siècle prit pour modèle celles déjà existantes dans les villages sévillans de Carmona et de Mairena del Alcor ${ }^{35}$. Jusqu'en 1972, la féria avait lieu sur le Prado de San Sebastián au sud-est de la ville pour être ensuite transférée dans le quartier de Los Remedios, de l'autre côté du Guadalquivir, afin de pouvoir s'agrandir et répondre à l'afflux croissant de visiteurs, en ces années d'élargissement de la fréquentation locale, nationale et internationale.

Le champ de foire actuel s'étend sur environ 500.000 mètres carrés répartis entre le Real de la Feria, l'enceinte de la féria proprement dite et la fête foraine attenante. Il faut ajouter à cette superficie les quelque 500.000 mètres carrés réservés au stationnement des véhicules pour lesquels sont prévus environ 20.000 emplacements ainsi que les espaces réservés aux divers services (postes de police, centre de secours de premiers soins, pompiers, assistance vétérinaire...). Le champ de foire est une petite ville. La féria actuelle n'est évidemment plus liée à la foire au bétail, qui néanmoins existe toujours et se déroule les trois jours précédant l'illumination rituelle de la portada et du campo de Feria. Chaque

\footnotetext{
${ }^{33}$ Pour donner un ordre de grandeur, chacune des fêtes regroupent entre 500.000 et 1 million de participants. La Semaine sainte de 1999 aurait compté 288.000 visiteurs dont 143.000 Espagnols et 145.000 étrangers pour un total de 676.000 participants. E. Aguilar Criado, « La economía de la fiesta », p. 210.

${ }^{34}$ Cette corrida opposa les taureaux de don Luís Taviel de Andrade aux toreros Juan Lucas Blanco et Manuel Díaz « El Lavi », laissant de côté les grandes figures de l'époque Curro Cúchares et Francisco Montes Paquiro. «El Lavi » fit partie des toreros qui ont tenté, par la suite, leur chance en Amérique.

${ }^{35}$ E. Aguilar Criado, « La economía de la fiesta », p. 206.
} 
année un concours est organisé pour l'édification de cette grande porte monumentale qui donne accès à l'enceinte du champ de foire et qui est illuminée le premier jour de la féria à minuit. Le campo de Feria conserve du champ de foire au bétail la division en allées, répartissant de part et d'autre les rangées de casetas, où naguère les négociants traitaient leurs affaires en observant à l'abri du soleil, les enclos à bestiaux.

Les casetas, ces fameuses tentes en toile rayées au couleur de Séville (rouge et blanc) et de l'Andalousie (rouge et vert), donnent désormais à nombre de férias leur identité visuelle bien au-delà de l'ancienne Bétique ${ }^{36}$. Les farolillos, ces ampoules électriques entourées de papier qui décorent les allées le jour et les éclairent la nuit, participent également de cette ambiance visuelle à laquelle s'ajoutent les calèches, les hommes en traje corto, les femmes en robe de gitane. La fête se déroule à l'intérieur des casetas qui sont privées dans leur immense majorité et dont le nombre est désormais supérieur à mille ${ }^{37}$. C'est ici qu'à toute heure, on danse et on chante des airs de sevillanas, sur une estrade en bois entourée de chaises, inspirée des tablaos du flamenco. C'est ici que l'on déguste la mazanilla, vin blanc sec de la région de Sanlúcar de Barrameda, généralement servie en demi-bouteille pour en garder la fraîcheur. Elle est fréquemment mélangée à du Seven Up par tous ceux qui souhaitent adoucir ce vin sec. Ce mélange, nommé rebujito, est quasiment devenu une deuxième boisson officielle des férias andalouses. Il est en quelque sorte l'équivalent méridional du Kalimutxo du Nord de l'Espagne, qui mélange le vin rouge et le Coca-Cola. Le premier très clair, le second très sombre, les deux terriblement sucrés. C'est ici que l'on mange des calamars, des poissons frits, du fromage à pâte dure, du jambon et du chorizo ibérico. Le plan intérieur des casetas et leur décor reproduit l'image d'une maison dans laquelle vous êtes invité... ou non. Au fond de cette maison, se situent les cuisines qui servent également de comptoir, audevant s'allonge une pièce qui prend des allures de salon cossu avec des meubles, des tableaux, des photos de famille, des tables et des chaises disposées autour d'une estrade. La première caseta librement ouverte au public fut celle de la mairie dans les années 1970, à la suite de la première élection démocratique. C'est à partir de cette période que des autorisations ont été accordées aux partis politiques, aux syndicats et à diverses associations pour ouvrir des casetas, élargissant ainsi considérablement la base sociale de la fête. Pendant longtemps la Féria est donc restée une fête fermée, organisée pour et par les élites locales, une vitrine de la bourgeoisie et de l'aristocratie sévillane qui se rendaient entre elles les invitations. La Féria s'est aujourd'hui démocratisée sans pour autant renoncer, d'une caseta à l'autre, à faire valoir son rang. Dans cette économie de la distinction, les taureaux occupent une place de choix.

\footnotetext{
${ }^{36}$ Buren n'aurait pas été plus strict pour définir les normes de ces rayures qui doivent mesurer 10 centimètres de large et être imprimées verticalement sur les deux faces de la toile.

${ }^{37}$ La féria de 2006 a compté 1.046 casetas dont 32 familiales, 500 partagées entre plusieurs familles, 313 entités, 180 " peñas ", 7 casetas municipales à entrée libre, 13 casetas des Services municipaux et la caseta de la mairie de Séville.
} 
Remarquons d'abord que la féria taurine dure plus longtemps que la Féria. Elle commence généralement quelques jours après le dimanche de Pâques qui marque la fin de la Semaine sainte et se termine le lendemain de la Féria. C'est elle qui dans le temps fait la liaison entre les processions de la Semaine sainte et les fastes de la Féria. La corrida du dimanche de Résurrection, qui est hors féria taurine, signifie à Séville le début de la saison tauromachique et constitue l'un des événements les plus prestigieux de la "planète des taureaux ", qui n'a sans doute pour seul équivalent en Espagne que la corrida de la Bienfaisance de Madrid. Le ban et l'arrière-ban du gratin sévillan s'y retrouvent dans une sorte de prologue maniériste de la féria d'avril. Certaines bonnes familles s'y rendent à cheval, les uns remontent la rive du Guadalquivir sur laquelle s'ouvre la porte principale des arènes, la porte du Prince, les autres patientent à l'ombre dans la rue opposée, la calle Adriano, de laquelle les arènes insérées dans le tissu urbain sont invisibles. Les arènes de Séville appartiennent à un ordre de chevalerie, la confrérie de la Maestranza créée en 1670. Cette confrérie intégra des chevaliers appartenant à des confréries plus anciennes, celle de San Hermenegido et celle de la Vierge du Rosaire, deux figures tutélaires de la cité. Ces chevaliers sont les descendants des nobles venus du Nord de l'Espagne pour reconquérir l'Andalousie maure. C'est cette même confrérie à qui fut confiée l'organisation exclusive des corridas. En 1733, la Maestranza construit une arène en bois, au lieu-dit El Baratillo du quartier de l'Arenal, qui est remplacée en 1763 par les arènes actuelles édifiées au même endroit. Cet héritage suffit à donner le ton et la mesure de ce que représentent les arènes de la Real Maestranza de Caballería de Sevilla : un sanctuaire de la tauromachie et un haut lieu de l'identité sévillane.

La féria taurine de Séville compte aujourd'hui une vingtaine de courses consécutives, ce qui en fait numériquement la deuxième féria la plus importante après celle de Madrid. La bonne société sévillane se rend à la Real Maestranza comme celle de Milan se rend à La Scala. Si la Féria s'est démocratisée, les corridas à Séville sont plutôt allées dans le sens d'un élitisme croissant, ne serait-ce que par les prix élevés des entrées. Mais là comme ailleurs, le public des arènes ne peut être réduit à une classe homogène de spectateurs. Les corridas de Séville attirent de nombreux aficionados venus de toutes parts et de nombreux touristes souvent regardés avec hauteur. Les premiers, quelle que soit leur origine géographique, ne se définissent jamais comme les seconds, comme si le goût et la connaissance de la tauromachie leur offraient une forme de naturalisation. Les néo-locaux s'empressent souvent d'afficher, avec plus ou moins de discrétion, qu'ils connaissent les lieux et qu'ils y viennent depuis des années : il ne faudrait pas passer pour un nouveau venu et surtout par pour un touriste. Pour cela, les places situées à l'ombre sont souvent convoitées, tout au moins celles qui ne sont pas réservées, d'année en année, par les abonnés sévillans. On aura compris que l'arène fonctionne comme un creuset d'observation et de reconnaissance des autres, entre le haut et le bas, entre l'ombre et le soleil, entre les aficionados d'ici et les aficionados d'ailleurs, qu'ils soient madrilènes, de plus en plus nombreux grâce à la liaison ferroviaire à grande vitesse, ou qu'ils viennent des quatre coins du monde. La corrida à Séville est-elle une fête ? Non, 
si l'on observe l'immense majorité des corridas qui se déroulent dans le plus grand silence, celui d'un public recueilli ou indifférent, mais qui s'abaisse très rarement à exprimer bruyamment son insatisfaction. Oui, si l'on tombe le jour où l'arène se livre à l'unisson d'un olé parfaitement synchrone qui rythme les instants de grâce d'un artiste inspiré. Il ne fait aucun doute que dans ce cas, qui en tauromachie ne peut jamais être prévu à l'avance, le spectacle est une fête avec les attributs qui classiquement la caractérisent : la liesse, l'effervescence, la démesure, la spontanéité.

Quoi qu'il en soit, la tauromachie est vécue comme un élément totalement indispensable à l'univers de la Féria. Les arènes constituent à partir de 18 h 30, le centre névralgique de la fête. Les bars et les restaurants alentour ne désemplissent pas. Les attelages venus du campo ferial se succèdent aux abords des arènes pour déposer leurs hôtes de marque. Ceux restés au campo ferial regarderont également la course retransmise à la télévision. La relation inverse n'est pas vraie puisque beaucoup d'aficionados n'affectionnent pas du tout l'ambiance du champ de foire et prévoient même de se rendre à Séville uniquement pour les jours de la féria taurine qui précèdent la semaine dite des farolillos, c'est-àdire la semaine de la Féria. Ils trouvent que la féria taurine est alors plus intime et la ville plus agréable que lorsque le campo férial, à l'extérieur du centre-ville, polarise toutes les attentions au point que beaucoup de rues de Séville sont alors totalement désertes. En effet, à ce moment-là, les rues en fêtes sont bien celles de cette ville éphémère constituée de toiles de tentes et de rues qui toutes portent des noms de matadors sévillans. C'est que la Séville utopique du campo férial ne cesse de jouer de la connivence avec l'univers tauromachique qui participe pleinement à l'identité sévillane et andalouse. Pour certains les liens entre fête et tauromachie à Séville vont encore au-delà, à l'image des analyses de Pedro Romero de Solís. Conformément à une interprétation sacrificielle de la tauromachie que l'on a déjà présentée, Pedro Romero de Solís voit dans la corrida du Dimanche de Résurrection le point d'orgue de la Semaine sainte qui ouvre sur le temps profane des réjouissances collectives de la Féria : «En réalité pour la sagesse inconsciente du peuple sévillan, le grand rite compensatoire, la grande cérémonie durant laquelle la mort de Dieu est surpassée par le triomphe de la Vie ne peut être une procession de plus sinon celle qui se produit lors de la corrida célébrée dans les arènes de la Maestranza, le Dimanche de Résurrection ${ }^{38}$ ". La tauromachie occupe à Séville une place visible toute l'année, même en dehors de la Féria qui en accentue la présence. La tauromachie constitue ici un élément à la fois autonome et intégré au contexte festif global.

\section{FÊTES TAURINES ET TERRITORIALITÉ}

Les trois exemples précédents montrent à quel point l'idée de fêtes taurines qui auraient partout la même place et la même signification ne résiste pas à

\footnotetext{
${ }^{38}$ P. Romero de Solís, « Sevilla: tauromaquia y religiosidad », p. 168.
} 
l'analyse des relations très variables entre la fête et la tauromachie. En se limitant au cas des férias taurines de Madrid, de Séville et de Pampelune, on observe déjà la pluralité des facteurs qui déterminent cette relation.

À Madrid, la féria taurine est la plus importante du monde, mais elle apparaît comme indépendante et éloignée des autres activités festives de la San Isidro. La taille de l'agglomération et la localisation périphérique des arènes contribuent à cela. À moins que son rôle de haut lieu de la tauromachie mondiale lui permette de se passer d'un enracinement local fortement territorialisé, à l'image de certaines activités d'excellence internationale qui dans le contexte de la métropolisation ont davantage de liens avec les villes de rang équivalent qu'avec leur environnement immédiat. Autrement dit, tout donne l'impression que la portée nationale et transnationale de la féria de la San Isidro n'a guère besoin de liens visibles entre fête et tauromachie pour s'assurer d'une influence réciproque, bénéfique et cumulative.

À Pampelune, on observe un stade très avancé de fusion entre la fête urbaine et l'activité taurine qui se présente sous la forme conjuguée de la tauromachie professionnelle et de la tauromachie participative. Cette articulation entre tauromachie participative et tauromachie professionnelle, qui s'inscrit spatialement dans l'arène et dans la rue, renforce l'omniprésence matérielle et symbolique de la tauromachie dans la fête urbaine. L'idée d'une fête taurine partagée par tous consacre une dimension égalitaire de la tauromachie à Pampelune, au-delà des clivages toujours présents entre culture savante et culture populaire. À Pampelune, la fête taurine navarraise apparaît comme une fête fédératrice, même si l'on doit aussi la considérer comme une fête distinctive par rapport aux autres façons de vivre la tauromachie.

À Séville, la tauromachie imprègne les références culturelles mobilisées par la fête urbaine, mais l'activité taurine ne peut être considérée comme la fête ellemême. La Féria fonctionne par la complémentarité des lieux de fête qui prennent la forme d'un réseau dont les deux nœuds principaux sont les arènes de la Maestranza et le campo ferial. La stratification sociale rendue visible par la fête, se joue dans la maitrise de l'espace festif et plus précisément dans la capacité à être présent dans l'un et l'autre lieu. Avoir une bonne place aux arènes (et parfois avoir une place tout court) et être invité dans de nombreuses casetas privées sont les signes d'une pleine et valorisante participation à la fête. Dans le système festif sévillan, l'accès à la tauromachie participe puissamment à la distinction sociale des élites. Cette participation est en outre relayée par les médias et les chaînes de télévision locales qui sont autorisés à entrer dans les arènes et dans les casetas. Sous cet angle, la Féria rend visible un pouvoir qui s'appuie sur des stratégies territoriales locales : être présent et être visible dans les lieux de centralités festives, qui le temps de la Féria se hissent au rang de centre du monde.

Taille de la ville, situation des arènes dans le tissu urbain, localisation des réjouissances taurines dans l'espace festif, rôle de la tauromachie comme facteur de distinction sociale et/ou de cohésion communautaire, voilà autant de facteurs qui contribuent à la diversité des relations entre fêtes taurines et territoire local. Â ces facteurs, s'ajoute la place ambivalente du jeu tauromachique lui-même. 
En tant que spectacle ordonné et réglementé, la tauromachie peut apparaître, à beaucoup d'égards, comme une activité anti-festive si l'on retient comme attributs distinctifs de la fête le tumulte, l'effervescence et le désordre. Sous cet angle, on perçoit aisément l'originalité et la dimension subversive du spectacle taurin de Pampelune en comparaison des spectacles taurins madrilènes et sévillans, où l'embrasement festif demeure l'exception. L'idée même d'une saison tauromachique, aux spectacles hebdomadaires, inscrits dans un temps régulier, répétitif, presque habituel, atténue l'idée d'une fête taurine perçue comme un espace-temps d'exception et de rupture avec le quotidien. Malgré cela, il n'est pas déraisonnable de penser que tout divertissement qui rassemble une foule dans un temps et un espace donné relève bien d'une activité festive. Enfin, la coïncidence d'un grand rassemblement avec le calendrier festif officiel place nécessairement le divertissement, qui par ailleurs peut avoir sa propre autonomie, sous le signe de la fête. La tauromachie participe ainsi à la création de lieux de centralités festives qui n'ont pas tous les mêmes caractéristiques.

Nous pensons que ces lieux de centralité festive ne fonctionnent pas indépendamment les uns des autres, ce qui nous permet de mettre en relation le territoire local avec des territoires plus englobants, à commencer par le territoire national. Les férias des principales villes taurines d'Espagne sont comme les vitrines des valeurs tauromachiques locales, provinciales ou régionales selon le rang occupé par la cité. Le déroulement de la saison tauromachique est rythmé par le cycle des férias qui chaque année se répète. Cette structure a pour effet une circulation dans le temps et dans l'espace d'une centralité tauromachique éphémère. Autrement dit, malgré l'importance inégale des férias, chacune peut assumer à tour de rôle, d'être le centre vers lequel converge l'attention des amateurs, centre qui transite au rythme d'un calendrier taurin relativement fixe. Malgré le poids écrasant des férias de Madrid et de Séville, et de façon secondaire de Bilbao, Pampelune, Valence... cette forme de centralité circulatoire empêche la constitution d'une version unique et normative des valeurs tauromachiques. Si l'on accepte de considérer que les valeurs véhiculées par les pratiques tauromachiques sont, au moins en partie, celles de la communauté locale qui leur donne forme et signification, alors la structure spatiale et temporelle de la saison tauromachique permet leur confrontation à distance. Ainsi se voit concrétiser dans le champ de la tauromachie, la rivalité entre les grandes villes d'Espagne. Les mêmes toreros circulent d'arènes en arènes, qui les acclament ou les accablent, en fonction de leur prestation. Nous sommes là en présence d'un système propre à la tauromachie qui est assez différent de celui qui existe dans de nombreux sports collectifs, proposant des confrontations entre une équipe locale et une équipe de visiteurs.

Le fait que la corrida relève davantage de la représentation artistique que du sport, ainsi que nous l'avons évoqué précédemment, trouve ici un prolongement significatif. Nous pensons que le torero apparaît comme un intercesseur d'une identification qui n'est pas de même nature que celle produite par les modèles compétitifs sportifs. Dans le sport, et nombreux rodéos américains sont ici concernés, l'exclusivité de l'appartenance territoriale est rendue expli- 


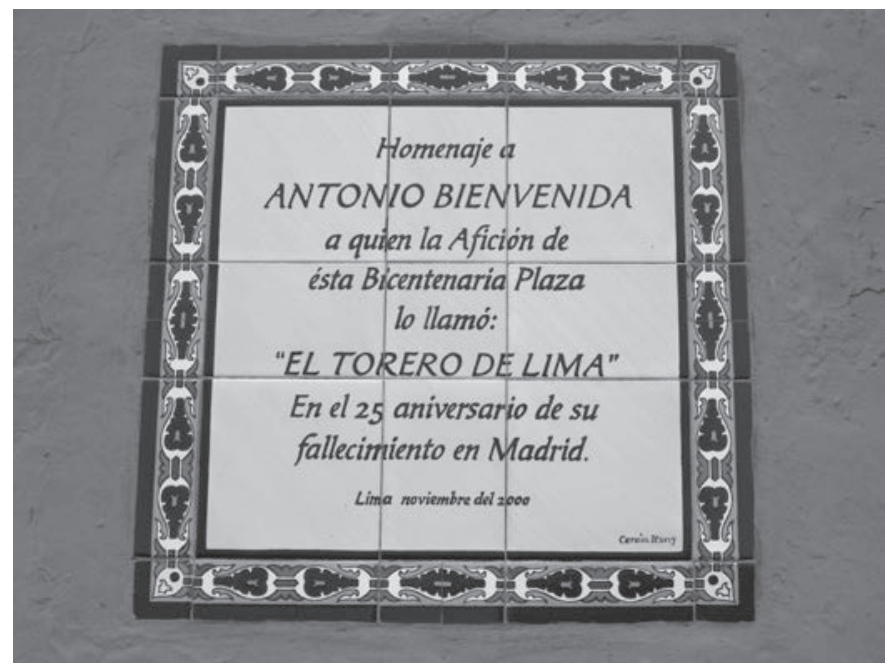

Fig. 47. - Antonio Bienvenida, «le torero de Lima». Hommage à Antonio Bienvenida que l'afición de cette arène bicentenaire appela « Le torero de Lima». Pour le $25^{\mathrm{e}}$ anniversaire de sa mort (Lima, 2005, cliché : Jean-Baptiste Maudet)

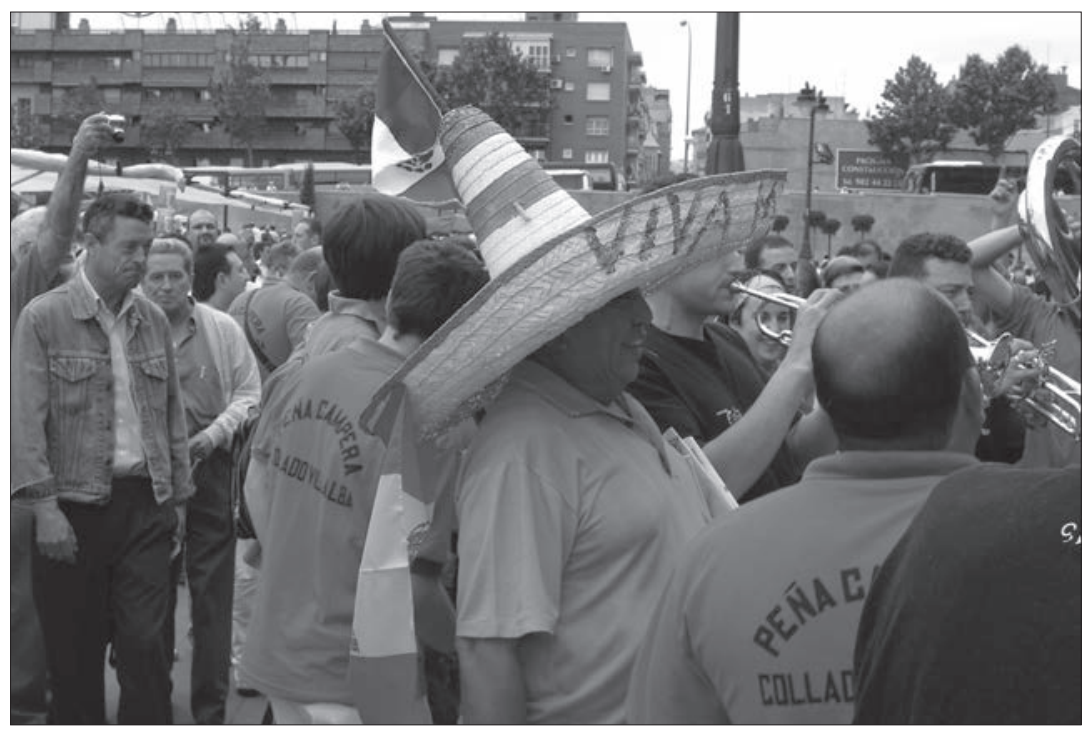

Fig. 48. - Signes identitaires ostensibles d'une hispanité syncrétique (Las Ventas). La Peña Campera de la ville de Collado Villalba joue devant les arènes de Madrid. Un aficionado mexicain dans une autocaricature des sentiments d'appartenance multiple, véhiculés par la corrida (Drapeau mexicain, Viva Mexico et drapeau espagnol). [Madrid, 2005, cliché : Jean-Baptiste Maudet]. 
cite par la formation d'équipes locales ou régionales, par le port d'une couleur renvoyant à un territoire défini. Les spectateurs soutiennent leur équipe, qu'elle perde ou qu'elle gagne, selon des critères parfaitement objectifs et comptables. La victoire d'une équipe sur une autre, et donc d'une ville sur une autre, d'une région sur une autre, ou d'un pays sur un autre, quand bien même serait-elle injuste, est indiscutable. $L^{\prime}$ « autre » est reconnu, soit dans sa victoire, soit dans sa défaite, et permet la différenciation heureuse ou malheureuse d'une identité locale. Dans la corrida, il n'y a pas d'équipe ou d'individu qui représenterait un lieu ou une identité locale exclusive. Il y a certes des toreros d'ici et des toreros d'ailleurs, mais leur rôle est différent de celui que peuvent jouer des équipes ou des individus représentant leur petite patrie. L'habit de lumière, interchangeable, alors même que l'identité des toreros est bien connue, les met en apparence sur un pied d'égalité. Selon nous, la corrida combine un chauvinisme avéré et un principe de reconnaissance de l'altérité qui est soumis à la subjectivité du triomphe plus qu'à l'objectivité de la victoire. Précisons. Lorsque vient à échouer un torero qui n'est pas du cru, il est renvoyé à son origine géographique, ce qui signifie dans le même temps un déni de son talent. Autrement dit, et en forçant le trait : " tu n'as pas triomphé, tu ne sais pas toréer, tu peux retourner chez toi où, d'ailleurs... personne ne sait toréer ». Mais s'il triomphe, le public se reconnaît en lui, quelle que soit son origine géographique, bien qu'il soit généralement plus facile d'y parvenir lorsque l'on est la figure locale. Le torero qui triomphe sort alors par la grande porte, ouverte sur la cité en signe d'adoption par la communauté. Le torero se voit comme naturalisé par le triomphe et la cité renforcée dans son identité, puisqu'il y a une valeur à avoir triomphé ici et pas ailleurs. Autrement dit : « tu sais toréer, tu as triomphé, tu es des nôtres... ainsi que tous les tiens ». Ce principe souffre sans doute une exception de taille, Madrid, qui n'a peut-être pas d'autre héros que son public lui-même, qui aime tant faire et défaire les hiérarchies.

Nous pensons que ce mécanisme, sinon propre à la corrida, du moins exacerbé par elle, aide à comprendre les relations territoriales complexes tissées entre les terres de corridas, qui rappelons-le sont les seules à s'étendre sur deux continents. C'est ainsi, parmi de très nombreux exemples au cours de l'histoire, que le grand matador espagnol Manolete a pu devenir « le torero de Mexico » et de bien d'autres villes, ou qu'Antonio Bienvenida a pu devenir le « torero de Lima » et de bien d'autres villes (voir fig. 47). À l'inverse, c'est ainsi que le torero colombien César Rincón devint, au début des années 1990, « le torero de Madrid », acclamé au cri de « ¡Viva Colombia! " par le public madrilène, qui à travers ce nouvel empereur entend jeter le discrédit sur les figures nationales.

Notre séjour à la féria de Quito en 2005, nous a permis d'étayer cette lecture. Le public, pour encourager le torero national Guillermo Albán, scandait "Ecuador, Ecuador, Ecuador ». Il fut très applaudi avant et après sa prestation honorable. La déroute du sévillan Morante de la Puebla s'est soldée par des " rentre chez toi, l'aéroport n'est pas loin » (les arènes de Quito sont situées à quelques centaines de mètres de l'aéroport Mariscal Sucre). En revanche, le triomphe du valencien Enrique Ponce a motivé une forte ovation qui a laissé 
échapper le fameux cri "Ecuador, Ecuador " lorsque ce dernier, d'un geste symbolique, a ramassé une poignée de sable de la piste, en signe d'adoption consentie par la terre d'accueil. Sous cet angle, la corrida peut alors être considérée comme un support d'identification territoriale locale, qui passe par l'expérience d'une altérité, rejetée ou adoptée, dans une combinaison renouvelée à chaque féria. La tauromachie tisse alors des liens entre des territoires qui partagent une passion commune habitée par des sentiments concurrentiels d'hispanité composite (voir fig. 48, p. 138). 
DEUXIÈME PARTIE

\section{LA CONFIGURATION}

DE L'ESPACE TAUROMACHIQUE 

Rendre compte de la configuration de l'espace tauromachique, c'est en premier lieu insister sur l'existence d'un espace fragmenté. Mais malgré le recoupement partiel des pratiques tauromachiques dans l'espace, leur inégale extension et le maillage des frontières territoriales, nous avançons l'idée qu'il existe un espace tauromachique relativement cohérent. Comme nous l'avons précisé en introduction, cette cohérence est double. Elle est d'abord le fruit d'une histoire, celle de la conquête du Nouveau Monde par les Espagnols et les Portugais qui font découvrir aux populations locales, les jeux taurins et les méthodes d'élevage de la péninsule Ibérique. La question tauromachique participe alors de l'un des bouleversements culturels les plus importants de notre histoire, né de la rencontre de l'Europe et de l'Amérique, qui constitue aussi l'une des étapes majeures de la mondialisation. Cette question participe également des enjeux scientifiques de "la Redécouverte de l'Amérique ", tels qu'ils ont été définis par Carmen Bernand et Serge Gruzinski à l'occasion du cinquième centenaire de la découverte du Nouveau Monde : « Par-delà le discours pluridisciplinaire qui se propose d'articuler anthropologie, histoire, sociologie, c'est dans une approche à géométrie variable que dorénavant nous sommes entraînés, approche qui s'adapte cas par cas aux questions du chercheur et à la spécificité de son objet $»^{1}$.

La cohérence d'un espace tauromachique au singulier est aussi le fruit d'un regard parfaitement contemporain qui, malgré les discontinuités de son organisation et derrière le cloisonnement des cultures taurines, fait apparaître des relations de sens et de fonctionnement entre les pratiques. C'est à travers la notion de coexistence spatiale que la nature de ces relations changeantes, selon les lieux et selon les pratiques considérées, peut être appréhendée. L'approfondissement de cette question traitée dans des cadres nationaux fera l'objet de la troisième partie. L'enjeu est pour l'instant de qualifier au mieux les limites et les discontinuités majeures de l'espace tauromachique, ainsi que les processus qui ont présidé à sa formation générale. Pour ce faire, nous analysons

\footnotetext{
${ }^{1}$ C. Bernand et S. Gruzinski, «La redécouverte de l'Amérique ».
} 
les limites de chacune des pratiques tauromachiques en lien avec les cadres juridiques et territoriaux nationaux de leur régulation (chap. IV). Puis nous retraçons les grandes étapes de genèse, de transformation, de diffusion, d'enracinement et de rejet des pratiques tauromachiques, qui donnent à cet espace tauromachique, du Sud-Ouest européen à l'Amérique, son contenu et sa géométrie (chap. v). 


\section{LIMITES ET DISCONTINUITÉS DE L'ESPACE TAUROMACHIQUE}

La question des limites et des discontinuités des pratiques tauromachiques pose le problème des décalages entre les frontières historiques de leur diffusion, les frontières légales des jeux aujourd'hui autorisés ou interdits, et les frontières réelles de leur extension. La question peut ainsi être appréhendée à travers les cadres juridiques qui autorisent, tolèrent ou interdisent la pratique de tel ou tel jeu taurin. En tant que spectacle, et en raison d'une interaction mettant en jeu l'intégrité physique des hommes et/ou des animaux, les jeux taurins impliquent fortement les pouvoirs publics. Sur ce plan, les divertissements tauromachiques européens ont suscité depuis toujours de vifs débats conduisant les autorités à exercer un contrôle sur les conditions de leur déroulement. Les rodéos, perçus comme étant issus de la sphère du travail et dont l'émergence en tant que spectacle moderne est plus tardive, n'ont généralement pas connu la longue histoire des interdictions à laquelle la corrida a été confrontée. Cela étant, les rodéos aujourd'hui n'échappent pas aux critiques formulées par les mouvements de protection animale, de mieux en mieux organisés à l'échelle mondiale. La division entre les pays où la corrida est autorisée et les pays où elle est interdite ne correspond à aucun découpage régional évident, pas plus en Europe qu'en Amérique. Les pays où les pratiques tauromachiques sont autorisées ont aujourd'hui un point commun fondamental : elles sont légalement exclues du champ d'application des lois de protection des animaux qui pourraient en condamner l'exercice.

\section{I. - LES LIMITES EUROPÉENNES \\ DES PRATIQUES TAUROMACHIQUES}

En Europe, les limites externes des pratiques tauromachiques sont celles de la corrida, ou plutôt, on observe qu'aucune autre pratique ne déborde son aire d'extension. Tout se passe comme si la corrida bornait l'espace tauromachique à l'intérieur duquel se distinguent des régions taurines aux profils différents. La seule exception européenne, que chacun pourra juger anecdotique ou non, serait les jeux taurins d'Interville qui montrent que d'un point de vue juridique 
rien n'empêcherait certaines pratiques de s'exporter ailleurs, en France et dans d'autres pays où le programme télévisé a déjà été imité. Les limites des jeux taurins résultent de la rencontre d'une délimitation d'un cadre juridique territorial, définissant une aire d'extension légale de telle ou telle pratique avec celle d'une aire culturelle de la passion taurine.

Précisons que la régulation des spectacles publics et des fêtes populaires n'entrent pas directement dans les compétences du droit communautaire européen même si les traités, par la voix de la politique agricole commune et de la réglementation sur « la protection et le bien-être des animaux », ont des conséquences sur les pratiques taurines ${ }^{1}$. Lors de la conférence intergouvernementale préparant le traité d'Amsterdam, les représentants espagnols ont fait valoir leurs arguments en faveur de la préservation des spectacles taurins. Associé à des intérêts convergents sur d'autres sujets que les questions taurines, le Protocole $n^{\circ} 10$ établit que l'Union et les États membres doivent tenir compte pleinement des exigences en matière de bien-être animal, en respectant à la fois les dispositions légales et les coutumes des États membres relatives aux rites religieux, aux traditions culturelles et au patrimoine régional. Les situations differrent dans les trois États membres concernés par la tauromachie : l'Espagne, le Portugal et la France.

\section{LE CADRE JURIDIQUE ET TERRITORIAL}

DE LA TAUROMACHIE EN ESPAgNE

En Espagne, l'ensemble des jeux taurins s'inscrit aujourd'hui dans un cadre législatif défini par une loi votée au Parlement le 10 avril 1991. Cette loi taurine, dite « loi Corcuera » du nom du ministre de l'Intérieur de l'époque, est entérinée par un décret royal le 10 mars 1992. Un « Règlement des spectacles taurins » permet l'application de cette loi. Cette loi taurine vient remplacer le règlement de 1962, adopté par décret, qui n'avait été complété qu'en 1982 pour légiférer et reconnaître officiellement l'existence des spectacles taurins populaires. Dans le cadre d'un transfert de compétences prévu dans la Constitution de 1978 et des attributions administratives définies dans la loi taurine, ce sont désormais les Communautés autonomes qui légifèrent en matière de spectacles taurins. Quand aucun règlement régional n'existe, la norme étatique a valeur supplétive $^{2}$. Soulignons qu'avant 1982, les jeux taurins populaires n'avaient aucune existence légale et ont toujours fait l'objet de nombreuses interdictions. Pour ne mentionner ici que les périodes les plus récentes, rappelons que tout au long $\mathrm{du} \mathrm{XIX}^{\mathrm{e}}$ siècle et du $\mathrm{Xx}^{\mathrm{e}}$ siècle, les interdictions de courir les taureaux dans la

\footnotetext{
${ }^{1}$ Sur cette question, voir le colloque CEDECE organisé par l'université de Limoges les 7 et 8 avril 2005 intitulé «Les animaux et les droits européens », en particulier les communications sur le thème de la corrida prononcées par les professeurs de droit Jean-Claude Gautron, Christine Hugon et Jacques Leroy. Nous remercions Bénédicte Langue pour nous avoir transmis le contenu de ces conférences.

${ }^{2}$ Pour un commentaire complet de la réglementation espagnole voir P. Plasencia, La fiesta de los toros.
} 
rue se sont multipliées : en 1833, en 1908 par ordre royal, renouvelé en 1924, puis en 1928 à l'initiative de Primo de Rivera, en 1932 sous la République, ainsi que dans le règlement taurin de 1962 sous le franquisme. Cette énumération montre, en retour, à quel point l'éviction de la tauromachie populaire fut, dans les faits, irréalisable. L'éloignement du pouvoir central résiste souvent assez mal aux diverses stratégies locales de contournement de la loi et de travestissement des jeux taurins qui ont continué, de fait, à se dérouler.

La plupart des Communautés autonomes, en s'appuyant sur tout ou partie du Règlement national de 1991, ont élaboré un règlement régional par l'adoption de mesures visant à légiférer spécifiquement tel ou tel aspect des spectacles taurins. Et ce sont les spectacles taurins populaires qui ont prioritairement fait l'objet des nouvelles réglementations régionales, étant donné le caractère trop général du texte de 1991 sur ce sujet. Seules les régions du Pays Basque, de la Navarre et, en 2007, de l'Andalousie ont adopté un règlement taurin complet. Sans forcément contredire les normes du règlement national, ces règlements témoignent d'une forte volonté de réappropriation régionale de la culture taurine. Â l'inverse, seules Les Canaries, dès 1991, ont interdit dans leur territoire la pratique de la corrida et de tout type de spectacle taurin. Contrairement à une idée fausse qui gagne du terrain, les corridas ne sont pas interdites en Catalogne bien qu'elles y soient menacées par un projet de loi déposé au Parlement. On trouve à l'origine de ce projet de loi une déclaration de rejet de la corrida adoptée par le conseil municipal de Barcelone en avril 2004 par 21 voix contre 15. De plus la Catalogne a déjà pris des mesures restrictives interdisant la construction de nouvelles arènes et interdisant l'entrée aux corridas aux mineurs de moins de 14 ans. En ce qui concerne les jeux taurins populaires (les corre bous), la Catalogne a également restreint les autorisations par une ferme application de la loi régionale 3/1988 sur la protection des animaux, qui n'accorde de dérogation qu'aux municipalités ayant constitué un solide dossier pour faire valoir l'ancienneté de la tradition.

Aussi existe-t-il une réglementation homogène sur l'ensemble du territoire pour ce qui est des spectacles professionnels et des réglementations régionales différentes pour ce qui est des festejos taurinos populares. Pour ces derniers, non seulement les réglementations diffèrent d'une région à l'autre, mais prévoient également des dispositions particulières pour un certain nombre de traditions et de fêtes taurines locales, répertoriées et validées par les autorités compétentes. Les différences régionales concernent principalement le traitement des bovins en piste et les modalités autorisées pour les affronter. La réglementation nationale prévoit l'abattage systématique du bétail ayant participé à un spectacle taurin. Cet abattage a lieu hors de la présence du public et dans les conditions sanitaires prévues par les réglementations nationales et européennes. Cette loi, qui ne manque pas de paraître cruelle à certains, a pour objectif de réduire les risques d'accidents provoqués par des animaux qui parviendraient à acquérir une expérience du combat, mettant alors excessivement en danger les nombreux participants inexpérimentés des jeux taurins populaires. Au cours de l'histoire, le danger que représente l'affrontement de bêtes déjà courues a entraîné de 
nombreuses interdictions, en particulier celles concernant les capeas de villages et autres lâchers de taureaux sur la voie publique. Certaines régions sont revenues sur ces principes pour entériner des pratiques locales reposant au contraire sur la sortie d'animaux aguerris au combat, permettant ainsi de programmer à moindre coût des spectacles par la réutilisation de bêtes louées. C'est le cas dans les régions du Pays Basque, de la Navarre, de La Rioja, de l'Aragon, de Valence et de la Catalogne, plus précisément de la province de Tarragone lors des corre bous et des bous capllaçats (taureau encordé). En vue d'accroître le danger et l'émotion des jeux taurins populaires, certaines régions comme l'Estrémadure et surtout la Communauté valencienne autorisent, en dehors des encierros, le lâcher de taureaux de combat aux cornes intactes (toros cerriles), pratique interdite ailleurs. Les taureaux appartiennent souvent à des élevages réputés, destinés aux corridas, que les habitants s'enorgueillissent alors d'affronter.

Les réglementations régionales détaillent également toute une série de mesures visant à interdire les mauvais traitements envers les animaux et en particulier toute forme d'agression à leur encontre. Partout il est formellement interdit d'utiliser ou de lancer des objets qui pourraient blesser les animaux. Ces pratiques sont aujourd'hui punies par la loi en dehors des rares cas autorisés de blessures du taureau par les participants (toros de Coria) ou de mise à mort collective (toro de la Vega de Tordesillas). Tous les amateurs impliqués dans l'organisation des jeux taurins populaires se félicitent que soient interdites ces pratiques, même si elles ont encore cours par endroits en toute illégalité. Dans le détail, les réglementations différentes d'une région à l'autre, en fonction de ce qui est défini comme mauvais traitement envers les animaux. Par exemple, la Communauté autonome de Madrid a interdit tous les toros embolados de fuego et tous les taureaux à la corde, n'autorisant que les encierros et les lâchers de bovins en place fermée, l'animal ne devant pas rester en piste plus de quinze minutes. Autre exemple, la Navarre et le Pays Basque interdisent aux participants de se saisir des animaux, à l'opposé de ce qui se fait couramment dans nombre de lâchers populaires au Portugal afin de réaliser des pegas. Enfin, tous les jeux taurins populaires font aujourd'hui l'objet d'une autorisation préalable qui est accordée par les services des spectacles publics des Communautés autonomes, vérifiant leur condition d'exécution.

\section{L'EXCEPTION PORTUGAISE \\ DES TERRES DE CORRIDAS}

$\mathrm{Au}$ Portugal, le règlement taurin actuel fut remis à jour par un décret du 29 novembre 1991, reprenant les caractéristiques des règlements antérieurs qui depuis 1928 interdisent la mise à mort des taureaux en piste, imposent que les cornes du taureau soient protégées et rendent obligatoire l'intervention finale des forcados pour chacun des taureaux combattus. La corrida espagnole à pied, pratiquée sans pique ni mise à mort publique, ne fait pas exception à la règle. Qu'il s'agisse d'une corrida portugaise (tourada) ou d'une corrida à l'espagnole, le taureau est donc abattu à l'issue du combat hors de la présence du 
public, selon les normes sanitaires en vigueur. Le règlement définit également les conditions et les prérogatives locales dans l'encadrement et le contrôle des jeux taurins populaires.

Si l'interdiction de la mise à mort publique du taureau apparaît bien comme une exception remarquable en terre de corridas, la loi n'en a pas totalement débarrassé les traditions taurines portugaises, ni dans le domaine des corridas professionnelles, ni dans le domaine de certaines fêtes populaires. Là aussi, la réitération des interdictions durant le $\mathrm{XIX}^{\mathrm{e}}$ siècle pourrait suffire à démontrer que cette évolution fut lente et souvent chaotique, même si les transgressions sont aujourd'hui exceptionnelles. Dans le domaine des fêtes taurines populaires, Luís Capucha rapporte l'existence de certains jeux à la corde dont l'aboutissement est l'immobilisation du taureau, suivie de sa mise à mort publique par un individu chargé de le sacrifier. La pratique étant interdite par la loi, l'auteur préserve l'anonymat de ce rituel clandestin. Dans le domaine de la tauromachie professionnelle, les corridas dites " intégrales », entendons avec pique et mise à mort, ont connu quelques dérogations officielles à Lisbonne et quelques précédents non officiels, à Moita et à Vila Franca de Xira en 1976 et 1977. Les corridas de Vila Franca de Xira ont alors donné lieu à des émeutes populaires lors de l'arrestation des toreros par les forces de l'ordre.

Mais la manifestation la plus remarquable d'une résistance à voir le jeu taurin se terminer par la mort de l'animal ne trouve pas meilleure expression que la polémique d'envergure nationale à propos de l'exception culturelle de Barrancos qui agita le pays pendant plus de quatre ans, de 1998 à 2002. Cette petite cité rurale située à quelques kilomètres de la province espagnole de Huelva avait l'habitude de tuer les taureaux à l'issue des corridas des fêtes, malgré l'interdiction de la loi. Le débat avait été lancé une première fois en 1969 à la suite d'une plainte déposée par un certain Dr Lino, soulignant que la législation portugaise n'était pas respectée à Barrancos ${ }^{3}$. En 1996, la polémique resurgit à la suite d'un reportage télévisé sur les corridas de Barrancos et d'une série de plaintes déposées dans la foulée par des associations de protections des animaux. À partir de là, divisant l'opinion publique, les médias ont largement relayé et amplifié le débat dont la portée s'est étendue au fonctionnement général de la société portugaise $^{4}$. Ce que l'on a appelé la « crise barranquenha » servit ainsi de prétexte pour débattre pendant quatre ans du pouvoir, de la loi, des sources du droit, des relations villes-campagnes, des relations entre les hommes et les animaux, des rapports entre culture savante et culture populaire, de l'idiosyncrasie portugaise et bien entendu de la fête des taureaux. Le parlement portugais a fini par voter en 2002 l'autorisation de célébrer des spectacles tauromachiques avec mise à mort dans les municipalités pouvant se prévaloir d'une telle tradition. Depuis, trente-deux municipalités ont engagé des procédures pour établir la légalité de la mise à mort, procédures qui pour l'instant n'ont pas abouti mise à part celle

\footnotetext{
${ }^{3}$ N. Franco, O porquê de Barrancos, p. 201.

${ }^{4}$ L. Capucha, « Barrancos en escena », pp. 433-434.
} 
de Barrancos. Cette nouvelle loi est assez proche des transformations législatives françaises du début des années 1950 visant alors à clarifier le statut des courses de taureaux, même si, à ce jour, les décrets d'application portugais ne semblent pas être favorables à une extension des autorisations ailleurs qu'à Barrancos.

\section{La France taurine coupée en deux}

La situation française est très originale comparée aux autres pays de corridas puisque son aire d'exercice légal coupe le territoire en deux. Ce phénomène ne manque pas d'étonner dans un État profondément marqué par la tradition juridique jacobine d'intégrité territoriale. S'il existe bien des corridas en France depuis la deuxième moitié du xix ${ }^{e}$ siècle, il faut attendre 1951 pour qu'elles soient reconnues légalement par une modification de la loi Grammont de 1850 qui condamne les mauvais traitements envers les animaux domestiques ${ }^{5}:$ « La présente loi (loi Grammont du 2 juillet 1850) n'est pas applicable aux courses de taureaux lorsqu'une tradition ininterrompue peut être invoquée $»^{6}$. Le nouveau texte prévoit par exception au principe de territorialité des lois (art. ${ }^{\text {er }}$ du Code civil) que la corrida n'est autorisée que dans une partie du territoire de la République $^{7}$. Ce texte est complété dès 1959 par un décret qui précise que la tradition, en plus d'être ininterrompue, doit être locale. Ces mesures ne visent ni la course landaise, ni la course camarguaise, qui en théorie pourraient être pratiquées sur la totalité du territoire français tant qu'aucune jurisprudence n'est établie pour définir le contraire. Les courses de taureaux dans les textes législatifs font référence aux corridas, également désignées par la formule " corrida dite à l'espagnole » dans les décisions judiciaires ${ }^{8}$.

Ces dispositions ne fixent donc pas d'autres frontières à la pratique de la corrida que celles délimitées par le champ d'application de la loi qui relève en dernière instance du droit pénal. Les différentes instances et tribunaux qui ont eu à apprécier les conditions d'application de la loi Grammont modifiée ont fini par développer une jurisprudence plutôt favorable à l'organisation des corridas par une interprétation spatiale et temporelle extensive de la notion de « tradition locale ininterrompue $»^{9}$. Jean-François Auby résume ainsi l'esprit de cette jurisprudence :

\footnotetext{
${ }^{5}$ Loi Grammont de 1850 : «Seront punis d'une amende de 5 à 15 francs et pourront l'être de un à cinq jours de prison, ceux qui auront exercé publiquement et abusivement de mauvais traitements envers les animaux domestiques. La peine de prison sera toujours appliquée en cas de récidive. L’article 483 sera toujours applicable ».

${ }^{6}$ La loi no 51.461 du 24 août 1951. Pour l'analyse de textes juridiques sur les spectacles tauromachiques en France nous suivons, pour l'essentiel, J.-F. Auby, Droit et finances de la tauromachie et E. De Monredon, La corrida par le droit.

${ }^{7}$ E. De Monredon, La corrida par le droit, p. 95.

${ }^{8}$ La Semaine Juridique, $\mathrm{n}^{\circ}$ 37, 1995, p. 332.

${ }^{9}$ J.-F. Auby, Droit et finances de la tauromachie, p. 57. Les instances et les tribunaux chargés de légiférer en la matière sont les autorités municipales, les autorités préfectorales, les tribunaux administratifs et les tribunaux répressifs.
} 
Il existe une tradition locale dès lors que les corridas ont lieu dans une ville de manière traditionnelle et qu'il existe, autour de celle-ci, une adhésion culturelle suffisante. Cette tradition s'applique non seulement aux communes mais également à leur bassin de vie (on aurait tendance à appeler cela « un bassin culturel »), qui englobe une zone ayant les mêmes caractéristiques socio-économiques et culturelles. Ce point, après avoir été contesté, semble établi ${ }^{10}$.

D'abord limitée au seul territoire communal, la notion de tradition locale s'est étendue à celle " d'ensemble démographique ${ }^{11}$. Cette extension s'adapte sans doute à une évolution de la société où le territoire communal peut de moins en moins être considéré comme une entité coupée de son entourage, à mesure qu'augmentent les mobilités spatiales de toutes natures. C'est ce que montre par exemple l'autorisation depuis le 25 octobre 1987 des corridas à Floirac, commune de l'agglomération bordelaise qui prend le relais d'une tradition interrompue le 9 juillet 1961, lorsque sont fermées les arènes de la commune du Bouscat à la suite de l'effondrement d'un escalier. C'est ce que montre encore l'autorisation depuis le 27 juin 2003 des corridas à Fenouillet, dans la banlieue de Toulouse, qui prend le relais des arènes du Soleil d'Or, dans le quartier de Saint-Cyprien, dont la tradition est interrompue en 1977. Le territoire de la corrida en France est donc un territoire en partie évolutif qui semble échapper à toute détermination géographique définitive. Pour délimiter cet étrange cas d'espace que représente un territoire que nous pourrions qualifier de « territoire à variabilité géographique jurisprudentielle », la cour d'appel de Toulouse, le 3 avril 2000, a considéré qu'il existait une forte tradition taurine « dans le Midi de la France, entre le Pays d'Arles et le Pays Basque, entre Garrigue et Méditerranée, entre Pyrénées et Garonne, en Provence, Languedoc, Catalogne, Landes et Pays Basque ». Cette décision entérine l'idée d'une aire culturelle française de la tauromachie qui échappe à la seule logique ponctuelle des lieux pris au sens restreint de la localité communale ou de son bassin de vie. Cela étant, à l'exception des quelques cas de nouvelles implantations d'arènes sur lesquelles les décisions juridiques doivent trancher au sujet de l'ancienneté de la tradition, de la nature de son interruption et de la délimitation spatiale de la dite tradition « locale », la majorité des lieux où se déroulent actuellement des courses de taureaux est depuis un demi-siècle stabilisée et ne fait l'objet d'aucune contestation. Lorsque les contestations existent, elles concernent, soit la programmation d'une course dans une nouvelle municipalité, soit de façon plus radicale, l'existence même de la corrida.

Retenons que l'espace tauromachique du Sud-Ouest européen ne fonctionne pas comme une unité homogène, puisqu'il est partagé en trois pays à l'intérieur desquels les courses de taureaux n'ont pas le même statut, ni ne suivent les

${ }^{10}$ J.-F. AubY, Droit et finances de la tauromachie, p. 57.

${ }^{11}$ Arrêt de la Cour suprême rendu le 27 mai 1972. 
mêmes réglementations dans les modalités d'affrontement entre les hommes et les bovins.

\section{II. — LES LIMITES AMÉRICAINES \\ DES PRATIQUES TAUROMACHIQUES}

En Amérique, la question est plus complexe ne serait-ce que par le nombre de pays concernés par les traditions tauromachiques passées et présentes. Comme nous l'avons évoqué dès l'introduction, il est possible de distinguer trois groupes de pays :

- des pays où il existe des corridas, diverses courses de taureaux populaires et des formes de rodéos ;

— des pays où seules existent des pratiques apparentées au rodéo ;

- des pays qui se caractérisent par une indigence des pratiques tauromachiques quelle qu'en soit la nature.

Cependant, l'ensemble des espaces ibéro-américains, à quelque degré que ce soit, a été concerné à un moment ou à un autre de son histoire par les pratiques tauromachiques. Sans entrer trop avant dans les processus qui ont présidé à la formation de l'espace tauromachique, rappelons simplement que c'est la conquête espagnole et portugaise qui introduit sur le continent les premiers chevaux et les premiers bovins domestiqués. Le bétail est introduit pour conquérir des territoires, pour constituer des réserves d'escale maritime et pour approvisionner en viande les villes coloniales, les mines et les plantations. Dans les nouvelles villes fondées, se multiplient rapidement les fêtes taurines des conquérants alors que l'élevage bovin extensif et l'ampleur du marronnage à l'échelle de l'immensité américaine donnent naissance à une très grande diversité de jeux taurins pratiqués par les populations indigènes, noires, métisses et créoles qui copient ou parodient l'usage des élites. Dans un contexte où le rodéo, sous une forme ou sous une autre, est une pratique autorisée dans la plupart des pays américains, c'est bien l'existence ou non de la corrida qui constitue le critère majeur de différenciation spatiale. Le statut des jeux tauromachiques au sein de ces pays est très variable, depuis les textes législatifs qui en régulent scrupuleusement la pratique, jusqu'aux closes dérogatoires qui les excluent du champ d'application des lois de protection animale. Certaines lois, où il n'est aucunement fait référence aux jeux taurins, sous-entendent qu'ils sont illégaux, mais les textes juridiques régulièrement remis à jour ne reprennent plus une interdiction qui a pu être prononcée dans un passé désormais lointain et qui n'aurait pas de sens à être réitérée explicitement lorsque la tradition s'est éteinte. Par souci de clarté, nous adoptons une distinction simple pour l'ensemble du continent entre les pays où la corrida est autorisée et les pays où elle a été interdite, malgré quelques cas plus complexes. 


\section{LA CORRIDA AUTORISÉE}

Les corridas sont autorisées au Mexique, au Venezuela, en Colombie, en Équateur, au Pérou et en Bolivie. Dans les petits pays d'Amérique centrale, nous n'avons pas trouvé de textes permettant de préciser le statut exact des pratiques tauromachiques, mais le fait que des corridas y soient occasionnellement programmées, dont certaines avec mise à mort, laisse supposer qu'elles y sont au moins tolérées ponctuellement. C'est le cas au Guatemala, au Costa Rica et au Panamá. Sont également autorisées dans ces pays de nombreuses formes de jeux taurins populaires sans mise à mort où les taureaux sont courus, toréés et montés. Ailleurs, la corrida est interdite, sauf quelques exceptions locales moyennant un ajustement du rituel qui vise à amoindrir la violence du spectacle. C'est le cas en Californie qui, s'il n'était question d'établir une classification par pays, pourrait rejoindre le groupe des terres de corridas... le sang et la mort publique du taureau en moins. Au sein des pays où la corrida est autorisée, les cadres juridiques et territoriaux de sa pratique diffèrent d'un État à l'autre.

Au Mexique, la loi Fédérale délègue aux États les conditions d'application de la législation sur la protection animale. La loi de protection animale de l'État de Mexico « interdit d'organiser des combats d'animaux et de donner en spectacle tout type de combat privé ou public incluant des animaux, à l'exception des corridas de toros, des charreadas et des combats de coqs, lesquels doivent se soumettre aux règlements et aux dispositions adéquates $»^{12}$. Il en va de même pour l'État d'Aguascalientes qui précise que «sont exclus de l'application de la loi de protection animale, les combats, les corridas de toros, les novilladas et les festivals taurins ainsi que les travaux des champs tels que les tientas nécessaires à l'élevage des taureaux de combat, de même que les charreadas, jaripeos, coleaderos et, en général, toutes les suertes du sport national ${ }^{13}$. Par sport national, il faut ici entendre toutes les activités qui touchent de près ou de loin à la charrería.

$\mathrm{Au}$ Pérou, la loi de protection animale souligne en des termes équivalents qu'elle ne s'applique pas " aux corridas de toros, combats de coqs et autres spectacles à caractère culturel reconnus par les autorités compétentes ${ }^{14}$. L'Institut national de la culture reconnaît sous le terme de " corrida de toros » les innombrables façons de courir les taureaux incluant toutes les tauromachies populaires. Parmi les «spectacles à caractère culturel » sont également inclus les combats entre taureaux (peleas de toros) de la région andine d'Arequipa ainsi que les turupukllay de la Yawar Fiesta. Le 27 juillet 2001, le parlement péruvien a adopté une Loi taurine nationale qui vise à réguler toutes les facettes de la tauromachie, depuis les normes administratives relatives au secteur du loisir, jusqu'à la promotion de la culture taurine, en passant par les droits et les devoirs des spectateurs, des toreros, des entrepreneurs de spectacles, des éleveurs ainsi que

\footnotetext{
${ }^{12}$ Loi de protection animale de l'État de Mexico du 20 octobre 1985, chap. I, article 7.

${ }^{13}$ Loi de protection animale de l'État d'Aguascalientes, article 29.

${ }^{14}$ Loi de protection animale $n^{\circ} 27265$, titre I : Disposition générale, $3^{\text {e }}$ alinéa.
} 
les régimes d'infraction. Le texte introductif exposant les motifs de la loi signale que, jusqu'à aujourd'hui, aucune loi spécifique ne protégeait ni les aficionados, ni les acteurs du spectacle, ni les autorités compétentes. Il est ensuite précisé que « ce projet comble un vide juridique en la matière puisque, jusqu'à présent, aucune norme de rang légal ne régulait cette activité régie par des dispositions administratives ou municipales $»^{15}$.

En Colombie, au même titre que les législations précédentes, l'article 7 de la loi ${ }^{\circ} 84$ de 1989 sur la protection animale précise qu'elle ne s'applique pas « au rodeo, au coleo, aux corridas, aux novilladas, aux becerradas et aux corralejas, ainsi qu'aux combats de coqs $»^{16}$. Depuis 2004, le congrès colombien a lui aussi décrété une Loi taurine nationale (loi 916) garantissant l'application d'un règlement taurin unique. L'article 1 précise que « les spectacles taurins sont considérés comme une expression artistique de l'être humain » et l'article 2 ajoute que « le règlement est applicable sur l'ensemble du territoire national ». La tauromachie quitte ainsi le cadre dérogatoire par une reconnaissance positive et institutionnelle de sa pratique.

Les cas de la Colombie et du Pérou contrastent avec celui de leur voisin équatorien où il n'existe pas de loi taurine de rang national. En 2004, un groupe de pression anti-taurin est parvenu à faire annuler les corridas prévues à Guayaquil. À la suite de cet événement, à l'occasion de la féria taurine de Quito, un groupe composé d'aficionados, de syndicats de toreros, d'éleveurs, de journalistes et de responsables d'associations taurines, rédigea un manifeste pour la défense de la tauromachie au niveau local et au niveau national. Le Manifeste taurin de Quito, du $1^{\text {er }}$ décembre 2005, s'inspire d'une ordonnance signée par le gouvernement métropolitain de la ville, le 12 décembre 2003, qui « reconnaît que les spectacles taurins à l'espagnole sont une tradition inaliénable des habitants de Quito et qu'il est du devoir de la municipalité de les promouvoir et de les diffuser en tant que biens culturels et éléments irrévocables de l'identité historique de Quito $»^{17}$. Le manifeste de 2005, inspiré du texte établi par la municipalité de Quito, souhaite :

1 - que soit défendu et respecté notre droit de citoyen d'accéder librement et sans agression d'aucun type aux arènes ;

\footnotetext{
${ }^{15}$ Soulignons qu'un premier projet de loi avait été déposé dès 1996 à l'initiative des parlementaires Oscar Martorell Flores et Harold Forsyth Mejia, dans une version assez proche du texte actuel rédigé par Marcial Ayaipoma Alvarado alors président du Congrès.

${ }^{16}$ Gazette de la Cour constitutionnelle, sentence C-1192/05, 22 novembre 2005, magistrat Dr. Rodrigo Escobar Gil, reprenant les termes du Statut national de protection des animaux. Disponible sur le site : http://www.secretariasenado.gov.co/leyes/SC119205.htm [réf. du 10 décembre 2006]. Loi 84 du 27 décembre 1989, section « exception à la loi : article 7 » : « sont exclus de la loi le rejoneo, le coleo, les corridas de toros, les novilladas, les corralejas, les becerradas et les tientas ainsi que les techniques et procédés utilisés lors de ces spectacles».

${ }^{17}$ Document $n^{\circ}$ IC-2003-406 du 20 octobre 2003, article IV-196, Conseil métropolitain de Quito. Version papier du document qui nous a été remise en main propre le $1^{\text {er }}$ décembre 2005.
} 
2 - que soit réaffirmé et diffusé le caractère culturel des fêtes taurines et que, en tant que telles, soit protégée une expression populaire enfouie dans les racines historiques de l'Équateur;

3 - que soit établi un engagement résolu des autorités pour promouvoir une activité culturelle qui génère des emplois et des bénéfices dans de nombreux secteurs de la société ;

Il est difficile de dire si ce texte sera le prologue d'une loi taurine nationale dans un pays où les contrastes régionaux de l'afición sont forts. Une chose est le caractère légal d'une pratique au niveau national, autre chose les autorisations locales nécessaires pour son déroulement. L'exemple de Guayaquil rappelle qu'en dépit des lois qui légifèrent sur la légalité d'une pratique, en l'absence de Loi nationale garantissant le déroulement effectif des spectacles taurins sur l'ensemble du territoire, c'est bien souvent au niveau de l'autorité municipale ou régionale qu'il convient de se situer pour savoir si les corridas peuvent, de fait, avoir lieu ou non. En Équateur, la ville de Cuenca constitue un exemple inverse, puisqu'une ordonnance municipale interdit, depuis 2004, les corridas avec mise à mort, ce qui dans les faits a provoqué leur disparition.

De même en Bolivie, depuis 1997, sans modification de la loi de protection animale qui tolère le déroulement des corridas, la ville de La Paz par une ordonnance municipale a interdit définitivement les courses de taureaux. Les arguments de l'ordonnance $\mathrm{n}^{\circ} 168 / 97$, approuvée en conseil municipal le 5 décembre 1997, concernent principalement des domaines d'ordre éthique et culturel. L'argument éthique repose sur la condamnation de la corrida en tant qu'activité contraire au respect de la vie animale. L'argument culturel repose sur la condamnation de la corrida comme produit historique de la colonisation. Le texte, par sa radicalité, mérite d'être cité.

L'activité taurine a été transmise à notre région par un effet de transculturation qui est en contradiction avec nos racines ethnoculturelles. Dans notre histoire culturelle préhispanique, riche en traditions, les corridas n'existent pas, en conséquence de quoi elles ne sont que l'empreinte du colonialisme espagnol et non la propre expression du peuple de la $\mathrm{Paz}^{18}$.

Le texte est d'un grand intérêt pour notre propos général, puisque le caractère authentique du « peuple de la Paz » est considéré comme une entité antérieure à l'époque coloniale, en lien direct avec l'histoire préhispanique et en contradiction totale avec les logiques métisses qui donnent forme aux identités culturelles du monde andin et, plus généralement, de l'Amérique latine. Dans le cas bolivien, le texte confirme la force identificatoire de l'Inca préhispanique en lien avec le développement des mouvements indigénistes. Rappelons, sans doute au

\footnotetext{
${ }^{18}$ Ordonnance $n^{\circ} 168 / 97$, préambule aux articles de l'ordonnance.
} 
grand dam du " peuple de la Paz » ainsi défini, que les courses de taureaux populaires continuent d'être pratiquées sous des formes diverses en d'autres lieux du pays. C'est le cas des jocheos du département amazonien de Beni où ceux pratiqués dans la ville de Trinidad ont acquis une certaine notoriété. Les déclarations opposées de Quito et de La Paz, justifiées par des arguments d'ordre culturel, illustrent bien la complexité des débats et les divergences d'opinion concernant la symbolique identitaire attachée aux pratiques taurines en Amérique latine. À elles seules, ces déclarations font la démonstration que la définition des traditions culturelles relève avant tout d'un projet socio-politique ou d'une «invention de la tradition » pour reprendre la célèbre formule de Hobsbawm et Ranger ${ }^{19}$.

Enfin, au Venezuela, jusqu'à l'adoption de la Loi taurine nationale le 14 décembre 2005, les courses de taureaux étaient jusqu'alors autorisées par une close dérogatoire au champ d'application des lois de protection animale et régulées par diverses réglementations propres à chaque arène inspirées du règlement espagnol de 1930. Le projet de loi rédigé en octobre 2005 possède deux objectifs clairs : réguler l'organisation des spectacles taurins et promouvoir les activités tauromachiques sur l'ensemble de la filière, dans le respect des principes fondamentaux et des valeurs universelles contenues dans la Constitution de la République bolivarienne du Venezuela. Ce texte souhaite combler un vide juridique au même titre que le texte adopté en Colombie une année avant. Parmi les mesures importantes, signalons que les spectacles taurins sont déclarés d'intérêt touristique national et qu'ils dépendent d'une Commission nationale des affaires taurines, sous tutelle du ministère du Tourisme. Remarquons également qu'au Venezuela, la législation interdit aux personnes de moins de quatorze ans d'assister aux spectacles taurins, en raison de leur caractère violent. L'histoire de la tauromachie au Venezuela se caractérise d'ailleurs par de nombreuses mesures visant, sinon à abolir la corrida, tout au moins à en réduire le niveau de violence. C'est ce que montrent le décret de 1894 qui « interdit formellement de donner la mort à des taureaux durant ou après un spectacle public » et le décret de 1899 qui « interdit la pique des taureaux afin d'éviter les lésions et la mort des chevaux $»^{20}$. La suite de l'histoire montre que ces décrets restèrent lettre morte, mais un récent projet de loi de protection animale, déposé en 2007, soit deux ans à peine après l'adoption de la Loi taurine nationale, entend revenir sur les acquis du texte de 2005.

Le caractère récent des lois taurines nationales et des mesures locales allant dans le même sens traduit une normalisation juridique des pratiques tauromachiques dans de nombreux pays latino-américains (le Pérou en 2001, la Colombie en 2004, le Venezuela en 2005 et la ville de Quito en 2003). Il y a plusieurs façons d'interpréter cette évolution. Elle semble en grande partie relever d'une volonté de protéger une tradition ancienne, menacée par un secteur de la

\footnotetext{
${ }^{19}$ E. Hobsbawm et T. Ranger, L'invention de la tradition.

${ }^{20}$ J.-M. Lomogodeuc « Taureaux et corridas au Venezuela », p. 83.
} 
société souhaitant la voir disparaître et par des groupes anti-taurins de mieux en mieux structurés et influents. Elle relève également d'une volonté de promouvoir une activité transversale de mieux en mieux perçue comme une ressource pour le territoire, notamment en matière de développement touristique.

\section{LA CORRIDA INTERDITE}

Dans les pays latino-américains où la corrida est interdite, il ne faudrait pas considérer le cadre législatif existant comme une sorte de carcan juridique qui empêcherait la tradition taurine de renaître sitôt l'interdit levé. Même si les dates d'interdiction de la corrida varient d'un pays à l'autre, on peut aujourd'hui admettre que dans la plupart d'entre eux la tradition s'est perdue. Aujourd'hui, aucun indice ne montre qu'il existe une réelle demande sociale ou un embryon d'offre qui serait susceptible de faire renaitre la tradition taurine des corridas. Cela étant, la disparition des traditions taurines a été souvent beaucoup moins rapide que ne le laisse croire l'histoire officielle de ces pays, intéressée à les enterrer dans un passé aussi lointain que possible. En outre, dans certains de ces pays comme le Chili, le Brésil ou Cuba, l'existence de jeux taurins tels que les rodéos montre que toutes les pratiques reposant sur un affrontement entre les hommes et les bovins ne sont pas disqualifiées. Au cours des périodes intermédiaires d'affaiblissement et de raréfaction des corridas, il n'est pas toujours évident de mesurer la part revenant à la volonté politique d'éradiquer les courses de taureaux et celle revenant à une évolution sociologique spontanée vers leur progressive extinction.

Les premiers pays à mettre un terme à la tradition des courses de taureaux sont l'Argentine et le Chili, dans le contexte troublé des guerres d'indépendance qui s'ouvre à partir de 1810. La déclaration officielle d'indépendance de l'Argentine date du 9 juillet 1816 et joue d'ailleurs un rôle crucial dans l'indépendance du Chili, définitivement acquise en 1821, grâce à l'appui des troupes de San Martín obligeant le vice-roi d'Espagne à quitter le Pérou ${ }^{21}$. Pour l'Argentine, la destruction des arènes en 1819 marque une rupture dans l'histoire taurine du pays. Le 16 janvier 1819, à la demande du maire de Buenos Aires, Díaz Vélez, au directeur suprême de la Confédération, Juan Martín de Puyrredón, l'autorisation de démolir les arènes est accordée afin d'employer les matériaux à des fins jugées plus utiles et, en particulier, à l'effort de guerre ${ }^{22}$. Les historiens retiennent généralement cette date pour fixer la disparition des jeux taurins en Argentine. En réalité, le premier décret officiel concernant les jeux taurins date du 4 janvier 1822. Il est signé par le gouverneur de Buenos Aires, Martín Rodríguez et déclare que les jeux taurins sont interdits dans toute la province sans autorisation préalable du chef de la police et sans que les taureaux n'aient les cornes

\footnotetext{
${ }^{21}$ Dans l'organisation de l'administration coloniale la capitainerie du Chili dépendait de la viceroyauté du Pérou.

${ }^{22}$ E. ERAusquin, « Un aspect méconnu du Buenos Aires d'antan », p. 181.
} 
épointées ${ }^{23}$. Nous conviendrons que la nuance est de taille entre une supposée interdiction radicale qui coïncide avec la geste indépendantiste et une probable tolérance, datant de 1822, qui à la différence de la prohibition choisit une régulation selon des normes. La réaffirmation régulière, tout au long du XIX ${ }^{e}$ siècle, des décrets interdisant la corrida sous toutes ses formes ne fait finalement que renforcer l'idée d'une disparition très progressive de la tauromachie, qui a dû continuer d'être pratiquée de façon non négligeable jusqu'à la fin du siècle. Le voyageur français Arsène Isabelle affirme que les corridas furent rétablies en 1832 pendant la dictature de Rosas, mais ajoute que les gens se lassèrent vite de ce «beau spectacle » dont ils condamnaient le « caractère barbare $»^{24}$. Même la première loi de protection animale qui date de 1891 ne semble pas mettre un terme définitif à sa pratique, si l'on en juge par les quinze corridas données en 1900 à Buenos Aires, probablement sans mise à mort ${ }^{25}$. Pour preuve plus récente, il n'est sans doute de meilleur exemple que l'intervention du président Perón auprès du Congrès, dans les années 1950, pour affirmer une nouvelle fois l'interdiction des spectacles taurins en raison de novilladas privées organisées par le Club El Ventorrillo et fréquentées par certains membres de son cabinet ${ }^{26}$.

Aujourd'hui, en Argentine, la loi du Code pénal 14.346 qui date du 27 octobre 1954 interdit tout type de spectacles pouvant entraîner de mauvais traitements envers les animaux. Les modalités d'application de cette loi ne semblent pas inclure les spectacles apparentés au rodéo pratiqués en terres de gauchos uniquement avec des chevaux et sous une forme peu professionnalisée. On remarquera aussi que l'ancienne tradition festive des yerras, encore pratiquée par endroits dans la Pampa, échappe à la loi, à moins que le lien plus immédiat avec les nécessités de l'élevage conduise à une tolérance ${ }^{27}$. Au cours de la yerra, les vachers à cheval, héritiers des gauchos, poursuivent le bétail à castrer ou à marquer, l'isolent et l'immobilisent à terre. Une fois le travail accompli, ils tuent un animal au poignard qui sera le plat principal du churrasco (viande grillée) organisé par le propriétaire de l'hacienda, se chargeant d'apporter les vins et d'inviter quelques convives de marque. C'est également en vertu de cette loi que le Mouvement argentin de protection animale, attaque régulièrement en justice la tradition du toreo de la vincha qui a lieu tous les 15 août dans le village de Casabindo, perdu dans la puna de la province septentrionale de Jujuy. Isolina Comas Haquim, responsable de la culture dans la province, a défendu la tradition en soulignant qu'il ne s'agissait en rien d'un spectacle susceptible de blesser le taureau, puisque le rituel consiste simplement à s'emparer de la vincha, sorte de foulard couvert de pièces d'argent fixé sur le frontal de l'animal, et à le toréer

\footnotetext{
${ }^{23}$ Ibid., p. 182.

${ }^{24}$ Cité par P. Verdevoye, « Corridas et Carnaval dans la presse argentine avant 1833 », p. 7.

${ }^{25}$ Loi de protection animale $\mathrm{n}^{\circ} 2786,25$ juillet 1891.

${ }^{26}$ F. López IzQuierdo, Los Toros del Nuevo Mundo, p. 319.

${ }^{27}$ Aude de Rouffignac, ingénieur agronome qui a travaillé à plusieurs reprises en Argentine nous a confirmé que les yerras se pratiquent avec des bovins et des ovins. Petit détail (croustillant), dans le cas des ovins les bêtes sont parfois castrées avec les dents, en souvenir de l'ancien temps.
} 
sans aucune effusion de sang ${ }^{28}$. On observe des pratiques équivalentes en Bolivie, au Pérou et en Équateur.

Au Chili, la première loi interdisant les courses de taureaux sur tout le territoire date du 15 septembre 1823 . Elle ne semble pas avoir été suivie d'effets immédiats, puisque le ministre de l'exécutif, Diego Portales, est contraint de remettre aux intendants, le 24 novembre 1835, une circulaire leur signalant que la dite loi n'était pas respectée, en conséquence de quoi le chef suprême de la Nation les rappelait à leur devoir ${ }^{29}$. La première loi de protection animale date de 1874. Il semble qu'au Chili, l'interdiction ait été par la suite relativement bien respectée, si l'on en juge par le peu d'informations faisant référence à la célébration de courses de taureaux. Il n'y aurait guère à signaler que deux spectacles taurins en 1950 et 1965. Le premier a lieu à l'initiative du torero valencien Jaime Marco «El Choni » et fut sévèrement dénoncé par la presse, en particulier par le journal El Mercurio de Santiago du Chili. Le second aurait eu lieu en 1965 dans le territoire chilien d'Arica et fut à la base d'une polémique durant laquelle la Société protectrice des animaux accusait ouvertement (et vertement) le ministre de l'Intérieur d'avoir laissé se dérouler une course de taureaux en dépit de sa stricte interdiction datant de 1823. L'événement fut démenti par le Ministère, promettant néanmoins d'ouvrir une enquête à ce sujet.

Rappelons que le rodéo chilien tient lieu de sport national officiel et donc ne tombe ni sous le coup de l'interdiction de 1823, ni sous le coup de la loi de protection animale (article 291 bis du Code pénal). Cette loi dans son ensemble est aujourd'hui fortement critiquée par de nombreuses associations de défense des animaux qui y voient une « loi de déprotection » en raison des faibles peines encourues. Il est intéressant de constater que lorsque les partisans du rodéo chilien sont attaqués sur le thème de la violence envers les animaux, ils veillent à ce que leur pratique soit bien distinguée de la corrida espagnole, condamnée pour son caractère sanglant. En outre, ils font remarquer que le règlement détermine très scrupuleusement les conditions d'affrontement entre les cavaliers et les bovins en vue d'éviter au maximum les mauvais traitements inutiles envers le bétail équin et bovin. En effet, le règlement sanctionne par des points de pénalité tous les chocs entre le cheval et le bovin, en dehors des limites où doivent avoir lieu le contact et l'impact stoppant la course du taureau (les remates ou la atajada). L'article 4 précise même que « la violence des remates n'est pas une exigence réglementaire, en conséquence de quoi, il convient de tous les comptabiliser $»^{30}$.

L'Uruguay se trouve dans une situation à peu près équivalente à celle du Chili, puisque la pratique du rodéo est autorisée alors que les corridas y sont interdites

\footnotetext{
${ }^{28}$ Clarin.com, 15 août 1998. Article de Laura Ballatore de la revue numérique Clarin.com disponible sur le site Internet : http://www.clarín.com/diario/1998/08/15/e-05201d.htm [réf. du 10 décembre 2006].

${ }^{29}$ F. López IzQuierdo, Los Toros del Nuevo Mundo, p. 328.

${ }^{30}$ Règlement officiel du Rodéo Chileno. Le remate est l'action finale de la atajada qui stoppe le bovin contre la paroi. Ce terme existe aussi dans le langage de la corrida pour désigner la dernière passe d'une série.
} 
depuis 1890. Mais en comparaison de ce qui s'est passé au Chili et en Argentine, l'interdiction intervient bien après l'indépendance du pays, acquise en 1828. À l'inverse des cas précédents, l'Indépendance semble ici favoriser au contraire un renouveau de la tauromachie dont la pratique avait quasiment disparu de 1796 à 1823, durant la période où l'Uruguay s'était retrouvé sous domination portugaise $^{31}$. Le 18 février 1885, l'inauguration officielle d'une nouvelle arène, la Plaza de la Villa de la Unión, pouvant accueillir 12.000 spectateurs et dont les travaux avaient commencé en 1854, traduit alors le contraste existant entre les deux pays situés de part et d'autre du Río de La Plata. C'est apparemment la vive polémique au parlement occasionnée par la mort du torero Joaquín Sanz « Punteret » dans les arènes de Montevideo, le 26 février 1888, qui servit de catalyseur à la suppression des corridas dans le pays ${ }^{32}$. À la suite de cette polémique, la loi 2017 interdit par décret les corridas : " le Sénat et la Chambre des représentants de la République orientale de l'Uruguay, réunis en Assemblée générale, déclarent qu’à partir du 31 mars 1890, le spectacle public désigné sous le nom de "corridas de toros" est interdit sur tout le territoire de la République " ${ }^{33}$. La loi de protection animale promulguée en 1918 confirme celle de 1890, en précisant que sont interdites « les parodies de corridas, quelle que soit leur forme et leur dénomination $»^{34}$. La formulation de la loi laisse supposer que des jeux taurins ont continué à se dérouler sous des aspects permettant de contourner la législation de 1890. Mais la preuve la plus manifeste du maintien des jeux taurins est offerte par l'inauguration des arènes du département de Colonia de Sacramento situé à l'ouest de Montevideo, le 9 janvier $1910^{35}$. Une loi est même prononcée en 1935 revenant sur l'interdiction de 1890 en permettant la célébration des courses de taureaux dans ce département. Il est précisé que les bénéfices doivent revenir, à part égale, au ministère de la Santé et à la municipalité de Colonia $^{36}$. Des corridas portugaises s'y déroulent jusqu'à la fin des années 1930, après quoi la tradition semble s'éteindre progressivement et les tentatives pour réintroduire les courses de taureaux en 1961 et en 1978 échouent.

Cuba partage avec l'Uruguay de connaître à La Havane la période d'apogée de la tauromachie moderne de la fin du XIX ${ }^{e}$ siècle en terre américaine. À Cuba, ont ainsi toréé d'illustres matadors espagnols comme Luis Mazzantini (1886), Rafael Guerra « Guerrita » (1887) ou Juan Jiménez «El Ecijano » (1897), auxquels il conviendrait d'ajouter la venue de nombreux toreros mexicains. Ici, c'est la guerre entre les États-Unis et l'Espagne débouchant sur l'indépendance de Cuba le 10 décembre 1898, qui joue un rôle clef dans la disparition de la

\footnotetext{
${ }^{31}$ F. López IzQuierdo, Los Toros del Nuevo Mundo, p. 339.

${ }^{32}$ Ibid., p. 340.

${ }^{33}$ Informations disponibles sur le site Internet de l'association Animanaturalis Uruguay : www. animanaturalis.org/modules.php?goto=Svst95_906 [réf. du 10 décembre 2006].

${ }^{34}$ Loi n ${ }^{\circ} 5657$, article 1.

${ }^{35}$ Informations disponibles sur le guide touristique en ligne de la Colonia de Sacramento : http://www.guiacolonia.com.uy/Turismo/Colonia/PzaToros.htm [réf. du 10 décembre 2006].

${ }^{36}$ Loi n ${ }^{\circ} 9483$, article 1.
} 
tauromachie. Les autorités militaires américaines, en la personne du chef d'ÉtatMajor des États-Unis, le général Adna R. Chafee, signent le 10 octobre 1899 l'ordre d'interdire les corridas à $\mathrm{Cuba}^{37}$. À Cuba, la République, proclamée en 1902, ne revient pas sur cette décision en accord avec le courant de modernisation du pays qui habite les élites et une majorité de l'opinion publique ${ }^{38}$. Par la suite, les nombreuses tentatives pour réimplanter durablement les corridas à Cuba ont toutes échoué, malgré quelques courses ponctuelles. Sont de celles-là, les corridas du 30 et 31 août 1947 à La Havane. Les corridas eurent lieu devant 20.000 spectateurs installés dans les tribunes du Estadio del Cerro inauguré en 1946, avec du bétail colombien de l'élevage Aguas Vivas, transporté par avion, et la présence des toreros mexicains Fermín Espinosa «Armillita Chico » et Silverio Pérez «El Faraón de Texcoco ». Conformément aux dispositions prises, les animaux ne furent ni banderillés, ni piqués, ni mis à mort. Il n'existe pas à Cuba de véritable loi pour la protection des animaux, mais les spectacles apparentés aux corridas demeurent interdits, comme le prouve l'annulation en 2000 d'un projet de festival taurin à l'initiative de la chambre de commerce de Málaga (Espagne). Rien n'empêche en revanche l'organisation de rodéos qui ont généralement lieu pendant les foires agricoles, la plus connue étant celle de La Havane (Feria Agropecuaria de La Habana) au cours de laquelle se déroule un rodéo international. Il est intéressant de constater que des concurrents d'autres nations américaines s'y produisent, y compris d'origine étasunienne. Depuis 1994, la Sociedad Cubana de Vaqueros de Rodeo s'est donnée comme projet de promouvoir les spectacles de rodéo et d'encourager par cet intermédiaire les relations avec les pays voisins, en particulier la Floride où l'on pratique le rodéo ainsi que le coleo notamment au sein de la communauté d'origine cubaine.

Au Brésil, José María de Cossío considère que la tradition des corridas fut assez faible à l'exception de quelques courses ayant eu lieu à Rio de Janeiro et à São Paulo en lien avec l'importante communauté espagnole qui s'y trouvait pendant la période coloniale. Après l'indépendance du Brésil en 1825, les styles espagnol et portugais semblent avoir coexisté sous l'Empire, puis, à partir de 1889, sous la République ${ }^{39}$. L'interdiction des corridas intervient assez tard en comparaison des autres pays, le 10 septembre 1924, à la suite d'une polémique où triomphèrent les partisans de sa suppression ${ }^{40}$. Comme partout, l'interdiction ne marqua pas un coup d'arrêt immédiat et définitif, à en croire le déroulement postérieur de certains spectacles plus ou moins inspirés des corridas espagnoles ou portugaises. Entre dans cette catégorie le spectacle intitulé d'un nom curieux "Temporada de Touradas Típicas Espanholas" (saison de touradas typiques espagnoles) qui s'est tenu à Belém de Pará en 1960. Le journal Pueblo en offrit un compte-rendu pour le moins cocasse. Le journal

\footnotetext{
${ }^{37}$ Ordre militaire 187, confirmé le 28 mai 1900 par l'ordre militaire 217. M. LunA-PARra, Toros en Cuba II, p. 13.

${ }^{38}$ M. Porcheron, «Las corridas de toros en Cuba», p. 127.

${ }^{39}$ F. López Izquierdo, Los Toros del Nuevo Mundo, p. 321.

${ }^{40}$ Ibid., p. 322. Décret no 16590 , article 5.
} 
raconte qu'après le combat mené par le torero espagnol Juan Bravo, un Portugais surnommé «El Indio Apache, les cheveux jusqu’aux épaules, surmontés d'un grand panache de plumes, tirait les taureaux par la queue jusqu'à les faire chuter pour ensuite s'asseoir sur les cornes de l'animal $»^{41}$. Cet avatar burlesque du coleo à pied ne manque pas de faire écho aux vaquejadas du Nordeste. Il ne faut pas réduire l'importance de ces formes de corridas bouffes et ne les juger que comme un épiphénomène rémanent d'une tradition tauromachique ensevelie. Nous en avons trouvé la trace jusque dans les années 1970 où s'illustrait un « torero clown » tel que Francisco « Garrafinho » qui connut à cette époque un certain succès ${ }^{42}$. Les vaquejadas, le rodeio d'inspiration nord-américaine et le rodeio crioulo ne tombent pas sous le coup de la Loi fédérale, de 1998, de protection de l'environnement ${ }^{43}$. Comme nous l'avons évoqué plus haut, la progression de l'engouement pour la vaquejada et les rodeios a même débouché sur leur reconnaissance comme activité professionnelle par la loi $\mathrm{n}^{\circ} 10.220 \mathrm{du}$ 11 avril 2001. En revanche, la pratique des farras do boi de l'État de Santa Catarina est officiellement condamnée, depuis le 3 juin 1997, à la suite de la décision rendue par le Tribunal suprême fédéral, venant clore une campagne de dénonciation commencée dix ans plus tôt $t^{44}$.

Les États-Unis constituent un cas atypique dans une géographie des corridas interdites, établie par pays, puisqu'elles sont autorisées en Californie sous une forme portugaise adoucie. En effet, les corridas californiennes désignées par l'appellation de bloodless bullfighting se caractérisent, comme leur nom l'indique, par l'absence de sang animal durant le spectacle. En 1960, le gouvernement américain avait pourtant interdit les courses de taureaux en adoptant des mesures de protection animale, alors que les californios, mexicains et descendants des Espagnols de la Haute Californie, organisaient encore des corridas notamment à Los Angeles. Il faut dire qu'avant que les États-Unis ne s'approprient les terres septentrionales du Mexique, des villes comme Los Angeles, ou Santa Fe et San Antonio au Texas, organisaient, au moins depuis le début du $\mathrm{xIX}^{\mathrm{e}}$ siècle, des courses de taureaux, au cours desquelles se mêlaient corrida à l'espagnole et jeux dérivés des travaux de l'élevage, jaripeo et coleo. En 1852, c'est même un Anglo-américain, un certain H. L. Kinney, rancher et ancien Texas Ranger, qui organise à Corpus Christi au Texas plusieurs jours de corridas avec au programme le matador Don Camarena et sa cuadrilla venus spécialement de Mexico $^{45}$. Dans le dernier quart du XIX ${ }^{\mathrm{e}}$ siècle, les interdictions répétées et le poids de la American Society for the Prevention of Cruelty to Animals (ASPCA) semblent avoir considérablement limité le maintien de la pratique dans le Sud précocement hispanisé ainsi que les tentatives d'introduction plus au nord.

\footnotetext{
${ }^{41}$ Ibid., p. 323.

42 J. A. Bezerra, « Anjas da arena ».

${ }^{43} \mathrm{~N}^{\circ} 9605$ de février 1998. L'article 32 de la section « crime contre la faune » définit les modalités d'application.

${ }^{44}$ Recours extraordinaire $\mathrm{n}^{\circ}$ 153.531-8/SC du Tribunal suprême fédéral.

${ }^{45}$ M. L. Le Compte, « The Hispanic Influence on the History of Rodeo », p. 28.
} 
Un article de 1933 de Kirke Mecham, nous éclairant sur ce thème, identifie en effet seulement quatre courses de taureaux avec mises à mort au cours de cette période : celles des 4 et 5 juillet 1884, à Dodge City, la célèbre ville du bétail, et celles des 24 et 25 août 1895, à Gillette, dans le district minier de Cripple Creek, situé dans les Grandes Plaines également, pour lesquelles ont été vendus plus de 3.000 billets à un public venu principalement de Colorado Springs et de Denver ${ }^{46}$. Au tournant du siècle, on note d'autres exemples, non exhaustifs, de courses de taureaux sans mise à mort, comme le 9 juillet 1901 à Omaa dans le Nebraska ou le 27 novembre 1902 à Kansas City dans le Missouri, toujours suivies de nombreuses protestations ${ }^{47}$. Kirke Mecham recense ensuite de 1922 à 1927, à partir du New York Times comme seule source, une douzaine de courses " modifiées », « simulées », « burlesques » ou bloodless, prouvant une nouvelle fois que la tauromachie, et ce jusqu'en Amérique du Nord, n'a pas disparu d'un seul coup sans de multiples adaptations et réemplois partiels ${ }^{48}$.

La seule adaptation ayant fini par aboutir, malgré une importante coupure historique et une origine exogène, est celle que l'on observe aujourd'hui en Californie, issue des traditions taurines venues avec les émigrés des Açores. Leur arrivée qui démarre au milieu du XIX ${ }^{\mathrm{e}}$ siècle s'accélère dans les années 1950, en particulier pour ceux provenant de l'île Terceira. Les jeux avec des taureaux ont alors pris suffisamment d'importance pour que le gouvernement de Californie renouvelle en 1957 l'interdiction formelle de pratiquer toute forme de spectacles tauromachiques ${ }^{49}$. Le gouvernement, devant la pression des citoyens issus de la communauté d'origine açorienne finit par céder en autorisant les spectacles taurins dans leur version portugaise, mais sans effusion de sang, et à la condition qu'ils soient liés à une fête religieuse ${ }^{50}$.

Que conclure de ce panorama des différents cadres juridiques en matière taurine sur le continent américain ? Il existe des frontières rigides entre les pays où la corrida est autorisée et les pays où elle est interdite. Historiquement, les interdictions gouvernementales ciblées sur les corridas ont eu tendance, dans le sillage des indépendances, à précéder les mesures législatives générales de protection des animaux. Dans certains pays, éradiquer les symboles coloniaux fut en cette période un argument autrement plus décisif que les questions de protection des animaux, qui pour autant n’ont pas été absentes des débats et des justifications puisque les corridas sont généralement partout dénoncées pour leur archaïsme. Cela étant, de nombreux pays qui avaient annoncé leur inter-

\footnotetext{
${ }^{46}$ K. Meснам, «The Bull Fight at Dodge ». J'ai pu retrouver une photo de la corrida de Gillette dans R. Bowe, Historical Album of Colorado, p. 28, sur laquelle on observe une femme toréant, vêtue à la mexicaine, accompagnée d'un picador.

${ }^{47}$ K. Meснам, « The Bull Fight at Dodge », p. 294.

${ }^{48}$ Ibid., p. 294.

${ }^{49}$ The New York Times, 27 juin 2001. Édition en ligne, article de Patricia Leigh Brown intitulé «In California Bullfights, the Final Deed Is Done With Velcro » disponible sur le site Internet : http://query.nytimes.com/gst/fullpage.html?sec=travel\&res [réf. du 10 juin 2007].

${ }^{50}$ A. Graves, The Portuguese Californians, pp. 158-164.
} 
diction ont continué, assez tard dans le XIX ${ }^{e}$ siècle, à célébrer des corridas et même à construire des arènes comme l'Argentine, l'Uruguay ou le Costa Rica. Aujourd'hui, nous pouvons considérer que les frontières de la corrida sont, dans leur ensemble, largement stabilisées et qu'il n'existe pas ou plus d'écart important entre ce que l'on pourrait appeler un espace taurin légal et un espace taurin réel qui pâtirait de législations restrictives. Les interdictions paraissent assez anciennes et les tentatives de réintroduction endogènes ou exogènes relativement marginales.

Si un tel écart a pu être perceptible au lendemain des interdictions qui ont partout mis du temps à être respectées, on assiste plutôt actuellement à un renversement, puisque des menaces croissantes pèsent sur la pérennité des courses de taureaux y compris dans des pays où la tradition n'a jamais été interrompue. Sous cet angle, le cas des États-Unis peut paraitre paradoxal puisqu'en Californie la corrida, sous une forme certes adaptée à la législation en vigueur, resurgit à partir des années 1950. Dans l'ensemble, les corridas sont de plus en plus souvent dénoncées par des groupes aux intérêts hétérogènes qui ont la certitude d'embrasser une cause juste et mondiale au-dessus de tout soupçon : protéger les animaux et en finir avec la barbarie. On observe que si la corrida échappe légalement à l'application des lois de protection animale dans certains pays, les rodéos y échappent quasiment partout. À l'échelle du continent, les rodéos sont entrés dans une phase d'institutionnalisation accélérée en dépit des critiques qui s'élèvent. L'Amérique est bien le continent de la coexistence spatiale de deux "planètes des taureaux ", celle de la corrida et celle du rodéo. 


\section{LA FORMATION DE L'ESPACE TAUROMACHIQUE}

Il ne s'agit pas de retracer une à une les évolutions des pratiques taurines, mais de repérer les grandes étapes de formation de l'espace tauromachique. Pour les jeux taurins du Sud-Ouest européen, la documentation et les travaux sont nombreux si l'on tient compte à la fois des approches transversales, des études régionales et locales ainsi que des recherches monographiques sur tel ou tel type de fête ou de spectacle taurin. Pour les jeux taurins de l'Amérique latine, le terrain est immense et les travaux plus fragmentaires. En l'état actuel des recherches, les travaux sont mieux développés pour les pratiques perçues dans la continuité des pratiques tauromachiques du Vieux Continent et pour les rodéos d'Amérique du Nord (États-Unis et Mexique) que pour ceux d'Amérique du Sud. Nous pouvons fixer dès à présent un cadre historique général, permettant de clarifier, sur le temps long, les grandes étapes de la formation de l'espace tauromachique pris dans son ensemble.

Partons du principe général que la formation de cet espace relève d'une logique de diffusion spatiale des pratiques taurines qui ne suivent pas toutes la même chronologie, ni ne connaissent la même expansion. En France, la terminologie employée en géographie pour qualifier les processus de diffusion spatiale est largement inspirée des travaux de Torsten Hägerstrand des années $1950^{1}$. La géographe Thérèse Saint-Julien propose une définition simple de la diffusion spatiale : «La notion de diffusion spatiale recouvre l'ensemble des processus qui concourent au déplacement, à la migration de l'innovation dans l'espace géographique et aux effets en retour que ces déplacements engendrent dans cet espace $»^{2}$. La diffusion spatiale selon Härgerstrand met en évidence l'existence de régularités temporelles et géographiques dans les processus de diffusion des innovations qui sont particulièrement sensibles à la structure hiérarchique des systèmes de peuplement. La diffusion progresse généralement en suivant le sens descendant de la hiérarchie urbaine et elle peut être

\footnotetext{
${ }^{1}$ T. HÄGERSTRAND, Innovation diffusion as a spatial process.

${ }^{2}$ T. SAint-Julien, « Diffusion spatiale ».
} 
freinée, transformée ou arrêtée par des «barrières spatiales ", c'est-à-dire des frontières physiques, étatiques, sociales, culturelles plus ou moins perméables. La diffusion qui correspond à la transmission et l'adoption progressive d'une innovation se réalise de trois façons, par « contagion » lorsqu'elle se déroule en continuité spatiale, par « extension » lorsqu'un centre est touché et qu'il permet une nouvelle contagion et par « relocalisation » lorsque des individus, ayant adopté l'innovation, se déplacent dans d'autres aires culturelles. Bien que les emprunts terminologiques réutilisés dans un autre contexte que celui de leur élaboration soient toujours à manier avec précaution, la portée des notions définies par Hägerstrand sont d'un intérêt certain pour présenter la formation de l'espace tauromachique. D'un point de vue théorique, Hägerstrand distingue quatre phases dans les processus de diffusion spatiale: « l'amorce de diffusion " à partir d'un foyer d'innovation, la phase dite "d'expansion », la phase de " condensation » et celle de «saturation».

Telle pourrait être décrite schématiquement la formation de l'espace tauromachique, à partir des grandes phases de la diffusion spatiale des innovations. Partons de l'idée que la véritable innovation en matière de pratiques taurines est l'apparition d'un spectacle moderne, codifié, réglé et payant, séparant les acteurs spécialistes des spectateurs, même si, comme on le développera, ce changement de nature du divertissement mérite discussion. Voici le scénario simplifié des différentes étapes de formation de l'espace tauromachique, que nous traitons ensuite séparément dans son cadre européen et américain :

— «l'amorce de diffusion » est la phase durant laquelle les premiers centres adoptent l'innovation. Historiquement, le premier spectacle moderne est la corrida pédestre. Elle est souvent qualifiée de corrida moderne par les historiens, dont la codification est établie au XVIII ${ }^{\mathrm{e}}$ siècle, en Basse Andalousie, même s'il existe alors d'autres creusets de décantation de la tauromachie, en particulier dans la région Basco-navarraise. Cette amorce de diffusion de la corrida moderne apparaît bien après l'épanouissement des courses de taureaux aristocratiques du $\mathrm{XVI}^{\mathrm{e}}$ et $\mathrm{XVII}^{\mathrm{e}}$ siècles, qui ont été exportées dans les grandes villes de l'administration coloniale (capitales des vice-royautés, des audiences et des capitaineries). Ces courses et le développement de l'élevage extensif de bovins font naître des jeux taurins de toutes natures, indépendants ou intégrés aux spectacles des élites urbaines;

— «la phase d'expansion " constitue le cour du processus de diffusion par la conquête de nouveaux centres. Ces centres seraient d'abord les villes de la Basse Andalousie, par " contagion ", puis rapidement Madrid et les grandes villes de la péninsule Ibérique, par " extension». Le processus se poursuit de proche en proche, des grandes villes vers les moyennes, durant tout le XIX ${ }^{e}$ siècle jusqu'à s'imposer comme l'évidence d'un phénomène d'envergure nationale. La phase d'expansion se prolonge en Amérique, encore timidement au milieu du XIx ${ }^{e}$ siècle, puis de 
façon accélérée pendant les deux dernières décennies du siècle, en phase avec le premier grand âge d'or de la tauromachie moderne en Espagne. Mais, cette phase d'expansion est ralentie, puis stoppée par l'interdiction de sa pratique dans nombre de nouveaux États américains. Ces interdictions peuvent intervenir avant la diffusion de la corrida moderne (Chili, Argentine) ou après avoir connu des heures de gloire (Uruguay, Cuba). En France, l'expansion se prolonge par «contagion » frontalière et par « délocalisation » à l'initiative d'entrepreneurs de spectacles à la conquête d'un nouveau marché. Les deux dernières décennies du XIX ${ }^{\mathrm{e}}$ siècle représentent également la principale phase d'expansion de la corrida, qui en retour contribue à moderniser les spectacles locaux : la course landaise et la course camarguaise ;

— « la phase de condensation » correspond à un temps d'homogénéisation de l'espace et de réduction des contrastes régionaux. Cette phase s'installe dans le sillage de l'expansion de la fin du XIX ${ }^{e}$ siècle. Les pays d'Amérique adoptent les codes de la nouvelle tauromachie, éliminant progressivement de l'arène les particularismes encore nombreux qui marquent les courses de taureaux du XvirI ${ }^{\mathrm{e}}$ siècle. Les particularismes qui survivent, quittent l'arène de la corrida moderne pour enrichir certaines formes de rodéos en gestation dans les grandes propriétés d'élevage de bovins. Les premiers rodéos modernes, pris au sens de spectacles commerciaux, apparaissent aux États-Unis à la fin du XIX ${ }^{\mathrm{e}}$ siècle, dans un contexte de régression des rodéos utilitaires, liée aux progrès de la Révolution industrielle, notamment en matière de transport. En France, la « phase de condensation » stabilise la distribution des corridas au niveau des régions de vieilles traditions taurines que sont la Gascogne et la Camargue. Au Portugal, les interdictions de mise à mort publique des taureaux sont réitérées depuis le premier tiers du XIX ${ }^{\mathrm{e}}$ siècle jusqu'en 1928. Elles créent l'existence d'une «barrière spatiale » qui entérine la bifurcation entre la corrida espagnole et la corrida portugaise ;

— « la phase de saturation » correspond au ralentissement et à l'arrêt de la diffusion. Cette phase marquerait la stabilisation des frontières et des limites des terres de corridas qui depuis évoluent faiblement même si, dans le détail, il existe des recompositions non négligeables. C'est durant cette phase que la plupart des rodéos acquièrent une forme codifiée, puis se modernisent selon les modèles institutionnels du sport, bien que les chronologies soient très différentes d'un pays à l'autre, et s'étendent de la fin du XIX $x^{e}$ siècle à nos jours. Quoi qu'il en soit la phase d'expansion des rodéos, en tant que spectacle moderne, est un phénomène $\mathrm{du} \mathrm{xx}^{\mathrm{e}}$ siècle, qui continue aujourd'hui à conquérir de nouveaux territoires, comme le montre la diffusion contemporaine du rodéo étasunien, au Mexique et au Brésil. La grande phase d'expansion de la corrida moderne est en revanche un phénomène de la fin du XIX ${ }^{\mathrm{e}}$ siècle. 


\section{I. - LA FORMATION}

\section{DE L'ESPACE TAUROMACHIQUE EUROPÉEN}

La charnière entre le dernier tiers $\mathrm{du} \mathrm{XIX}^{\mathrm{e}}$ siècle et le premier tiers du $\mathrm{Xx}^{\mathrm{e}}$ siècle est une période fondamentale dans la stabilisation de l'espace tauromachique européen. Certes, il s'agit alors d'une géographie encore mouvante : certains spectacles sont déjà codifiés telles que la corrida espagnole et la corrida portugaise, d'autres n'ont pas tout à fait fini de l'être, telles que les courses landaises et camarguaises, d'autres encore n'existent toujours pas (les courses de recortadores), mais il s'agit de transformations et d'ajustements spatiaux dont les conditions sont alors largement établies.

Dans son travail sur les tauromachies européennes, Frédéric Saumade a montré à partir d'une analyse structuraliste des formes et des techniques taurines que la diffusion de la corrida moderne a contribué à fixer les codes des autres spectacles tauromachiques par une «dynamique d'engendrement réciproque ${ }^{3}$. Cette mise en relation des formes tauromachiques constitue, à nos yeux, une grille de lecture irremplaçable pour la compréhension générale de la formation de l'espace tauromachique européen. Conformément à une prise en compte élargie des pratiques tauromachiques qui tient au principe de notre définition préliminaire, nous reviendrons sur le problème des origines de la tauromachie et nous questionnerons l'innovation que constitue le spectacle tauromachique moderne. Nous retracerons ensuite les rythmes et les étapes de la diffusion de la corrida moderne, leurs conséquences sur la formation de l'espace tauromachique et sur la stabilisation d'une coexistence spatiale des jeux taurins.

\section{QUELLES SONT LES ORIGINES}

DE LA TAUROMACHIE?

Nous pensons que la difficulté de répondre à cette question repose paradoxalement sur la juxtaposition de deux très bonnes intuitions historiques. La première est d'inscrire la tauromachie dans une histoire longue des rapports entre les hommes et les bovins, avec les risques précédemment évoqués de forcer les traits de la continuité. La seconde est de considérer que dans l'histoire des spectacles, la tauromachie telle que nous la connaissons aujourd'hui apparaît à une période donnée comme un fait social radicalement nouveau, avec le risque de forcer les traits de la rupture.

Au sujet de l'Espagne, la voie suivie par l'historien Bartolomé Bennassar est de considérer qu'il existe des « siècles obscurs » pour lesquels on ignore l'origine précise des jeux taurins, mais qu'à partir du $\mathrm{XIV}^{\mathrm{e}}$ et $\mathrm{XV}^{\mathrm{e}}$ siècle, une " moisson documentaire » suffisante existe qui " atteste de manière incontestable, [...] la rencontre entre les fêtes de toute sorte (cérémonies et fastes royaux, fêtes religieuses et votives) et les jeux ou les rites tauromachiques » permettant d'en

\footnotetext{
${ }^{3}$ F. SAumade, Les tauromachies européennes, p. 178.
} 
retracer l'histoire de façon satisfaisante ${ }^{4}$. Dès le $\mathrm{Xv}^{\mathrm{e}}$ siècle nous savons que coexistent en Espagne une tauromachie chevaleresque dont les témoignages abondent et une tauromachie populaire à pied, parfaitement autonome, pour laquelle les données sont plus disparates.

La tauromachie à cheval est organisée par la haute aristocratie et par les villes comme une grande parade ostentatoire de la noblesse en armes, à l'occasion de laquelle les chevaliers font étalage de leur vaillance et de leur richesse. Parmi ces villes, mentionnons Madrid qui célèbre des « corridas royales » et des « corridas de la ville » ainsi que Valladolid, Saragosse, Tolède Séville, Pampelune. D’un point de vue technique, la lanzada, le coup de lance porté à l'arrêt, est la technique la plus courante au $\mathrm{XVI}^{\mathrm{e}}$ siècle. Les cavaliers sont accompagnés d'hommes à pied participant aux manœuvres et détournant l'attention du taureau, si leur maître venait à être désarçonné. Dans ce cas, pour venger l'affront, le taureau doit être tué à l'épée. Les taureaux se refusant au combat ont les tendons coupés et sont livrés aux chiens. La corrida équestre connaît elle-même des évolutions. La technique de la lanzada est supplantée par celle du rejón pratiquée en mouvement, plus apte à faire briller les qualités équestres du cavalier et dont s'inspire le travail actuel des rejoneadores. La technique est également adoptée au Portugal. Jusqu'au milieu du XvII ${ }^{\mathrm{e}}$ siècle, la noblesse court les taureaux selon les mêmes règles de part et d'autre de la frontière. Les spécialistes s'accordent à considérer que la bifurcation commence avec la Restauration portugaise en 1648, qui met fin à soixante-dix années d'union dynastique. L'année 1648 n'est cependant qu'une date symbolique puisque la séparation des formes tauromachiques n'est pas consommée avant le premier tiers du XVIII ${ }^{\mathrm{e}}$ siècle, au moment où émerge la tauromachie pédestre espagnole. Au-delà des frontières de la péninsule Ibérique, c'est cette tauromachie équestre qui est exportée en Amérique latine par la noblesse et pratiquée aux XVI ${ }^{\mathrm{e}}$ et $\mathrm{XVII}^{\mathrm{e}}$ siècles en Italie, à Rome, à Sienne et à Naples, introduite par les Borgia.

La tauromachie pédestre de cette époque, pratiquée indépendamment du spectacle de la noblesse incluant des auxiliaires à pied, est moins bien connue. Les recherches menées par Araceli Guillaume-Alonso montrent que la tauromachie pédestre populaire n'est en aucun cas postérieure à la tauromachie équestre nobiliaire qui jouit tout simplement d'une meilleure visibilité dans les sources 5 . Nous avons déjà évoqué à plusieurs reprises les courses taurines populaires mentionnées dès le $\mathrm{xI}^{\mathrm{e}}$ siècle en Estrémadure par Álvarez de Miranda. Selon le marquis de San Juan Piedras Albas, l'existence de professionnels rémunérés en Navarre, en la présence des matatoros chargés de mettre un terme au jeu sanguinaire en achevant l'animal, serait également repérable dès le $\mathrm{XI}^{\mathrm{e}}$ siècle et totalement irréfutable à partir du XIII ${ }^{\mathrm{e}}$ siècle ${ }^{6}$. Quelle que soit l'ancienneté de ces pratiques, elles sont de toute évidence d'une très grande diversité, dans leur

\footnotetext{
${ }^{4}$ B. Bennassar, « Histoire de la tauromachie », p. 5.

${ }^{5}$ Sur les hommes à pied (gardes, soldats, laquais) dans le toreo à cheval, voir A. GuillaumeAlonso, La tauromaquia y su génesis, pp. 143-153.

${ }^{6}$ Ibid., p. 161.
} 
gestuelle, leur motivation et leur finalité : jeux taurins organisés lors des fêtes agricoles et religieuses, jeux taurins improvisés lors du passage des troupeaux près des lieux d'habitation jusqu'aux abattoirs.

Ce qui est généralement considéré comme la première interdiction des jeux taurins date du Código de las Siete Partidas d'Alphonse X en 1263 qui frappe d'infamie, dans tout le royaume de Castille, le fait d'être rémunéré pour combattre des taureaux. D'un point de vue social, le loisir aristocratique qui est financièrement désintéressé ne tombe pas sous le coup de la condamnation, posant ainsi les bases de sa reconnaissance. Le caractère édifiant de la tauromachie chevaleresque dans le contexte de la Reconquête se voit renforcé par le rôle stratégique des troupeaux de bovins. En raison des déplacements fréquents de population, l'activité agricole est perturbée, conférant aux troupeaux de bovins une valeur inestimable pour nourrir les populations ${ }^{7}$. Combattre les taureaux, les chasser, les maîtriser assoit le pouvoir des seigneurs dans la sphère socio-économique la plus vitale.

D'un point de vue territorial le royaume de Navarre a longtemps gardé une part d'autonomie vis-à-vis de la Castille, ce qui pourrait expliquer le développement précoce d'un embryon de tauromachie professionnelle dans les contrées septentrionales, dont Pampelune constitue dès le $\mathrm{xvI}^{\mathrm{e}}$ siècle un centre important ${ }^{8}$. De cette partition territoriale est née une première controverse historiographique autour de la formation de la corrida moderne, dont les uns affirment qu'elle a pour origine le Nord de l'Espagne, et les autres qu'elle est andalouse. Ainsi formulée, la controverse relève en partie d'une question mal posée, puisque la corrida moderne ne peut pas se limiter au seul statut professionnel des protagonistes. Ce qui est sûr, c'est que des toreros du Nord apparaissent dans les courses données à Madrid au XVII ${ }^{e}$ siècle au côté de toreros locaux. Au XVI ${ }^{\mathrm{e}}$ siècle, à Pampelune, la mise à mort des bêtes dans les courses du Nord est loin d'être systématique, puisqu'il est prévu des soins pour une partie des taureaux, reconduits après la fête dans des pâturages à l'extérieur de la ville9. L'une des finalités essentielles de la course de taureaux en Navarre réside déjà dans le fait de jouer avec le taureau, de le courir, de le tromper, ce que confirmerait une appellation courante des protagonistes de l'époque sous le nom de corredor de toros (coureur de taureaux) ${ }^{10}$. La place croissante de la mise à mort des taureaux ne s'impose à Pampelune qu'à la fin du XviI siècle, sans doute en raison des interactions croissantes entre la tauromachie de la noblesse et la tauromachie plébéienne.

En Basse Andalousie, les preuves sont nombreuses pour pouvoir affirmer l'existence d'un autre foyer de codification de la tauromachie, parfaitement contemporain, qui pour la plupart des historiens est responsable des transformations décisives de la tauromachie du $\mathrm{XvI}^{\mathrm{e}}$ et $\mathrm{du} \mathrm{XvII}^{\mathrm{e}}$ siècle vers la corrida

\footnotetext{
${ }^{7}$ A. García-Baquero González et alii, Sevilla y la fiesta de los toros, p. 34.

${ }^{8}$ L. del Campo, Pamplona y toros.

${ }^{9}$ A. Gulllaume-Alonso, La tauromaquia y su génesis, p. 166.

${ }^{10}$ Ibid., p. 170.
} 
moderne. Les travaux de l'historien Antonio García-Baquero ont mis en lumière une pratique de la tauromachie à pied comme spectacle dans les abattoirs de Séville, dès la deuxième moitié du $\mathrm{XVI}^{\mathrm{e}}$ siècle $^{11}$. Les toitures des abattoirs servaient alors de gradins improvisés et exigeaient de fréquentes réparations, et cela malgré les interdictions, fréquemment réitérées par les autorités, de toréer dans ces lieux. Les premiers toreros des arènes de la Maestranza de Séville au $\mathrm{XVIII}^{\mathrm{e}}$ siècle seront issus des abattoirs du quartier de San Bernardo.

Une chronologie comparable peut être établie pour les jeux taurins en France, ou plutôt dans les régions du Sud de la France où l'on avait la quasi-certitude de trouver dans les archives les preuves de leur ancienne existence. L'étude critique de l'histoire de la course camarguaise et de ses sources menées par Évelyne Duret en vient à la conclusion qu'il y aurait un grand intérêt à " prendre comme champ d'investigation une zone plus large que l'aire géographique de la course camarguaise $»^{12}$. Nous avons précédemment signalé l'existence de jeux taurins en divers lieux européens qui se sont maintenus jusqu'au XIX ${ }^{\mathrm{e}}$ siècle, fréquemment en lien avec l'activité des abattoirs, lieu charnière entre le monde agricole et le monde urbain. Nombreuses sont les monographies sur les villes taurines françaises réalisées par l'Union des bibliophiles taurins de France qui mentionnent, sous l'Ancien Régime l'existence de jeux taurins dans les foires ou à proximité des abattoirs : Marseille, Bordeaux, Toulouse, Paris, Arles, Nîmes, Bayonne $^{13}$. À Bordeaux, un arrêté est pris en 1565 interdisant aux bouchers de faire courir les bœufs et les vaches à travers la ville ${ }^{14}$.

En Camargue, les plus anciennes mentions font état de jeux taurins à Arles, dès le $\mathrm{XII}^{\mathrm{e}}$ ou le $\mathrm{XIII}^{\mathrm{e}}$ siècle, lors de la foire qui se tenait la veille de la Pentecôte $^{15}$. Les mentions de jeux taurins sont fréquentes pour Arles et la Camargue dès le $\mathrm{XVI}^{\mathrm{e}}$ siècle, se multiplient dans la région de Nîmes et les bourgs du Gard méridional à partir du XVIII ${ }^{\mathrm{e}}$ siècle et s'étendent à Avignon et ses environs au $\mathrm{XIX}^{\mathrm{e}}$ siècle $^{16}$. Les historiens s'accordent à considérer que les jeux sont nés sur place, dans les campagnes aux alentours d'Arles, en lien avec la présence des bovins élevés au XIII ${ }^{\mathrm{e}}$ siècle en nombre important. Dès le Moyen Âge, ces jeux débordent le cadre des cours de mas ruraux et sont pratiqués dans les rues de petites villes proches lors des fêtes locales ou pour célébrer la visite de hauts personnages. L'exemple le plus fameux est la course de taureaux donnée en 1564, lors du tour de France du jeune Charles IX et de Catherine de Médicis.

\footnotetext{
${ }^{11}$ A. García-Baquero GonzÁlez, «El macelo sevillano ».

12 É. Duret, «La course camarguaise, aspects historiques ».

${ }^{13}$ P. Berdoues, Histoire de la tauromachie à Toulouse ; J.-P. DarracQ, Histoire taurine de VicFezensac; M. Figère, Les arènes de Lunel et la corrida ; L.-G. LACroix, Histoire de la tauromachie en Arles; A. Lafront, Histoire de la tauromachie à Bordeaux; H. Marc, Histoire taurine de la ville de Béziers ; J.-L. Rouyre, Un siècle de corridas à Beaucaire; ID., La tradition taurine à Tarascon; C. Sabathí, Histoire de la tauromachie à Céret.

${ }^{14}$ A. Lafront, Histoire de la tauromachie à Bordeaux, p. 9.

${ }^{15}$ L.-G. Lacroix, Histoire de la tauromachie en Arles, p. 24.

${ }^{16}$ É. Duret, «La course camarguaise, aspects historiques », p. 63.
} 
Dans le Sud-Ouest, la plus ancienne mention de jeux taurins est celle découverte par Claude Pelletier dans les archives de Bayonne datant de 1289, qui interdit aux bouchers de lâcher les bêtes dans la ville pour les faire courir. De même, les témoignages abondent dans les sources à partir du Xvi ${ }^{\mathrm{e}}$ siècle concernant de nombreuses courses de vaches, bœufs et taureaux lors des fêtes patronales ou de l'envoi des animaux à l'abattoir (Bazas, Saint-Sever, Mont-deMarsan, Bayonne, Dax, Salies-de-Béarn $)^{17}$. Les troupeaux de bovins des marais landais auraient fourni une partie du bétail pour la boucherie et les jeux taurins, en particulier les vaches à demi-sauvages, dites "marines ", de la zone côtière des Landes de Gascogne qui furent ensuite croisées avec les bazadaises ${ }^{18}$.

Dans la France de l'Ancien Régime, les mesures d'interdiction des jeux taurins prennent de l'ampleur à partir du XviI ${ }^{\mathrm{e}}$ siècle. À la demande du parlement de Bordeaux impuissant à faire interdire les courses de taureaux à Bazas, Louis XIII les condamne par lettres patentes. Louis XIV en 1648 abolit solennellement toutes les courses dans le diocèse d'Aire où sont visées les courses de Montde-Marsan, Saint-Sever, Montaut, Hagetmau, Grenade, Cazères, Castandet. Ces interdictions se poursuivent sous Louis XV qui finit par concéder en 1757 une ordonnance royale autorisant les courses à la seule condition qu'elles aient lieu hors des agglomérations, dans des espaces clos par des barrières. Le maréchal duc de Richelieu, gouverneur militaire de Guyenne, revient sur cette ordonnance en tentant de faire disparaître tout type de courses à partir de 1766, avant de céder sept ans plus tard, en reconnaissant ne pas pouvoir lutter contre « le goût dominant et si général des peuples d'Armagnac » pour les courses ${ }^{19}$. Dans le Sud-Est, se déroule un scénario équivalent, Louis XIV interdit les courses à Beaucaire en 1655, à la suite d'une plainte déposée par les Capucins. À Arles, sans davantage de succès, un arrêté du Parlement interdit en 1722 les courses improvisées par la foule sur le passage des bêtes vers les abattoirs, à la demande de la Ferme de la boucherie qui se plaint de l'état dans lequel se trouve le bétail à l'arrivée. Il semble bien que les valeurs absolutistes françaises de réduction à l'obéissance échouent à supprimer ces particularismes locaux.

Si nous rappelons ces aspects bien connus des historiens spécialistes de la question, c'est pour prévenir les excès qui consisteraient, sous prétexte que la corrida moderne est un phénomène social nouveau, à faire débuter l'histoire de la tauromachie au XVIII ${ }^{\mathrm{e}}$ siècle. Qu'il n'y ait pas de méprise, ici ne sont pas visés les auteurs à qui l'on doit précisément la mise en valeur d'une rupture importante dans l'histoire des spectacles, rupture jusqu'alors insuffisamment lisible, mais les écrits qui font démarrer l'histoire de la corrida en France au XVIII ${ }^{\mathrm{e}}$ voire au $\mathrm{XIX}^{\mathrm{e}}$ siècle, sans mise en contexte rigoureuse. Sur ce point, l'ouvrage d'Élisabeth Hardouin-Fugier, Histoire de la corrida en Europe du XVIII siècle

\footnotetext{
${ }^{17}$ M. Laforcade, La tauromachie dans le Sud-Ouest de la France, pp. 36-40.

${ }^{18}$ J.-Y. PuYo et M. DAUGA, « La course landaise, sport-spectacle », p. 17. Les auteurs précisent que les dernières « marines » ont été abattues par les militaires français de la base de Biscarosse dans les années 1950 car elles occasionnaient des accidents de la circulation.

${ }^{19}$ A. Lafront, Histoire de la corrida en France, p. 8.
} 
au $\mathrm{XXI}^{e}$ siècle, malgré l'abondance des sources utilisées, nous semble offrir une perspective historique très contestable. L'auteur considère en effet que l'implantation de la corrida en France s'est faite dans le cadre d'une «longue hostilité méridionale à la corrida (1853-1894) ». À aucun moment elle ne prend au sérieux l'hypothèse selon laquelle l'existence d'une passion taurine préexistante ait pu favoriser l'implantation de la corrida dans le Sud de la France même si les jeux taurins autochtones et la course espagnole ont pu être en concurrence ${ }^{20}$. Or il semble bien que la corrida n'aurait pu s'implanter durablement en France sans la présence de ces jeux taurins.

\section{LE SPECTACLE TAUROMACHIQUE}

COMME INNOVATION

Tous les historiens s'accordent aujourd'hui à considérer que la corrida moderne, dans l'histoire des jeux taurins, correspond à une transformation sociale importante. La Basse Andalousie du XVIII ${ }^{\mathrm{e}}$ siècle et en particulier Séville ont joué un rôle crucial dans cette histoire, tant du point de vue de la définition des techniques que du point de vue d'une conception d'ensemble de la fête taurine. Les auteurs du livre Sevilla y la fiesta de los toros affirment « qu'au cours des vingt années qui vont de 1730 à 1750, Séville assista dans son arène de la Maestranza au développement complet du processus d'invention des corridas modernes ${ }^{21}$. On retrouve à l'origine de cette évolution la convergence de deux facteurs favorables : l'existence d'un vivier d'hommes expérimentés dans le maniement du bétail issu des abattoirs sévillans et le privilège d'organiser des corridas à buts caritatifs, accordé en 1729 par Philippe V à la Maîtrise royale de cavalerie de Séville (Real Maestranza de Caballería de Sevilla).

À la suite de la guerre de Succession, avec l'arrivée sur le trône d'Espagne d'un Bourbon peu intéressé au début de son règne par les courses de taureaux, la noblesse finit de s'éloigner de la pratique du toreo à cheval, qui, déjà en déclin, ne fait que décroître encore tout au long du Xviri ${ }^{\mathrm{e}}$ siècle. Comme le remarque Araceli Guillaume-Alonso, par un nouvel éclairage, il ne faudrait pas oublier que pendant le règne précédent, celui de Philippe IV, en raison des nombreux combats livrés sur le champ de bataille européen, les chevaux deviennent une denrée rare, ce qui explique aussi que les nobles prennent moins volontiers le risque de les perdre pour des jeux taurins ${ }^{22}$. Pourtant, les hommes à cheval ne disparaissent pas de la piste. Ils sont remplacés par les varilargueros, des cavaliers issus des travailleurs du bétail au service de la noblesse, qui deviennent pour un temps les nouveaux héros des courses de taureaux. Ils n'occupent pas longtemps les devants de la scène, puisqu'à la fin des années 1730, dans ce qui était alors appelé la corrida de varilargueros, la mise à mort à pied était déjà

\footnotetext{
${ }^{20}$ É. Hardouin-Fugier, Histoire de la corrida en Europe, p. 135.

${ }^{21}$ A. García-Baquero González et alii, Sevilla y la fiesta de los toros, p. 82.

${ }^{22}$ A. Guillaume-Alonso, « Toros y sociedad », p. 125.
} 
devenue l'événement dramatique fondamental de la cérémonie ${ }^{23}$. Le surnom de celui qui passe pour avoir inventé la technique de mise à mort dite du volapié, Juan Rodríguez « Costillares » (de costillas : côtelettes), fait clairement allusion à la corporation professionnelle dont nombre de toreros sont issus.

Malgré son rôle indéniable, Séville ne peut pas être considérée comme le seul laboratoire de codification de la corrida moderne, ni même peut-être comme le premier. Rappelons d'abord que la Maestranza de Ronda bénéficie d'un privilège identique en 1739 , soit dix ans après, et joue au milieu du siècle un rôle équivalent. Ronda revendique fièrement son statut de berceau de la tauromachie moderne avec comme argument des plus convaincants d'abriter alors la célèbre dynastie des Romero qui commence avec Francisco (1700-1763), le grand père de Pedro Romero, à qui la tradition attribue souvent le privilège d'avoir le premier utilisé la muleta et l'épée pour tuer les taureaux en piste. Selon les recherches récentes de Guillermo Boto Arnau, c'est Cadix qui devrait être considérée comme le premier centre d'invention de la corrida à pied, près d'un quart de siècle avant Séville ${ }^{24}$. Pour montrer le rôle de Cadix dans la formation de la tauromachie moderne et son antériorité sur Séville, l'auteur s'appuie sur le nombre de courses qu'on y célèbre, en particulier pour financer la construction des remparts, et sur le nombre de toreros d'origine gaditane au milieu du XviII ${ }^{\mathrm{e}}$ siècle. Hors de l'Andalousie, il est avéré que d'autres villes jouent en parallèle un rôle important, telles que Pampelune et Bilbao où l'on observe, dès le début des années 1730 , la performance de cuadrillas à pied ${ }^{25}$. À la fin des années 1730, à Madrid et à Valence, l'organisation des spectacles taurins est confiée aux hôpitaux et aux institutions de bienfaisance ${ }^{26}$. Nous sommes donc dans la première phase d'une innovation qui se crée à partir de foyers multiples en cours d'éclosion. Jusqu'à la fin du siècle, on peut considérer qu'il existe deux zones principales à l'intérieur desquelles les échanges et les contacts se multiplient : une zone méridionale andalouse où s'affirment les arènes de Séville, Cadix, Ronda, Cordoue, Grenade, Málaga et une zone plus septentrionale où s'affirment les arènes de Madrid, Salamanque, Valence, Saragosse, Pampelune, Bilbao. Ce clivage reprend une délimitation bien connue des aficionados qui sépare l'Espagne, à partir de Despeñaperros, en deux régions taurines. À la fin du siècle, toutes ces arènes sont plus ou moins acquises aux normes tauromachiques andalouses qui définissent les principales techniques et mettent en ordre le spectacle : il est organisé en trois tiers de cinq minutes chacun, le protagoniste central est le torero à pied qui met à mort le taureau, le travail des picadors héritiers des varilargueros et le travail des banderilleros lui sont subordonnés, enfin cape, pique à cheval, banderilles, muleta, épée deviennent les seuls instruments utilisés. Un texte confirme ces avancées : La

\footnotetext{
${ }^{23}$ A. García-Baquero GonzÁlez et alii, Sevilla y la fiesta de los toros, p. 74.

${ }^{24}$ G. Boto-Arnau, « Cádiz y la aparición del toreo moderno ».

${ }^{25} \mathrm{~L}$. del Rey, « Los festejos taurinos ».

${ }^{26}$ R. CABrera Bonet, « Transformación y continuidad del espectáculo taurino madrileño »; A. Shubert, A las cinco de la tarde, p. 29.
} 
Tauromachie ou l'Art de toréer de Pepe Illo, publié en 1796, qui entend en exposer les règles et les suertes (techniques et gestes). Au cours de cette évolution, par sa position géographique et son rôle de capitale, il est difficile de ne pas faire de Madrid une charnière centrale de la modernisation du spectacle, et de la confrontation des styles régionaux. Parfois mise en retrait des histoires taurines attentives à souligner la part des codifications régionales, c'est bien Madrid qui entérine les évolutions majeures et consacre les normes pour l'ensemble du territoire.

Dans ce processus, la véritable innovation sociologique réside dans la dimension commerciale acquise par les spectacles taurins. L'apparition des arènes permanentes, spécifiquement construites pour le spectacle taurin, marque une étape décisive de ce processus. La professionnalisation des toreros légitime la séparation des acteurs et des spectateurs. Même si la police montée est encore chargée de dégager la piste occupée par la foule, l'intervention spontanée du public, qui plonge ses racines dans les jeux taurins populaires, est progressivement reléguée à une partie finale séparée du spectacle. Le divertissement taurin a changé de nature, il est différent des fêtes taurines de la noblesse qui sont cependant encore célébrées occasionnellement jusqu'au début du $\mathrm{xx}^{\mathrm{e}}$ siècle, et différent des fêtes taurines où la foule en piste joue un rôle important.

On considère généralement que les premières arènes sont en bois, de forme rectangulaire, inspirées des places urbaines de la Renaissance où se déroulaient les corridas chevaleresques, puis s'arrondissent au milieu du XVIII ${ }^{\mathrm{e}}$ siècle, pour atteindre la forme circulaire des plazas de toros que nous connaissons aujourd'hui et qui consacrent l'autonomie du spectacle. Les premières arènes en bois construites par la Maestranza en 1733 sur le mont Baratillo sont en effet rectangulaires, ensuite remplacées sur le même site par des arènes rondes. Dans le détail, il y aurait probablement des ajustements à apporter à cette évolution générale, car les plus anciennes arènes circulaires, en ouvrage de maçonnerie, sont celles relativement modestes de Béjar dans la province de Salamanque, construites en 1711 et celles, également circulaires, de Campofrío (Huelva), construites en 1718. Avant que ne s'affirment définitivement les édifices circulaires autonomes et spécifiquement bâtis pour le spectacle taurin, sont construits des places mixtes d'habitation et de spectacle, de forme polygonale, qui témoignent d'une transition architecturale d'une grande valeur patrimoniale : les arènes hexagonales d'Almadén (Ciudad Real) édifiées en 1752, celles octogonales de La Carolina (Jaén) en 1767. Les premières grandes arènes permanentes sont celles de Madrid édifiées en 1749, possédant encore des parties en bois, celles de Séville dont les travaux commencent en 1759, celles de Saragosse en 1764, de Ronda en 1785, d'Aranjuez en 1797. Notre travail de recensement des arènes en Espagne permet d'établir qu'une vingtaine d'arènes, en ouvrage de maçonnerie, ont été construites avant 1800. À cette même époque des éleveurs de bovins commencent à se spécialiser afin d'approvisionner les spectacles en bétail. Comme nous l'avons souligné précédemment, ce sont les castes de la Basse Andalousie qui finissent par s'imposer au fur et à mesure de la professionnalisation du spectacle taurin. Selon Bartolomé Bennassar, le prix des taureaux 
de combat entre 1730 et 1800 a été multiplié en moyenne par six, alors que celui des produits agro-pastoraux a été multiplié par deux ${ }^{27}$.

Au tournant du siècle, il ne fait plus aucun doute que tous les éléments du spectacle moderne sont réunis. Il s'agit d'un divertissement payant, aux codes définis, qui se déroule dans un lieu scénique spécifique. Pour l'historien Adrian Shubert, à qui l'on doit une étude détaillée de l'histoire sociale de la tauromachie en Espagne, il se pourrait bien que la corrida fût le premier divertissement commercial de l'Occident, qui annonce les grands loisirs de masse avec près d'un siècle d'avance ${ }^{28}$. Selon l'auteur, «ses équivalents étrangers sont le baseball professionnel aux États-Unis ainsi que le football et le criquet en Grande-Bretagne qui apparaissent dans les années 1870, un siècle après que la tauromachie est déjà fermement établie en Espagne $»^{29}$.

GraphiQue 1. - Construction des arènes en Espagne

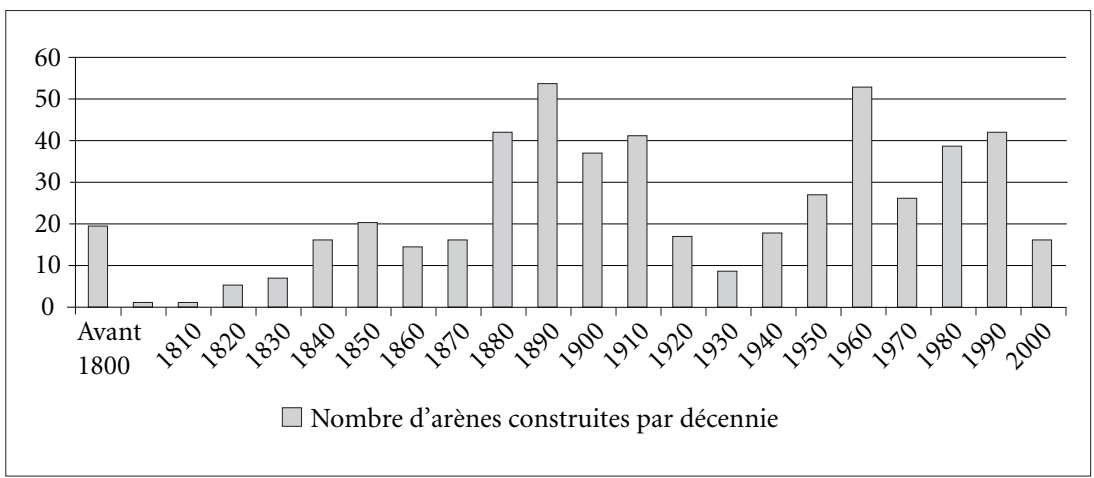

Au Portugal, à partir du milieu du xviII ${ }^{\mathrm{e}}$ siècle, l'épointage des cornes permettant de limiter les accidents se généralise. L'historiographie portugaise rapporte que le public semble progressivement préférer la technique des cavaliers portugais qui protègent leur monture à celle des varilargueros, puis des picadors où les chevaux sont éventrés ${ }^{30}$. À la codification de Pepe-Illo pour la tauromachie pédestre espagnole répond celle du marquis de Marialva pour la tauromachie équestre portugaise. À cette époque, il n'y a aucune restriction à la mise à mort des taureaux.

En France, ni la course landaise, ni la course camarguaise, ne possèdent alors les caractéristiques d'un spectacle moderne. Les éléments de modernisation du $\mathrm{XVIII}^{\mathrm{e}}$ siècle sont dus aux conséquences des réglementations qui essayent d'im-

\footnotetext{
${ }^{27}$ B. Bennassar, « Histoire de la tauromachie », p. 35.

${ }^{28}$ A. Shubert, «El toreo en la historia española », p. 25.

${ }^{29}$ Ibid., p. 10.

${ }^{30}$ J.-M. de Cossío, Los Toros, t. IV, p. 35.
} 
poser que les jeux se déroulent dans des enclos dissociés des places publiques et situés à l'extérieur des agglomérations. Cette transformation suffit néanmoins à faire quelque peu évoluer les pratiques. Dans le Sud-Est, la cocarde est intégrée aux jeux taurins lors des fêtes révolutionnaires ${ }^{31}$. Les cirques romains de Nîmes et d'Arles sont respectivement déblayés de leurs habitations en 18121813 et entre 1825 et 1837 , pouvant dès lors accueillir des courses ${ }^{32}$. Beaucaire construit des arènes en 1818. Dans le Sud-Ouest, Dax construit dès 1784 des arènes permanentes ${ }^{33}$. De plus, les techniques s'affinent par la généralisation des pistes fermées et le terme "écarteurs », pour désigner les protagonistes du jeu, apparaît dès le Premier Empire ${ }^{34}$. La corde qui n'est pas alors systématique apparaît au XVIII ${ }^{\mathrm{e}}$ siècle ${ }^{35}$. Cela n'empêche en rien les jeux taurins participatifs de toutes sortes d'avoir encore lieu au cœur du tissu urbain. Il faut attendre le $\mathrm{XIX}^{\mathrm{e}}$ siècle et la diffusion en France de la corrida moderne pour que ces évolutions s'accélèrent.

\section{LA DIFFUSION DE LA CORRIDA MODERNE}

a) La diffusion en Espagne

Au début du XIX ${ }^{\mathrm{e}}$ siècle, de nombreux événements freinent l'expansion territoriale du nouveau spectacle qui se trouve en réalité dans une situation critique jusqu'au début des années 1830. D’une part, la génération des grandes figures pionnières de la tauromachie moderne, Pedro Romero, "Costillares » et « Pepe Illo » disparaît avec le XVIII ${ }^{\mathrm{e}}$ siècle finissant. D’autre part, en 1805 est entérinée par le Conseil de Castille l'une des tentatives d'interdiction des jeux taurins les plus importantes depuis la bulle papale De Salute Gregis Dominici prononcée par Pie V en 1567. Carlos IV dans la Novísima Recopilación reprend les mesures prises par son père Carlos III dans la Pragmática Sanción de 1785 qui, en n’interdisant que partiellement les jeux taurins, s'étaient révélées inefficaces ${ }^{36}$. En dépit de cette interdiction, les corridas se maintiennent en Andalousie plus éloignée du pouvoir central, ce qui contribue, au moins en partie, à favoriser la décantation des normes méridionales par rapport aux autres styles régionaux. L'ironie de l'histoire veut qu'après l'invasion napoléonienne ce soit un roi français, en la personne de José Bonaparte, le frère de Napoléon, qui autorise à nouveau les courses de taureaux. Après avoir assisté à une course de taureau au Puerto de Santa María, il organise la reconstruction d'une arène à Madrid, financée par la mairie au titre pompeux des « Accords de Madrid du 28 avril », pour la

\footnotetext{
${ }^{31}$ F. Saumade, Des sauvages en Occident, p. 227.

32 É. Duret, « La course camarguaise, aspects historiques », p. 61.

${ }^{33}$ J.-Y. Puyo et M. DAugA, « La course landaise, sport-spectacle », p. 16.

${ }^{34}$ B. Traimond, Les fêtes du Taureau, p. 10.

${ }^{35}$ M. Dauga et J.-Y. Puyo, « Les écarteurs landais entre mythe et réalité », p. 192.

${ }^{36}$ P. Plasencia, La fiesta de los toros, pp. 16-17.
} 
célébration d'une corrida le 14 mai $1811^{37}$. Après la Restauration de 1814, les milieux favorables aux courses de taureaux obtiennent du roi Ferdinand VII une ordonnance créant, en 1830, la première école de tauromachie à Séville. Malgré sa fermeture trois ans après, cette école permit de former une nouvelle génération de toreros dont Francisco Montes « Paquiro » à qui l'on doit la deuxième grande Tauromaquia. À travers ce texte, « Paquiro » passe pour le codificateur presque définitif de la corrida dont les modalités sont détaillées depuis l'habit de lumière jusqu'à la construction optimale des arènes. Dans cette période troublée par la première guerre Carliste, plusieurs villes du Nord, situées dans la région la plus affectée par le conflit, Bilbao, Pampelune, Saragosse, ne programment des courses de taureaux que très irrégulièrement ${ }^{38}$.

De nombreux critères sont réunis pour que la phase d'expansion de la corrida moderne confirme le poids des acteurs andalous. D’après les calculs d'Adrien Shubert, plus de $80 \%$ des toreros nés avant 1815, dont on connaît le lieu de naissance, sont issus des provinces andalouses ${ }^{39}$. Le graphique 2 permet d'apprécier l'accélération de la diffusion spatiale du spectacle moderne à travers le nombre d'arènes construites, qui décolle à partir des années 1840 .

GraphiQue 2. - Évolution du nombre d'arènes en Espagne

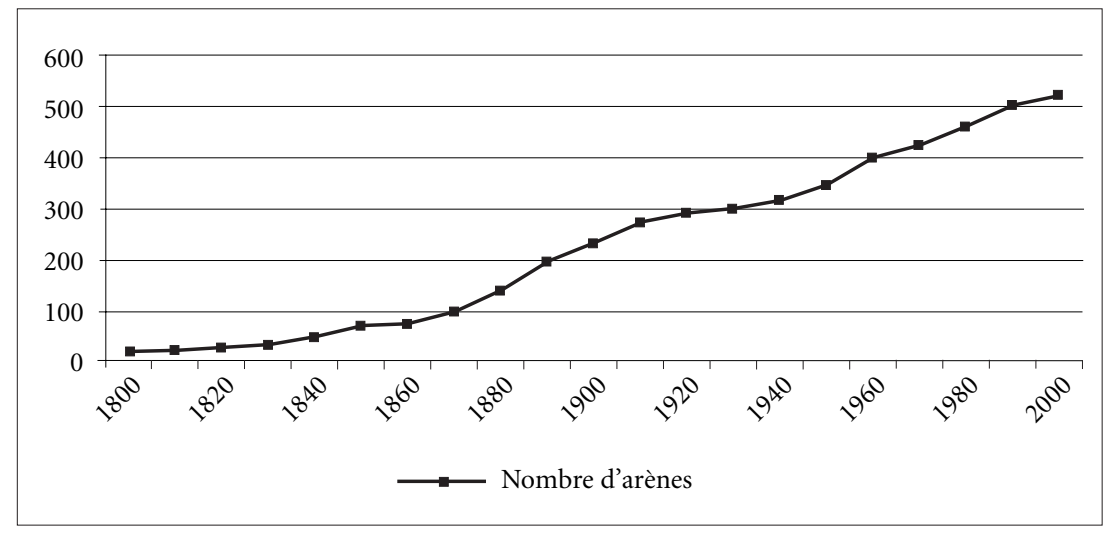

Les arènes demeurent pour nous le meilleur marqueur matériel du processus de diffusion spatiale de la corrida moderne. Si nos recherches contribuent à mieux cerner l'histoire de la tauromachie en Espagne, c'est bien à travers l'élaboration d'un recensement précis des arènes, permettant d'apprécier les rythmes de formation de l'espace tauromachique du XVIII ${ }^{\mathrm{e}}$ siècle à nos jours. La construction des arènes s'accélère encore à partir des années 1880 où la majo-

\footnotetext{
${ }^{37}$ J.-F. Batté, Politique et tauromachie, p. 18.

${ }^{38}$ B. Bennassar, " Histoire de la tauromachie », p. 19.

${ }^{39}$ A. Shubert, A las cinco de la tarde, p. 80.
} 
rité des grandes villes d'Espagne se dotent d'arènes permanentes dont les plus importantes dépassent désormais fréquemment le seuil des 10.000 places. À cette densification de la trame taurine, correspond une diminution du nombre des toreros d'origine andalouse, qui montre l'épanouissement d'un divertissement d'envergure nationale sur un plan territorial, réalité plus tangible que l'appellation controversée de fiesta nacional. Cela étant, la part des acteurs andalous demeure forte. En effet, plus de $45 \%$ des toreros nés entre 1875 de 1895 sont encore d'origine andalouse et $15 \%$ d'origine sévillane ${ }^{40}$. De 1860 à 1895, le nombre de corridas par an passe de 400 à 700. Tous les spécialistes se rejoignent pour faire de la période qui va de la fin des années 1860 au milieu des années 1885 un premier âge d'or de la tauromachie auquel les toreros Lagartijo et Frascuelo prennent une part active. Contrairement à une idée reçue, le tourisme constitue dès la fin du $\mathrm{XIX}^{\mathrm{e}}$ siècle une composante importante de l'extension de la tauromachie. La construction du réseau ferré augmente l'aire d'attraction des courses de taureaux autour des principales villes desservies et fait le succès des grandes arènes. À cette époque, nombre d'entre elles sont d'ailleurs construites sur des terrains à proximité des gares, ce que l'on peut encore observer à Bilbao ou à Valence. À partir des années 1860, les compagnies ferroviaires affrètent spécialement des trains lors des principales férias, les revues taurines se développent, les publicités exploitent largement l'iconographie et la rhétorique des corridas. Dans cette phase d'expansion des corridas, beaucoup réclament l'abolition des courses de taureaux, question qui, à de nombreuses reprises, est débattue par le pouvoir législatif. En 1876, le marquis de San Carlos, cherchant une voie progressive à leur disparition, proposa dans un premier temps d'interdire la construction de nouvelles arènes, le temps de trouver des mesures transitoires vers la suppression définitive de la tauromachie. Devant la résistance des défenseurs de la tauromachie, il pouvait encore déclarer : « En 1875, 101 arènes étaient en activité ; il me semble que ce chiffre est suffisant pour que les aficionados puissent rendre un culte à leur spectacle favori. En outre, évitons que ce genre de spectacles se propage dans les provinces où il n'a pas encore pénétré $»^{41}$. En 1900, un quart de siècle plus tard, le nombre de ces arènes a doublé et il n'y a guère que la Galice qui reste en marge de ce processus.

En cette fin de siècle, la tauromachie est devenue un spectacle de masse, et partage la société entre ses inconditionnels et ses détracteurs pour qui la corrida cumule les vices et les retards d'une nation décadente. Elle semble avoir gagné la faveur des masses, alors qu'elle scandalise une partie des élites éclairées qui dénoncent l'immoralité d'un spectacle donnant libre cours à la contestation de l'autorité, qui incite à la promiscuité des deux sexes et qui encourage l'expression des bas instincts. En outre, le spectacle est accusé d'appauvrir l'économie agricole, de retarder l'industrialisation et de donner une image archaïque de l'Espagne en Europe. Cependant, on remarque qu'aucun projet d'interdiction n'a longtemps

\footnotetext{
${ }^{40}$ Ibid., p. 80.

${ }^{41}$ J.-F. BAtté, Politique et tauromachie, p. 58.
} 
résisté à l'engouement populaire ou à l'intérêt politique d'y céder, en ce siècle finissant où les masses s'affirment comme un acteur social de premier plan.

b) La diffusion hors des frontières espagnoles

La diffusion de la corrida espagnole hors de ses frontières nationales est freinée au Portugal par les premiers interdits concernant les pratiques taurines. En 1836, l'année où Montes publie en Espagne sa Tauromaquia, un décret royal interdit au Portugal les spectacles taurins, et non la mise à mort comme cela est souvent répété chez ceux qui entendent démarquer le plus tôt possible les spécificités de la tauromachie portugaise par rapport à la tauromachie espagnole. Le décret souligne que les corridas sont " un spectacle barbare et impropre des nations civilisées » et entend supprimer ce qui « empêche et retarde le perfectionnement moral de la nation portugaise $»^{42}$. Ce décret du 19 septembre 1836 est révoqué en juin 1837 par une loi sur les spectacles publics autorisant les courses de taureaux, mais les soumettant à un contrôle étatique. Dès le milieu du siècle, l'habitude est prise d'embouler les cornes et de garder les taureaux pour plusieurs courses, ce qui ne rend plus leur mise à mort systématique. Ces évolutions divergentes par rapport à la norme espagnole participent néanmoins du développement de la tauromachie en tant que spectacle moderne, comme en témoigne la construction de nombreuses arènes dans les années 1890. En effet, plus de la moitié des arènes existant en 1900 ont été construites durant la dernière décennie du siècle (voir annexe II [CD-ROM]). À l'image de Lagartijo et Frascuelo en Espagne, Victorino Froes devient en cette fin de siècle, le cavalier emblématique de la consécration populaire du spectacle et de sa formalisation classique. En outre, l'emboulement des cornes en vue de civiliser le spectacle favorise l'affirmation de la tauromachie pédestre des forcados, fondée sur le corps à corps.

En France, si l'on excepte le précèdent de la course "à la mode d'Espagne » organisée en 1701 à Bayonne en l'honneur du futur roi Philippe V, l'histoire de la corrida espagnole commence au début des années 1850 . Les premières corridas intégrales ont eu lieu, dans cette même ville, les 21, 22 et 24 août 1853, dans le quartier Saint-Esprit qui, étant situé sur la rive droite de l'Adour, dépend à cette époque du département des Landes. Ces premières courses déplacent la presse parisienne qui couvre l'événement. Il convient d'être précis sur les conditions d'apparition de la corrida espagnole en France, tant les interprétations simplistes déforment les faits. Le quartier Saint-Esprit organisait déjà depuis de nombreuses années des courses de taureaux pour ses fêtes et en 1852, pour la première fois une équipe de toreros espagnols venus de Guipúzcoa alterna avec les représentants habituels du jeu landais ${ }^{43}$. Ces courses sont qualifiées d'hispano-landaises et connaissent un certain succès pendant près d'un demi-siècle. Cette même année 1852, de nouvelles courses identiques

\footnotetext{
${ }^{42}$ S. CARVALHo, « As tradiçoes, as leis e a constituição deveriam ser para manter e cumprir ».

${ }^{43}$ A. Lafront, Histoire de la corrida en France, pp. 18-19.
} 
sont programmées, cette fois conclues par la mise à mort de six taureaux. Animée par l'ambition de mieux faire l'année suivante, la commission des fêtes de Saint-Esprit traite avec un imprésario navarrais lui accordant d'organiser des courses moyennant une redevance. À peine l'accord signé, le Ministre interdit les courses. Le conseil municipal envoie, contre l'avis du préfet, deux délégués à Paris pour solliciter le retrait de cette mesure. Sans qu'on puisse le prouver ni le démentir, il est probable que le Ministre se laissa infléchir, pensant être agréable à la nouvelle impératrice Eugénie de Montijo, en accordant finalement une autorisation de dix ans. La famille impériale, qui choisit Biarritz comme résidence d'été, assista aux corridas en 1856 et 1857 et d'une manière générale contribua, pendant le Second Empire, à rendre l'Espagne à la mode.

En 1853, la même année que les premières corridas de Bayonne, une cuadrilla espagnole emmenée par le modeste torero-entrepreneur Basilio González se produit à Nîmes, Arles, Marseille, Avignon offrant un spectacle très éloigné de l'orthodoxie taurine, qui intégrait en deuxième partie des protagonistes du jeu camarguais et des jeux participatifs. Jusque dans les années 1880, mises à part Bayonne qui de 1853 à 1862 organise des corridas intégrales puis Nîmes et Mont-de-Marsan en 1865, les courses inspirées de la tauromachie espagnole qui se développent en ces années-là possèdent un caractère très hétérodoxe. En témoignent les pérégrinations du gaditan Pablo Mesa, à partir de 1866, qui exportent un spectacle taurin des plus syncrétiques, à Périgueux, Agen, Poitiers, puis au Havre. À partir des années 1880, une dizaine de villes du Sud de la France commencent à programmer des courses de plus en plus approchantes des canons espagnols et avec une plus grande régularité : Nîmes, Bordeaux, Mont-de-Marsan, Dax, Toulouse, Cauterets, Céret, Béziers, Montpellier, Avignon, Arles, Beaucaire et Marseille. C'est aussi le cas de Bayonne, à partir de 1885, après avoir connu une interruption qui durait depuis 1862. Ajoutons à cette liste, des arènes montées à Paris à partir de 1889, baptisées « La Gran Plaza de Toros de Boulogne ", édifiées près de la rue Pergolèse. Elles contenaient 22.000 places et offrirent, de 1889 à 1893, un total de 129 courses de taureaux sans pique et sans mise à mort. La première année qui accueillit de grands toreros tels que Guerrita, Frascuelo, Lagartijo, Mazzantini, fut un succès. Les suivantes, sans la présence de grandes figures, furent un échec qui mit en faillite l'entreprise. À partir des années 1892-1893, la mise à mort des taureaux prend de l'importance dans les villes du Sud, alors même que les protestations s'élèvent en vertu de la violation de la loi Grammont. En ces mêmes années, apparaît dans le Sud-Est un spectacle appelé « courses de quadrille », création du français Étienne Boudin dit le « Pouly » de Beaucaire, intégrant des techniques de jeux camarguais, espagnols et landais, s'achevant par un simulacre de mise à mort.

L'introduction chaotique, mais néanmoins progressive de la corrida espagnole, entraîne une diversification des pratiques taurines et une pluralité de situations locales, plus ou moins en marge de la légalité. C'est la dimension politique prise par les courses de taureaux à partir de 1894 qui constitue le grand tournant historique de l'enracinement des corridas espagnoles en France. Depuis 1881, le pouvoir central s'inquiète de la propagation des courses de tau- 
reaux de tout type et rappelle aux préfets que les corridas espagnoles avec mise à mort sont condamnées par la loi. Les premiers conflits surviennent au début des années 1891 entre les préfets et les municipalités dont les maires sont élus depuis 1884 : échanges de lettres sans lendemain, promesses non tenues, menaces non suivies d'effet, arrêtés d'expulsion de toreros prononcés, tantôt respectés, tantôt différés au lendemain de la corrida, préfets indifférents. Le manque de fermeté du pouvoir central profite aux amateurs de corridas jusqu'à ce qu'en 1894, le président du Conseil Charles Dupuy interdise la dixième corrida de la saison nîmoise. Les villes de Bayonne et de Mont-de-Marsan encouragent Nîmes à résister : cette dernière organise alors une corrida de protestation en compensation de celle qui a été supprimée. Une foule nombreuse se réunit et Frédéric Mistral, figure de proue du Félibrige, accepte de siéger à la tribune d'honneur à côté du maire. La fronde des villes taurines méridionales est lancée, se transformant rapidement en une rivalité politico-territoriale entre Paris et le Midi. L'arrêt de la cour de Cassation de 1895 confirmant l'application de la loi Grammont aux corridas n'y fait rien. Les conflits se multiplient, les lois sont partout bafouées sans que le pouvoir central ne parvienne à endiguer la popularité d'un spectacle qui participe désormais de l'affirmation d'une identité régionale. C'est le cas à Caudéran (Bordeaux) en 1896 où la corrida prend fin au cri de «Vive le Midi, vive ses libertés! », repris en cœur par la foule réclamant La Marseillaise. Cette période voit se multiplier les expériences d'exportation de la corrida en dehors de la zone méridionale où les jeux taurins s'étaient renforcés au cours des $\mathrm{XVII}^{\mathrm{e}}$ et XVIII ${ }^{\mathrm{e}}$ siècles. Citons les expériences lointaines de Lyon en 1894, Dijon en 1899, Limoges en 1897 et 1899, Roubaix de 1899 à 1901, mais aussi des tentatives plus proches des foyers taurins, à Bagnères-de-Luchon de 1899 à 1907, ou à Casteljaloux (Lot-et-Garonne) de 1896 à 1908. Aucune de ces expériences n'a entraîné un enracinement local de la corrida espagnole qui finalement ne déborde guère l'aire d'extension des jeux taurins préexistants. Cela explique sans doute qu'une tolérance de fait ait fini par s'établir.

S'il faut effectivement replacer l'histoire de l'introduction de la corrida en France dans un mouvement progressif d'une quarantaine d'années, qui n'a jamais ressemblé à une implantation fulgurante, il n'en demeure pas moins qu'on assiste au développement d'une adhésion grandissante de la part des populations locales, sans laquelle il serait difficile d'expliquer les nombreux épisodes qui témoignent d'un rapport de force avec le pouvoir central. Sur ce point, l'argumentation d'Élisabeth Hardouin-Fugier nous semble contradictoire. Elle affirme que dans les années 1860-1870, il existe un «profond désintérêt de la population méridionale pour la corrida espagnole, voire une opposition $»^{44}$. Pour cette période, cette lecture peut encore être l'une des interprétations possibles d'une introduction lente et chaotique de la tauromachie espagnole qui dure jusqu'au début des années 1880. Mais elle affirme, à la même page, que « depuis la loi du 5 avril 1884, les maires sont élus localement » et que «soumis à la pression

\footnotetext{
${ }^{44}$ É. Hardouin-Fugier, Histoire de la corrida en Europe, p. 136.
} 
électorale locale, ils sont pris en otage entre leurs électeurs et le pouvoir central » et d'ajouter : " telle pourrait être une des explications du laxisme gouvernemental à venir envers les corridas $»^{45}$. Par qui les maires seraient-ils donc soi-disant " pris en otage ", si cette « longue hostilité méridionale à la corrida (18531894) » était avérée ? De quelle « pression électorale » s'agirait-il, s'il existait un tel «profond désintérêt de la population méridionale, voire une opposition »? Pourquoi alors ne pas reconnaître simplement qu'à partir des années 1880, qui correspondent à la phase de croissance historique de la tauromachie espagnole, les populations locales s'y intéressent de plus en plus? La contradiction de l'argumentation aurait pu passer pour un point de détail si la responsabilité n'était pas renvoyée « aux immigrés espagnols, déjà très nombreux dans la France du Sud, [qui] ont largement soutenu de leur présence la poussée économique des imprésarios espagnols à la conquête du débouché français », comme s'ils avaient alors constitué le seul public fervent et les seuls instigateurs de la tauromachie. Il n'est pas question de remettre en cause l'importance de l'immigration espagnole dans le Sud de la France qui fut le fruit d'un exode économique à partir du XIX ${ }^{\mathrm{e}}$ siècle, ni de minimiser leur participation réelle au succès des corridas. En outre, nous convenons avec l'auteur que cette période représente une mise à l'épreuve de la loi Grammont face à laquelle le gouvernement n'a pas su faire respecter la loi au sujet des corridas, pourtant clarifiée en 1895. Cela étant, les propos nous semblent on ne peut plus contestables, lorsqu'il est précisé, pour clore le chapitre, que la loi Grammont « arrachée depuis trop peu de temps à un anthropocentrisme millénaire, [est] habilement exploitée par des Espagnols jadis conquistadors $»^{46}$. Cette phrase met un terme à une partie intitulée, dans le même ordre d'idée, «Les conquistadors à l'assaut de l'or rouge français ${ }^{47}$. Et que dire de l'évocation d'une corrida organisée à Enghien en 1900, quand l'auteur déclare que "le public est composé de bouchers et d'hispanophones ", tant les termes choisis paraissent d'eux-mêmes disqualifier le propos ${ }^{48}$. Quelle que soient les opinions personnelles des chercheurs qui s'intéressent à la corrida, opinions éminemment respectables dans leur diversité, elles ne méritent pas d'entacher l'analyse critique d'un ouvrage scientifique, sauf à considérer que telle n'est pas exclusivement l'ambition de cet ouvrage.

\section{LA STABILISATION}

DE L'ESPACE TAUROMACHIQUE

Les évolutions du $\mathrm{xx}^{\mathrm{e}}$ siècle marquent globalement la stabilisation de l'espace tauromachique à l'intérieur duquel les pratiques continuent d'évoluer. En

\footnotetext{
${ }^{45}$ Ibid., p. 136.

${ }^{46}$ Ibid., p. 147.

${ }^{47}$ Ibid., p. 132.

${ }^{48}$ Ibid., p. 144.
} 
Espagne, le «Désastre » de 1898 que constitue la perte des dernières colonies (Puerto Rico, Cuba, Les Philippines) plonge le pays dans une profonde crise politique et morale. De nombreux écrivains et intellectuels, réunis sous le nom de " génération de 98 ", dénoncent les maux d'un pays qui, à leurs yeux, est archaïque et déchu. La tauromachie ne leur inspire généralement qu'aversion et d'aucuns observent que le jour même où la flotte espagnole coule sous le feu des navires nord-américains, la foule insouciante n’a d'autre préoccupation que d'applaudir le torero «Guerrita » aux arènes de Madrid ${ }^{49}$. Les critiques nombreuses des tribunes anti-taurines de la fin du Xix ${ }^{\mathrm{e}}$ siècle ne faiblissent pas, alors même que les courses connaissent une légère récession dans la décennie 1900, après 20 ans de forte croissance de la tauromachie. Mais de 1913 à 1920 s'imposent en Espagne deux toreros « Joselito » et Juan Belmonte dont la confrontation devait révolutionner l'art taurin et transformer le regard des élites à l'endroit d'un spectacle décrié en maintes occasions. Tous les spécialistes taurins considèrent généralement que Joselito fut le parfait aboutissement d'un siècle de tauromachie classique et Belmonte fut la figure révolutionnaire d'une nouvelle esthétique. Il est souvent répété que Belmonte substitue au toreo de jambe, exigeant de grandes qualités athlétiques, un toreo de bras, exigeant une parfaite maitrise de la muleta pour parvenir non plus seulement à détourner la charge du taureau, mais à la conduire. Belmonte lui-même définit cette nouvelle conception de l'art taurin : "J'ai conçu le toreo comme l'antithèse de la lutte, de la brusquerie, de la violence, de la rapidité. Pour moi - ce "moi" artistique que nous possédons tous au-dedans de nous-mêmes, que certains parviennent à extérioriser et d'autres non - j'ai éprouvé le toreo comme cadence, rythme, suavité, lenteur $»^{50}$. Ce tournant marque une esthétisation des valeurs de la tauromachie recentrée sur le travail artistique de la muleta.

À propos de ce long processus de professionnalisation et de transformation de la tauromachie, l'anthropologue Manuel Delgado Ruiz juge très révélateur le souci constant de vouloir en perfectionner la valeur artistique ${ }^{51}$. Considérer la tauromachie comme un art et non comme une fête, si l'on admet que l'art concerne d'abord le plaisir esthétique et intériorisé de celui qui en jouit, c'est l'assimiler à une activité qui n'est pas immédiatement fonctionnelle au niveau collectif. Selon l'auteur, en s'assurant d'une meilleure reconnaissance artistique auprès des élites, la tauromachie professionnelle perd en grande partie la fonction sociale des festivités taurines populaires. On assiste alors à une désactivation du rite tauromachique premier. Nous pensons que la connivence toujours présente entre la fête et la tauromachie traduit peut-être la volonté de conserver ou de recréer a posteriori certaines vertus des jeux traditionnels participatifs. Ces vertus sont celles qui, selon Norbert Elias et Éric Dunning, sont à l'opposé des principes fondateurs du sport moderne qui émergent à la fin du xix ${ }^{e}$ siècle

\footnotetext{
${ }^{49}$ C. Serrano, «Entre l'amour et la réprobation des siens », p. 72.

${ }^{50}$ Cité par B. Bennassar, Histoire de la tauromachie, p. 73.

${ }^{51}$ M. Delgado Ruiz, «El toreo como arte o cómo se desactiva un rito ».
} 
et dont la caractéristique est précisément de n'avoir « ni fonction rituelle, ni finalité festive $»^{52}$.

Il est évident que cette évolution esthétisante prépare aussi la transformation que constitue en 1928 , le port obligatoire du caparaçon, à l'essai à Nîmes depuis le début du siècle, et imposé par Primo de Rivera en Espagne, pour que les chevaux cessent d'être éventrés en piste sous la charge des taureaux. Mais les milieux artistiques et intellectuels de l'époque n'ont pas attendu 1928 pour exalter les valeurs de la tauromachie, courant qui, loin d'être unanime, constitue néanmoins un retournement historique important. La génération 27, García Lorca, Alberti, Valle-Inclán, Bergamín, rompt avec la tradition d’un élitisme éclairé jugeant de ce qui convient au peuple, par la mise en valeur du génie propre des créations populaires auquel sont associés la tauromachie et le flamenco ${ }^{53}$. D'un point de vue spatial, on peut penser que la révolution belmontiste entraîne une légère densification du réseau d'arènes, mais ne modifie pas une géographie qui est établie pour l'essentiel. Pour voir une augmentation significative du nombre d'arènes et de spectacles en tout genre, il faut attendre le développement du tourisme dans les années 1960, puis les années 1980, contrairement à une idée reçue qui fait de cette dernière période une époque de crise de la tauromachie.

Au Portugal, tout se passe comme si chaque mutation importante de l'univers tauromachique espagnol trouvait un écho de l'autre côté de la frontière. Avec un décalage chronologique par rapport à l'Espagne, la décennie 1920 correspond à une période de croissance du nombre de spectacles et de constructions de nouvelles arènes en Estrémadure et en Alentejo. Ces années sont également considérées comme un âge d'or de la tauromachie nationale après la décennie antérieure, plutôt placée sous le signe d'une décadence de l'art taurin. En 1921, un premier décret interdit la mise à mort des taureaux. Cette coïncidence temporelle laisse penser que le nouvel âge d'or de la tauromachie espagnole, suscité par la révolution belmontiste, n'a pas laissé indifférent les amateurs portugais, les autorités ayant plutôt tendance à vouloir freiner cet engouement. Le décret n'est pas véritablement respecté ou de façon très aléatoire principalement par manque de sanctions dissuasives. Mais dans le même temps, la confrontation de João Branco Núncio et Simão da Veiga renouvelle l'esthétique de la corrida portugaise en des termes comparables à ce que représente la rivalité entre « Joselito » et Belmonte pour l'Espagne. En outre, ces deux cavaliers ont fréquemment toréé de l'autre côté de la frontière, dans les années 1920-1930, à l'époque où se développe à nouveau en Espagne une tauromachie équestre, sous l'impulsion du capitaine de cavalerie cordouan Antonio Cañero. En Espagne, Antonio Cañero est volontiers considéré comme le père fondateur du rejoneo espagnol, qui se serait " réfugié » dans le campo andalou depuis que la noblesse a cessé de le pratiquer $^{54}$. Cette version de l'histoire taurine n'insiste guère sur le rôle tenu par

\footnotetext{
${ }^{52}$ N. Elias et E. Dunning, Sport et civilisation, p. 15.

${ }^{53}$ C. Serrano, «Entre l'amour et la réprobation des siens », p. 74.

${ }^{54}$ J.-M. de Cossío, Los Toros, t. I, p. 319.
} 
les cavaliers portugais en ces années 1920-1930, qui obtiennent de nombreux contrats sur le territoire espagnol. Les écrits de l'époque montrent que cette rivalité trouve un point d'orgue lorsque la tauromachie à cheval espagnole est « réinventée ». En 1927, le critique taurin portugais Pepe Luis compile dans son ouvrage Touros de morte em Portugal toute une série de commentaires qui accablent le «pseudo cavaleiro toureiro D. Antonio Cañero », traité de "Capitaine Fracaso » s'illustrant par la pantomime ${ }^{55}$. L'auteur accuse Cañero de dégrader la grandeur de la Festa Nacional (portugaise) et va jusqu'à le rendre en partie responsable de la menace d'interdiction stricte et totale de la mise à mort qui plane au milieu des années 1920, tant ses prestations sont critiquables. Il répond en cela aux arguments des Espagnols qui affirment à l'inverse que "Cañero représente le torero à cheval dans toute son arrogance, son authentique majesté et son traditionnel flamenquisme ", comparé au cavalier portugais Simão da Veiga "professeur chargé de grave et froide suffisance $»^{56}$. En 1928, l'année où est imposé en Espagne le caparaçon pour protéger les chevaux, la loi portugaise interdit de façon définitive la mise à mort publique des taureaux.

En présence d'un symbole social aussi distinctif que l'homme à pied ou l'homme à cheval, et d'un symbole politique aussi discriminant que l'autorisation de mise à mort ou son interdiction, les arguments sont nombreux pour penser en termes d'irréductible opposition les différences entre la tauromachie espagnole et la tauromachie portugaise. Cette opposition est effectivement devenue une image courante des représentations collectives. Dans les années 1950, José María de Cossío y voyait le résultat d'une rivalité historique entre les deux nations et mettait en avant le besoin " obsessionnel» des Portugais de se différencier de l'Espagne ${ }^{57}$. L'« obsession » n'est peut-être pas le mot juste, mais cette mise en relation vaut sans doute mieux que les nombreuses théories essentialistes qui font de la tauromachie le reflet du caractère national, sans aucune prise en compte de l'altérité. L'analyse de Frédéric Saumade observe que la tauromachie portugaise (tourada) et la tauromachie espagnole (corrida) s'opposent en termes de techniques d'affrontement, de représentation du taureau, d'origine sociale des officiants et d'évolution des "dynamiques spectaculaires » et de conclure « qu'avec les codes qui la caractérisent, la tourada peut être comprise comme une éclatante mise en scène des ancestrales relations conflictuelles entre les deux nations ibériques $\aleph^{58}$. Nous montrons plus loin, ainsi que nous l'avons proposé dans un article détaillé, qu'à la tauromachie comme expression des rivalités nationales s'ajoute aussi celle d'une commune appartenance à la péninsule Ibérique ${ }^{59}$.

En France, la diffusion de la corrida espagnole à partir des années 1850 contribue à la refonte des jeux locaux et à leur modernisation en spectacle

\footnotetext{
${ }^{55}$ Pepe Luis, Touros de morte em Portugal, p. 43. Le nom commun fracaso, en espagnol, signifie échec, dont l'homonymie évoque le célèbre héros de Théophile Gautier.

${ }^{56}$ Pepe Luis, Touros de morte em Portugal, p. 51.

${ }^{57}$ J.-M. de Cossío, Los Toros, t. IV, p. 35.

${ }^{58}$ F. SAUMAde, Les tauromachies européennes, p. 48.

${ }^{59}$ J.-B. MAUDET, « Tauromachie et géopolitique en péninsule Ibérique ».
} 
tauromachique à part entière. Pour Auguste Lafront, la période de l'Entre-deuxguerres est une phase de consolidation de la corrida espagnole, malgré la guerre d'Espagne et les fluctuations houleuses des cycles économiques. Cette consolidation favorise une légère extension de la géographie taurine au début des années 1920, avec l'ouverture de nouvelles arènes à Toulouse (1921), Béziers (1921) et Céret (1922). Le Franc Poincaré et la baisse de la peseta au début des années 1930 relancent à nouveau le nombre de courses en raison d'un équilibre des termes de l'échange favorable à la France. Malgré les transformations qualitatives importantes liées à la révolution belmontiste et l'adoption du caparaçon, ces évolutions ne transforment pas de façon fondamentale la trame taurine existante. Si la trame taurine ne subit que quelques retouches, les jeux taurins régionaux dont la pratique s'était diversifiée au contact du jeu espagnol se modernisent. Après une période de mixité des pratiques (courses hispano-provençales, courses hispano-landaises), qui n'a pas empêché dans le même temps une superposition des corridas espagnoles, des courses mixtes, des jeux landais et des jeux camarguais, les formes taurines méridionales finissent par s'individualiser.

Selon Frédéric Saumade, cette individualisation des tauromachies européennes répond à une logique structurale d'opposition des codes sexuels, ludiques, eschatologiques et sociologiques qui témoignent d'un processus historique de diffusion de la corrida andalouse, hors de son foyer d'origine. Dans cette organisation générale, la course camarguaise prend le contre-pied des codes tauromachiques espagnols en ce qui concerne les techniques, le rôle et la place des officiants, ou encore le sort, le statut, la race et les qualités recherchées du taureau idéal. La course landaise, elle aussi en opposition avec de nombreux aspects de la corrida espagnole, lui emprunte néanmoins certains traits : le lexique, du bétail pour effectuer des croisements, le gilet des toreros. Elle cultive néanmoins des particularismes remarquables comme la réintroduction de la corde en tant qu'élément du jeu d'arène. À propos de la course landaise, les analyses de Bernard Traimond rejoignent celles de Frédéric Saumade, lorsqu'il développe un système d'oppositions techniques, sociales et symboliques, entre la corrida espagnole et la course landaise, tout en faisant de la course landaise " une adaptation de la mode des jeux taurins selon le modèle espagnol, aux savoirs, aux moyens financiers et aux possibilités techniques et culturelles chalossaises et armagnacaises $»^{60}$.

Historiquement, insistons sur le caractère progressif des transformations des courses régionales, sur les différents rythmes de leur modernisation, sur les réponses distinctes à la diffusion de la corrida et sur des évolutions qui obéissent aussi à des raisons propres de la société locale. Le terme "écarteur » (landais) est utilisé dès le Premier Empire alors que le terme de raseteur n'apparaît qu'en 1883 dans la presse ${ }^{61}$. Le mot écarteur remplace alors le terme de "jouteur ", fréquemment utilisé jusque dans les années 1820 dans Le journal des Landes et

\footnotetext{
${ }^{60}$ B. Traimond, Les fêtes du Taureau, p. 11.

${ }^{61}$ Ibid., p. 10.
} 
la Gazette des Landes ${ }^{62}$. Le terme de course « landaise » daterait d'une confrontation avec les spectacles camarguais, lors des Fêtes du Soleil organisées en 1887 à Paris $^{63}$. Le terme de course " camarguaise » n'apparaitt qu'en 1970, un siècle plus tard, remplaçant celui de course "libre ", utilisé depuis le milieu du $\mathrm{XIX}^{e}$ siècle pour la différencier des jeux pratiqués avec des taureaux encordés ${ }^{64}$. La Fédération de la course landaise est créée en 1953 et agréée par le Ministère en 1973. Celle de la course camarguaise est créée en 1975. Dans la course landaise, les premières transformations notables d'un point de vue technique fixant des normes gestuelles se font sentir à partir des années 1820, avant l'introduction en France de la corrida espagnole. La feinte en tant que technique codifiée serait apparue en 1831 et aurait remplacé toute une série de gestes et de techniques d'affrontement les plus divers : maittriser les animaux pour les seller, pour les monter, pour les mettre à terre, exécuter un " paré ", pratiquer "l'aiguillade ", poser des « lances " (banderilles) et lâcher les chiens ${ }^{65}$. Entre 1821 et 1886, les feintes, les écarts et les sauts deviennent les principales techniques d'affrontement du bétail, débarrassées des autres modalités du combat. Dans les années 1850 , les premiers croisements avec du bétail ibérique sont effectués. Il est rapporté, par l'histoire officielle, que les croisements commencent en raison du succès obtenu par Jean Chicoy à Magescq qui eut le courage d'écarter les taureaux navarrais ayant servi en 1852 lors des fêtes de Saint-Esprit dont nous avons parlé6. Enfin, si la corde apparaît dès le XviII ${ }^{e}$ siècle pour tenter d'éviter des blessures très nombreuses, l'épointage des cornes n'apparaît qu'au milieu du XIX ${ }^{e}$ siècle. D'abord ne sont sciées que les pointes des cornes des animaux jugés trop dangereux, mais l'intérêt du jeu semble alors décroître aux yeux du public $^{67}$. Vers 1885 , on cesse de scier les pointes pour protéger la corne par une boule de cuir. Cet usage est d'abord réservé aux bêtes ayant déjà tué, puis il se généralise en 1905 pour le bétail jugé particulièrement dangereux ${ }^{68}$. En 1905, en raison de ces transformations, le journal spécialisé La Course Landaise annonce en gros titre : «la course landaise est finie ${ }^{69}$. Dans les années 1880, la course landaise s'exporte dans les arènes espagnoles, ou tout au moins, on retrouve certains de ses acteurs dans le Nord du pays, à Saint-Sébastien, à Saragosse et même à Madrid en 1887.

\footnotetext{
62 Les Amis du musée de la vie rurale en Pays landais, La course landaise, jeu-spectacle traditionnel, pp. 5-7.

${ }^{63}$ B. Traimond, Les fêtes du Taureau, p. 10.

${ }^{64}$ F. SAumade, Les tauromachies européennes, p. 151.

${ }^{65} \mathrm{P}$. Bordes, La course landaise, p. 17. Le paré consiste à attendre la bête lancée pour lui faire détourner la tête au moment attendu de l'impact. L'aiguillade est le harcèlement du bétail à l'aide d'un batôn pointu. M. DAugA, " La course landaise et le musée de Bascons », p. 26.

${ }^{66}$ Les Amis du musée de la vie rurale en Pays landais, La course landaise, jeu-spectacle traditionnel, p. 8.

${ }^{67}$ M. Dauga et J.-Y. PuYo, « Les écarteurs landais entre mythe et réalité », p. 192.

${ }^{68}$ Ibid., p. 192.

${ }^{69}$ Ibid., p. 200.
} 
Les recherches menées par Marylis Dauga ont montré qu'à partir des années 1880 , les courses s'exportent en dehors du «territoire historique » des jeux landais, correspondant au Sud des Landes et à l'Ouest du Gers. L'extension se déroule principalement dans les départements limitrophes de la Gironde, du Lot-et-Garonne et des Hautes-Pyrénées, puis ponctuellement dans une série de villes plus éloignées : Orléans, Paris, Limoges, Nantes, Rennes, Vichy, Aixles-Bains, Grenoble, Lyon, Laval, Rennes, Montpellier, Collioure, Céret ${ }^{70}$. Ce mouvement se rétracte au tournant du siècle. Nous pensons, comme pour les corridas, qu'il est lié à une curiosité diffuse pour les jeux taurins en général, qui faiblit rapidement en dehors des zones d'ancienne tradition.

Pour ce qui est des courses camarguaises, les croisements avec du bétail ibérique commencent en 1869, à l'initiative de l'éleveur Joseph Yonnet, et se généralisent chez les manadiers provençaux et seulement quelques manadiers languedociens ${ }^{71}$. Les techniques taurines semblent fixées avant la fin du XIX $^{\mathrm{e}}$ siècle entre les années 1893 et 1897 , en partie sous l'influence du poète, manadier et marquis Folco de Baroncelli ${ }^{72}$. La défense de la " race d'Oc » et de ses coutumes, dans le sillage du Félibrige, contribua au retour en grâce des taureaux de $"$ pur-sang Camargue ${ }^{73}$. C'est aussi à cette époque que les raseteurs deviennent des professionnels, différenciés des simples participants, par l'adoption de la tenue blanche et du crochet ${ }^{74}$.

$\mathrm{Au}$ début $\mathrm{du} \mathrm{xx}^{\mathrm{e}}$ siècle, les trois courses taurines en France sont fixées dans leur forme et leur statut de spectacle commercial moderne, de même que les limites géographiques de leur extension. Il est fort probable que la tolérance croissante dont bénéficient les spectacles landais et camarguais, à partir des années 1870-1880, soit due, au moins en partie, à la cristallisation autour de la corrida des contestations issues du pouvoir central et des associations de protection des animaux. Dans le Sud-Ouest européen, il semble bien que l'existence de plusieurs foyers tauromachiques, leur relative proximité spatiale dans un territoire de circulation ancien, et l'affirmation d'une forme moderne conquérante proposant une synthèse entre culture savante et culture populaire, aient contribué à les renforcer toutes.

\section{II. — LA FORMATION}

\section{DE L’ESPACE TAUROMACHIQUE AMÉRICAIN}

La question de la formation de l'espace tauromachique américain ne se pose pas dans les mêmes termes que celle de l'espace tauromachique européen. La

\footnotetext{
${ }^{70}$ M. DAUGA, 1965-1985 : La course landaise entre tradition et modernité, pp. 24-27.

${ }^{71}$ F. SAUmade, Les tauromachies européennes, p. 158.

${ }^{72}$ Ibid., p. 158. Pour les dates É. Duret, « La course camarguaise, aspects historiques ", p. 76, d'après le témoignage du manadier Marcel Mailhan.

${ }^{73}$ F. SAUmade, Les tauromachies européennes, p. 159.

${ }^{74}$ Ibid., p. 159.
} 
raison tient tout entière dans le simple fait qu'il n'existe pas de bovins domestiques, ni de chevaux, avant la conquête du Nouveau Monde par les Européens ${ }^{75}$. Les pratiques tauromachiques du continent américain ont donc toutes comme substrat la conquête du Nouveau Monde, les fêtes taurines importées de la péninsule Ibérique et les jeux dérivés de l'élevage bovin extensif, quand bien même ces pratiques intègrent des principes et des représentations préexistant à la conquête. Afin de fixer les principaux éléments qui président à la formation de l'espace tauromachique américain, observons l'introduction du bétail dans le Nouveau Monde, l'introduction des jeux taurins par la Conquête, la diffusion de la corrida moderne, et l'apparition des rodéos.

La genèse des jeux taurins en Amérique racontée par les adeptes de rodéo ou de la course de taureaux suit deux voies différentes. Les amateurs de corridas insistent généralement sur les premiers bovins débarqués avec les conquistadors, ceux que Christophe Colomb introduit sur l'île Española (actuelle Saint-Domingue) lors de son second voyage en 1493, puis sur les premières courses de taureaux aristocratiques données une trentaine d'années après, enfin sur une histoire régénérée par l'arrivée des premières cuadrillas espagnoles au $\mathrm{XIX}^{e}$ siècle. Les amateurs de rodéo, qu'il s'agisse des charreadas au Mexique, des toros coleados en Colombie et au Venezuela, des vaquejadas au Brésil ou du rodeo chileno au Chili insistent généralement, non pas sur les premiers bovins introduits, mais sur les haciendas d'élevages déjà constituées. Et, dans le cas des rodéos pétris d'esprit aristocratique que sont la charreada et le rodeo chileno, il est généralement fait référence à l'introduction des premiers chevaux de race par les conquérants. Au Mexique, les chevaux de la charreada sont ainsi placés dans la filiation des 14 chevaux débarqués par Hernán Cortés en 1519. Au Chili, les chevaux du rodeo chileno sont les descendants des 75 chevaux arrivés avec le conquistador Pedro de Valdivia en 1540, qu'il ne faut bien sûr pas confondre avec ceux introduits par Mendoza en Argentine quatre ans plus tard. Entre l'histoire officielle des rodéos et celle des courses de taureaux, bien peu de recoupements sont établis. Pourtant, des imbrications et un passé commun existent avant que les bifurcations entre corrida et rodéo ne s'affirment dans le cadre d'histoires nationales.

\section{L'introduction du bétail dans le Nouveau Monde}

Parmi les premières recherches sur l'introduction du bétail dans le Nouveau Monde figurent celles du géographe Pierre Deffontaines, dans les années 1950, qui en a mesuré toute l'importance pour l'histoire du continent. L'article intitulé "L'introduction du bétail en Amérique Latine », publié en 1957, est une source d'informations d'une grande valeur pour comprendre la formation de l'espace

\footnotetext{
${ }^{75}$ Le continent ne connaissait pas le cheval, même si un ancêtre a existé pendant la préhistoire, et ne connaissait que des espèces sauvages de bovidés, en Amérique du Nord : le bison des Plaines et le bœuf musqué du Nord canadien. X. de Planhol, Le paysage animal.
} 
tauromachique ${ }^{76}$. Pierre Deffontaines commence par citer un proverbe cher à «l'Espagnol, homme du cheval », qui caractérise une " psychologie » du cavalier méprisant la vie du piéton laboureur : " on ne touche à la terre que quand on est mort ${ }^{77}$. Cette caractéristique distingue fondamentalement la colonisation espagnole de celle menée par les colons en Amérique du Nord.

Les premiers centres de reproduction des chevaux pour répondre aux besoins de la conquête apparaissent dès le début du XvI ${ }^{\mathrm{e}}$ siècle, d'abord dans les grandes Antilles, au Nicaragua, à Santa Marta en Colombie, puis près de Quito, de Lima, autour de Tucumán et de Santiago du Chili, qui, dès le Xvir ${ }^{e}$ siècle, est l'une des meilleures régions d'élevage de chevaux et de mulets. En un siècle, ne pas posséder un cheval est considéré comme la preuve de la plus grande pauvreté, tant cet animal est devenu bon marché et omniprésent sur le continent. Ajoutons qu'officiellement les Indiens n'ont pas le droit de monter à cheval. La place du cheval distingue l'Amérique espagnole de l'Amérique du Nord anglo-française qui est plutôt le continent de la navigation fluviale et aussi de l'Amérique portugaise restée fidèle au cabotage et au développement, sur la terre ferme, des chars à bœufs. En revanche, malgré des stratégies différentes d'occupation du Nouveau Monde, Espagnols et Portugais pratiquent dès qu'ils le peuvent des « lâchers de bêtes " qui étaient d'ailleurs rendus obligatoires par les règlements de navigation. Cet acte constituait une forme d'occupation du sol, de prise de possession par le bétail, d'appropriation territoriale par les animaux, avant même l'installation des hommes. Il a ainsi précédé les hommes dans la colonisation, leur présence conférant un droit de propriété par la preuve qu'on y a lâché des animaux Les quelque mille villes fondées en trois siècles dont plus de deux cents au terme du $\mathrm{XVI}^{\mathrm{e}}$ siècle n'auraient pu connaître un tel développement sans le bétail $^{78}$. Ce bétail permettait également un ravitaillement des escales maritimes comme le pratiquaient les Espagnols aux Canaries et les Portugais aux Açores, à Madères, aux îles du Cap Vert, au Cap, au Mozambique, en Angola, à Madagascar, à Goa. On a vu, dans certains cas, qu'il existe encore aujourd'hui des jeux taurins dans ces régions. De même, du bétail était progressivement abandonné sur le continent, au fur et à mesure de la progression, expliquant qu'en moins d'un siècle on trouve des bovins du Mexique au Chili et jusque dans les secteurs les plus reculés, tels les llanos de l'Orénoque, le Chaco paraguayen et les pampas argentines.

Pendant longtemps, la date de ces « lâchers » de bovins et leurs auteurs étaient commémorés. Les premiers lâchers eurent lieu en 1509, lors de la fondation de la ville de Darién près de Panamá, en 1516 en Colombie, en 1519 à Coro au Venezuela, en 1524 dans les llanos de l'Orénoque, et à partir de 1551 dans les Andes d'où les bêtes débordent rapidement vers le versant oriental, llanos de Bolivie, Chaco et pampa. Sur le littoral atlantique du Nord du Brésil, les lâchers

\footnotetext{
${ }^{76}$ P. Deffontaines, « L'introduction du bétail en Amérique latine ».

${ }^{77}$ Ibid., p. 5.

${ }^{78}$ C. Bataillon, J.-P. Deler et H. Théry, Amérique latine, pp. 11-12 ; C. Bernand et S. GruZINSKI, « La redécouverte de l'Amérique », p. 24.
} 
de bêtes commencent en 1530 à Olinda (Pernambuco), en 1531 à Bahia, en 1534 à São Vicente, mais la pénétration à l'intérieur des terres est plus lente. Dans le Sud du Brésil, l'introduction est plus tardive et provient probablement des animaux de la Banda Oriental (actuel Uruguay) en nombre important au $\mathrm{xVIII}^{\mathrm{e}}$ siècle $^{79}$. En Amérique du Nord, les travaux de François Chevalier ont montré la formation de grands domaines dans le Nord et le Nord-Ouest du Mexique à partir du $\mathrm{XvI}^{\mathrm{e}}$ siècle et leur lien avec le développement de l'activité minière ${ }^{80}$. Dans le Texas, qui appartenait alors à la Nouvelle-Espagne, la légende veut que l'un des premiers «lâchers de bêtes " ait été constitué des chevaux et des bovins de Francisco Vásquez de Coronado, à la recherche des Sept Villes d'or de Cibola, qui furent dispersés par un violent orage ${ }^{81}$.

Cette légende permet d'approcher une autre réalité fondamentale de l'introduction du bétail sur le continent américain : le retour des bêtes à l'état sauvage. Jean-Pierre Digard dans un article de 1992, intitulé « Un aspect méconnu de l'histoire de l'Amérique : la domestication des animaux », met en lumière toute l'importance des phénomènes de marronnage. L'auteur affirme que «l'histoire du bétail en Amérique (on pourrait même dire : l'histoire de l'Amérique ») n'aurait pas été ce qu'elle est sans le marronnage. Ce phénomène est à mettre en lien avec le développement de l'élevage bovin extensif dont les bêtes, qui se multiplient naturellement, sont mal contrôlées et s'échappent dès qu'un conflit ou des troubles désorganisent le régime de l'hacienda. Par exemple, au début des troubles qui agitent le Texas de 1830 à 1848, et qui aboutissent à son indépendance prise au dépens du Mexique, environ $80 \%$ du bétail des 100.000 têtes de la région retournent à l'état sauvage ${ }^{82}$. Le nouvel État hérite ainsi de l'un des plus grands troupeaux du continent. L'action de rassembler périodiquement les bêtes, le rodeo est alors le seul moyen de contrôler un tant soit peu le marronnage. Les Anglo-Saxons du Texas rebaptiseront la pratique cow hunt, littéralement la " chasse à la vache ", avant de remplacer cette expression par le round-up, plus proche du mot d'origine espagnol rodeo ${ }^{83}$. À côté des explications qui tiennent à la nature même des animaux et des milieux, le marronnage apparaît comme un phénomène typique de la colonisation pastorale des nouveaux mondes, dans un contexte de faible densité ${ }^{84}$.

Dans ce contexte, nous pensons que la re-domestication plus ou moins continue dans le cadre de l'élevage extensif participe d'un phénomène d'appropriation territoriale commun à l'ensemble du continent. N'oublions pas que le terme espagnol pour désigner le bétail, el ganado, vient de ce qui a été gagné, lo

\footnotetext{
${ }^{79}$ P. Deffontaines, «L'introduction du bétail en Amérique latine », pp. 11-13.

${ }^{80}$ F. Chevalier, La formation des grands domaines au Mexique, pp. 118-133.

${ }^{81}$ P. JACQUIN, Le cow-boy. Un Américain entre le mythe et l'histoire, p. 37. Les cités de Cibola sont des villes mythiques du Nord du Mexique dont la légende racontait qu'elles étaient construites en or. On notera que le nom Cibola vient d'un terme espagnol ancien, cíbolo, qui désignait les bisons.

${ }^{82}$ P. JACQUIN, Le cow-boy. Un Américain entre le mythe et l'histoire, p. 55.

${ }^{83}$ Ibid., p. 121.

${ }^{84}$ J.-P. Digard, « Un aspect méconnu de l'histoire de l’Amérique », p. 263.
} 
ganado. Les rodeos et les round-up dans leur version utilitaire sont des moments essentiels pour ce transfert de richesses qui, dans le même temps, affirme le pouvoir des possédants et leur emprise territoriale. Observons aujourd'hui que la plupart des régions où il existe de fortes traditions de spectacles de rodéo correspondent à d'anciennes contrées reconnues entre le $\mathrm{XVI}^{\mathrm{e}}$ et le XVIII $\mathrm{I}^{\mathrm{e}}$ siècle pour l'importance de leurs troupeaux de bovins et dont certaines le sont encore. Nous pensons que les spectacles du rodéo ne sont pas totalement dégagés de cette entreprise d'appropriation territoriale s'exprimant aujourd'hui par de nouveaux codes puisés dans les registres des fêtes sportives qui leur offrent le cadre social et spatial de leur continuité.

\section{Les JeUX TAURINS DE LA CONQUÊTE}

Les premières courses de taureaux n'apparaissent pas immédiatement avec la découverte du Nouveau Monde pour la simple raison que le bétail est d'abord une denrée protégée, afin de ne pas compromettre la multiplication des grands troupeaux nécessaires à la conquête et à la fondation des villes. Pour cette raison, les premiers taureaux tués sont comptés. Les jeux taurins à pied ont démarré en même temps que le toreo à cheval, l'absence de mise à mort systématique ayant pu contribuer à leur développement. Les premières courses de taureaux apparaissent autour de 1530 et se développent ensuite comme les réjouissances naturelles de la noblesse conquérante. Ils accompagnent d'autres divertissements tels que les courses de chevaux également très prisées. Avant que ne soient organisées les premières fêtes dans lesquelles sont inclus des jeux taurins, Argote de Molina rapporte en 1582 que les premiers animaux combattus sont les bovins marrons des Îles caribéennes chassés à l'aide des desjarretaderas (les demi-lunes $)^{85}$. Cette pratique existait en péninsule Ibérique où elle a pu être en vigueur du $\mathrm{Xv}^{\mathrm{e}}$ au début du $\mathrm{XIX}^{\mathrm{e}}$ siècle, confirmant une relation ancienne entre la chasse et la tauromachie dans les divertissements de l'aristocratie ${ }^{86}$.

La chronologie exacte des premières courses de taureaux du Nouveau Monde diffère quelque peu selon les auteurs. D'après Nicolás Rangel, la première corrida est celle du 13 août 1529 à Mexico en l'honneur de saint Hippolyte, qui est le jour de la chute de la capitale aztèque Tenochtitlán ${ }^{87}$. Francisco López Izquierdo retient l'année 1526 où des courses de taureaux auraient eu lieu pour célébrer le retour d'Hernán Cortés de Las Hibueras (actuel Honduras) ${ }^{88}$. Dès 1535, il est établi que la venue du vice-roi est célébrée par trois jours de corridas incluant la participation de 100 taureaux $^{89}$.

Voilà réunies les deux justifications, religieuse et politique, des premières courses de taureaux, qui tout en réjouissant les foules, participent à l'édifica-

\footnotetext{
${ }^{85}$ Cité par Á. López Cantos, Juegos, fiestas y diversiones en la América española, p. 159.

${ }^{86}$ F. Flores Arroyuelos, Correr los toros en España, p. 77.

${ }^{87}$ N. Rangel, Historia del toreo en México, p. 7.

${ }^{88}$ F. López-Izquierdo, Los Toros del Nuevo Mundo, p. 48.

${ }^{89}$ N. Rangel, Historia del toreo en México, p. 8.
} 
tion des populations récemment conquises. D’ailleurs, le nouveau pouvoir fait en sorte que l'ordre traditionnel autochtone assiste au spectacle ${ }^{90}$. La course de Mexico faisant d'emblée de la tauromachie un acte d'affirmation politique est rapidement suivie d'autres manifestations qui progressivement s'étendent aux différents centres et relais de pouvoirs de l'Amérique espagnole. En 1530, une corrida est organisée par l'autorité municipale de Santiago de los Caballeros au Guatemala pour célébrer le saint patron de la ville. S'ensuit une course de taureaux en 1532 à Acla, en Nouvelle-Grenade (actuelle Colombie), cinq ans avant la fondation de Santafé de Bogotá ${ }^{91}$. Puis les courses atteignent la viceroyauté du Pérou, dont la première se déroule le 29 mars 1540 à Lima, durant laquelle aurait combattu Francisco Pizarro en personne. C'est ce que soutient le grand intellectuel péruvien Ricardo Palma, alors même que le conquistador aurait été âgé de plus de 70 ans, si tel avait été le cas ${ }^{92}$. Il semble en réalité que ce fût son frère Fernando qui participa à la corrida. Quoi qu'il en soit, à partir de 1559, les corridas de Lima étaient fixées à quatre dates du calendrier : l'Épiphanie, la Saint-Jean, la Saint-Jacques (Santiago) et l'Ascension ${ }^{93}$. À partir de 1555 , les informations dont on dispose permettent de garantir que Santiago du Chili célèbre par des corridas la Saint-Jean, la Saint-Jacques et la Virgen de Agosto (Vierge d'août). À la même période, des courses sont organisées à Puerto Rico et à Cuba. Au rythme de la Conquête, des courses sont également données à Potosí en 1555, dix ans après les premières extractions d'argent du Cerro Rico, à la Paz en 1562, et à Villa Rica de Nirga, dans l'actuel Venezuela, le 20 janvier 1567, jour de la Saint-Sébastien pour se protéger des flèches des Indiens, avant même que ne soit fondée Caracas la même année. Des courses sont ensuite données à Córdoba en Argentine en 1573, et en 1609 à Buenos Aires ${ }^{94}$. La course au Venezuela donnée en vue de se protéger des flèches des Indiens est instructive en ce qu'elle montre l'apparition de corridas votives comme celles qui eurent lieu à La Havane en 1569 en l'honneur de saint Simon, consacré pour l'occasion saint intercesseur contre les fourmis qui avaient envahi la ville ${ }^{95}$.

L'histoire de l'implantation des courses de taureaux ne fonctionne pourtant pas de façon linéaire. De même qu'en Espagne, la bulle De salute gregis dominici de novembre 1567, promulguée par le pape Pie $\mathrm{V}$, a contribué à ralentir le développement des corridas dans les nouvelles extensions du royaume d'Espagne. Officiellement, elles furent interdites jusqu’à ce qu'en 1595, le pape Clément VIII revînt sur la bulle antérieure. Dans les faits, il n'y a guère que dans les capitales

\footnotetext{
${ }^{90}$ D. Fournier, « Corrida, charreada et jaripeo », p. 188.

${ }^{91}$ P. Rodríguez, « La fiesta de toros en Colombia », p. 165.

${ }^{92}$ H. López Martínez, Plaza de Acho, p. 23 ; Á. López Cantos, Juegos, fiestas y diversiones en la América española, p. 160.

${ }^{93}$ F. Iwasaki Cauti, « Toros y sociedad en la Lima colonial », p. 313 ; R. Palma, Tradiciones peruanas, p. 46.

94 Á. López Cantos, Juegos, fiestas y diversiones en la América española, p. 162 ; F. LópezIzQuierdo, Los Toros del Nuevo Mundo, p. 317.

${ }^{95}$ Á. López Cantos, Juegos, fiestas y diversiones en la América española, p. 161.
} 
des vice-royautés, Mexico (Nouvelle-Espagne) et Lima (Nouvelle-Castille) où elles furent empêchées. Dans le cas du Pérou, de nombreuses courses sont données à Cuzco en pleine période d'interdiction. Comme le montre la chronologie précédente, loin des vice-royautés et des archevêchés de Lima et de Mexico, l'interdiction n'est pas respectée. Une fois de plus la distance au pouvoir central est une donnée fondamentale pour comprendre la formation de l'espace tauromachique qui, dans ses périphéries, atteste toujours de quelques particularismes.

D'un point de vue technique, le toreo pratiqué par la noblesse en arme possède les mêmes caractéristiques que celui de la métropole, le rejón s'ajoutant à la pratique de la lanzada qui ici reste en vigueur plus longtemps. Très tôt, les fêtes aristocratiques incluent des lâchers de taureaux auxquels participent les populations locales. C'est ainsi que les indigènes deviennent rapidement des protagonistes actifs du jeu taurin. À Lima, ils participent aux corridas dès les années 1560. Les Indiens étaient alors connus pour planter sur les taureaux les moharras ou mojarras, des sortes de banderilles ${ }^{96}$. La technique consistait à attendre la charge du taureau, en restant assis ou couché au sol, afin de planter les banderilles à son passage. L'appellation d'Indiens mojarreros pour les désigner atteste d'un début de spécialisation de leur fonction, même si les témoignages de l'époque insistent surtout sur celle de s'enivrer le plus possible avant d'oser défier le taureau ${ }^{97}$. On peut remarquer que la technique actuelle des banderilleros des fiestas de corralejas du Nord de la Colombie correspond assez précisément à cette description. À Mexico, en 1554, dans une correspondance de l'archevêque à l'Empereur, il est fait allusion au nombre d'Indiens tués «comme des bêtes » pendant les courses de taureaux ${ }^{98}$. De même en 1597, un prélat de Guadalajara se plaint que les Indiens commencent à courir les taureaux régulièrement, ce qui laisse entendre un développement précoce de jeux taurins populaires, indépendants des fêtes de la noblesse ${ }^{99}$. L'historien Ángel López Cantos nous livre ensuite une information des plus intéressantes. Dans la région de San Miguel de Allende, on apprend qu'à la même époque des Indiens se sont spécialisés dans les jeux taurins et se désignent eux-mêmes lazadores, picadores ou avec d'autres surnoms qui soulignent une qualification technique ${ }^{100}$. En Nouvelle-Grenade (Colombie), sans que l'on sache ni pourquoi, ni dans quelles conditions, les Indiens Coyima, Natagaima et Ataco ainsi que les Noirs ont, à la même époque, acquis une certaine renommée dans les exercices taurins que ce soit à Santafé de Bogotá, Cali, Medellín ou Cartagena ${ }^{101}$.

Finalement, dans un premier temps qui pourrait être celui du Xvi ${ }^{e}$ siècle et du $\mathrm{XVII}^{\mathrm{e}}$ siècle, on observe ainsi une différence fondamentale entre la tauromachie européenne et la tauromachie américaine : l'intégration précoce des indi-

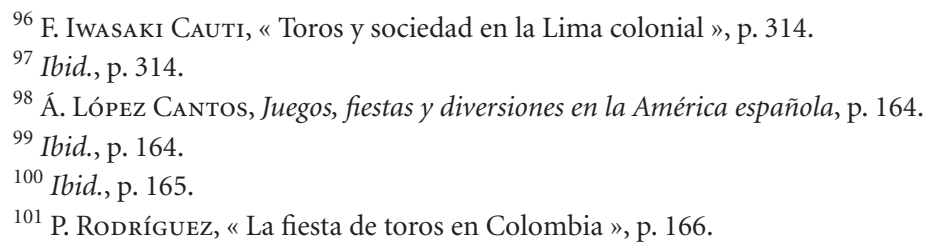


gènes aux jeux taurins de l'aristocratie, durant lesquels ils interviennent en des moments choisis comme des protagonistes de premier plan. Notons également, dès le début $\mathrm{du} \mathrm{XVII}^{\mathrm{e}}$ siècle, que des récompenses financières étaient accordées aux hommes à cheval et aux hommes à pied, ce qui confirme sinon une professionnalisation plus précoce de certains groupes d'acteurs populaires, tout au moins l'existence officialisée de toreros indiens ${ }^{102}$. Il semble que cet intéressement économique ait pu faire naître de nouvelles formes de jeux taurins permettant d'étalonner les gains en fonction des risques, afin de rehausser les spectacles. En Bolivie, l'intérêt moindre suscité par l'organisation de taureaux emboulés, probablement pour réduire le nombre de victimes, fut compensé par la possibilité de gagner des pièces d'or ou d'argent fixées à la base des cornes. La technique consistait à s'avancer vers le taureau, à le saisir par la queue afin de le faire tomber et à s'emparer des pièces ${ }^{103}$. Ce jeu se rapproche des jocheos des toros actuels du département de Beni et du toreo de la vincha de Casabindo en Argentine.

D'un point de vue technique, il est possible de repérer quelques nouveautés, même si l'époque en Espagne de même qu'en Amérique est encore à la diversité gestuelle et aux inventions taurines de toutes sortes, de telle façon qu'il serait imprudent de forcer le trait des différences. En revanche, au XviII ${ }^{e}$ siècle, l'évolution des jeux taurins américains, éloignés des transformations qui se jouent sur le Vieux Continent, donne jour à des différences incontestables.

Les caractères distinctifs des corridas américaines du XviII ${ }^{\mathrm{e}}$ siècle relèvent d'un certain nombre d'innovations et de détournements techniques. On les retrouve parfois dans les gestuelles des rodéos modernes qui ainsi dans leur codification ont puisé non seulement à la source des techniques d'élevage, mais aussi à celle des jeux taurins importés et réinventés. Les prémices du coleo pédestre évoquées dans le cas de la Bolivie se retrouvent très affirmées au XviII ${ }^{\mathrm{e}}$ siècle en Colombie où, à Caracas, le jour de la Vierge, les édiles lâchent un taureau poursuivi par un grand nombre de cavaliers ${ }^{104}$. Le plus rapide l'attrape par la queue pour le mettre à terre.

La plus célèbre des innovations est décrite par le voyageur Emmanuel Witz en 1754 qui assiste, à Madrid, à la performance d'un certain Mariano Cevallos réussissant l'exploit, incongru pour les Espagnols, d'arrêter au lasso un taureau, de l'immobiliser, de le seller, pour finalement le tuer d'un coup de dague. Celui qui fut surtout rendu célèbre par les eaux-fortes de Goya, nommé Mariano Cevallos, «El Indio » (L'Indien) ou "El famoso americano » (le célèbre Américain), demeure pourtant une figure assez mystérieuse de la tauromachie de l'époque dont l'analyse pourrait renverser quelques perspectives.

La plupart des auteurs en font un Indien argentin, alors qu'il est très certainement un Noir péruvien, comme le montrent les écrits du grand homme de lettres péruvien Aurelio Miró Quesada. Mariano Cevallos est un esclave appar-

\footnotetext{
${ }^{102}$ D. Fournier, « Corrida, charreada et jaripeo », p. 189.

103 Á. López Cantos, Juegos, fiestas y diversiones en la América Española, p. 168.

${ }^{104}$ Ibid., p. 169.
} 
tenant à don Joseph de Cevallos Ribera y Dávila, comte de Santa Ana de las Torres ; Noir, car les Indiens ne peuvent pas être esclaves ${ }^{105}$. C'est accompagné de son maître que Mariano Cevallos voyagea en Espagne au milieu du XviII ${ }^{\mathrm{e}}$ siècle. Le 16 juin 1769, la liberté lui est accordée par son maître, mais le document attestant de sa liberté précise qu'il lui est interdit de toréer à pied ou à cheval et que s'il affronte à nouveau des taureaux, il retournera à sa condition d'esclave. En dépit de cette interdiction, Mariano Cevallos continua de toréer à Pampelune en 1775, à Madrid en 1776 et en 1777 où il fut accompagné de Costillares et Pepe-Illo, puis à nouveau à Pampelune de 1778 à 1780, pour finalement mourir, victime de sa témérité, à Tudela (Navarre). En cette période de codification de la tauromachie en Espagne, il n'est pas exclu que Mariano Cevallos fût le torero venu de Lima dont parle José Tixera dans Las fiestas de Toros, ouvrage publié en 1802. Dans cet ouvrage, l'auteur affirme que ce torero exécuta à Cadix une surprenante mise à mort à un temps, anticipant sur la technique du volapié qui deviendra la norme ${ }^{106}$. Quelle que soit la véracité de l'anecdote, elle démontre que la tauromachie américaine a pris part à la définition de la tauromachie espagnole, ne serait-ce que par la découverte de nouvelles techniques, même si elles sont le plus souvent rejetées. L'Espagne reste le foyer émetteur dominant des échanges transatlantiques, mais il existe en sens inverse des influences ponctuelles, triées au sein d'un répertoire varié que la tauromachie en Amérique a contribué à enrichir très tôt. Cela ne manquera pas d'être confirmé par la participation de grands toreros américains à l'histoire de la corrida moderne.

Bien après les aventures de "El Indio », la tradition des monteurs Noirs dans les arènes de la capitale péruvienne s'est poursuivie. Elle survit au moins jusqu'au début du $\mathrm{xx}^{\mathrm{e}}$ siècle où l'on pouvait encore voir un taureau non seulement encordé, mais monté par José de la Columna « Papito », que le capeador à cheval Juan Francisco Céspedes toréait. Toréer à la cape, du haut de son cheval, constitue une autre nouveauté américaine très en vogue à partir du XVIII ${ }^{\mathrm{e}}$ siècle. D'après Pedro de Zabala, qui en 1831 publie à Madrid un ouvrage intitulé Escuela de Caballería conforme a la práctica observada en Lima, cette technique serait née de l'impossibilité d'appliquer aux taureaux criollos le traitement des picadores et des varilargueros, tel qu'il existe alors en Espagne ${ }^{107}$. L'explication paraît plausible jusqu'au moment où l'auteur explique que ce n'est pas à cause du manque de bravoure des taureaux locaux, comme nous aurions pu le penser, mais au contraire parce que leur violence et leur férocité font qu'ils seraient morts sous la pique dont ils ne voudraient plus se défaire ${ }^{108}$. Dans le même ordre d'idée, l'érudit péruvien Ismael Portal ajoute que toréer les taureaux à la cape, du haut du cheval, permettait de les fatiguer avant l'épreuve des piques ${ }^{109}$. Nous penchons plutôt pour l'idée qu'une bravoure inégale des taureaux de l'époque

\footnotetext{
${ }^{105}$ A. Miro Quesada Sosa, Temas Taurinos, pp. 19-29.

${ }^{106}$ C. Sillero, « Los toros y el patrimonio cultural ».

${ }^{107}$ H. López Martínez, Plaza de Acho, p. 44.

${ }^{108}$ ID., Tauromaquia limeña en el siglo XIX, p. 25.

${ }^{109}$ ID., Plaza de Acho, p. 45.
} 
rendait la charge naturelle des bêtes vers les montures très aléatoire, obligeant les cavaliers à les inciter à charger par la vision de la cape en mouvement. L'absence de pique pendant la plus grande partie du XIX ${ }^{e}$ siècle au Pérou nous incite à trouver cette version plus crédible, bien qu'elle soit moins élogieuse au sujet de la bravoure des taureaux locaux. Quoi qu'il en soit, la compétence des capeadores est attestée dès le milieu du XviII ${ }^{\mathrm{e}}$ siècle, ainsi que leur origine, puisqu' ils seraient pour la plupart des Noirs esclaves, travaillant dans les haciendas à proximité de Lima $^{110}$. La vice-royauté du Pérou aurait donc donné naissance à des cavaliers taurins qui ne sont pas des descendants des chevaliers conquistadors, mais des cavaliers plébéiens toréant au nom de leur seigneur, à l'image des varilargueros en Espagne que l'histoire a ici rapidement relégués à un rôle secondaire.

$\mathrm{Au}$ Mexique, une forme populaire de tauromachie à cheval et la monte des taureaux avaient intégré les courses de taureaux officielles. Ces techniques naissent dans les haciendas coloniales en devenant le divertissement des péons indigènes dont la codification et la mise en spectacle finira par caractériser les jeux taurins et équestres mexicains : la charreada et le jaripeo. Très précocement, les indigènes pratiquent la monte des taureaux, toréent à cheval en utilisant la cape (le sarape : couverture de laine des vachers) et manifestent une dextérité particulière dans l'usage des lassos ${ }^{111}$. On peut remarquer que la figure taurine du lazador n'apparaît pas au Pérou. Dans son ouvrage Historia du toreo en México, Nicolás Rangel observait qu'à la fin du XviI ${ }^{\mathrm{e}}$ siècle, précisément lors d'un procès du 30 août 1662, il était fait référence à la compétence des Indiens dans les exercices taurins à cheval. L'auteur s'interroge sur l'usage du terme vaquear alors employé et se demande s'il est utilisé pour désigner « l'exécution des techniques de lazar, manganear, pealar y colear pratiquées sur les bêtes, c'està-dire, les techniques de notre jaripeo ", avant de conclure qu'il n'y a guère de doute à avoir à ce sujet ${ }^{112}$. Frédéric Saumade dans le cas du Mexique éclaire définitivement l'origine de ces techniques, depuis «l'énigme de la monte " jusqu'à l'usage de la corde (du lasso), interprétée dans les arcanes mêmes de la pensée méso-américaine $\mathrm{e}^{113}$.

Observons qu'il existe une différence importante entre le Mexique et le Pérou. Le développement de l'élevage bovin et des corridas en terre américaine a engendré dans le cas du Mexique l'élaboration de jeux taurins et taurinoéquestres individualisés que nous classons aujourd'hui dans la famille des rodéos, la charreada et le jaripeo. Dans le cas du Pérou, les innovations taurines intégrées aux corridas de la vice-royauté, n'ont pas abouti à l'élaboration de jeux institutionnalisés appartenant actuellement à la catégorie des rodéos, ce qui est finalement une exception parmi les grands pays de corridas puisque le Mexique, la Colombie et le Venezuela en possèdent. Pour autant, les innova-

\footnotetext{
${ }^{110}$ Ibid., p. 52.

${ }^{111}$ F. Saumade, Maçatl, pp. 275-276.

112 N. Rangel, Historia del toreo en México, p. 49.

${ }^{113}$ F. Saumade, « Animal de rente, animal de loisir ».
} 
tions coloniales péruviennes n'ont pas totalement disparu. En effet, pendant tout le $\mathrm{XIX}^{\mathrm{e}}$ siècle, la technique consistant à toréer à cheval au Pérou est appelée la «suerte nacional » (la technique ou le geste national) dans laquelle s'illustrent de grands cavaliers, véritables idoles du peuple. De plus, sur l'Altiplano andin, s'est conservée au moins jusqu'au dernier tiers du $\mathrm{xx}^{\mathrm{e}}$ siècle, la figure indigène du monta-toro (le monte-taureau) prenant part à certaines fêtes taurines populaires. Enfin, les jeux consistant à décrocher des récompenses fixées sur des taureaux concernent encore de nombreuses pratiques andines, en Équateur (colcha), au Pérou (enjalma et moña), en Bolivie dans le jocheo et jusqu'en Argentine, dans le cas du toreo de la vincha à Casabindo.

\section{LA DIFFUSION DE LA CORRIDA MODERNE}

L'innovation que constituent les corridas modernes semble bien avoir été reçue comme telle par les nations en devenir de l'Amérique espagnole, malgré une longue période d'imbrication avec les pratiques qui ont grandi pendant toute la période coloniale. Les premières arènes construites spécifiquement pour la tauromachie apparaissent quasiment en même temps qu'en métropole et la forme canonique circulaire est définitivement acquise dès la fin du XVIII ${ }^{\mathrm{e}}$ siècle. À Mexico, dans la vice-royauté de la Nouvelle-Espagne, une première arène octogonale dont les plans datent de 1770 est remplacée en 1788 par une arène circulaire : la Real Plaza de Toros de San Pablo ${ }^{114}$. À Lima, dans la vice-royauté du Pérou, les transformations sont plus précoces puisqu'une première arène circulaire, construite en 1756, est remplacée par une seconde, inaugurée en 1766 et qui demeure actuellement en place : la célèbre plaza de toros de Acho ${ }^{115}$. Généralement le critère le plus souvent utilisé pour dater l'émergence des corridas modernes en Amérique latine est la venue des premières cuadrillas espagnoles. Ce critère favorise sans doute à l'excès l'image d'une discontinuité très forte entre ce qui serait une ancienne tauromachie et une tauromachie moderne, alors même qu'en dépit des phases de changements accélérés, la tauromachie possède aussi ses lourdes inerties et ses rythmes lents. Dans ce domaine, les toreros de la métropole n'ont bien évidemment pas attendu que la corrida soit un spectacle commercial codifié pour tenter leur chance en Amérique. Les guerres d'indépendance semblent avoir partout créé une rupture tantôt définitive, tantôt temporaire, marquant le retour de nombreux toreros espagnols en métropole ou, pour un temps, leur plus grande discrétion en terre américaine. En fonction des dates des Indépendances et des régimes juridiques adoptés à l'égard des courses de taureaux, les toreros espagnols qui partent de nouveau vers l'Amérique emportent dans leur bagage une tauromachie située à des stades différents de son évolution, lors d'une période pour laquelle, d'une Tauromaquia à l'autre, de celle de Pepe-Illo (1796) à celle de Paquiro (1836), on sait l'importance des

${ }^{114}$ F. HaLcón, « Las plazas de toros de los virreinatos de América », p. 713.

${ }^{115}$ H. López Martínez, Plaza de Acho, p. 57. 
changements opérés. Le témoignage de l'érudit aficionado Leopoldo Vázquez dans son ouvrage América taurina, publié en 1898, offre un regard très instructif pour notre propos :

Non seulement au Mexique mais aussi au Chili, au Pérou, en Uruguay et dans les autres pays de l'Amérique que domina l'Espagne, depuis sa découverte jusqu'aux premiers années de ce siècle où ces nations obtinrent leur indépendance, il est possible de dire que le toreo à pied était encore dans son enfance jusque vers 1830, époque où quelques toreros Espagnols voyagèrent en diverses régions d'Amérique, avides, plus que de gloire, de nouveaux horizons où faire fortune ${ }^{116}$.

Ces pionniers sont généralement des seconds couteaux venus de leur Andalousie natale qui, rappelons-le, fournit encore à l'époque la majorité des toreros espagnols. Parmi les précurseurs, figure le torero Bernardo Gaviño, originaire de Puerto Real (Cadix), qui s'embarque en 1829 pour Montevideo. De là, il rejoint La Havane en 1831, puis Mexico en 1834. Après plusieurs années sans torero espagnol, consécutives à la période de forte instabilité politique des indépendances, la nouvelle tauromachie venue d'Espagne retrouve celle qu'elle avait laissée à la fin du XviII ${ }^{e}$ siècle, où se mélangeaient, parmi d'autres usages, le rejoneo, la lanzada, les nombreux exercices pratiqués par les Indiens et les Noirs, et des lâchers de taureaux emboulés ouverts à la participation collective. D'un point de vue technique, Leopoldo Vázquez concède à la tauromachie américaine une supériorité dans les exercices équestres :

Le seul toreo que les enfants de cette terre, qu'ils soient gauchos des Andes, Indiens, Péruviens, Chiliens ou Mexicains, portent plus haut que les Espagnols est la tauromachie à cheval ${ }^{117}$.

Il juge en outre cruciale la période durant laquelle se retirent les protagonistes espagnols pour laisser la place « aux techniques des champs que les natifs introduisent dans l'arène $»^{118}$. Parmi ces techniques, il mentionne le maniement du lasso pour enlacer les bêtes comme un exercice faisant partie du spectacle, ou servant à les retirer lorsqu'elles sont inaptes au combat. Il évoque également les pratiques équestres du coleo, des banderilles et du travail à la cape, ainsi que la monte des taureaux, ce qui n'exclue pas qu'ils soient dans le même temps toréés ${ }^{119}$.

Le retour des toreros espagnols s'accélère dans le dernier tiers du siècle et impose progressivement les normes de la corrida moderne : l'habit de lumière,

\footnotetext{
${ }^{116}$ L. VÁzQUez, América taurina, p. 31.

${ }^{117}$ Ibid., p. 34.

${ }^{118}$ Ibid., p. 47.

${ }^{119}$ Ibid., p. 47.
} 
l'usage de l'épée qui remplace le poignard, la réintroduction des banderilles et de façon plus aléatoire, la pique ${ }^{120}$. Ils participent aussi à la formation des toreros locaux recrutés dans leur cuadrilla. Des années 1890 jusqu'à la première guerre mondiale, dans la période de maturité et de plein épanouissement de la corrida moderne en Espagne, la filière s'élargit. Non seulement des débutants et des toreros modestes sont du voyage, espérant, toujours plus nombreux, trouver outre-Atlantique un vaste marché à conquérir ou une seconde chance, mais aussi d'importantes figures de la tauromachie espagnole, car les grandes arènes d'Amérique, principalement Mexico, Lima, La Havane, Montevideo, proposent des contrats très attractifs. Selon Adrian Shubert, sur une période qui s'étend des années 1880 jusqu'aux années 1920, l’Amérique paye les grands toreros du moment jusqu'à deux fois et demie ce qu'ils gagnent en Espagne pour un contrat équivalent ${ }^{121}$. À la fin du siècle, l'espace tauromachique européen est connecté à l'espace tauromachique américain, et les terres de corridas outre-Atlantique sont perçues comme un El Dorado qui, comme souvent, est un mirage pour la plupart et un formidable accès à la richesse pour quelques-uns. Quelles que soient les fortunes individuelles, les arènes du haut niveau de la hiérarchie qui demeurent en place à la fin du siècle, au premier rang desquelles figurent Mexico et Lima, commencent à former un réseau de hauts lieux qui structurent un espace tauromachique transatlantique. Les capitales jouent à cet égard un rôle crucial dans la diffusion de la corrida moderne au cour des terres taurines américaines.

$\mathrm{Au}$ Mexique, l'historien Benjamín Flores Hernández, nous rapporte qu’au $\mathrm{XVIII}^{\mathrm{e}}$ siècle, en marge des corridas officielles calquées sur le modèle des fêtes royales de la métropole, se sont développées des courses de taureaux pour financer la construction d'ouvrages publics ${ }^{122}$. Le maillage des villes taurines à la fin du XVIII ${ }^{e}$ siècle est bien plus développé au Mexique qu'ailleurs en Amérique. En témoigne par exemple le nombre de villes à avoir programmé des courses de taureaux en l'honneur de l'intronisation de Carlos IV à partir de 1790 : Mexico, Durango, Papantla, Veracruz, Patzcuaro, Guanajuato, Tehuantepec, Aguascalientes, Tabasco, Valladolid, Chilpa, Zamora, San Luis de la Paz, León, Guadalajara, Mérida, Campeche, Real de Catorce et Puebla ${ }^{123}$. Ajoutons de nombreuses cités où des courses informelles, en marge des festivités royales, sont données avec des taureaux emboulés ou aux cornes épointées pour limiter le nombre de blessures parmi les populations locales qui participent activement aux réjouissances taurines. Au moment de l'Indépendance, en 1821, le Mexique est déjà un pays où les jeux taurins ont acquis une forte popularité dans toutes les couches de la société et sur une base territoriale étendue. Enrique Guarner, dans son ouvrage Historia del toreo en México, indique qu'il existe alors un double clivage territorial qui sépare, d'une part les courses de Mexico de celles des autres villes, et d'autre part

${ }^{120}$ Ibid., p. 41.

${ }^{121}$ A. Shubert, A las cinco de la tarde, p. 40.

122 B. Flores Hernández, « Organización de corridas de toros en la Nueva España ».

${ }^{123}$ Ibid., pp. 499-500. 
la tauromachie urbaine de la tauromachie rurale ${ }^{124}$. Dans les premiers termes de l'opposition, les éléments espagnols sont toujours mieux représentés que dans les seconds où les jeux inspirés par l'élevage occupent une plus grande place : poursuivre le taureau, l'enlacer à cheval, pratiquer l'acoso y derribo, les toros de cola (coleo), la mise à mort au couteau ${ }^{125}$. Les troubles consécutifs à l'Indépendance rendent les courses de taureaux rares jusqu'en 1835, date qui inaugure une période allant jusqu'en 1885 que l'auteur baptise : «école mexicaine du toreo ». De 1822 à 1833 , les courses sont interdites par les autorités qui souhaitent faire oublier les symboles de la domination espagnole. À partir du milieu des années 1830 , surgissent des « cuadrillas régionales » qui exercent leur domination sur certaines zones du Mexique, sans aucune concurrence extérieure, comme s'il s'agissait d'un quadrillage du territoire en fiefs taurins séparés. Cette période marque une certaine stagnation dans l'évolution des pratiques liées à la faiblesse des échanges et de la mobilité spatiale des toreros ${ }^{126}$. C'est à cette même époque que s'épanouit une tauromachie à cheval, mexicaine, largement inspirée des travaux des champs. Selon la périodisation historique de l'auteur, l'année 1835 correspond à l'arrivée au Mexique du premier représentant espagnol de la tauromachie «moderne » dont l'histoire retient le nom : Bernardo Gaviño. L'année 1885 correspond à l'arrivée massive de toreros venus d'Espagne à la conquête du marché mexicain. Entre ces deux dates, un intermède d'une vingtaine d'années interdit les corridas dans le District Fédéral de Mexico, de 1867 à 1886, renforçant probablement partout ailleurs la part de la tauromachie à cheval, autochtone, très prisée en dehors de la capitale. Selon Daniel Medina de la Serna, ce qui à cette époque sauve finalement les corridas au Mexique, c'est la structure fédérale de la République qui permit que l'interdiction voulue par Benito Juárez en 1867 ne s'étende à l'ensemble du pays que pendant une seule année ${ }^{127}$.

L'itinéraire géographique et professionnel de Bernardo Gaviño mérite qu'on s'y attarde. Gaviño est né le 20 août 1813, à Puerto Real (Cadix). Orphelin de père, il est élevé par l'évêque Francisco Javier Cienfuegos qui devient archevêque de Séville. Gaviño, qui n’a pas dix ans, le suit pour être placé dans un séminaire. Dès 1825 , avec ses amis, il torée parfois des bêtes, tuées dans la cour du cloître. Il s'enfuit souvent pour aller aux abattoirs, parrainé par le torero Juan León auprès duquel il apprend le métier. L'archevêque juge cette afición naissante funeste et l'envoie en prison quinze jours. À sa sortie, il intègre une cuadrilla qui sillonne la Basse Andalousie, mais menacé par l'archevêque, il finit par s'enfuir en Amérique. C'est pour cette raison qu'il aurait rejoint Montevideo en 1829, où il torée pendant deux ans, puis part pour La Havane en 1831, avant de rejoindre Mexico en 1834. À Mexico, il aurait été à cette période un torero sans rival ${ }^{128}$. Il meurt en 1887 à Texcoco des suites d'un coup de corne, non sans avoir transmis à

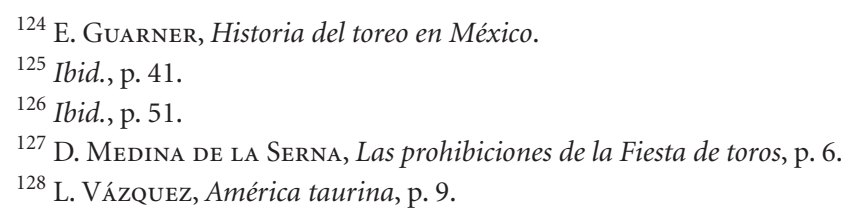


de nombreux toreros locaux, engagés dans sa cuadrilla, les techniques apprises dans son Andalousie natale.

Le premier grand torero mexicain retenu par l'histoire est Ponciano Díaz qui passe pour maîtriser en tout point la tauromachie équestre autochtone, alors connue sous le terme de jaripeo, et les principales techniques de la tauromachie à pied espagnole. À lui seul, il peut être considéré comme un personnage charnière entre l'ancienne tauromachie mexicaine, qui de façon autonome prendra les traits de la charreada, et de la nouvelle, calquée sur le modèle dominant de la corrida espagnole de la fin du XIX $^{\mathrm{e}}$ siècle. L'iconographie retiendra d'ailleurs deux Ponciano Díaz : l'un en costume de charro, présenté lasso en main, l'autre en habit de lumière, présenté épée en main ${ }^{129}$. Ponciano, qui grandit dans l'hacienda d'Atenco spécialisée dans les taureaux de combat, commence à toréer en 1873, alors âgé de 15 ans. En 1878, c’est précisément dans la cuadrilla de Bernardo Gaviño qu'il est engagé comme banderillero et reçoit l'alternative de ses mains en 1879, à Puebla. En 1889, il s'embarque pour l'Espagne. Il se présente dans les arènes de Madrid où il suscite une forte impression en plantant les banderilles à cheval, technique qui, comme on l'a vu, est courante dans la tauromachie mexicaine du XIx ${ }^{\mathrm{e}}$ siècle. Observons d'ailleurs que la pose de rejones courts à deux mains est pratiquée dans le rejoneo actuel, sans qu'il soit fait référence à une possible origine américaine de la technique.

Enfin, la dernière étape du processus d'implantation de la corrida moderne au Mexique repose sur l'arrivée de grandes vedettes espagnoles. C'est le torero Mazzantini qui incarne le mieux ce tournant. Mazzantini foule les arènes de Mexico en 1887, mettant un terme à vingt ans d'interdiction. À l'inverse de Ponciano auquel s'identifient les classes moyennes et populaires, Mazzantini attire l'attention des élites hispanophiles qui attendent sa venue avec impatience. La fin des années 1880 marque une période de forte rivalité entre les deux tauromachies, celle de Ponciano et celle de Mazzantini. Au premier échec de ce dernier, des émeutes s'emparent de la ville, des boutiques tenues par des Espagnols sont saccagées au cri : "ique mueran los gachupines! " (mort aux Espagnols) ${ }^{130}$. Loin d'exprimer un refus de la corrida, nous pensons que ces poussées d'animosité nationalistes confirment l'assimilation de la tauromachie espagnole comme fait culturel transversal à partir duquel se déclinent des rivalités d'ordre social et identitaire. L'interdiction de courte durée, de 1914 à 1919, à la suite de la Révolution mexicaine ne remet pas en cause cet enracinement comme le prouve la venue de quasiment tous les grands matadors espagnols de l'histoire à partir des années 1920 .

Un scénario presque analogue peut être envisagé pour le Pérou. Avec l'Indépendance, tous les toreros espagnols ont dû quitter le pays en raison de la politique répressive menée par Bernardo de Monteagudo, ministre de San

\footnotetext{
129 A. Villatoro, Antología taurina mexicana, pp. 127-128.

${ }^{130}$ Gachupin est un terme péjoratif désignant les Espagnols. E. GuARner, Historia del toreo en México, p. 66.
} 
Martín, à l'exception, dit-on, de l'un d'entre eux, un certain Vicente Tirado ${ }^{131}$. C'est à partir de cette époque que la technique de la cape à cheval prit une grande importance jusqu'à la fin du siècle, au point d'être nommée la suerte nacional, ainsi que le travail des monteurs de taureaux. Dans les programmes du milieu du $\mathrm{XIX}^{\mathrm{e}}$ siècle, qui au Pérou étaient annoncés sous forme de strophes rimées, les listines, les noms des taureaux ainsi que leur élevage d'origine apparaissaient en tête d'affiche, puis venaient le nom des capeadores a caballo et, en dernier, celui des matadors et autres toreros de la cuadrilla ${ }^{132}$. La première cuadrilla espagnole débarque à Lima le 30 décembre 1848, après avoir signé un contrat à Cadix par l'intermédiaire du capitaine de navire chargé de la liaison Callao-Cadix, un certain Quintín Quilaver. Deux matadors (Carlos Rodríguez et Antonio Romero Pastor) sont engagés ainsi que quatre banderilleros, tous andalous de Málaga et de Cadix. Ils toréent entre douze et quatorze corridas jusqu'au mois de juillet 1849 et tous restèrent au Pérou, sans jamais revoir leur terre natale. Finalement, ils ne font que s'inscrire dans les migrations économiques de l'époque vers les Amériques. Les comptes-rendus des courses d'alors parlent d'une vive concurrence entre les toreros espagnols et les capeadores à cheval qui, à Lima, sont d'authentiques idoles, tels que Monteblanco et Arredondo. Mais le bilan de cette rivalité débouche sur une incontestable évolution qui progressivement impose les canons de la corrida moderne espagnole. En témoignent la réincorporation des banderilles, l'adoption de l'habit de lumière et le port de la montera par les toreros locaux. La dernière étape de ce processus est l'apparition de toreros péruviens rompus aux nouvelles techniques, dont le premier à se faire un grand nom est le matador Ángel Valdez dit « El Maestro » qui commença à toréer en 1857, soit moins de dix ans après l'arrivée de la première cuadrilla. Il se présenta à Madrid en 1883, quelques années avant le Mexicain Ponciano Díaz. Ángel Valdez fait partie de ces figures qui ont incontestablement favorisé l'enracinement de la corrida moderne en Amérique en position d'idoles nationales capables de soutenir la comparaison avec les toreros espagnols. À partir de 1885, en plein âge d'or de la tauromachie en Espagne, le Pérou connaît lui aussi une arrivée croissante de toreros venus de la Péninsule dont certains, tels " Cuatrodedos ", puis « Faíco », finissent d'imposer à l'afición le goût de la corrida à l'espagnole. Comme signe incontestable de cette évolution au cours du premier tiers du $\mathrm{xx}^{\mathrm{e}}$ siècle, la technique des capeadores à cheval est définitivement remplacée par celle des picadors. En dépit de traditions taurines actuelles assez différentes, on observe un fort parallélisme de l'implantation des corridas modernes dans les deux anciennes vice-royautés.

Retenons que la fin du $\mathrm{XIX}^{\mathrm{e}}$ siècle est une période d'homogénéisation de l'espace tauromachique américain qui s'aligne sur les codes de la tauromachie espagnole. L'émergence d'un spectacle taurin d'envergure transatlantique, unifié dans ses codes et assimilé comme un élément du patrimoine local par une partie de la société, n'empêche en rien le développement et l'individualisation d'autres jeux taurins marqués par une plus forte autochtonie. En revanche, il

\footnotetext{
${ }^{131}$ H. López Martínez, Plaza de Acho, p. 95.

${ }^{132}$ Ibid., p. 59.
} 
semble difficile de trouver une explication générale entièrement satisfaisante qui permettrait de comprendre pourquoi la corrida s'est maintenue dans tel pays plutôt que dans tel autre. On peut constater que les terres peuplées, situées au cœur de l'organisation impériale de la conquête (le Mexique et le Pérou) sont restées d'importantes terres de corridas. On peut également constater que la fragmentation nationale en territoires de petite taille a plutôt joué dans le sens d'une disparition ou de leur faible développement. La relation entre les dates des Indépendances, les premières interdictions des courses de taureaux et la diffusion de la corrida sous sa forme moderne ne permet pas de tirer de conclusion générale. Certains États ont en effet interdit les courses de taureaux après avoir connu l'avènement des corridas modernes, ce qui confirme que la transformation et la modernisation du spectacle n'ont pas été des critères suffisants pour expliquer son enracinement définitif. Cet argument invalide les lectures finalistes de la tauromachie qui pensent que certains pays ont abandonné les courses de taureaux parce qu'ils n'en connaissaient pas encore la véritable forme, sans quoi ils se seraient rendus à l'évidence et au bien fondé d'un art supérieur. L'abandon des corridas dans les pays du Cône Sud (Argentine, Chili, Uruguay) semble lié à deux facteurs. Notons d'une part que les indépendances menées par des libéraux acquis aux idées progressistes des révolutions françaises et américaines n’ont pas été suivies dans ces pays d'une reconquête espagnole du pouvoir perdu, comme ce fut le cas au Pérou, en Équateur, en Colombie et au Venezuela. D’autre part, ces pays ont connu une très forte immigration européenne à partir de la fin du $\mathrm{XIX}^{\mathrm{e}}$ siècle, beaucoup plus diversifiée que les flux anciens à dominante espagnole. Il en va de même au Brésil. Cet élément démographique a fortement joué sur la disqualification de la corrida espagnole. Enfin, on peut remarquer que là où les corridas ont été conservées, les Indiens et les Noirs se sont très tôt réappropriés la figure du taureau et la culture taurine (Mexique, Pérou, Équateur, Colombie). On notera également qu'au Chili et en Argentine, l'éradication des cultures indigènes a été très marquée. Cette piste nous semble fort intéressante en ce qu'elle renverse quelques perspectives. En effet, il est possible de considérer que les corridas se sont enracinées non pas tant en raison d'une permanence hispanophile comme certains se limitent à le penser, qu'en raison d'un métissage précoce de la culture taurine, qui aurait permis une confrontation directe entre corrida espagnole et jeux taurins locaux. Autrement dit, l'implantation de la corrida en Amérique latine ne s'explique pas tant par le maintien d'un héritage figé de « l'ExtrêmeOccident ", que par l'existence d'un champ culturel tauromachique local qui concerne un éventail social élargi, différencié et contrasté ${ }^{133}$.

\section{Pour Une NOUVElle histoire Des Rodéos}

L'histoire de la formation des rodéos et de leur emprise territoriale se construit en trois étapes fondamentales. La première résulte des contacts entre les sociétés

${ }^{133}$ L'expression «Extrême-Occident » pour désigner le prolongement culturel occidental en Amérique est empruntée à A. Rouquié, Amérique latine. Introduction à l'Extrême-Occident. 
locales et les colons ibériques à partir de l'introduction du bétail et des jeux taurins importés de la métropole. La deuxième relève d'une réappropriation territoriale du code des rodéos dans le cadre des États-nations indépendants. La troisième est celle d'une modernisation des rodéos selon les modèles institutionnels et spectaculaires du sport.

La première étape entrerait parfaitement dans le cadre de ce que George Foster a baptisé dans les années 1960 une "culture de la conquête ». Selon l'auteur, cette "culture de la conquête» dépend d'un processus de sélection de traits culturels qui aboutit à une uniformisation relative de la culture hispano-américaine. Notre étonnement face à l'existence de pratiques relativement proches, apparentées au rodéo, et qui s'étendent de l'Amérique du Nord au Cône Sud, nous ramène à cette lecture. L'originalité du modèle d'acculturation proposé par George Foster réside dans le fait que la sélection est opérée conjointement par les Indiens et par les Espagnols au sein d'une culture nouvelle pour tous. Selon l'auteur, tout se joue dans les premières années de contact, déterminant les principaux traits de la « culture de la conquête » qui ensuite demeure relativement imperméable aux apports postérieurs ${ }^{134}$. S'il est un élément survalorisé par la culture taurine de la conquête, c'est bien la place du cheval. Au sein des courses de taureaux, cet animal s'impose longtemps comme un élément central, alors même qu'il est relégué à un rôle subalterne dans la codification espagnole de la corrida moderne. Au sein des rodéos, il occupe une place prépondérante, soit sous la forme des chevaux sauvages qu'il faut dompter, soit sous la forme d'un partenaire indispensable à la maîtrise les bovins, soit à travers la monte des bovins qui renvoie, de façon détournée, à son absence. La place importante du cheval dans les tauromachies américaines peut ainsi être interprétée comme l'une des spécificités de la "culture de la conquête ».

Dans le domaine de l'élevage, l'article de Charles Julian Bishko, datant de 1952, sur l'héritage péninsulaire dans la formation de l'élevage extensif latinoaméricain demeure une référence indispensable, à laquelle il faut ajouter les approfondissements apportés plus récemment par le géographe Terry Jordan ${ }^{135}$. Charles Julian Bishko fait du bouvier médiéval castillan « l'ancêtre des vaqueros, vaqueiros, gauchos, huasos et llaneros " et repère que dans les conditions matérielles de leur exécution ainsi que dans la répartition des tâches, les rodéos prolongent certaines méthodes d'élevage de la péninsule Ibérique existant au $\mathrm{XV}^{\mathrm{e}}$ siècle ${ }^{136}$. Selon l'auteur, les costumes doivent beaucoup aux charros de la région de Salamanque, la pique utilisée par les llaneros vénézuélien et les sertanejos brésiliens hérite des instruments des vaqueros de Castille et d'Alentejo, alors que le lasso et la pratique agraire du rodeo seraient d'invention améri-

\footnotetext{
${ }^{134}$ G. Foster, La cultura tradicional en España y América. Sur l'intérêt de l'approche théorique de la lecture de George Foster voir C. BERnAND et S. Gruzinski « La redécouverte de l'Amérique ».

${ }^{135}$ C. J. Bishко, « The peninsular background »; T. Jordan, North American Cattle-Ranching Frontiers.

${ }^{136}$ C. J. Bisнко, « The peninsular background », pp. 506 et 509.
} 
caine $^{137}$. On peut observer aujourd'hui que le coleo vénézuélien et la vaquejada brésilienne ont, en quelque sorte, abandonné la pique qui a jadis été utilisée par les travailleurs du bétail, llaneros et sertanejos. Terry Jordan ne remet en cause, ni la prépondérance, ni l'antécédence de l'héritage ibérique analysé dans sa double composante : celle des élevages bovins des basses terres de la Marisma en Andalousie et celle des élevages bovins des plateaux de la Meseta occidentale en Estrémadure ${ }^{138}$. Il enrichit néanmoins le modèle en intégrant aussi le rôle joué par les deux autres régions outre-Atlantique qui connaissaient également des formes extensives d'élevage de bovins : l'Europe du Nord-Ouest et en particulier les Highlands britanniques ainsi que l'Afrique de l'Ouest et en particulier le Sahel ${ }^{139}$.

En dépit du grand intérêt de ces approches, ce sont les recherches initiées par Dominique Fournier et par Frédéric Saumade sur les tauromachies mexicaines qui constituent le maillon manquant permettant de sortir d'une conception des rodéos qui ne seraient que la transformation américaine des méthodes de l'élevage extensif. Malgré les divergences d'interprétation qui existent entre eux, c'est en intégrant la part revenant aux courses de taureaux dans les processus de codification du jaripeo et de la charreada que les auteurs montrent qu'il est nécessaire de considérer l'ensemble des tauromachies comme un tout faisant système.

Dominique Fournier, en 1995, dans un article pionnier sur cette thématique, fait du taureau un outil efficace d'acculturation des structures sociales, politiques et religieuses du Nouveau Monde ${ }^{140}$. Selon l'auteur, "dans le Mexique encore influencé par l'expansionnisme culturel aztèque, le sacrifice taurin aurait, dans une certaine mesure, servi de substitut au sacrifice humain après une transformation résultant de la confrontation violente de deux États constitués, de deux formes d'élaboration idéologique fort distinctes ${ }^{141}$. L'un des points clés de l'argumentation réside dans la correspondance entre les deux rituels des nécessités matérielles et idéelles de leur exécution, des logiques d'édification du pouvoir, des lieux de mise en scène et des calendriers qui permettent de comprendre l'efficacité de la substitution ${ }^{142}$. Selon l'auteur, durant la période coloniale, le taureau, sacrificateur dans le jaripeo et objet de sacrifice dans la corrida, autorise un contact ambigu avec la figure du diable et du dieu de la religion officielle. Cette interprétation est prolongée, en 1999, par un article davantage attentif à caractériser la nature des identités véhiculées par les différents jeux taurins qui nous fait entrer dans la logique mexicaine du métissage culturel ${ }^{143}$. Cet article montre qu'à chaque pratique taurine correspond schématiquement une strate

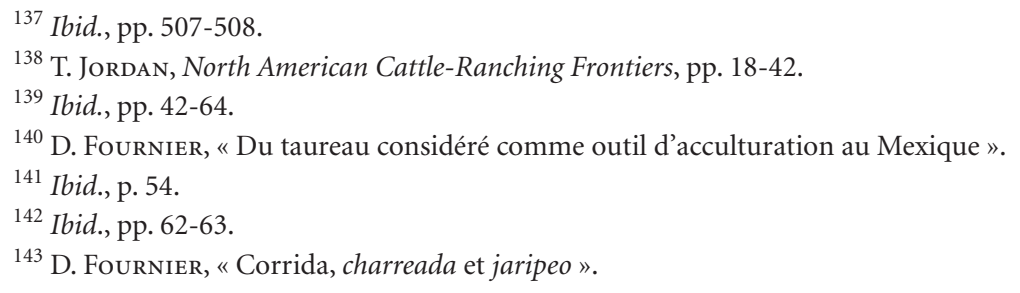


particulière de la société mexicaine : la corrida renvoie à l'idéologie du Mexique colonial, la charreada à une idéologie métisse des grands propriétaires terriens et des groupes urbains dominants, le jaripeo renvoie aux communautés rurales dominées, qu'elles soient métisses ou indigènes. La monte des taureaux est alors identifiée à « une forme d'inversion de la corrida formelle de l'origine » où le taureau est ici utilisé comme monture, et le monteur comme victime du sacrifice ${ }^{144}$.

Frédéric Saumade résout différemment « l'énigme de la monte » en renonçant à la logique d'une substitution sacrificielle et en écornant la figure du taureau-diable comme vecteur symbolique de l'acculturation, chère à Dominique Fournier ${ }^{145}$. Fidèle à son modèle explicatif développé pour appréhender les tauromachies européennes, il ramène les tauromachies mexicaines à une transformation des techniques espagnoles de maniement du bétail, utilisées dans les courses de taureaux et dans les élevages bovins. S'il conserve l'idée de la monte des taureaux comme un rite d'inversion de la tauromachie équestre espagnole et principal vecteur de transformation des pratiques, il refuse sa lecture sacrificielle et ajoute à la grammaire tauromachique mexicaine une interprétation fouillée de l'utilisation de la corde, la reata d'ixtle. La monte du taureau est ici interprétée dans un double registre. Elle est une forme d'inversion de l'homme à cheval espagnol et relève aussi d'un principe existant dans la culture préhispanique à travers les figures humaines des porteurs de marchandises (les tlamemes) et des porteurs de dieux (les teomama). La monte du taureau s'inscrit alors dans une ambiguïté constitutive de la pensée méso-américaine qui assimile en une même entité les figures contradictoires du monteur et de la monture, ainsi que celles de l'homme et de l'animal. La corde est analysée comme la réinterprétation mexicaine d'un instrument sacré préhispanique utilisé dans la chasse, dans la guerre et dans les sacrifices permettant d'intégrer l'élevage extensif ibérique aux représentations mentales indigènes.

La technique du coleo à cheval, qui demeure l'une des modalités techniques des rodéos ludiques parmi les plus prisées du monde latino-américain, n’a pas retenu pour l'instant la même attention. Observons qu'elle est identifiée dans la Tauromachie de Pepe-Illo (1796) comme une technique "brillante " mais rarement exécutée ${ }^{146}$. En revanche, elle n'apparaît plus dans la Tauromachie de Montes (1836) autrement que comme un recours à la technique pédestre de mancornar (attraper les taureaux par les cornes), se saisir de la queue permettant alors de se mettre hors d'atteinte des cornes. Dans la corrida espagnole actuelle, attraper le taureau par la queue sans nécessité impérieuse de sauver un homme à terre est sifflé par le public et représente un manque de respect pour l'animal. Dans la corrida portugaise, une fois la pega correctement exécutée, la queue est tenue par le forcado qui relâche le taureau en dernier, se laissant ainsi traîner quelques instants par l'animal. Sur le continent américain, le coleo ne

\footnotetext{
${ }^{144}$ Ibid., p. 192.

${ }^{145}$ F. SAUMADE, « Animal de rente, animal de loisir ».

${ }^{146}$ Pepe-Illo, La Tauromaquia, p. 93.
} 
fait pas partie des exercices du rodéo étasunien. La technique n'est guère utilisée que par les cowboys protector pour sauver les monteurs désarçonnés. Dans le rodéo chilien, elle est explicitement interdite et pénalisée par le règlement, ce qui laisse penser que bien qu'elle ne figure pas parmi les gestes actuels autorisés, elle a dû faire partie des techniques employées pour stopper le bétail dans sa course. Étant donné l'aire d'extension à la fois vaste et discontinue du coleo, il nous paraît difficile de croire à l'existence d'un foyer unique d'apparition de la technique. Dans le cadre du Mexique, Guadalupe Silva Corcuera affirme que les Llanos de Apan dans l'État d'Hidalgo furent la zone d'invention du coleo, qui aurait été exécuté pour la première fois par un picador espagnol ${ }^{147}$. Cette version, qui tient de la légende, fait du coleo une pratique inventée en terre américaine par un protagoniste de la corrida espagnole, mettant ainsi l'accent sur les origines ibériques des jeux apparentés aux rodéos qui habituellement sont plutôt représentés sous les traits de pratiques issues du génie local. Au Brésil, le grand spécialiste du folklore, Luis da Câmara Cascudo, considère que la technique de la coleada a été préférée par les vaqueiros du Nordeste en raison d'un couvert végétal qui aurait empêché le maniement d'ustensiles encombrants, dont feraient partie la garrocha et le lasso, progressivement abandonnés ${ }^{148}$. L'explication matérielle justifiant la technique du coleo par les conditions du milieu physique local ne nous semble guère convaincante. Ici transparaît plus sûrement un processus idéologique de réappropriation territoriale de la technique pour faire d'elle une forme autonome et autochtone. Ce processus est caractéristique de la réinvention des rodéos dans le contexte post-colonial des États-nations indépendants. En cela, l'affirmation des rodéos en tant que jeux codifiés, séparés de la pratique utilitaire et représentatif d'une culture régionale ou nationale, est une étape fondamentale de la différenciation des territoires tauromachiques américains.

Tous les rodéos proviennent ainsi d'une transformation des pratiques de l'élevage extensif, descendant du régime des haciendas coloniales, pratiques qui sont réinvesties par un imaginaire territorial tendant à les individualiser en autant d'expressions culturelles locales et indépendantes les unes des autres. Le poids des courses de taureaux comme contre-modèle de la formalisation des jeux taurino-équestres américains a joué un rôle important dans ce processus. Il est tentant de considérer que les courses de taureaux n'ont pas pu être absentes des représentations bovines et équines de la «culture de la conquête ». Cela étant, la différenciation des rodéos les uns par rapport aux autres, au sein d'une évolution strictement panaméricaine, semble avoir davantage joué dans le résultat final que les logiques de distinction avec le spectacle espagnol. En la matière, tout se passe comme si le rodéo étasunien, en tant que forme hégémonique, prenait le relais de la tauromachie espagnole dans l'influence exercée sur la codification définitive des rodéos américains.

${ }^{147}$ G. Silva Corcuera, « La charrería. Representación simbólica de lo mexicano », p. 11.

${ }^{148}$ L. da CÂmara CAscudo, A vaquejada nordestina e sua origem, p. 27. 
Par ailleurs, aucun argument simple ne permet d'expliquer l'absence ou la présence des jeux apparentés au rodéo au sein de ce vaste espace américain. Si l'existence d'une région ancienne d'élevage extensif semble partout une condition nécessaire (États-Unis, Mexique, Colombie, Venezuela, Brésil, Chili), elle n'est pas une condition suffisante. C'est ce que montre le cas de l'Argentine et de l'Uruguay qui possèdent l'une des figures vaqueras parmi les plus connues, le gaucho de la Pampa, mais qui n'est pas reliée à la pratique d'un rodéo d'envergure et qui, lorsqu'il est pratiqué, occulte la figure taurine au sein de la trilogie élémentaire homme-cheval-bovin. Si la combinatoire ville-campagne, associant à une arène urbaine de corrida une hacienda périurbaine d'élevage, permet de comprendre l'émergence de certains jeux taurins, elle n'explique pas pourquoi ils finissent, ici par disparaître (les monteurs de taureaux et le capeador à cheval au Pérou), et là par être consolidés sous la forme de rodéos indépendants (Mexique, Colombie, Venezuela). La présence de la corrida ne semble pas non plus expliquer l'affirmation du rodéo moderne comme s'il s'était agi d'un face à face entre deux traditions, l'une espagnole, l'autre autochtone, dont la confrontation eût été favorable à un développement mutuel. En témoignent les rodéos du Chili et du Brésil qui se sont consolidés malgré la disparition de la corrida. Les éléments de réponse sont à préciser à travers des chronologies très différentes selon les pratiques et selon les pays.

À la différence de la stabilisation des terres de corridas qui se joue entre la fin $\mathrm{du} \mathrm{XIX} \mathrm{X}^{\mathrm{e}}$ siècle et le début du $\mathrm{Xx}^{\mathrm{e}}$ siècle, la géographie du rodéo, qui connaît l'essentiel de ses transformations durant le $\mathrm{xx}^{\mathrm{e}}$ siècle, est encore en mouvement aujourd'hui. Quoi qu'il en soit, l'institutionnalisation des rodéos et leur transformation en spectacles modernes ont toujours quelque chose à voir avec la construction territoriale des États-nations et l'affirmation de figures vaqueras, garantes d'un lien essentialiste entre la terre et le territoire. Pour ces raisons rapidement esquissées, nous intégrons la transformation des rodéos et l'évolution de leur spatialité à une analyse des dynamiques récentes, pays par pays, que nous présentons en troisième partie. 
TROISIÈME PARTIE

ESPACES ET TERRITOIRES

DE LA TAUROMACHIE 

L'analyse menée sur l'unité et la diversité des pratiques tauromachiques a montré la pluralité des critères permettant de les différencier et de les rapprocher. Elle a pour cela précisé les éléments communs d'une forme à l'autre. Elle a aussi mis en relief les éléments qui en sont exclusifs afin d'établir des degrés de parenté formelle entre ces pratiques, en dépit des codifications, qui les cloisonnent en des jeux autonomes, et de leur institutionnalisation dans des cadres territoriaux généralement séparés. Après avoir esquissé à grands traits la configuration générale de l'espace tauromachique, ses limites et les étapes historiques de sa formation, il s'agit désormais d'approfondir l'étude de la spatialité contemporaine des pratiques taurines en faisant apparaître, d'une part, la géographie particulière de chacune d'elles et, d'autre part, les relations territoriales qu'elles entretiennent les unes avec les autres.

Il existe plusieurs figures géométriques de la relation territoriale entre les pratiques tauromachiques. On peut les regrouper sous l'angle des différentes modalités de coexistence spatiale qu'elles entretiennent à différentes échelles : l'éloignement dans un même cadre territorial, la juxtaposition, le chevauchement partiel, la superposition, la coprésence. Ces figures ne suffisent pourtant jamais à rendre compte du cloisonnement culturel entre les pratiques tauromachiques. En effet, des pratiques peuvent coexister sur un même espace sans qu'il n'existe de fortes relations qui les unissent, comme pourrait par exemple le matérialiser un même public commun à plusieurs pratiques. C'est bien la distance géographique et sociale plus ou moins grande entre les pratiques qu'il s'agit alors de mesurer. Certaines sont reliées par des lieux d'articulation tels que des villes aux pratiques plurielles, des fêtes qui les conjuguent ou des arènes uniques qui les accueillent. Quoi qu'il en soit, nous pensons que dans le cadre des États-nations, les modalités de coexistence entre les pratiques tauromachiques participent toujours de l'agencement territorial et identitaire du pays, de ses différenciations régionales, de ses relations villes-campagne, de ses relations centre-périphéries. Au niveau géographique supérieur, les pratiques tauromachiques offrent également une lecture des relations territoriales entre la péninsule Ibérique et l'Amérique, en particulier à travers l'existence d'un espace tauromachique transatlantique de la corrida espagnole. Au sein d'un ensemble Sud-Ouest européen, les pratiques tauromachiques participent à leur tour à l'articulation des territoires autour de l'Espagne, en position centrale, 
et matérialisent des dynamiques transfrontalières avec le Portugal et la France. De même en Amérique, les pratiques tauromachiques articulent des relations territoriales entre les États-Unis et le reste du continent, entre des pays voisins (États-Unis - Mexique; Colombie-Venezuela), mais aussi entre catégories socioethniques spatialisées (Blancs, Métis, Indiens et Noirs).

Dans cette perspective, nous analysons la structure des espaces tauromachiques et leurs dynamiques récentes. D'une part, il s'agit de rendre compte de la répartition des jeux et des spectacles, de la répartition des arènes, de la répartition des élevages, des dynamiques régressives, progressives ou stabilisées des pratiques. D’autre part, il s'agit de mettre en évidence la façon dont les pratiques tauromachiques participent aux dynamiques territoriales des régions qui les supportent, aux dynamiques internes propres à chaque pays et à l'ensemble des dynamiques transfrontalières. Les questions sont traitées dans un cadre européen (chap. vi et viI) et dans un cadre américain (chap. VIII), en veillant toujours à rendre compte des relations croisées entre les espaces nationaux observés successivement. 


\section{LA STRUCTURE DES ESPACES TAUROMACHIQUES EUROPÉENS}

\section{I. — L'ESPACE TAUROMACHIQUE ESPAGNOL}

\section{Pour Une RÉgIONALISATION DES PRATIQUeS TAURINES}

En Espagne, les données chiffrées relatives au nombre de spectacles taurins sont plus fiables que dans beaucoup d'autres pays. Les variations d'une source à l'autre ne tiennent souvent qu'à des différences de classification ${ }^{1}$.

Comme nous l'avons signalé, le règlement taurin espagnol distingue huit types de spectacles que nous avons pris soin de séparer en spectacles de la tauromachie professionnelle et jeux taurins à participation collective. Au sein des spectacles de la tauromachie professionnelle, les statistiques de la CCNAT ne prennent en considération que les spectacles dits majeurs, c'est-à-dire tous les spectacles incluant des courses piquées, à pied, et/ou des courses de rejoneo, à cheval : corridas de toros, festejos de rejones (corridas de rejones con toros, rejones con novillos), novilladas con picadores et otros festejos (festejo mixto, festival con picadores). Le Ministère s'en justifie en déclarant que ces spectacles du haut de la hiérarchie sont les plus significatifs de ce que représente la tauromachie en Espagne ${ }^{2}$. Par conséquent, ces statistiques-là ne tiennent pas compte des spectacles dits mineurs (novilladas sans picadors, becerradas et toreo comique), ni des festejos populares. Depuis la loi taurine de 1991, les chiffres de ces deux dernières catégories ne sont plus enregistrés de façon systématique au niveau national en raison du partage des compétences entre l'État et les Communautés autonomes en matière de spectacles taurins. Aujourd'hui seules les Communautés autonomes comptabilisent l'ensemble des autorisations accordées pour le déroulement des spectacles publics. Le tableau 2 (pp. 216-219) offre, pour

\footnotetext{
${ }^{1}$ Nous avons utilisé quatre sources principales d'informations : les données de Mundotoro (société de journalisme spécialisée dans la corrida), celles de la CCNAT affiliée au ministère de l'Intérieur (Commission consultative nationale des affaires taurines), celles communiquées par José María Sotomayor (journaliste et documentaliste taurin) et celles des services responsables des jeux et des spectacles publics de chacune des Communautés autonomes.

${ }^{2}$ Ministerio del Interior, Estadísticas de la Temporada 2003, p. 3.
} 


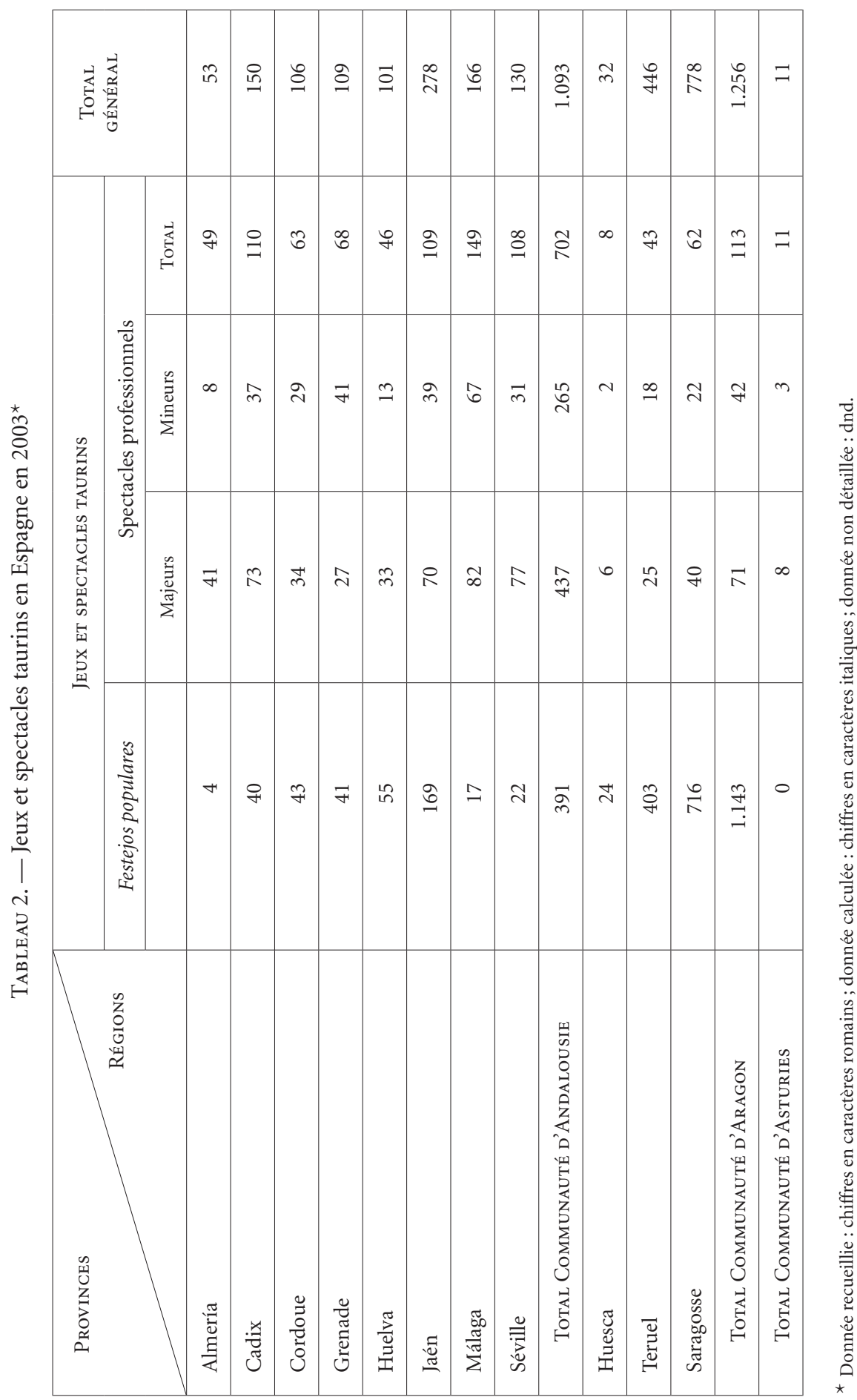




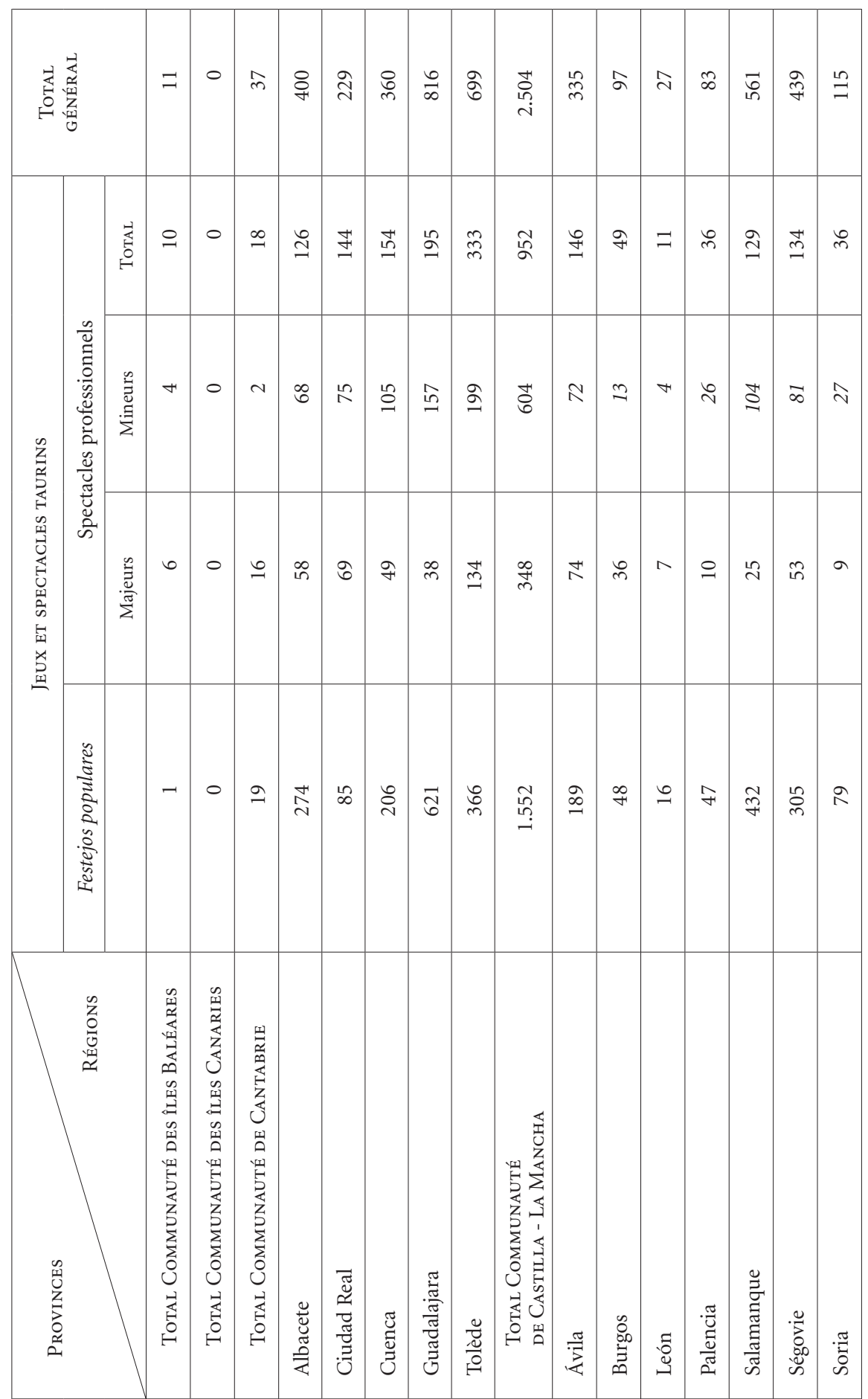




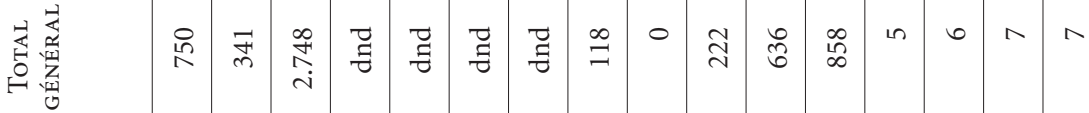

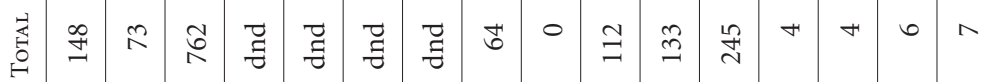

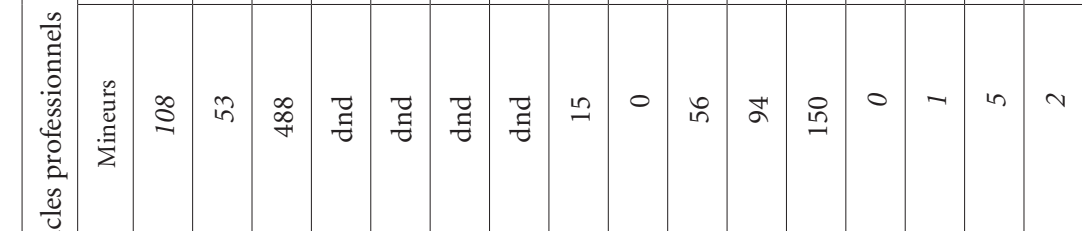

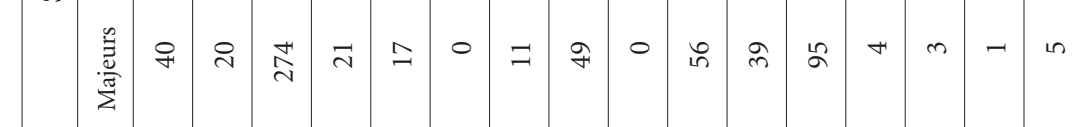

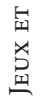

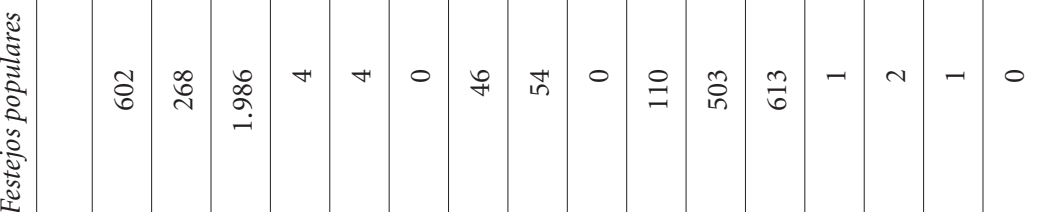

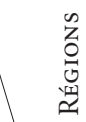

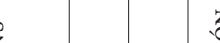

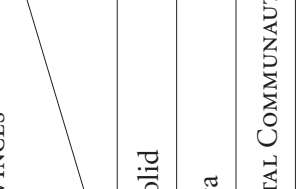

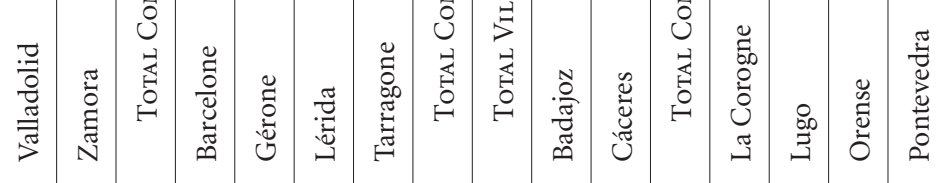




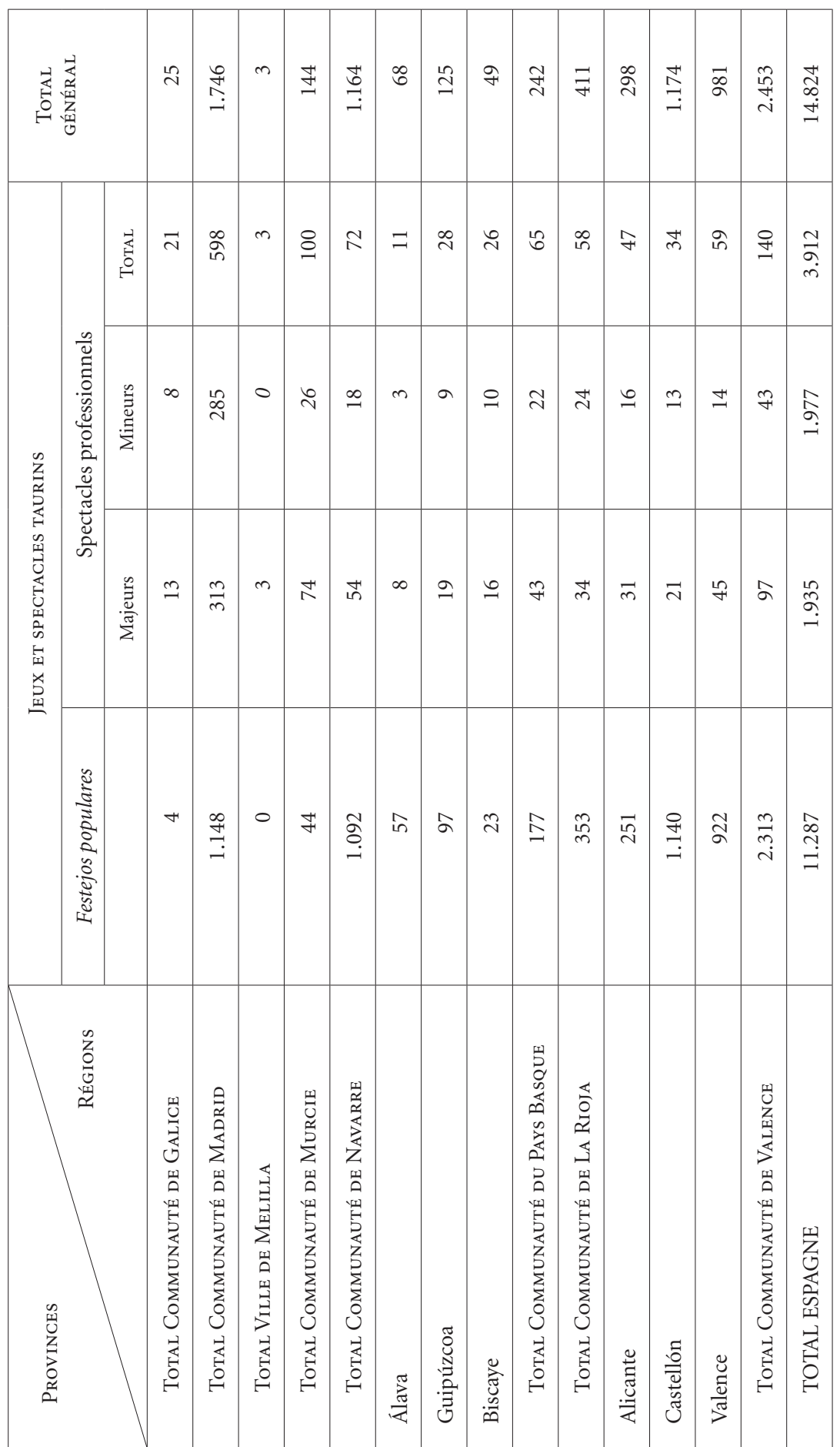


l'année 2003, un récapitulatif de l'ensemble des spectacles taurins majeurs, mineurs et populaires qui se sont déroulés en Espagne ${ }^{3}$.

Retenons d'emblée un ordre de grandeur significatif. En Espagne, plus de 15.000 spectacles et jeux taurins se déroulent chaque année. Sur ce total, environ 4.000 spectacles taurins relèvent de la tauromachie professionnelle $(2.000 \mathrm{spec}-$ tacles majeurs et 2.000 spectacles mineurs) et un peu plus de 11.000 relèvent des divers jeux taurins populaires. En réalité, le chiffre concernant la tauromachie participative doit être réévalué à la hausse puisque dans certaines régions, en particulier dans la Communauté valencienne et en Aragon, les chiffres avancés correspondent au nombre d'autorisations journalières accordées par les services en charge des spectacles publics. Or plusieurs types de jeux et de spectacles taurins se succèdent au cours d'un même " jour de taureau » dans ces régions où la tauromachie participative a une grande importance. Dans la Communauté valencienne, une autorisation de bous al carrer comprend fréquemment un encierro matinal, des lâchers de vaches et des taureaux dans l'après-midi ainsi qu'un toro de fuego en soirée. Les quelque 2.300 autorisations accordées dans les provinces de Castellón et de Valence représentent ainsi un nombre réel de festejos populares de l'ordre de 7.000 spectacles, faisant de cette zone le foyer taurin le plus important d'Espagne au regard de la tauromachie participative. Il en va de même en Aragon où les quelque 1.000 autorisations accordées par les pouvoirs publics correspondent au moins au double de spectacles taurins populaires ${ }^{4}$. Partant de ce principe, le nombre réel de festejos populares en Espagne est plus proche des 17.000 que des 11.000. Le chiffre total des manifestations tauromachiques, toutes pratiques confondues, dépasse donc le seuil des 20.000 spectacles par an. Ce bilan global révèle toute l'importance des pratiques tauromachiques populaires dont l'étude spatiale et quantitative n'a fait l'objet d'aucune recherche approfondie à l'échelle du pays.

Les cartes 2 et 3 permettent d'examiner la distribution spatiale par province des deux grandes formes de pratiques tauromachiques, professionnelle et participative, en 2003 (voir cartes 2 et 3, pp. 222 et 223). Avant de les analyser séparément, formulons une remarque préliminaire. La répartition des spectacles de la tauromachie professionnelle est plus homogène que celle des jeux taurins populaires qui accuse de plus forts contrastes régionaux. Les deux formes de spectacles possèdent une emprise territoriale différente : les spectacles taurins professionnels sont plus nombreux au centre et au sud du pays qu'au nord et à l'est alors que les spectacles taurins populaires sont mieux représentés au centre, au nord et à l'est du pays qu'au sud.

\footnotetext{
${ }^{3}$ L'élaboration de ce tableau a nécessité un travail de collecte d'information étalé sur plusieurs années auprès des services de chacune des Communautés autonomes en charge des spectacles taurins. Il a nécessité, en raison de séries incomplètes, un assemblage de données disparates qui, pour les jeux taurins populaires (festejos populares), n'a pu se faire qu'en complétant les données partielles de 2003 par des données de 2004.

${ }^{4}$ La proportion a été vérifiée à l'aide du fichier papier exhaustif transmis par le service en charge des spectacles publics.
} 


\section{a) Spectacles majeurs et mineurs}

Il n'existe aucune étude menée au niveau national qui prenne en compte la totalité des spectacles de la tauromachie professionnelle. La raison en est simple. Soit les approches statistiques de la tauromachie sont menées au niveau national et ne comptabilisent que les spectacles dits majeurs, soit elles prennent en compte l'ensemble des spectacles majeurs et mineurs, mais sont alors menées à un niveau régional, tout au moins pour les rares régions, l'Andalousie et Madrid, pour lesquelles ces études existent ${ }^{5}$.

Les spectacles taurins, mineurs et majeurs, sont présents dans toutes les Communautés autonomes à l'exception notable des Canaries où la tauromachie est interdite depuis 1991. La tauromachie est également présente dans toutes les provinces sauf celle de Lérida en Catalogne. Au seul point de vue de la répartition, et même s'il existe des écarts provinciaux et régionaux importants, l'appellation de fiesta nacional ne serait pas totalement inappropriée. L'écart entre les régions administratives n'est pas très significatif étant donné le nombre très variable de provinces au sein de chacune d'elles. En effet, il semble peu pertinent de comparer des données entre les Communautés autonomes mono-provinciales (les Asturies, les Baléares, la Cantabrie, La Rioja, Madrid, la Navarre et Murcie) et celles qui en comptent plusieurs, en particulier la région de Castilla-León et l'Andalousie. Remarquons cependant que ni l'Andalousie, qui s'accorde fréquemment ce rang, ni Castilla-León, qui compte le plus de provinces, n'occupent la première place au nombre de spectacles de la tauromachie professionnelle. C'est la région de Castilla - La Mancha qui peut s'en prévaloir avec 952 spectacles répartis sur cinq provinces, suivie de l'autre Castille avec 762 spectacles et de l'Andalousie avec 702 spectacles.

L'analyse de la répartition des spectacles à l'échelon provincial permet une approche beaucoup plus fine. Par notre expérience de la géographie taurine espagnole, le seuil des vingt spectacles par an nous semble assez significatif pour isoler les provinces où la tauromachie professionnelle constitue un phénomène socioculturel négligeable. La seule entorse à ce découpage est le cas du Pays Basque qui possède des territoires provinciaux beaucoup moins étendus que la moyenne. Pour cette raison, ils n'ont pas été désolidarisés, sans quoi le Pays Basque serait exclu à tort des régions où la tauromachie a quelque importance. À l'inverse, il n'est pas possible de définir un seuil à partir duquel la tauromachie professionnelle constituerait un phénomène socioculturel de premier ordre qui reposerait seulement sur le nombre total de spectacles, sans le détail qualitatif de leur composition, ainsi que d'autres paramètres, qu'ils soient matériels tels que le nombre d'arènes et d'élevages, ou plus immatériels et difficilement quantifiables telle que l'afición.

\footnotetext{
5 À l'époque de l'enquête, seules deux Communautés autonomes élaborent un véritable rapport statistique de synthèse sur les spectacles taurins: l'Andalousie (Dirección GENERAL de Espectáculos Públicos y Juego de la Junta de Andalucía, Estadísticas taurinas de la Comunidad Autónoma de Andalucía) et la région de Madrid, par l'intermédiaire du Collège des vétérinaires (Colegio OfiCIAL de Veterinarios de Madrid, Memoria espectáculos taurinos de la Comunidad de Madrid).
} 


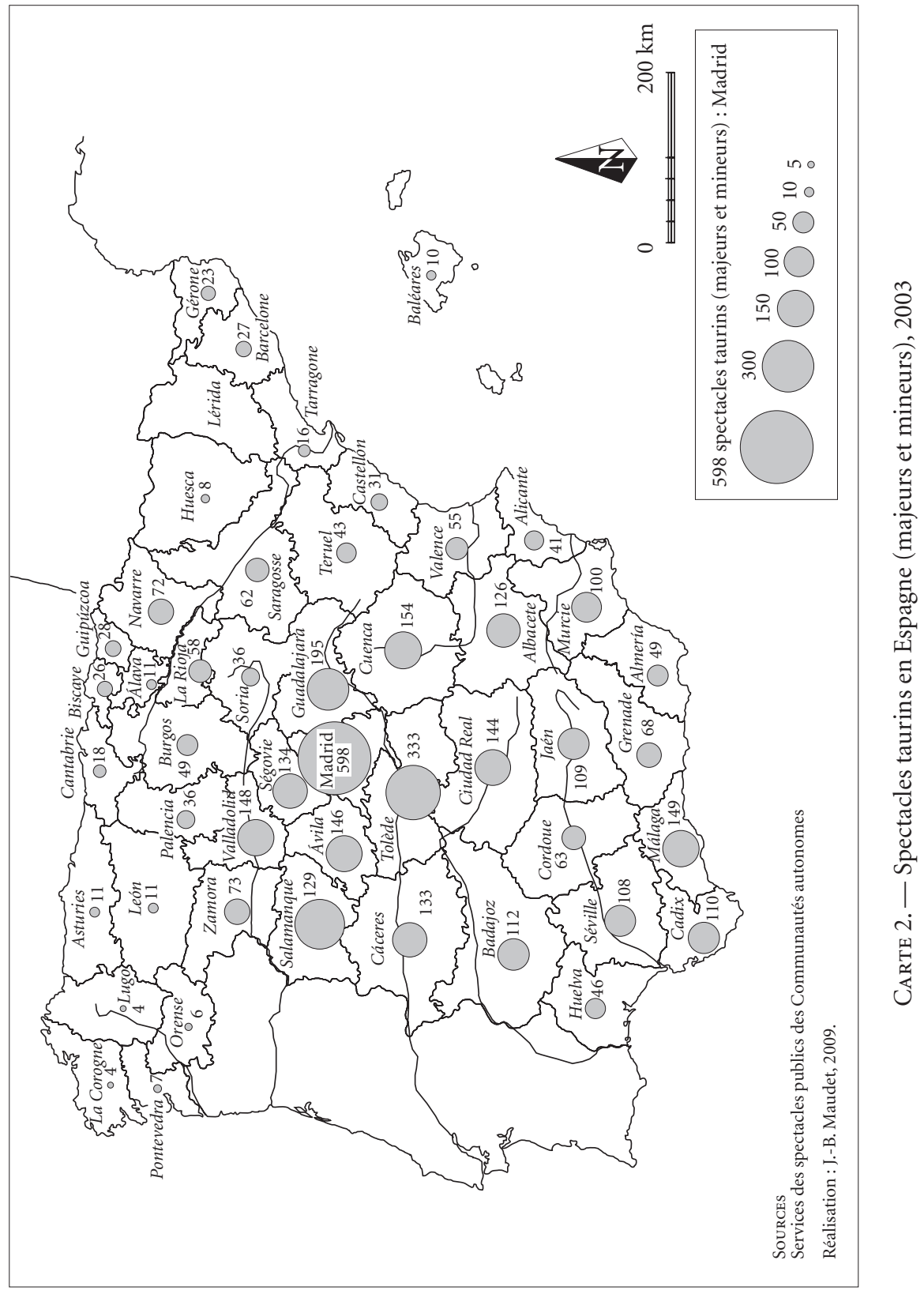




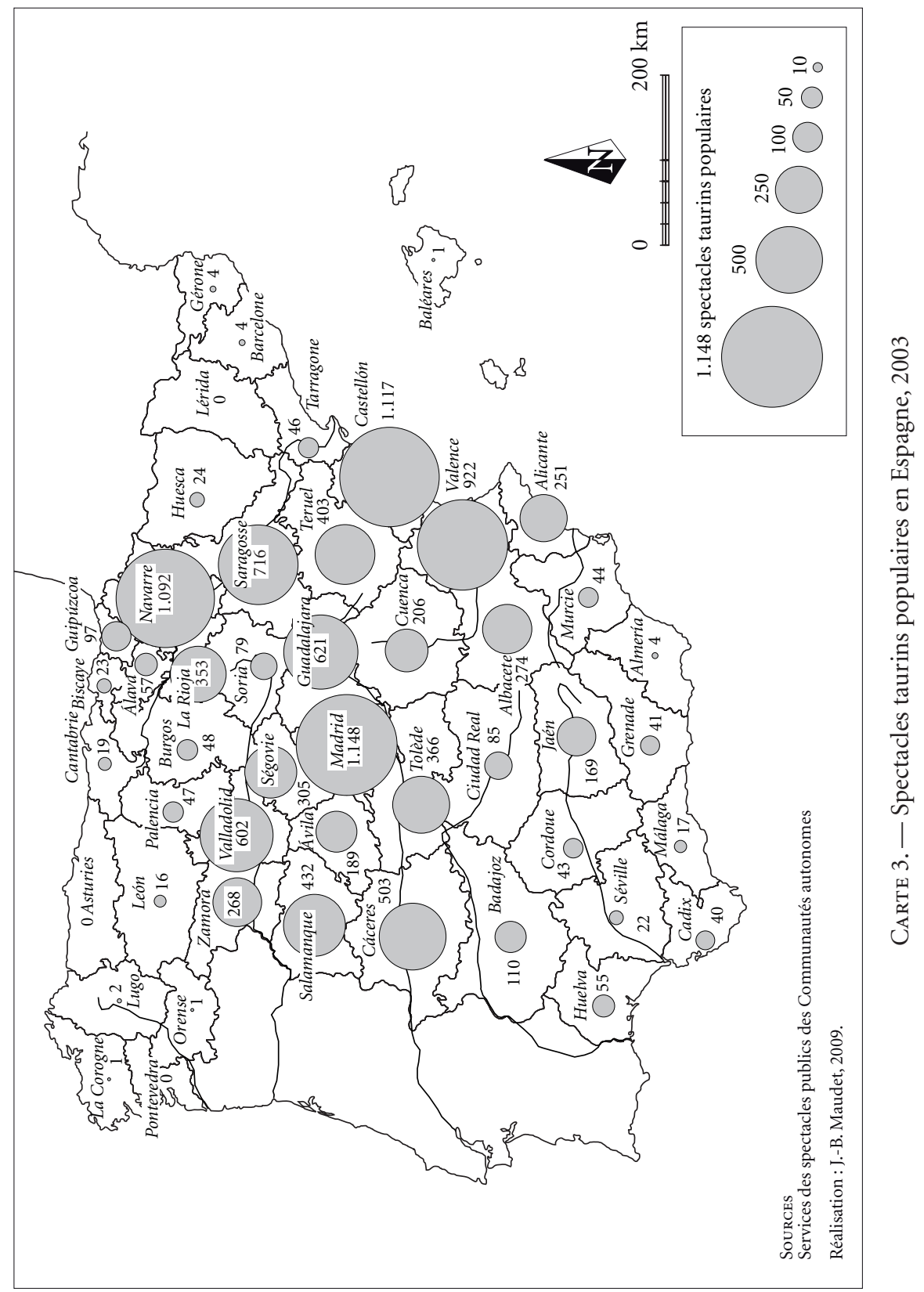


La moyenne nationale du nombre de spectacles par province tourne autour de 80 . Elle monte à une centaine si l'on ne tient pas compte des provinces où se déroulent moins de 20 spectacles. Le poids de la Castille apparaît alors très significatif avec de nombreuses provinces qui programment autour de 150 spectacles et plus, à commencer par Madrid avec un record national de 598 spectacles. Suivent Tolède (333), Guadalajara (195), Cuenca (154), Valladolid (148), Ávila (146), Ciudad Real (144). Ailleurs, seule Málaga, en Andalousie, se hisse à ce niveau, en raison de l'importance prise par la tauromachie dans les stations balnéaires. On trouve ensuite de nombreuses provinces qui programment un nombre de spectacles supérieur à la moyenne avec une répartition plus éclatée : les deux provinces d'Estrémadure, Cáceres (133) et Badajoz (112), quatre provinces andalouses, Cadix (110), Jaén (109), Séville (108), la dernière province de Castilla - La Mancha, Albacete (126), deux provinces de Castilla-León, Ségovie (134) et Salamanque (129), et enfin la province de Murcie (100). Cette répartition confirme que les espaces où les spectacles taurins sont rares sont situés dans le Nord et plus précisément en ses extrémités nord-ouest (Galice, Asturies, Cantabrie, León) et nord-est (Catalogne et Haut Aragon). Le rien de nouveauté vient de l'Est, qui d'ordinaire n'est pas perçu comme une région où la tauromachie professionnelle est faiblement développée, sans doute en raison du rayonnement des arènes de Castellón et surtout de Valence, alors que le nombre total de spectacles à l'échelle régionale est en réalité assez faible. Enfin, on mesure, d'un point de vue quantitatif, tout le poids de la Castille, ce qui peut paraître surprenant au regard des représentations dominantes de l'espace tauromachique marquées par un fort tropisme andalou.

L'espace tauromachique vu sous l'angle des spectacles majeurs, les seuls qui comptent véritablement aux yeux des aficionados et des professionnels, change de physionomie (voir carte 4, p. 225). À partir des données du ministère de l'Intérieur, nous pouvons en détailler la composition (voir graphique 3, p. 226) ${ }^{6}$.

En dépit de l'image dominante d'une tauromachie espagnole fondamentalement pédestre, on observe que le rejoneo, la forme espagnole de la tauromachie à cheval, est présent dans plus d'un spectacle sur cinq. Ce spectacle est en forte progression depuis le début des années 1990 et constitue une valeur très sûre pour les organisateurs de féria en termes de fréquentation du public. C'est un phénomène que l'on observera dans beaucoup d'autres pays et qui témoigne d'une transformation importante de la culture taurine, liée à la progression d'un intérêt pour les tauromachies équestres. En revanche, la corrida portugaise, dans l'intégralité de son cérémonial, faisant alterner le travail des cavaliers et celui des forcados, reste ici un spectacle très rare. Les statistiques de 2004 pointent un zéro dans une colonne intitulée non sans quelque provocation : corrida de rejoneo a la usanza portuguesa. Cela ne veut pas dire que les cavaliers

\footnotetext{
${ }^{6}$ Pour l'année 2004, Mundotoro a recensé 1.967 courses. Sur la base des données recueillies auprès des Communautés autonomes, nous avons recensé un total de 1.935 spectacles majeurs. Précisons, au sujet des spectacles de rejoneo, que 142 ont été des spectacles de taureaux adultes et 218 des spectacles de novillos.
} 


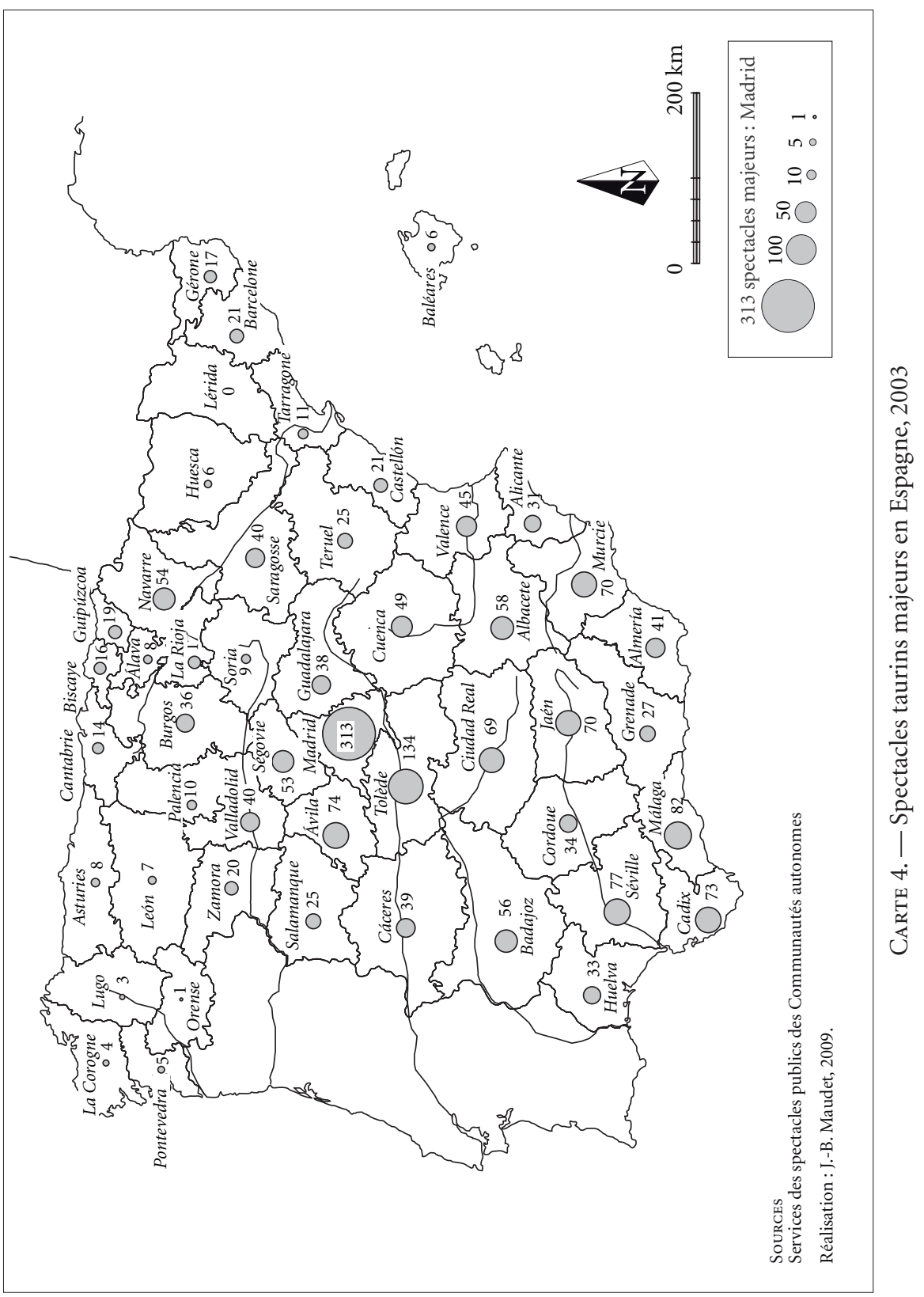


portugais ne viennent pas toréer en Espagne. Certains, bien intégrés au marché espagnol, sont à l'affiche des corridas de rejoneo des plus grandes férias. Leur prestation se terminant alors par la mise à mort du taureau, exercice dans lequel il est de bon ton de dire qu'ils n'excellent pas.

\section{GraphiQue 3. - Spectacles majeurs en 2004 (Espagne)}

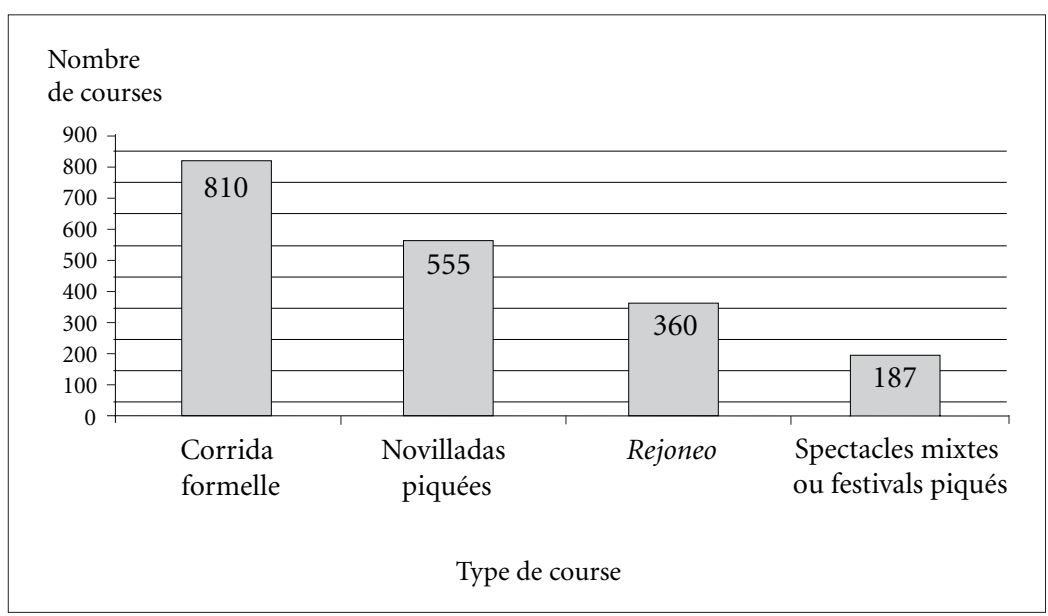

La moyenne des spectacles majeurs tourne autour de 40 par province. Elle s'élève à 50 spectacles si l'on exclut les provinces où nous avons avancé l'idée d'une importance négligeable du fait taurin. Remarquons que l'Andalousie remonte dans la hiérarchie puisqu'elle possède quatre provinces à programmer plus de 70 spectacles sur les neuf que possède l'Espagne : Málaga (82), Séville (77), Cadix (73) et Jaén (70). En revanche, la Castille perd de son importance relative malgré Madrid, nettement en tête avec 313 courses, suivie par Tolède (134), Ávila (74) et Ciudad Real (69). Par conséquent, l'assise territoriale des spectacles majeurs se déplace vers le Sud du territoire. Pour autant, la place de la Castille centrale n'est pas véritablement remise en cause dans cette hiérarchie. Madrid et ses provinces limitrophes concentrent plus de $30 \%$ des spectacles majeurs.

La place de la Castille dans le bilan global de la tauromachie professionnelle est due en grande partie aux provinces dont le nombre de spectacles mineurs est supérieur à celui des spectacles majeurs. Cette situation, très rare ailleurs, est systématique en Castilla - La Mancha et largement dominante en Castilla-León. Dans certaines provinces, le nombre de spectacles mineurs représente plus du double du nombre des spectacles majeurs. C'est le cas à Salamanque (108 contre 25), à Guadalajara (157/38), à Valladolid (108/40), à Cuenca (105/49), à Zamora (53/20) et dans une seule autre province en dehors de la Castille, à Cáceres (94/39). Ces écarts témoignent d'un circuit taurin de deuxième catégorie très étoffé. Ces spectacles sont à mettre en relation avec la proximité de zones d'élevages de taureaux de combat : les élevages du campo charro de la province 
de Salamanque, ceux du Nord de la province madrilène et dans une moindre mesure ceux de la province de Cáceres. À l'inverse de ce modèle, les provinces andalouses, et en particulier Séville et Cadix, sont celles qui présentent les plus forts taux de spectacles majeurs. Observons qu'il s'agit aussi d'une zone importante d'élevages de taureaux de combat qui fournit à la fois le circuit taurin de première catégorie de toute l'Espagne et une grande part du marché local. Cela explique en partie l'épanouissement conjoint des élevages et des spectacles majeurs de la Basse Andalousie.

Parmi les spectacles majeurs, s'il ne fallait retenir que les corridas de toros (courses de taureaux de quatre ans révolus), on constaterait à nouveau le renforcement du poids relatif des provinces de la Basse Andalousie. Pour l'année 2003, quatre provinces andalouses ont proposé plus de 30 corridas (Málaga, Cadix, Séville, Jaén) sur les huit provinces espagnoles dans ce cas. Les quatre autres sont Madrid, Murcie, Ciudad Real et Tolède. On l'aura compris, dans une logique qui est celle d'une régionalisation qualitative des spectacles taurins et en dehors de toutes différenciations entre les arènes, plus les spectacles sont importants dans la hiérarchie de l'afición et plus l'Andalousie s'affirme au côté d'un noyau central madrilène, de moindre extension, mais de plus forte densité. La place de Madrid est d'autant plus affirmée que la centaine de novilladas non piquées, classées dans les spectacles mineurs, qui ont lieu chaque année dans la région, affiche souvent une qualité et un volume de taureaux qui n'ont rien à envier à de nombreux spectacles majeurs présentés ailleurs. La région de Madrid avec ses quelque 600 spectacles taurins majeurs et mineurs, c'est-à-dire plus d'un quart du total espagnol, s'affirme comme un foyer de densité tauromachique inégalé.

b) Les spectacles taurins populaires

Il s'agit assurément de l'aspect le plus méconnu et le moins étudié de la tauromachie espagnole qui jusqu'à une date récente passait largement inaperçu du plus grand nombre, comme s'il était question d'une pratique sinon clandestine, du moins en partie occultée. C'est que les autorités politiques ont pendant longtemps tenté de les supprimer pour des raisons qui ont d'ailleurs évolué selon les époques. Rappelons qu'il faut attendre le 10 mai 1982 pour que les festejos taurinos populares soient officiellement reconnus et autorisés. La reconnaissance institutionnelle a aussitôt entraîné une différenciation territoriale des réglementations et des évolutions divergentes concernant le maintien, le développement ou au contraire la régression des spectacles taurins populaires en Espagne.

Tous les jeux taurins populaires étant soumis à une autorisation préalable de la part des pouvoirs publics de chacune des régions autonomes, nous avons pu recenser le nombre de festejos populares (voir tableau 2, pp. 216-219). Comme nous l'avons évoqué précédemment, la localisation dominante des pratiques tauromachiques populaires possède une assise territoriale plus septentrionale et orientale que celle des spectacles de la tauromachie professionnelle. La distribution provinciale des jeux taurins populaires accuse des contrastes beaucoup plus forts que celle des spectacles majeurs et mineurs (voir carte 3, p. 223). En effet, dans certaines provinces ces pratiques se comptent en milliers (Madrid, Cas- 
tellón, La Navarre, Valence) alors qu'elles ne dépassent pas la vingtaine dans une douzaine d'entre elles. La corrélation globale entre tauromachie professionnelle et tauromachie participative est assez forte, dans le sens où aucune province dont le nombre de spectacles majeurs est très faible ne possède un nombre élevé de festejos populares qui en feraient un foyer taurin d'importance. La distribution des provinces où les festejos populares sont rares recoupe donc assez largement celle où la tauromachie apparaît, d'une manière générale, comme un phénomène négligeable, à savoir les extrémités nord-est et nord-ouest de la Péninsule précédemment identifiées. Le grand changement de la géographie des spectacles vue sous l'angle des jeux populaires est l'affirmation massive du Levant et du Nord. Les provinces de Castellón et de Valence, relativement discrètes dans la hiérarchie des spectacles professionnels, apparaissent ici au contraire comme un foyer de première importance avec respectivement 1.117 et 922 « jours de taureaux populaires». Il en va de même avec la Navarre (1.092) et, dans une moindre mesure, les provinces aragonaises de Saragosse (716) et de Teruel (403), ainsi que celle de La Rioja (353). Se dessine ainsi un arc de forte densité taurine qui suit la vallée de l'Èbre et bifurque vers le Sud par le Maestrazgo, en direction des pays valenciens. Ce foyer est d'autant plus important que les données chiffrées, comme nous l'avons indiqué, sous-évaluent le nombre réel de manifestations en Aragon et dans la région de Valence.

Parmi les éléments qui confirment les tendances lourdes de la répartition des spectacles professionnels, on observe que Madrid, avec 1.148 festejos populares, continue d'être une région de forte densité taurine, de même que les deux Castilles dans leur ensemble. Cependant, dans le détail, les hiérarchies internes sont bousculées. Guadalajara avec 621 festejos populares s'affirme comme la province la plus taurine de Castilla - La Mancha et Tolède (366) consolide son poids. Valladolid avec 602 festejos populares creuse l'écart avec les autres provinces de Castilla-León alors que Salamanque (432) et Ségovie (305) remontent dans la hiérarchie. Un phénomène comparable apparaît en Estrémadure. Alors que Badajoz est en tête quant au nombre de spectacles majeurs (56 contre 39), elle passe au second plan par l'importance des spectacles mineurs à Cáceres (94 contre 56) et se voit largement dépassée quant au nombre de festejos populares (503 contre 110). Enfin, l'Andalousie dans son ensemble voit son importance diminuer considérablement avec un total de 391 festejos populares. Notons que l'Andalousie rassemble les seules provinces où le nombre de festejos populares soit inférieur aux spectacles majeurs et mineurs. Seules la province de Jaén et, dans une bien moindre mesure, celle de Huelva font exception à cette règle andalouse de la tauromachie. Cette anomalie au regard de la plupart des profils taurins des autres provinces espagnoles appelle une interprétation. Faut-il conclure à la faiblesse intrinsèque de la tauromachie participative ou bien émettre l'hypothèse que toréer sur le modèle canonique de la corrida demeure la forme d'expression tauromachique la plus populaire ? La réponse est complexe, mais redonne à l'Andalousie toute son originalité au sein de l'espace taurin espagnol puisque les spectacles du haut de la hiérarchie (437) sont toujours mieux représentés que ceux du bas, qu'ils soient mineurs (265) ou populaires (391). 
GraphiQue 4. - Encierros et vaquillas en Castille

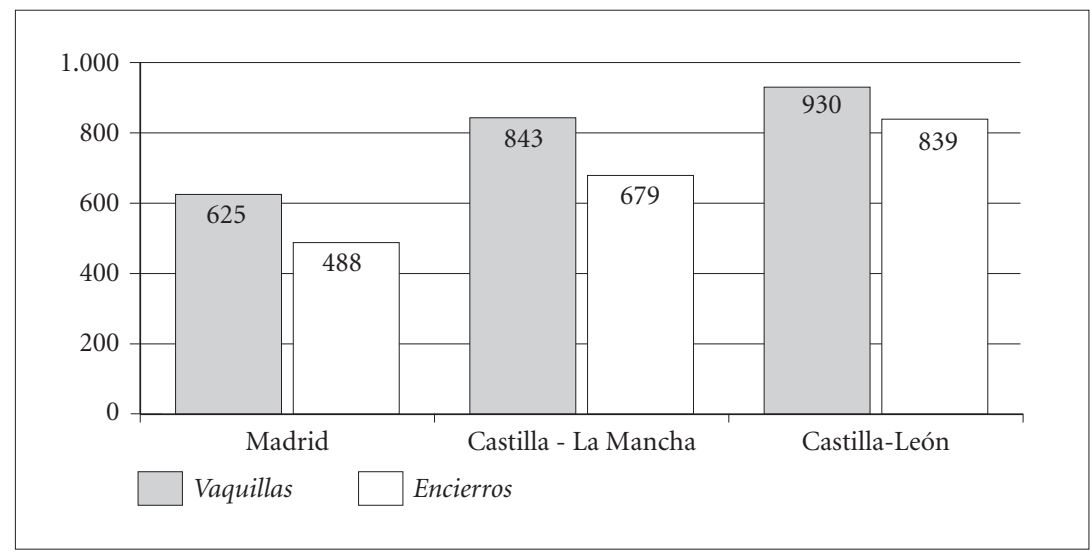

Graphique 5. - Encierros et vaquillas en Castille par province

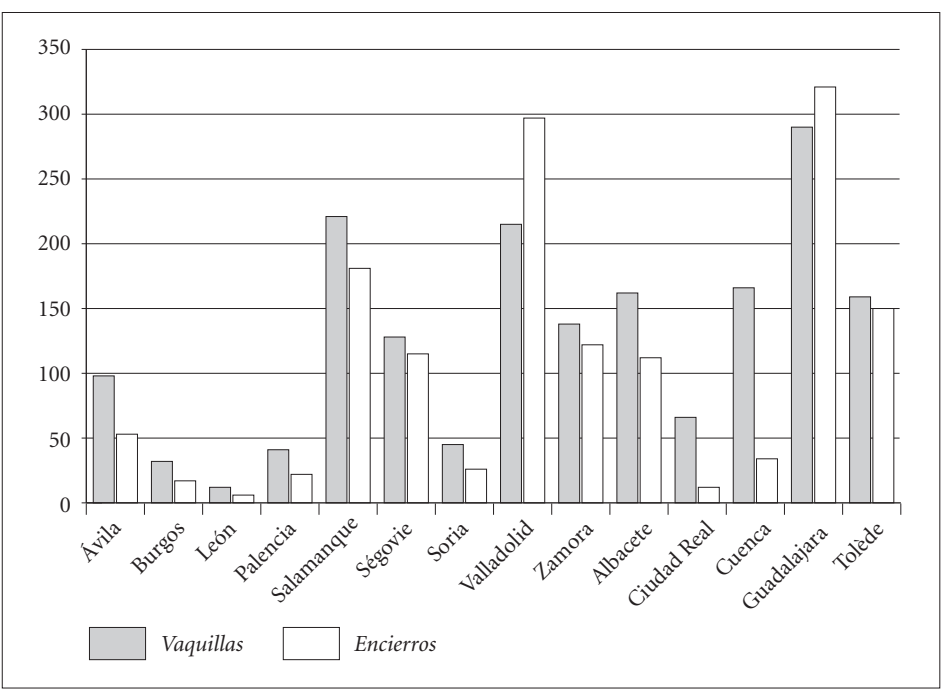

Un aspect important de la diversité des spectacles taurins échappe en grande partie à ce bilan global ainsi présenté : le nombre et la répartition des concours de recortadores. Le nombre de concours de recortadores dépasserait les $500 \mathrm{spec}$ tacles par an, chiffre qu'il est difficile d'établir avec exactitude, car ils sont classés tantôt dans les spectacles mineurs, tantôt dans les spectacles populaires, dont le détail est rarement donné. Seules les données de quelques régions nous permettent de préciser localement leur nombre. Dans l'année 2004, se sont déroulés 108 concours de recortadores en Castilla-León, 123 en Castilla - La Mancha, 
9 dans La Rioja et 9 dans la région de Murcie. Manquent ici les espaces qui concentrent plus de la moitié des spectacles de recortadores : le cours moyen de l'Ėbre au niveau de la frontière entre La Rioja, la Navarre et l'Aragon, et les provinces du Levant, en particulier celles de Castellón et de Valence.

Les données détaillées de certaines régions nous permettent d'affiner encore l'analyse quant à la nature précise des festejos populares programmés. Nous l'avons vu, exception faite du cas particulier des spectacles de recortadores souvent comptés avec les festejos populares, cette catégorie se partage en deux grandes formes de jeu : les encierros et les lâchers de bovins en lieu clos fréquemment désignés sous l'appellation générique de vaquillas. La distribution des encierros attire plus particulièrement notre attention en ce qu'ils représentent la forme ludique, sociale et scénographique la plus éloignée des corridas formelles. Les données dont nous disposons ne nous permettent pas d'en dessiner la géographie avec la même précision que celle des festejos populares dans leur ensemble. Cependant nous possédons assez d'informations pour pouvoir affirmer que le Sud de l'Espagne se trouve marginalisé bien plus encore que ne l'a révélé la répartition des festejos populares dans leur totalité. En effet, les encierros sont bien rares en Andalousie. Le journaliste Ángel Cervantes, dans son ouvrage Las rutas del toro en Andalucía recense 217 festivités où l'on donne des jeux taurins populaires ${ }^{7}$. Sur ce total, seulement 22 programment des encierros dont 11 dans la province de Jaén qui, il est vrai, concentre $43 \%$ des festejos populares andalous. Même augmenté du nombre de jours de fêtes où il existe la possibilité de voir ces encierros se répéter quotidiennement, leur nombre total et leur proportion au regard des autres jeux taurins populaires paraît très faible en comparaison des autres régions. Pour autant, leur exercice n'est pas réservé, loin s'en faut, à la seule Navarre. En particulier, le nombre d'encierros en Castille est très élevé comme le montrent les graphiques 4 et 5 (p. 229).

Pour les deux provinces qui possèdent le plus grand nombre de festejos populares, Guadalajara et Valladolid, le nombre d'encierros est plus élevé que celui des vaquillas. Guadalajara compte 321 encierros pour 290 vaquillas et Valladolid compte 297 encierros pour 215 vaquillas. Ces chiffres confirment que l'encierro traduit une dimension populaire de la tauromachie, au double sens de son succès et de son caractère participatif. Nous ne connaissons malheureusement pas le nombre exact d'encierros pour les autres provinces du Nord et du Levant, riches en jeux taurins populaires. Tous les observateurs confirment leur importance en Navarre. Il faut imaginer leur nombre élevé, si l'on en croit un bilan global communiqué par la CCNAT pour l'année 1996 qui chiffre les encierros en Espagne à 7.260 contre 6.530 vaquillas, soit la pratique tauromachique populaire la plus importante entre toutes. Cependant notre expérience du labyrinthe statistique des pratiques tauromachiques espagnoles nous incite à la plus grande

\footnotetext{
${ }^{7}$ Á. Cervantes et J. E. Moreno, Las rutas del toro en Andalucía, p. 143. Ce travail complète une liste antérieure établie par Pedro Romero de Solís qui en dénombrait 158. P. Romero DE Solís, «Las fiestas de toros en Andalucía ». Francisco López Izquierdo en 1993 en recensait 172. F. López IzQUiERDo, Fiesta Nacional.
} 
prudence quant à l'utilisation de chiffres globaux sans connaître les modalités précises de comptage.

Que conclure de cette analyse détaillée de la distribution géographique des spectacles et des jeux taurins populaires? Il existe visiblement une différenciation Sud-Ouest / Nord-Est dans la répartition des types des manifestations tauromachiques qui semble diviser l'Espagne en quatre zones :

- l'Andalousie qui se caractérise, en dépit de la diversité des pratiques tauromachiques, par la prééminence des spectacles majeurs ;

- la Castille qui se caractérise par un profil plus équilibré entre tauromachie professionnelle et tauromachie participative, avec Madrid comme principal foyer de densité tauromachique ;

— un arc Nord-Est qui s'étend de la Navarre au Levant caractérisé par l'importance de la tauromachie participative ;

- les deux extrémités Nord-Est et Nord-Ouest de la Péninsule (la Galice, les Asturies, la Catalogne) qui se caractérisent par la faiblesse de la tauromachie.

Cette analyse ne peut suffire. La compréhension du fonctionnement de l'espace tauromachique exige qu'on aborde la répartition et la différenciation des spectacles en fonction des lieux de mise en scène qui entretiennent entre eux des relations hiérarchiques et réticulaires.

\section{UN ESPACE HIÉRARCHISÉ :}

ARÈNES ET AUTRES LIEUX DE SPECTACLES

a) Différenciation et recensement des arènes

Il existe de nombreux critères différenciant les arènes espagnoles. Le critère le plus évident est la taille des arènes, plus précisément, leur contenance. Elle varie grandement entre une petite arène de village composée d'un simple muret, pouvant accueillir une centaine de spectateurs dont la plupart reste debout et une arène dite monumentale pour laquelle le seuil des 10.000 personnes est communément atteint. La plus grande arène d'Espagne est celle de Las Ventas à Madrid avec environ 24.000 spectateurs. La forme est un autre critère. Même si le cercle est la forme canonique de l'arène ibérique, il existe bien d'autres formes d'arènes, irrégulières, polygonales, rectangulaires. Les formes quadrangulaires et polygonales correspondent généralement à des stades intermédiaires qui précèdent l'affirmation définitive de la forme circulaire. D'une manière générale, la taille, les formes et les styles architecturaux offrent un vaste champ de recherche permettant de retracer les étapes de leurs évolutions déterminées par des principes de construction, des modes esthétiques, des nécessités scénographiques, la consécration sociale d'un spectacle de masse 
et la recherche d'une rentabilité accrue ${ }^{8}$. Le dernier stade de cette évolution se traduit aujourd'hui par l'augmentation du nombre d'arènes couvertes et polyvalentes qui aux yeux de beaucoup d'aficionados portent atteinte à l'authenticité du spectacle. Et d'aucuns de s'insurger contre ces nouvelles arènes «qui ressemblent à des volières, qui résonnent comme des piscines municipales où l'on ne pourra bientôt même plus allumer son cigare en paix »". Soulignons par là, en dépit des complaintes inhérentes à toute innovation, que l'on continue en ce début de $\mathrm{XxI}^{\mathrm{e}}$ siècle à bâtir des arènes.

L'une des difficultés à dater les plazas de toros et à les recenser avec précision réside dans le fait que certaines places urbaines servent d'arènes et ont d'ailleurs été édifiées pour pouvoir accueillir un spectacle, qu'il soit tauromachique ou non. Le modèle de la plaza mayor est sur ce point le meilleur témoignage d'une telle intention urbanistique. En 1992, l'historien Francisco López Izquierdo tente de recenser les arènes en Espagne dans un ouvrage de référence : Plazas Mayores y de toros ${ }^{10}$. C'est pour insister sur l'originalité de cet édifice que l'auteur a choisi ce titre curieux. Il s'en justifie aux premières lignes de l'introduction :

Peut-être que ce titre surprend. C'est le plus logique. Je m'explique. Les fêtes taurines se déroulaient autrefois sur les Plazas mayores. Et quand les premières Plazas ad hoc pour combattre les taureaux ont été édifiées, celles-là ont, la plupart du temps, cessé de fonctionner comme arènes taurines, mais dans de nombreux villages modestes, elles ont continué jusqu'à aujourd'hui à les célébrer en leur sein ${ }^{11}$.

La plaza mayor de Chinchón est l'illustration parfaite et la plus connue de cette configuration (voir fig. 49). Cette place à arcades, du Xvi siècle, est constituée de maisons de trois étages à balcons : les jours de taureaux, elle se change en arène d'environ 5.000 places. De gros pavés non fixés sont retirés pour recevoir les madriers de bois permettant d'agencer la structure. Une barrière sépare la piste des gradins aujourd'hui métalliques qui rejoignent la base des balcons. Cet exemple, en dehors de sa valeur historique qui témoigne de l'évolution architecturale des plazas de toros, éclaire les réelles difficultés à recenser de manière exhaustive les arènes espagnoles. Faut-il considérer que Chinchón possède une arène ? Si oui, elle est invisible en dehors des jours de spectacles, et les balcons ne deviennent des loges que pour ceux qui l'ont vu montée. Pour ceux-là, place publique et arènes sont indissociablement liées et il va de soi que Chinchón possède une arène. Pour les autres, ils visiteront Chinchón sans que rien n'indique sa présence. Quoi qu'il en soit, les arènes bâties temporairement dans le tissu

\footnotetext{
${ }^{8}$ Citons, parmi d'autres, un article érudit et richement illustré : A. Demas et J. LeCCiA, « Architecture et tauromachie».

${ }^{9}$ Témoignage recueilli à l'Albero après une corrida, sous la pluie, où la question des arènes couvertes est naturellement venue sur le comptoir.

${ }^{10}$ F. López Izquierdo, Plazas Mayores y de toros.

${ }^{11}$ Ibid., p. 9.
} 


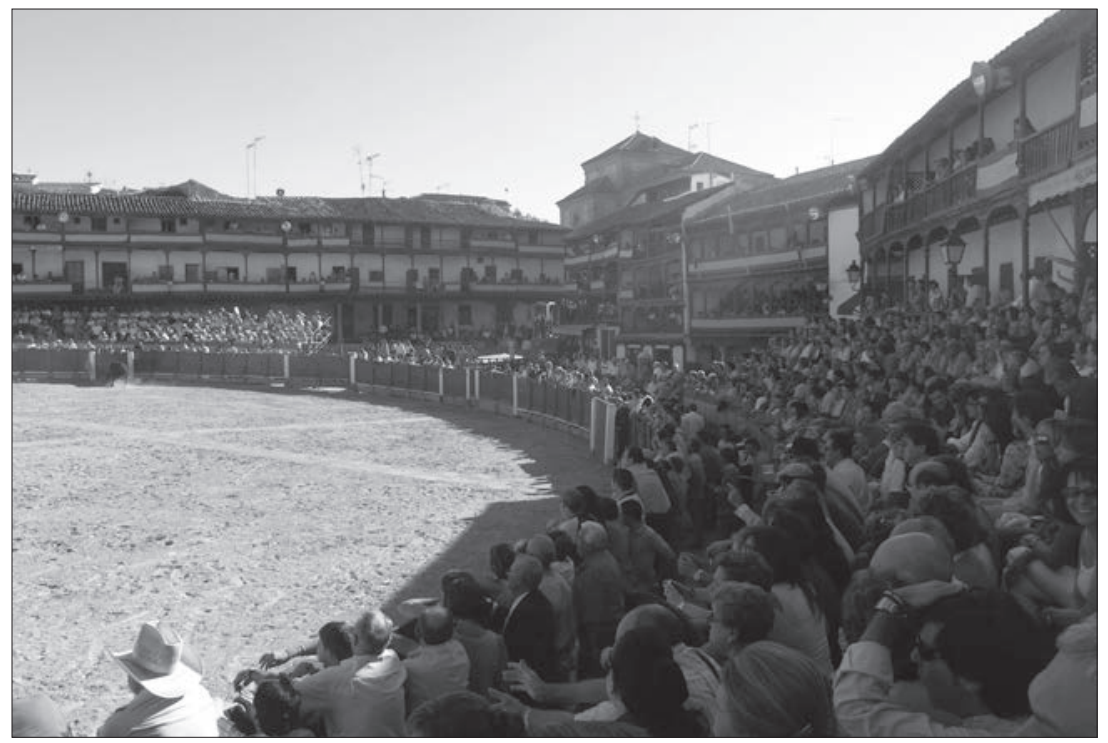

Fig. 49. - Plaza de toros de Chinchón. Arènes de Chinchón montées pour le spectacle. Balcons servant de loges aux couleurs de l'Espagne. Pavés recouverts de sable. Contre-piste envahie par le public adossé à la barrière (Chinchón, 2006, cliché : Jean-Baptiste Maudet).

urbain ouvert par la place publique constituent une catégorie particulière de lieux de mise en scène éphémère et périodique de la tauromachie.

Plus prosaïquement, la CCNAT distingue officiellement quatre types de « $\mathrm{Pla}$ $z a s$ de toros et autres enceintes aptes au déroulement de spectacles taurins » : les arènes permanentes, les arènes non permanentes, les arènes transportables et les autres enceintes. Le premier type correspond aux édifices permanents, construits en dur spécifiquement pour le spectacle taurin. Le deuxième type correspond aux édifices et enceintes qui, bien que n'ayant pas comme principale fonction d'être un lieu de spectacle taurin, sont habilités à le devenir temporairement comme Chinchón (Madrid) ou Navas de Tolosa (Jaén). Le troisième type correspond aux arènes dites portatiles, démontables et transportables, métalliques ou en bois, qui répondent aux exigences d'hygiène et de sécurité établies par le règlement. Un cas limite d'arène est représenté par les arènes démontables, soit qui ne voyagent pas, car elles appartiennent à la municipalité, soit qui ne se démontent plus, leur induration ne permettant plus de les distinguer véritablement des arènes permanentes. Le quatrième type correspond aux enceintes de toute nature qui répondent aux normes autorisant les capeas pour « la promotion et le divertissement de l'afición » et les arènes des écoles taurines. Les arènes privées des élevages destinées aux tientas ne dépendent pas du règlement. Il y en a presque autant qu'il existe d'élevages. Cette diversité n'est évidemment pas faite pour simplifier un recensement exhaustif.

Le règlement taurin définit ensuite des catégories d'arènes qui impliquent des normes différentes pour le déroulement légal du spectacle concernant les condi- 
tions architecturales et le poids minimal des taureaux. Cette classification qui est établie en fonction du nombre et du type de spectacles, mais aussi en fonction de facteurs historiques, introduit une hiérarchie qualitative entre les arènes. Pour les implications spatiales de cette hiérarchie, l'article 23 qui justifie cette classification mérite qu'on s'y attarde. Pourront être classées en première catégorie les arènes des capitales de provinces et des villes qui célèbrent plus de quinze spectacles par an, parmi lesquels au moins dix doivent être des corridas de toros. À ce jour, font partie des arènes de première catégorie les arènes de Barcelone (la Monumental, la Plaza Arenas n'existant plus en tant que telle), de Bilbao, de Cordoue, de Las Ventas à Madrid, de Pampelune, de Saint-Sébastien, de Séville, de Saragosse et de Valence. Seront considérées comme arènes de deuxième catégorie, les arènes des capitales de provinces n'appartenant pas à la première catégorie, ainsi que celles classées par les organismes compétents, c'est-à-dire le ministère de l'Intérieur par ses délégations régionales et la CCNAT. Font actuellement partie des arènes de deuxième catégorie celle d'Algésiras, d'Aranjuez, de Carabanchel à Madrid, de Carthagène, Gijón, Jerez de la Frontera, Linares, Mérida, Puerto de Santa María, Plasencia et celles de toutes les capitales de provinces à l'exception de Lugo, qui n'en possède pas, et de Cadix, qui n'en possède plus ; particularité gaditane dans une région pourtant éminemment taurine. Les autres arènes seront incluses dans la troisième catégorie, les non permanentes et les transportables, puis la quatrième, les autres enceintes. Pourquoi l'emploi du futur ? À la demande des mairies, la classification peut être modifiée par le ministère de l'Intérieur, en fonction de " la tradition, du nombre et de la catégorie des spectacles ». Cette hiérarchie est donc en partie évolutive.

La question élémentaire " combien existe-t-il d'arènes en Espagne ? » est un casse-tête que seules les simplifications outrancières résolvent en un chiffre. Pour y répondre nous avons croisé un nombre important de sources :

— la liste « officielle » de la CCNAT dont nous avons pu vérifier que la précision des données est très variable d'une région à l'autre. Par exemple, la liste est d'une grande fiabilité pour les arènes de Castilla - La Mancha, alors qu'elle est très incomplète pour celles de Castilla-León. Pour preuve, la province de Zamora n'est créditée que d'une seule arène, celle de la capitale ;

- le recensement effectué par José María Sotomayor qui a collaboré aux listes proposées dans les différentes éditions du Cossío, listes qui de son propre aveu, ne sont pas exhaustives et ne précisent pas toujours s'il s'agit d'une arène permanente en dur ou d'une arène démontable ;

- l'ouvrage de Francisco López Izquierdo précédemment cité qui a l'avantage de compiler les données de plusieurs ouvrages en particulier le Gran diccionario tauromáquico de José Sánchez de Neira publié en 1896, le Diccionario geográfico de España en 17 volumes (1956-1961) et l'ouvrage de Luis Pinto Maeso, publié en 1981, Plazas de toros de España ;

— les listes publiées par l'UCTL; 
— des monographies régionales ${ }^{12}$;

- nos travaux antérieurs sur la question et de nombreuses vérifications directes auprès des municipalités ou à l'aide d'images satellitaires et de photographies aériennes ${ }^{13}$.

À l'issue de cette étude, nous proposons deux réponses complémentaires. La première réponse souhaite rendre compte du nombre d'arènes permanentes construites en dur et encore existantes. Cette réponse aborde la question sous l'angle de l'arène en tant qu'infrastructure pérenne de la tauromachie et marqueur architectural de sa territorialité (voir carte 6, p. 237). Cette réponse se heurte à une difficulté de taille. Si le recoupement des sources utilisées permet de recenser, de façon assez satisfaisante, les arènes qui ont été construites, en revanche, elles ne précisent pas toujours si elles demeurent en place et si elles servent encore. Dans certains cas, les arènes demeurent, mais les spectacles se déroulent dans une arène démontable qui répond mieux aux normes exigées par la réglementation en vigueur. Là où il est mentionné qu'il existe une arène en fonctionnement, rien ne permet alors de distinguer s'il s'agit de l'arène en dur ou de l'arène démontable qui est utilisée.

La seconde réponse entend recenser les lieux de spectacles, que ces spectacles aient lieu dans des arènes permanentes ou démontables (voir carte 5, p. 236). Pour l'Espagne, nous avons limité l'étude aux données de la CCNAT sur les spectacles majeurs, détaillées par municipalité pour l'année 2003. L'annexe I (CD-ROM), contient des informations complémentaires sur les arènes, leur capacité, leur année de construction et certains particularismes.

b) Répartition et foyers de densités

de la tauromachie professionnelle

La carte 6 (p. 237) représente 558 arènes permanentes, une fois éliminées celles que nous pensons aujourd'hui détruites. Sauf erreur, les arènes détruites ne figurent pas sur la carte, mais continuent d'apparaître dans les tableaux de recensement. Sur ces 558 arènes permanentes, 432 ont fonctionné en 2003 pour la programmation d'un spectacle majeur. Cette même année, 833 communes ont programmé au moins un spectacle majeur. Le nombre d'arènes démontables qui ont fonctionné s'élève ainsi à environ 400. Ce type d'arène augmente alors de moitié le nombre total d'arènes en fonctionnement avec un roulement d'environ $25 \%$ tous les 5 ans. La trame taurine n'est donc pas totalement figée.

\footnotetext{
12 R. Aspiazu, Plazas de toros de Bizkaia ; N. Largo Aguado, Toros: Castilla - La Mancha ; A. Maestro Sanjuan, Las plazas de toros de Aragón; F. J. Orgambides Gómez, Las plazas de toros en la provincia de Cádiz; Á. Cervantes et J. E. Moneno, Las rutas del toro en Andalucía.

${ }^{13}$ J.-B. MAudet, Pour une géographie de la "planète des taureaux »; ID., "Les territoires de la planète des taureaux »; ID., Géographie taurine du Sud-Ouest européen. Vérifications des arènes à partir du fonds de photographies aériennes de la Casa de Velázquez, complétées par des images satellitaires disponibles sur le site Internet : www.googlemap.es.
} 


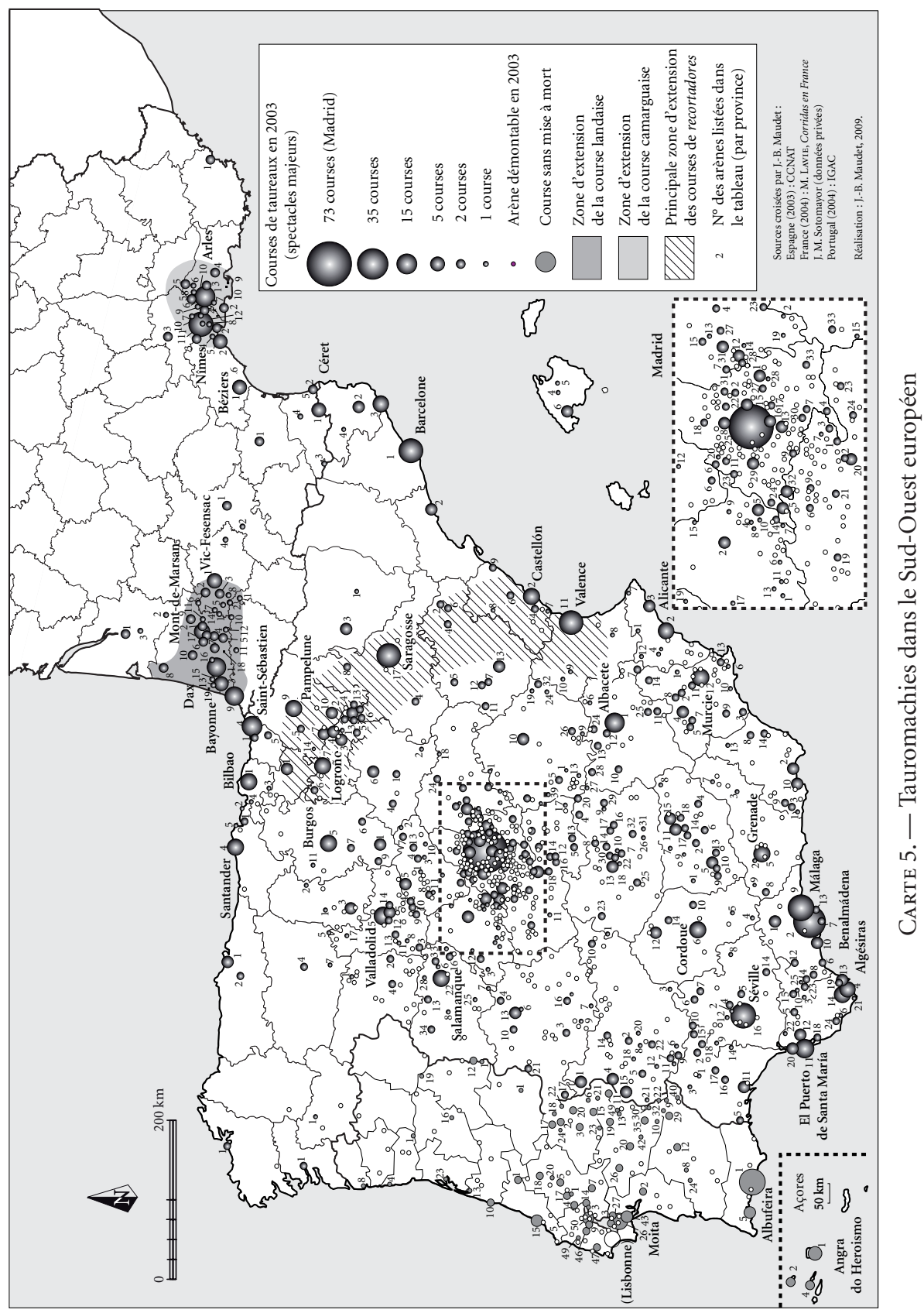




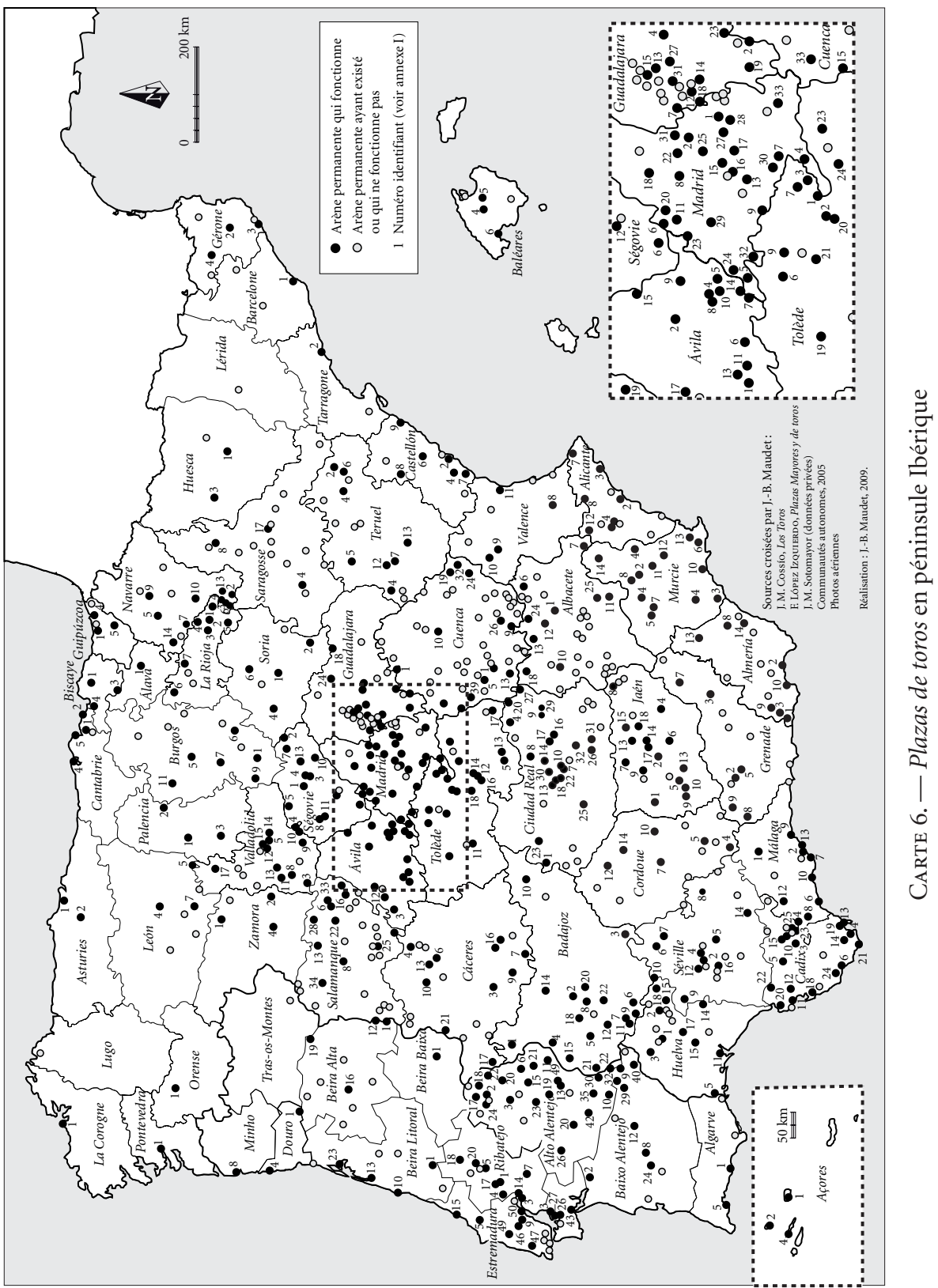


Leur contenance est distribuée de la façon suivante : une cinquantaine possède une contenance supérieure à 10.000 places, 150 arènes contiennent entre 10.000 et 5.000 places, les arènes restantes possèdent moins de 5.000 places. Observons enfin que les $3 / 4$ des spectacles se déroulent dans des arènes de troisième catégorie (environ $40 \%$ dans des arènes permanentes et près de $35 \%$ dans des arènes démontables).

La distribution hiérarchique des spectacles est globalement conforme à une loi rang-taille :

- 7 arènes proposent plus de 20 spectacles, généralement des arènes de première catégorie ;

— 15 arènes offrent entre 10 et 20 spectacles, généralement des arènes de capitales provinciales ;

- 60 arènes offrent entre 5 et 10 spectacles ;

- 324 arènes offrent entre 2 et 5 spectacles ;

— 427 arènes offrent 1 spectacle, ce qui représente un peu plus de $50 \%$ des communes taurines.

On reconnaît dans cette distribution spatiale hiérarchisée des lieux de spectacles une organisation répondant assez clairement à la théorie des "lieux centraux » d'August Lösch et Walter Christaller ${ }^{14}$. Le géographe Jean-Pierre Augustin avait déjà insisté sur la fécondité de la théorie des "lieux centraux" appliquée aux équipements de type sportif ${ }^{15}$. En cela, il reprend les travaux de John Bale proposant une " théorie des lieux sportifs " qui répond à une organisation hiérarchique de type "christallerienne ${ }^{16}$. Appliquée à l'espace tauromachique, elle se résume ainsi : la fonction d'une arène est de fournir un loisir marchand, le spectacle tauromachique. Chaque arène est au centre d'un arrière-pays taurin plus ou moins vaste. Les arènes les plus importantes, celles des grandes villes, peu nombreuses, plus espacées, programment des spectacles professionnels du haut de la hiérarchie, généralement regroupés en féria, destinés à des seuils de populations élevés et des aires d'influences élargies. Les arènes de rang inférieur, plus petites, s'adressent à des seuils de population plus réduits, des aires d'influences moins larges et programment, avec une fréquence moindre, des spectacles taurins moins coûteux. Il existe ainsi pour la tauromachie une sorte de loi rang-taille qui fait correspondre à la hiérarchie des arènes et des divertissements taurins celle de la trame urbaine. Cette organisation hiérarchique participe alors puissamment à la différenciation entre les arènes des grandes villes, celles des petites et celles des bourgs ruraux. Voilà pour la théorie qui, bien évidemment, ne fait que définir les tendances lourdes de

\footnotetext{
${ }^{14}$ W. Cristaller, Die Zentralen Orte; A. Lösch, Die raumlische Ordnung.

${ }^{15}$ J.-P. Augustin, Sport, géographie et aménagement, p. 80.

${ }^{16}$ J. BALE, Sport and Place.
} 
l'organisation hiérarchique de l'espace tauromachique permettant de mesurer les écarts, les variations régionales et les particularismes.

Les cartes 5 et 6 (pp. 236 et 237) permettent également d'affiner notre approche de la géographie des courses de taureaux en individualisant de petits pays taurins de forte densité d'arènes et/ou de spectacles. La place de la Castille centrale entre la Sierra de Guadarrama et les Monts de Tolède apparaît très clairement avec une véritable couronne de forte densité d'arènes et de spectacles autour de la capitale. Un important foyer d'arènes apparaît entre les affluents de rive droite du Tage que sont le Tajuña et le Henares, prolongé à l'aval par un important foyer compris entre la Sierra de Gredos et les Monts de Tolède. Au cour de cet ensemble on aura reconnu la vallée du Tiétar qui dans le milieu taurin porte le nom de « la vallée de la terreur », réputée pour l'importance de l'afición locale et la dureté de son bétail dont les novillos sortent ici plus armés que beaucoup de taureaux ne le sont ailleurs ${ }^{17}$. D'autres foyers importants se détachent parmi lesquels la région proche de Séville, riche en arènes et en spectacles, les nombreuses arènes de la Sierra de Aracena à Huelva qui se prolongent vers Badajoz, le linéaire d'arènes à proximité de la frontière portugaise, l'important foyer de spectacles en rive droite du Segura entre Murcie et Alicante. Au regard de cette répartition, il n'existe aucun déterminisme géographique simple de type naturel ou humain qui pourrait rendre compte de la localisation des foyers de densités. Devançons la construction de l'analyse en constatant qu'il existe en revanche une certaine corrélation entre la localisation des élevages et celle des importants foyers de spectacles. Pour l'exprimer de façon volontairement minimaliste, là où grandit le taureau se trouvent l'afición et le spectacle, ce qui ne veut pas dire qu'il n'existe pas de spectacles et d'afición en dehors des régions d'élevage de taureaux de combat. Cela implique que la répartition des spectacles est beaucoup plus dispersée que la localisation concentrée des élevages.

\section{c) Ubiquité et concentration des jeux taurins populaires}

Les logiques d'organisation spatiale de la tauromachie populaire sont différentes de celles des courses de taureaux, à toutes les échelles d'observation. Le premier point, déjà évoqué, concerne l'utilisation dominante de la voie publique qui permet de pratiquer la tauromachie populaire en dehors de tout équipement spécialisé. Néanmoins, il ne faut pas négliger le rôle des arènes de troisième catégorie, démontables ou non, fréquemment utilisées pour les lâchers de bovins à participation collective. Grâce à la possibilité d'employer des arènes démontables, on observe que l'absence d'arène en dur n'est pas une explication satisfaisante permettant de comprendre pourquoi les taureaux sont affrontés dans la rue. Ainsi il s'agit bien d'une décision positive, généralement prise dans la continuité de la tradition, et non d'une contrainte due au manque d'équipement.

Parmi les rares données dont nous disposons sur cet aspect, celles de l'Andalousie sont instructives. Sur l'année 2005, 35 \% des festejos populares se sont

\footnotetext{
${ }^{17}$ Sur ce sujet voir, « El valle del terror », 6 Toros 6, n 7, 1992, p. 12.
} 
déroulés dans des arènes (135 spectacles populaires sur 385). La proportion est inversée dans la province de Cáceres où plus de $70 \%$ des festejos populares se sont déroulés dans des arènes (369 spectacles populaires sur 503). Ces arènes sont à plus de $80 \%$ des arènes démontables ce qui signifie que les lieux de mise en scène de la tauromachie populaire échappent largement à la trame pérenne des arènes permanentes. Le cas de Cáceres, bien qu'il ne soit pas exclu que le mode de comptage sous-estime le déroulement réel d'une partie des festejos populares sur la voie publique, permet aussi de nuancer l'opposition qui fait de la tauromachie participative une tauromachie de rue et de la tauromachie professionnelle une tauromachie d'arène. Soit, comme nous l'avons envisagé, la tauromachie de rue est à prendre au sens sociologique du terme plus qu'au sens géographique, quel que soit le lieu de sa pratique, soit, il faut accepter un changement de regard sur la réalité de nombreuses petites arènes du monde rural. L'arène de troisième catégorie de nombreuses bourgades n'est parfois qu'un prolongement spatial de la rue et de l'espace public, et demeure un lieu ouvert, accessible à tous pendant les fêtes taurines comme pendant le reste de l'année lorsqu'il s'agit d'arènes permanentes. L'arène est une aire de jeu ouverte et libre. Dès lors, il faut admettre une représentation tout autre de l'arène qui dans ce cas n'est pas perçue comme le symbole de la tauromachie professionnelle, mais comme le lieu de la mise en scène tauromachique sous ces différentes facettes. À l'inverse, la piste des arènes dans les grandes villes n'est généralement pas ouverte au public, mise à part quelques exceptions ponctuelles, et demeure le lieu exclusif de l'expression professionnelle de la tauromachie. Cette vision permet de relativiser un certain regard urbain sur la réalité rurale du fait taurin qui ne peut en aucun cas faire de l'arène la marque d'une acculturation urbaine descendante, la marque du spectacle de la ville qui se serait installé à la campagne. Une autre culture du taureau se dessine, profondément rurale, où l'arène demeure un lieu de pratiques collectives et communautaires.

L'importance de la voie publique comme espace de mise en scène tauromachique est généralement liée au nombre d'encierros pratiqués, sauf dans le cas particulier des pays valenciens où le bous al carrer tend à devenir une forme tauromachique à part entière. Le rayonnement du modèle pamplonais de l'encierro appauvrit sans doute la perception de la diversité des encierros espagnols. Nombre d'entre eux se déroulent sur des parcours mixtes, du champ jusqu'au bâti continu de l'urbain. Les données recueillies dans la région de Castilla La Mancha nous permettent d'apporter des précisions. Dans la province de Guadalajara, 82 encierros sur 321 sont des encierros qui se déroulent à travers champs, sur plusieurs kilomètres parcourus à cheval, avant d'atteindre les abords de la ville ou du village où le bétail est ensuite conduit à pied. On observe également ce phénomène à Albacete dans des proportions encore plus fortes avec 44 encierros ruraux sur 112, les autres se déroulant le long de parcours considérés comme exclusivement urbains. Nous ne possédons pas ces chiffres pour les provinces de Castilla-León, mais nous savons la pratique très développée à Valladolid, Salamanque et Zamora. Dans son ensemble, cette pratique différencie fortement les encierros de Castille de ceux de Navarre, qui eux se déroulent à 
pied dans leur immense majorité, y compris lorsqu'il s'agit d'encierros pratiqués en dehors du tissu urbain comme à Falces. Il en va de même pour les encierros valenciens même s'il existe ici de célèbres exceptions telles que l'encierro à cheval de Segorbe (Castellón), classé comme spectacle d'Intérêt touristique national. Les modalités techniques de l'encierro ne dépendent aujourd'hui d'aucune détermination géographique telle que le relief ou la distance aux élevages.

Ce n'est pas de l'encierro que les pays valenciens tirent leur originalité relative aux lieux de mise de scène de la tauromachie participative. Les bous al carrer, les taureaux de rues, sont sans doute la pratique qui pousse le plus loin la transformation de la voie publique en arène. Cette transformation n'est jamais une simple reproduction des caractéristiques architectoniques de l'arène. Certes, le pavé est souvent recouvert de sable pour offrir au bétail une meilleure adhérence. Certes, des gradins sont souvent installés tout autour de la piste pour assurer une mise en spectacle du jeu. Certes, la porte des boxes individuels où sont enfermés les taureaux s'ouvre comme s'il s'agissait de la porte du toril. Cela étant, à l'opposé de la piste plane et dégagée de tout obstacle, l'espace de jeu des bous al carrer intègre toujours des éléments urbanistiques tels que des bancs, des fontaines ou des arbres. Mieux, s'ils n'existent pas dans l'espace de jeu, ils sont ajoutés en installant des tables, des bancs, des marches d'escaliers montantes et descendantes. Le jeu matérialise ainsi un lieu de mise en scène original qui, loin de masquer la rue et la place publique sous l'artifice de l'arène interchangeable, en reconstitue les attributs. La tauromachie dans la rue porte ici les valeurs de l'espace public.

La répartition des jeux taurins populaires suit une autre logique que celle répondant aux caractéristiques théoriques des lieux centraux. Ici le nombre de spectacles en un même lieu échappe aux règles d'une distribution quantitative proportionnée par la répartition et la taille des noyaux de peuplement. Les forts contrastes de la répartition provinciale des jeux taurins populaires comparée à celle des spectacles majeurs en faisaient déjà la démonstration. Si certaines grandes villes des provinces riches en spectacles taurins populaires programment un nombre important de manifestations, beaucoup de villes moyennes et petites en offrent un nombre équivalent voire supérieur. Ce nombre dépasse souvent celui des spectacles programmés lors des plus grandes férias d'Espagne. C'est le cas précisément de nombreuses communes situées dans les deux zones où l'on a souligné l'importance de la tauromachie populaire : Madrid, les rives de l'Èbre et les pays valenciens (voir carte 7, p. 242). En 2003, dans un espace compris entre les capitales provinciales de Valence et de Castellón, une quinzaine de municipalités ont célébré chacune plus de vingt spectacles taurins populaires. Observons ce phénomène pour l'année 2003. La municipalité de Vall de Uxó (40.000 habitants) qui s'est autoproclamée " capitale du taureau » au milieu des années 1990 possède un record avec 79 jours de taureaux. Chaque quartier possède un comité des fêtes et des associations d'amateurs, les " peñas » (penyes en valencien) qui organisent un ou plusieurs lâchers de taureaux dans l'année. La seule portion du territoire espagnol comparable est la vallée de l'Èbre entre Logroño et Saragosse, partagée entre La Rioja, la Navarre 


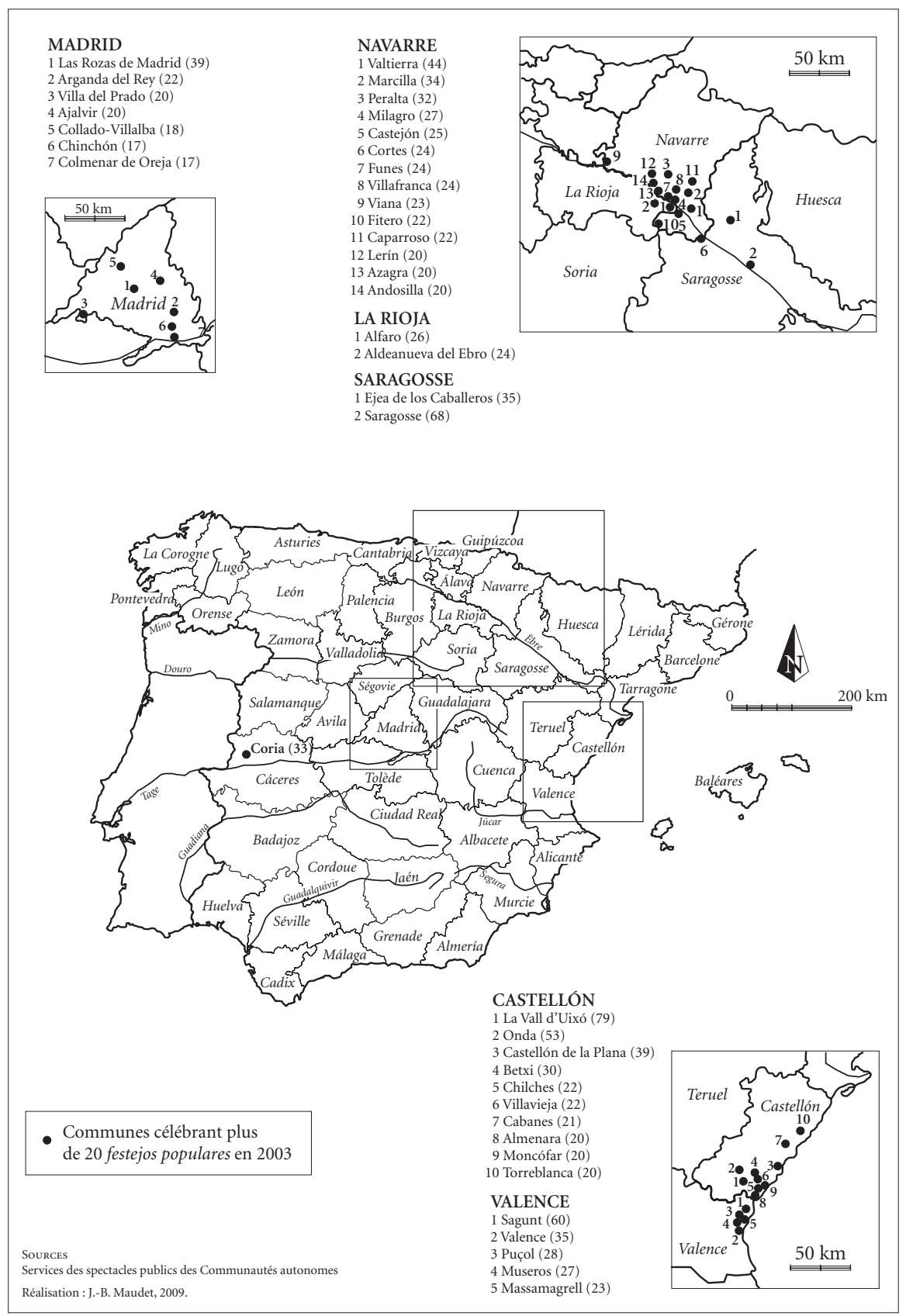

Carte 7. - Foyers denses de festejos populares 
et l'Aragon. Pour la même année 2003, une vingtaine de communes y ont programmé plus de vingt spectacles populaires. À l'exception de Madrid, mais dans une moindre mesure, aucune autre région ne concentre autant de municipalités programmant des spectacles populaires en si grand nombre. En 2003, Madrid compte 7 communes à avoir programmé plus de 15 festejos populares : Ajalvir (20), Arganda del Rey (22), Chinchón (17), Collado Villalba (18), Colmenar de Oreja (17), Las Rozas de Madrid (39), Villa del Prado (20). Ailleurs seuls se distinguent quelques cas isolés, comme Coria dans la province de Cáceres avec 33 festejos populares. Cette cartographie donne une vision très différente des habituelles représentations de l'espace tauromachique, focalisées sur le monde de la corrida.

Une autre façon de rendre compte de l'importance de la tauromachie est d'observer la couverture territoriale du fait taurin en Espagne à partir du rapport entre le nombre de communes programmant des spectacles taurins et le nombre de communes de la province. Dans les régions où les jeux taurins populaires sont nombreux, ces pratiques sont très largement responsables d'un taux de couverture territorial élevé. Pour certaines provinces nous avons pu calculer précisément le taux de municipalités concernées par la tauromachie. Castellón figure en tête des provinces les plus taurines avec $96 \%$ des municipalités qui proposent au moins une manifestation tauromachique par an, toutes pratiques confondues. Un tel classement fait suivre la Communauté autonome de Madrid avec $76 \%$ des municipalités, Saragosse avec $50 \%$, Teruel avec $44 \%$, Valence avec $42 \%$, la Navarre avec $33 \%$, Alicante et La Rioja avec $30 \%$.

Étant donné le plus faible développement quantitatif des tauromachies populaires dans le Sud de l'Espagne comparé à celui des provinces du Centre, du Nord et du Levant, on aurait pu s'attendre à y trouver un taux de couverture très faible. La réalité est tout autre en raison d'un maillage municipal beaucoup plus fin dans le Nord de l'Espagne que dans le Sud. Par comparaison les huit provinces andalouses comptent 769 municipalités, alors que les trois provinces aragonaises en comptent à elles seules 825 . De ce fait et malgré la faiblesse méridionale apparente de la tauromachie participative, le taux de recouvrement est partout supérieur à $20 \%$ des communes. Cadix, qui ne compte il est vrai que 44 communes, arrive en tête avec $90 \%$ des municipalités à avoir programmé un spectacle taurin, qu'il relève de la tauromachie professionnelle ou de la tauromachie participative. Jaén, dont on a souligné le particularisme au sein de l'ensemble andalou, occupe le deuxième rang avec un taux aux environs des $80 \%$. Suivent Cordoue, Málaga, Huelva et Séville avec des taux compris entre 35 et $55 \%$, soit des taux comparables avec ceux de Saragosse, Teruel, Valence et la Navarre. Grenade et Almería occupent les dernières places avec des taux compris entre 20 et $30 \%$. Murcie fait partie des provinces intermédiaires avec $51 \%$ de ses communes qui ont programmé un spectacle ou un jeu taurin (soit 23 municipalités sur 45). Le Sud, en dépit d'un nombre de spectacles nettement inférieur à celui du Nord, peut ainsi se prévaloir d'un taux de municipalités taurines beaucoup plus élevé que ne le laisserait croire la seule prise en compte de données brutes, qui ne seraient pas rapportées au maillage territorial. 
Ces mesures partielles semblent ne pas invalider l'estimation proposée par Sigfredo Ortuño Pérez selon laquelle la tauromachie serait présente "dans 3.500 municipalités sur les quelque 8.000 que compte l'Espagne », soit environ $43 \%$ des territoires communaux ${ }^{18}$. Il est seulement regrettable qu'aucune source ni aucun mode de calcul ne soit explicité pour valider cette estimation. En Espagne, quel loisir collectif peut se prévaloir d'une telle couverture territoriale ? Le football très probablement, encore serait-il intéressant de préciser dans quelle proportion afin d'établir des comparaisons détaillées entre ces deux loisirs.

\section{Les haUts LIEUX DE L'ESPACE TAURIN}

L'analogie d'un espace tauromachique organisé par la théorie des lieux centraux demeure une observation incomplète de l'organisation spatiale du fait taurin, même lorsque celle-ci met en relief des foyers de forte densité taurine qui s'affranchissent largement des régularités territoriales. La première nuance à apporter est d'ordre phénoménologique : l'importance des spectacles telle qu'elle est vécue par ses adeptes est en partie indépendante de cette hiérarchie, au sommet comme à la base. Avec un ou deux spectacles dans l'année, la tauromachie peut être omniprésente dans la vie de la cité et collectivement plus efficiente et significative que la féria taurine d'une grande ville. Autrement dit, en dehors des hiérarchies internes de valorisation des spectacles, la taille des cités et la perception culturelle de la tauromachie en leur sein jouent un rôle primordial dans l'importance sociale du fait taurin. Dans nombre de villages les deux édifices les plus imposants sont l'église et l'arène : chacune d'elles suffit parfois à contenir toute la population. Dans ces villages, la participation à la fête taurine regroupe parfois quasiment tous les habitants, à qui s'ajoutent ceux qui n'y vivent pas, mais qui viennent repeupler le pueblo d'origine, les jours de fêtes.

De même, l'approche hiérarchique des espaces taurins resterait désincarnée si elle ne rendait pas compte de l'idiosyncrasie des lieux et, en particulier, des quelques hauts lieux de spectacles dont dépend le fonctionnement de la saison tauromachique. Au-delà du nombre de spectacles, certaines arènes et certaines corridas possèdent en effet des formes de valorisation qualitative plus complexes. Certes, les hauts lieux de la tauromachie professionnelle correspondent, pour l'essentiel, à des arènes du haut de la hiérarchie en termes de catégorie, de capacité et de jours de programmation. Cependant, beaucoup de grandes arènes proposant une programmation riche d'un point de vue quantitatif ne comptent pratiquement pas dans le déroulement général de la saison. Quelques arènes ont en revanche un style, un rôle et un pouvoir qui rehaussent leur importance au-delà de tout critère quantitatif.

De ce point de vue, il est possible de considérer que l'espace tauromachique espagnol est structuré autour de deux pôles principaux, complémentaires et

\footnotetext{
${ }^{18}$ S. Ortuño PÉrez, «Ecología y economía de las explotaciones de ganado de lidia », p. 171.
} 
concurrents : Madrid et Séville. Ces deux villes commandent les deux régions les plus taurines d'Espagne pour ce qui est des spectacles majeurs : la Castille et l'Andalousie. Elles constituent pour les toreros en quête de reconnaissances artistiques et financières, à un moment ou à un autre de leur carrière, le point de passage incontournable. Madrid et Séville forment un centre bicéphale dont dépendent plus ou moins toutes les périphéries. Madrid constitue le centre structurel, économique et décisionnel où convergent toutes les organisations du mundillo pour la féria de la San Isidro. Séville représente le centre historique et culturel de la tauromachie dont la Maestranza perpétue les valeurs avec élégance. Leur histoire et leur localisation dans le tissu urbain offrent un écho significatif à cette répartition des fonctions de centralité. Comme nous l'avons détaillé précédemment, à Madrid, les arènes de Las Ventas sont éloignées du centre ancien et se trouvent désormais à quelques mètres de l'échangeur autoroutier de la M 30. Ces arènes ont remplacé les arènes de la Puerta de Alcalá à la fin des années 1920 qui, comme leur nom l'indique, étaient déjà en situation urbaine périphérique. À Séville, les arènes de la Real Maestranza sont situées au cour du centre historique, à quelques pas de la Torre de Oro, entre la rive du Guadalquivir et la cathédrale (la Giralda). On peut voir à travers ce contraste une correspondance métaphorique des fonctions de centralité qu'elles incarnent. Las Ventas par sa centralité organisationnelle, constitue la tête de réseaux de l'espace tauromachique qui contrôle la circulation des toreros, des capitaux et des contrats. Las Ventas apparaît bien comme une «bourse des valeurs » pour reprendre l'expression de Bartolomé Bennassar, notion qui revient sous la plume de Jacques Durand qui la considère comme une "maxibourse de la tauromachie ${ }^{19}$. Sa localisation changeante au sein du tissu urbain n'a sûrement qu'une importance secondaire pour exercer cette fonction. En revanche, la Maestranza, afin de maintenir sa centralité culturelle et historique sur le monde taurin, ancre son rayonnement iconographique et sa légitimité dans la longue durée. La Real Maestranza de Caballería construite sur le mont Baratillo en 1761, en plus d'être une arène de première importance dans le déroulement de la saison, est un « lieu de mémoire » dont la localisation immuable et le maintien d'un style assurent le prestige mondial. De nombreuses arènes dans le monde s'en sont d'ailleurs inspirées comme nous le verrons par la suite. On comprend, d'ores et déjà, que la relation de concurrence indéniable entre Las Ventas et La Maestranza constitue également une relation de complémentarité qui assure une véritable maîtrise du fonctionnement territorial de l'espace tauromachique espagnol et mondial.

Cela étant, Madrid et Séville ne sont pas les seuls centres de l'espace tauromachique. Au moins deux arènes peuvent prétendre peser d'un poids réel sur l'organisation générale de la saison. Il s'agit de deux arènes septentrionales : Bilbao et Pampelune. Ces arènes sont en effet considérées comme des bastions d'une conception de la tauromachie selon laquelle la primauté est donnée aux taureaux qui se doivent d'être parfaitement présentés, imposants et bien

\footnotetext{
${ }^{19}$ B. Bennassar, Histoire de la tauromachie, p. 166 ; J. Durand, Chroniques taurines, p. 129.
} 
armés pour le combat. Dans le détail, ces deux arènes ont un rôle différent et incarnent deux modes de participation au fonctionnement de l'espace tauromachique. Toutes deux se distinguent des canons de la tauromachie dominante par la présentation de taureaux réputés difficiles, mais Bilbao reste inscrite dans la norme du spectacle, alors que Pampelune par son exubérance s'en démarque. En effet, les éleveurs envoient dans ces arènes les spécimens les plus volumineux et aux cornes les plus développées. De leur côté, les arènes veillent à programmer majoritairement des élevages difficiles, c'est-à-dire des élevages d'encastes réputés pour leur bravoure, dont la noblesse ne le cède jamais à l'agressivité et à une certaine forme d'âpreté. Ces taureaux sont qualifiés par le jargon taurin de taureaux «durs », « sérieux » ou de «toro toro » comme si la redondance faisait exister toutes les qualités en puissance de l'animal. Ces adversaires redoutés mettent en lumière la capacité méritoire des matadors à les dominer. Cette distinction, très classique en tauromachie, oppose les arènes dites toristas (celles qui accordent la primauté au sérieux des taureaux) aux arènes dites toreristas (celles qui accordent la primauté au sens artistique des toreros). Dans sa version quelque peu caricaturale, les arènes toristas construiraient leur réputation sur des taureaux exigeants, alors que les arènes toreristas ne mettraient rien audessus de l'expression artistique du torero, les premières exigeraient la présence combative du matador autoritaire, les secondes attendraient que le maestro, touché par la grâce, révèle la dimension esthétique du toreo. Quoiqu'en partie fondée, cette distinction mérite d'être relativisée. La qualité du spectacle dépend toujours d'un équilibre qui intègre les deux aspects du problème et il $\mathrm{y}$ a quelque flagornerie à présenter les choses en termes d'alternative irréconciliable. Tous s'accordent à penser que le spectacle parfait est la synthèse de ces deux conceptions : un taureau brave, puissant et armé, dominé par un grand artiste courageux.

Quelle que soit la validité de cette différenciation éthique et esthétique sur la nature même de la tauromachie, il est important d'observer qu'elle fonde une opposition territoriale. Les arènes du Nord sont considérées comme le centre de la conception torista alors que les arènes du Sud sont considérées comme le centre de sa conception torerista. Un célèbre dicton taurin le revendique, chargé de ce que le mépris andalou a de plus impertinent et savoureux : "Au Sud de Despeñaperros on torée, au Nord on travaille $»^{20}$. Dans cette opposition stylistique, Séville tient le rôle de centre de l'esthétique torerista alors que Pampelune et Bilbao jouent le rôle de bastion de l'éthique torista. Dans ce partage de la géographie taurine, Madrid est du côté des arènes toristas en raison du niveau d'exigence dans le choix des taureaux, mais l'histoire montre qu'elle a toujours su consacrer les grands artistes, à commencer par le plus sévillan d'entre eux, Curro Romero ${ }^{21}$. Madrid incarne à nos yeux un arbitrage idéal et sans doute

\footnotetext{
${ }^{20}$ Despeñaperros est un défilé de la Sierra Morena qui permet de passer de l'Andalousie à la Castille et qui est devenu l'un des principaux marqueurs territoriaux pour situer l'Andalousie dans sa position méridionale par rapport au reste de l'Espagne.

${ }^{21}$ Curro Romero (naissance : 1933 à Camas; alternative : 1959 à Valence ; retraite : 2000).
} 
impossible entre ce que les conceptions torista et torerista auraient de meilleur à donner. Le résultat de cette exigence est étonnant et quelque peu paradoxal. Les véritables héros de Madrid sont peut-être l'arène elle-même et son public, avant que d'être les toreros ou les taureaux. Quoi qu'il en soit, cette opposition n'en révèle pas moins un rapport de force qui, historiquement, a toujours tourné en faveur d'une esthétique torerista dominante. Ce ne sont pas les taureaux qui ont commandé l'organisation générale et l'évolution de la tauromachie, mais bien les artistes et leur capacité à transformer l'équilibre des forces entre les intérêts divergents des différents acteurs du spectacle taurin. En outre, il paraît clair que les toreros capables de transformer les codes artistiques tels que Belmonte, Manolete, «El Cordobés », Paco Ojeda ou José Tomás ont toujours joui d'une valorisation supérieure à celle des belluaires athlétiques, aussi talentueux et méritants qu'ils soient. En tauromachie, il y a une esthétique de la grâce qui relègue en arrière-plan celle du mérite et du courage. C'est sans doute une des raisons profondes qui explique le succès de la corrida et sa diffusion au-delà d'une culture locale, non pas qu'elle soit un art, catégorie si difficile à circonscrire, mais qu'elle ait su constamment évoluer vers la recherche d'une émotion esthétique toujours plus grande ayant su correspondre au désir de distinction d'une partie des élites. Et en matière taurine, ce sont les élites qui construisent les solidarités horizontales les plus fortes dont les portées spatiales sont les plus étendues, au-delà de toute appartenance territoriale.

Si les bastions résistent, ce n'est donc pas dans le refus de l'esthétique torerista dominante, mais dans le refus d'une dérive esthétisante et chorégraphique, servie par des taureaux qui ne seraient que des partenaires conciliants. Remarquons que des garanties minimales sur le poids et la présentation des taureaux apportées par les arènes de première catégorie les mettent en grande partie à l'abri d'une dérive torerista. Autrement dit, la catégorie des arènes introduit une hiérarchie et une norme qui dans les faits relativisent considérablement l'opposition territoriale Nord-Sud. Ainsi Séville, bien qu'elle s'affiche comme le centre d'une esthétique torerista, présente des taureaux plus " sérieux » qu'une arène septentrionale de deuxième catégorie. De plus, les arènes que l'on dit toristas programment les mêmes toreros vedettes, à quelques exceptions près, que les arènes dites toreristas. Et si la différence se fait effectivement sur le choix majoritaire de taureaux « durs », ils n'empêchent pas la présence de taureaux réputés pour leur noblesse, imposés par les matadors influents que toute arène importante se doit de programmer. À l'inverse, la plupart des arènes dites toreristas réservent toujours une partie de leur programmation aux taureaux « durs » combattus par ceux qui acceptent souvent de les affronter, faute de mieux, à moins qu'ils en aient fait une spécialité rentable. L'exemple le plus emblématique est la traditionnelle corrida de Miura qui termine la féria taurine de Séville ${ }^{22}$. La différenciation de l'opposition entre arènes toristas et arènes toreristas est donc à nuancer de même que sa traduction géographique. Bien qu'en partie fondée,

\footnotetext{
${ }^{22}$ Miura est un élevage fondé en 1842 et réputé pour le caractère dangereux de ses taureaux.
} 
il existe dans l'inertie de cette historique opposition un écart entre les faits et les représentations. Les représentations qui forcent le trait à l'échelle de l'Espagne privilégient un partage régional Nord-Sud de la géographie taurine, en occultant la différenciation non territoriale entre les catégories d'arènes. Ce décalage de la représentation sur les faits témoigne à nos yeux d'un enjeu fondamental du fonctionnement des identités territoriales selon lequel la manifestation d'une identité commune passe par l'expression d'une altérité radicale. Nous reviendrons sur cette clé de lecture qui selon nous permet de comprendre une grande partie des différenciations sociogéographiques des espaces taurins.

S'il existe de hauts lieux secondaires de l'espace tauromachique, à commencer par les capitales régionales ou provinciales, ils ne remettent jamais en cause l'organisation générale de cet espace structuré par les centres précédemment cités. Certaines arènes trouvent une place de choix dans cette hiérarchie du fait d'une spécialisation taurine qui, sans transformer véritablement le déroulement de la saison, les distingue aux yeux des amateurs comme des lieux importants. Salamanque occupe une place distinctive, car c'est la capitale d'une région très riche en élevages de taureaux de combat. Jerez de la Frontera ajoute à la tradition des élevages braves celle de l'équitation taurine. Ronda fait valoir son statut de haut lieu de l'histoire taurine en exploitant son image de berceau originel de la tauromachie moderne grâce à la glorieuse dynastie des Romero qui y a vu le jour. Castellón profite d'être la première grande féria qui ouvre la saison tauromachique, même si Olivenza s'est récemment installée sur ce créneau commercial avantageux. Saragosse s'impose en octobre comme la dernière féria importante de l'année. Cenicientos, dans la région de Madrid, trouve sa place en tant que bastion torista. Comme dans tout processus distinctif, certaines arènes sont à l'inverse montrées du doigt et incarnent ce que la tauromachie a de plus critiquable aux yeux des connaisseurs. C'est le rôle joué par les arènes de plages, en particulier celles du littoral méditerranéen. Arrivent en tête de cette catégorie les arènes des stations et des villes balnéaires de la Costa Blanca (Benidorm), de la Costa del Sol (Marbella, Torremolinos) ou encore celles de la Costa Brava (San Feliu de Guíxols, Lloret de Mar). Ces arènes sont alors chargées de tous les maux et de toutes les perversions de l'art tauromachique: des taureaux imprésentables aux cornes " trafiquées " pour en réduire le danger, des toreros de seconde zone ou en bout de course qui s'accordent un dernier moment de gloire gagné sur l'ignorance d'un public inculte, des spectateurs étrangers qui n'y connaissent rien et applaudissent, sans aucun jugement critique, la moindre vulgarité tapeà-l'œil. Ces arènes symbolisent la tauromachie pour "guiri », cette appellation dépréciative du touriste généralement stigmatisé sous l'aspect d'un " toutou » d'Europe du Nord aux cheveux blonds et aux yeux clairs, portant short et chaussettes blanches remontées, qui, n'étant pas Espagnol, serait incapable de faire la différence entre un bon et un mauvais spectacle. Là encore, avant même de s'interroger sur la fiabilité de la description des arènes de plage, et une fois retirés les relents de racisme ordinaire propre à la dénonciation du nouvel envahisseur touristique, la distorsion entre les faits et les représentations traduit les mêmes enjeux identitaires : être distingué sociologiquement et territorialement. 
Les hauts lieux de la tauromachie participative sont de nature différente. Certes il faut noter l'émergence de quelques grandes villes qui progressivement s'imposent comme les nouveaux centres de la tauromachie populaire. Il s'agit, en particulier, des capitales de provinces situées dans les zones de forte densité des festejos populares (Saragosse, Pampelune, Valence, Castellón, Madrid) qui organisent de plus en plus régulièrement de grands événements autour des tauromachies populaires. C'est par ces villes que progresse d'ailleurs la professionnalisation des concours de recortadores. Elles développent ainsi leur fonction de centralité dans un espace en cours de structuration comme nous le montrons dans la partie consacrée aux dynamiques spatiales. Cela étant, elles sont encore mal identifiées en tant que centres, sauf aux yeux des semi-professionnels et des passionnés de festejos populares. En tous les cas, cette activité ne fait pas ombrage aux corridas données dans les mêmes villes. Les cités les mieux associées à la tauromachie populaire dans l'imaginaire collectif sont plutôt de petites villes qui créent l'événement à partir d'une spécificité tauromachique locale. C'est souvent une forme tauromachique rare et ancienne dans un cadre festif d'exception qui favorise leur reconnaissance à l'échelle nationale. Certaines municipalités ont d'ailleurs parfaitement su tirer parti de leur patrimoine taurin en obtenant le label des fêtes d'Intérêt touristique national. C'est le cas des encierro de Ampuero (Cantabrie), de Medina del Campo (Valladolid), de Cuéllar (Segovia), de Almodóvar del Campo (Ciudad Real), de Segorbe (Castellón) et de Blanca (Murcie). C'est le cas du toro de la Vega de Tordesillas (Valladolid), des fiestas de Nuestra Señora y San Roque à Peñafiel (Valladolid), du carnaval de Ciudad Rodrigo (Salamanque), du toro enmaromado de Benavente (Zamora), des fiestas de San Mateo de Cuenca, des fiestas de San Juan de Coria (Cáceres), des festividades de San Vicente Ferrer de la Vall de Uxó (Castellón), du bou al mar de Dénia (Alicante), toro ensogado de Beas de Segura (Jaén). D’autres ne relèvent pas de cette classification, mais jouissent néanmoins d'une grande renommée : le toro ensogado de Rubielos de Mora (Aragon), le toro de júbilo de Medinaceli (Soria), le toro capllaçat de Amposta (Cataluña), le corre bou de Cardona (Cataluña), le toro de cuerda de Gaucín (Málaga). Ces hauts lieux de la tauromachie populaire s'éparpillent sur l'ensemble du territoire y compris dans des régions qui ne font pas figure de foyer tauromachique important comme la Cantabrie et la Catalogne. En dehors du fait qu'il s'agisse, dans la majorité des cas, de bourgades ou de petites villes inscrites dans des espaces à dominante rurale, il n'existe pas de raisons évidentes pour expliquer leur localisation.

\section{L'ÉLEVAGE BRAVE}

L'élevage brave constitue un élément fondamental de l'organisation spatiale du fait taurin. Il faut insister sur l'originalité d'une activité qui relève d'un élevage hautement spécialisé dans la production d'un animal de loisir et qui s'inscrit également dans le circuit de l'élevage extensif consacré à la production de viande. Rappelons qu'après chaque spectacle, le règlement oblige à la mise à mort du bétail. Cette mise à mort publique dans le cas des spectacles majeurs et 
mineurs est intégrée au rituel tauromachique. Dans le cas des spectacles taurins populaires, l'abattage du bétail est théoriquement obligatoire, mais se déroule sans la présence du public, soit à l'abattoir, soit dans les dépendances de l'arène prévues à cet effet. Observons que cette règle admet des exceptions en Navarre et dans la région de Valence où il existe une tradition de lâchers de bétails qui sont courus à plusieurs reprises. Partout lorsque l'animal est abattu, la viande est aussitôt conditionnée selon les normes sanitaires définies par le règlement afin de réintégrer le circuit commercial. Il en va de même pour la viande des animaux qui, à divers stades de leur croissance, ne sont pas retenus par les éleveurs pour participer à un spectacle taurin. Depuis une dizaine d'années, l'originalité de cette forme d'élevage est au cœur d'une réflexion de mieux en mieux étayée par une recherche scientifique pluridisciplinaire, réunissant vétérinaires, généticiens, agronomes, économistes, historiens, sociologues ${ }^{23}$. Aussi des progrès considérables ont-ils été réalisés dans la connaissance des taureaux de combat qui est restée longtemps dominée par une mythologie romantique faisant volontiers de cet animal le descendant direct du taureau primitif et sauvage. Limitons-nous ici aux données susceptibles d'en éclairer la géographie actuelle.

Au début de l'année 2004, l'Espagne compte 1.128 élevages de taureaux de combat regroupant quelque 160.000 têtes de bétail ${ }^{24}$. Chaque année, environ 35.000 bêtes sont commercialisées dont $30 \%$ de taureaux et de novillos pour des spectacles majeurs, le reste étant réparti entre les animaux participant aux spectacles mineurs et aux fêtes taurines populaires, ainsi que ceux envoyés directement à l'abattoir. En 2003, 11.501 bêtes ont été combattues dans des spectacles majeurs, dont 7.977 dans des arènes de troisième catégorie (démontables incluses ${ }^{25}$. Chaque élevage peut posséder plusieurs propriétés, plusieurs unités d'exploitation appelées fincas. Antonio López Martínez, dans son ouvrage sur l'histoire et l'économie des taureaux de combat en Espagne, a recensé, autour de l'année 2000, quelque 1.464 fincas qui se consacrent à l'élevage brave ${ }^{26}$. Ces données nous permettent de rendre compte de leur répartition provinciale (voir carte 8 ).

Les 1.464 fincas d'élevages braves sont réparties au sein de 33 provinces sur les 49 que compte l'Espagne. Plus des 3/4 de l'élevage brave se concentrent dans les neuf provinces qui en comptent le plus. La province de Salamanque arrive en tête avec 271 fincas, suivie de Séville avec 182 fincas, suivie de trois provinces possédant plus de 110 fincas (Madrid, Cadix et Jaén), de trois provinces qui approchent les 100 fincas (Tolède, Badajoz et Cáceres) et de la province de Huelva avec 69 fincas. Cette répartition témoigne d'une localisation dominante des élevages de taureaux de combat dans un quart sud-ouest du pays. Cette zone inclut Salamanque avec 18,5\% des élevages, les provinces d'Estrémadure

\footnotetext{
${ }^{23}$ Parmi de nombreuses initiatives scientifiques qui s'intéressent aux taureaux de combats, citons les journées d'études organisées chaque année par l'université publique de Navarre à Pampelune.

${ }^{24}$ MAPA (ministère de l'Agriculture, de la Pêche et de l'Alimentation).

${ }^{25}$ Ministerio del Interior, Estadísticas de la Temporada 2003, p. 90.

${ }^{26}$ A. L. López Martínez, Ganaderías de lidia y ganaderos, p. 63.
} 


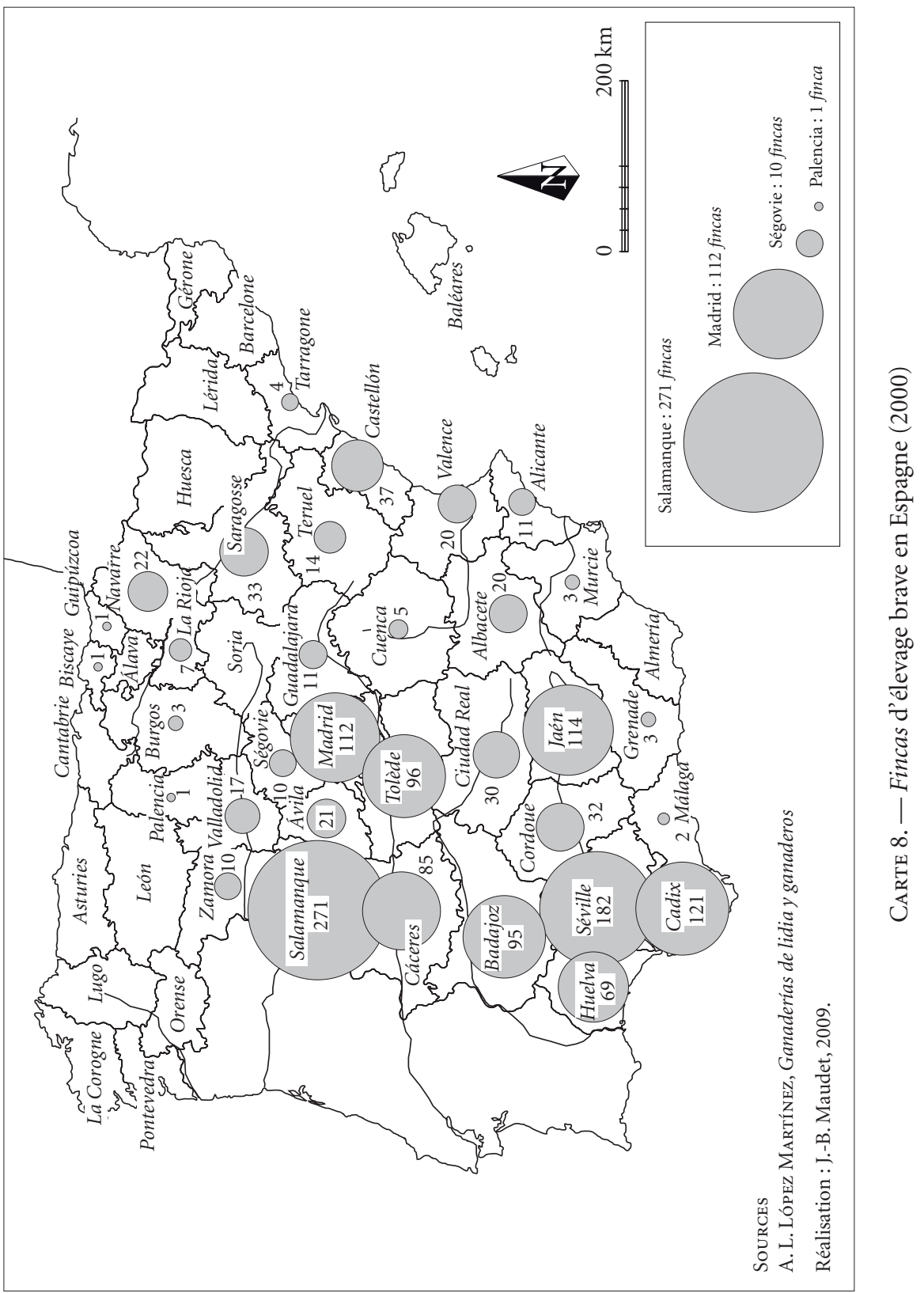


(Cáceres et Badajoz : 12,29 \%), les provinces de la Basse Andalousie (Huelva, Séville et Cadix : 25,4 \%) ainsi que celle de Jaén (7,7 \%), en amont du Guadalquivir. Selon Sigfredo Ortuño Perez, cet élevage occupe actuellement en Espagne environ 435.000 ha, ce qui impliquerait, selon le recensement du Ministère, un rapport de 2,7 ha par tête de bétail ${ }^{27}$. Ce rapport moyen est conforme à l'estimation avancée par Luis Ruiz Abad concernant la surface nécessaire par animal qu'il estime entre 1 et 6 hectares en fonction de la qualité nutritive des pâturages $^{28}$. L'Andalousie arrive en tête des superficies consacrées à l'élevage avec environ 250.000 ha et quelque 500 élevages de taureaux de combat ${ }^{29}$. L'Andalousie est suivie par la région de Castilla-León qui possède 60.000 ha consacrés aux taureaux braves dont 45.000 dans la seule province de Salamanque, riche de ses 200 élevages. Viennent ensuite l'Estrémadure qui possède 55.000 ha et quelque 130 élevages, puis Castilla - La Mancha (50.000 ha), suivie de Madrid (10.000 ha) pour une cinquantaine d'élevages. Ce dernier chiffre est important dans un contexte où l'extension urbaine et le compartimentage de l'espace lié au développement des voies de communication exercent une forte pression sur l'élevage extensif de la province.

À partir d'un échantillonnage de 20 élevages de taureaux de combat, de tailles distinctes et répartis en différents endroits de la péninsule Ibérique, Antonio Purroy et Marina Grijalba, ingénieurs agronomes et professeurs à l'université de Navarre, ont fixé les caractéristiques économiques et techniques moyennes d'une exploitation ${ }^{30}$. En incluant le bétail nécessaire aux différentes opérations de l'élevage, l'exploitation moyenne est formée de 748 bovins dont 253 vaches reproductrices pour une superficie de 715 ha. La valeur moyenne à l'hectare de cette exploitation est de 7.407 euros. Cette valeur moyenne n'est pas très significative tant le prix de la terre varie. Les prix peuvent descendre à 6.000 euros/ha dans certains secteurs de Castille et d'Estrémadure et monter à plus de 90.000 euros/ha dans l'aire urbaine madrilène ${ }^{31}$. L'exploitation moyenne compte quatre employés salariés et vend 161 bêtes par an pour une valeur de 308.943 euros. Sur cette somme, 268.200 euros proviennent des bêtes vendues pour des spectacles, dont 23 bêtes ont moins de 3 ans ( 1.800 euros/tête) et 42 ont plus de 3 ans (5.400 euros/tête). Un quart des revenus provient des subventions de la politique agricole commune à hauteur de 94.747 euros, venant s'ajouter aux ventes du bétail. Ces subventions proviennent principalement des primes par tête et des primes à l'extensification, étant entendu que l'élevage brave ne dispose d'aucune mesure spécifique dans le cadre de la PAC sinon celles prévues pour la viande bovine ${ }^{32}$. Sur l'échantillon des vingt exploitations étudiées, seulement neuf sont bénéficiaires, les autres rééquilibrent leur renta-

\footnotetext{
27 S. Ortuño Pérez, « Ecología y economía de las explotaciones de ganado de lidia », p. 170.

${ }^{28}$ L. Ruiz Abad, « La economía de las ganaderías de lidia », p. 14.

${ }^{29}$ S. Ortuño Pérez, « Ecología y economía de las explotaciones de ganado de lidia », p. 169.

${ }^{30}$ M. Grijalba et A. Purroy, «Estudio técnico-económico de las ganaderías », p. 39.

${ }^{31}$ L. Ruiz ABAD, « La economía de las ganaderías de lidia », p. 18.

${ }^{32}$ I. Bardaji Azcárate, « La aplicación de la Política Agrícola Común en el ganado de lidia ».
} 
bilité par les revenus d'activités agropastorales connexes et parfois d'activités agrotouristiques. Pour certains, la rente foncière, le grand prestige de l'éleveur de taureaux de combat et le cadre de vie idyllique offert par la finca, suffisent à compenser ce que le bilan comptable annuel affiche comme déficitaire. Si les subventions de la PAC n'étaient pas versées, beaucoup d'exploitations seraient soit contraintes de fermer, soit tenues d'augmenter les prix de l'offre.

Cette exploitation moyenne ne doit évidemment pas masquer les écarts existant d'un élevage à l'autre, car il existe en réalité plusieurs marchés du taureau de combat. Les élevages haut de gamme sont inscrits dans leur immense majorité dans l'association de l'Unión de Criadores de Toros de Lidia. Un lot de six taureaux d'un élevage très réputé peut être vendu autour de 150.000 euros, soit quatre fois et demi le prix moyen des bêtes de plus de trois ans. Luis Ruiz Abad, bien qu'il reconnaisse la difficulté d'obtenir des sources fiables en la matière estime que l'achat d'une corrida (un lot de six taureaux de quatre ans révolus) est compris dans une fourchette de prix allant de 9.000 à 72.000 euros, une novillada piquée dans une fourchette allant de 7.200 à 18.000 euros, et une novillada non piquée autour de 7.200 euros $^{33}$. Notons également que les grands élevages, ceux possédant le plus de terres et le plus de bêtes, ont une rentabilité généralement supérieure à celle des plus petits.

Cette variation reflète la hiérarchie entre les différents élevages de taureaux de combat. Les 1.128 élevages de taureaux braves existant en 2004 sont répartis en quatre associations, l'UCTL (362 élevages), l'AGL (403), le GLU (162), l'AEGRB (201). La première citée, la Unión de Criadores de Toros de Lidia est la plus ancienne et la plus prestigieuse des associations d'éleveurs. Elle fut créée en 1905 et avait jusqu'au début des années 1930 le monopole de l'approvisionnement des taureaux pour les corridas. Elle réunit actuellement les plus grands noms de l'élevage brave et l'immense majorité de ceux qui sont à l'affiche des principales férias espagnoles et françaises. Cette association ne regroupe pas le plus grand nombre d'élevages, mais bien le plus grand nombre d'animaux de combat. En retenant le critère du nombre de mâles marqués, inscrits au registre généalogique, l'UCTL arrive en tête avec 18.726 bêtes, loin devant l'AGL (8.933), le GLU (1.912) et l'AEGRB (2.646). Les membres de l'association possèdent ainsi près de $60 \%$ du bétail brave ${ }^{34}$. Si l'on observe la participation aux spectacles majeurs, la domination de l'UCTL est encore plus criante puisque les 3/4 d'entre eux se déroulent avec du bétail qui en est issu (1.591 spectacles sur 2.125, France incluse). Enfin, il en est de même avec la superficie consacrée à l'élevage brave puisque l'UCTL regroupe, en Espagne, 296.781 ha, soit près de 70 \% de la superficie totale estimée à environ $435.000 \mathrm{ha}^{35}$. Les autres associations regroupent donc des exploitations beaucoup plus petites, en taille et en nombre de bêtes, qui fournissent principalement un bétail destiné soit aux spectacles mineurs, soit aux spectacles populaires. Les élevages des rives de l'Èbre et de la région de

\footnotetext{
${ }^{33}$ L. Ruiz ABAD, « La economía de las ganaderías de lidia », p. 30.

${ }^{34}$ MAPA, 2004.

${ }^{35}$ UCTL, chiffres de 2005.
} 
Valence à destination des jeux populaires et des courses de recortadores s'inscrivent dans ce contexte-là. Les vaches sont utilisées pour les concours d'anneaux, les taureaux pour les concours de sauts et de recortes libres. Les rives de l'Ëbre au carrefour entre l'Aragon, la Navarre et La Rioja concentrent une trentaine d'élevages qui font renaître l'ancienne lignée de la caste navarraise, exclue des spectacles majeurs, mais hautement valorisée dans les différents jeux de la tauromachie populaire. Citons quelques noms parmi les plus importants : Arnillas, Ozcoz, Domínguez, Arriazu, Laparte. Rappelons, comme nous l'avons déjà signalé, que les taureaux des élevages de l'UCTL sont de plus en plus prisés pour les bous al carrer et les concours de recortadores.

Les chiffres détaillés, fournis par l'UCTL, permettent d'affiner l'approche de la répartition des élevages de combat, tout au moins de ceux de cette association d'éleveurs qui produisent plus de $60 \%$ du bétail, fournissent les $3 / 4$ des bêtes combattues dans les spectacles majeurs et possèdent les $3 / 4$ des surfaces des élevages braves. Les cartes 9 et 10 (pp. 256 et 257) permettent de confirmer la localisation dominante méridionale et occidentale des élevages du haut de la hiérarchie taurine. En 2005, un siècle après sa création, l'UCTL regroupe 364 élevages, répartis en 303 élevages dits titulaires et 61 élevages dits aspirants, amenés à devenir membres à part entière. Notons que ce total ne regroupe pas seulement des élevages espagnols au nombre de 335, mais compte également 26 élevages portugais, principalement situés dans le Haut Alentejo, région limitrophe de l'Estrémadure espagnole et trois élevages français. Deux provinces, Salamanque et Séville, sont nettement en tête avec respectivement 71 et 64 élevages. Vient ensuite un second groupe de provinces avec Cadix (34 élevages), Badajoz (31) et Cáceres (29). Pour ce qui est des superficies consacrées à l'élevage, une seule province se détache largement des autres, Séville avec 62.673 ha. Salamanque occupe la deuxième place avec 39.199 ha, suivie par deux provinces à plus de 30.000 ha (Jaén et Cadix), et trois provinces à plus de 20.000 ha (Huelva, Badajoz, Cáceres). L’inversion de la hiérarchie entre Salamanque et Séville au regard des superficies consacrées à l'élevage renforce l'idée d'un tropisme tauromachique andalou où les grands noms des taureaux de combat se conjuguent au prestige des fincas latifundiaires. Un travail ancien, mais d'une valeur inégalée, permet d'approfondir encore la question de la localisation de ces élevages. Il s'agit d'un rapport du ministère de l'Agriculture espagnol, publié en 1980, intitulé : Geografía española del toro de lidia. Cette étude offre l'avantage d'affiner l'analyse en deçà de l'échelon provincial grâce à un travail statistique et cartographique détaillé. Il nous permet également de rendre compte des évolutions historiques de la géographie de l'élevage sur plus de vingt-cinq ans. En effet, les chiffres de 1978 recensent un total de 613 élevages braves. Cependant le nombre de fincas, qui aujourd'hui s'élève à 1.464, était, en 1978, au nombre de 1.342. Le nombre d'élevages, c'est-à-dire de propriétaires d'un fer, a ainsi augmenté beaucoup plus vite que le nombre réel d'exploitations. Le nombre d'unités d'exploitation par élevage a donc globalement diminué, même si des tendances contraires existent localement. Quoi qu'il en soit, l'inertie de la structure agraire est plus importante que ne le laisse 
croire le doublement du nombre d'élevages en trente ans et permet ainsi une utilisation encore pertinente des principales données de la répartition et de la localisation des élevages contenues dans cette étude. Les cartes proposées dans cet ouvrage révèlent une répartition marquée par une forte concentration à différentes échelles et en particulier aux échelles intraprovinciales et municipales rarement prises en considération.

Parmi les provinces les plus riches en élevages braves, une différence peut être observée en fonction de la dispersion intraprovinciale des exploitations. Deux cas de figures peuvent être distingués. Les provinces comme Jaén et Madrid ont une faible dispersion provinciale d'élevages. Dans la province de Jaén, seulement $17 \%$ des communes possèdent des élevages et dans la province de Madrid, $18 \%$. Les communes concernées par l'élevage ont un indice de concentration moyenne d'élevages de 6 exploitations par commune taurine à Jaén et 3,5 à Madrid. L'autre cas de figure est celui présenté par les provinces de Cadix, de Séville et de Salamanque qui ont une dispersion provinciale d'élevages nettement plus élevée. Dans la province de Cadix, 50 \% des communes possèdent des exploitations de taureaux de combat et dans celle de Séville, ce taux s'élève à $30 \%$. Salamanque possède un taux intermédiaire avec $40 \%$ des communes qui possèdent des élevages de taureaux de combat. Les communes concernées par l'élevage possèdent un indice de concentration moyenne d'élevages de 6,25 exploitations par commune taurine à Cadix, 4 à Séville et 3 à Salamanque. Dans ces provinces, l'élevage de taureaux de combat est une réalité économique, sociale et paysagère quotidienne.

À l'échelle locale, toutes les communes possédant plus de 10 exploitations peuvent être considérées comme des sous-ensembles importants au sein desquels l'élevage du taureau de combat est à la fois une activité économique majeure et un élément paysager hautement caractéristique. Certaines de ces communes appartiennent à des provinces fortement marquées par l'élevage brave sur une grande partie de leur territoire. C'est le cas de Jerez de la Frontera dans la province de Cadix avec 31 exploitations, même s'il s'agit d'une immense commune étendue sur plus de 140.000 ha. C'est le cas également de Buenamadre dans la province de Salamanque avec 16 exploitations et de Puebla del Río dans la province de Séville avec 15 exploitations. D’autres communes appartiennent à des provinces globalement moins taurines que les précédentes. Citons le cas de Baños de la Encina à Jaén avec 38 exploitations sur une superficie municipale de 40.000 ha, ce qui constitue un record en valeur absolue et en valeur relative. En effet, Baños de la Encina est la commune espagnole qui concentre le plus d'exploitations de taureaux de combat et qui regroupe plus du tiers des exploitations de la province. Citons également dans cette catégorie, Colmenar Viejo à Madrid avec 18 exploitations, Olivenza à Badajoz avec 17 exploitations, Navamorcuende à Tolède avec 13 exploitations. Enfin, certaines de ces communes représentent des centres isolés au sein de provinces beaucoup moins dotées en nombre d'élevages, telles que Alfera de Carles à Tarragone avec 13 exploitations sur les 20 recensées dans la province et El Espinar à Ségovie avec 10 exploitations sur les 14 recensées. Notons, d'après les chiffres proposés par Antonio Luis 


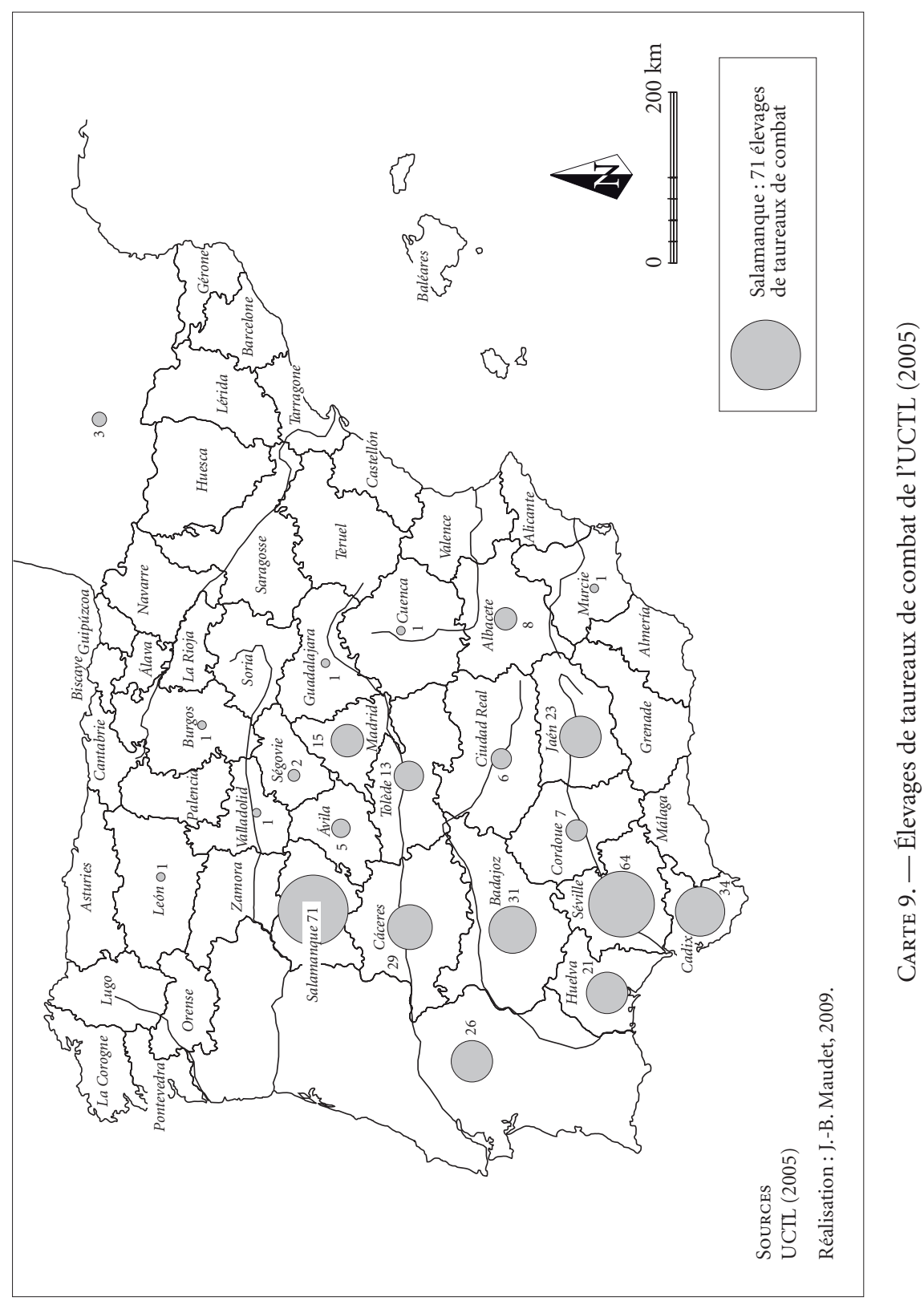




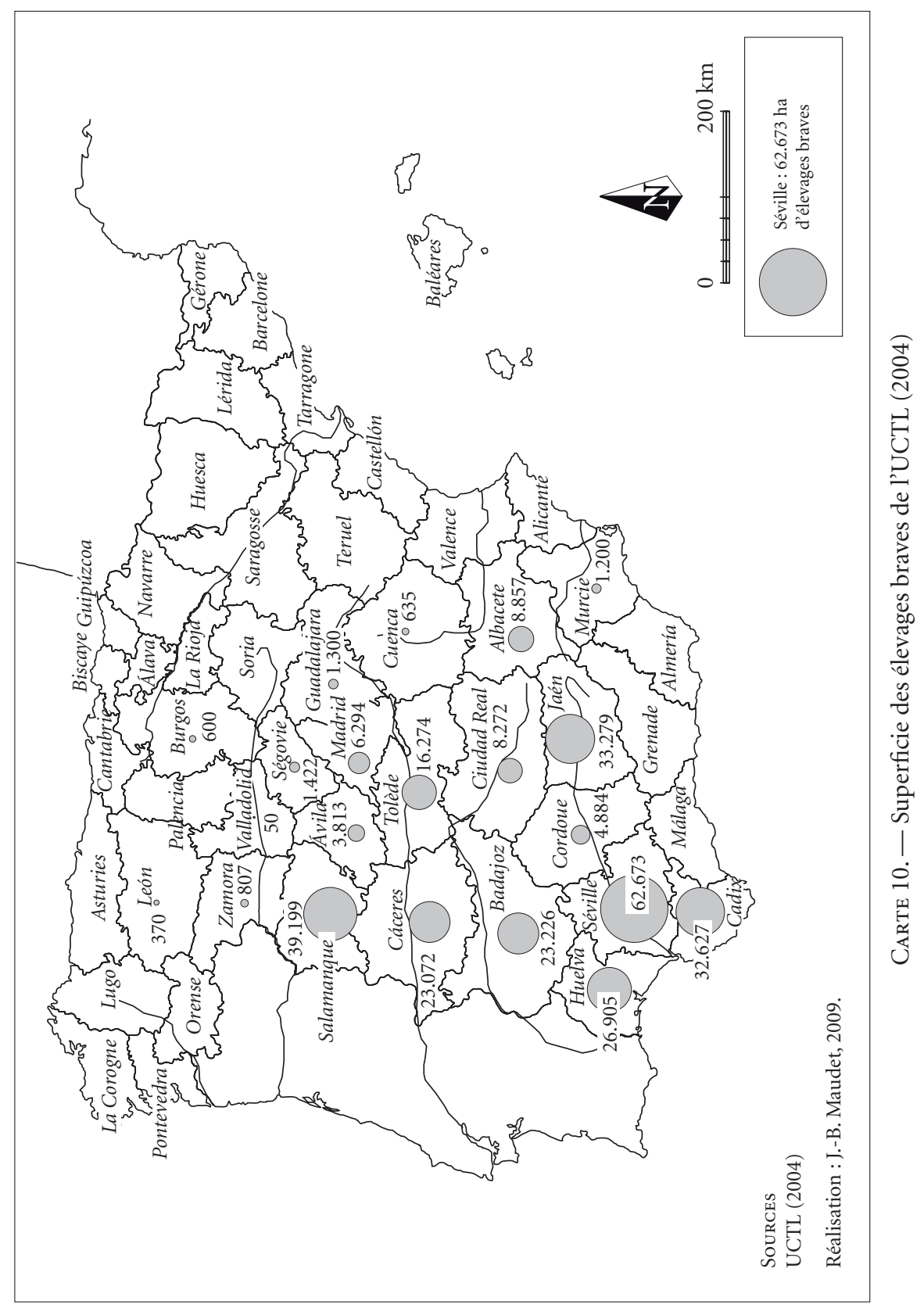


López Martínez, que le nombre actuel d'exploitations dans ces deux dernières provinces aurait décru.

En dehors du quadrillage politico-administratif, il est possible d'analyser la localisation des élevages de taureaux au regard des milieux géographiques. Tous les éleveurs sont extrêmement sensibles au problème de l'approvisionnement en eau du bétail. C'est sans doute une des raisons pour lesquelles il existe un lien lexical fort entre les élevages de taureaux et le réseau hydrographique. Au $\mathrm{XVI}^{\mathrm{e}}$ siècle, au cours de corridas royales, l'habitude était de mentionner la provenance géographique du bétail, ce qui suffisait alors à distinguer des morphotypes de taureaux différents. Toros jarameños et toros tarifeños, qui provenaient respectivement des rives du Jarama au Nord de Madrid et des plaines marécageuses du littoral de Tarifa ont alors connu de grandes heures. Aujourd'hui les taureaux sont désignés par le nom de l'éleveur et leurs caractéristiques sont identifiées à partir de l'encaste dominant, prenant le pas sur toutes considérations territoriales. Autrement dit, le déterminisme du sang prime sur tout déterminisme géographique, même si à une autre échelle beaucoup d'éleveurs affirment que les terrains montagneux renforcent les pattes des taureaux. Cependant, et sans contradiction avec ce que nous venons d'énoncer, certains fleuves et affluents, à défaut de correspondre comme autrefois à des caractéristiques zootechniques individualisées, évoquent de hauts lieux de la géographie du taureau de combat. Certains cours d'eau acquièrent ainsi dans une géographie des représentations une forte légitimité pour découper l'espace de l'élevage brave en « fleuves taurins » au premier rang desquels figure assurément le Guadalquivir. Il semble que cet objet pseudo-géographique, manipulé à satiété par nombre d'aficionados érudits, résiste mal à l'analyse détaillée d'une implantation des élevages qui suivrait la logique axiale des fleuves. Cependant, il constitue une représentation importante de l'espace taurin dont il n'est pas exclu qu'elle plonge ses racines dans un mythe méditerranéen qui associe au taureau, en de nombreuses contrées le fleuve et la fertilité.

Quelle que soit la validité d'un découpage de la géographie du taureau de combat par bassin versant, les exploitations ne sont pas réparties de façon aléatoire le long des vallées, mais semblent au contraire occuper des tronçons longitudinaux particuliers du réseau hydrographique : les zones humides des embouchures et les interfluves d'amont. Dans le bassin du Douro, apparaissent des foyers de forte concentration d'élevages le long des affluents de rive droite du Tage, précisément les vallées du Tormes, de l'Hebra, du Yeltes et de l'Águeda qui descendent de la Sierra de Gata et de la Sierra de Peña de Francia. Dans la littérature taurine, le Yeltes porte souvent le nom de Guadalquivir charro afin de mettre en valeur l'extrême densité des élevages braves qu'il accueille. Dans le bassin du Tage, les élevages se concentrent au Nord de Madrid, à l'amont des vallées du Jarama, toujours célèbre pour ses taureaux, du Manzanares et du Guadarrama qui descendent de la Sierra du même nom. Ajoutons, avec une plus forte dispersion, la vallée du Tiétar. Sur la rive gauche du Tage, notons la présence d'un foyer de forte concentration dans les Monts de Tolède, depuis longtemps considérés comme une importante zone d'élevage. Le Guadalquivir est le fleuve le plus asso- 
cié à l'activité tauromachique et participe en cela au tropisme taurin andalou qui domine les représentations collectives. Deux zones principales concentrent les élevages de taureaux, les marismas du Guadalquivir et les affluents de rives droites correspondant aux vallées de la Jándula, du Guarrizas et du Guadalén qui descendent de la Sierra Morena dans la partie nord de la province de Jaén. Entre ces deux zones, s'égrène un chapelet discontinu d'élevages qui longent le fleuve, témoignant assurément d'un dynamisme important dans des espaces de fortes concurrences agricoles. Ajoutons à cet ensemble, en discontinuité avec le Guadalquivir, la partie avale du Guadalete et du petit fleuve côtier le Barbate. Enfin, la confluence estuarienne de l'Odiel et du Tinto dans la province de Huelva ainsi que le delta de l'Èbre dans la province de Tarragone, constituent des foyers secondaires de concentration d'élevages braves. Pour ce qui est du delta de l'Èbre, la plupart des élevages ont aujourd'hui quitté la zone deltaïque, concurrencée par l'urbanisation et le développement des activités agricoles.

Tous les observateurs s'accordent à considérer que les élevages de taureaux possèdent une très haute valeur environnementale liée au fonctionnement écosystémique des zones de pâturages et en particulier des dehesas ou montes adehesados. Ces milieux sont devenus parmi les plus emblématiques du paysage de la péninsule Ibérique. Qu’ils soient arborés ou non, ils se localisent principalement dans le Sud-Ouest de la Péninsule, en Alentejo au Portugal, à Salamanque, en Estrémadure, en Castilla - La Mancha et en Andalousie occidentale. Leur extension est estimée à 9 millions d'hectares. Le maintien d'un élevage extensif sur ces milieux fragiles contribue à la stabilité de l'écosystème ${ }^{36}$. En revanche, une intensification pastorale et une introduction excessive d'espèces ovines, caprines, porcines ou bovines fragilisent l'ensemble du système. En effet, le surpâturage entraîne la raréfaction des plantes possédant la plus forte valeur nutritive et peut causer, lorsqu'il s'intensifie, la disparition de la couverture herbacée, voire arbustive augmentant alors considérablement les risques d'érosion. Les taux de fertilité et le poids du bétail diminuent alors. À l'inverse, un souspâturage entraîne l'apparition d'espèces ligneuses qui augmentent les risques d'incendie et renforcent la concentration locale et la sédentarité du bétail. Les éleveurs cherchent toujours à éviter cette situation de concentration qui multiplie les risques de blessures pour les animaux de combat pouvant perdre alors toute valeur économique.

\section{II. — L'ESPACE TAUROMACHIQUE FRANÇAIS}

\section{Trois SPECTACLES POUR DEUX RÉGIONS TAURINES}

L'originalité de l'espace tauromachique français réside dans la coexistence de trois spectacles taurins autonomes : la tauromachie d'origine espagnole, la

\footnotetext{
${ }^{36}$ C. Hernández Díaz-Ambrona, « Ecología del pastoreo con ganado de lidia », p. 128.
} 
course landaise et la course camarguaise (voir carte 5, p. 236). Il existe de même qu'en Espagne des variations mineures dans le recensement des spectacles, liées principalement aux méthodes de classification. La tauromachie de type espagnol représente environ 200 spectacles par $\mathrm{an}^{37}$. L'Espagne programme donc vingt fois plus de spectacles de ce type que la France. Les chiffres de José María Sotomayor permettent une comparaison des spectacles majeurs entre les deux pays ${ }^{38}$.

GraphiQue 6. - Spectacles majeurs en Espagne et en France (2003)

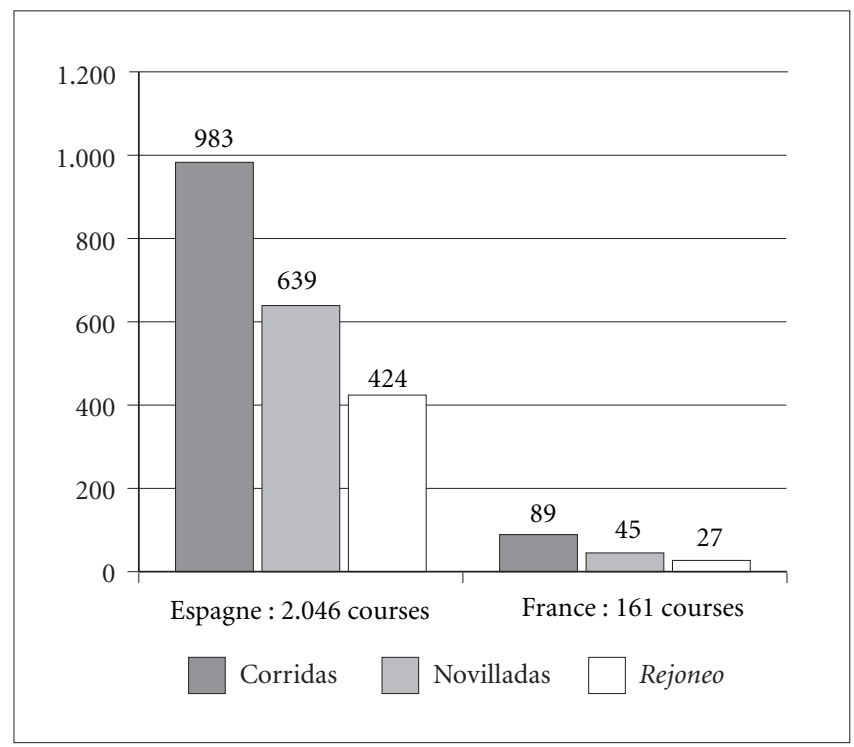

L'Espagne programme 12 fois plus de spectacles majeurs que la France. Les spectacles mineurs, environ une cinquantaine, représentent, en France, le quart des spectacles apparentés à la tauromachie espagnole ${ }^{39}$. Ils sont donc, en proportion, moins nombreux que leur équivalent espagnol, ce qui témoigne d'un marché secondaire beaucoup moins étoffé. Un élément apparemment anecdotique au regard des chiffres globaux est la plus forte proportion des corridas portugaises en France, comparée à celles célébrées en Espagne. En 2003, sur les 27 courses classées dans la catégorie du rejoneo, 9 sont des corridas portugaises sans mise à mort avec l'intervention finale des forcados. Cette proportion contraste avec la rarissime occurrence des corridas portugaises en Espagne. La

\footnotetext{
${ }^{37}$ Pour la saison 2003, José María Sotomayor a recensé 161 spectacles majeurs quand Mundotoro en compte 159 et la revue Tendido, 151.

${ }^{38} \mathrm{~J}$. M. Sотомауов, Estadísticas taurinas.

${ }^{39}$ Pour l'année 2004, Marc Lavie, co-fondateur de d'ATIDE (Asociación Taurina Internacional de Documentalistas y Estadísticos), recense 151 spectacles majeurs et 51 spectacles mineurs, soit un total de 202 spectacles taurins relevant de la tauromachie espagnole. M. Lavie, Corridas en France 2004.
} 
tauromachie à cheval en France accorde donc une place plus grande aux spectacles portugais, en particulier en Camargue où la tauromachie équestre connaît des expressions variées.

Les tauromachies camarguaise et landaise proposent un nombre de spectacles bien supérieur. Le nombre de courses camarguaises tourne autour des $900 \mathrm{spec}-$ tacles par an, et celui des courses landaises avoisine les 600 spectacles $^{40}$. De même que pour la tauromachie espagnole, il existe des catégories de spectacles permettant de différencier le haut niveau réunissant l'élite des raseteurs et des écarteurs. Pour chacune des courses, le haut niveau de la compétition représente environ 150 courses dans l'année ${ }^{41}$. Toutes pratiques tauromachiques confondues, la France programme ainsi autour de 1.700 spectacles d'arènes par an, ce qui revalorise l'importance des jeux taurins sur le sol national par rapport à la seule prise en compte des courses de type espagnol. L'écart est, en tous les cas, beaucoup moins important que ne le pensent généralement Espagnols et Français, qu'ils s'intéressent ou non à la tauromachie. Le cloisonnement des cultures taurines en France, cloisonnement qui n'est d'ailleurs pas totalement hermétique, et l'hégémonie médiatique exercée par le monde de la corrida, sont en partie responsables de cette minoration du patrimoine tauromachique français.

De même qu'en Espagne, le poids des jeux taurins populaires élargit considérablement la base des pratiques tauromachiques. Ces jeux sont plus nombreux dans le Sud-Est que dans le Sud-Ouest. Bien que cette explication ne soit pas entièrement satisfaisante, la raison de ce contraste tient en partie au fait que la Camargue est une importante région d'élevages de taureaux de combat, alors que les élevages sont peu nombreux dans le Sud-Ouest. Les chiffres annoncés par la Fédération française de course camarguaise témoignent d'une densité des pratiques tout à fait exceptionnelle. Les différents jeux et manifestations taurines à participation collective s'élèveraient à environ 8.000 manifestations par an, réparties entre les abrivados (2.000), les bandidos (2.000), les encierros camarguais (2.000) et les quelque 2.000 à 3.000 taureaux piscines, toro-ball et «courses de nuits ». Si l'on ajoute à cette liste, les quelque 2.000 ferrades annuelles, cet espace apparaît bien comme l'un des foyers taurins les plus denses du Sud-Ouest européen. Dans le Sud-Ouest, la Fédération française de courses landaises signale que chaque année sont officiellement enregistrées une centaine de courses intervaches. Programmées dans de nombreuses fêtes locales des Landes, du Gers et des Pyrénées Atlantiques, elles sont en réalité assurément plus nombreuses. Les courses de vaches sont quotidiennes lors des fêtes de Bayonne et de Dax. Ajoutons aux courses de vaches, les toro-piscines des stations balnéaires littorales qui s'étendent d'ailleurs bien plus au nord que la zone d'extension de la course landaise. Par ailleurs, une vingtaine d'encierros se

\footnotetext{
${ }^{40}$ Les données officielles de la FFCC comptabilisent 921 spectacles pour l'année 2004. Les données officielles de la FFCL comptabilisent 597 spectacles en 2006.

${ }^{41}$ Sont comptabilisées les courses camarguaises comptant pour le trophée des As (122 en 2006) et les courses landaises comptant pour les challenges Landes-Béarn et Armagnac ainsi que les concours landais (154 courses formelles et 7 concours landais pour 2006).
} 
déroulent désormais chaque année, calqués sur le modèle navarrais, mais pratiqués généralement avec des vaches ${ }^{42}$.

La tauromachie espagnole s'étend sur une frange méridionale. Douze départements sont concernés par ces spectacles, même si plus de $85 \%$ d'entre eux se déroulent en réalité sur six départements. Ils forment deux ensembles discontinus : à l'ouest, les départements des Landes, des Pyrénées Atlantiques et du Gers, à l'est les départements de l'Hérault, du Gard et des Bouches-du-Rhône. Au milieu apparaît un foyer intermédiaire de moindre importance dans les Pyrénées Orientales qui avec 8 spectacles se hissent quasiment à la hauteur du Gers (10), des Pyrénées Atlantiques (11) ou de l'Hérault (12).

La répartition des courses landaises et camarguaises est beaucoup plus concentrée. Notons que les Landes et la Camargue, quelles qu'en soient les définitions adoptées, ne regroupent pas la totalité des pratiques éponymes. Cette distorsion entre l'appellation des jeux et leur emprise spatiale a des conséquences sur la nature des sentiments d'appartenance territoriale véhiculés par les pratiques. Si on s'attache aux limites départementales, les courses camarguaises sont pratiquées dans quatre départements du midi méditerranéen, l'Hérault, le Gard, les Bouches-du-Rhône et le Vaucluse. L'essentiel de la pratique est néanmoins circonscrit entre la basse Provence et le Languedoc oriental. L'espace, mieux vaudrait-il dire le territoire, situé entre les villes de Montpellier, Marseille et Avignon, est appelé par les afeciounados le "triangle sacré ", ces villes demeurant en marge de la pratique taurine et de sa représentation géographique. La course landaise concerne six départements du Sud-Ouest, les quatre départements aquitains et deux départements de Midi-Pyrénées (Gers et Hautes-Pyrénées), même si l'immense majorité des spectacles se déroulent au nord de l'Adour, entre le sud-est landais et l'ouest du Gers. La répartition de l'ensemble des spectacles taurins détermine un recoupement partiel des pratiques séparé en deux ensembles régionaux disjoints : course espagnole et course landaise dans le Sud-Ouest et course espagnole et course camarguaise dans le Sud-Est. Entre ces deux ensembles les relations et les mobilités spatiales sont faibles y compris pour la tauromachie espagnole qui, à l'ouest comme à l'est, regarde plutôt vers l'Espagne.

\section{ArÈnes et haUtS LIEUX TAURINS}

Cette organisation se retrouve dans la répartition, l'utilisation et les caractéristiques mêmes des arènes. Compter les arènes existant en France pose des difficultés analogues à celles détaillées pour l'Espagne. Aucune étude, ni aucune institution ne les ont recensées dans leur totalité, chaque passion taurine n'ayant généralement d'yeux que pour ses propres temples ou sa propre région. Il existe néanmoins des études et des comptages partiels, de plus ou moins grande qualité. Pour les arènes du Sud-Est nous avons principalement utilisé les données d'un Annuaire des arènes Provence-Languedoc ${ }^{43}$. Pour les arènes du Sud-Ouest, le

\footnotetext{
${ }^{42}$ La FFCL a enregistré, en 2006, 22 encierros répartis sur 15 communes.

${ }^{43}$ G. Arnaud, Annuaire des arènes Provence-Languedoc.
} 
travail le plus fouillé a été réalisé par le Conseil d'architecture, d'urbanisme et de l'environnement des Landes, publié sous le titre : "Arènes de la course landaise et de la corrida $»^{44}$.

Notre recensement compte 270 arènes en France, chiffre global qu'il est nécessaire de détailler (voir annexe III [CD-ROM] et cartes 11 et 12, pp. 264 et 265). Les unes, les moins nombreuses, programment exclusivement des courses espagnoles. D'autres programment dans des proportions variables courses espagnoles et courses landaises ou camarguaises, le jeu local pouvant être dominant ou bien exceptionnel. Enfin, les dernières programment exclusivement des courses landaises ou camarguaises. Sur ces 270 arènes, une trentaine sont, soit des arènes démontables, soit des places de villages mixtes servant à la fois de place urbaine et d'arène éphémère. Durant ces cinq dernières années, 72 municipalités ont programmé des courses de type espagnol. Mais un certain nombre d'entre elles n'ouvrent pas leur porte chaque année. Le nombre des arènes qui proposent chaque année un spectacle de type espagnol est de l'ordre de la cinquantaine. En 2003, le nombre de "villes taurines », ainsi nommées dans la presse spécialisée dans la corrida et dans l'association qui rassemble ces villes, était de 53, contre 49 en 2004. Parmi ces arènes, certaines programment également des courses landaises (28 d'entre elles), d'autres des courses camarguaises (24 d'entre elles), quelques-unes programment les trois jeux taurins. Seulement 20 arènes sont exclusivement des lieux de courses espagnoles. La distinction entre les arènes de courses espagnoles et les autres arènes n'est donc pas toujours pertinente. Ainsi l'arène de Dax est à la fois un haut lieu de la corrida et de la course landaise, de même que les arènes d'Arles un haut lieu de la corrida et de la course camarguaise. Il existe 164 arènes de courses landaises homologuées par la Fédération pour accueillir les écarteurs et les coursières. Il en existe en réalité environ 180, mais certaines ne sont plus utilisables. Les arènes en dur de courses camarguaises sont moins nombreuses avec 89 pistes. Mais chaque année, il faut compter autour d'une centaine de lieux de spectacles, car certaines communes ont recours à des arènes démontables ou aménagent la place du village prévue à cet effet ${ }^{45}$.

Les cartes 11 et 12 (pp. 264 et 265) rendent compte de leur répartition et des modalités de coexistence spatiale des spectacles. La densité des arènes atteint localement des niveaux très élevés. Tel est le cas pour la course landaise, dans ce que Marylis Dauga nomme le «territoire historique » formé par la Chalosse, le Tursan et le Marsan ${ }^{46}$. Le département des Landes regroupe à lui seul 133 arènes permanentes, dont 80 se situent au sud d'une ligne Dax - Mont-de-Marsan - Gabarret. À une échelle plus fine le canton de Mugron, où vivent environ 5.300 Chalossais, compte une douzaine d'arènes permanentes. Le CAUE l'affirme sans ambages : "On peut considérer que la Chalosse est le lieu de plus forte concentration de sites tauromachiques dans le monde ${ }^{47}$.

\footnotetext{
${ }^{44}$ CAUE des LAndes, Arènes de la course landaise et de la corrida.

${ }^{45}$ En 2005, 97 communes ont programmé au moins une course camarguaise.

${ }^{46}$ M. Dauga, « L'âge d'or de la course landaise », p. 71.

${ }^{47}$ CAUE des Landes, Arènes de la course landaise et de la corrida, p. 17.
} 


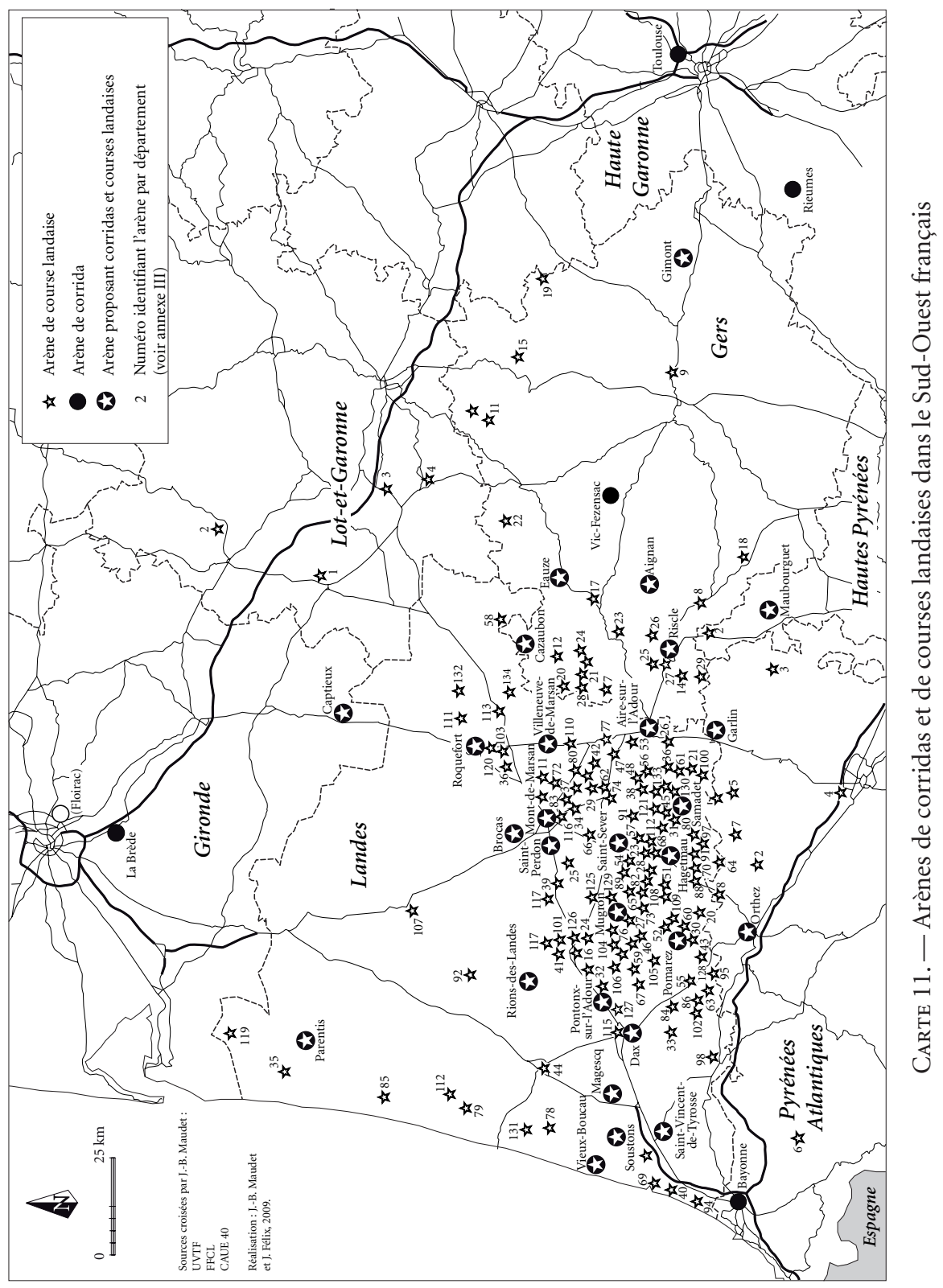




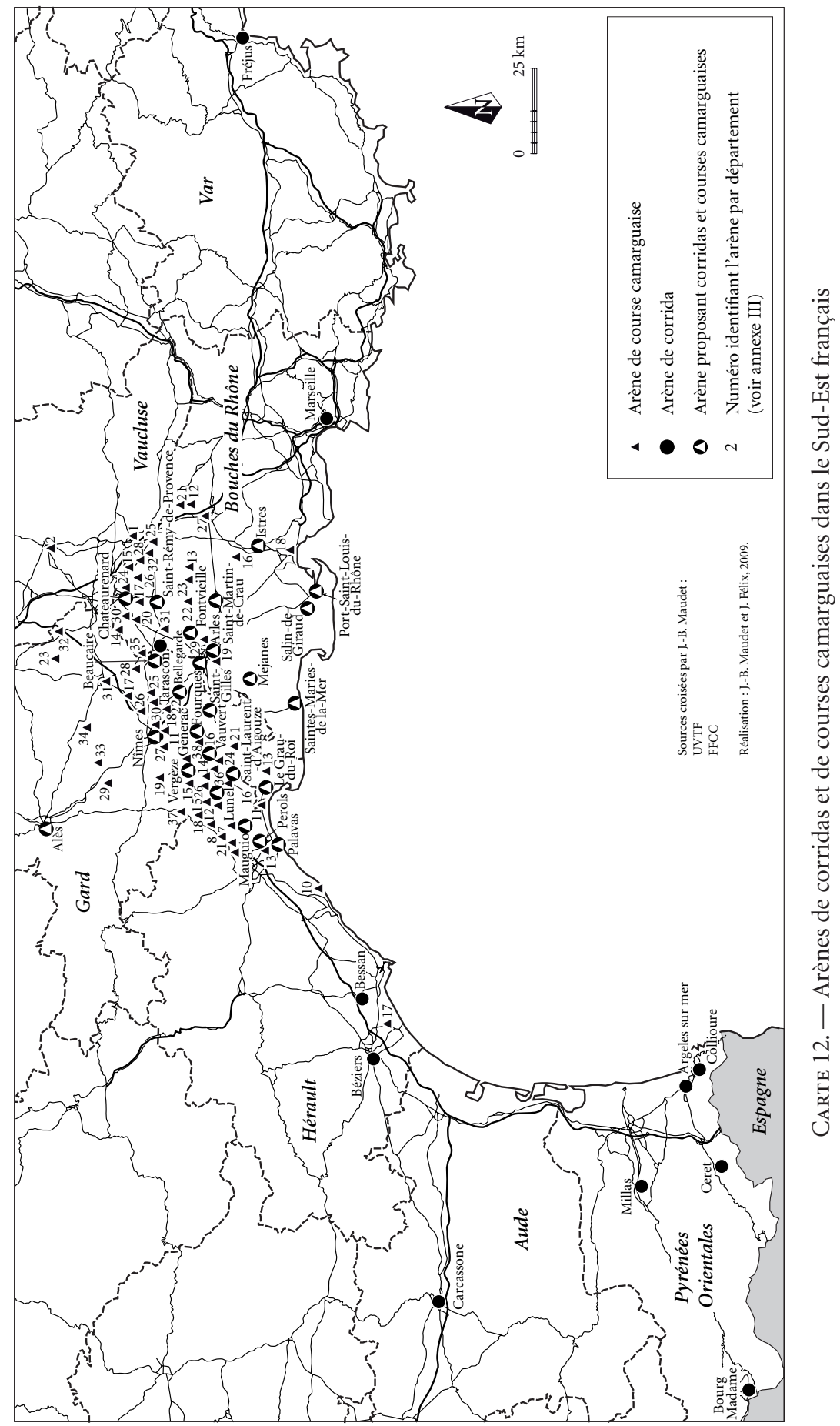


Une corrélation semble pouvoir être établie entre la forme des arènes, leur taille, la dominante des spectacles programmés et la trame du peuplement. Une règle simple suffit à l'exprimer : plus l'arène est grande et urbaine, plus elle programme de courses espagnoles. Plus l'arène est petite et rurale, plus elle tend à servir principalement de lieux de mise en scène de la tauromachie landaise ou camarguaise. Cette relation confirme l'idée d'une emprise majoritairement rurale des courses landaises et des courses camarguaises. Le caractère rural de ces courses s'exprime également par la valorisation symbolique des hauts lieux de leur pratique. Certes, les plus grandes villes du système urbain des régions considérées accueillent des courses de première importance. Le plus prestigieux et le plus ancien des « concours landais », qui est l'épreuve reine de la course landaise, se tient à Dax. Un autre se déroule à Mont-de-Marsan, un troisième à Aire-surAdour. Cela étant, les petites villes de Nogaro, Pomarez et Hagetmau complètent cette liste avec des concours de haute valeur. Pomarez, la seule arène couverte de la région, accueille la finale du Boléro d'or. Depuis 18 ans, elle organise le festival Art et Courage, remplaçant les vaches encordées par des taureaux sans corde, venus de France, d'Espagne et du Portugal. Cet événement est devenu l'un des rendez-vous les plus appréciés des coursayres. Mieux, Pomarez est appelé « la Mecque de la course landaise ", traduisant toute l'importance du haut lieu aux yeux des adeptes. La religiosité cesse d'être une simple métaphore à Bascons, où se dresse, à proximité des arènes, la chapelle de Notre-Dame de la Course Landaise ainsi que le musée de la course landaise, signes on ne peut plus explicites d'une géographie symbolique organisée dans l'intimité de la ruralité profonde.

Les arènes de courses landaises se distinguent également d'un point de vue architectural en raison des nécessités scénographiques liées à la logique axiale du jeu. L'étude du CAUE des Landes fournit, sur ce thème, des informations d'une grande précision permettant d'établir une typologie architecturale des arènes : l'arène semi-permanente, la tribune arène ou arène hispano-landaise, les amphithéâtres de gradins orientés, les amphithéâtres couverts en bois, les arènes de bourg à l'espagnole, les plazas espagnoles. Quatre formes principales peuvent être retenues : les arènes circulaires, oblongues, en fer à cheval et rectangulaires. À la circularité des arènes de corrida s'oppose la disposition axiale des arènes de courses landaises où la vache encordée charge dans le sens de la longueur. Le cercle et la piste longitudinale sont respectivement les formes idéales de la codification normative des jeux taurins espagnols et landais. Les deux autres formes sont des formes intermédiaires de compromis. Les formes angulaires et axiales des pistes sont mieux marquées à mesure que l'on descend dans la hiérarchie urbaine. Historiquement la forme en fer à cheval apparaît au moment où sont programmées, à la fin du XIX ${ }^{\mathrm{e}}$ siècle, des pratiques mixtes dites hispano-landaises. La forme oblongue apparaît plus tardivement et traduit un stade plus avancé d'intégration de la logique scénographique de la tauromachie espagnole. Les arènes constituent ainsi à la fois des marqueurs de diffusion de la tauromachie espagnole et d'assimilation syncrétique des pratiques.

Un développement analogue peut être mené au sujet de la course camarguaise. Arles et Nîmes accueillent les finales importantes de la compétition. La finale du 
biòu d'or qui récompense le meilleur taureau cocardier de l'année se tient en alternance dans ces deux arènes gallo-romaines. Mais les hauts lieux de la course camarguaise, ceux identifiés comme les lieux qui révèlent l'esprit de la course et l'authenticité de la passion taurine, sont des cités rurales de Petite Camargue : Le Cailar, également surnommé " la Mecque de la course camarguaise », Marsillargues et Saint-Laurent-d'Aigouze. Ajoutons Vauvert, Aimargues, Aigues-Vives, Lansargues et Lunel, situées dans le berceau de la tradition taurine camarguaise. Le nombre de courses dans ces villages et petites villes est d'ailleurs bien supérieur à celui des grandes cités. Observons pour l'année 2004 que les villes comme Montpellier, Nîmes et Arles ont programmé respectivement 3, 4 et 16 courses. Marsillargues en a programmé 28 et Saint-Laurent-d'Aigouze 33. À Marsillargues, l'arène n'est autre que la place du village et le toril est inséré dans le bâti, à droite du Café Le Français. La Petite Camargue est à beaucoup d'égards pour la course camarguaise ce que la Chalosse est à la course landaise. Notons également parmi les municipalités à offrir le plus de spectacles, celle du Grau-du-Roi avec 22 courses qui est la seule station balnéaire gardoise et qui profite à plein de la complémentarité entre bouvine et activité touristique.

Arles occupe néanmoins un rôle important dans l'espace matériel et symbolique de ce dispositif en raison de son emprise historique sur les activités du delta, et figure à ce titre parmi les lieux majeurs de la culture camarguaise. En témoigne, chaque premier mai, la réunion de la Confrérie des gardians sur le Boulevard des Lices que rejoignent les Arlésiennes en costume et quelques groupes folkloriques. En témoigne également le trophée de la Cocarde d'Or disputé le premier lundi de juillet depuis 1928 et qui a longtemps alimenté la rivalité entre Provençaux et Languedociens. À l'inverse d'une passion taurine vécue de façon intimiste et rustique dans les villages, Arles multiplie les signes de son rayonnement sur le territoire de la bouvine par une mise en scène plus cérémoniale.

Enfin, la Fédération impose également sa propre hiérarchie en distinguant les pistes en fonction de leur taille et du nombre de raseteurs ainsi autorisés à concourir. La Fédération distingue ainsi les « grandes pistes », les « moyennes » et les « petites », qui d'une certaine façon reprennent une catégorisation proche de celle existant pour les arènes en Espagne. Les grandes pistes, qui possèdent une capacité d'accueil supérieure, sont aussi celles qui programment le plus de spectacles. Les grandes pistes sont celles d'Alès, Arles, Beaucaire, Béziers, Châteaurenard, Le Grau-du-Roi, Les Saintes-Maries-de-la-Mer, Lunel, Nîmes, autant d'arènes dont la taille permet aussi de recevoir des courses espagnoles. Conformément au règlement espagnol, le diamètre d'une arène permanente ne doit en théorie pas être inférieur à 45 mètres pour que puisse se dérouler une corrida, et si le ruedo possède des angles, aucun côté ne doit être inférieur à 20 mètres. Beaucoup de petites pistes se trouvent ainsi disqualifiées.

Les hauts lieux français de la corrida s'organisent à partir d'une trame urbaine plus lâche. L'Union des villes taurines françaises s'est appliquée, sur le modèle espagnol, à différencier des catégories d'arènes. En France, les arènes dites de première catégorie, sont celles de Bayonne, Dax, Mont-de-Marsan, Vic-Fezensac pour le Sud-Ouest et Arles, Béziers et Nîmes pour le Sud-Est. Chaque année, 
ces arènes sont en tête des statistiques taurines avec un avantage pour Nîmes et Arles qui généralement offrent plus de quinze spectacles par an, suivies de Bayonne et Dax qui programment entre 10 et 15 spectacles par an. Les autres arènes sont classées en deuxième catégorie dont les normes techniques et réglementaires correspondent aux arènes espagnoles de troisième catégorie. Nîmes peut être considérée comme l'arène la plus importante en nombre de spectacles et en réputation acquise au-delà des Pyrénées. Nîmes a d'ailleurs été élue la meilleure arène de la saison 2006 par l'influente revue taurine 6 Toros 6 qui chaque année décerne diverses récompenses. La majesté de son amphithéâtre gallo-romain, que l'on retrouve également à Arles, participe de cette réputation. Beaucoup de toreros évoquent à ce propos l'émotion particulière à toréer dans ces lieux chargés d'histoire : entendre le contraire serait surprenant lors d'entretiens rapides qui souvent ne font qu'accoucher les représentations des journalistes eux-mêmes. Bayonne jouit d'un prestige historique qui lui confère une place à part. Comme nous l'avons souligné, elle est considérée comme la première ville française à avoir accueilli une corrida en août 1853 et joua un rôle important, pendant les dix années qui ont suivi, dans l'introduction progressive du spectacle espagnol en France.

Une autre différenciation des arènes françaises doit être mise en relief. Elle reprend l'opposition espagnole entre les arènes toristas et les arènes toreristas, qui en France ne possède aucune traduction géographique évidente. Les arènes sont globalement acquises à l'esthétique dominante qui est celle d'une passion torerista qui se réjouit d'inviter les plus grandes vedettes de la tauromachie actuelle. Cependant, l'afición française est riche de nombreux rendez-vous toristas, soit que l'arène sache alterner entre les corridas « dures » et les corridas de toreros vedettes, soit qu'elle s'érige en bastion radical du taureau " sérieux ». En témoignent deux indices très révélateurs. Parmi les cinq élevages qui ont obtenu le plus de contrats en France en 2004 figurent quatre fers toristas : Victorino Martín, Miura, Cebada Gago et l'élevage portugais de Palha, situation totalement impensable en Espagne ${ }^{48}$. De même, les deux matadors à avoir obtenu le plus de contrats en France sont des toreros spécialisés dans ce genre de bétail, l'Espagnol «El Fundi » et le Français Stéphane Fernandez Meca. Quoique très respectés par la profession, ces matadors ne peuvent pas être considérés comme des toreros vedettes qui généralement dès que leur statut le leur permet évitent de multiplier les affrontements avec de tels adversaires. Les villes de Béziers, Céret et Vic-Fezensac ont bâti leur réputation sur la programmation de taureaux « durs ». Dans le cas de Vic, la féria de la Pentecôte offre une alternative à la féria de Nîmes, ce dont s'enorgueillit une partie du public, fière d'avoir choisi son camp et accablant de tous les maux les courses de Nîmes. D'autres villes taurines comme Aignan, Aire-sur-Adour, Hagetmau, Parentis, Saint-Vincent-de-Tyrosse, Alès, Saint-Martin-de-Crau,

\footnotetext{
${ }^{48}$ On retrouve la même domination des élevages toristas pour les novilladas avec parmi les cinq élevages à avoir obtenu le plus de contrats : Fuente Ymbro, La Quinta et deux élevages français Gallon et Hubert Yonnet.
} 
programment également des corridas ou des novilladas propres à satisfaire l'afición torista et remplissent leur arène sur ce critère. À l'opposé, les arènes de Palavas ou des Saintes-Marie-de-la-Mer prolongent le littoral méditerranéen outrancièrement torerista en de nombreuses étapes. Les membres les plus radicaux de l'afición torista, qui s'accordent les lauriers d'une éthique puriste et intransigeante, dénigrent volontiers les spectacles qui se tiennent ailleurs y compris dans les arènes les plus connues s'abaissant à toutes les compromissions afin d'afficher un torero vedette : bétail faible, aux cornes réduites, issu d'élevages propres à faire briller artificiellement les artistes capricieux, sans prise de risque excessive. Pour fustiger la standardisation d'un bétail élevé sur mesure, ces aficionados dressent une liste noire des arènes qui consomment du « Mac Domecq », en référence aux élevages issus des encastes de la célèbre famille Domecq, dont les produits seraient à la tauromachie ce que les hamburgers sont au Guide Michelin. Une nouvelle fois, cette discrimination, qui peut avoir dans certaines occasions sa part de vérité, en dit sans doute davantage sur les processus de différenciation identitaire que sur la qualité générale des élevages issus de cette branche. À l'inverse beaucoup d'aficionados considèrent cette afición radicale comme « les ayatollahs » d'une tauromachie de principe et de règlement, aveuglés par leur esprit de clocher et ne jugeant les taureaux que par leur belle carrosserie et leur dangerosité ${ }^{49}$.

Retenons d'un point de vue géographique que la répartition des goûts ne relève ici d'aucun découpage évident à la différence de ce que l'on observe en Espagne. On remarque cependant que les arènes d'afición torista correspondent souvent à des cités où la société locale est très investie dans la programmation des spectacles taurins ou bien possède des moyens efficaces de régulation. C'est le cas en particulier à Vic-Fezensac, petite ville gersoise de 4.000 habitants, tard venue dans le cercle fermé des villes taurines en organisant sa première corrida en 1932 et sa première féria en 1966. À Vic, le club taurin, organisateur de la féria en lien avec les entrepreneurs de spectacles espagnols Chopera, fixe avant toute chose le choix des taureaux, le reste passe après. Cette stratégie s'est révélée payante et la féria de Vic fait chaque année le plein.

Les représentations de la géographie taurine française vue depuis l'Espagne sont riches d'enseignement. Beaucoup d'Espagnols ignorent l'existence de la tauromachie dans le Sud-Ouest français, à l'exception de Bayonne, et ont d'abord à l'esprit les arènes du Sud-Est de la France en particulier Nîmes et Arles. Cette image de la géographie taurine française s'accompagne parfois d'une autre déformation, répandue chez de nombreux spectateurs occasionnels, selon laquelle il n'y aurait pas de mise à mort du taureau en France. La raison réside sans doute en grande partie dans le fait que peu d'aficionados espagnols voyagent en France pour voir des corridas. Elle tient peut-être aussi à l'existence des courses landaises et camarguaises qui, même mal connues de l'autre côté des Pyrénées, brouillent l'image des caractères précis de la tauro-

\footnotetext{
${ }^{49}$ L'emploi du terme ayatollah n'est pas une liberté métaphorique de notre part, mais bien un terme souvent utilisé par les aficionados en question.
} 
machie en France. Du côté des professionnels, l'immense majorité s'accorde à reconnaître la très grande qualité de l'afición française, sa grande ferveur et son niveau d'exigence élevé. Certains lui reprochent néanmoins, à demi-mot, d'être souvent très intellectualisée, reprenant au passage quelques clichés sur le poids d'un héritage cartésien qui conditionnerait les aficionados français à apprendre d'abord leurs émotions dans les livres. Intéressante discrimination qui en retour fait de l'aficionado espagnol celui qui aurait la tauromachie dans le sang. Ces différences organisent un imaginaire qui sépare une afición innée qui serait celle des Espagnols, d'une afición acquise qui serait celle des Français, en rapport avec la diffusion spatiale de la corrida espagnole hors de ses frontières nationales d'origine.

\section{Les ÉleVAges de TAUREAUX : L'HÉGÉmonie CAMARguAise}

En France, il existe plusieurs types d'élevages de bovins qui répondent à chacune des tauromachies existantes. La plupart des taureaux de corrida en France sont des bêtes importées d'Espagne et dans une mesure non négligeable du Portugal. Néanmoins, il existe aussi des taureaux braves élevés en France, une quarantaine d'élevages réunis au sein de l'Association des Éleveurs français de taureaux de combat. Cette association est détentrice de la gestion du Livre généalogique de la race brave et gestionnaire des intérêts de la filière. La plupart de ces élevages, une trentaine, est située entre Crau, Camargue et pourtour des Alpilles. Les élevages restants sont éparpillés dans le Sud-Ouest entre les Landes, les Pyrénées Atlantiques et le Gers. Ces élevages sont destinés presque exclusivement au marché français, en particulier à celui des novilladas dont on a souligné l'importance pour de nombreuses arènes de deuxième catégorie. Certains ont acquis une bonne réputation aux yeux des aficionados français tels que les Tardieu, les Yonnet ou les Gallon. Les produits de l'élevage français ont du mal à s'exporter en Espagne en raison d'un protectionnisme de fait qui laisse d'autant moins de chance à leur concurrent que ces élevages n'ont pas acquis une réputation sur le temps long. En France, ils représentent environ 1/5 du marché national.

Ces élevages sont beaucoup moins nombreux que les manades de taureaux en Camargue. Elles sont actuellement entre 130 et 140 dont 117 enregistrées au sein de la Fédération. Les élevages sont principalement situés entre Fos-sur-Mer et l'étang de l'Or avec d'importantes concentrations sur les communes d'Arles, des Saintes-Maries-de-la-Mer, du Cailar. On compte actuellement sur cette zone quelque 6.000 taureaux de race brave et 15.000 taureaux Camargue ${ }^{50}$. Deux associations rassemblent l'essentiel des manadiers : l'Association des manadiers de taureaux de Camargue et l'Association des éleveurs de taureaux de race Camargue. L'activité ne regroupe qu'une trentaine de gardians salariés, l'essentiel du contingent des bouviers étant fourni par des gardians amateurs, soit quelque 2.000 cavaliers qui offrent leur aide gratuitement. Cela montre parfaitement

\footnotetext{
${ }^{50}$ J. Deffontaines, Taureau de Camargue, p. 6.
} 
l'imbrication complexe dans la culture de la bouvine entre la sphère des loisirs et la sphère du travail, que l'on retrouve en particulier dans les ferrades à michemin entre le jeu et la nécessité agronomique.

La viande du «Taureau de Camargue » bénéficie depuis 1996 d'une Appellation d'origine contrôlée qui garantit une meilleure rentabilité. Ce statut lui a assuré un certain succès pendant la crise de la vache folle. Cette appellation regroupe sans distinction les deux races de taureaux de combat, le taureau Camargue et le taureau de race brave. Certains propriétaires élèvent les deux races telle la famille Yonnet au Mas de la Bélugue. C'est à cette famille que l'on doit l'introduction en Camargue des premiers reproducteurs espagnols de race brave, à l'initiative de Joseph Yonnet, au domaine de Faramond, en 1869, dont le but à l'époque était de croiser les taureaux camarguais et espagnols. Dans les années 1950, cette famille fut à nouveau pionnière, cette fois dans l'élevage de taureaux de pure race espagnole à partir de bêtes issues de l'élevage portugais Pinto Barreiros ${ }^{51}$. Le cahier des charges de l'AOC précise que ce ne sont jamais les taureaux participant effectivement aux spectacles taurins que le consommateur retrouve dans son assiette puisque ceux-là sont exclus de l'appellation. Cependant les critères de sélection et d'élevage du bétail doivent correspondre à la vocation des jeux taurins. La charge pastorale maximale est de 1,5 ha par tête au sein de l'aire délimitée par l'AOC. Les taureaux doivent rester six mois de l'année sur les zones humides du delta. Du mois de janvier au mois d'avril, les taureaux pâturent sur les sansouires, des terres pauvres et salées, recouvertes l'hiver par la mer, où quelques graminées poussent au printemps. Du mois d'avril au mois de septembre les taureaux sont dans les marais d'eau douce. Le peu d'herbage disponible en raison de la concurrence d'activités pour l'occupation du sol, telles que la riziculture, la saliculture ou le tourisme, oblige les manadiers à alimenter leurs bêtes en fourrage et/ou à les déplacer l'hiver vers les garrigues des Costières, des Alpilles et des contreforts des Cévennes. Le Parc naturel régional de Camargue loue des zones de pâturage à de nombreux éleveurs dans des conditions qui évitent le surpâturage. De même que pour les marismas du Guadalquivir et avec des nombreuses ressemblances écosystémiques, les élevages de taureaux participent à l'équilibre environnemental, paysager et économique de la région. L'élevage des chevaux Camargue est le corollaire indispensable de l'élevage bovin et des jeux précédemment décrits tels que l'abrivado et la bandido. La vente de la viande, autrefois considérée comme un déshonneur par de nombreux manadiers, représente aujourd'hui jusqu'à $40 \%$ des revenus d'un élevage ${ }^{52}$. Soulignons également que 60 à $70 \%$ des bêtes d'un élevage ne sont pas aptes à la course et qu'un manadier renouvelle chaque année environ $50 \%$ de son troupeau ${ }^{53}$. L'AOC est donc devenue indispensable à la viabilité de la plupart des élevages.

\footnotetext{
${ }^{51}$ Sur la famille Yonnet et leur rôle dans l'élevage des taureaux de combat depuis un siècle et demi voir la revue Toros, $\mathrm{n}^{\circ} 1663,11$ octobre 2001, pp. 1-7.

52 J. Deffontaines, Taureau de Camargue p. 16.

${ }^{53}$ Conseil Économique et Social du Languedoc-Roussillon, Le rôle et l'impact du taureau, p. 16.
} 
Les élevages de bétail pour la course landaise sont appelés comme leurs homologues espagnols ganaderias, mais ils sont d'une tout autre nature. Il existe en 200718 ganaderias situées dans le «territoire historique » de la course landaise, totalisant environ 1.200 bêtes. Rappelons que dans la course landaise ce sont les ganaderias qui s'attachent les services d'une équipe d'écarteurs (la cuadrilla) pour une durée d'un an. Il ne faut pas exagérer l'affirmation souvent répétée selon laquelle ces ganaderias se contenteraient d'acheter des bêtes en péninsule Ibérique et ne s'emploieraient donc pas aux tâches de la reproduction. Certes, les éleveurs les plus importants achètent chaque année une centaine de vaches en Espagne, issue en particulier de la région de Salamanque. Cependant, la moitié des vaches naissent aujourd'hui sur place ce qui confirme l'émergence d'une filière d'élevage complète.

\section{III. — L'ESPACE TAUROMACHIQUE PORTUGAIS}

\section{Un Clivage Nord-Sud}

$\mathrm{Au}$ Portugal, en raison de la cohabitation originale de la corrida espagnole et de la corrida portugaise, la comparaison avec le reste des chiffres européens est rendue plus délicate. D’une manière générale, les chiffres concernant les spectacles taurins au Portugal, issus de sources françaises et espagnoles, sont à observer avec une grande prudence, car le pays n'est généralement pas regardé en tenant compte de la spécificité de ses tauromachies. Pour effectuer une comparaison précise du nombre de spectacles entre l'Espagne et le Portugal, il est impératif de détailler les chiffres bruts, comme dans le graphique 7 ci-dessous, puisque les formes tauromachiques n'ont pas de stricte équivalence de part et d'autre de la frontière.

D'après le Syndicat national des toreros portugais, il faut compter pour l'année 2004 quelque 300 spectacles taurins. Les chiffres du Syndicat portugais, à la différence des chiffres fournis par le Ministère espagnol, prennent en compte l'ensemble des spectacles taurins, majeurs et mineurs. Les 300 spectacles au Portugal sont donc à mettre en rapport avec les quelque 4.000 spectacles enregistrés en Espagne. Pour la même année, l'Inspection générale des affaires culturelles (l'IGAC), en charge de la régulation des manifestations taurines, estime le total à 287 spectacles. En dépit des variations comptables, l'Espagne programme environ dix fois plus de spectacles que le Portugal, pour une population espagnole environ quatre fois supérieure à la population portugaise. S'il fallait ne retenir pour le Portugal que les spectacles comparables aux spectacles majeurs espagnols et français, il conviendrait de soustraire du total les spectacles équivalant aux novilladas non piquées, aux becerradas et au toreo comique. Ce calcul est délicat puisque la pique est interdite au Portugal. Aussi décidons-nous de maintenir la totalité des novilladas dans la catégorie des spectacles majeurs, alors qu'en Espagne les novilladas non piquées appartiennent à la catégorie des spectacles mineurs. En revanche, les variedades taurinas et garraidas dont le règlement précise qu'il s'agit 
de bouvillons combattus par des amateurs ou des débutants, peuvent être considérées comme des spectacles mineurs. Les 1.912 spectacles majeurs en Espagne doivent alors être comparés aux 243 spectacles portugais d'une catégorie à peu près équivalente, avec toutes les réserves dues à la spécificité des formes tauromachiques. Après cette correction faite, les spectacles majeurs pour la période allant de 2000 à 2004 peuvent être comparés (voir graphique 8, p. 275).

GraphiQue 7. — Spectacles taurins au Portugal (2004)

\section{Nombre}

de courses

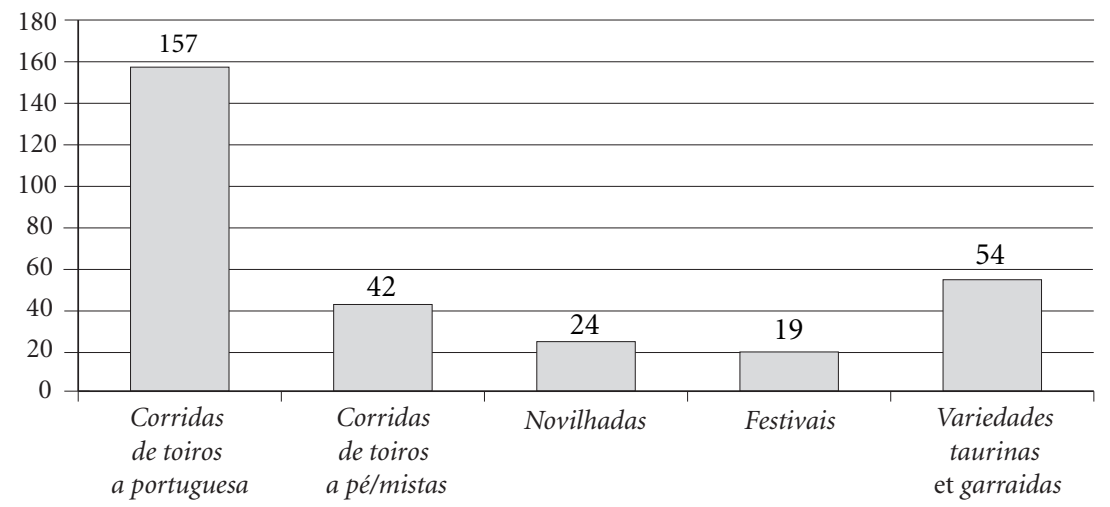

Type de courses

L'Espagne programme donc environ sept fois plus de spectacles majeurs que le Portugal. Si, au sein des spectacles majeurs, on ne tient compte que des spectacles du haut de la hiérarchie équivalant aux corridas qui incluent des taureaux de quatre ans révolus, il convient alors de comparer les 952 corridas côté espagnol avec les 199 corridas côté portugais, soit cinq fois plus de corridas de taureaux de quatre ans en Espagne qu'au Portugal ${ }^{54}$.

Au-delà des ajustements nécessaires à la comparaison quantitative des pratiques, il faut insister sur l'originalité de la mixité des pratiques tauromachiques au Portugal. Les matadors à pied, quelle que soit leur nationalité, sont présents lors d'un tiers des spectacles ${ }^{55}$. La pratique tauromachique d'origine espagnole, incarnée par la présence du matador, n'est donc, en aucun cas, à considérer comme une forme marginale en terre portugaise, même si elle demeure mino-

\footnotetext{
${ }^{54}$ Côté espagnol : 810 corridas formelles et 142 spectacles de rejoneo avec des taureaux ; côté portugais : 157 touradas ou corridas de toiros a portuguesa et 42 corridas de toiros a pé et corridas de toiros mistas incluant matador, cavaliers et forcados.

${ }^{55}$ Cette évaluation tient compte des corridas à pied, des corridas mixtes ainsi que des novilladas et des festivals qui, sauf exception, sont aussi des spectacles mixtes.
} 
ritaire comparée aux touradas. Cependant, remarquons que la tauromachie à pied programmée comme seule et unique pratique du spectacle tauromachique demeure très rare. En 2004, on compte seulement deux corridas ne présentant que des hommes à pied, les quarante autres spectacles étant des corridas mixtes. Par conséquent, les spectacles qui laissent seuls à l'affiche les toreros à pied, sans qu'ils ne soient accompagnés de cavaliers, sont très rares. Et même lorsque l'occasion se présente, comme à Moita do Ribatejo, rappelons que l'intervention des forcados à chaque taureau est une obligation réglementaire et ampute la norme espagnole de son acte final. Les seules exceptions, d'un intérêt géographique primordial pour comprendre les relations taurines entre l'Espagne et le Portugal, sont les courses de taureaux intégrales de Barrancos, autorisées depuis 2002.

La répartition des spectacles taurins au Portugal est déséquilibrée puisque la moitié Sud du pays regroupe $75 \%$ des spectacles, la moitié Nord $20 \%$ et l'archipel des Açores $5 \%$. Pour ce calcul, nous prenons comme unité territoriale le découpage historique des provinces portugaises. Conformément à la partition classique Nord-Sud du pays proposée par le grand géographe Orlando Ribeiro, nous incluons dans le Nord, les provinces du Minho, Douro, Trás-os-Montes e Alto Douro, Beira Alta, Beira Littoral, Beira Baixa et dans le Sud, les provinces du Ribatejo, d'Estrémadure, de l'Alto Alentejo, du Baixo Alentejo ainsi que de l'Algarve $^{56}$. En dépit de l'inégale superficie des provinces rendant les comparaisons délicates à cette échelle, l'Estrémadure, comptant presque un quart des spectacles, arrive en tête.

Pour ce qui est des jeux taurins populaires, nous disposons de trop rares données chiffrées pour présenter un bilan global satisfaisant. Au terme de notre enquête au Portugal, nous sommes néanmoins parvenus à la conviction qu'ils pouvaient être environ dix fois supérieurs au nombre de spectacles taurins officiellement enregistrés. Cette proportion témoigne sans doute autant du faible développement de la tauromachie professionnelle que de l'importance des jeux taurins populaires. Il y aurait ainsi autour de 3.000 spectacles taurins populaires par an au Portugal.

Nous avons souligné précédemment la diversité de ces jeux. Les travaux réalisés par Luís Capucha permettent une régionalisation relativement précise ${ }^{57}$. Au Nord, il existe de nombreux rituels faisant intervenir du bétail, mais ils prennent rarement la forme de jeux proprement tauromachiques. Les vacas de fuego du Douro Litoral ont les mêmes caractéristiques que les toros de fuego en France, réalisés avec de faux taureaux portés par des hommes. Il existe des cérémonies et des processions où les bovins interviennent comme élément du rituel, qu'ils soient bénis ou qu'ils prennent part au cortège, mais ils ne font pas l'objet de pratique tauromachique. De même, les chegas de bois du Barroso, conformément à notre définition préliminaire de la tauromachie, en sont exclues. Luís Capucha considère qu'il s'agit d'une zone où la référence aux bovins est principalement mythologique.

\footnotetext{
${ }^{56}$ O. Ribeiro, Portugal, o Mediterrâneo e o Atlântico.

${ }^{57}$ L. CAPUCha, « Mosaico de las fiestas de toros en Portugal ».
} 
En revanche, à la frontière entre l'Espagne et les provinces de Beira Alta et Beira Baixa, s'individualise un espace taurin d'une grande originalité, correspondant peu ou prou à la partie orientale de la Sierra da Estrela. C'est là, et nulle part ailleurs, qu'un groupe de villages organise les capeias raianas, parmi lesquels nous avons recensé les villages de Foios, Quadrazais, Soito, Alfaites, Aldeia do Bispo, Forcalhos, Lageosa, Aldeia Velha et Aldeia da Ponte. À Aldeia da Ponte, où se tient chaque année la finale opposant les différents villages, les habitants ont eux-mêmes financé l'arène et participé à sa construction.

Graphique 8. - Spectacles majeurs en Espagne et au Portugal (2000-2004)

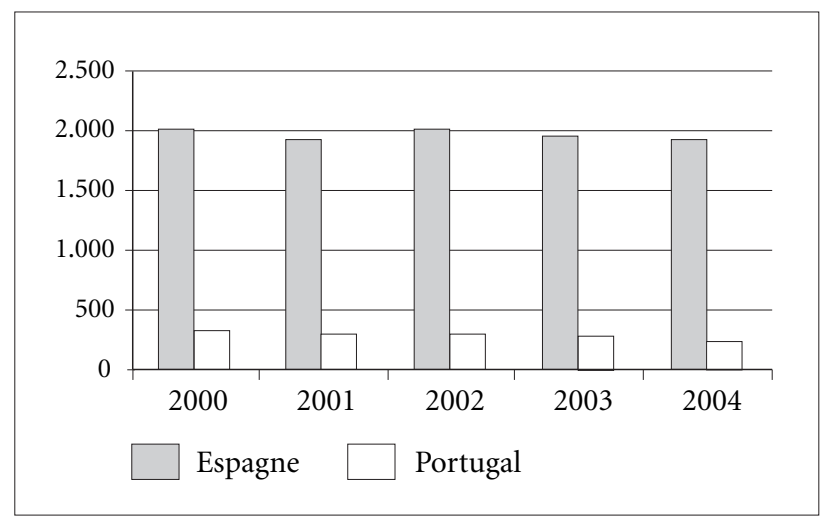

Plus au Sud, le nombre de jeux taurins s’intensifie avec des spécificités locales qui témoignent d'une importante fragmentation des rituels. Les encerros se multiplient dans la partie centrale de la frontière. Les esperas gagnent en importance autour de Lisbonne et dans le Ribatejo où les champs et les espaces urbains sont proches. Ce type de jeu populaire est fréquent à Santarém, Azambuja, Arruda, Benavente, Cartaxo, Samora, Moita, Montijo, Alcochete, Vila Franca de Xira. Selon Luis Capucha, les touradas a la alentejana (lâchers individuels de bovins en lieu clos) sont présentes dans « tous les villages de l'Alentejo à de rares exceptions » et les pamplonas sont nombreuses dans une zone comprise entre Lisbonne et Mondugo ${ }^{58}$.

Notons également l'existence de jeux taurins populaires suivis du sacrifice public du taureau. L'identité des municipalités concernées n'est pas révélée par l'auteur pour éviter leur éventuelle condamnation. Ces villages sont situés sur la rive gauche du Guadiana dans le Bas Alentejo. Le taureau est tué à l'aide d'un couteau, la choupa, après avoir été attaché à une corde et immobilisé contre une pierre de l'arène. L'animal est ensuite découpé et la viande vendue entre les habitants du village. Il convient de noter que Barrancos se situe précisément dans cette zone. La proximité géographique des rituels taurins, si l'on accepte de considérer qu'ils témoignent d'une signification sociale voisine, accrédite ici

${ }^{58}$ L. CAPUCHA, « Mosaico de las fiestas de toros en Portugal ». 
fortement la thèse de la dimension sacrificielle des courses de taureaux, même si d'autres éclairages complémentaires sont possibles.

Enfin, en matière de jeux taurins populaires, nous ne pouvons omettre le développement très original des touradas à corda sur l'île Terceira aux Açores. En 2004, ont été programmées 254 touradas à corda réparties entre les deux unités territoriales de l'̂̂le : 146 dans le concelho de Angra do Heroismo et 108 dans celui de Praia à la faveur d'une constante émulation.

\section{FAIBLE HiÉRARCHIE ET PARTICULARISME DES ARÈNES}

Il existe environ une centaine d'arènes en dur au Portugal. Nous en avons recensé 116, dont on ignore l'état pour une vingtaine d'entre elles et dont il ne fait aucun doute qu'une quinzaine est aujourd'hui détruite (voir annexe II [CD-ROM]). Sur cette centaine d'arènes en dur, 65 ont fonctionné en 2004, complétées par les quelque 74 communes qui ont eu recours à des arènes démontables. Ainsi cette année-là, 139 municipalités ont proposé un spectacle taurin. La proportion des arènes démontables utilisées au Portugal est plus forte qu'en Espagne. Le nombre de spectacles donnés dans des arènes en dur, demeure néanmoins légèrement supérieur à ceux donnés dans des arènes démontables. En effet, l'IGAC estime qu'en 2005, 60 \% des spectacles se sont déroulés dans des arènes en dur. Les arènes démontables manifestent souvent un enracinement plus superficiel et plus irrégulier du spectacle taurin. Sur la période allant de 2001 à 2005, par rapport à 2003 qui est l'année de référence pour ce calcul, 70 communes supplémentaires ont eu recours à des arènes démontables pour programmer au moins un spectacle. Il existe donc un volet important de communes pour lesquelles le jour de taureau ne s'inscrit pas dans une régularité annuelle et demeure tout à fait exceptionnel. Cette observation contraste avec ce qui se passe en France où le renouvellement des villes taurines est très faible en raison des conditions légales d'autorisation des spectacles qui dépend de l'ancienneté locale de la tradition. C'est une différence fondamentale de l'organisation des espaces taurins entre la péninsule Ibérique et la France, évolutive au Sud des Pyrénées, figée au Nord.

Au Portugal, la dichotomie entre un Sud taurin et un Nord qui l'est beaucoup moins, se voit matérialisée par le maillage et la hiérarchie des lieux de spectacles. Le règlement taurin portugais distingue des catégories d'arènes qui associent l'ancienneté de la tradition et le nombre de spectacles, de même qu'en Espagne. Les $3 / 4$ des arènes de première et de deuxième catégories sont situées dans la moitié Sud du pays. Dans cette moitié Sud, les huit arènes de première catégorie sont regroupées dans seulement trois districts équivalant aux deux provinces historiques Ribatejo et Estrémadure situées dans l'orbite immédiate de la capitale. Cette zone peut être considérée comme le cour de l'espace tauromachique portugais du point de vue des spectacles. Jusqu'à la réouverture des arènes de Lisbonne en 2007, l'armature taurine générale a été privée de sa tête pendant cinq ans. Malgré le retour de la tauromachie à Lisbonne après ce long intermède, les arènes de la capitale qui comptent aujourd'hui 11.000 places n'ont 
jamais été les plus grandes du Portugal. Les arènes de Santarém les dépassent avec 13.600 places. Par comparaison, rappelons qu'en Espagne, il existe une cinquantaine d'arènes de plus de 10.000 places alors qu'elles ne sont que deux au Portugal. Les nouvelles arènes de Lisbonne confirment néanmoins leur rang et restent une référence historique pour la tauromachie à cheval. Il est intéressant de constater que c'est un cavalier espagnol, Pablo Hermoso de Mendoza qui eut l'honneur d'inaugurer les nouvelles arènes, sans nul doute en raison de la suprématie sans frontière qu'il exerce depuis une dizaine d'années sur la discipline. Il était accompagné de deux matadors, l'Espagnol «El Juli », l'un des plus prestigieux toreros du moment et le Portugais Victor Mendes dont la carrière professionnelle a pris fin au milieu des années 1990. Cette course d'inauguration témoigne à elle seule d'une tauromachie portugaise placée sous le signe de la mixité. Elle révèle également, et c'est une hypothèse que nous vérifierons plus avant, que la culture taurine au Portugal véhicule un double sentiment identitaire, le premier fondé sur la revendication d'une identité nationale, le second fondé sur l'appartenance commune à la péninsule Ibérique, articulant ainsi une subtile dialectique entre frères ennemis et nations sœurs.

Plus surprenant encore sont les résultats d'une analyse du nombre de spectacles par arène. On observe qu'avec seulement quatre spectacles dans l'année, une arène se classe dans les dix premières. Cela confirme le faible développement de la tauromachie professionnelle en comparaison des programmations en Espagne et même en France. Une dizaine d'arènes programme ensuite trois spectacles, vingt-cinq autres deux spectacles et $65 \%$ des arènes offrent un seul et unique spectacle. La hiérarchie entre les arènes est donc faiblement développée et aucune arène ne propose de véritable saison tauromachique. Il existe une base très large de cités où la tauromachie n'a d'autre expression qu'une course annuelle, avec, on l'a vu, un renouvellement important des lieux de spectacles. En outre, les arènes qui proposent le plus de courses ne sont pas les plus grandes villes. Signe exemplaire d'un Nord faiblement taurin, la ville de Porto, malgré la construction de plusieurs arènes dans le dernier tiers du XIX ${ }^{\mathrm{e}}$ siècle, ne programme plus de spectacles depuis le milieu du $x^{e}$ siècle. À l'extrême sud du territoire, La Albufeira, en tête des statistiques, doit son record à la programmation estivale de courses de taureaux de piètre qualité à destination d'une importante clientèle touristique de passage en Algarve. Pour de nombreux aficionados portugais, les spectacles tauromachiques de l'Algarve sont une mise en scène folklorique de la tradition dénuée de toute dimension artistique. En cela, l'Algarve prolonge le chapelet discontinu d'arènes de plage qui court du Languedoc à l'Andalousie. Moita do Ribatejo s'affirme ensuite comme l'arène qui propose le plus de spectacles. On sait qu'elle est aussi celle où la tauromachie espagnole revêt une grande importance, de même qu'à Vila Franca de Xira qui se classe en quatrième position. Moita et Vila Franca de Xira sont deux petites villes voisines qui constituent des hauts lieux de la tauromachie au Portugal en général et de la tauromachie espagnole en particulier. Enfin, Angra de Heroismo sur l'île Terceira des Açores occupe la troisième place. Elle constitue un lieu taurin d'importance, insulaire et très excentré par rapport au reste du terri- 
toire national. Nombreux sont ainsi les lieux taurins d'importance à afficher de forts particularismes, qu'ils tiennent de leur situation, littorale et touristique, insulaire et distante, ou bien qu'ils se démarquent de la norme tauromachique nationale par un penchant affirmé pour la tauromachie espagnole.

Pour nuancer la rigidité de l'opposition Nord-Sud de l'espace tauromachique portugais, remarquons le rôle tenu par les arènes démontables en grand nombre dans la moitié Nord du pays. Elles relativisent la domination méridionale de l'implantation des arènes en dur. Grâce aux arènes démontables, les provinces du Minho et du Trás-os-Montes, acquièrent quelque importance et comptent chaque année une vingtaine de lieux de spectacles pour une trame permanente composée de seulement deux arènes. Du point de vue de la répartition géographique, l'appellation traditionnelle qui désigne la tauromachie au Portugal sous les traits de la Fête Nationale, s'arroge une plus forte légitimité, même si, on l'aura compris, ce n'est pas de ce côté-là qu'il faut chercher les raisons de cet usage. La dichotomie Nord-Sud de l'espace taurin portugais se double d'un autre clivage qui oppose cette fois-ci le littoral et l'intérieur. En effet, depuis le Nord du Portugal jusqu'à l'embouchure du Tage, se succèdent des villes taurines d'importance telles que Viana do Costelo, Póvoa do Varzim, Figueira da Foz, Nazaré, au-delà desquelles cette opposition perd de sa lisibilité dès que l'on pénètre en Estrémadure où la densité des lieux taurins s'étoffe.

\section{L'ÉLEVAGE BRAVE COMME GAGE D'INTÉGRATION}

La participation la plus évidente du Portugal au fonctionnement de l'espace tauromachique européen repose en grande partie sur ses élevages de taureaux de combat, anciens et de très bonne réputation. Nombre d'entre eux rivalisent avec les meilleurs élevages espagnols, spécialement dans le domaine des taureaux « durs ». L'Association portugaise des éleveurs de taureaux de combat recense 94 élevages dont 26 sont également inscrits à la section portugaise de l'Unión de Criadores de Toros de Lidia. Ajoutons à cela les treize ganaderias de l'île Terceira qui fournissent le bétail pour les courses professionnelles et les touradas à corda.

L'image du "fleuve taurin » est souvent reprise à propos du Tage autour duquel se répartissent les élevages braves du Ribatejo, en particulier ceux de la plaine alluviale de la Léziria. Ici les hauts lieux de l'élevage brave sont les alentours de Vila Franca de Xira, d'Alenquer, de Samora Correia et de Coruche. Cette zone riche en élevages se poursuit dans les milieux de montado du Haut Alentejo, équivalent des montes adehesados espagnols, jusqu'à la frontière avec la province de Badajoz. Historiquement, certains élevages ont précocement été intégrés au marché espagnol, d'abord les Palha dès 1883, puis les Oliveiras Irmãos, les Murteira Grave ou les Passanha. Aujourd'hui vingt-six élevages font partie de la prestigieuse UCTL témoignant d'une bonne pénétration du marché taurin espagnol et français pour les meilleurs d'entre eux. Cependant pour l'année 2004, pas un élevage portugais ne se classe parmi les vingt élevages qui ont été les plus programmés en Espagne alors qu'en France, les Palha occupent 
la cinquième place ${ }^{59}$. Le fait que les taureaux s'exportent mieux en France qu'en Espagne tient tout autant du bon développement du marché torista en France que de la vive concurrence exercée en Espagne par les élevages du cru. Soulignons qu'au début du $\mathrm{xx}^{\mathrm{e}}$ siècle, les taureaux portugais s'exportaient mieux qu'aujourd'hui chez le voisin Ibérique. En 1920, en Espagne, 110 Palha ont été combattus, seul Miura faisait alors mieux avec 140 taureaux ${ }^{60}$. Ces chiffres confirment la transformation des corridas orientée vers une esthétisation des valeurs du toreo tendant à réduire la part des taureaux « durs » qui ne s'adaptent pas à la nouvelle donne du marché. Pour les Palha, cette tentative d'adaptation s'est traduite dans les années 1930 par un apport de sang neuf visant à " anoblir » ou " adoucir » la caste originelle des taureaux composée du patrimoine génétique des Concha y Sierra et des Miura. Malgré cette évolution, l'élevage conserve son nom qui a la saveur d'un grand cru à la redoutable réputation.

${ }^{59}$ A. Piccamils Ruiz, Dietario taurino, p. 276 ; M. Lavie, Corridas en France 2004, p. 169.

${ }^{60}$ J. Durand, Libération, ${ }^{\circ}$ 7502, jeudi 23 juin 2005, p. 31. 



\section{LES DYNAMIQUES RÉCENTES DES ESPACES TAUROMACHIQUES EUROPÉENS}

La tauromachie en Europe est-elle une pratique en progression ou en déclin ? Cette question appelle des réponses qui tiennent compte de la diversité des pratiques et d'une différenciation géographique précise. Dans l'ensemble, il est possible de considérer que les pratiques tauromachiques sont en augmentation, en dépit de dynamiques contradictoires, en particulier en Espagne, qui contribuent à une différenciation croissante des régions taurines. En outre, cette augmentation spatialement sélective contribue à une redéfinition du paysage taurin européen et à l'affirmation de dynamiques transfrontalières jusqu’alors inédites.

\section{I. - CROISSANCE DES PRATIQUES \\ ET DIFFÉRENCIATION DES ESPACES TAURINS ESPAGNOLS}

En Espagne, les spectacles de la tauromachie professionnelle et les jeux taurins populaires sont entrés dans une phase de stabilisation après une période de progression qui court de la fin des années 1980 au début des années 2000. Cette période correspond également à une augmentation des contrastes entre les régions taurines espagnoles.

\section{EXPANSION ET RESTRUCTURATION}

DE LA TAUROMACHIE PROFESSIONNELLE

a) L'augmentation des spectacles

Une enquête réalisée en 2006 par l'institut de sondage ICSA Gallup révélait que $69 \%$ des Espagnols ne portaient aucun intérêt aux corridas. Ce chiffre est à peu près stable depuis une dizaine d'années. Il s'inscrit néanmoins, sur un temps plus long, dans une nette décroissance de l'intérêt porté aux corridas qui, selon les mêmes sources, auraient intéressé $55 \%$ de la population espagnole au début des années 1970, autour de $50 \%$ au début des années 1980 et $30 \%$ au début des années 1990. Les résultats de l'enquête font en outre apparaitre des 
différences géographiques qui confirment les tendances lourdes de la répartition des spectacles : un plus fort désintérêt pour les corridas en Catalogne et en Galice où il atteint $80 \%$ de la population contre $63 \%$ dans le reste du pays. À en croire ce sondage, cette évolution semble indiquer un irrémédiable déclin de la tauromachie. Or ces résultats ne sont pas en corrélation avec une baisse analogue du nombre de spectacles. Au contraire, ce nombre n’a jamais été aussi élevé et la tauromachie n'a sans doute jamais connu, en valeur absolue, autant de spectateurs. Pour les années 1990, 1991 et 1996, nous disposons d'un chiffre officiel qui fait état du nombre de spectacles taurins ${ }^{1}$. Le tableau ci-dessous rend compte de l'augmentation des spectacles de 1990 jusqu'aux évaluations de 2003 que nous avons nous-mêmes établies.

Tableau 3. - Croissance des spectacles taurins en Espagne (1990-2003)

\begin{tabular}{|c|c|c|c|c|}
\hline \multicolumn{1}{|c|}{ ANNÉE } & 1990 & 1991 & 1996 & 2003 \\
\hline $\begin{array}{l}\text { Spectacles taurins } \\
\text { majeurs et mineurs }\end{array}$ & 3.439 & 3.533 & 2.952 & 4.000 \\
\hline $\begin{array}{l}\text { Spectacles taurins } \\
\text { populaires }\end{array}$ & 11.130 & 12.020 & 13.790 & 17.000 \\
\hline \multicolumn{1}{|c|}{ ToTAL } & 14.569 & 15.553 & 16.742 & 21.000 \\
\hline
\end{tabular}

Cette évolution globale à la hausse masque des recompositions internes entre les pratiques. En 1990, le Conseil des vétérinaires d'Espagne annonçait un total de 14.569 spectacles, toutes pratiques confondues. Sur ce total, le nombre de festejos populares représente 11.130 spectacles et le nombre de spectacles de la tauromachie professionnelle s'élève à 3.439. Ce dernier chiffre comprend 1.162 spectacles majeurs et 2.277 spectacles mineurs.

Le grand changement des années 1990 tient à la diminution du nombre de spectacles mineurs, en particulier des spectacles comico-taurins et des becerradas, qui passent de 2.227 spectacles en 1990 à 1.313 spectacles en 1996. C'est principalement cette diminution qui fait chuter le bilan de la tauromachie professionnelle, alors que le nombre de spectacles majeurs n'a cessé d'augmenter sur la même période, passant de 1.162 spectacles en 1990 à 1.639 spectacles en 1996, pour approcher les 2.000 spectacles aujourd'hui. À partir du milieu

\footnotetext{
${ }^{1}$ Pour les années 1990-1991, il s’agit d'une étude du docteur Antonio Borregón Martínez, président du Consejo de Veterinarios de España, publié dans le quotidien Diario de Navarra, reproduite dans l'ouvrage E. De Marichalar, Le souffle dans le dos. Pour l'année 1996, il s'agit de données fournies par la CCNAT.
} 
des années 1980, nombre de villages et de petites villes, qui jusqu'alors ne célébraient qu'une novillada, passent au format au-dessus en organisant une corrida, signe direct du développement économique répercuté dans la sphère des loisirs. L'un des phénomènes marquants de cette croissance est aussi l'augmentation du nombre de spectacles à cheval, à partir de la fin des années 1980 . De 193 spectacles de rejoneo en 1990, ils atteignent un total de 372 en 1996 et se stabilisent ensuite autour des 350 spectacles. Mis à part les changements endogènes du secteur professionnel, cette évolution témoigne d'une transformation sociologique de fond liée à un engouement plus général pour l'équitation et une nouvelle place acquise par le cheval dans nos sociétés. Cette évolution est conforme aux analyses de Jean-Pierre Digard sur la position intermédiaire de la race équine dans le « système domesticatoire occidental » actuel, selon lequel le cheval a cessé d'être un animal de rente, attaché au travail des champs ou à la boucherie, pour appartenir à la catégorie des animaux de loisirs auprès d'un plus large éventail sociologique ${ }^{2}$.

Cette augmentation générale du nombre de spectacles est d'autant plus remarquable qu'elle vient après une période de reflux que beaucoup d'observateurs avaient analysée comme la chronique d'une fin proche. Des années 1970 jusqu'en 1981, le nombre de corridas retombe au niveau atteint à la fin des années 1950 avec moins de 400 courses dans l'année. À partir de 1982, une période de croissance s'amorce, d'abord lente et irrégulière jusqu'en 1988 (500 spectacles), puis de plus en plus rapide. La décennie 1990-2000 est bien celle d'une croissance accélérée du nombre de corridas qui passent de plus de 600 spectacles en 1993 à plus de 900 en 1998. Depuis ce nombre s'est stabilisé dans une fourchette comprise entre 850 et 950 corridas par an. Cette décennie est également marquée par la construction d'une quarantaine d'arènes. Ce phénomène avait été anticipé lors de la décennie antérieure qui a également compté une quarantaine de nouveaux édifices, ce qui aurait pu être interprété comme les premiers signes d'un renouveau. Par comparaison, dans les années 1970 moins d'une trentaine d'arènes avait été construite, alors que plus d'une cinquantaine d'arènes avait vu le jour dans les années 1960. Ces chiffres de construction d'arènes doivent être néanmoins tempérés puisqu'il est souvent très difficile de mettre en regard dans le même temps les arènes qui sont abandonnées.

Une étude de Borregón Martínez, pour les années 1990 et 1991, permet également d'en savoir plus sur le nombre de spectateurs que rassemblerait la tauromachie professionnelle. Les années 1990 et 1991 auraient enregistré respectivement un total de 41.839 .783 et 42.676 .578 spectateurs payants. Selon l'Union nationale des entrepreneurs taurins espagnols, l'année 1989 aurait rassemblé 39.102.601 spectateurs payants. Les estimations communiquées par l'Union nationale des spectacles taurins pour l'année 1996 sont de l'ordre de 60 millions de spectateurs payants, que l'on peut compléter par celles transmises personnellement pour l'année 1997 par Juan Manuel Moreno Menor, secrétaire général

\footnotetext{
${ }^{2}$ J.-P. Digard, Les français et leurs animaux, p. 69.
} 
de l'Union, qui estime le nombre de spectateurs payants à 65 millions. Selon ces critères, on observe une nette progression dans les années 1990 du nombre de spectateurs. Nous restons cependant très perplexes quant à la fiabilité de ces données. Pour l'année 1996, on ne voit pas par quel miracle les 2.952 spectacles de la tauromachie professionnelle auraient pu rassembler 60 millions de spectateurs, ce qui ferait une moyenne supérieure à 20.000 spectateurs par course. Même la moyenne plus basse d'environ 12.000 spectateurs par course que l'on obtient pour l'année 1990 nous paraît très excessive, ne serait-ce qu'en raison de la capacité des arènes. Nous trouvons encore plus étonnant que ces chiffres, qu'une simple division suffit à mettre en doute, n'aient jamais été critiqués. Le rapport de 2004 de la direction générale des spectacles publics d'Andalousie sur la tauromachie semble confirmer notre jugement sur le caractère fantaisiste des données nationales. En effet, pour l'année 2004, il y aurait eu en Andalousie quelque 1.836.887 spectateurs, estimation justifiée par un calcul par province et par type d'arènes, qui limite fortement la marge d'erreur. Ce chiffre ramené au nombre de spectacles de la tauromachie professionnelle en Andalousie cette année-là, soit 702 spectacles, implique une moyenne de 2.617 spectateurs par course. Sur la base de cette moyenne extrapolée au nombre total de spectacles pour l'année 2004, on obtient un nombre de spectateurs compris entre 10 et 11 millions. En dépit du caractère approximatif de cette extrapolation, le décalage nous semble suffisamment important pour dénoncer une affirmation répétée parfois dans les médias selon laquelle la tauromachie représenterait un nombre de spectateurs équivalent à celui du football professionnel. Si tel est le cas, le résultat est juste avec un raisonnement qui est faux. Mais une telle masse de spectateurs ne pourrait être approchée qu'en comptabilisant le public et les participants des jeux taurins populaires pour lesquels il n'existe guère de données fiables. Donnons un exemple qui permettrait de rendre crédible un nombre total de spectateurs bien supérieur à une dizaine de millions : en 2004, les cinq encierros de San Sebastián de los Reyes, dans la province de Madrid, ont rassemblé 18.500 personnes $^{3}$.

b) Les raisons de la croissance

Cette croissance relève de plusieurs facteurs que l'on peut résumer de la façon suivante. Après une crise de la tauromachie qui éclate au grand jour pendant la transition démocratique, les éléments précurseurs d'un renouveau se mettent progressivement en place. Ils conjuguent les effets d'une conjoncture extratauromachique favorable au développement des loisirs et d'un dynamisme endogène de l'industrie culturelle des spectacles taurins. La progression générale du niveau de vie, le développement des temps de loisirs, la croissance des mobilités, la massification du tourisme national et international, sont à mettre au compte des éléments conjoncturels extra-tauromachiques favorables. Cette évolution de fond explique en grande partie l'augmentation du nombre de spec-

\footnotetext{
${ }^{3}$ Bous al carrer, $n^{\circ} 42$, octobre 2004, p. 18.
} 
tacles taurins dans des régions où il n'y en avait pas (ou peu) et l'allongement des férias dans beaucoup d'arènes d'importance. Pour ce qui est du dynamisme endogène des industries culturelles de la tauromachie, dégageons deux évolutions majeures. D'une part, on observe un regroupement des spectacles en férias en vue d'accroître l'attractivité de chacun des événements taurins par leur coïncidence dans le temps. Cette évolution s'amorce dès les années 1950, mais s'accentue considérablement dans les années 1980. D'autre part, on observe une tendance à la concentration verticale et horizontale de la filière taurine. Autrement dit, la direction des arènes, la gestion de la carrière des toreros et parfois le contrôle des élevages de taureaux sont rassemblés entre les mains de quelquesuns. Certains trusts taurins s'illustrent par l'étendue de leur pouvoir possédant la gestion simultanée de nombreuses arènes. Cette transformation fait croître l'offre tauromachique, parfois au-delà de la demande réelle, tout en restant rentable sur l'ensemble de la filière.

Pour certains aficionados cette évolution s'est faite au détriment de la qualité des spectacles taurins en généralisant des programmations standardisées. Beaucoup d'aficionados qui possèdent un abonnement dans une arène se sentent alors piégés par une programmation d'ensemble dont ils sont captifs pour être sûrs de pouvoir assister aux quelques affiches qui les intéressent réellement. Mais il faut aussi reconnaître que cette rationalisation de la gestion a permis à de nombreuses férias de renaître, comme par exemple celle de Saragosse dans les années 1980 ou de redémarrer sur la base d'une féria étoffée comme à SaintSébastien à la fin des années 1990.

L'augmentation des spectacles tient aussi à l'engouement pour certaines figures de la tauromachie. Les unes ultra-médiatisées ont été dénigrées par nombre d'aficionados, mais ont attiré les foules, il est vrai parfois davantage devant leur poste de télévision que dans les arènes elles-mêmes. Tel fut le cas de Jesulín de Ubrique au milieu des années 1990 qui fut l'un des supports privilégiés des retransmissions télévisées de courses de taureaux sur les chaînes Antena 3 et Tele 5. D'autres toreros de la période sont reconnus pour leur qualité, leur génie ou l'espoir d'un geste historique qu'ils portent en eux et assurent aux organisateurs de spectacle une arène comble. En particulier, la croissance des spectacles des années 1980 ne peut se comprendre sans l'influence d'un torero d'exception, Paco Ojeda, qui non seulement attire à nouveau les foules sur son seul nom, mais développe aussi une transformation esthétique du toreo dont s'inspireront nombre de ses contemporains et de ses poursuivants.

Enfin, l'apparition des écoles de tauromachie marque également une évolution importante. Il existait bien le précédent historique de l'École de tauromachie de Séville établie par l'ordonnance royale du 28 mai 1830 sous le règne de Ferdinand III, mais elle ferme dès 1833 à la mort du roi. Jusqu'à la fin des années 1970, la formation et le repérage des futurs espoirs se faisaient soit par la pratique spontanée des maletillas qui attiraient l'attention dans les capeas de villages, soit par les liens familiaux avec des personnalités influentes insérées dans le milieu professionnel. Manuel Benítez «El Cordobés », dont les écrivains Dominique Lapierre et Larry Collins ont raconté l'histoire dans Ou tu porteras mon deuil, 
est sans doute l'un des derniers exemples, en tous les cas le plus emblématique, du torero parti de rien pour arriver au sommet ${ }^{4}$. Le développement des écoles de tauromachie ne semble pas avoir remis en cause le rôle déterminant des liens familiaux et des protecteurs influents pour percer. Il n'empêche que depuis l'ouverture de la première école de tauromachie à Madrid en 1976, une quinzaine d'autres ont ouvert leurs portes et transforment les conditions de recrutement des jeunes toreros. L'existence d'une filière taurine institutionnalisée a contribué à faire augmenter le nombre d'aspirants toreros. En 1999, plus de 4.000 professionnels taurins étaient inscrits sur les registres officiels ${ }^{5}$.

Revenons sur la concentration des pouvoirs entre les mains des entrepreneurs de spectacles, tant cette logique est importante pour comprendre le fonctionnement actuel de l'espace tauromachique. Cette concentration contribue à une modification de l'offre marchande. Le haut niveau de la tauromachie professionnelle se résume à quelques arènes importantes qui conditionnent les spectacles de la saison suivante et les hiérarchies entre les toreros. Cette logique d'organisation est le résultat d'une concurrence et d'une complémentarité entre entrepreneurs de spectacles qui contrôlent les arènes importantes. Au sein du mundillo, ils constituent une communauté relativement réduite. Ils forment entre eux une structure oligarchique par un système d'alliance complexe et ancien. Certains entrepreneurs, les plus puissants et les plus influents contrôlent des intérêts cumulatifs sur l'ensemble de la filière : propriétaires d'élevages, fondés de pouvoirs de toreros, organisateurs de spectacles dans plusieurs arènes et parfois même, propriétaires privés de ces mêmes arènes. Citons quelques noms de familles historiques : Canoera, Balaña, Lozano, Chopera. Ils répartissent leurs intérêts économiques en fonction du type de spectacle à programmer qui diffère selon la catégorie des arènes, selon les particularismes des sociétés " taurophiles", selon que l'afición s'affirme torista ou torerista et selon l'existence d'un torero du cru. Les caractéristiques des spectacles taurins dépendent ainsi des intérêts partiellement convergents des différents acteurs de la culture taurine : ceux de l'afición, ceux des municipalités propriétaires des arènes, ceux des gérants de l'arène, ceux des toreros, ceux des éleveurs.

Dans un article d'une portée théorique très éclairante, Michel Favory analyse ce fonctionnement à partir d'une lecture inspirée des théories de Boltanski et de Thevenot sur la justification publique : «En théorie, on peut considérer que tout spectacle taurin est le résultat de compromis et d'accords obtenus entre le monde de l'opinion (publics, journalistes, associations), celui de l'inspiration (toreros professionnels et élevages), le monde marchand et le monde civique $»^{6}$.

\footnotetext{
${ }^{4}$ Les maletillas, du mot maleta : valise, sont des personnages importants de la culture taurine qui faisaient montre de leur talent de village en village, afin de se faire remarquer et, pour certains, devenir toreros dans des spectacles professionnels.

${ }^{5}$ Dont 386 matadores de toros, 537 matadores de novillos con picadores, 1.221 matadores de novillos sin picadores, 96 rejoneadores de toros, 99 rejoneadores de novillos, 866 banderilleros de toros, 374 banderilleros de novillos, 280 picadores de toros, 146 picadores de novillos, 36 toreros cómicos.

${ }^{6} \mathrm{M}$. FAVORY, « Les bestiaires et l'espace », p. 5.
} 
Si l'on accepte de considérer que l'une des évolutions du fonctionnement de l'espace tauromachique est la constitution de trusts verticaux, cet équilibre peut se résoudre à une confrontation triangulaire des intérêts de la société marchande (les entrepreneurs de spectacles), de la société civile (les spectateurs) et des pouvoirs publics à la fois régulateurs et bénéficiaires de l'activité tauromachique. Dans cet équilibre, les grands entrepreneurs ont acquis un rôle prépondérant dont dépend une grande partie des dynamiques actuelles.

L'exemple le plus représentatif de ce phénomène est probablement celui de la famille Chopera que la presse taurine nomme parfois le clan Chopera, avec un mélange d'admiration et de méfiance face à ce que l'accumulation du pouvoir inspire souvent. En 1989, Manolo Chopera (originaire de Saint-Sébastien) est responsable de la gestion des arènes d'Almería, Badajoz, Logroño, Madrid, Tarazona, Talavera de la Reina, Tolède, Tudela, Soria ainsi qu'en France, des arènes de Bayonne, Mont-de-Marsan, Hagetmau. Cette même année, il est le fondé de pouvoir des matadors Antoñete, Manzanares, Ortega Cano et Juan Mora. Les cousins de Chopera, surnommé les Choperitas, ont la gestion des arènes d'Albacete, Cintruénigo, Murcie, Palencia, Salamanque, Valladolid, Vitoria et sont les fondés de pouvoir de Miguel Báez «Litri » et de Rafi Camino. Les Chopera possèdent également des intérêts économiques dans plusieurs élevages, dont celui de Martínez Elizondo qui porte leur véritable nom de famille. Cette influence s'étend indirectement à tous les acteurs de la tauromachie, tant leur pouvoir de négociation s'avère incontournable. Cette année-là, les Chopera contrôlaient la gestion de deux arènes de première catégorie, dont Madrid, la «bourse des valeurs taurines » et un quart des arènes de deuxième catégorie. Certaines années, les Chopera interviennent dans plus de 150 corridas. Manolo Chopera, que d'aucuns considèrent comme le plus grand imprésario taurin du $\mathrm{xx}^{\mathrm{e}}$ siècle, meurt en 2002 après avoir eu la satisfaction de bâtir en 1998 les nouvelles arènes de Saint-Sébastien, sa ville natale. Parmi les héritages importants que l'espace tauromachique doit à cet homme, on peut noter qu'il contribua à généraliser le transport des bêtes en camion. Cette évolution permit à de nombreuses arènes de s'affranchir de la proximité des élevages ou du tracé des lignes ferroviaires participant ainsi à un élargissement et à une densification de l'armature taurine. Malgré la mise à concours régulière des adjudications pour obtenir la gestion des arènes, les fils et cousins Chopera conservent aujourd'hui une très grande partie de cet héritage en ajoutant qu'ils sont désormais propriétaires des arènes de Saint-Sébastien, de celles de Logroño, copropriétaires de celles de Salamanque, de Badajoz et de Almendralejo. Leur emprise sur le fonctionnement de l'espace tauromachique est donc déterminante. Malgré son ampleur, cette emprise n'est pas pour autant déterritorialisée puisque leur pouvoir s'enracine prioritairement dans le Nord de l'Espagne, d'où ils sont originaires, à partir d'un réseau polynucléaire et transfrontalier qui s'étend jusqu'en France.

La croissance des spectacles de la tauromachie professionnelle, caractéristique des années 1990 avant de se stabiliser dans les années 2000, est donc le fruit d'une combinaison complexe de facteurs exogènes et endogènes. Cette croissance n'est évidemment pas uniforme sur l'ensemble du territoire. 
LES CONTRASTES GÉOGRAPHIQUES

DE LA CROISSANCE ET DU DÉCLIN

Cette croissance connaît des variations régionales importantes. Parmi les transformations les plus notables, signalons que le nombre de spectacles professionnels en Estrémadure a été multiplié par trois en vingt ans. Cette croissance supérieure à la moyenne nationale est à mettre en lien avec une augmentation importante du nombre d'élevages dans la région et avec la proximité du Portugal qui alimente un marché taurin transfrontalier favorable aux arènes espagnoles. En témoigne notamment le renouveau des arènes d'Olivenza qui utilisent à merveille les avantages de leur situation géographique frontalière conjugués aux avantages d'une féria de printemps qui ouvre la saison taurine. Nous reviendrons sur ces aspects dans l'étude des effets frontières liés aux discontinuités des espaces tauromachiques.

La province de Madrid fait également partie de celles qui enregistrent une croissance importante du nombre de spectacles. La construction de nouvelles arènes est un indice très révélateur de cette dynamique. Depuis le début des années 1980, plus d'une quinzaine d'arènes ont été construites. Le phénomène concerne tout autant l'agglomération madrilène que les autres villes de la province. Trois villes de la couronne sud de l'agglomération madrilène, dont la population a fortement augmenté au cours de ces trente dernières années en raison d'une importante mobilité intra-nationale et d'un étalement urbain de la capitale, ont récemment ouvert leurs portes : Móstoles en 1995, Leganés en 1997 et Getafe en 2004. Il n'est pas anodin que les origines géographiques de nombre de ces nouveaux urbains soient l'Andalousie, l'Estrémadure et la Castille, trois régions de fortes traditions taurines. Les enquêtes que nous avons menées sur place en 2005 ont révélé que l'afición locale se voit reconnue et consolidée par l'ouverture de ces arènes, tout en offrant une diversification de l'offre à l'échelle de l'aire urbaine madrilène. Cette configuration tend à renforcer les logiques ici cumulatives de l'offre et de la demande. Il en va de même de la réouverture en 2000, à Madrid même, de la célèbre arène de Vista Alegre dans le quartier de Carabanchel.

Enfin, la densification du réseau d'arènes littorales est également un phénomène important à souligner. Elle démarre dès les années 1960 en lien avec l'ouverture touristique du pays, donc bien avant la croissance des spectacles de la décennie 1990. Parmi la cinquantaine d'arènes construite dans les années 1960, une dizaine accompagne le développement des hauts lieux du tourisme balnéaire espagnol : Ibiza en 1960, Fuengirola, Lloret de Mar, Benidorm en 1962, Marbella en 1965, Benicassim en 1965, Benalmádena et Torremolinos en 1968. La plupart de ces arènes ne sont plus aujourd'hui des pôles tauromachiques actifs, à l'exception notable de Benalmádena, dans la province de Málaga, qui semble avoir fait de la tauromachie l'un des points forts de son offre touristique. En programment de 30 à 40 spectacles taurins par an, elle dépasse le nombre de spectacles programmés dans n'importe quelle arène andalouse y compris généralement dans celle de Séville. Les spectacles sont évidemment d'une tout 
autre qualité. À l'inverse en juin 2005, le maire de Lloret de Mar, Xavier Crespo, annonçait que les arènes allaient être détruites pour construire un parking souterrain, une place publique et un centre sportif.

À l'inverse de la tendance d'ensemble, certaines régions se caractérisent par le déclin des spectacles taurins à commencer par les Canaries qui les interdisent en 1991. Mais le phénomène le plus marquant est sans doute l'évolution de la Catalogne. Depuis une vingtaine d'années, les mesures qui tentent de faire disparaître la culture taurine s'accélèrent. En 1988, une loi de protection des animaux soutenue par les nationalistes de Convergència i Unió (CiU) limite la pratique de la tauromachie populaire, interdit la construction de nouvelles arènes, interdit l'utilisation d'arènes démontables comme celles d'Hospitalet ou de Figueras. En 2003, l'entrée dans les arènes est interdite aux enfants de moins de 14 ans. En avril 2004, le conseil municipal de Barcelone adopte par 21 voix contre 15 une déclaration de rejet de la corrida, déclarant la ville " anti-taurine ». Cette déclaration vise à faire interdire les pratiques tauromachiques en Catalogne par le gouvernement autonome. Avant Barcelone, certaines municipalités avaient déjà manifesté leur rejet de la tauromachie par des textes émanant des conseils municipaux : Tossa de Mar en 1989, Vilamacolum et la Vajol en 1991, Calonge en 1997. Après Barcelone une trentaine de municipalités ont fait de même, entraînées par la tête de pont $^{7}$. Certaines arènes ont récemment fermé leurs portes, celles de Gérone, de Lloret et de Las Arenas de Barcelone. Certes, à Barcelone, les arènes de la Monumental fonctionnent encore et proposent une saison tauromachique, mais elles sont sauf exception remplies au quart de leur contenance. Barcelone et, à travers sa capitale, l'ensemble de la Catalogne sont désormais au cour d'une opposition entre ceux qui condamnent la tauromachie et ceux qui la défendent en position très minoritaire. Du côté de ceux qui la condamnent, des motifs différents semblent regrouper les forces militantes autour de deux arguments principaux : d'une part l'argument nationaliste qui affirme que la tauromachie, marqueur ranci d'une Espagne unitaire et passéiste, n'est pas une tradition catalane, d'autre part l'argument « animalitaire » qui fustige une pratique considérée comme barbare et sadique. Les deux arguments convergent vers une conception de la modernité devant faire de Barcelone une ville catalane et civilisée. Du côté de ceux qui la défendent, les arguments s'articulent autour du poids de Barcelone dans l'histoire de la tauromachie, autour de l'ancienneté de la tradition taurine en Catalogne, notamment reliée aux élevages de taureaux du delta de l'Èbre, et autour d'un argument qui défend plus généralement l'importance universelle des valeurs culturelles et artistiques de la tauromachie. Ces arguments sont aujourd'hui réunis par l'intermé-

\footnotetext{
${ }^{7}$ Torelló, Calldetenes, Olot, Ripoll, Tavertet, Manlleu, Granollers, Valls, Molins de Rei, Sant Feliu de Llobregat en 2004, Bellpuig, Abrera, Sitges, Sant Cugat, Banyoles en 2005, Cerdanyola, Sant Andreu de la Barca, Mollet del Vallès, Teià, Sant Quirze de Besora, Gironella, Cabrera de Mar, Biure de l'Alt Empordà, Sant Iscle de Vallalta, Guissona en 2006 (le recensement de ces villes est exhaustif jusqu'en 2006).
} 
diaire d'une «plate-forme de défense de la Fiesta (taurine)», mise en place à l'initiative de la Fédération des entités taurines de Catalogne, à la suite des déclarations du conseil municipal de Barcelone rejetant la tauromachie. Cette plate-forme entend unir les efforts des milieux professionnels, de l'afición, de nombreux intellectuels, artistes et personnalités médiatiques pour expliquer et affirmer l'importance de la tauromachie afin de mieux la défendre des attaques croissantes qu'elle subit. Soulignons que le retour très attendu du matador José Tomás, après cinq ans d'absence, a eu lieu, le 17 juin 2007, dans les arènes de Barcelone, pleines à craquer pour l'occasion. Il s'agissait pour le monde professionnel et les aficionados, au-delà de l'euphorie suscitée par le retour du torero dans des arènes où il a maintes fois triomphé, de poser un acte symbolique permettant de démontrer que même les arènes de Barcelone pouvaient être trop petites. Cette corrida, qui était en une de nombreux journaux de la presse nationale, a donné lieu à d'importantes manifestations antitaurines à Barcelone, à la hauteur de l'événement.

La comparaison avec le Pays Basque est riche d'enseignements pour notre propos. En dépit d'une tradition tauromachique ancienne et d'une tout autre importance qu'en Catalogne, le rêve monolithique et unitaire de l'Espagne franquiste a comme nationalisé un héritage autrefois perçu sans appartenance identitaire exclusive. Claude Pelletier dans un ouvrage au tirage confidentiel parle du « grand malentendu » entre les Basques et la tauromachie ${ }^{8}$. L'utilisation plus ou moins directe du folklore taurin par le franquisme a eu des effets importants sur la désaffection des arènes au Pays Basque et le rejet de la tauromachie. Franco, contrairement à une idée reçue qui accentue volontiers les caractères de l'instrumentalisation politique de la tauromachie, ne se montrait guère aux arènes. Mais au Pays Basque, il s'y montrait chaque année lors de la corrida de l'Assomption à Saint-Sébastien, sa seule sortie taurine régulière avec la corrida annuelle de la Bienfaisance à Madrid. Comment ne pas interpréter cette présence comme un défi lancé au peuple basque, si l'on rappelle en parallèle que Bilbao avait pour habitude de donner une course le jour anniversaire de l'entrée des troupes nationalistes dans la capitale biscayenne ? Pour une immense majorité, l'annuelle " corrida de libération » avait tout d'une provocation, de même que la présence de Franco à Saint-Sébastien. La tauromachie était franquiste, point final. Les conséquences se font sentir dès avant la transition démocratique. La gestion des arènes de Bilbao et de Vitoria s'avère difficile. Les arènes privées du « Chofre » à Saint-Sébastien sont vendues en 1973 pour faire place à un ensemble immobilier et ne sont pas remplacées, le climat d'insécurité politique n'attirant pas les nouveaux investisseurs. Zarauz, Cestona, Fontarabie, Mondragón démolissent leurs arènes. À Tolosa, pendant la transition, on ironise en affirmant que la ville possède les arènes les plus vastes du monde en dépit de leurs modestes dimensions, pour la simple raison que l'on ne parvient jamais à les remplir !

\footnotetext{
${ }^{8}$ C. Pelletier, La tauromachie et les Basques.
} 
Même si la plupart des arènes de petites villes n'ont pas rouvert leurs portes, les années 1990 et 2000 ont quelque peu changé la donne. L'événement le plus marquant de cette évolution est la construction des arènes d'Illumbe à SaintSébastien en 1998, à l'initiative de la famille Chopera qui aussitôt lance une féria d'importance. Les vieux aficionados de Saint-Sébastien qui ont connu les arènes $\mathrm{du}$ Chofre en plein centre-ville, à quelques pas de la plage, affirment volontiers que les nouvelles arènes d'Illumbe manquent de charme : arène couverte, intérieur froid, sièges baquets, turbines d'aération, acoustique assourdissante. Elles sont attenantes à un cinéma multiplexe et à une galerie marchande animée par une musique d'ambiance et le va-et-vient des escalators. Les arènes sont situées à l'écart du centre-ville, près du stade de foot en bordure d'autoroute. Mais les canons architecturaux et urbanistiques modernes qui font des arènes d'Illumbe un des premiers "malls taurins », ont les mêmes avantages que leurs congénères commerciaux périurbains. Une meilleure accessibilité permet à la clientèle venue de France et d'ailleurs de se rendre aux arènes sans entrer dans Saint-Sébastien et un vaste parking règle en partie la question du stationnement. De plus, le spectacle a lieu quelle que soit la météo, facteur non négligeable en Guipúzcoa, ce qui permet assurément d'élargir l'aire de chalandise. En 2006, Vitoria, dernière grande ville en date à inaugurer un édifice flambant neuf, a aussi fait le pari d'une arène couverte et polyfonctionnelle afin d'ouvrir les lieux à d'autres types de spectacles et d'obtenir une rentabilité que la tauromachie seule ne suffirait probablement pas à garantir.

\section{RENAissanCE ET MUTATION}

\section{DES TAUROMACHIES POPULAIRES}

Il s'agit de l'évolution la plus remarquable de l'espace tauromachique espagnol allant à contre-courant de nombreuses prédictions qui pensaient que les tauromachies populaires allaient progressivement disparaître avec le déclin du monde rural traditionnel. La croissance explosive des jeux taurins populaires montre qu'il n'en est rien. D'après la CCNAT, le nombre global de festejos taurinos populares depuis 1987 aurait été multiplié par deux. Nos calculs attestent plus rigoureusement d'une augmentation inférieure, proche de $50 \%$ de 1990 à 2003 (voir tableau 3, p. 282). Là où nous disposons de données plus précises, nous pouvons mettre en évidence la mesure exacte de cette croissance.

En Castilla-León, le nombre de festejos taurinos populares est passé de 1.408 en 1997 à 2.109 en 2004, soit une augmentation moyenne de 100 spectacles par an. Dans le cas de la Communauté autonome de Madrid, le nombre de spectacles taurins populaires est passé de 485 en 1995 à 1.148 en 2004. Pour la région de Castilla-León, le décollage se fait à partir de l'année 1997, suivie de deux années qui enregistrent successivement une augmentation de plus de 250 spectacles. Comment expliquer ce décollage rapide? Nous avons constaté qu'il correspond précisément à l'application des normes du 28 avril 1997 régulant les conditions d'autorisation des spectacles taurins populaires. Il en va de même à Madrid et de façon encore plus saisissante. 
Graphique 9. - Augmentation des festejos populares dans la Communauté de Madrid

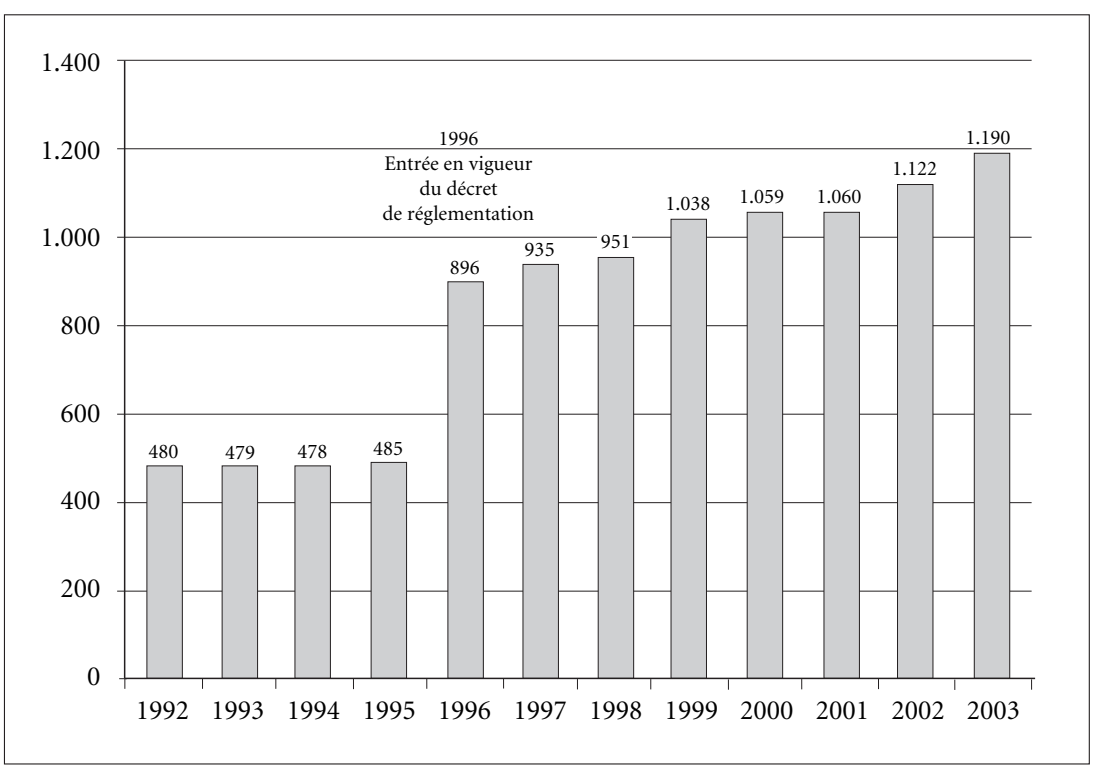

Le décollage s'opéré entre 1995 et 1996. Le nombre de spectacles passe alors de 485 à 896 . Ce décollage rapide correspond, ici aussi, à l'entrée en vigueur, en 1996, du décret régional définissant les normes d'application de la loi taurine au sujet des spectacles populaires. Ainsi établissons-nous un lien entre l'augmentation des jeux taurins populaires et leur régulation, clarifiant les conditions de leur exécution, la responsabilité des différents acteurs engagés, et les systèmes d'assurances afférents.

On retrouve cette même logique en Aragon, en Navarre et dans la Communauté valencienne où la réglementation et l'institutionnalisation des pratiques taurines populaires ont entraîné une augmentation du nombre de spectacles. Ceci explique sans doute en partie un décollage plus précoce du nombre de festejos taurinos populares dans la région de Valence qui adopte les premières réglementations dès 1988 dans un sens permissif, au moment même où le voisin catalan légifère en un sens très contraignant. Dans la région de Valence, les dix dernières années sont celles d'une consolidation et d'un enracinement des pratiques dont l'essentiel de la croissance est acquis dès le milieu des années 1990. Le cas de la province d'Alicante montre que le nombre d'autorisations a sensiblement diminué depuis, ce qui restreint la zone la plus dynamique aux provinces de Valence et de Castellón, en particulier la région comprise entre les deux capitales provinciales. En Castille, avec une entrée en vigueur plus tardive des réglementations, la croissance se poursuit à un rythme plus soutenu, mais qui montre là aussi les premiers signes d'une stabilisation. 
Comme évoqué plus haut, cette progression des festejos taurinos populares est allée de pair avec l'émergence, depuis la fin des années 1970, d'une nouvelle forme tauromachique en cours de professionnalisation : les concours de recortadores. Depuis les rives de l'Ėbre, d'où la mise en spectacle serait originaire, les concours de recortadores se sont développés dans les pays du Levant dès les années 1990, en Castille à la charnière des années 1990-2000, puis aujourd'hui en Andalousie. Nous avons déjà expliqué la difficulté à mesurer précisément leur nombre en raison d'une prise en compte institutionnelle encore très insatisfaisante. Nous disposons néanmoins de chiffres fiables pour certaines régions, détaillés sur le graphique ci-dessous. En Castilla-León, leur nombre est passé de 7 spectacles en 1997 à 64 en 2004. Dans la région de Madrid, pour les mêmes dates, leur nombre est passé de 29 spectacles à 123 .

\section{GraphiQue 10. - Augmentation des concours de recortadores (Madrid et Castilla-León)}

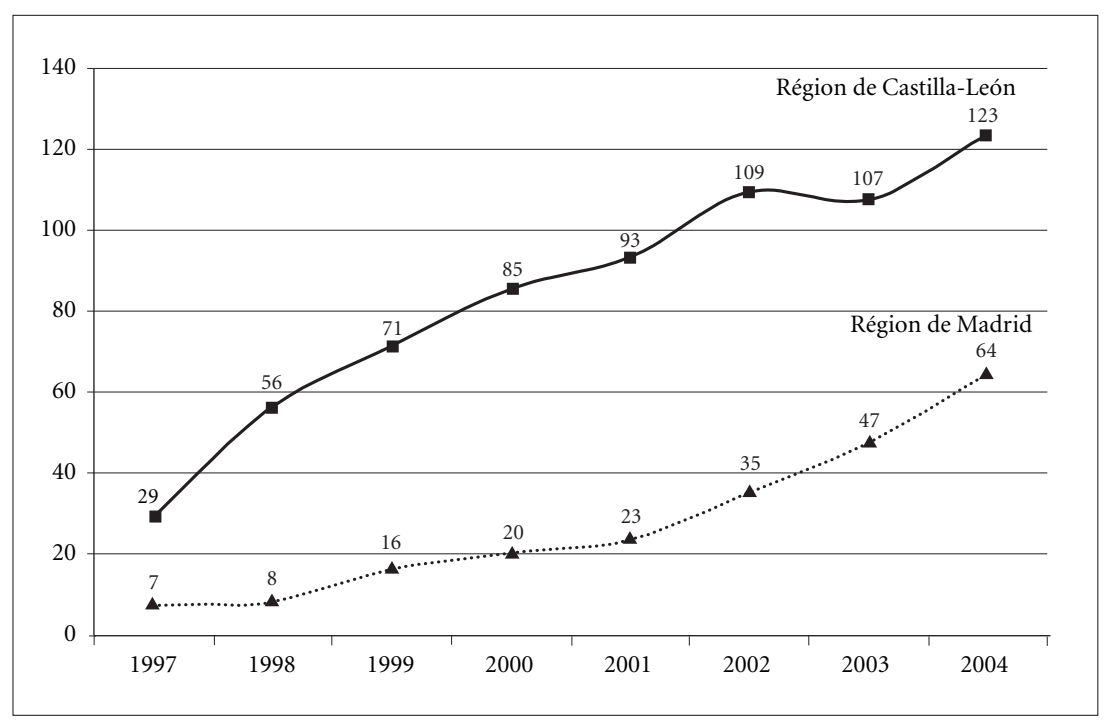

En 2003, ce sont les arènes de Las Ventas qui programment, pour la première fois de leur histoire, un concours de recortadores. En 2005, c'est au tour des arènes de Séville suivies en 2006 par celles de Málaga, de Jaén et de Jerez. Les concours de recortadores sous leur forme du concours d'anneaux, caractéristique de l'Èbre moyen, et sous leur forme des recortes libres et des sauts, caractéristiques des pays valenciens, sont donc sortis de leur berceau. Le simple fait de voir annoncés des concours de recortadores à Madrid et à Séville, dans les deux arènes les plus prestigieuses de la "planète des taureaux ", confirme une importante mutation du fait social taurin. En dépit du cloisonnement des passions taurines, puisse notre étude servir à rendre visible cette mutation aujourd'hui à l'œuvre. Si cette mutation passe encore inaperçue pour beaucoup, rappelons que les concours de recortadores 
ont déjà atteint les 500 spectacles annuels, soit davantage que les spectacles de la tauromachie à cheval dont on a pourtant souligné la vitalité. S'il s'agissait de les considérer comme des spectacles majeurs, ce qui ne tardera pas si le processus de professionnalisation se poursuit, ils représenteraient déjà un spectacle professionnel sur cinq. Pour l'instant, les organisateurs de spectacles semblent être les grands bénéficiaires de cette évolution puisque la programmation d'une course de recortadores est relativement peu onéreuse et remplit convenablement les gradins. Le témoignage du recortador Ramón Bellver «El Blanco » permet de comprendre que ce sont les hommes en piste qui pour l'instant pâtissent de cet équilibre du marché :

Aujourd'hui les concours sont en train de devenir un spectacle de masse et commencent à brasser beaucoup d'argent. Il y a des arènes comme Valence qui font le plein à des prix qui pourraient ensuite être répercutés sur les gains des recortadores. Il faut que les choses s'équilibrent, celui qui n'a pas la chance de remporter un prix rentre chez lui avec 70 ou 100 euros. C'est lamentable lorsque les organisateurs ont empoché 10 à 15 millions [...] aucun d'entre nous ne vit professionnellement de sa passion, on a tous un travail : mécanicien, peintre en bâtiment, électricien, conducteur, etc. ${ }^{9}$.

Ce témoignage nous renvoie à la modernisation de la tauromachie en Espagne à l'époque où les toreros commençaient à affirmer leur statut de professionnels. Il est possible d'y voir l'éclosion d'une nouvelle tauromachie qui pour l'instant suit un cheminement géographique inverse. De même que la tauromachie d'origine andalouse s'est hier diffusée sur l'ensemble du territoire, du Sud vers le Nord, de même on observe aujourd'hui, dans un parcours inverse, une progression des concours de recortadores, du Nord vers le Sud et de la périphérie vers le centre. Faut-il y voir une revanche de la tauromachie du Nord et des périphéries, historiquement éclipsée par les codes andalous? Quoi qu'il en soit, le fait qu'il s'agisse d'un spectacle dans lequel la vue du sang est absente n'est sans doute pas étranger à sa rapide expansion. Il est fort possible qu'il attire, en plus des adeptes des jeux taurins, une frange de population que la tauromachie classique rebute. Enfin, l'argument économique est également un élément important pour expliquer ce succès.

Ce nouvel engouement pour la tauromachie populaire trouve son point d'aboutissement dans la création de férias spécialisées. Dans les années 1980, cette tauromachie obtient droit de cité dans les arènes de Pampelune, Saragosse, Castellón, Valence, c'est-à-dire dans les capitales de provinces déjà riches en festejos taurinos populares. Deux types de spectacles y sont organisés. D’une part, des jeux taurins à participation collective et des exhibitions de pratiques qui ressuscitent des traditions locales, revues et corrigées pour leur mise en scène dans une arène. D'autre part, des concours de recortadores perçus comme l'aboutissement professionnel de ce que la tauromachie populaire de rue porte en elle. Si l'on met de côté le cas particulier des encierros de Pampelune, c'est à Saragosse que l'on doit

\footnotetext{
${ }^{9}$ Bous al carrer, $\mathrm{n}^{\circ} 49$, mai 2005, p. 7.
} 


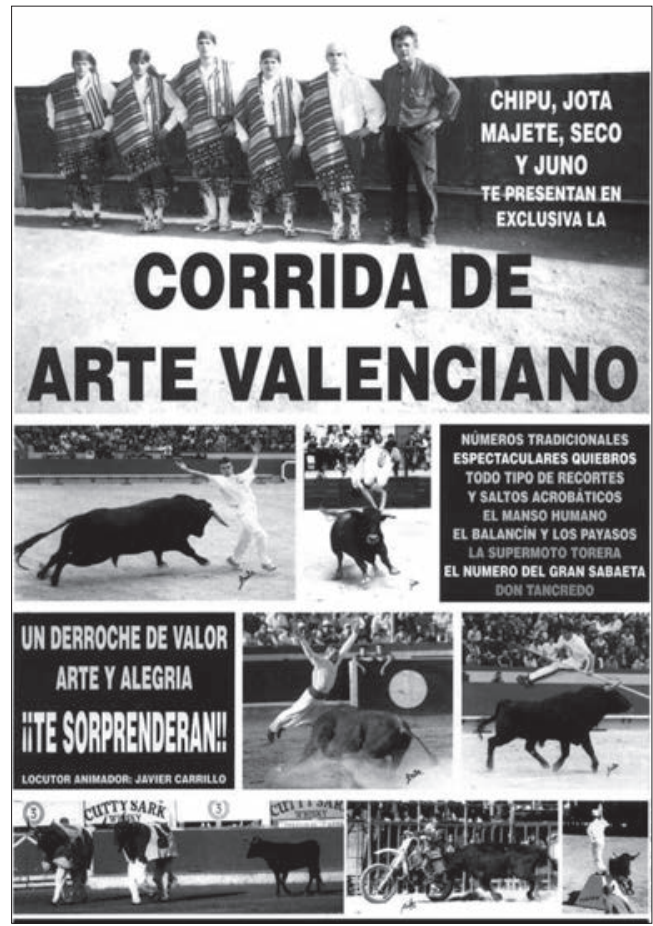

Fig. 50. - Corrida d'art valencien. Patrimonialisation régionale des courses de recortadores. La tenue blanche est abandonnée pour revêtir un habit traditionnel valencien (collection de l'auteur).

les premières initiatives. En 1982, des vaquillas sont programmées en matinée, complétées en 1984 par un concours de recortadores et des concours de roscaderos auxquels s'inscrivent plus de trente participants ${ }^{10}$. À l'initiative de ce projet, on trouve l'éleveur aragonais José María Arnillas qui est aussi l'un des codificateurs des concours de recortadores sous leur forme professionnelle, et Julián Nieto, organisateur de spectacles taurins populaires, à l'époque conseiller municipal de Saragosse. Ajoutons le rôle de la famille Lozano qui dirige alors les arènes et cherche les moyens d'en relancer la dynamique. Vingt ans après, Saragosse organise une double féria taurine, composée de douze spectacles majeurs donnés l'après-midi et de dix-sept festejos populares donnés en matinée. Saragosse organise à ce jour la plus importante féria de festejos taurinos populares suivie de celle de Castellón.

La liste des manifestations programmées lors de la féria de Saragosse en 2005 est riche d'enseignements. Le programme des réjouissances fut le suivant : huit vaquillas, un toro de fuego, un concours de roscaderos, un concours de cortes libres pour le trophée "Ciudad Zaragoza ", un défi hispano-landais, un concours de recortadores de anillas, un concours de taureaux emboulés et un spectacle

\footnotetext{
${ }^{10}$ Bous al carrer, $\mathrm{n}^{\circ} 45,1^{\mathrm{er}}$ janvier 2005, p. 36.
} 
comico-taurin. Les arènes jouent leur rôle de centre régional de la tauromachie populaire, en programmant des pratiques représentatives de la diversité du patrimoine tauromachique du Nord de l'Espagne. Elles mettent également en avant une spécificité du cours moyen de l'Èbre par l'organisation du concours de roscaderos. Elles affirment aussi leur pouvoir de centralité et leur rayonnement par la mise en jeu d'un trophée de la ville de Saragosse, qui d'année en année prend de la valeur. Enfin, elle tisse un lien avec les traditions françaises par la tenue d'un concours hispano-landais, en l'occurrence un concours où s'affrontent des recortadores espagnols et des écarteurs landais spécialisés dans les techniques des sauts.

On pourrait mener une analyse analogue avec les férias des villes de Pampelune, de Castellón ou de Valence. Il y a plusieurs nouveautés dans cette évolution. On assiste, d'une part, à l'évidente professionnalisation des concours de recortadores qui s'accompagne d'une autonomisation des disciplines internes : certains recortadores se spécialisent dans les concours d'anneaux, les autres dans les cortes libres, les autres dans les sauts. D'autre part, on observe un processus de redéfinition de la culture taurine, orientée vers une patrimonialisation territoriale. D'une tauromachie rassemblée sous l'appellation commune de concours de recortadores, on passe à une affirmation croissante de spécialités régionales. Apparaissent ainsi sur les affiches de promotion des appellations qui témoignent de ce processus : « corridas d'art valencien », " défis à la caste navarraise » ou « corridas d'art madrilène » (voir fig. 50, p. 295). Tout se passe alors comme si certaines identités régionales trouvaient à travers une réappropriation de la tauromachie populaire, les moyens d'une différenciation territoriale. Sur ce point, il est très symptomatique que le concours de recortes de Séville qui s'est tenu dans les arènes de la Maestranza en 2005 ait porté le nom, passablement ronflant, de Certamen Goyesco del Arte del Recorte con toros en puntas ${ }^{11}$. Associer l'événement à la tauromachie de Goya, tauromachie du Nord maintes fois représentée dans ses eaux-fortes, c'est la renvoyer à son origine septentrionale et à son origine archaïque, que l'art du toreo andalou aurait permis de dépasser. Ce n'est pas la dimension contemporaine des concours de recortadores qui est mise en avant, mais au contraire l'image d'une tauromachie des origines que la Maestranza toise et se remémore sur un mode passéiste.

\section{II. — STABILITÉ DES PRATIQUES \\ ET DES ESPACES TAURINS FRANÇAIS}

\section{STABILISATION ET ENRACINEMENT}

DE LA TAUROMACHIE ESPAGNOLE

Le dynamique récente des corridas en France semble avoir suivi le rythme global des évolutions espagnoles : une augmentation du nombre de spectacles

\footnotetext{
${ }^{11}$ Traduction libre qui en rend difficilement l'esprit : Événement goyesque de l'art du recorte exécuté sur des taureaux non épointés.
} 
jusque dans les années 1990 et une accentuation du regroupement des spectacles en féria qui se fait sentir dès l'après guerre. En quarante ans, du début des années 1960 à la fin du siècle, le nombre de spectacles majeurs a doublé : 64 spectacles en 1960, 81 en 1980, 133 en 1990. Après un léger reflux au milieu des années 1990, ce total s'est stabilisé entre 115 et 130 spectacles par an, avec un record historique de 89 corridas en 2003, confirmant la bonne santé des courses du haut de la hiérarchie. La tauromachie à cheval connaît elle aussi une progression remarquable puisque l'on est passé de courses encore épisodiques à la fin des années 1980 à une quinzaine de spectacles depuis la fin des années 1990. Certains aficionados s'interrogent d'ailleurs sur les conséquences de cette évolution qui confirme la marginalisation déjà très entamée de la figure du picador et l'émergence d'un nouveau public venu à la tauromachie par la culture équestre ${ }^{12}$.

Le point d'inflexion des évolutions géographiques de la tauromachie en France est marqué par l'entrée en vigueur de la modification de la loi Grammont en 1950 qui clarifie et normalise les conditions de la légalité des spectacles taurins. Elle n'explique pas la croissance, mais en détermine les modalités spatiales. Le milieu du siècle se caractérise par la fermeture de nombreuses arènes : Avignon en 1948, Marseille en 1951, Carcassonne en 1954 après une brève existence. Cette tendance se prolonge dans les années 1960 qui enregistrent de nouvelles fermetures d'arènes dans de grandes villes telles que Bordeaux (Le Bouscat) en 1961, Montpellier en 1962 puis Toulouse dans la décennie suivante, en 1975. La France connaît à la charnière des années 1970-1980 un creux analogue à celui vécu en Espagne qui s'explique en grande partie par les liens entre le marché taurin français et le milieu professionnel d'outre-Pyrénées.

Le nombre de spectacles se remet à croître au milieu des années 1980, puis aboutit progressivement à un élargissement et une densification du réseau d'arènes existantes. C'est souvent le choix d'une arène démontable qui est fait, ouvrant ses portes une fois par an. La Gironde retrouve timidement sa tradition en 1985 par des spectacles taurins à Captieux. Puis la région bordelaise renoue avec la tauromachie espagnole grâce à l'ouverture, en 1987, des arènes de Floirac $^{13}$. Suivent l'ouverture d'arènes à La Brède en 1999 visant à capter la même clientèle. En 2003, les deux villes sont candidates à la construction d'arènes en dur et se livrent bataille afin d'obtenir le projet ${ }^{14}$. Il finit par achopper en raison du retrait des investisseurs espagnols. Depuis, la décision de fermer les arènes de Floirac a été prise, à cause d'une rentabilité aléatoire et en vue d'utiliser le terrain à des fins immobilières dans un contexte de forte pression urbaine. Les années 2000 déplacent les transformations de la carte taurine plus à l'Est. Bourg-Madame installe une arène démontable en 1998. Puis en 2002, c'est au tour de Carcassonne et de Rieumes après de longs démêlés juridiques entre les

\footnotetext{
${ }^{12}$ Toros, $\mathrm{n}^{\circ}$ 1531, 12 novembre 1998, p. 16.

${ }^{13}$ Sur les arènes de Floirac, voir J.-P. CALLÈde, «L'affaire des corridas de Floirac »; ID., « Les corridas : passions taurines et modernisation urbaine».

${ }^{14}$ Sud-Ouest, 23 septembre 2003, p. 2-2.
} 
instigateurs du projet et les associations anti-taurines multipliant les procédures pour faire interdire les spectacles. En 2003, Toulouse inaugure une nouvelle féria dans sa banlieue de Fenouillet qui depuis connaît un vif succès ${ }^{15}$.

Plus que jamais, le succès des spectacles semble garanti par le regroupement des « jours de taureaux » en féria, comme l'indique la stratégie de l'immense majorité des arènes françaises. À l'inverse, cela rend plus difficile le maintien de corridas isolées. Cette rationalisation de la gestion des arènes est le fruit d'une recomposition des bases entrepreneuriales du mundillo français. L'augmentation du nombre de spectacles correspond à l'affirmation d'une nouvelle génération d'entrepreneurs de spectacles tels que Simon Casas, Robert Margé ou Alain Lartigues. Ce dernier, ancien avocat de Bayonne, s'est lancé dans la carrière d'organisateur de spectacles en 1988. Il gère désormais les arènes de Soustons, Parentis, Roquefort, Orthez, La Brède. En 2002, il confie à un journaliste du magazine économique Objectif Aquitaine que la gestion des arènes se fait de façon globale. Il faut accepter de perdre de l'argent dans l'organisation de certaines courses pour entretenir la passion des aficionados, qui se répercute sur une autre course qui sera plus rentable. On retrouve l'idée d'une organisation réticulaire de l'espace tauromachique. Cette stratégie a d'autant plus d'importance que lorsque le spectacle est alléchant, beaucoup de passionnés ne comptent pas les kilomètres. À Vic-Fezensac, à la fin des années 1980, 27 \% des spectateurs venaient d'une distance supérieure à 300 kilomètres $^{16}$.

La fin des années 1970 constitue un tournant important de l'histoire des toreros français durant laquelle apparait une génération fermement décidée à se faire une place dans le métier. Ce phénomène est l'un des signes tangibles de l'enracinement de la tauromachie. Des années 1970 au début des années 1980, sept matadors nîmois prennent l'alternative : Robert Pilès en 1971, fils d'un ancien novillero espagnol émigré en France, Bernard Dombs « Simon Casas » en 1975, Jacques Brunet « Jaquito » et Frédéric Pascal en 1976, Christian Montcouquiol « Nimeño II » (1977), frère cadet d'Alain Montcouquiol « Nimeño I » qui, lui, ne prit pas l'alternative, "Chinito de Francia » et Christian Lesur en 1978, et Curro Caro (1981). Ajoutons à cette liste le Lyonnais Patrick Varin qui fit ses premières armes dans le Sud-Est (1979) et Richard Milian, né à Canohès, dans les Pyrénées Orientales (1981). La veine nîmoise n'est pas épuisée si l'on prend acte de l'alternative de Stéphane Fernandez Meca en 1989. Depuis 1989, treize autres toreros français ont pris l'alternative, à un rythme qui depuis 1997 dépasse une alternative par an. Parmi les matadors récemment promus, deux d'entre eux ont réussi à pénétrer le marché espagnol, l'Arlésien Jean-Baptiste Jalabert «Juan Bautista ( 1999) et surtout le biterrois Sébastien Castella (2000) qui se hisse actuellement parmi les meilleurs matadors en activité comme aucun

\footnotetext{
${ }^{15}$ Le projet a vu le jour grâce à l'association d'aficionados Toro Tolosa et au groupe entrepreneurial RMDC composé de deux anciens rugbyman, Didier Lacroix du Stade Toulousain et le treiziste Carlos Zalduendo, le R et le M étant les initiales de l'entrepreneur taurin Robert Margé, directeur des arènes de Béziers et de son associé Michel Bonnafé.

${ }^{16}$ B. Bennassar, Histoire de la tauromachie, p. 197.
} 
autre torero français n’y était jusqu'alors parvenu. En 2004, Sébastien Castella a toréé 52 corridas dont 31 en Espagne, 13 en France et 8 en Amérique latine, ce qui le place au treizième rang parmi les matadors qui ont le plus toréé cette saison-là. En 2006, il est élu meilleur matador de l'année, toutes nationalités confondues, par la revue 6 Toros 6 . Sur les 69 matadors à avoir foulé les arènes françaises lors des 88 corridas de l'année 2004, 11 sont français. Quatre d'entre eux figurent parmi les dix premiers toreros à avoir signé le plus de contrats en France $^{17}$. La France constitue donc pour les toreros français une terre où il est plus facile d'être engagés, en lien avec une afición locale fière de les voir toréer au côté des grands noms espagnols. Certaines arènes françaises telles que Nîmes, Arles, Béziers servent également d'école de tauromachie.

\section{L'INFLATION DES PRATIQUES TAURINES}

\section{CAMARGUAISES ET DES MANADES}

Les courses camarguaises ont également connu une forte augmentation depuis le début des années 1980 : 625 courses en 1984, 762 en 1994, jusqu'au chiffre exceptionnel et isolé de 921 en 2004, qui se stabilise ensuite autour des 800 courses annuelles. Selon la Fédération, la saison 2004 a compté environ 250.000 spectateurs ${ }^{18}$. Le passage officiel de la "course libre » au sport de la « course camarguaise » par l'agrément ministériel de la Fédération en 1975 a favorisé l'augmentation du nombre de compétitions et leur déclinaison en catégories permettant de rationaliser la carrière des " tenues blanches » et celle des bêtes en piste. Dès 1977, la Fédération crée les courses de promotion (aujourd'hui dites de protection) qui permettent aux raseteurs débutants d'affronter de jeunes taureaux. En 1988, les vaches sont autorisées à participer à ce type de course, offrant ainsi la possibilité aux manadiers d'effectuer, par la même occasion, un travail de sélection.

Dans le détail, observons que l'année 1995 marque un léger coup d'arrêt de l'augmentation des spectacles en raison de l'entrée en vigueur d'une nouvelle réglementation sur la taxation des primes d'engagement par l'URSSAF, au même titre que toutes les rémunérations du travail. Si ces années correspondent à une augmentation du nombre de spectacles, elles ne se traduisent pas par une dilatation de l'espace d'exercice. Au contraire, certaines arènes, en situation marginale dans le "triangle sacré », ferment leur porte. Ainsi, dans le département du Gard, une dizaine d'arènes ferment entre le milieu des années 1970 et le milieu des années 1990 : Dions (1974), Saint-Hippolyte du Fort (1978), Canaules (1979), Goudargues et Quissac (1982), Combs (1985), Calvisson (1989), SaintGeniès-de-Malgoirès (1994), même si dans ce dernier cas la culture taurine n'a pas quitté les lieux puisqu'un festival du film taurin et camarguais s'est monté en 1998. Si la dynamique spatiale des courses camarguaises est allée vers une

\footnotetext{
17 Tendido, $\mathrm{n}^{\circ}$ 148, janvier-février 2005, p. 16.

${ }^{18}$ FFCC : 2004 (249.549 spectateurs), 2005 (263.653 spectateurs).
} 
concentration dans la zone du «triangle sacré », il n'en va pas de même de toutes les pratiques taurines.

De ce point de vue, le changement le plus remarquable est l'extension géographique de la zone des bandido et des abrivado, autrefois pratiquées dans les villes et les villages situés à proximité des élevages. Hier calquée sur la géographie des manades, cette extension adopte aujourd'hui celle de la répartition des courses et, plus largement, de toutes les fêtes de la région attachées de près ou de loin à la culture du taureau. Cette évolution est d'autant plus intéressante que les années 1970 avaient marqué une régression de la pratique en raison du développement du réseau viaire ${ }^{19}$. Le point de départ de cette renaissance, qui s'est ensuite révélée explosive, semble dater des années 1980, quand Paul Coulomb et le manadier Philippe Cuillé ont l'idée d'instaurer un concours d'abrivado à Vauvert. L'enthousiasme suscité par cette nouvelle manifestation aurait entraîné depuis une multiplication des abrivado dans de nombreuses villes ${ }^{20}$. Observons également de façon plus marginale d'un point de vue quantitatif, mais tout aussi important sur un plan sociologique, qu'en 2005 le préfet de l'Hérault autorise, sous certaines conditions, le retour du taureau à la corde qui avait pratiquement disparu, si ce n'est à Baillargues, Saint-Geniès, Lansargues, Lunel-Viel, Candillargues et dont la pratique avait été interdite. Le taureau à la corde d'Eyragues, dans les Bouches-du-Rhône, avait semble-t-il contourné la difficulté en rebaptisant la pratique sous le nom d'encierro à l'eyraguaise.

La croissance des spectacles et surtout de la tauromachie participative a été le moteur de l'augmentation du nombre d'élevages, qui ont pratiquement triplé en vingt ans passant d'une cinquantaine en 1977 à près de 150 dès la fin des années 1990. Pour nuancer cette croissance explosive, précisons que beaucoup de manades ne sont constituées que de quelques têtes de bétail, élevées pour le statut valorisant de manadier, et louées pour différentes manifestations. Ces manades connaissent des recompositions spatiales importantes parmi lesquelles la sortie hors de la zone traditionnelle d'élevage de la Crau, de la Camargue et de la Petite Camargue vers les garrigues intérieures et les zones lagunaires du Languedoc. Cette évolution se fait dans un contexte de diminution des zones d'herbages naturelles. D'après Alain Tamisier, en 1984, sur les 145.000 ha du triangle formé par Arles, Grau-du-Roi et Fos ne persistaient que 57.000 ha de milieux naturels, dont 15.000 ha d'étangs, 18.000 de marais, 13.000 de sansouires et 3.000 de pelouses ${ }^{21}$. Les élevages étaient alors répartis sur environ 20.000 ha, sur les quelque 145.000 que totalisent Crau, Camargue et Petite Camargue. Pour un nombre total d'élevages plus élevé, les manades disposeraient aujourd'hui de 15.000 ha en partie stabilisés par le Parc naturel régional. Il n'en demeure pas moins des risques évidents de surpâturage, localement atteint.

\footnotetext{
${ }^{19}$ L.-G. Lacroix, Histoire de la tauromachie en Arles, p. 42.

${ }^{20}$ Arène, no 64, 1995, p. 12 cité par R. KeErLe, Sport et territoires, p. 419.

${ }^{21}$ Cité par Le Conseil économique et social du Languedoc-Roussillon, Le rôle et l'impact du taureau, p. 9.
} 


\section{STAGNATION ET OUVERTURE}

DE LA COURSE LANDAISE

Les dynamiques récentes de la course landaise se caractérisent par un «âge d'or » qui court du milieu des années 1960 au milieu des années 1980, qui fait suite à une difficile sortie de l'après-guerre. La création de la Fédération française de course landaise en 1953, vingt ans avant l'agrément du Ministère, fut l'un des instruments qui permit à la course de refaire surface dans les nouveaux habits du sport moderne. Cet âge d'or est suivi d'une période de reflux où le nombre global de courses diminue tout en stabilisant le nombre de courses formelles et de concours landais correspondant au plus haut niveau de la discipline. Entre 1965 et 1980, les courses mixtes passent de 159 à 452 et les courses formelles de 102 à 158, le nombre total de courses ayant ainsi été multiplié par plus de deux en 15 ans $^{22}$. Sur la même période, la trame des arènes se densifie avec 24 constructions d'arènes et 13 rénovations. Cette époque correspond également à un renouvellement et un rajeunissement des pratiquants dont $90 \%$ sont originaires de la Chalosse ${ }^{23}$. Le dynamisme de l'activité taurine s'est alors traduit par une extension spatiale de l'aire géographique de la course landaise, plus spécialement des courses mixtes, vers les stations balnéaires du littoral : Hendaye, Saint-Jean-de-Luz, Vieux-Boucau, Biscarosse, Capbreton. Cette extension touche aussi ponctuellement des régions éloignées du Sud-Ouest : la Vienne, Les Côtes-du-Nord, Les Yvelines et l'Aube ${ }^{24}$. Cet engouement pour les courses mixtes n'est pas sans rapport avec le succès populaire des jeux télévisés d'Intervilles, créés en 1962, qui offrent à l'affrontement ludique entre l'homme et les «vachettes » une audience nationale. À la période faste que traversent les courses sérieuses, incarnée par l'immense popularité de deux écarteurs gitans, «Ramuntcho » et "Ramuntchito », les frères Christian et Guillaume Vis de leur état civil, répond un développement encore plus rapide des courses mixtes et des toro-piscines. Bien que le développement des courses sous une forme ludique ait suscité des critiques de la part des amateurs de courses sérieuses, il permit également des rentrées d'argent complémentaires pour les professionnels, en particulier pour les ganadères louant leurs bêtes. Le ganadero Joseph Labat est le premier à se lancer dans l'aventure en créant une véritable entreprise de spectacles proposant des " courses de plages », clés en main.

Le dynamisme général du marché taurin s'essouffle au milieu des années 1980. Cela se traduit avant tout par une chute des courses mixtes qui passent de 452 courses en 1980 à 381 en 1986 et 154 en 1998. La chute du nombre de spectacles est accompagnée d'une contraction de l'espace d'exercice de la tauromachie dans les limites historiques qu'il possédait au début des années 1960. La décrue des courses formelles, quoique sensible, fut moins marquée, se stabilisant

\footnotetext{
22 J.-Y. Puyo et M. DAugA, « La course landaise, sport-spectacle », p. 20.

${ }^{23}$ M. DAugA, «L'âge d'or de la course landaise », p. 72.

${ }^{24}$ Ibid., p. 88.
} 
rapidement autour des 150 courses et ce jusqu'à nos jours. Aujourd'hui, l'institutionnalisation de type fédéral, considérant la course comme une pratique sportive professionnelle avec les obligations fiscales qui en découlent, semble bloquer le renouveau de la pratique, même si les années 2005 et 2006 marquent une augmentation sensible du nombre de courses.

Observons dans cette séquence que le reflux des courses landaises coïncide avec une augmentation des courses espagnoles dans le Sud-Ouest. S'il fallait ne retenir qu'un exemple, celui de Dax est très significatif. Au début des années 1980, les arènes de Dax offraient plus de courses landaises que de courses espagnoles, tendance qui s'est totalement inversée depuis. On notera également que cette période est aussi celle de l'apparition des encierros de type espagnol dans le Sud-Ouest, comme si la tauromachie participative commençait aussi à regarder de l'autre côté des Pyrénées. Certains coureurs d'encierros ont fini par fonder une modeste association en 1995, l'Association française des coureurs d'encierros (AFCE) qui « a pour but de faire connaître l'encierro, son histoire, ses dangers et ses techniques, afin d'éviter les accidents et faire renaître dans le Sud-Ouest cette vieille tradition $»^{25}$.

Enfin, il faut souligner avec intérêt que certains écarteurs et sauteurs landais, comme par exemple Didier Laplace, David Casarin ou Nicolas Vergonzeanne, se produisent de plus en plus en Espagne, en s'inscrivant aux concours de recortadores. En effet, le développement des courses de recortadores en Espagne constitue potentiellement un très large débouché professionnel pour les écarteurs dont les techniques tauromachiques sont assez proches. Mieux les techniques de la course landaise participent à l'élaboration des codes de la course de recortadores en pleine mutation. Nous sommes désormais loin des concours de recortadores où la plupart des épreuves consistaient à enfiler des anneaux aux cornes des vaches suivant le modèle arago-navarrais. Les écarts et les sauts se généralisent et deviennent des épreuves à part entière et trouvent un plein épanouissement dans les concours des pays valenciens. La façon dont étaient réalisés les quiebros en Espagne au début des années 1980, nous incite à penser que l'écart dans le dos, les pieds joints, ainsi que le pratiquent les écarteurs landais, a fortement influencé les techniques actuelles des recortadores. Ce qui est probable pour les quiebros semble avéré pour les sauts. Alberto de Jesús, directeur du magazine Bous al Carrer et ancien champion d'Espagne de recortadores, nous a confirmé qu'il a vu faire les premiers sauts de l'ange au-dessus du taureau par des Français et que la pratique a rapidement été imitée et assimilée. Aujourd'hui cette pratique est visible jusque dans la rue, lors des bous al carrer où la chaussée est recouverte de sable.

Ce détail apparemment anecdotique permet de mieux comprendre les processus de codification des pratiques tauromachiques que nous croyons s'établir, aujourd'hui comme hier, à partir d'influences géographiques élargies. Dans les modalités de mise en forme et de diffusion des spectacles taurins, les innovations même ténues et les transferts individuels ont une grande importance. À n'en pas

\footnotetext{
${ }^{25}$ Toros, $\mathrm{n}^{\circ} 1631,6$ juillet 2000, p. 2.
} 
douter ces formes seront définitivement fixées techniquement et spatialement dans quelques années. En retour, il n'est pas impossible que le Sud-Ouest s'ouvre plus largement aux concours de recortadores. Dans les Landes, Pouillon, dont le panneau en forme de taureau planté à l'entrée des arènes affiche en lettres blanches "Pouillon canton coursayre ", organise depuis 2000 une course de recortadores par $\mathrm{an}^{26}$.

De façon moins anecdotique, il est intéressant de constater que dans le SudOuest, les courses landaises renouent avec l'affrontement de taureaux de combat libres (sans corde), à l'image des concours de sauts et de cortes pratiqués en Espagne. Cette pratique dans les Landes avait disparu progressivement au début du $\mathrm{xx}^{\mathrm{e}}$ siècle. Le concours "Art et Courage » de Pomarez qui se tient depuis 18 ans est organisé sur ce principe, de même que la récente Nuit du Toro à Dax, le 8 septembre 2006, opposait onze taureaux à sept écarteurs et quatre sauteurs. Ces événements montrent ainsi la réversibilité des principes taurins dans le cadre d'une évolution des pratiques, montrant qu'il ne s'agit en rien de pratiques figées. On observera en particulier que l'utilisation de la corde dont Frédéric Saumade fait la marque d'un contre modèle tauromachique archaïsant qui fonde la spécificité du jeu landais, apparaît ici comme un élément suppressible. Voilà la preuve d'une grande capacité d'adaptation des pratiques tauromachiques qu'aucun principe irrévocable ne paraît définir. C'est aussi ce que montre historiquement le port obligatoire du caparaçon pour protéger les chevaux. Il s'agissait alors d'une transformation radicale des règles du jeu qui pour beaucoup d'observateurs de l'époque devait finir par faire disparaître la corrida, vidée de sa substance. Quoi qu'il en soit, cette forme d'échanges et de dialogue d'une pratique à l'autre, et d'une région périphérique à l'autre, confirme l'existence d'un espace tauromachique européen dans lequel la contraction générale des « distances-temps » et l'augmentation des mobilités favorisent une accélération des évolutions.

\section{III. - FLUCTUATIONS ET BLOCAGES \\ DE LA TAUROMACHIE AU PORTUGAL}

\section{Le réÉquilibrage Nord-Sud de L'espace taurin}

L'évolution du nombre de courses au Portugal est beaucoup plus chaotique qu'ailleurs et semble correspondre à un rythme propre qui témoigne assez bien du relatif isolement du marché taurin du reste de la péninsule Ibérique et d'une certaine fragilité de la professionnalisation des spectacles. L'année 1986 a atteint les 333 courses, puis jusqu'à 1997 seule l'année 1993 a dépassé la barre des 300 spectacles. La période de 1997 à 2003 se caractérise par une réelle augmentation qui ensuite s'essouffle pour retomber aux quelque 300 courses de la fin des années 1980. Certains voient dans cette fluctuation les symptômes d'une crise larvée de la tauromachie au Portugal. D’autres observateurs plus modérés

\footnotetext{
${ }^{26}$ La revue Barrera Sol, n 49, 15 juin 2001, y consacre un numéro spécial.
} 
pensent que le nombre de spectacle est en concordance avec les lois de l'offre et de la demande. Cette fluctuation des chiffres masque en réalité une indéniable extension géographique des courses de taureaux. En effet, le nombre de communes ayant programmé des spectacles taurins a augmenté du milieu des années 1990 à nos jours et de façon plus régulière que le nombre de spectacles.

Tableau 4. - Évolution et distribution des spectacles taurins au Portugal

\begin{tabular}{|l|c|c|c|c|c|}
\hline Apectacle & 1996 & 1997 & 1998 & 1999 & 2004 \\
\hline $\begin{array}{l}\text { Nombre de } \\
\text { communes taurines }\end{array}$ & 88 & 109 & 113 & 141 & 139 \\
\hline $\begin{array}{l}\text { Arènes fixes } \\
\text { ayant fonctionné }\end{array}$ & 57 & 52 & 69 & 65 \\
\hline $\begin{array}{l}\text { Arènes démontables } \\
\text { ayant fonctionné }\end{array}$ & 255 & 330 & 313 & 366 & 297 \\
\hline $\begin{array}{l}\text { Nombre de } \\
\text { spectacles taurins }\end{array}$ & & & 72 & 74 \\
\hline
\end{tabular}

Le Nord est le principal bénéficiaire de cette évolution grâce aux arènes démontables venues renforcer, à partir des années 1980, un réseau d'arènes plus lâche que dans le Sud. Ailleurs, elles ont densifié le réseau existant. Ce rééquilibrage partiel de l'opposition Nord-Sud de l'organisation de l'espace tauromachique portugais s'est accompagné d'une accentuation de la littoralisation des spectacles due en grande partie à l'essor du tourisme. Au Nord, le tourisme a revitalisé une tradition ancienne dans les cités littorales de Viana do Castelo, Póvoa do Varzim, Figueira da Foz. Au Sud, il a renforcé le poids de l'Algarve décrié par certains comme un front pionnier taurin sans âme. En cela les arènes d'Albufeira, en tête des statistiques taurines grâce à l'offre de programmes volontairement plus courts afin de ne pas lasser les néophytes, ont quelques points communs avec celles de Benalmádena dans la province de Málaga.

Pour ce qui est des tauromachies à participation collective, le Portugal n'a pas connu la croissance explosive des jeux taurins populaires de l'Espagne. Elles étaient déjà fort développées et fort diversifiées dans un pays où la tauromachie professionnelle n'a pas passionné l'ensemble des couches sociales, comme la corrida a pu le faire un temps en Espagne. Le fait qu'elle n'ait pas connu de révolution aussi radicale que celle qui a marqué en Espagne le passage de la tauromachie aristocratique à la tauromachie du peuple peut être considéré comme un élément explicatif. Cela ne suffit sûrement pas à expliquer la variété des pratiques tauromachiques populaires qui font du Portugal un important conservatoire de formes anciennes telles que les taureaux encordés, les sacrifices 
de taureaux ou encore la capeia raiana. Le formidable essor des touradas à corda sur l'île Terceira contraste donc avec l'évolution du reste du pays. Autour de 250 aujourd'hui, elles ne dépassaient pas la cinquantaine dans les années 1960. Cet essor se double d'un intérêt soutenu pour les courses de taureaux dans les arènes d'Angra de Heroismo qui, malgré leur construction récente en 1984, se hissent parmi les premières arènes portugaises en nombre de spectacles. Le dynamisme de la tauromachie aux Açores a entraîné une rationalisation du secteur de l'élevage brave par la formation, en 2000, d'une Association des éleveurs de taureaux de tourada à corda de l'Ile Terceira et San Jorge. Ce dynamisme a aussi contribué à un meilleur encadrement des pratiques par l'adoption d'un Règlement des touradas à corda, en 2003, entériné par la Région Autonome. On observe que l'augmentation de la pratique a nécessité une meilleure réglementation qui a clarifié et normalisé les conditions de programmation des jeux taurins favorisant en retour leur croissance.

\section{LES RECOMPOSITIONS INTERNES DE L'AFICIÓN}

Derrière ces évolutions et ces dynamiques spatiales se cache une transformation plus fondamentale de l'espace tauromachique portugais, causé non seulement par le développement remarquable de la tauromachie à cheval chez le voisin espagnol, mais aussi par le désir croissant de voir se dérouler au Portugal des corridas intégrales, avec piques et mise à mort. En effet, la forte progression du rejoneo en Espagne n'a pas été sans conséquence au Portugal. On a vu que dans les années 1920-1930, la rivalité taurine des nations sœurs se cristallise autour de la tauromachie à cheval, le Portugal se sentant dépossédé d'un art qui lui serait propre. Dans les années 1940, le critique taurin espagnol Don Ventura pouvait encore affirmer :

Il est évident que jusqu'à ce que la tauromachie à pied s'implante en Espagne, la tauromachie à cheval était le spectacle traditionnel depuis le Moyen Âge. Celle-ci a été maintenue intacte au Portugal et ne se manifeste en Espagne que par la présence du picador, exception faite de ces dernières années où interviennent — très rarement - quelques rejoneadores qui offrent au public une tauromachie à cheval inspirée du campo andalou, laquelle diffère grandement du caractère aristocratique qui donne forme à ce que vous appelez «l'Art de Marialva » ${ }^{27}$.

Trente ans plus tard, dans les années 1970-1980, les rejoneadores espagnols dépassaient le nombre de cavaleiros portugais. Depuis les années 1990, le développement de la tauromachie à cheval en Espagne est tel que nombre de cavaliers portugais ont compris l'intérêt d'intégrer le marché espagnol et avec lui, sa projection latino-américaine. Mais la rivalité se prolonge. Dans la revue portugaise Tauromaquia, une aficionada témoigne :

\footnotetext{
${ }^{27}$ Don Ventura, Solidaridad taurina Hispano-lusitana, pp. 2-3.
} 
Dans les années 1960, je me rappelle que très peu de rejoneadores espagnols parvenaient à toréer sur les terres portugaises. Toréer au Campo Pequeno était pour eux comme obtenir un doctorat [...]. J'admire profondément le peuple espagnol. Tout ce qui vient d'eux est meilleur ! Nous les Portugais, nous pensons exactement le contraire ${ }^{28}$.

Il n'en demeure pas moins que les arènes les plus importantes de la tauromachie à cheval en termes de carrière, de rémunération et de nombre de spectacles sont désormais situées en Espagne, même si le Campo Pequeno de Lisbonne demeure une référence. Cette évolution peut être interprétée comme un transfert de centralité. L'invitation de Pablo Hermoso de Mendoza pour l'inauguration des arènes du Campo Pequeno de Lisbonne est également une reconnaissance de la qualité technique et esthétique atteinte par le rejoneo espagnol même si, pour autant, elle ne remet pas totalement en cause la valeur et la part d'autonomie des critères portugais.

Une autre évolution touche cette fois à la place de la tauromachie à pied qui a considérablement augmenté depuis les années 1970. Elle traduit aujourd'hui par une revendication de plus en plus affirmée, sans qu'elle soit unanime, d'une transformation de la loi qui permettrait le déroulement des corridas intégrales. Rappelons qu'en 2004, une quinzaine de matadors espagnols sont venus toréer au Portugal au côté des sept matadors portugais en activité. Les premiers matadors portugais apparaissent à la fin des années 1940. Comme en France, ce sont les années 1970 qui marquent un véritable décollage. Dans ces années, une dizaine de matadors portugais toréaient : Diamentino Viseu, Manuel Dos Santos, José Trincheira, José Julio, Armando Soares, Amadeu dos Anjos, Mario Coelho, José Falcão et Ricardo Chibanga, ce dernier originaire du Mozambique. Dans les années 1980, apparaît la figure de Victor Mendes, dont la carrière est une référence puisqu'il est resté en haut de l'affiche, dans les arènes du monde entier, pendant plus de quinze ans. Le bilan de sa longue carrière de 1981 à 1998 montre bien l'obligation qu'il y a à franchir les frontières pour pouvoir vivre de sa profession au Portugal : sur les 1.055 corridas auxquelles il a participé, 678 ont eu lieu en Espagne, 150 en France, 129 en Amérique et seulement 98 au Portugal, dans son propre pays. Au total, le Portugal a vu naître 30 matadors d'alternative. Aujourd'hui le Portugal ne possède pas de matador capable de rivaliser avec les meilleurs toreros espagnols, situation qui est tout à fait inverse en France. Cette situation éclaire le fait que de nombreux aficionados portugais militent en faveur de la légalisation des corridas « intégrales » dans leur pays. Pour eux, l'autorisation de la mise à mort entraînerait un développement rapide de la tauromachie à pied, considérée comme constitutive du patrimoine portugais, en vue de combler un sous-développement taurin dont la législation est tenue pour responsable. Plusieurs associations affichent ouvertement ce désir comme le Clube taurino da Moita (Pró-touros de morte), fondé en 1999 (voir fig. 51, p. 308).

\footnotetext{
${ }^{28}$ Tauromaquia, 3 septembre 2003, p. 16. Le Campo Pequeno est le nom des arènes de Lisbonne.
} 
Le débat autour des corridas intégrales au Portugal est de plus en plus vif. Les propos de l'un des organisateurs de spectacles parmi les plus expérimentés du Portugal, Manuel Gonçalves, actuel gestionnaire des arènes de Moita, en laissent apparaître la complexité en des termes où se mêlent des considérations éthiques, identitaires et économiques :

Soyons directs. Je suis portugais fier de l'être et convaincu que la corrida portugaise est un spectacle beaucoup plus diversifié et attirant. Le public du monde entier adhère plus facilement à la corrida portugaise qu'à la corrida espagnole. Évidemment, en tant qu'aficionado et en termes commerciaux, je peux vous dire que j'aimerais être le premier entrepreneur de spectacles à organiser des corridas avec mise à mort au Portugal ${ }^{29}$.

Curieuse distorsion de la rationalité économique qui trahit sans doute un rapport de force entre les acteurs de chacune des pratiques tauromachiques. Pourquoi serait-il plus rentable d'organiser des corridas avec mise à mort alors que la corrida portugaise est considérée comme un spectacle plus attrayant ? La réponse est sans doute à chercher du côté des cavaliers portugais qui n'auraient pas grand intérêt à voir se développer les corridas intégrales sur le territoire national, les mettant en concurrence directe avec les toreros à pied. Dans une certaine mesure, le discours transnational de la tauromachie à pied se heurte aux intérêts portugais de la tauromachie à cheval qui ne tirerait aucun avantage à voir s'effacer la frontière. Cette frontière n'est donc pas une simple limite entre deux cultures taurines opposées, elle est aussi un rempart juridique permettant à la tauromachie portugaise dominante de protéger ses intérêts.

Les blocages et les forces contradictoires du système taurin portugais ont été révélés par la périphérie du système : les courses de taureaux du petit village de Barrancos, situé à quelques kilomètres de l'Espagne. Pendant près de quatre ans, de 1998 à 2002, le pays a été plongé dans une polémique d'envergure nationale à propos de ce qu'il est alors convenu d'appeler la «crise barranquenha ». Le débat avait été lancé une première fois en 1969 à la suite d'une plainte déposée par un certain Dr Lino soulignant que la législation portugaise n'était pas respectée à Barrancos qui en toute impunité faisait mettre à mort les taureaux par des toreros espagnols lors de ses fêtes ${ }^{30}$. La polémique resurgit à la suite d'un reportage télévisé sur les corridas de Barrancos en 1996 et d'une série de plaintes déposées dans la foulée par des associations de protections des animaux. À partir de là, les médias ont largement relayé et amplifié le débat divisant l'opinion publique dont la portée s'est étendue au fonctionnement général de la société portugaise ${ }^{31}$.

La crise barranquenha servit ainsi de prétexte pour débattre, pendant quatre ans, du pouvoir, de la loi, des sources du droit, des relations villes-campagnes,

\footnotetext{
${ }^{29}$ Tauromaquia, 3 septembre 2003, p. 17.

${ }^{30}$ N. Franco, O porquê de Barrancos, p. 201.

${ }^{31}$ L. CAPUCHA, «Barrancos en escena ».
} 


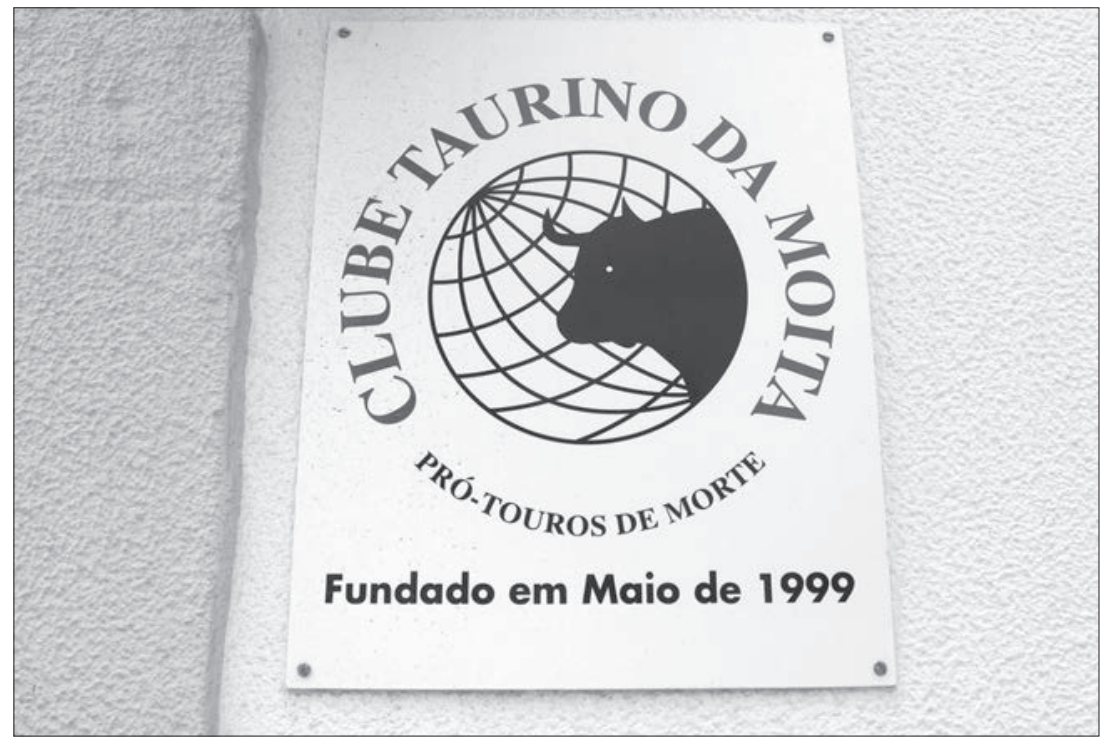

FIg. 51. - Club taurin de Moita en faveur des corridas intégrales. Les aficionados de Moita affichent leur volonté de faire changer la loi

(Moita, 2005, cliché : Jean-Baptiste Maudet).

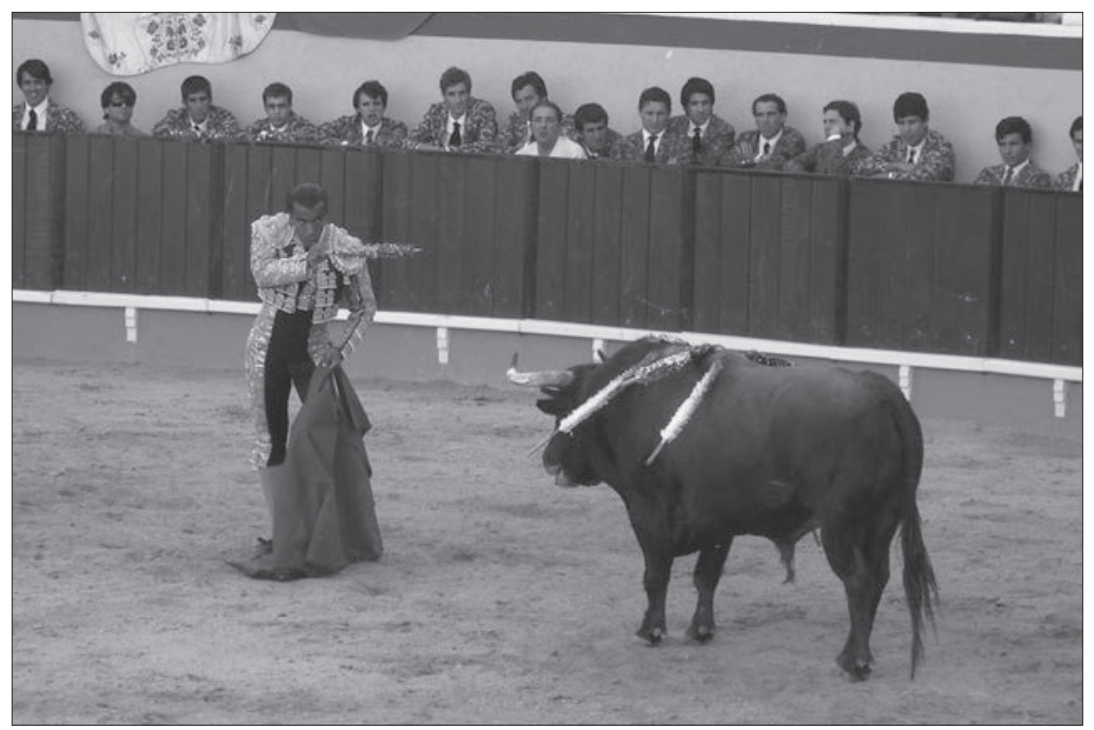

Fig. 52. - Simulacre de mise à mort avec une banderille au Portugal.

Le matador portugais José Luís Gonçalves simulant la mise à mort du taureau avec une banderille, avant de laisser entrer en piste les forcados

(Vila Franca de Xira, 2005,cliché : Jean-Baptiste Maudet). 
des relations entre les hommes et les animaux, des relations entre culture savante et culture populaire, de l'idiosyncrasie portugaise et bien entendu de la fête des taureaux ${ }^{32}$. Après la démission de deux ministres, l'intervention du président de la République Jorge Sampaio en faveur d'un déblocage législatif de la situation et les réactions enflammées de certains habitants de Barrancos menaçant de fouler au pied leur carte d'identité nationale, le parlement portugais a fini par voter, en 2002, l'autorisation de célébrer des spectacles tauromachiques avec mise à mort, dans les municipalités pouvant se prévaloir d'une ancienne tradition $^{33}$. Depuis lors, trente-deux municipalités ont engagé des procédures afin d'établir la légalité de la mise à mort, procédures qui, pour l'instant, n'ont pas abouti, l'exception ne demeurant effective qu'à Barrancos. Le Portugal apparaît bien comme un pays taurin où les évolutions actuelles sont à même de transformer radicalement le panorama des pratiques tauromachiques.

\section{IV. — L'ARTICULATION TRANSFRONTALIÈRE DES ESPACES TAURINS}

\section{LA « FRONTIÈre TAURine » FranCe-Espagne : LE DÉSIR D’HISPANITÉ}

Au début des années 1980, le torero André Viard posait ouvertement une interrogation provocatrice dans un petit ouvrage intitulé Corrida française? La question ne portait pas sur l'assimilation évidente de la tradition des courses de taureaux au patrimoine culturel des régions du Sud de la France. Cette question visait à dénoncer la main mise des professionnels espagnols sur l'organisation des spectacles taurins en France, pour leur plus grand bénéfice. Bien qu'André Viard reconnût alors quelques exceptions dans le Sud-Est, il persistait dans son jugement pour affirmer : "La France taurine est bel et bien une colonie espagnole, qui s'apparente d'ailleurs pour les bienheureux colons à un El Dorado moderne $»^{34}$. Derrière cette dénonciation de l'impérialisme économique espagnol au-delà des Pyrénées est posée la question de l'intégration des marchés taurins ainsi que de l'articulation transfrontalière des espaces français et espagnols à partir du fait culturel tauromachique.

Certains éléments de ce constat pessimiste ont objectivement évolué. Comme on l'a vu, il existe davantage d'élevages, de mieux en mieux appréciés pour leur qualité. Les toreros français ne sont plus les parents pauvres de la tauromachie dans leur propre pays et exportent brillamment leur talent au-delà des Pyrénées et de l'Atlantique. Le fait de voir deux toreros français programmés lors de la très prisée corrida de la presse à Madrid en 2007 aurait été impensable il y a encore quelques années. Certains entrepreneurs de spectacles français,

${ }^{32}$ Ibid., pp. 433-434.

${ }^{33}$ Vote du Parlement 115 voix pour, 91 contre, 9 abstentions.

${ }^{34}$ A. VIard, Corrida française?, p. 60. 
tel Simon Casas sont désormais fermement établis en Espagne et possèdent la confiance des adjudicateurs. Il s'en est fallu d'un rien qu'il ne remporte la gestion des arènes de Madrid lors des deux derniers concours, un rien que l'intéressé attribue à des pratiques frauduleuses dans l'évaluation de son dossier par la Communauté autonome propriétaire des lieux, faisant l'objet d'un recours en justice. Enfin, l'Union des villes taurines de France (UVTF) tente de participer de plus en plus à la régulation des activités tauromachiques de façon autonome. L'une des mesures affichées, pas toujours évidente à appliquer, est l'exclusion du marché français des acteurs qui enfreindraient le règlement. Si ces évolutions ont contribué à quelque peu rééquilibrer les termes de l'échange, la France dépend encore largement des organisateurs de spectacles espagnols pour monter ses férias. C'est plus souvent le cas dans le Sud-Ouest que dans le Sud-Est où la formation plus étoffée du mundillo semble jouer en faveur d'une plus grande autonomie dans les négociations.

La frontière nationale n'implique ici aucune transformation dans la pratique des courses de taureaux, dans un espace soumis à une libre circulation de tous les protagonistes en jeu. Seule est menacée épisodiquement la circulation des taureaux, lorsque des mesures visant à endiguer les risques de maladie de la vache folle ou de la langue bleue dressent des barrières sanitaires. Cette mobilité des acteurs et des spectateurs de la tauromachie est responsable de relations transfrontalières plus ou moins intenses entre l'Espagne et la France. Cependant, les deux grandes régions taurines françaises ne sont pas dans la même situation visà-vis du voisin espagnol. Si l'espace tauromachique du Sud-Ouest est contigu à l'espace espagnol, celui du Sud-Est est plus éloigné et isolé. La frontière ellemême peut-être découpée en plusieurs sections inégalement concernées par les dynamiques spatiales engendrées par l'activité tauromachique.

La zone frontière occidentale qui met en contact le Sud-Ouest français avec le Pays Basque et la Navarre est la section la plus active. Les relations transfrontalières concernent d'abord les professionnels taurins, éleveurs et toreros, indispensables aux férias du Sud-Ouest. Dans cette région, les entrepreneurs espagnols contrôlent une grande partie de la programmation des spectacles en tant que prestataires de services auprès de nombreuses arènes, qu'elles soient gérées en régie municipale ou par l'intermédiaire d'une société. On retrouve ici le rôle incontournable des Chopera qui en 2006 possèdent des intérêts dans l'organisation des spectacles à Bayonne, Mont-de-Marsan, Hagetmau, VicFezensac, Orthez. Les relations transfrontalières sont aussi celles de l'intense mobilité des spectateurs, plus importantes vers l'Espagne, de la part du public français, que vers la France, de la part du public espagnol. Cette mobilité est néanmoins sélective et se dirige principalement vers les arènes de première catégorie et de deuxième catégorie : d'abord celles de Bilbao en Biscaye, celles de Pampelune en Navarre et bien évidemment la plus proche et la plus francoespagnole des arènes, celles de Saint-Sébastien. De façon secondaire, sont aussi concernées les arènes de Saragosse en Aragon, celles de Logroño dans La Rioja, et celles de Vitoria en Álava. L'afición ne se déplace ensuite au-delà de l'Èbre que pour rejoindre généralement les férias de Madrid, de Séville, ou de Valence. La 
mobilité spatiale joue à deux échelles, celle d'un espace taurin régional perçu comme proche et celle d'un espace plus lointain justifié par le rang et la qualité des spectacles. Cette mobilité régionale existe également pour la tauromachie populaire si l'on observe les nombreux coureurs d'encierros français qui rejoignent les rangs des courses navarraises. L'encierro de Pampelune recrute des adeptes venus du monde entier sans doute comme aucun autre jeu taurin n'y parvient, contribuant ainsi à faire de la San Fermín la plus internationale des férias. Les encierros de Tafalla et de Falces sont également des rendez-vous prisés des encierristes français. En retour, cette passion des encierristes français contribue à faire renaître dans le Sud-Ouest cette ancienne pratique sous les traits du modèle navarrais. La tauromachie sous ses deux espèces, professionnelle et participative, fait ainsi exister un territoire taurin transfrontalier.

La course landaise n'attire pas les spectateurs espagnols, mais certains écarteurs landais s'impliquent de plus en plus dans l'essor des concours de recortadores. Le mouvement commence à se faire sentir du côté des ganadères qui présentent des vaches en Espagne, comme celles issues de l'élevage Les Armagnacaises. En outre, les ganadères achètent depuis longtemps des vaches navarraises, même si les achats de bétail ne se limitent pas au voisinage proche, étant donné que les achats en provenance du campo charro de Salamanque demeurent majoritaires. Si la corrida recompose un territoire transfrontalier, les territoires de la course landaise et de la course arago-navarraise demeurent juxtaposés même s'il existe désormais quelques signes forts d'un accroissement des relations. Le spectacle le plus jeune (les courses de recortadores) ouvre les perspectives d'une revitalisation du spectacle le plus ancien (les courses landaises). À l'inverse, le spectacle le plus ancien participe à la codification du nouveau venu.

Plus généralement, les férias du Sud-Ouest s'inspirent du modèle des férias navarraises. Ce rapport d'imitation participe à la remontée septentrionale d'une hispanité syncrétique perceptible au-delà des spectacles taurins dans la gastronomie, la musique, les tenues vestimentaires. Mais c'est bien le thème tauromachique qui articule entre eux ces formes d'assimilation. La tenue blanche de Pampelune rehaussée par un foulard et une ceinture rouge, devenue quasiment la tenue officielle du festayre se retrouve aux fêtes de Bayonne, de Dax, de Montde-Marsan. À Bayonne, le rouge et blanc dans les tenues vestimentaires ont pris le dessus sur d'autres couleurs, en particulier le bleu, qui passe pour être la couleur de la ville, ou même le vert dans son association avec le rouge comme couleurs du Pays Basque. La ville de Bayonne s'est ouvertement inspirée de la fête de Pampelune dans ses codes. Les villes de Bayonne et de Pampelune sont jumelées et la corrida des fêtes se tient le dimanche, journée officiellement placée sous le signe de la capitale navarraise. Il est intéressant de constater que les relations transfrontalières liées à la tauromachie véhiculent davantage l'image d'un territoire régional composite formé par la Gascogne, la Navarre, la Biscaye et Guipúzcoa que celle d'un territoire unifié par l'Euskadi, absent des références de la culture taurine. La territorialité taurine semble mieux fonctionner comme une structure feuilletée par des acteurs en réseaux plutôt que comme des espaces et des aires culturelles qui s'imbriquent hiérarchiquement les uns aux autres. Autrement dit, 
alors que le Pays Basque est spatialement au cœur des relations taurines transfrontalières franco-espagnoles, il en est étonnamment absent en tant qu'entité culturelle unifiée par la tauromachie. Les jeux de pelote, de force, de navigation, la randonnée, la gastronomie et les chants, participent bien plus à la cohésion territoriale transfrontalière du Pays Basque que les pratiques tauromachiques.

À la différence de la frontière atlantique, la zone frontalière catalane met face à face deux espaces dont les dynamiques tauromachiques sont divergentes. L'une, la Catalogne française, sur une partie de son territoire en particulier la vallée du Vallespir, revendique l'enracinement de sa culture tauromachique, l'autre, la Catalogne espagnole, semble faire beaucoup pour la taire et s'en débarrasser. Il existe un différentiel paradoxal du dynamisme des activités taurines de part et d'autre de la frontière. Du côté espagnol, les arènes de Barcelone, malgré la crise qu'elles traversent, demeurent des arènes de première catégorie qui offrent plus de spectacles que les arènes de Céret, arènes de deuxième catégorie française, qui affichent haut et fort leur dynamisme et leur existence par une culture du bastion torista. Le simple fait que l'on chante l'hymne catalan Els segadors dans les arènes de Céret montre toute la distance qui sépare les terres taurines catalanes de part et d'autre de la frontière. La tauromachie joue ici dans une affirmation de la frontière qui sépare deux versions de la Catalogne, par ailleurs unies par de fortes relations socioculturelles. On remarquera là, une belle inversion territoriale. La Catalogne française est une périphérie méridionale du territoire national où la tauromachie participe d'une différenciation identitaire avec un Nord au contour flou. La Catalogne espagnole constitue un centre moteur de l'économie nationale, dans un contexte d'autonomie régionale affirmé où la tauromachie, disons plutôt sa négation, participe d'une différenciation identitaire avec le reste de l'Espagne. La tauromachie est-elle catalane ? On l'aura compris, la tauromachie n'est donc porteuse d'aucune valeur territoriale intrinsèque. Elle n'est porteuse de valeur territoriale qu'en fonction d'un lieu pris dans un contexte national et frontalier plus englobant. Sur ce point, la démarche multiscalaire du géographe est irremplaçable.

Enfin, le Sud-Est taurin centré sur une Camargue plus éloignée de l'Espagne se caractérise par une plus grande autonomie du fonctionnement de son espace taurin. Néanmoins il n'est aucunement autarcique ni autosuffisant pour les corridas. On retrouve une nouvelle fois l'influence des Chopera, partenaires importants pour la gestion des arènes de Nîmes, de Béziers et de Fréjus. L'afición du Sud-Est se caractérise par une plus forte mobilité vers les arènes espagnoles que vers les arènes du Sud-Ouest français. Les mobilités dépendent de la distance et du pouvoir d'attraction des férias. Barcelone, la plus proche est fréquemment visitée, sans doute moins que les arènes de Madrid et de Séville. La féria de Séville a d'ailleurs servi de référence dans les années 1980 pour inspirer les formes du renouvellement de la féria de Nîmes. La féria de Nîmes demeure néanmoins un amalgame singulier d'influences composites. Les fanfares que le Sud-Ouest nomme « bandas » s'appellent ici " peñas », les bars associatifs ouverts par les «peñas » dans le Sud-Ouest s'appellent ici « bodegas » et il n'existe aucune tenue de fête attitrée. À un étonnement feint, faisant part de mon admiration pour la 
qualité de l'afición du Sud-Est par rapport à celle du Sud-Ouest afin d'obtenir des informations, un spectateur arlésien m'a offert une leçon synthétique de géographie culturelle comparée : "Ah oui, ici l'afición est belle, et au moins on n'est pas déguisé, mais ici on bouffe mal, on chante faux et on a mal au cul à force de monter sur les chevaux $»$.

\section{LA « Frontière taurine » ESPAgne-Portugal :}

ENTRE FRÈRES ENNEMIS ET NATIONS SEEURS

La frontière taurine qui sépare l'Espagne et le Portugal est l'une des discontinuités les plus marquées de l'espace tauromachique dont on a déjà révélé certains aspects. L'observation des dynamiques tauromachiques entre le Portugal et l'Espagne offre un éclairage particulier de «l'articulation des territoires en péninsule Ibérique $»^{35}$.

Comme nous l'avons signalé, de part et d'autre de la frontière, il existe une version officielle de l'histoire taurine qui fait volontiers l'apologie de la tauromachie comme pratique emblématique de la nation. À partir du xıx ${ }^{\mathrm{e}}$ siècle, en Espagne comme au Portugal, l'expression Fiesta Nacional, est employée pour désigner le spectacle tauromachique. Les traditions tauromachiques y sont présentées comme l'aboutissement artistique d'une tauromachie immémoriale à laquelle l'idiosyncrasie nationale aurait donné forme et signification. En outre, en dehors d'un certain parallélisme des histoires déjà mentionné, chaque version analyse à sa manière l'enracinement de la tradition sur le temps long. Le Portugal insiste sur la très grande continuité de la tauromachie aristocratique au-delà des modifications formelles et juridiques. Pour certains, l'interdiction de la mise à mort et le gainage des cornes ont même permis de débarrasser la tauromachie de sa dramaturgie macabre et archaïque. L'Espagne, quant à elle, valorise une tauromachie inventée par le peuple, un peuple qui s'empare de la fête, du sens de l'honneur et dépossède la noblesse d'une part de la violence légitime. Il est intéressant de remarquer que la relecture des histoires taurines portugaise et espagnole de la tauromachie correspond, dans une certaine mesure, à deux lieux communs des historiographies nationales respectives. Pour le Portugal, la continuité de la pratique tauromachique trouve une correspondance dans la continuité exceptionnelle de l'identité nationale, construite dans le cadre d'une frontière qui est fixée avec la Castille dès le XIII ${ }^{\mathrm{e}}$ siècle. Pour l'Espagne, l'idée que la tauromachie «nationale » est une invention du peuple trouve une correspondance dans le raccourci développé notamment par Menéndez Pidal ou Ortega y Gasset, selon lequel en Espagne «c'est le peuple qui a tout fait ». Le Portugal et l'Espagne offrent donc deux modes de valorisation de la culture taurine qui reprennent respectivement des éléments constitutifs de la construction identitaire nationale.

\footnotetext{
${ }^{35}$ Il s'agit d'un thème de recherche qui a occupé, en 1998, les IVe journées d'études du CENPA (Centre d'études Nord du Portugal - Aquitaine) et dans lequel la tauromachie pourrait offrir un éclairage singulier. F. GuICHARD (dir.), Articulation des territoires dans la péninsule Ibérique.
} 
Nous avons vu que les pratiques tauromachiques portugaises et espagnoles, sous leur forme de la tourada et de la corrida, reflètent à la fois l'autonomie d'un destin national et la rivalité entre les frères ennemis. Mais les évolutions récentes des pratiques tauromachiques qui s'accélèrent depuis un demi-siècle révèlent aussi l'affirmation d'un autre type de rapports entre les pays voisins qui n'a jamais totalement disparu. En effet, à la tauromachie considérée comme expression des rivalités nationales se superpose une autre représentation qui exprime la commune appartenance à la péninsule Ibérique. Ce sentiment de fraternité passe par un discours taurin transnational qui efface les frontières entre l'Espagne et le Portugal.

Ce discours est le fruit de l'évolution des relations diplomatiques entre le Portugal et L'Espagne ainsi que de l'évolution des pratiques tauromachiques réinterprétées sous cet angle. À la suite de la deuxième guerre mondiale, dans un contexte géopolitique de rapprochement entre les deux pays, la tauromachie offre une occasion supplémentaire de resserrer les liens. La conférence prononcée au théâtre Tivoli de Lisbonne en 1947 par le critique taurin espagnol Don Ventura, intitulée Solidaridad Taurina Hispano-Lusitana ${ }^{36}$, véhicule clairement l'idée que la tauromachie est un facteur de cohésion : " Je me sens relié à vous par la Raza et par le principe de contiguïté territoriale et psychologique $^{37}$ ». La passion pour les fêtes du taureau, " puissamment enracinée chez les Portugais et chez les Espagnols [...] fait la démonstration que le courage et la dextérité sont le patrimoine exclusif de la Raza ibérique ${ }^{38} »$. Selon l'auteur, cette solidarité passe par la tauromachie à pied : « le Portugal établit désormais une collaboration autrefois inexistante en nous offrant un vivier d'aspirants matadors qui feront croître la solidarité taurine entre les «taurophiles » des deux pays ${ }^{39}$ ". Du côté portugais, les aspirations sont identiques. En témoigne la conférence intitulée Panorama del toreo en Portugal, prononcée en 1949 par le critique Jaime Saraiva Lima à l'Ateneo de Madrid en présence du ministre de l'Agriculture espagnol et de l'ambassadeur du Portugal : "Nous nous rassemblons pour cultiver et admirer l'Art qui nous enthousiasme le plus : la Fiesta Nacional ${ }^{40} »$. On s'accordera à reconnaître l'ambiguïté d'une telle déclaration qui réunit en un seul " art » et en une seule "fête nationale », la tauromachie portugaise et la tauromachie espagnole. Il est vrai que la tauromachie portugaise a retrouvé de son éclat grâce aux cavaliers Simão de Veiga et à João Nuncio, mais il faut surtout expliquer la satisfaction de l'orateur par l'apparition des deux premiers grands matadors de l'histoire portugaise Diamantino Viseu et Manuel Dos Santos, dont nous avons parlé. Et Jaime Saraiva Lima

\footnotetext{
${ }^{36}$ Don Ventura, Solidaridad taurina Hispano-lusitana.

${ }^{37}$ Ibid., p. 2. Rappelons que la Raza désigne sous un même ensemble une communauté « hispanique » constituée de l'Espagne, du Portugal et des pays latino-américains, définie par des critères historiques, linguistiques et culturels.

${ }^{38}$ Don Ventura, Solidaridad taurina Hispano-lusitana, p. 4.

${ }^{39}$ Ibid., p. 45.

${ }^{40}$ J. Saraiva Lima, Panorama del toreo en Portugal, p. 10.
} 
d'ajouter : « Je crois que l'interdiction de tuer les taureaux ne durera pas longtemps au Portugal ${ }^{41} »$.

Aujourd'hui, dans un tout autre contexte qui fait suite à la démocratisation du Portugal et de l'Espagne, et à leur entrée dans l'Union européenne, le recoupement partiel des formes tauromachiques et l'hégémonie taurine espagnole exercée sur les termes de l'échange entre les deux pays, contribuent à les rapprocher à nouveau. Ainsi aux discours taurins qui font et défont les frontières de la péninsule Ibérique, s'ajoute une représentation holiste de la culture tauromachique qui célèbre son unité par-delà ses clivages. Il en résulte une différenciation de la «frontière tauromachique » en plusieurs zones.

Nous pouvons segmenter la frontière tauromachique en quatre zones transversales du nord au sud, pour lesquelles les dynamiques transfrontalières recouvrent des réalités diverses.

La zone frontière Galice / Minho - Trás-os-Montes est marquée par l'insignifiance des relations liées aux spectacles taurins dans une aire culturelle que l'on pourrait qualifier d'aire " a-tauromachique ", si l'on excepte les arènes de Viana do Castelo et la traditionnelle vaca das cordas de Ponte de Lima. Par ailleurs, cette zone frontière se caractérise par d'intenses relations linguistiques, économiques et sociales. Ici la tauromachie ne constitue, ni un véritable champ de représentations, ni un facteur de recomposition territoriale.

La zone frontière qui comprend la province de Salamanque et l'Alto Douro - Beira Alta ne se caractérise pas non plus par d'intenses relations liées aux pratiques taurines. Pour autant, ces pratiques n'y sont pas absentes. Il existe un fort différentiel en termes d'activités taurines de part et d'autre de la frontière. La province de Salamanque est totalement intégrée au circuit professionnel international et tourne le dos au Portugal. Elle regroupe à elle seule un quart des élevages espagnols de taureaux de combat et a vu naître de nombreux matadors au cours de l'histoire. Du côté portugais, exception faite des arènes démontables qui programment épisodiquement des spectacles taurins, on remarque une certaine faiblesse de la tauromachie professionnelle caractéristique de la moitié nord du Portugal. En revanche, nous avons signalé, l'importance et l'originalité de la pratique de la corrida do forcao ou capeia raiana, autour de Sabugal, à proximité de la ligne frontière. La coutume veut que l'on fasse venir les taureaux de l'autre côté de la frontière. Le qualificatif de la pratique elle-même, capeia raiana (de raia : la frontière), met l'accent sur la dimension territoriale du rituel taurin. Autrefois volés aux Espagnols, les taureaux sont aujourd'hui achetés dans les nombreux élevages de la province de Salamanque. Fernando Teixeira analyse cette pratique comme la trace d'un antagonisme frontalier ancien, localisé dans une zone régulièrement soumise aux raids des cavaliers espagnols au $\mathrm{XIV}^{\mathrm{e}}$ siècle et plusieurs fois employée comme couloir d'invasion vers l'ouest ${ }^{42}$. Aujourd'hui le rite resserre les liens

\footnotetext{
${ }^{41}$ Ibid., p. 35.

${ }^{42}$ F. Teixera, «A corrida do forção ».
} 
d'une communauté dont la plupart a émigré en Espagne ou en France, et qui revient au pays lors des fêtes estivales. C'est aussi l'occasion de maintenir une cohésion entre villages voisins à partir d'une rivalité taurine lors du concours annuel de capeia raiana. Le jeu renvoie donc à la double composante de la frontière, à la fois ligne de rupture et zone périphérique. Du côté espagnol, le carnaval de Ciudad Rodrigo, à quelques kilomètres de la frontière, offre un autre témoignage exprimant l'isolement périphérique des confins de Castille sur la base d'un rite rural, collectif et carnavalesque qui s'oppose en tout point à la tauromachie urbaine, professionnelle et si sérieuse de Salamanque.

La troisième zone frontière Haut Alentejo - Estrémadure est une zone d'intenses dynamiques transfrontalières hispano-portugaises du point de vue des pratiques taurines. La ville frontalière de Badajoz sert d'interface entre la tauromachie portugaise et espagnole. La féria de Badajoz accueille un public portugais important et veille à programmer dans l'arène des confrontations hispano-portugaises. Lors de la corrida du 18 juillet 2005 à Badajoz, à défaut de connaitre le nombre précis de spectateurs portugais, nous avons compté, sur le parking attenant, une voiture portugaise pour trois voitures espagnoles. De nombreux toreros portugais ont d'ailleurs pris leur « alternative " à Badajoz qui joue ainsi le rôle de porte d'entrée vers le marché taurin espagnol : Antonio dos Santos (24 juin 1952), José Simões (24 juin 1967), Mario Coelho (27 juin 1967), José Falcão (23 juin 1968), Manuel Moreno (22 juin 1985), Rui Bento Vasques (25 juin 1988), José Luís Gonsalves (10 mars 1994) et Pedrito de Portugal (26 juin 1994). Le Portugal est mieux représenté dans ces arènes qu'ailleurs en Espagne. Il en va de même pour les arènes voisines de Cáceres, Mérida, Almendralejo, Zafra ou Jerez de los Caballeros. Il n'est pourtant pas possible d'en faire une règle absolue comme en témoigne la féria d'Olivenza où la place de la tauromachie espagnole et des professionnels espagnols ne semble en rien répercuter l'influence de la frontière. Il est d'ailleurs curieux de constater que cette cité, hautement symbolique en raison des litiges frontaliers auxquels elle a donné lieu, n'ait fixé aucune pratique tauromachique qui puisse en rendre compte de quelque manière. Cela en dit long sur l'attrait de la tauromachie espagnole dans cette zone et sur la position de force de l'offre sur la demande. Grâce aux choix judicieux du directeur des arènes José Cutiño, la féria d'Olivenza depuis le début des années 1990 a su se situer sur un créneau efficace : une féria haut de gamme de début de saison qui réunit des toreros très prestigieux attirant un public élargi dont de nombreux Portugais. Dans une entrevue accordée à la revue 6 Toros 6 , José Cutiño analyse ainsi la composition de son public :

Il y a un noyau majoritaire d'aficionados locaux et aussi des villages les plus taurins de la province de Badajoz, ceux de la capitale, ceux de Mérida, Zafra, de toute la terre de Barros, qui peuvent représenter jusqu'à $50 \%$ de l'arène. Ensuite, $30 \%$ doivent être Portugais, la majorité du continent, mais aussi de l'île Terceira. Les $20 \%$ restant sont composés d'aficionados de Séville, de Madrid, de Valence, du Nord de l'Espagne et 
aussi des Français de Mont-de-Marsan et de Bayonne, et cette année vient au complet le Club taurin de Londres ${ }^{43}$.

Sur le plan du développement économique et du point de vue de la vitalité des activités taurines, c'est bien l'Estrémadure, où le nombre de spectacles taurins a été multiplié par trois en vingt ans, qui profite le plus de cette situation. De nombreuses ganaderias d'Andalousie, de Madrid et de Salamanque y transfèrent désormais leur activité. On comprend pourquoi la promotion des activités taurines est inscrite officiellement au programme de la Junte d'Estrémadure. La région exploite donc une situation frontalière doublement avantageuse : la réserve d'espaces libres des dehesas pour les ganaderias et la proximité du public portugais privé de corridas intégrales dans son pays.

Le cas du village de Barrancos bien que situé plus au sud, peut être inclus dans cette zone. Il en va de même des municipalités qui ont déposé des demandes allant dans le sens d'une autorisation de la mise à mort des taureaux. La plupart de ces communes sont situées à proximité de la frontière telles que Aldeia da Luz, Monsaraz, Santo Aleixo da Restauração. Barrancos n'est pas simplement le reflet du rayonnement de la tauromachie espagnole sur la zone frontalière. En réalité, cette zone dite de " la Contienda " relève depuis des siècles d'un espace communautaire hispano-portugais, des confins flous, laissés en marge de la linéarisation moderne des frontières. Le tracé de la frontière n'y fut fixé qu'en 1926. Ainsi, célébrer un spectacle avec mise à mort renvoie, certes, à la proximité culturelle avec l'Espagne, mais c'est aussi, pour une région parmi les plus pauvres d'Europe, un moyen de signifier son existence et de défier par la transgression l'autorité centrale, lointaine et forcément coupable de quelque chose.

L'extension des interactions spatiales du Portugal et de l'Espagne ne se limite pas aux régions frontalières. C'est l'ensemble de l'espace taurin portugais, en particulier le cour de cet espace (région de Lisbonne, Ribatejo, Haut Alentejo) qui entretient des relations intenses avec les grandes cités taurines d'Espagne. On l'a vu, les interactions liées au monde de l'élevage sont anciennes. Plus récentes sont les dynamiques spatiales liées aux flux réguliers d'aficionados portugais qui se rendent de plus en plus facilement aux férias de Séville et de Madrid grâce au progrès des transports. Les clubs taurins les plus actifs comme ceux de Moita do Ribatejo et de Vila Franca de Xira y participent en organisant des voyages presque chaque année. Cela n'est bien sûr pas incompatible avec la volonté de voir se développer des corridas intégrales sur le sol portugais qui sont identifiées comme partie intégrante de la tradition et de l'identité locale. À Vila Franca de Xira, patrie des matadors José Julio, Mario Coelho, José Falcão, Antonio de Portugal, Victor Mendes et Rui Bento Vasques, la corrida intégrale a d'ailleurs connu des précédents en 1927, 1976 et 1977 donnant lieu à des émeutes de la population lors de l'arrestation des toreros. Les dynamiques territoriales de cette zone relèvent bien d'une logique d'organisation réticulaire des espaces tau-

\footnotetext{
${ }^{43} 6$ Toros $6, \mathrm{n}^{\circ}$ 608, mardi 21 février 2006, p. 11.
} 
romachiques qui s'affranchit des frontières. Autrement dit, la discontinuité de l'espace - une frontière tauromachique héritée de la tradition et maintenue par la différence de législation - engendre des dynamiques transfrontalières qui établissent de nouvelles continuités territoriales. Roger Brunet avait exprimé cette idée sur un plan théorique en affirmant que, dans le fonctionnement de l'espace humain, la discontinuité était l'une des conditions de la réintroduction de la continuitét ${ }^{44}$. Les arènes frontalières jouent assurément un rôle de synapses organisant les contacts entre pays voisins aux cultures taurines différentes, ce que nous aurons également l'occasion d'observer au sujet de la frontière entre les États-Unis et le Mexique.

Enfin, la quatrième zone frontière Algarve-Huelva est un espace de rapide transformation. Le pont construit sur le Guadiana a changé les conditions de circulation des hommes et des activités dans un secteur littoral à forte densité, marqué par le tourisme de masse. Cependant, le développement récent des spectacles taurins du côté portugais - ce dont témoignent les arènes de la Albufeira - semble davantage accompagner la diversification locale de l'offre touristique que le renforcement des relations transfrontalières liées à la tauromachie. À Huelva, du côté espagnol, la tradition taurine est plus ancienne. Elle acquit même une grande importance dans la Sierra de Aracena à la fin du $\mathrm{XIX}^{\mathrm{e}}$ siècle, en lien avec le développement de l'économie du liège, qui permit à de nombreux villages de construire des arènes ${ }^{45}$. Aujourd'hui, d'un point de vue tauromachique, Huelva reste dans l'ombre de la province voisine, Cadix, qui entretient de fortes relations avec Séville.

La frontière Espagne-Portugal vue à travers la tauromachie, nous offre ainsi un riche éventail de configurations spatiales : l'indifférence des régions septentrionales, les vestiges d'une marche militaire, les particularismes des marges oubliées, et des synapses de reconnections des territoires taurins.

\footnotetext{
${ }^{44}$ R. BRUnet et alii, « La discontinuité en géographie », p. 303.

${ }^{45}$ J. L. Pérez de lama Halcón, « Una arquitectura popular. Las plazas de toros de la Sierra de Aracena ».
} 


\section{STRUCTURE ET DYNAMIQUES DES ESPACES TAUROMACHIQUES AMÉRICAINS}

La présence de la corrida est l'un des critères les plus efficaces pour différencier les pays d'Amérique possédant des traditions tauromachiques au sein d'un vaste ensemble où le rodéo, dans des versions diverses, est la marque dominante d'une culture commune. La corrida tisse de façon très évidente des liens économiques, historiques et culturels entre l'Europe et l'Amérique. Les rodéos constituent une pratique panaméricaine qui pourrait se passer de toute référence à la corrida espagnole, s'il n'était aussi question de comprendre leur filiation historique et d'analyser les relations territoriales qu'ils contribuent à façonner en raison de leur dissociation et de leur coexistence. De plus, nombre de pratiques tauromachiques populaires mettent à mal la distinction catégorielle entre les rodéos et la corrida qui, au niveau des spectacles professionnels, apparaît pourtant sans ambiguïté. Certaines pratiques populaires maintenues dans un état de codification élémentaire se jouent des clivages apparemment incontestables entre corrida et rodéo. Il en résulte une organisation territoriale des pratiques complexe où se côtoient des frontières franches, des frontières floues, des coexistences et des continuums allant jusqu'à rendre invisible le passage d'un jeu taurin à l'autre.

L'espace tauromachique américain est appréhendé à l'échelle des Étatsnations qui demeurent les cadres spatiaux les plus appropriés pour analyser les relations territoriales véhiculées par les pratiques tauromachiques. Une nouvelle fois, afin de pallier ce que les analyses nationales laisseraient à la marge, l'attention est portée sur la question des frontières qui apparaissent comme de véritables supports matériels et idéologiques de différenciation : les frontières des pratiques tauromachiques qui ne respectent guère les cadres territoriaux et les frontières des pays qui participent en retour aux discontinuités de l'espace tauromachique. Enfin, l'évolution récente de certaines pratiques tauromachiques et de leur ancrage territorial, loin de représenter de simples retouches au sein d'une géographie héritée d'un cadre ancien, manifestent au contraire un important dynamisme en phase avec les transformations géopolitiques contemporaines. 


\section{I. — L'ESPACE TAUROMACHIQUE MEXICAIN : CORRIDA, CHARREADA, JARIPEO ET RODEO}

Le Mexique est un pays taurin aux pratiques multiples, souvent liées les unes aux autres de façon complexe. Deux spectacles codifiés dominent les pratiques et les représentations : la corrida et la charreada. La pratique fondée sur la monte des taureaux est ramifiée entre plusieurs jeux et spectacles : jaripeo, rodéo nord-américain et charreada dans laquelle la monte n'est qu'un exercice parmi d'autres. Au cœur du plateau central mexicain où se situent les principaux foyers de peuplement, la forte densité des pratiques tauromachiques délimite le cadre de leur coexistence spatiale et le terrain principal de leur articulation. Avec l'éloignement du centre apparaissent des profils régionaux distincts où les pratiques tauromachiques se combinent différemment. Ces profils révèlent alors des clivages nord-sud et est-ouest, des logiques centre-périphéries et des formes d'organisation spatiale qui tiennent aux dynamiques transfrontalières et à la place des États-Unis.

\section{La CORRIDA Au Mexique}

OU LE GÉANT TAURIN DE L'AMÉRIQUE LATINE

L'Institut National des Statistiques (INEGI) publie chaque année un bulletin récapitulatif des activités culturelles, sportives et récréatives qui se sont déroulées dans le pays ${ }^{1}$. Dans ce document, les activités qui intéressent notre sujet sont réparties entre, d'un côté, les courses de taureaux et de l'autre, les charreadas et jaripeos, sans que l'on puisse d'aucune manière les différencier. Cette source d'information officielle, en apparence inestimable, se révèle décevante en raison d'une collecte incomplète des données. Pour l'année 2003, L'INEGI déclare que se sont déroulées 301 courses de taureaux (majeures et mineures confondues), réparties dans 14 États sur les 32 entités fédératives existantes. Dans ce classement, s'illustrent les États de Jalisco, Distrito Federal, Querétaro, Aguascalientes et Zacatecas qui concentreraient $65,4 \%$ des programmations. Le bilan général fait état de 830.733 spectateurs payants dont 530.073 assistants aux corridas de toros.

Tous ces chiffres sont contestables mais offrent une ébauche acceptable de la forte concentration des spectacles taurins dans la zone centrale du pays. Cependant ces données doivent être revues à la hausse et l'aire d'extension de la pratique, élargie. En ne comptabilisant pourtant que les spectacles majeurs, là où les statistiques officielles enregistrent tous les types de courses de taureaux, les données de Mundotoro doublent les chiffres de l'INEGI (voir graphique 11, p. 322).

Grâce aux informations transmises par Luis Ruiz Quiroz, considéré comme l'un des meilleurs spécialistes des questions statistiques touchant aux courses de taureaux au Mexique, nous pouvons détailler ces chiffres. En 2005, parmi les spec-

\footnotetext{
${ }^{1}$ INEGI, Estadísticas de Cultura, Cuaderno no 8, 2003.
} 


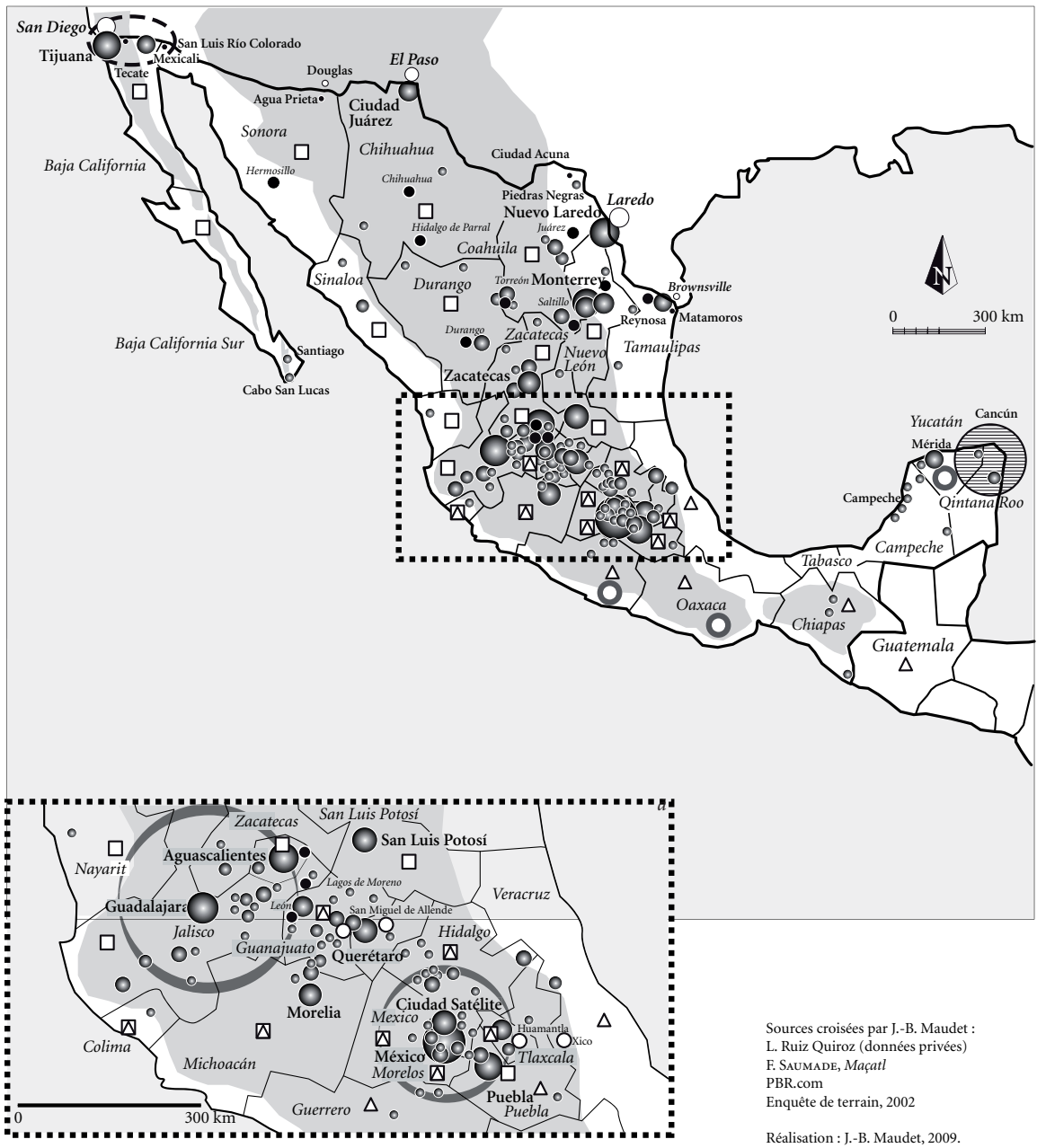

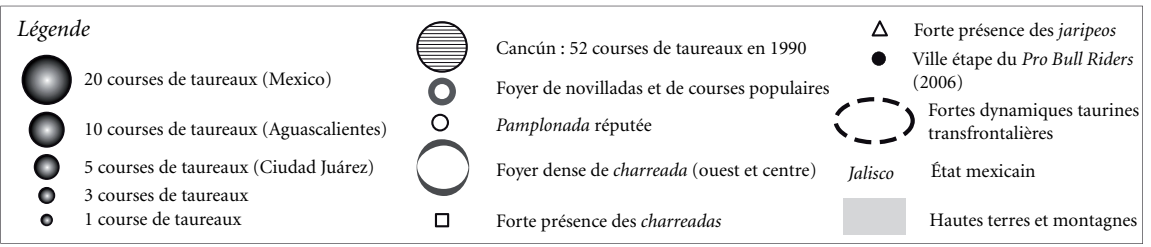

Carte 13. - Tauromachies au Mexique 
tacles majeurs se sont déroulées 355 corridas. Ce total est inférieur à la moyenne des années 1980 qui est de 415 corridas, sans que l'on puisse pour autant parler actuellement de véritable crise de la tauromachie au Mexique ainsi que l'écrivent parfois les médias. Ici les analyses des medias oscillent souvent sans nuance entre l'irrémédiable disparition de la tauromachie ou son incommensurable importance, ces jugements ne s'appuyant jamais sur des données quantitatives étayées.

GraphiQue 11. - Spectacles taurins majeurs au Mexique (2001-2005)

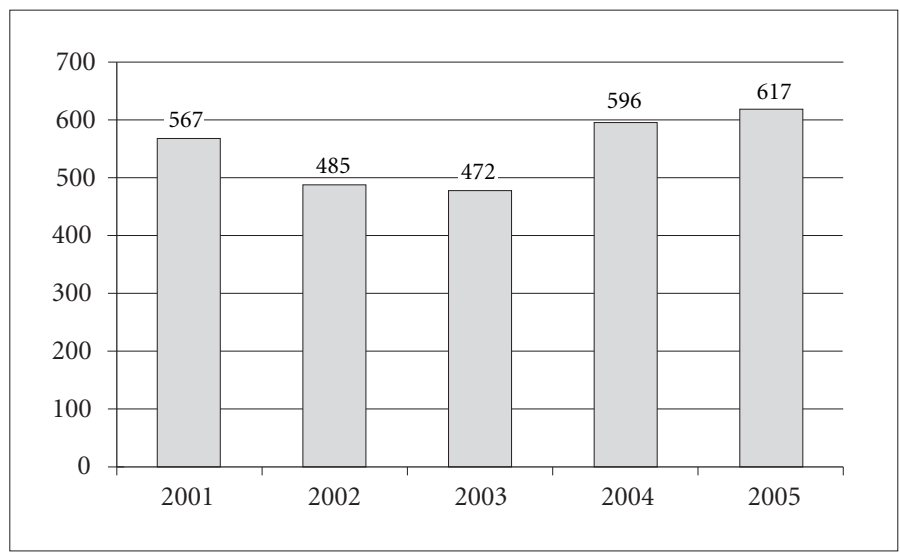

L'une des particularités à souligner dans ce bilan est la place importante de la tauromachie à cheval. Le plus souvent, elle est associée à la tauromachie à pied puisque dans un tiers des spectacles un rejoneador est à l'affiche avec des matadors (117 courses sur 355). Aussi est-il moins étonnant de trouver en tête des professionnels taurins Espagnols venant toréer au Mexique un rejoneador : le navarrais Pablo Hermoso de Mendoza. En 2002, avec 65 contrats, il bat le record du nombre de courses toréées au Mexique par un Européen lors d'une même année, record jusqu'alors détenu par le torero à pied «El Cordobés » avec 50 contrats. Ce détail confirme le succès plus général des pratiques taurinoéquestres au Mexique. De l'aveu du cavalier : «Il y a beaucoup de personnes qui viennent voir mes corridas et qui ne sont pas des aficionados, ils y viennent par leur passion pour le cheval, la charrería et l'équitation $»^{2}$. Voici un premier témoignage qui atteste d'une certaine perméabilité entre corrida et charreada, à l'opposé d'une conception qui en ferait des passions étanches.

Pour une comparaison sommaire entre les deux géants taurins européens et américains observons que le Mexique programme quatre fois moins de spectacles majeurs que l'Espagne et trois fois moins de corridas de toros. Le nombre annuel de spectateurs doit avoisiner les 2 millions sans compter les spectacles

\footnotetext{
${ }^{2}$ Aplausos, $\mathrm{n}^{\circ}$ 1281, lundi 15 avril 2002, p. 27.
} 
mineurs sur lesquels nous avons peu d'informations. Sur ce point, une indication d'une grande valeur nous est donnée dans l'ouvrage de Luis de Tabique de 1955 intitulé Guía de América taurina ${ }^{3}$. L'auteur y recense pas moins de $2.701 \mathrm{spec}-$ tacles taurins classés en trois catégories : 215 corridas de toros, 704 novilladas avec du bétail de caste et 1.782 avec du bétail créole et de demi-caste. Malgré l'ancienneté de ces données, elles font apparaître toute l'importance des spectacles mineurs marginalisés par les statistiques des aficionados qui permettent ainsi de restituer une plus juste image du poids de la tauromachie au Mexique. Dans les années 1950, il est fort probable que le nombre de spectacles taurins ait été plus important au Mexique qu'en Espagne.

La carte 13 (p. 321) rend compte de la répartition des spectacles majeurs en 2004. Observons d'abord que sont concernés par la tauromachie davantage d'États que les 14 recensés par l'INEGI. La répartition des spectacles est dans l'ensemble conforme aux densités de population et à la trame du peuplement. En effet, elle fait apparaître tout le poids des petits États du plateau central, qui sont aussi les plus peuplés, et le poids du doublet Mexico-Jalisco ${ }^{4}$. Dans cette partie centrale, de Guadalajara à Puebla en passant par Mexico, se concentrent près de $80 \%$ des spectacles et $70 \%$ des arènes permanentes du pays. C'est aussi dans cet espace que l'on observe les manifestations les plus connues d'encierros, les pamplonadas inspirées du modèle navarrais : à Huamantla (Tlaxcala), à San Miguel de Allende (Guanajuato), dans le quartier de Santa Ana à Querétaro, à Xico (Veracruz). Cet ensemble de forte densité taurine se prolonge vers le nord par les États de San Luis Potosí, de Zacatecas, de Cohuila et surtout de Nuevo León, autour de sa capitale Monterrey, qui est la troisième plus grande ville du pays. En 2005, l'État du Nuevo León se situe au troisième rang au nombre de courses de taureaux avec 36 spectacles, derrière Mexico (État de Mexico + Mexico DF : 105) et Jalisco (98). La hiérarchie des villes taurines est conforme à celle de la trame urbaine. Voilà peut-être l'un des signes les plus tangibles d'une envergure nationale de la corrida.

Grâce aux indications précieuses de Luis de Tabique, il convient néanmoins d'élargir l'aire d'extension des spectacles taurins aux États du Sud, à forte composante " indigène ", qui offrent un nombre insoupçonné de courses mineures données avec du bétail de demi-caste, du bétail créole ou des zébus ${ }^{5}$. Ces spectacles sont généralement passés sous silence ou ignorés par les observateurs focalisés sur l'orthodoxie taurine. Les États du Guerrero, de Oaxaca et du Yucatán offraient en 1955 près de 700 courses de ce type, témoignant d'une très grande popularité qui s'est prolongée en partie jusqu'à nos jours. Pour le Yucatán, Luis de Tabique fait certainement référence aux nombreuses courses

\footnotetext{
${ }^{3}$ L. de TABIQUe, Guía de América taurina, p. 13.

${ }^{4}$ Rappelons que les petits États du plateau central sont nés historiquement de la fragmentation des « gros » : Aguascalientes, Colima, Nayarit issus du Jalisco, Guanajuato né du Michoacán, Querétaro, Hidalgo, Guerrero et Morelos né du México.

${ }^{5}$ Le terme « indigène » est couramment utilisé dans toute l'Amérique latine pour remplacer le terme dévalorisé d'Indien.
} 
composées d'une partie conventionnelle avec des toreros locaux et d'une partie plus informelle où le public affronte lui-même les taureaux, sans mise à mort du bétail. Quoi qu'il en soit, il est important de ne pas circonscrire la pratique de la tauromachie au seul plateau central et aux États du Nord, mais de l'étendre aux territoires du Sud, existant sous une forme plus populaire et participative. Cette tauromachie est généralement invisible pour ceux qui n’en observent que la partie urbaine et professionnelle.

Il existerait sur le territoire mexicain quelque 300 arènes permanentes en activité. En 1992, Miguel Luna Parra et Luis Ruiz Quiroz en recensaient 291, dont une vingtaine avait été construite depuis les années 1970, plaçant en tête les États de Jalisco et de Mexico avec chacun une quarantaine d'arènes ${ }^{6}$. Les capitales de ces États, Guadalajara et Mexico, sur la base d'une délimitation de leur aire métropolitaine, possèdent respectivement 5 et 17 arènes, pour des populations équivalentes à 5 et 20 millions d'habitants. D'autres grandes agglomérations possèdent ainsi plusieurs arènes : trois à Ciudad Juárez (Chihuahua), trois à Monterrey (Nuevo León), trois à Querétaro et deux à Tijuana (Baja California). En outre, ces arènes ont généralement de grandes capacités d'accueil : l'immense Plaza México de la capitale avec plus de 45.000 places qui possède, au sol, les dimensions d'un stade de foot, les arènes Playas de Tijuana avec 22.000 places et les arènes du Nuevo Progreso de Guadalajara avec 20.000 places.

Une fois de plus, les indications de Luis de Tabique laissent supposer que leur nombre est beaucoup plus élevé, ou tout au moins le nombre des lieux de spectacles. En effet, il recensait en 1955, 217 arènes fixes et pas moins de 493 arènes « improvisées » offrant des spectacles avec régularité 7 . Ángel Villatoro, dans son ouvrage Antología taurina mexicana de 1986 en recense 774, sans aucune précision sur le type d'arènes répertorié ${ }^{8}$. Remarquons que sur les 217 arènes fixes mentionnées par Luis de Tabique, 107 sont qualifiées d'ouvrages de maçonnerie, 91 d'ouvrages mixtes (bois et maçonnerie) et 19 sont des ranchos de charros utilisés pour les corridas. Si le nombre d'arènes improvisées est important dans les États traditionnellement considérés comme les plus taurins (58 à Jalisco, 30 à Mexico), elles sont beaucoup plus nombreuses dans les États du Sud, que ce soit le Guerrero ( 8 arènes fixes pour 72 arènes improvisées) ou le Yucatán ( 2 arènes fixes pour 76 arènes improvisées). Pour ce qui est du Yucatán, la tradition de novilladas et de courses populaires données dans des arènes improvisées demeure vive. Elle est revitalisée dans certains lieux par le développement du tourisme, mais l'essentiel de la pratique correspond encore à des courses de villages qui attestent d'une véritable tradition tauromachique populaire. On observe ainsi que la différenciation des spectacles taurins entre corridas formelles et novilladas populaires recoupe en partie le clivage ethnique et territorial entre le Mexique central et les États du Sud aux communautés indigènes importantes.

\footnotetext{
${ }^{6}$ M. Luna Parra et L. Ruíz Quíroz, Análisis y registro de las plazas de toros de México, pp. 16 et 24.

${ }^{7}$ L. de TABique, Guía de América taurina, p. 11.

${ }^{8}$ Á. Villatoro, Antología taurina mexicana, pp. 373-382.
} 
La carte 13 (p. 321) fait également apparaître toute l'importance de la frontière avec les États-Unis où se sont fixées des arènes actives, au premier rang desquelles celles de Tijuana, puis dans une moindre mesure, celles de Mexicali, Ciudad Juárez, Nuevo Laredo. Si l'on ajoute les arènes proposant des spectacles mineurs telles que Tecate, San Luis Río Colorado, Agua Prieta, cette implantation fait figure de chapelet distendu par l'immensité de la frontière ${ }^{9}$. Ce chapelet d'arènes témoigne d'une logique d'organisation spatiale liée à la discontinuité des pratiques entre le Mexique où la corrida est autorisée et les États-Unis où elle est interdite sous sa forme conventionnelle. Il en résulte une interface attractive rythmée par les villes doublons de la frontière : Tijuana - San Diego ; Agua Prieta - Douglas ; Ciudad Juárez - El Paso ; Nuevo Laredo - Laredo ; Reynosa-Matatoros - Brownsville. Dans les années 1950, Ciudad Juárez célébrait une cinquantaine de courses de taureaux par an. Cela étant, mis à part Tijuana qui invite encore les plus grands matadors du moment et Ciudad Juárez, la plupart de ces arènes ne proposent aujourd'hui que des spectacles de faible tenue, comme si l'effet frontière s'était émoussé, soit au profit de mobilités spatiales de plus grandes envergures vers des villes taurines mieux consolidées, soit que l'attirance exotique pour le bullfighting ait quelque peu passé. On notera que le bien nommé ex-matador mexicain Eliseo Gómez «El Charro » a pris, à la fin des années 1990, la direction des arènes de Mexicali afin de programmer quelques courses pendant l'hiver. Le manque de rentabilité l'a ensuite amené à réduire ses ambitions. Il propose désormais des pamplonadas dans la ville voisine de Tecate $^{10}$. Nul doute que les nombreux adeptes américains de la San Fermin y préparent leur course afin de ne pas perdre le souffle et la juste distance nécessaire pour affronter les taureaux, à des milliers de kilomètres de la calle Estafeta ${ }^{11}$.

À l'image des arènes frontalières, les nombreuses arènes du littoral sont également des lieux de spectacles à destination d'une clientèle internationale majoritairement venue d'Amérique du Nord. C'est le cas des arènes de Santiago et de Cabo San Lucas en Basse Californie et des nombreuses arènes littorales de la péninsule du Yucatán. En la matière, la palme revient aux arènes de Cancún, aujourd'hui en sommeil, qui peuvent être considérées comme le modèle outrancier de l'arène de plage. Inaugurée en 1989, elle a programmé de 1990 à 1993, sans aucune interruption, une course par semaine, atteignant ainsi le record de 52 corridas dans l'année ${ }^{12}$. Il s'agissait néanmoins de spectacles plus courts qu'une corrida formelle pour un public généralement néophyte et différent chaque semaine. Dès l'année 1994, le spectacle eut moins de succès et laissa sa place à un spectacle mixte incluant la sortie d'un taureau

\footnotetext{
${ }^{9}$ Nous avons repéré l'existence de ces arènes grâce à un article de D. PiERCE, dans Toros, nº 1570, 29 janvier 1998.

10 Toros, $\mathrm{n}^{\circ} 1570,29$ janvier 1998.

${ }^{11}$ La calle Estafeta est la plus célèbre rue de l'encierro de Pampelune.

${ }^{12}$ Par comparaison, qui n'en est pas une en raison de la qualité des spectacles, les arènes de Madrid ont programmé durant ces années-là un total de 45 corridas.
} 
combattu à l'espagnole et des exercices charros. C'est ce spectacle que l'Union taurine nîmoise a dû observer lors de son voyage au Mexique en 1996. En voici le compte rendu simplifié :

Le spectacle débute par la venue de cavaliers portant fièrement le drapeau de diverses nations [...] suivie d'exhibitions de maniement de lasso, de combats de coqs, de danses typiques mexicaines (celle des plumes ou de l'ananas) permettant d'attendre la sortie d'un seul toro afeité et très maigre affronté par un torero bien modeste. [...] Le seul intérêt de cette course réside dans l'originalité de l'arrastre. [...] On admire ici deux ou trois cavaliers habillés en costume charro et montés sur de beaux chevaux venant attraper au lasso le toro pour le traîner rapidement vers le desolladero ${ }^{13}$.

En observant les photographies accompagnant l'article, il nous semble que c'est ce que la fusion des genres tauromachiques offre peut-être de plus appauvri pour les spectateurs, qui d'ailleurs désertent les gradins. Il faut placer Cancún au plus bas de l'échelle des hiérarchies internes du monde de l'afición.

Dans cette hiérarchie, les grandes villes et les capitales fédérales ont un incontestable rayonnement : Guadalajara, Aguascalientes, Monterrey, Querétaro, Puebla, San Luis Potosí. À n'en pas douter, le Mexique est le pays d'Amérique latine qui possède le plus d'arènes de rang international où toréent chaque année les meilleurs toreros européens. L'arène de Mexico se détache entre toutes. Il n'y en a pas de plus importante en taille, ni en réputation, dans toute l'Amérique latine. De l'extérieur, ces dimensions sont impressionnantes et doublent quand on y pénètre. Le niveau de la rue vous fait entrer à mi-hauteur de l'arène, creusée en contrebas sur trente-neuf rangées supplémentaires. C'est là que le placier, sûr de son coup, vous glisse une phrase sans doute mille fois répétée : "El más impresionante es el olé » (le plus impressionnant, c'est le olé). Les arènes sont parfois surnommées, en raison de leur profondeur et de leur verticalité, l'entonnoir ou le cratère, terme sans doute mieux adapté à la géomorphologie mexicaine. Ce sont les seules arènes avec celles de Madrid dans lesquelles les toreros passent le rite de passage de la confirmation d'alternative la première fois qu'ils y toréent.

Symboliquement, ce rituel instaure une polarité transatlantique entre le Vieux Continent et le Nouveau Monde qui fait de la Plaza México l'homologue latinoaméricaine de Las Ventas. La corrida la plus prestigieuse est celle du 5 février qui correspond à la date d'inauguration des arènes en 1946. La date relativement récente de la dernière version des arènes de Mexico ne doit pas faire oublier que la ville a connu quelque 45 arènes au cours de son histoire, depuis les débuts de vice-royauté à nos jours. Ce chiffre démontre à lui seul la force de l'enracinement culturel de la tauromachie dans la capitale. Toréer devant le public de

\footnotetext{
${ }^{13}$ Toros, n 1569, 2 janvier 1998. L'arrastre désigne le retrait des taureaux de l'arène. Le desolladero est la cour où les taureaux morts sont conditionnés afin de pouvoir commercialiser la viande.
} 
Mexico est une expérience qui marque tous les toreros conscients du prestige et de la renommée des lieux. Pour autant, les arènes de Mexico traversent une période difficile en partie due au maintien de son rang d'arène de temporada et à l'absence de véritable féria. Si la programmation d'une saison tauromachique renforce la réputation d'une arène, elle ne permet pas de retirer les bénéfices du regroupement des spectacles. Pour beaucoup d'observateurs autorisés, le rang des arènes de Mexico relève d'un prestige historique qui ne se traduit plus dans les faits par l'existence d'un événement taurin visible et fédérateur.

À la différence de Mexico, les arènes d'Aguascalientes tirent leur renommée nationale et internationale de la féria taurine d'avril. Elle se déroule pendant la Feria Nacional de San Marcos et en constitue l'une des attractions principales. Il s'agit d'une des férias taurines les plus anciennes puisque dès l'année 1830, deux ans après la création de la foire mercantile, le programme n'incluait pas moins de dix courses de taureaux. Comme l'annoncent aujourd'hui les encarts publicitaires, la Feria Nacional de San Marcos est devenue la « féria du Mexique ${ }^{14}$. Nombreux aficionados pensent de même pour la féria taurine qui en est inséparable. Ce phénomène révèle un enjeu territorial plus général qui dans l'imaginaire collectif national fait de la zone occidentale du plateau central la région de ce que l'identité mexicaine a de plus représentatif. Cette région, aux limites proches de l'ancienne audience de Nouvelle-Galice, correspond également, comme on le verra, au cœur du territoire de la charrería où coexistent ainsi deux images de la mexicanité. L'une est exprimée par la corrida, une manifestation culturelle transatlantique qui instaure un rapport de concurrence et de complémentarité entre le Mexique et la Madre Patria (la Terre Mère qui renvoie à l'Espagne), l'autre est exprimée par la charreada, une manifestation taurine plus représentative de l'autochtonie mexicaine.

La répartition des élevages recoupe en partie les foyers de forte densité taurine, en particulier dans l'État de Guanajuato, du Querétaro, de Jalisco et de Zacatecas. Il faut noter dans ce bref repérage de l'implantation des quelque 300 élevages braves mexicains, la discrétion de l'État de Mexico. Mexico et Mexico DF sont des zones de spectacles et de consommation, mais ne sont pas les zones importantes d'élevages braves qui forment une ceinture plus extérieure. Un autre élément remarquable de ce repérage est la place de l'État de Tlaxcala qui, bien qu'il soit le plus petit État mexicain, est celui qui possède le plus grand nombre d'élevages inscrits à l'Association des éleveurs de taureaux de race brave, avec 41 ganaderias. L'État de Tlaxcala, abrite parmi les plus célèbres élevages du Mexique : Coaxamalucan, Piedras Negras, Rancho Seco, José María Arturo Huerta, Soltepec, Juan Huerta, La Laguna, Montecristo y Zotoluca. Fort de cette tradition, l'État de Tlaxcala, au début des années 2000, a développé un programme pionnier de rationalisation, de mise en valeur et de promotion de l'élevage brave. Le projet est alors géré par le vétérinaire mexicain Javier Arriola Bueno, qui a pendant longtemps travaillé dans les élevages des alentours de Jerez de la Frontera en

\footnotetext{
${ }^{14}$ Slogan publicitaire : «Viens à Aguascalientes et tu vérifieras pourquoi la Feria de San Marcos est la féria du Mexique ».
} 
Espagne $^{15}$. Souvent les parcours individuels soulignent l'intensité des liens tissés de part et d'autre de l'Atlantique autour de cette commune passion.

Enfin, s'il est un domaine qui justifie que le Mexique soit qualifié de géant américain de la tauromachie, c'est bien celui du nombre et de la réputation des toreros. En 2004, environ 140 matadors d'alternative ont toréé au Mexique dont 130 de nationalité mexicaine ${ }^{16}$. Le nombre total de matadors dans l'histoire du Mexique est également très éloquent. En 1992, Luis Ruiz Quiroz a dressé une liste de 433 matadors auxquels il est possible d'ajouter les 89 matadors qui ont pris l'alternative de 1992 à 2004, soit un total de $522^{17}$. Ces chiffres sont beaucoup plus élevés que ceux des autres pays latino-américains et témoignent du développement du marché taurin intérieur qui pourrait se suffire à lui-même, s'il n'était question d'autre chose en jeu que de la seule performance des protagonistes. Cette autre chose, c'est l'existence d'une hispanité complexe, un lien culturel entre l'Espagne et le Mexique, que la tauromachie permet d'exprimer. Observons qu'aucun pays mieux que le Mexique n'est parvenu à exporter ses toreros, non seulement dans les autres pays taurins d'Amérique latine, mais aussi en Espagne, à l'image de Rodolfo Gaona et Luis Freig dans les années 1910-1920, de "Armillita chico » à la fin des années 1920 ou de Carlos Arruza qui fut le grand rival de Manolete au milieu des années 1940. La veine des toreros mexicains à la conquête de l'Espagne est actuellement tarie en dépit de leur qualité.

Les relations taurines entre l'Espagne et le Mexique ont toujours été assez chaotiques, entrecoupées d'embellies et de crises profondes, rythmées par des politiques de quotas, officiels ou officieux, pratiquées de part et d'autre. En 1931 avait été négocié un accord hispano-mexicain autorisant de part et d'autre une proportion de toreros étrangers de $10 \%$. Mais au milieu des années 1930, la presse espagnole n'hésite pas à parler de véritable « invasion taurine mexicaine ", leur contrat étant passé de 31 en 1925 à 340 en 1935. Le débat fut complexe, car beaucoup de toreros mexicains venaient, à cette époque, dans les bagages de l'agent et entrepreneur de spectacles Espagnol, Domingo "Dominguín », qui co-dirigeait également les arènes de Mexico. Ces accords furent rompus en 1936, renégociés en 1944, à nouveau rompus dans les années 1950, occasionnant des périodes de blocus et de libre-échange. Observons dans l'évolution des relations taurines hispano-mexicaines, le rôle particulier de Barcelone qui a accueilli et présenté de très nombreux toreros mexicains.

Pendant longtemps, les arènes de Barcelone ont servi pour eux de porte d'entrée en péninsule Ibérique comme si l'accès au marché taurin espagnol n'était rendu possible que par sa périphérie. Ce fut sans doute aussi l'un des moyens pour Barcelone de marquer sa différence, ici dans la plus pure tradition de son

\footnotetext{
${ }^{15}$ Je le remercie de m'avoir reçu, de m'avoir présenté le programme de revalorisation de la culture taurine et de m'avoir fait mieux connaître la réalité de l'élevage brave au Mexique.

${ }^{16}$ Données transmises par Luis Ruiz Quiroz.

${ }^{17}$ Ángel Villatoro en 1986 propose une courte bibliographie de 488 toreros mexicains. Á. VilLATORo, Antología taurina mexicana, pp. 71-271.
} 
cosmopolitisme et de son ouverture, tout en restant dans le champ tauromachique. Comme nous l'avons vu, Barcelone se caractérise actuellement par un courant souhaitant sortir de ce champ de références communes avec le reste de l'Espagne, par des mesures d'interdiction, justifiées en partie par une certaine idée du repli identitaire sur des valeurs qui seraient fondamentalement catalanes. Ce qu'il est intéressant de constater, c'est que cette différence était jadis marquée dans le champ tauromachique, par une proximité avec une altérité lointaine : le Mexique.

\section{Terre ET NATION DE LA CHARREADA}

Dans la charreada, les symboles d'une certaine exaltation nationale sont ostensiblement mis en scène. Le défilé des équipes ne laisse aucun doute sur cette intention. Elles paradent drapeau mexicain en main au son de la Marcha Zacateca, considérée comme un deuxième hymne national. Depuis 1933, la charreada est officiellement reconnue comme sport national et l'habit charro, réglementaire pour les compétitions, suffit à incarner partout dans le monde l'un des stéréotypes mexicains les plus célèbres.

L'anthropologue Cristina Palomar considère que les charros résultent d'une condensation d'attributs moraux et physiques qui symbolisent un idéal national ${ }^{18}$. Ils acquièrent ce statut dans les années 1920 et 1930, quand l'État post-révolutionnaire déploie toutes les stratégies possibles de consolidation et de légitimation pour unifier la nation et obtenir la paix sociale. Rien d'étonnant à ce que les charros rallient alors des figures historiquement contradictoires. Le charro par un processus de construction idéologique tient tout à la fois du contremaître de l'hacienda et du propriétaire aristocrate, du Chinaco de la fin du $\mathrm{XIX}^{\mathrm{e}}$ siècle, ce policier rural chargé de faire respecter l'ordre au temps de Benito Juárez, et du héros en arme de la Révolution mexicaine. Comment mieux conjuguer les tensions sociopolitiques en une même icône fédératrice et patriote ? Comme le montre Frédéric Saumade, le charro est aussi le produit du «bon » métissage de l'élite post-coloniale, issu d'une alliance vertueuse entre le créole et l'Indien mythique préhispanique à l'opposé de l'indigène réel, abâtardi par l'acculturation ${ }^{19}$. Dans cette perspective, l'auteur constate que la métaphore de l'attachement dans l'utilisation du lasso (la reata) et le choix racial des montures révèlent les stratégies matrimoniales endogamiques des charros. À travers le charro, au-delà de son symbolisme national officiellement fédérateur, s'exprime aussi la permanence oligarchique d'une élite.

En 2003, d'après les données de l'INEGI, charreadas et jaripeos ont enregistré la présence de 343.209 spectateurs. Ce chiffre a très peu de valeur, car rien ne permet de différencier les formes de jeu et encore moins de prendre en compte la charreada en tant qu'activité sportive de loisir, en dehors des

\footnotetext{
${ }^{18}$ C. Palomar, « El papel de la charrería como fenómeno cultural », p. 83.

${ }^{19}$ F. Saumade, Maçatl, p. 61.
} 
spectacles. La Fédération nationale de Charreada nous livre un bon indicateur pour en mesurer toute l'importance. À l'échelle du pays, elle déclare posséder le plus grand nombre de licenciés parmi les sports inscrits à la Confédération sportive nationale mexicaine. En dépit des limites des données de l'INEGI, les données officielles placent en tête, au nombre de spectateurs, les États de Jalisco, de Aguascalientes et du Michoacán. Ces données suffisent à mettre en avant un aspect important de la géographie de la charreada évoqué précédemment : le poids de la région occidentale du Mexique. Cependant, l'engouement pour la charreada se prolonge également plus au nord, dans les vastes territoires des États de Zacatecas, de Durango, de Sinaloa et Nuevo León. Dans cette géographie de la charreada, Jalisco occupe une place à part. C'est l'État du Mexique qui possède le plus grand nombre d'associations charras, le plus grand nombre de lienzos charros, les équipes qui ont le plus souvent remporté les championnats nationaux et, dit-on, les meilleurs charros individuels répartis dans les associations de beaucoup d'autres États mexicains. Il y aurait aujourd'hui plus de 900 associations charras enregistrées en sein de la Fédération mexicaine de Charrería, dont 116 dans le seul État de Jalisco ${ }^{20}$. Ce chiffre est en augmentation puisqu'elles étaient au nombre de 650 en 1993, même si cette évolution résulte plus d'une fragmentation des associations que d'une réelle croissance de la pratique. Jalisco se targue également d'avoir vu naître la première association de charros en 1921. Cela étant, comme pour toutes les données quantitatives du pays, la ville tentaculaire de Mexico impose sa masse. L'agglomération est le siège de la puissante Fédération nationale et compte environ trois cents lienzos où viennent s'entraîner et se divertir les adeptes ${ }^{21}$.

Au-delà des données quantitatives, derrière Jalisco, plusieurs États se disputent une place de choix pour mettre en valeur la haute importance de la culture charra. L'État d'Hidalgo prétend détenir le deuxième plus grand nombre d'associations du pays et n'est pas en reste pour affirmer que la charreada y est née, précisément dans la zone de Llanos de Apan « où les indigènes apprirent à monter à cheval au temps de la colonie et développèrent une série de techniques dans le maniement du gros bétail ${ }^{22}$. De même Frédéric Saumade, dans son enquête dans l'État Tlaxcala, rapporte que les charros locaux sont persuadés que la charreada est née sur leur terre, étant donné que les indigènes de cet État ont été les premiers à être autorisés à monter à cheval comme récompense de l'alliance passée avec Cortés contre l'empire Aztèque ${ }^{23}$. Bien entendu, les habitants de Jalisco ne laissent aucun doute planer quant à l'origine locale de la charreada qui aurait été inventée par les petits rancheros de la zone de Los Altos de Jalisco. L'État d'Aguascalientes fait lui aussi de nombreux efforts pour affirmer sur ses terres l'enracinement de la charreada. En 1998, la ville en partenariat

\footnotetext{
${ }^{20}$ C. PAlomar, « El papel de la charrería como fenómeno cultural », p. 92.

${ }^{21}$ F. SAUmade, Maçatl, p. 37.

${ }^{22}$ V. M. Rodríguez García, « La charrería, cuestión de enfoque », p 332.

${ }^{23}$ F. Saumade, Maçatl, p. 28.
} 
avec l'Association de Charros d'Aguascalientes s'est lancée dans l'ambitieux projet de rénovation de la Villa Charra, vaste complexe férial autour d'un lienzo charro qui fut terminé à temps pour accueillir en 2004 le Congreso y Campeonato Nacional Charro ${ }^{24}$. La Villa Charra "Profesor J. Refugio Esparza Reyes", du nom de l'initiateur de la construction du premier lienzo charro de la ville en 1948, est désormais un complexe de grande qualité, pouvant accueillir plus de 6.000 spectateurs et de très nombreux professionnels.

Au total, si l'on précise que la charreada est plus rare dans les États du Sud, on observe que son assise territoriale recoupe assez largement l'aire d'extension des corridas conventionnelles marquée par le poids de la zone centre et ouest du pays. Mais ici l'ouest et le centre du cœur territorial mexicain occupent une position différente au regard des pratiques taurines et de leur combinaison. Dans le champ de la charrería, l'occident mexicain fait valoir d'être le berceau de la pratique, la zone de plus grande ferveur et le principal foyer d'activité face à Mexico, qui doit d'abord son importance à son rôle de capitale du pays et à son imposante masse humaine. Dans le champ de la corrida, Mexico et son arène font valoir un prestige ancien et une incontournable centralité face à un occident mexicain riche en spectacles, en élevages et en férias de renom. Nous pensons que le champ tauromachique élargi à ses deux composantes exprime une rivalité interne au sein de chaque pratique et une rivalité entre les pratiques qui fait écho à la traditionnelle concurrence entre le centre du Mexique et l'occident mexicain, entre Mexico et Guadalajara, entre la première ville du pays et la deuxième, entre la capitale et la province. À travers des pratiques festives associées explicitement à l'idée d'une certaine représentation de l'identité nationale, cette rivalité touche à la nature au moins duale de la "mexicanité " ${ }^{25}$. L’une est portée par la charreada, marquée par une forme d'autochtonie exclusive pour laquelle la province, moins cosmopolite, tire souvent quelques avantages. L'autre est portée par la corrida, marquée par un lien culturel fondateur avec la péninsule Ibérique, pour lequel la capitale, souvent mieux intégrée à l'histoire des mondialisations, garantit la permanence. Dans cet équilibre, c'est la figure du charro, exclusivement mexicaine, qui se charge d'incarner le plus clairement la « mexicanité » et place ainsi l'occident mexicain au centre de cette représentation.

Les statistiques de l'INEGI sur l'utilisation des lieux de spectacles indiquent indirectement qu'un nombre non négligeable de plazas de toros sert à la réalisation de spectacles charros, en dépit des modèles scénographiques qui les distinguent théoriquement, et qu'à l'inverse, peu de lienzos charros, accueillent les acteurs de la tauromachie espagnole. En effet, un quart des spectacles charros recensés par l'INEGI ont lieu dans des plazas de toros, alors que moins de $5 \%$

\footnotetext{
${ }^{24}$ Cet événement qui a lieu chaque année dans une ville différente doit être considéré comme une sorte de grande finale des compétitions charras dont le titre laisse comprendre qu'il s'agit aussi d'une manifestation culturelle.

${ }^{25}$ Le terme, rarement utilisé en français, est d'usage courant au Mexique pour désigner ce qui relève de l'identité mexicaine : la mexicanidad.
} 
des courses de taureaux se déroulent dans des lienzos charros. Ne concluons pas pour autant à une évidente proximité culturelle des pratiques dont témoignerait l'utilisation du même lieu de spectacle. D'autres preuves d'une articulation ponctuelle des univers culturels sont plus probantes, telle que l'ouverture du spectacle de la corrida par un cavalier charro, parfois au côté d'un alguazil en costume espagnol ou, plus souvent, le remplaçant. Dans son Historia de la charrería de 1941, José Álvarez de Villar précise « que savoir toréer est une qualité inhérente à tout bon ranchero, la science tauromachique est comprise parmi les activités de celui qui se conçoit bon cavalier charro ${ }^{26}$. Au XIX ${ }^{e}$ siècle, les premières exhibitions de charros avaient lieu au cours des intermèdes des corridas et certaines personnalités de l'époque, tel le célèbre Ponciano Díaz, officiait selon les deux techniques ${ }^{27}$. Ángel Villatoro dit de celui qui est considéré comme le premier grand torero de l'histoire mexicaine " qu'il aimait utiliser le vêtement du charro et exécuter les exercices du jaripeo, dans lesquels il excellait $»^{28}$. $\mathrm{Au} \mathrm{xx}^{\mathrm{e}}$ siècle, nombre de lienzos charros ont, par la suite, servi à accueillir des courses de taureaux : le Rancho Grande de la Villa propriété de la Asociación de Charros de la Villa, le Rancho La Tapatía propriété de la Unión de Charros Metropolitanos, Le Rancho El Hormiguero propriété de la Agrupación de Charros, le Rancho El Charro, appartenant à la très influente Asociación Nacional de Charros $^{29}$. Les témoignages d'une utilisation régulière datent du milieu des années 1950 et attestent d'une afición des charros pour la corrida qui n’hésitent pas à en programmer eux-mêmes.

L'inverse est encore plus remarquable puisque l'INEGI prétend qu'un quart des spectacles charros ont lieu dans des arènes. Malheureusement cette statistique ne donne pas davantage de détails sur le type de spectacle précis dont il s'agit. Faut-il y voir la preuve que la charreada s'oriente par l'intermédiaire scénique des arènes de corrida vers une ouverture à un public plus large ? Quoi qu'il en soit, à en croire l'analyse de Cristina Palomar l'évolution de la charreada sur le modèle des sports-spectacles organisés en compétitions et en exhibitions payantes, bouleverse les codes habituels liés à une culture du club fermé. L'auteur déclare sur ce point qu’à se définir comme un sport, la charrería cesse d'être quelque chose de traditionnel pour devenir un rituel de la modernité, un rituel sportif qui produit des vainqueurs et des vaincus à travers un système qui n'est plus seulement symbolique, mais réel et matériel $^{30}$. Autrement dit, il semble que la pérennité du mythe du charro comme symbole identitaire de la nation idéalisée passe aujourd'hui en partie par cette adaptation.

\footnotetext{
${ }^{26}$ Cité par F. López Izquierdo, Los Toros del Nuevo Mundo, p. 204.

${ }^{27}$ E. Guarner, Historia del toreo en México, pp. 42-43 ; F. Saumade, Maçatl, p. 38.

${ }^{28}$ A. Villatoro, Antología taurina mexicana, p. 127. L'auteur ne donne ici aucune précision sur la nature des exercices regroupés sous le terme de jaripeo.

${ }^{29}$ F. Garibay Anaya et M. Luna Parra, México se viste de luces, p. 58.

${ }^{30}$ C. Palomar, «El papel de la charrería como fenómeno cultural », p. 90.
} 


\section{JARIPEOS PROFESSIONNELS ET RODÉO NORD-AMÉRICAIN :} COUSINS MONTÉS L'UN CONTRE L'AUTRE?

Selon Frédéric Saumade, la diffusion géographique du jaripeo se limite au milieu rural ou suburbain de quelques États du centre, de l'ouest et du sud du pays à forte composante indienne : Guerrero, Michoacán, Guanajuato, Colima, Veracruz, Oaxaca, Chiapas, jusqu'au Guatemala ${ }^{31}$. On peut observer, par rapport à la localisation des courses de taureaux mineures dans les régions à forte composante indienne, que l'État du Yucatán, où elles sont nombreuses, est absent de cette liste comme si les deux modalités populaires du jeu taurin s'évitaient. D'après ce que nous savons des courses de taureaux populaires du Yucatán, il n'est pas rare de voir des taureaux montés dans la séquence participative du spectacle ce qui pourrait plutôt signifier qu'ici le jaripeo n'est pas séparé de l'action de toréer. On retrouve l'idée, déjà relevée, d'une relative indistinction des techniques taurines dans nombre de pratiques à caractère participatif, ou tout au moins, d'une plus grande perméabilité des registres. Ce détail suffit à rappeler que l'animal monté est aussi toréé de façon élémentaire par des hommes à pied, point important qu'il ne faut pas sous-estimer en dépit de l'originalité technique de la monte ${ }^{32}$. Le jaripeo rural est aujourd'hui en recul ou plutôt, comme le montre Frédéric Saumade, en mutation sous la forme du jaripeo ranchero professionnel. Cette mutation qui passe par une mise en spectacle beaucoup élaborée que le jaripeo rural est fortement influencée par le rodéo nord-américain.

Le rodéo nord-américain s'est diffusé dès la fin des années 1960 dans les États du Nord du Mexique en contact avec les États-Unis. Dans les années 1980, plusieurs entreprises mexicaines commencent à programmer des spectacles de rodéos professionnels, d'abord dans les États septentrionaux de Chihuahua et de Cohuila, puis plus au sud dans des villes comme Querétaro et San Luis Potosí. Aujourd'hui, comme l'affirme Cuernos Chuecos, une importante entreprise de rodéo du Mexique, il existe des spectacles dans toutes les grandes villes. Les spectacles de cette compagnie, et elle n'est pas la seule à faire ainsi, annoncent les rodéos sous l'appellation « rodeo estilo americano ». Juan Carlos Hachity, principal promoteur de cette entreprise, s'en justifie ainsi :

Le rodéo a commencé au Mexique par la monte des taureaux. Il s'est professionnalisé aux États-Unis grâce aux infrastructures et aux sponsors. C'est aujourd'hui le sport extrême le plus spectaculaire au monde. La différence c'est que nous sommes au Mexique. Il s'agit d'un show que nous faisons pour les Mexicains. Si nous l'appelons rodéo "style américain », c'est en raison des règles en vigueur aux États-Unis ${ }^{33}$.

\footnotetext{
${ }^{31}$ F. Saumade, Maçatl, p. 32 ; ID., « Du taureau au dindon. Domestication du métissage ». Dans cet article l'auteur propose une liste avec quelques différences mineures : Morelos, Mexico et le DF, Hidalgo, Puebla, Veracruz, Guerrero, Michoacán, Colima, Guanajuato et Oaxaca.

${ }^{32}$ Nous ferons le même type de remarque lors de l'analyse du turupukllay andin.

${ }^{33}$ Propos recueillis par le journal en ligne Noti-Arandas le 4 janvier 2007, disponible sur le site Internet : http://notiarandas.com/noticia.asp?id=7071 [réf. du 10 juin 2007].
} 
Le jaripeo "professionnel » dont le sens du show s'inspire désormais du rodeo estilo americano dans sa musique et ses codes vestimentaires, n'en conserve pas moins avec fierté ses particularismes : l'utilisation des éperons à crochets et l'absence de chronomètre alors que le rodeo américain utilise des éperons sans crochets et limite la monte à huit secondes. Ainsi deux spectacles de monte coexistent. Ils partagent des principes communs et une mise en scène très proche, mais ne sont pas confondus. Le jaripeo demeure plus rural et s'enracine dans les régions où le jeu paysan était prisé. Le rodeo estilo americano est plus urbain dans ses implantations. Dans cette transformation l'animal monté n'est plus toréé.

Mais le rôle des États-Unis ne se limite pas à l'influence exercée par le modèle dominant sur les évolutions du jaripeo et du rodéo mexicain. Plutôt, cette influence ne passe pas seulement par l'adoption des codes nord-américains, mais aussi par le développement de l'industrie américaine du rodéo en terres mexicaines, et ailleurs comme on le verra. S'il est un élément quelque peu novateur, permettant d'enrichir les travaux qui nous précèdent sur les jeux taurins mexicains, c'est bien cet aspect-là de la question. En 2006, la ville de Chihuahua est choisie comme étape mexicaine du Pro Bull Riders (PBR), qui est à l'heure actuelle le principal circuit mondial de spectacle de rodéo fondé spécifiquement sur la monte des taureaux. Ce circuit est organisé depuis les États-Unis où le PBR a son siège, dans l'État du Colorado. La répartition des 11 villes étapes mexicaines du PBR témoigne d'une localisation septentrionale dominante en continuité avec l'espace nord-américain du rodéo (voir cartes 13 et 14, pp. 321 et 336) ${ }^{34}$. Les compétitions ont généralement lieu dans les arènes de corrida ou dans les lienzo charros. Lizzette Solorio Martínez, directrice de la Oficina de Convenciones et Visitantes de l'État de Chihuahua, ne cache pas sa fierté qu'une "petite » ville comme Chihuahua puisse participer au PBR et attribue ce succès au formidable engouement que connaît le rodéo dans la région :

C'est un grand honneur de pouvoir faire venir le PBR dans notre État [...] On est désormais en compétition avec des villes importantes comme Guadalajara et Monterrey, mais la vraie culture vaquera est à Chihuahua ${ }^{35}$.

Il ne fait guère de doute que la position septentrionale de l'État de Chihuahua à proximité des États-Unis et son fort attachement à la tradition de l'élevage extensif soit pour notre interlocutrice un gage d'authenticité de la culture vaquera. Il existe donc une superposition de trois systèmes possédant chacun une emprise territoriale bien définie. Le jaripeo ranchero «professionnel» repose sur une tradition rurale ancienne en pleine modernisation sous l'influence des codes américains. Frédéric Saumade parle à son sujet d'un « retour d'acculturation ", à l'image d'un retour de flamme, afin d'éclairer le parcours inverse du transfert culturel de la monte des taureaux de l'Amérique du Nord vers le Mexique qui conserve néanmoins de forts particularismes. Le rodeo estilo ame-

\footnotetext{
${ }^{34}$ Les villes étapes du PBR sont : Saltillo, Monterrey, Hermosillo, Reynosa, Durango, Aguascalientes, Hidalgo de Parral, Torreón, Juárez, León, Lagos de Moreno.

${ }^{35}$ El Diario Lealtad de Chihuahua, 20 août 2006.
} 
ricano produit par des entreprises mexicaines reproduit à l'identique ce qui fait le succès du rodéo américain en insistant, non sans quelques paradoxes, sur la dimension extrême du sport et sur le caractère familial du spectacle, enrichi par un sens du show visant à combler tous les publics. Enfin, le dernier est un sportspectacle d'exportation produit par les États-Unis qui fait circuler les acteurs du haut niveau de la monte sur la base territoriale d'un réseau professionnel de grandes villes mises en concurrence. Ce que perd le jaripeo rural dans cette évolution c'est l'ambiguïté technique d'un jeu qui cumule la monte des taureaux et l'action élémentaire de toréer qui se voit définitivement marginalisée par les codes nord-américains du spectacle de la monte.

\section{II. - L'ESPACE TAUROMACHIQUE ÉTASUNIEN : RODÉO, CHARREADA ET «CORRIDA SOFT»}

S'il est une région généralement exclue des terres taurines, c'est bien l'Amérique du Nord $^{36}$. Entre l'Amérique anglo-saxonne et l'Amérique latine, la juxtaposition de ce qui est donné comme deux civilisations et deux aires culturelles fondamentalement différentes suffit à tracer la limite au-delà de laquelle, en ce qui concerne la tauromachie, le regard n'est pas même porté. L'intégration du rodéo à la problématique tauromachique replace cet espace au cour de la réflexion. Refuser les délimitations qui découpent traditionnellement l'espace par aire culturelle ou par la géographie ethnocentrique d'une seule pratique, nous permet de recoudre l'espace tauromachique transatlantique dans sa cohérence profonde. Rappelons que le territoire actuel des États-Unis s'est historiquement agrandi, à partir du début du XIX ${ }^{\mathrm{e}}$ siècle, de possessions françaises et mexicaines : l'achat de l'immense Louisiane (plus de 2 millions de $\mathrm{km}^{2}$ ) à la France en 1803, l'annexion du Texas en 1845 qui s'était séparé du Mexique en 1836 à la suite d'un soulèvement soutenu par les États-Unis, puis l'acquisition au détriment du territoire mexicain, après deux années de guerre de 1846 à 1848, de l'actuelle Californie, Arizona, Nevada, Colorado, Nouveau-Mexique. Notre hypothèse régionale est de considérer que les pratiques tauromachiques, et pas seulement le rodéo nord-américain, contribuent à faire de la zone frontière plus une couture culturelle qu'une coupure territoriale, sans en effacer pour autant l'évidence des clivages. Les jeux taurins nord-américains, réduits dans l'imaginaire collectif à un rodéo aux contours flous, sont en réalité enrichis de la charreada pratiquée par une importante communauté mexicaine et de courses de taureaux, en Californie, adaptées à une législation sur la protection des animaux interdisant toute effusion de sang.

\section{RODÉO ET BULL RIDING À L'ASSAUT DU CONTINENT}

Aux États-Unis, la pratique du rodéo est bien plus qu'un sport, puisqu'elle est aussi une exaltation du cow-boy et de l'Ouest américain, deux ferments

\footnotetext{
${ }^{36}$ Une partie des informations sur les États-Unis a pu être approfondie grâce à l'obtention du programme ANR « Torobullmexamerica » que nous menons en partenariat avec Frédéric Saumade depuis 2008.
} 

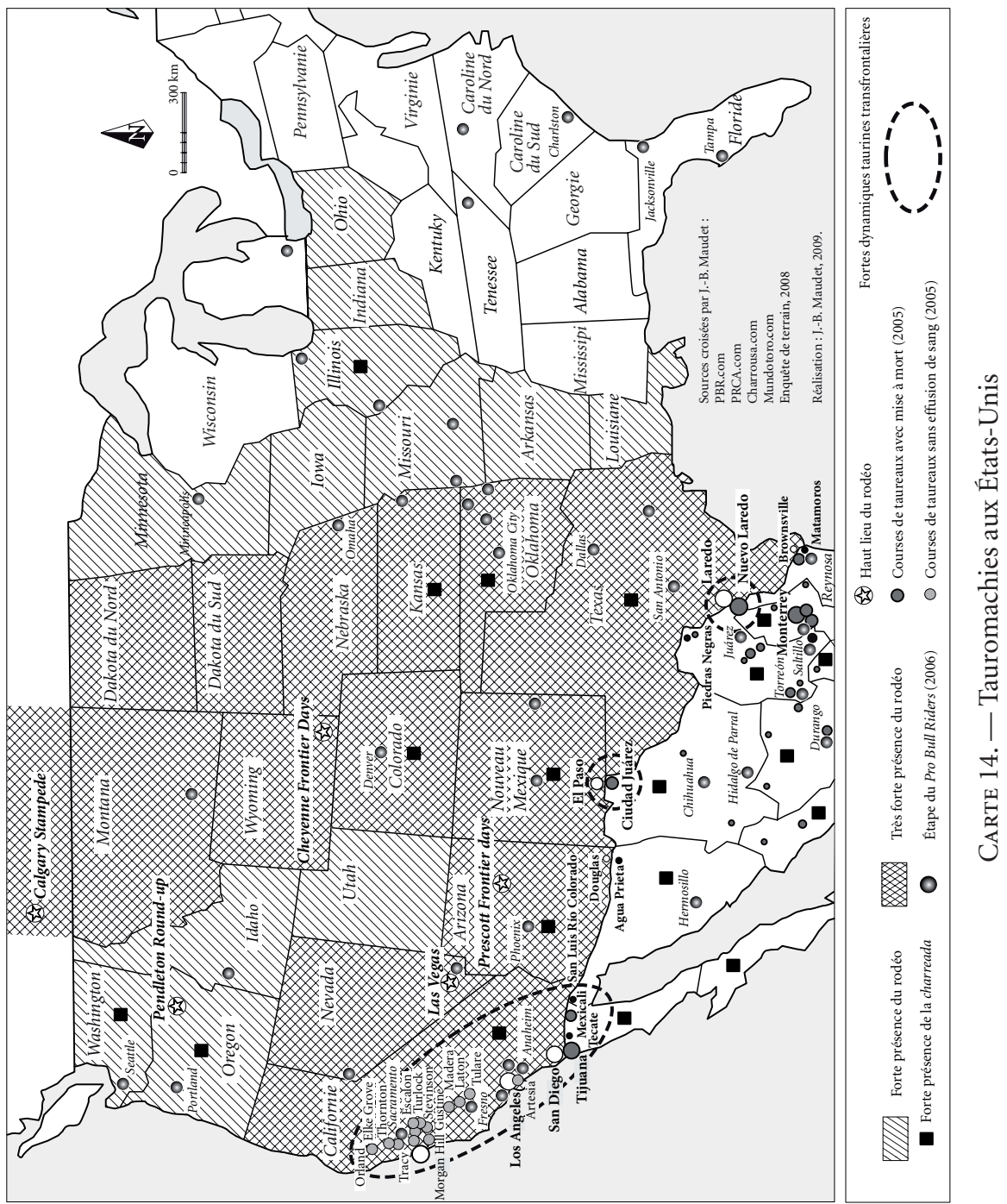

○

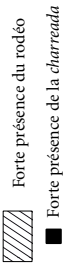


identitaires de la nation. L'historien Philippe Jacquin a montré comment le mythe de l'Ouest se développe dans une Amérique coloniale agraire qui se désagrège au cours du $\mathrm{XIX}^{\mathrm{e}}$ siècle, bousculée par une rapide expansion économique, humaine et spatiale :

L'histoire du cow-boy s'enracine dans le plus profond de la Conquête des Amériques. Il naît à la frontière des deux expériences coloniales, l'hispanique et l'anglo-saxonne, et grandit dans leurs affrontements et le succès de l'Amérique anglo-saxonne du $\mathrm{XIX}^{\mathrm{e}}$ siècle, pour marquer enfin l'effacement de l'Amérique métissée. L'élaboration du mythe du cow-boy a été menée à la fois comme une quête d'identité nationale et comme une lutte symbolique pour imposer un nouveau système de valeurs dans un pays en plein bouleversement, un véritable processus d'américanisation ${ }^{37}$.

Évidemment, ce seul mythe ne suffit pas à résumer le processus de construction identitaire de la nation. En outre, le cow-boy, à l'image de tout mythe institué est aussi le repoussoir d'un héroïsme suranné, raillée sur son propre sol. Avec des différences, remarquons d'emblée que le Mexique et les États-Unis possèdent chacun une figure tutélaire de l'imaginaire national qui relève $\mathrm{du}$ monde de l'élevage bovin et des jeux taurino-équestres.

La géographie du rodéo américain est d'une appréhension complexe en raison d'un nombre important de disciplines, de circuits et d'associations professionnelles qui, comme dans de nombreux sports aux États-Unis, ne sont pas unifiés. Fixons quelques repères pour comprendre les traits généraux de son organisation spatiale. L'organisation professionnelle la plus ancienne et la plus importante est la PRCA (Professional Rodeo Cowboys Association), prenant la suite de la Cowboys Turtle Association créée en 1936 à l'issue d'un mouvement de protestation des cow-boys désirant défendre leur droit face aux entrepreneurs de spectacles. Son siège est situé à Colorado Springs au sud de Denver dans l'État du Colorado. La PRCA coordonne différents événements et circuits professionnels dont le Wrangler Tour, le Dodge National Circuit Finals Rodeo subdivisé en 12 circuits géographiquement séparés et le Heartland Series encourageant la professionnalisation des rodéos organisés dans de petites villes. L'assise territoriale du rodéo correspond globalement à l'Ouest, au sens américain du terme, qui démarre dans les Grandes Plaines, mais tout le territoire est concerné par sa pratique (voir carte 14). Les États où les rodéos sont les plus nombreux suivent deux axes : l'un d'orientation est-ouest qui correspond à la frontière avec le Mexique (Californie, Nevada, Arizona, Nouveau-Mexique, Texas), l'autre d'orientation méridienne qui prolonge le premier vers le Nord, le long du piémont oriental des Rocheuses et des Grandes Plaines (Lousiane, Texas, Oklahoma, Kansas, Colorado, Nebraska, Wyoming, Dakota, Montana et l'Alberta au Canada). Ajoutons la Floride, quelque peu séparée de cet ensemble,

\footnotetext{
${ }^{37}$ P. JACQUIN, Le cow-boy. Un américain entre le mythe et l'histoire, p. 219.
} 
mais très amatrice du genre. Dans ces États, les rodéos, en plus d'être des spectacles professionnels, sont parfois une pratique semi-récréative de la sphère socio-économique du ranch ayant lieu dans la continuité des nécessités de l'élevage (marquage, castration, remue du bétail...) ${ }^{38}$.

Aujourd'hui la PRCA compte environ 7.000 compétiteurs, organise quelque 650 rodéos annuels et répartit des gains en compétitions à hauteur de 40 millions de dollars issus de la billetterie, des droits de télévision et des nombreux sponsors (Wrangler, Dodge, Coors, US Smokless Tobacco Co., Justin Boot) ${ }^{39}$. En 1987, d'après James Hibdon du département économique de l'Université de l'Oklahoma, la PRCA était présente dans 41 États américains, comptait 5.342 compétiteurs affiliés (soit $85 \%$ des professionnels) et organisait 637 rodéos $^{40}$. Au début des années 1980, le nombre de spectateurs était estimé à 14 millions et dépasse aujourd'hui les 24 millions $^{41}$. La PRCA organise des compétitions qui proposent tous les exercices de la pratique classique du rodéo. Les adeptes du rodéo comptent quelques grands rendez-vous tels que le Cheyenne Frontier Days (Wyoming) célébré depuis 1888, le Prescott Frontier Day (Arizona) qui date aussi de 1888 et a déposé la marque du " plus vieux rodéo du monde » ("The World's Oldest Rodeo »), le Pendleton Round-Up (Oregon) célébré depuis 1910, le Calgary Stampede (Alberta, Canada) depuis 1912, le California Rodeo Salinas (Californie) et le San Antonio Stock Show and Rodeo (Texas). Le rendez-vous le plus important pour les professionnels est le Wrangler National Finals Rodeo, conçu comme une grande finale annuelle réunissant les compétiteurs qui ont gagné le plus d'argent pendant l'année. Dans le monde du rodéo, l'argent apparaît bien comme l'unité de mesure la plus objective du talent. D'abord organisé à Oklahoma City, cette phase finale se tient à Las Vegas depuis 1985. Las Vegas inscrit incontestablement la tradition dans une sorte d'hypermodernité sociale et économique à l'américaine. Tous ces grands rendez-vous annuels ont la saveur d'une fête nationale où l'hymne américain est chanté et le drapeau glorifié. Mieux, les rodéos se déroulent souvent sur un arrière-fond commémoratif d'appropriation territoriale, actant de la progression de la frontier et de l'aboutissement de la conquête de l'Ouest.

L'une des évolutions récentes les plus marquantes du rodéo nord-américain est la création, en 1992, du Pro Bull Riders (PBR) que nous avons évoquée à propos du Mexique. Ainsi que nous l'avons remarqué, cette organisation donne corps professionnellement à un rodéo fondé uniquement sur la monte des taureaux, modalité qui n’a cessé de gagner en importance depuis la fin des années 1970. Le PBR, en pleine croissance, compte aujourd'hui 700 compétiteurs affiliés, répartit des gains à hauteur de 10 millions de dollars et possède des filiales au Canada, au Mexique, au Brésil et en Australie. La finale se tient depuis 1994

\footnotetext{
${ }^{38}$ E. A. LaWrence, Rodeo. An anthropologist looks, p. 84. Pour une analyse poussée des diverses terres de ranch dans l'Ouest américain voir P. STARRs, Let the Cowboy Ride, en particulier au sein des États de New Mexico, Texas, Nebraska, Wyoming et Nevada.

${ }^{39}$ PRCA, 2006.

${ }^{40}$ J. Hibdon, « The economics of rodeo cowboys », p. 239.

${ }^{41}$ E. Atwood Lawrence, Rodeo. An anthropologist looks, p. 4 ; PRCA, 2006.
} 
à Las Vegas qui est de ce fait devenue en quelques années le centre incontesté du rodéo nord-américain professionnel, national et international. Cette activité ne fait qu'accroître le potentiel d'attraction de cette capitale du tourisme, du jeu et du divertissement. En 2003, face au succès du Pro Bull Riders, la PRCA a réagi en créant, elle aussi, une compétition de rodéo exclusivement fondée sur la monte des taureaux, le Xtreme Bulls Tour, confirmant l'importance croissante acquise par cet exercice parmi ceux du rodéo traditionnel. Sa professionnalisation aux États-Unis s'est considérablement accrue ces vingt dernières années. Sur la base d'une modalité technique unique, cette professionnalisation pose les fondements d'un sport panaméricain fortement associé à la culture des États-Unis lui assurant un leadership économique sur la pratique. On a vu, au Mexique, dans quelle mesure cette évolution participe d'un processus complexe de néoacculturation et de résistance des modèles autochtones.

Mais les évolutions du rodéo nord-américain ne s'arrêtent pas là. À partir des années 1980, émerge une nouvelle forme de jeux taurins jusqu'alors marginale qui, d'une certaine manière, referme la boucle des transformations américaines de la tauromachie. Reprenons le parcours de ces transformations. En faisant passer au premier plan la monte des taureaux, les bull riders deviennent les nouveaux héros et la pièce maîtresse du rodéo nord-américain. Avec eux, gagne en importance la figure des rodeo clowns. Les rodeo clowns sont des cow-boys débraillés, grimés à la manière d'un clown de cirque, dont le rôle est de protéger les bull riders de la charge et des ruades des taureaux lorsque ceux-ci sont désarçonnés. Autrefois, dans le cadre des rodéos classiques, ils assuraient également des intermèdes entre les différentes épreuves en offrant des tours comiques et participaient du caractère familial annoncé par le show. Les rodeos clowns ont toujours bénéficié du plus grand respect de la part des cow-boys dans la mesure où leur habileté permet d'éviter les accidents. L'évolution professionnelle du rodéo nordaméricain est allée dans le sens d'une spécialisation des rôles entre le rodeo clown comique et le rodeo clown protecteur. Chaque année, la PRCA les met à l'honneur, en invitant les meilleurs rodeo clowns à participer aux grandes finales de rodéos et récompense le plus performant d'entre eux par un trophée individuel.

Mais le PBR va désormais plus loin dans cette démarche en organisant une compétition qui leur est exclusivement réservée, le World Bullfighting Championship, où les rodeo clowns, cette fois seuls en piste, disposent de 70 secondes pour réaliser des acrobaties avec un taureau, des sauts, des contacts et des esquives en utilisant un tonneau. Les spectacles de ce genre se développant, les rodeo clowns préfèrent être appelés bullfigthers et la tenue de certains d'entre eux s'éloigne de plus en plus de celles de leur congénère burlesque. Le jeu n'est alors pas très éloigné, dans son principe, de n'importe quel autre lâcher de bovins où il s'agit simplement de défier l'animal, au corps à corps, dans une arène. L'individualisation du jeu nécessite un animal adapté qui ne soit pas seulement le taureau choisi pour la force de ses ruades. Pour être plus spectaculaire, cet exercice a besoin de taureaux qui chargent l'homme parmi lesquels figurent des brahmas et surtout des taureaux de corridas achetés soit au Mexique, soit aux éleveurs californiens d'origine açorienne. 
Comment interpréter cette nouvelle transformation du rodéo qui, en passant par la valorisation exclusive de la monte, place au centre du jeu les auxiliaires, pour finalement se rapprocher des composantes élémentaires des courses de taureaux du vieux continent? Faut-il y voir la promotion d'une parodie devenue sérieuse ou un retour aux sources d'un affrontement minimal entre l'homme à pied et le taureau ? L'analyse de cette évolution témoigne de la complexité des processus de transformation des pratiques taurines qui, les contenant toutes en puissance, confirme l'importance des choix culturels dans les modalités d'affrontement privilégiées. Elle témoigne aussi d'une ambiguïté permanente de la tauromachie entre les exercices académiques et les exercices bouffons, et d'une articulation originale entre la culture savante et la culture populaire. Certaines figures historiques du rodéo, tout comme de la corrida, témoignent de cette ambiguïté structurelle.

Dans l'histoire du rodéo, certains rodeo clowns ont acquis une popularité d'envergure nationale à l'instar de Slim Pickens, de son vrai nom Louis Burton Lindley, né en 1919 en Californie, à l'instar de Quail Dobbs, né en 1941 au Texas ou encore de Flint Rasmussen il y a quelques années. Le parcours de ce dernier est intéressant si l'on observe qu'il s'est éloigné des compétitions de la PRCA pour privilégier celles du Pro Bull Riders où son rôle se trouve accru et mieux valorisé par la spécialisation qu'il propose. Ces personnages ont toujours eu une place ambivalente dans la mesure où ils sont à la fois des éléments salvateurs indispensables au bon déroulement du spectacle et, dans le même temps, des figures parodiques du cow-boy idéal. En la personne de Slim Pickens, la parodie ne peut être poussée plus loin, puisqu'il s'agit de l'acteur qui, dans le film de Stanley Kubrick, Docteur Follamour, chevauche à la manière des bull riders non pas un taureau, mais la bombe atomique de la scène finale. L'irrévérence envers le mythe du cow-boy, chez un cinéaste aussi subtil que Stanley Kubrick, peut-elle être plus explosive ? D'une certaine façon, la tauromachie espagnole a également produit des figures subversives à travers le toreo comique qui, loin de n'être qu'une dérision de la corrida, a également formé des toreros sérieux et inventé des passes reprises dans le registre académique. Dans le détail, la passe du pendule qui deviendra la pedresina (du nom du matador Pedrés), la manoletina mise à l'honneur par Manolete ou encore le saut de la grenouille de «El Cordobés », qui lui n'est pas entré dans le registre classique, existaient dans le toreo comique. Aux États-Unis, le passage des rodeo clowns aux bullfighters exprime ce va-et-vient ambivalent de la culture taurine d'un registre à l'autre. Mieux, sur le plan des relations culturelles transatlantiques, il exprime aussi une rivalité latente entre tauromachie ibérique et tauromachie anglo-saxonne, puisque ce clown devenu bullfighter porte le même nom que le héros de la tauromachie espagnole, écornant dans le show à l'américaine sa gravité rituelle. Et par une proximité frontalière plus immédiate, ne raille-t-il pas aussi la tauromachie du voisin mexicain?

Il est d'ailleurs un jeu pratiqué par les rodeo clowns qui fait explicitement référence à cette rivalité : le poker mexicain. Le jeu consiste à lâcher un taureau dans l'arène où se tiennent des joueurs de poker parfaitement immobiles, assis à une table. Le principe ludique est le même que celui mis en scène par la figure de Don Tancredo en Espagne, ce clown blanc, immobile, dressé sur un tabouret 
de cirque, que le taureau frôle sans le voir. Le rodeo clown du poker mexicain doit attirer l'animal vers la table pour qu'il la renverse, provoquant l'hilarité du public, le gagnant étant le dernier « mexicain » à rester assis. Le bullfighter et le bullfighting, appelé aussi free style bullfighting, désignent donc, dans l'univers taurin de la corrida aux États-Unis, le matador et la course de taureaux, alors qu'ils désignent, dans l'univers du rodéo, le rodeo clown et la nouvelle épreuve qui lui est désormais réservée. Il est alors tentant de voir dans ce rodeo clown bullfighter une figure taurine qui tout à la fois subvertit le héros de la tauromachie européenne et s'en rapproche. Il est trop tôt pour savoir quel sera le statut définitif du bullfighter aujourd'hui en pleine évolution, mais l'atténuation de sa dimension comique dans le cadre d'un jeu autonome, face à des taureaux de combat, qui remplacerait la valorisation du rire par celle de la prise de risque extrême, témoignerait sans équivoque, de la naissance d'un nouveau sport tauromachique.

Il est difficile d'affirmer que ces parallèles transatlantiques soient porteurs d'une signification tout à fait claire, mais on ne peut omettre de mentionner que l'autre façon de désigner la tauromachie comique en Espagne est la charlotada, inspirée du personnage inventé par l'acteur américain Charlie Chaplin au début du $\mathrm{xx}^{\mathrm{e}}$ siècle. La première " charlotade " proprement dite, où l'on observe un torero en costume de charlot, serait une invention barcelonaise de 1916, que l'on doit à l'entrepreneur de spectacles Eduardo Pagès ${ }^{42}$. Une nouvelle fois Barcelone s'illustre par son anticonformisme taurin. Cette formule eut un grand succès dans toute l'Espagne et s'exporta au Mexique. Son plus digne représentant mexicain fut Mario Moreno Reyes "Cantinflas » né en 1911. En tant que torero comique, il se présenta dans de nombreuses arènes d'Amérique latine et, par ailleurs, fut un grand acteur de comédie pour lequel Charlie Chaplin (le vrai), auquel il était comparé, avait la plus haute estime. Sans vouloir à tout prix forcer la logique de ces significations croisées entre rodeo clown et charlotada, il est manifeste que l'interface entre le Mexique et les États-Unis s'est traduite par une influence de la corrida sur la formation du rodéo nord-américain. Le parcours des transformations laisse apparaître le rôle clef de la frontière dans les processus de différenciation. Le rodéo s'épanouit aux États-Unis par l'acquisition territoriale des terres mexicaines septentrionales d'élevage extensif où il est ensuite transformé et mis en sport à la sauce américaine (entendons étasunienne). La figure du torero et du torero clown, que l'on retrouvera d'ailleurs au Brésil, n'a donc cessé d'accompagner ces métamorphoses, de la corrida aux jeux taurins mexicains, des jeux taurins mexicains au rodéo nord-américain, du rodéo nord-américain à l'individualisation du bull riding, et aujourd'hui du bull riding à l'autonomie du free style bullfighting qui par ses propriétés formelles élémentaires se rapproche à nouveau de la corrida. Les pratiques taurines de la Mexamérique n'ont cessé d'évoluer de façon interdépendante.

\footnotetext{
${ }^{42}$ R. Bérard (dir.), La Tauromachie. Histoire et dictionnaire, p. 909.
} 


\section{LA CHARREADA}

OU LA NOSTALGIE MEXICAINE EN PAYS COW-BOY

En lien avec l'importante communauté mexicaine des États-Unis, la pratique de la charreada manifeste un attachement au pays d'origine et à ses traditions, même quand il ne s'agit pas, pour beaucoup de ses adeptes, du pays natal ${ }^{43}$. Sur les 900 associations affiliées à la Fédération nationale des Charros du Mexique, une centaine est située aux États-Unis. La Fédération des Charros de Los Angeles dit posséder le plus grand nombre d'associations après les États de Jalisco et d'Hidalgo. Aux États-Unis, la Californie et le Texas seraient les deux États qui en comptent le plus, auxquels viennent s'ajouter l'Arizona, le Nouveau-Mexique et l'État pourtant lointain de l'Illinois en lien avec l'importante communauté mexicaine qui y réside. Les États frontaliers américains et mexicains constituent donc une zone de chevauchement du rodéo nord-américain, du nord vers le sud, et de la charreada, du sud vers le nord. Mais cette frontière n'est évidemment pas symétrique. En terre mexicaine, le rodéo nordaméricain pénètre par les voix de la transformation du jaripeo professionnel, par la croissance de spectacles qui en sont l'exacte réplique et par la formation d'un réseau transnational de compétitions. En terre nord-américaine, la pratique de la charreada s'est enracinée avec l'immigration mexicaine, qui en respecte le plus possible les codes et l'étiquette même si sont interdits les éperons à crochets et, désormais dans la plupart des États concernés, les manganas, l'utilisation du lasso pour piéger les pouliches au galop (Arizona, Californie, Florida, Illinois, Maine, Nebraska, New Mexico, Oklahoma, Texas). Aux ÉtatsUnis, la professionnalisation de la charreada date des années 1970, en lien avec le mouvement de revitalisation de l'héritage hispanique mené par les Chicanos $^{44}$. Il existe désormais une Union des associations charras qui organise son propre circuit de compétitions couronnées par le Congreso y Campeonato Nacional de Norteamérica, pendant étasunien du Congreso y Campeonato Nacional de México dont nous avons déjà parlé. Comme au Mexique, la compétition est abrégée sous l'appellation « Le National ». En 2003, « Le National » nord-américain s'est déroulé dans la ville frontalière d'El Paso au contact de Ciudad Juárez, sous le titre «Nacionales del Campeonato Charro José Cuervo Tradicional ». José Cuervo Tradicional est une marque de tequila qui sponsorise l'événement. Ce sponsor qui mise sur l'image du Mexique authentique a notamment permis, en 2003, l'inauguration d'un nouveau lienzo charro de grande qualité à El Paso, le Rancho Charros 100X. En 2007, c'est la ville frontalière de Tecate qui est choisie pour « Le National » mexicain, confirmant une sorte de bornage du territoire de la charreada qui utilise sciemment la frontière comme un élément d'intégration.

C'est que les compétitions de charreadas sur le sol étasunien ne fonctionnent pas en vase clos puisque les meilleures équipes du « National » nord-américain

\footnotetext{
${ }^{43}$ K. Mullen Sands, Charrería mexicana.

${ }^{44}$ O. NÁjera-Ramírez, « Engendering Nationalism ».
} 
ont l'honneur de participer au « National » mexicain. Les équipes défilent alors avec le drapeau américain et l'habit charro, revendiquant fièrement une double appartenance identitaire. Il n'y a aucune contradiction à cela, mais simplement l'expression d'une fierté d'être citoyen américain et mexicain de cœur. Ce détail concrétise une propriété du fonctionnement de l'identité qui n'est jamais parfaitement identique en fonction des lieux et des contextes où elle se manifeste. L'existence d'une communauté mexaméricaine qui puise notamment dans la charreada sa visibilité se traduit également par des formes de jumelages transfrontaliers entre associations. Un bon exemple de ce phénomène est le jumelage qui existe depuis 1988 entre l'Association des Charros de San Antonio (Texas) et celle des Charros de San Juan del Río dans l'État du Querétaro ${ }^{45}$. Il se traduit par un voyage annuel d'un lienzo charro à l'autre où sont resserrés les liens d'amitié par la charreada. Des confrontations sont organisées, on y danse et on y torée en habit de charro, confirmant une nouvelle fois la porosité des passions entre la charreada et la tauromachie espagnole. Les charros de San Juan del Río sont fiers d'être en lien avec une puissante association qui a souvent gagné sa place au « National» mexicain et de leur côté, les charros de San Antonio sont fiers d'être en lien avec l'une des plus anciennes associations mexicaines, puisqu'il s'agirait de la plus vieille association du pays après celle des charros de Jalisco fondée en 1921. En 2007, lors du voyage des charros de San Antonio à San Juan del Río, Raúl Gaona, le président honoraire de l'association texane, annonçait la création d'un club d'histoire du Mexique « visant à récupérer culturellement ce qui, géographiquement, ne peut pas l'être ${ }^{46}$. Derrière la charreada et l'expression du sentiment d'appartenir au Mexique, affleurent parfois les fantasmes d'un irrédentisme.

Aux États-Unis comme au Mexique, charreada et rodéo nord-américain, qui possèdent certains exercices identiques et une origine commune ne se mélangent pas plus que ne se confondent aujourd'hui le charro et le cow-boy. Ils ont pourtant coexisté en la personne du vaquero, un travailleur du bétail antérieur à la construction idéologique des cavaliers nationaux. Il est certain que l'acquisition des terres mexicaines par les colons américains qui précèdent l'agrandissement territorial des États-Unis est une inflexion majeure dans ce processus de différenciation. De 1830 à 1848, les conflits au Texas auraient fait retourner à l'état sauvage $80 \%$ des cent mille têtes de bétail qu'il abritait, ouvrant sur toute la frontière une période de banditisme et de troubles sociaux autour de l'appropriation des bêtes ${ }^{47}$. Le terme de « cow-boy » apparaît dans ce contexte pour désigner d'abord des hors-la-loi, parmi lesquels les colons américains dénoncent, bien entendu, les Mexicains et des Indiens. Philippe Jacquin retrace ainsi l'évolution du terme :

En fait, le mot cow-boy n'est pas né au Texas. [...] En Irlande, vers l'an 1000, les bergers sont appelés « cow-boys » et le terme demeure jusqu'à

\footnotetext{
${ }^{45}$ Informations disponibles sur le site Internet de l'association de charros de la ville de San Antonio : http://www.sanantoniocharros.org [réf. du 10 juin 2007].

${ }^{46}$ El Sol de San Juan del Río, 30 juillet 2007.

${ }^{47}$ P. JACQUIN, Le cow-boy. Un américain entre le mythe et l'histoire, p. 55.
} 
l'invasion anglaise de l'île et aux violences du XvII ${ }^{\mathrm{e}}$ siècle. Mais lorsque les bergers irlandais persécutés s'expatrient en Amérique, ils se gardent bien de conserver ce nom. Dans les colonies américaines, le gardien de bétail, tant à pied qu'à cheval, est plutôt appelé cowkeepers. Pourtant, le mot " cow-boy » revient au moment de la Révolution américaine de 1776 pour désigner les loyalistes, les colons favorables à la Couronne britannique, parce qu'ils dérobent le bétail et détruisent les récoltes des insurgés. Encore au XIX ${ }^{\mathrm{e}}$ siècle, le mot est toujours employé en référence à son héritage historique. [...] Il n'est pas surprenant qu'un terme entaché des pires vilenies, le vol de chevaux, la trahison, le meurtre, ait retrouvé une certaine vitalité dans le Texas des années 1830 avec les exactions des jeunes cavaliers ${ }^{48}$.

Il faut attendre la fin du XIX ${ }^{e}$ siècle pour que le cow-boy acquière ses lettres de noblesse et se transforme en représentant de l'Amérique triomphante nécessairement blanche et patriote. Cette invention s'affirme au moment même où, après 1890, le cow-boy traditionnel n'est déjà plus qu'une légende en raison des progrès de la Révolution industrielle et de l'essor du chemin de fer, permettant de se passer de plus en plus de la «piste » du bétail ${ }^{49}$. Le glissement sémantique du cow-boy s'opère au rythme de la conquête de l'Ouest. Du renégat, il devient prolétaire du troupeau, il qualifie ensuite celui qui « prend la piste » pour accompagner les bêtes depuis les pâturages vers une gare de bétail, puis il désigne tous les travailleurs du ranch pour enfin incarner tout Américain de l'Ouest sur un cheval. Ce qui est important pour notre propos c'est la progressive occultation de l'héritage hispanique et mexicain au profit d'une valorisation d'une ascendance qui serait purement étasunienne. Pourtant les estimations concernant l'origine des travailleurs du bétail confirment qu'au moins $45 \%$ d'entre eux se recrutaient chez les Mexicains, les Noirs et les Indiens ${ }^{50}$. Dès la fin des années 1880, le chemin de fer, la multiplication des clôtures, puis l'interdiction aux troupeaux de « prendre la piste » transforment le travail des cow-boys et renforcent leur sédentarisation.

Après avoir nourri la croissance des grandes villes de l'Est, les cow-boys en nourrissent l'imaginaire. On a vu que les premiers spectacles de rodéos naissent dans la décennie des années 1880 au cours desquelles beaucoup de cow-boys cherchent les moyens d'une reconversion, sans tout à fait renoncer aux caractères extérieurs de leur genre de vie. Le mythe de l'Ouest fait figure d'antidote face à la rapidité des transformations dues à la Révolution industrielle et à l'afflux migratoire d'origine européenne. L'Ouest devient une réserve de grands espaces, de nature, mais aussi de valeurs idylliques et patriotiques forgées par la bourgeoisie anglo-saxonne pour maintenir une cohésion sociale et identitaire au sein d'une Amérique dont la population double de 1870 à 1900. La première

\footnotetext{
${ }^{48}$ Ibid., p. 57.

${ }^{49}$ P. Starrs, Let the Cowboy Ride.

${ }^{50}$ P. JACQUIN, Le cow-boy. Un américain entre le mythe et l'histoire, p. 109.
} 
tournée du Wild West Show de Buffalo Bill démarre dans les villes de l'Est en 1873. Bien que l'historiographie dominante ait gommé cet aspect, l'importance de l'héritage hispanique dans les techniques du rodéo est largement assumée dans les premiers Wild West Show au point que Buffalo Bill en personne pouvait apparaître en tenue de charro $^{51}$. Il en va d'ailleurs de même dans la plupart des rodéos de la fin du XIX ${ }^{\mathrm{e}}$ siècle, au point qu'en 1898, le Wild West Show et le Cheyenne Frontiers Days fusionnent au sein d'un même spectacle. L'Ouest se vend bien, ainsi que les rodéos, mais progressivement, et la littérature puis le cinéma ne feront que forcer encore le trait, ils évoluent vers l'image d'un cow-boy aux antipodes des rustres vaqueros, des Indiens, des Mexicains et des métis dont les méfaits sont invariablement réparés par l'adresse et la droiture morale de ces nouveaux chevaliers. Le cow-boy n'est plus l'héritier des vaqueros mexicains, mais des valeurs morales de l'Amérique protestante. Le rodéo n'est plus héritier des traditions vaqueras hispaniques, mais du ranch de l'Ouest. La frontière n'est plus une zone aux influences culturelles multiples, mais la métaphore de la victoire de la civilisation nord-américaine sur l'Ouest sauvage. Autrement dit, la conquête de l'Ouest et la frontier au sens de Frederick Turner ont définitivement remplacé, dans l'imaginaire, la conquête du Sud et le déplacement de la frontière politico-administrative avec le Mexique ${ }^{52}$.

Nous pensons que la charreada et le rodéo nord-américain véhiculent ainsi deux images différentes du fonctionnement de la frontière entre le Mexique et les États-Unis. La charreada, activité communautaire et traditionnelle, est attachée à l'idée d'une continuité culturelle mexicaine au-delà de la rupture territoriale. Le rodéo, sport individuel et moderne, est attaché à l'idée d'une rupture culturelle États-Unis - Mexique qui s'accommode néanmoins d'une étanchéité sélective de la frontière et d'un terrain favorable à l'expansionnisme de la pratique vers le sud. Cela étant, il ne faudrait pas réduire le rodéo à la seule émanation sportive du mythe du cow-boy, car il est en fait traversé par des courants et des conceptions divergentes. Malgré la puissance du mythe, les cow-boys réels du rodéo n'ont jamais tout à fait ressemblé à ce qu'ils incarnaient dans le regard de l'Amérique. Preuve en est que les Indiens et les Hispanos n'ont jamais quitté le fil de l'histoire réelle et forment un contingent non négligeable dans une profession à risque qui promet une rapide ascension sociale. Cette réalité est aussi davantage conforme à l'image que la frontière actuelle entre le Mexique et les États-Unis donne à voir : une zone multiculturelle d'échanges.

\section{LA FRONTIÈRE EXOTIQUE}

ET LES «CORRIDAS SOFT » LUSO-CALIFORNIENNES

On aurait pu croire les corridas absentes des États-Unis et considérer la « taurophilie » d'un Hemingway, d'un Orson Wells ou d'un James Dean comme des

\footnotetext{
${ }^{51}$ M. L. Le Compte, « The hispanic influence on the history of rodeo ».

${ }^{52}$ F. Turner, « The significance of the frontier».
} 
exceptions anglo-saxonnes qui confirment la règle d'une Amérique moyenne puritaine et trop attachée aux droits des animaux pour en tolérer la pratique. Et pour tous ceux chez qui le puritanisme s'accompagne de sa nécessaire transgression, les arènes frontalières permettent de succomber au vice honteux. N'oublions pas que Tijuana, dont la réputation sulfureuse n'est pas à faire, possède des arènes situées sur la ligne frontière, à proximité de San Diego et à moins de 250 kilomètres de Los Angeles. L'étude détaillée des faits montre comme toujours une réalité plus complexe.

Soulignons d'abord l'existence de clubs taurins à New York, Los Angeles, Chicago, San Francisco, Boston pour ne citer que les plus importants parmi une quinzaine. Ils se réunissent périodiquement au sein de NATC (National Association of Taurine Clubs of the United States of America) qui date de 1963 et organisent de fréquents voyages en péninsule Ibérique et au Mexique. Aux arènes de Tijuana, on peut observer une statue du torero mexicain Carlos Arruza érigée par les aficionados de Los Angeles avec la dédicace suivante : "In memory of the Mexican Cyclone Carlos Arruza, from the aficion of the United States erected by las Peñas Taurinas de California ${ }^{53}$. Le club taurin de New York, qui compte environ 150 membres, privilégie chaque année la féria de Séville et la féria de Pampelune, comme si, loin de l'Espagne, les plaisirs de l'afición souhaitaient profiter de l'éventail le plus large possible de ce que l'art taurin donne à voir. Outre-Atlantique, l'aura de la San Fermín popularisée par Hemingway dépasse largement le cercle des aficionados et Pampelune figure dans le semis des destinations à ne pas manquer, si l'on se trouve en Europe début juillet. Enfin, il existe depuis une dizaine d'années une Académie de tauromachie à San Diego qui propose des cours et des stages afin d'apprendre "l'art du toreo ». On ne sera donc pas étonné de compter quelques matadors américains depuis Harper B. Lee né au Texas en 1884, jusqu’à Dennis Borba qui prend son « alternative » en 1986, en passant par Sydney Franklin, qui triomphe à Séville en 1929, John Fulton et Robert Ryan qui prend son « alternative » à Tijuana comme beaucoup de ses compatriotes moins connus ${ }^{54}$.

Mais la tauromachie ibérique aux États-Unis ne se réduit pas à l'existence d'un petit groupe d'aficionados cosmopolites, à quelques épigones d'Hemingway et à une frontière commune avec le géant taurin d'Amérique. Sans doute à la surprise de ceux qui voient dans la tauromachie la manifestation d'une pratique archaïque en proie à un inexorable déclin, la très moderne Californie s'affirme depuis une quinzaine d'années comme un foyer tauromachique émergent. D'avril à octobre, pour une vingtaine de spectacles, les arènes, principalement situées dans la Central Valley et en particulier dans sa partie sud de la San Joaquin Valley, font le plein : Orland, Elke Grove, Thornton, Tracy, Escalon, Stevinson, Gustine, Morgan Hill, Madera, Laton, Tulare, Artesia. Comme

\footnotetext{
${ }^{53}$ F. Garibay Anaya et M. Luna Parra, México se viste de luces, p. 35. Traduction : À la mémoire du Cyclone mexicain Carlos Arruza, de la part de l'afición des États-Unis, érigé par les Peñas taurines de Californie.

${ }^{54}$ M. DARrieumerlou, « Toreros Yankees au Mexique ».
} 
on l'a vu précédemment, les spectateurs sont, dans l'immense majorité, originaires de la communauté des Açores qui compterait actuellement pas moins de 350.000 membres en Californie. Les spectacles prennent la forme des corridas mixtes portugaises, réunissant la prestation des cavaliers et des forcados ainsi que celle des matadors à pied. De la même façon qu'au Portugal, il n'y a pas de mise à mort du taureau et, de surcroît, toute effusion de sang est exclue du programme, conformément à la législation californienne. Il n'y a donc pas de piques et les banderilles sont fixées par un système d'attache en velcro sur un tapis collé à la glu sur le dos de l'animal. Les spectacles intègrent le cadre des fêtes religieuses de la communauté açorienne exilée depuis plusieurs générations et, à ce titre, participent des fêtes de l'immigration qui visent à faire resurgir le pays lointain. Là encore, il ne s'agit pas d'une revendication exclusive de l'identité açorienne, mais bien d'un double sentiment d'appartenance américain et portugais. Les spectacles s'ouvrent sur les deux hymnes nationaux, chantés tête découverte et la main sur le cœur, pendant que défilent, côte à côte, les drapeaux des États-Unis et du Portugal. Cela étant, c'est bien la langue portugaise qui est très majoritairement utilisée lors des festivités davantage que dans le cadre professionnel où la mixité des origines est plus forte.

Les premiers jeux taurins réalisés à l'initiative de la communauté açorienne de Californie semblent remonter aux années 1920. Le témoignage documenté le plus ancien fait état d'une corrida qui a eu lieu le 15 septembre 1925 au Fairgrounds de Tulare dans la San Joaquin Valley ${ }^{55}$. Le journaliste de The Portuguese Tribune, José Ávila, affirme qu'en 1926, existaient déjà quatre arènes en Californie à San Pablo, Tracy, Tulare et Los Banos. Un article d'Anna Hadwick Gayton, publié en 1948, qui décrit le déroulement de la fête de Lady of Miracles à Gustine, nous laisse penser que la tradition pourrait même être plus ancienne ou, en tous les cas, qu'elle est déjà bien implantée dans les années $1930^{56}$. La fête est également appelée Festa da Serrata, transposition directe de la fête du village de Serrata située sur l'île Terceira, en l'honneur de Nossa Senhora dos Milagros. La première édition est organisée en 1932, à l'initiative de M. Souza, membre du Portuguese-American club of Gustine, de retour d'un voyage au pays. Ce club a pour vocation première de rassembler les Portugais de la région et d'en faire de bons Américains en créant un lien avec l'Église catholique de Californie. La fête se compose d'une procession dominicale accompagnant six statues de la vierge, suivie d'un repas collectif, puis du retour des statues à l'église le lendemain matin. Une autre procession accompagne une vache laitière décorée et le soir a lieu une course de taureaux. L'auteur parle déjà des courses traditionnelles du lundi soir comme d'un sport très populaire, ce qui laisse entendre qu'en 1932 la pratique n'est pas une nouveauté. L'unique photographie représentant la course de taureaux montre un animal encordé, lâché dans une arène et couru par la foule dans la plus proche tradition des touradas à corda des îles Terceira. Les

55 A. Graves, The Portuguese Californians, p. 160. L'auteur s'appuie sur un article inédit d'Adrienne Alston de 1996.

${ }^{56}$ A. Hadwick Gayton, «The festa da Serrata ». 
journaux locaux portugais attestent en 1947 que la Festa da Serrata de Gustine est déjà très connue en Californie, et « les Portugais, tous les Portugais, assistent à la fête de Gustine pour soulager la nostalgie du pays au-delà des mers ${ }^{57}$.

Quelles qu'aient été les modalités des jeux taurins de l'époque, les deux étapes fondamentales de modernisation de la pratique sont, d'une part, la loi de 1957 faisant entrer le bloodless bullfighting dans la légalité et, d'autre part, les premières importations de taureaux de combat mexicains au milieu des années 1970 qui remplacent les Brahmas, Herefords et autres spécimens croisés alors couramment utilisés. C'est pourquoi la plupart des témoignages recueillis sur place font véritablement démarrer l'histoire des courses de taureaux californiennes avec les achats de taureaux venus du pays voisin qui se succèdent à partir de 1974, à l'initiative de Manuel Correia, Manuel de Sousa et Franck Borba. Ce tournant des années 1970 entraîne la multiplication des spectacles et la construction de nouvelles arènes. Il est intéressant de constater que la première corrida avec des taureaux mexicains, qui se tient à Visalia le 10 mai 1975, considérée en quelque sorte comme la première corrida bloodless formelle, se déroule avec des matadors également mexicains, donc sans la présence de cavaliers et de forcados portugais qui ne feront leur apparition que dans les années 1980. Ce sont donc les éleveurs de taureaux, aujourd'hui une dizaine, qui sont à l'origine de la délocalisation de la tauromachie portugaise en Californie, éleveurs qui se sont lancés dans cette aventure après avoir acquis de solides bases économiques dans l'élevage laitier, la grande affaire de l'émigration açorienne de la Central Valley, et parfois aussi dans l'élevage à viande.

Aujourd'hui, les courses de taureaux continuent d'avoir lieu le lundi soir et des images pieuses continuent d'être associées au défilé d'ouverture. La tauromachie est incontestablement en voie de consolidation, mais dans la discrétion, afin d'attirer le moins possible l'attention des associations de protection des animaux. Malgré cela, il n'est nullement question de repli, puisque la tauromachie s'ouvre sur l'extérieur, bien entendu en invitant des professionnels venus d'Europe, mais aussi d'Amérique latine, qu'ils soient matadors ou forcados à l’image des équipes récemment constituées de Mazatlán ou de Querétaro.

\section{III. — L'ESPACE TAUROMACHIQUE COLOMBIEN : CORRIDA, COLEO ET CORRALEJAS}

La Colombie possède deux types de pratiques tauromachiques professionnelles : les courses de taureaux et les toros coleados. À celles-ci s'ajoute une pratique populaire originale : les fiestas en corralejas. À grands traits, la répartition des pratiques taurines présentées sur la carte 16 (p. 352) fait apparaître une tripartition territoriale conforme aux grands ensembles de la géographie colombienne. Les corridas occupent principalement les hautes terres des cor- 
dillères et des vallées intramontagnardes, une caractéristique que l'on retrouve pour tous les pays de corridas de l'Amérique andine (voir carte 15, p. 350). Les toros coleados occupent les basses terres des llanos orientaux. Les fiestas en corralejas se concentrent sur la Costa Atlántica. À la répartition géographique des pratiques qui ferait croire à une grande simplicité de l'organisation, répond un profond brassage socio-ethnique des publics et des acteurs à l'image d'un pays très métissé.

\section{LES CORRIDAS :}

\section{UNE PRATIQUE DYNAMIQUE, DES FÉRIAS RÉPUTÉES}

Les courses de taureaux enregistrent actuellement une croissance importante. Selon Mundotoro, les corridas ont dépassé le seuil des cent spectacles depuis 2004, atteignant le chiffre exact de 111 courses. Le journaliste taurin Julián Parra Díaz nous a confirmé que se déroulaient chaque année en Colombie quelque 450 spectacles taurins en comptant les corridas, les novilladas piquées et non piquées ainsi que les festivals. Ces estimations en font, sur un plan quantitatif, le deuxième pays de corridas le plus important d'Amérique latine, après le Mexique. Cette position n'est pas démentie d'un point de vue qualitatif, tant la Colombie compte d'arènes et de férias renommées. Les cordillères et les vallées intramontagnardes concentrent la plupart des villes taurines réparties selon deux grands axes. Au centre, les villes taurines se succèdent le long des interfluves du Cauca et de la cordillère centrale : Cali, Armenia, Ibagué, Cartago, Pereira, Manizales, Medellín. À l'Est, elles se répartissent le long d'une cordillère orientale plus épaisse, formée de hauts plateaux qui se prolongent plus au nord : Bogotá, Sogamoso, Duitama, Bucaramanga. Ces deux sous-ensembles concentrent plus de $90 \%$ des corridas et $60 \%$ d'entre elles se déroulent dans la douzaine de villes mentionnées. L'importance des Andes colombiennes se voit confirmée à partir de l'étude historique que nous avons menée sur les arènes du pays. Nous avons trouvé trace de l'existence de 184 arènes fixes ou démontables qui, depuis les années 1950, ont fonctionné avec une plus ou moins grande régularité (voir annexe IV $[\mathrm{CD}-\mathrm{ROM}])^{58}$. Plus de $75 \%$ des arènes ainsi répertoriées, qui sont à la fois la mémoire et le présent de la géographie taurine colombienne, appartiennent à la zone andine avec en tête le vaste département de Cundinamarca (une quarantaine d'arènes) qui s'étend du fleuve Magdalena au piémont oriental de la cordillère. En dehors de la zone andine, les villes taurines de quelque importance correspondent à de grandes villes du littoral caribéen, Cartagena de Indias et Santa María. Apparaissent également deux villes importantes pour notre propos : celle de Sincelejo, haut lieu des fiestas en corralejas et celle de Villavicencio, ville de contact entre les Andes et les Llanos, qui peut être considérée comme la

\footnotetext{
${ }^{58}$ Nous avons croisé plusieurs sources : L. de TABique, Guía de América taurina; J. M. de Cossío, Los Toros; A. Lopera, Colombia, tierra de toros; Pepe Alcázar, Plazas de toros de Colombia ; Mundotoro.com ; Portaltaurino.com ainsi que les informations aimablement transmises par le journaliste taurin Julián Parra Díaz.
} 


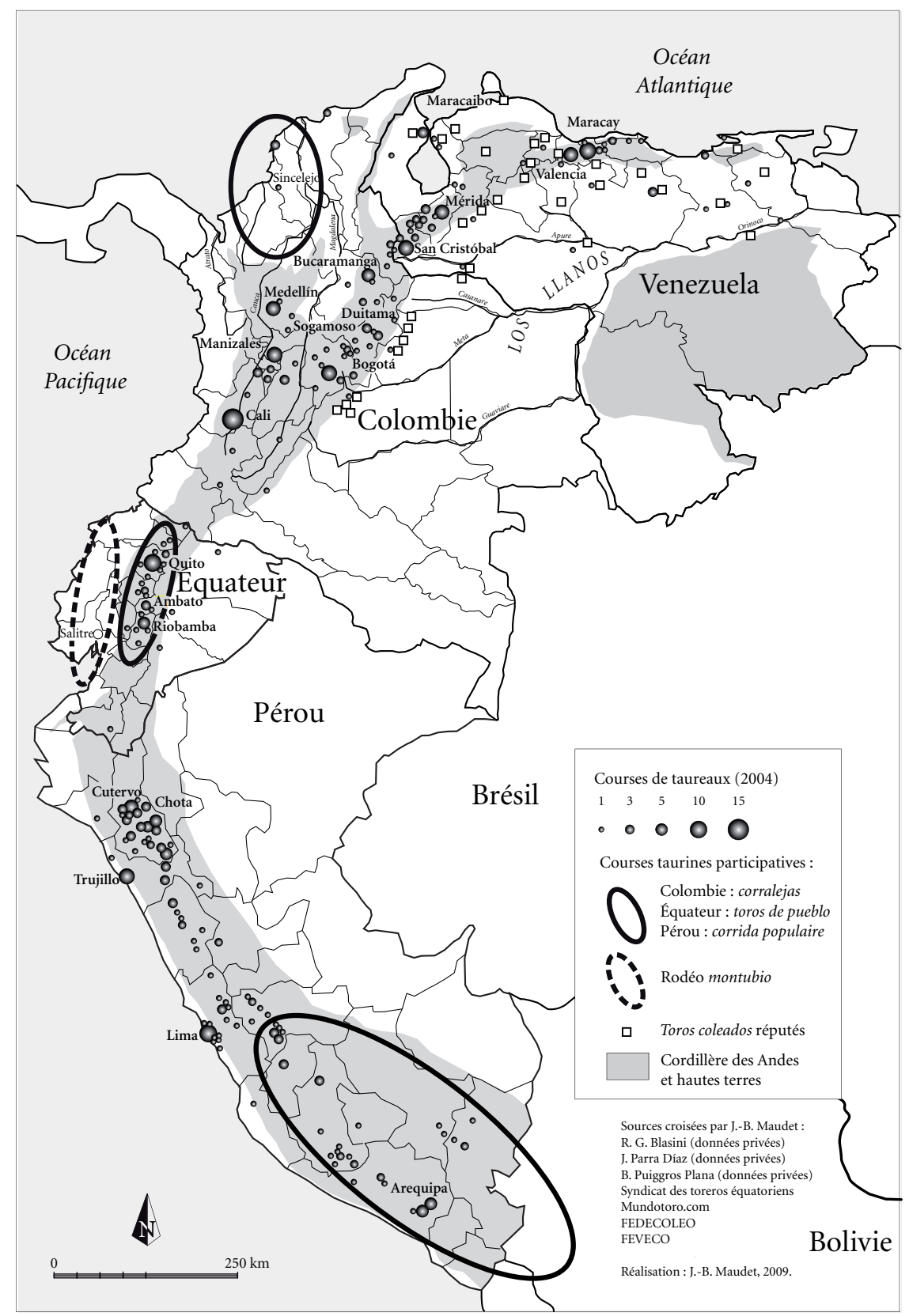

CARte 15. - Tauromachies en Amérique andine 
capitale des toros coleados. Ces deux villes ont une place à part dans la géographie des pratiques car elles sont des lieux de coexistence des cultures taurines qui, par leur intermédiaire, relient leur territoire respectif. La bordure orientale des Andes a fixé d'autres villes taurines telles qu'Aguazul dans le département de Casanare et Florencia plus au sud, dans le département amazonien de Caquetá. La situation de contact peut être envisagée plus largement d'un point de vue territorial à propos du doublet formé par Sogamoso-Aguazul, ville des Andes et ville des Llanos, qui répète sur un mode mineur le doublet Bogotá-Villavicencio. Sogamoso, également appelée " la porte du Llano », vit en connivence avec la culture llanera. De là lui viendrait peut-être son goût pour les chevaux qui se répercute dans les programmes taurins où les rejoneadores sont fréquemment à l'affiche. Une nouvelle fois, il n'est pas déraisonnable de penser que l'attrait pour les chevaux et les exercices équestres servent de pont entre la passion des courses de taureaux et des différentes formes de rodéos.

Les arènes les plus importantes en nombre de corridas correspondent globalement aux grandes métropoles du pays, en tous les cas pour les trois plus importantes : Cali, Bogotá, Medellín. La hiérarchie taurine inverse une partie de la hiérarchie urbaine puisque Cali est assurément la capitale de la tauromachie colombienne. Elle possède les plus grandes arènes (18.000 places), programme le plus grand nombre de corridas avec une quinzaine de courses par an et organise la féria la plus réputée. Beaucoup d'aficionados considèrent aujourd'hui la feria de la Macarena de Cali comme la plus importante d'Amérique latine. En 2007, un sondage réalisé par Radio Caracol, radio de forte audience en Colombie, auquel ont répondu par internet 6.720 personnes, classait ainsi les arènes du pays ${ }^{59}$. À la question «Quelle ville en Colombie possède les meilleurs programmes pour cette saison ? ", Cali remporte $30 \%$ des suffrages, Cartagena : $25 \%$, Bogotá : $20 \%$, Duitama $11 \%$, Manizales $8 \%$, Medellín $5 \%$, Bucaramanga $1 \%$.

Manizales occupe une place à part dans ce panorama, car elle figure régulièrement au deuxième rang des arènes qui programment le plus de spectacles avec une ville qui n'atteint pas les 400.000 habitants. Le cas de Manizales est intéressant pour notre étude, d'abord car il s'agit d'une ville jeune fondée en 1849 et non d'une ville coloniale qui connut le précédent des courses de taureaux aristocratiques. Le premier spectacle taurin a lieu en 1897 et les premières arènes en dur, après une douzaine d'arènes provisoires, sont terminées en 1951, construites à l'initiative d'un groupe de notables aficionados qui fondent une société anonyme Plaza de Toros de Manizales. En 1952, le maire signe un décret créant la féria annuelle de Manizales qui prend corps en 1955. Parmi les organisateurs, un homme, Oscar Hoyos Botero, fasciné par la féria de Séville entend faire de Manizales une sorte de réplique de l'ambiance andalouse. La première fait participer des groupes de chanteurs andalous, on se déplace en calèche, on installe des tentes à l'image des casetas du champ de foire sévillan et l'on

\footnotetext{
${ }^{59}$ Résultat du sondage mis à jour le 18 juillet 2007 disponible sur le site Internet de la radio : http://www.caracol.com.co/encu/index.asp?t=V\&id=803 [réf. du 30 juillet 2007].
} 


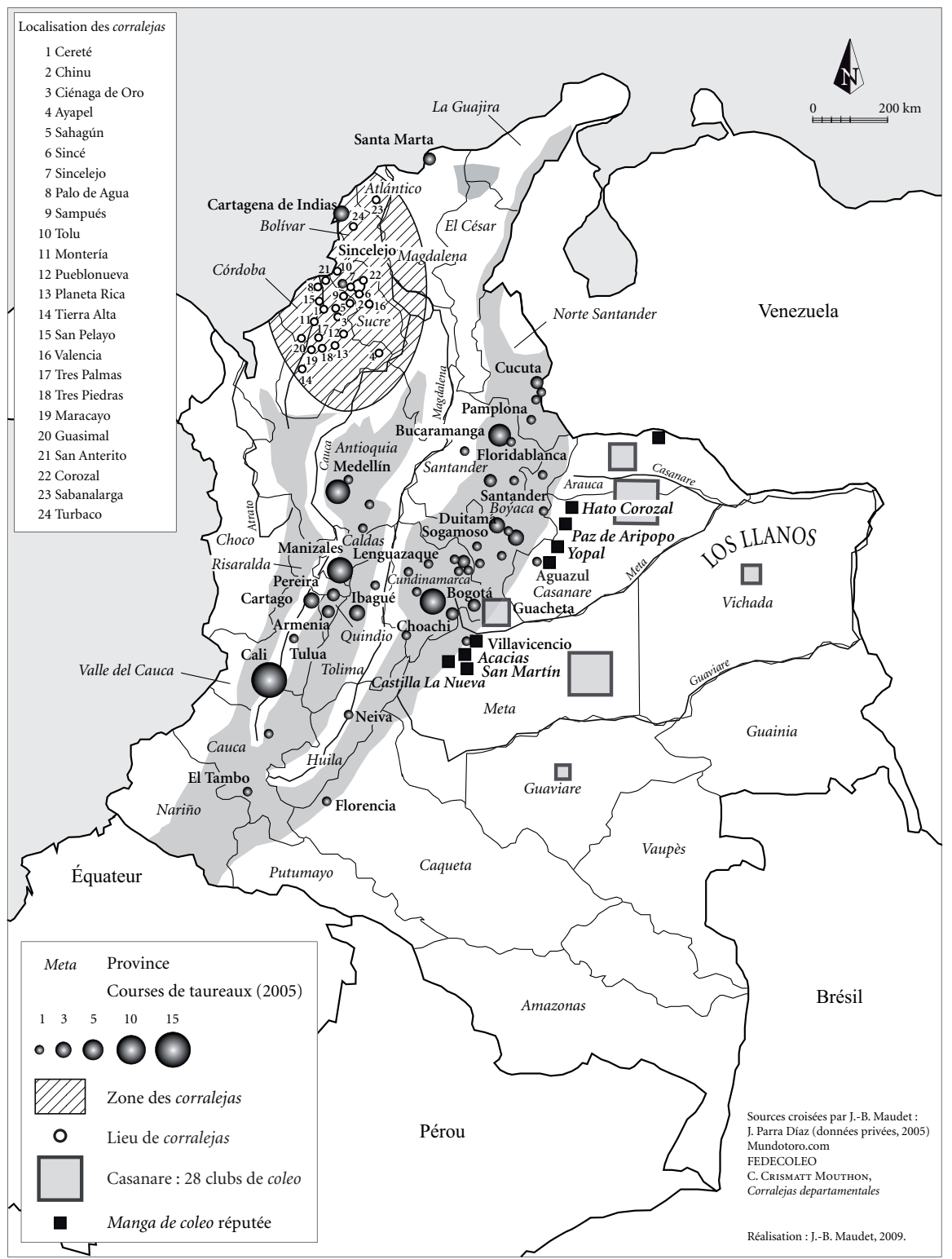

Carte 16. - Tauromachies en Colombie

boit de la Manzanilla ${ }^{60}$. La féria crée également en 1957 un pasodoble «Féria de Manizales », du compositeur Juan Mari Asins, sur lequel le poète Guillermo

${ }^{60}$ A. Lopera, Colombia, tierra de toros, p. 62. 
González Ospina écrit des paroles à la gloire de la " perle del Ruiz » ${ }^{61}$. Elle crée aussi un trophée, d'abord une caisse de vieux rhum de Caldas, puis une réplique de la cathédrale de Manizales. Le pasodoble de Manizales devient rapidement non seulement l'hymne taurin de la féria, mais de toutes les férias colombiennes. Cet air est également repris dans les arènes du monde entier comme le détail d'une connivence sans frontières, joué d'autant plus fréquemment qu'un torero colombien est à l'affiche. À bon droit, par ces initiatives, la féria de Manizales est considérée par nombre d'écrivains et journalistes taurins comme la mère des férias taurines dont se sont inspirées les autres férias de Colombie, mais également celle de Quito en Équateur et celle de San Cristóbal au Venezuela ${ }^{62}$. Parmi les événements importants, il faut noter en 1968, après plusieurs années de mauvaise gestion, le sauvetage de la féria par Manolo Chopera, qui commence par lier les dates des courses à la féria de Cali, dont il est également l'organisateur, afin de faciliter l'engagement des toreros espagnols qui groupent ainsi leur contrat. En 1976, la féria de Manizales est jumelée avec celle de Valence en Espagne, favorisant à cette occasion les échanges commerciaux entre les deux pays et les invitations réciproques à y participer. La presse de l'époque relaie les déclarations du maire de Valence, Miguel Ramón Izquierdo, très satisfait de voir " qu'un motif aussi enthousiasmant que les Fêtes servent à resserrer encore les liens d'union entre deux villes de langue et de culture hispanique ${ }^{63}$. La féria participe aujourd'hui à la reconnaissance nationale et internationale de la ville. Manizales souhaite véhiculer l'image d'une ville ouverte sur le monde, identifiée par un grand événement. La place du café et le rôle stratégique de Manizales dans l' " eje cafetero » (axe du café) sont de mieux en mieux intégrés aux thématiques de la féria.

L'ambiance dans les arènes de Manizales est beaucoup plus festive que celle des arènes de Bogotá davantage empêtrée dans la responsabilité de la représentation nationale, ce qui n'est jamais simple dans un pays aux fortes tensions politiques. L'écrivain colombien et aficionado Antonio Caballero affirme que les différences entre les arènes sont beaucoup plus marquées en Colombie qu'en Europe en raison de la composition ethnique du pays, majoritairement métis et mulâtre, mais qui compte également des minorités blanches, noires et indiennes ${ }^{64}$. Si nous émettons quelques réserves à accepter l'idée que « la passion taurine existe de la même façon dans toutes les régions ", nous nous rangeons volontiers au constat selon lequel, dans l'ensemble, en Colombie, cette passion ne connaît guère de clivage ethnique et qu'elle est aussi forte dans « des régions très indiennes comme Boyocá, que très blanche comme Antioquia ou très noires comme la Côte ${ }^{65}$. Pour ce qui est de la Côte, les données quantitatives et la spécificité des corralejas montrent dans le détail que ce jugement est à nuan-

\footnotetext{
${ }^{61}$ La ville est à proximité du volcan le Nevado del Ruiz, tristement célèbre pour son éruption de 1985.

${ }^{62}$ Pepe Alcázar, Plazas de toros de Colombia, p. 47.

${ }^{63}$ Ibid., p. 51.

${ }^{64}$ A. Caballero, « Le condor et le taureau », p. 27.

${ }^{65} \mathrm{Ibid}$., p. 27.
} 
cer. À propos de Bogotá, où l'on chante l'hymne national en ouverture, l'auteur rapporte qu'on va aux corridas faire de la politique et que les présidents ne s'y risquent généralement qu'en début de mandat pour ne pas avoir à essuyer les affronts du public. On retrouve là l'idée d'une analogie récurrente dans tous les pays entre l'arène politique et l'arène de corrida qui agit comme une caisse de résonance ou un baromètre de la situation sociopolitique du moment mesurant la popularité des gouvernants. Cet aspect rend toujours les arènes de la capitale politique différentes des arènes de provinces.

Sur la période 2000-2005, les quatre premières villes taurines, Cali, Bogotá, Manizales, Medellín, ont programmé un tiers des corridas formelles. Elles sont les seules à avoir proposé chaque année plus de cinq corridas. La trame des villes taurines qui offrent de nombreux spectacles s'épuise rapidement par rapport à celle du Mexique. Entre cinq et dix arènes selon les années offrent plus de trois spectacles par an (Bucaramanga, Cartagena de Indias, Duitama, Ibagué, Sogamoso, Cartago, Armenia). Ces villes correspondent à des centres secondaires de tradition plus ou moins ancienne. Dans certaines villes, les spectacles sont en pleine croissance comme à Bucaramanga depuis que ses arènes, véritable joyau de l'architecture taurine, ont été rénovées en 2003. Dans d'autres, la tradition s'est essoufflée ou n'a jamais vraiment pris comme dans la grande métropole de Barranquilla. Il est étonnant malgré sa population millionnaire de constater que la Monumental del Caribe enregistre une faible activité taurine. Il est possible aussi que le très célèbre Carnaval de Barranquilla n'ait pas laissé beaucoup de place au taureau en chair et en os, alors même qu'il est omniprésent dans les masques et les danses ${ }^{66}$. Après ce réseau de villes réputées pour leur tradition taurine, une dizaine d'autres programment une à deux corridas par an, les dernières ne sont que des lieux taurins épisodiques, même si les courses de taureaux sont toujours accueillies avec succès. Au total, sur l'année 2004, plus de $65 \%$ des cités taurines n'ont offert qu'une seule course confirmant le caractère exceptionnel du «jour de taureau ». Loin d'être la preuve de l'indigence des pratiques taurines nous pensons qu'il s'agit au contraire d'un signe de leur enracinement dans le calendrier avec lequel on ne transige pas.

La hiérarchie des villes taurines se double d'une césure dans la saison tauromachique. La plupart des arènes importantes programment leurs courses entre décembre et mars, lors de ce qu'il est convenu d'appeler la temporada grande (la grande saison) : Cali, Bogotá, Manizales, Medellín, Duitama, Bucaramanga, Armenia. Lors de cette " saison haute » de la tauromachie, les grandes figures européennes font le voyage aux Amériques et participent à toutes les grandes férias. Ce fonctionnement saisonnier manifeste une complémentarité transatlantique dont profite l'ensemble des terres de corridas outre-Atlantique. On distingue la temporada grande de la petite saison, temporada chica, qui se déroule autour des mois de juin et juillet en particulier à l'occasion des fêtes de San Pedro et San Pablo dans les villes de Sogamoso, Aguazul, Ibagué, Espinal.

\footnotetext{
${ }^{66}$ On rencontre de telles pratiques dans toute l'Amérique latine, du Mexique (los toritos) au Brésil (traditions du bumba meu boi du Nordeste ou du boi de mamão dans le Sud).
} 
C'est lors de ces fêtes que la proportion de toreros colombiens est maximale, presque exclusive. Les deux saisons taurines correspondent à deux modes de fonctionnement de l'espace tauromachie. La haute saison, espace-temps mis en continuité avec la tauromachie internationale, est un moment de confrontation et de rencontre entre les toreros nationaux et les toreros européens (entendons espagnols dans leur immense majorité). La basse saison est un temps de repli sur le marché local qui est aussi vécu comme un temps de préparation et de sélection en vue de soutenir la comparaison à venir avec les toreros espagnols. Pour autant, cette confrontation ne prend pas les traits d'une simple opposition entre nations, ce qui différencie la corrida des pratiques sportives et véhicule un tout autre rapport au territoire.

Parmi les autres particularités de l'afición colombienne, il convient de remarquer son grand intérêt pour la tauromachie à cheval. Cela provient sans aucun doute d'une passion colombienne pour les chevaux et en particulier pour le cheval de race Paso Fino. Ce qui a été évoqué pour Sogamoso se voit confirmé à Medellín. Medellín est la capitale du cheval Paso Fino et le berceau de la tauromachie à cheval en Colombie. Chaque année à Medellín a lieu l'une des plus grandes chevauchées d'Amérique latine où quelque 10.000 cavaliers paradent lors de la Feria de las Flores. C'est aussi le lieu d'une des plus grandes foires internationales du cheval. Le premier rejoneador colombien est une cavalière qui toréa au début des années 1950, Ana Beatriz Cuchet, fille d'un torero espagnol parti faire sa vie en Amérique. Elle ouvre une lignée de rejoneadores colombiens adulés du public à l'image d'Oscar Oky Botero et dont beaucoup sont nés à Medellín ou dans la province d'Antioquia (Oky, Antonio Vargas, Alberto Uribe Sierra, Andrés Vélez, Carlos Roldán). Répétons-le, culture équestre et culture taurine possèdent en Amérique latine de nombreuses connexions, alors que l'Europe généralement les sépare davantage.

La Colombie possède trois associations professionnelles d'éleveurs de taureaux de combat qui regroupent une soixantaine d'élevages : ASTOLCO, ASOLIDIA et ASOBRAVO. Ces associations, comme toutes les autres organisations du même type, se sont fixées comme objectif de garantir et de promouvoir la race des taureaux de combat. La plus prestigieuse, ASTOLCO, regroupe 24 élevages braves dont quasiment la moitié est située dans la province de Cundinamarca, les autres ne s'écartant que rarement de la zone andine. Il s'agit d'un cheptel relativement récent puisque les premiers exemplaires de taureaux de caste sont arrivés sur le sol colombien en 1923, en provenance des élevages espagnols de Santa Coloma et de Veragua à destination de l'élevage Mondoñedo à Mosquera situé dans las sábanas de Bogotá ${ }^{67}$. Le sang Santa Coloma fut rapidement privilégié par l'achat de nouveaux reproducteurs en 1929. Avant que les taureaux de race ibérique n'intègrent les élevages colombiens, des taureaux de race locale étaient donc combattus, des taureaux dits criollos ainsi que des zébus dont le comportement adéquat restait très aléatoire. Certains criollos avaient acquis des

\footnotetext{
${ }^{67}$ A. Lopera, Colombia, tierra de toros, p. 276.
} 
caractéristiques morphologiques et comportementales qui les distinguaient en fonction des régions géographiques où ils étaient élevés, tels que les toros llaneros (des llanos orientaux), toros costeros (de la Costa Atlántica), toros caucanos (de la vallée du Cauca). L'importation des reproducteurs espagnols, puis mexicains, a progressivement recentré la zone d'élevage des taureaux utilisés pour les courses espagnoles sur la zone des Andes, dans les provinces connaissant les plus fortes densités de spectacles. Les élevages braves colombiens ont progressivement remplacé les taureaux importés d'Espagne et du Mexique, allant même jusqu'à exporter des bêtes à leur tour vers des férias étrangères, en particulier vénézueliennes. Afin de protéger le marché intérieur, des mesures protectionnistes ont même été prises puisque les trois associations d'éleveurs ont signé un accord en 1998 qui visait à boycotter les arènes colombiennes ayant recours à des taureaux importés d'Espagne.

Pour ce qui est des professionnels de l'arène, il existe un accord signé en 2004 entre les syndicats des toreros colombiens (UNDETOC) et différentes organisations espagnoles en vue de réguler le marché6 ${ }^{6}$. Outre des mesures administratives touchant au statut social des professionnels, cet accord prévoit que les matadors espagnols et colombiens pourront occuper deux tiers du cartel seulement dans les arènes de première catégorie ${ }^{69}$. Les toreros subalternes colombiens et espagnols sont autorisés à toréer librement dans toutes les arènes d'Espagne et de Colombie au service d'un matador étranger, à condition qu'il $y$ ait au moins un Espagnol ou un Colombien à l'affiche. Mais les Colombiens en Espagne ne pourront pas occuper plus du tiers du cartel. Les organisations colombiennes négocient la proportion des toreros espagnols pouvant toréer lors d'une course et définissent des contreparties pour pénétrer le marché espagnol connu pour sa fermeture. L'UNDETOC est à l'origine d'un autre accord qui intéresse cette fois les relations entre pays d'Amérique latine. Le 18 octobre 2001, à Bogotá est créée une Fédération internationale des toreros américains, la FITA. Cette fédération définit les bases d'un accord de libre-échange taurin entre la Colombie, le Mexique, le Pérou et l'Équateur, manquant à l'appel le Venezuela qui finalement intègre la FITA le 4 mars 2003. L'un des objectifs de la FITA est de garantir « une fiesta brava qui soit davantage nôtre et témoigne d'une identité américaine ». Ce type d'accord entend défendre les intérêts des toreros américains dans les différentes férias face aux toreros espagnols qui souvent dictent leur loi aux entrepreneurs de spectacles. La consolidation de la filière taurine s'accompagne ainsi d'une vision d'ensemble du marché qui tient compte des équilibres entre l'Europe et l'Amérique. La position hégémonique du monde du spectacle espagnol ne laisse pas d'alimenter les débats, puisque les toreros espagnols semblent indispensables au déroulement d'une grande féria digne de ce nom tout en posant clairement le problème du rôle et de la place des toreros

\footnotetext{
${ }^{68}$ Cet accord hispano-colombien m’a été remis en mains propres par le syndicat des toreros équatoriens qui entend s'en inspirer.

${ }^{69}$ Le cartel (l'affiche), désigne couramment dans le vocabulaire les protagonistes qui sont au programme.
} 
nationaux. À nos yeux, porter le débat sur le terrain de l'identité ne témoigne pas tant d'un rapport d'équivalence simple entre la nationalité des toreros et l'appropriation de la fête que d'une imbrication des questions identitaires et des questions économiques.

Ces initiatives montrent le dynamisme et la bonne santé actuelle de la tauromachie en Colombie. Ce pays continue d'ailleurs à produire de nombreux matadors d'alternative. L'histoire en compte environ 80 dont une trentaine depuis le début des années 1990. Beaucoup ont connu la gloire dans leur pays, quelques-uns l'ont étendue aux terres de corridas d'Amérique latine (« Joselillo de Colombia », Pepe Cáceres), très peu l’ont atteinte en Espagne (César Rincón). L'un d'entre eux a joué un rôle clé dans le développement de la tauromachie en Colombie : Edgar Zúñiga Villarquirán dit « Joselillo de Colombia ». Il prit l’alternative en Espagne en 1953 et connut de nombreux succès au Venezuela, au Pérou et surtout au Mexique. Il devint ensuite le plus important entrepreneur de spectacles du pays et dirigea ou co-dirigea les arènes de Cali, Bogotá, Medellín. Il fut à l'origine des férias de Cartagena, de Armenia, Calarcá, Pereira et Bucaramanga ${ }^{70}$. Le cas relativement récent de César Rincón, considéré comme un des grands matadors de l'histoire, mérite également d'être souligné et confirme sans doute le dynamisme de la tauromachie en Colombie, si l'on accepte de considérer que le succès d'un torero ne surgit pas de sa seule individualité, mais d'un marché taurin qui le fait éclore. César Rincón triompha aux arènes de Las Ventas à Madrid le jour même de sa présentation le 21 mai 1992. Il sortira quatre fois de suite par la grande porte d'une arène qui se rend au cri de " $i$ Viva Colombia, Viva Colombia! ». De retour chez lui, il est reçu en véritable héros et se voit nommé par les autorités «Ambassadeur itinérant de la Colombie».

\section{LES TOROS COLEADOS DES LLANOS ORIENTAUX}

La pratique des toros coleados s'étend principalement sur les llanos orientaux et constitue un trait culturel emblématique de la région. Cette pratique renvoie sans doute plus qu'aucune autre à la terre qui l'a vu naître, une caractéristique qu'elle partage avec les vaquejadas du Sertão dans le Nordeste brésilien. Ce que les Colombiens appellent les llanos orientaux, qui recouvrent quelque $230.000 \mathrm{~km}^{2}$, sont une partie d'un ensemble géographique plus vaste nommé, avec une majuscule, les Llanos, qui s'étendent entre la Colombie et le Venezuela. Les limites des Llanos sont comprises entre le piémont des Andes orientales et le massif ancien des Guyanes, entre la rive gauche du Guaviare, qui correspond approximativement à l'écotone forêt amazonienne-savane et l'embouchure de l'Orénoque qui draine l'ensemble du bassin. Cet espace possède une forte personnalité au sein du territoire national, à la fois immensité naturelle des savanes peuplées de bétail en semi-liberté, périphérie lointaine malgré une filière bovine anciennement intégrée et marge troublée des contre-sociétés conflictuelles.

\footnotetext{
${ }^{70}$ A. Lopera, Colombia, tierra de toros, p. 164.
} 
La FEDECOLEO est la Fédération qui réglemente et organise la pratique des toros coleados en club ainsi que les compétitions. Son siège est situé à Villavicencio, la plus grosse ville en bordure des llanos avec 330.000 habitants, qui peut être considérée comme la capitale colombienne du coleo. Villavicencio est une ville du piémont qui pendant longtemps ne fut qu'une agglomération d'haciendas d'élevage également désignées sous le nom de ranchos ou de hatos dont la toponymie témoigne encore. Villavicencio est aussi le relais de l'influence de Bogotá sur l'arrière pays intérieur et un gros bourg rural pourvoyeur de viande ${ }^{71}$. Les compétitions de coleo sont organisées sur la base de ligues provinciales, de trophées qui en opposent les équipes, d'une ligue nationale et des tournois individuels. Les provinces de Casanare et du Meta, qui sont les plus peuplées des llanos colombiens, rassemblent $65 \%$ des clubs de coleo affiliés à la Fédération (55 clubs sur 85 ). Les mangas de coleo les plus réputées et dans lesquelles sont organisées les compétitions les plus importantes sont situées dans ces provinces : Yopal, Paz de Aripopo, Hato Corozal, Aguazul, Tauramena dans le Casanare, Villavicencio, Castilla la Nueva, San Martín, Acacias dans le Meta (voir carte 16, p. 352). Ajoutons la ville de Arauca dans le département du même nom. Le département de Cundinamarca possède également une dizaine de clubs de coleo liés aux migrations des habitants des llanos vers la capitale. Il existe d'autres structures associatives plus modestes dispersées dans les hatos d'élevages qui animent les divertissements d'une société encore profondément rurale et très attachée au genre de vie défini par le pastoralisme semi-extensif. Quelque 80 rencontres de coleo sont organisées chaque année par la Fédération. Chacune dure généralement deux jours permettant d'associer aux joies de la compétition celles des activités festives (retrouvailles, grillades, bals, concerts). Le joropo llanero est une musique et une danse typique de la région dont les adeptes affirment qu'il dérive du flamenco mis à la sauce des llanos. Certains gestes tels que le jeu de pied des zapateos confirmeraient cette forme de réappropriation du taconeo (coup de talon) du flamenco.

Les toros coleados possèdent tous les attributs du divertissement régional dans ce qu'il peut avoir de représentatif de la culture et de l'identité locale. Ils sont circonscrits spatialement à une région naturelle et culturelle relativement bien délimitée. Ils transposent, sur un mode ludique et sportif, les techniques de l'économie pastorale de la région, fondées sur le maniement du bétail. Les toros coleados sont vécus comme un ferment de l'identité llanera et rassemblent autour des fêtes des moments d'intense sociabilité. On peut considérer que nous sommes en présence d'une pratique qui célèbre un « genre de vie » au sens le plus vidalien du terme : celui du llanero correspondant à un type social aussi défini, mais assurément moins connu, que celui du gaucho en Argentine.

Il est alors intéressant d'observer que ce "genre de vie », célébré sous un mode à la fois idyllique, nostalgique et festif, a souffert de nombreuses perturbations récentes. Les transformations sociétales des llanos émergent à partir des années

\footnotetext{
${ }^{71}$ L. Bolívar et E. Elisur, Geografía apureña.
} 
1970 par le développement des conflits armés et des cultures illicites de marijuana et de coca. Les facteurs externes sont l'augmentation de la consommation de produits stupéfiants en Europe et aux États-Unis ainsi que la répression de la production et du trafic au Mexique à partir de 1975. Les cultures se développent à grande vitesse dans les provinces des llanos, en particulier celles de Guaviare, de Meta et de Vichada au rythme de l'emprise des organisations criminelles ${ }^{72}$. Le deuxième élément de transformation sociétale intervient dans les années 1980 et 1990 avec le développement de l'activité pétrolière dans le piémont. Il intensifie les mouvements migratoires et l'emprise économique du centre sur la périphérie. La manne pétrolière provoque également des graves dysfonctionnements de gouvernance régionale, liés à une corruption des appareils d'État. Les provinces de l'Arauca et du Casanare deviennent alors d'importantes zones de conflits sociaux et armés. Enfin, les années 1990 correspondent également à l'expansion des FARC qui profitent des faiblesses et de la désorganisation du pouvoir central dans les llanos avec comme résultat une déstructuration du tissu rural ${ }^{73}$.

Or, l'institutionnalisation visant à promouvoir et développer le coleo date précisément de cette période d'accélération des transformations sociales et territoriales. Comme nous l'avons noté, la Fédération est fondée en 1994 et le coleo reconnu comme sport par le Comité olympique national en 1998. Voici l'un des objectifs clairement affichés par la Fédération, au-delà des questions de réglementation et d'organisation des compétitions : "Pourquoi le faisons-nous ? Avec le sport du coleo, on parvient à se diriger vers la paix et on reconstruit le tissu humain ». Un des slogans de la Fédération répétés en de maintes occasions, telle la prière incantatoire des actes officiels, l'affirme à satiété : « À travers le sport du coleo, nous contribuons à unir davantage les peuples à la recherche de la paix ». La foi, clairement exprimée, en les vertus cathartiques du sport et sa mission de service public peut-elle trouver une justification plus nécessaire?

Dans cette dynamique, qui est aussi celle d'une articulation territoriale, certaines villes jouent un rôle important telle que Villavicencio, la ville du piémont. Villavicencio, la plus occidentale des villes du coleo (et la plus orientale des villes des courses de taureaux) tisse un lien entre les Andes et les Llanos, entre nation et région, entre centre et périphérie. Aguazul dans une situation géographique comparable, tient une place identique. Lors du festival Alma Sabanera (âme de la Savane), qui se tient en novembre, ont lieu conjointement des courses de taureaux et des tournois de coleo. Ajoutons que Villavicencio est le siège de compétitions internationales de coleo telles que les « rencontres de la confraternité colombo-vénézuelienne » ou encore le Mondial de Coleo qui a fêté en 2006 ses dix ans. L'édition de 2005 a regroupé 200 coleadores venus du Mexique, des États-Unis, du Costa Rica, du Panamá, du Canada, du Venezuela et de la Colombie. Aux liens internes à la Colombie, s'ajoutent ainsi des solidarités nationales et panaméricaines. Les compétitions se sont étalées sur cinq jours et ont ras-

\footnotetext{
72 J. Fierra PAtiÑo, « Llanos orientales ».

${ }^{73}$ R. VArgas Meza, Las FARC, la guerra y la crisis del Estado.
} 
semblé 50.000 personnes. Les prix à hauteur de 25 millions de pesos (environ 8.500 euros) sont financés par l'Institut du tourisme du département du Meta ${ }^{74}$. Le Mondial de Coleo, malgré son jeune âge est désormais considéré comme l'un des six événements touristiques les plus attractifs du pays avec le Carnaval de Barranquilla, le Festival del Bambuco de Neiva, la féria de Manizales, la féria de Cali, le Festival de la leyenda Vallenata de Valledupar. Trois de ces événements festifs sont liés aux cultures taurines colombiennes.

\section{LES CORRALEJAS DES SÁBANAS DE BOLÍVAR}

La fiesta en corralejas, pourtant directement inspirée des codes de la tauromachie espagnole par la présence de ses capeadores, banderilleros et garrocheros, est passée inaperçue aux yeux des amateurs de corrida, et très peu d'écrits en témoignent. Elle est absente du dictionnaire de Claude Popelin et l'historien Francisco López Izquierdo dans son ouvrage Los Toros del Nuevo Mundo y consacre trois lignes : «En Colombie se déroulent les corralejas, sorte de capeas données le long de la côte et durant lesquelles interviennent, à pied et à cheval, toréant des zébus et du bétail criollo, les hommes du peuple ${ }^{75}$. Cette brève évocation est victime d'une double approximation. L'une est sociale et ne rend pas compte de la semi-professionnalisation de nombreux acteurs des corralejas, élément que nous avons déjà évoqué. L'autre est géographique et élude toute délimitation spatiale précise du phénomène. Cette imprécision récurrente au sujet des fiestas en corralejas mérite de clarifier les sources d'informations existantes. Notre connaissance des fiestas en corralejas s'appuie sur divers matériaux. Alberto Lopera y consacre un chapitre dans son livre Colombia tierras de toros où il rapporte en de larges extraits les propos d'un ouvrage de référence, tiré à très peu d'exemplaires et totalement introuvable en Europe : El Mundo de las corralejas de Juan Santana Vega. Il existe également un court article signé Julio Olaciregui : « La fiesta en corralejas dans le Nord de la Colombie, ou le sacrifice bouffon ». Nous avons pu étayer ces écrits par divers documents iconographiques : un documentaire de Jean-Louis Cavalier et diverses séquences vidéo mises en ligne ${ }^{76}$. Enfin, soulignons l'apport d'un mémoire de recherche dirigé par le professeur Carlos Crismatt Mouthon de l'université de Córdoba en Colombie ${ }^{77}$. Le croisement de ces divers matériaux nous permet de restituer avec une certaine précision la géographie et les enjeux de cette pratique locale mal connue.

Les corralejas sont généralement associées à la région des Caraïbes colombiennes appelée Costa Atlántica qui se compose de sept départements continentaux : Atlántico, Bolívar, César, Córdoba, La Guajira, Magdalena et Sucre (voir carte 16, p. 352). Cet ensemble constitue une mosaïque ethnique et

\footnotetext{
${ }^{74}$ Instituto de Turismo del Meta, 2005. Informations disponibles sur le site Internet de l'Institut du tourisme du département de Meta : http://turismometa.gov.co/index.php [réf. du 10 juin 2007].

${ }^{75}$ F. López Izquierdo, Los Toros del Nuevo Mundo, p. 73.

${ }^{76}$ J.-L. Cavalier, Fiesta en corralejas.

${ }^{77}$ C. Crismatt Mouthon (dir.), Corralejas departementales.
} 
paysagère complexe. Les corralejas occupent en réalité une partie de ce qu'il était convenu d'appeler autrefois les sábanas de Bolívar (les savanes de Bolívar), partagées entre les départements de Córdoba, Sucre, Bolívar, Magdalena et César. La carte 16 (p. 352) montre que les corralejas sont concentrées plus précisément dans les départements de Córdoba et de Sucre, le long du fleuve côtier Sinu, situé à l'ouest de la dépression du Mompós qui est la réplique structurale du lac de Maracaibo, comblé ici par les apports convergents des grands cours d'eau du versant atlantique. Des témoignages attestent également de l'existence de fiestas en corralejas dans les départements de Bolívar, Magdalena et Atlántico bien qu'elles y soient plus rares aujourd'hui. À l'image de la relation que nous avons précisée entre le coleo, les llanos et les llaneros, il existe entre les correlejas, las sábanas et les sabaneros une correspondance forte entre un jeu taurin, une unité paysagère et culturelle, et un type socio-ethnique défini. La « carte culturelle des Caraibes colombiennes » qualifie les sabaneros, parmi huit types de caribéens colombiens, en des termes qui peuvent nous sembler quelque peu désuets, mais précisant explicitement les caractères de cette correspondance :

Ils [les sabaneros] vivent dans les sábanas de Bolívar, César, Sucre et Magdalena. [...] Ils sont porteurs de chants de vaquerías et la nuit, ils offrent à leurs enfants et à leurs femmes des vers et des chansons sur la vie quotidienne. Bien que la terre où ils vivent ne soit pas la leur, ils s'en sentent possesseurs comme des propriétaires [...] Les chants de vaquerías et les fiestas en corralejas sont les éléments culturels intrinsèques du sabanero cordouan et de quelques zones de Sucre, Bolívar, jusque dans les départements de César et Magdalena ${ }^{78}$.

Les auteurs font une distinction entre les sabaneros, les costeños (Barranquilla, Santa Marta, Cartagena), les montañeros, en contact avec le piémont andin, et les amfibios, au creux de la dépression marécageuse. L'un des éléments importants de cette description est la référence à la question agraire qui fait du sabanero non pas un propriétaire terrien, mais un employé ou un fermier de ces terres dont il se sent possesseur par son travail. Les chants de vaquerías font évidemment référence aux activités de l'élevage bovin et du mode de vie qui lui est attaché.

La capitale des corralejas est la ville de Sincelejo, où, chaque année, a lieu à partir du 20 janvier la plus importante fiesta en corralejas de la région, en honneur du Dulce Nombre de Jesús. On l'a dit, quelque 40 taureaux sont combattus chaque jour par des milliers de personnes qui s'enferment dans la corraleja, le rhum coule à flot, au son des porros, ces orchestres de cuivres qui animent sans discontinuer la fougue des participants, les plus téméraires, les plus chanceux, les plus habiles. Les taureaux proviennent des éleveurs de la région qui louent leur bétail aux organisateurs. Ce sont généralement les éleveurs qui engagent les manteros et les banderilleros semi-professionnels chargés d'animer le spectacle

\footnotetext{
${ }^{78}$ G. E. Rodríguez Navarro et alii, Mapa cultural del Caribe Colombiano, pp. 143-147.
} 
ainsi que les cavaliers garrocheros dont la plupart travaillent pour eux. Ils payent également une partie du rhum et des fanfares dont le coût est partagé avec l'entreprise qui construit la corraleja. Une fois construite, l'entreprise en possède l'exploitation économique en percevant un droit d'entrée à la piste et dans les gradins. Aujourd'hui l'entrée en piste coûte 6.000 pesos (environ 2 euros) et l'entrée aux gradins 25.000 pesos (environ 8,5 euros). Pour beaucoup, le seul moyen de participer à la fête est d'entrer en piste. Les grands propriétaires terriens, les édiles et leurs invités s'installent dans des loges réservées. Dans une région de forte pauvreté rurale en butte à de récurrentes guérillas, les corralejas se veulent le signe de la paix sociale, entre les éleveurs propriétaires terriens et les travailleurs du bétail, entre les milices armées privées et les guérilleros.

Mais ce désir de paix sociale affiché s'exprime dans un climat de fête démesurée et de violence qui laisse apparaître toute l'intensité des tensions sociopolitiques. Ce fut le cas à la fin des années 1960 dans la ville de Montería, capitale du département de Córdoba et également surnommée, « la perle du Sinú » ou « capitale de l'élevage ». En 1969, lors des correlejas de Montería, la fête dégénéra en jets de pierres et de bouteilles vers les gradins, les taureaux furent lynchés et tués en piste. Ces actes de violence furent répétés lors des corralejas de 1970, les toits des gradins furent brûlés et les taureaux ne durent leur survie qu'aux participants qui parvinrent à les protéger des agressions sans borne de certains groupuscules en piste. En 1971, des taureaux sont à nouveau tués, leurs viscères jetés de rage vers les gradins et les loges des possédants. Les taureaux tués cet après midi-là appartenaient au riche propriétaire Martín Vargas, nouveau venu dans la région qui possédait également des bêtes dans les llanos orientaux. Ces actes de violence mirent fin aux corralejas dans la ville de Montería ${ }^{79}$. Le « Festival du Fleuve Sinu » visant à remplacer les corralejas fut alors lancé autour d'une expression pacifiée des traditions de Córdoba, telles que les musiques et les danses des porros, mais il n'eut pas le succès attendu. Les uns n'iraient pas sans les autres : les chants de vaquerías ne raconteraient rien sans les correlejas. Ce qui déborde parfois sous la forme d'une révolte contre les possédants traduit la force des tensions sociales par une rupture avec les normes fragiles de la pratique taurine. Le jeu lui-même ne traduit sans doute pas autre chose. La répartition des rôles entre taureau, homme à cheval et homme à pied peut être interprétée dans ce sens. Les garrocheros engagés par les propriétaires de bétail poursuivent en troupe le taureau qui fuit pour leur échapper. À l'inverse, les hommes à pied dont la plupart sont des anonymes, mis à part quelques manteros et banderilleros engagés, provoquent l'animal qui cette fois ne les fuit pas, mais les charge, provoquant de nombreux accidents. Le taureau se trouve ainsi au cours du même jeu, l'animal chassé par les hommes à cheval et l'animal chasseur pour les hommes à pied. Comme on l'observe dans d'autres formes andines de tauromachies populaires, la fiesta en correlejas n'est réussie que si le sang humain a coulé. Comment ne pas $\mathrm{y}$ voir aussi en deçà de la réconciliation festive et d'une possible interprétation

\footnotetext{
${ }^{79}$ C. Crismatt Mouthon (dir.), Corralejas departementales.
} 
sacrificielle, une image des conditions de vie inégalitaires dans un monde rural périphérique et marginalisé.

Après la fin des correlejas à Montería qui par son rang de petite ville-centre souhaite rompre avec les inerties sociohistoriques du monde rural, les fiestas en corralejas gagnèrent de l'importance à Sincelejo, l'autre ville de la région et capitale du département de Sucre. Sincelejo devient le grand rendez-vous régional des fiestas en corralejas jusqu'en 1980 où un drame d'une tout autre nature mit fin à la fête. Le 20 janvier 1980, huit des trente-deux sections des gradins de l'arène s'écroulèrent causant la mort de 250 personnes, plus d'un millier selon certains témoignages. La catastrophe de Sincelejo eut un retentissement national, preuve pour quelques observateurs de l'époque de l'anachronisme d'une fête païenne punie elle-même de sa propre barbarie. Les corralejas n'ont alors continué que dans des enceintes plus modestes, celles de nombreux villages pour qui la catastrophe de Sincelejo n'avait rien entamé de la popularité de la fête. Les hauts lieux des fiestas en corralejas se nomment alors Sincé, Sampués (Sucre), Planeta Rica, Pueblo Nuevo, Cotorra, San Pelayo, Ciénaga de Oro, Chinú, Colomboy (Córdoba). Il faut attendre 1998 pour que la capitale de Sucre renoue avec ses fiestas en corralejas. Inis Amador Paternina, l'un des organisateurs responsables du retour des corralejas à Sincelejo, et Horacio Contreras, l'inspecteur en charge de contrôler la construction de la corraleja, affirment que « la fête ne peut pas mourir malgré la tragédie, c'est la tradition religieuse, c'est la vie de Sucre, unie à sa culture et à son économie ${ }^{80}$. On retrouve autour des corralejas cette alliance des composantes culturelle et matérielle de la vie locale. Les six jours de fête représenteraient une activité économique de 10.000 millions de pesos et généreraient 2.000 emplois, activité dont la ville ne pourrait plus se passer. Dernier épilogue de la consolidation des fiestas en corralejas de Sincelejos, elles sont désormais intégrées aux routes touristiques de la Costa Atlántica. C'est en effet la destination finale des trois routes touristiques pour découvrir la région Caraïbe, mises en place par le ministère du Commerce, de l'Industrie et du Tourisme dans le cadre de son programme "Vive Colombia, Viaja por ella " (Vis la Colombie, voyage à travers elle). Les corralejas de Sincelejo, que les médias européens et nord-américains commencent à relayer comme l'une des fêtes les plus exotiques du continent, sont devenues par l'intermédiaire du tourisme l'une des opportunités de désenclavement d'une région qui demeure marquée par une forte marginalité. Cette opportunité cherche à renverser les héritages d'une situation intermédiaire mal desservie entre les villes littorales de Cartagena, Barranquilla et Santa Marta et les métropoles andines. Du point de vue de l'afición, les corralejas offrent l'image d'une fête primitive, une tauromachie de la périphérie, entre les fêtes civilisées des Andes et du littoral. L'originalité de Sincelejo est de posséder à la fois la tradition des corralejas et des corridas de taureaux, occupant un rôle et une position analogue à celle de Villavicencio entre Andes et Llanos. D'après

${ }^{80}$ Colombia Universal, 28 janvier 2005. 
Pepe Alcázar, « de même que les arènes de Sogamoso sont nées de la grande afición des llaneros pour leur chevaux et leur taureaux, de même Sincelejo intégra à ses coutumes cette tragicomédie des corralejas qui donna naissance aux arènes ${ }^{81}$. À Sincelejo se superposent ainsi deux fêtes taurines qui s'opposent et se complètent, chacune offrant en quelque sorte à l'autre, à l'échelle locale, le miroir de sa distinction. Mais à l'échelle nationale, les corridas de Sincelejo sont parfaitement insignifiantes et anonymes, alors que les fiestas en corralejas identifient fortement la ville dans tout le pays.

\section{IV. — L'ESPACE TAUROMACHIQUE VÉNÉZUÉLIEN : COLEO ET CORRIDA}

Le Venezuela est l'autre grand pays du coleo où il ne se contente pas d'être un divertissement régional comme en Colombie, mais une fête et un sport national pratiqué dans tous les États vénézuéliens (voir carte 17, p. 365). À quelques exceptions locales près, les corridas traversent quant à elles une période de faible dynamisme. Pour ces deux raisons, le Venezuela et la Colombie forment une sorte de miroir inversé. Les corridas sont essentiellement localisées dans les grandes villes des cordillères andine et centrale. Les deux pratiques coexistent ainsi sur une partie du territoire, mais n'en demeurent pas moins deux univers faiblement articulés. Ici transparaît sans doute mieux qu'en Colombie, l'image d'une corrida fortement attachée à la fête espagnole et d'un coleo davantage représentatif d'une fête nationale.

\section{LES CORRIDAS :}

UN PRESTIGE ANCIEN, UNE PRATIQUE EN DÉCLIN

Le Venezuela est généralement considéré comme un pays où les grandes heures de la tauromachie sont révolues, en particulier celles des grands toreros de réputation mondiale, tel César Girón qui triompha en Espagne dans les années 1950. Malgré quelques férias d'importance, beaucoup d'observateurs soulignent la mauvaise passe dans laquelle se trouvent les courses de taureaux au Venezuela. Elles ne semblent plus déplacer les foules et font régulièrement l'objet de critiques, internes au monde taurin et extérieur à lui. Les chiffres qui nous ont été transmis par le journaliste taurin Raúl Gordon Blasini donnent sur la période 2001-2004 une moyenne annuelle de 95 spectacles taurins. Les statistiques de Mundotoro, affichent pour la même période une moyenne annuelle de 62 spectacles majeurs.

À la différence des autres terres de corridas d'Amérique latine, les spectacles majeurs ne sont pas relayés par un important volet de courses taurines populaires, plus ou moins conventionnelles et plus ou moins subversives, qui

\footnotetext{
${ }^{81}$ Pepe Alcázar, Plazas de toros de Colombia, p. 163.
} 


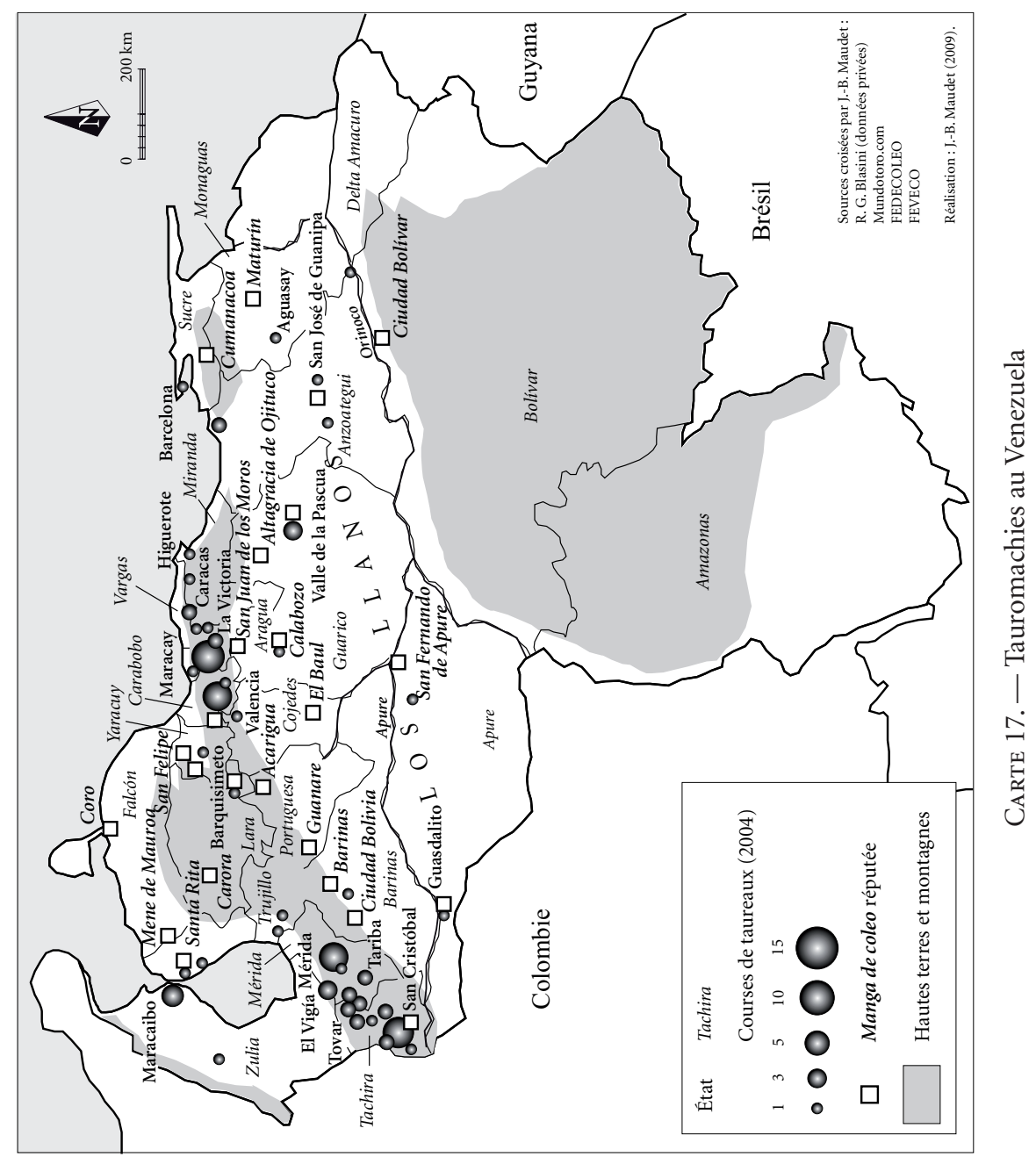


élargit la base de leur popularité. La corrida reste une affaire de la ville, plutôt de la grande ville et d'une élite réduite. Pour autant il ne faut pas conclure à un irrémédiable déclin de la pratique puisque le nombre de spectacles, malgré des variations interannuelles plus importantes qu'ailleurs, reste stable sur les deux dernières décennies. En outre, le Venezuela possède trois férias d'importance, celles de San Cristóbal, de Mérida et de Valencia, où se rendent les grandes figures de la tauromachie mondiale. Les arènes de ces villes taurines possèdent d'ailleurs des dimensions très impressionnantes : celles de Valencia ont longtemps possédé 26.000 places, celles de San Cristóbal contiennent 22.000 spectateurs et celles de Mérida 17.500. Preuve de leur dynamisme, les arènes de Valencia ont été entièrement rénovées en 1998 et repensées pour accueillir également d'autres types de spectacles. Pour des raisons de confort, leur contenance a été réduite à 15.000 places. Les arènes se trouvent au milieu d'un parc de 70 ha qui possède d'autres installations fériales dont une piste de coleo. On peut observer le contraste entre les transformations des arènes de Valencia et le sort du Nuevo Circo de Caracas qui ferme ses portes en 1997, mais dont la pérennité architecturale est néanmoins assurée par l'Institut du patrimoine qui a fait de l'édifice un Bien d'intérêt culturel. Son propriétaire Rafael Branger avouait en ces années que l'édifice, inauguré en 1919, coûtait moins cher fermé qu'ouvert au public pour offrir des courses de taureaux. Après cinq années d'absence, de 1998 à 2004, des corridas sont à nouveau programmées dans la capitale et ont désormais lieu dans la salle de spectacle du Poliedro de Caracas. La métropolisation de la capitale n'est pas allée dans le sens d'un développement corrélatif d'une offre de loisir taurin de rang international. Un âge d'or de la tauromachie situé dans les années 1950, une capitale qui n'est plus une ville taurine d'importance et un écroulement de la monnaie depuis le milieu des années 1980, accentuent l'impression générale du déclin vénézuélien de la passion taurine.

La répartition des arènes et des spectacles en 2004 fait clairement apparaître le poids des cordillères dans la localisation des courses de taureaux (voir carte 17, p. 365). Les arènes sont principalement réparties en deux ensembles géographiques disjoints : la cordillère de Mérida et la cordillère littorale. Les États de cordillère de Mérida (Táchira, Mérida, Trujillo) et ceux de la cordillère littorale (Carabobo, Aragua, Miranda, Distrito Federal de Caracas) concentrent $80 \%$ des courses de taureaux. Cette répartition donne une plus-value relative à la densité taurine de la cordillère de Mérida, car près de $50 \%$ de la population vénézuélienne se situe dans la cordillère littorale. Entre San Cristóbal et Mérida, on observe une forte concentration d'arènes permettant de délimiter un pays taurin local de première importance à l'échelle de la zone andine, tout à fait comparable à celui qui s'étend en Colombie entre les départements de Cundinamarca et de Boyaca. Dans cette région se concentrent également nombre d'élevages. Sur les trente-deux élevages de race brave que compte le pays, une dizaine se situe entre les capitales de Mérida et du Táchira. Cette région est aussi celle où l'héritage colonial est le plus visible dans l'architecture et les traditions. La place des courses de taureaux confirme ici la présence de cet héritage. 
Nous avons retrouvé la trace d'une centaine d'arènes au Venezuela dont une cinquantaine fonctionne régulièrement. Sur la centaine d'arènes existantes et qui ont existé dans un passé proche, le poids des Andes vénézuéliennes et de la cordillère centrale n'est pas remis en question puisqu'elles rassemblent $70 \%$ d'entre elles (voir annexe V [CD-ROM]). La cordillère de Mérida, a elle seule, compte 49 arènes. Cela étant, on observe que les llanos et la costa ne sont pas des zones taurines négligeables, ce que confirme encore aujourd'hui l'existence de courses de taureaux dans ces espaces. Sur la côte, Maracaibo et les villes de sa région métropolitaine telles que Ciudad Ojeda et Santa Rita forment un foyer taurin secondaire. Dans l'immensité des llanos se dispersent quelques lieux de courses de taureaux, généralement dans des villes de relative importance : Guasdalito (Apure), Valle de la Pascua (Guárico), San José de Guanipa (Anzoátegui), Aguasay (Monagas). Les arènes démontables aident à ce redéploiement des spectacles taurins à l'instar des arènes « La Maestranza de Barcelona » qui circulent dans l'État d'Anzoátegui. Enfin, le milieu des années 1960 correspond à une période de dynamisme des activités taurines avec notamment l'inauguration de nombreuses arènes et non des moindres : La Monumental de San Cristóbal en 1965, la Alternativa de Barquisimeto et la Monumental de Mérida en 1967, la Monumental de Valencia, les arènes de Ciudad Bolívar et de Puerto Cabello en 1968. Ces années correspondent à une période de stabilité politique, de démocratisation et d'augmentation de la rente pétrolière qui permettent de traduire dans la pierre ce que la période faste de la décennie antérieure avait semé comme afición.

Le marché taurin n'est sans doute pas assez étoffé pour distinguer les arènes de temporada des arènes de féria. Remarquons néanmoins que Valencia et Maracay possèdent plusieurs rendez-vous taurins et ajoutent à leur féria des corridas régulièrement programmées dans l'année. Valencia célèbre une Corrida de la municipalité en mars, une course en juin et une féria de trois ou quatre courses en novembre, la Feria de la Naranja (la féria de l'Orange). Maracay célèbre une Corrida de la municipalité en février, une féria de trois ou quatre courses en mars pour la San José et plusieurs courses d'octobre à novembre. Pour cette raison, on considère souvent ces deux villes comme les plus taurines dans la mesure où il existe des rendez-vous réguliers en dehors des férias, qui témoignent de la présence d'une afición locale permanente. Ajoutons que Valencia et Maracay possèdent chacune une école taurine de même que Mérida et San Cristóbal. Ces deux dernières villes présentent un profil différent qui se rapproche davantage du modèle des arènes de féria. La Feria del Sol de Mérida, créée en 1969, également appelée « le Carnaval taurin d'Amérique », constitue l'une des plus importantes dates festives du Venezuela. Mérida, ville de 200.000 habitants également nommée la "ciudad de los caballeros ", est très réputée pour son architecture coloniale et constitue l'un des centres touristiques les plus importants du pays $^{82}$. La Feria del Sol est une puissante image de marque de la ville durant

\footnotetext{
${ }^{82}$ La traduction nécessairement imparfaite de caballeros ne peut retranscrire les connotations hispaniques du mot qui renvoient tout à la fois à l'homme opposé à la femme, à l'homme à cheval, à la noblesse du chevalier et au gentleman.
} 
laquelle sont négociées de nombreuses affaires extra-taurines. À San Cristóbal, la Feria Internacional de San Sebastián joue un rôle analogue et peut se prévaloir de l'origine ancienne d'une foire de l'époque coloniale. Les ferias y fiestas de San Sebastián changent de nom et deviennent la féria internationale de SaintSébastien en 1965 avec l'inauguration des arènes de la Monumental de Pueblo Nuevo et du complexe férial baptisé « Pavillon de la Colombie et du Venezuela ». C'est à cette époque que la tauromachie devient l'une des principales vitrines de la Féria. Pour revenir à la féria de Valencia, observons qu'elle s'inspire très largement du modèle sévillan. On y retrouve un champ de foire où se succèdent les casetas qui font la renommée des férias andalouses, mais on passe abondamment des musiques sévillanes mélangée à de la salsa. On y retrouve aussi la porte illuminée qui marque le seuil d'entrée du champ de foire. Les références à l'Espagne et à l'Andalousie y sont omniprésentes.

Dans l'histoire de la tauromachie vénézuélienne nombreux sont les matadors originaires de Maracay ce qui vaut à la ville d'être un lieu important de transmission de la passion taurine : Pedro Pineda, les cinq frères Girón, Iván Rodríguez Vázquez, José Nelo " Morenito de Maracay », Alfonso Rondón, Alfredo Sánchez, Manolito Rodríguez, Carlos Rodríguez « El Mito », Juan Alfaro. Le Venezuela a produit environ 115 matadors d'alternative, chiffre plus élevé qu'en Colombie qui est néanmoins en train de rapidement combler son retard sur son voisin ${ }^{83}$. La figure de César Girón se détache entre toutes en raison de l'importante carrière qu'il fit en Espagne dans les années 1950, en ouvrant six fois la grande porte des arènes de Las Ventas à Madrid, en coupant par deux fois deux oreilles et la queue dans les arènes de La Maestranza de Séville en 1954. Aujourd'hui les arènes de Maracay portent le nom de celui par qui s'est épanouie l'afición vénézuélienne. Au Venezuela, il est difficile de ne pas voir les matadors qui l'ont suivi comme les héritiers de César Girón. En 2004, sur les 60 matadors à avoir toréé au Venezuela, on compte 27 Vénézuéliens, 18 Espagnols, 6 Colombiens, 5 Mexicains, 1 Équatorien, 1 Péruvien, 1 Portugais et 1 Français. Il s'agit donc d'une terre taurine largement pénétrée par les toreros des autres pays, ce qui ne manque pas de signifier, au-delà d'une idée flatteuse qui serait celle d'un carrefour taurin international, que le pays manque de toreros du cru.

Aujourd'hui, les courses de taureaux sont menacées au Venezuela par un projet de loi déposé à l'Assemblée nationale, en discussion depuis 2007, qui propose que « toute production de spectacles privés ou publics, tels que cirques, fêtes populaires, fêtes taurines, qui par nature peuvent occasionner à un animal douleurs, humiliations, vexations, souffrances, tortures, mauvais traitement ou mort, doivent être supprimés définitivement, sanctionnés par la présente loi devant les organismes compétents $»^{84}$. Un article du texte précise que "les corridas et les toros coleados sont autorisés, sans torturer, martyriser, ni tuer les taureaux, ainsi que les autres animaux prenant part aux spectacles. [...]

\footnotetext{
${ }^{83}$ F. López Izquierdo, Los Toros del Nuevo Mundo, pp. 309-313, et données personnelles de Raúl Gordon Blasini.

${ }^{84}$ El Nacionalista de Guárico, 4 mai 2007.
} 
Ces spectacles continueront de faire partie de la mosaïque vénézuélienne des fêtes traditionnelles seulement s'ils respectent rigoureusement les dispositions établies par cette loi ${ }^{85}$. Il va de soi que ce projet est rejeté en bloc par les professionnels taurins et les aficionados qui tentent de faire entendre leur voix en organisant des débats. Pour cela, ils ont créé une plate-forme de défense de la fiesta brava, sur le modèle de celle mise en place en Espagne pour tenter de protéger la tauromachie à Barcelone. À l'Assemblée nationale, les rangs sont divisés. Luis Tascón, député de l'État de Táchira, pourtant considéré comme l'un des États les plus unanimement taurins du pays, est paradoxalement à l'origine de ce projet de loi. Ainsi confie-t-il ces commentaires au journaliste Humberto Márquez de l'agence de presse IPS en avril 2007 :

Nous savons que c'est difficile d'accepter cette loi qui touche à une fibre culturelle très ancienne que nous héritons de l'Empire romain, mais si nous voulons préserver la planète, nous devons aller contre la culture qui consiste à faire souffrir les animaux, qui fait que l'espèce humaine affronte d'autres espèces. La loi s'oppose à des intérêts économiques parce qu'il s'agit de spectacles élitistes, pour les riches, le peuple ne va pas aux corridas, ni aux toros coleados, ni aux combats de coqs, ce ne sont pas des passions populaires, le basebol est populaire et surtout le football ${ }^{86}$.

Et Tascón d'ajouter : « la violence inutile envers les animaux contredit l’idéal socialiste ». Il semble que les erreurs historiques quant à l'origine des jeux taurins, qu'il s'agisse de la corrida ou des toros coleados n'ont d'égal que l'angélisme simplificateur des préoccupations environnementales. Quant à l'analyse de ce qui est populaire, elle reste très difficile à mener dans un pays où depuis vingt ans la croissance des inégalités et la décroissance des revenus par habitant est constante faisant du Venezuela le pays où le taux de pauvreté est le plus important d'Amérique latine ${ }^{87}$. Si la corrida peut à beaucoup d'égards être considérée comme un spectacle cher et élitiste, cette affirmation est irrecevable pour les toros coleados et les combats de coqs. Enfin, pour ce qui est de "l'idéal socialiste », dans le contexte vénézuélien, nous laisserons trancher Hugo Chávez qui aura le fin mot de l'histoire. À la vision de Luis Tascón s'oppose celle du député de l'État de Guárico, Eustoquio Contreras, qui affirme que «si le peuple ne va pas jouer au golf, il accompagne les fêtes des toros coleados et les combats de coqs » et critique les collègues de Tascón en soutenant qu'il existe partout de nombreuses formes de violences envers les animaux et d'ajouter : « comment savent-ils que tuer une vache ou un cochon pour les manger ne fait, en revanche, pas partie

\footnotetext{
${ }^{85}$ Ibid.

${ }^{86}$ Propos recueillis par le journaliste Humberto Márquez pour l'Agence de Presse IPS (Inter Press Service) dans un article intitulé « Peligran las tradicionales corridas de toros », daté du 26 avril 2007, mis en ligne sur le site Internet de l'agence $:$ http://ipsnoticias.net/nota.asp?idnews $=40770$ [réf. 10 juin 2007].

${ }^{87}$ F. RODRíGUEZ, « Factor shares and ressource booms ».
} 
de ces violences ». Le débat prend des formes assez semblables à celui que nous connaissons dans le Sud-Ouest européen.

LES TOROS COLEADOS : JEU DES LLANOS, SPORT NATIONAL ET MYTHE DU LLANO

Les llanos vénézueliens s'étendent sur sept États, désignés sous l'appellation des États llaneros: Apure, Barinas, Portuguesa, Cojedes, Guárico, Anzoátegui, Monagas. Ils couvrent environ $30 \%$ du territoire et contiennent $10 \%$ de la population. C'est dans ces États que la pratique du coleo est la plus développée, mais à la différence de la Colombie, il existe des clubs, des mangas de coleo et des associations fédérales dans la plupart des États extérieurs aux llanos (voir carte 15, p. 350). Les mangas de coleo réputées et celles qui accueillent les compétitions importantes sont réparties sur le territoire de façon plus homogène que les grandes férias et arènes de courses de taureaux concentrées dans les cordillères. La Fédération veille d'ailleurs à ce qu'un maximum d'États participe aux compétitions.

En 2007, le calendrier prévu par la Fédération programme des compétitions dans une quinzaine d'États, précisément dans les villes de San Cristóbal (Táchira), de Valle de la Pascua (Guárico), Maturín (Monaguas), Papélon (Portuguesa), Ciudad Bolívar (Bolívar), Barinas (Barinas), Guanare (Portuguesa), Cunamacoa (Sucre), Ciudad Bolivia (Barinas), San Juan de los Moros (Guárico), Coro (Falcón), San Fernando de Apure (Apure), San Felipe (Yaracuy), Guasdalito (Apure), San Carlos (Cojedes) [voir carte 17, p. 365]. Pour la Fédération, la déclaration officielle de 1981 qui fait du coleo un «sport criollo et national » n'est pas un vœu pieux. Elle se traduit par une distribution territoriale des compétitions en rapport avec la dimension spatiale de cet objectif. En outre, depuis 1997, les coleadores doivent porter des chemises de différentes couleurs pour identifier leur provenance, dans les compétitions par équipes, comme dans les compétitions individuelles. La pratique du coleo évolue vers une structure compétitive qui intègre les enjeux de l'identification, de la différenciation et de l'appropriation territoriale du sport.

Aujourd'hui, tous les États participent au classement du championnat et les huit premiers sont qualifiés pour la coupe de la Fédération. En 2006, les États qualifiés par ordre de classement étaient ceux du Guárico, de Portuguesa, de Monagas, de Trujillo, du Distrito Capital de Caracas, de Cojedes de Carabobo et de Yarucuy, soit des États appartenant aux trois grands ensembles territoriaux du pays : la Costa, la Sierra et les Llanos. L'État du Guárico est considéré comme le meilleur et l'un des plus fervents. Jesús Aguilera, baptisé « coleador du millénaire » (excusez du peu) ou "général du coleo ", qui a gagné onze titres individuels de champion national et deux championnats du monde, est originaire de l'État de Guárico qui en fait tout un symbole. On peut observer que l'État de Guárico est situé au sud de la cordillère centrale avec laquelle il a longtemps fonctionné de pair lorsque les découpages coloniaux avaient pensé l'organisation territoriale en unité transversale au milieu géographique, de la Costa aux Llanos. L'État de Portuguesa fait également bonne figure en termes de prestige et de palmarès. En 
tout, la Fédération déclare 20.000 compétiteurs et 60.000 pratiquants amateurs, quelque 1.500 clubs, 1.200 mangas de coleo et, s'il fallait finir de démontrer l'importance de la pratique, plus de 300 journées de coleo par semaine.

Ces chiffres en disent assez pour faire du coleo au Venezuela un spectacle de masse. Dans les villes, le coleo demeure une activité réservée à une élite pouvant financer l'entretien des chevaux, dans les llanos où il existe une culture équestre plus populaire, le coleo touche une base sociale plus large. On y pratique couramment des toros coleados populaires lors des fêtes patronales. La Fédération fait également valoir qu'il s'agit du sport qui attire le plus de monde lors des Jeux nationaux, depuis 1961, sans nous donner aucun chiffre à ce sujet. Quoi qu'il en soit, le travail de la Fédération depuis sa création en 1959 a incontestablement permis le développement et une meilleure visibilité de la pratique. Marco Barrios, directeur général de l'Association d'information et de promotion «torocoleados » qui réunit plus de 20.000 membres à travers le Venezuela et qui est elle-même organisatrice de rencontres, mesurait ainsi, à la fin de l'année 2003, tout le chemin parcouru :

Je me sens très fier de la tâche réalisée jusqu’à présent, la communauté (toroscoleados) est parvenue à créer une véritable tribune d'opinion qui autrefois n'existait pas, elle a permis que le monde du coleo se connaisse davantage et elle a contribué à ce que des individus qui jusqu'à 2003 confondaient une course de taureaux avec une après-midi de coleo, assistent à d'innombrables journées de toros coleados et deviennent des fans ${ }^{88}$.

Il est significatif que les progrès de l'institutionnalisation du coleo à partir des années 1960 marquent un âge d'or des courses de taureaux qui, elles, entameront par la suite un déclin relatif, comme si les chronologies se croisaient dans une pente inverse.

Le coleo représente davantage encore. Derrière l'institutionnalisation d'un sport national apparaissent les inflexions des identités territoriales. Le coleador est la figure sportive héritière du llanero, l'habitant des llanos. Le llanero est par définition au bon coleador au même titre que l'homme de l'Ouest américain doit être un bon cavalier. Les llaneros au Venezuela sont un symbole fort de l'histoire nationale. Ils tirent leur prestige ambigu du rôle militaire qu'ils ont tenu dans les guerres de libération, faisant basculer les batailles d'un côté à l'autre en fonction de leur ralliement. Dans l'historiographie du Venezuela, les llaneros sont des protagonistes de premier plan de la geste indépendantiste du $\mathrm{xIX}^{\mathrm{e}}$ siècle $^{89}$. Les llaneros participent activement à la guerre dans un camp comme dans l'autre et les llanos sont le théâtre principal des opérations qui voient finalement triom-

\footnotetext{
${ }^{88}$ Propos de l'éditorial de 24 décembre 2003 tenu par Marco Barrios, directeur de l'Association de promotion du coleo et entreprise des spectacles Toroscoleados.com. Disponible sur les site Internet de l'association : http://toroscoleados.com $/ \mathrm{html} /$ modules.php?name $=$ News\&file=print\&sid=\&à [réf. du 10 juin 2007].

${ }^{89}$ V. RAGO, « Llano y llanero ».
} 
pher les Patriotes sur les Réalistes fidèles à la Couronne d'Espagne. Les llaneros sont dans leur immense majorité des pardos, terme qui regroupe les non-blancs, principalement des Métis, des Noirs descendants des esclaves et des Indiens qui représentent en tout $2 / 3$ de la population vénézuelienne. Un personnage clef de l'indépendance du Venezuela qui concentre en une même substance les caractères de la nationalité héroïque, des llanos et des llaneros, est le général en chef José Antonio Páez surnommé "le centaure des llanos». Il passe pour avoir su rassembler les forces indomptées et anarchiques des llaneros autour d'un destin patriotique $^{90}$. Il consomme la séparation avec la Grande-Colombie et est élu président constitutionnel du Venezuela en 1831. C'est à cette époque que les llaneros passent au premier plan de l'historiographie et que les llanos (au pluriel et en minuscule) deviennent le Llano (au singulier et avec une majuscule) ${ }^{91}$. Le Llano s'impose comme une terre mère patriotique et soude l'association entre les llaneros et l'imagerie nationale. Dans la langue des troupes de llaneros, il est intéressant de souligner que se battre contre les Espagnols se disait alors « aller aux taureaux $»^{92}$. Bien qu'il soit difficile de tirer toutes les conséquences de cette expression, il témoigne d'un arrière-fond tauromachique où les Espagnols et les courses de taureaux sont clairement associés.

Au-delà des guerres d'indépendance, la rudesse des éléments naturels du Llano, à commencer par les taureaux sauvages et les caïmans, deviennent les nouveaux stéréotypes d'une identité vénézuélienne forgée grâce à la vaillance insoumise d'un peuple à cheval. La position des pardos se trouve rehaussée, en tous les cas pour ceux issus du métissage. L'affrontement avec la nature du Llano devient un élément fédérateur de leur identité sans doute moins problématique que leur origine ethnique et géographique diverse, de même que leur rôle ambivalent dans l'histoire nationale. Comme dans le cas des cow-boys de l'Ouest, des vaqueiros du Nordeste et des gauchos de la Pampa, on observe que la référence à la nature locale, déterminante et pourvoyeuse d'identité, est une des voies du consensus social et identitaire. Elle permet en quelque sorte de (re)-naturaliser des groupes sociaux qui ont pu être, dans leur histoire, des composantes marginales et mal intégrées. L'historien Hobsbawm, dans ses recherches sur les bandits, voit dans la singularité des costumes de ces figures sociales la trace de cette dissidence :

Le costume du vaquero, c'est-à-dire du vacher mexicain, qui est devenu dans les Westerns le costume classique du cow-boy et les styles plus ou moins équivalents des gauchos et des llaneros dans les plaines d'Amérique du Sud, des bétyars dans la putza hongroise, des majos et des flamencos en Espagne, sont dans le monde occidental, des symboles analogues d'insoumission $^{93}$.

\footnotetext{
${ }^{90}$ T. Clément, « Puissance et souveraineté ».

${ }^{91}$ V. Rago, «Llano y llanero », p. 4.

92 T. CLÉment, « Puissance et souveraineté ».

${ }^{93}$ E. Новsвашм, Les bandits, p. 28.
} 
Dans le cas du Venezuela, et comme on le verra pour le Brésil, cette évolution participe d'un mythe de la démocratie raciale, qui court jusqu'à aujourd'hui, selon lequel il existe une égale reconnaissance théorique des groupes ethniques et des couleurs de peau. Pour autant, le prestige des llaneros n'est pas sans être accompagné d'une crainte toujours vive à leur égard, car ils ont à plusieurs reprises participé à des foyers d'insurrection contre le pouvoir central ${ }^{94}$. Il va sans dire que dans l'histoire du coleo, José Antonio Páez est considéré comme le plus grand coleador du XIx ${ }^{\mathrm{e}}$ siècle dont le fils a dit qu'il fut « le premier cavalier d'Amérique du Sud et le plus parfait llanero de la République $»^{95}$. Dans ce contexte, l'appellation du coleador Jesús Aguilera " général du coleo » est très probablement une évocation déguisée du général José Antonio Páez, surnommé « centaure des llanos ", sans quoi on ne verrait guère toutes les raisons de l'emprunt à la rhétorique militaire. Le coleo actuel est donc riche de cette histoire nationale qui fait du coleador un descendant du llanero, faisant du sportif un descendant du guerrier.

On l'a vu, de même que les corridas, la pratique du coleo est aujourd'hui visée par le projet de loi de protection animale déposé à l'Assemblée nationale. Nous avons évoqué les positions d'un député de l'État de Guárico, Eustoquio Contreras, défavorable à ce projet de loi. Le gouverneur de l'État de Guárico, Eduardo Manuitt et le président de la Fédération de Coleo, Víctor Manuel Sarmiento, ont également fait connaître aux autorités leur inquiétude par une déclaration qui souligne que la Fédération, les maires, les gouverneurs, les associations, les professionnels, les employés agricoles liés à la pratique du coleo étaient unis pour défendre cette " activité sportive et de travail ${ }^{96}$. Parmi les points importants de cette déclaration pour notre propos, il convient de noter que le coleo n'est absolument pas perçu en continuité avec le monde des corridas, le gouverneur de l'État de Guárico allant jusqu'à affirmer qu'il n'était pas en désaccord avec cette loi, mais qu'elle ne pouvait être appliquée au coleo qui ne fait que reproduire le travail des champs et d'ajouter « cette loi abrupte relève d'une confusion avec les courses de taureaux $»^{97}$. Aux dernières nouvelles, Hugo Chávez, originaire des llanos, digne descendant de la "pardocratie ", guide du socialisme vénézuelien et défenseur de la nation, a garanti à la Fédération de coleo, par l'intermédiaire du vice-président de la République bolivarienne du Venezuela, Jorge Rodríguez, que « tant qu'il serait président le coleo national ne disparaîtra pas ${ }^{98}$. Hugo Chávez n’a fait aucune allusion aux courses de taureaux. Pour ce qui est du monde du coleo, il semble bien que les garanties personnelles apportées par Hugo Chávez aient désamorcé le projet de rassembler une " armée » de 15.000 coleadores aux portes de l'Assemblée nationale ainsi que projetaient de le faire les instances fédérales du coleo. Cet acte n'aurait pas manqué d'évoquer le rôle historique des llaneros entre geste patriotique et rébellion.

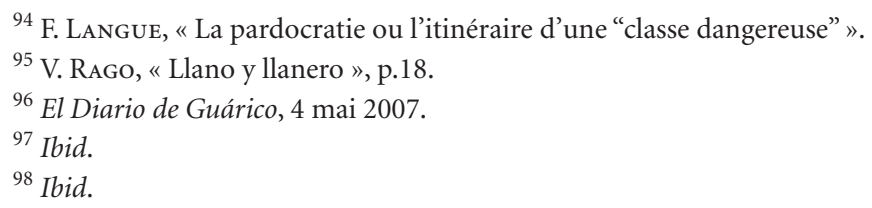




\section{V. - L'ESPACE TAUROMACHIQUE ÉQUATORIEN : CORRIDA, TOROS DE PUEBLO ET RODEO MONTUBIO}

L'Équateur a quasiment une valeur paradigmatique pour comprendre l'organisation et les dynamiques des espaces taurins des pays andins. Les villes taurines d'importance sont situées dans les bassins andins d'altitude, encadrées par deux cordillères où se multiplient les formes tauromachiques mineures et populaires (voir carte 18). Les pratiques tauromachiques relevant des courses de taureaux, sous une forme conventionnelle ou réinventée, concernent surtout la Sierra, même si les évolutions actuelles tendent à les faire déborder dans l'Oriente amazonien au rythme de sa colonisation. La Costa, quant à elle, témoigne d'une modalité locale de rodéo assez originale, le rodéo montubio, qui semble cumuler de nombreuses formes de subversions ludiques. La tauromachie participe nettement aux différenciations territoriales entre Costa, Sierra et Oriente comme si la taille réduite du pays contribuait à clarifier l'organisation.

Corrida, toros de PUEBLO ET RODEO CHAGRA :

LA DÉClinAison TAURINE DES ANDES ÉQUATORIENNES

D’un point de vue tauromachique, l'Équateur est un pays qui connaît actuellement d'importantes transformations. Les observateurs de la " planète des taureaux » en font un nain tauromachique qui ne doit sa renommée qu'à la Feria de Jesús del Grand Poder de Quito durant laquelle défilent au côté de quelques Équatoriens, les plus grands toreros du moment. Pour l'année 2004, les statistiques de Mundotoro ou de Portaltaurino enregistrent un total de 22 spectacles majeurs dont la moitié reviendrait aux seules arènes de Quito. Cette macrocéphalie taurine qui se justifie en termes de réputation est pourtant loin de représenter l'activité tauromachique réelle du pays. Lors de notre séjour sur place, la Unión de Toreros del Ecuador, en la personne de son président, le picador Hernán Tapia, estimait le nombre de spectacles majeurs à une soixantaine par an. Crayon en main, Hernán Tapia insiste pour nous expliquer qu'il est difficile de compter les courses comme en Espagne, puisque à cette soixantaine de corridas formelles, s'ajoute une quarantaine de courses mixtes qui comptent la participation de matadors et de novilleros. Au total, il faudrait compter de 200 à 250 courses de taureaux si l'on ajoute les spectacles composés d'une partie formelle courte et d'une partie ouverte au public, à l'image de ce que l'on a repéré au Yucatán. Ces courses populaires dont la plupart se déroule dans des arènes démontables sont parfois confondues avec les toros de pueblo (taureaux de village) improvisés dans des arènes de fortune. La déclinaison des pratiques taurines depuis les formes les plus conventionnelles jusqu'aux jeux les plus informels ne facilite pas un décompte précis. Sur la carte 18 (p. 375), pour tenter de ne pas fausser les comparaisons, nous avons conservé la série des spectacles majeurs et fait apparaître les villes taurines où nous sommes assurés de la programmation régulière de spectacles taurins avec picadors grâce aux informations de l'Union des toreros. 


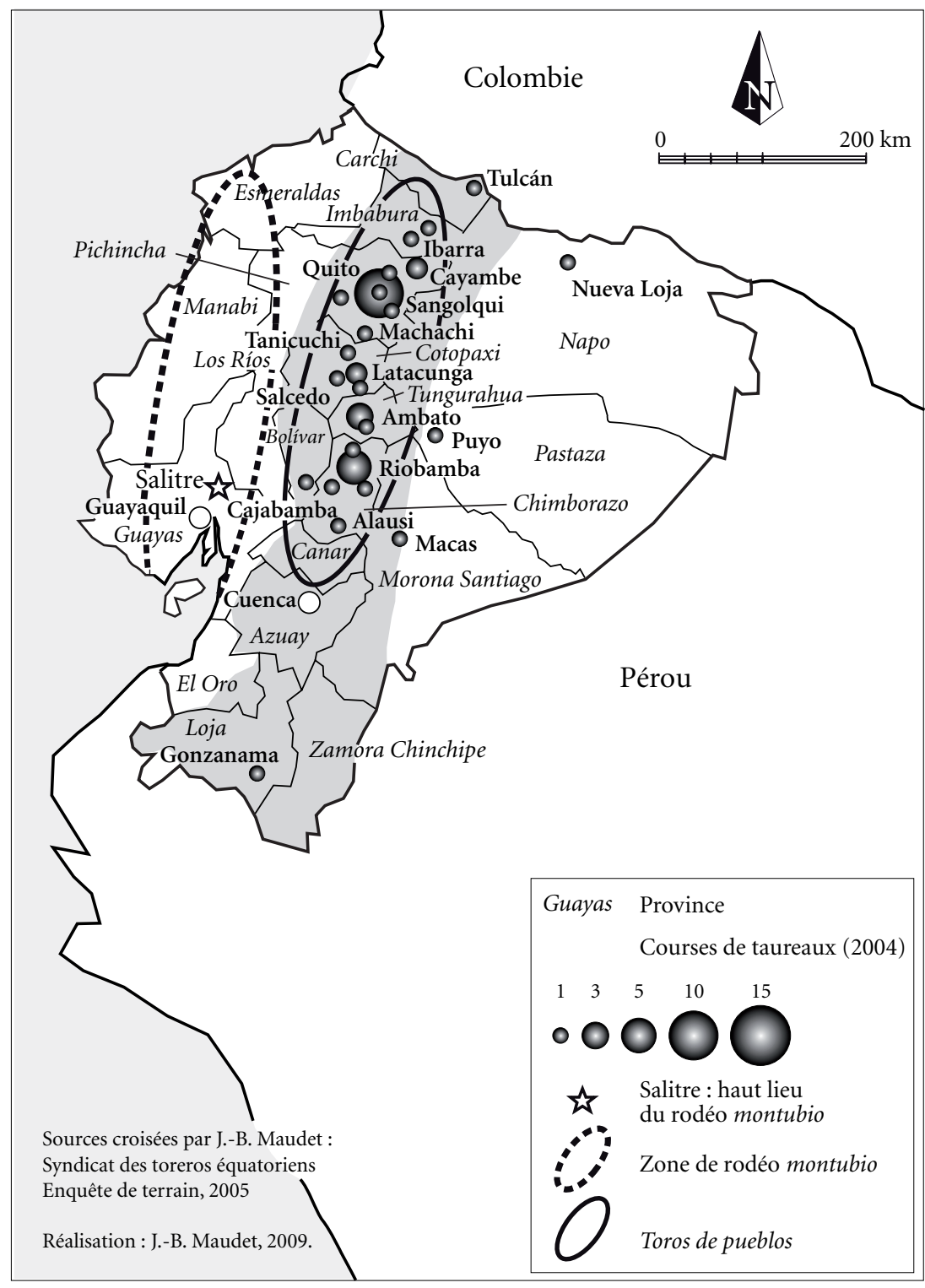

CARTE 18. - Tauromachies en Équateur

Il existe dans le pays une vingtaine d'arènes en dur. Le règlement sur les spectacles taurins de 1978 prévoit de les classer en fonction de leur contenance. Les arènes de plus de 12.000 spectateurs sont classées en première catégorie. Il n'en existe qu'une : les arènes de Quito avec 14.700 places. Les arènes de plus de 8.000 spectateurs sont classées en deuxième catégorie : Riobamba (11.000), 
Ambato (11.000) et Guayaquil (9.500). Les suivantes sont classées en troisième catégorie parmi lesquelles, pour ne citer que les plus grandes : Cuenca (7.500) aujourd'hui inactive, Ibarra (5.000), Salcedo, Tanicuchi et Cayambe (3.500). La répartition des arènes et des spectacles fait clairement apparaitre une organisation méridienne de l'espace tauromachique équatorien qui correspond à l'alignement des bassins intramontagnards d'altitude. Ces bassins sont séparés par des seuils à moins de 3.500 mètres et enchâssés entre deux cordillères parallèles dont les lignes de crêtes sont distantes de moins de 50 kilomètres. Le long de la route centrale qui traverse le pays de la frontière colombienne à la frontière péruvienne s'égrène donc l'immense majorité des arènes et des lieux de spectacles : Tulcán, Ibarra, Cotacachi, Cayambe, Pomasqui, Quito, Sangolqui, Machachi, Tanicuchi, Latacunga, Salcedo, Ambato, Guano, Riobamba, Chambo, Cajabamba, Alausi, Cuenca et Gonzanamá. Dans les arènes citées ont lieu plus de $80 \%$ des spectacles taurins. Cette organisation linéaire se double d'un clivage Nord-Sud puisque les provinces méridionales de Cañar, Azuay et Loja comptent peu d'arènes et de spectacles en comparaison des provinces de la moitié Nord. C'est aussi dans les Andes équatoriennes du Nord que se situent les principaux élevages de taureaux de combat puisque $80 \%$ d'entre eux sont concentrés dans les provinces de Pichincha, Cotopaxi, Tungurahua, Chimborazo, installés à une altitude qui varie entre 2.800 et 3.500 mètres $^{99}$. Le pays possède aujourd'hui quarante élevages inscrits dans l'Association des éleveurs de taureaux de combat d'Équateur qui assurent les besoins du marché national, à quelques exceptions près pour la féria de Quito qui parfois fait appel à des taureaux colombiens ${ }^{100}$.

À propos de la moitié nord des Andes équatoriennes, le géographe Jean-Paul Deler observe que « depuis plusieurs siècles, dans ce domaine étroit et fondamentalement anisotropique, la conjonction d'héritages pré-hispaniques, coloniaux et républicains a donné naissance à un véritable modèle d'organisation de l'espace, fondé sur une adéquation presque parfaite entre le bassin andin, unité naturelle aux aptitudes agricoles ouvertes grâce au gradient d'altitude, la province, circonscription administrative de premier rang de l'État, et l'aire d'attraction d'une ville centre, autour de laquelle gravite la vie économique et sociale locale $»^{101}$. Cette partie des Andes rassemble un tiers des Équatoriens sur à peine $5 \%$ du territoire avec des densités rurales couramment comprises entre 100 et 200 habitants au $\mathrm{km}^{2}$. Dans chacun de ces bassins se répète une même organisation spatiale des pratiques taurines pouvant tenir lieu de modèle taurin de la Sierra équatorienne septentrionale. Ce modèle a quelque valeur plus généralement pour les Andes :

— une capitale provinciale possédant une arène en dur et une féria taurine de trois à quatre jours de taureaux ;

\footnotetext{
${ }^{99}$ Estimation calculée à partir des chiffres et des adresses des fincas aimablement communiqués par l'Asociación de Criadores de Ganado de Lidia del Ecuador.

${ }^{100}$ En 2005, à Quito a été programmé l'élevage colombien Las Ventas del Espíritu Santo, appartenant au matador César Rincón.

${ }^{101}$ C. Batalllon, J.-P. Deler et H. Théry, Amérique latine, p. 269.
} 
- une à deux villes satellites proposant une à deux courses, dans des arènes en dur ou des arènes démontables, avec l'apparition de courses populaires et de toros de pueblo;

- des bourgs ruraux de fonds de bassin, bâtissant des arènes en bois ou louant des arènes démontables, pour de modestes courses et des toros de pueblo ;

— de nombreux villages d'altitude en bordure de l'étage des páramos où augmente la proportion de toros de pueblo à mesure que s'affirment les communautés indigènes et où apparaît la figure du chagra, paysan métis et gardien des troupeaux de bovins d'altitude des haciendas latifundiaires, au contact des deux mondes.

Cette organisation rend compte de la hiérarchie et de la différenciation graduelle des pratiques en fonction d'une opposition entre l'urbain et le rural, entre fond de bassin et hautes terres, entre le centre et la périphérie.

Pichincha, la province de la capitale semble exacerber ce modèle. Au centre, Quito possède les plus grandes arènes du pays et la seule féria de rang international. À une trentaine de kilomètres au Nord, se trouve la ville de Cayambe de 55.000 habitants qui possède des arènes fixes (3.500 places) et qui organise une féria de deux à quatre courses par an. À moins de dix kilomètres au sudest de Quito dans la vallée du Chillo, se trouve la ville de Sangolqui (70.000) qui dans l'orbite immédiate de Quito a connu une croissance récente, en partie due à l'afflux de population indienne. À la fin des années 1990, des arènes en dur de 3.000 places sont construites pour organiser une féria taurine de deux ou trois courses. Cela n'empêche en rien la construction d'une arène en bois, sur la place centrale, pour accueillir, une douzaine de jours durant, des toros de pueblo auxquels participent plusieurs milliers de personnes. Le plus grand rendez-vous de toros de pueblo à moins de 10 kilomètres de la féria de Quito rend compte d'un contraste très explicite qui exprime les oppositions complémentaires entre la tauromachie du Centre et la tauromachie des périphéries, entre la tauromachie urbaine et la tauromachie rurale, entre la tauromachie élitiste et la tauromachie populaire, entre une tauromachie Métis-créole et une tauromachie Métis-Indien. Lors de ces toros de pueblo, il est très fréquent de voir le bétail monté et toréé dans un mélange des genres populaires qui combinent à loisir les modalités ludiques.

Enfin, Machachi, à la tête d'un canton rural d'altitude de 25.000 habitants, organise une féria d'une course mixte et d'une novillada. Les courses sérieuses sont ici suivies d'une course comique et d'un toro-gol avec la participation du public (match de foot avec du bétail). À Machachi se déroule également le Paseo Procesional del Chagra organisé par l'Association de la confrérie du Chagra (ACOCHA). Le chagra est le nom porté dans les Andes du Nord par les paysans. Selon l'association, le défilé a pour fonction de représenter l'image populaire du chagra comme l'essence même des traditions métisses ${ }^{102}$. Il s'agit d'un défilé

${ }^{102}$ Revista Terra Incógnita, 18 juillet 2002. 
à cheval des chagras en habit traditionnel, le chapeau chacarero, le poncho, la huasca (branche utilisée pour guider les bêtes), les martingalas (bottes que portent les hommes en armes). Depuis peu, le défilé s'est enrichi de jeux consistant à poursuivre et manœuvrer des taureaux qui annoncent l'émergence d'un rodéo chagra présenté sous une forme ludique afin de faire revivre ou connaître le travail des bouviers. Depuis 2002, l'ACOCHA organise un nouvel événement, la "Fiesta del Toro del Páramo », qui consiste à lâcher des taureaux les uns après les autres le long d'une avenue barricadée sur environ 800 mètres. Les taureaux sont courus et toréés par des chagras à pied. Gustavo Moya, le président de l'association affirme qu'il ne s'agit pas d'une copie des corridas de San Fermín à Pampelune et d'ajouter : "Nous célébrons la mémoire de nos ancêtres chagras qui accompagnaient le bétail depuis les páramos jusqu’au village $»^{103}$.

Machachi organise évidemment des toros de pueblo, moyen par lequel s'affirme aussi l'identité chagra. À Machachi, on conserve la tradition de fixer des colchas sur le dos de l'animal. Les colchas sont des toiles tissées contenant des billets ou des pièces attachées au taureau que le public tente d'arracher. Les artistes peintres qui présentent leurs ouvres au marché de Quito disent que la coutume était très fréquente lors de la célébration du Corpus Christi dans les villages des communautés indigènes. L'anthropologue Paulo de Carvalho Neto, dans les années 1960, signale cette forme de jeu à Alausi dans le Sud de la province du Chimborazo ${ }^{104}$. Augusto Goicochea Luna signale la pratique encore existante au Pérou dans les années 1960, le terme de enjalma remplaçant celui de colcha. Enfin, le toro de la vincha dans la province de Jujuy en Argentine reprend cette même modalité.

Que l'on s'éloigne de la Sierra du Nord et l'organisation spatiale du fait taurin prend une physionomie quelque peu différente, les marges, qu'elles soient montagnardes ou non, accusant des dynamiques contradictoires. La partie Sud de la Sierra, bien que moins taurine, connaît une activité non négligeable, comme peut en témoigner l'inauguration des arènes de Gonzanamá en 2005, dans la province méridionale de Loja. Selon Hernán Tapia, grâce aux arènes démontables, le Sud (Azuay et Loja) rattrape partiellement son retard. L'arène démontable baptisée La Macarena, dont il est propriétaire, part chaque année en tournée dans le Sud pour une vingtaine de dates, de mars à novembre. À chaque date l'arène est généralement louée pour une course de taureaux et une course populaire ou un rodéo montubio. Il existe actuellement six arènes démontables qui sillonnent le pays et étoffent la trame des cités taurines. Ces arènes se nomment la Macarena, la Albán (du torero et imprésario Guillermo Albán), la Ecuatoriana, la Sevillana, la Vista Alegre et la Virgen del Cisne. Malgré cela, observons que Cuenca, la troisième ville du pays avec 500.000 habitants ne programme plus de courses de taureaux depuis que la nouvelle ordonnance municipale sur la protection des animaux domestiques et sauvages est entrée en vigueur fin 2004. La loi de

\footnotetext{
${ }^{103}$ Hoy, 29 novembre 2006.

${ }^{104}$ P. de Carvalho Neto, « Un caso de mestizaje cultural».
} 
protection qui visait également à résoudre les problèmes de contagion de rage canine interdit désormais la mise à mort des taureaux ainsi que toute effusion de sang, ce qui dans la pratique, s'est traduit par la disparition des corridas ${ }^{105}$. Les arènes démontables jouent également un rôle important dans la diffusion des courses de taureaux dans le piémont amazonien, en particulier à Puyo et Macas, capitales respectives des provinces orientales de Pastaza et de Morona-Santiago. Ici, le développement des courses de taureaux dans un secteur où elles étaient rares auparavant est le fait d'une colonisation du piémont oriental à partir des provinces de la Sierra, de la progression des fronts pionniers, de l'émergence de l'économie du pétrole et de la croissance de l'urbanisation qui participe à une transformation territoriale d'ensemble.

La Costa et surtout Guayaquil qui est la plus grande ville du pays avec 2,3 millions d'habitants, brillent par leur absence dans cette géographie des courses de taureaux. La «Perle du Pacifique » possède bien des arènes sur la commune excentrée de Durán, construites à la fin des années 1990, mais les spectacles se font rares alors que les manifestations anti-taurines gagnent en importance. En 2004, les corridas prévues ont même été annulées en raison, dit-on, de l'ampleur des manifestations. Ces manifestations reçurent cette année-là, le soutien de Silvana Ibarra, chanteuse romantique des années 1990, figure incontournable de la " presse people » et députée de la province de Guayas (Guayaquil) au Parlement national. Elle défraya la chronique en affirmant qu'elle obtiendrait un projet de loi d'interdiction de la tauromachie. En 2005, à l'initiative de l'entrepreneur et torero guayaquileño Guillermo Albán, les spectacles ont timidement repris dans la salle de spectacle polyvalente du Coliseo Voltaire Paladines Polo. Mais l'affluence reste fragile et les craintes d'une récupération de la question taurine à des fins électorales inquiètent de plus en plus les professionnels et les aficionados. C'est qu'en l'absence de Loi taurine nationale qui en garantirait la pratique, comme elle existe dans d'autres pays, le spectacle demeure exclusivement soumis aux autorisations municipales.

Le sort de Guayaquil et de Cuenca contraste avec celui de la capitale, Quito, où a été signée, en 2003, une ordonnance municipale qui engage les autorités dans la défense et la promotion des activités taurines ${ }^{106}$. À Quito la tradition taurine n'apparaît pas menacée et les professionnels taurins ont conscience que le meilleur rempart demeure la programmation d'une féria d'excellence qui associe les meilleurs toreros du moment et les figures locales de tauromachie équatorienne. La féria de Quito compte actuellement parmi les grandes dates du calendrier taurin américain. Dès l'arrivée à l'aéroport, de grands panneaux publicitaires annoncent les corridas de la féria comme nous ne l'avons vu dans aucune autre ville taurine. L'idée d'une féria taurine de rang international à Quito est née à la fin des années 1950 avec le projet de construire de nouvelles arènes plus grandes que celles de Las Arenas qui, avec ces 2.500 places,

${ }^{105}$ El Mercurio, 7 décembre 2004.

${ }^{106}$ Conseil Métropolitain de Quito, $n^{\circ}$ IC-2003-406 du 20 octobre 2003. 


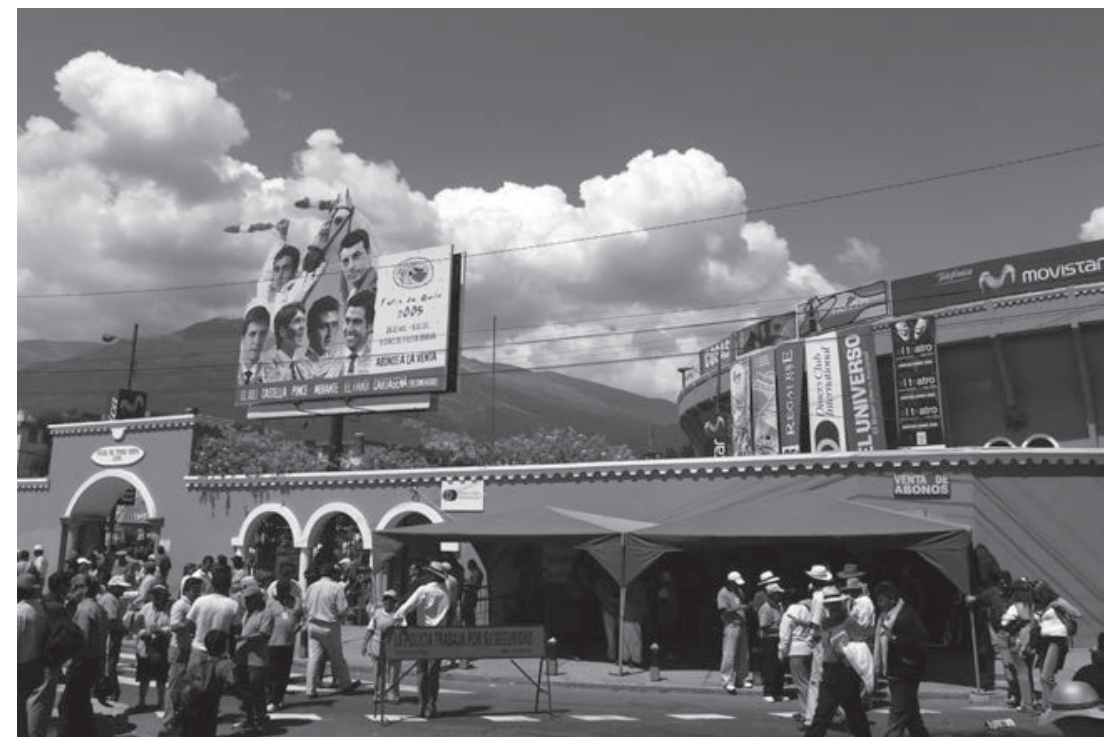

Fig. 53. - Plaza de toros de Quito (extérieur). Les toreros de la vieille Europe sont à l'affiche : «El Fandi », Andy Cartagena, «El Juli », Sébastien Castella, Enrique Ponce, Morante de la Puebla (Quito, 2005, cliché : Jean-Baptiste Maudet).

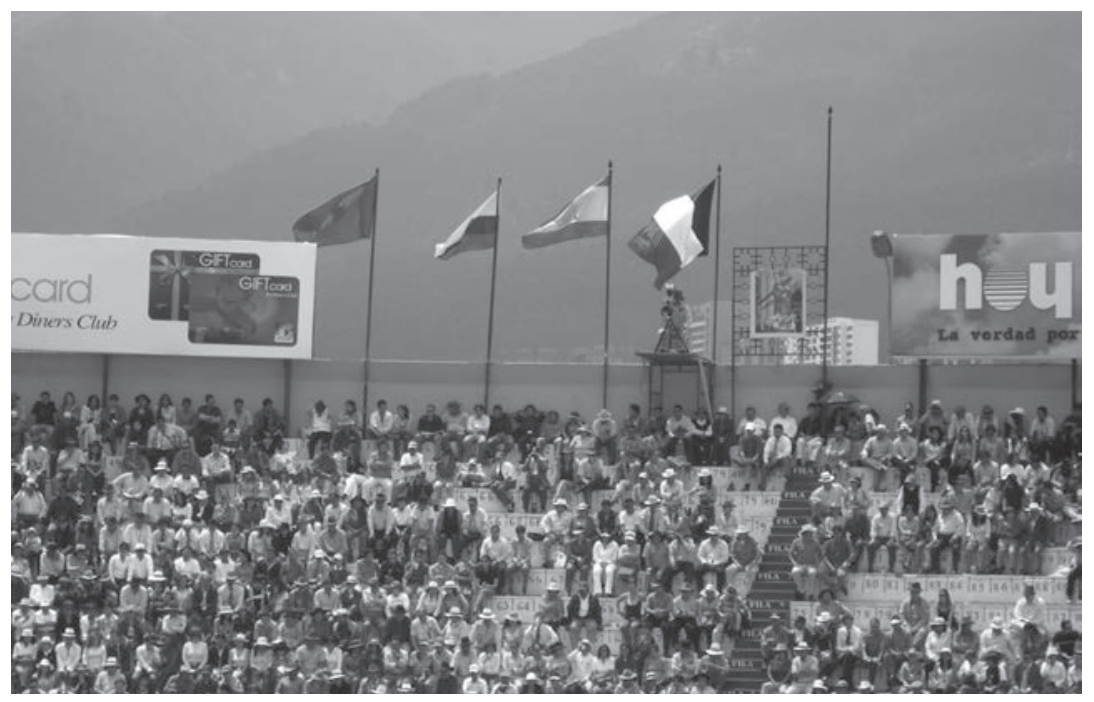

FIg. 54. - Plaza de toros de Quito (intérieur). Quatre drapeaux flottent sur les arènes : les drapeaux de Quito, de l'Équateur, de l'Espagne et de la France (Quito, 2005, cliché : Jean-Baptiste Maudet). 
permettaient difficilement d'attirer les grands toreros de l'époque. Le projet émane d'un groupe d'aficionados influents de la ville parmi lesquels des journalistes taurins, des directeurs de journaux (El Comercio, Últimas Noticias), le comte de Urquijo alors ambassadeur d'Espagne à Quito, Marco Tulio González, le président de la compagnie d'aviation « Ecuatoriana de Aviación », le directeur de la banque Pichincha et le maire de Quito. Les nouvelles arènes, contenant désormais près de 15.000 places, furent bâties en 1960 dans le quartier nord de la ville près de l'aéroport. La Féria est lancée la même année en vue de créer une étape supplémentaire dans la tournée des toreros espagnols en Amérique du Sud. À l'époque, entre Lima, Cali, Bogotá et Caracas, Quito souhaite se faire une place. La féria taurine baptisée Jesús del Gran Poder se greffe alors aux fêtes de Quito créées en 1958 pour célébrer l'anniversaire de la fondation de la ville. Fêtes et féria sont aujourd'hui indissociables.

C'est aussi la féria de Quito qui, d'une certaine manière, est responsable par son succès de la modernisation et du développement des élevages équatoriens. De 1960 à 1972, les élevages équatoriens représentaient environ 50 \% du bétail torée lors de la Féria ${ }^{107}$. En 1973, les arènes passent sous la direction d'entrepreneurs espagnols faisant chuter la demande en bétail national. Ils privilégient alors des bêtes importées d'Espagne et du Mexique qui sont exigées par les grands matadors de l'époque. En 1975, le pourcentage de bêtes équatoriennes toréées tombe à $5 \%$. En réaction à une évolution les menaçant, les éleveurs équatoriens fondent leur association professionnelle en 1977 qui, avec l'appui du ministère de l'Agriculture, obtient l'autorisation d'importer du bétail espagnol. S'ouvre alors une nouvelle époque pour les élevages remplaçant le bétail criollo par des produits issus de caste espagnole. En quelque sorte, la féria de Quito a tiré vers le haut l'ensemble de la filière taurine.

Aujourd'hui, tous les professionnels taurins équatoriens, éleveurs et toreros, cherchent à faire partie de la Féria. En 2005, il existait très précisément 67 professionnels de l'arène inscrits à l'Union des toreros dont 16 matadors d'alternative, 5 rejoneadores et 8 novilleros. La féria taurine de Quito a l'image d'une féria dynamique, enthousiaste et populaire auprès des jeunes. Des concerts sont organisés à l'issue des courses dans l'enceinte fériale où le public poursuit la fête une grande partie de l'après-midi. La féria attire les grands sponsors nationaux et internationaux qui personnalisent des campagnes publicitaires en relation avec la tauromachie. Tout ce qui se passe pendant la féria est relayé par les groupes de presse et les chaînes de télévision qui en font un événement national. Fort de ce succès, Quito s'est même dotée, depuis 2005, d'une deuxième arène avec la réouverture de la Plaza Belmonte située dans le centre historique de la ville. Les arènes avaient été inaugurées en 1920, puis elles sont tombées en désuétude en raison de la dégradation du centre ancien. Elles retrouvent aujourd'hui leur fonction dans le cadre du projet de rénovation urbaine du quartier de Tolia, en vue de renforcer les équipements culturels du centre. Les programmations de

${ }^{107}$ P. Espinosa et J. P. Espinosa, El toro de lidia en el Ecuador, p. 70. 
2006, dans le cadre des fêtes de Quito, se sont articulées autour d'un projet original, un Festival Taurino Nocturno Flamenco réunissant deux matadors andalous appartenant par excellence à la catégorie des toreros artistes : Morante de la Puebla et Javier Conde. Ils étaient accompagnés pendant leur prestation par un groupe flamenco espagnol. Une procession de la Vierge de Triana a même été organisée se terminant dans les arènes. Il est difficile de trouver hommage plus baroque à la tauromachie andalouse à plusieurs milliers de kilomètres de son berceau. Les affinités culturelles avec l'Espagne véhiculées pas la tauromachie sont ici manifestes.

\section{Loin DES ANDES, LOIN DU CEUR :}

LE RODÉO MONTUBIO

Les contrastes entre Guayaquil et Quito du point de vue des activités tauromachiques s'ajoutent aux nombreuses différences historiques et culturelles entre les deux grandes métropoles équatoriennes. À travers cette dyarchie urbaine et le pouvoir polarisant des deux métropoles, se jouent également les différences entre la Costa et la Sierra. Si les Andes sont caractéristiques de l'enracinement des courses de taureaux déclinées sous toutes leurs formes, la Costa témoigne du développement d'une forme locale de rodéo profondément marquée par la ruralité : le rodéo montubio.

Le terme de montubio possède plusieurs définitions. La plus simple est celle de paysan de la côte équatorienne qui en fait le pendant littoral des chagras montagnards. La plus élaborée fait du montubio une communauté sociale et ethnique caractéristique des provinces littorales issue d'un métissage entre Blancs, Noirs et Indiens. Les montubios habitent les provinces de Guayas, Los Ríos, Manabí et dans une moindre mesure les provinces plus excentrées de El Oro, au sud, et de Esmeraldas, au nord. Le terme possède une troisième définition, que l'on pourrait qualifier d'externe, qui fait du montubio un personnage grossier et rustre, tout ce que le cul-terreux ou le bouseux portent de stigmates en comparaison d'une urbanité blanche idéale. Cette définition fait pencher le montubio du côté du métissage le moins valorisé l'Indien et le Noir. Montubio et chagra sont deux catégories ethno-territoriales périphériques, qui se rejoignent dans leur distance au centre, celui d'une identité métisse idéale, comme blanchie par l'urbanité. Autrement dit, montubio et chagra sont aussi des termes utilisés par les classes dominantes pour désigner le Métis, trop indien, trop noir ou trop rural, les confondant ainsi dans une même identité. Enfin, il existe une définition interne du montubio qui en fait une communauté d'hommes d'honneur, libres, joyeux et robustes, vivant près de la nature, loin des corruptions du progrès. Dans une version érudite, selon Ubaldo Gil de l'université Eloy Alfaro de Manabí, les montubios seraient également les héritiers de la culture indigène manteña qui «ne s'est jamais soumise à la conquête espagnole, qui ne fut jamais esclave comme la culture andine, qui maîtrisait la navigation et le commerce, qui créa des hiérarchies sociales non pas pyramidales, mais horizontales reposant sur un cacique et ses conseillers, privilégiant non pas le sang et les liens 
familiaux, mais la connaissance et l'habileté de chacun ${ }^{108}$. On aura compris que la culture montubia est en quête de reconnaissance et de respectabilité au sein d'une identité nationale qui l'a jusqu'à présent fortement dévalorisée, alors même que le pays s'est construit autour d'une complémentarité entre la Costa et la Sierra, et qu'il s'est individualisé face aux voisins, Colombiens et Péruviens, à partir d'une organisation bipolaire Guayaquil-Quito. Rappelons que l'une des premières volontés des indépendantistes de Guayaquil, le 9 octobre 1820, fut de libérer la partie andine de l'audience de Quito au cri de « Guayaquil pour la patrie ! » par la formation d'une armée de la côte appelée «Unité protectrice de Quito » qui aboutira, en 1822, à la victoire de Pichincha sur les troupes Réalistes fidèles à la Couronne d'Espagne. Aujourd'hui, l'historien Wellington Paredes Ramírez, spécialiste des questions qui touchent au rôle et la place de Guayaquil dans le processus de construction nationale, parle à propos des montubios de la Costa d'une « ethnie socioculturelle invisible ${ }^{109}$.

Elle commence à acquérir une reconnaissance politique par l'intermédiaire du décret exécutif $\mathrm{n}^{\circ} 1394$ du 30 mars 2001 signé par le président constitutionnel de la République Gustavo Noboa Bejarano reconnaissant la création du Conseil national du développement du peuple Montubio de la côte équatorienne et des zones subtropicales du littoral (CODEPMOC) dont le siège principal est situé à Guayaquil. Cette évolution est à mettre en lien avec les mouvements indigénistes sociaux qui se développent plus généralement en Amérique latine. L’un des premiers combats du mouvement est une lutte lexicale qui entend modifier la définition du dictionnaire académique, calqué sur le Diccionario de la Real Academia Española dans lequel persistent les connotations péjoratives associées à l'adjectif montubio.

Les événements récents montrent que le débat n'est pas simple. Même si Guayaquil est au cour de la culture montubia, la ville de Salitre (50.000 habitants) qui a des allures de gros bourg rural spécialisé dans l'élevage bovin, située à une trentaine de kilomètres au Nord, s'affirme depuis le début des années 1950 comme un centre dynamique de cette culture en marche. Ici même aurait eu lieu, en 1953, le premier véritable spectacle de rodéo montubio organisé par l'Institut du folklore, du tourisme et de la culture de la ville. Salitre perpétue cette tradition en organisant de nombreux rodéos et des actes symboliques en lien avec la culture montubia parmi lesquels la fête du Montubio célébrée le 12 octobre, date de l'arrivée de Christophe Colomb sur le continent. En 2005, par une ordonnance municipale, le maire de Salitre, Julio Alfaro Mieles, a déclaré la ville «Capitale Montubia de l'Équateur ». Une plainte est aussitôt déposée par Luis Alvarado Macías, en qualité de procureur des membres du canton de Salitre, condamnant cette appellation en déclarant que " par caprice, par précipitation et par manque de bon sens, le conseil municipal de Salitre a promulgué une ordonnance municipale diffamante qui porte atteinte aux citoyens, les qualifiant

${ }^{108}$ El Comercio, 14 mars 2002. Conférence prononcée à la Maison de la culture équatorienne.

${ }^{109}$ W. Paredes Ramírez, Indios, problema indígna, regionalidad y localidad. 
du vocable péjoratif de montubio $»^{110}$. Le Tribunal constitutionnel a tranché la question modifiant légèrement l'article 2 de l'ordonnance municipale qui « exigeait » que toutes les institutions cantonales signent « Salitre, Capital Montubia del Ecuador». Faible modification, cette formule est désormais facultative. La réaction du président de la Fondation régionale de la culture Montubia, Sergio Cedeño Amador, est très instructive pour notre propos général. Il appuie la décision du maire et fait du qualificatif de montubio un honneur, « de même qu'être appelé charro au Mexique, llanero au Venezuela, gaucho en Argentine ou huaso au Chili $»^{111}$. Parmi ces qualificatifs, admettons qu'ils n'ont pas tous la même reconnaissance nationale, ni internationale. En revanche, et il faudrait ajouter les cow-boys pour les États-Unis et les vaqueiros pour le Brésil, ils ont tous traversé des phases de marginalisation avant d'être instrumentalisés dans la construction d'une iconographie nationale en quête de héros. Le montubio et le chagra demeurent, à ce jour, des figures périphériques, régionales et marginalisées bien qu’elles soient engagées sur le chemin d'une meilleure reconnaissance historique, politique et culturelle. Observons aussi que leur rodéo, offrant un spectacle vivant de leur culture respective, se situe à un stade très élémentaire d'institutionnalisation et plus encore de professionnalisation.

Le rodeo montubio et la tradition des amorfinos (échange de versification improvisée) sont considérés comme les manifestations culturelles les plus emblématiques de la communauté socio-ethnique des montubios. Les techniques du rodéo montubio apparaissent assez différentes de celles des autres rodéos latino-américains. Le rodéo montubio n'est pas totalement codifié et intègre des éléments nouveaux qui, aux yeux mêmes des montubios, ne sont pas caractéristiques d'une pratique authentique telle que la présence des clowns toreros ici identifiés au rodéo nord-américain. Les exercices les mieux codifiés où s'affrontent des équipes formées par hacienda d'élevage, sont :

- le caracoleo consistant à faire tourner en girouette le cheval sur les pattes arrière ;

- la monte des chevaux "chúcaros » (chevaux sauvages) pratiquée avec des éperons parfois chaussés directement sur les pieds nus ;

- les piales consistant à attraper au lasso les chevaux avec les pieds ou les yeux bandés pratiqués par les hommes et les femmes ;

— la maitrise de toretes, du bétail bovin inoffensif attrapé au lasso et parfois monté.

Les exercices avec le bétail bovin occupent une place marginale par rapport aux chevaux domestiques et sauvages. En dehors de toute référence aux enjeux

\footnotetext{
${ }^{110}$ El Universo, 17 juin 2006.
}

${ }^{111}$ El Universo, 24 juin 2006. 
identitaires nationaux, nous pensons que cette pratique met également en avant une culture populaire et ancienne de l'hacienda d'élevage dans un contexte régional marqué par l'importance de l'agriculture commerciale d'exportation. Elle revendique également les valeurs d'une culture rurale, de plein-vent et de pieds nus, dans une région dominée par la capitale industrielle de Guayaquil. Il existe une rivalité Salitre-Guayaquil pour s'arroger le statut officiel ou officieux de la capitale montubia. Ce n'est sans doute pas un hasard si les arènes de Guayaquil (Durán) commencent à programmer de plus en plus souvent des exhibitions de rodéo montubio, alors même que les corridas régressent. Enfin, le rodéo montubio exprime l'existence culturelle de la Costa dans un pays traditionnellement dominé par la Sierra. Peut-être aussi valorise-t-il la pratique d'un rodéo fondamentalement équestre dans un espace tauromachique national dominé par la figure du taureau.

\section{VI. — L'ESPACE TAUROMACHIQUE PÉRUVIEN : CORRIDA ET «TAUROMACHIES ANDINES »}

Dans l'immensité du Pérou, la diversité des pratiques tauromachiques semble se démultiplier. Leur répartition et leur profil reprennent quelques grandes logiques qui président à l'organisation territoriale du Pérou : une tripartition Côte-Andes-Selva, une dichotomie entre un Pérou du Sud, andin et indien, et un Pérou du Nord, moins indien et plus littoral, enfin un cloisonnement en cellules plus ou moins coalescentes (voir carte 19, p. 387). Vers le Nord, la Côte et les villes, s'affirment les corridas formelles, vers le Sud, les hautes terres et les communautés paysannes, apparaissent leurs transformations les plus excentriques. Comprendre l'organisation de la géographie taurine du Pérou n'a été possible qu'avec l'aide de nombreux informateurs ${ }^{112}$.

\section{LES CORRIDAS OU LES AMBIGUÏTÉS DE LA FÊTE CRÉOLE}

La plupart des journalistes spécialisés dans les questions taurines au Pérou évaluent le nombre de spectacles à environ 500 par an. Certains parlent de 700 spectacles annuels et n'hésitent pas à dire, comme s'il s'agissait d'une ren-

\footnotetext{
112 Je remercie particulièrement Pablo Gómez de Barbieri, Bartolomé Puiggros Plana et Claudio Sillero. Le premier est journaliste taurin à $E l$ Correo et nous a très aimablement et patiemment décrit le fonctionnement et l'organisation de cet immense pays taurin. Le deuxième, pendant longtemps ingénieur agronome, puis éleveur de taureaux de combat, est responsable depuis 1967 de la rubrique taurine du journal $\mathrm{El} \mathrm{Comercio.} \mathrm{Il} \mathrm{prit} \mathrm{la} \mathrm{suite} \mathrm{à} \mathrm{la} \mathrm{tête} \mathrm{de} \mathrm{cette} \mathrm{prestigieuse} \mathrm{rubrique} \mathrm{d'une}$ figure incontournable de l'afición péruvienne Manuel Solari « Zeño Manué », qui est à l'origine de la création de la Feria Taurina del Señor de los Milagros de Lima en 1947. Le troisième est l'auteur de nombreux reportages de presse écrite et de télévision sur la tauromachie au Pérou dans une perspective ouverte à ses multiples formes et transformations. Je remercie également le professeur d'anthropologie de l'université Catholique de Lima, Juan Ossio de m'avoir longuement reçu ainsi que le professeur de sociologie, Fanni Muñoz.
} 
gaine pour mieux prouver l'importance du fait social taurin, que la tauromachie attire plus de monde que le football. Sous la plume de Bartolomé Puiggros, on retrouve même l'idée d'un déterminisme topographique qui rendrait difficile la pratique du football dans de nombreux villages montagnards et pentus, alors que la tauromachie serait encore possible et incarnerait en cela, mieux que le football, la véritable Fiesta Nacional.

La reprise de ce thème, déjà évoqué pour l'Espagne, témoigne plus de la revendication d'une passion vécue comme fortement enracinée dans tous les recoins de la géographie péruvienne que d'une réelle évaluation comparée des pratiques. Au-delà d'une explication déterminée par la topographie, qui n'est pas acceptable en l'état, la répétition de ce thème n'est finalement que le reflet d'une sousévaluation assez systématique de la tauromachie de la part de médias dominants qui ne s'intéresseraient qu’à la passion footballistique des grandes villes. S’il est un élément tangible à retenir en dehors de toute considération topographique, c'est l'existence d'arènes dans les villages les plus reculés. Quant à ceux qui manqueraient d'espace plan, il est en effet assez remarquable d'observer l'ingéniosité locale à construire des arènes dans les endroits les plus inattendus. Un terrain est dégagé dans le versant afin de délimiter un ruedo. À l'aval est construit un muret de pierre contre lequel s'appuient des loges de toile et de bambou grossier, montées au moment des fêtes. La pente amont sert de gradin naturel.

Sur environ 500 spectacles annuels, Bartolomé Puiggros, en 2005, nous a donné la répartition précise des spectacles que l'on peut considérer comme majeurs, soit 158 courses distribuées dans 80 arènes $^{113}$. Sur ces courses moins d'une cinquantaine répond aux critères espagnols d'une corrida formelle. Les variations interannuelles sont assez faibles, les mêmes cités taurines offrant, à peu de chose près, le même nombre de spectacles chaque année. Il existerait au Pérou plus de 300 arènes permanentes. Il doit certainement en exister davantage puisqu'en 1980, le ministère de l'Agriculture et de l'Alimentation, en ne comptant que celles existant dans les 3.030 communautés paysannes officiellement enregistrées, recensait 289 arènes permanentes ${ }^{114}$. Nombreuses d'entre elles doivent cependant ne plus être actives. Nous avons retrouvé la trace de 156 arènes fixes à partir des sources croisées de Luis de Tabique, José María de Cossío, Francisco López Izquierdo et de nos recherches sur place (voir annexe VI [CD-ROM]). Cela nous laisse penser qu'il existe un volet important d'arènes élémentaires, matérialisées par une simple limite au sol ou un muret, et qui ne prennent véritablement forme que le jour du spectacle.

La répartition des arènes et des spectacles respecte grossièrement la distribution d'ensemble du peuplement entre un Pérou occidental " plein », celui des Andes et du littoral et un Pérou oriental « vide », celui de l'Amazonie forestière. La localisation du fait taurin est majoritairement andine, complétée par quelques grandes villes littorales. En Amazonie, les témoignages de fêtes taurines

\footnotetext{
${ }^{113}$ Les données de Mundotoro annoncent un total de 46 courses.

${ }^{114}$ F. MuÑoz, Cultura popular andina: el Turupukllay, p. 102.
} 


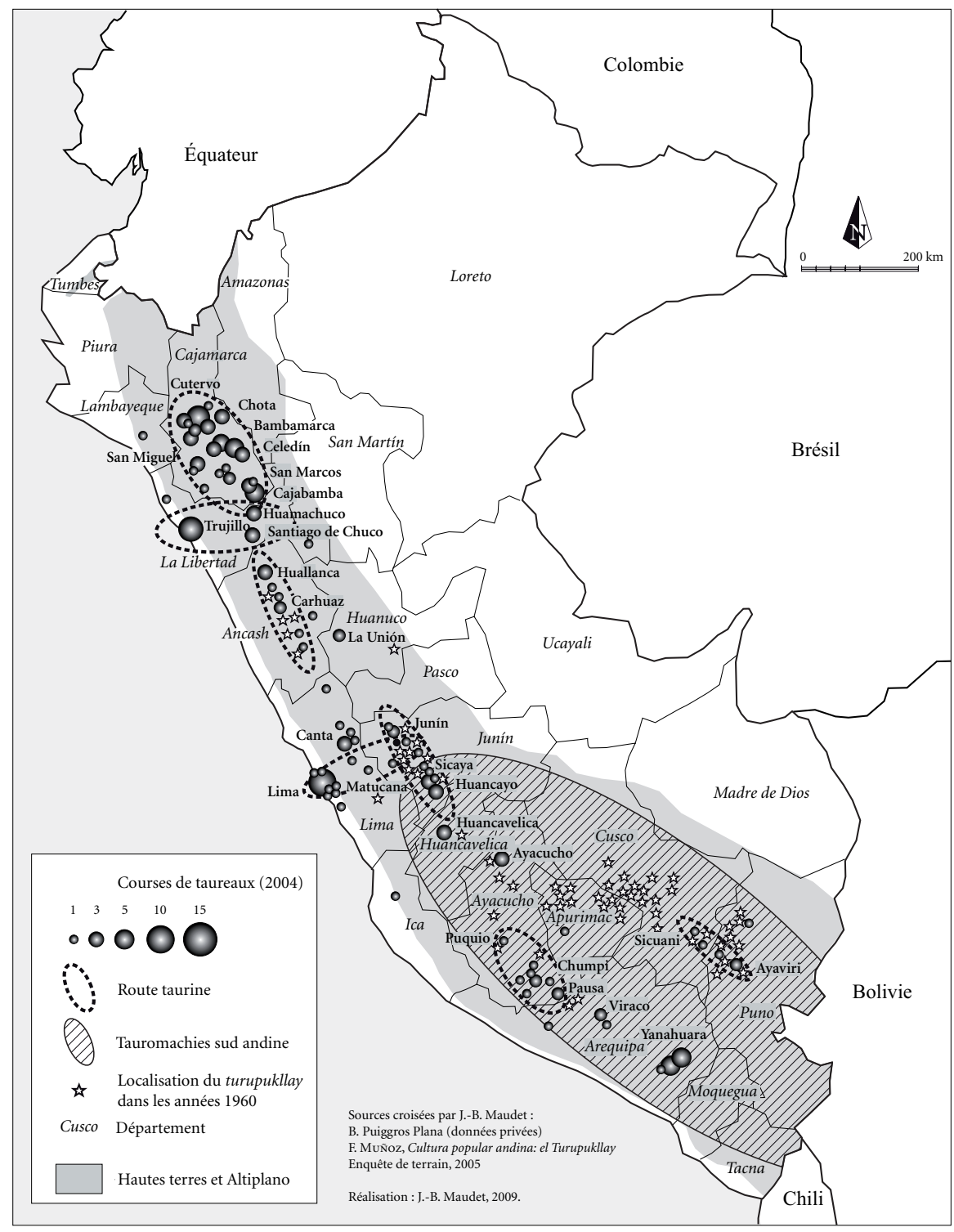

Carte 19. - Tauromachies au Pérou

sont rares. Il y en aurait occasionnellement à Iquitos. À première vue, cette répartition fait apparaître trois ensembles de densité taurine décroissante du Nord au Sud. Le Pérou du Nord est la zone de plus forte densité taurine autour des départements andins de Cajamarca et de La Libertad, prolongée par la capitale littorale de Trujillo. Cet ensemble concentre environ $45 \%$ des spectacles majeurs 
péruviens. Le département de Cajamarca, à juste titre considéré depuis toujours comme le plus taurin du pays, concentre à lui seul $35 \%$ des spectacles majeurs et $28 \%$ des arènes dans lesquelles ils sont programmés. Le Pérou central est une zone de densité moins forte, composée des départements de Junín et de Lima, qui répètent la structure précédente entre une Sierra riche en spectacles et une Costa marquée ici par le poids considérable de l'agglomération capitale. Les départements de Lima et de Junín concentrent un quart des spectacles majeurs et un quart des arènes en fonctionnement. L'agglomération de Lima en comptant les villes satellites nées d'un important mouvement migratoire qui démarre dès les années 1930, concentre à elle seule $20 \%$ des spectacles majeurs du pays. Ce pourcentage est moins élevé que la part de la population nationale qui y est concentrée puisque environ un péruvien sur trois y réside. Le Pérou du Sud, dont la compacité du foyer est moins lisible est une zone de plus faible densité où les arènes et les spectacles se dispersent entre l'Altiplano et les versants de la cordillère occidentale dans une région où le littoral, étroit et désertique, ne compte pas de villes importantes. Ce vaste Pérou du Sud, dilaté par l'épaisseur de l'Altiplano, étendu au département de Arequipa, Ayacucho, Apurimac, Cuzco et Puno, concentre $20 \%$ des spectacles majeurs et $25 \%$ des arènes dans lesquelles ils ont lieu. Cette région, au-delà de toute comptabilité officielle, est une zone très riche en courses de taureaux populaires qui échappent aux statistiques.

La répartition des spectacles en trois ensembles transversaux reprend une segmentation classique du territoire péruvien entre le Pérou du Sud, intérieur, montagnard, marqué par un fort enclavement et l'importance des communautés indiennes, et un Pérou du Nord, marqué par la disparition de l'Altiplano, une meilleure ouverture et une plus forte proportion de Blancs et de Métis. Entre les deux, s'étend un Pérou intermédiaire aux contours flous, polarisé par l'exemplaire macrocéphalie de la capitale, servant de charnière entre ces deux Pérou fortement individualisés. Chacun de ces ensembles est plus ou moins dominé par l'une des trois villes péruviennes les plus peuplées, Lima (8 millions d'habitants), Arequipa (800.000 habitants), Trujillo (500.000 habitants) et chacune de ces villes possède des arènes importantes.

La répartition des élevages de taureaux de combat ne fait que renforcer le poids des départements septentrionaux et de la région de Lima. Il existe 43 élevages de taureaux de combat appartenant à deux associations. Le Comité Nacional de Criadores de Ganado de Lidia qui regroupe 30 ganaderias et la Asociación de Criadores de Ganado de Lidia del Perú qui en regroupe 8, alors que 5 sont sans affiliation. Sur ce total, 13 ganaderias, soit $30 \%$, sont situées dans le département de Lima, qui est celui qui en possède le plus, et 25 élevages, soit près de $60 \%$, sont situés dans les départements septentrionaux de Cajamarca (11), La Libertad (8) et Lambayeque (6). Le Pérou du Sud est pauvre en élevages de taureaux de caste, puisque Arequipa et Ayacucho en possèdent chacun un et Puno en possède deux. En revanche ces régions comptent des élevages de demi-caste qui interviennent dans les nombreuses courses populaires. Ces élevages situés dans les hautes terres de Puno et de Cuzco ont pendant longtemps été désignés sous l'appellation de « taureaux arequipeños » en raison 
d'une complémentarité des terroirs avec le département d'Arequipa servant de pays d'embouche.

Les outrances de la géographie péruvienne, en particulier son immensité et son cloisonnement, permettent de comprendre certains aspects de l'organisation spatiale du fait taurin et du fonctionnement de ses hiérarchies. À ce titre, nous pouvons faire du Pérou un cas idéal pour comprendre l'organisation d'un marché taurin en fonction des différentes variables qui le structurent, parmi lesquelles deux nous semblent déterminantes : les moyens de transports et la taille des arènes. La moyenne approximative de la contenance des arènes péruviennes est d'environ 2.500 places, à l'exclusion des plus grandes : Lima (13.000), Chota (11.000), Cutervo (8.500), Trujillo (6.500), Celedín (6.000), Miraflores (Arequipa 4.000). L'organisation d'une corrida formelle avec six taureaux de quatre ans coûte au minimum 30.000 euros dont la moitié est consacrée à l'achat du bétail. Pour qu'un entrepreneur de spectacles rentre dans ses frais en organisant une corrida formelle dans une arène de 2.500 places, il lui faut établir, en dehors de tout bénéfice et en tablant sur une arène pleine, un prix d'entrée moyen de 12 euros, sans compter les taxes. À ce prix, en dehors des villes où l'offre est toujours susceptible de rencontrer pleinement la demande, beaucoup d'arènes ne feraient pas le plein. Les moyens de réduire les coûts sont multiples : abaisser l'âge du bétail, acheter du bétail de demi-caste, employer des toreros au rabais, soit qu'il s'agisse de jeunes toreros souhaitant percer, soit qu'il s'agisse de toreros locaux dont la réputation ne leur permet pas de gagner davantage. Par toreros locaux, nous ne faisons pas allusion à des toreros latino-américains, ni même nationaux, mais bien souvent à des toreros dont la renommée ne dépasse pas le département et parfois même la vallée. Ce mécanisme général valable partout est exacerbé par l'immensité et le cloisonnement du pays qui rend les déplacements, soit très longs, ceux effectués par la route ou par le chemin de fer, soit coûteux, ceux effectués par avion. Il en résulte la nécessité pour tous les acteurs de la tauromachie de grouper au mieux les contrats dans le temps et dans l'espace tout en respectant les calendriers traditionnels des fêtes.

D'un point de vue spatial, on observe que les arènes sont organisées en amas plus ou moins linéaires qui correspondent au tracé d'une route. L'espace taurin peut ainsi être divisé en " routes taurines » qui forment des cellules plus ou moins coalescentes où les spectacles sont regroupés dans le temps (voir carte 19, p. 387). Ces routes sont longitudinales ou transversales à la structure du relief et épousent les bassins de vie compartimentés du Pérou. Le foyer taurin de la Sierra du Nord est organisé à partir de la route qui relie la capitale littorale du département de la Libertad, aux hautes terres de la vallée du Marañón. Se succèdent ainsi le long d'une même route, les arènes de Trujillo, Huamachuco, Cajabamba, San Marcos, Cajamarca, Hualgayoc, Bambamarca, Chota, Llama, Huambos, Cochabamba, Cutervo, Socota. D'un point de vue temporel, les courses se concentrent dans leur immense majorité entre les mois de juin et juillet. En juin, la San Juan Bautista, patron de nombreuses cités de Cajamarca, est fêtée par des courses de taureaux. C'est le cas en particulier à Chota et Cutervo qui sont considérées comme les deux arènes les plus importantes 
de la géographie taurine péruvienne après celles de Lima. Elles engagent pour l'occasion au côté des meilleurs toreros péruviens quelques toreros étrangers, généralement peu connus ou en début de carrière. Dans le département de Cajamarca, la San Juan Bautista est également fêtée à Llama, Huambos et San Pablo. Il n'est pas inintéressant de constater qu'à San Pablo, on fête la San Juan Bautista ce qui montre peut-être toute l'importance des effets d'entraînements due à la concentration dans le temps et dans l'espace des activités taurines. En juillet, la Virgen del Carmen est fêtée à Bambamarca dont les arènes ont été inaugurées en 2000 ainsi qu'à Celedín et beaucoup d'autres villages proches très attachés à leur « jour de taureau ». À elle seule, cette route de Cajamarca, si l'on tient compte de la totalité des courses de taureaux, regrouperait environ 150 spectacles par an. L'entrepreneur de spectacles Marco Latorre émet une opinion très optimiste sur le devenir des fêtes taurines dans le département de Cajamarca, lors d'un entretien accordé à la revue péruvienne Fiesta Brava :

Les fêtes patronales sont célébrées avec une ou deux courses, jusque dans les villages cajamarquinos les plus petits et les plus éloignés de la ville. [... I Il $\mathrm{y}$ a de nouveaux éleveurs, de nouvelles et impressionnantes arènes qui sont construites par les habitants eux-mêmes. Il y a de plus en plus de monde qui fait preuve d'une bonne culture taurine y compris dans les villages. Mais il est parfois impératif de sortir du strict règlement qui est fait pour les arènes bien établies et tenir compte des usages et des coutumes des villages ${ }^{115}$.

Dans la région de Cajamarca, la culture taurine et l'intensité de son expression sont inséparables d'une complémentarité entre la ville et la campagne.

Il existe au moins cinq autres routes taurines qui répondent à une organisation comparable. Dans le département de Ancash, le long de la route qui passe entre la Cordillère Noire et le Parc national de la Cordillère Blanche se succèdent du sud au nord, les arènes de Ticapampa, Recuay, Huaraz, Carhuaz, Ranrahirca, Yungay, Caraz, Huallanca. Un grand nombre de courses est donné durant le mois de septembre pour célébrer El Señor de la Misericordia (Recuay), La Virgen de las Mercedes (Ancash), El señor de los Milagros (Ranrahirca). Ces deux routes structurent deux cellules quasiment coalescentes avec un déphasage dans le temps, permettant à nombre de toreros locaux de se déplacer d'une vallée à l'autre. Une troisième route s'individualise clairement dans le département de Junín en arrière de la Cordillère Occidentale : Huancayo, Sicaya, Orcatuna, Concepción, Huasa Huasi, Apata, Jauja, Oraya, Junín. Les arènes de Orcaya font la jonction avec la route transversale en direction de la capitale joignant les arènes de Lima, Matucana, Yauli, Oraya, Tarma et Palca.

Dans l'orbite de Lima, le cas intéressant de la féria de Canta, à mi-chemin entre la fête religieuse et la fête civile commémorative, mérite d'être évoqué. À Canta se déroulent les fêtes du Mariscal Chaperito (Maréchal Chaperito) qui chaque année offrent trois corridas. L’enfant Chaperito était un nom donné autrefois à

${ }^{115}$ Fiesta Brava, n $^{\circ}$ 26, 2005, p. 3. 
Jesús par les habitants de la région. Pendant la guerre du Pacifique qui oppose le Chili au Pérou, les troupes chiliennes en route vers Lima étaient sur le point d'assiéger Canta. L'armée chilienne depuis les hauteurs aperçut une longue troupe qu'elle prit pour le regroupement des forces patriotiques et décida de se détourner de Canta. En réalité, la troupe n'était autre que la procession religieuse de la vierge de la Nativité et de l'enfant Chaperito. Les habitants de Canta élevèrent l'enfant au rang de Maréchal qui ne sort plus en procession sans son uniforme.

Les routes du Pérou du sud apparaissent moins clairement en raison d'une proportion plus forte de spectacles mineurs et de courses non répertoriées par les professionnels, mais le fonctionnement est analogue. Dans le département de Ayacucho, en contrebas de la Cordillère de Huanzo, se succèdent sur une vingtaine de kilomètres, les arènes de Pullo, Chumpi, Caracora, Chaviña, donnant corps à la route de la province de Parinacochas, prolongée plus au nord par les arènes de Puquio. Enfin, une dernière route s'individualise sur l'Altiplano le long d'un axe sacré de la civilisation Inca, reliant le lac Titicaca à Cuzco, où se succèdent les arènes de Ayaviri, Macari, Matangani, Sicuani.

Cette lecture de l'espace tauromachique fait donc apparaître une articulation entre une logique aréale fondée sur la proximité des lieux de spectacles entre eux et une logique réticulaire fondée sur l'existence de quelques arènes de rang supérieur. Ces arènes, parmi lesquelles figurent Chota, Cutervo, Trujillo et Arequipa, programment des courses de réputation nationale où circulent les meilleurs élevages, les meilleurs toreros péruviens et quelques toreros étrangers, généralement situés dans le ventre mou des hiérarchies nationales. À Trujillo, la féria aux allures très espagnoles est complétée par l'organisation d'encierros à la mode navarraise, appelés comme au Mexique pamplonadas. Trujillo pendant les fêtes ressemble à une sorte d'Espagne composite où les femmes en robes de sévillanes côtoient des hommes habillés en blanc portant le foulard et la ceinture rouge emblématiques de la San Fermin. Comment ne pas voir en Trujillo et sa riche architecture coloniale l'image d'une Amérique espagnole qui sélectionne au sein d'un vaste répertoire quelques traits culturels emblématiques de la Mère Patrie.

Enfin, les arènes de Acho à Lima semblent totalement échapper aux contraintes organisationnelles liées aux vastes dimensions de l'espace péruvien par une forte intégration au marché taurin international. Dans le cas de Lima, on peut même considérer que ses arènes sont déconnectées de la trame taurine péruvienne, car elles apparaissent plus comme un élément du réseau des grandes arènes latino-américaines que comme la capitale des arènes du pays, dont la fonction serait de célébrer les grands noms de la tauromachie nationale. Cette analyse rejoint étroitement les problématiques contemporaines au sujet des enjeux de la métropolisation et en particulier des liens croissants entre les métropoles de rang supérieur au détriment d'une certaine cohésion territoriale nationale. Cette caractéristique en fait une arène différente des autres grandes arènes latino-américaines et c'est, à mon sens, une des raisons importantes de la crise latente qu'elles traversent. Développons l'argument.

Les arènes de Acho jouissent d'un immense prestige qui tient à plusieurs raisons. D'abord ce sont des arènes anciennes qui historiquement, au même titre 
que les arènes de Mexico, s'inscrivent dans la continuité des plazas de toros de la vice-royauté et sont assez fières de le revendiquer. Mais à la différence des arènes actuelles de Mexico construites en 1946, les arènes de Acho datent de 1766 et s'enorgueillissent d'être contemporaines des plus anciennes arènes des grandes villes espagnoles. En effet parmi les grandes arènes, seules les précédentes celles de Séville qui datent de 1761 et celles de Saragosse qui datent de 1764. Malgré d'importants travaux en 1945 qui réduisent le diamètre de la piste à 60 mètres permettant d'augmenter leur contenance à 13.000 spectateurs, les arènes n'ont jamais changé de localisation. Elles se situent dans le quartier de Rimac, aujourd'hui fortement paupérisé, ce qui n’a jamais constitué un argument pour les déplacer ou envisager d'en construire d'autres ailleurs. Pour ce qui est de leur permanence jamais remise en cause comme signe de leur grandeur, les arènes de Lima se rapprochent des arènes de Séville, alors que les arènes de Mexico, par leur taille et leur localisation urbaine changeante, se rapprocheraient davantage des arènes de Madrid. Revendiquer le titre des plus anciennes arènes d'Amérique leur confère une aura dont ne se lassent pas de parler les aficionados de Lima.

Parmi les thèmes récurrents de ces conversations figurent d'autres ressemblances entre Séville et Lima : une conception de la tauromachie que les aficionados liméens s'attachent à rapprocher de celle qui règne à la Maestranza, mais aussi, évoquée sur le ton de la nostalgie, une certaine atmosphère urbaine, propre à la capitale péruvienne de la "belle époque ", qui était alors comparable à l'atmosphère de Séville. Cette analogie a probablement pour origine, au moins partiellement, les propos du matador Juan Belmonte retranscrits par Manuel Chávez Nogales : "Lima était comme Séville. J'étais émerveillé d'être allé si loin pour me retrouver comme dans mon propre quartier. Parfois je rencontrais dans la rue des gens qui m'étaient tellement familiers et des visages tellement connus que j'avais envie de les saluer ${ }^{116}$. En outre, les arènes de Acho ont vu défiler les plus grands matadors de l'histoire de la tauromachie, tout au moins ceux du $\mathrm{xx}^{\mathrm{e}}$ siècle à nos jours : Joselito (pour qui Acho fut la seule arène américaine où il se présenta), Belmonte, Rafael «El Gallo », Ignacio Sánchez Mejías, Bienvenida, Manolete, Dominguín, Ordóñez, «El Cordobés », Curro Romero, Paco Ojeda...

À partir de 1946 est créée la féria d'octobre, une année avant la création de la féria de la San Isidro madrilène. La féria taurine de Lima est ensuite baptisée Feria del Señor de los Milagros en hommage au sein patron de la ville. L'affiche inaugurale était alors composée de l'Espagnol Manolete, du Mexicain Luis Procuna et du Péruvien Alejandro Montani. Au xxe siècle, le Pérou a connu quelques bons toreros (Adolfo Rojas " El Nene », Miguel López « Trujillano », Alejandro Montani, Rafael «Trujillano ») mais finalement aucun grand nom de la tauromachie d'une envergure comparable au Vénézuelien César Girón, aux Mexicains Rodolfo Gaona, «Armillita » et Carlos Arruza, ni au Colombien César Rincón. Le prestige des arènes semble donc ne pas avoir fait la grandeur

${ }^{116}$ H. López Martínez, Plaza de Acho, p. 198. Citant les propos de Manuel Chávez Nogales. 
des toreros. On peut se demander pourquoi le Pérou est le seul grand pays taurin américain à ne pas avoir produit un grand matador international. On en revient à la particularité des arènes de Acho, très accueillantes pour les grandes figures de la tauromachie mondiale, mais historiquement peu enclines à faire valoir la concurrence entre toreros étrangers et toreros nationaux, indépendamment d'une qualité qui serait moindre.

Lors de la féria de Lima 2005 à laquelle nous avons assisté, sur les vingt toreros engagés seuls cinq toreros péruviens étaient à l'affiche et tous étaient des novilleros dont deux prenaient l'alternative, le rejoneador Jorge Enrique Piraquive et le torero à pied Fernando Roca Rey, fils d'une famille d'éleveurs. La faible représentation des Péruviens en piste est-elle seulement due à l'absence de bons toreros? Certes les apprentis qui peuvent s'entraîner avec du bétail de caste sont au Pérou de rares privilégiés et les corridas de Acho entendent conserver leur rang en ne programmant que les meilleurs toreros. Cependant, on pourrait être surpris de constater l'absence du seul torero péruvien de renom qui démarre avec succès une carrière internationale : Juan Carlos Cubas. D’après nos informateurs, il n'a pas été programmé, car lors des négociations, les entrepreneurs de spectacles ont refusé de débourser plus de 5.000 euros, quand des toreros espagnols comme « El Juli » et Enrique Ponce, les plus cotés, négocient leur contrat à quelque 100.000 euros. Pour compenser les quotas réglementaires de toreros péruviens, les arènes préfèrent organiser durant l'année d'autres spectacles incluant des toreros nationaux qui n'attirent pas foule. Existerait-il un divorce entre l'afición péruvienne et les représentants tauromachiques natifs, à l'image d'une identité nationale introuvable? Existerait-il une mentalité élitiste profondément coloniale dans l'afición taurine de Lima qui n'accorde finalement leur place aux toreros nationaux que de mauvaise grâce? Nous le croyons et l'approfondissement de nos recherches semble le confirmer, ou tout au moins, montrer que les mentalités évoluent très lentement.

Il faut attendre 1968 pour que l'Association des toreros du Pérou, nouvellement reconnue comme entité syndicale par le Ministère, obtienne de la part des organisateurs de la Féria l'obligation d'inclure un torero péruvien. Ce changement est une conséquence dans le domaine taurin d'une transformation radicale de la société péruvienne dans son ensemble, à partir du mouvement nationaliste révolutionnaire porté par le général Velasco qui arrive au pouvoir à cette même date. Une autre conséquence, qui laissa un mauvais souvenir dans le mundillo local, fut en 1969 la collectivisation des terres et le regroupement en coopérative de la plupart des 28 élevages de taureaux de combat alors existant. Contrairement à une idée reçue, cette réforme agraire n'eut, dans un premier temps, aucune conséquence négative sur la production du bétail brave. C'est la parcellisation des terres consécutive à la réforme agraire de 1982 à 1985 qui constitua un réel danger de voir disparaître les élevages de taureaux de combat. La disparition de nombreux élevages du Sud du Pérou date de cette époque, alors qu’ils parvinrent à mieux se reconstituer dans le Nord et le département de Lima.

Revenons à nos toreros. De la date de réinauguration des arènes de Acho en 1947 à la date de l'obtention d'un quota de toreros péruviens pour la Féria 
en 1968, seuls trois noms étaient parvenus à fouler le sol de Acho : Alejandro Montani, Rafael Santa Cruz et Humberto Valle. De 1968 à 2005, vingt-cinq matadors d'alternative péruviens ont torée à Acho, beaucoup d'entre eux toréant plusieurs années consécutivement. Il y a certes eu un changement important à la suite des transformations sociétales de la fin des années 1960, pour autant il ne s'agit guère d'un changement radical, puisque la programmation de toreros nationaux demeure très faible, à la différence des autres grandes arènes d'Amérique latine ${ }^{117}$.

Dans un raisonnement d'un racisme édifiant, certains aficionados de Lima (dont ne font pas partie nos informateurs) m'ont expliqué, sans le moindre embarras, qu'il fallait avoir du sang espagnol pour bien toréer... au moins la moitié. On ne peut exprimer plus violemment l'idée selon laquelle, pour une certaine élite créole, la tauromachie, sous-entendue la vraie, celle de Lima et de nulle part ailleurs au Pérou, est un art qui lui serait exclusivement réservé. Lorsque Antonio Garland écrit en 1948 dans son ouvrage Lima y El Toreo que Acho est « l'archive du criollismo » entre deux envolées confondantes d'hispanophilie exacerbée, admettons que dans la bouche de nos interlocuteurs, il s'agisse de la version la plus poussiéreuse et la moins intégratrice des identités plurielles péruviennes ${ }^{118}$. Comme le montre la suite de notre propos, cette version ne fait que démontrer l'amplitude des sentiments d'appartenance véhiculés par la tauromachie et n'expose en rien les valeurs qu'elle porterait intrinsèquement et en dehors de toute mise en contexte précis.

La place particulière de Acho dans l'espace national n'explique sans doute pas la multiplication des arènes et des spectacles autour de la capitale dans les pueblos jóvenes témoignant d'une urbanisation galopante de la couronne extérieure de l'agglomération. Les spectacles sont donnés dans des arènes démontables et attirent une population urbaine qui a récemment migré vers la ville, parmi laquelle de nombreux Indiens et Métis venus des angles morts de la géographie péruvienne. À Surco ou à San Juan de Lurigancho, ces courses de taureaux semblent constituer un moment festif d'intégration sociale pour des populations fraîchement déracinées et d'origines géographiques diverses. Les nouveaux urbains qui réussissent offriraient même des courses de taureaux lorsqu'ils retournent au pueblo d'origine en signe d'ascension sociale. Ils prennent alors le rôle des anciens mayordomos et capitanes de toros, chargés jadis d'organiser à tour de rôle les fêtes taurines dans les communautés andines ${ }^{119}$.

Il nous plaît de croire que la tauromachie participe ici à " la Lima » des périphéries décrite par Jean-Paul Deler : «C'est la Lima où est peut-être en train de naître la nation péruvienne, où les Andes rejoignent enfin la côte, quand régionalismes et particularismes se mêlent et s'enrichissent sans se renier ${ }^{120}$.

\footnotetext{
${ }^{117}$ Pour la petite histoire que l'on peut relier avec les pages précédentes, le premier entrepreneur qui eut l'obligation réglementaire d'intégrer un torero péruvien n'est autre que Manolo Chopera.

${ }^{118}$ A. Garland, Lima y el toreo, p. 163.

119 A. Goicochea Luna, Tauromaquia andina, p. 170.

${ }^{120}$ C. Bataillon, J.-P. Deler et H. Théry, Amérique latine, p. 289.
} 


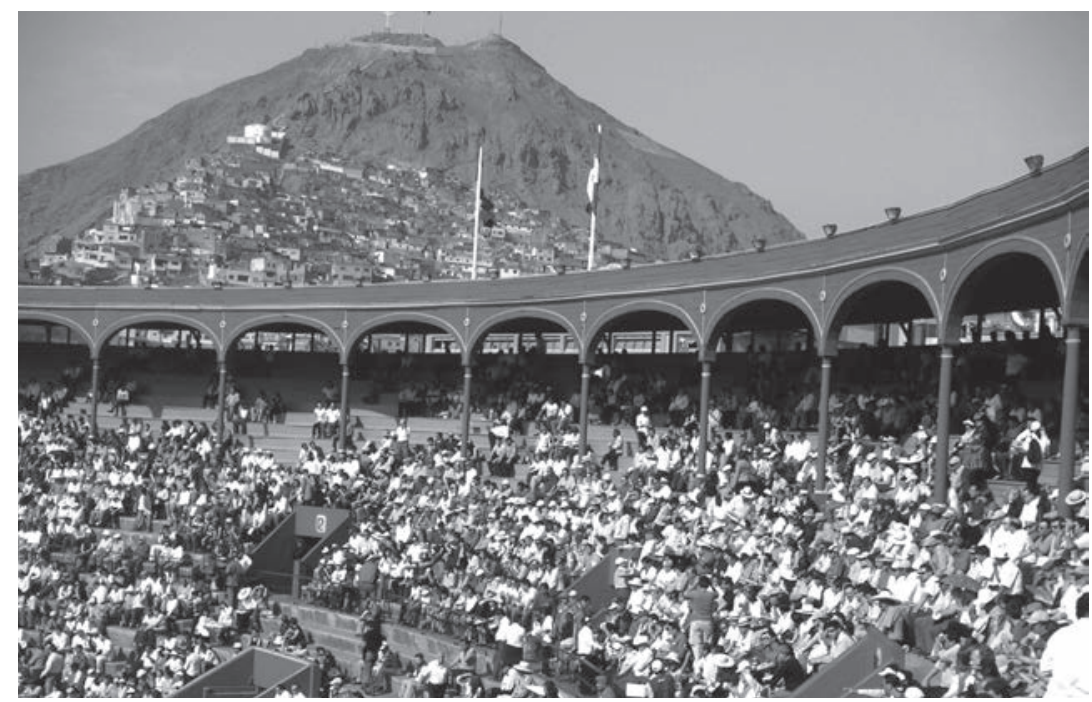

FIG. 55. - Plaza de toros de Lima. Les drapeaux espagnols et péruviens flottent sur les arènes de Lima

(Lima, 2005, cliché : Jean-Baptiste Maudet).

On ne résiste pas, en guise de transition, à remarquer que l'une de ses arènes démontables s'appelle El turupukllay.

\section{«TAUROMACHIES ANDINES » ET TURUPUKLLAY}

Le turupukllay, également connu sous le nom de Yawar Fiesta qui donne son titre au roman de José María de Arguedas, est généralement placé au centre des tauromachies andines péruviennes comme s'il en incarnait la plus pure expression. La vision de cette créature hybride, moitié taureau moitié condor, a de très bonnes raisons de fasciner. Le rituel du turupukllay a donné lieu à de nombreux travaux anthropologiques. Parmi les recherches récentes les plus approfondies figurent les travaux de Fanni Muñoz, de Penelope Harvey et d'Antoinette Molinié $^{121}$. Ces travaux ont en commun la critique d'une interprétation fréquemment véhiculée selon laquelle la figure du turupukllay serait une métaphore du métissage conflictuel entre un élément andin et autochtone, représenté par le condor, et un élément hispanique et exogène, représenté par le taureau. C'est aujourd'hui l'interprétation dominante des guides touristiques, des agences de voyages, de certains reportages et de nombreux Péruviens qui se prêtent volontiers à la diffuser, quand ils ne la déforment pas pour en faire rien de moins que la revanche de

${ }^{121}$ F. MuÑoz, Cultura popular andina: el Turupukllay; F. MuÑoz, « La fiesta del Turupukllay en el mundo andino »; P. HARvey, « Jugando por la Identidad y la tradición »; ID., « El toro y el cóndor en lidia »; A. Molinié, « Le taureau aux ailes de condor ». 
l'Inca sur l'Espagnol. C'est que la Yawar Fiesta a généralement lieu lors des Fiestas Patrias, qui célèbrent, le 28 juillet, le jour de l'indépendance du Pérou.

La position de Fanni Muñoz n'est pas tant de considérer que la conception faisant du turupukllay l'expression d'un métissage conflictuel est fausse, mais d'insister sur la pluralité des interprétations en fonction des groupes d'acteurs investis dans le rituel. Dans le cadre traditionnel des communautés paysannes, les ayllus, le turupukllay est originellement vécu comme un rituel propitiatoire d'offrande à la Pachamama pour garantir la fertilité de la terre par le sang versé. Et par les hasards curieux de l'histoire, il a fallu que le 28 juillet tombe durant la période de la célébration de la divinité locale du Wamani, protectrice des récoltes, facilitant ainsi la surimpression des fêtes civiques et des fêtes calendaires. L'interprétation du turupukllay comme signe d'un métissage conflictuel gagne de l'importance chez les mistis et les cholos, c'est-à-dire les élites locales métisses et les migrants urbains de retour au village, alors que celle de l'offrande à la Pachamama demeure dominante chez de nombreux paysans indiens ${ }^{122}$. Penelope Harvey insiste sur l'ambivalence des significations qui régulent la position des classes socio-éthniques mistis, cholos, indios dans leur rapport au pouvoir de l'État et au pouvoir de la Nature ${ }^{123}$. Antoinette Molinié, dans le sens de Fanni Muñoz, souligne que cette forme de totémisme national, faisant de chaque animal un représentant des cultures qui formeraient la nation péruvienne, est une représentation totalement étrangère à la culture andine traditionnelle. L'une des raisons des plus convaincantes est que le taureau est identifié à une entité parfaitement autochtone ${ }^{124}$. Sans entrer plus avant dans la richesse de ces interprétations, les auteurs s'accordent à mesurer toute la distance qui sépare le turupukllay des corridas conventionnelles. Il n'y a pas lieu de contester cette évidence mais, conformément à notre méthode générale, de s'interroger sur la place actuelle occupée par cette pratique dans la mesure où la culture andine est en proie à de profonds remaniements.

D'abord soulignons qu'il n'existe pas un turupukllay, et les auteurs en conviennent, mais un ensemble de pratiques voisines qui, dans le détail peuvent suivre un déroulement différent. Ces détails ne sont pas des moindres, puisque dans certains cas le taureau est tué, dans d'autres, taureau et condor sont relâchés dans leur espace d'origine respectif, la puna et le ciel de la cordillère, enfin il arrivait autrefois que le condor soit battu à mort si le taureau est blessé, pratique qui semble avoir disparu. Observons également que le rôle et la place des toreros sont également changeants : certains turupukllay laissent une part active à la participation populaire, d'autres font exclusivement intervenir des toreros locaux ou venus de la capitale en habits de lumière. Ensuite soulignons qu'il existe des turupukllay... sans condor, et les auteurs n'insistent généralement pas beaucoup sur cet aspect. N'oublions pas que dans le roman d'Arguedas, la

\footnotetext{
${ }^{122}$ F. MuÑoz, « La fiesta del Turupukllay en el mundo andino », p. 318.

${ }^{123}$ P. Harvey, « Jugando por la Identidad y la tradición », p. 229.

${ }^{124}$ A. Molinié, « El toro y el cóndor en lidia », p. 502.
} 
Yawar Fiesta mise en scène dans le village de Puquio se déroule sans la présence du condor et n'en demeure pas moins placé sous le signe d'une lutte entre les cultures et entre les classes. Le terme quechua turupukllay, construit à partir de la racine turu : taureau et pukllay signifie simplement « jeu du taureau » ou " jeu taurin » et peut être considéré comme synonyme andin de course taurine. Le turupukllay avec condor est donc une modalité andine des courses de taureaux parmi d'autres manifestations. L'enjeu n'est pas de minimiser l'importance du rituel du turupukllay avec condor, bien au contraire, mais de ne pas le soustraire d'un ensemble de pratiques d'une plus grande diversité.

Pour évoquer cette diversité, Angusto Goicochea Luna parle de «tauromachies andines ", expression qui est reprise par Fanni Muñoz dans son travail qui rend compte de la pluralité des manifestations tauromachiques de la Sierra péruvienne ${ }^{125}$. Augusto Goicochea Luna mentionne l'existence de nombreux jeux taurins, certains consistant à s'emparer d'attributs fixés sur le taureau, la enjalma et la moña, à l'image des colchas des toros de pueblo équatoriens, d'autres intègrent des jeux de monte du taureau dont nous avons pu vérifier qu'ils s'inscrivent encore dans le déroulement de nombreuses courses taurines populaires où il est également question de toréer l'animal. Aujourd'hui que représentent les turupukllay avec des condors en comparaison des autres pratiques taurines? Sans nul doute une manifestation irremplaçable, mais très minoritaire d'un point de vue quantitatif et en forte régression en comparaison des autres formes de jeux taurins populaires.

D’après Fanni Muñoz, au milieu des années 1980, la pratique du turupukllay se réduit déjà à quelques provinces des départements du Sud de la Sierra péruvienne : Ayacucho et Apurimac. Sur la base d'une enquête réalisée par Arguedas au milieu des années 1940 conservée dans les archives du musée national de la Culture, Fanni Muñoz estime qu'à la fin des années 1960, les corridas avec des condors se déroulaient dans une dizaine de départements essentiellement dans une moitié sud des Andes et en particulier les départements de Junín, Ayacucho, Apurimac, Cusco, Puno (voir carte 19, p. 387). On observe que les départements dans lesquels le turupukllay a fortement régressé sont parmi les régions qui ont été partiellement désenclavées, soit par la métropolisation de la région capitale, comme celui de Junín, soit par l'activité touristique comme l'axe sacré de Puno à Cuzco. Dans le département de Junín, la localisation du turupukllay d'hier est celle des zones rurales qui aujourd'hui sont dans l'orbite de la route taurine connectée à Lima. Les Métis qui donnent des courses populaires ou les cholos de retour au village tendent à aligner les codes des jeux taurins populaires sur le modèle dominant des villes. Il n'en demeure pas moins que les significations plurielles du rituel taurin puissent continuer de coexister. Dans la désormais célèbre ville taurine de Puquio, la dernière corrida avec condor s'est tenue le jour de l'inauguration des arènes lors de la féria de mai 1971. Le condor de ses

${ }^{125}$ F. Muñoz, Cultura popular andina: el Turupukllay, p. 79. 
ailes aurait coupé le sourcil du novillero Antonio Navarro ${ }^{126}$. Le sang a coulé, le rite est respecté.

La raréfaction du turupukllay avec condor révèle les signes d'une transformation de la société péruvienne par le levier de l'urbanisation et des migrations internes entre la capitale et l'intérieur. Elle montre aussi les limites d'une opposition radicale entre culture taurine urbaine et culture taurine rurale qui, dans les faits, se relaient et se déclinent en de nombreuses formes intermédiaires. En élargissant l'observation à l'ensemble des pratiques taurines, cette évolution révèle également que le nombre de courses de taureaux n'a sans doute jamais été aussi élevé dans les Andes. Il existe une évidence démographique à ce constat. Les Andes péruviennes, malgré le déclin de leur importance relative dans la population globale, passant de $70 \%$ dans les années 1930 à environ $35 \%$ en 2000, sont plus peuplées aujourd'hui qu'hier, avec huit millions d'habitants vivant entre 2.000 et 4.500 mètres, et un taux d'urbanisation qui progresse. Cette évolution de fond ne suffit pas à expliquer la régression du turupukllay qui tient aussi, de façon beaucoup plus brutale, à la déstructuration du tissu rural et de la culture locale par la guérilla du Sentier Lumineux, et tout simplement, à la raréfaction des condors, d'ailleurs protégés par le gouvernement. Dans certains cas isolés, le tourisme permet le maintien du turupukllay comme produit commercial, sous la forme d'un néo-turupukllay amputé de ses ambiguïtés constitutives. Cette promotion valorise, dans une simplification qui se veut sans doute pédagogique, la version du combat inlassablement répété entre l'Inca et l'Espagnol. Nous les cherchons, et l'Inca, et l'Espagnol, nous ne les trouvons plus que dans l'œil de celui qui les crée. Les statues récentes du turupukllay édifiées dans certaines municipalités témoignent d'ailleurs de cette évolution en ne représentant plus que le taureau et le condor, sans l'homme toréant cet animal hybride. Or cet homme est traditionnellement l'Indien réel, exclu de cette nouvelle représentation, le même qui continue en revanche de participer à des turupukllay sans condor. Au Pérou, la pureté du sang, de l'Inca, de l'Espagnol, du taureau et du « vrai » torero, ainsi que la question du métissage, n'en finissent pas de hanter les représentations identitaires attachées aux pratiques taurines.

\section{VII. - L'ESPACE TAUROMACHIQUE BRÉSILIEN : VAQUEJADA, RODEIO ET FARRA DO BOI}

Le Brésil est généralement exclu des pays taurins, car les corridas y ont disparu. L'intérêt de les inclure ne réside pas seulement dans le souci de compléter le panorama des rodéos américains, mais aussi d'approfondir la compréhension des relations spatiales et culturelles à l'échelle continentale entre rodéo et corrida, à travers un pays où cette dernière est absente (voir carte 20, p. 400). Il s'agit aussi de rendre compte de l'existence d'un type de courses de taureaux d'ori-

\footnotetext{
${ }^{126}$ Fiesta Brava, n 23, 2004, p. 10.
} 
gine portugaise, les farras do boi (les fêtes du taureau), passées inaperçues des observateurs de la chose taurine, alors même qu'elles témoignent d'une pratique hautement significative pour appréhender de façon contemporaine les enjeux et les tensions entre la tradition et la modernité, entre la culture locale et la culture nationale brésilienne. Comme nous l'avons évoqué, cette lacune est dans le même temps assez révélatrice à la fois d'un regard taurin focalisé excessivement sur l'Espagne et d'un cloisonnement géographique des études scientifiques qui, sans doute pour des raisons linguistiques, mettent insuffisamment en lumière les comparaisons et les problématiques communes entre l'Espagne et le Portugal.

Le Brésil constitue assurément le géant du rodéo de l'Amérique du Sud par le nombre de spectacles, leur popularité et les différentes formes qu'ils prennent. On peut considérer qu'il existe au moins trois types de rodéos dont deux se situent à un stade avancé de professionnalisation. L'un, la vaquejada, qui est une forme de coleo, possède un fort ancrage régional dans le Nordeste, même s’il existe des parques de vaquejada et des professionnels dans de nombreux autres États. L'autre, appelé couramment rodeio, est une activité en pleine croissance dont les ferments de pratiques autochtones se sont totalement moulés dans les nouveaux codes du rodéo nord-américain. Enfin, il existe un rodeio crioulo (rodéo créole) lié à la culture gaucha du Rio Grande do Sul qui prend davantage les traits d'une fête champêtre que d'un sport professionnel. Le rodeio crioulo revendique un fort attachement à la culture traditionnelle et populaire du sud du Brésil, alors que le rodeio d'inspiration nord-américaine est un formidable exemple d'un rapide transfert culturel sur un terrain favorable qui affirme sans aucun complexe sa modernité. Quant à la vaquejada, elle se situe dans un entredeux qui entend concilier l'héritage d'une tradition régionale et d'une pratique sportive moderne, tout en affirmant la volonté d'en ériger la pratique en symbole identitaire de rang national.

\section{LA VAQUEJADA NORDESTINA : DU SERTÃo AU SERTÃo}

La vaquejada comme les autres formes de rodéo étudiées est issue des pratiques de l'élevage extensif particulièrement développées dans la région du Nordeste. Maurice Le Lannou considère le Nordeste comme « la zone de bétail la plus ancienne, s'étendant sur la caatinga du sertão au-delà de l'agreste ${ }^{127}$. À partir du XVII ${ }^{\mathrm{e}}$ siècle dans le cadre de ce que l'on nomme couramment le « cycle du sucre ", les élevages bovins de l'intérieur fournissent la viande nécessaire aux plantations et aux ports littoraux. La pratique professionnelle de la vaquejada démarre véritablement à la fin des années 1980 et transforme une activité ludique typique des fêtes rurales du Nordeste en sport rémunéré. La

\footnotetext{
${ }^{127}$ M. Le Lannou, Le Brésil, p. 159. D’après Jean Orrechioni, le sertão représente « les contrées incultes, sauvages, éloignées de la côte, des centres de population et par conséquent des foyers de civilisation ». J. OrReChioni, "Contribution à l'étude du mot sertão ", p. 655. La caatinga est une formation xérophile de brousse à épineux que l'on distingue localement du campo (savane herbeuse) et du cerrado (savane arborée). L'agreste est la zone cultivable.
} 


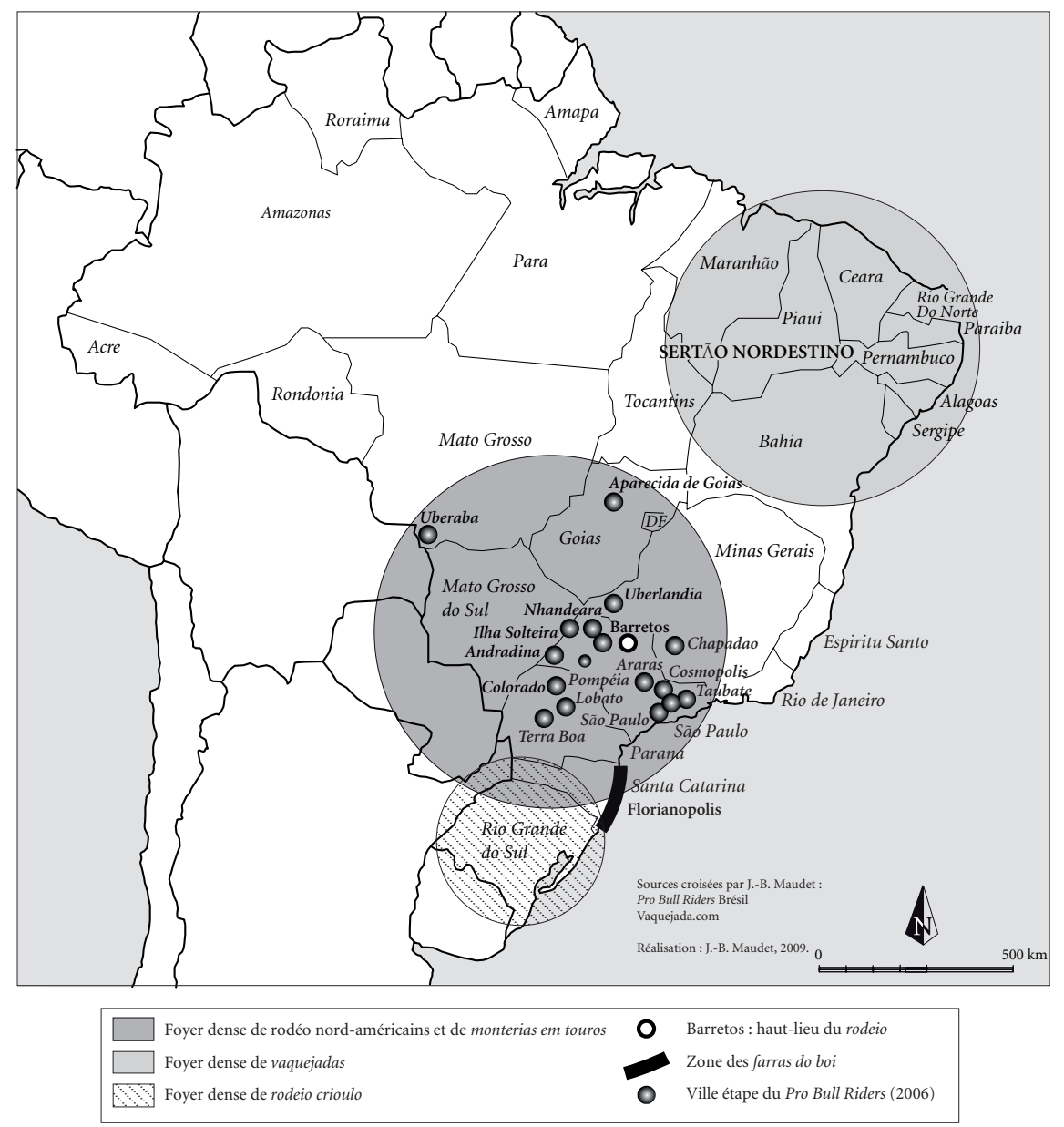

CARte 20. - Tauromachies au Brésil

piste de vaquejada la plus ancienne dont nous ayons eu connaissance est le Parque J. Galdino à Surubim dans l'État de Pernambuco construit au milieu des années $1940^{128}$. Et la plus ancienne vaquejada organisée comme une exhibition sportive serait celle de la vaquejada de Serrinha (Bahia) dans les années 1950. La vaquejada est aujourd'hui organisée en circuits ou en championnats sur des bases territoriales emboîtées, sans aucune logique de maillage uniforme des compétitions à l'échelle nationale. Il existe des circuits que l'on pourrait qualifier d'inter-districts regroupant quelques villes, comme par exemple le circuit Maranguape-Guaiúba dans l'État de Ceara créé en 2004. Il existe des circuits par 
État, dont les deux plus importants sont le Circuito Pernambuco de Vaquejada et le Cicuito Bahia de Vaquejada. Il existe des circuits entre États tels que le Circuito Nordeste de Vaquejada qui s'étend d'octobre à novembre, organisant des compétitions dans des communes situées dans les États de Pernambuco, de Paraiba et de Alagoas. Enfin, il existe des circuits nationaux tels que le Circuit ABQM de Vaquejada (Association brésilienne des éleveurs de chevaux Quarto de Milhas) ou le Circuit national Mastruz com Leite qui joue aussi le rôle de canal de diffusion de la culture musicale actuelle du Nordeste dans le reste du Brésil ${ }^{129}$. Quelle que soit la complexité de l'organisation des compétitions, le Nordeste représente bien le cœur du territoire de la vaquejada, la région de son origine, celle où se trouvent le plus de pistes et de vaiqueiros enregistrés professionnellement.

Comme nous l'avons indiqué, en 2001, les vaqueiros des vaquejadas et les peaes des rodéos sont reconnus comme sportifs professionnels par une $\operatorname{loi}^{130}$. La reconnaissance institutionnelle du vaqueiro ne s'arrête pas à son statut professionnel puisque, en 2004, il est officiellement élevé au rang de symbole de la culture nationale. Il ne s'agit pas de célébrer directement le sportif, ni d'ériger la vaquejada en sport officiel, mais de valoriser la figure du vaqueiro par une loi qui institue une Fête nationale en son honneur, chaque année, le 29 août ${ }^{131}$. La justification du projet de loi présenté par le député Nazareno Fonteles est riche d'enseignement sur les mécanismes d'instrumentalisation nationale d'un élément culturel régional. Ainsi présente-t-il son argument :

Cette proposition de loi n'a d'autre raison que de rendre hommage au vaqueiro, figure représentative de la culture brésilienne, spécialement du sertão nordestino. Issu de la fusion de diverses races, il a pour compagnon le bétail, le cheval et la musique, caractères qui en font un représentant légitime de la culture populaire brésilienne. Célébré par Euclides da Cunha, dans «Os Sertões », le vaqueiro, dans sa manière d'affronter les obstacles du sertão, les longues périodes de sécheresse qui culminent par les intenses remues du bétail à travers les régions les plus inhospitalières de la caatinga nordestina, est une représentation d'un peuple qui lutte et qui vit dans le dépassement des difficultés que le climat et que le sol imposent ${ }^{132}$.

Les éléments principaux mis en avant à travers la figure du vaqueiro sont le métissage racial et le "genre de vie » déterminé par les conditions écolo-

\footnotetext{
${ }^{129}$ Mastruz com Leite est un groupe musical très populaire au Brésil associant des instruments de la musique traditionnelle du Nordeste (zabumba, triangulo, sanfona) et des instruments modernes. Ils organisent une partie de leur tournée en fonction du calendrier des vaquejadas et incarnent le succès populaire de la musique nordestina labellisée par l'entreprise A Somzoom. En 2000, cette dernière aurait vendu 2,5 millions de disques au Brésil.

${ }^{130}$ Loi n $^{\circ} 10.220 \mathrm{du} 11$ avril 2001. Le terme de peão synonyme de péon désigne les acteurs du rodéo.

${ }^{131}$ Loi n $^{\circ} 3.986,2004$.

${ }^{132}$ Projet de loi Fête nationale du Vaqueiro. Disponible sur le site Internet : www.camara.gov.br/ sileg/integras/235523.pdf ; El Diario de Guárico, 4 mai 2007.
} 
giques arides du sertão. Mais l'hagiographie du vaqueiro repose avant tout sur le dépassement des contraintes environnementales comme symbole héroïque du peuple brésilien, c'est-à-dire plus sur les relations hommes-milieu que sur tout autre mise en contexte historique à caractère social, dans une région pourtant marquée depuis toujours par de fortes inégalités. Dans un esprit comparable, la suite du discours invoque des précédents historiques locaux qui mettent en valeur les cavaliers de la caatinga, tels que le premier défilé des vaqueiros tenu le 29 août 1944, dans l'État du Piaui et qui justifie la date choisie pour la fête de rang national. Organisée à União depuis plus d'un demi-siècle, cette fête régionale est la plus importante réunion de vaqueiros de la région, qui rassemble chaque année près de mille cavaliers et qui, bien entendu, est désormais couronnée par des épreuves de vaquejada insérées dans le circuit professionnel. União est également la ville où s'est formée, en 1984, la première association syndicale de vaqueiros pour défendre leurs droits professionnels. Remarquons qu'il n'y a pas de fusion totale entre le vaqueiro professionnel et le vaqueiro traditionnel, mais il est évident, à l'image des llaneros du Venezuela, qu'il existe entre eux une relation qui fait de la vaquejada un formidable support de leur reconnaissance mutuelle. La vaquejada comme vitrine contemporaine du vaqueiro contribue ainsi à replacer au cœur de l'iconographie brésilienne un Nordeste longtemps marginalisé d'un point de vue socio-économique.

La référence à l'œuvre d'Euclides Da Cunha nous semble fondamentale pour comprendre toute la portée territoriale de cette loi, en lien avec la pratique de la vaquejada. Le roman de Da Cunha, Os Sertoes, est considéré comme l'un des grands chef-d'œuvre de la littérature brésilienne qui certes évoque admirablement les contraintes liées au milieu géographique des sertoes du Nordeste, mais qui est aussi une œuvre sociopolitique qui analyse la guerre des Canudos de 1897. Les Canudos forment un mouvement de révolte messianique auquel se rallient nombreux vaqueiros du sertão ayant pour seule activité l'élevage extensif des bovins, au service de propriétaires absentéistes. La révolte est réprimée dans le sang par la jeune République. Euclides Da Cunha montre toute la fracture entre un monde rural archaïque, fortement métissé, rudimentaire et violent, et un Brésil urbain, civilisé, littoral et d'influence européenne. Mais dès le début du $\mathrm{xx}^{\mathrm{e}}$ siècle, la réception de l'œuvre de Euclides Da Cunha transforme le sertão nordestino en un espace géographique et culturel : le Sertão (avec une majuscule). Cette évolution est proche dans son principe, de ce que nous avons observé au Venezuela pour les llanos transformés en terre patriotique : le Llano.

Dans le cas brésilien, le Sertão acquiert les caractéristiques d'un milieu qui a forgé, à l'écart des influences pernicieuses de la civilisation européenne, un peuple vaillant et authentique dont «la société rude, libre et forte des vaqueiros» est la meilleure incarnation ${ }^{133}$. Le roman de Euclides Da Cunha devient l'un des piliers des mouvements régionalistes et l'un des fondements d'une identité nationale affichant officiellement les vertus raciales du métissage généralisé. À

${ }^{133}$ E. Da Cunha, Hautes Terres. La guerre de Canudos, p. 90. 
travers la promotion du vaqueiro et de la vaquejeda, c'est ce même idéal sousjacent qui est mis en avant par le Brésil actuel. La vaquejada serait en cela une émanation de ce que le mythe sociopolitique du Sertão offre à l'identité nationale, alors même que la vaquejada en se professionnalisant tend à exclure les travailleurs du bétail du Nordeste ne pouvant participer aux compétitions réservées aux classes dominantes. Le vaqueiro professionnel récupère ainsi les valeurs du vaqueiro traditionnel en devenant l'un des symboles culturels de l'identité nationale qui n'a plus de populaire que la captation d'un héritage régional valorisé. Ce processus ne doit pas faire oublier, malgré tout, qu'il existe encore des vaquejadas populaires, non professionnelles, et que le public continue de recouper plus largement l'éventail des classes sociales.

À nos yeux la technique même de la vaquejada peut être interprétée dans cette perspective. Parmi les innombrables travaux de l'un des plus grands spécialistes de la culture brésilienne, Luís da Câmara Cascudo, existe un court essai consacré à cette question : A vaquejada nordestina e sua origem ${ }^{134}$. L'auteur affirme que la technique est d'origine espagnole : «Disparue en Espagne, la technique perdure dans les terres américaines parmi les populations pastorales anciennement colonisées ${ }^{135}$. Il précise également que la technique de faire chuter les bovins par la queue s'est popularisée à l'intérieur du Nordeste « en raison de la nature de la végétation de la caatinga [...] n'offrant pas d'espace libre pour lancer le lasso ${ }^{136}$. Les épineux de la caatinga auraient ainsi empêché l'utilisation du lasso. Cette explication déterminée par la nature du couvert végétal mériterait une investigation approfondie, à la faveur de laquelle on découvrirait, à n'en pas douter, d'autres éléments de réponse plus convaincants. En particulier, elle permettrait sûrement d'expliquer pourquoi la garrocha employée dans la technique dite de l'acoso y derribo n'a pas été davantage retenue. Mais une fois de plus, trouver les raisons de la culture dans les déterminations de la nature semble être surtout un formidable vecteur d'appropriation territoriale locale, alors que la technique du coleo concerne l'ensemble du continent. Quoi qu'il en soit, en justifiant la technique par une nécessité du milieu, la vaquejada se voit renforcée dans son rôle d'expression culturelle du Sertão.

Mais la spécificité technique de la vaquejada brésilienne moderne par rapport aux autres jeux taurins du coleo se situe sur un autre plan. À la différence des coleos, pratiqués au Mexique, à Cuba, au Venezuela ou en Colombie, la vaquejada est une technique collective, alors qu'elle est une technique individuelle ailleurs. Rappelons-le, dans la vaquejada brésilienne le bovin est poursuivi par deux cavaliers possédant chacun leur rôle, le bate esteira chargé de guider l'animal dans sa course et de passer la queue au puxador qui le fait chuter. Cette relation d'entraide et de subordination hiérarchique peut être interprétée comme celle qui régule les relations de pouvoir des anciennes fazendas entre

\footnotetext{
${ }^{134}$ L. da CÂmara Cascudo, A vaquejada nordestina e sua origem.

135 Ibid., p. 31.

${ }^{136}$ Ibid., p. 27.
} 
les ouvriers vaqueiros et le contremaître voire même, par son intermédiaire, le propriétaire. Les vaqueiros travaillent, les propriétaires latifundiaires en tirent les bénéfices au moment du rodéo. Cette ritualisation à travers la vaquejada des rapports socio-économiques issus du système latifundiaire peut aussi témoigner d'une passation de pouvoir entre le vaqueiro régional traditionnel et le vaqueiro national professionnel, entre le vaqueiro métis et noir plongé dans l'anonymat du travailleur de l'ombre et le vaqueiro blanc, dressé sur le cheval de la reconnaissance sociale. Cette interprétation se voit renforcée, dans un autre contexte, par l'analyse du rodéo chilien qui, quoique différent dans sa technique, possède certains points communs avec la vaquejada.

\section{RODÉO NORD-AMÉRICAIN ET RODEIO CRIOULO :}

MODERNITÉ YANKEE CONTRE TRADITION GAUCHA

La vaquejada coexiste avec une autre forme de rodéo inspirée directement des codes nord-américains. À l'image du rodéo professionnel aux États-Unis, il existe aujourd'hui plusieurs organismes et associations qui en régulent la pratique. La croissance du rodéo est sans exagération l'une des transformations les plus importantes de la sphère des loisirs de ces vingt dernières années au Brésil. Elle a d'ailleurs très probablement contribué à l'institutionnalisation de la vaquejada et à sa reconnaissance comme tradition brésilienne de rang national. Les rodéos professionnels rassembleraient quelque 24 millions de spectateurs annuels et les compétitions concerneraient près de 1.300 communes principalement situées dans les États de São Paulo, le sud de Minas Gerais, Mato Grosso, Goias et le nord de Paraná ${ }^{137}$. Un tel phénomène de société mériterait évidemment des analyses plus fouillées, mais ces chiffres suffisent à faire du rodeio un loisir de masse de première importance dont certains disent qu'il concerne déjà plus de spectateurs que le football. Nous ne revenons pas sur cette obsession comparative entre football et tauromachie qui révèle une tension entre sport moderne et sport traditionnel ainsi qu'entre sport national-universel et sport national-autochtone.

D'après nos évaluations approximatives, les rodéos au Brésil, sous toutes leurs formes, sont plus nombreux qu'aux États-Unis, même si l'Amérique du Nord reste la référence professionnelle et un El Dorado pour les champions qui se consacrent à la monte des taureaux. La victoire lors de la finale du PBR de Las Vegas en 2007 du Brésilien Adriano Moraes, qui est le premier professionnel de bull riding à avoir gagné trois titres mondiaux, laisse apparaître une rivalité naissante dans le champ du rodéo entre les États-Unis et le Brésil. Au Brésil, avec encore plus d'acuité qu'aux États-Unis, la monte est devenue l'épreuve reine. La Confédération nationale de rodéo en est la principale organisatrice. Elle réalise chaque année deux grands circuits nationaux : le Circuito Nacional de Rodeio qui ne compte que des épreuves de monte des taureaux et le Campeonato de

\footnotetext{
${ }^{137}$ Federaçao Nacional de Rodeio Completo.
} 
Rodeio Brasileiro qui est divisé en deux catégories principales, l'une réservée à la monte des taureaux, l'autre réservée à la monte des chevaux avec la selle cutiano, modalité spécifiquement brésilienne ${ }^{138}$. Malgré l'existence d'une modalité brésilienne de monte des chevaux, c'est bien la monte des taureaux selon les règles nord-américaines qui domine actuellement la pratique.

D’après tous les commentaires des milieux professionnels qui retracent l'histoire du rodéo nord-américain au Brésil, tout démarre à Barretos considéré comme sa capitale et son lieu de (re)naissance. Barretos est une ville d'environ 100.000 habitants, située dans l'État de São Paulo à 438 kilomètres de la capitale éponyme. Barretos organise actuellement un événement majeur du rodéo brésilien, le Circuit national de Barretos, dont la finale se déroule lors de la prestigieuse Festa do Peão de Boiaderos de Barretos ( «la fête du vacher »). La première Festa do Peão de Boiaderos de Barretos est créée en 1956 par l'association Os Independentes qui souhaite alors créer un événement attractif renouvelant les festivités de la ville. Voici l'histoire racontée par l'association de rodéo Os ndependentes:

Lors de cette décade (les années 1950), le rodéo, qui est venu remplacer les cavalhadas qui symbolisaient la lutte des Chrétiens contre les Maures, était déjà devenu l'attraction principale des fêtes réjouissant les spectateurs qui s’identifiaient à un événement mélangeant le sport avec le travail quotidien des fazendas ${ }^{139}$.

Insister sur le remplacement des fêtes des Moros y Cristianos par un jeu issu de la vocation économique locale ne peut pas exprimer plus clairement un désir de rompre avec l'héritage de la péninsule Ibérique et d'entrer dans une modernité festive qui serait celle du sport-spectacle propre au rodéo. Conformément à cette rupture spatiale et temporelle, la désignation de la fête sous l'appellation de Peão de Boiaderos exprime la volonté de valoriser le caractère autochtone des pratiques ludiques et la condition des travailleurs du bétail. Cette volonté se voit concrétisée par l'organisation d'une compétition de rodéo fondée, dans un premier temps, sur la monte des chevaux. L'association prétend qu'il s'agit là de la première fête du genre de toute l'Amérique latine. Détail intéressant pour notre propos, il est précisé que la première fête se tient dans une enceinte nommée Recinto Paulo de Lima Correa qualifiée de "velho picadeiro de circo de tourada ", soit une ancienne arène de course de taureaux. La fête de Barretos donne là un exemple hautement significatif d'une transition, en un même lieu, entre les courses de taureaux tombées en désuétude et la pratique d'un rodéo local, qui lui-même va évoluer vers un rodéo nord-américain. Barretos offre ainsi l'exemple d'un relais dans le champ de la culture taurine entre la corrida et le rodéo. Cette transition entre deux cultures taurines offre une image éloquente du relais, dans le champ de la

\footnotetext{
${ }^{138}$ La selle cutiano est une selle différente de la selle américaine, plus allongée, fixée à l'avant du cheval, qui serait originaire du Mato Grosso.

${ }^{139}$ Informations disponibles sur le site de l'association Os Independentes : www//http : independentes.com.br/2006/link.php?xopcao=0101 [réf. du 10 juin 2007].
} 
culture taurine, entre deux pouvoirs hégémoniques sur le continent, celui de la péninsule Ibérique à celui des États-Unis. Le caractère exemplaire de cette transition mérite qu'on en détaille la séquence chronologique.

Signe de l'attachement de la fête du Peão de Boiaderos à la culture locale, elle est déclarée d'utilité publique par l'État de São Paulo en 1964 et l'hymne national retentit à partir de 1966 lors de l'ouverture de la compétition. Les années 1960 marquent une phase importante de patrimonialisation nationale qui passe également par l'invitation de monteurs étrangers, en particulier argentins, paraguayens et uruguayens. Le logo de la compétition est aujourd'hui orné de quatre drapeaux, celui du Brésil, celui des États-Unis, celui du Paraguay et celui de l'Uruguay. Barretos à l'époque s'inscrit comme la capitale du rodéo au Brésil et dans l'ensemble du Cône Sud.

Il faut attendre la décennie suivante pour que soit introduite la monte des taureaux. La première exhibition, hors compétition, se tient en 1973, annoncée comme une première nationale. Il est précisé que «comme il n'existait pas de monteurs de taureaux, ce sont les monteurs de chevaux qui se risquèrent dans cet exercice ». Dès 1974, l'éleveur João Gargalaque responsable de la première exhibition organisa une série de spectacles à São Paulo avec un monteur masqué du nom de Toninho Cabeludo. En 1979, la monte des taureaux est introduite au rang de compétition autonome et en 1982, les monteurs réclament une égalité des gains avec les monteurs de chevaux, obtenue dès l'année suivante. Dans les années 1980, la fête de Barretos devient le plus grand événement country du pays, couronnée en 1989 par l'inauguration d'un nouveau stade de rodeio construit par Oscar Niemeyer avec une capacité de 35.000 places. Le développement de la monte des taureaux dans les années 1980 marque son acculturation définitive selon les codes nord-américains. Aujourd'hui, les spots publicitaires pour la fête de Barretos se focalisent presque exclusivement sur cette modalité. Beaucoup d'observateurs affirment que l'engouement brésilien pour la mode vestimentaire country, partie de São Paulo, est largement lié au succès des rodéos de Barretos. En 1993, Barretos ajoute une nouvelle compétition, le premier Festival international de rodéo qui accueille des monteurs étasuniens, canadiens et mexicains. L'année suivante, le Festival intègre l'ensemble de modalités techniques du rodéo nord-américain, nommé « rodéo complet». En 1995, la Festa do Peão de Boiaderos où se concentrent toutes les compétitions sur une dizaine de jours entre dans le Guiness Book comme la plus importante manifestation de rodéo au monde. En 1996 est créée la Fédération nationale de rodéo complet qui s'aligne sur la réglementation nord-américaine de la $\mathrm{PRCA}^{140}$. Cette adoption des règles nord-américaines entraîne le développement des jeux des rodeo clowns, dont on a parlé pour les États-Unis, qui sont appelés ici « clowns » ou tout simplement toureiros.

N'y voyons pas le fruit d'un simple transfert, car les nouveaux clowns prennent le relais des anciennes troupes de toreros qui ont existé au Brésil jusqu’à

${ }^{140}$ Le rodeio completo au Brésil compte huit exercices : cutiano, sela americana (saddle bronc), bareback, bulldog, laço de bezerro, laço em duplas, três tambores, montaria em toura. 
la fin des années 1960 dans un style mixte de tourada portugaise et de course comique, souvent associées à des spectacles de cirques. Le plus étonnant est de constater que dans certaines familles de toureiros, en une génération, la vocation familiale est passée presque naturellement d'une pratique à l'autre, de la tourada au rodeio. C'est le cas chez les Damasceno où le père, Francisco dit «Garrafinho » fut connu comme toureiro de tourada alors que les fils Antonio Carlos dit «Django » et Deusicler dit « Meio Quilo » forment aujourd'hui l'une des paires de clowns de rodéo les plus admirées. Ils ont tous deux fait leurs premières armes dans le cirque de leur père et aujourd'hui les fils de « Meio Quilo » rêvent d'embrasser la carrière non pas de clown de rodéo, mais de monteurs de taureaux ${ }^{141}$. Ainsi sur trois générations est-on passé dans la famille Damasceno des avatars brésiliens de la tauromachie portugaise au rodéo d'inspiration étasunienne.

En 1997, le Festival international de Barretos est intégré comme étape mondiale du Pro Bull Riders, l'entreprise du Colorado élargissant ainsi son réseau continental à l'Amérique du Sud. Aujourd'hui, il existe au Brésil 26 étapes intégrées aux compétions du PBR, deux fois plus qu'au Mexique. Le vainqueur du PBR Brasil, entre autres modalités de participation des professionnels brésiliens aux compétitions mondiales, se voit directement qualifié pour la finale de Las Vegas. Faut-il considérer Barretos, capitale brésilienne du rodeio comme le cheval de Troie du rodéo nord-américain ? Quoi qu'il en soit, on perçoit mieux les enjeux territoriaux qui séparent la vaquejada et le rodeio, l'une représentative d'une autochtonie taurine récemment promue comme symbole national, l'autre représentatif d'une puissante acculturation nord-américaine en phase avancée d'assimilation dans l'aire dont la région urbaine de São Paulo. Dans cette relation se reflète une part des enjeux identitaires et territoriaux du Brésil dans la mondialisation.

Le rayonnement du rodéo nord-américain est tel qu'il atteint désormais la région du Rio Grande do Sul, autre grande région historique de l'élevage extensif des bovins. La programmation de rodéo selon les modalités nordaméricaines à Forqueta ou Caxias est le signe qu'il est en passe de vaincre une résistance de taille en ces terres de «gauchismo ». D’après María Eunice Souza Maciel, le gauchismo désigne le processus de construction et d'affirmation de l'identité culturelle fondée sur l'appartenance régionale au Rio Grande do Sul autour de la figure du gaucho ${ }^{142}$. Actuellement le Mouvement traditionaliste gaucho qui regroupe deux millions de participants actifs, revendique le fait d'être le plus grand mouvement de culture populaire du monde. Le mouvement créé en 1948 à Porto Alegre par des étudiants originaires des zones de pâturages du Rio Grande do Sul prône les valeurs traditionnelles de la vie dans les fazendas latifundiaires d'élevage ${ }^{143}$. Le rodéo ne pouvait rester en marge de

141 J. A. Bezerra, "Anjas da arena ».

${ }^{142}$ M. E. de Souza Maciel, Le gaúcho brésilien.

${ }^{143}$ R. George Oliven, « À la recherche des origines perdues ». 
ces traditions, réinventées sur un mode spectaculaire pour mettre en scène une part essentielle de la culture gaucha. C'est dans ce cadre qu'il faut comprendre la multiplication des rodeios crioulos (rodéos créoles) dont la qualification suffit à démontrer qu'ils ne doivent pas être confondus avec les rodéos de São Paulo. Dans le même temps leur identification reste dépendante du contremodèle dominant, car rien ne servirait de qualifier un rodéo d'ici s'il n'existait un rodéo d'ailleurs. Les deux principales épreuves du rodeio crioulo sont la monte des chevaux sauvages et l'immobilisation des bovins au lasso, alors que la monte des bovins en est exclue ou très rare. Le Rodeio Crioulo Internacional de Osorio créé en 1980 est l'un des grands événements de la communauté. Il réunit des participants liés pour l'essentiel à des Centres traditionalistes gauchos dont certains se sont formés dans d'autres États du Sud (Santa Catarina, Paraná) ou plus loin encore de la terre d'origine. Il réunit également des participants venus d'Argentine et d'Uruguay, resserrant les liens d'une grande communauté gaucha.

Cela étant, le développement et la professionnalisation du rodeio crioulo ne sont pas comparables avec ceux de la vaquejada du Nordeste ou du rodeio nord-américain. La progression du rodéo nord-américain anime au sein de la communauté gaucha un sentiment de rejet envers une pratique qui serait à l'opposé des valeurs du Rio Grande do Sul. Se dresse en toile de fond, la menace confuse d'une mondialisation qui mettrait en danger ces valeurs, alors que le succès actuel de leur mouvement culturel en est aussi une manifestation. Antonio Augusto Fagundes, l'un des folkloristes en vue de la culture gaucha s'exprimait ainsi, en 1999, à propos de ce qu'il nomme avec mépris le rodeio country:

Les « rodéo country» n'ont rien de sertanejo et encore moins de brésilien. De la façon dont ils se présentent, ils sont une agression culturelle pour le Brésil en général et pour le Rio Grande do Sul en particulier. L'utilisation qu'ils font d'éléments qui sont nôtres ne masque pas un esprit exclusivement mercantile ${ }^{144}$.

Selon l'auteur, la fête de Barretos qui est d'abord née pour être une fête typiquement sertaneja s'est rapidement américanisée sans aucun respect pour les traditions locales et de conclure : "Nous n'avons pas besoin de singer les Américains [...] Dehors la monte des taureaux, dehors le chapeau Stetson, dehors le rodéo country ». Le " rodéo country» ne sera pas mis à la porte comme en témoigne d'ores et déjà son haut degré d'assimilation à la culture brésilienne. En revanche, depuis peu, le rodeio crioulo a entamé un processus pour une meilleure reconnaissance culturelle directement liée à l'opposition latente entre Barretos et Osorio, entre « rodéo country» et rodéo gaucho. En 2002, le rodéo est officiellement institué par le gouvernement de l'État du Rio Grande do Sul comme une composante de sa culture populaire et en 2006 une loi en régule tous les aspects

\footnotetext{
${ }^{144}$ A. Augusto Fagundes, « Rodeio Country, Nao! ».
} 
techniques et administratifs placés sous l'autorité directe du puissant Mouvement Traditionaliste Gaucho ${ }^{145}$. Le rodéo au Brésil constitue bien un champ d'affrontement des iconographies régionales, nationales et nord-américaines.

\section{FARras do boi À SANTA Catarina :}

\section{L'ISOLAT ARCHAÏQUE CONTRE « L'ILÉITÉ » AÇORIENNE}

Entre São Paulo et le Rio Grande do Sul se trouve le petit État de Santa Catarina qui connaît l'existence d'une pratique taurine d'origine açorienne ne figurant dans aucun ouvrage portant sur la tauromachie. Dans une perspective diffusionniste, les farras do boi manifestent une migration des pratiques taurines, d'abord de la péninsule Ibérique vers les Açores, puis de l’Archipel vers le continent américain. L'État de Santa Catarina et la Californie sont deux exemples de cette filiation qui montrent un fort attachement de la culture açorienne à la tauromachie, même s'il existe d'autres foyers de migrations qui n'ont pas donné lieu au maintien des pratiques taurines. À l'image des courses de taureaux de Barrancos au Portugal, les farras do boi (fêtes du taureau) de Santa Catarina n'ont connu de publicité qu'à partir du moment où les médias ont relayé une polémique entre les défenseurs de la tradition séculaire et les associations de protection des animaux dénonçant la cruauté de telles pratiques. Il s'en est suivi une criminalisation de la fête qui la fit évoluer. La pratique des farras do boi ne concerne pas l'ensemble de l'État de Santa Catarina, mais seulement son littoral et l'île de Florianopolis où se trouve concentrée la communauté açorienne qui, pendant longtemps, a vécu de la complémentarité de la pêche, de l'agriculture et de l'élevage.

Derrière le vocable de farra do boi qui cristallise les enjeux du problème existe une diversité de pratiques taurines. Les fêtes du taureau et les jeux autour desquels elles s'organisent portaient autrefois différents noms : boi de campo, boino-campo, boi-na-vara, boi-no-laço, boi-no-arame, boi solto, brincadeira do boi ou simplement boi ${ }^{146}$. Techniquement ces différentes pratiques pouvaient être regroupées selon deux modalités : les taureaux à la corde (boi-na-vara, boi-nolaço, boi-no-arame) et les taureaux lâchés en liberté dans les champs ou dans les rues (boi-no-campo, boi-solto). Ces derniers, une fois rattrapés et maîtrisés, étaient mis à mort et leur viande était ensuite distribuée entre les membres de la communauté. À cela s'ajoute une tradition de danse avec un masque de taureau, le Boi de Mamão, qui existe sous d'autres noms dans de nombreuses régions du Brésil, en particulier le Nordeste, d'où elle serait originaire, et l'Amazonie (Bumba-meu-boi, Boi-Bumba $)^{147}$. Les bovins, présents dans l'économie locale

\footnotetext{
${ }^{145}$ Assemblée législative de l'État du Rio Grande do Sul, Loi 11. 719/02 et Loi 79/06. Disponible sur le site Internet : https://www.al.rs.gov.br/ag/NOTICIAS.ASP ?txtIDMATERIA=152957\&txtId TipoMateria $=3$.

${ }^{146}$ E. P. LACERDA, As Farras do Boi no litoral de Santa Catarina.

${ }^{147}$ Le mamão est une sorte de grosse papaye avec laquelle était autrefois fabriqué le masque du taureau.
} 
sans que l'État de Santa Catarina n'ait jamais été considéré comme un grand foyer d'élevage, servaient dans le travail des champs en tant que force de traction, dans le transport et la consommation carnée. Les jeux étaient réalisés avec des taureaux achetés à des éleveurs qui sélectionnaient pour l'occasion des exemplaires farouches. Une origine probable du boi-na-vara serait la domestication des bovins pour qu'ils apprennent progressivement à obéir à l'homme. L'animal est attaché par le cou à une corde dont l'autre extrémité est reliée à la pointe supérieure d'un bambou fixé dans la terre (la vara). Le jeu consiste à s'approcher de l'animal en jouant de la flexibilité du bambou, celui-ci ne pouvant pas aller au-delà d'un point où il se sent étranglé. Ce jeu encore pratiqué au milieu du $\mathrm{xx}^{\mathrm{e}}$ siècle semble avoir totalement disparu.

Sans que l'on puisse le prouver, nous pensons qu'il y a une parenté lointaine avec les touradas a vara larga au Portugal pour lesquelles la partie en bois ou en bambou a disparu, alors même que sa trace persiste dans son appellation. Si tel est le cas, il faudrait envisager que l'une des origines possibles de certains jeux de corde portugais ne soit autre que la domestication des bovins pour le travail des champs et le transport de charges. Attacher le cou comme c'est le cas dans les touradas à corda de l'île Terceira, n'a peut-être pas la même origine qu'attacher les cornes, comme c'est le cas dans les divers taureaux encordés espagnols. Il est alors intéressant d'insister sur le fait que l'attelage des bovins pour le travail de la terre et pour le transport, sans être une exclusivité brésilienne, fut plus développée en Amérique portugaise qu'en Amérique espagnole où les chars à bœufs étaient plus rares que l'emploi des chevaux et des mules ${ }^{148}$.

Depuis une trentaine d'années, il n'existe plus de distinction effective des pratiques qui sont regroupées sous l'appellation de farra do boi héritière du boi no campo. La farra do boi, célébrée lors de la Semaine sainte, à Noël ou lors de la fête du saint patron, appartenait de toute évidence à la famille des pratiques sacrificielles liées à la consommation exceptionnelle de viande. L'animal était lâché dans la nature, poursuivi parfois plusieurs jours, puis ramené au village où il était tué et consommé lors d'un grand banquet populaire. Les farras do boi décrites par Eugenio Pascele Lacerda cadrent parfaitement avec les analyses de Pedro Romero de Solís sur la structure sacrificielle ternaire des fêtes taurines $^{149}$. Sur le littoral de Santa Catarina, le rituel ne semble avoir subi aucune dissociation de la séquence festive entre l'affrontement, la mise à mort et le banquet depuis la fin du XIX ${ }^{\mathrm{e}}$ siècle où apparaissent les premiers témoignages écrits jusqu'au milieu des années 1970.

Les farras do boi se transforment avec la progression de l'urbanisation et l'ouverture touristique du littoral, responsables du recul des traditions rurales et d'une ouverture des sociétés locales au regard collectif de la société civile brésilienne. Ce qui était encore décrit, il y a une trentaine d'années, de façon dépassionnée comme un folklore local, devient à partir des années 1980 syno-

\footnotetext{
${ }^{148}$ P. Deffontaines, «L'introduction du bétail en Amérique latine », p. 18.

${ }^{149}$ E. P. LACERDA, As Farras do Boi no litoral de Santa Catarina.
} 
nyme de violence intolérable dans les médias nationaux. La polémique semble débuter à partir d'une campagne menée en 1987 par un certain Dagomir Marquezzi dans le journal $O$ Estado de São Paulo qui n'hésite pas à parler de " tumeur nationale » pour dénoncer les farras do $b o i^{150}$. À la suite de plusieurs actions en justice lancées par des associations de protection animale, le gouverneur de l'État de Santa Catarina prend la décision d'interdire les farras do boi en 1988. Il revient sur sa décision face à la mobilisation des farristas à condition qu'elles aient lieu dans des enceintes fermées et sans mauvais traitement envers les animaux. Une dizaine d'années s'écoulent entre tolérance et répression, respect ponctuel des nouvelles règles et reprises des anciennes habitudes. En 1997, le gouvernement de l'État de Santa Catarina crée une commission d'étude pour faire de la farra do boi une activité culturelle moyennant un meilleur encadrement des pratiques et la valorisation d'autres activités reliées à cette tradition en particulier le boi-de-mamão. La même année, le projet est rejeté par une délibération du Tribunal suprême fédéral qui interdit les farras do boi sur le territoire catarinense et le parlement adopte une nouvelle loi sur la protection des animaux $^{151}$. De nos jours, les farras de boi semblent se scinder en deux, d'un côté des jeux d'arènes tolérés où l'animal est couru comme dans n'importe quel lâcher de bovins en lieu clos et des farras do boi clandestines où les taureaux sont lâchés dans la nature provoquant parfois des affrontements entre farristas et forces de l'ordre. Dans les deux cas, il est certain que l'on assiste à une transformation du rituel et de sa signification sociale. Le résultat de cette polémique est la cristallisation de l'identité açorienne autour de la farra de boi, alors même que dans les années 1970 elle n'était pas liée dans les consciences à un quelconque héritage d'une appartenance historique à l'archipel ${ }^{152}$. Nous pensons que ce qui demeure un isolat archaïque pour les uns, représente pour les autres, la manifestation d'une "iléité » açorienne mieux affirmée, reliée à un réseau communautaire international en plein essor.

\section{VIII. — L'ESPACE TAUROMACHIQUE CHILIEN : UN RODEO CHILENO SINON RIEN}

Le rodeo chileno est le seul type de rodéo à être qualifié, dans son propre pays, par un adjectif qui rende compte explicitement de sa nationalité. Ce simple détail peut recevoir deux interprétations. Il peut signifier la précision d'une différence vis-à-vis des autres rodéos ou d'un modèle qui serait dominant, en l'occurrence le rodéo nord-américain, avec lequel il n'apprécie guère d'être confondu. Il peut aussi signifier, dans le même temps, la revendication d'une pratique emblématique de l'identité chilienne. L'institution, creée en 1961, qui en régule la pratique,

${ }^{150}$ Disponible sur le site Internet de l'association « SOS animal » : http://sosanimalmg.com.br.

${ }^{151}$ Tribunal suprême fédéral, 153.531-8/SC. Loi fédéral n ${ }^{\circ}$ 9.605/1998.

${ }^{152}$ E. P. LaCerda, O Atlântico Açoriano, p. 146. 
s'intitule bien la Federación del Rodeo Chileno et non la fédération chilienne de rodéo. Tout confirme un processus cohérent et volontaire de patrimonialisation du rodéo chilien pour qu'il incarne une certaine idée de la chilenidad ${ }^{153}$.

La Fédération du Rodéo Chilien a pris historiquement le relais de l'Association des éleveurs de chevaux criollo chileno, créée en 1946, avec pour double mission de promouvoir l'élevage de chevaux de pure race et de diffuser la pratique du rodéo. Dans un esprit comparable à l'analyse de Frédéric Saumade pour le Mexique sur la relation entre le choix des chevaux du rodéo et les stratégies matrimoniales et identitaires, il semble bien qu'au désir de conserver la pureté de cheval de race criolla chilena réponde la distinction d'une identité de classe, nous pourrions dire de caste, de l'élite créole. Sur ce thème, l'Association n'oublie jamais de souligner que le cheval criollo chileno descend des 75 chevaux qui accompagnaient Pedro de Valdivia en 1540, le conquérant et père mythique de la nation. C'est ensuite l'isolement géographique des hautes vallées centrales du pays qui aurait donné au cheval de pur-sang chilien, « race la plus pure de toute l'Amérique latine ", ses qualités exceptionnelles de force et d'endurance. La création de la Fédération permet d'accélérer le processus de patrimonialisation du rodeo chileno qui met en scène l'excellence de ces chevaux.

En 1962, le rodéo chilien est reconnu sport officiel, affilié au Comité olympique du Chili. La Fédération compte aujourd'hui environ 9.000 membres répartis entre 273 clubs, eux-mêmes regroupés en associations provinciales. Il y aurait quelque 200 arènes appelées medialunas pouvant accueillir des spectateurs. Il est curieux de constater que le mot utilisé pour désigner l'arène reprend le nom de l'instrument utilisé par les cavaliers pour sectionner les tendons des bêtes, la medialuna (la demi-lune), utilisée jadis à la guerre et à la chasse. Le Championnat national de rodéo qui est la grande finale qui couronne l'ensemble des compétitions, se tient en avril dans la ville de Rancagua (région O'Higgins) au centre du territoire. La sélection des équipes se fait par un système qui ajoute au mérite une forme de péréquation nationale pour que soient représentées toutes les régions du pays, divisées en trois entités Zone Nord, Zone Centre, Zone Sud. Les provinces de Aisén et de Magallanes, les deux régions méridionales les plus éloignées du Centre, comptent obligatoirement une équipe présente par dérogation du règlement. Cette close dérogatoire révèle assez bien le souci de représentation nationale qui déroge à la pure logique de l'excellence sportive. Dans la Medialuna Monumental de Rancagua, également appelée la Medialuna nationale, une fois par an depuis 1949, sont annulées les distances de cette "folle géographie » autour d'un événement central qui se veut fédérateur. Le règlement impose l'habit traditionnel du huaso à tous les participants y compris à ceux venus de régions où il existe d'autres costumes représentatifs d'une l'identité régionale. Le costume huaso se compose, du chapeau type cordouan à ailette réglementaire d'un minimum de sept centimètres, de la chaqueta corta (veste courte qui n'est pas sans rappeler la coupe du traje corto andalou), le faja

\footnotetext{
${ }^{153}$ La chilenidad au même titre que la mexicanidad est un terme couramment utilisé pour exprimer ce qui serait le propre de l'identité chilienne.
} 
(ceinture aux couleurs du Chili), le chamanto (poncho chilien), le pantalon de toile ouvert à la base, les bottes et les éperons. Le cavalier huaso qui remporte la finale devient le champion du Chili qui est également désigné par le titre de «El Chileno » (Le Chilien). En l'absence de toute autre forme de pratiques taurines, aucune tauromachie ne semble mieux participer à la construction d'une identité nationale univoque. Derrière cette construction idéologique qui fait du cavalier huaso, le champion de la « chilénité » et de Rancagua sa capitale apparaît, en creux, la formation de l'État-nation chilien et sans doute aussi de ses traumatismes.

Il y a une raison géographique et historique à faire se dérouler la grande finale de rodéo à Rancagua, située à proximité de Santiago du Chili et à michemin entre les confins septentrionaux du désert d'Atacama chilien et de ceux de la Terre de Feu. On peut se demander pourquoi ne pas avoir choisi la capitale pour cette fonction. L'explication tient selon nous au rôle historique de Rancagua dans l'histoire du Chili, et à la symbolique sociale du rodéo chilien qui cadre mal avec les excès modernes de l'urbanité. Rancagua est un haut lieu de l'histoire de l'indépendance du Chili, connu pour avoir été le théâtre du Désastre de Rancagua, période qui s'étend de 1814 à 1817 où les troupes royalistes espagnoles rétablissent leur domination sur le Chili. Certains indépendantistes ont pu fuir Rancagua comme le général O’Higgins. mais beaucoup sont morts dans cette période d'épuration politique. Lors de la deuxième indépendance, le Libérateur O'Higgins en fait la ville martyre de l'identité nationale, qualifiée dès 1818 de ville "Loyale et Nationale ». Dans le langage courant, la ville connaît comme autre surnom ceux de Historique Ville, de Capital du Rodéo et de Ville Huasa.

Dans l'historiographie nationale, le Chili n'a pu être libéré que grâce au ralliement des masses populaires et en particulier des paysans huasos de la Vallée Centrale. À l'origine, les huasos de la Vallée Centrale ne sont pas ces cavaliers tirés à quatre épingles, portant le chapeau cordouan, la couverture et la ceinture aux couleurs du Chili, montés sur un cheval de pure race créole. À l'origine, ils ne sont pas non plus ces cavaliers qui depuis 1931 guident le défilé militaire célébrant l'indépendance du pays. À l'image de ce que nous avons mentionné pour les protagonistes d'autres rodéos, le mot huaso signifie d'abord un homme rustre et métissé, journalier vagabond, vaquant d'hacienda en hacienda, ou attaché à l'une d'entre elles, tel que l'évoquent les écrits du célèbre romancier chilien Alberto Blest Gaña ${ }^{154}$. Les écrits sur les huasos font état d'une étymologie obscure du terme liée à un mot quechua ou mapuche qui signifierait le dos, renvoyant directement à la figure du cavalier ${ }^{155}$. Le huaso est d'abord perçu comme un être dangeureux, au même titre que les premiers cow-boys de l'Ouest américain, les llaneros du Venezuela et les vaqueiros révoltés du Nordeste, en raison de sa marginalité et de son indifférence au sort de la nation naissante. Mais

\footnotetext{
${ }^{154}$ M. Fraysse, « Alberto Blest Gaña et Balzac ».

${ }^{155}$ L. Thomas, El Huaso. Ensayo de Antropología Social ; R. León Echaíz, Interpretacíon Histórica del Huaso Chileno.
} 
vers les années 1850, les huasos deviennent, de toute évidence, les forces nécessaires à la construction agricole du pays et des hommes indispensables pour mener la guerre du Pacifique contre le Pérou ${ }^{156}$. Le géographe Jean Bordes dans sa thèse secondaire portant sur les structures agraires de la vallée du Pangue (Chili Central) montre que cette période est cruciale dans le développement des haciendas d'élevage et la normalisation des règles entre hacendados propriétaires et inquilinos (ouvriers agricoles) travaillant à leur service ${ }^{157}$. D'après un rapport statistique de 1875 du département de Melpilla situé dans la Vallée Centrale faisant état des contrats de travail des haciendas, l'inquilino doit posséder sa propre monture pour le travail du rodeo ${ }^{158}$. Les huasos sont donc des travailleurs du bétail possédant leur propre cheval d'où l'intérêt qu'ils représentent pour les classes dirigeantes dès lors qu'il est question de défendre le territoire. Reste à expliquer, dans une perspective chère à Hobsbawm comment la figure rurale du «bandit social », marquée par le métissage avec l'Indien, s'est finalement transformée en représentante des classes dominantes de la nation, blanche et urbaine. Des éléments de réponse se trouvent inscrits dans le rituel même du rodéo à la manière de ce que nous avons établi pour la vaquejada brésilienne.

Le rodéo utilitaire organisé comme un temps périodique du travail des champs est toujours une démonstration de pouvoir de l'hacendado. On peut considérer que le rodéo affirme d'abord un pouvoir de transformation du milieu et d'appropriation de l'espace. En effet, en rassemblant et en marquant le bétail, la richesse se voit transférée du monde libre et sauvage au monde domestiqué appartenant au propriétaire terrien. Le rodéo affirme ensuite le pouvoir du patron qui dirige ses travailleurs et qui, exceptionnellement, sort de son seul rôle de propriétaire pour prendre part aux opérations, célébrant une sorte d'égalitarisme dans l'action et jouant alors pleinement son rôle d'éleveur de bétail. Selon l'anthropologue Juan Carlos Skewes de l'université Australe du Chili, attaché à l'idée que le rodéo chilien est une évocation de l'ancien temps, il est aussi une ritualisation des systèmes de domination caractéristiques du régime de l'hacienda ${ }^{159}$. Il est intéressant de constater que les règles du rodéo moderne insistent précisément sur cet aspect puisqu'il existe une répartition des rôles entre les deux cavaliers (la collera) en apparence identiques. L'un dirige et oriente l'animal pour que l'autre puisse rester en contact avec le bovin par l'intermédiaire de son cheval, afin de le stopper dans sa course le moment venu. On retrouve la même logique que dans la vaquejada au Brésil. Ainsi, il s'agit à la fois d'une valorisation du travail collectif, mais aussi d'un rapport de subordination où l'on peut voir l'héritage des huasos inquilinos au service du contremaître et du propriétaire devenant alors huasos hacendados, les seuls à pouvoir prétendre, dans la version moderne et sportive, au titre de « El Chi-

\footnotetext{
${ }^{156}$ G. Salazar et J. Pinto, Historia contemporánea del Chile, t. II.

157 J. Bordes, Les Structures agraires de la vallée du Puangue, chap. III : " La descendance des grandes haciendas ».

${ }^{158}$ Ibid., p. 119.

${ }^{159}$ J. C. Skewes, «El Rodeo, una metáfora del Tiempo Viejo ».
} 
leno ». Les réformes agraires et les évolutions historiques du Chili contemporain ont certes transformé le cadre de l'hacienda héritière du monde colonial. Mais on peut penser que la création du sport moderne permet d'en élargir la portée. Alors, le rodéo chilien n'est plus la seule évocation nostalgique du modèle d'organisation de l'hacienda, mais la tentative de transposer symboliquement l'équilibre traditionnel des pouvoirs dans le cadre territorial de l'État-nation. On comprend mieux l'accumulation de la symbolique nationale autour de cette activité. Le cavalier huaso devient donc le garant d'un système de valeurs qui assure la domination de la ville sur le monde rural, du centre sur les périphéries et qui finalement symbolise une cohésion territoriale projetée par les élites. La répartition des clubs sur l'ensemble du territoire témoigne d'un marquage d'une chilenidad officielle. Même la convocation dérogatoire des provinces du Sud au Championnat national s'éclaire. Cette région ne constitue-t-elle pas la marche frontière la plus tardivement intégrée, et non sans féroce répression, en raison de la résistance des Mapuches ? Derrière le lissage de l'organisation de l'espace taurin et la figure exemplaire du cavalier huaso qui n'est concurrencé par aucune autre forme tauromachique, se dessinent l'histoire et la géographie d'une cohésion territoriale difficile, marquée par des événements violents. La violence est sans doute d'abord celle de l'appropriation d'un espace géographique atypique, étiré comme nul autre au monde, passant du littoral à la haute montagne en quelques kilomètres. La violence est surtout celle d'une construction nationale douloureuse : rejet du métissage, radicalité de la société de classe, dictature, massacre des Mapuches.

Aujourd'hui, chaque 19 septembre pour célébrer l'Indépendance nationale, le président de la République reçoit des mains d'un cavalier huaso du vin qui circule parmi les membres du cabinet jusqu'au commandant en chef des forces armées. En 2006, la loi de 1968 qui célébrait la fête du Huaso (Día del Huaso), le deuxième vendredi d'octobre, est remplacée par une nouvelle loi, adoptée par 84 voix contre 1, qui institue désormais ce jour le premier septembre, c'est-àdire au début du « Mois de la Patrie ». Sergio Ojeda, l'un des députés portant le projet de loi, se réjouit de cette nouvelle loi: « Nous croyons que c'est le devoir de tout peuple de conserver ce qui est autochtone, de fortifier l'identité et son style de vie original, de valoriser ce qui est à nous, ce qui est vital pour renforcer l'âme nationale ${ }^{160}$.

Enfin, au regard de cette analyse, on ne peut éviter de souligner que le rodéo chilien a quelques points communs avec la charreada au Mexique. Il s'agit d'une pratique hautement représentative de l'identité nationale qui évoque la place de l'hacienda comme cellule sociale paternaliste. Il existe un costume folklorique officiel intégré dans la réglementation du jeu. Ce sont deux pratiques taurinoéquestres centrées sur le cheval et sur sa race. Dans les deux cas, les valeurs collectives affirmées dans la pratique sportive de loisir renvoient à un groupe social dominant lié au corps armé. À l'opposé des valeurs individualistes et de

\footnotetext{
${ }^{160}$ Diario del Congreso Chileno, 13 juillet 2006. Disponible sur le site Internet : www.camara.cl/ diario/noticia.asp?vid/19985.
} 
l'esprit de lucre du rodéo nord-américain, la pratique valorise l'idée d'un otium aristocratique, source de prestige qui s'exprime en amont d'un pouvoir économique non moins réel. La grande différence entre ces deux pratiques, réside dans l'articulation de la charreada avec les autres jeux taurins coexistant sur son territoire, alors que le rodéo chilien ne connaît aucune autre forme taurine dérivée, transformée ou concurrente, la première faisant écho à la nation métisse, la seconde relevant de sa négation.

Retenons de ce parcours américain que l'extension de chacune des pratiques tauromachiques recouvre des aires de taille très variables. La corrida s'étend d'un continent à l'autre et peut, à ce titre, être considérée comme la seule véritable forme tauromachique d'envergure transatlantique. Le rodéo concerne la quasi-totalité du continent américain bien qu'il soit individualisé sous des formes tantôt communes à plusieurs pays (le rodéo nord-américain) tantôt spécifique d'un seul. En cela, le rodéo apparaît comme une forme taurine panaméricaine aux multiples ramifications. Certaines pratiques s'individualisent en des aires plus réduites qui souvent recoupent des régions marquées par une forte homogénéité historique et culturelle, parfois même paysagère : le coleo des Llanos vénézuélo-colombiens, la vaquejada du Sertão, à l'image dans une certaine mesure de la course landaise en Gascogne, de la course camarguaise en Camargue. Ces pratiques ne connaissent pas toutes les mêmes dynamiques. La plupart demeurent cantonnées dans des frontières relativement stables, certaines connaissent une expansion spatiale récente (rodéo nord-américain), d'autres sont en voie de régression (turupukllay andin, farra do boi du littoral de Santa Catarina). À ces différences propres à l'étendue de chacune des pratiques taurines et à leur dynamique spatiale s'ajoutent des différences liées à la patrimonialisation des pratiques taurines, plus ou moins reconnues et mises en scène comme des traits culturels constitutifs de l'identité locale. Nombre d'entre elles sont instrumentalisées comme symbole culturel national au-delà de la diversité interne des pratiques taurines régionales et face à des pratiques taurines étrangères ou extérieures. Il y a donc un usage interne et externe au pays, dans les logiques d'institutionnalisation des jeux taurins dont la représentativité peut être discordante avec l'aire d'extension réelle de leur pratique. 


\section{LA TAUROMACHIE COMME MARQUEUR TERRITORIAL}

De toutes les interprétations existantes sur ce que sont les jeux taurins, sur leur place et leur rôle dans les sociétés qui les produisent, celle qui paraît désormais la plus évidente à nos yeux fait de la tauromachie un formidable marqueur territorial. L'expression est à prendre au sens premier et plein : les jeux taurins bornent l'espace, tracent des frontières, font vivre des différences culturelles, créent des hauts lieux d'identification, véhiculent des sentiments d'appartenance territoriale d'échelle locale, régionale, nationale et transnationale. Toutes les pratiques ne le font-elles pas? Sans doute, si ce n'est que la tauromachie offre un champ commun de références pour des territoires très vastes témoignant d'une mondialisation partielle et fondamentalement transatlantique. Si ce n'est aussi que ces jeux, qui constituent une exception dans le champ des loisirs modernes et des relations homme-animal, alimentent des débats sociaux et territoriaux conflictuels. Si ce n'est enfin, qu'en de nombreuses régions taurines et à l'échelle de certains États-nations, ces jeux représentent l'expression culturelle la plus emblématique de ce qui serait propre à tel ou tel territoire.

Au sein de cette aire culturelle de la tauromachie qui tient à la fois d'un héritage historique ancien et d'une réadaptation permanente des jeux taurins aux évolutions sociales et politiques, nous pensons que, de proche en proche ou d'échelle en échelle, tous les marqueurs territoriaux qu'ils véhiculent sont en relation les uns avec les autres. Ces relations se tissent à partir de quelques configurations territoriales qui suffisent à donner à l'ensemble une certaine cohérence et un fonctionnement commun : les hauts lieux des pratiques, les espaces de coexistence de cultures taurines différenciées et les frontières taurines. Avant de mettre en relief les propriétés de ces configurations territoriales, rassemblons ce que la géographie particulière de chacune des pratiques tauromachiques révèle en termes de lien entre les hommes et leur territoire.

\section{I. - LES DYNAMIQUES CENTRE-PÉRIPHÉRIES \\ DE L'ESPACE TAUROMACHIQUE}

L'étude détaillée de la structure et des dynamiques de l'espace tauromachique a fait apparaître des logiques d'organisation qui se répètent. Malgré des emprises 
territoriales très différentes d'une pratique à l'autre, l'organisation spatiale des faits taurins possède quelques invariants : des densités inégales entre le centre et les périphéries de la pratique ainsi que la coexistence de la tauromachie professionnelle et de la tauromachie participative.

Quelles que soient les pratiques considérées et leur extension spatiale, il est possible de repérer des zones d'activité de plus forte densité qui ont tendance à correspondre, soit au berceau originel de la pratique, soit aux zones de diffusion les plus anciennes. Pour ce qui est de la corrida, l'Espagne est de loin le pays taurin possédant l'activité la plus importante. En son sein, l'Andalousie et la Castille centrale commandées par Madrid et Séville qui ont joué un rôle crucial dans la codification du spectacle moderne, demeurent aujourd'hui les foyers de plus forte densité. Ces deux villes sont aussi les centres organisationnels et iconographiques des terres de corridas qui fonctionnent comme des périphéries, plus ou moins intégrées, plus ou moins autonomes. La corrida portugaise, qui en comparaison passerait pour une pratique régionale de rang inférieur, témoigne elle aussi d'une extension transatlantique, certes plus modeste, mais bien réelle, par l'intermédiaire des Açores et de sa projection californienne. Le cœur de la pratique et la zone de plus forte densité demeure la région urbaine de Lisbonne et les rives du Tage. Enfin, la Chalosse pour la course landaise, la Petite Camargue pour la course camarguaise, les rives arago-navarraises de l'Èbre prolongées par les pays valenciens pour les courses de recortadores, sont comme les noyaux durs et les terres denses de ces cultures taurines respectives.

Les pratiques tauromachiques en Amérique obéissent à des logiques comparables. Pour la corrida, le plateau central mexicain et la cordillère andine par lesquels s'est réalisée la colonisation de l'Amérique espagnole, demeurent les foyers denses de la corrida outre-Atlantique. Conformément aux caractères premiers de la colonisation, l'Amérique est d'abord taurine par ses villes. Tous les grands relais du pouvoir espagnol ont été, à un moment donné de leur histoire, des villes taurines. Le rodéo nord-américain, connu dans le monde entier au même titre que la corrida, possède son cour et sa zone de plus forte activité dans l'Ouest qui l'a vu naître, à savoir la frontière, hier mexicaine, et les Grandes Plaines traçant « la piste » du bétail vers le Nord-Est. La frontière entre les États-Unis et le Mexique, dans sa fluctuation historique, est la grande zone de contact qui révèle, à travers le prisme de la tauromachie, ce que l'Amérique anglo-saxonne a de latine dès les premiers temps. Au Mexique, la charreada qui véhicule l'un des stéréotypes les plus reconnaissables de l'identité mexicaine, possède son foyer le plus dense dans le centre occidental du pays, la région historique de Jalisco, prolongée par les terres d'élevage extensif du Nord. Les toros coleados au Venezuela et en Colombie, s'épanouissent dans les Llanos, d'où ils sont originaires, et multiplient les zones d'intense activité le long du piémont andin, au contact des terres de corrida. La vaquejada au Brésil conserve le cour de sa pratique dans le Nordeste, cette région déshéritée en marge de la construction économique contemporaine du pays, mais qui demeure une terre d'identification nationale. Enfin, le rodéo chilien des cavaliers huasos, très offi- 
ciellement instrumentalisé comme ciment identitaire de la nation, possède le cour de sa pratique et ses lieux de commandement autour de Santiago, Rancagua et la Vallée Centrale.

Dans les zones de forte densité taurine et plus généralement là où la tauromachie professionnelle acquiert quelque importance, il n'est pas rare qu'elle soit accompagnée de jeux taurins à participation collective. Tout se passe alors comme si l'une n'allait pas sans l'autre, dans un rapport ambivalent de concurrence et de complémentarité, entre les spectacles de l'élite et les spectacles populaires, entre les spectacles urbains et les spectacles ruraux, entre les spectacles codifiés d'envergure nationale ou régionale et les spectacles locaux, parfois uniques en leur genre. Ainsi fonctionnent les festejos taurinos populares de la région de Madrid, les entradas et largadas de la région de Lisbonne, les abrivado et bandido en Camargue, les rodéos populaires de l'Ouest, les toros coleados ruraux des Llanos, les vaquejadas non professionnelles du Nordeste, le jaripeo des paysans mexicains du plateau central... Ces pratiques possèdent aussi leur propre logique de localisation affirmant l'existence de cultures taurines locales, maintenue dans une sorte de vocation communautaire : encierros navarrais, bous al carrer des pays valenciens, abrivado, bandido, encierros camarguais, capeias raianas de la frontière portugaise, fiestas en corralejas de la Costa Atlántica colombienne, turupukllay des Andes méridionales péruviennes, toros de pueblo de la Sierra équatorienne, rodéo montubio de la Costa équatorienne, jocheos du département bolivien de Béni. La tauromachie participative dit toujours quelque chose de son rapport à la tauromachie dominante : la reconnaissance d'un attachement à la culture taurine englobante mais sous les traits d'activités spécifiques dans le cadre d'identités culturelles locales et le désir de ne pas s'en faire déposséder. C'est de cette dialectique entre tauromachie professionnelle et tauromachie participative, tauromachie du centre et tauromachie périphérique, tauromachie des villes et tauromachies des champs, que nombre de fêtes taurines, intégratives des deux composantes, tirent leur originalité.

\section{II. - TAUROMACHIES}

\section{ET IDENTITÉS TERRITORIALES}

Les pratiques tauromachiques professionnelles possèdent une organisation spatiale hiérarchisée. La hiérarchie des lieux taurins peut être spontanée dans le cas des corridas espagnoles et portugaises, c'est-à-dire liées principalement à la trame du peuplement et au poids variable de la culture taurine locale, ou relever partiellement d'une organisation territoriale régulée par une autorité ou un projet politique. Dans le cas des corridas, on a souligné l'originalité des processus d'identification territoriale véhiculés par la mise en réseau des férias. Chaque féria occupe à tour de rôle les devants de la scène tauromachique, observée sur place et à distance par tous les passionnés. Chaque rendez-vous taurin, à son échelle et selon l'importance de l'événement, constitue pour la 
communauté locale qui se reconnaît dans ce spectacle, une occasion de parler de ses valeurs, de parler de celles des autres, de parler d'un ici comparé à un ailleurs. Le déroulement de la saison fait que les rendez-vous taurins sont toujours, à quelque degré, un discours sur le territoire, ou tout au moins, sur des cultures territorialisées : " D'où vient ce torero ? Qu'a-t-il fait à Madrid ? La féria de Séville a-t-elle été bonne cette année ? Quels sont les toreros qui partent en Amérique ? » Mieux, la dimension festive inhérente aux pratiques taurines renforce ce discours, en ce que la fête est toujours une affirmation des identités locales qui passe par une ouverture aux autres, invités à y participer, à la contempler, mais aussi invités parfois et non sans quelque paradoxe, à s'y sentir exclus.

Les mécanismes ne sont pas exactement les mêmes pour les pratiques tauromachiques régulées dans le cadre d'organisations sportives, si ce n'est le rôle de la fête qui semble l'un des invariants du loisir taurin. La régulation des pratiques sur les modèles du sport délimitent généralement des cadres locaux, régionaux et nationaux, emboîtés les uns dans les autres et reliés entre eux de façon pyramidale par des compétitions, depuis la pratique amateur jusqu'au plus haut niveau. Ce principe d'organisation, qui relie la base au sommet, crée un lien d'identification sociale fort entre les spectateurs, les amateurs, les passionnés, les pratiquants et les héros, pouvant être envisagés dans un continuum. Il crée aussi des liens d'identification territoriale par l'intermédiaire d'acteurs et d'équipes représentant des clochers, des villes, des régions au sein d'un cadre national. Les toros coleados au Venezuela et en Colombie, ainsi que le rodeo chileno au Chili représentent sans doute les modèles les plus parfaits de cette régulation spatiale de la pratique tauromachique qui, dans les pays cités, confinent au volontarisme politique d'unifier les territoires à la nation. Ce modèle d'organisation est très souvent associé à des calendriers festifs traditionnels qui garantissent l'enracinement de la pratique. Dans le cas de la course landaise et de la course camarguaise, cette superposition des fêtes de village et de la compétition sportive de haut niveau témoigne d'une adaptation originale d'un loisir ancien à la modernité, pour tenter d'en accroître l'attractivité sans en perdre les valeurs traditionnelles. Ce grand écart crée souvent des tensions et n'assure en aucun cas la réussite du modèle. Voilà une façon originale de mettre en relation les territoires, puisque chaque rendez-vous local et ponctuel s'inscrit dans un temps saisonnier linéaire et progressif, celui de la compétition, associant différents lieux. Cette mise en relation des territoires par les modèles d'organisation sportive témoigne alors d'une articulation entre niveaux géographiques, parfois du local au national, puisque chaque ville ou village participe à une trame compétitive quadrillant l'espace. Dans le détail, la mise en place annuelle de cette organisation est toujours l'occasion de négocier et de dialoguer pour satisfaire au mieux des intérêts pouvant être divergents entre des pouvoirs locaux, des contribuables, des associations sportives, des clubs taurins, des professionnels et des amateurs.

Sous cet angle, la tauromachie participe doublement à la construction territoriale des régions qui la produisent. Elle participe, d'une part, à l'indivi- 
dualisation des régions taurines par rapport à celles qui ne partagent pas les mêmes formes de loisirs. La région ou le pays se démarque d'autant mieux de son voisin qu'il ne connaît pas d'activité tauromachique ou bien qu'il pratique une autre forme. C'est sans doute le sens des différenciations panaméricaines des rodéos construites dans le cadre d'États-nations indépendants. Les pratiques partagent une appartenance à la famille du rodéo, mais dans des formes singulières qui renvoient aux spécificités nationales. Souvent ces formes renvoient également aux caractéristiques géographiques et paysagères du pays pour en prouver l'autochtonie, comme si les milieux avaient déterminé l'individualisation des jeux taurins en dehors de toute référence aux processus de diffusion spatiale et de transformations réciproques des pratiques. C'est également le sens de la patrimonialisation des figures vaqueras si proches les unes des autres et pourtant toujours pensées dans une éclatante singularité nationale : le cowboy américain, le charro mexicain, le llanero vénézuélien, le vaqueiro brésilien, le huaso chilien et le gaucho argentin. Ainsi apparaissent les mécanismes de la diffusion des traits culturels, ceux de leur transformation qui est généralement guidée par des enjeux de différenciation territoriale au sein d'un champ commun de références, et ceux de leur assimilation locale soutenue par des discours essentialistes sur la culture.

D'autre part, les pratiques tauromachiques contribuent, au sein de leur aire d'extension, à faire vivre des rivalités, des concurrences et des complémentarités territoriales. L'écrivain Bernard Manciet raconte que le public de Mont-de-Marsan, insatisfait des bêtes et des écarteurs, n'a rien à crier de plus tendre que l'insulte « à Dax, à Dax $»^{1}$. Au-delà des rivalités locales, s'exprime souvent une complémentarité des oppositions qui révèle un sentiment d'appartenance commun d'un niveau supérieur. D'une certaine manière, la course landaise, derrière son appellation en trompe-l'œil, fait exister une Gascogne tiraillée entre plusieurs pays (Hautes Landes, Marsan, Tursan, Chalosse, Gers). De même, la course camarguaise, au-delà du delta, fait exister la Camargue comme territoire d'appartenance entre Languedoc oriental et Provence occidentale. Dans un autre contexte, les toros coleados font exister comme un tout le territoire culturel des Llanos, coupé en deux par les constructions nationales du Venezuela et de la Colombie, sur les cendres du projet bolivarien de la Grande-Colombie.

Cette forme d'emboîtement des territoires qui marquerait à telle échelle spatiale une opposition et qui, à telle autre échelle d'un niveau supérieur, exprimerait une appartenance commune, n'est pas sans rappeler le célèbre modèle théorisé par l'anthropologue Evans-Pritchard à propos des Nuer. Sous le nom de "modèle segmentaire ", Evans-Pritchard a mis en évidence que l'opposition ou la complémentarité des identités était relative à des niveaux d'organisation socio-spatiale. S'il fallait se risquer à une application des apports théoriques de ce modèle à l'organisation spatiale et historique de l'aire culturelle de la tauro-

\footnotetext{
${ }^{1}$ J. Valat, Pour la beauté du geste, la course landaise, p. 14.
} 
machie, nous pourrions formuler comme suit, un système d'oppositions et de complémentarités des territoires taurins :

- l'Andalousie torerista s'oppose au Nord torista ;

- le centre de l'espace taurin espagnol acquis à la tauromachie formelle (Andalousie-Castille) s'oppose aux périphéries taurines de l'Espagne (Pays Basque, Navarre, Aragon, Levant) où se développent des tauromachies parallèles ;

-l'Espagne taurine en tant que pays dominant de la culture s'oppose aux terres taurines extérieures et périphériques (Portugal, France, Amérique);

- le Sud-Ouest européen taurin, acquis au modèle de la corrida et des tauromachies pédestres, s'oppose à l'Amérique taurine, dominée par les rodéos et les pratiques taurino-équestres.

Au-delà même du caractère très simplifié de cette ébauche, la limite évidente de cette formulation aux vues des analyses que nous avons menées, repose sur la dimension transnationale de la corrida espagnole tissant des liens de proximité entre les pays qui précisément ne répondent pas à ce genre d'organisation territoriale emboîtée. En revanche, nous retenons le principe que la conscience d'une identité commune passe par l'expérience d'une altérité voisine, pensée comme radicale.

\section{III. — DE LA SPÉCIFICITÉ D’UNE DIFFUSION TAUROMACHIQUE}

Cette organisation d'ensemble nécessite de s'interroger sur une particularité des processus de diffusion et de développement des pratiques tauromachiques, notamment pour celles qui ont acquis une extension transnationale. Les processus de diffusion spatiale des pratiques tauromachiques n'ont généralement pas abouti à une délocalisation du cœur de l'activité qui demeure fortement ancré dans sa terre d'origine. Ils n'ont pas entraîné non plus une inversion des rapports quantitatifs de leur développement entre territoire d'origine et nouveaux territoires. L'espace d'origine de chacune des pratiques taurines continue à jouer un rôle important dans les représentations, dans les hiérarchies entre les lieux et tient souvent une place dominante en termes d'activité. Quelques pays attestent néanmoins de rééquilibrages importants : le poids du Mexique pour la corrida, celui plus récent du Brésil pour le rodéo nord-américain et, d'une manière plus générale, la place des États-Unis dans la famille des rodéos, pourtant née de l'Amérique espagnole.

Ces éléments suffisent à constater une différence importante entre la diffusion des pratiques tauromachiques et celle de certains sports modernes pour lesquels la terre d'origine ne constitue plus une référence iconographique, ni n'occupe une place dominante dans la pratique. Il n'est qu'à voir le football né du système sportif anglais, diffusé à l'échelle internationale sans que l'Angle- 
terre, ni même le monde anglo-saxon, n'occupe une place privilégiée. Dans le cas de la corrida, voir le drapeau espagnol flotter au-dessus d'une arène française, portugaise, mexicaine, colombienne, vénézuélienne, équatorienne ou péruvienne souligne clairement cette originalité. La corrida, tout en étant assimilée au patrimoine respectif des différents pays où elle est pratiquée, marque dans le même temps, un lien privilégié avec l'Espagne. Dans le football, on imaginerait mal voir flotter un drapeau anglais au-dessus du Stade de France, du stade Santiago Bernabéu à Madrid ou de l'Estadio Azteca à Mexico, afin de souligner une quelconque relation avec la terre d'origine de ce sport universel, même si le nom de certains clubs et une partie du vocabulaire ont gardé la trace d'une genèse anglo-saxonne. Le football est devenu une caisse de résonance des identités nationales sous la forme de compétitions sportives par équipes, ce que n'est pas la corrida, qui par un autre mécanisme mettant en scène les relations territoriales, contribue plutôt à mesurer une proximité avec l'Espagne. En cela, la corrida apparaît comme le produit d'une diffusion qui atteste d'un équilibre original entre une acculturation avancée et une assimilation incomplète qui partout pose la question de la distance culturelle avec l'Espagne, y compris dans les régions où elle n'existe plus.

\section{IV. — LE RAYONNEMENT DES HAUTS LIEUX TAURINS}

Les hauts lieux de chacune des pratiques possèdent des rayonnements de portées spatiales différentes, locale, nationale, internationale, qui débordent souvent assez largement l'aire d'extension réelle de la pratique. La métaphore géographique du rayonnement permet de résoudre l'idée d'un cloisonnement parfaitement étanche des passions taurines qui sont ainsi mises en relation, quand bien même les aires d'extension ne se recoupent pas. Autrement dit, le rayonnement des hauts lieux des pratiques tauromachiques élève nécessairement le degré de leur coexistence spatiale que la représentation cartographique tend à figer dans des limites bornées. Ce fonctionnement de la spatialité permet la démultiplication des relations entre les pratiques, même si le grand clivage à l'échelle globale reste celui entre les courses de taureaux, généralement pensées dans le cadre d'une culture ibérique, et les différentes formes de rodéos, pensées dans le cadre d'une culture américaine.

Ces hauts lieux, à l'extérieur de la passion taurine, agissent comme des territoires de références, plus ou moins flous, qui organisent des cartes mentales élémentaires des jeux taurins non reliés entre eux comme des phénomènes comparables. Pour la tauromachie espagnole citons Madrid, mais surtout Séville et Pampelune qui symbolisent la culture taurine dans le monde entier. Au-delà de ces villes, citons plus largement l'Andalousie qui concentre les stéréotypes simplifiés de l'imagerie taurine : les élevages de taureaux, les arènes de la Maestranza, les toreros gitans sur fond de culture flamenca et de Guadalquivir. La tauromachie portugaise est souvent mieux connue dans les particularités de sa pratique, une corrida à cheval sans mise à mort, que dans ses hauts lieux : le 
Campo Pequeno à Lisbonne, Vila Franca de Xira, Moita, Santarém, les rives du Tage et l'Alentejo. En France, Nîmes, Arles et la Camargue sont généralement mieux associés à la culture taurine que le Sud-Ouest, et la course camarguaise est mieux connue que la course landaise. L'impact des paysages camarguais joue sûrement dans cette inégale reconnaissance des pratiques taurines. Pour le rodéo nord-américain, pour lequel la curiosité européenne ne dépasse généralement pas celle du stéréotype du cow-boy de l'Ouest, le Texas s'impose comme un espace mythique de référence. Les autres formes de rodéo sont généralement mal connues, et beaucoup, en Europe, ignorent même qu'il existe des rodéos latino-américains.

À l'intérieur de chacune des passions taurines, les espaces tauromachiques structurés par leurs hauts lieux ont une tout autre configuration. Ces hauts lieux puisent leur légitimité dans deux registres : celui du lieu historique et celui des couronnements périodiques de la pratique professionnelle. Pour la corrida, le maillage est dense en Espagne et se simplifie au-delà : Madrid, Séville, Pampelune, Bilbao, Valence et quelques autres capitales de provinces, en France, Nîmes, au Portugal, Lisbonne, puis outre-Atlantique, Mexico, Cali, Quito, Lima. Parmi ces hauts lieux, Madrid, Séville et Pampelune, servent fréquemment de modèles ou de contre-modèles au sein des terres de corrida, pour les goûts tauromachiques (torista-torerista), pour les formes tauromachiques (corridas-encierros) et dans les types de fêtes qu'ils engendrent (féria de Séville - San Isidro - Sanfermines). Pour le rodéo américain, Las Vegas, capitale fonctionnelle choisie pour la rentabilité des spectacles, centralise nombre de finales sans éclipser pour autant certains hauts lieux historiques de la pratique : Cheyenne Frontier Days, Prescott Frontier Days, Pendleton Round-up, Calgary Stampede au Canada. Cette liste peut être complétée par le nouveau grand centre d'Amérique du Sud du rodéo nordaméricain : Barretos dans l'État de São Paulo et son immense stade construit par Niemeyer. Pour la charreada, rivalisent Mexico et Guadalajara, mais il n'y a pas de lieu fixe des grandes finales puisqu'elles se déplacent chaque année, manifestant l'existence d'une centralité circulatoire. Pour les toros coleados, au Venezuela et en Colombie, les calendriers professionnels organisent également une rotation des grands rendez-vous sur l'ensemble du territoire. Au Brésil, la structure fédérale du pays contribue à une démultiplication des circuits professionnels, comme le montre l'exemple de la vaquejada dont le cour reste le Nordeste. À l'inverse le rodéo chilien sacralise l'excellence sportive du meilleur cavalier huaso, prenant le titre d'El Chileno, en un même lieu, la ville de Rancagua, non loin de Santiago, qui cumule les fantasmes de la centralité identitaire. Les pratiques tauromachiques plus modestes possèdent aussi leurs hauts lieux qui correspondent généralement à leur berceau d'origine ou à la zone de plus fervente passion : la Chalosse pour le course landaise, la Petite Camargue pour le course camarguaise, le département de Córdoba sur la Costa Atlántica colombienne pour les fiestas en corralejas, l'île de Florianopolis pour les farras da boi du littoral de Santa Catarina au Brésil. Derrière les choix de la territorialisation de la pratique taurine, qu'elle s'opère selon le modèle sportif ou non, qu'elle favorise l'intégration des différentes parties du territoire national ou non, qu'elle privilégie la centralité 
fixe à la centralité circulatoire, il est bien souvent possible de lire les enjeux de la géopolitique interne des pays concernés.

\section{V. — LES LIEUX DE COEXISTENCE \\ DES PRATIQUES TAUROMACHIQUES}

Si le rayonnement des hauts lieux taurins participe indirectement aux relations entre les pratiques tauromachiques, l'existence d'espaces où elles coexistent atteste d'une relation beaucoup plus directe. Pour autant, nous l'avons vu, la notion de coexistence spatiale nécessite d'être qualifiée pour être significative. En effet, elle peut être envisagée à différentes échelles selon des configurations spatiales très diverses qui vont des régions taurines séparées au sein d'un même pays, au partage d'une même arène, plus rarement d'un même spectacle. Et quand bien même, deux pratiques tauromachiques auraient-elles en commun un nombre significatif d'arènes, comme c'est le cas pour la corrida et les concours de recortadores en Espagne, pour la corrida et les courses landaises et camarguaises en France, ou encore dans une bien moindre mesure, la corrida et la charreada au Mexique, cela ne dit pas grand-chose sur l'existence d'une passion commune. Cela étant, le partage du lieu de mise en scène, l'existence de deux passions taurines dans une même ville ou dans une même région rendent très significatives les relations qu'elles entretiennent. Selon les contextes historiques, politiques, selon les acteurs investis, selon les contextes de l'énonciation, ces relations varient de l'indifférence au respect mutuel, de la dénonciation d'une pratique radicalement autre à la reconnaissance d'une passion commune. Le temps des fêtes, dans une ville ou dans un village, en ce qu'il est un moment de rassemblement et de démonstration exacerbée des marques de l'identité, apparaît toujours comme un moment important pour remettre en jeu l'équilibre de ces relations. Au fil de l'analyse, nous avons repéré certaines villes jouant à ce titre un rôle crucial dans l'expression plurielle des pratiques taurines et par conséquent dans la mise en relation des identités territoriales qu'elles incarnent.

En Espagne, nous avons souligné, la correspondance entre l'arc dessiné par les spectacles taurins populaires et les régions septentrionales et orientales marquées par une identité forte. Les fêtes taurines de Pampelune, Saragosse, Castellón et Valence à travers la corrida expriment le partage d'une passion commune avec le reste de l'Espagne. Mais à travers la spécificité des formes tauromachiques populaires, dont certaines présentent un stade avancé de patrimonialisation régionale, elles expriment aussi leur singularité et leur différence. Dans les cas mentionnés, l'expression de la singularité régionale ne sort pas du champ tauromachique, qui finalement constitue une base commune de différenciation acceptée par tous. À Barcelone, nous l'avons vu, c'est le champ tauromachique lui-même qui est de plus en plus contesté. En France, dans le Sud-Ouest, ce sont les villes de Dax et de Mont-de-Marsan, hauts lieux de la corrida et de la course landaise, qui jouent un rôle crucial dans l'articulation des pratiques tauromachiques et des territorialités auxquelles elles 
renvoient. De même dans le Sud-Est, Nîmes et plus encore Arles, tiennent un rôle équivalent dans l'expression des liens entre corrida et course camarguaise. Au Portugal, les villes de Vila Franca de Xira et de Moita ainsi que quelques cités de la frontière de moindre importance, à l'image de Barrancos, témoignent des relations complexes entre corrida espagnole et corrida portugaise, jouant un rôle clé dans la compatibilité des passions généralement données comme antithétiques.

De même en Amérique, certaines villes occupent une place à part dans la cohésion d'ensemble de l'espace tauromachique, par la coexistence en leur sein de pratiques tauromachiques différenciées. La zone frontière entre les ÉtatsUnis et le Mexique multiplie les modalités de coexistence entre la corrida, le rodéo nord-américain, le jaripeo ranchero professionnel et la charreada, pour ce qui demeure l'une des « coutures » de l'espace tauromachique la plus dynamique qui soit. L'agglomération de Los Angeles pourrait à elle seule incarner cette imbrication des cultures taurines (club taurin de la corrida espagnole, bloodless bullfigting, jaripeo, charreada, rodéo...). Au Mexique, Mexico, Guadalajara et Aguascalientes s'affirment à la fois comme des centres de la culture charra et des lieux de corridas de première importance. En Colombie, où les aires d'extension des jeux taurins ne se chevauchent guère, les villes où ils coexistent sont rares, à l'inverse de ce que l'on observe au Mexique. À ce titre Villavicencio, située sur le piémont oriental de la cordillère, se distingue par sa fonction de capitale nationale du coleo et sa féria de corridas. La ville intérieure de Sincelejo, appartenant à la région de la Costa Atlántica, occupe une position comparable : elle est à la fois le centre le plus important des fiestas en corralejas et possède l'une des rares arènes de corridas de cette zone rurale paupérisée. Au Venezuela, le recoupement spatial de ces deux pratiques tauromachiques est beaucoup plus important, de sorte qu'il existe des villes de corridas où le coleo n'est pas négligeable (Maracay, Valencia, San Cristóbal) et des villes de coleo où les corridas ont quelque importance (Valle de la Pascua, Barinas). En Équateur, les villes périphériques des capitales provinciales mélangent volontiers les genres, celui de la corrida, des toros de pueblo et du rodéo chagra. Guayaquil rivale de Quito, semble décidée à abandonner la corrida, trop andine, au profit du rodéo montubio, plus en accord avec sa conception de l'identité littorale. Enfin, au Pérou, dans de nombreuses villes de la Sierra méridionale, coexiste une corrida des plus canoniques avec les multiples formes acquises par les " tauromachies andines ", de la corrida populaire à la pratique aujourd'hui rare du turupukllay.

Compte-tenu de la diffusion transatlantique de la corrida et de son antériorité historique sur les autres pratiques tauromachiques, c'est le plus souvent à travers elle que se joue la plupart des questions posées par leur coexistence spatiale. La corrida apparaît ainsi comme une sorte de clé de voûte de l'espace tauromachique à partir de laquelle s'est organisée l'architecture générale des pratiques. Outre-Atlantique, le rodéo étasunien, joue sans doute un rôle comparable qui s'affirme depuis un demi-siècle, puisqu'il apparaît comme un modèle dominant et spatialement conquérant, dont l'influence se fait sentir y compris dans les 
régions où il n'est pas directement présent. Il y a d'ailleurs quelques parallèles qu'il est intéressant d'établir entre la corrida et le rodéo nord-américain. Ces pratiques incarnent les deux archétypes d'affrontement entre les hommes et les bovins, les plus connus au monde : les jeux taurins méditerranéens pour la corrida espagnole et le rodéo américain pour le bull riding étasunien. Dans les deux cas, les archétypes sont d'une grande imprécision par rapport à la réalité des pratiques. De plus, ce sont deux pratiques fortement marquées par la symbolique nationale du pays qui les a vu naître dans leur forme moderne. La corrida a longtemps été pensée comme incarnant les valeurs de "l'homme espagnol », de même que le rodéo incarnerait celles des Yankees. Enfin, leur répartition est en partie le résultat de deux phases d'expansion hégémonique, chacune inscrite dans un temps fort de la mondialisation occidentale. Pour l'Espagne, celui de la conquête de l'Amérique à partir du $\mathrm{xvI}^{\mathrm{e}}$ siècle qui importe les jeux taurins outre-Atlantique. Pour les États-Unis, celui d'un impérialisme continental plus ou moins déguisé, qui démarre dès le milieu du XIX ${ }^{\mathrm{e}}$ siècle et s'affirme tout au long du $\mathrm{xx}^{\mathrm{e}}$ siècle, dont les idées libérales ont d'ailleurs contribué à rejeter une partie de l'héritage ibérique. En cela, le XIX ${ }^{\mathrm{e}}$ siècle est un siècle charnière, car dans un contexte d'affirmation forte des identités nationales, l'expansion de la corrida s'achève au moment où démarre l'individualisation des rodéos nationaux. Les rodéos incarnent ainsi une sorte de tournant tauromachique qui ancre la problématique des affrontements entre les hommes et les bovins dans un contexte panaméricain, venant rejouer localement avec les héritages composites de l'hispanité.

\section{VI. — LES FRONTIËRES TAURINES}

Enfin, une dernière configuration territoriale nous semble jouer un rôle de première importance dans la cohésion d'ensemble de l'espace tauromachique : les frontières taurines. Les frontières taurines sont à la fois les limites de l'extension des pratiques et celles des États territoriaux participant à la discontinuité de la mosaïque taurine. Dans le contexte d'une régulation nationale des pratiques, les frontières entre les États créent des coupures, plus ou moins visibles au sein de l'espace tauromachique. Celles qui concernent la corrida espagnole sont les plus visibles, puisque la pratique est explicitement interdite dans certains pays où elle fut, un temps, autorisée. Dans ce domaine, la frontière taurine entre l'Espagne et le Portugal coupe la péninsule Ibérique en deux. En France, c'est le pays lui-même qui est coupé en deux par une frontière légale de la tauromachie. Cette frontière constitue à la fois une exception remarquable dans le droit français et une exception parmi les pays de corridas, qui généralement ne connaissent pas de césure interne aussi radicale. En Amérique, la frontière taurine entre les États-Unis et le Mexique marque également une rupture fondamentale de l'espace tauromachique, délimitant les confins septentrionaux des terres de corridas. Les frontières en Amérique du Sud ne matérialisent pas une telle coupure, en raison d'une localisation des corridas qui suit l'axe de la cor- 
dillère andine. La Bolivie apparaît comme un pays de transition au-delà duquel la corrida est interdite et a totalement disparu (Brésil, Chili, Argentine, Uruguay, Paraguay). Mais la frontière n'est jamais simple coupure. En Europe, on a vu à quel point les frontières taurines de la corrida, dans leurs dimensions économique, juridique, historique et culturelle étaient le support de relations de voisinage complexes. De même entre le Mexique et les États-Unis, on a souligné le rôle particulier de la frontière taurine de la corrida, engendrant sur son tracé des relations transfrontalières dissymétriques.

Les frontières des rodéos sont de natures beaucoup plus variées. La raison tient d'abord à l'existence d'une famille de pratiques attachées à des principes et des techniques en partie communes. Les frontières des techniques ne correspondent donc pas à celles des cadres nationaux de régulation sportive des jeux. Trois techniques principales donnent forme aux rodéos : la monte des chevaux et des taureaux, l'usage du lasso et le coleo. Sous cet angle, la monte des taureaux est commune à trois grandes nations du rodéo : les États-Unis, le Mexique et le Brésil. L'usage du lasso se retrouve aux États-Unis, au Mexique et dans les régions où des rodéos sont en cours de normalisation, à l'image du rodéo montubio en Équateur et du rodeio crioulo au Brésil. Enfin, le coleo rapproche le Mexique, Cuba, le Venezuela, la Colombie et le Brésil. Seul le Chili paraît isolé par une technique singulière. Quant à l'Argentine, elle est exclue de la géographie des jeux taurino-équestres qui demeurent assez rudimentaires en comparaison de ce que représente la figure vaquera du gaucho dans l'iconographie nationale.

Bien que les spectacles soient le plus souvent régulés dans des cadres nationaux, il existe des relations transfrontalières non négligeables générées par les jeux taurins. Les plus visibles sont celles engendrées par le bull riding nordaméricain dans le cadre du Pro Bull Riders qui est désormais implanté au Canada, au Mexique, au Brésil et en Australie. Deux pratiques permettent ensuite d'intenses relations bilatérales. C'est d'une part, la charreada entre le Mexique et les États-Unis servant de trait d'union mexaméricain. C'est d'autre part, les toros coleados, entre le Venezuela et la Colombie, célébrant l'appartenance commune à un territoire culturel partagé : les Llanos. Enfin, il existe également quelques compétitions internationales de coleo qui rassemblent des participants venus de toute l'Amérique. Cette modalité est peut-être la mieux à même d'incarner un rodéo latino-américain unifié qui serait opposé au bull riding dominé par l'Amérique du Nord.

Quelles que soient les pratiques considérées, la frontière taurine apparaît comme un espace de premier plan dans la cohésion de la mosaïque taurine. La raison tient dans l'ambivalence même de la frontière en tant qu'objet géographique : la frontière crée une rupture spatiale qui, dans le même temps, crée de la continuité territoriale. Voilà tout le paradoxe, nous pourrions même dire toute l'ironie géographique de la frontière telle qu'elle a été perçue sur un plan théorique par Roger Brunet et, avant lui, par Jean Gottmann dans La signification $d u$ territoire ${ }^{2}$. On pourrait même renverser quelques perspectives, par

\footnotetext{
${ }^{2}$ J. Gottmann, The Significance of Territory.
} 
une acception extensive de la frontière au sens d'accélération des discontinuités territoriales, en envisageant que nombre de pratiques taurines sont nées d'une expérience de la frontière. On a vu pour le rodéo nord-américain, comment l'imaginaire de la frontier, celle de la progression de la conquête de l'Ouest, a fini par remplacer la frontière territoriale, celle de l'annexion des terres mexicaines. En France, la corrida s'est enracinée dans une proximité frontalière avec l'Espagne, en faisant remonter vers le nord la culture hispanique qui elle-même a contribué à donner forme aux « fêtes du taureau » dans le Sud-Ouest et le SudEst, qu'elles soient structurées autour de la corrida, de la course landaise ou de la course camarguaise. Les courses de recortadores arago-navarraises ont grandi à la périphérie du territoire espagnol dans une frontière historique du royaume de Castille et ont profité à leur tour de l'expérience landaise dans l'introduction de certaines techniques. Les « corridas soft » de la Californie sont nées, grâce à la communauté des Açores, à proximité du Mexique, et le free style bullfighting du rodéo nord-américain utilise les taureaux de corrida du voisin.

S'il est un problème géographique aussi fondamental que difficile à résoudre, c'est bien celui touchant à la question : «pourquoi là et pas ailleurs ? " En tenant compte des différenciations spatiales et des transformations de la culture, les interrogations se multiplient : "pourquoi de telle façon ici et de telle autre façon ailleurs? " Les questions sur les causes premières de la localisation et des discontinuités du territoire incitent le géographe à multiplier les points de vue historique, sociologique, anthropologique, juridique, économique, psychologique. Elles l'obligent surtout à tenter d'en coordonner et d'en peser la valeur explicative. Et le point de vue géographique ? Nous assumons qu'il soit une réflexion circulaire sur les rapports entre les hommes et leur territoire, comme cause et conséquence des phénomènes sociaux. 



\section{BIBLIOGRAPHIE}

\section{I. - OUVRAGES ET ARTICLES}

Aguilar Criado, Encarnación, "La economía de la fiesta ", dans José Hurtado SÁnchez (dir.), Nuevos aspectos de la religiosidad sevillana. Fiesta, imagen, sociedad, Séville, Ayuntamiento de Sevilla, 2002, pp. 199-232.

Alvarez, Sandra, Tauromachie et flamenco : polémiques et clichés (Espagne, fin du XIX $X^{e}$ - début XX $X^{e}$ siècles), Paris, l'Harmattan, 2007.

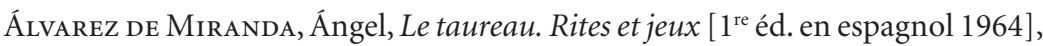
Portet-sur-Garonne, Loubatières, 2003.

Álvarez del Villar, José, Raíces de la tauromaquia charra, México, Editorial Texto e Imagen, 1973.

Amselle, Jean-Loup, Branchements. Anthropologie de l'universalité des cultures, Paris, Flammarion, 2001.

Argote de Molina, Gonzalo, Discurso sobre la montería, Madrid, Casariego, 1991.

Armaignac, Henry, Viaje por las pampas argentinas, Buenos Aires, Eudeba, 1974.

Arnaud, Gilles, Annuaire des arènes Provence-Languedoc, Sommières, 2001.

Aspiazu, Roberto, Plazas de toros de Bizkaia, Bilbao, Diputación Foral, 1991.

Auby, Jean-François, Droit et finances de la tauromachie, Bordeaux, Éditions Sud-Ouest, 2000.

Augustin, Jean-Pierre, Sport, géographie et aménagement, Paris, Nathan, coll. « Fac. Géographie », 1995.

Augusto Fagundes, Antonio, "Rodeio Country, Nao! », Zero Hora, 1999 : http://www.paginadogaucho/com.br/deba/rcn.htm [réf. du 10 juin 2007].

Bale, John, Sport and Place. A Geography of Sport in England, Scotland and Wales, Londres, C. Hurst-Company, 1982.

Baratay, Éric, Et l'homme créa l'animal. Histoire d'une condition, Paris, Odile Jacob, 2003.

Baratay, Éric, et Hardouin Fugier, Élisabeth, La corrida, Paris, Presses Universitaires de France, coll. «Que sais-je? » (568), 1995. 
Bardaji Azcárate, Isabel, «La aplicación de la Política Agrícola Común en el ganado de lidia ", dans Economía de la ganadería de lidia en España, dossier publié dans le no 3 (2005) de la Revista del Instituto de Estudios Económicos, pp. 71-82.

BARTH, Frederik, Ethnic groups and boundaries: the social organisation for cultural difference, Boston, Little Brown, 1969.

Bataillon, Claude, Deler, Jean-Paul, et Théry, Hervé, Amérique latine, Paris, Belin, coll. « Géographie universelle » 1991.

Batté, Jean-François, Politique et tauromachie. De Charles Quint à Juan Carlos, Nîmes, Union des bibliophiles taurins de France, 1993.

Bennassar, Bartolomé, Histoire de la tauromachie. Une société du spectacle, Paris, Desjonquères, 1993.

— «Histoire de la tauromachie », dans Robert BÉRARD (dir.), La Tauromachie. Histoire et dictionnaire, Paris, Robert Laffont, 2003, pp. 3-106.

BérARD, Robert, (dir.), La Tauromachie. Histoire et dictionnaire, Paris, Robert Laffont, 2003.

Berdoues, Pierre, Histoire de la tauromachie à Toulouse, Nîmes, UBTF, 1978.

Berdoulay, Vincent, "Les idéologies comme phénomènes géographiques ", Cahiers de géographie du Québec, 29(77), 1988, pp. 205-216.

Berdoulay, Vincent, et Entrikin, Nicholas, «Lieu et sujet. Perspectives théoriques ", L'Espace géographique, 2, 1998, pp. 111-121.

Bernand, Carmen, et Grunzinski, Serge, "La redécouverte de l'Amérique ", L'Homme, 122-124(32), 1992, pp. 7-38.

Bernard, Vincent, "La iglesia y los toros ", dans Antonio García-Baquero GonzÁlez et Pedro Romero de Solís (éd.), Fiestas de toros y sociedad. Actas del Congreso Internacional (Sevilla, 26 de noviembre - 1 de diciembre de 2001), Séville, Fundación Real Maestranza de Caballería de Sevilla - Universidad de Sevilla, 2003, pp. 337-354.

Bernié-Boissard, Catherine, « Nîmes en Feria : une ville sous la ville », dans ID. (dir.), Tauromachies et identités locales, Paris, L'Harmattan, 2004, pp. 41-56.

— " Le public des courses de taureaux : une contre-distinction ? ", dans ID. (dir.), Tauromachies, sport, culture, regards croisés sur les publics, Paris, L'Harmattan, 2006, pp. 25-50.

Bernié-Boissard, Catherine, (dir.), Tauromachies et identités locales, Paris, L'Harmattan, 2004.

— Tauromachies, sport, culture, regards croisés sur les publics, Paris, L’Harmattan, 2006.

Bezerra, José Augusto, «Anjas da arena », Revista Globo Rural, 2000 : http:// globorural.globo.com/edic/169/rep_rodeio1.htm.

BIRD Rose, Deborah, Reports from a Wild Country: Ethics for Decolonisation, Sydney, UNSW Press, 2004.

Bisнко, Charles Julian, "The peninsular background of latin american cattle ranching », Hispanis American Historical Review, 32(4), 1952, pp. 491-515. 
Bolívar, Lares, et Elisur, Emilio, Geografía apureña, San Fernando de Apure, 2003.

Bonnemaison, Joël, et Cambrezy, Luc, " Le lien territorial entre frontières et identités », Le territoire. Géographie et cultures, [Paris], 20, 1996, pp. 7-19.

Bordes, Jean, Les Structures agraires de la vallée du Puangue (Chili central), thèse de doctorat soutenue en 1957 à l'université de Bordeaux (inédite).

Bordes, Pierre, La course landaise. Des origines à nos jours, Pau, Princi Négue, 2003.

Boto-Arnau, Guillermo, « Cádiz y la aparición del toreo moderno », Revista de Estudios Taurinos, 22, 2006, pp. 75-98.

Bowes, Richard, Historical álbum of Colorado 1859-1959, Denver, Richard Bowes Publisher and Editor, 1959.

Bromberger, Christian, Foot-ball, la bagatelle la plus sérieuse du monde, Paris, Bayard, 1998.

Brunet, Roger, François, Jean-Christophe, et Grasland, Claude, " La discontinuité en géographie : origines et problèmes de recherche ", L'Espace géographique, 4, 1997, pp. 297-308.

Brunnschweiler, Dieter, The Llanos frontier of Colombia, Michigan, Latin American Studies Center - Michigan State University, 1972.

Caballero, Antonio, «Le condor et le taureau ", dans Jean Ortiz (dir.), Tauromachies en Amérique latine, Anglet, Atlantica, 2004, pp. 23-33.

Cabrera Bonet, Rafael, "Transformación y continuidad del espectáculo taurino madrileño en el primer tercio del siglo XVIII ", dans Annie MoliniÉBertrand, Jean-Paul Duviols et Araceli Guillaume-Alonso (coord.), Des taureaux et des hommes. Tauromachie et société dans le monde ibérique et ibéro-américain, Paris, Presses de l'université de Paris-Sorbonne, coll. «Iberica " (12), 1999, pp. 113-120.

Caillois, Roger, L’homme et le sacré [1950], Paris, Gallimard, 1994.

— Les jeux et les hommes [1958], Paris, Gallimard, 2000.

CALLÈDE, Jean-Paul, «L'affaire des corridas de Floirac : la production de la tradition tauromachique ", Jeux et enjeux du taureau. Cahiers ethnologiques, 11, 1990, pp. 103-136.

— «Les corridas : passions taurines et modernisation urbaine ", Jeux et enjeux du taureau II. Cahiers ethnologiques, 17, 1995, pp. 101-124.

CÂmara Cascudo, Luís da, A vaquejada nordestina e sua origem, Natal, Fundaçao José Augusto, 1976.

Campo, Luis del, Pamplona y toros (siglo XVIII), Pampelune, La Acción Social, 1972.

CAPucha, Luís, «O campo da tauromaquia », Sociología-Problemas e Prácticas, 5, 1988, pp. 149-170.

— «Tauromaquia e identidades culturais locais », Sociologia-Problemas e Praticas, 8, 1990, pp. 139-145.

— "Touros e touradas ", dans Portugal Moderno, Enciclopedia Tematica, Lisbonne, Pomo, 1991, pp. 90-102. 
- "O espelho quebrado: versus e reversus nas tauromaquias populares ", Mediterrâneo, 5-6, 1995, pp. 33-56.

- "Mosaico de las fiestas de toros en Portugal ", dans Pedro Romero DE Solís (coord.), Las fiestas populares de toros. Demófilo, [Séville], 25, 1998, pp. 285-311.

— « Historias da tauromaquia em Portugal: cavaleiros, forcados, matadores e festas populares ", dans Annie Molinié-Bertrand, Jean-Paul Duviols et Araceli Guillaume-Alonso (coord.), Des taureaux et des hommes. Tauromachie et société dans le monde ibérique et ibéro-américain, Paris, Presses de l'université de Paris-Sorbonne, coll. "Iberica " (12), 1999, pp. 135-148.

— «Barrancos en escena, o una metáfora del Portugal de hoy », dans Antonio García-Baquero González et Pedro Romero de Solís (éd.), Fiestas de toros y sociedad. Actas del Congreso Internacional (Sevilla, 26 de noviembre 1 de diciembre de 2001), Séville, Fundación Real Maestranza de Caballería de Sevilla - Universidad de Sevilla, 2003, pp. 431-448.

Caro Baroja, Julio, Le Carnaval, Paris, Gallimard, 1979.

CARvalho, Sérgio, "As tradiçoes, as leis e a constituição deveriam ser para manter e cumprir » : http://toureio.no.sapo.pt/falando/as_traicoes.htm [réf. du 17 août 2006].

Carvalho Neto, Paulo de, "Un caso de mestizaje cultural: los toros en el folklore ecuatoriano, sus raices hispánicas ", Revista de Indias, 95-96(24), 1964, pp. 221-228.

Casares, Julio, Diccionario ideológico de la lengua española, Barcelone, Gustavo Gili, 1994.

Castanet, Jean-Louis, Campo Bravo. Le guide des ganaderias, s. l., Lit. Danona, 1995.

Castillo Martos, Manuel, "Toros en el Altiplano andino (1550-1650). Una aproximación a su historia ", dans Antonio García-BAQuero González et Pedro Romero de Solís (éd.), Fiestas de toros y sociedad. Actas del Congreso Internacional (Sevilla, 26 de noviembre - 1 de diciembre de 2001), Séville, Fundación Real Maestranza de Caballería de Sevilla - Universidad de Sevilla, 2003, pp. 211-234.

CAUE des LAndes, Arènes de la course landaise et de la corrida, Mont-deMarsan, CAUE 40 - Le Festin, 2000.

Cervantes, Ángel, et Moreno, José Enrique, Las rutas del toro en Andalucía, Séville, Fundacíon José Manuel Lara, coll. « Rutas Culturales », 2004.

Chartier, Roger, Avant propos, dans Norbert Elias et Éric Dunning, Sport et civilisation, la violence maîtrisée, Paris, Fayard, 1986.

Chevalier, François, La formation des grands domaines au Mexique. Terre et société auX XVI et $X V I I^{e}$ siècles, Paris, Institut d'Ethnologie, coll. " Travaux et mémoires » (54), 1952.

Clement, Thibaud, "Puissance et souveraineté : sur l'institutionnalisation des armées patriotes », Bulletin de l'Institut Pierre Renouvin, 7, 1999 : http://ipr. univ-paris1.fr/spip.php?article49. 
Cossío, José María de, Los Toros. Tratado histórico-técnico, Madrid, EspasaCalpe, 1943-1997 (11 vol.) ; 1995 (2 vol.).

Crépin, Olivier, "La région toulousaine : un territoire de tradition taurine à la géographie variable et incertaine ", Sud-Ouest européen, 20, 2005, pp. 21-35.

Crismatt Mouthon, Carlos, (dir.), Corralejas departementales, mémoire de recherche soutenu en 1998 à l'Universidad de Córdoba, Colombie (inédit).

Cristaller, Walter, Die Zentralen Orte in Suddeutschland, Inéna, Fisher, 1933.

Da Cunha, Euclides, Hautes Terres. La guerre de Canudos, Paris, Métaillé, 1997.

Dalla Bernardina, Sergio, L'éloquence des bêtes. Quand l'homme parle des animaux, Paris, Métaillé, 2006.

DarracQ, Jean-Pierre, Histoire taurine de Vic-Fezensac, Nîmes, UBTF, 1978.

Darrieumerlou, Miguel, "Toreros yankees au Mexique », dans Jean Ortiz (dir.), Tauromachies en Amérique latine, Anglet, Atlantica, 2004, pp. 59-66.

DAUGA, Marylis, 1965-1985: la course landaise entre tradition et modernité, mémoire de DEA soutenu en 2000 à l'université de Pau et des Pays de l'Adour.

— « La course landaise et le musée de Bascons », Le Festin, 42, 2002, pp. 25-33.

— "L'âge d'or de la course landaise : l'adaptation d'une pratique culturelle à la modernité ", dans Michel PApy et Christian Thibon (éd.), Chalosse, l'esprit des lieux entre mémoires et histoires, Éditions Gascogne, Orthez, 2002, pp. 69-91.

Dauga, Marylis, et Puyo, Jean-Yves, "Les écarteurs landais entre mythe et réalité, $\mathrm{XIX}^{\mathrm{e}}-\mathrm{XX}^{\mathrm{e}}$ siècle ", dans Christian Desplat (dir.), L'homme du Midi, sociabilités méridionales, Paris, CTHS, 2003, pp. 185-219.

De Marichalar, Emmanuel, Le souffle dans le dos, Biarritz, J. et D. éditions, 1997.

De Monredon, Emmanuel, La corrida par le droit, Nîmes, Union des bibliophiles taurins de France, 2001.

Deffontaines, Julie, Taureau de Camargue. Un produit, un paysage, Paris, Les éditions de l'Épure, 2005.

Deffontaines, Pierre, "L'introduction du bétail en Amérique latine ", Les Cahiers d'Outre-Mer, 37(10), 1957, pp. 5-22.

Delgado Linacero, Cristina, El toro en el Mediterráneo: análisis de su presencia y significado en las grandes culturas del mundo antiguo, Madrid, Egartorre, 1996.

— «El toro y el Mediterráneo: simbolismo de una cultura », dans Boletín de Loterías y Toros (1991-2001). 10 años de pensamientos, Cordoue, Asociación Boletín de Loterías y Toros, 2001, pp. 221-226.

Delgado Ruiz, Manuel, "El toreo como arte o cómo se desactiva un rito », Taurología, 1, 1989, pp. 32-38.

Delmas, Alain, et Leccia, Jacques, "Architecture et tauromachie. Eupalinos entre dans l'Arène ", dans Tauromachie. Regards croisés, Mont-de-Marsan, Éditions Jean Lacoste, 2002, pp. 189-212. 
Desveaux, Emmanuel, et Saumade, Frédéric, "Relativizar el sacrificio o el cuadrante taurómaco ", dans Pedro Romero de Solís (coord.), Las fiestas populares de toros. Demófilo, [Séville], 25, 1998, pp. 235-244.

Di Meo, Guy, (dir.), La géographie en fêtes, Paris, Ophrys, 2001.

Diccionario de la Real Academia Española, 2007 [édition en ligne : http://buscon. rae.es/draeI/].

Diccionario geográfico de España, Madrid, 1956-1961 (17 vol.).

Digard, Jean-Pierre, L'Homme et les animaux domestiques. Anthropologie d'une passion, Paris, Fayard, 1990.

— «Un aspect méconnu de l'histoire de l'Amérique : la domestication des animaux », L'Homme, 122-124(32), 1992, pp. 253-270.

- Les Français et leurs animaux. Ethnologie d'un phénomène de société, Paris, Fayard, 1999.

— "Les Européens du Sud-Ouest, les animaux et la tauromachie », dans La corrida au $\mathrm{XXI}^{e}$ siècle : pourquoi ?, Nîmes, Cercle universitaire d'études et de médiation des cultures et pratiques taurines - Arts-Culture-Foi - Les Amis de Pablo Romero, 2007, pp. 35-49.

Domínguez Rojas, Jesús, "Los toros de lidia en la economía », dans Economía de la ganadería de lidia en España, dossier publié dans le no 3 (2005) de la Revista del Instituto de Estudios Económicos, pp. 83-98.

Don Ventura, Solidaridad taurina Hispano-lusitana (1846-1946), Lisbonne, 1947.

Drain Mothré, Michel, «Les identités territoriales du Portugal », Lusotopie. Portugal une identité sur la longue durée, 2, 2002, pp. 159-163.

Dumazedier, Joffre, Vers une civilisation des loisirs?, Paris, Seuil, 1962.

Dupuy, Pierre, Guide des férias : France, Espagne, Portugal, Paris, La Manufacture, 1990.

- Toros en Camargue, Nîmes, Éditions Toros, 1995.

- Histoire et tauromachie de Lascaux à nos jours, Pau, Cairn, 2006.

Durand, Jacques, Humbles et phénomènes, Lagrasse, Verdier, 1995.

- Chroniques taurines, Paris, De Falois, 2003.

Durand, Jacques, et Maigne, Jacques, L'habit de lumière. Voyage en tauromachie, Paris, Ramsay, 1985.

Duret, Évelyne, "La course camarguaise, aspects historiques ", dans Claude Martel et Jean-Noël Pelen (éd.), L'homme et le taureau en Provence et en Languedoc, Grenoble, Glénat, 1990, pp. 61-78.

Duvignaud, Jean, Fêtes et civilisations [1973], Arles, Actes Sud, 1991.

Elias, Norbert, et Dunning, Éric, Sport et civilisation, la violence maîtrisée [1986], Paris, Fayard, 1994.

Enciclopedia Universal Ilustrada Europeo-americana, Madrid, Espasa-Calpe, 1926 (72 vol.). 
Erausquin, Estela, « Un aspect méconnu du Buenos Aires d'antan : la corrida (1609-1819) », dans Annie Molinié-Bertrand, Jean-Paul Duviols et Araceli Guillaume-Alonso (coord.), Des taureaux et des hommes. Tauromachie et société dans le monde ibérique et ibéro-américain, Paris, Presses de l'université de Paris-Sorbonne, coll. «Iberica » (12), 1999, pp. 175-186.

Espinosa Serrano, Patricio, et Espinosa Vela, José Patricio, El toro de lidia en el Ecuador. Apuntes históricos, Quito, Éd. David Andrade Aguirre, 2001.

Esteban Gordo, Emilio, «Los festejos populares. Un mercado en expansión en una época difícil para las fiestas de toros ", dans Economía de la ganadería de lidia en España, dossier publié dans le no 3 (2005) de la Revista del Instituto de Estudios Económicos, pp. 239-248.

Fabaron, Jean-Pierre, «Répartition des jeux taurins dans le monde : essai de classification ", Jeux et enjeux du taureau. Cahiers ethnologiques, 11, 1990, pp. 35-48.

FAvory, Michel, «Les bestiaires et l'espace, raisons géographiques de la passion taurine dans le sud-ouest européen », Sud-Ouest Européen, 8, 2000, pp. 5-14.

FÉdÉRATION FRANÇAISE DE COURSE LANDAISE, La course landaise. Les 50 ans de la Fédération, Dax, Aquitaine Presse, 2003.

Ferrao, João, "Portugal, três geografias em recombinação », Lusotopie, Portugal une identité sur la longue durée, 2, 2002, pp. 151-158.

Ferras, Robert, Saint-Julien, Thérèse, et Pumain, Denise, France, Europe du Sud, Paris, Hachette, coll. « Géographie universelle », 1990.

Ferry, Luc, Le nouvel ordre écologique. L'arbre, l'animal et l'homme, Paris, Grasset, 1992.

Fierro Patiño, Javier, "Llanos orientales: de los hermosos atardeceres al conflicto armado ", Revista Javeriana, Bogotá, 2006 : http://javierfierro.files. wordpress.com/2007/06/llanos-orientales.pdf.

Figère, Maurice, Les arènes de Lunel et la corrida, Nîmes, UBTF, 2001.

Flanet, Véronique, et Veilletet, Pierre, Le peuple du toro, Paris, Herné, 1986.

Flores Arroyuelo, Francisco, Correr los toros en España. Del monte a la plaza, Madrid, Biblioteca Nueva, 1999.

Flores Hernández, Benjamín, "Organización de corridas de toros en la Nueva España del siglo xviII y primeros años del XIX », Anuario de Estudios Americanos, 61(2), 2004, pp. 491-515.

Foster, George, La cultura tradicional en España y América [ $1^{\text {re }}$ éd. en anglais 1960], Séville, Signaura Demos, 2003.

Foucher, Michel, L'invention des frontières, Paris, FED, 1986.

- L'obsession des frontières, Paris, Perrin, 2007.

Fournier, Dominique, « Du taureau considéré comme outil d'acculturation au Mexique », L'Homme, 136, 1995, pp. 53-73. 
- «Corrida, charreada et jaripeo. Identité taurine mexicaine et métissage culturel ", dans Annie Molinié-Bertrand, Jean-Paul Duviols et Araceli Guillaume-Alonso (coord.), Des taureaux et des hommes. Tauromachie et société dans le monde ibérique et ibéro-américain, Paris, Presses de l'université de Paris-Sorbonne, coll. « Iberica » (12), 1999, pp. 187-200.

- "Les spectacles tauromachiques naissent aussi de l'histoire ", Ethnologie française, 1(31), 2001, pp. 169-172.

Fournier, Sébastien, Bernié-Boissard, Catherine, et Michel, Jean-Pierre, Tauromachie, sport, culture. Regards croisés sur les publics, Paris, L'Harmattan, 2006.

Franco, Noberto, O porquê de Barrancos, Lisbonne, Amareleja, 2000.

Fraysse, Maurice, "Alberto Blest Gaña et Balzac », Cahiers du monde hispanique et luso-brésilien (Caravelle), 20, 1977, pp. 117-134.

Fredriksson, Kristine, American Rodeo: From Buffalo Bill to big business, College Station, Texas A\&M University Press, 1984.

Fuente Frechoso, Miguel Ángel de la, et Moral Hurtado, Ana del, "Reflexiones en torno a una imagen: el toro en la cultura ibérica », dans Boletín de Loterías y Toros (1991-2001). 10 años de pensamientos, Cordoue, Asociación Boletín de Loterías y Toros, 2001, pp. 215-220.

Gagnol, Laurent, Pour une géographie nomade. Perspectives anthropogéographiques à partir de l'expérience des Touaregs Kel Ewey (Aïr-Niger), thèse doctorale de géographie soutenue en 2009 à l'université Joseph Fourier, Grenoble I (inédite).

GARAT, Isabelle, "La tauromachie dans la vie bayonnaise : de la présence à l'omniprésence ", dans Catherine BErnié-Boissard (dir.), Tauromachies et identités locales, Paris, L'Harmattan, 2004, pp. 27-40.

García-Baquero González, Antonio, «El macelo sevillano y los orígenes de la tauromaquia moderna », Taurología, 2, 1990, pp. 38-44.

García-Baquero González, Antonio, Romero de Solís, Pedro, et Vázquez Parladé, Ignacio, Sevilla y la fiesta de los toros [1980], Séville, Ayuntamiento de Sevilla, Biblioteca de Temas Sevillanos (5), 1994.

García-Baquero González, Antonio, et Romero de Solís, Pedro, (éd.), Fiestas de toros y sociedad. Actas del Congreso Internacional (Sevilla, 26 de noviembre - 1 de diciembre de 2001), Séville, Fundación Real Maestranza de Caballería de Sevilla - Universidad de Sevilla, 2003.

Garibay Anaya, Federico, et Luna Parra, Miguel, México se viste de luces. Un recorrido histórico por el territorio taurino de nuestro país, Guadalajara, Ágata Editores, 2001.

Garland, Antonio, Lima y el toreo, Lima, Librería Internacional del Perú, 1948.

Gavira Rivero, Natividad, «La fiesta taurina al otro lado del Atlántico. Estudio de "Toros y Deportes", semanario de actualidad gráfica ", dans Boletín de Loterías y Toros (1991-2001). 10 años de pensamientos, Cordoue, Asociación Boletín de Loterías y Toros, 2001, pp. 206-213. 
George Oliven, Ruben, «À la recherche des origines perdues, Le mouvement traditionaliste gaucho au Brésil », Études rurales, 163-164, 2002 : http:// etudesrurales.revues.org/document117.html.

Gilbert-Lacroix, Louis, Histoire de la tauromachie en Arles, Nîmes, Union des bibliophiles taurins de France, 1977.

Goicochea Luna, Augusto, Tauromaquia andina, Madrid, Ediciones Cultura Hispánica, 1966.

Gómez Castañeda, Juan, «La ganadería de lidia y su papel en la tauromaquia actual. Un ensayo socioeconómico », dans Economía de la ganadería de lidia en España, dossier publié dans le no 3 (2005) de la Revista del Instituto de Estudios Económicos, pp. 224-238.

Gottmann, Jean, The Significance of Territory, Charlottesville, University Press of Virginia, 1973.

Graves, Alvin, The Portuguese Californians: Inmigrants in Agriculture, San Jose, Portuguese Heritage Publications of California, 2004.

Grijalba, Marina, et Purroy, Antonio, «Estudio técnico-económico de las ganaderías de toros de lidia ", dans Antonio Purroy (éd.), V Jornadas sobre Ganado de Lidia (Pamplona, 24-25 de noviembre de 2006), Pampelune, Universidad Pública de Navarra, 2006, pp. 33-59.

Guarner, Enrique, Historia del toreo en México, México, Diana, 1979.

Guichard, François, "Portugal-Espagne : frontières, identités nationales et stratégies européennes », Lusotopie, 1-2, 1994, pp. 35-50.

Guichard, François, López Trigal, Lorenzo, et Marrou, Louis, (coord.), Itinerarios transfronterizos en la Península Ibérica, Zamora, Fundación Rei Afonso Henriques, 2000.

Guichard, François, (dir.), Articulation des territoires dans la péninsule Ibérique. IV journées d'études Nord du Portugal - Aquitaine (Bordeaux-Pessac, 19-21 novembre 1998), Bordeaux, Maison des Pays Ibériques, 2001.

Guillaume-Alonso, Araceli, La tauromaquia y su génesis, Bilbao, Ediciones Laga, 1994.

- "Contre la corrida. Essai de typologie des positions anti-taurines $\left(\mathrm{xVI}^{\mathrm{e}}\right.$ XVII ${ }^{\mathrm{e}}$ siècles) ", dans Annie Molinié-Bertrand, Jean-Paul Duviols et Araceli Guillaume-Alonso (coord.), Des taureaux et des hommes. Tauromachie et société dans le monde ibérique et ibéro-américain, Paris, Presses de l'université de Paris-Sorbonne, coll. «Iberica » (12), 1999, pp. 13-22.

— «Toros y sociedad", dans Antonio Purroy (éd.), V Jornadas sobre Ganado de Lidia (Pamplona, 24-25 de noviembre de 2006), Pampelune, Universidad Pública de Navarra, 2006, pp. 121-131.

Hadwick Gayton, Anna, « The festa da Serrata », Western Folklore, 3(7), 1948, pp. 251-265. 
Hägerstrand, Torsten, Innovation diffusion as a spatial process, Chicago, Chicago University Press, 1953.

Halcón, Fátima, "Las plazas de toros de los virreinatos de América », dans Antonio García-Baquero González et Pedro Romero de Solís (éd.), Fiestas de toros y sociedad. Actas del Congreso Internacional (Sevilla, 26 de noviembre - 1 de diciembre de 2001), Séville, Fundación Real Maestranza de Caballería de Sevilla - Universidad de Sevilla, 2003, pp. 707-718.

Hancock, Claire, et Staszak, Jean-François, « L'animal au zoo, enjeu de géographie politique. Le zoo de Mexico, de Moctezuma à l'écologie ", Espaces et sociétés, 110-111, 2002, pp. 87-110.

Hardouin-Fugier, Élisabeth, Histoire de la corrida en Europe du XVIII au XXI $I^{e}$ siècle, Paris, Connaissances et Savoirs, 2005.

Harvey, Penelope, «Jugando por la identidad y la tradición. Las corridas de toros en el sur andino ", dans Tradición y modernidad en los Andes, Cusco, Centro Bartolomé de Las Casas, 1992.

Hernández, Benjamín, « Organización de corridas de toros en la Nueva España del siglo XVIII y primeros años del XIX ", Anuario de Estudios Americanos, 61(2), 2004, pp. 491-515.

Hernández Díaz-Ambrona, Carlos, «Ecología del pastoreo con ganado de lidia en las dehesas ", dans Economía de la ganadería de lidia en España, dossier publié dans le no 3 (2005) de la Revista del Instituto de Estudios Económicos, pp. 101-140.

Hibdon, James, « The economics of rodeo cowboys ", The Social Science Journal, 3(26), 1989, pp. 237-248.

Новsваwм, Éric, Les bandits [1969], Paris, La Découverte, 1999.

Hobsbawm, Éric, et Ranger, Terence, L'invention de la tradition [1983], Paris, Éditions Amsterdam, 2006.

Hubert, Henri, et Mauss, Marcel, « Essai sur la nature et la fonction du sacrifice ", Année sociologique, 2, 1899, pp. 29-138.

Instituto Nacional de Estadística, Geografía e Informática, Estadísticas Culturales, México, INEGI, 2005.

Iwasaki Cauti, Fernando, "Toros y sociedad en la Lima colonial », Anuario de Estudios Americanos, 49, 1992, pp. 313-329.

JACQUIN, Philippe, Le cow-boy. Un Américain entre le mythe et l'histoire, Paris, Albin Michel, 1992.

— Vers l'Ouest. Un nouveau monde, Paris, Gallimard, 2006.

Jordan, Terry, North American Cattle-Ranching Frontiers: Origins, diffusion and differentiation, Albuquerque, University of New Mexico Press, 1993. 
KeErle, Régis, Sport et territoires. Contribution à une géographie du pouvoir. Une géographie sociale du champ sportif, thèse doctorale de géographie soutenue en 2002 à l'université Paul Valéry, Montpellier III (inédite).

- "La tauromachie camarguaise en quête d'un territoire ", dans Catherine Bernié-Boissard (dir.), Tauromachies et identités locales, Paris, L'Harmat$\tan , 2004$, pp. 59-73.

Lacerda, Eugenio Pascele, As Farras do Boi no litoral de Santa Catarina, mémoire universitaire soutenu en 1994 à l'Universidade Federal de Santa Catarina, Florianopolis (inédit).

- O Atlântico Açoriano. Uma antropologia dos contextos globais e locais da açorianidade, thèse doctorale d'anthropologie sociale soutenue en 2003 à l'Universidade Federal de Santa Catarina, Florianopolis (inédite).

Lacroix, Louis-Gilbert, Histoire de la tauromachie en Arles, Nîmes, UBTF, 1977

LAForcade, Michel, La tauromachie dans le Sud-Ouest de la France, s. l., Imprimerie Delteil, 1984.

Lafront, Auguste, Histoire de la corrida en France. Du Second Empire à nos jours, Paris, Julliard, 1977.

- Histoire de la tauromachie à Bordeaux, Nîmes, Union des bibliophiles taurins de France, 1982.

Lago, Thomas, El Huaso. Ensayo de Antropología social, Santiago de Chile, Ediciones de la Universidad de Chile, 1953.

Langaro, Carmen, « Rodeio crioulo: a festa maior dos gaúchos », Revista Geográfica Universal, 113, 1984, pp. 53-58.

LANGue, Frédéric, "La pardocratie ou l'itinéraire d'une "classe dangereuse" dans le Venezuela des XVIII et XIX ${ }^{\mathrm{e}}$ siècle », dans Nuevo Mundo Mundos Nuevos, 2005 : http://nuevomundo.revues.org/document643.html.

Largo Aguado, Natividad, Toros: Castilla - La Mancha, Ciudad Real, Junta de la Comunidad de Castilla - La Mancha, 1991.

Larrea Apezteguía, José Luis, et Napal Lecumberri, Saturnino, El encierro de Pamplona y sus protagonistas, Pampelune, Evidencia Médica, 2005.

LaVIE, Marc, Corridas en France 2004, Anglet, Atlantica, 2004.

Lawrence, Elisabeth Atwood, Rodeo. An anthropologist looks at the Wild and the Tame, Chicago, University of Chicago Press, 1984.

Le Compte, Mary Lou, "The Hispanic Influence on the History of Rodeo, 1823-1922 », Journal of Sport History, 12(1), 1985, pp. 21-38.

Le Lannou, Maurice, Le Brésil, Colin, Paris, 1955.

Legemaate, Marco, "Ilustración y los toros ", dans Boletín de Loterías y Toros (1991-2001). 10 años de pensamientos, Cordoue, Asociación Boletín de Loterías y Toros, 2001, pp. 35-37.

LeIris, Michel, Miroir de la tauromachie, Montpellier, Fata Morgana, 1981.

Lemogodeuc, Jean-Marie, "Taureaux et corridas au Venezuela. Évolutions de la fiesta brava ", dans Jean Ortiz (dir.), Tauromachies en Amérique latine, Anglet, Atlantica, 2004, pp. 79-86. 
León Echaíz, René, Interpretación Histórica del Huaso Chileno, Buenos Aires, Francisco de Aguirre, 1971.

Les Amis du musée de la vie rurale en Pays landais, La course landaise, jeuspectacle traditionnel, Laluque, 2006, pp. 5-7 [brochure].

Lopera, Alberto, Colombia, tierra de toros, Madrid, Espasa-Calpe, 1989.

López Campillo, Évelyne, "Essence et inexistence de l'Espagne », dans Carlos Serrano (dir.), Nations en quête de passé. La péninsule Ibérique (XIX ${ }^{e}$ $X X^{e}$ siècles), Paris, Presses de l'univérsité de Paris-Sorbonne, 2000, pp. 227-233.

López Cantos, Ángel, Juegos, fiestas y diversiones en la América española, Madrid, Mapfre, 1992.

LóPez IzQuierdo, Francisco, Plazas Mayores y de toros, Madrid, Ergatorre, 1992.

— Los Toros del Nuevo Mundo (1492-1992), Madrid, Espasa-Calpe, 1992.

- Fiesta Nacional (calendario de los toros tradicionales y populares), Madrid, Agualarga, 1996.

López Martínez, Antonio Luis, Ganaderías de lidia y ganaderos. Historia y economía de lidia en España, Séville, Fundación Real Maestranza de Caballería de Sevilla, Universidad de Sevilla, 2002.

- "Los orígenes de las ganaderías de toros en España ", dans Antonio García-Baquero González et Pedro Romero de Solís (éd.), Fiestas de toros y sociedad. Actas del Congreso Internacional (Sevilla, 26 de noviembre - 1 de diciembre de 2001), Séville, Fundación Real Maestranza de Caballería de Sevilla - Universidad de Sevilla, 2003, pp. 377-390.

— « La empresa ganadera de lidia en España. Principales etapas de su evolución histórica ", dans Economía de la ganadería de lidia en España, dossier publié dans le no 3 (2005) de la Revista del Instituto de Estudios Económicos, pp. 193-224.

López Martínez, Héctor, Plaza de Acho. Historia y tradición (1766-1944), Lima, Fondo Editorial del Congreso del Perú, 2005.

- Tauromaquia limeña en el siglo XIx. Testimonios de extranjeros, Lima, Héctor López Martínez Editor, 2006.

Lösch, August, Die raumlische Ordnung der Wirstschaft, Iéna, Fische, 1940.

LujÁn, Néstor, Historia del toreo, Barcelone, Destino, 1954.

Luna Parra, Miguel, Toros en Cuba II. Auge, independencia y prohibición, México, Bibliófilos Taurinos Mexicanos, 1999.

Luna Parra, Miguel, et Ruiz Quiroz, Luis, Análisis y registro de las plazas de toros de México, México, Unión de Bibliófilos Taurinos de México, 1992.

Maestro Sanjuan, Alberto, Las plazas de toros de Aragón, Saragosse, Diputación Foral, 1991.

MarC, Henri, Histoire taurine de la ville de Béziers, Nîmes, UBTF, 1985.

Martel, Claude, "Du vocabulaire des "gens de bouvine" à celui de la course landaise : première approche lexicographique ", Jeux et enjeux du taureau II. Cahiers ethnologiques, 17, 1995, pp. 7-25. 
Martel, Claude, et Pelen, Jean-Noël, (éd.), L’homme et le taureau en Provence et en Languedoc, Grenoble, Glénat, 1990.

Maudet, Jean-Baptiste, Pour une géographie de la "planète des taureaux ", mémoire de maîtrise de géographie soutenu en 1998, à l'université Paris IV - Sorbonne (inédit).

— «Les territoires de la planète des taureaux », Géographie et cultures, 30, 1999, pp. 3-23.

- Géographie taurine du Sud-Ouest européen, mémoire de DEA de géographie soutenu en 2001 à l'université Paris IV - Sorbonne (inédit).

— « La organización funcional del espacio taurino. Apuntes de geografía taurina », dans Antonio García-BAQuero GonzÁlez et Pedro Romero de Solís (éd.), Fiestas de toros y sociedad. Actas del Congreso Internacional (Sevilla, 26 de noviembre - 1 de diciembre de 2001), Séville, Fundación Real Maestranza de Caballería de Sevilla - Universidad de Sevilla, 2003, pp. 490-494.

— «Tauromachie et géopolitique en péninsule Ibérique : la frontière Espagne/ Portugal depuis l'arène ", Mélanges de la Casa de Velázquez, nouvelle série, 33(1), 2006, pp. 259-281.

- «Le taureau marque son territoire (festivités taurines et identités territoriales du Sud-Ouest européen à l'Amérique latine) ", Annales de Géographie, 650, 2006, pp. 361-387.

- "Le renouveau des fêtes taurines en Espagne, nouveau contexte sociopolitique, nouveaux enjeux géographiques ", dans La fête au présent. Mutations des fêtes au sein des loisirs. Actes du colloque (Nîmes, 14-16 septembre 2006), Paris, L'Harmattan, 2009, pp. 61-74.

Mecham, Kirke, "The Bull Fight at Dodge », Kansas Historical Quarterly, 2(3), 1933, pp. 294-308.

Medina de la Serna, Daniel, Las prohibiciones de la Fiesta de toros en el Distrito Federal, México, Bibliófilos Taurinos de México, 1990.

Merle, Alexandra, «Tauromachie et identité nationale dans les mentalités espagnoles et étrangères à l'époque moderne ", dans Annie Molinié-BERTrand, Jean-Paul Duviols et Araceli Guillaume-Alonso (coord.), Des taureaux et des hommes. Tauromachie et société dans le monde ibérique et ibéro-américain, Paris, Presses de l'université de Paris-Sorbonne, coll. « Iberica » (12), 1999, pp. 37-50.

Ministerio de Agricultura, Geografía española del toro de lidia, Madrid, Neografis, Servicios de Publicaciones Agrarias, 1980.

Miró Quesada Sosa, Aurelio, Temas Taurinos, Lima, El Comercio S. A., 1997.

Molinié, Antoinette, «El toro y el cóndor en lidia: una corrida en los Andes peruanos », dans Antonio García-BAQuero González et Pedro Romero de Solís (éd.), Fiestas de toros y sociedad. Actas del Congreso Internacional (Sevilla, 26 de noviembre - 1 de diciembre de 2001), Séville, Fundación Real Maestranza de Caballería de Sevilla - Universidad de Sevilla, 2003, pp. 495-514.

- "Le taureau aux ailes de condor. L'invention indigéniste d'une chimère andine ", Ethnologie française, 39(1), 2009, pp. 123-131. 
Molinié-Bertrand, Annie, Duviols, Jean-Paul, et Guillaume-Alonso, Araceli, (coord.), Des taureaux et des hommes. Tauromachie et société dans le monde ibérique et ibéro-américain, Paris, Presses de l'université de ParisSorbonne, coll. « Iberica » (12), 1999.

Montes, Francisco, Tauromaquia completa o sea el Arte de torear en plaza tanto a pie como a caballo [1836], Madrid, Turner, 1994.

Morales, Javier, "Fiestas de toros en la Comunidad de Madrid », dans Antonio García-Baquero González et Pedro Romero de Solís (éd.), Fiestas de toros y sociedad. Actas del Congreso Internacional (Sevilla, 26 de noviembre 1 de diciembre de 2001), Séville, Fundación Real Maestranza de Caballería de Sevilla - Universidad de Sevilla, 2003, pp. 401-414.

Moreira, Adriano, "A tensão ibérica », dans Hipólito de la Torre Gómez et Antonio José Telo (coord.), La mirada del otro. Percepciones luso-españolas desde la historia, Mérida, Editora Regional de Extremadura, 2002, pp. 25-33.

Mullen Sands, Kathleen, Charrería Mexicana: An Equestrian Folk Tradition, Tucson, University of Arizona Press, 1993.

MuÑoz, Fanni, Cultura popular andina: el Turupukllay (corrida de toros con cóndor), mémoire de recherche en sciences sociales soutenu en 1984, à la Pontificia Universidad Católica del Perú, Lima (inédit).

— « La fiesta del Turupukllay en el mundo andino », dans Pedro Romero DE Solís (coord.), Las fiestas populares de toros. Demófilo, [Séville], 25, 1998, pp. 313-327.

Musée des cultures taurines, Rites éternels. L'homme et le taureau dans le monde, catalogue d'exposition (Nîmes, musée des cultures taurines, 20 mai 17 octobre 2004), Sommières, Romains Page éditions, 2004.

Nájera-Ramírez, Olga, " Engendering Nationalism: Identity, Discourse, and the Mexican Charro ", Anthropological Quarterly, 67(1), 1994, pp. 1-14.

Nemésio, Vitorino, Mau tempo no canal, Lisbonne, Bertrand, 1994.

Nieto Manjón, Luis, Términos taurinos, Madrid, Espasa-Calpe, 1987.

Nogueira, Néia, Festa do Peão de Boiadeiro, São Paulo, Icone Editora, 1989.

Nora, Pierre, Les lieux de mémoire, t. I : La République, Paris, Gallimard, 1984.

Olaciregui, Julio, « La fiesta en corralejas dans le nord de la Colombie, ou le sacrifice bouffon », dans Jean OrTiz (dir.), Tauromachies en Amérique latine, Anglet, Atlantica, 2004, pp. 99-108.

Orgambides Gómez, Francisco Javier, Las plazas de toros en la provincia de Cádiz, Cádiz, Tántalo, 1996.

Orrechioni, Jean, "Contribution à l'étude du mot sertão ", Bulletin de la faculté des lettres de Strasbourg, 8-9, 1967, pp. 651-657.

Ortiz, Jean, (dir.), Tauromachies en Amérique latine, Anglet, Atlantica, 2004.

Ortuño Pérez, Sigfredo, «Ecología y economía de las explotaciones de ganado de lidia en las dehesas españolas ", dans Economía de la ganadería de lidia en España, dossier publié dans le no 3 (2005) de la Revista del Instituto de Estudios Económicos. 
Palma, Ricardo, Tradiciones peruanas, Madrid, Aguilar, 1957.

Palomar, Cristina, "El papel de la charrería como fenómeno cultural en la construcción del Occidente de México ", Revista Europea de Estudios Latinoamericanos y del Caribe, 76, 2004, pp. 83-98.

Paredes Ramírez, Wellington, Indios, problema indígena, regionalidad y localidad: la posición desde la costa y Guayaquil, Guayaquil, Archivo Histórico del Guayas, 2000.

Pelletier, Claude, La tauromachie et les Basques (ou le grand malentendu), Saint-Sébastien, imprimerie Lorea, 1981.

Pepe Alcázar, Plazas de toros de Colombia, Madrid, Agualarga, 1997.

Pepe Luis, Cañero nunca existiu, Lisbonne, Paleta d'Ouro, 1925.

— Touros de morte em Portugal, Lisbonne, Paleta d'Ouro, 1927.

Pepe-Illo, La Tauromaquia [1796], Grenade, Editoriales Andaluzas Unidas, Biblioteca de la Cultura Andaluza (5), 1984.

Perez de Lama Halcón, José Luis, "Una arquitectura popular. Las plazas de toros de la Sierra de Aracena ", dans Pedro Romero de Solís (coord.), Las fiestas populares de toros. Demófilo, [Séville], 25, 1998, pp. 115-152.

Picamills Ruiz, Antonio, Dietario taurino 2005, Madrid, Dietario Taurino, 2005.

Pinto Maeso, Luis, Plazas de toros de España, Madrid, Pinmas, 1981.

Pitt-Rivers, Julian, « Le sacrifice du taureau », dans Le temps de la réflexion IV, Paris, Gallimard, 1983, pp. 281-297.

Planhol, Xavier de, Le paysage animal. L'homme et la grande faune : une zoogéographie historique, Paris, Fayard, 2004.

Plasencia, Pedro, La fiesta de los toros. Historia, régimen jurídico y textos legales, Madrid, Trotta, 2000.

Popelin, Claude, La Tauromachie [1970], Paris, Seuil, 1994.

Porcheron, Michel, «Las corridas de toros en Cuba, esquisse de l'histoire d'un échec ", dans Jean Ortiz (dir.), Tauromachies en Amérique latine, Anglet, Atlantica, 2004, pp. 109-141.

Purroy, Antonio, (éd.), V Jornadas sobre Ganado de Lidia (Pamplona, 24-25 de noviembre de 2006), Pampelune, Universidad Pública de Navarra, 2006.

Puyo, Jean-Yves, et Dauga, Marylis, "La course landaise, sport-spectacle confronté au défi de sa pérennisation ( $\mathrm{xIX}^{\mathrm{e}}$ et $\mathrm{xx}^{\mathrm{e}}$ siècle) ", Sud-Ouest européen, 8, 2000, pp. 15-22.

Raffestin, Claude, «Élements pour une théorie des frontières », Diogène, 134, 1986, pp. 3-21.

RAGo, Victor, «Llano y llanero: contribución al estudio del forjamiento de una imagen », Boletín Antropológico, [Mérida] 45, 1999, pp. 1-21.

Ramírez Barreto, Ana Cristina, "Defendiendo animales, redefiniendo tradiciones. Cómo charros y charras en California enfrentan las acciones legales contra eventos de la charreada", dans Actas de Cultura y Política. IX Congreso de Antropología de la Federación de Asociaciones de Antropología del Estado Español, Barcelone, Insituto Catalán de Antropología, 2002. 
- El juego del valor. Varones, mujeres y bestias en la charrería en Morelia (19232003), thèse de doctorat, soutenue en 2005 à l'université Michoacana de San Nicolás de Hidalgo (inédite).

Rangel, Nicolás, Historia del toreo en México, México, Cosmos, 1980.

Renard, Jean-Pierre, (dir.), La géographie et les frontières, Paris, L'Harmattan, 1997.

Rey, Alain, (dir.), Dictionnaire culturel en langue française, Paris, Le Robert, 1998.

Rey, Laura del, "Los festejos taurinos y su repercusión económica y social en el Bilbao del siglo xviII », dans Annie Molinié-Bertrand, Jean-Paul Duviols et Araceli Guillaume-Alonso (coord.), Des taureaux et des hommes. Tauromachie et société dans le monde ibérique et ibéro-américain, Paris, Presses de l'université de Paris-Sorbonne, coll. « Iberica (12), 1999, pp. 101-111.

— «Toros en Bilbao en los siglos xvi y Xvir. Del festejo popular al espectáculo », dans Antonio García-Baquero González et Pedro Romero de Solís (éd.), Fiestas de toros y sociedad. Actas del Congreso Internacional (Sevilla, 26 de noviembre - 1 de diciembre de 2001), Séville, Fundación Real Maestranza de Caballería de Sevilla - Universidad de Sevilla, 2003, pp. 235-246.

Ribeiro, Orlando, Portugal, o Mediterrâneo e o Atlântico, Lisbonne, Sá da Costa Editora, 1945.

Rivière, Claude, Les rites profanes, Paris, Presses Universitaires de France, 1995.

Rodríguez, Francisco, "Factor shares and ressource booms: Accounting for the Evolution of Venezuelan Inequality », Working Paper, [World Institute for Developpment Economics Research], 205, 2000.

Rodríguez, Pablo, " La fiesta de toros en Colombia, siglos XVI-XIX », dans Annie Molinié-Bertrand, Jean-Paul Duviols et Araceli GuillaumeAlonso (coord.), Des taureaux et des hommes. Tauromachie et société dans le monde ibérique et ibéro-américain, Paris, Presses de l'université de ParisSorbonne, coll. «Iberica » (12), 1999, pp. 165-174.

Rodríguez Becerra, Salvador, «Poder y fiestas populares con toros en Andalucía », dans Pedro Romero de Solís (coord.), Las fiestas populares de toros. Demófilo, [Séville], 25, 1998, pp. 71-88.

Rodríguez García, Víctor Manuel, «La charrería, cuestión de enfoque », dans El deporte nacional en Aguascalientes y la Villa Charra Prof. J. Refugio Esparza Reyes, Estado de Aguascalientes, 2004, pp. 331-333.

Rodríguez Navarro, Guillermo Enrique, Serje de la Ossa, Marguarita Rosa, et Rey Sinning, Edgar, Mapa cultural del Caribe Colombiano: la unidad en la diversidad, Santa Marta, Corpes, 1993.

Romero De Solís, Pedro, « La tauromachie comme ensemble sacrificiel », Informations sur les sciences sociales, [Paris], 31, 1992, pp. 531-550.

— «La dimensión sacrificial de la tauromaquia popular», dans ID. (coord.), Las fiestas populares de toros. Demófilo, [Séville], 25, 1998, pp. 245-260.

- "Las fiestas de toros en Andalucía. Definición, tipología y catálogo », dans ID. (coord.), Las fiestas populares de toros. Demófilo, [Séville], 25, 1998, pp. 261-284. 
— «El toro de Osborne y la identidad cultural », dans Annie Molinié-Bertrand, Jean-Paul Duviols et Araceli Guillaume-Alonso (coord.), Des taureaux et des hommes. Tauromachie et société dans le monde ibérique et ibéro-américain, Paris, Presses de l'université de Paris-Sorbonne, coll. «Iberica » (12), 1999, pp. 359-376.

- "Corpus Christi y tauromaquia. Una fiesta bajo el signo de la muerte sacrificial ", dans Antoinette Molinié, Celebrando el cuerpo de Dios, Lima, Pontificia Universidad Católica del Perú (PUCP), 1999, pp. 87-127.

— «Público de toros y opinión pública », dans Boletín de Loterías y Toros (19912001). 10 años de pensamientos, Cordoue, Asociación Boletín de Loterías y Toros, 2001, pp. 174-176.

— «Una luz sobre la época oscura de la tauromaquia », dans Boletín de Loterías y Toros (1991-2001). 10 años de pensamientos, Cordoue, Asociación Boletín de Loterías y Toros, 2001, pp. 58-68.

— «Sevilla: tauromaquia y religiosidad », dans José Hurtado Sánchez (éd.), Nuevos aspectos de la religiosidad sevillana. Fiesta, imagen, sociedad, Séville, Ayuntamiento de Sevilla, 2002, pp. 165-198.

— « La tauromaquia: un ritual de conversión del animal en alimento », dans Antonio García-Baquero González et Pedro Romero de Solís (éd.), Fiestas de toros y sociedad. Actas del Congreso Internacional (Sevilla, 26 de noviembre - 1 de diciembre de 2001), Séville, Fundación Real Maestranza de Caballería de Sevilla - Universidad de Sevilla, 2003, pp. 537-542.

Romero de Solís, Pedro, (coord.), Sacrificio y tauromaquia en España y América, Séville, Universidad de Sevilla, coll. « Tauromaquia », 1996.

— Las fiestas populares de toros. Demófilo, [Séville], 25, 1998.

Romero de Solís, Pedro, (éd.), Antropología de la tauromaquia. Obra completa taurina de Julian Pitt-Rivers, Séville, Fundación de Estudios Taurinos, 2002.

Romero-Miura Giménez, Fausto, La nación del toro, Roquetas de Mar por la Puerta Grande, Almería, Ayuntamiento de Roquetas de Mar, 2002.

Rose, Sonia, « Tauromaquia y sociedad en el Perú virreinal: las fiestas de Lima por el nacimiento de Baltasar Carlos », dans Annie Molinié-Bertrand, Jean-Paul Duviols et Araceli Guillaume-Alonso (coord.), Des taureaux et des hommes. Tauromachie et société dans le monde ibérique et ibéro-américain, Paris, Presses de l'université de Paris-Sorbonne, coll. « Iberica » (12), 1999, pp. 151-164.

Rouquié, Alain, Amérique latine. Introduction à l'Extrême-Occident, Paris, Éditions du Seuil, coll. «Points Essais » (373), 1998.

Rouy re, Jean-Louis, La tradition taurine à Tarascon, Nîmes, UBTF, 1988.

— Un siècle de corridas à Beaucaire, Nîmes, UBTF, 1994.

Ruiz AвAD, Luis, « La economía de las ganaderías de lidia: los puntos clave de su rentabilidad ", dans Economía de la ganadería de lidia en España, dossier publié dans le no 3 (2005) de la Revista del Instituto de Estudios Económicos, pp 13-32.

Sabathié, Claude, Histoire de la tauromachie à Céret, Nîmes, UBTF, 1993.

Saint-Julien, Thérèse, "Diffusion spatiale ", dans Encyclopédie de géographie, Paris, Economica, 1995, pp. 559-581. 
Salazar, Gabriel, et Pinto, Julio, Historia contemporánea del Chile, Santiago, Lom, 1990 (2 vol.).

SÁnchez de NeIra, José, El toreo: gran diccionario tauromáquico, Madrid, Turner, 1988.

Santana Vega, Juan, El mundo de las corralejas [1896], Montería, Caja de Previsión Social de Córdoba, 1986.

Saraiva Lima, Jaime, Panorama del toreo en Portugal, Lisbonne, 1950 [conférence prononcée dans le salon des actes de l'Ateneo de Madrid, le 11 juillet 1949].

Saumade, Frédéric, Des sauvages en Occident. Les cultures tauromachiques en Camargue et en Andalousie, Paris, Maison des Sciences de l'Homme, 1994.

— « Deux formes d'opposition à la corrida espagnole : les courses portugaises et camarguaises », Mediterrâneo, [Lisbonne], 5-6, 1995, pp 109-120.

- Les tauromachies européennes, Paris, Éditions du CTHS, 1998.

- " Frontières, contrastes géographiques et rites d'inversion (Andalousie, Camargue, Catalogne, Landes, Navarre, Portugal) », dans Annie MoliniéBertrand, Jean-Paul Duviols et Araceli Guillaume-Alonso (coord.), Des taureaux et des hommes. Tauromachie et société dans le monde ibérique et ibéro-américain, Paris, Presses de l'université de Paris-Sorbonne, coll. "Iberica » (12), 1999, pp. 203-214.

— «L'obsession sacrificielle (réponse à la note critique de Dominique Fournier, "Les sepctacles naissent aussi de l'histoire", relative à l'ouvrage de Frédéric Saumade Les tauromachies européennes. La forme et l'histoire, un approche anthropologique »), Ethnologie française, 31(2), 2001, pp. 337-341.

— «Du taureau au dindon. Domestication du métissage dans le Nouveau Monde mexicain ", Études rurales, 157-158, 2001, pp. 107-140.

— «Una metamorfosis tauromáquica en México: el toro, entre venado y guajalote », dans Antonio García-Baquero González et Pedro Romero de Solís (éd.), Fiestas de toros y sociedad. Actas del Congreso Internacional (Sevilla, 26 de noviembre - 1 de diciembre de 2001), Séville, Fundación Real Maestranza de Caballería de Sevilla - Universidad de Sevilla, 2003, pp. 543-554.

- «Animal de rente, animal de loisir. L'énigme de la monte du taureau au Mexique », Antropozoologica, 39(1), 2004, pp. 61-72.

- Maçatl. Les transformations mexicaines des jeux taurins, Bordeaux, Presses Universitaires de Bordeaux, 2008.

Saumade, Frédéric, et Desvaux, Emmanuel, « Relativiser le sacrifice ou le quadrant tauromachique ", Gradhiva, 16, 1994, pp. 79-84.

Serrano, Carlos, "Entre l'amour et la réprobation des siens ", dans Véronique Flanet et Pierre Veilletet, Le peuple du toro, Paris, Herné, 1986, pp. 63-75.

— "De l'habit de lumière à l'Espagne noire ", dans Annie Molinié-Bertrand, Jean-Paul Duviols et Araceli Guillaume-Alonso (coord.), Des taureaux et des hommes. Tauromachie et société dans le monde ibérique et ibéro-américain, Paris, Presses de l'université de Paris-Sorbonne, coll. « Iberica » (12), 1999, pp. 51-62. 
Shubert, Adrian, A las cinco de la tarde. Una historia social del toreo [ $1^{\text {re }}$ éd. en anglais 1999], Turner, Real Maestranza de Caballería de Ronda, 2002.

- «El toreo en la historia española », dans ID., De toros, toreros y tendidos, Valence, Centro Francisco Tomás y Valiente, 2004, pp. 5-29.

Silva Corcuera, Guadalupe, "La charrería. Representación simbólica de lo mexicano ", Tips de Aeroméxico, 1, 1997, p. 11.

Skewes, Juan Carlos, "El Rodeo, una metáfora del Tiempo Viejo ", Revista Austral de Ciencias Sociales, 2, 1998, pp. 69-80.

Slatta, Richard, "The gaucho in Argentina's quest for national identity ", Canadian Review of Studies in Nationalism, 21(1), 1985, pp. 99-122.

- Cowboys of the Americas, New Haven, Yale University Press, 1990.

Souza Maciel, María Eunice de, Le gaúcho brésilien. Identité culturelle dans le sud du Brésil, thèse de doctorat d'ethnologie soutenue en 1994 à l'université Paris V- René Descartes (inédite).

Starrs, Paul, Let the Cowboy ride. Cattle ranching in the American West, Baltimore, Hopkins University Press, 1998.

Tabique, Luis de, Guía de América Taurina, México, Édition de l'auteur, 1955.

Teixera, Fernando, "A corrida do forção », Mediterrâneo, [Lisbonne], 5-6, 1995, pp 23-32.

Thомas, Keith, Dans le jardin de la nature. La mutation des sensibilités en Angleterre à l'époque moderne, Paris, Gallimard, 1985.

Traimond, Bernard, Les fêtes du Taureau, Bordeaux, L'Atelier de l'Almanach Éditions, 1996.

Turner, Frederick Jackson, "The significance of the Frontier in American history ", dans David Weber et Jane Rush, Where Cultures Meet Frontiers in Latin American History [1893], Wilmington, Jaguar Books, 1994, pp. 1-18.

Valat, Jacques, Pour la beauté du geste, la course landaise, Portet-Sur-Garonne, Loubatières, 1993.

Vargas Meza, Ricardo, Las FARC, la guerra y la crisis del Estado, Bogotá, Centro para la Investigación y la Educación Popular (CINEP), 1998.

VÁzQuEz, Leopoldo, América taurina, Madrid, Librería de Victoriano Suárez, 1898.

Verdevoye, Paul, « Corridas et carnaval dans la presse argentine avant 1833 », Cahiers du monde hispanique et luso-brésilien (Caravelle), 10, 1968, pp. 5-16.

VIALles, Noémie, Le sang et la chair. Les abattoirs des pays de l'Adour, Paris, MSH, 1987.

VIARD, André, Corrida française? Plaidoyer en faveur d'un statut de la tauromachie en France, Paris, Hots, 1983.

— Le Mythe du Taureau, s. l., éd. Torero, 1986.

- Comprendre la corrida, Anglet, Atlantica, 2001.

Villatoro, Ángel, Antología taurina mexicana, Madrid, Ergatorre - Biblioteca Nueva, 1986. 
Woerner, Gail, Fearless Funnymen: the history of rodeo clown, Austin, Eaken Press, 1993.

WolfF, Francis, "Qui est le taureau ? Les représentations de l'animal dans les discours et les pratiques tauromachiques contemporaines ", dans Annie Molinié-Bertrand, Jean-Paul Duviols et Araceli Guillaume-Alonso (coord.), Des taureaux et des hommes. Tauromachie et société dans le monde ibérique et ibéro-américain, Paris, Presses de l'université de Paris-Sorbonne, coll. « Iberica » (12), 1999, pp. 23-37.

- Philosophie de la corrida, Paris, Fayard, 2007.

\section{II. — DOCUMENTS INTERNES ET DONNÉES PRIVÉES}

Colegio Oficial de Veterinarios de Madrid, Memoria espectáculos taurinos de la Comunidad de Madrid, Madrid, 2004 [brochure].

Conseil ÉCONOMIQUe et SOCIAL DU LANGUEdoc-Roussillon, Le rôle et l'impact du taureau et de la filière taurine en Languedoc-Roussillon [rapport du 16 septembre 1997].

Dirección General de Espectáculos Públicos y Juego de la Junta de Andalucía, Estadísticas taurinas de la Comunidad Autónoma de Andalucía, Séville, Junta de Andalucía, 2005.

Ministerio del Interior, CCNAT, Estadísticas de la temporada, 2003.

Sотомауов, José María, Estadísticas taurinas, 1991-2003.

\section{III. -DOCUMENTS AUDIO ET AUDIOVISUELS}

Cavalier, Jean-Louis, Fiesta en corralejas (52 minutes), Production Film d'ici, 1985.

Sillero, Claudio, Los toros en el Perú, Télévision nationale du Pérou, 1990.

- Los toros y el patrimonio cultural [conférence prononcée le 20 novembre 2003 au congrès iberoamericain des Aficionados Taurins].

\section{IV. — JOURNAUX ET REVUES}

6 Toros 6, $\mathrm{n}^{\circ} 7,1992 ; \mathrm{n}^{\circ} 608,2006$.

Aplausos, $\mathrm{n}^{\circ}$ 1281, avril 2002.

Barrera Sol, n 49, juin 2001.

Bous al Carrer, $\mathrm{n}^{\circ}$ 42, octobre 2004 ; n 45, janvier 2005.

Colombia Universal, 28 janvier 2005.

Diario del Congreso Chileno, 13 juillet 2006.

El Comercio, 14 mars 2002.

El Diario de Guárico, 4 mai 2007. 
El Diario Lealtad de Chihuahua, 20 août 2006.

El Nacionalista de Guárico, 4 mai 2007.

El Nuevo Diario, 9 avril 2005.

El País, 21 juillet 1996.

El Sol de San Juan del Río, 30 juillet 2007.

El Universo, 17 juin 2006.

Fiesta Brava, n 23, 2004 ; n² 26, 2005.

Hoy, 29 novembre 2006.

La Nación, 4 janvier 2004.

Libération, 16 mai 2001 ; 23 juin 2005.

Revista Terra Incógnita, 18 juillet 2002.

Sud-Ouest, p. 2-2, 23 septembre 2003.

Tauromaquia, $\mathrm{n}^{\circ}$ 6, septembre 2004 .

Tendido, n 148, janvier-février 2005.

The New York Times, 27 juin 2001.

Tierramérica, Inter Press Service, 26 avril 2007.

Toros, $\mathrm{n}^{\text {os }} 1267-1268$, décembre $1985 ; \mathrm{n}^{\circ} 1570$, janvier $1998 ; \mathrm{n}^{\circ} 1531$, novembre $1998 ; n^{\circ} 1569$, janvier $1998 ; n^{\circ} 1570$, janvier $1998 ; n^{\circ} 1631$, juillet 2000 ; $\mathrm{n}^{\circ} 1662$, septembre $2001 ; \mathrm{n}^{\circ} 1663$, octobre $2001 ; \mathrm{n}^{\text {os }} 1667-1668$, décembre 2001.

Zero Hora, 15 mai 1999. 

RÉSUMÉS 



\section{RÉSUMÉ}

Ce travail part d'une redéfinition des pratiques tauromachiques fondée sur des critères matériels et débarrassée au mieux des présupposés idéologiques qui ont pendant longtemps encombré son examen critique. Si l'on accepte de considérer que le taureau des pratiques tauromachiques est un animal générique, qu'il soit veau, vache, taureau ou bœuf, et en deçà des différents systèmes de valeurs engendrés par la race, le genre et les transformations subies par l'animal, alors toutes les pratiques qui mettent en scène un affrontement réel entre les hommes et les bovins sont, à nos yeux, tributaires d'une même analyse. Ainsi l'éventail des pratiques tauromachiques et leur extension géographique s'élargissent considérablement, en rupture avec les nombreuses approches hispano-centrées du fait taurin. Les pratiques tauromachiques intègrent alors les diverses courses taurines du Sud-Ouest européen et leur exportation en terres américaines, ainsi que l'immense famille des rodéos nord et sud-américains, généralement exclus de cette catégorie. Ces pratiques se déclinent ensuite entre des jeux taurins codifiés, plus ou moins professionnalisés, et des jeux taurins à participation collective très divers. Ces derniers sont d'une grande plasticité formelle et sont en constante évolution au point de brouiller les distinctions apparemment bien tranchées entre jeux professionnels et jeux populaires, jeux d'arènes et jeux de rues, tauromachies européennes et tauromachies américaines, corrida et rodéo.

Dans le champ des loisirs modernes, les pratiques tauromachiques constituent une forme d'exception dans la mesure où il n'existe guère d'autres spectacles publics au cours desquels les hommes se mesurent en des corps à corps réels et violents avec des animaux. Les risques humains et l'utilisation des animaux en font un sujet particulièrement polémique. Ils en font aussi un sujet éminemment politique si l'on accepte de considérer que le contrôle de la violence est une préoccupation élémentaire de la cité. Les pratiques tauromachiques posent le problème très concrètement par la délimitation même des régions où elles sont autorisées et par la délimitation des lieux de mise en scène de ces affrontements qui bien souvent transfigurent les espaces publics.

Enfin, dans de nombreuses régions et parfois même à l'échelle des États-nations, ces pratiques représentent l'expression culturelle la plus emblématique de ce qui serait propre à tel ou tel territoire : course landaise, course camarguaise, corrida portugaise, rodéo chilien, rodeo montubio, charreada mexicaine, vaquejada nordestina... Cette individualisation territoriale des pratiques taurines est d'autant plus intéressante, d'un point de vue géographique, que la répartition des techniques d'affrontement ne la recoupe 
que très imparfaitement, puisqu'en effet certains spectacles et certaines gestuelles sont communs à plusieurs régions. La culture apparaît ici moins comme une émanation essentialiste du génie local que comme un moyen d'appropriation et de redécoupage de l'espace. Ce problème renvoie le géographe à la question fondamentale du fonctionnement de la territorialité et de la capacité des sociétés à fonder leur propre culture au sein d'un éventail des traits culturels partagés par d'autres. Ainsi, au sein d'une aire transatlantique, les pratiques tauromachiques contribuent à borner l'espace, tracer des frontières, faire coexister des différences culturelles, créer des hauts lieux d'identification et véhiculer des sentiments d'appartenance territoriale. Les pratiques tauromachiques sont alors considérées comme des marqueurs territoriaux qui héritent de la diffusion, de la transformation et des métissages de la culture entre l'Europe et l'Amérique. Les pratiques tauromachiques, généralement mobilisées dans un discours sur l'identité et sur la tradition, forment ainsi un champ commun de distinction territoriale.

Le recensement, la description et la cartographie de ces pratiques permettent ainsi de poser les bases d'une nouvelle réflexion sur leur diffusion et leur transformation, alors même que beaucoup de ces jeux n'ont pas été reliés entre eux de façon significative, ni sur le plan formel, ni sur le plan historique, ni sur le plan territorial. Ces pratiques constituent un répertoire de formes matérialisant des relations homme-bovin-cheval géographiquement variables. Elles expriment des identités territoriales qui se chevauchent, se recoupent ou s'excluent, vécues dans des régions aux cultures taurines plus ou moins imbriquées les unes aux autres. Ainsi observe-t-on un « espace tauromachique » en mosaïque qui témoigne d'un processus de construction territoriale discontinu où se relaient deux temps forts de la mondialisation occidentale : l'impérialisme ibérique, contemporain de la colonisation du Nouveau Monde à partir du $\mathrm{XVI}^{\mathrm{e}}$ siècle et une hégémonie étasunienne en Amérique, qui démarre dès le milieu du XIX ${ }^{\mathrm{e}}$ siècle dans un contexte d'affirmation des États-nations.

L'analyse des différents jeux taurins, de leur signification sociétale et de leur répartition spatiale exprime, en premier lieu, un fort contraste géographique entre l'Europe et l'Amérique. L'Europe, dans son extrémité sud-ouest formée par l'Espagne, le Portugal et une partie du Sud de la France, est dominée par la corrida espagnole. L'Amérique est dominée par la pratique des divers rodéos, plus ou moins dépendants de l'influence hégémonique du modèle spectaculaire étasunien sur l'ensemble du continent. Cette opposition fondamentale, qui est aussi celle d'un face à face entre l'homme à pied et l'homme à cheval, se double de solidarités transatlantiques dues à l'enracinement de la culture taurine ibérique en terres américaines. En effet, la corrida y est pratiquée dans de nombreux pays : au Mexique, en Colombie, au Venezuela, en Équateur, au Pérou et depuis peu en Californie sous une forme portugaise remaniée qui exclue toute effusion de sang. Les jeux taurins traduisent également des clivages au sein même du continent américain entre Amérique latine et Amérique anglo-saxonne, entre Amérique hispanophone et Amérique lusophone, entre pays andins et pays du Cône Sud ; autant de différenciations qui sont alimentées par la subtile grammaire des diverses formes de rodéos. Elles sont fondées sur l'articulation de trois modalités techniques principales : la monte des taureaux et des chevaux sauvages, l'usage du lasso et le renversement des bovins, à cheval, en les attrapant par la queue. Les rodéos consacrent, à l'échelle continentale, la figure du centaure dont la patrimonialisation a toujours quelque chose à voir 
avec la construction des États-nations : cow-boys états-uniens, charros mexicains, llaneros vénézuéliens, vaqueiros et gauchos brésiliens, huasos chiliens, gauchos argentins. Enfin, les jeux taurins traduisent un clivage entre un Sud-Ouest européen marqué par une hispanité composite et le reste de l'Europe. À une échelle plus fine, les pratiques tauromachiques révèlent des rapprochements et des différenciations géopolitiques internes entre des régions aux caractéristiques propres : Andalousie, Castille, Levant, Landes, Camargue, Alentejo-Ribatejo...

L'enracinement ou le refus des jeux taurins, les limites de leur extension spatiale ainsi que leurs transformations répondent dans leur ensemble à des logiques identitaires de distinctions territoriales. Ces logiques, au sein de notre espace étude, posent partout la question de la distance culturelle avec l'Espagne et/ou les États-Unis. Cette distance s'observe à travers les rejets, les syncrétismes et les métissages qui finalement donnent forme et signification à la majorité des pratiques taurines. La corrida, tout en étant assimilée au patrimoine respectif des différents pays où elle est pratiquée, traduit, dans le même temps, un lien privilégié avec l'Espagne, attestant d'un équilibre original entre une acculturation avancée et une incomplète assimilation. En Amérique, rejetée dans certains pays et pérennisée dans d'autres, la corrida se stabilise dans ses frontières actuelles au cours d'un long $\mathrm{XIX}^{\mathrm{e}}$ siècle, au moment même où s'opère l'individualisation des rodéos nationaux. Les rodéos incarnent ainsi une sorte de tournant tauromachique qui ancre la problématique des affrontements homme-bovin dans un contexte panaméricain dominé par les États-Unis, venant rejouer avec les héritages de l'hispanité. Cette analyse permet de mieux comprendre sur quoi repose la cohésion d'une aire culturelle. En dépit des vides où la tauromachie n'est rien, la cohésion, les différences et les discontinuités internes de cette mosaïque reposent sur les interactions entre trois types de configurations spatiales : les hauts lieux de la culture taurine, les zones de coexistence des pratiques et les zones de dynamiques transfrontalières.

Les hauts lieux des pratiques tauromachiques, qu'ils soient économiques ou iconographiques, produisent des polarisations et des mobilités spatiales débordant largement l'aire d'extension des pratiques concernées (les corridas de Séville, les encierros de Pampelune, les rodéos de Las Vegas). Les zones de coexistence des pratiques taurines favorisent leur concurrence et leur complémentarité lorsque celles-ci s'inscrivent dans des oppositions fécondes entre tauromachie rurale et tauromachie urbaine, entre tauromachie régionale et tauromachie nationale, entre tauromachie des périphéries et tauromachie du centre. Ces zones sont généralement structurées par des villes charnières, têtes de réseaux, marquées par la coexistence de plusieurs cultures taurines. La coexistence peut s'affirmer au sein d'une même ville, au sein d'un même quartier, au sein d'une même arène et, dans certains cas relevant d'un syncrétisme qui confine à la fusion des genres, au sein d'un seul et même spectacle. Dans le Sud-Ouest européen, pour retenir quelques exemples représentatifs, citons Dax (corrida et course landaise), Arles (corrida et course camarguaise), Pampelune (corrida et encierro), Saragosse et Valence (corrida et course de recortadores). En Amérique, citons Los Angeles en Californie et San Antonio au Texas (charreada et rodéo), Mexico et Guadalajara (charreada et corrida), Villavicencio en Colombie ou Barinas au Venezuela (toros coleados et corrida). Enfin, les "frontières taurines ", formées par les contacts et les ruptures entre les limites de pratiques et entre les frontières politiques, sont le siège d'intenses 
dynamiques territoriales suscitées par la culture taurine : mobilités transfrontalières, effets différenciateur ou unificateur de la discontinuité frontalière, effacement ou mise en scène idéologique de la frontière, réminiscence d'irrédentisme... Ces dynamiques transfrontalières produites par la culture taurine agissent comme des coutures territoriales entre frères ennemis et nations sœurs (Espagne-Portugal, États-Unis - Mexique, Venezuela-Colombie). Ce travail propose ainsi une analyse des modalités de cohésion et d'imbrication des aires culturelles, susceptible d'enrichir la réflexion sur les processus de diffusion et de transformation de la culture sur le temps long de la mondialisation. D’une manière plus générale, il propose une démarche d'analyse de la différenciation culturelle territorialisée. 


\section{RESUMEN}

Este trabajo parte de una redefinición de las prácticas taurinas basada en criterios materiales y despejada, lo más posible, de los apriori ideológicos que durante tiempo han impedido su examen crítico. Si se acepta considerar que el toro de las prácticas taurinas es un animal genérico, que sea utrero, vaca, toro o buey, y por encima de los distintos sistemas de valores engendrados por la raza, el género y las transformaciones del animal, entonces todas las prácticas que ponen en escena un enfrentamiento efectivo entre hombre y bovino son, para nosotros, tributarias de un mismo análisis. De esta forma, el abanico de las prácticas taurinas y su extensión geográfica se amplían considerablemente, en ruptura con los análisis del hecho taurino centrados casi exclusivamente en el mundo ibérico. Las prácticas taurinas incluyen no sólo las diversas corridas de toros del Suroeste europeo y su exportación en tierras americanas, sino también la inmensa familia de rodeos norte y suramericanos, generalmente excluidos de esta categoría. Después, estas prácticas se componen de juegos taurinos codificados, más o menos profesionalizados, y de juegos taurinos muy variados con participación colectiva. Estos últimos son de una gran plasticidad formal y están en constante evolución, de tal manera que borran parcialmente las distinciones entre juegos profesionales y juegos populares, juegos de arena y juegos de calle, tauromaquias europeas y tauromaquias americanas, corrida y rodeo.

En el campo del ocio moderno, las prácticas taurinas constituyen una excepción puesto que casi no existen otros espectáculos públicos durante los cuales los hombres se miden cuerpo a cuerpo real y violento con los animales. Los riesgos humanos y la utilización de los animales hacen de dicha práctica un objeto de polémica. Es también un tema sumamente político si se considera que el control de la violencia es una preocupación elemental de la sociedad cívica. Las prácticas taurinas plantean el problema de manera muy concreta por las delimitaciones mismas de las regiones donde son legales y por las delimitaciones de los lugares de puesta en escena de estos enfrentamientos que muy a menudo transfiguran el espacio público.

Finalmente, en muchas regiones, e incluso a veces a escala de los Estados-nación, estas prácticas representan la expresión cultural más emblemática de lo que sería lo propio de tal o tal territorio: corrida landesa, corrida camarguesa, corrida portuguesa, rodeo chileno, rodeo montubio, charreada mexicana, vaquejada nordestina... Esta individualización territorial de las prácticas taurinas es tanto más interesante desde el punto de vista geográfico cuanto que la repartición de las técnicas de enfrentamiento no le corres- 
ponde claramente. En efecto, varias regiones tienen en común ciertos espectáculos y ciertas actitudes corporales. Aquí la cultura no aparece como una emanación esencialista del genio local, sino como medio de apropiación y regionalización del espacio. El problema remite al geógrafo a la cuestión fundamental del funcionamiento de la territorialidad y a la capacidad para hacer suya culturas compartidas. Así dentro de un área cultural transatlántica, las prácticas taurinas contribuyen a delimitar el espacio, trazar fronteras, hacer coexistir diferencias culturales, crear altos lugares de identificación y mediatizar sentimientos de pertenencia territorial. Las prácticas taurinas son consideradas como marcadores territoriales que proceden de la difusión, de la transformación y de los mestizajes de la cultura entre Europa y América. Las prácticas taurinas, generalmente movilizadas en un discurso sobre la identidad y la tradición, forman así un campo común de distinción territorial.

El censo, la descripción y la cartografía de estas prácticas permiten plantear una nueva reflexión sobre su difusión, su transformación y sus interrelaciones, en un contexto en el cual muchos de estos juegos no habían sido relacionados los unos con los otros de manera significativa, ni a nivel formal, ni a nivel histórico, ni a nivel territorial. Estas prácticas constituyen un repertorio de formas que materializan las relaciones geográficamente variables entre hombre, bovino y caballo. Expresan identidades territoriales que se superponen, se recortan y se excluyen, vividas en regiones cuyas culturas taurinas se combinan. Así se observa la existencia de un «espacio taurino» formando un mosaico que traduce un proceso de construcción territorial discontinuo en el que se relevan dos tiempos cumbres de la mundialización occidental: el imperialismo ibérico contemporáneo de la colonización de América a partir del siglo xvi y una hegemonía estadounidense en América, que empieza a mitad de siglo xix en un contexto de afirmación de los Estados-nación.

El análisis de los distintos juegos taurinos, de su significación social y de su repartición espacial traduce, en primer lugar, un importante contraste geográfico entre Europa y América. Europa, en su extremidad suroeste formada por España, Portugal y una parte del sur de Francia, está dominada por la corrida española. Mientras América esta dominada por la práctica de diversos rodeos, más o menos dependientes de la influencia hegemónica del modelo estadounidense en materia de espectáculos. A esta oposición fundamental, que también corresponde a una rivalidad entre el hombre a pie y el hombre a caballo, se añade solidaridades transatlánticas expresadas por el enraizamiento de la cultura taurina ibérica en tierras americanas. En efecto, la corrida sigue siendo practicada en muchos países: México, Colombia, Venezuela, Ecuador, Perú $\mathrm{y}$, desde hace poco, California bajo una forma portuguesa incruenta. Los juegos taurinos expresan también las divisiones dentro del continente americano, entre la América latina y la anglosajona, entre la América hispanohablante y la lusohablante, entre países andinos y países del Cono Sur; cuántas diferencias alimentadas por la sutil gramática de las diversas formas de rodeo. Los rodeos se basan en la articulación de tres modalidades técnicas principales: la monta de toros y de caballos, la utilización del lazo y el coleo, es decir, el derribo de los bovinos, a caballo, cogiéndolos por la cola. Los rodeos consagran a escala continental la figura del centauro cuya patrimonialización ha tenido siempre que ver con la construcción des los Estados-nación: cowboys americanos, charros mexicanos, llaneros venezolanos, vaqueros y gauchos brasileños, huasos chilenos, gau- 
chos argentinos. También, los juegos taurinos traducen una división entre un suroeste europeo marcado por una hispanidad plural y el resto de Europa. A escala más fina, las prácticas taurinas revelan acercamientos y diferenciaciones geopolíticas internas entre regiones que tienen características propias: Andalucía, Castilla, Levante, Las Landas, La Camarga, Alentejo-Ribatejo.

El enraizamiento o el rechazo de los juegos taurinos, los límites de su extensión espacial y sus transformaciones obedecen, en su conjunto, a lógicas identitarias de distinciones territoriales. Estas lógicas, en nuestro espacio de estudio, plantean por todas partes el problema de la distancia cultural con España y/o con Estados Unidos. Esta distancia se observa a través de los rechazos, los sincretismos y los mestizajes que, al fin y al cabo, dan forma y significación a la mayoría de las prácticas taurinas. La corrida, al mismo tiempo que representa una parte del patrimonio de cada país en el que se desarrolla, traduce también una relación privilegiada con España que aparece como el testimonio de un equilibrio original entre una aculturación avanzada y una asimilación incompleta. En América, rechazada en ciertos países y mantenida en otros, la corrida se estabiliza en sus fronteras actuales durante un largo siglo XIX, al mismo tiempo que ocurre la individualización de los rodeos nacionales. Los rodeos reflejan una especie de giro tauromáquico que ancla la problemática de los enfrentamientos hombre-bovino en un contexto panamericano dominado por Estados Unidos, superponiéndose a las herencias de la hispanidad. Al final, la cohesión, las diferencias y las discontinuidades internas de este mosaico, a pesar de los vacíos donde la tauromaquia no es nada, dependen de las interacciones entre tres configuraciones espaciales: los altos lugares de la cultura taurina, las zonas de coexistencia de las prácticas y las zonas de las dinámicas transfronterizas.

Los altos lugares de las prácticas, ya sean económicos o iconográficos, producen polaridades y movilidades espaciales que desbordan ampliamente el área de extensión de dichas prácticas (las corridas de Sevilla, los encierros de Pamplona, los rodeos de Las Vegas). Las zonas de coexistencia de las prácticas taurinas favorecen su competencia y su complementariedad cuando se desarrollan en el marco de oposiciones fecundas entre tauromaquia rural y tauromaquia urbana, tauromaquia regional y tauromaquia nacional, tauromaquia de las periferias y tauromaquias del centro. Generalmente lo que da estructura a estas zona son ciudades bisagra, cabezas de redes, marcadas por la copresencia de varias culturas taurinas. La co-presencia puede expresarse a nivel de una misma ciudad, de un mismo barrio, de una misma plaza de toros, y, en ciertos casos de sincretismo raya en la fusión de los géneros, a nivel de un mismo espectáculo. En el Suroeste europeo, para subrayar algunos ejemplos representativos, se puede mencionar Dax (corrida española y corrida landesa), Arles (corrida española y corrida camarguesa), Pamplona (corrida y encierro), Zaragoza y Valencia (corrida y concurso de recortadores). En América, se puede mencionar Los Ángeles en California y San Antonio en Texas (charreada y rodeo), México y Guadalajara (charreada y corrida), Villavicencio en Colombia o Barinas en Venezuela (toros coleados y corrida). Finalmente, «las fronteras taurinas», formadas por los contactos y rupturas entre los límites de las prácticas y entre las fronteras políticas, son la sede de intensas dinámicas territoriales suscitadas por la cultura taurina: movilidades transfronterizas, efectos diferenciador o unificador de la discontinuidad fronteriza, supresión o puesta en escena ideológica de la frontera, 
reminiscencia de irredentismo... Estas dinámicas transfronterizas producidas por la cultura taurina actúan como suturas territoriales entre hermanos enemigos y naciones hermanas (España-Portugal, Estados Unidos - México, Venezuela-Colombia). Este trabajo propone un análisis de las modalidades de cohesión y de interpenetración de las áreas culturales susceptible de enriquecer la reflexión sobre los procesos de difusión y de transformación de la cultura en el tiempo largo de la mundialización. De manera más general, propone un método de análisis de las diferenciaciones culturales territorializadas. 


\section{SUMMARY}

This study starts with a redefinition of bullfighting practices founded on material criteria and as far as possible stripped of the ideological assumptions that have for so long hampered any critical examination. If we agree to consider the bull in bullfighting practices a generic animal — be it a calf, cow, bull or ox and without going into the different value systems engendered by breed, gender and the transformations wrought in the animal- then all practices that entail a real confrontation between man and bovine can, we believe, be analysed in the same way. This considerably enlarges the range of bullfighting modes and their geographical spread and diverges from the numerous Hispano-focused approaches to bullfighting. Bullfighting practices then embrace the various forms of bullfight in South-Western Europe and their exportation to the New World, including the great variety of North and South American rodeos, which are generally excluded from this category. These practices may then be divided into highly-structured and more or less professionalised bullfights and the large variety of mass-participation events. The latter present great flexibility of form and are constantly evolving to the extent of blurring the ostensibly well-defined distinctions between professional and popular bullfights, bullring events and street events, European bullfighting and American bullfighting, corrida and rodeo.

In the field of modern leisure, bullfighting practices are an exception inasmuch as there are practically no other public shows in the course of which men engage in real, violent hand-to-hand combat with animals. This is a particularly controversial subject because of the personal risks involved and the use of the animals. It is also an eminently political subject if we consider that the control of violence is a primary concern of civic life. Bullfighting practices pose the problem in a particular way in view of the circumscription of the regions where they are authorised and the circumscription of the places where such events are staged, given that they frequently entail the alteration of public spaces.

The fact is that in many regions - and sometimes even at the level of the United Nations - these practices are seen as the most emblematic cultural expression of what distinguishes this land or that: course landaise, course camarguaise, corrida (Portugal), rodeo (Chile), rodeo montubio (Ecuador), charreada (Mexico), vaquejada nordestina (Brazil), etc. This identification of bullfighting practices with particular locations is all the more interesting from a geographical standpoint in that the modes of fighting follow the same pattern only very roughly, for in fact certain types of show and 
certain types of movement are common to more than one region. Culture in this context appears less a manifestation of the local geist than a means of appropriating and redistributing space. For the geographer this problem leads back to the fundamental question of territorial behaviour and the capacity to interiorise shared cultures. For instance, in a transatlantic context, bull fighting practices serve to mark spatial boundaries, draw frontiers, force different cultures to coexist, create poles of identification and embody feelings of territorial belonging. Bullfighting practices are then viewed as territorial markers bequeathed by the spread, transformation and intermingling of European and American cultures. Thus bullfighting practices, which are generally raised in the context of a discourse on identity and tradition, constitute a common field of territorial differentiation.

By listing, describing and mapping these practices we can lay down the basis for a new debate on how they have spread, how they have changed and how they interrelate, albeit many of these types of fight have never been linked to any significant extent formally, historically or geographically. These practices cover a range of different man/bovine/ horse permutations which vary geographically and express territorial identities which straddle, overlap or are absent from regions with bullfighting traditions that are more or less interlinked. What emerges then is a mosaic-patterned "bullfighting area" that has seen a discontinuous process of territory-building in two successive eras of Western globalisation: Iberian imperialism, contemporary with the colonisation of the New World starting in the 16th century, and US hegemony in America commencing in the mid-19th century in a context of consolidation of nation-states.

The most obvious result of an analysis of the different modes of bullfighting, their societal significance and their spatial distribution is the strong geographical contrast between Europe and America. In the south-western corner of Europe formed by Spain Portugal and part of southern France, the Spanish-style corrida predominates. The predominant practice in America is the rodeo of various kinds, all more or less subject to the hegemonic influence of the spectacular US model in the continent as a whole. This basic difference, which also evokes the difference between the bullfighter on foot and the bullfighter on horseback, sits side-by-side with transatlantic survivals due to the depth of Iberian cultural roots in the Americas. In fact Spanish-style bullfighting - the corridais practised in many countries: Mexico, Colombia, Venezuela, Ecuador, Peru, and lately in California in a bloodless Portuguese version. There are also divides in bullfighting forms on the American continent, between Latin America and Anglo-Saxon America, between Spanish-speaking and Portuguese-speaking America, between the Andean countries and the Southern Cone - in addition to the distinctions deriving from the subtle grammar of variations in rodeo format. These are essentially elaborations of three main modes: bull- and bronco-riding, lassoing, and horseback steer tailing. Rodeos are a continentwide evocation of the figure of the centaur, which has consistently been appropriated in connection with the construction of the nation-state: US cowboys, Mexico's charros, Venezuela's llaneros, Brazil's vaqueiros and gauchos, Chile's huasos, Argentina's gauchos. And bullfighting modes reflect a divide between a Hispanic-oriented South-Western Europe and the rest of Europe. On a smaller scale, bullfighting practices reveal internal geopolitical similarities and differences between regions with their own characteristics: Andalusia, Castile, Levante, Landes, Camargue, Alentejo-Ribatejo, etc. 
Whether bullfights are generally accepted or rejected, their geographical limits and their variations —all these are largely functions of the identitary logic of territorial differentiation. In the context of this study, that logic generally poses the question of cultural distance with Spain and/or the USA. That distance can be measured through the exclusions, amalgamations and cross-formations that give form and meaning to most bullfighting practices. The corrida, while forming part of the heritage of each of the countries where it is practised, still represents a special tie with Spain, reflecting a singular balance between advanced acculturation and incomplete assimilation. In America the corrida, which was rejected in some countries and firmly established in others, attained its present stable frontiers in the long course of the 19th century, at the same time as rodeos acquired individual national forms. Rodeos thus embody a species of bullfighting watershed in which the problematics of man/bovine confrontations became set in a Pan-American context dominated by the USA but influenced by Hispanic traditions. This analysis helps to understand the foundation on which the cohesion of a cultural area rests. Leaving aside the blank areas where bullfighting has no roots, the cohesion, the differences and the internal breaks in this mosaic are products of interactions between three types of spatial configuration: the meccas of bullfighting culture, the areas where different practices coexist, and the areas of cross-border influence.

The meccas of bullfighting practice, whether economic or emblematic, produce polarisations and spatial shifts which stretch well beyond the confines of the practices concerned (corridas in Seville, encierros in Pamplona, rodeos in Las Vegas). Areas where different bullfighting practices coexist encourage competition and complementariness by generating fruitful oppositions of rural bullfighting versus urban bullfighting, regional bullfighting versus national bullfighting, or bullfighting on the periphery versus bullfighting at the centre. Such areas are generally structured around pivotal towns, district capitals, where more than one bullfighting culture coexist. Such coexistence may occur within the same township, the same quarter, or even the same bullring, in some cases becoming amalgamated, with different modes in one and the same show. Some representative examples in south-western Europe include Dax (corrida and course landaise), Arles (corrida and course camarguaise), Pamplona (corrida and encierro), Zaragoza and Valencia (corrida and concurso de recortadores). In America we can cite Los Angeles in California and San Antonio in Texas (charreada and rodeo), Mexico City and Guadalajara (charreada and corrida), Villavicencio in Colombia or Barinas in Venezuela (toros coleados and corrida). In short, the "bullfighting boundaries" drawn by the convergences and disparities between practices and between political frontiers generate intense territorial dynamics within the field of bullfighting culture: cross-border mobility, differentiating or unifying effects of frontier discontinuity, effacement or highlighting of the notion of the frontier, echoes of irredentism, etc. These cross-border dynamics generated by bullfighting culture act as a species of territorial links between enemy brothers and sister nations (Spain-Portugal, USA-Mexico, Venezuela-Colombia). This study, then, offers an analysis of the modes of cohesion and overlapping of cultural areas, with a view to enriching the discussion of the processes whereby culture spreads and is transformed in the long-term process of globalisation. And in a more general way it proposes a procedure for analysis of territorialised cultural differentiation. 



\section{GLOSSAIRE}

ABRIVADo : " arrivée », en Camargue, désigne l'arrivée des taureaux guidés par les gardians à cheval qui les conduisent depuis les prés jusqu'aux arènes avant la course camarguaise. L'abrivado est devenue un jeu et un spectacle à part entière, indépendant des courses (voir bandido).

ACOSO Y DERRIBO : "harcèlement et action de faire chuter ", dans les élevages de taureaux braves, désigne l'épreuve pratiquée à cheval qui a pour but de tester les jeunes taureaux à champ ouvert pour mesurer leur bravoure. La technique consiste à faire chuter l'animal dans sa course avec une garrocha (longue pique en bois) jusqu'à ce qu'il décide d'attaquer la monture plutôt que de fuir.

Afeciounado : " amateur, passionné ", en Camargue, désigne les aficionados, les amateurs de courses camarguaises.

Afeitado: " rasé ", en Camargue, désigne l'épointage des cornes du taureau. Dans la corrida espagnole, à l'exception des courses de rejoneo et des festivals, la pratique est interdite. Elle est un cas de fraude qui est loin d'être exceptionnel dès que les moyens pour contrôler le respect du règlement faiblissent.

AFICIÓN : «passion ", dans le monde de la corrida, désigne un goût passionnel pour la tauromachie et la bonne connaissance de ses principes. Désigne par extension l'ensemble des aficionados.

Aficionado : " amateur, passionné ", dans le monde de la corrida, désigne l'amateur de courses de taureaux.

AFICIONADO PRÁCTICO : dans le monde de la corrida, désigne l'amateur pratiquant occasionnellement la tauromachie lors de fêtes organisées par des passionnés ou lors de tientas privées.

Aiguillade : harcèlement du bétail à l'aide d'un bâton pointu. Pratique fréquente jusqu'au $\mathrm{XIX}^{\mathrm{e}}$ siècle lors du passage des troupeaux en déplacement à proximité des habitations.
ALGUAZIL : dans la corrida, désigne le cavalier représentant les forces de l'ordre dans l'arène, chargé d'appliquer le règlement taurin, sous l'autorité de la présidence technique de la course.

Alternative : dans la corrida, désigne le rite d'investiture signifiant le passage du rang de novillero à celui de matador de toros affrontant des taureaux de quatre ans révolus.

AnILLAS : dans les courses de recortadores, désigne les anneaux que les protagonistes doivent enfiler sur les cornes des vaches.

APIÑADERO : dans le rodéo chilien, nom donné à la plus petite section des arènes servant aussi d'enclos et coulisses pour les cavaliers qui attendent leur tour. La plus grande section est la cancha : le terrain, la piste.

Apoderado: dans le monde de la corrida, désigne le fondé de pouvoir représentant le matador.

ArÈne : édifice construit pour le jeu tauromachique reprenant certains canons architecturaux du modèle romain (voir plaza de toros).

ATAJADA : dans le rodéo chilien, désigne le blocage du bovin dans sa course par le cavalier huaso contre la paroi de l'arène.

Attrapaires : en Camargue, hommes à pied surgis du public essayant d'attraper les taureaux lors des abrivado et des bandido et de les faire sortir du troupeau conduit par les gardians.

AtTRIbUts PRIMÉs : dans la course camarguaise, désigne la cocarde, les glands et les ficelles fixés sur le frontal des taureaux représentant des primes.

Banderillero : dans la corrida espagnole, désigne le subalterne du matador qui a pour fonction de planter les banderilles et d'aider à mener le combat. Dans la corrida portugaise, désigne le banderillero accompagnant les cavaliers, en dépit de leur nom, ne servent qu'à replacer ponctuellement le taureau ou à protéger le cavalier et le cheval en cas de chute. 
BANDIDO : en Camargue, selon un principe proche de celui de l'abrivado, les bandido se déroulent après la course camarguaise pour reconduire les taureaux aux élevages.

BECERRADA : dans le monde de la corrida, course de taureaux pour les débutants ou pour les amateurs, se déroulant avec des taureaux de moins de deux ans, sans pique et le plus souvent sans mise à mort.

BECERRO : jeune taureau de moins de deux ans.

BıòU : " bœuf ", terme provençal, désignant le taureau protagoniste des courses camarguaises.

BOI : « bœuf ", terme portugais, désignant les bovins des farras do boi (fêtes du bœuf ou du taureau) au Brésil.

Bou : "bœuf ", terme catalan et valencien, désignant les bovins des courses de rue en Catalogne (corre bou) et dans la région de Valence (bous al carrer).

BOUS AL CARRER : " taureaux dans la rue ", expression catalane et valencienne, désignant les courses de rue à participation collective (Terres de l'Ėbre, Castellón, Valence, Alicante).

BOUS AL MAR : "taureaux dans la mer ", expression catalane et valencienne, désignant les courses de rue à participation collective, pratiquées sur le littoral, consistant à attirer l'animal dans la mer ou à le faire tomber du quai (exemple : Dénia et Peñíscola dans la région de Valence).

BOU CAPLLAÇAT : "bœuf encordé ", en Catalogne, et en particulier dans les Terres de l'Èbre de la province de Tarragone, jeu taurin à participation collective fondé sur l'utilisation d'un taureau encordé (voir toro ensogado).

BOU DE CORDA : appellation valencienne du jeu taurin à participation collective fondé sur l'utilisation d'un taureau encordé (voir toro ensogado).

BOU ENSOGAT : " taureau encordé ", appellation valencienne et catalane du jeu taurin populaire fondé sur l'utilisation d'un taureau encordé (voir toro ensogado).

BouRgino : "corde ", bourgine ou taureau à la bourgine, en Camargue, désigne le jeu taurin à participation collective fondé sur l'utilisation d'un taureau encordé, aujourd'hui interdit, il se maintient localement sous le nom d'encierro (exemple : l'encierro à l'eyraguaise).

Bouvine : en Camargue, désigne l'espèce bovine et par extension l'ensemble du milieu taurin de la course camarguaise, terme fréquemment utilisé dans les expressions : pays de bouvine, monde de la bouvine.

BRAVo : "sauvage ", dans le monde de la corrida, désigne la combativité de l'animal et son instinct offensif. En France, on parle d'élevage brave ou de taureau brave pour désigner, par ce terme, la race des taureaux de combat et leur combativité individuelle contingente (voir manso).
BRonCA : dans le monde de la corrida, colère des spectateurs qui se manifeste par des sifflets, des huées et parfois le jet d'objets en piste, à l'encontre de l'éleveur, de la présidence ou des toreros dont la prestation ou l'attitude scandalise.

BRONCO : "sauvage ", en Amérique latine, cheval non domestiqué qu'il s'agit de monter dans de nombreuses formes de rodéo.

BULL RIDERS : " monteur de taureau », dans le rodéo nord-américain, désigne le protagoniste du bull riding.

BULL RIDING : action de monter un taureau à cru. Dans le règlement du rodéo nord-américain, il convient de résister pendant 8 secondes aux ruades de l'animal. L'exercice peut être intégré à l'ensemble des exercices du rodéo nord-américain ou pratiqué en tant que jeu et spectacle autonome, notamment dans le cadre des compétitions organisées par le Pro Bull Riders et le Xtrem Bulls de la PRCA.

BULLFIGHT : en anglais, corrida ou de tauromachie. Dans le rodéo nord-américain, désigne aussi depuis peu, le jeu spécifique des rodeo clowns, en tant que protagoniste principal, qui affrontent le taureau. Le terme bullfighting est utilisé dans cette double acception pour désigner la corrida et le spectacle des rodeo clowns.

BULLFIGHTER : désigne le torero des corridas ibériques et, depuis peu, les rodeo clowns du rodéo nord-américain dans les jeux dont ils sont les protagonistes exclusifs.

BURLADERO : dans l'arène de corrida, abri en chicane placé devant la barrière séparant la piste de la contre-piste pour que les hommes puissent circuler facilement et se protéger sans avoir à sauter la barrière.

CADALFAS : en Espagne, désigne les larges grilles métalliques verticales permettant un passage relativement aisé pour les hommes qui souhaitent circuler entre l'intérieur et l'extérieur de l'espace de mise en scène taurine, tout en empêchant le taureau de s'en échapper. Souvent utilisées dans les tauromachies à participation collective de la région de Valence.

CALA DE CABALLO : dans la charreada, désigne l'exercice équestre consistant à galoper le plus vite possible et à faire arrêter d'un coup le cheval dans une zone déterminée.

CALF ROPING : dans le rodéo nord-américain, capture d'un veau au lasso. Un veau est lâché sur la piste à l'ouverture d'une barrière. Il est poursuivi par un cavalier qui l'attrape au lasso, puis descend de son cheval afin de retourner le veau pour lui lier trois pattes en un temps record.

CALLEJón : dans l'arène, désigne la contre-piste de protection et couloir de circulation pour les protagonistes des courses de taureaux. 
CAmpana : "cloche ", dans le coleo venezuélien et colombien, désigne une modalité de chute du taureau qui se retrouve les quatre pattes en l'air.

CAMpinos : au Portugal, désigne les bouviers à cheval travaillant dans les élevages de taureaux. Ils prennent part aux jeux taurins des entradas et des picarias. Équivalent portugais des gardians camarguais.

CАMPO : « champ », en Espagne, désigne aussi la campagne, en particulier celle où vivent les taureaux de combat. Espace sociologique du monde taurin associé à l'élevage brave.

CANCHA : "terrain, piste ", dans le rodéo chilien, désigne la plus grande section de l'arène où se déroulent les exercices taurins notés. La plus petite partie est l'apiñadero.

CAPEA : dans le monde de la corrida, désigne une course taurine populaire, caractéristique des villages dépourvus d'arène permanente où participaient autrefois amateurs et toreros aspirants. Ce terme désigne parfois des courses d'amateurs organisées dans des arènes.

CAPEADOR A CABALLO : intervenant à cheval maniant la cape, caractéristique, pendant longtemps, d'une technique utilisée au Pérou et désignée sous le nom de suerte nacional (geste ou technique nationale).

CAPEIA RAIANA : " capea de la frontière " ou capeia arraiana ou corrida do forcao, jeu portugais pratiqué à proximité de la frontière avec la province de Salamanque en Espagne, consistant à contenir collectivement les attaques d'un taureau à l'aide d'un instrument en bois, composé de pieux et de rondins, appelé le forcao.

CAPOTE : cape utilisée dans les courses de taureaux par les auxiliaires du matador et par le matador lui-même soit pour placer le taureau en position d'être piqué, soit pour exécuter des figures esthétiques lors du tercio de piques, soit pour protéger les banderilleros lors du tercio de banderilles.

CARACOLEO : dans le rodéo montubio, en Équateur, désigne l'exercice équestre consistant à faire tourner en girouette le cheval sur les pattes arrière.

Cartel : "affiche ", dans le monde de la corrida, désigne par extension le programme de la course, le nom des toreros et des taureaux à l'affiche.

CASTA : " caste, race ", terme espagnol, race ou pedigree du taureau. Désigne aussi sa qualité de taureau de combat que lui confere sa race. Pour un taureau en piste, indépendamment de son élevage d'origine, la caste désigne ses qualités de bravoure et de combativité dont il fait montre.

CAVALEIRO : " cavalier, chevalier », terme portugais, désigne le cavalier des corridas portugaises et protagoniste principal du spectacle.

Cazérienne (Marche) : dans le monde de la course landaise, désigne l'hymne joué en début et fin de course.
CHAGRA : en Équateur, désigne un paysan de la sierra. Le rodéo chagra est le jeu taurin encore mal dégagé de la pratique utilitaire pratiqué par les paysans, Indiens et Métis, gardiens des troupeaux de bovins en altitude.

Charlotada : " charlotade ", terme espagnol, désigne la course comique de taureaux qui renvoie à la figure burlesque de Charlot. Jeu qui s'affirme au début des années 1920. La pratique d'une tauromachie burlesque en marge des spectacles sérieux et esthétiques concerne l'ensemble des terres taurines.

CHARREADA : spectacle taurino-équestre mexicain, regroupant des exercices de maniement du lasso (la reata), d'habilité équestre et de maîtrise dans la monte des bovins et des chevaux non dressés. La charrería désigne l'activité des charros au sens large, du jeu au mode de vie.

ChARro : nom des paysans de la région espagnole de Salamanque du campo charro. Cet adjectif est à l'origine du mot mexicain désignant les cavaliers charros ou les charros devenus un type social emblématique de la mexicanité.

Chega de bOIS : au Portugal, combat entre taureaux, organisé dans les montagnes du Tras-os-Montes (exemple : la région du Barroso).

CHÚCARO : "sauvage ", en Équateur, le cheval chúcaro désigne le cheval non dressé qu'il s'agit de monter dans de nombreux rodéos, équivalent de bronco au Mexique et bronc aux États-Unis.

Cocardier : dans le monde de la course camarguaise, désigne le taureau présenté dans l'arène.

Colcha : en Équateur, désigne l'étoffe parfois richement ornée, fixée sur le dos du taureau, pouvant constituer une prime pour celui qui l'attrape.

COLEADOR : terme latino-américain, désignant le cavalier du coleo devant attraper la queue du bovin pour le faire tomber dans sa course. Le terme est également utilisé pour désigner les coleadores pédestres dans certaines pratiques tauromachiques populaires.

COLEAR : faire chuter le bovin en l'attrapant par la queue. Aujourd'hui, en tant que technique spectaculaire, le coleo se pratique généralement à cheval.

Coleo : substantif du verbe colear. Désigne le spectacle des toros coleados au Venezuela et en Colombie. C'est aussi la technique utilisée dans la vaquejada au Brésil. La technique existe dans la charreada sous le nom de colas parmi d'autres exercices.

COLLERA : " collier ", dans le rodéo chilien, désigne une équipe de deux cavaliers chargés de prendre un taureau en tenaille et de le conduire au galop autour de la piste, afin de le bloquer contre la paroi de l'arène en des endroits déterminés permettant de gagner des points. 
Concours de RECORTAdores : spectacle taurin espagnol recouvrant plusieurs modalités d'affrontement entre les hommes et les bovins : concours d'anneaux, consistant à enfiler des anneaux sur les cornes de la vache, concours de cortes ou de recortes libres, consistant à pratiquer des feintes et des quiebros sur des taureaux, concours de sauts consistant à sauter par-dessus l'animal pour éviter sa charge.

Cordier : sur l'île Terceira aux Açores, et dans la communauté açorienne de Californie, protagoniste des touradas à corda, chargé de tenir l'animal encordé. Il s'agit généralement d'employés travaillant dans les élevages bovins.

CORRAL : terme espagnol, désigne la cour, l'enclos, l'enceinte de taille réduite où sont gardés les bovins dans les élevages ou dans les arènes en attendant leur sortie en piste.

CorRaleja : vient du mot corral. En Colombie, désigne l'enceinte du jeu taurin de la Costa Atlántica et le jeu lui-même. L'expression la plus souvent employée pour parler du jeu est la fiesta en corralejas (la fête dans les corralejas).

CORRE BOU : terme catalan, désigne la course de taureaux à participation collective.

CORREDOR DE TOROS : " coureur de taureaux ", terme utilisé en Espagne dès le XVI ${ }^{\mathrm{e}}$ siècle pour qualifier certains protagonistes à pied des courses de taureaux.

Corrida : " course » (de taureaux), employé seul, désigne la course de taureaux formelle espagnole, avec des taureaux de quatre ans et des matadors d'alternative. Terme défini le plus souvent dans les dictionnaires comme « art de combattre les taureaux dans l'arène ». Il peut être précisé, s'il s'agit d'une corrida de toros, d'une corrida de novillos (taureaux de moins de quatre ans), d'une corrida de rejoneo (corrida à cheval).

CORTE : " coupure ", dans les concours de recortadores, désigne la feinte ou le quiebro permettant d'éviter, au dernier moment, d'être pris par le taureau (voir recortadores).

Coso : terme espagnol utilisé par la presse spécialisée dans les corridas comme synonyme d'arène. En Amérique latine, le terme est également employé pour désigner la piste d'autres pratiques tauromachiques telles que les mangas de coleo ou les lienzos charros. Diccionario de la Real Academia Española: " une place, site ou tout autre lieu circonscrit où sont courus et combattus des taureaux et où sont célébrées d'autres fêtes publiques ".

Costado : "flanc", dans le coleo en Colombie et au Venezuela, désigne une modalité de chute des taureaux consistant à coucher le bovin sur le flanc. Le costado est moins bien noté que la campana.

COUP DE BARRIÈre : dans la course camarguaise, désigne l'action d'éclat du taureau cocardier qui frappe de son poitrail la barrière délimitant la piste, en propulsant le raseteur hors de l'aire de jeu.
Coursayre : passionné de course landaise.

Course camarguaise : tauromachie camarguaise consistant à pratiquer des rasets sur un taureau cocardier afin d'en décrocher les attributs primés.

Course formelle : course landaise conforme aux règles orthodoxes de la discipline, comptant le plus généralement pour les championnats officiels.

COURSE LANDAISE : tauromachie landaise consistant à pratiquer des écarts et des sauts sur une vache encordée, lancée à pleine vitesse.

Course miXte : course landaise comportant une partie formelle et une partie comique ou ludique à participation collective.

COW HUNT : " chasse à la vache ", ancien nom nordaméricain du rodéo utilitaire, remplacé ensuite par le mot round up, le terme rodéo étant employé généralement pour désigner le spectacle.

Cuadrilla : dans la corrida espagnole, désigne l'ensemble formé par le matador et son équipe de subalternes qui l'assistent dans le combat, composée des banderilleros, des picadors et du valet d'épée.

DESJARRETADERA : instrument de guerre et de chasse de la renaissance, formé d'une longue pique se terminant par une lame en croissant de lune qui servait à couper les tendons des montures ou des proies. Également appelée demi-lune (medialuna). Fut utilisée dans les jeux taurins jusqu'au début du XIX siècle.

DUENDE : "esprit, inspiration », dans le monde de la corrida et du flamenco désigne un état d'inspiration comme si les artistes étaient visités par un esprit ou touchés par la grâce.

Dur : adjectif qui dans la corrida se dit des taureaux provenant d'élevages réputés pour donner des taureaux au comportement particulièrement âpre, dangereux et difficile à manœuvrer dans le combat. Proche du taureau dit "sérieux " ou de « respect».

ÉCART : dans la course landaise, désigne la figure consistant à laisser passer la vache au creux des reins par une esquive rotative effectuée quasiment sur place.

ÉCARTEUR : dans la course landaise, protagoniste principal exécutant des écarts pour éviter la charge du bétail.

ЕмвоLADOR : " embouleur ", terme espagnol, dans le jeu taurin consistant à embouler le taureau, désigne les membres d'une équipe d'emboladores devant fixer sur la tête d'un taureau immobilisé contre un madrier en bois, la structure métallique supportant des boules inflammables, afin ensuite d'y mettre feu, puis de libérer l'animal lâché dans les rues. Dans les pays valenciens, le taureau embolado est un taureau de fuego. Il constitue aujourd'hui, dans le pays valencien, une pratique ludique à part entière qui fait l'objet de concours chronométrés. 
Емвоulé : dans le monde de la course landaise et camarguaise, se dit d'un taureau ou d'une vache dont les cornes sont munies à leur extrémité d'une boule ou d'une gaine de cuir afin de les rendre moins dangereuses.

EMPRESA : " entreprise ", dans le monde de la corrida le terme renvoie aux entreprises organisatrice de courses de taureaux possédant la gestion et parfois la propriété des arènes.

ENCASTE : désigne la souche généalogique de laquelle est issu le taureau de combat (voir casta).

ENCERRO : équivalent portugais d'encierro. Terme d'usage moins fréquent que ceux d'entrada, d'espera ou de largada qui désignent des pratiques proches.

ENCIERRO : du verbe enfermer : «encerrar ». Ce terme désigne la conduite des taureaux aux arènes pour les y enfermer avant la course. Plus généralement, désigne tout lâcher de taureaux à travers les rues d'un village ou d'une ville ou à travers champs. L'exemple le plus connu d'encierro est celui de Pampelune. En Camargue, le mot désigne une course de taureaux à participation collective, donnée sur une place fermée par des barricades, sans qu'il ne s'agisse aucunement de conduire les bêtes d'un lieu à un autre.

Enjalma : au Pérou, étoffe richement décorée, fixée sur le dos du taureau.

ENTRADA : " entrée », au Portugal, désigne l'arrivée des taureaux dans la ville sous la conduite des campinos. Terme qui a existé en Espagne avant qu'il ne disparaisse au profit du terme d'encierro.

ESPERA : " attente ", au Portugal, équivalent de l'entrada.

ESPONTÁNEO : dans le monde de la corrida, individu du public qui spontanément se jette en piste pour montrer son désir et son aptitude à toréer ou tout simplement son courage. Cette pratique est interdite, mais, par le passé, elle offrait souvent l'occasion de faire remarquer son talent.

FAENA : " travail ", dans la corrida, désigne l'ensemble des passes de muleta par lesquelles le matador met en valeur son talent d'artiste à travers sa capacité technique et esthétique à dominer son adversaire, avant de le préparer à recevoir le coup d'épée final.

FARPA : équivalent portugais du rejón espagnol, désigne une sorte de banderille plantée par le cavalier sur le dos du taureau lors des corridas à cheval.

FARRA DO вOI : au Brésil, "fête du bœuf ou du taureau ", jeu taurin populaire pratiqué sur le littoral de l'État de Santa Catarina par les membres de la communauté originaire des Açores. La pratique est aujourd'hui interdite mais se poursuit localement de façon clandestine ou quelque peu transformée.
FE (DI вIÒU) : "foi », dans le monde de la course camarguaise, désigne la passion pour les taureaux et plus généralement pour la culture liée à la course camarguaise sous toutes ses formes.

FER : marque de l'élevage, inscrite au fer rouge sur la cuisse des veaux pour authentifier leur origine.

FERIA : "foire ", dans le monde de la corrida désigne la séquence festive de quelques jours à plusieurs semaines au cours de laquelle sont rassemblés les spectacles taurins.

Ferrade : dans le monde de la course camarguaise, opération qui consiste à marquer au fer les bovins. Dans de nombreux pays de traditions taurines, cette opération fait l'objet d'une fête champêtre organisée par l'éleveur.

FESTEjo : terme espagnol, désigne le spectacle ou la fête taurine.

Festejos taURinos populares : «spectacles taurins populaires », nom donné dans le règlement taurin espagnol pour rassembler les pratiques tauromachiques à participation collective et parfois les exhibitions de jeux taurins autres que la corrida tels que les concours de recortadores.

Festival : dans le monde de la corrida, course de taureaux non rémunérée, autorisant l'épointage des cornes, qui est généralement organisée à des fins caritatives.

FIESTA : « fête », dans la culture taurine le terme suffit souvent à désigner la tauromachie, également utilisé dans les expressions fiesta taurina, fiesta brava, fiesta nacional.

FIESTA BRAVA : expression fréquemment utilisée en Amérique latine pour désigner la fiesta taurina, la tauromachie.

FIGURA : " figure », dans le monde de la corrida, désigne un torero vedette.

FINCA : "propriété », dans le monde de la corrida désigne la propriété de l'éleveur de taureaux et statistiquement une unité d'exploitation d'un élevage brave qui peut en posséder plusieurs (voir ganaderia).

FORCADO : terme portugais issu du mot fourche, dans la corrida portugaise, désigne les membres regroupés en équipe de huit forcados qui réalisent en piste la pega, consistant à recevoir la charge de l'animal au corps à corps en vue de l'immobiliser totalement.

GANADERIA : "élevage de bétail », dans le monde de la corrida, désigne l'élevage de taureaux de combat. Dans le monde de la course landaise, désigne le troupeau de bêtes présenté lors d'un spectacle.

GARDIAN : bouvier des élevages camarguais.

GARRAIADA : au Portugal, désigne un lâcher de bovins et les jeux taurins à participation collective généralement pratiqués avec du petit bétail.

GARROCHA : longue pique utilisée par les bouviers à cheval andalous, équivalent du pampilho des campinos portugais. 
Garrocheros : en Colombie, dans les fiestas en corralejas de la Costa Atlántica, désigne les cavaliers poursuivant les taureaux dans l'arène. Ce sont généralement des employés des élevages de la région.

Gaucho : cavalier de la Pampa argentine. Désigne aussi les habitants de la région brésilienne du Rio Grande do Sul attachés à la culture des terres d'élevage.

Hierra : de fer, hierro. Au Panamá, désigne souvent le coleo. En Argentine, généralement écrit yerra, prend le sens de ferrade.

HUASo : au Chili, désigne le cavalier du rodéo chilien et une figure emblématique de la chilenidad ( « chilénité »).

JARIPEO : au Mexique, désigne le jeu populaire ou professionnel fondé sur la monte des taureaux. Pendant longtemps parfait équivalent du terme de charreada.

JARIPEO RANCHERO PROFESSIONNEL : au Mexique, désigne le spectacle professionnel de jaripeo fondé sur la monte des taureaux, influencé par les codes du bull riding nord-américain.

JINETE : «cavalier », au Mexique, désigne le monteur de taureaux du jaripeo.

JINETEADA DE TOROS : dans la charreada et le jaripeo, désigne l'exercice consistant à monter à cru sur le dos du taureau jusqu'à ce que l'homme soit expulsé ou que le taureau cesse de se débattre.

JocHeO : en Bolivie, désigne un type de jeu taurin à participation collective, caractéristique de la région amazonienne, pouvant associer lâcher de taureaux, monte et toreo élémentaire.

LANZADA : en Espagne, technique taurine ancienne existant dès le Moyen Âge, consistant à tuer un taureau d'un coup de lance.

LARGADA : au Portugal, après les entradas ou esperas, jeu qui consiste à lâcher un taureau dans l'enceinte du parcours et plus généralement lâcher de bovins.

LAZADOR : terme latino-américain souvent employé pendant la période coloniale pour désigner ceux qui manient le lasso dans les élevages ou lors des fêtes taurines.

LLANERO : de plaine : llano, au Venezuela et en Colombie, adjectif désignant l'appartenance aux llanos. Désigne aussi les habitants de la région des Llanos colombo-vénézuéliens qui s'étendent sur la partie intérieure des deux pays.

LLANOS : "plaines ", avec une majuscule désigne la grande plaine drainée par l'Orénoque entre le Venezuela et la Colombie.

LIDIA : " combat ", dans la corrida, désigne aussi la stratégie du matador face au taureau.

Lienzo : dans la charreada, le lienzo charro désigne une partie de la piste où se déroulent les exercices et plus généralement désigne l'enceinte spécialisée du jeu, alors synonyme de rancho de charreada.
MAESTRO : " maître ", dans la corrida, désigne le matador.

Manade : en Camargue, désigne le troupeau de bovins et par extension les élevages de taureaux camarguais.

MANCORNAR: attraper le taureau par les cornes.

MANGA : "manche ", dans les toros coleados du Venezuela et de la Colombie, désigne la piste du jeu taurin. Dans les élevages, désigne le couloir final en forme d'entonnoir où l'on précipite les bêtes pour les faire entrer dans les enclos.

MANGANA : dans la charreada, désigne les exercices de maniement du lasso pour attraper les bovins ou les chevaux.

MANSO : "domestique, domestiqué ", dans la corrida cet adjectif désigne le bétail fuyard et insuffisamment courageux, à l'opposé du taureau brave qui charge pour se défendre. Il désigne aussi le bétail domestique n'appartenant pas à la race des taureaux de combat (voir bravo).

Manteros : de manta : couverture, toile, en Colombie, dans les fiestas en corralejas, protagonistes maniant la cape, la muleta ou toute autre étoffe faisant l'affaire. Les manteros sont souvent engagés au coup par coup par les organisateurs des corralejas pour animer le spectacle.

MATAdor : " tueur ", dans la corrida, désigne le torero d'alternative à la tête d'une cuadrilla. Équivalent de maestro (voir torero).

Matatoros : en Espagne, dans les courses taurines populaires, à partir du $\mathrm{XI}^{\mathrm{e}}$ et XIII ${ }^{\mathrm{e}}$ siècle, désigne l'individu engagé par les organisateurs de la fête taurine pour tuer le taureau et mettre un terme au jeu.

Media CASTA : "demi-caste ", adjectif qualifiant les bovins résultant d'un croisement entre animaux de caste et autres types de bovins ( créoles » ou zébus).

Medialuna : dans le rodéo chilien, désigne le nom de l'arène. Homonyme de l'instrument de guerre et de chasse nommé demi-lune.

MoJIGANGa : en Espagne, désigne une catégorie ancienne de jeux taurins burlesques et fantaisistes dans l'esprit du toreo comique.

MoÑA : au Pérou, désigne une étoffe ressemblant à une cocarde, fixée sur le front des bovins et qui peut constituer une prime à décrocher.

Monta-toro : "monte-taureau ", au Pérou, désigne le protagoniste des jeux taurins populaires qui monte les taureaux.

MONTERA : dans la corrida, chapeau noir porté par les toreros.

Montubio : en Équateur, désigne le paysan de la côte qui en fait le pendant littoral du chagra de la Sierra. Désigne plus largement une communauté sociale et ethnique caractéristique du littoral, issue d'un métissage entre Blancs, Noirs et Indiens. Adjectif qualifiant le rodéo caractéristique de cette zone. 
Movimiento a la rienda: dans le rodeo chileno, désigne l'exercice de dextérité équestre visant à évaluer la qualité du dressage, du cheval et du cavalier.

Muleta : dans la corrida, désigne l'étoffe rouge fixée sur un bâton, qui sert de leurre pour guider les charges du taureau.

MundiLlo : « petit monde » de la tauromachie, regroupant toreros, anciens toreros, éleveurs, apoderados, directeurs d'arène, journalistes spécialisés, aficionados, etc.

Noblesse : dans le monde de la corrida, désigne la qualité du taureau qui répond avec franchise aux sollicitations des toreros, sans vice et sans détour intempestifs. Métaphore du comportement loyal au combat caractéristique des valeurs de la noblesse en armes (voir bravo).

Novillada : dans le monde de la corrida, désigne la course donnée avec des taureaux de moins de quatre ans (novillo).

Novillero : dans le monde de la corrida, désigne le jeune torero qui affronte des novillos avant de devenir matador.

Pampilho : au Portugal, désigne la lance utilisée par les campinos dans les élevages de taureaux de combat, également appelée vara de campinar, équivalent de la garrocha des vaqueros andalous et du trident des gardiens camarguais.

PAmplonada : au Mexique, nom courant des encierros faisant référence à ceux de Pampelune.

PArÉ : dans la course landaise, désigne une ancienne technique consistant à attendre la bête lancée à pleine vitesse jusqu'à lui faire détourner la tête au moment de l'impact.

PARQUE DE VAQUEJADA : au Brésil, désigne le parc, l'enceinte où se déroule la vaquejada.

PASEO : dans la corrida, présentation et défilé des cuadrillas marquant l'ouverture du spectacle.

PASODOBLE : musique de corridas qui accompagne l'ouverture du spectacle et les moments les plus artistiques de la faena du matador.

PAsse : dans la corrida, désigne toutes figures exécutées à l'aide de la cape ou de la muleta consistant à faire passer le taureau en déviant sa charge et en le guidant (voir faena).

PEÃo : équivalent portugais du mot espagnol peón. Au Brésil, désigne un employé journalier à pied. Dans le rodeio brésilien désigne le protagoniste du jeu.

$P_{E G A}$ : dans la corrida portugaise, désigne la phase réalisée par les forcados consistant à recevoir la charge du taureau en vue de l'immobiliser.

PEGAR : dans la corrida portugaise, verbe désignant l'exécution de la pega.

PELEA DE TOROS: " combat de taureaux», au Pérou, désigne les combats entre taureaux caractéristique de la région d'Arequipa.
Peña taurina : dans le monde de la corrida, désigne le club, le cercle ou l'association dans laquelle se réunissent les aficionados.

PIAL: dans la charreada, désigne le piégeage des pattes du bétail avec la corde.

Picador : dans le monde de la corrida, désigne le membre de la cuadrilla, chargé de piquer le taureau avec la pique.

Pique : dans la corrida, instrument utilisé par les picadors. La pique désigne aussi la phase du combat où elle prend place.

PlazA DE TOROS : équivalent espagnol d'arène, désigne l'enceinte spécifiquement construite pour le spectacle tauromachique attestant de son autonomisation à partir du XVIII ${ }^{\mathrm{e}}$ siècle.

PortátIL: "portable», se dit des arènes démontables.

PRETAL : corde utilisée dans les rodéos pour ceinturer l'animal au niveau du bassin ou du poitrail.

PUXADOR : au Brésil, dans la vaquejada, désigne parmi les deux cavaliers lancés à la poursuite de l'animal, celui qui attrape le taureau par la queue pour le faire chuter dans sa course.

QuAdrille : du mot espagnol cuadrilla, utilisé dans l'expression course de quadrille, désigne le jeu taurin mixte apparu en France à la fin du $\mathrm{XIX}^{\mathrm{e}}$ siècle, mélangeant les techniques de la corrida espagnole, de la course camarguaise et de la course landaise.

QuiEbro : en Espagne, désigne une feinte pour éviter la charge du taureau, consistant à tromper le taureau en lui faisant anticiper son déplacement dans une direction qui est à l'opposé de celle que l'homme prendra au dernier moment.

QUINCHA : dans le rodéo chilien, désigne une sorte de butoir rembourré, autrefois en paille, situé dans la zone réservée au blocage du taureau contre la paroi (la atajada).

RASET : dans la course camarguaise, désigne une technique pour décrocher les attributs primés, consistant à provoquer le taureau pour le faire charger, puis à choisir une direction en arc de cercle permettant de croiser la course du taureau sans se faire prendre.

RASETER : dans la course camarguaise, action consistant à effectuer des rasets.

RASETEUR : protagoniste de la course camarguaise effectuant les rasets.

REATA : dans la charreada, lasso en corde d'ixtle des cavaliers charros.

RECORTADOR : protagoniste des concours de recortadores.

RECORTAR : dans les concours de recortadores, action d'effectuer des recortes, feintes et quiebros trompant au dernier moment la charge de l'animal qui croit atteindre sa proie alors qu'elle se dérobe. 
RECORTE : action consistant à citer l'animal de face, à décrire un arc de cercle en courant pour rejoindre sa course, puis, à l'instant de l'impact attendu, à esquiver la charge en cambrant le dos afin de sortir, au pas, hors de portée des cornes.

REDONDEL : dans le monde de la corrida, désigne la piste, moins fréquent que ruedo.

REJÓN : dans la corrida à cheval, désigne une sorte de banderille que les cavaliers à cheval, les rejoneadores, plantent sur le dos du taureau.

REJONEO : En Espagne, l'art de planter les rejones et, plus généralement, désigne la corrida à cheval dans son ensemble.

Remate : du verbe rematar : terminer. Dans la corrida ce terme désigne la dernière passe d'une série. Dans le rodéo chilien, désigne le contact qui stoppe le bovin contre la paroi (la quincha), équivalent de atajada.

RoDEIO : traduction brésilienne du rodéo, désigne le rodéo fondé essentiellement sur la monte des taureaux dont les codes et les règles sont calqués sur le bull riding.

RODEIO CRIOULO : "rodéo créole ", au Brésil, rodéo faiblement institutionnalisé, lié à la culture gaucha du Rio Grande do Sul, prenant les traits d'une fête champêtre.

RODEO : du verbe rodear : encercler tourner. Action de rassembler les bêtes périodiquement dans les élevages extensifs de bovins, pour les marquer, les compter ou les conduire. Sous le terme de rodéo est rassemblé l'ensemble des jeux américains provenant de façon plus ou moins directe des jeux taurino-équestres de l'époque coloniale et des méthodes de l'élevage extensif de bovins. Ils reposent, tout ou partie, sur la maitrise de la monte des taureaux et des chevaux, sur les exercices équestres, l'usage du lasso, la course-poursuite d'un animal et son immobilisation.

Rodéo : le terme rodéo désigne généralement dans les dictionnaires français le seul rodéo nord-américain.

RODEO AMERICANO : ou rodeo estilo americano, au Mexique, désigne les spectacles de rodéo professionnel, calqués sur les règles du rodéo nord-américain. À ne pas confondre avec le jaripeo ranchero professionnel qui, bien qu'influencé par les codes du bull riding, possède des règles quelque peu différentes, notamment une durée de monte qui n'est pas limitée à 8 secondes pour la considérer comme valide.

Rodéo CHAGRA : en Équateur, rodéo des chagras, consiste pour les éleveurs de bétail à partir dans la montagne pour rassembler les bêtes laissées sur les hauteurs du páramo et à redescendre aux enclos celles devant être castrées, marquées ou vendues. Désigne également les jeux élémentaires issus de cette pratique utilitaire (voir chagra).
Rodeo chileno : au Chili, désigne le rodéo fondé sur la course-poursuite n'utilisant ni la technique du coleo, ni lasso, pour stopper l'animal, mais le seul usage du poitrail du cheval pour bloquer le taureau contre la paroi de la piste en une zone déterminée.

RODEO CLOWN : aux États-Unis, désigne le protagoniste du rodéo nord-américain chargé de venir en aide aux bull riders en cas de chute et d'assurer le spectacle pendant les intermèdes par des tours comiques avec ou sans bétail. Les rôles se spécialisant, le rodeo clown reste attaché à la partie comique du spectacle alors que ceux qui protègent les bull riders tendent à être désignés par le terme de cowboy protector et ceux faisant l'objet d'un spectacle spécifique par celui de bullfighter.

Rodéo MONTUBIO : en Équateur, rodéo des montubios, désigne une forme de rodéo principalement équestre, marquée par une faible présence réelle des bovins (voir montubio).

ROSCADERo : en Espagne, jeu pratiqué en Aragon consistant à recevoir la charge d'une vache ou d'un taureau dans un grand panier en osier et en bois, tenu à plusieurs, utilisé d'habitude dans les travaux agricoles.

RoUND UP : aux États-Unis, désigne le rodéo utilitaire, remplaçant à la fin du XIX ${ }^{\mathrm{e}}$ siècle le terme de cow hunt (chasse aux vaches).

RUEDO : dans le monde de la corrida, désigne la piste des arènes ou terrain circulaire délimité par des barrières. Par métonymie, désigne aussi l'arène.

SADDLE BRONC RIDING : dans le rodéo étasunien, désigne la monte des chevaux sauvage sellés.

Sokamuturra : en Basque, désigne le taureau à la corde.

STEER ROPING : dans le rodéo nord-américain, consiste à attraper un taureau adulte au lasso depuis son cheval pour lui lier trois pattes ensemble. Équivalent du calf roping mais avec un bétail plus âgé.

STEER WRESTLING : dans le rodéo nord-américain, consiste à attraper à cheval, un jeune bovin, à le saisir par les cornes en pleine course, à mains nues, en sautant de son cheval pour le retourner au sol et l'immobiliser. La technique s'appelait autrefois le bulldogging, considérée comme une invention d'un cow-boy afro-américain de la fin du XIX ${ }^{\mathrm{e}} \mathrm{du}$ nom de Bill Picket.

Suelta : en Espagne, désigne un lâcher de bovins.

SUERTE : en Espagne, terme polysémique qui désigne le hasard, la chance et, dans le contexte taurin, sans que ces significations disparaissent, désigne également une passe du torero ainsi qu'une phase du combat.

Suerte nacional : au Pérou, désigne une technique d'affrontement dite nationale, consistant à toréer à la cape depuis le cheval (voir capeador a caballo). 
TAU : dans le monde de la course camarguaise, désigne un jeune taureau non castré que l'on réserve également pour les « courses de nuit».

TAUROMAQUia : « tauromachie», dans le monde de la corrida, désigne aussi les ouvrages codifiant l'art du toreo.

TEAM ROPING : dans le rodéo nord-américain, capture d'un veau au lasso, exécutée à deux cavaliers. Un bouvillon, lâché en piste à l'ouverture d'une barrière, est poursuivi par deux cavaliers, l'un lui attrape au lasso les cornes, avant que l'autre lui attrape au lasso une patte, l'animal se trouvant ainsi immobilisé en un temps record.

TEMPORADA : «saison ", dans le monde de la corrida, désigne la période de l'année où se déroulent les spectacles, approximativement de mars à octobre. Le terme est passé dans la langue taurine française. L'expression existe aussi dans le langage de la course camarguaise.

Tendido : dans le monde de la corrida, désigne le secteur de places divisant les gradins d'une arène.

TERCIO : «tiers », dans la corrida, désigne chacune des trois phases qui se succèdent au cours de l'affrontement d'un taureau : Tiers de piques incluant le travail à la cape, tiers de banderilles et tiers de mise à mort incluant le travail à la muleta.

TERNA EN EL RUEDO : dans la charreada, désigne l'exercice effectué par trois cavaliers charros devant maîtriser un taurillon à l'aide de leur lasso, en moins de 6 minutes. L'un d'entre eux attrape la tête, les autres piègent au sol les pattes de l'animal.

Tienta : dans le monde de la corrida, épreuve de sélection à laquelle sont soumis les jeunes bovins destinés à la reproduction dans les élevages de taureaux de combat.

TOREO : en Espagne, art et manière de toréer.

ToREO COMIQue : désigne une catégorie de jeux taurins qui se caractérisent par leur dimension burlesque.

TOREO DE LA VINCHA : en Argentine, désigne le jeu de la province de Jujuy, consistant à s'emparer de la vincha, sorte de foulard accroché à l'animal, couvert de pièces d'argent. Par prolongement, il désigne aussi le fait de toréer cet animal, sans effusion de sang.

TORERISTA : dans le monde de la corrida, désigne la partie de l'afición qui porte son attention essentiellement sur le torero et qui accorde la primauté à la dimension artistique de son travail (voir torista).

Torero : nom générique donné à tout homme affrontant les taureaux dans l'arène, utilisé pour la corrida et ses dérivés, mais aussi parfois pour les protagonistes de la course landaise.

TORISTA : dans le monde de la corrida, désigne la partie de l'afición qui porte son attention essentiellement sur le taureau et qui accorde la primauté à la qualité combative et au sérieux des taureaux en piste (voir torerista).
Toros : «taureaux », En Espagne, l'expression « los toros ", de même qu'en France, l'expression « les taureaux », suffisent, dans de nombreuses régions taurines, à désigner le jeu taurin lui même.

TOROS A LA TICA : « taureau à la mode du Costa Rica », Au Costa Rica, désigne un type de lâchers de bovins qui se caractérise par un mélange des formes : courir les taureaux, les monter, les toréer de façon élémentaire.

TORO CERRIL : en Espagne, désigne le taureau aux cornes intactes (non protégées et non épointées) lâché dans les jeux taurins à participation collective. Se pratique principalement dans les régions de Valence et d'Estrémadure.

Toros ColEAdos : au Venezuela et en Colombie, nom du rodéo fondé sur la technique du coleo, consistant à faire chuter le taureau dans sa course en l'attrapant par la queue.

TOROS DE COLA : en Amérique latine, autre expression pour désigner le coleo.

TORO DE CUERDA : en Espagne, jeu taurin populaire fondé sur l'utilisation d'un taureau encordé (voir toro ensogado).

Toro DE FUEGo : en Espagne, équivalent du taureau embolado. En France, armatures chargées de pétards et de fusées, prises en charge par un ou deux individus qui reproduisent la furie d'un véritable taureau lâché au milieu de la foule.

Toro de REPARo : au Mexique, dans le jaripeo ranchero professionnel, désigne les taureaux destinés à être montés, choisis pour leur agressivité.

TORO ENMAROMADO : jeu taurin à participation collective fondé sur l'utilisation d'un taureau encordé.

TORO ENSOGADO : en Espagne, jeu taurin à participation collective fondé sur l'utilisation d'un taureau encordé. De nombreuses expressions existent pour nommer ce jeu selon les langues et les régions (toro de cuerda, toro enmaromado, bou capllaçat, bou de corda, bou ensogat, bourgine, tourada à corda).

Toro-PIscine : en France, course de vaches populaire, généralement estivale et fréquente dans les stations balnéaires, intégrant une piscine comme élément ludique.

TOROS DE PUEBLO : « taureaux de village ", en Équateur, nom donné aux jeux taurins à participation collective dans la Sierra.

TOURADA : au Portugal, désigne couramment la corrida portugaise qui est aussi appelée corrida de touros.

TOURADA À CORDA : aux Açores, désigne le type de jeux taurins à participation collective reposant sur la présence d'un taureau encordé tenu par des cordiers.

ToURADA À VARA LARGA : au Portugal, désigne couramment les lâchers de bovins.

Touradas a ALENTEJANA : au Portugal, dans l'Alentejo, désigne couramment les lâchers de bovins en lieux clos.

TRIDEnT : en Camargue, désigne la pique des gardians, terminée par un embout métallique à trois pointes. 
Turupukllay : au Pérou, fête taurine des Andes reposant sur la présence d'un taureau sur le dos duquel est fixé un condor, qui est ensuite toréé de façon élémentaire.

VAQUeIRo : au Brésil, désigne les bouviers et une figure emblématique des travailleurs du bétail du Sertão. Dans la vaquejada, nom donné aux cavaliers pratiquant le coleo.

VAQUEIRO DE ESTEIRO : dans la vaquejada, parmi les deux cavaliers lancés à la poursuite du bovin, désigne celui chargé de faire courir l'animal en ligne droite, le rabattant vers le second, appelé puxador, afin de lui transmettre la queue.

VAQUEJADA : au Brésil, rodéo de la région du Nordeste fondé sur l'exercice du coleo.

VAQUería : en Amérique latine, désigne les activités et la culture des vaqueros.

VAQUERO : de vaca (vache), en Espagne et en Amérique latine, désigne le bouvier qui conduit et garde le bétail, équivalent du cow-boy nordaméricain. Par extension culture vaquera, culture liée à l'univers des vaqueros à cheval.

VAQUILLA : en Espagne, appellation très courante des jeux taurins populaires fondés sur un lâcher de bovins.

VARIEDAdes taurinas : au Portugal, désigne des spectacles taurins mixtes, populaires et comiques relativement informels.

VARILARGUEROS : en Espagne, au XVIII ${ }^{e}$ siècle, cavaliers issus des élevages bovins au service de la noblesse, qui deviennent pour un temps les nouveaux héros des courses de taureaux en tant que picador.

VOLAPIÉ : dans la corrida, technique de mise à mort du taureau consistant, en face à face, à planter l'épée par-dessus les cornes, à proximité du cœur de l'animal, en détournant sa tête à l'aide de la muleta.

YAWAR FIESTA : "fête du sang ", au Pérou, dans les Andes, équivalent du turupukllay. Titre du roman éponyme de José María de Arguedas. 


\section{TABLE DES CARTES}

Carte 1. - Les pratiques tauromachiques du Sud-Ouest européen à l'Amérique

Carte 2. - Spectacles taurins en Espagne

(majeurs et mineurs), 2003

CARTe 3. - Spectacles taurins populaires

en Espagne, 2003

Carte 4. - Spectacles taurins majeurs en Espagne, 2003

Carte 5. - Tauromachies dans le Sud-Ouest européen

Carte 6. - Plazas de toros en péninsule Ibérique

Carte 7. - Foyers denses de festejos populares

Carte 8. - Fincas d'élevage brave en Espagne (2000)

Carte 9. - Élevages de taureaux de combat de l’UCTL (2005) 256

Carte 10. - Superficie des élevages braves de l'UCTL (2004) 257

CARTE 11. - Arènes de corridas et de courses landaises dans le Sud-Ouest français

CARTE 12. - Arènes de corridas et de courses camarguaises dans le Sud-Est français

CARte 13. - Tauromachies au Mexique 
Carte 14. - Tauromachies aux États-Unis 336

CArTe 15. - Tauromachies en Amérique andine 350

CArte 16. - Tauromachies en Colombie 352

CARTE 17. - Tauromachies au Venezuela 365

CARTE 18. - Tauromachies en Équateur 375

CArte 19. - Tauromachies au Pérou 387

Carte 20. - Tauromachies au Brésil 400 


\section{TABLE DES FIGURES}

FIg. 1. — Corrida espagnole, Ávila, Espagne, 2007

Fig. 2. - Corrida espagnole, Madrid, Espagne, 2007

Fig. 3. — Corrida espagnole, picador, Espagne, 2007

Fig. 4. - Rejoneo, Espagne, 2007

Fig. 5. - Concours de recortadores, Espagne, 2008

FIG. 6. - Concours de recortadores, salto, Espagne 2008

FIG. 7. - Concours de recortadores, Espagne, 2008

FIg. 8. - Corrida portugaise, Vila Franca de Xira, Portugal, 2005

FIG. 9. - Corrida portugaise, Vila Franca de Xira, Portugal, $2005 \quad 18$

FIG. 10. - Course camarguaise, France

FIg. 11. - Course camarguaise, France

Fig. 12. — Course landaise, Amou, France, 2008

Fig. 13. - Course landaise, Amou, France, 2008

FIg. 14. - Rodéo nord-américain, bull riding,

Californie, États-Unis, 2009

Fig. 15. - Rodéo nord-américain, calf roping,

Californie, États-Unis, 2009 
FIG. 16. - Rodéo nord-américain, free style bullFighting,

Californie, États-Unis, 2009

FIg. 17. — Bloodless bullFighting, Californie, États-Unis, 2009

Fig. 18. - Jaripeo, Morelia, Mexique, 2007

FIG. 19. - Charreada rurale, coleada, Jalisco, Mexique, 2007

FIg. 20. - Toros coleados, Venezuela, 2008

FIG. 21. - Rodeo chileno, Chili

FIG. 22. - Suelta de vaquilla, Pampelune, Espagne

FIg. 23. - Encierro, Pampelune, Espagne

Fig. 24. - Encierro à cheval, Cuéllar, Espagne

Fig. 25. - Toro embolado, Communauté valencienne, Espagne

FIg. 26. — Largada, Vila Franca de Xira, Portugal, 2005

FIg. 27. - Tourada à corda, Riverdale, Californie, États-Unis, 200825

Fig. 28. - Abrivade, Aigues-Mortes, France

FIg. 29. — Bandide, Saint-Laurent-d'Aigouze, France 26

FIg. 30. - Capeia arraiana, Aldeia Velha, Portugal, 2008 26

FIg. 31. — Garraiada, Aldeia Velha, Portugal, 2008 27

FIG. 32. - Monta de toros, El Salvador, 2007

Fig. 33. — Fiestas en corralejas, Sincelejo, Colombie, 2008

Fig. 34. - Fiestas en corralejas, Sincelejo, Colombie, 2008

Fig. 35. - Turupukllay, Ccollurqui, Pérou, 2007

Fig. 36. - Toros de pueblo, Équateur, 2007

Fig. 37. - Taureau des arènes de Vila Franca de Xira (Portugal) 
FIg. 38. - Taureau à l'entrée du Cailar (France)

FIg. 39. - Taureau à l'entrée des arènes

du Puerto de Santa María (Espagne)

FIg. 40. - Taureau de la fête du Corpus Christi

sur lequel est fixée la colcha

FIg. 41. - Taureau de la fête du Corpus Christi, torée à l'aide d'un poncho dans une arène élémentaire

FIg. 42. - Bouée décorative, Vierge du Bon Voyage

FIG. 43. - Bouée décorative, corrida de toros...

FIg. 44. - Plaza de toros de Madrid

Fig. 45. - Plaza de toros de Séville

FIG. 46. - Plaza de toros de Pampelune

FIg. 47. - Antonio Bienvenida, «le torero de Lima»

FIG. 48. - Signes identitaires ostensibles

d'une hispanité syncrétique (Las Ventas)

Fig. 49. - Plaza de toros de Chinchón

FIG. 50. — Corrida d'art valencien

FIG. 51. - Club taurin de Moita

en faveur des corridas intégrales

FIG. 52. - Simulacre de mise à mort avec une banderille au Portugal

FIg. 53. - Plaza de toros de Quito (extérieur) 380

FIG. 54. — Plaza de toros de Quito (intérieur)

FIG. 55. — Plaza de toros de Lima 



\section{TABLE DES GRAPHIQUES}

Graphique 1. - Construction des arènes en Espagne 176

Graphique 2. —Évolution du nombre d'arènes en Espagne $\quad 178$

Graphique 3. - Spectacles majeurs en 2004 (Espagne) 226

GraphiQue 4. - Encierros et vaquillas en Castille 229

GraphiQue 5. - Encierros et vaquillas en Castille par province 229

GraPhiQue 6. - Spectacles majeurs en Espagne et en France (2003) 260

GraphiQue 7. — Spectacles taurins au Portugal (2004) 273

Graphique 8. - Spectacles majeurs en Espagne et au Portugal (2000-2004)

Graphique 9. - Augmentation des festejos populares dans la Communauté de Madrid 292

GraphiQue 10. - Augmentation des concours de recortadores (Madrid et Castilla-León) 293

GraPhique 11. - Spectacles taurins majeurs au Mexique (2001-2005) 



\section{TABLE DES TABLEAUX}

TABleau 1. - Les pratiques tauromachiques 9

TABleau 2. - Jeux et spectacles taurins en Espagne en $2003 \quad 216$

Tableau 3. - Croissance des spectacles taurins

en Espagne (1990-2003) 282

Tableau 4. - Évolution et distribution des spectacles taurins au Portugal 



\section{INDEX}

Açores : 6, 73, 103, 163, 191, 274, 276-277, 305, 347, 409, 418, 429.

Aguascalientes : 153, 201, 320, 323, 326-327, 330-331, 334, 426.

Alentejo : 66, 185, 206, 254, 259, 274-275, 278, 316$317,424$.

Andalousie : 76, 99, 103, 113, 133-134, 147, 166, 170, 173-175, 177, 200, 202-203, 207, 221-231, 239, 245-246, 252, 259, 277, 284, 288, 293, 317, 368, $418,422-423$.

Andes : 63, 98, 100, 121, 200, 349-351, 356-357, 359$363,367,374,376-377,379,382,385-386,394$, 397-398, 419.

Aragon : 7, 72, 148, 220, 224, 228, 230, 235, 243, 249, 254, 292, 295, 310, 422.

Arles : 151, 171-172, 177, 181-182, 206, 263, 266-270, 298-300, 424, 426.

Argentine : 8, 12, 91, 97, 157-158, 160, 164, 167, 190191, 194, 196, 199, 205, 210, 358, 378, 384, 408, 428.

Asturies : 221, 224, 231.

Australie : 6, 12, 338, 428 .

Barcelone : 6, 113, 147, 234, 289-290, 312, 328-329, 341, 369, 425.

Barrancos : 149-150, 274-275, 307, 309, 317, 409, 426.

Barretos : 400, 405-408, 424.

Bayonne : 6, 60, 93, 99-100, 120, 171-172, 180-182, 261, 267-269, 287, 298, 310-311, 317.

Béziers : 171, 181, 187, 267-268, 298-299, 312.

Bilbao : 120, 124, 137, 174, 178-179, 234, 245-246, $290,310,424$

Bogotá : 6, 194-195, 349, 351, 353-358, 381.

Bolivie : 8, 12, 63, 94, 100, 153, 155, 159, 191, 196, $199,419,428$.

Brésil : 2, 6-8, 12, 58, 62, 64, 72, 80, 90-91, 100, 102, $110,157,161,167,190-192,205-207,209-210$, $338,341,354,357,373,384,398-410,414,418$, 421-422, 424, 428.

Buenos Aires : 157-158, 194.

Cali : 6, 195, 349, 351, 353-354, 357, 360, 381, 424.
Californie : 6, 8, 10, 20, 104, 153, 162-164, 325, 335$340,342,345-348,409,418,429$.

Camargue : 66, 76, 79, 96-97, 100, 104, 167, 171, 189, 261-262, 267, 270-271, 300, 312, 416, 418-419, 421, 424.

Canada : 5, 8, 10, 61, 190, 337-338, 359, 406, 424, 428.

Cantabrie : 221, 224, 249.

Caracas : 6, 194, 196, 366, 370, 381.

Castellón : 113, 224, 241, 248-249, 294-296.

Castille : 170, 177, 206, 221-231, 234-235, 239-240, 245 246, 252, 259, 288, 291-293, 313, 316, 418, 422, 429.

Catalogne : 104, 147-148, 151, 221, 224, 231, 249, 282, 289-290, 312.

Chili : 2, 5-6, 8, 12, 23, 40-41, 61-62, 64, 69, 71-72, $80,81,86,93,97,110,157,159-160,167,190-191$, 194, 200, 205, 209-210, 384, 391, 404, 411-416, 418, 420-421, 424, 428.

Colombie : 2, 6, 8, 11, 40, 58, 62, 72-74, 78-80, 90-91, $94,100,102,110,121,139,153-154,156,161,190-$ 191, 194-196, 198, 205, 210, 214, 348-364, 366, $368,370,372,376,383,392,403,416,418-421$, 423-424, 426, 428.

Costa Rica : 8, 11, 39, 62-63, 91, 100, 153, 164, 359.

Cuba : 8, 11, 41, 62, 91, 100, 157, 160-161, 167, 184, $194,403,428$.

Dax : 6, 120, 172, 177, 181, 261, 263, 266, 267-268, 302-303, 311, 421, 425.

El Salvador : 10, 27.

Équateur : 2, 6, 8, 11, 29, 74, 78, 80, 94, 98, 100-102, 119-120, 153-155, 159, 199, 205, 350, 353, 356, $368,374-383,397,419,423,426,428$.

Espagne : 2, 6-7, 9, 15-17, 23-24, 36-38, 40-42, 45, 48, $57,72,74,76,78,79,83-84,89,96-99,104,108-109$, $112-114,119-120,125,133-134,137,146,157,160-$ 161, 167-170, 173-182, 184-187, 194, 196-204, 213, 215-262, 266-270, 272-282, 287, 289-290, 294-299, 302-307, 309-318, 322-324, 327-329, 340-341, 346, 353, 356-357, 364, 368-369, 372-374, 380-383, 386, 391, 399, 403, 418, 422-425, 427, 429. 
Estrémadure : 79, 148, 169, 185, 207, 224, 228, 250, $252,254,259,274,276,278,288,316-317$.

États-Unis : 6, 8, 10, 20-21, 25, 57, 61, 80, 160-162, $164-165,167,176,210,214,318,320,325,333-$ $348,359,384,404,406,418,422,426-428$.

France : 6, 8-9, 18-19, 25-26, 57, 60, 64-66, 78, 82, $96,99,104,11,146,150-151,165,167,171-173$, 176-177, 180-183, 186-189, 214, 253, 260-272, 274, 276-279, 287, 291, 296-299, 306, 309-310, 316, $335,380,422-425,427,429$.

Galice : 80, 179, 224, 231, 282, 315.

Gascogne : 167, 172, 311, 416, 421.

Guadalajara (Mexique) : 98, 195, 201, 323-324, 326, $331,334,424,426$.

Guatemala : 8, 10, 39, 100, 153, 194, 333.

Guayaquil : 154-155, 376, 379, 382-383, 385, 426.

Jerez de la Frontera : 234, 248, 255, 293, 327.

La Havane : 160-161, 194, 200-202.

La Rioja : 7, 148, 221, 228, 230, 241-243, 254, 310.

Landes : 37, 86, 103, 151, 172, 180, 187-189, 261-266, $270,303,421$.

Las Vegas : 338-339, 404, 407, 424.

Levant : 7, 104, 228-231, 243, 293, 422.

Lima : 6, 73, 84, 96, 109-110, 138-139, 191, 194-195, 197-199, 201, 204, 290, 381, 386, 388-395, 397, 424.

Lisbonne : 117, 149, 275-277, 306, 314, 317, 418-419, 424.

Madrid : 6, 16, 48, 75-76, 84, 103, 113-114, 121-127, $134,136-139,148,166,169-170,174-175,177$, $184,188,196-197,203-204,221,224,226-229$, $231,233-234,241,243,245-252,255,258,284$, 286-288, 290-293, 309-310, 312, 314, 316-317, 325-326, 357, 368, 392, 418-420, 423-424.

Maracay : 367-368, 426.

Medellín : 6, 195, 349, 351, 354-355, 357.

Mexico : 6, 102, 139, 153, 162, 193-195, 199-202, 323$324,326-328,330-331,392,423-424,426$.

Mexique : 2, 6-8, 10, 21-22, 39-40, 52, 57, 62-63, 72, $74,80,91-92,96,98,100,110,153,162,165,167$, 190-192, 198, 200-203, 205, 207-210, 214, 318, $320-335,337-339,341-343,345-346,349,354$, $356-357,359,381,384,391,403,407,412,415$, 418, 422, 425-429.

Moita : 99, 114-116, 149, 274-275, 277, 306-308, 317, $424,426$.

Monterrey : 323-324, 334.

Montevideo : 160, 200-202.

Mont-de-Marsan : 172, 181-182, 263, 266-267, 287, $310,317,421,425$.

Murcie : 221, 224, 227, 230, 239, 243, 249, 287.
Navarre : 7, 96, 103, 127-128, 147-148, 169-170, 197, $221,228,230-231,240-243,250-252,254,292$, 310-311, 422 .

Nicaragua : 8, 11, 39, 62, 83, 100, 191.

Nîmes : 6, 43, 111, 118, 171, 177, 181-182, 185, 266269, 299, 312, 424, 426.

Pampelune : 4, 23, 75, 77, 95-96, 99, 117, 121, 123 $124,127-131,136-137,169-170,174,178,197$, 234, 245-246, 249-250, 294, 296, 310-311, 325, $346,378,423-425$.

Panamá : 8, 11, 39, 62, 91, 100, 153, 191, 359.

Paraguay: 8, 12, 100, 191, 406, 428.

Paris : 65, 171, 181-182, 188-189.

Pays Basque : 147-148, 151, 221, 290, 310-312, 422.

Pérou : 6-8, 12, 28, 49-50, 74, 78, 84, 96, 100, 109$110,121,153-154,156-157,159,194-195,198-200$, 203-205, 210, 350, 356-357, 378, 385-398, 414, 426.

Portugal : 6-7, 9, 18, 25-27, 37, 50, 72, 78-79, 82, 95-96, 99, 104, 113-118, 121, 146, 148-150, 167, $169,176,180,185,186,214,236,259,266,270$, 272-279, 288, 303-309, 313-318, 347, 399, 409-410, $422,424,426-427$.

Rancagua : 412-413, 419, 424.

Ribatejo : 93, 114, 117, 274-278, 317.

Ronda : 174-175, 248.

Saint-Sébastien : 188, 234, 285, 287, 290-291, 310.

Salamanque : 79, 81, 84, 98, 174-175, 206, 224, 226-229, 240, 248-252, 254-256, 259, 272, 287, 311, 315-317.

Santiago du Chili : 159, 191, 194, 413, 419, 424.

Saragosse : $120,169,174-175,178,188,228,234,241$ 243, 248-249, 285, 294-296, 310, 392, 425.

Séville : 6, 76, 77, 109, 113-114, 121-122, 131-137, 169, 171, 173-175, 178, 202, 224, 226-227, 234, 239, 243, 245-247, 250, 252, 254-255, 285, 288, 293, 296, 310, $312,316-318,346,351,368,392,418,420,423$.

Sincelejo : 27, 102, 349, 361, 363-364, 426.

Tijuana : 324-325, 346.

Trujillo (Pérou) : 96, 387-389, 391.

Uruguay : 8, 159-160, 164, 167, 192, 200, 205, 210, 406, 408, 428.

Valence : 94, 99, 112-113, 137, 148, 174, 179, 220, 224, 228, 230, 234, 241-243, 246, 249, 250, 254, 272, 292, 294, 296, 310, 316, 353, 424-425, 428.

Valencia (Venezuela) : 366-368, 426.

Venezuela : 6, 8, 11, 22, 40, 57, 62, 72, 78, 80, 90, 91, $110,153,156,190-191,194,198,205,210,214$, $350,353,356-357,359,364-373,384,402-403$, $413,418,420-421,424,426,428$.

Vila Franca de Xira : 18, 25, 82, 93, 99, 116, 118, 121, 149, 275, 277-278, 308, 317, 424, 426.

Villavicencio : 349, 351, 358-359, 363, 426. 


\section{TABLE DES MATIÈRES}

Préface de Jean-Robert Pitte

Abréviations

INTRODUCTION

I. - Une aire culturelle de la tauromachie 1

La singulière diversité des jeux taurins $\quad 1$

Tauromachies transatlantiques $\quad 2$

Fête et tauromachie 4

L'espace tauromachique 5

Géographie, sciences humaines et tauromachie 13

II. — La diversité tauromachique en images 14

PREMIÈRE PARTIE

JEUX TAURINS D'EUROPE ET D'AMÉRIQUE

CHAPITRE PREMier. - Définitions et représentations

de la tauromachie $\quad 35$

I. - Tauromachie et corrida 36

La corrida : un art tauromachique au sommet de toutes les hiérarchies? 36

La tauromachie à l'épreuve du relativisme culturel 39

II. — Tauromachie et rites taurins 41

La tauromachie : « rite éternel »? 41

Les vertiges de l'histoire taurine 44 
III. — Les jeux taurins 49

La recherche d'une définition minimale $\quad 49$

Les divergences d'interprétation de la tauromachie 51

La tauromachie entre l'art, le sport et les jeux traditionnels $\quad 55$

IV. — La tauromachie dans le cadre des relations homme-animal 59

Le paradigme de la «course» $\quad 59$

Pratiques tauromachiques, violence et domestication 63

Chapitre II. — Unité et diversité des pratiques tauromachiques 69

I. - Tauromachie professionnelle - tauromachie participative 70

Une opposition fondamentale dans le registre des divertissements $\quad 70$

$\begin{array}{ll}\text { Quand la tauromachie mélange les genres } & 73\end{array}$

II. — Les taureaux des jeux taurins $\quad 75$

Taureaux bravos - taureaux mansos; taureaux de race - taureaux croisés $\quad 75$

$\begin{array}{lr}\text { Les sociétés «taurophiles » } & 81\end{array}$

III. — Jeux d'arènes $\quad 85$

$\begin{array}{ll}\text { Les jeux d'arènes du Sud-Ouest européen } & 87\end{array}$

Les rodéos de l'Amérique $\quad 90$

IV. - Jeux de rue 93

Encierros... 95

... et autres lâchers de bovins $\quad 98$

Chapitre iII. — La tauromachie sous le signe de la fête 107

I. — Fêtes et tauromachies : une relation à interroger 108

La tauromachie entre fête et spectacle 108

Calendriers taurins et calendriers festifs 111

II. — Tauromachies festives et festivités taurines 114

La tauromachie, cause ou conséquence de la fête 114

Trois exemples d'imbrication : Madrid, Pampelune, Séville 121

a) Madrid : la San Isidro et la sanisidro (121)

b) Pampelune : San Fermin et sanfermines (127)

c) Séville : la Féria et la féria taurine (131)

Fêtes taurines et territorialité 


\section{DEUXIÈME PARTIE}

\section{LA CONFIGURATION DE L'ESPACE TAUROMACHIQUE}

Chapitre iv. - Limites et discontinuités de l'espace tauromachique 145

I. - Les limites européennes des pratiques tauromachiques 145

Le cadre juridique et territorial de la tauromachie en Espagne 146

L'exception portugaise des terres de corridas $\quad 148$

La France taurine coupée en deux $\quad 150$

II. — Les limites américaines des pratiques tauromachiques 152

La corrida autorisée $\quad 153$

$\begin{array}{ll}\text { La corrida interdite } & 157\end{array}$

Chapitre v. — La formation de l'espace tauromachique 165

I. — La formation de l'espace tauromachique européen 168

Quelles sont les origines de la tauromachie? 168

Le spectacle tauromachique comme innovation 173

$\begin{array}{ll}\text { La diffusion de la corrida moderne } & 177\end{array}$

a) La diffusion en Espagne (177)

b) La diffusion hors des frontières espagnoles (180)

La stabilisation de l'espace tauromachique

II. — La formation de l'espace tauromachique américain 189

L'introduction du bétail dans le Nouveau Monde $\quad 190$

Les jeux taurins de la Conquête 193

La diffusion de la corrida moderne $\quad 199$

Pour une nouvelle histoire des rodéos 205

TROISIÈME PARTIE

ESPACES ET TERRITOIRES DE LA TAUROMACHIE

Chapitre Vi. - La structure des espaces tauromachiques européens

I. - L'espace tauromachique espagnol

Pour une régionalisation des pratiques taurines

a) Spectacles majeurs et mineurs (221)

b) Les spectacles taurins populaires (227) 
Un espace hiérarchisé : arènes et autres lieux de spectacles

a) Différenciation et recensement des arènes (231)

b) Répartition et foyers de densités de la tauromachie professionnelle (235)

c) Ubiquité et concentration des jeux taurins populaires (239)

Les hauts lieux de l'espace taurin

L'élevage brave

II. - L'espace tauromachique français

Trois spectacles pour deux régions taurines $\quad 259$

Arènes et hauts lieux taurins $\quad 262$

Les élevages de taureaux : l'hégémonie camarguaise 270

III. — L'espace tauromachique portugais 272

Un clivage Nord-Sud $\quad 272$

Faible hiérarchie et particularisme des arènes 276

L'élevage brave comme gage d'intégration 278

Chapitre vir. - Les dynamiques récentes

des espaces tauromachiques européens $\quad 281$

I. - Croissance des pratiques et différenciation des espaces taurins espagnols $\quad 281$

Expansion et restructuration de la tauromachie professionnelle $\quad 281$

a) L'augmentation des spectacles (281)

b) Les raisons de la croissance (284)

Les contrastes géographiques de la croissance et du déclin 288

Renaissance et mutation des tauromachies populaires 291

II. - Stabilité des pratiques et des espaces taurins français 296

Stabilisation et enracinement de la tauromachie espagnole 296

L'inflation des pratiques taurines camarguaises et des manades 299

Stagnation et ouverture de la course landaise $\quad 301$

III. - Fluctuations et blocages de la tauromachie au Portugal 303

Le rééquilibrage Nord-Sud de l'espace taurin 303

Les recompositions internes de l'afición 305

IV. — L'articulation transfrontalière des espaces taurins 309

La «frontière taurine » France-Espagne : le désir d'hispanité 309 
La «frontière taurine » Espagne-Portugal :

entre frères ennemis et nations sœurs

Chapitre viII. - Structure et dynamiques

des espaces tauromachiques américains

I. - L'espace tauromachique mexicain :

corrida, charreada, jaripeo et rodeo 320

La corrida au Mexique ou le géant taurin de l'Amérique latine $\quad 320$

Terre et nation de la charreada

Jaripeos professionnels et rodéo nord-américain : cousins montés l'un contre l'autre?

II. - L'espace tauromachique étasunien :

rodéo, charreada et « corrida soft»

Rodéo et bull riding à l'assaut du continent

La charreada ou la nostalgie mexicaine en pays cow-boy

La frontière exotique et les « corridas soft» luso-californiennes

III. - L'espace tauromachique colombien : corrida, coleo et corralejas

Les corridas : une pratique dynamique, des férias réputées

Les toros coleados des llanos orientaux

Les corralejas des sábanas de Bolívar

IV. - L'espace tauromachique vénézuélien : coleo et corrida

Les corridas : un prestige ancien, une pratique en déclin

Les toros coleados: jeu des llanos, sport national et mythe du Llano

V. - L'espace tauromachique équatorien : corrida, toros de pueblo et rodeo montubio

Corrida, toros de pueblo et rodeo chagra: la déclinaison taurine des Andes équatoriennes

Loin des Andes, loin du cœur : le rodéo montubio

VI. - L'espace tauromachique péruvien : corrida

et « tauromachies andines »

Les corridas ou les ambiguïtés de la fête créole

« Tauromachies andines » et turupukllay

VII. - L'espace tauromachique brésilien : vaquejada, rodeio et farra do boi

La vaquejada nordestina : du sertão au Sertão 
Rodéo nord-américain et rodeio crioulo : modernité yankee contre tradition gaucha

Farras do boi à Santa Catarina : l'isolat archaïque contre « l'iléité » açorienne

VIII. — L'espace tauromachique chilien :

un rodeo chileno sinon rien

Conclusion. - La tauromachie comme marqueur territorial

I. - Les dynamiques centre-périphéries de l'espace tauromachique

II. - Tauromachies et identités territoriales

III. - De la spécificité d'une diffusion tauromachique

IV. - Le rayonnement des hauts lieux taurins

V. - Les lieux de coexistence des pratiques tauromachiques

VI. — Les frontières taurines

Bibliographie

RÉsumÉs

Résumé

455

Resumen

Glossaire

Table des cartes

Table des figures

Table des graphiques

Table des tableaux

Index

Table des matières

CD-ROM : ANNEXES

I. - Arènes et spectacles en Espagne (2003)

II. — Arènes et spectacles au Portugal (2004)

III. - Arènes en spectacles en France (2004)

IV. - Arènes et spectacles en Colombie (2001-2004)

V. - Arènes et spectacles au Venezuela (2004)

VI. — Arènes et spectacles au Pérou (2004) 
BIBLIOTHÈQUE DE LA CASA DE VELÁZQUEZ

VOLUME 46

\section{JEAN-BAPTISTE MAUDET \\ TERRES DE TAUREAUX \\ LES JEUX TAURINS \\ DE L'EUROPE À L'AMÉRIQUE}

ANNEXES

$\mathrm{C} \Lambda \mathrm{S} \Lambda \mathrm{DE}$ VELÁZQVEZ

MADRID 2010 
I. — Arènes et spectacles en Espagne (2003) 4

II. - Arènes et spectacles au Portugal (2004) 61

III. — Arènes et spectacles en France (2004) 74

IV. - Arènes et spectacles en Colombie (2001-2004) 93

V. — Arènes et spectacles au Venezuela (2004) 101

VI. - Arènes et spectacles au Pérou (2004) 105 


\section{Abréviations}

$A$ : arène d'existence ancienne ; AE : arène existante en $1950 ; \mathrm{CC}$ : course camarguaise ; CE : course espagnole ; CL : course landaise ; D : détruite ; $\mathrm{F}$ : arène fixe ; $\mathrm{FB}$ : arène fixe en bois; $\mathrm{FD}$ : arène fixe en dur ; ID : numéro d'identification des arènes par province, région ou département sur les cartes ; $\mathrm{M}$ : mauvais état ; $\mathrm{P}:$ arène démontable ; $\mathrm{PB}:$ arène démontable en bois ; PF : démontable fixée ; R : reconstruction ou rénovation; SM 2003 : nombre de spectacles majeurs en 2003. 
ANNEXES

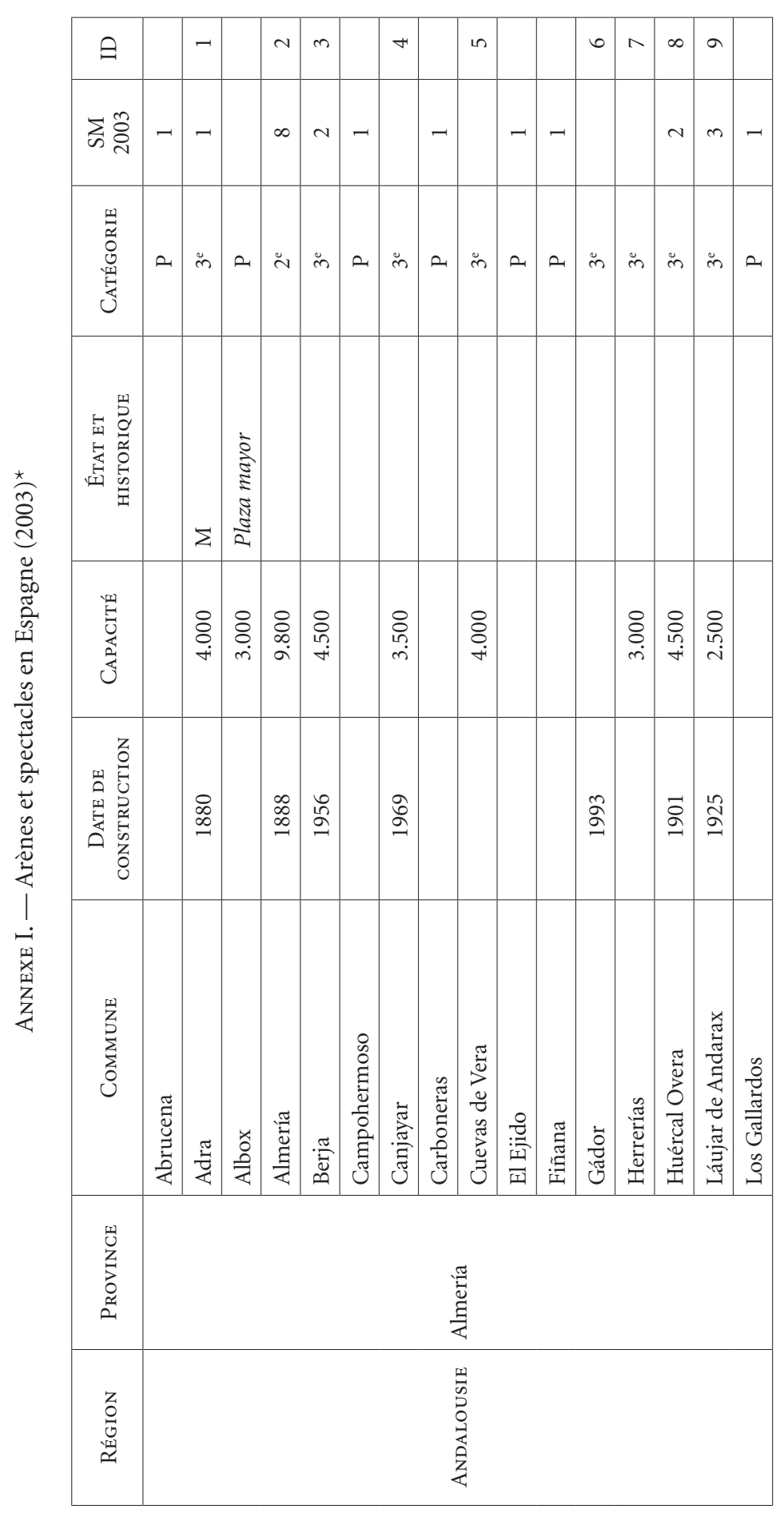

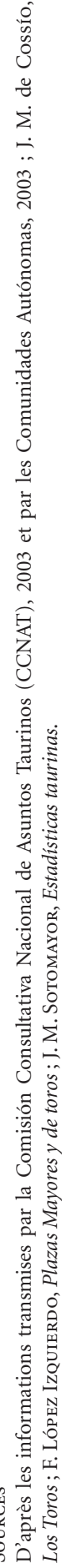




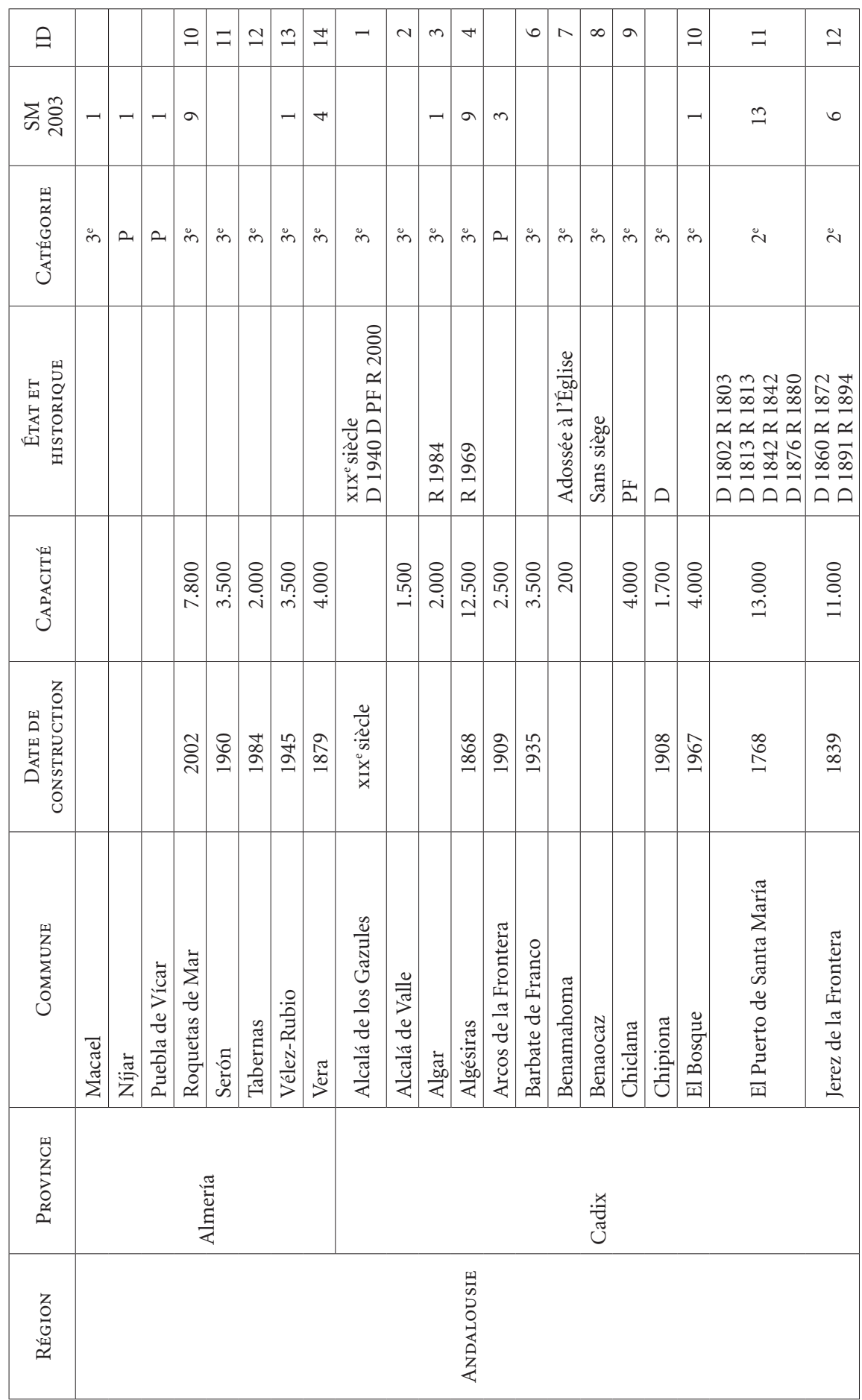




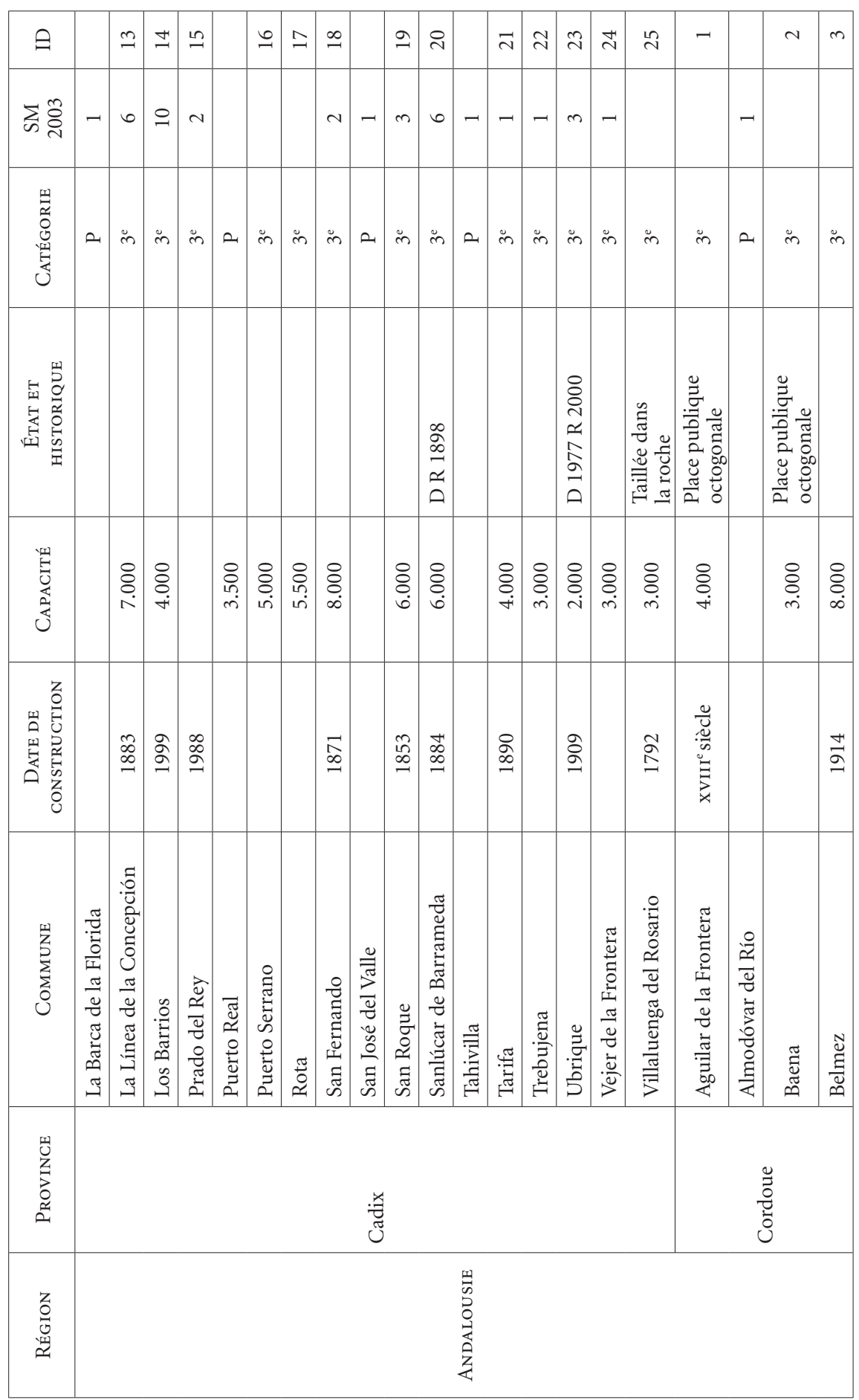









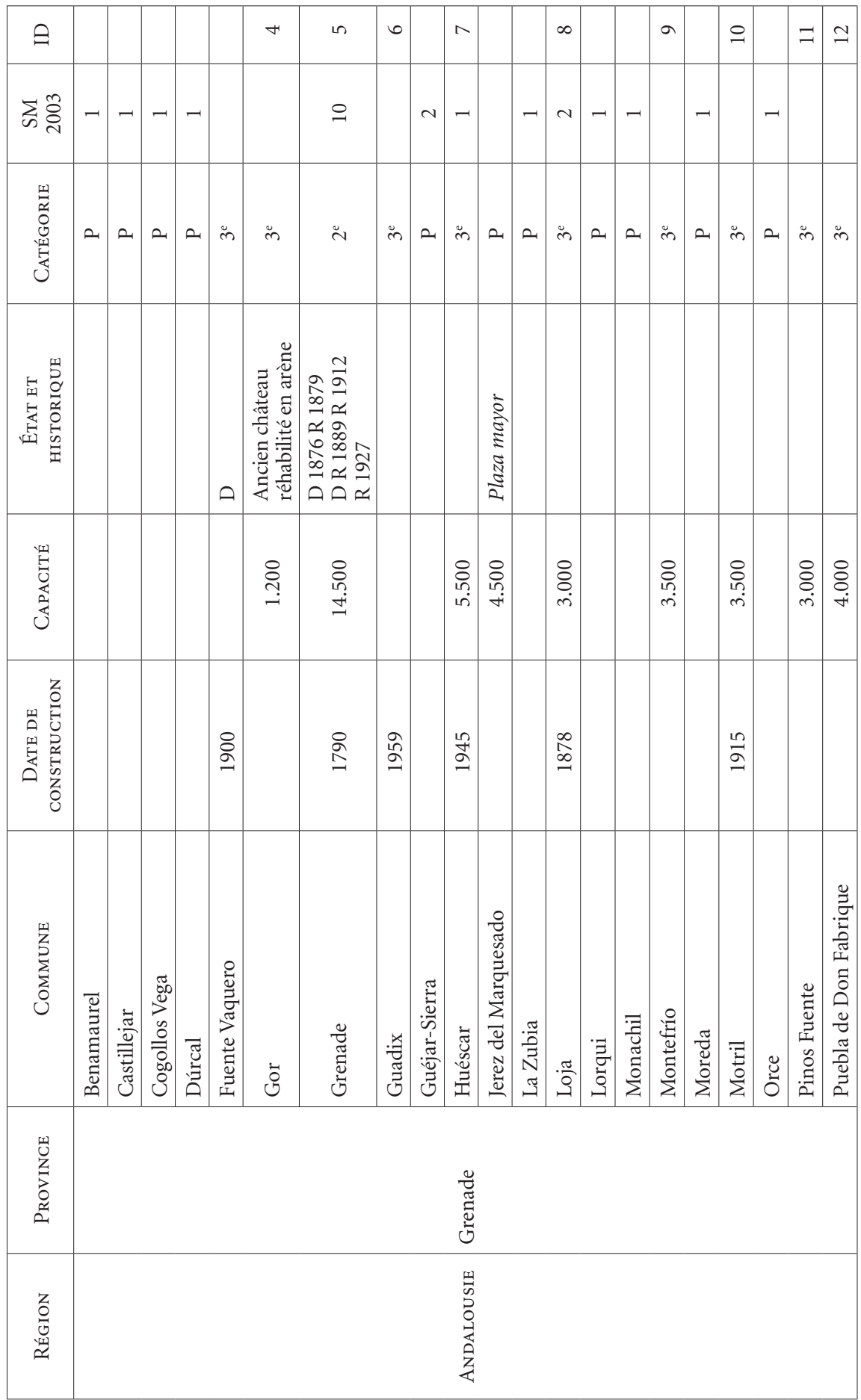




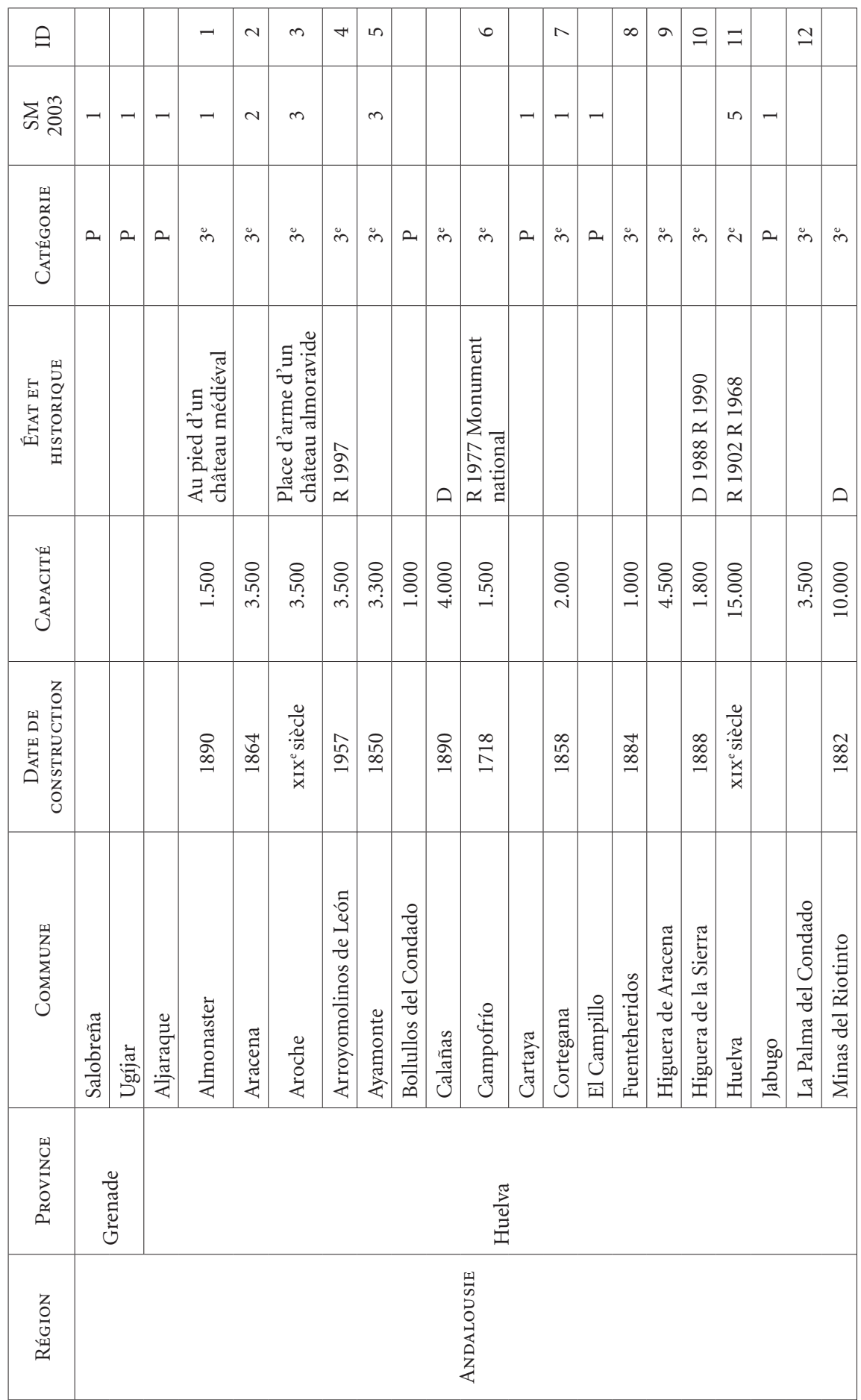




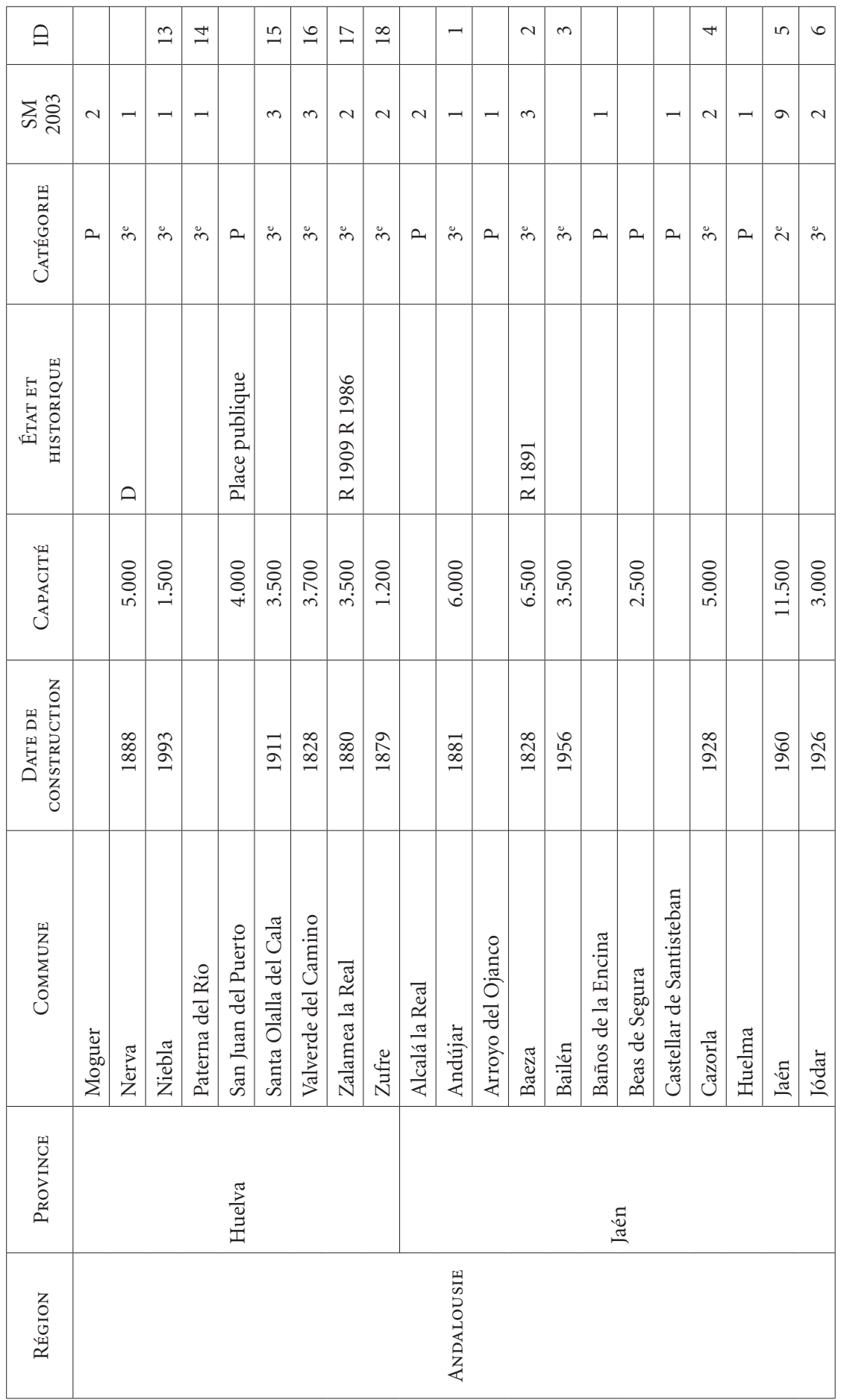


ANNEXES

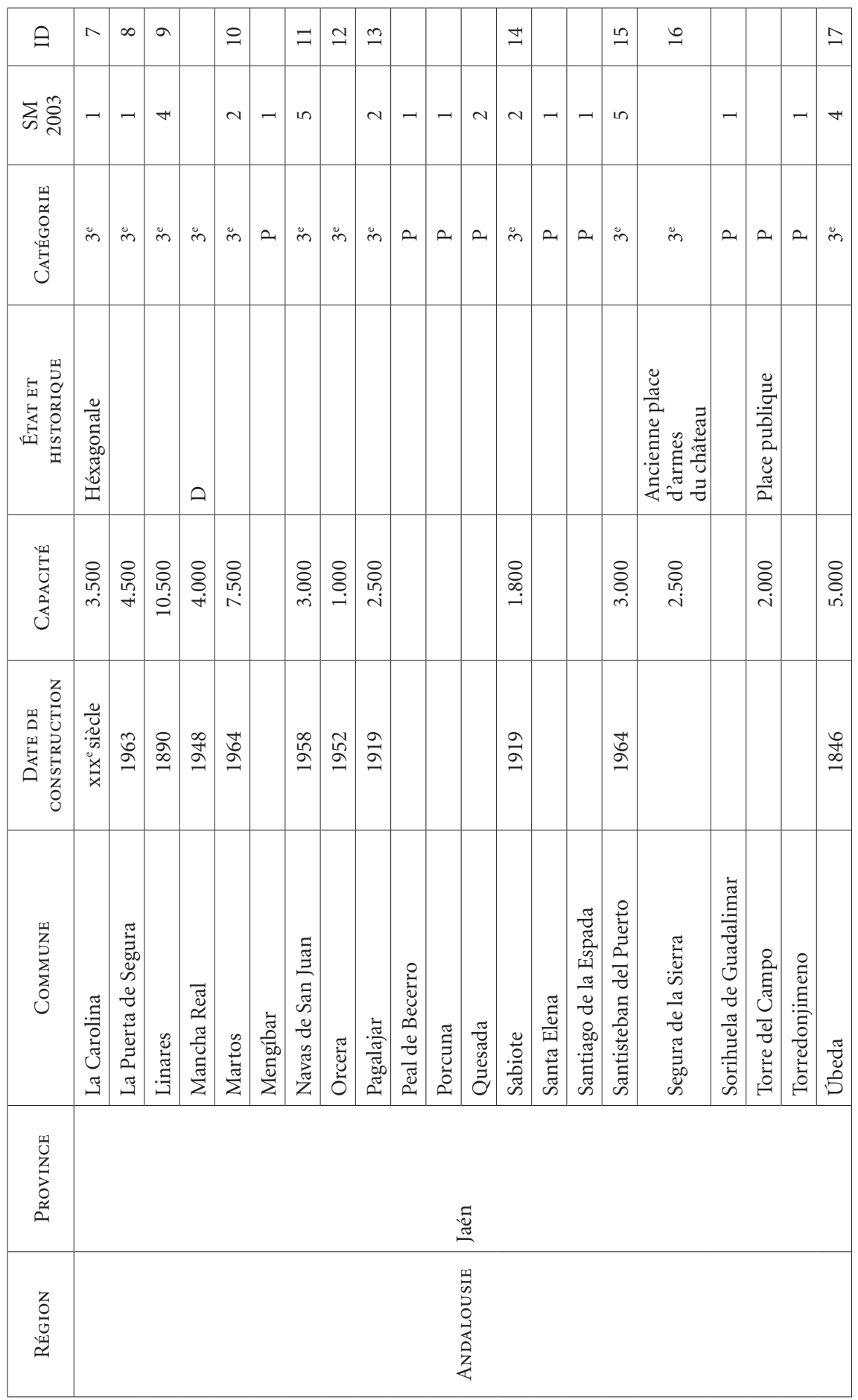




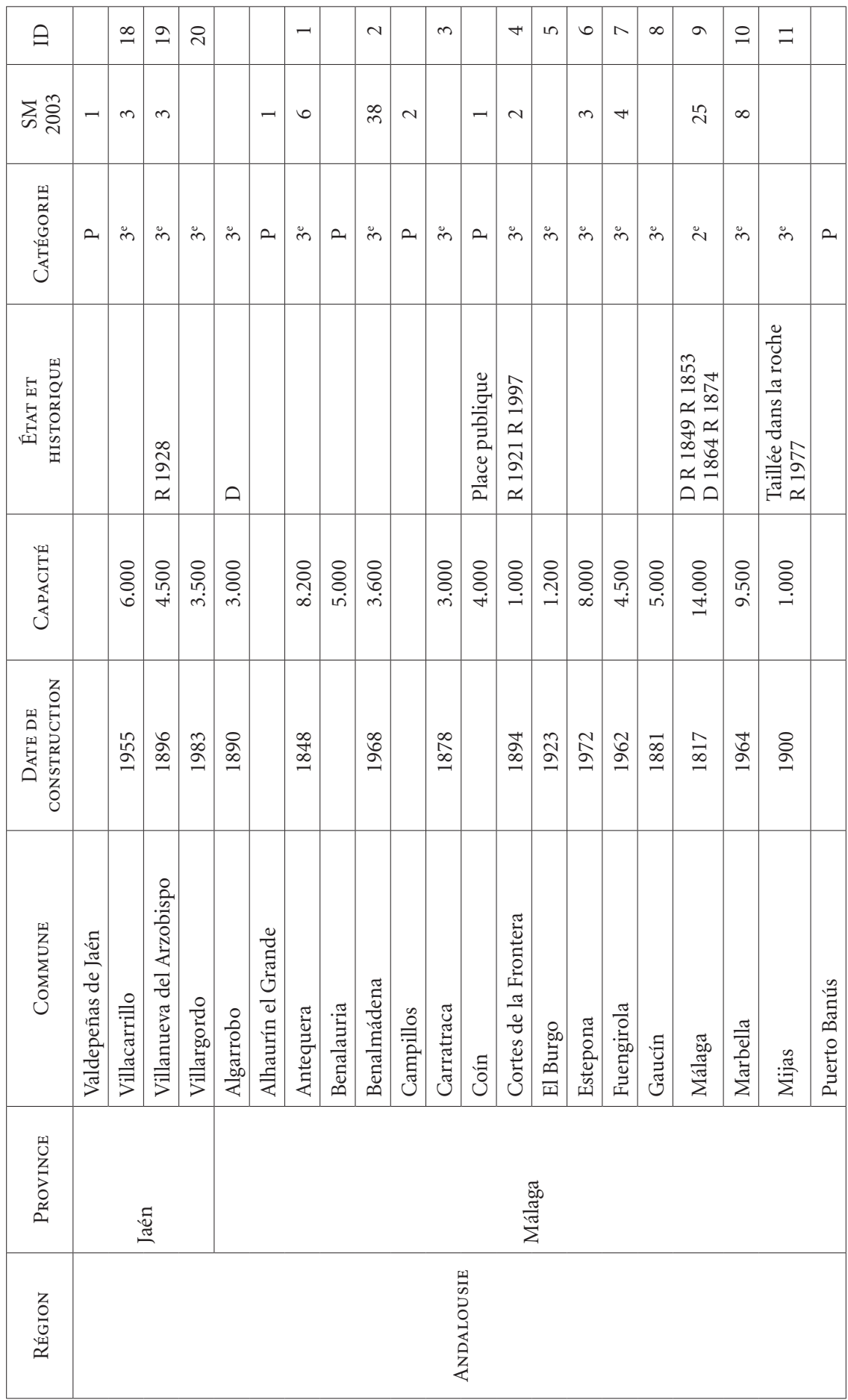




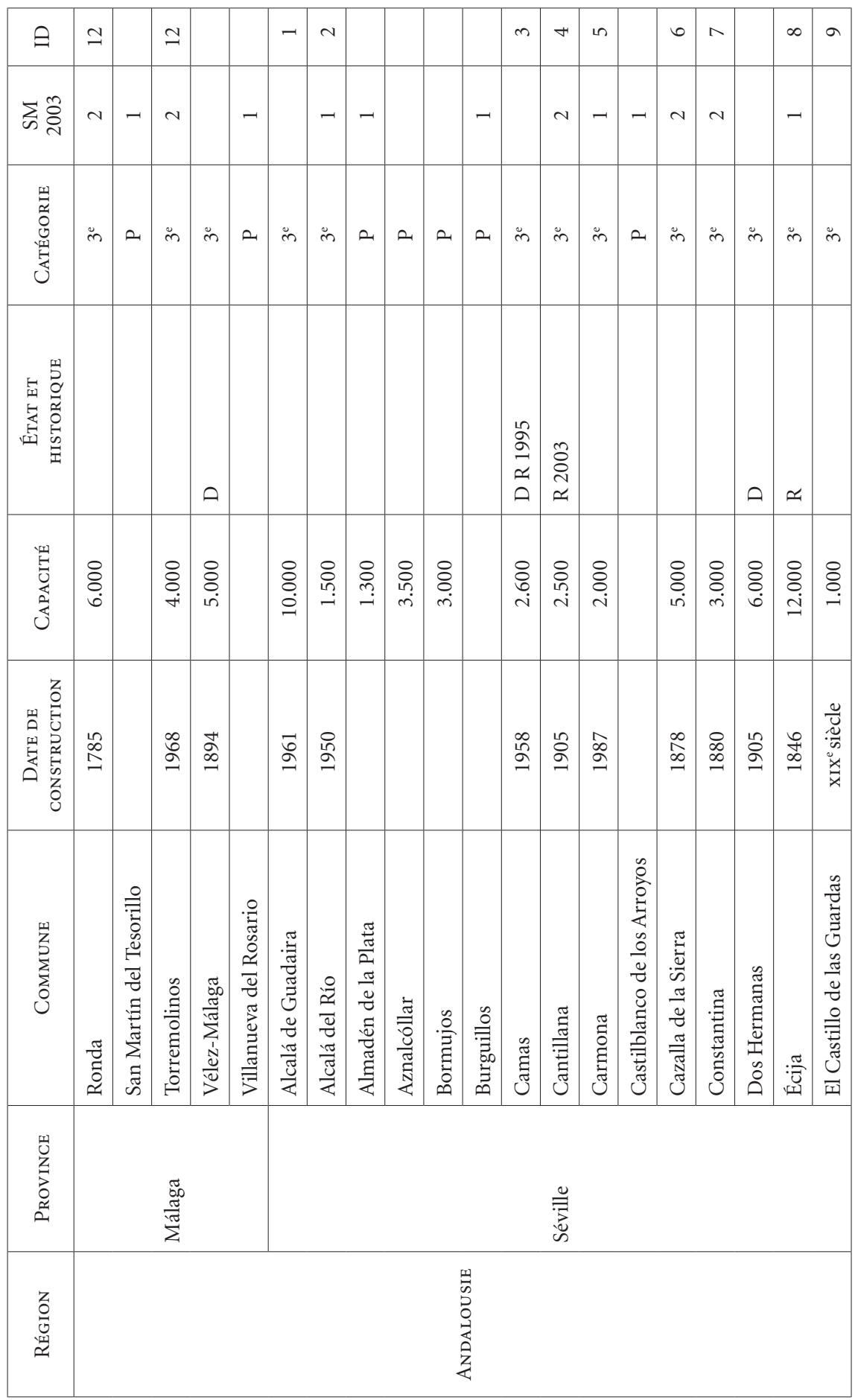




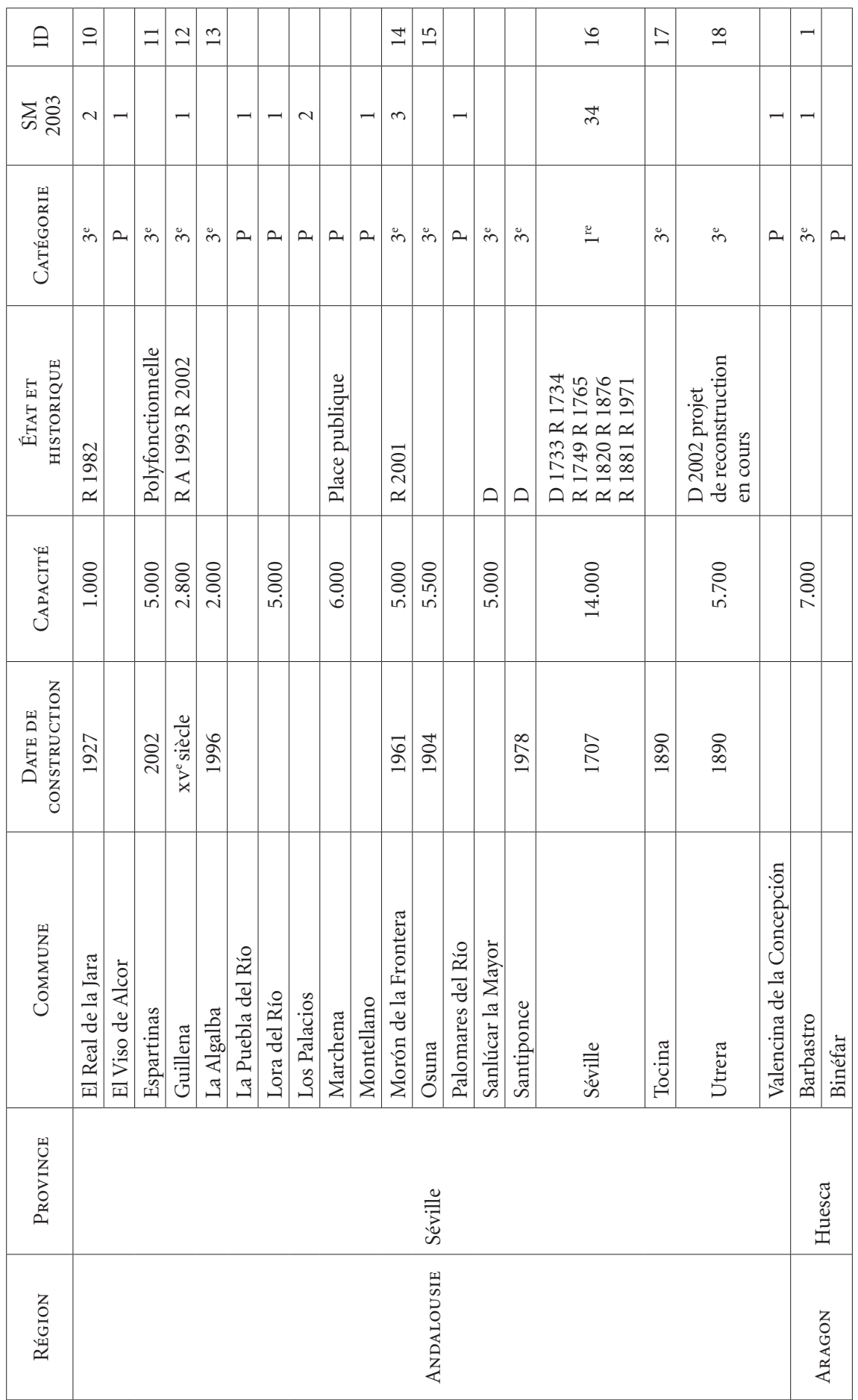




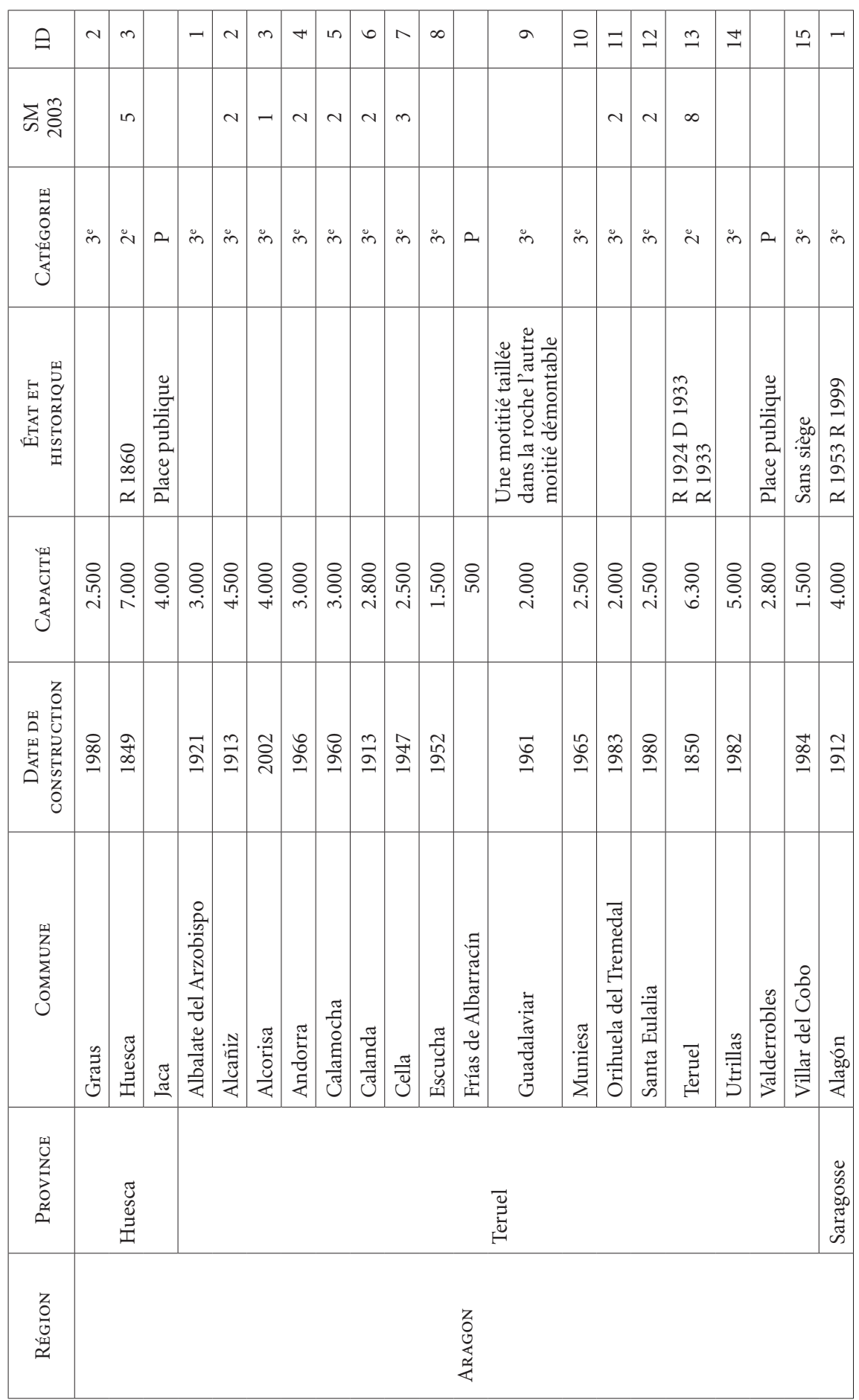




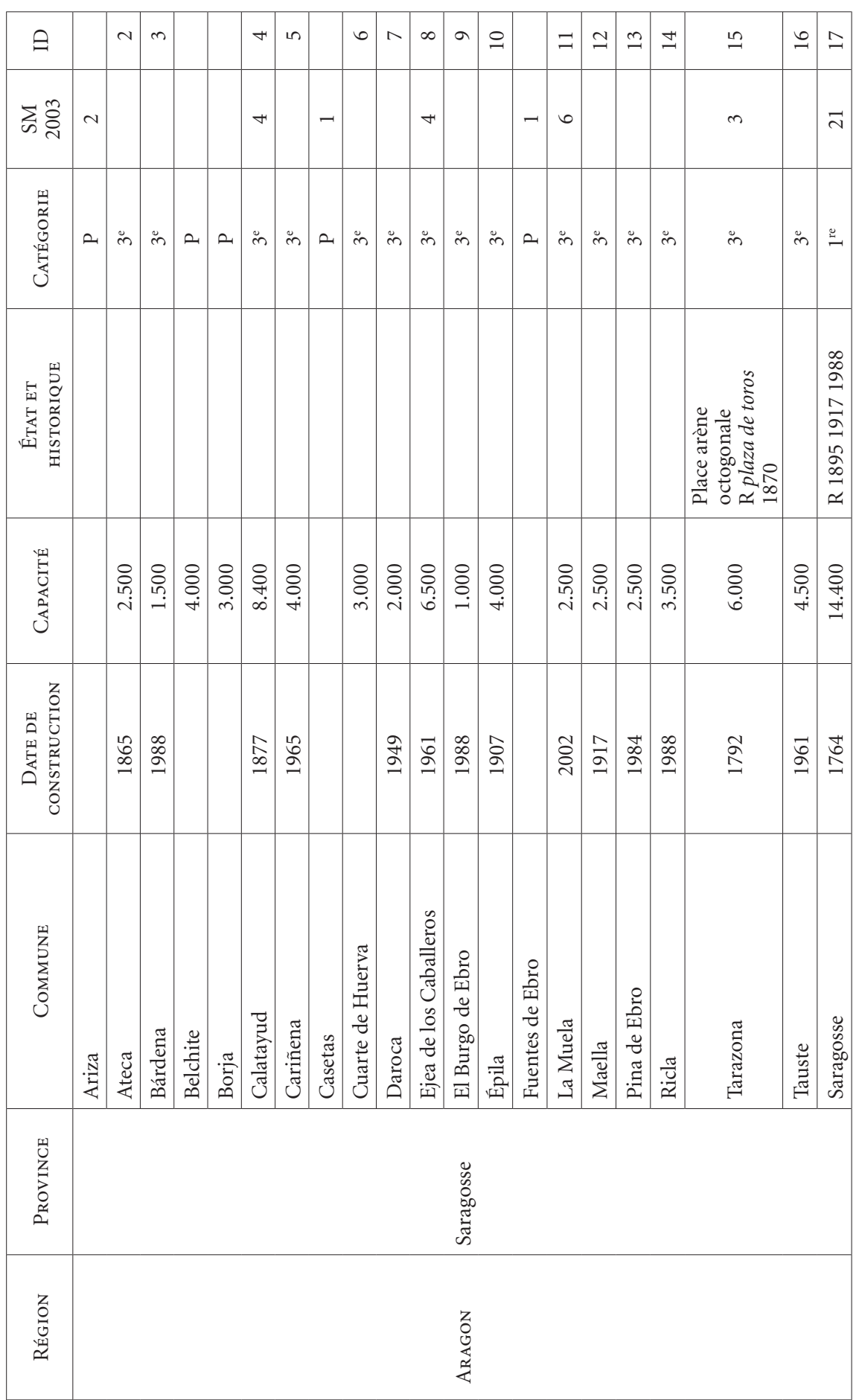




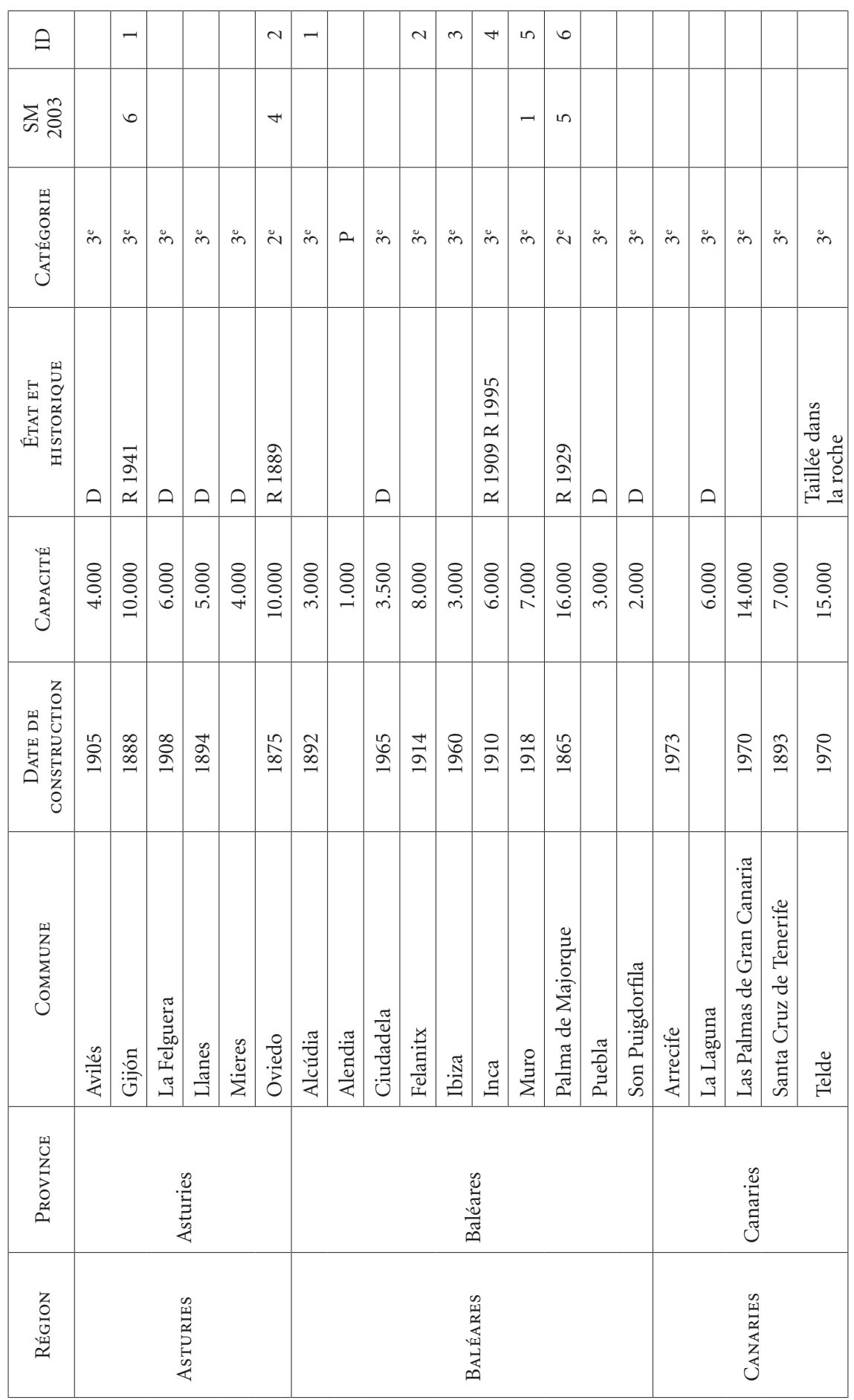




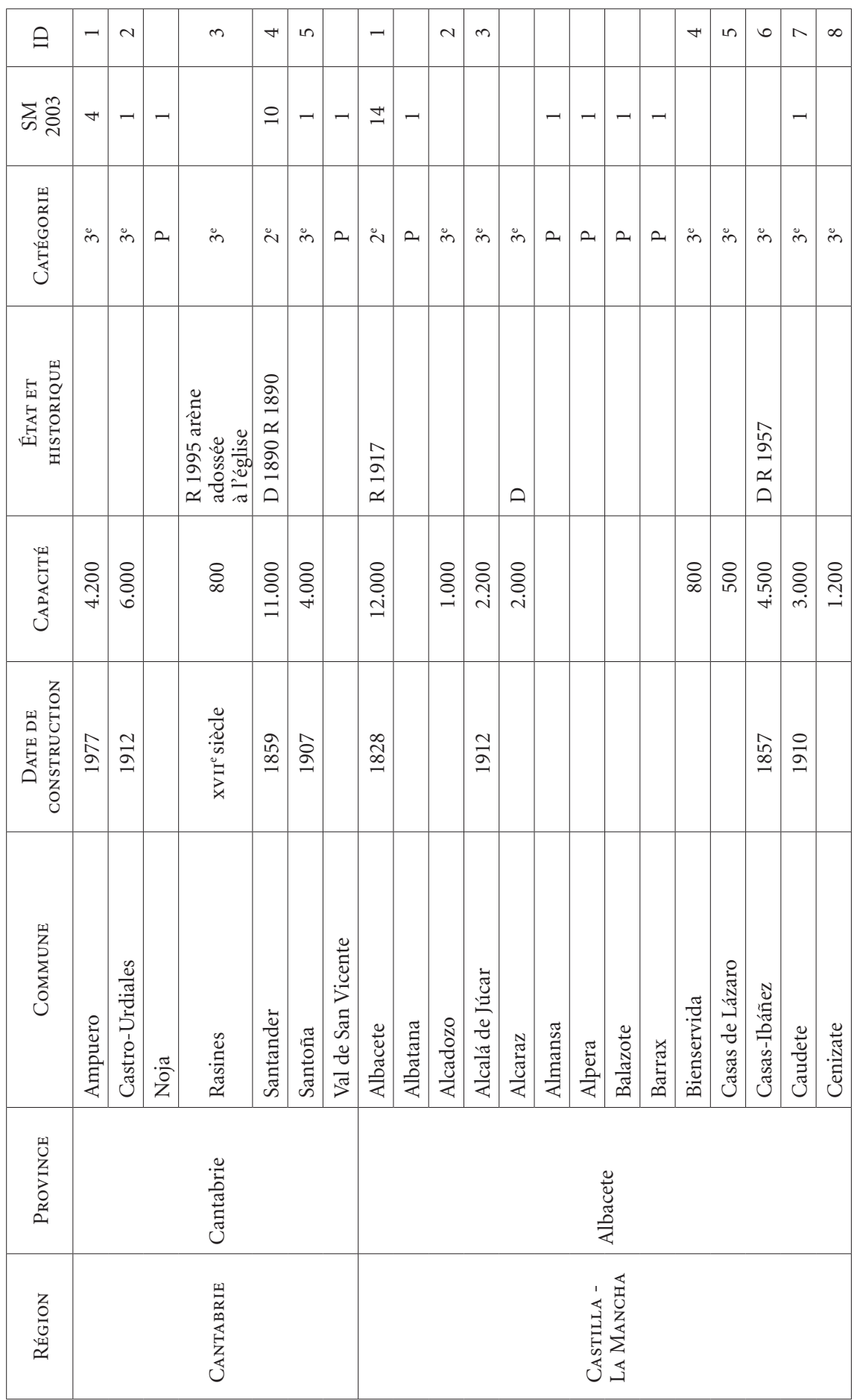




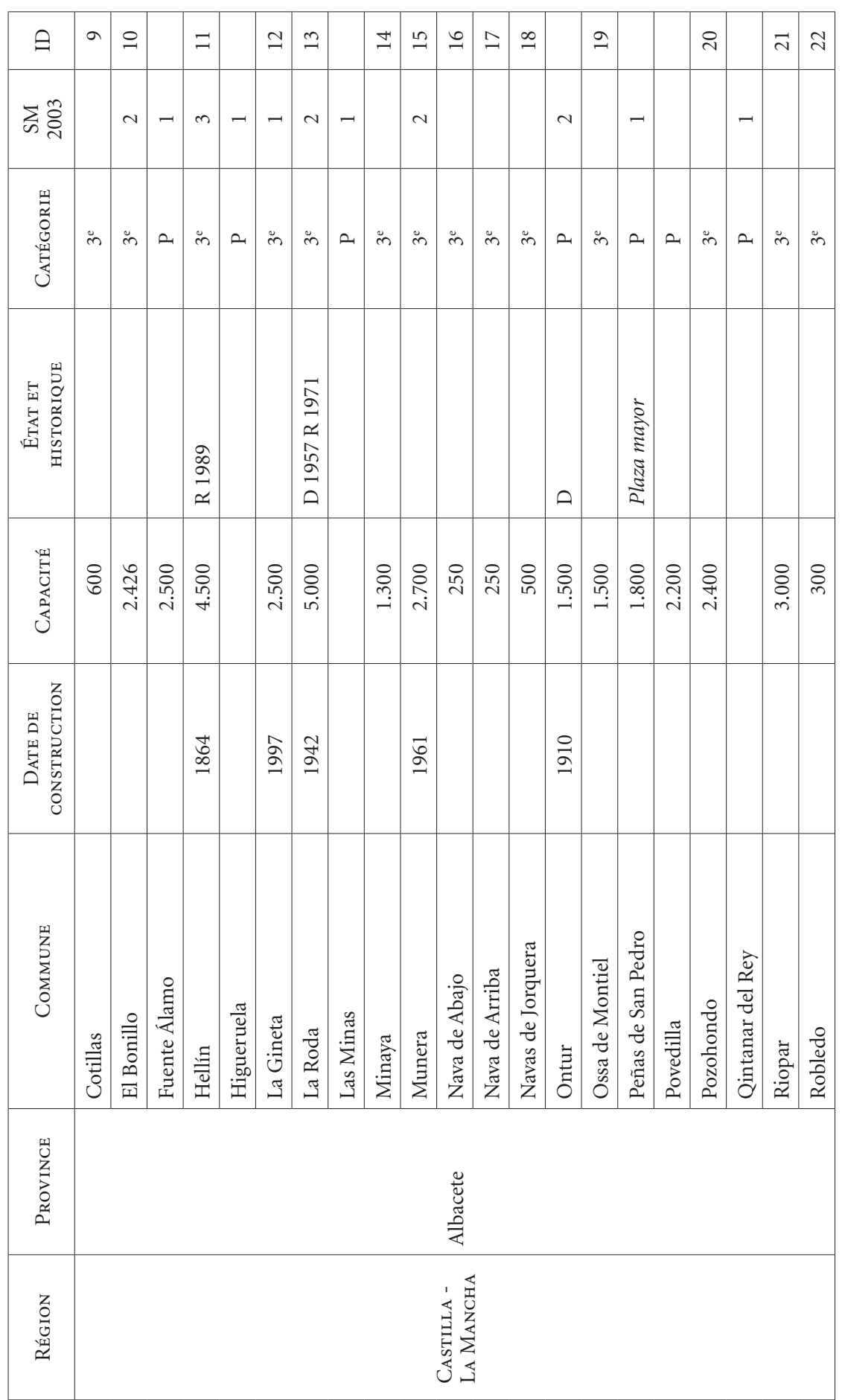




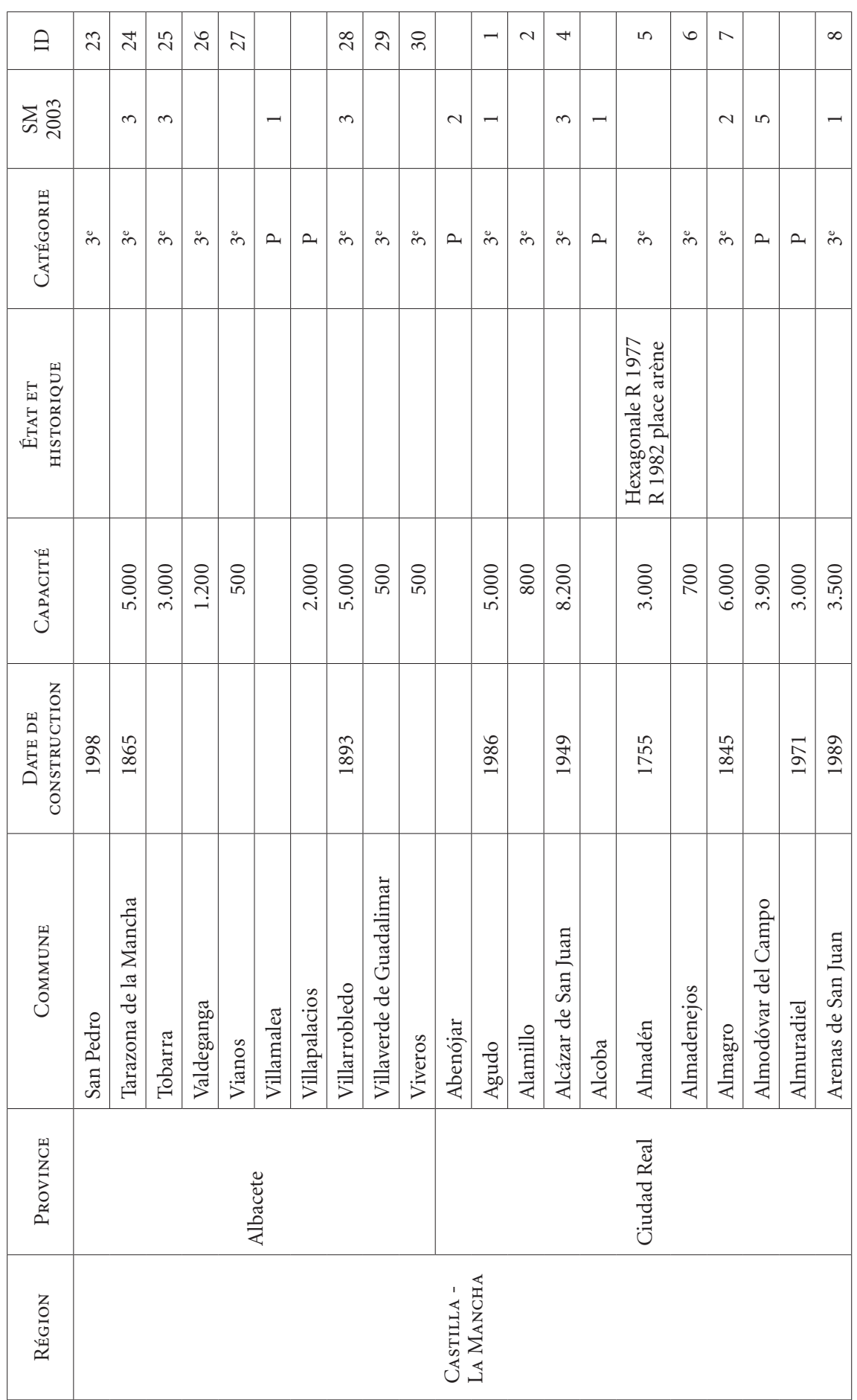


ANNEXES

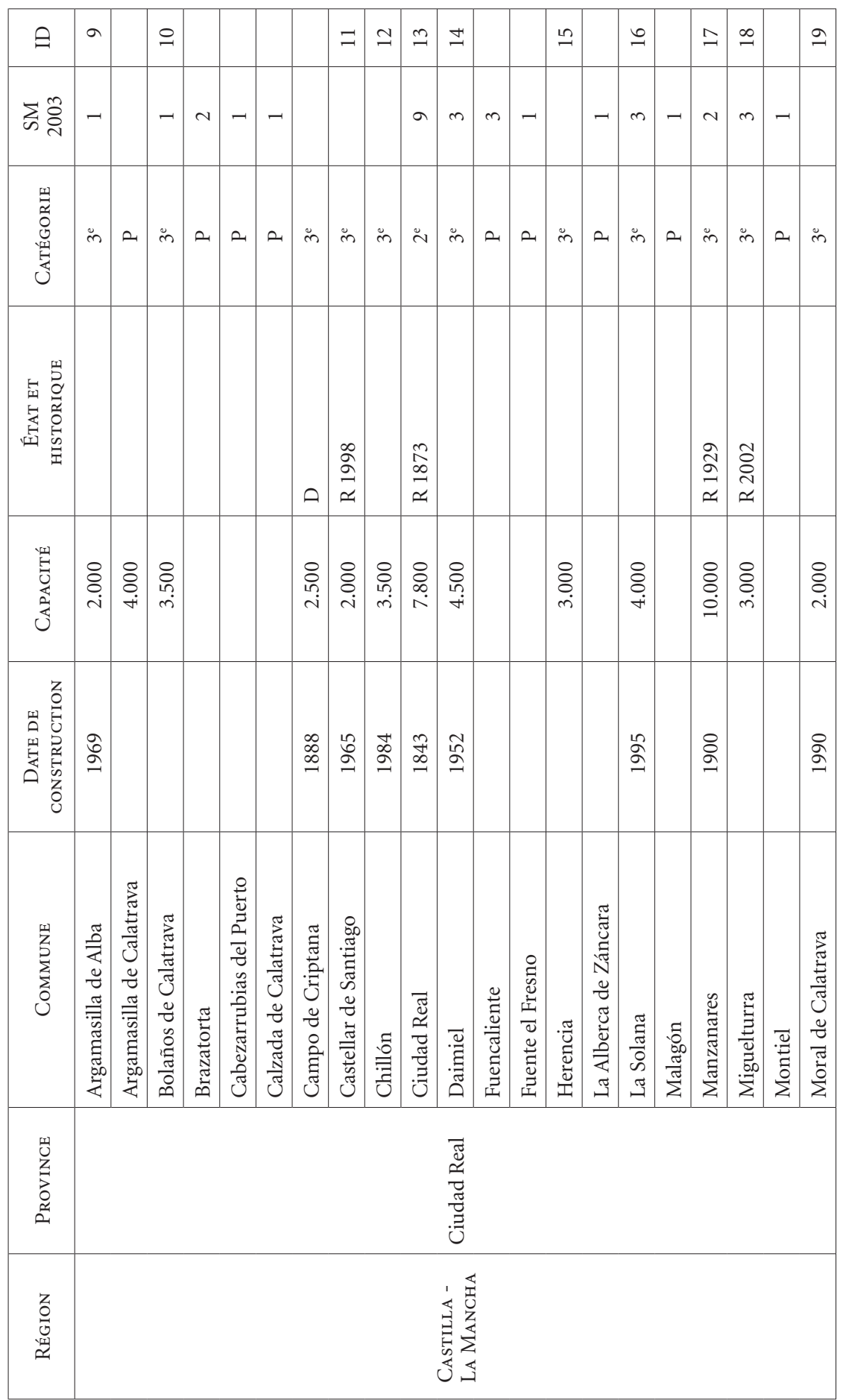




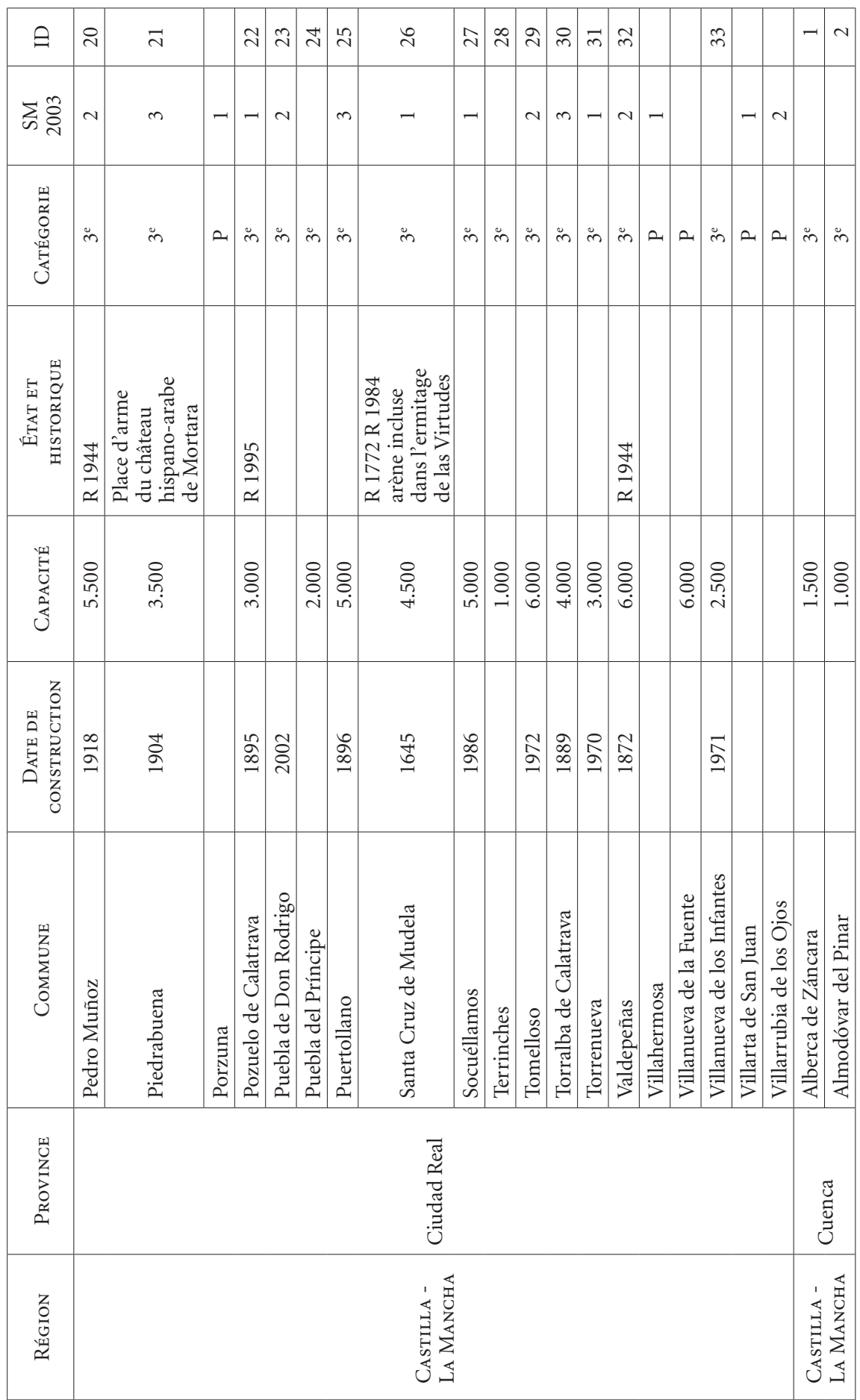


ANNEXES

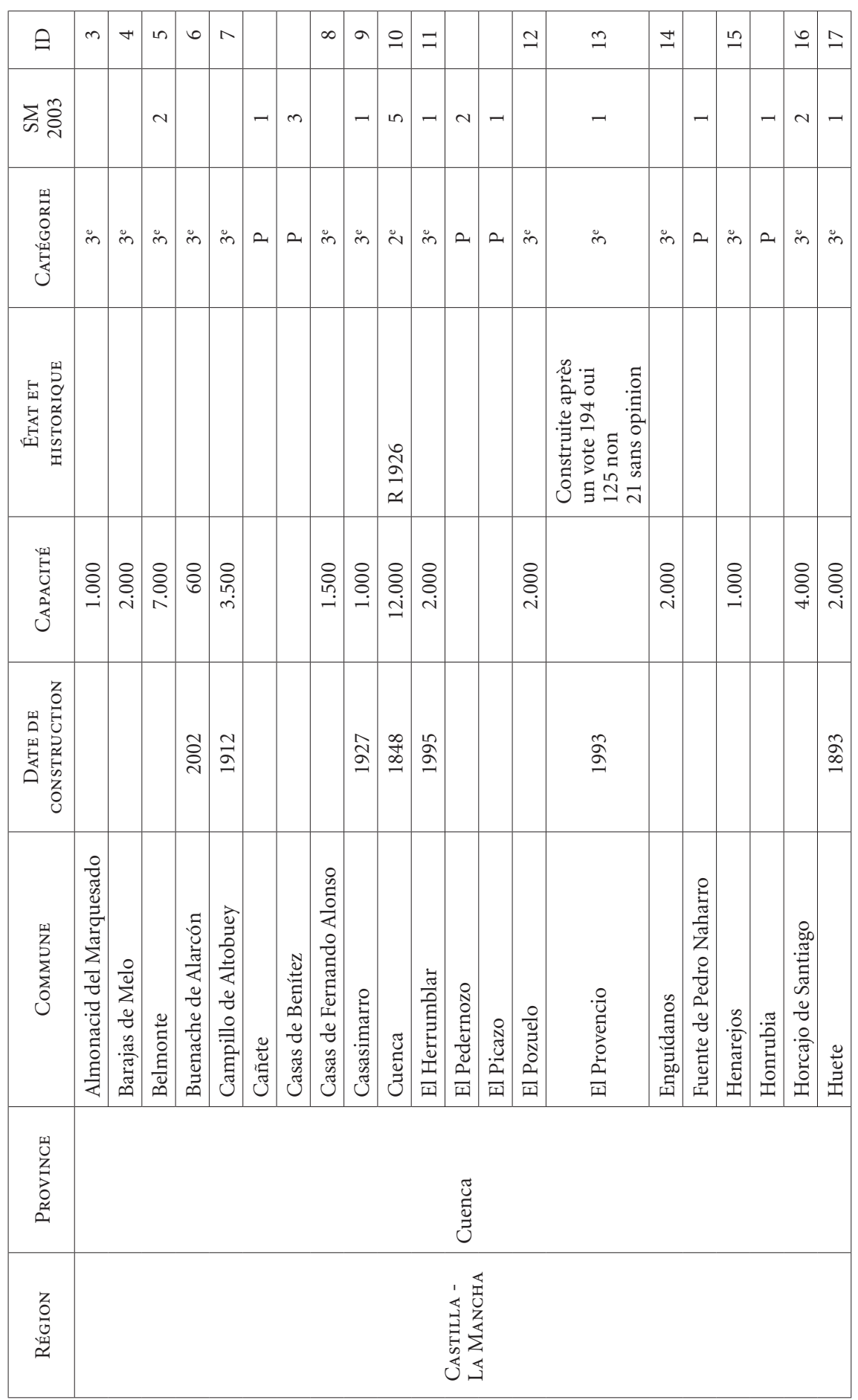




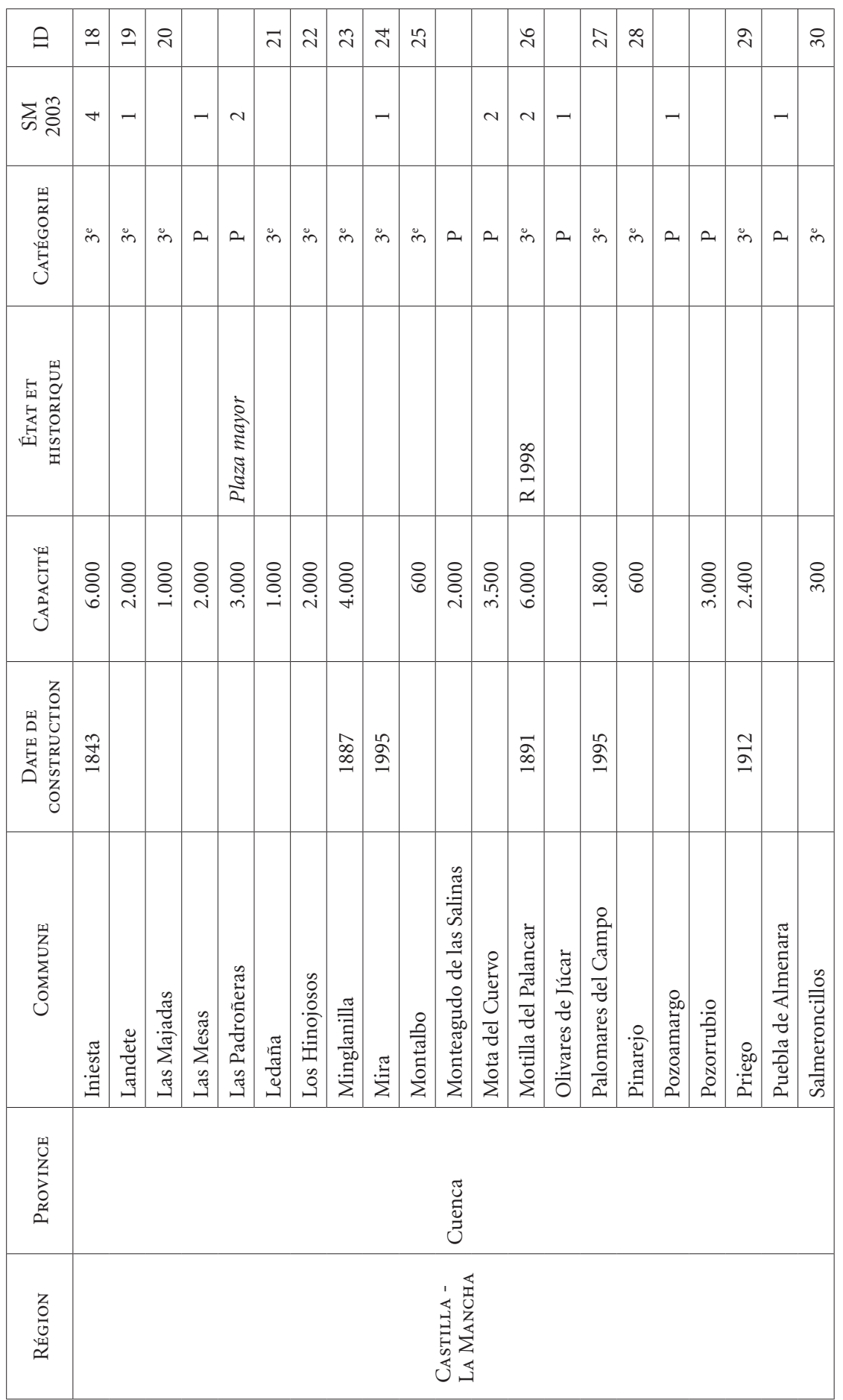




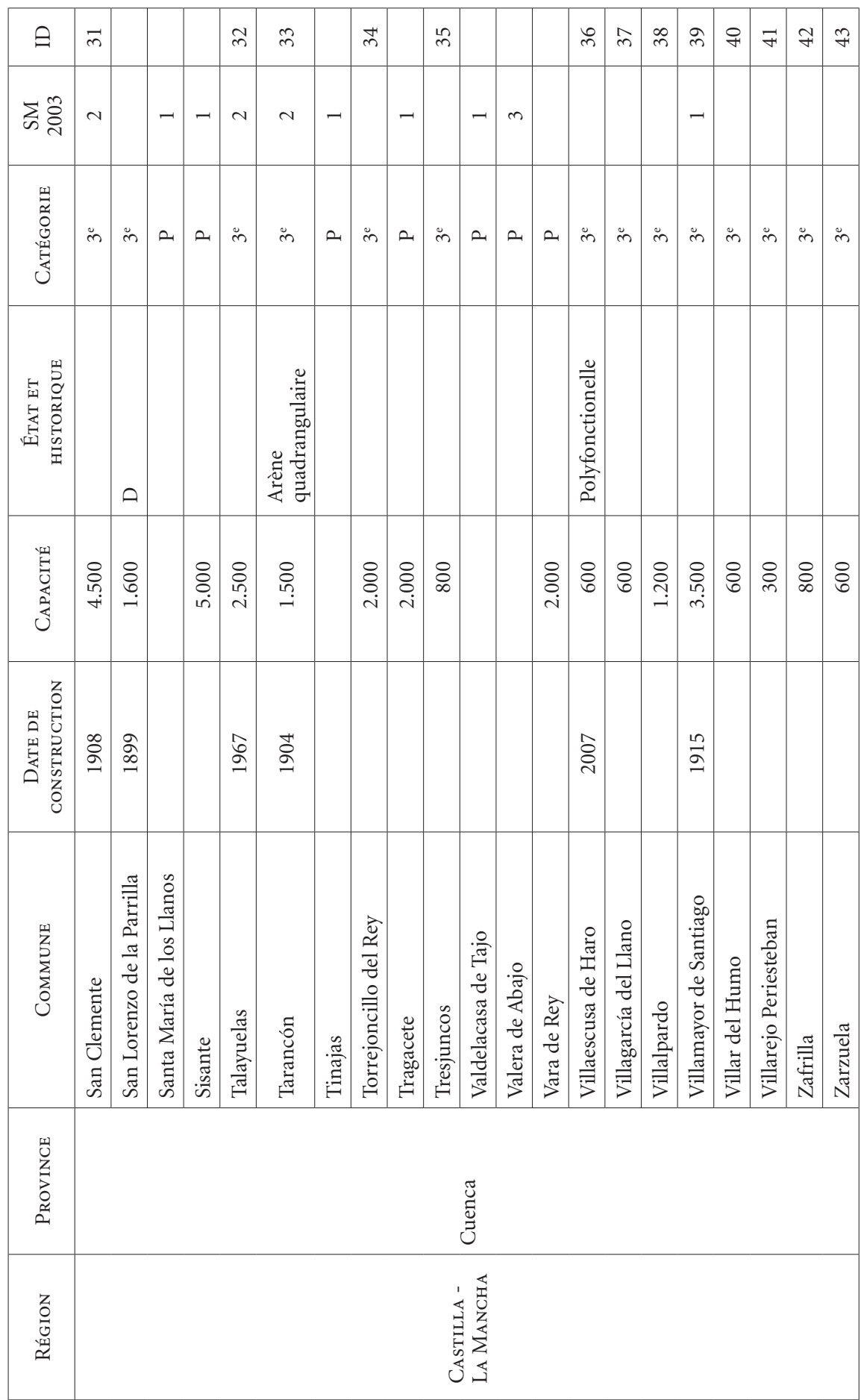




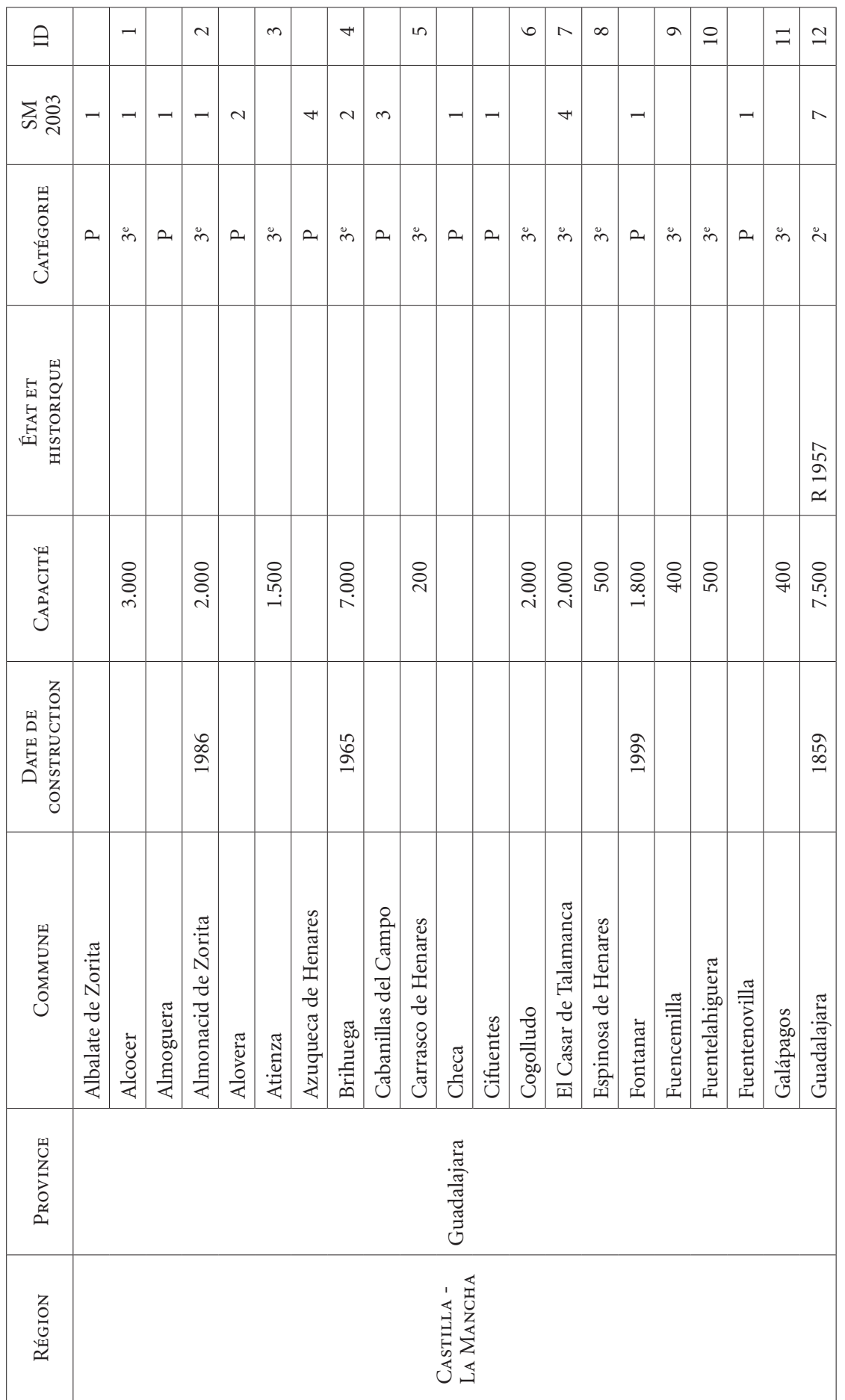




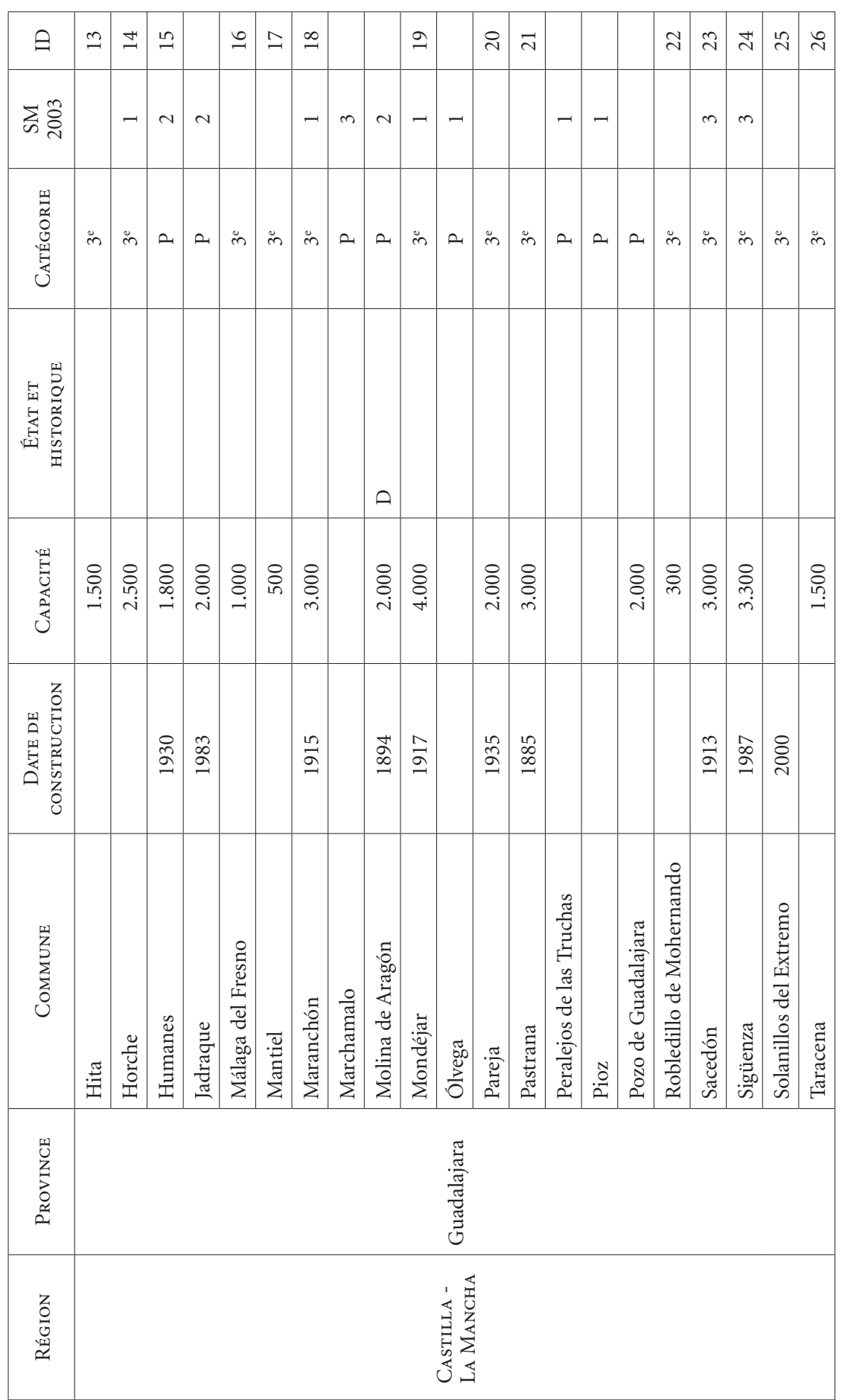




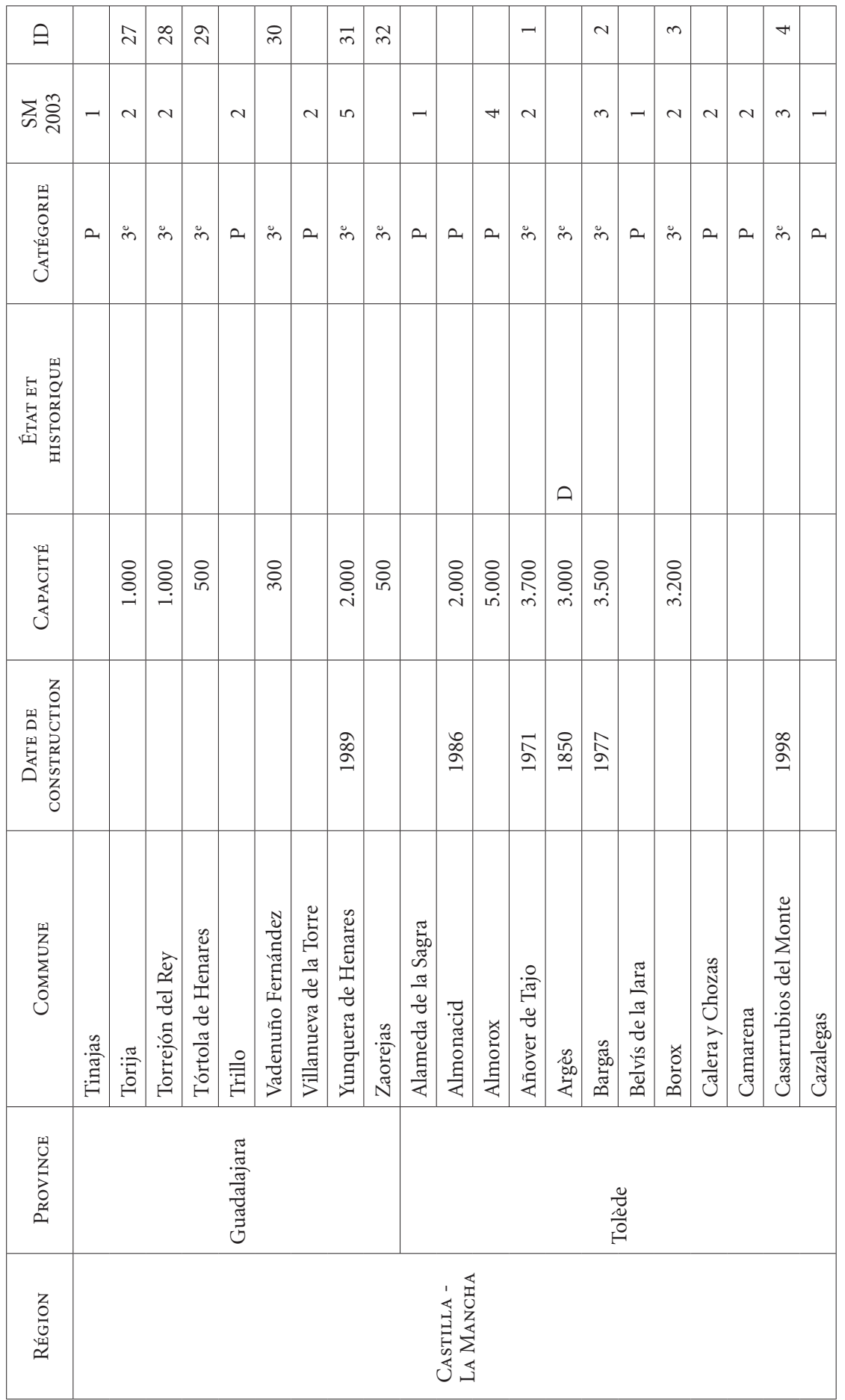




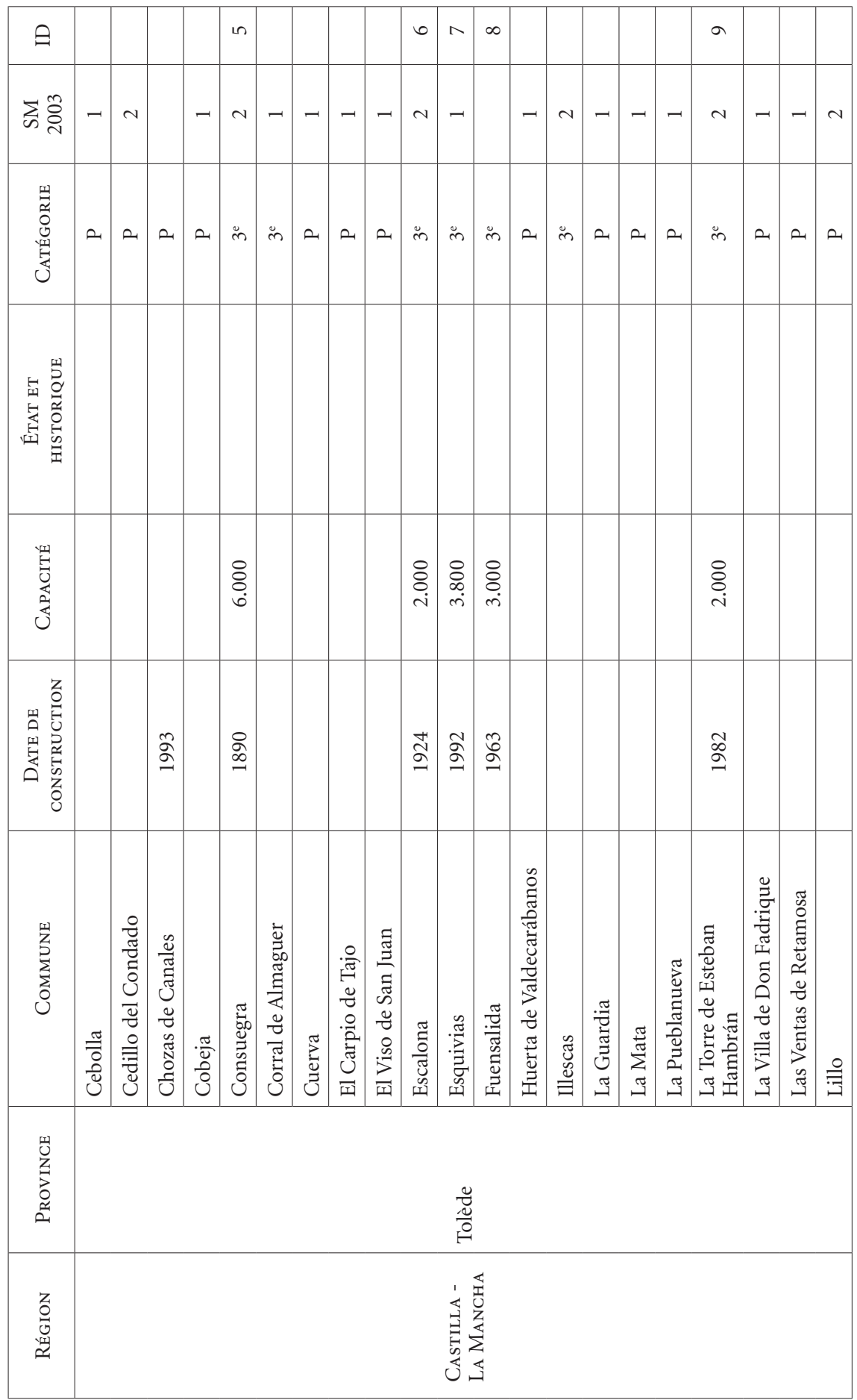




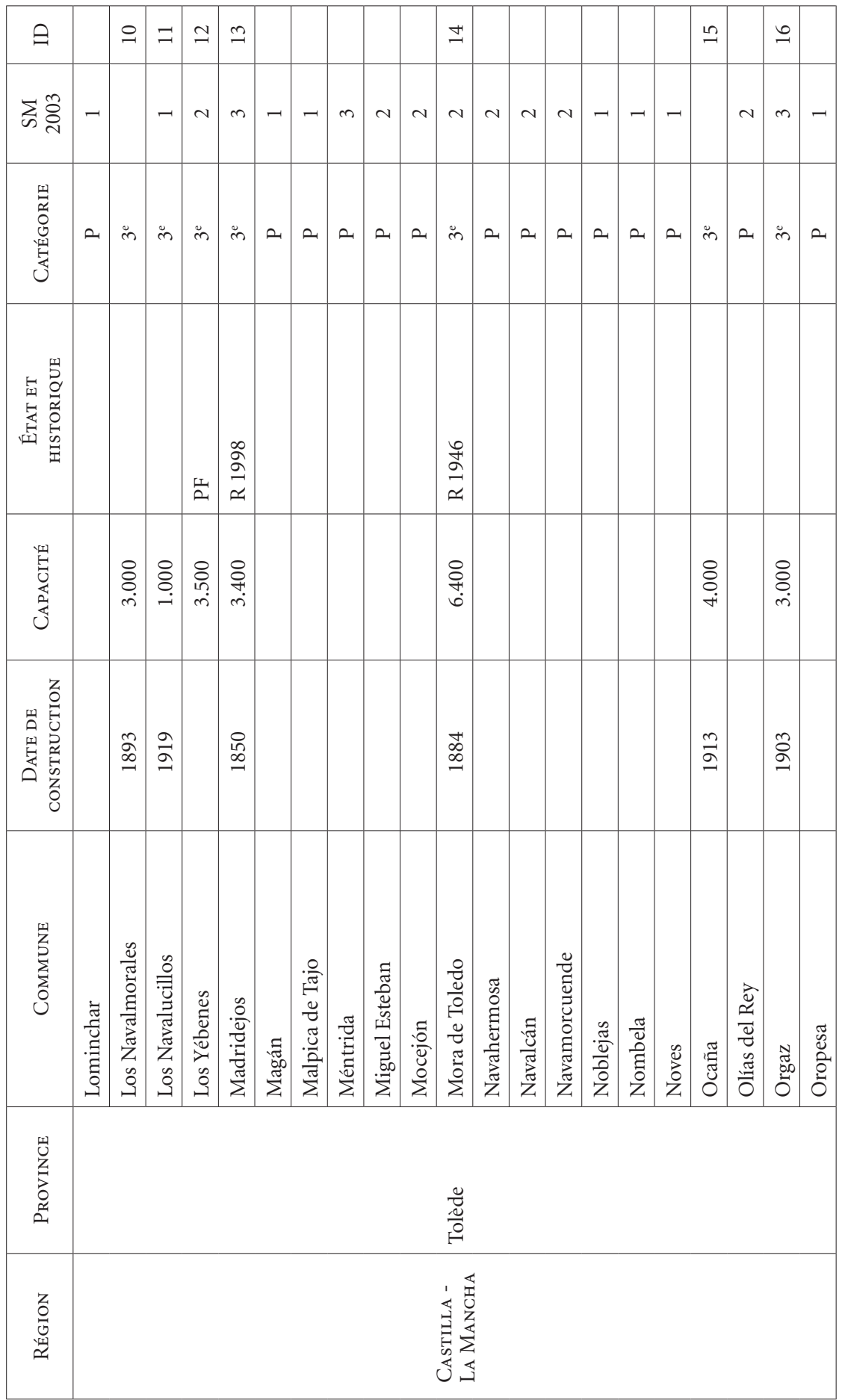




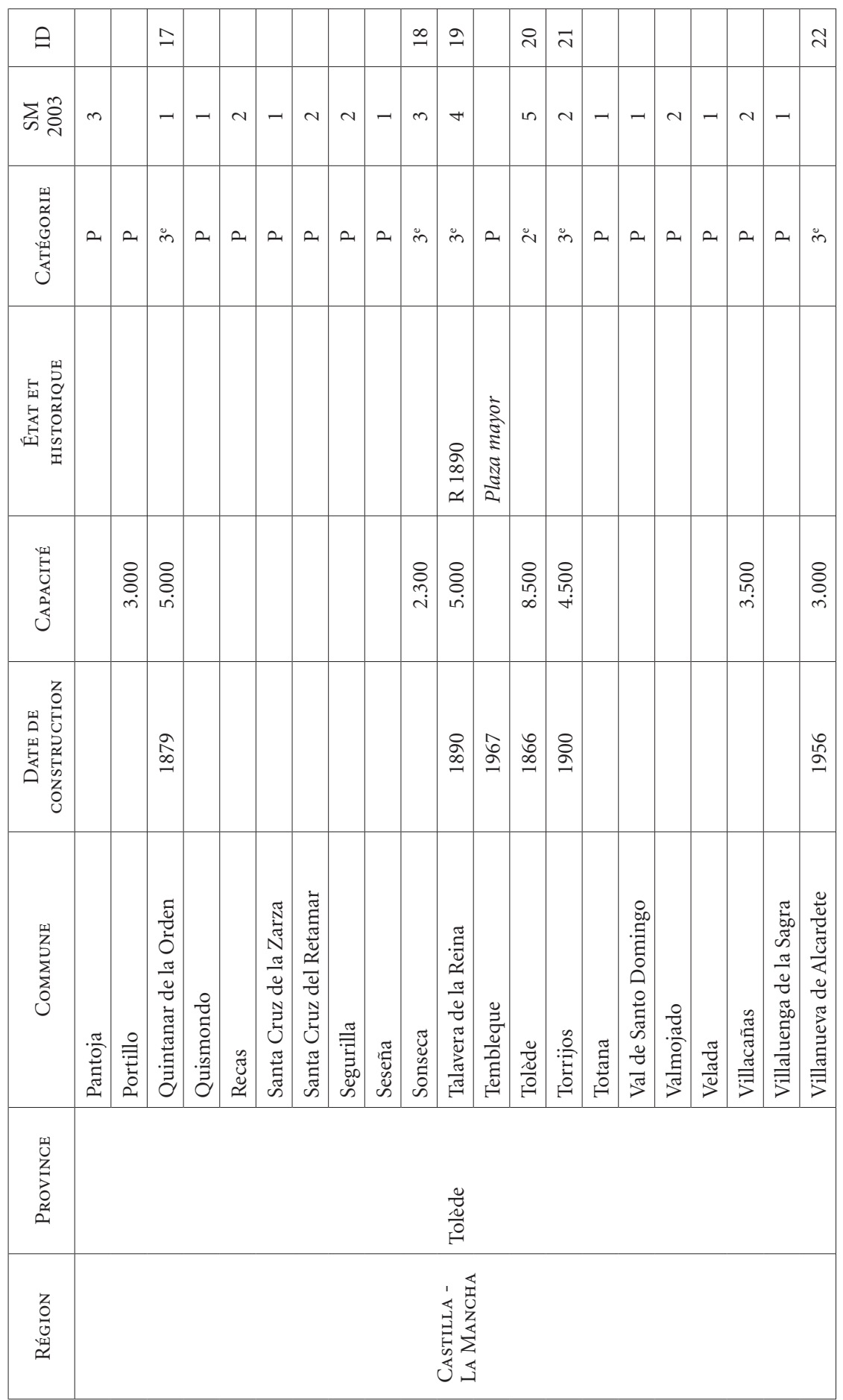




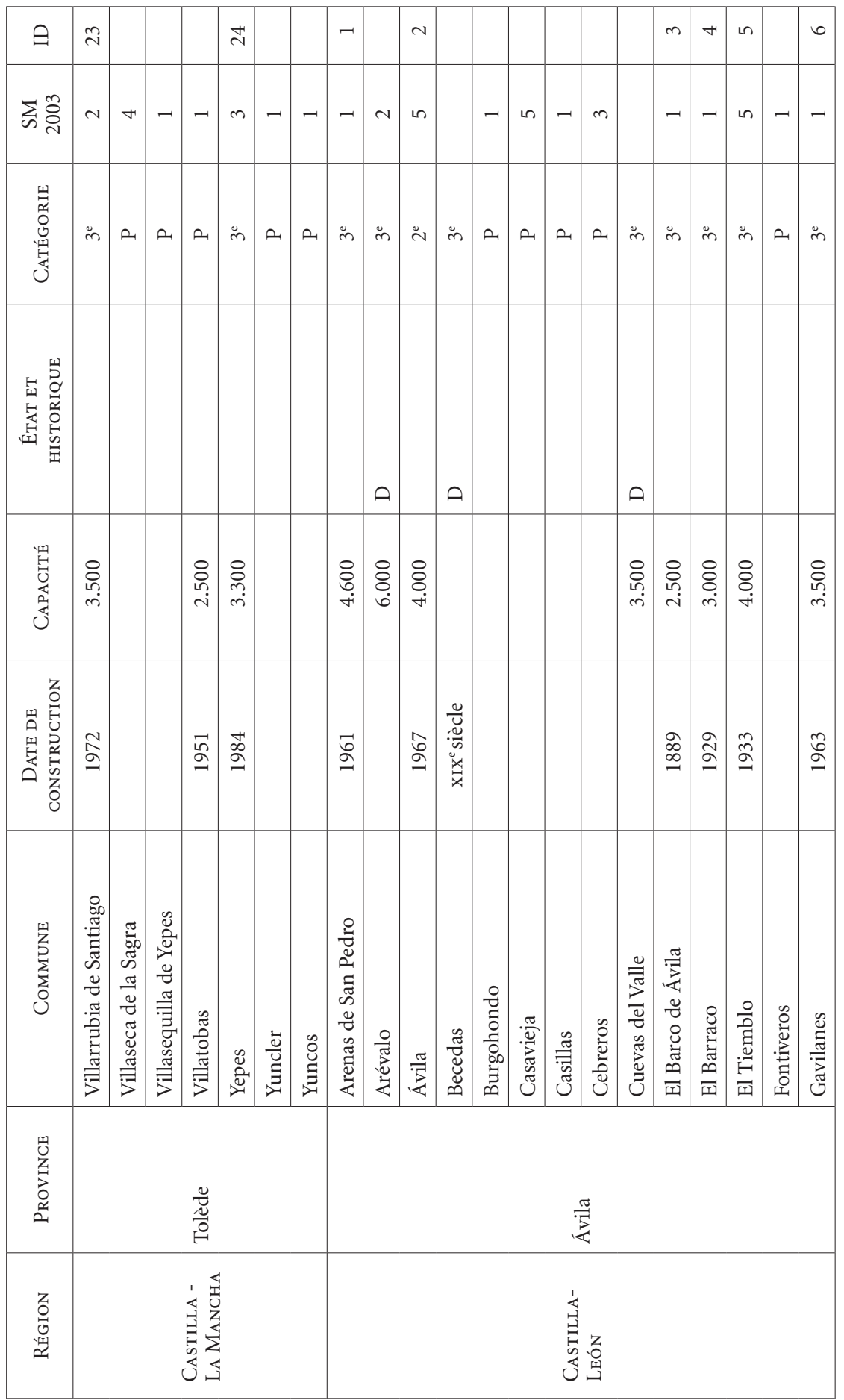




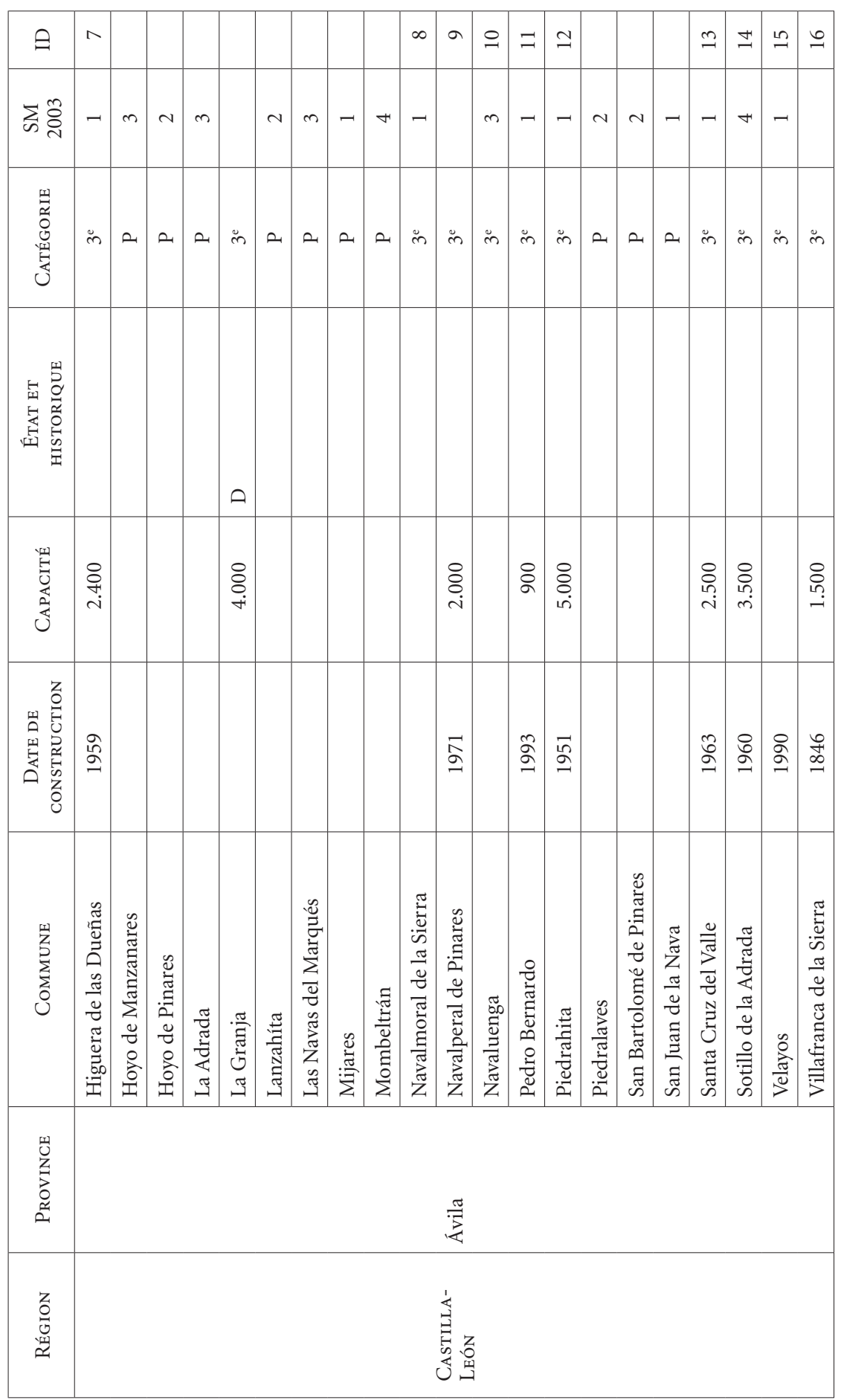




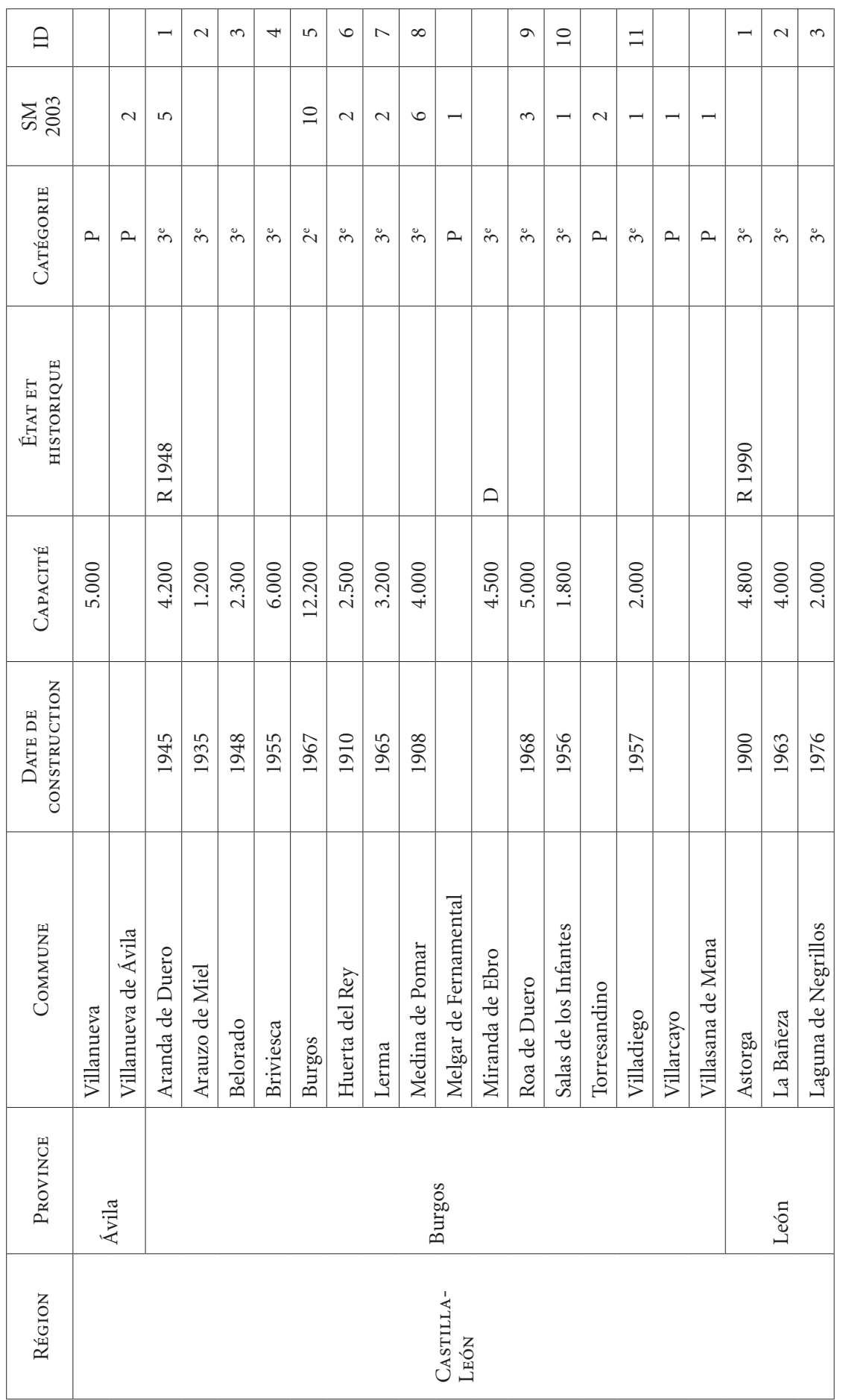




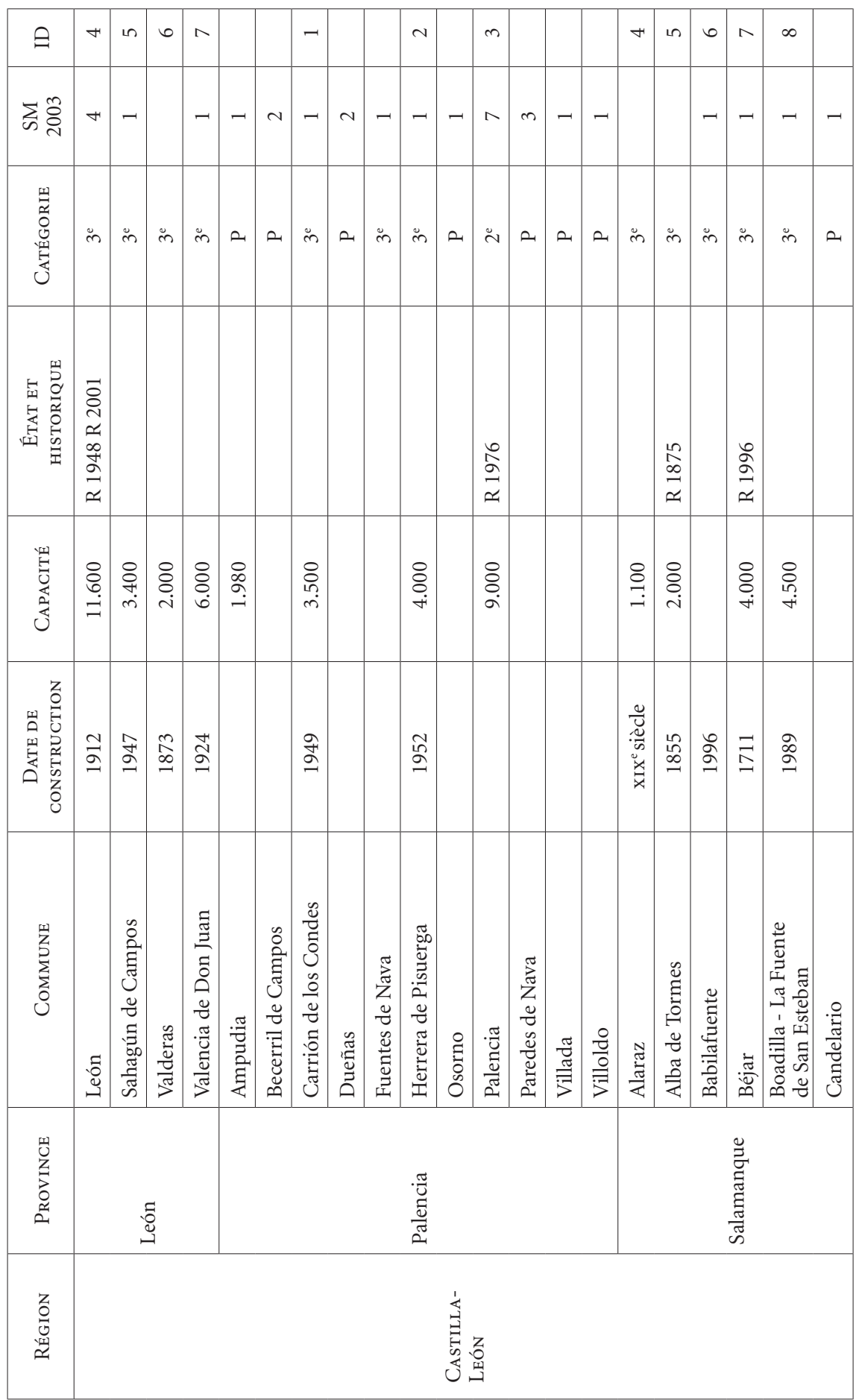




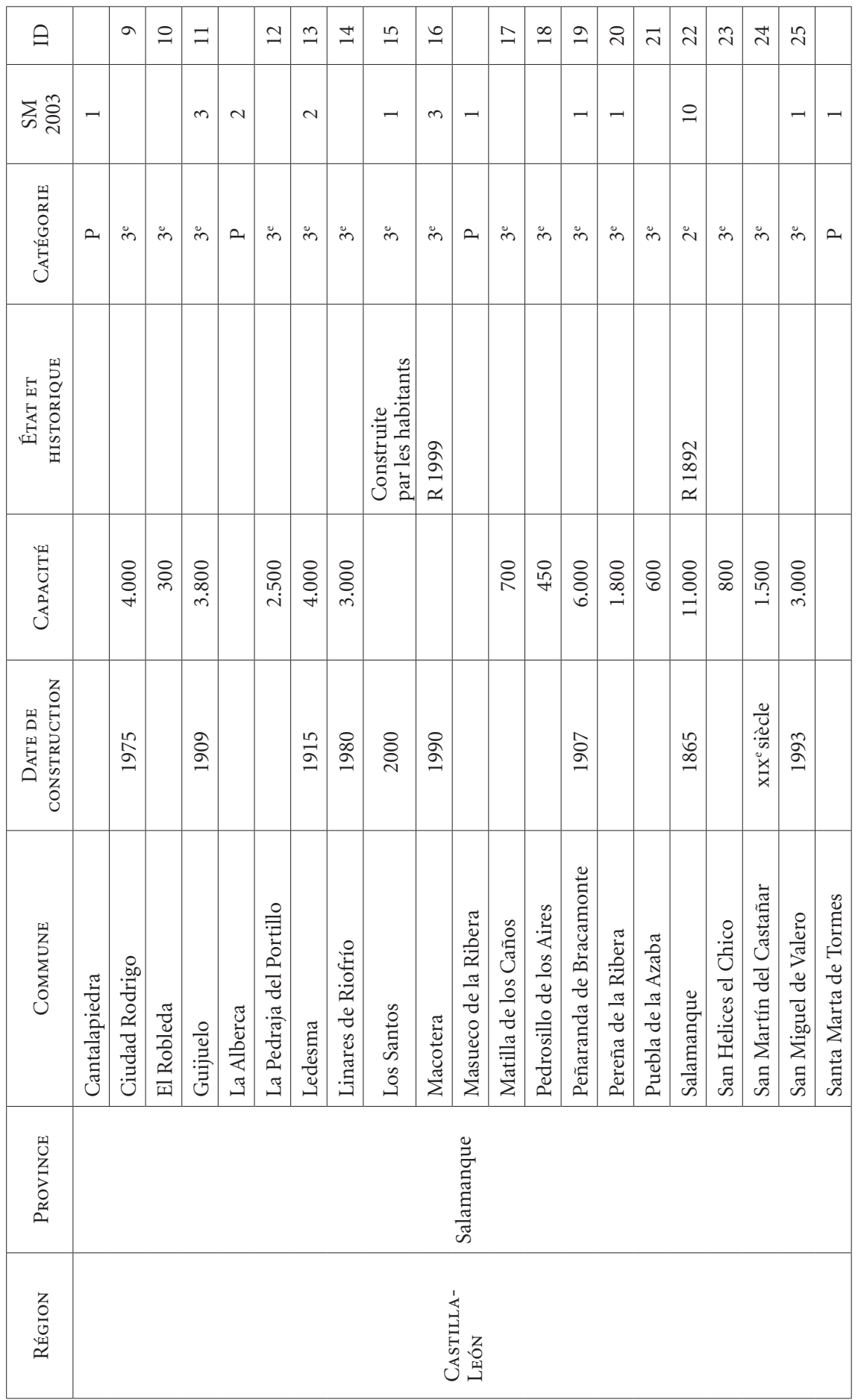




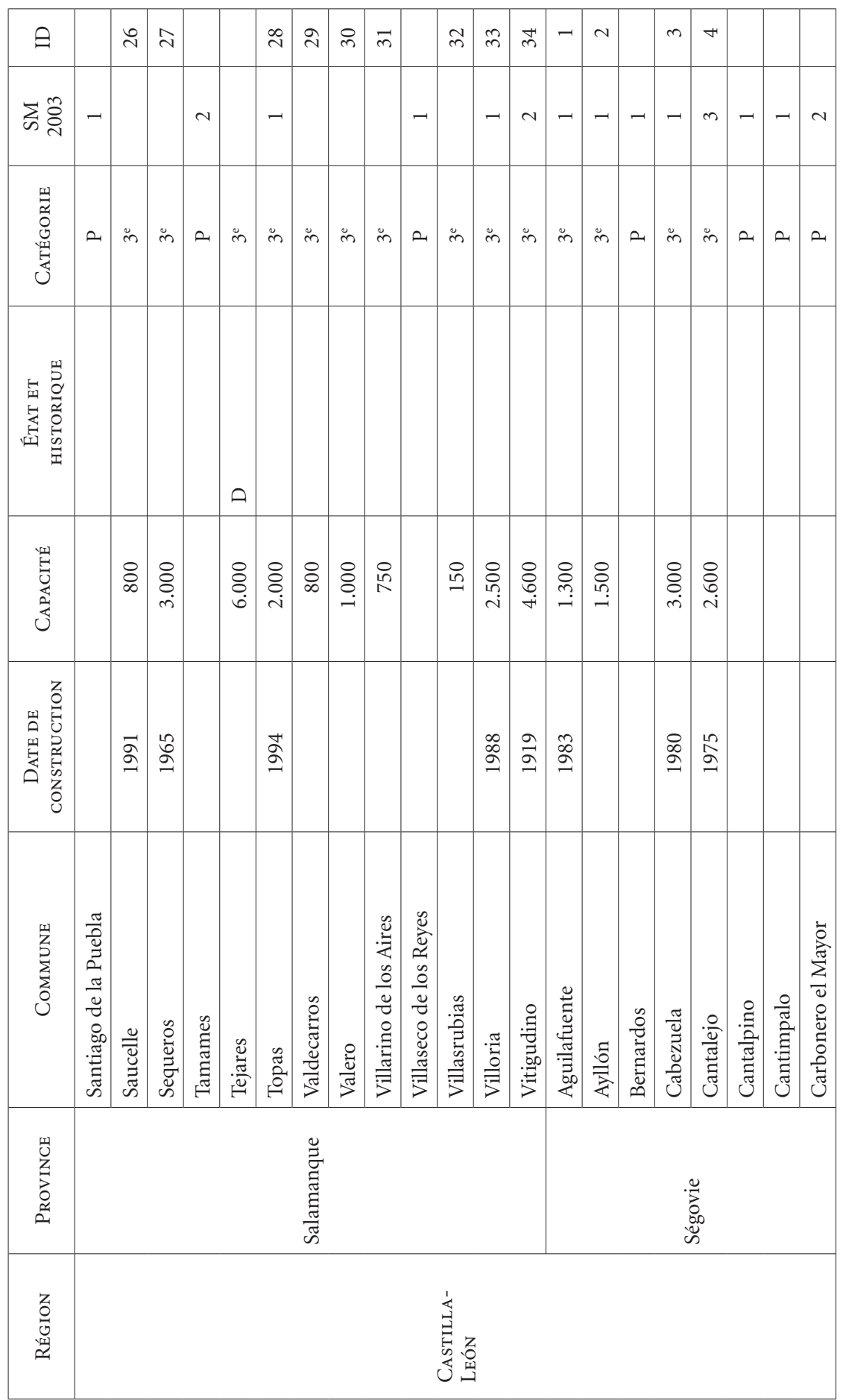




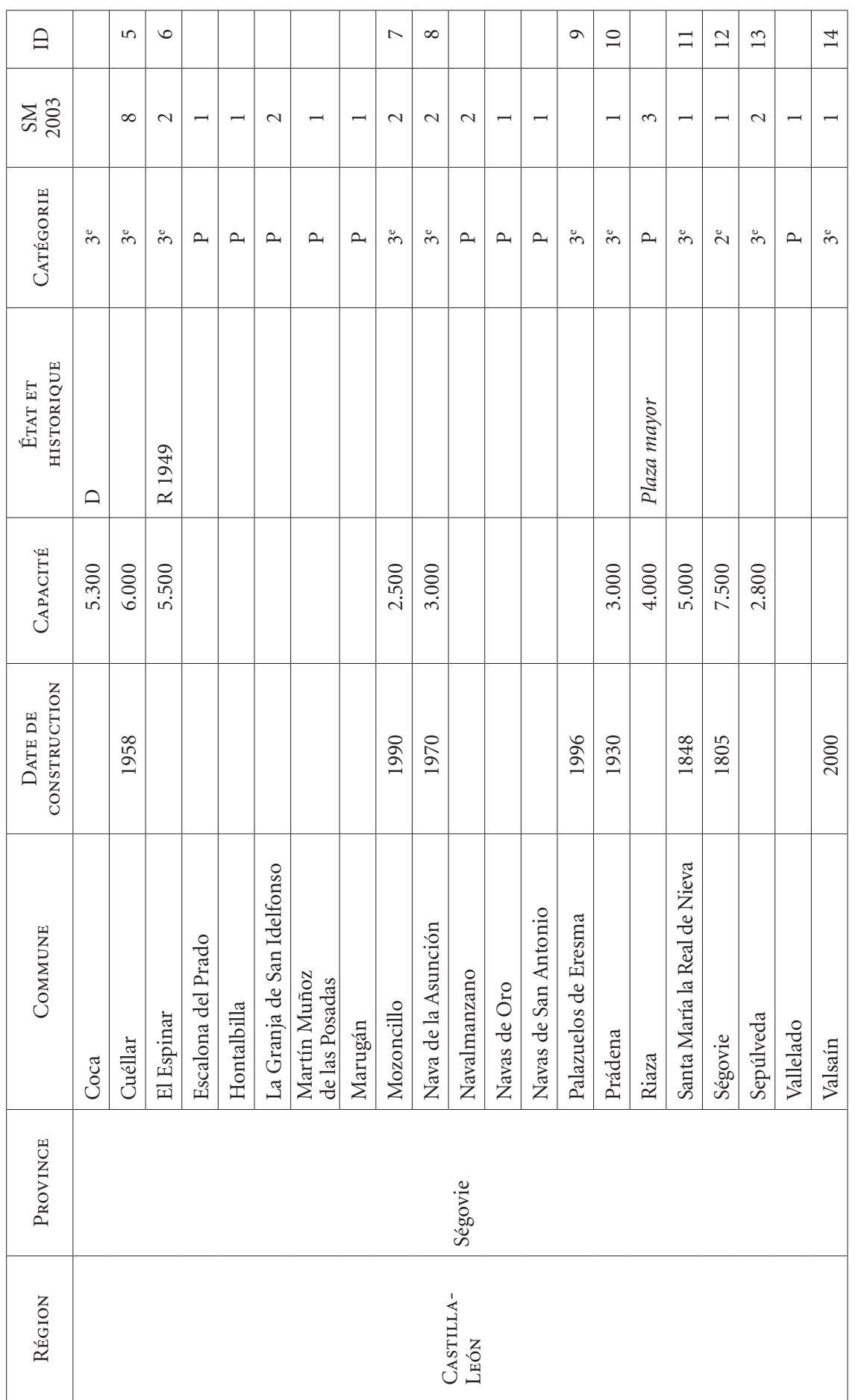




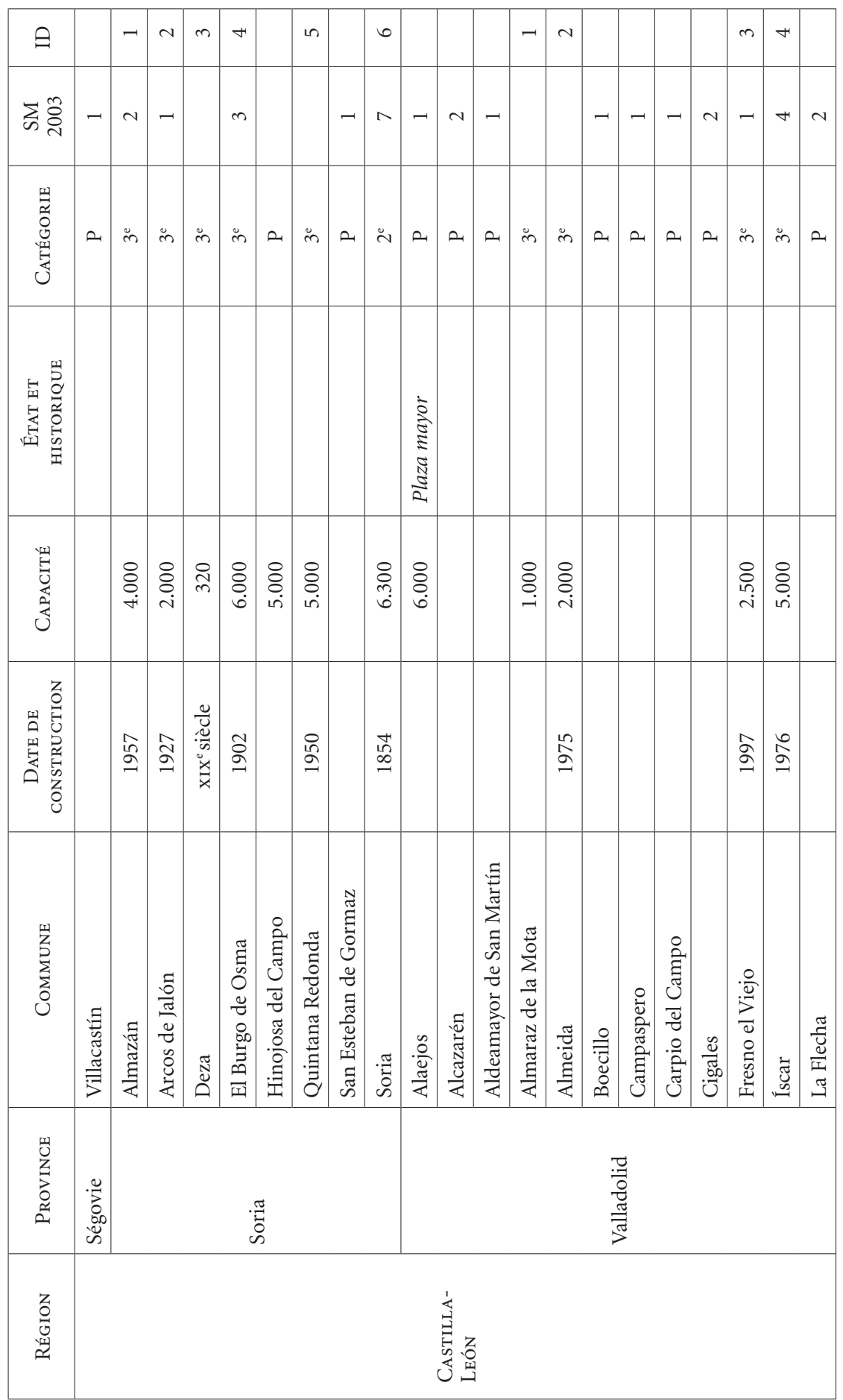




\begin{tabular}{|c|c|c|c|c|c|c|c|c|c|c|c|c|c|c|c|c|c|c|c|c|c|}
\hline$\theta$ & in & & 0 & $\wedge$ & & & & $\infty$ & $a$ & $\stackrel{ }{-}$ & & $=$ & & $\simeq$ & 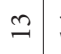 & $\Xi$ & & $\stackrel{10}{\longrightarrow}$ & ㄴ. & & $\simeq$ \\
\hline 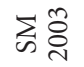 & in & $\sim$ & & & + & - & & - & $\sim$ & $m$ & -1 & - & -1 & $N$ & + & $n$ & - & $\simeq$ & & - & - \\
\hline 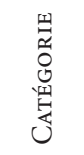 & $\dot{m}$ & $a$ & $\ddot{m}$ & $\dot{m}$ & $\ddot{m}$ & $\infty$ & $\ddot{m}$ & $\ddot{m}$ & $\ddot{n}$ & $\ddot{n}$ & $a_{1}$ & $\ddot{m}$ & $a_{1}$ & $\dot{m}$ & in & $\ddot{m}$ & $a_{1}$ & 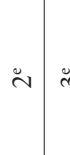 & $\ddot{m}$ & $\infty$ & $\ddot{m}$ \\
\hline 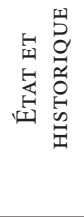 & & & & 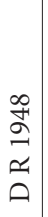 & & & D & & & & & & & & & & & $\begin{array}{l}\not \\
\infty \\
\vec{\sim} \\
\infty \\
\infty \\
\infty \\
\vec{\simeq} \\
\simeq\end{array}$ & & & \\
\hline 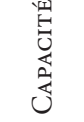 & $\begin{array}{l}8 \\
\vdots \\
0\end{array}$ & & 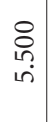 & $\begin{array}{l}8 \\
\infty \\
\sigma \\
\sigma\end{array}$ & & & 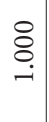 & & 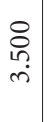 & $\begin{array}{l}\stackrel{8}{ } \\
\text { ri }\end{array}$ & & $\begin{array}{l}\stackrel{8}{0} \\
\text { i }\end{array}$ & & \begin{tabular}{l}
8 \\
\multirow{2}{n}{} \\
$i$
\end{tabular} & 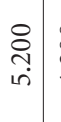 & 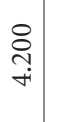 & & 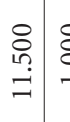 & ه̊ & & $\begin{array}{l}\stackrel{8}{\circ} \\
\text { in } \\
+\end{array}$ \\
\hline 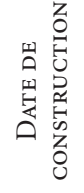 & & & $\begin{array}{l}\stackrel{a}{b} \\
\infty \\
0\end{array}$ & $\stackrel{\varrho}{\infty}$ & & & & $\stackrel{\infty}{2}$ & $\begin{array}{l}\text { ปे } \\
\curvearrowright\end{array}$ & گે & & $\begin{array}{l}\hat{\infty} \\
\stackrel{=}{二}\end{array}$ & & $\begin{array}{l}\curvearrowright \\
\curvearrowright\end{array}$ & 亏ิ & స్ & & $\begin{array}{l}\stackrel{+}{\infty} \\
\infty \\
\infty\end{array}$ & & & \\
\hline 崩 & 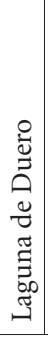 & 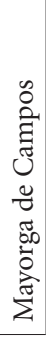 & 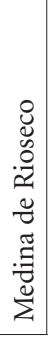 & 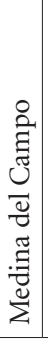 & $\begin{array}{l} \\
\frac{8}{0} \\
\frac{0}{0} \\
\frac{\pi}{2}\end{array}$ & 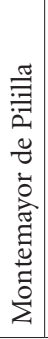 & 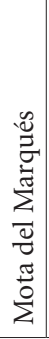 & 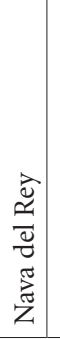 & 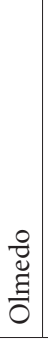 & 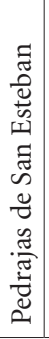 & 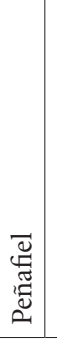 & $\stackrel{0}{\stackrel{0}{0}}$ & 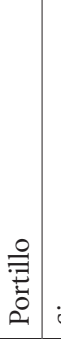 & 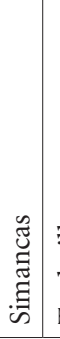 & 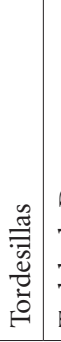 & 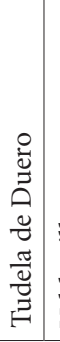 & 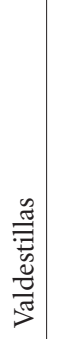 & 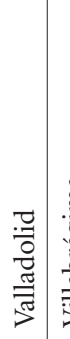 & 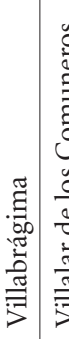 & 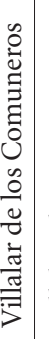 & 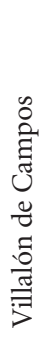 \\
\hline 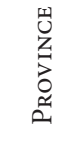 & & & & & & & & & & & 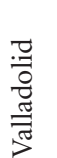 & & & & & & & & & & \\
\hline 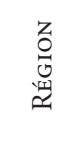 & & & & & & & & & & & 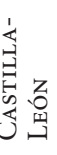 & & & & & & & & & & \\
\hline
\end{tabular}




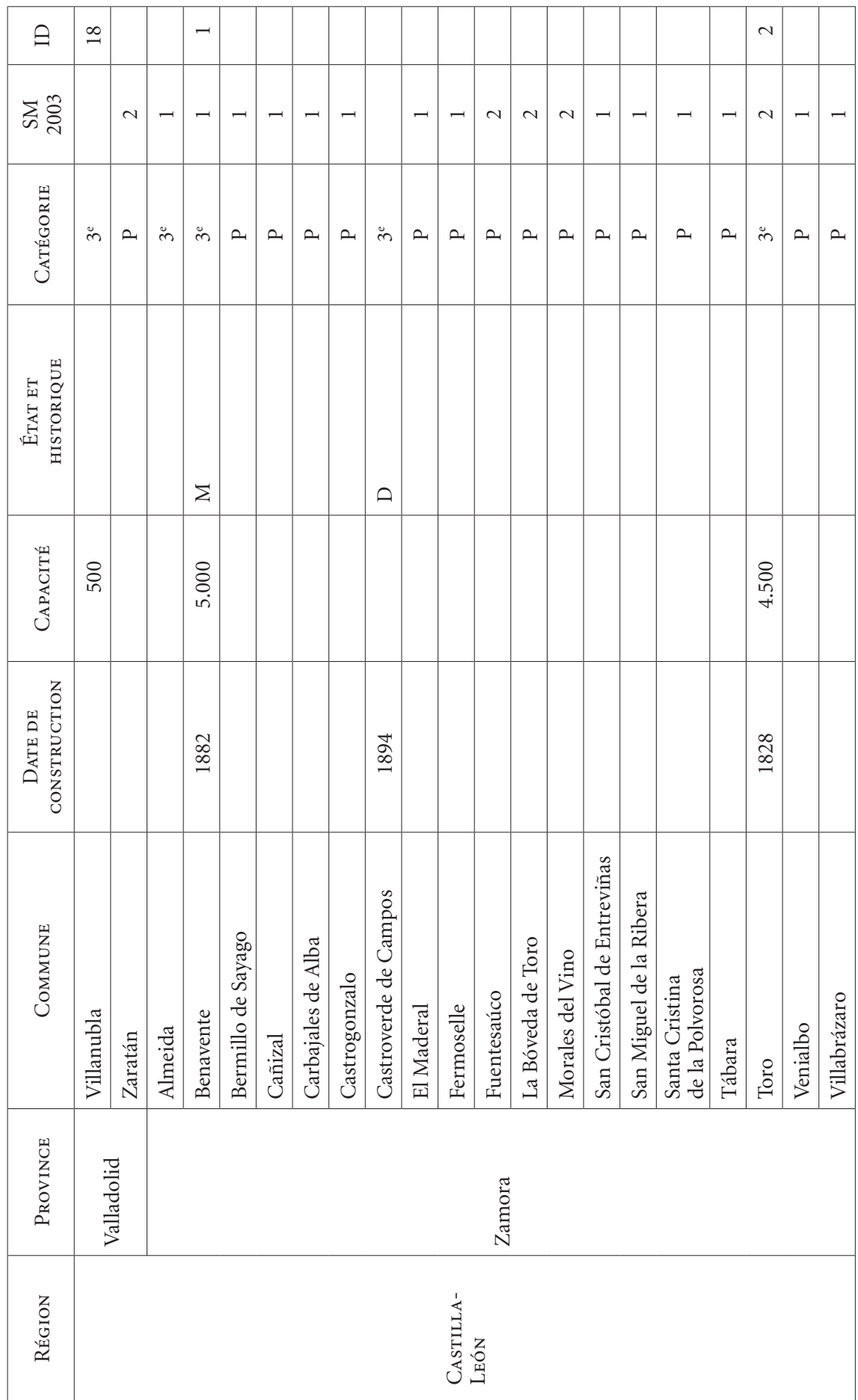




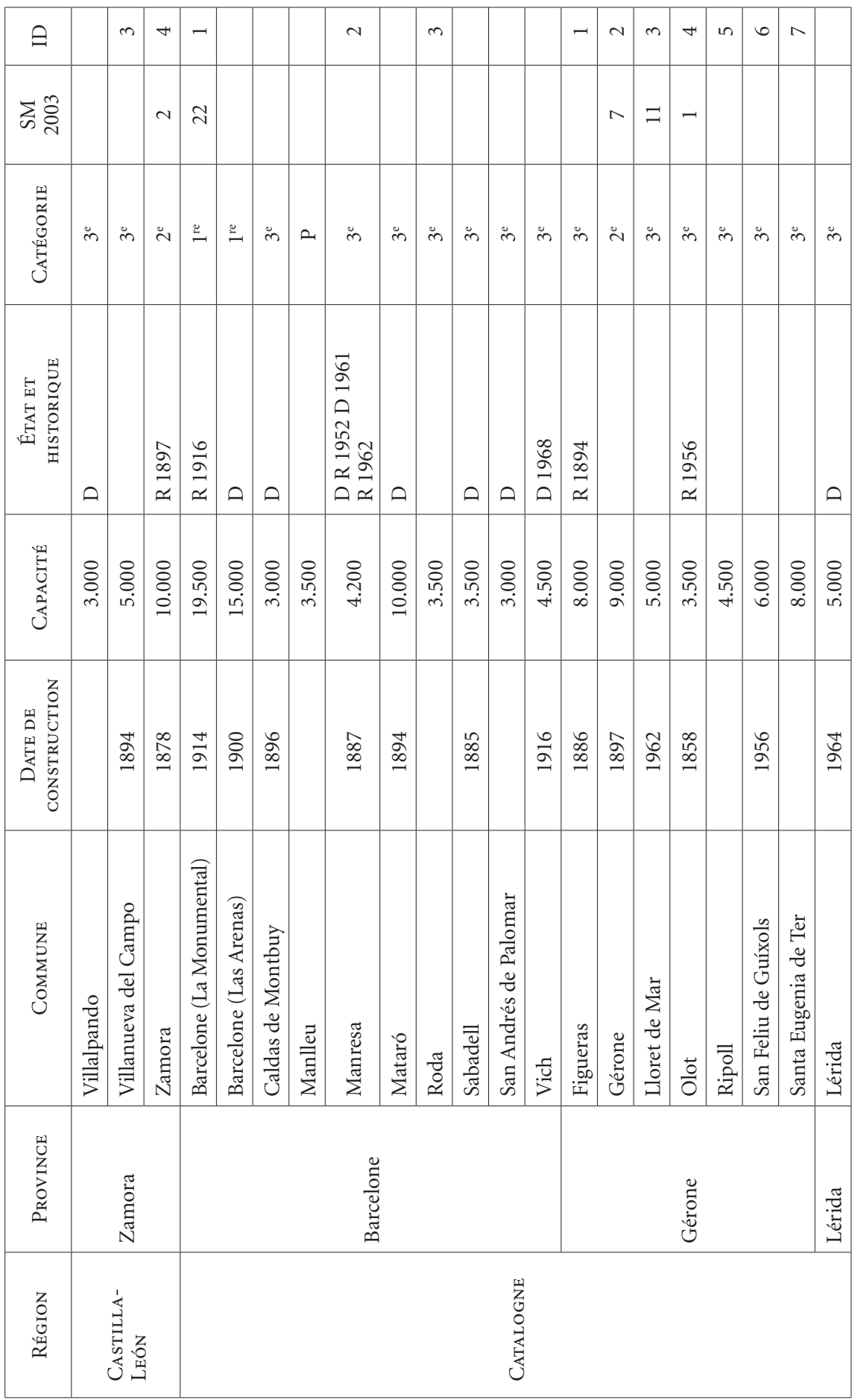




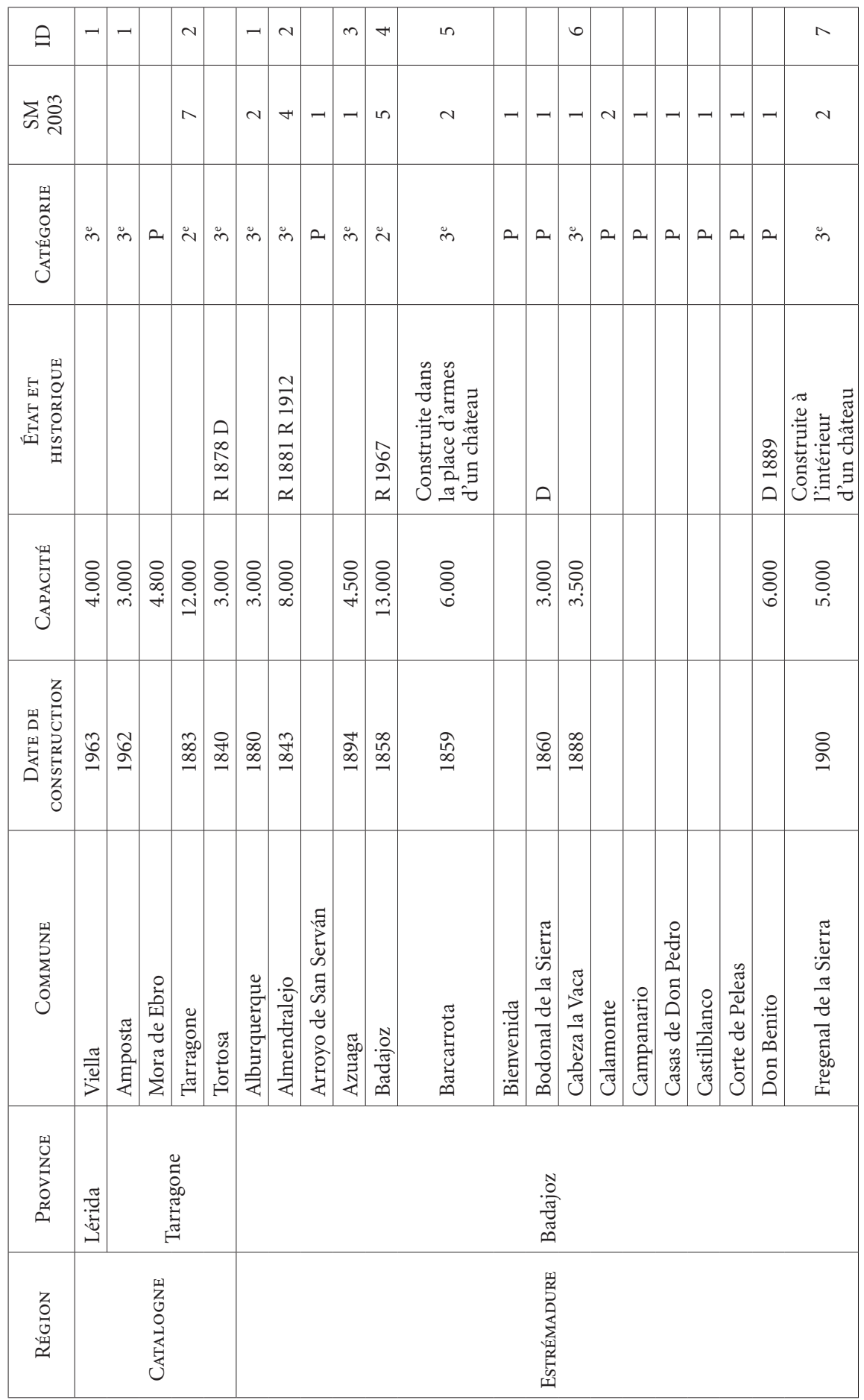




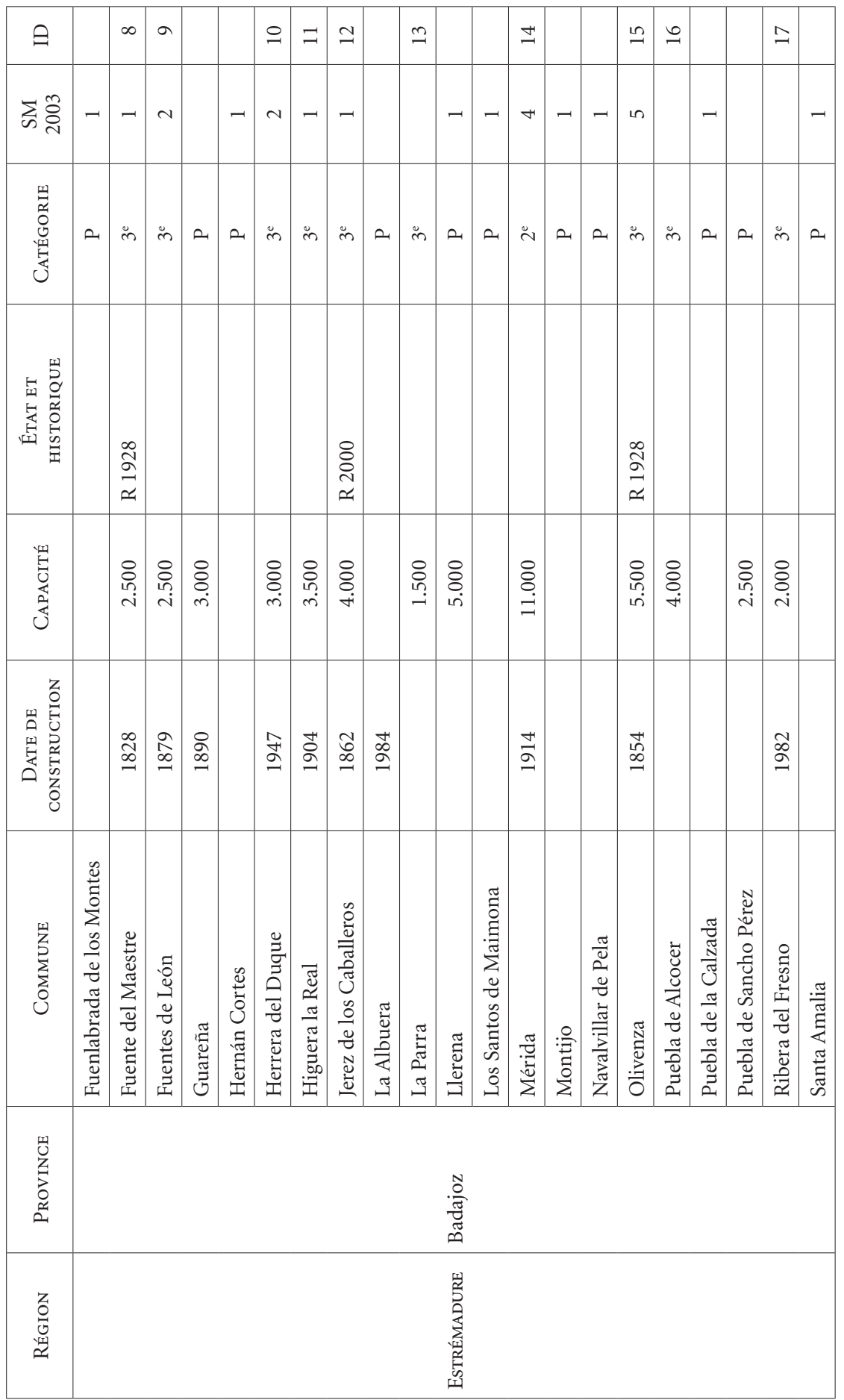


ANNEXES

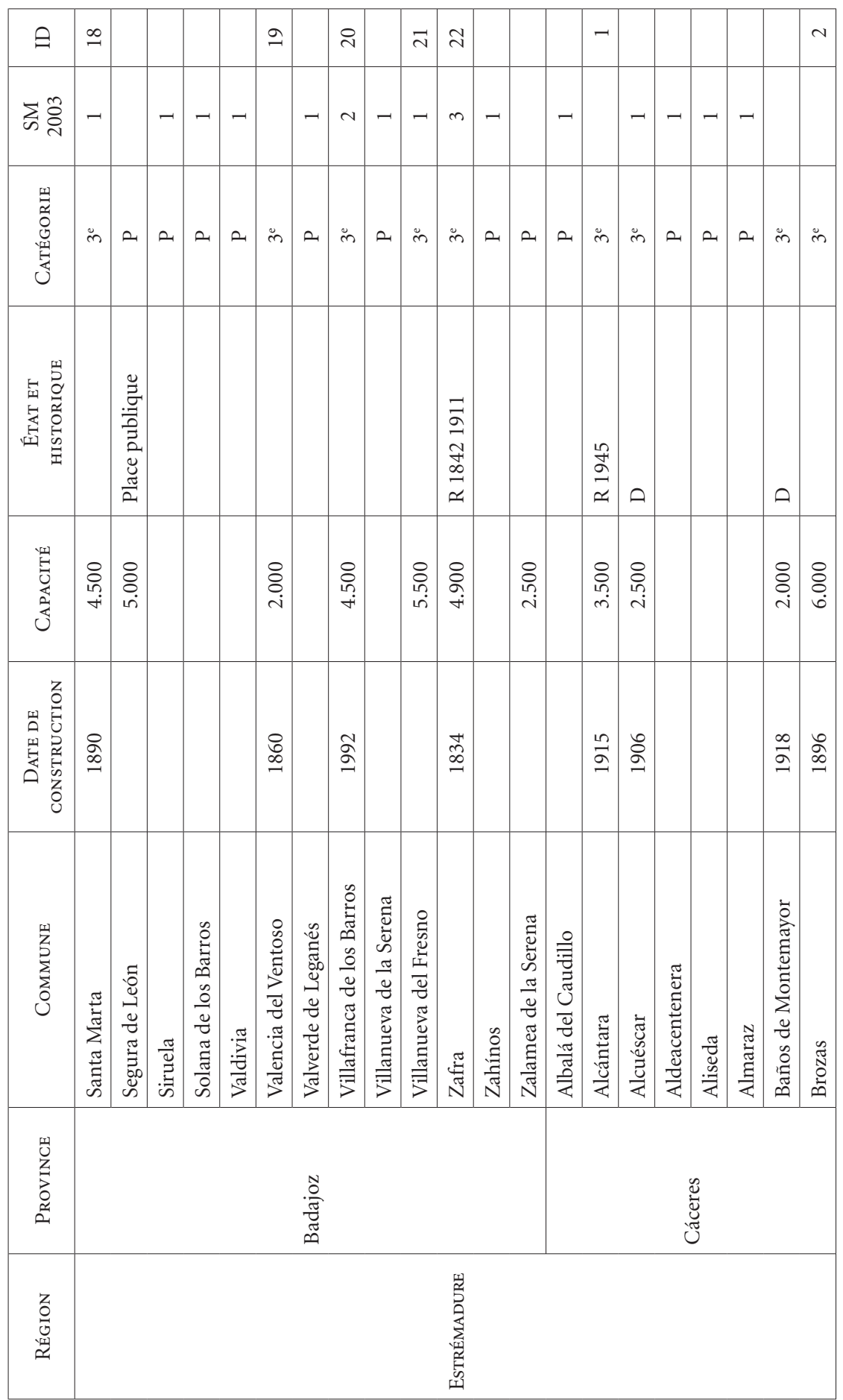




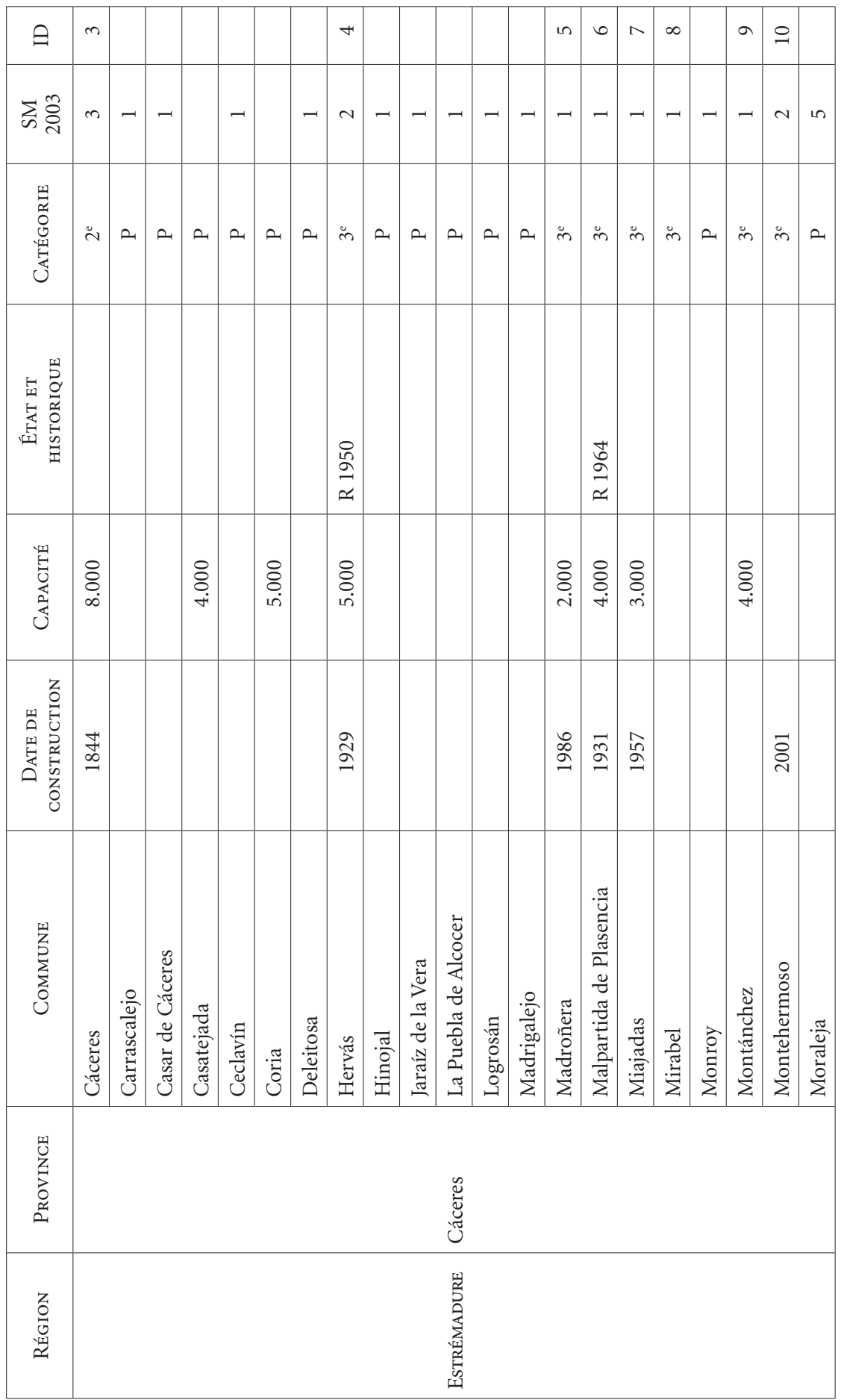




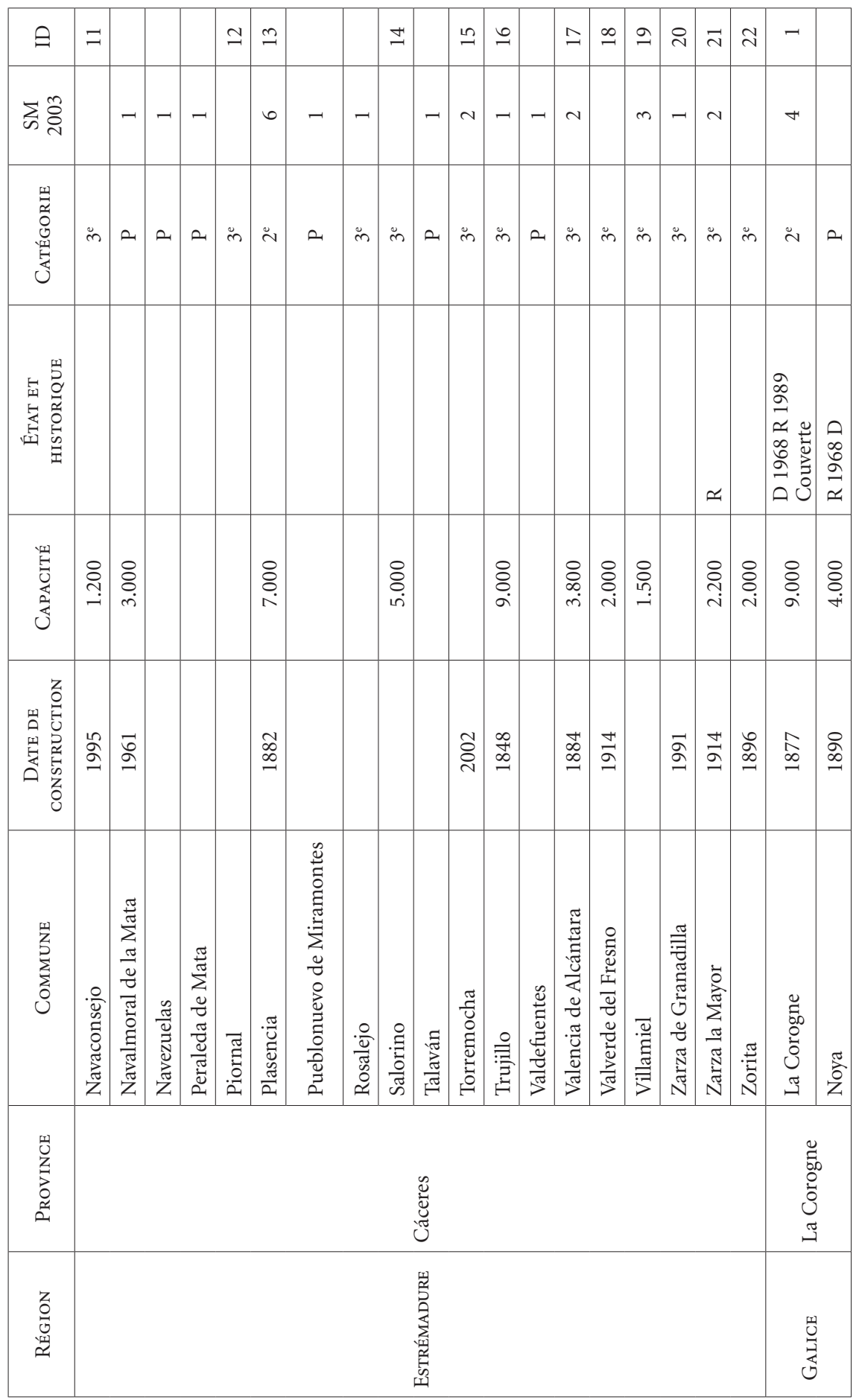




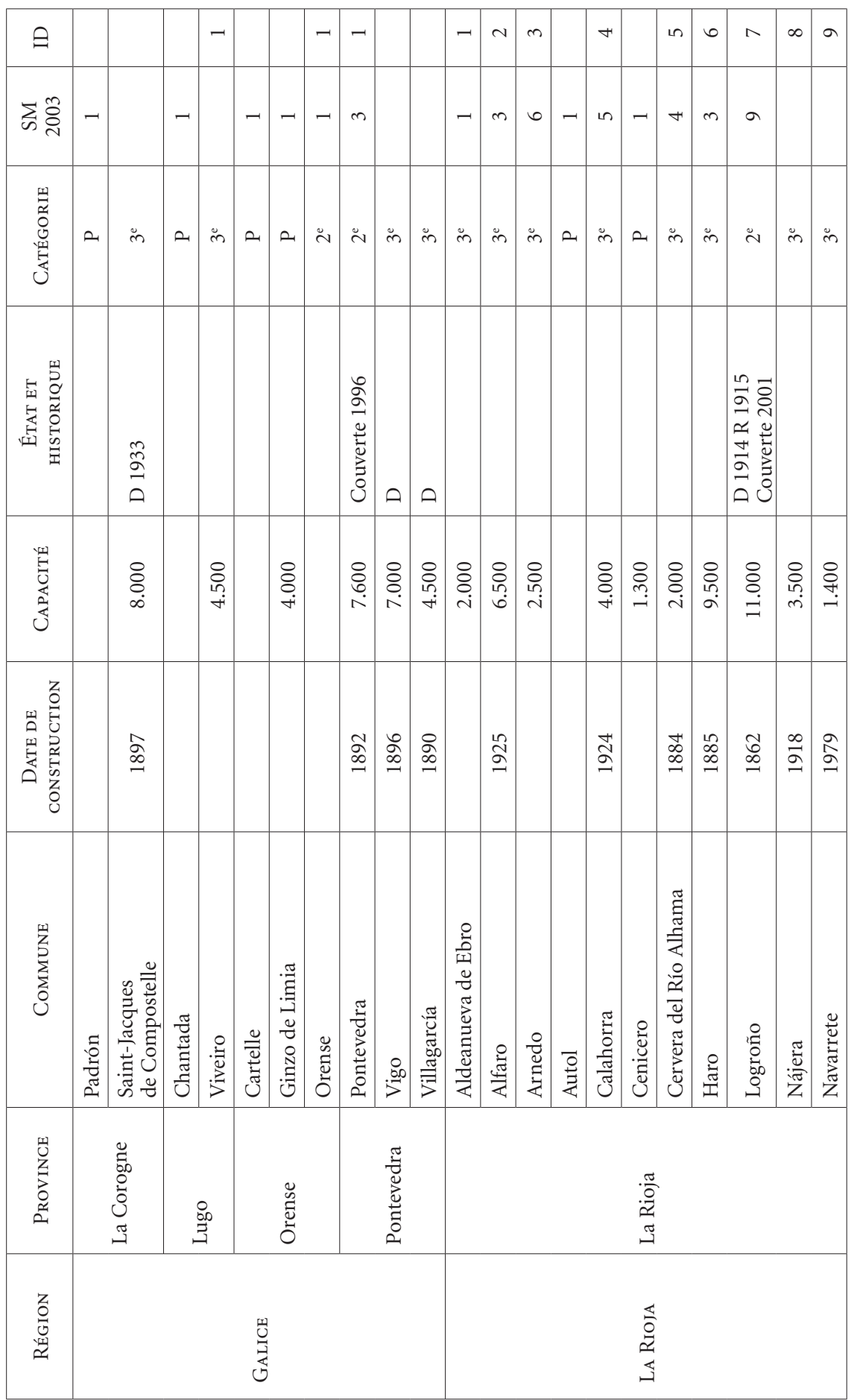




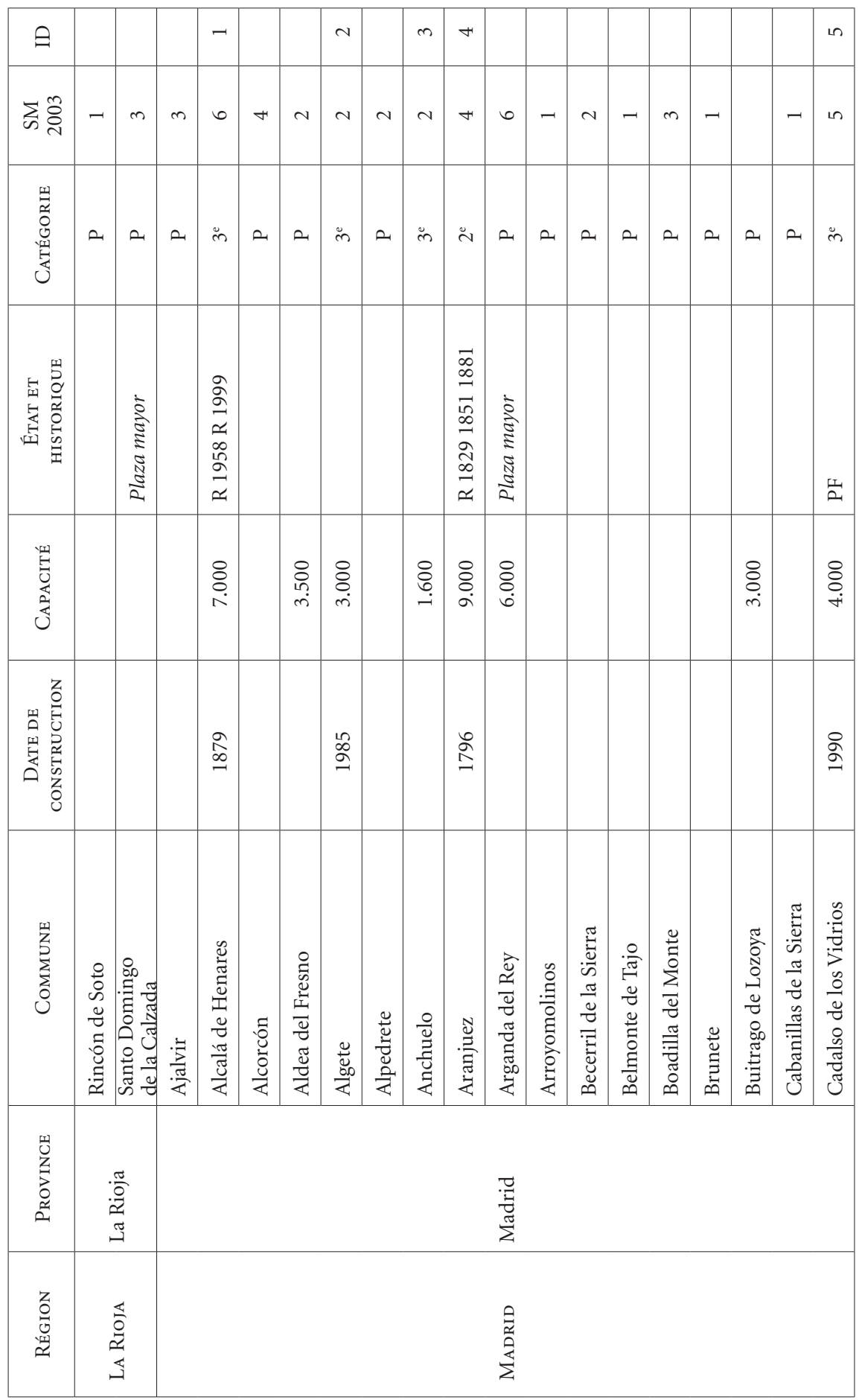




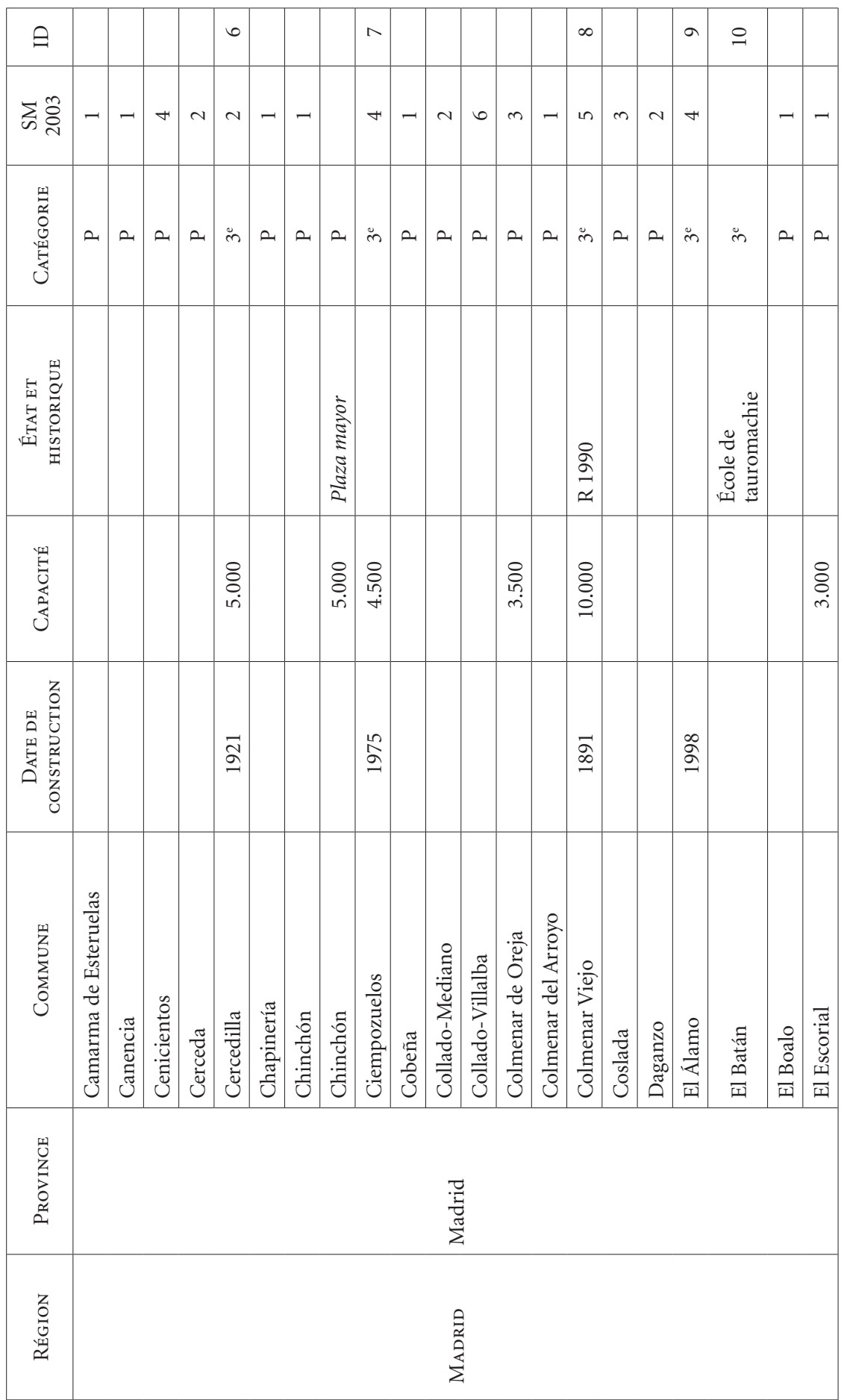


ANNEXES

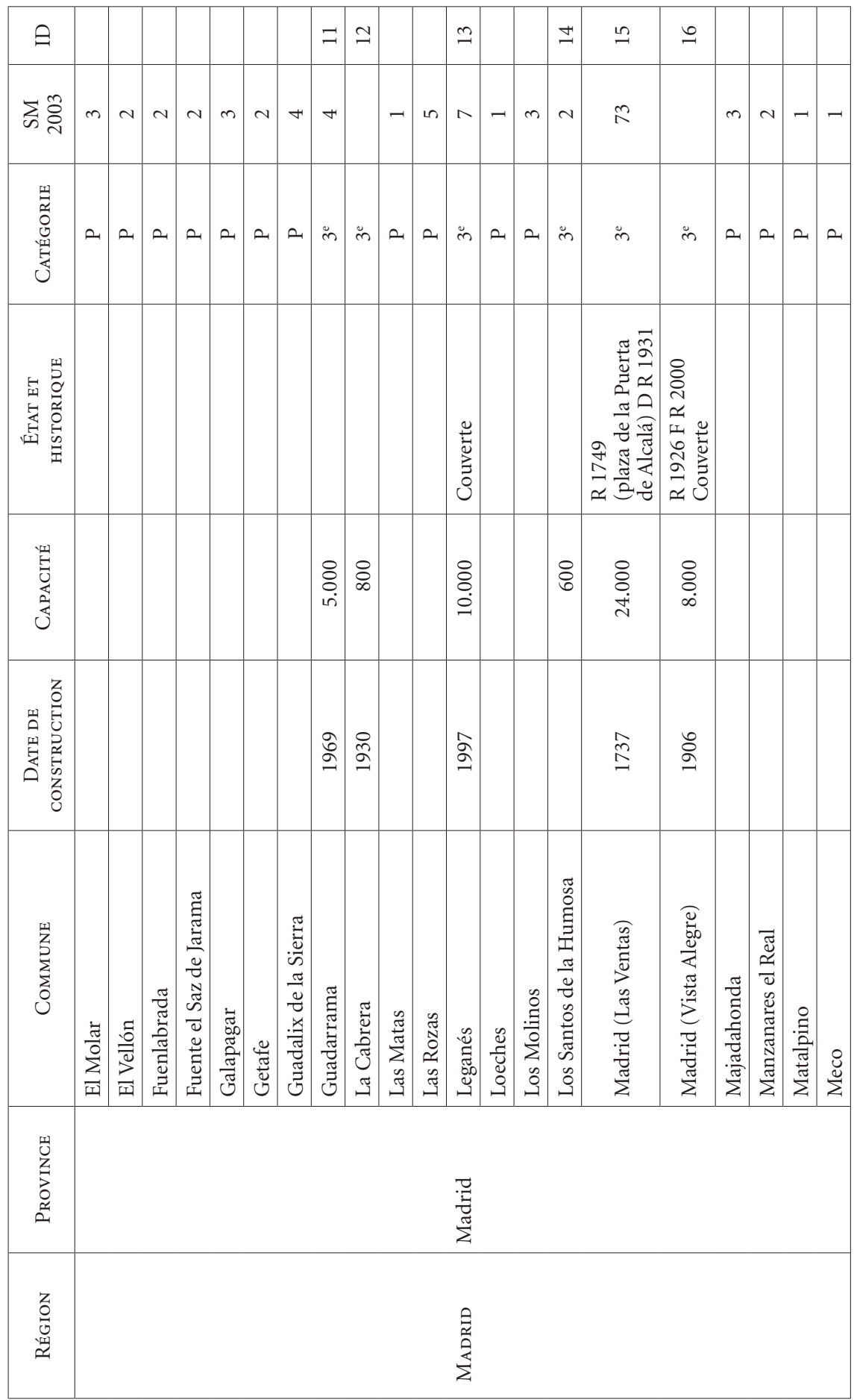




\begin{tabular}{|c|c|c|c|c|c|c|c|c|c|c|c|c|c|c|c|c|c|c|c|c|c|}
\hline$\theta$ & $\beth$ & $\infty$ & & & & $\curvearrowright$ & 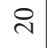 & & & & & & & $\vec{\sim}$ & & & & & & & \\
\hline$\sum_{s} \underset{0}{0}$ & $m$ & + & - & $\theta$ & $\sim$ & 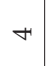 & - & 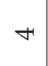 & $\neg$ & - & -1 & $m$ & - & & $\sim$ & $n$ & 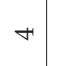 & -1 & - & $m$ & \\
\hline 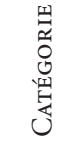 & $\dot{m}$ & $\ddot{n}$ & $\infty$ & $\infty$ & 2 & $\ddot{m}$ & $\ddot{m}$ & $\infty$ & $\infty$ & 2 & $a$ & $\ddot{m}$ & $a_{1}$ & in & $\infty$ & $a$ & $a_{1}$ & $n_{1}$ & ar & $\infty$ & $a_{1}$ \\
\hline 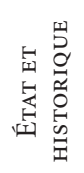 & & & & & & & & & & & & & & & & & & & & & \\
\hline 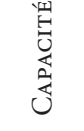 & 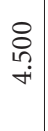 & $\begin{array}{l}8 \\
\vdots \\
\dot{r}\end{array}$ & & & & $\begin{array}{l}8 \\
\stackrel{0}{0} \\
+ \\
+\end{array}$ & $\begin{array}{l}8 \\
\text { in } \\
\text { nn }\end{array}$ & & & & & & & $\begin{array}{l}8 \\
\stackrel{1}{ } \\
i\end{array}$ & & & & & & & $\underset{\dot{m}}{\stackrel{一}{\circ}}$ \\
\hline 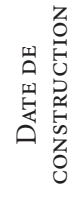 & $\begin{array}{l}\infty \\
\stackrel{2}{\sigma}\end{array}$ & $\stackrel{\partial े}{\circ}$ & & & $\begin{array}{l}\text { むั } \\
\varrho\end{array}$ & $\stackrel{n}{\alpha}$ & 옥 & & & & & & & 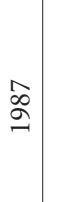 & & & & & & & \\
\hline 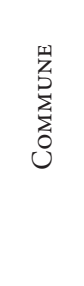 & 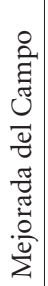 & 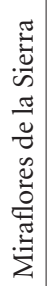 & 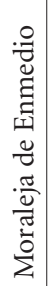 & 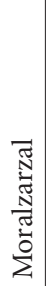 & 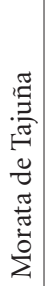 & $\begin{array}{l}\frac{0}{0} \\
\frac{0}{0} \\
\frac{0}{0} \\
\stackrel{0}{\Sigma}\end{array}$ & 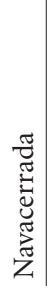 & 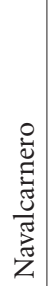 & 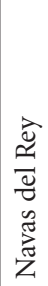 & 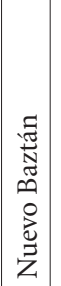 & 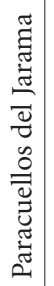 & $\frac{\pi}{\square}$ & 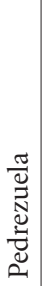 & 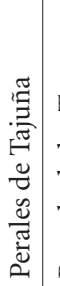 & 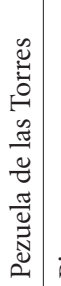 & . & 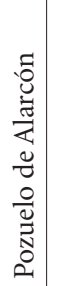 & & 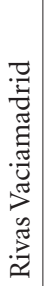 & 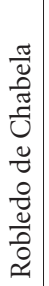 & 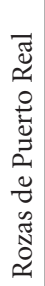 \\
\hline 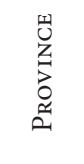 & & & & & & & & & & & $\begin{array}{l}\vec{Z} \cdot \vec{Z} \\
\stackrel{\vec{Z}}{Z}\end{array}$ & & & & & & & & & & \\
\hline 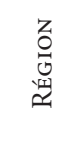 & & & & & & & & & & & 党 & & & & & & & & & & \\
\hline
\end{tabular}




\begin{tabular}{|c|c|c|c|c|c|c|c|c|c|c|c|c|c|c|c|c|c|c|c|c|}
\hline$\theta$ & $\widetilde{N}$ & & $\ddot{\sim}$ & & $\stackrel{\sim}{\sim}$ & $\stackrel{\llcorner 2}{\sim}$ & $\stackrel{\sim}{\sim}$ & & & & $\widehat{\sim}$ & & & & $\stackrel{\infty}{\sim}$ & & શે & 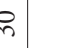 & $\vec{m}$ & \\
\hline 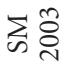 & $N$ & $m$ & $\sim$ & $N$ & + & $\infty$ & & - & $m$ & $m$ & + & - & $N$ & $n$ & - & -1 & in & \begin{tabular}{l|l}
$*$ \\
S
\end{tabular} & $n$ & \\
\hline 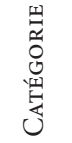 & in & $\infty$ & in & $a$ & $\dot{m}$ & $\ddot{m}$ & $\ddot{n}$ & $\infty$ & $a$ & $a$ & in & $a$ & 2 & $a$ & $\ddot{n}$ & a & m & $n=0$ & $\ddot{m}$ & a \\
\hline 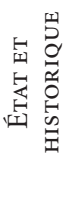 & & & & & $\begin{array}{l}\vec{\sigma} \\
\approx \\
\widetilde{a} \\
\check{\sigma} \\
\widetilde{\simeq}\end{array}$ & & & & & & & & & & & & 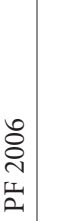 & & & \\
\hline 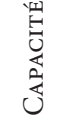 & ¿̊̊. & & 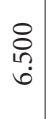 & & $\begin{array}{c}8 \\
\vdots \\
\text { in }\end{array}$ & $\begin{array}{c}8 \\
i \\
i n \\
\end{array}$ & \& & & 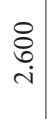 & & $\begin{array}{c}8 \\
0 \\
i n \\
\text { in }\end{array}$ & & & & & & 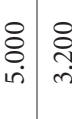 & 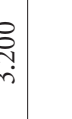 & $\begin{array}{l}8 \\
8 \\
i n n \\
+ \\
\end{array}$ & \&े \\
\hline 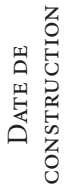 & बे & & $\stackrel{\tilde{n}}{\Omega}$ & & $\begin{array}{l}\vec{\infty} \\
\infty \\
\stackrel{-}{*}\end{array}$ & $\overrightarrow{0}$ & & & & & $\stackrel{2}{\sigma}$ & & & & হ & & ร & S. & ลे & \\
\hline \begin{tabular}{l} 
M \\
\multicolumn{2}{|c}{} \\
$\sum_{2}$ \\
0 \\
0
\end{tabular} & 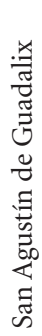 & 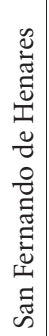 & 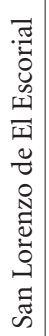 & 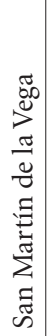 & 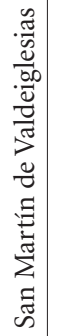 & 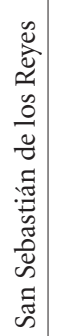 & 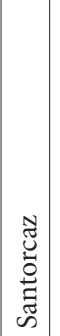 & 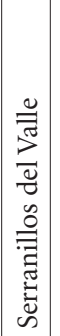 & 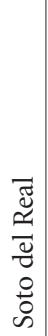 & 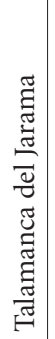 & 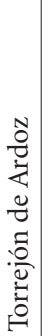 & 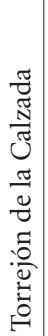 & 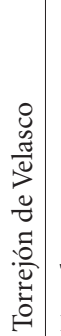 & 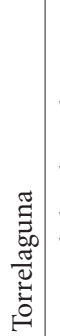 & 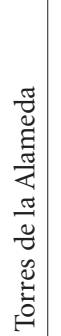 & 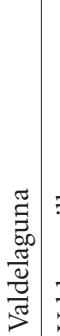 & 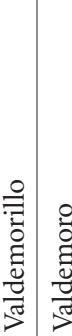 & 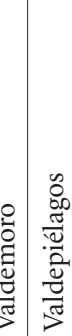 & 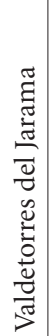 & $\frac{\pi}{\frac{\pi}{0}} \frac{0}{\pi}$ \\
\hline 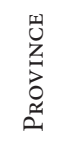 & & & & & & & & & & & 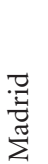 & & & & & & & & & \\
\hline 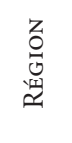 & & & & & & & & & & & $\begin{array}{l}\text { 党 } \\
\text { 芒 }\end{array}$ & & & & & & & & & \\
\hline
\end{tabular}




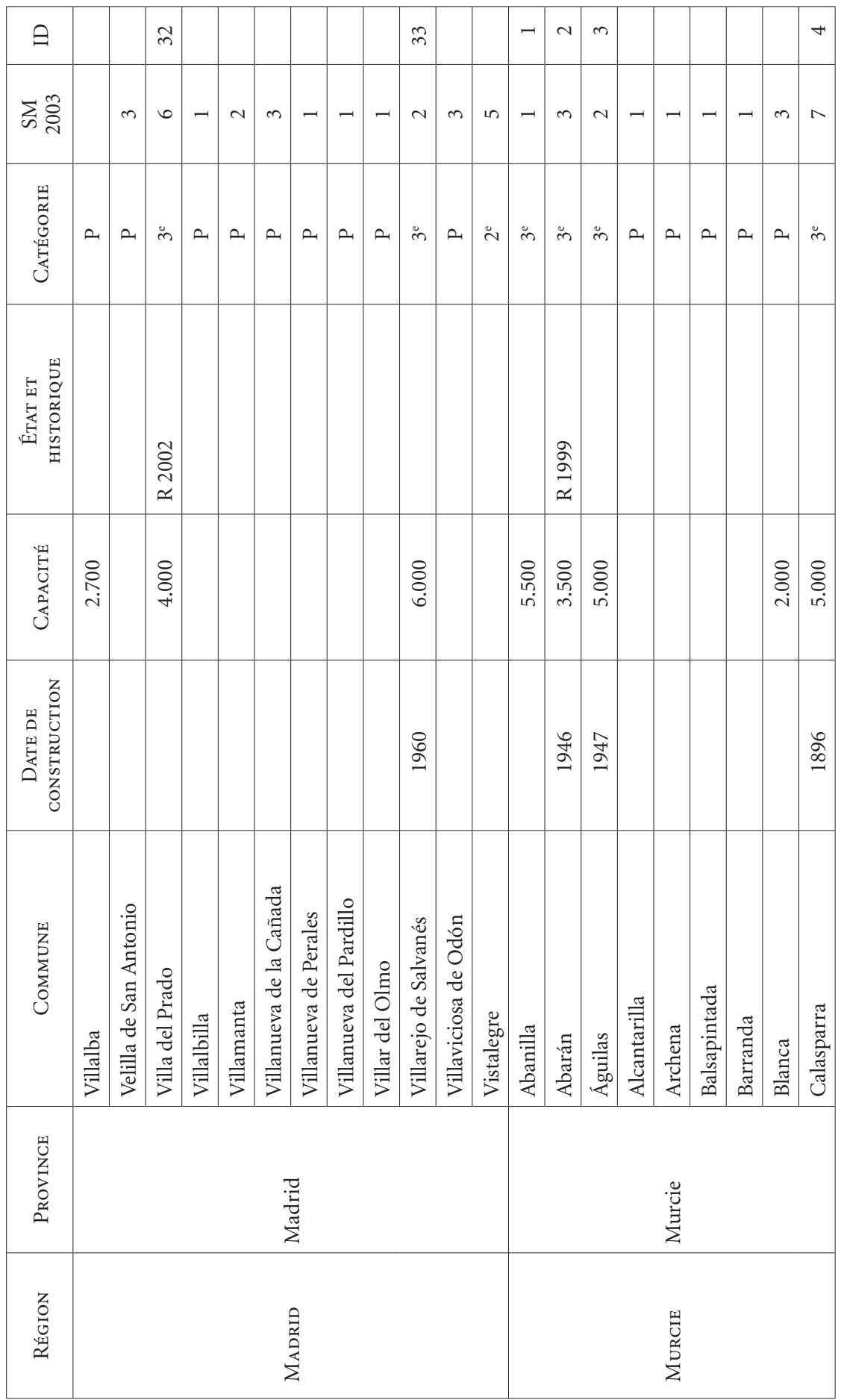




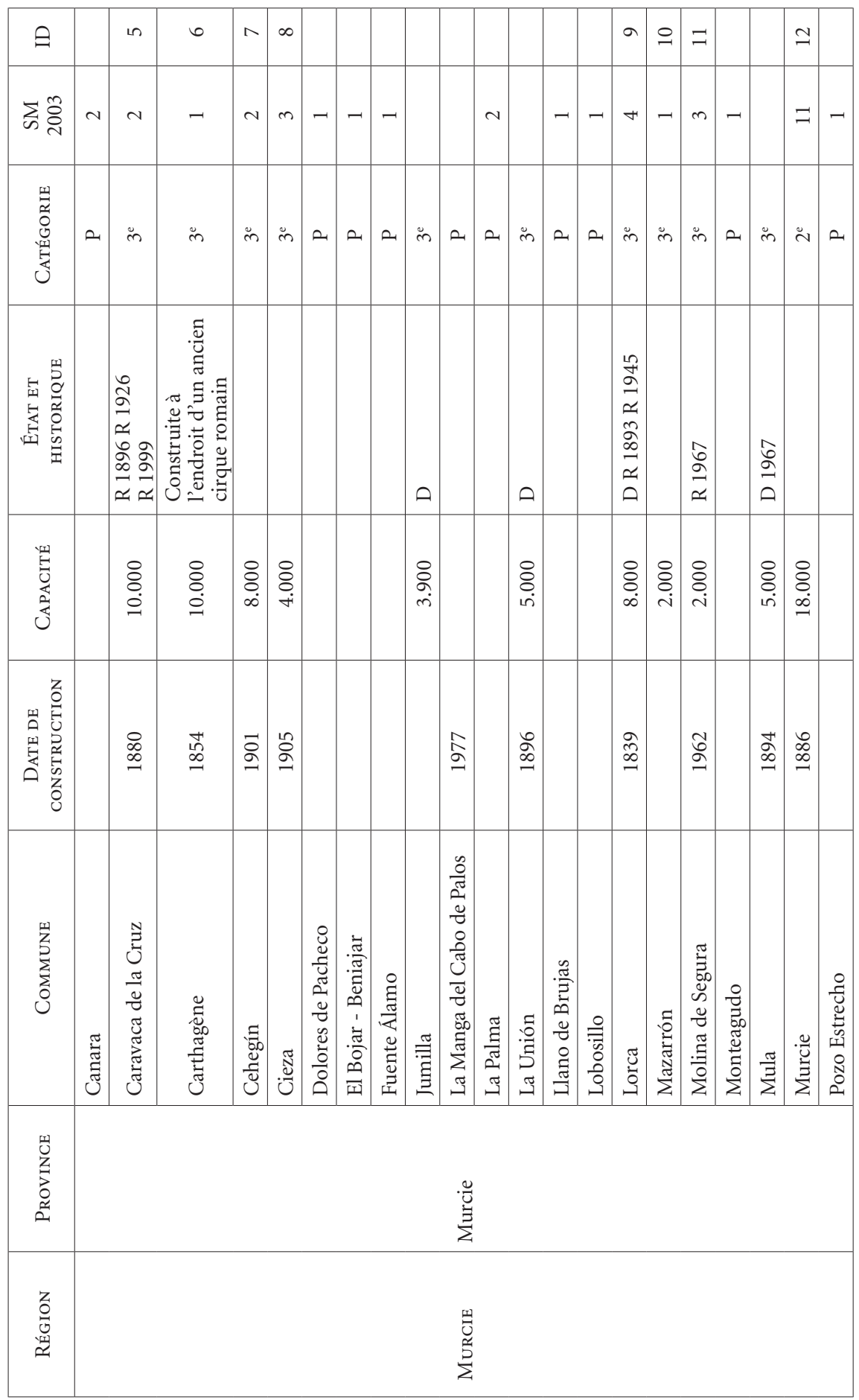




\begin{tabular}{|c|c|c|c|c|c|c|c|c|c|c|c|c|c|c|c|c|c|c|c|c|}
\hline$\theta$ & & & & 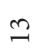 & & $\Xi$ & & -1 & $\sim$ & & $m$ & 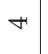 & 10 & 0 & $\wedge$ & $\infty$ & & a 0 & & $=$ \\
\hline$\sum_{\infty} \stackrel{m}{0}$ & - & $N$ & - & $N$ & - & $n$ & $\sim$ & & + & - & $m$ & in & $m$ & $m$ & in & & $-\neg$ & $\bigcirc$ in & & H \\
\hline 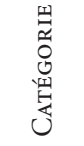 & 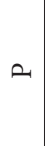 & $a$ & a & m & $\infty$ & $\ddot{m}$ & $a$ & $\ddot{m}$ & $\dot{m}$ & 2 & $\ddot{m}$ & $\ddot{m}$ & $\dot{m}$ & $\ddot{m}$ & in & $\ddot{m}$ & $a$ & $\stackrel{2}{*}$ & or & m \\
\hline 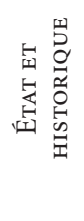 & & & & & & $\begin{array}{l}\bar{\sigma} \\
\stackrel{\sigma}{\simeq}\end{array}$ & & & & & & & & $\begin{array}{l}\stackrel{+}{\circ} \\
\stackrel{\Xi}{\simeq 4}\end{array}$ & & & & 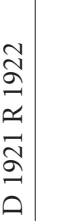 & & \\
\hline 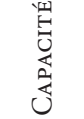 & & & & $\begin{array}{l}\stackrel{8}{0} \\
\dot{r}\end{array}$ & & $\begin{array}{l}8 \\
\text { on } \\
\text { h. }\end{array}$ & & 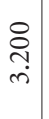 & $\begin{array}{l}8 \\
\stackrel{0}{0} \\
i\end{array}$ & & 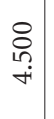 & 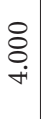 & $\begin{array}{l}8 \\
\stackrel{8}{n} \\
\dot{i n} \\
+\end{array}$ & $\begin{array}{l}\stackrel{8}{8} \\
\stackrel{+}{+}\end{array}$ & $\begin{array}{l}8 \\
\vdots \\
i\end{array}$ & $\begin{array}{l}8 \\
8 \\
0 \\
0\end{array}$ & & 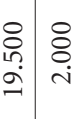 & $\begin{array}{l}\delta \\
\vdots \\
i\end{array}$ & $\begin{array}{l}\stackrel{8}{0} \\
\stackrel{\sim}{ }\end{array}$ \\
\hline 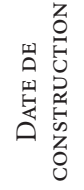 & & & & $\stackrel{?}{2}$ & & $\begin{array}{l}\text { Oे } \\
\text { - }\end{array}$ & & 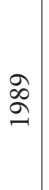 & 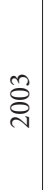 & & $\begin{array}{l}\infty \\
\stackrel{2}{\varrho}\end{array}$ & $\begin{array}{l}\widetilde{\infty} \\
\infty \\
\sim\end{array}$ & $\stackrel{0}{\sigma}$ & $\stackrel{m}{\sigma}$ & $\bar{\Xi}$ & $\stackrel{\widehat{ે}}{\widehat{\Xi}}$ & & $\begin{array}{lll}0 \\
\infty \\
\infty\end{array}$ & & $\stackrel{2}{\curvearrowright}$ \\
\hline 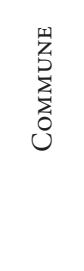 & 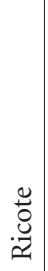 & 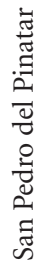 & 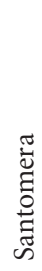 & 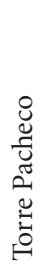 & 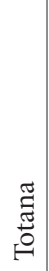 & $\frac{\pi}{\tilde{u}}$ & $\begin{array}{l}\stackrel{0}{0} \\
\overrightarrow{7} \\
0 \\
\frac{0}{2} \\
\frac{8}{4}\end{array}$ & 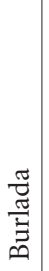 & 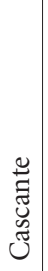 & : & 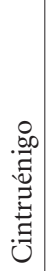 & 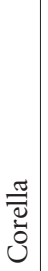 & 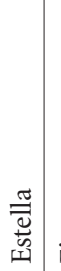 & 忞 & 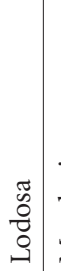 & 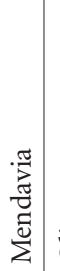 & $\stackrel{.0}{0}$ & 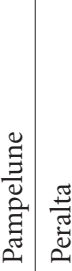 & 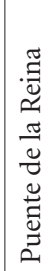 & 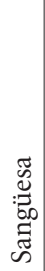 \\
\hline 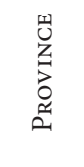 & & & & & & & & & & & & & & 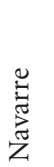 & & & & & & \\
\hline 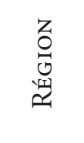 & & & & 然 & & & & & & & & & & 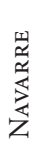 & & & & & & \\
\hline
\end{tabular}




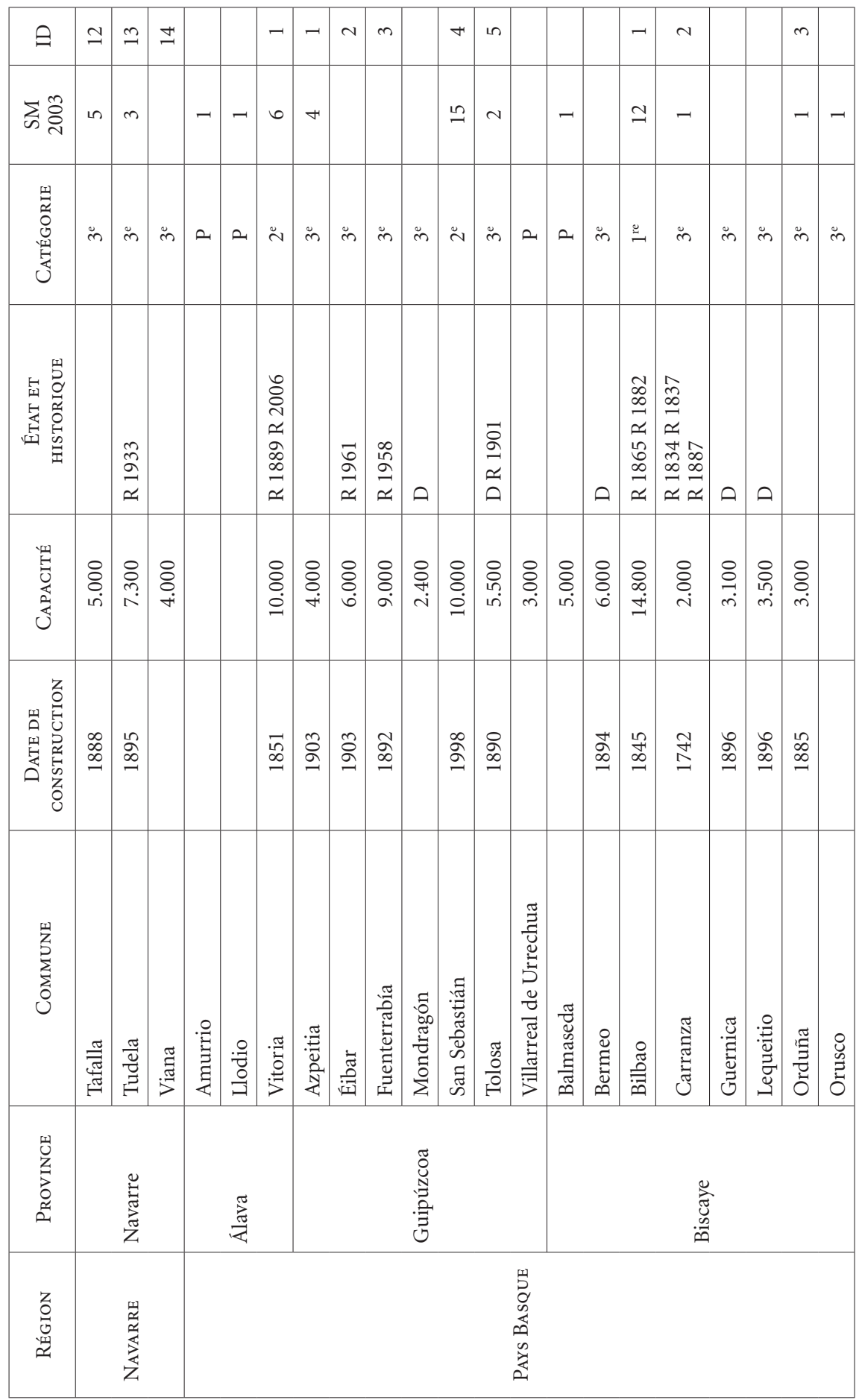




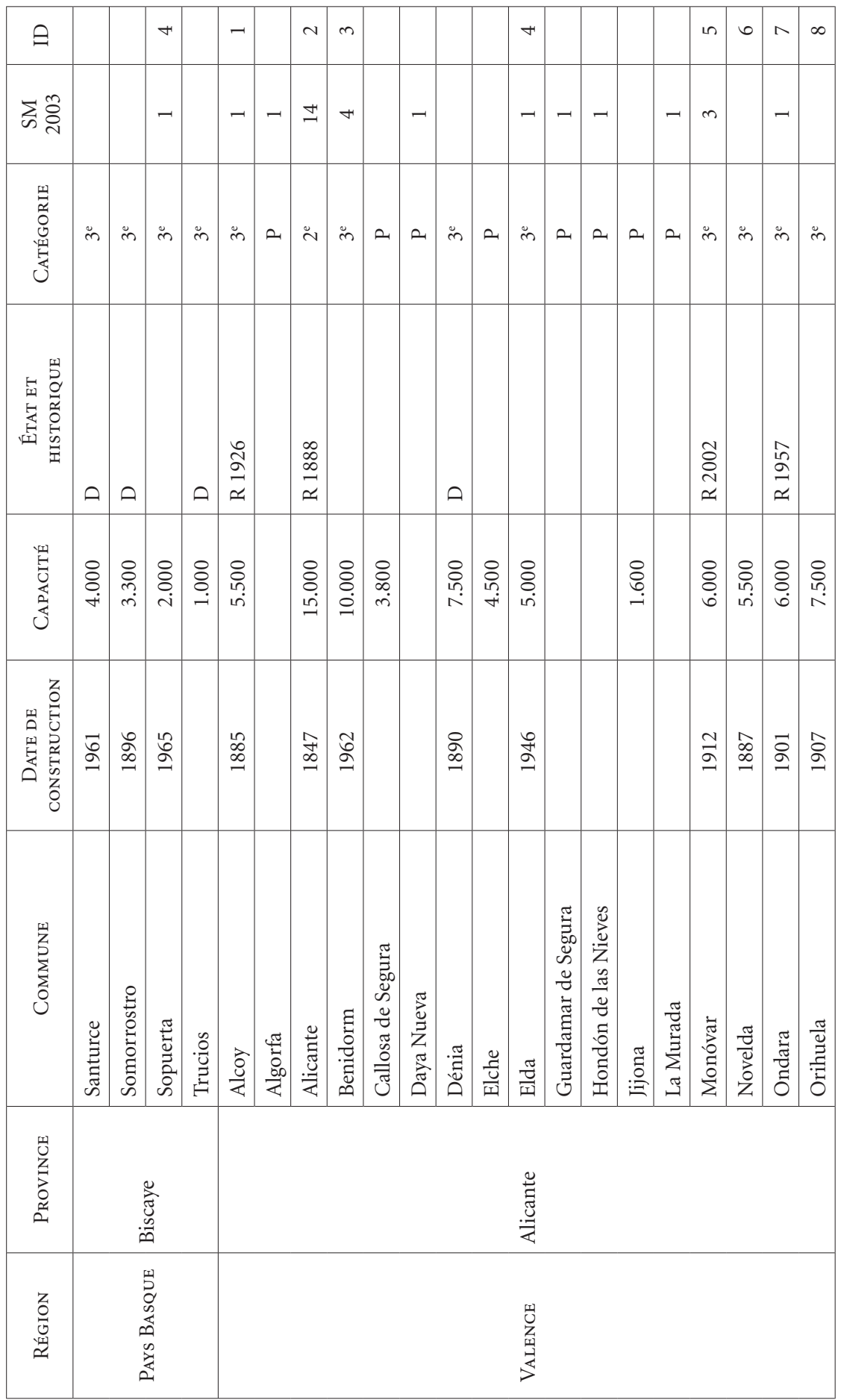




\begin{tabular}{|c|c|c|c|c|c|c|c|c|c|c|c|c|c|c|c|c|c|c|c|c|c|}
\hline$\theta$ & & & $a$ & & $\stackrel{0}{ }$ & $\exists$ & $\underset{\sim}{\sim}$ & & & $\neg$ & $\sim$ & $n$ & & 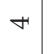 & in & & 0 & $\wedge$ & $\infty$ & $a$ & \\
\hline 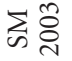 & - & - & & - & & & $\neg$ & $\sim$ & - & & 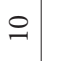 & & - & $\sim$ & & - & $\sim$ & - & - & $r$ & \\
\hline 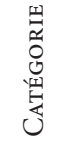 & a & D & $\dot{m}$ & $\infty$ & $\ddot{m}$ & $\ddot{m}$ & $\dot{m}$ & $\infty$ & 2 & $\ddot{m}$ & $\ddot{\sim}$ & $\ddot{m}$ & $a_{1}$ & $\ddot{m}$ & $\ddot{m}$ & 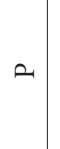 & m & $\ddot{m}$ & $\ddot{m}$ & $\ddot{m}$ & $\ddot{m}$ \\
\hline 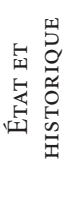 & & & & & & & 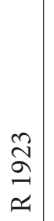 & & & & & & & & & & & & & $\begin{array}{l}\vec{\sigma} \\
\stackrel{\infty}{\simeq}\end{array}$ & $\theta$ \\
\hline 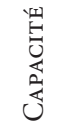 & & & $\begin{array}{c}8 \\
\vdots \\
\dot{m} \\
\text {. }\end{array}$ & & ¿̨ి & $\begin{array}{l}8 \\
\vdots \\
\vdots \\
0\end{array}$ & \begin{tabular}{l}
8 \\
8 \\
\hdashline \\
\hdashline
\end{tabular} & & & $\begin{array}{l}\mathscr{8} \\
\infty \\
\dot{r}\end{array}$ & $\begin{array}{l}8 \\
\dot{0} \\
\ddot{2}\end{array}$ & $\begin{array}{l}8 \\
\Xi \\
\dot{r}\end{array}$ & & & $\begin{array}{l}8 \\
\stackrel{0}{0} \\
\text { in }\end{array}$ & & & 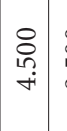 & $\begin{array}{c}8 \\
\stackrel{1}{0} \\
\infty \\
\infty\end{array}$ & $\begin{array}{l}\stackrel{8}{0} \\
\stackrel{\infty}{\circ}\end{array}$ & $\underset{+}{\stackrel{7}{\rightleftarrows}}$ \\
\hline 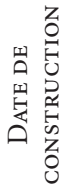 & & & $\begin{array}{l}\widehat{\infty} \\
\infty \\
\infty \\
\sim\end{array}$ & & & ڤे & 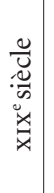 & & & $\begin{array}{l}\stackrel{0}{0} \\
2\end{array}$ & $\begin{array}{l}\infty \\
\infty \\
\infty \\
\infty\end{array}$ & ఫே & & 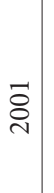 & & & 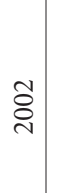 & & $\stackrel{\Re}{\sigma}$ & 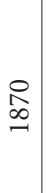 & \\
\hline 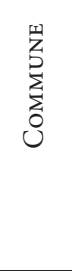 & $\begin{array}{l}\circ \\
\stackrel{0}{0} \\
. \\
0\end{array}$ & $\begin{array}{l}\frac{8}{8} \\
\overline{0} \\
\frac{0}{0} \\
\approx\end{array}$ & 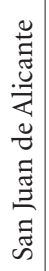 & 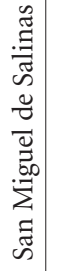 & 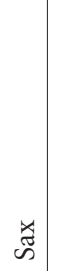 & 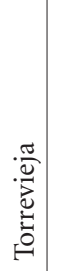 & $\begin{array}{l}\frac{\pi}{0} \\
\stackrel{\overrightarrow{0}}{5}\end{array}$ & $\underset{\frac{\pi}{Z}}{\stackrel{\Xi}{Z}}$ & $\begin{array}{l}\overrightarrow{\tilde{y}} \\
\stackrel{\tilde{u}}{0}\end{array}$ & 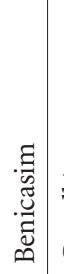 & 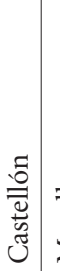 & $\begin{array}{l}\frac{\pi}{\vec{J}} \\
\stackrel{0}{0} \\
\stackrel{0}{\Sigma}\end{array}$ & $\frac{\ddot{g}}{\vec{z}}$ & $\begin{array}{l}\frac{\pi}{\pi} \\
0 \\
0\end{array}$ & 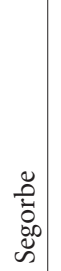 & 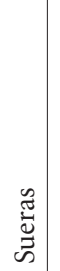 & 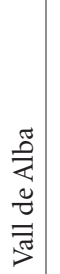 & 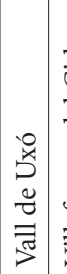 & 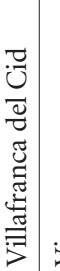 & 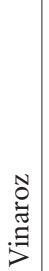 & $\frac{\pi}{\frac{\pi}{4}}$ \\
\hline 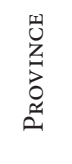 & & & & 节 & & & & & & & & & & $\frac{\Xi}{0}$ & & & & & & & $\frac{\ddot{\breve{~}}}{\frac{\pi}{\pi}}$ \\
\hline 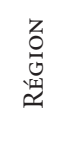 & & & & & & & & & & & 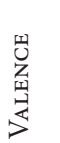 & & & & & & & & & & \\
\hline
\end{tabular}




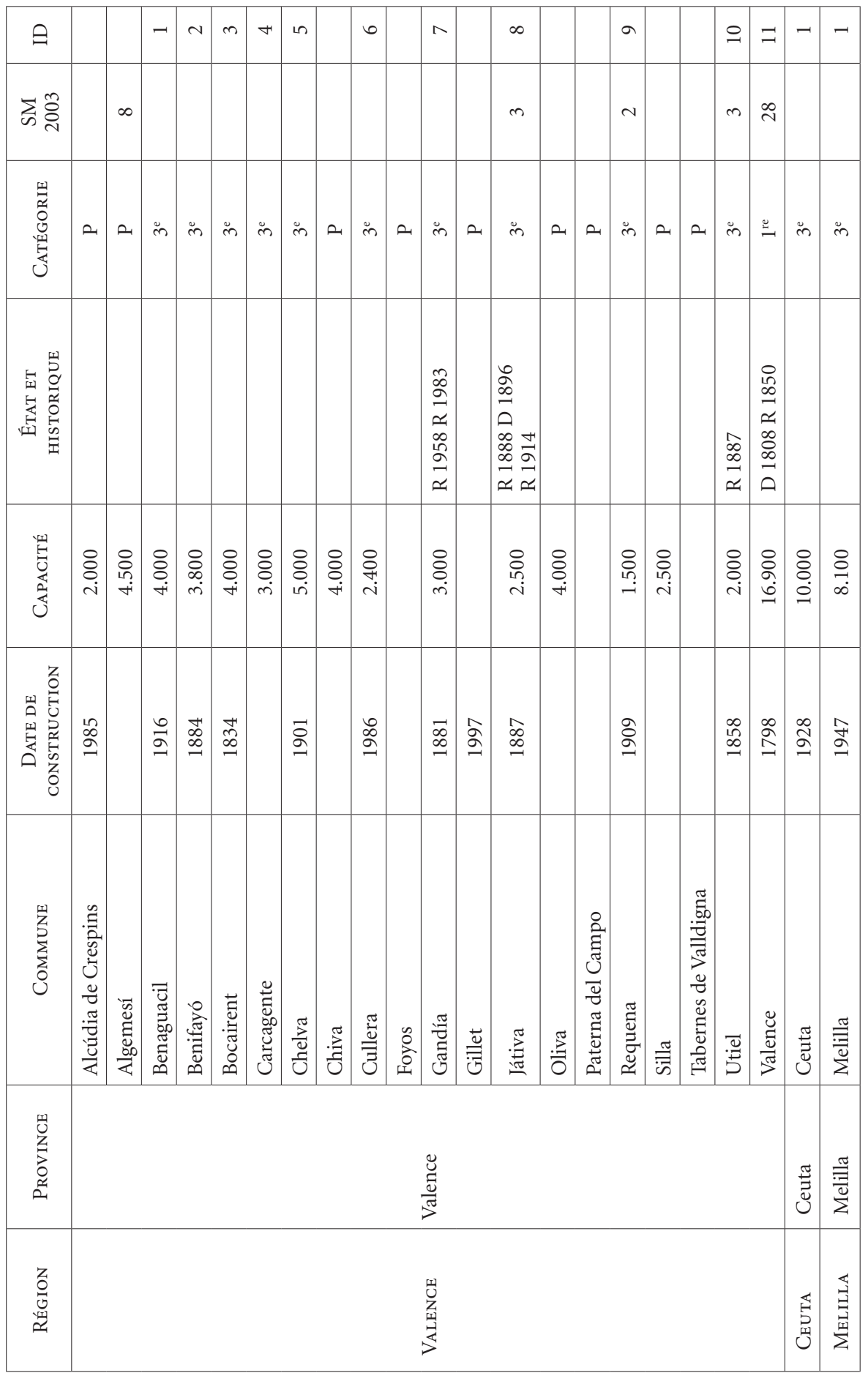




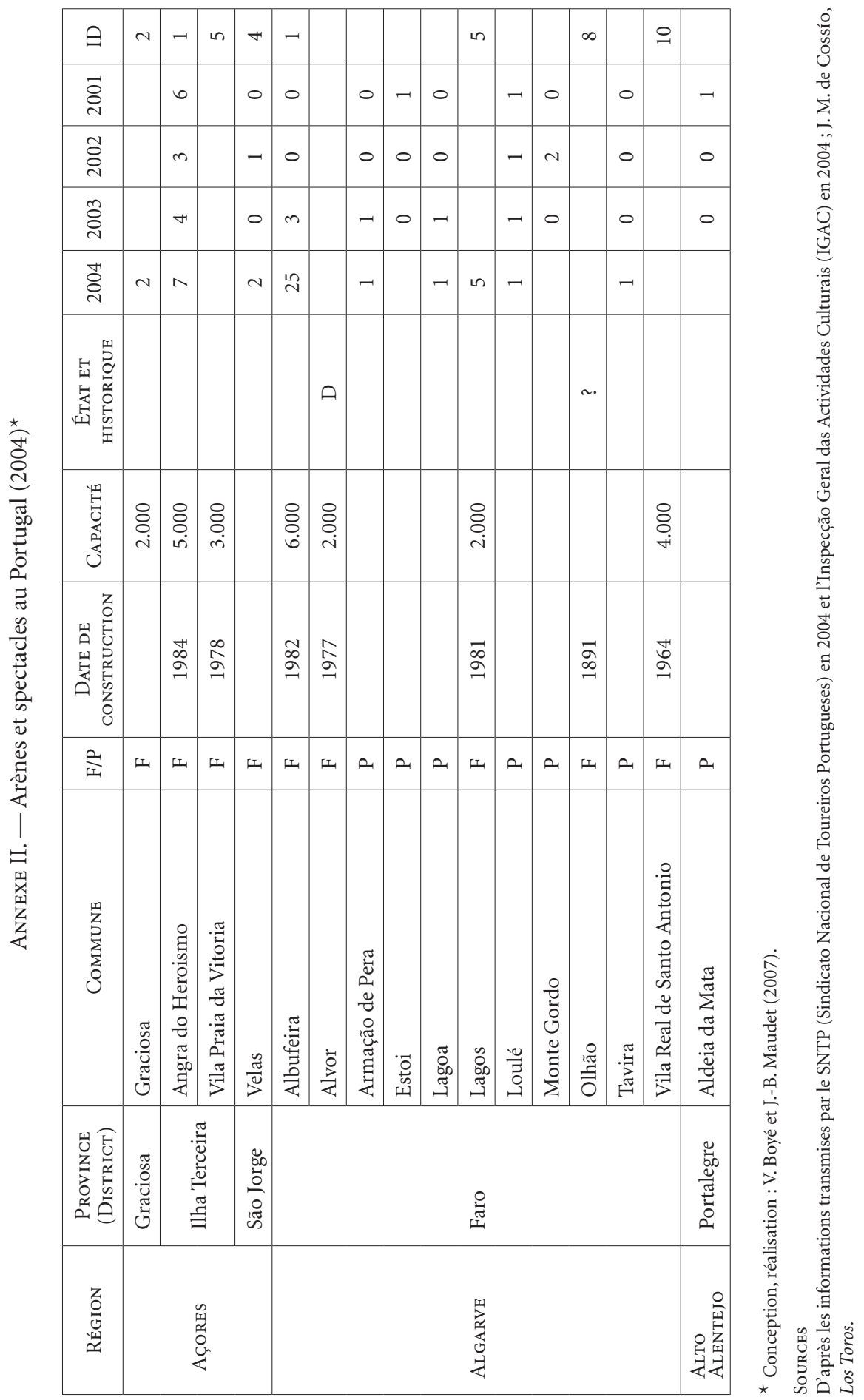




\begin{tabular}{|c|c|c|c|c|c|c|c|c|c|c|c|c|c|c|c|c|c|c|c|}
\hline$\theta$ & $\sim$ & $n$ & 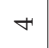 & 0 & 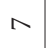 & $\infty$ & & & $=$ & & 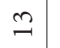 & & $\stackrel{20}{2}$ & $\stackrel{0}{0}$ & $\beth$ & $\stackrel{\infty}{-}$ & & $\stackrel{\sim}{\sim}$ & $\vec{\sim}$ \\
\hline 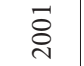 & - & - & - & $\sim$ & - & $\neg$ & 0 & & - & 0 & & - & $\neg$ & & 0 & 0 & - & 0 & 0 \\
\hline ণ్రి & - & $\sim$ & 0 & 0 & 0 & 0 & 0 & & 0 & - & & 0 & $\sim$ & & 0 & 0 & - & - & - \\
\hline ᄋి & $N$ & $\sim$ & - & - & 0 & 0 & 0 & & 0 & 0 & & - & $\sim$ & & $N$ & 0 & 0 & $N$ & 0 \\
\hline 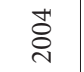 & $\sim$ & $\sim$ & & - & & & $\sim$ & & $\sim$ & & & & $\sim$ & & $m$ & - & & $m$ & - \\
\hline 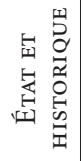 & & & & & $\sim$ & & & & 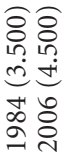 & & & & & & & & & & \\
\hline 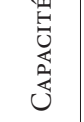 & 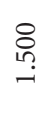 & 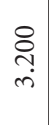 & 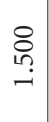 & $\begin{array}{l}\stackrel{8}{0} \\
\text { ñ. } \\
i\end{array}$ & & 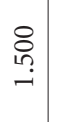 & & & $\begin{array}{l}8 \\
\stackrel{0}{n} \\
+ \\
+\end{array}$ & & 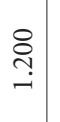 & & $\begin{array}{l}\stackrel{8}{0} \\
\stackrel{i}{i}\end{array}$ & $\underset{\substack{0 \\
\hdashline}}{ }$ & 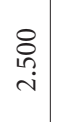 & 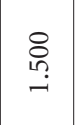 & & $\begin{array}{l}8 \\
\vdots \\
\dot{i n}\end{array}$ & 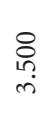 \\
\hline 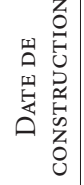 & & & & 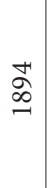 & $\stackrel{n}{\stackrel{n}{\infty}}$ & & & $\stackrel{\infty}{2}$ & $\begin{array}{l}H \\
\stackrel{2}{二}\end{array}$ & & $\stackrel{\infty}{\stackrel{\varpi}{二}}$ & & $\stackrel{\infty}{\stackrel{\sim}{\beth}}$ & $\stackrel{\aleph}{\varrho}$ & Әे & گૂ & & $\begin{array}{l}\underset{\Delta}{\Delta} \\
\stackrel{-}{\sigma}\end{array}$ & $\stackrel{\substack{\alpha \\
\infty}}{ }$ \\
\hline$\frac{\partial}{\mid r}$ & 山 & I & 山 & 山 & 山 & 山 & 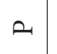 & 山 & س & a & 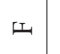 & $\infty$ & 山 & 山 & 山 & $\omega$ & $a_{1}$ & 山 & 山 \\
\hline $\begin{array}{l}\text { س } \\
2 \\
\sum_{2} \\
0 \\
0\end{array}$ & 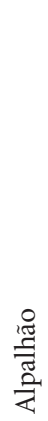 & 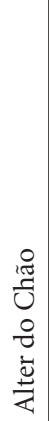 & 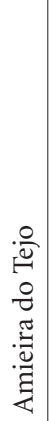 & 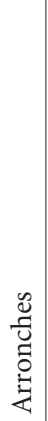 & . 릴 & 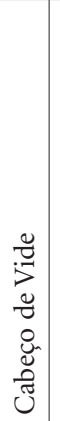 & 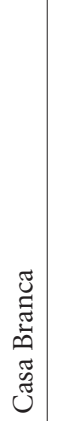 & 苞 & 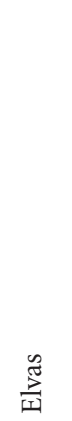 & 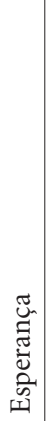 & 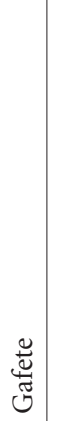 & 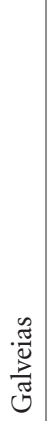 & 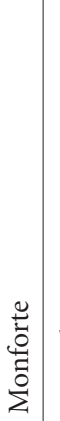 & 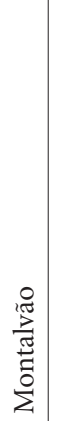 & 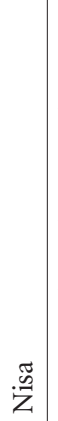 & 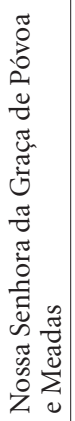 & $\begin{array}{l}\overrightarrow{0} \\
0 \\
\tilde{y} \\
\tilde{y} \\
\tilde{0} \\
0\end{array}$ & 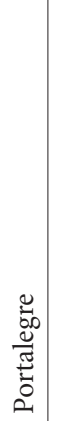 & 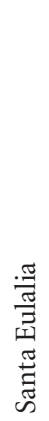 \\
\hline 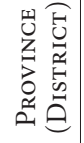 & & & & & & & & & & 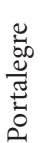 & & & & & & & & & \\
\hline 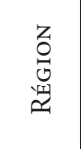 & & & & & & & & & & 兽 & & & & & & & & & \\
\hline
\end{tabular}




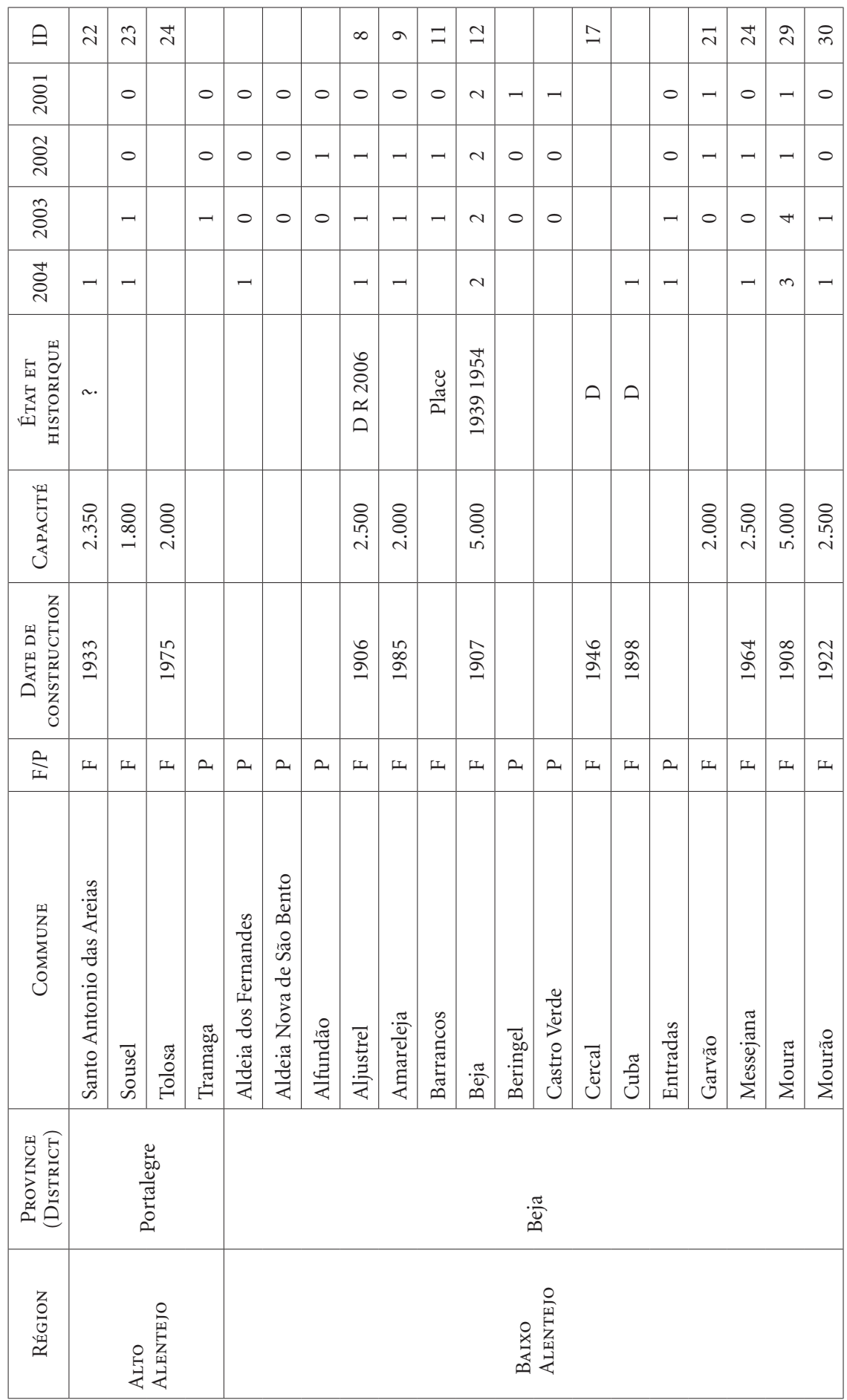




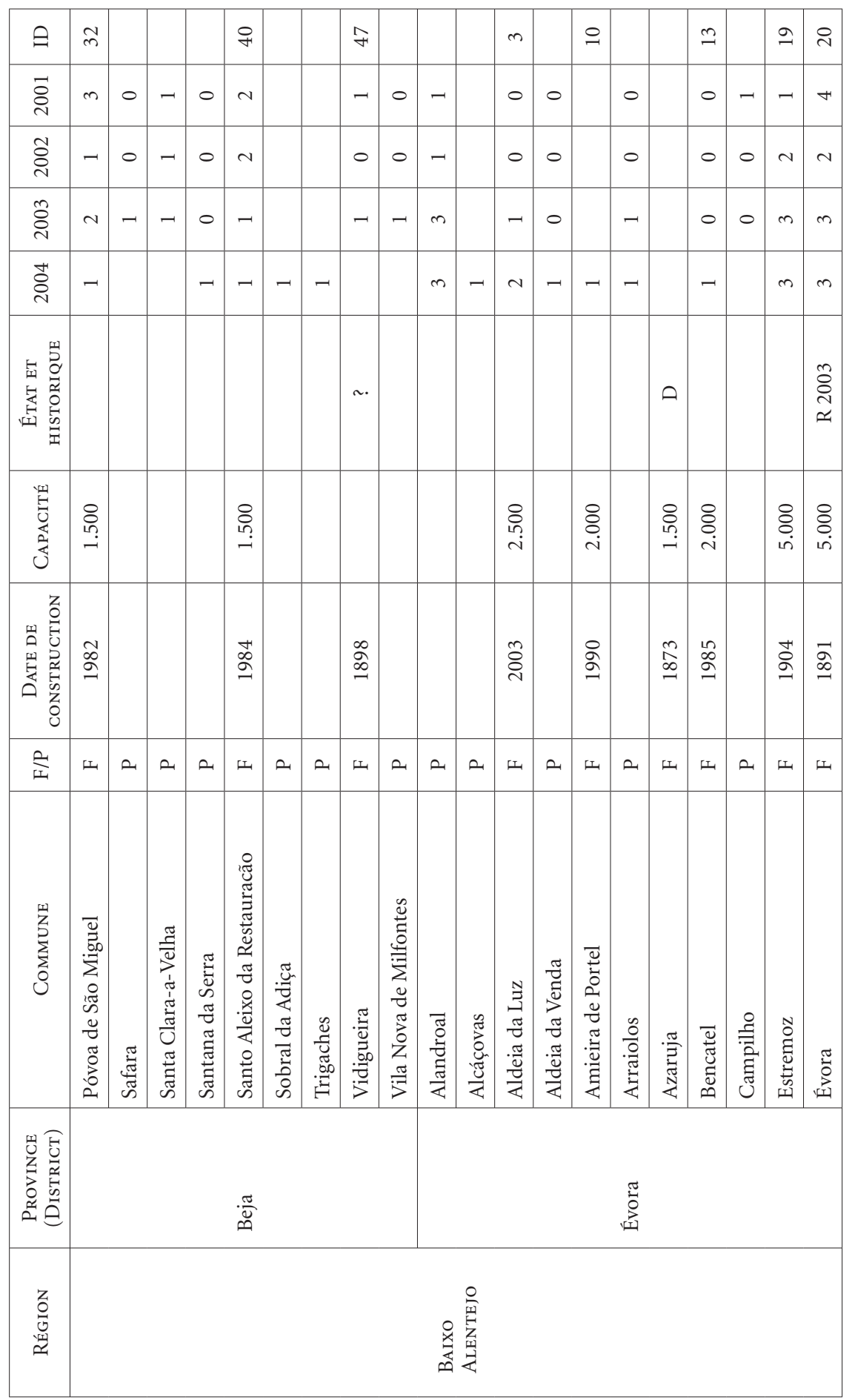




\begin{tabular}{|c|c|c|c|c|c|c|c|c|c|c|c|c|c|c|c|c|c|c|c|c|}
\hline 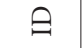 & $\widetilde{\sim}$ & & & $\stackrel{\sim}{\sim}$ & & & & $m$ & $\stackrel{n}{n}$ & & & F & & & g) & & - & & $\infty$ & \\
\hline ఫ్ & 0 & 0 & & - & - & 0 & - & 0 & 0 & 0 & - & - & 0 & -1 & - & $\rightarrow$ & 0 & 0 & & - \\
\hline రి & 0 & 0 & & - & 0 & 0 & 0 & 0 & $\sim$ & 0 & 0 & 0 & 0 & 0 & - & 0 & 0 & 0 & & 0 \\
\hline ڤ్ & - & - & & $n$ & 0 & 0 & 0 & - & 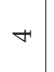 & - & - & - & 0 & 0 & - & 0 & - & 0 & & 0 \\
\hline 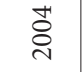 & - & & - & $\sim$ & & - & & - & 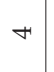 & & $\neg$ & - & & & - & & - & & & \\
\hline 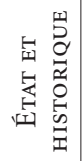 & & & & 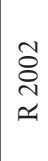 & & & & & & & & & & & $\begin{array}{l}\hat{\infty} \\
\curvearrowright \\
\widetilde{a} \\
\infty \\
2 \\
\infty \\
-1\end{array}$ & & & & $\sim$ & O \\
\hline 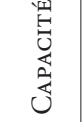 & 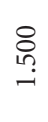 & & & $\begin{array}{l}\stackrel{8}{0} \\
\text { m. }\end{array}$ & & & & 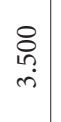 & 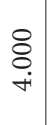 & & & $\begin{array}{l}8 \\
\dot{m} \\
\dot{m}\end{array}$ & & & 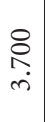 & & $\begin{array}{l}\stackrel{8}{0} \\
\stackrel{+}{+}\end{array}$ & & & \\
\hline 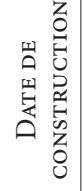 & $\begin{array}{l}\stackrel{2}{\Omega} \\
\stackrel{2}{-}\end{array}$ & & & $\begin{array}{l}\infty \\
\infty \\
\infty \\
-1\end{array}$ & & & & $\stackrel{\text { ڤิ }}{=}$ & $\stackrel{\llcorner}{\Omega}$ & & & 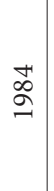 & & & $\stackrel{\infty}{\stackrel{\infty}{\infty}}$ & & 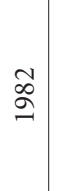 & & & \\
\hline$\frac{2}{|r|}$ & 山 & $\infty$ & a & 山 & ar & $a$ & 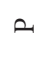 & 工 & 出 & $\infty$ & $\infty$ & $\omega$ & 2 & $\infty$ & 山 & 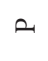 & I & $a$ & 山 & 工 \\
\hline 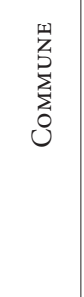 & 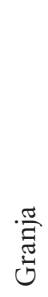 & 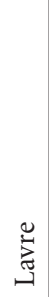 & 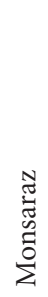 & 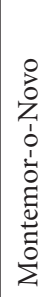 & $\begin{array}{l}\stackrel{0}{0} \\
\stackrel{0}{0} \\
\stackrel{0}{0} \\
\dot{0}\end{array}$ & $\stackrel{\widetilde{\sigma}}{\tilde{c}}$ & 荀 & 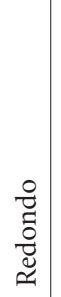 & 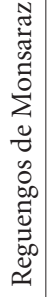 & 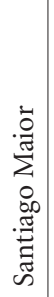 & 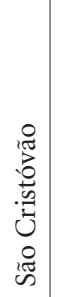 & 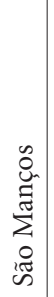 & 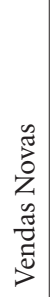 & 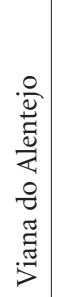 & 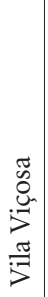 & 号 & 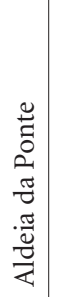 & $\begin{array}{l}0 \\
\stackrel{0}{\tilde{J}} \\
\tilde{\Xi} \\
ن\end{array}$ & $\begin{array}{l}\cdot \frac{\pi}{0} \\
\stackrel{0}{0} \\
0 \\
0 \\
0\end{array}$ & 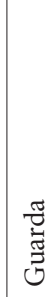 \\
\hline 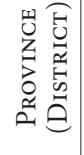 & & & & & & & & 可 & & & & & & & & & & $\begin{array}{ll}\pi \\
0\end{array}$ & & \\
\hline 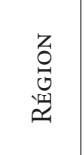 & & & & & & & & 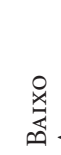 & & & & & & & & & & 焉 & 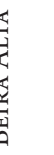 & \\
\hline
\end{tabular}




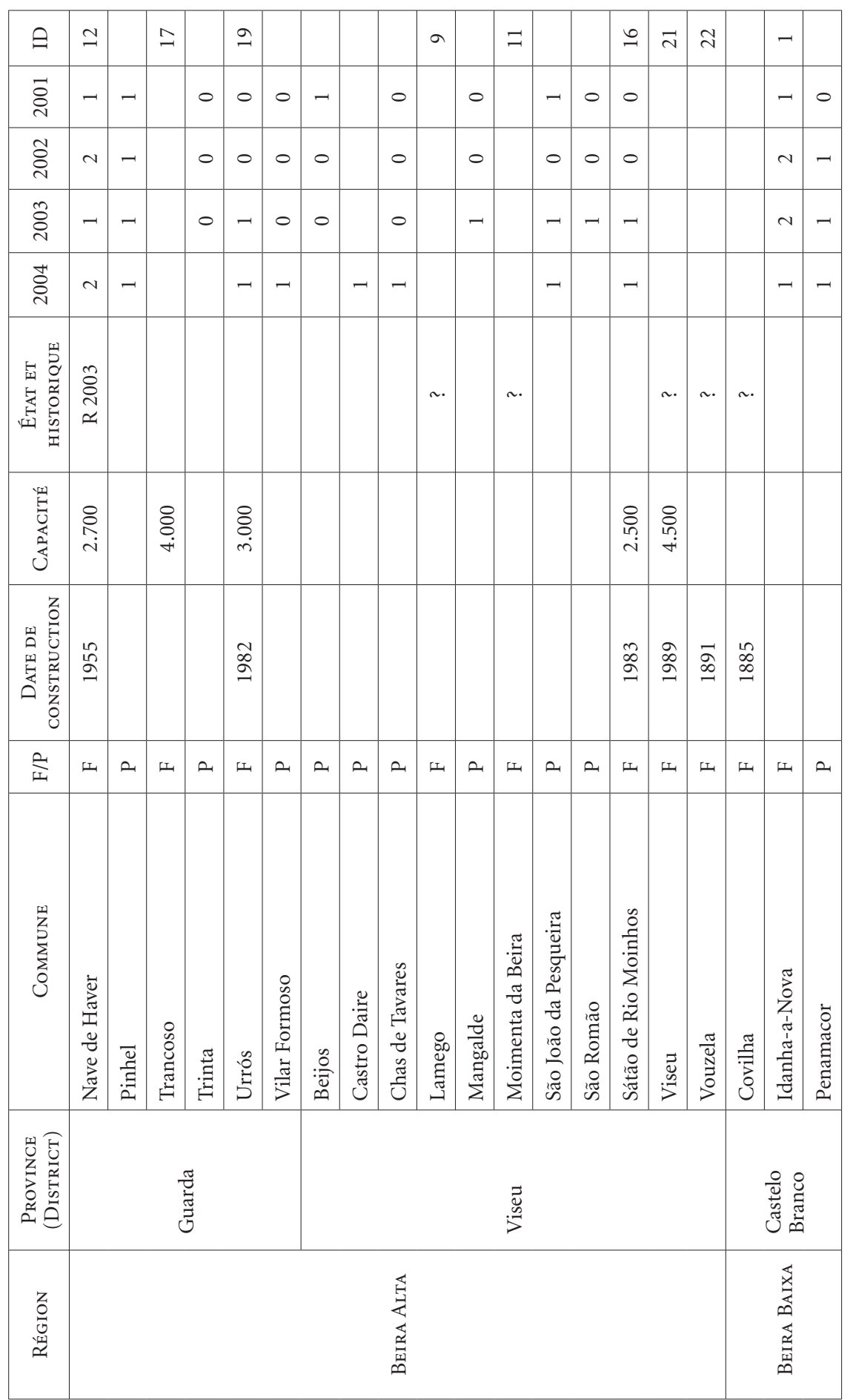




\begin{tabular}{|c|c|c|c|c|c|c|c|c|c|c|c|c|c|c|c|c|c|c|c|}
\hline$\theta$ & & & $\infty$ & & 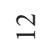 & & & $\ddot{\sim}$ & & & $\wedge$ & $ㅇ$ & & & & & - & & \\
\hline ¿্ঠి & 0 & 0 & & - & & - & 0 & & -1 & 0 & - & $n$ & 0 & 0 & 0 & 0 & $\rightarrow$ & 0 & 0 \\
\hline రి & 0 & - & & - & & 0 & - & & 0 & 0 & 0 & - & $N$ & - & 0 & - & $\sim$ & 0 & 0 \\
\hline ڤి & 0 & - & & 0 & & 0 & - & & 0 & 0 & 0 & $N$ & 0 & 0 & 0 & - & $n$ & 0 & - \\
\hline 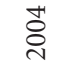 & & & & & & & & & - & - & & $\sim$ & & & - & - & $\forall$ & $\sim$ & \\
\hline 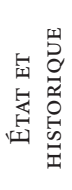 & & & 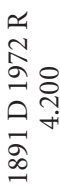 & & $\sim$ & & & $\tilde{\tilde{L}}$ & & & & & & & & & & & \\
\hline 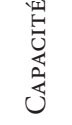 & & & $\underset{+}{\stackrel{\leftrightarrow}{+}}$ & & & & & 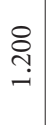 & & & 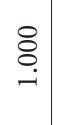 & $\begin{array}{l}8 \\
0 \\
0 \\
0\end{array}$ & & & & & $\begin{array}{l}8 \\
\text { in } \\
\text { in }\end{array}$ & & \\
\hline 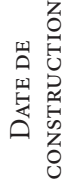 & & & $\underset{\infty}{\sigma}$ & & & & & ๙ิ & & & ڤి & 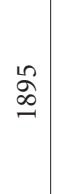 & & & & & & & \\
\hline$\frac{2}{|r|}$ & a & 2 & 山 & 2 & 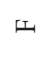 & 2 & $\infty$ & $a_{1}$ & $a_{1}$ & 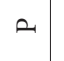 & 山 & 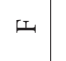 & 2 & a & $\infty$ & $\infty$ & $\omega$ & $\infty$ & o \\
\hline 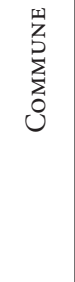 & 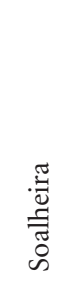 & 号 & 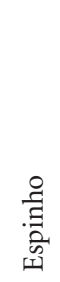 & 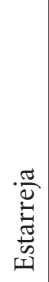 & $\frac{\underset{\tilde{J}}{\tilde{J}}}{\stackrel{\vec{J}}{\tilde{J}}}$ & 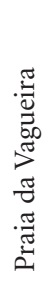 & 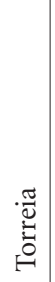 & : & 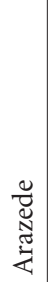 & 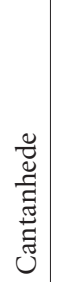 & $\begin{array}{l}\frac{\pi}{\tilde{0}} \\
\vec{\Xi} \\
\dot{0}\end{array}$ & 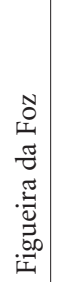 & 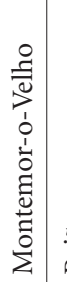 & $\frac{?}{\pi}$ & 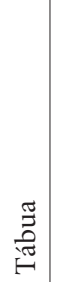 & 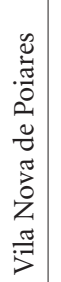 & $\begin{array}{l}3 \\
\overline{2} \\
\end{array}$ & 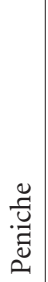 & वृ \\
\hline 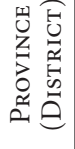 & 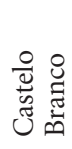 & & & & 竞 & & & & & & & $\frac{\pi}{\tilde{\Xi}}$ & & & & & & : & \\
\hline 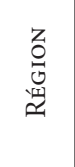 & 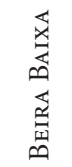 & & & & & & & & & 壹 & & & & & & & & & \\
\hline
\end{tabular}




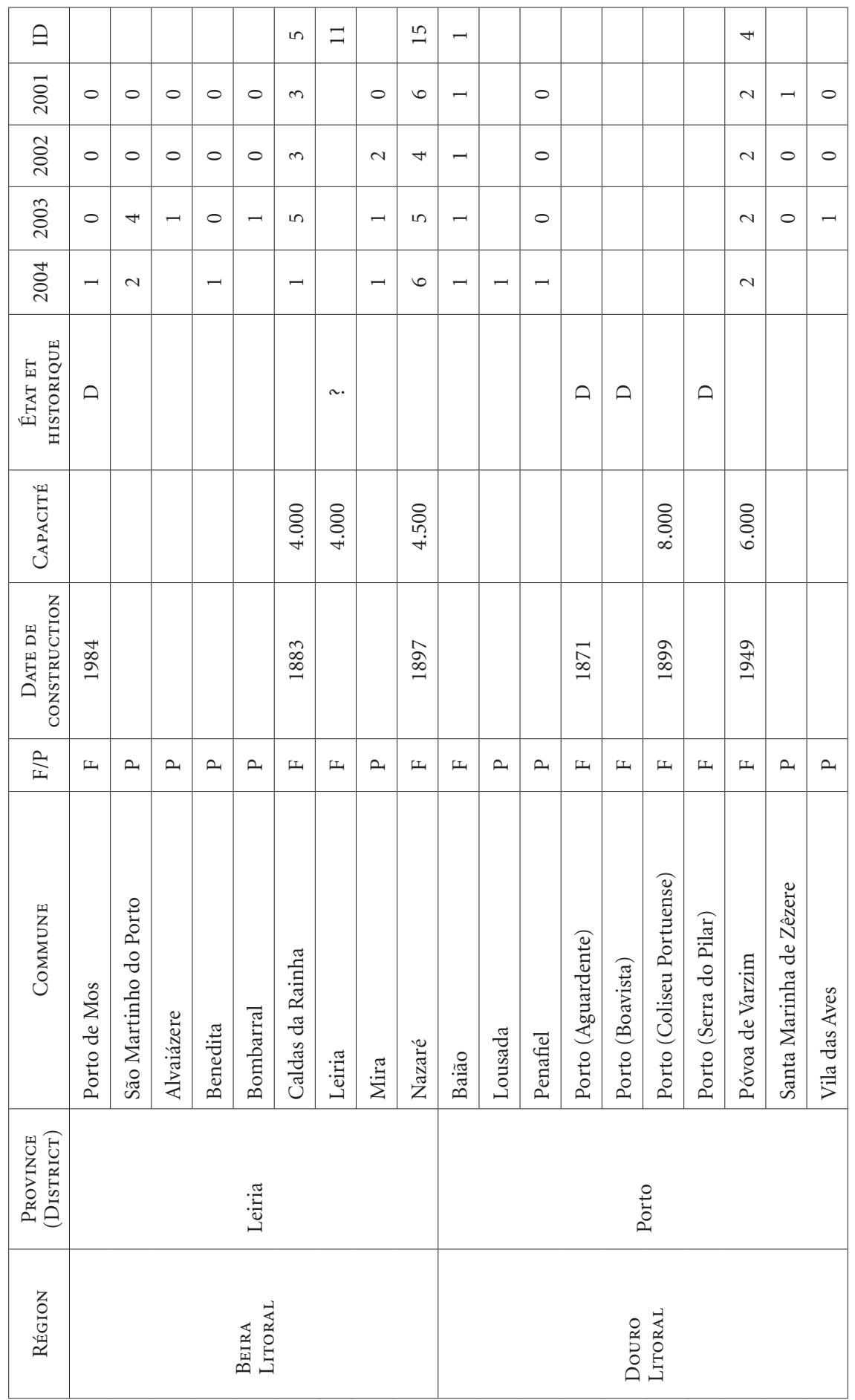


ANNEXES

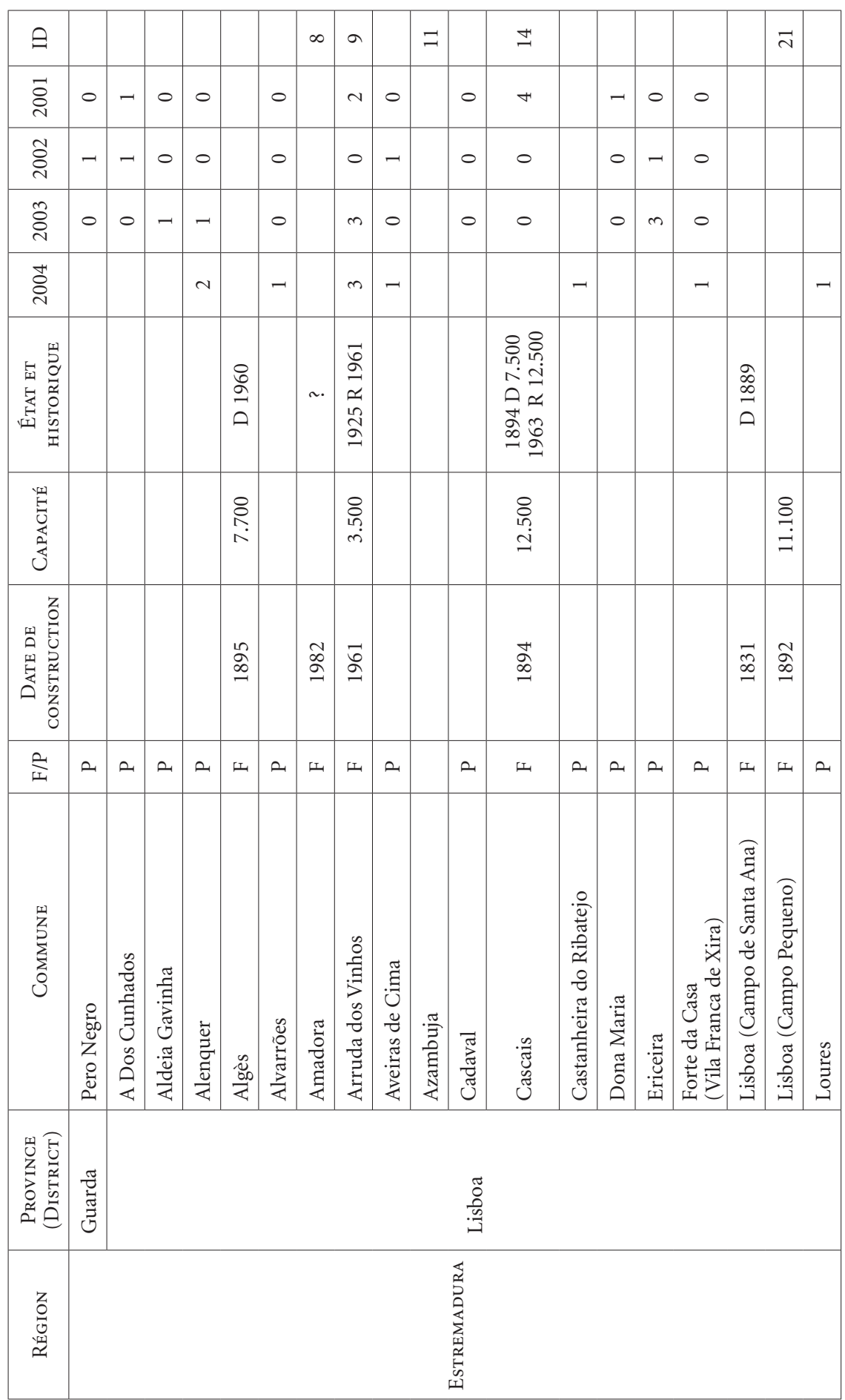




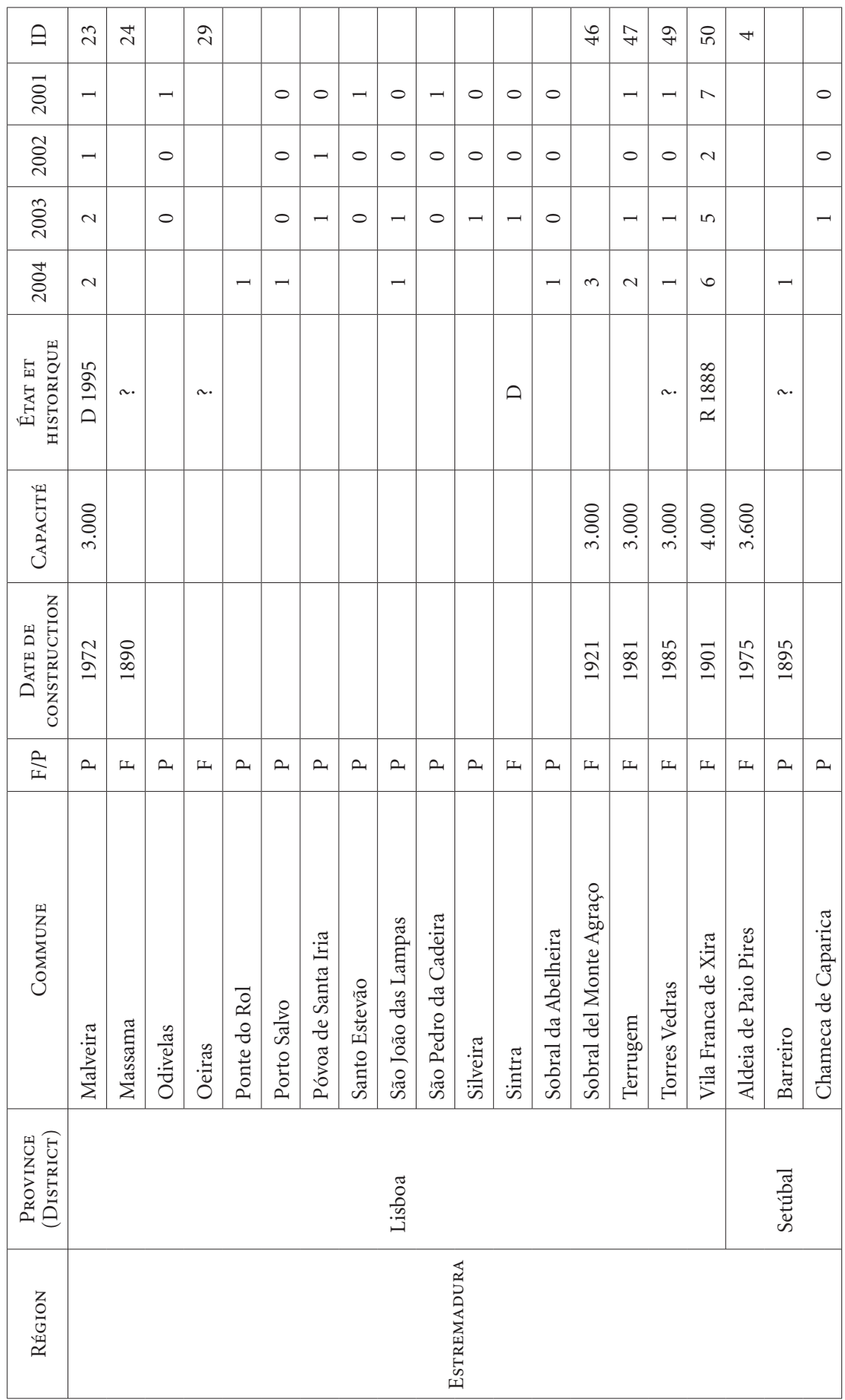




\begin{tabular}{|c|c|c|c|c|c|c|c|c|c|c|c|c|c|c|c|c|c|c|c|c|}
\hline$\theta$ & & & $\stackrel{\sim}{\sim}$ & $\widehat{\triangleq}$ & & & & & & & $\mathscr{F}$ & & & $\sim$ & $n$ & & - & & & H \\
\hline శ్రి & 0 & 0 & $\wedge$ & in & - & 0 & 0 & 0 & 0 & 0 & in & - & - & $\sim$ & $r$ & 0 & & 0 & 0 & \\
\hline ర్రి & 0 & 0 & $\wedge$ & $r$ & 0 & 0 & 0 & 0 & 0 & 0 & $\sim$ & 0 & 0 & $\neg$ & $m$ & 0 & & 0 & 0 & \\
\hline ஜి & 0 & 0 & 6 & $H$ & 0 & - & 0 & - & - & - & in & - & 0 & $\sim$ & $N$ & - & & 0 & 0 & \\
\hline 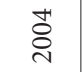 & - & - & 으 & H & - & & & - & - & & in & - & & $\sim$ & $m$ & - & & - & - & - \\
\hline 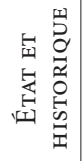 & & & & & & & & & & & & & & 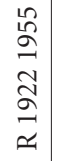 & & & $\sim$. & & & $\underset{\text { 足 }}{\stackrel{a}{a}}$ \\
\hline 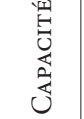 & & & 点 & 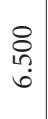 & & & & & & & $\begin{array}{l}8 \\
\text { in } \\
\text { in }\end{array}$ & & & 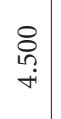 & $\begin{array}{l}8 \\
\stackrel{+}{+} \\
+ \\
+\end{array}$ & & $\begin{array}{l}\stackrel{8}{0} \\
\stackrel{+}{+}\end{array}$ & & & $\begin{array}{l}8 \\
\stackrel{8}{1} \\
\end{array}$ \\
\hline 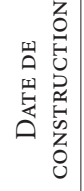 & & & ڤૂ ૂૂ & & & & & & & & $\begin{array}{l}\infty \\
\infty \\
\infty\end{array}$ & & & ర్ర & $\overrightarrow{\widetilde{\sigma}}$ & & $\begin{array}{l}\stackrel{L}{2} \\
\stackrel{\infty}{2}\end{array}$ & & & \\
\hline$\frac{2}{\mid r}$ & a & a & 山 & 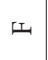 & ar & 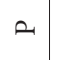 & 2 & $a$ & $a$ & ar & 山 & $a$ & $a$ & 山 & 山 & a & $\omega$ & $a$ & $a$ & a \\
\hline 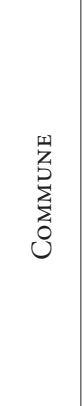 & 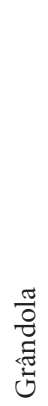 & $\frac{\mathscr{u}}{0 . \bar{\pi}}$ & 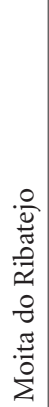 & $\begin{array}{l}: \stackrel{0}{:} \\
\stackrel{0}{0} \\
\stackrel{0}{*}\end{array}$ & 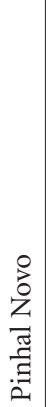 & 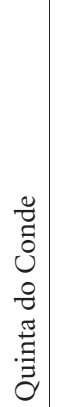 & 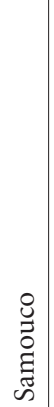 & 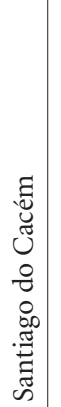 & 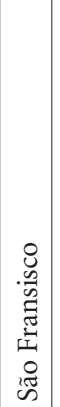 & $\begin{array}{l}\overrightarrow{\tilde{x}} \\
\vec{n} \\
\tilde{n}\end{array}$ & 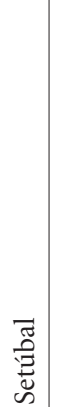 & 胥 & 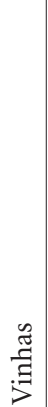 & 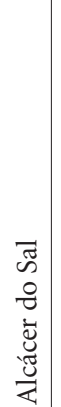 & 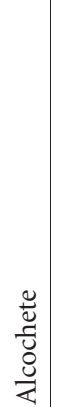 & 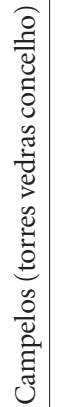 & 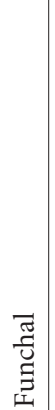 & & 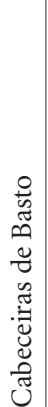 & 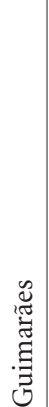 \\
\hline 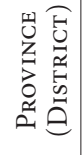 & & & & & & & & 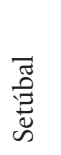 & & & & & & & & 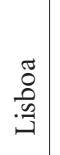 & 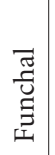 & & 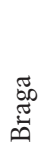 & \\
\hline 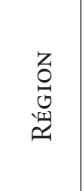 & & & & & & & & 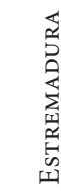 & t: & & & & & & & & 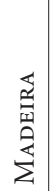 & & $\begin{array}{l}\stackrel{0}{\pi} \\
\stackrel{\mathbb{Z}}{\mathbf{Z}}\end{array}$ & \\
\hline
\end{tabular}


ANNEXES

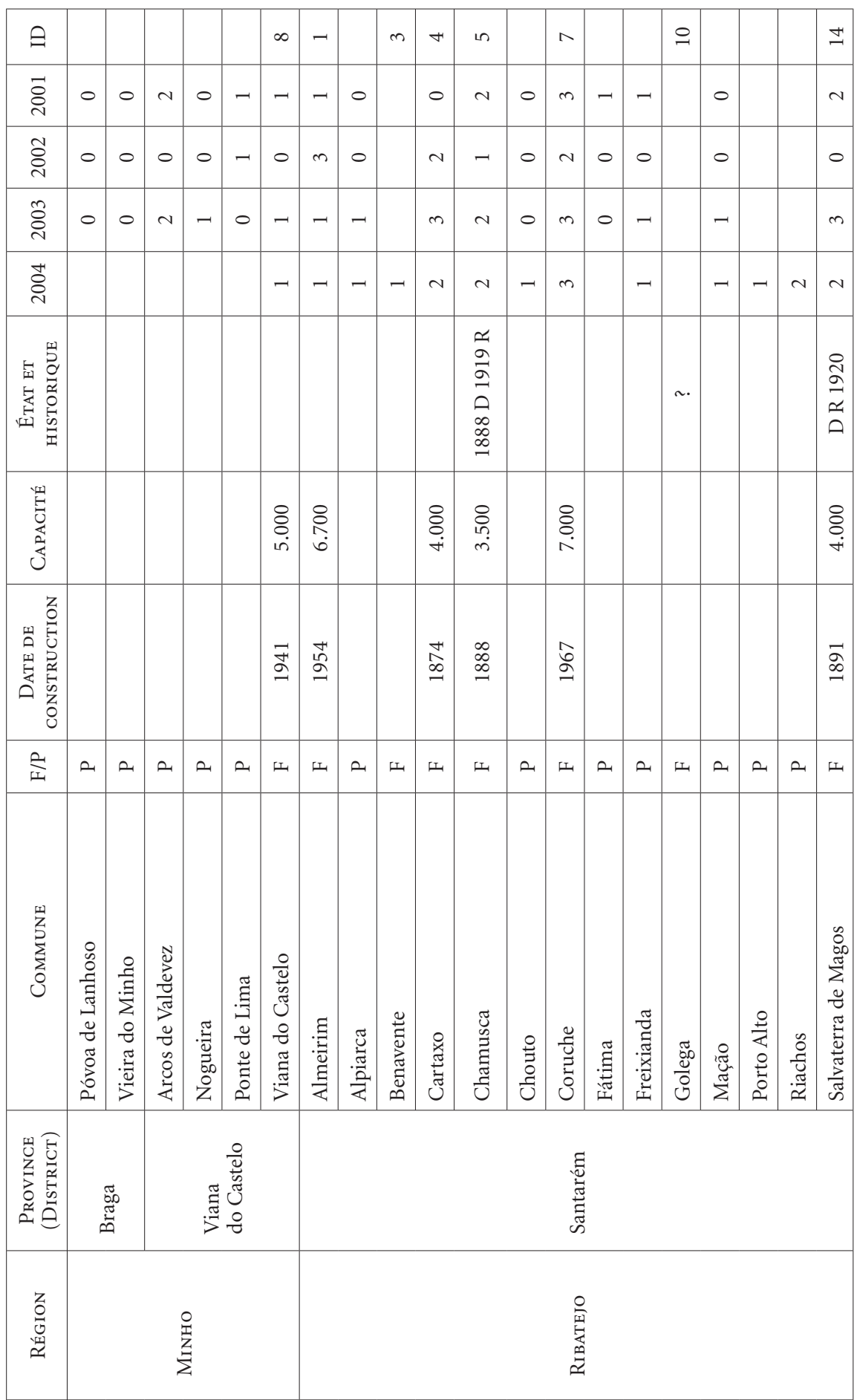


ANNEXES

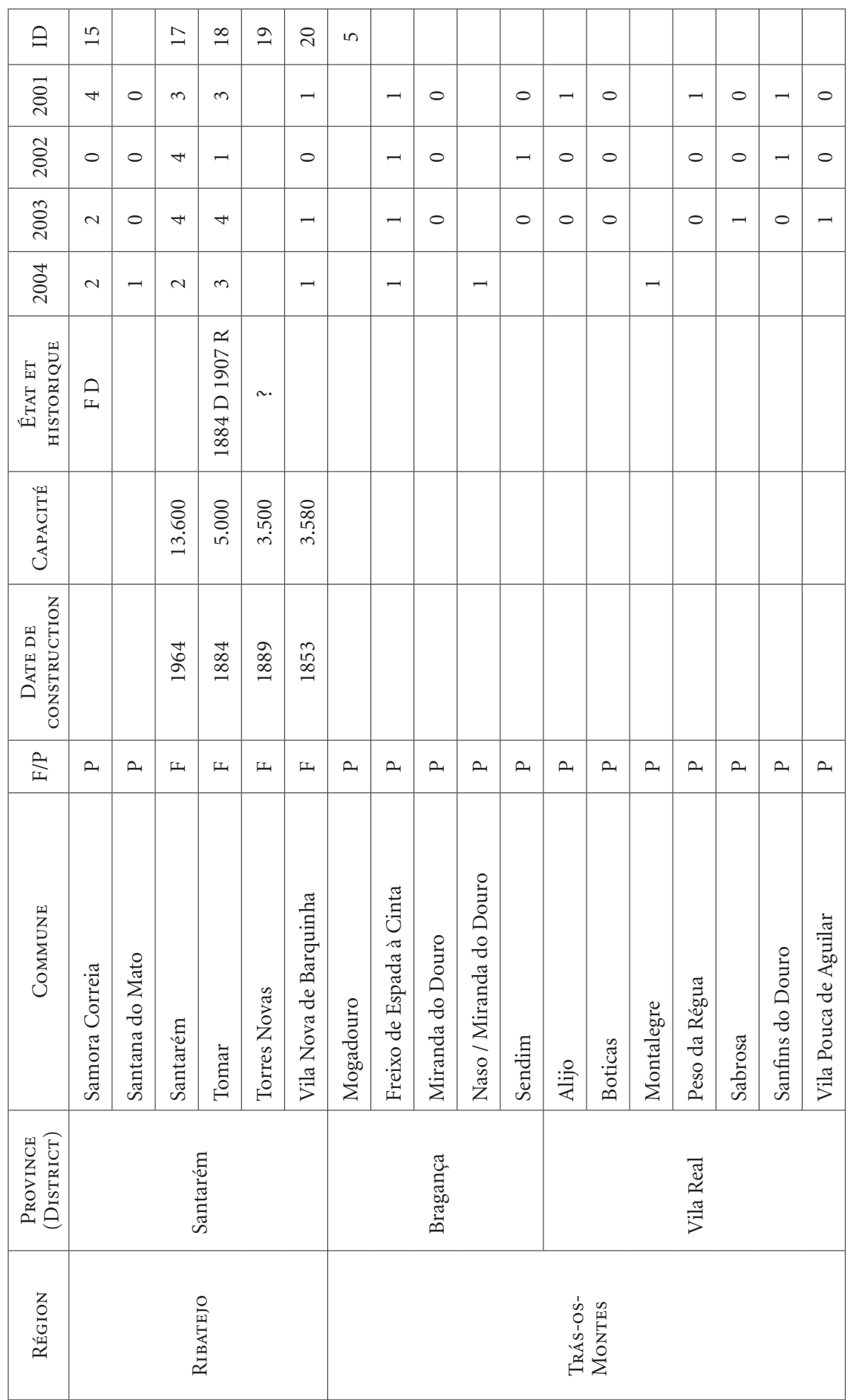




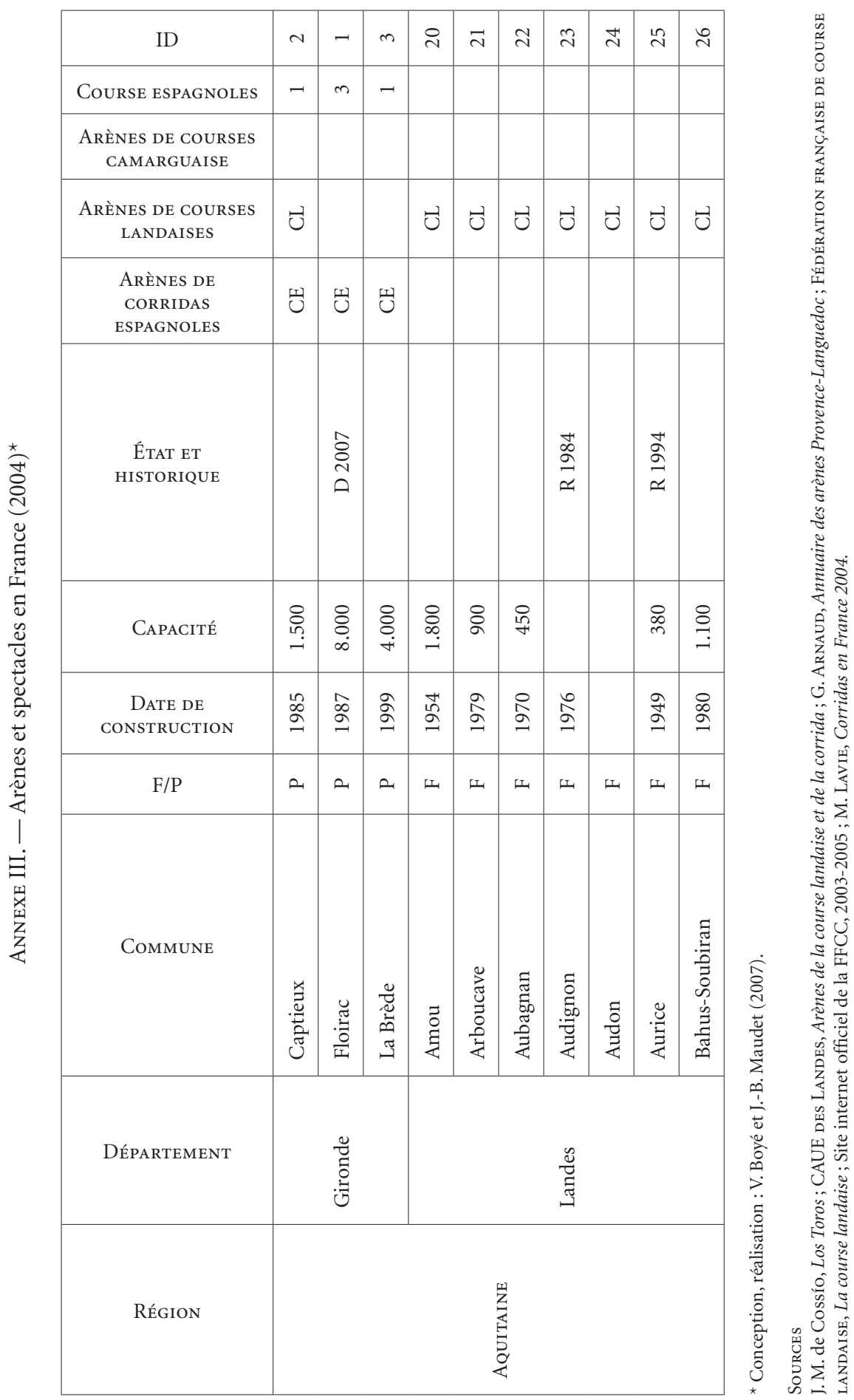




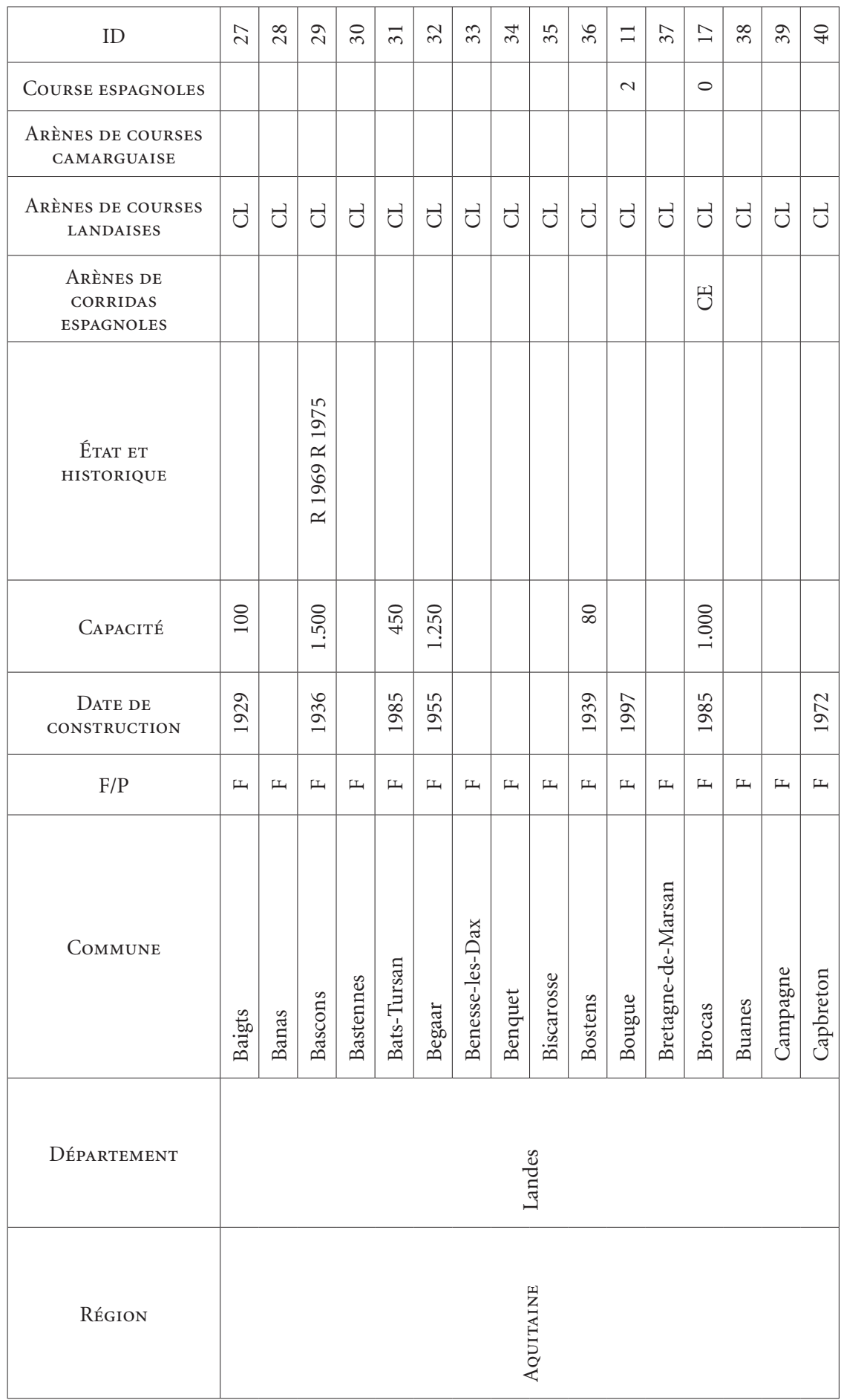




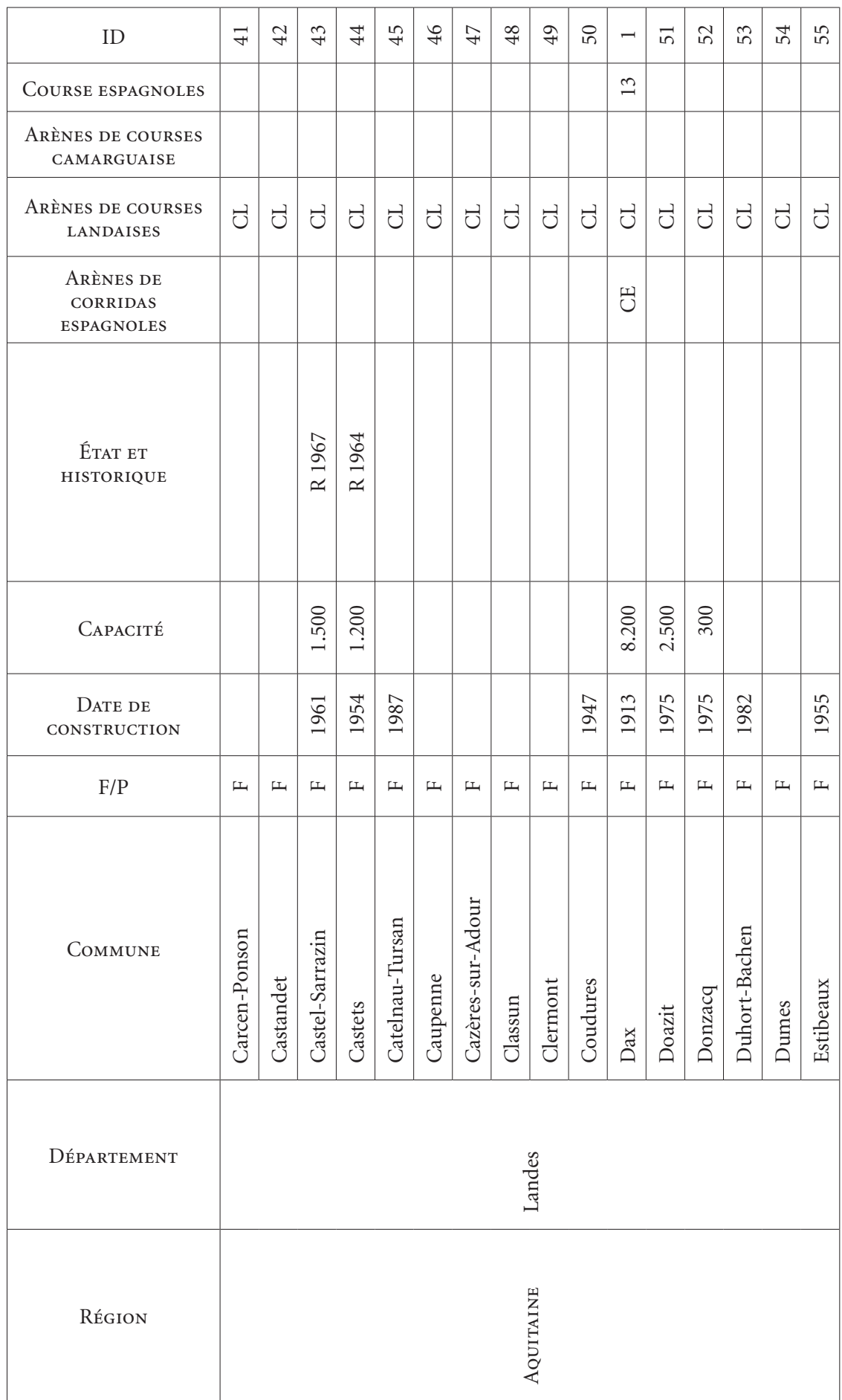




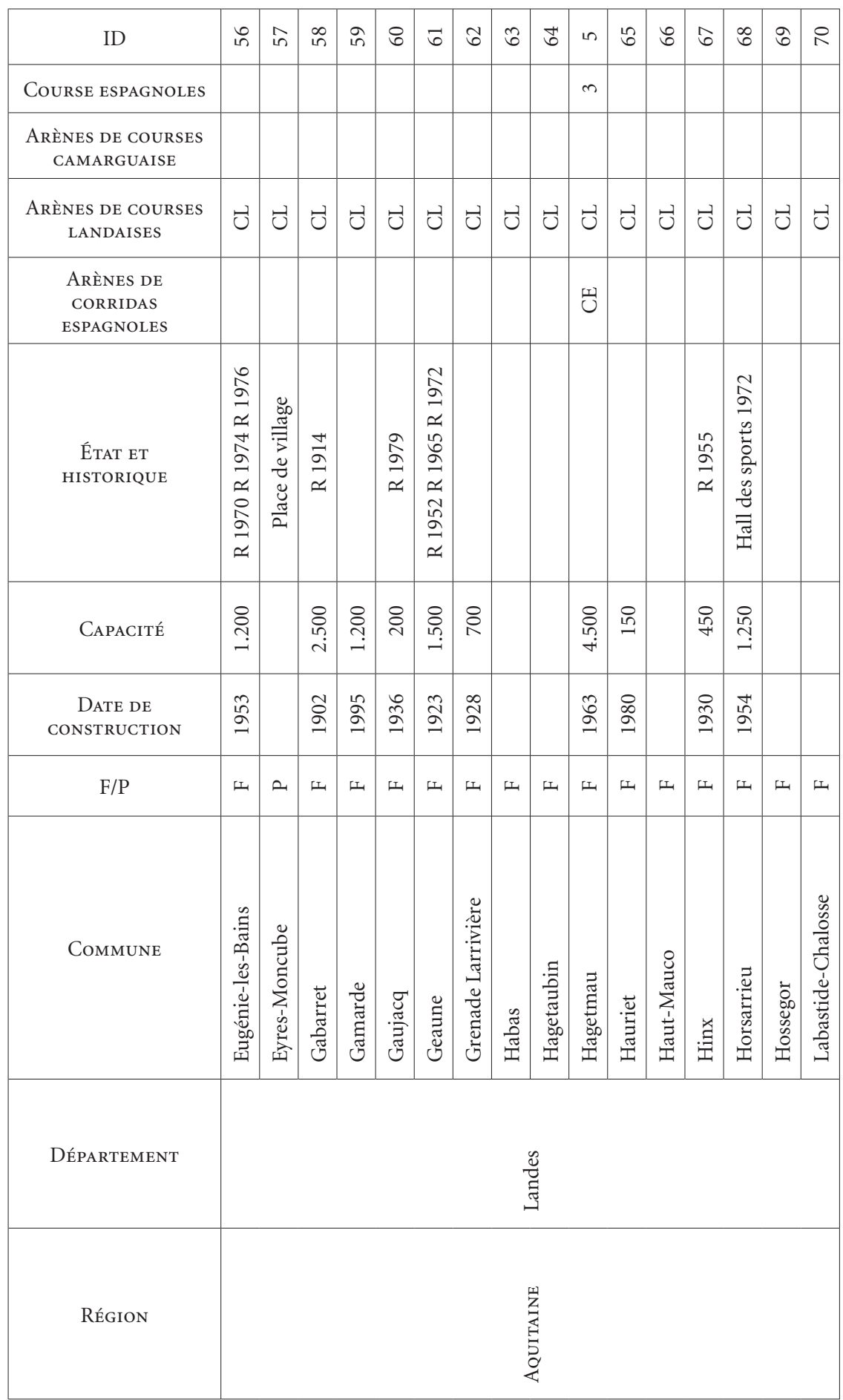




\begin{tabular}{|c|c|c|c|c|c|c|c|c|c|c|c|c|c|c|c|c|}
\hline ID & के & 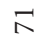 & $\mathrm{N}$ & $\cong$ & 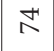 & $\stackrel{2 n}{\wedge}$ & $\stackrel{2}{1}$ & $\curvearrowright$ & $\stackrel{\infty}{\wedge}$ & 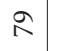 & $\stackrel{2}{2}$ & $\infty$ & $\infty$ & $\infty$ & $\infty$ & $\infty$ \\
\hline Course espagnoles & & & & & & & & & & & $\neg$ & & & & & \\
\hline $\begin{array}{l}\text { ARÈNES DE COURSES } \\
\text { CAMARGUAISE }\end{array}$ & & & & & & & & & & & & & & & & \\
\hline $\begin{array}{c}\text { ARÈNES DE COURSES } \\
\text { LANDAISES }\end{array}$ & U & $\vec{U}$ & 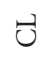 & $\vec{U}$ & 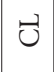 & U & $\bar{U}$ & $\Xi$ & $\theta$ & $\Xi$ & 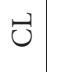 & $\Xi$ & $\vec{J}$ & U & 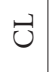 & $\theta$ \\
\hline $\begin{array}{l}\text { ARÈNES DE } \\
\text { CORRIDAS } \\
\text { ESPAGNOLES }\end{array}$ & & & & & & & & & & & 피 & & & & & \\
\hline $\begin{array}{l}\text { ÉtAT ET } \\
\text { HISTORIQUe }\end{array}$ & & & & & $\stackrel{\substack{\infty \\
ٌ}}{\simeq}$ & & & & & & ¿্ণ & & & & & \\
\hline CAPACItÉ & & & & $\stackrel{\infty}{\infty}$ & ஓ & & ஓ् & & & 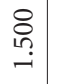 & 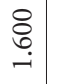 & 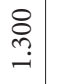 & & 点 & & \\
\hline $\begin{array}{c}\text { DATE DE } \\
\text { CONSTRUCTION }\end{array}$ & & & & $\stackrel{\Re}{\hat{\sigma}}$ & $\stackrel{\infty}{\Omega}$ & & ڤి & & & $\stackrel{\hat{2}}{2}$ & $\stackrel{\infty}{\stackrel{\infty}{二}}$ & ڤి & बू & $\stackrel{\infty}{2}$ & & \\
\hline $\mathrm{F} / \mathrm{P}$ & 工 & 工 & 厌 & 坞 & 山 & 山 & 工 & 山 & 山 & 山 & 山 & 山 & $\omega$ & 山 & 山 & 工 \\
\hline CoMmune & 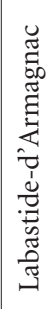 & 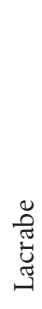 & 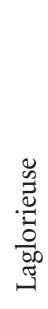 & 矛 & 莺 & 蔦 & 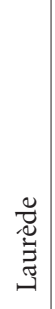 & 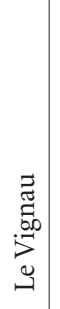 & こ్ & 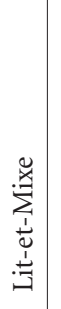 & 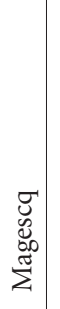 & 壱 & $\stackrel{\Xi}{\stackrel{\Xi}{\Xi}}$ & $\frac{2}{\sqrt{a}}$ & 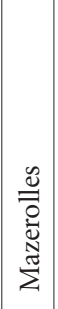 & 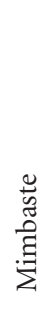 \\
\hline DÉPARTEMENT & \multicolumn{16}{|c|}{ 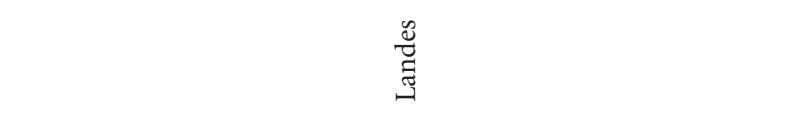 } \\
\hline RÉGION & & & & & & & & $\begin{array}{l}\frac{1}{2} \\
\frac{3}{5} \\
\vdots \\
0\end{array}$ & & & & & & & & \\
\hline
\end{tabular}




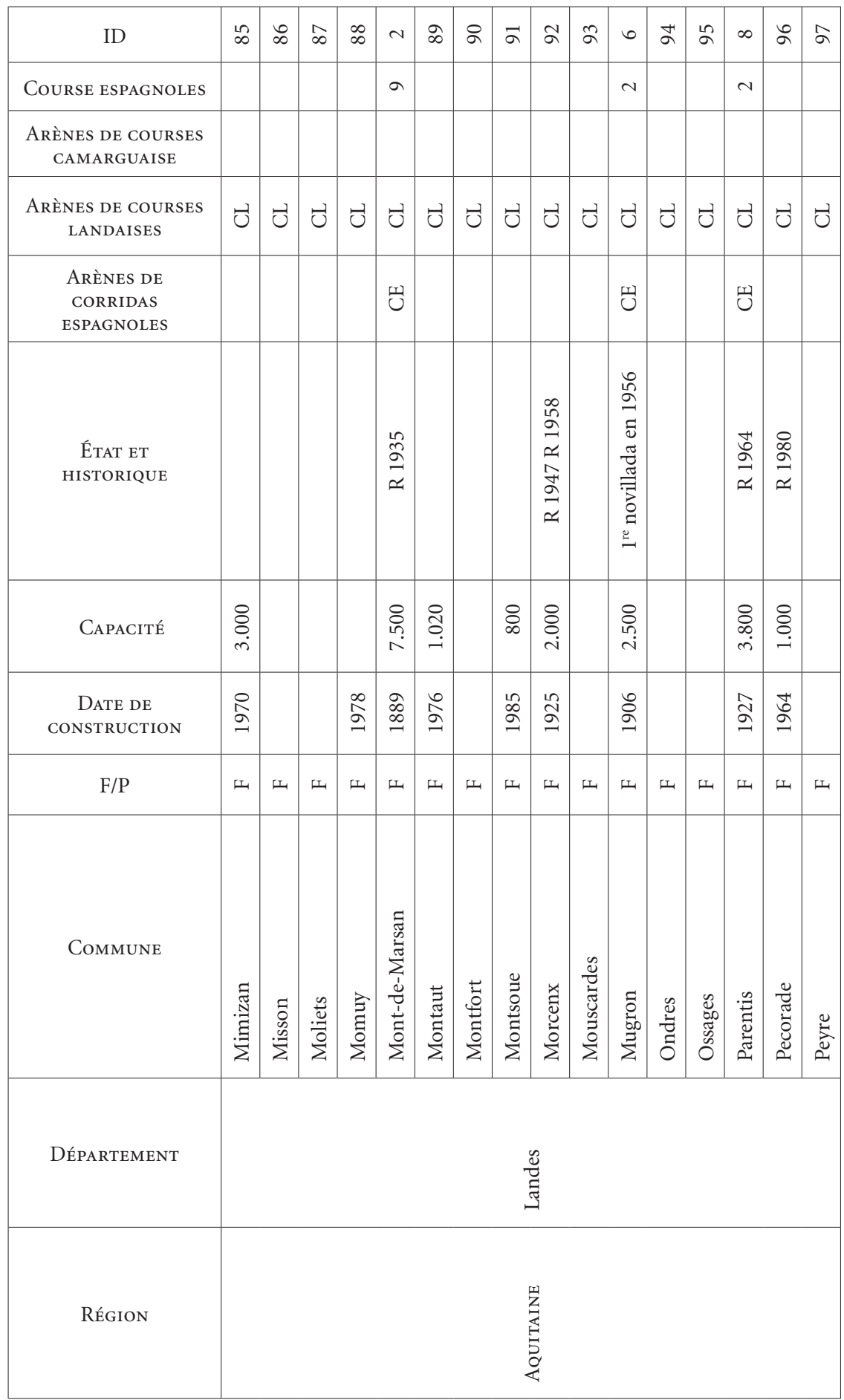




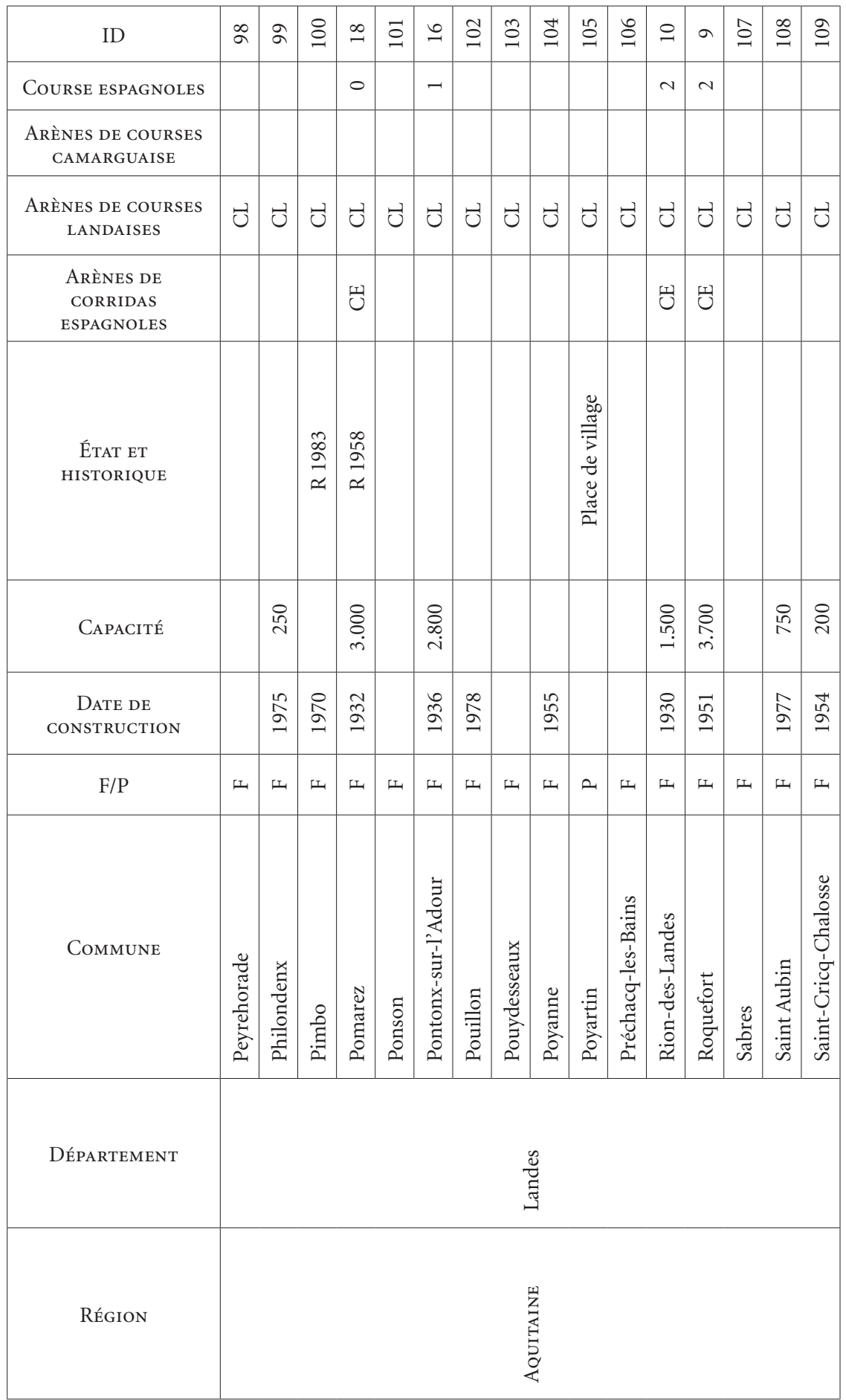




\begin{tabular}{|c|c|c|c|c|c|c|c|c|c|c|c|c|c|c|c|}
\hline ID & $\stackrel{\varrho}{=}$ & $\exists$ & $\stackrel{\beth}{\Xi}$ & $\stackrel{\varrho}{=}$ & $\stackrel{\Xi}{\Xi}$ & $\stackrel{20}{=}$ & $\wedge$ & $\stackrel{0}{=}+$ & n & $气$ & $\stackrel{\infty}{=}$ & $\cong$ & $\stackrel{\vartheta}{\exists}$ & $\stackrel{\Xi}{\approx}$ & $\vec{\beth}$ \\
\hline Course espagnoles & & & & & & & N & + & in & & & $\neg$ & & & \\
\hline \multicolumn{16}{|l|}{$\begin{array}{l}\text { ARÈNES DE COURSES } \\
\text { CAMARGUAISE }\end{array}$} \\
\hline $\begin{array}{l}\text { ARÈNES DE COURSES } \\
\text { LANDAISES }\end{array}$ & 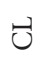 & $\vec{U}$ & $\theta$ & 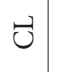 & 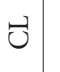 & $\theta$ & $\vec{U}$ & $\theta$ & $\vec{U}$ & $\theta$ & $\theta$ & $\theta$ & 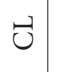 & $\vec{U}$ & $\dot{U}$ \\
\hline $\begin{array}{l}\text { ARÈNES DE } \\
\text { CORRIDAS } \\
\text { ESPAGNOLES }\end{array}$ & & & & & & & પ્ & 凹ુ & 던 & & & 빈 & & & \\
\hline $\begin{array}{l}\text { ÉTAT ET } \\
\text { HISTORIQUE }\end{array}$ & $\frac{\widetilde{\sigma}}{2}$ & & & & $\begin{array}{l}\stackrel{0}{2} \\
\stackrel{2}{a} \\
\simeq\end{array}$ & & 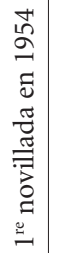 & & 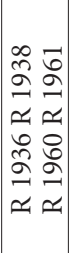 & & & $\frac{N}{\stackrel{N}{a}}$ & & $\begin{array}{l}\stackrel{\infty}{\infty} \\
\stackrel{2}{\simeq}\end{array}$ & \\
\hline CAPACitÉ & 孚 & 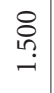 & & 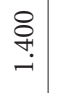 & 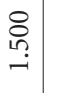 & & 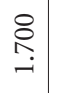 & $\begin{array}{l}8 \\
\infty \\
\dot{m}\end{array}$ & $\begin{array}{l}8 \\
\text { in } \\
\text { in }\end{array}$ & 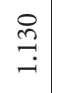 & $\stackrel{\infty}{\infty}$ & $\underset{\substack{\infty \\
\infty}}{\infty}$ & & $\begin{array}{l}8 \\
\text { กุ } \\
\end{array}$ & \\
\hline $\begin{array}{c}\text { DATE DE } \\
\text { CONSTRUCTION }\end{array}$ & $\stackrel{\infty}{\stackrel{\infty}{\sigma}}$ & $\stackrel{2}{2}$ & & $\stackrel{\infty}{\circ}$ & ลิ & & $\stackrel{\Re}{2}$ & $\vec{a}$ & $\stackrel{\text { ڤ̆ }}{=}$ & 今 & $\stackrel{\infty}{2}$ & 은 & & $\stackrel{\circ}{2}$ & $\stackrel{2}{\Omega}$ \\
\hline $\mathrm{F} / \mathrm{P}$ & 山 & 工 & 山 & 山 & 山 & 山 & 厌 & 山出 & 山 & 出 & 工 & 山 & 工 & 工 & 工 \\
\hline Commune & 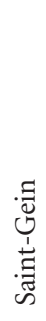 & 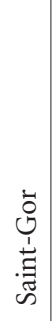 & 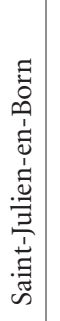 & 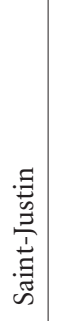 & 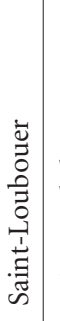 & 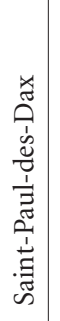 & 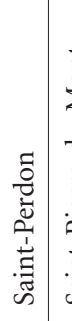 & 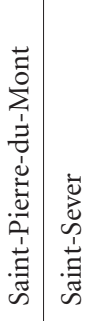 & 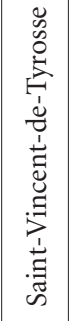 & 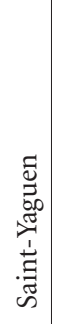 & 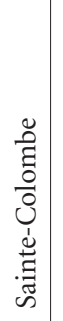 & 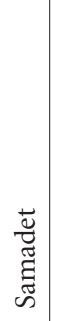 & 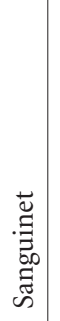 & 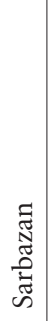 & 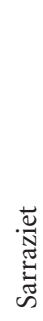 \\
\hline DÉPARTEMENT & & & & & & & & $\begin{array}{l}\mathscr{U} \\
\tilde{\Xi} \\
\tilde{\Xi}\end{array}$ & & & & & & & \\
\hline RÉGION & & & & & & & & 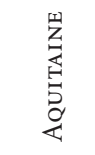 & & & & & & & \\
\hline
\end{tabular}




\begin{tabular}{|c|c|c|c|c|c|c|c|c|c|c|c|c|c|c|c|c|}
\hline ID & $\tilde{\Xi}$ & $\tilde{\Xi}$ & $\stackrel{\stackrel{H}{J}}{ }$ & $\stackrel{2}{\cong}$ & 2 & 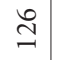 & $\widehat{\Xi}$ & $\stackrel{\infty}{\simeq}$ & 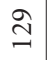 & 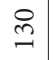 & $\vec{n}$ & $\cong ี$ & $\stackrel{m}{n}$ & 2 & $\Xi$ & - \\
\hline Course espagnoles & & & & & - & & & & & & & & & 0 & - & \\
\hline \multicolumn{17}{|l|}{$\begin{array}{l}\text { ARÈNES DE COURSES } \\
\text { CAMARGUAISE }\end{array}$} \\
\hline $\begin{array}{l}\text { ARÈNES DE COURSES } \\
\text { LANDAISES }\end{array}$ & 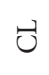 & U & $\theta$ & $\vec{U}$ & 0 & $\vec{U}$ & 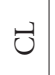 & $\vec{U}$ & $\theta$ & 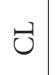 & U & $\theta$ & $\vec{U}$ & $\vec{U}$ & $\vec{U}$ & $\vec{U}$ \\
\hline $\begin{array}{l}\text { ARÈNES DE } \\
\text { CORRIDAS } \\
\text { ESPAGNOLES }\end{array}$ & & & & & 凹ુ & & & & & & & & & 버 & 너 & \\
\hline $\begin{array}{l}\text { État ET } \\
\text { HISTORIQue }\end{array}$ & & & & & 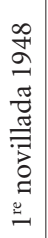 & & & & & & & $\begin{array}{l}\stackrel{\infty}{\varrho} \\
\stackrel{\sim}{\simeq}\end{array}$ & & & 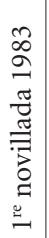 & \\
\hline CAPACITÉ & & 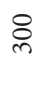 & $\stackrel{\circ}{2}$ & 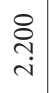 & 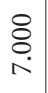 & & 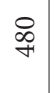 & 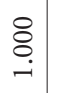 & 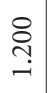 & 孚 & & $\stackrel{8}{\infty}$ & 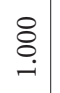 & $\underset{+}{\stackrel{\leftrightarrow}{+}}$ & $\begin{array}{l}8 \\
\vdots \\
i \\
i\end{array}$ & \\
\hline $\begin{array}{c}\text { DATE DE } \\
\text { CONSTRUCTION }\end{array}$ & & $\hat{\Omega}$ & & $\stackrel{\infty}{2}$ & $\stackrel{む}{\Xi}$ & & $\begin{array}{l}\hat{\infty} \\
\infty \\
\sim\end{array}$ & $\stackrel{0}{\stackrel{0}{2}}$ & $\stackrel{\triangleright}{\stackrel{2}{二}}$ & $\stackrel{2}{2}$ & & $\stackrel{\circ}{\stackrel{2}{a}}$ & $\stackrel{a}{\stackrel{a}{a}}$ & むั & $\vec{\sigma}$ & \\
\hline $\mathrm{F} / \mathrm{P}$ & 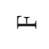 & 原 & 厌 & 工 & 工 & 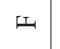 & 工 & 工 & 垊 & 山 & 圷 & 山 & 山 & 丰 & 山 & 山 \\
\hline Commune & 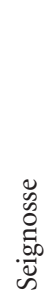 & 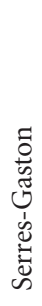 & 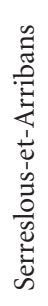 & 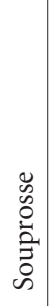 & 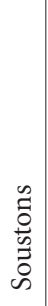 & 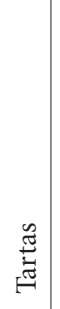 & 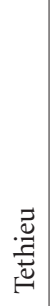 & $\stackrel{\Xi}{\equiv}$ & 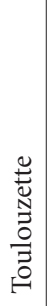 & 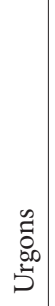 & 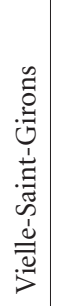 & 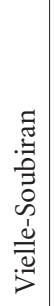 & 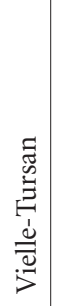 & 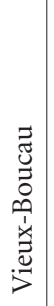 & 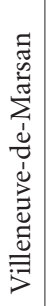 & 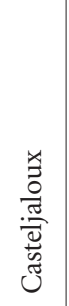 \\
\hline DÉPARTEMENT & & & & & & & & 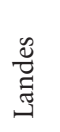 & & & & & & & & 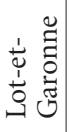 \\
\hline RÉGION & & & & & & & & 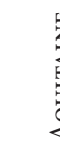 & 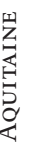 & & & & & & & \\
\hline
\end{tabular}




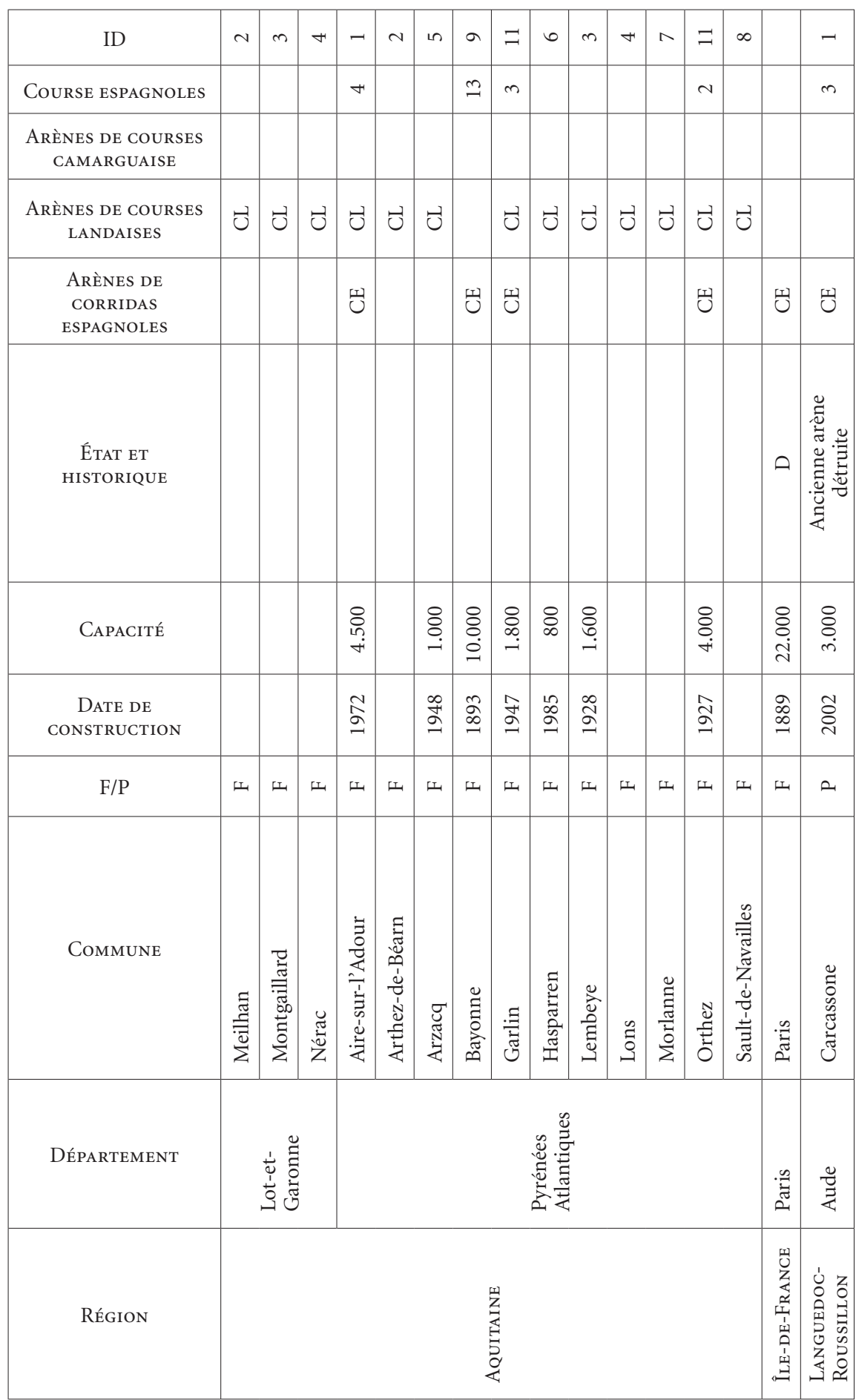




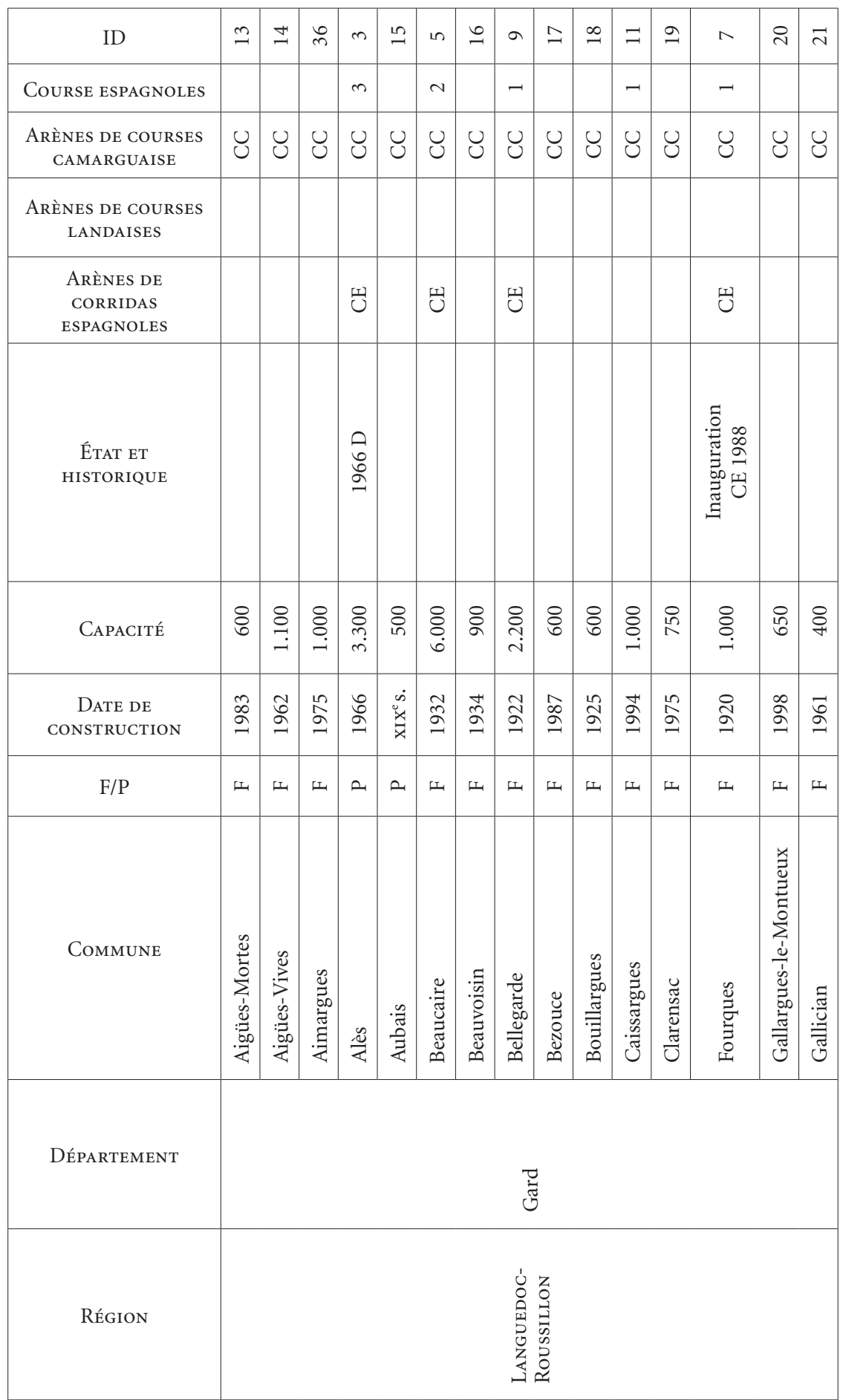




\begin{tabular}{|c|c|c|c|c|c|c|c|c|c|c|c|c|c|c|c|c|}
\hline ID & ส & 으 & $\ddot{\sim}$ & $\stackrel{\sim}{\sim}$ & $\sim$ & $\stackrel{\stackrel{n}{N}}{ }$ & $\stackrel{\sim}{\sim}$ & $\widehat{\triangleq}$ & $\stackrel{\infty}{\sim}$ & ঐે & - & in & $\vec{m}$ & $\pi$ & $\tilde{m}$ & H \\
\hline COURSE ESPAGNOLES & & - & & & H & & & & & & ป & & & & & $m$ \\
\hline $\begin{array}{l}\text { ARÈNES DE COURSES } \\
\text { CAMARGUAISE }\end{array}$ & ن & ن & U & U & & U & $\cup$ & U & $\cup$ & $\cup$ & U & U & U & U & $\cup$ & U \\
\hline $\begin{array}{c}\text { ARÈNES DE COURSES } \\
\text { LANDAISES }\end{array}$ & & & & & $\vec{U}$ & & & & & & & & & & & \\
\hline $\begin{array}{c}\text { ARÈNES DE } \\
\text { CORRIDAS } \\
\text { ESPAGNOLES }\end{array}$ & & 던 & & & 벙 & & & & & & 떠 & & & & & 펀 \\
\hline $\begin{array}{c}\text { ÉTAT ET } \\
\text { HISTORIQUE }\end{array}$ & & & & & & & & & & & 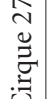 & & & & & \\
\hline CAPACITÉ & 号 & \& & \& & : & $\begin{array}{l}8 \\
\dot{m}\end{array}$ & $\underset{-}{8}$ & 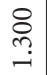 & 总 & $\stackrel{\infty}{\circ}$ & $\stackrel{\circ}{n}$ & 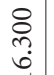 & $\underset{\infty}{\varnothing}$ & 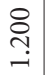 & ஓ & 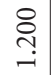 & 용 \\
\hline $\begin{array}{c}\text { DATE DE } \\
\text { CONSTRUCTION }\end{array}$ & สู & $\begin{array}{l}\infty \\
ٌ\end{array}$ & $\stackrel{\infty}{\Omega}$ & 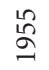 & $\stackrel{\circ}{\circ}$ & گે & 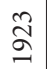 & $\stackrel{2}{2}$ & $\stackrel{2}{\Xi}$ & $\stackrel{\infty}{\stackrel{\infty}{2}}$ & $\stackrel{\substack{\infty \\
\infty}}{\infty}$ & $\overline{\check{\sigma}}$ & $\stackrel{2}{2}$ & $\stackrel{2}{\varrho}$ & $\stackrel{\infty}{2}$ & $\stackrel{\infty}{\sim}$ \\
\hline $\mathrm{F} / \mathrm{P}$ & 山 & 山 & 山 & 山 & 山 & 山 & 山 & 山 & 山 & 山 & 山 & 山 & $山$ & 山 & 山 & 山 \\
\hline Commune & 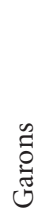 & 苞 & 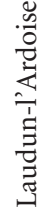 & 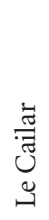 & 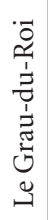 & $\begin{array}{l}\overrightarrow{\mathcal{g}} \\
\text { है } \\
\text { 志 }\end{array}$ & 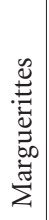 & 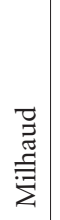 & $\begin{array}{l}\underset{\Xi}{\Xi} \\
\stackrel{\Xi}{\Sigma}\end{array}$ & 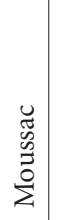 & $\begin{array}{l}\stackrel{\mathscr{Z}}{\Xi} \\
\text { Z } \\
\text { Z }\end{array}$ & 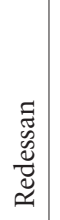 & 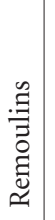 & 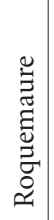 & 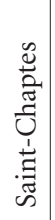 & 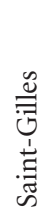 \\
\hline DÉPARTEMENT & & \multicolumn{15}{|c|}{ تृ } \\
\hline RÉGION & & & & & & & & 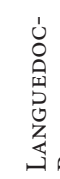 & 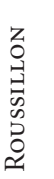 & & & & & & & \\
\hline
\end{tabular}




\begin{tabular}{|c|c|c|c|c|c|c|c|c|c|c|c|c|c|c|c|}
\hline ID & $\infty$ & $\hat{n}$ & Hे & $\stackrel{m}{m}$ & $\simeq$ & 6 & $\stackrel{\infty}{m}$ & $n$ & 6 & - & $\infty$ & $a$ & 으 & $=$ & $\simeq$ \\
\hline CoURSE ESPAGNOLES & - & & & & 0 & $\sim$ & $\sim$ & & & $\wedge$ & & & & & \\
\hline $\begin{array}{l}\text { ARÈNES DE COURSES } \\
\text { CAMARGUAISE }\end{array}$ & $\cup$ & U & U & $\cup$ & $\cup$ & $\cup$ & $\cup$ & U & & & U & U & $\cup$ & $\cup$ & $\dot{U}$ \\
\hline \multicolumn{16}{|l|}{$\begin{array}{l}\text { ARÈNES DE COURSES } \\
\text { LANDAISES }\end{array}$} \\
\hline $\begin{array}{c}\text { ARÈNES DE } \\
\text { CORRIDAS } \\
\text { ESPAGNOLES }\end{array}$ & ஸુ & & & & ખ્ & 던 & & & 더 & 너 & & & & & \\
\hline $\begin{array}{l}\text { ÉtAT ET } \\
\text { HistoriQue }\end{array}$ & & $\frac{\nwarrow}{\curvearrowright}$ & & & & & & & & $\begin{array}{l}\overrightarrow{\widetilde{\Omega}} \\
\widetilde{\simeq}\end{array}$ & & & & & \\
\hline CAPACitÉ & 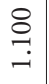 & 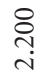 & 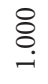 & 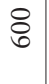 & 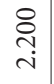 & 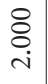 & ¿ & $\stackrel{\infty}{-\infty}$ & & 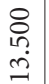 & ஓ & 总 & 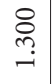 & 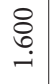 & $\stackrel{8}{8}$ \\
\hline $\begin{array}{c}\text { DATE DE } \\
\text { CONSTRUCTION }\end{array}$ & $\begin{array}{l}\stackrel{2}{\circ} \\
\stackrel{二}{二}\end{array}$ & $\stackrel{\aleph}{\Omega}$ & $\stackrel{\infty}{\sigma}$ & $\begin{array}{l}\stackrel{\infty}{2} \\
\stackrel{2}{2}\end{array}$ & $\stackrel{\mathscr{0}}{2}$ & ڤ్ & बू & $\stackrel{\infty}{2}$ & & $\underset{\infty}{\infty}$ & 苋 & $\stackrel{\widehat{\infty}}{\varrho}$ & $\stackrel{+}{\circ}$ & ๙ू & హ。 \\
\hline $\mathrm{F} / \mathrm{P}$ & $\infty$ & 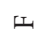 & $\omega$ & 山 & $\omega$ & 山 & 山 & 山 & $a$ & $\omega$ & 山 & a & 山 & 山 & 山 \\
\hline Commune & 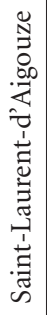 & 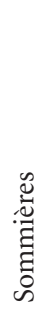 & $\stackrel{\mathscr{N}}{s}$ & 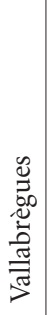 & 槖 & 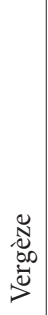 & 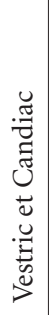 & 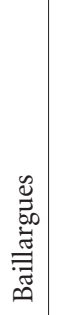 & 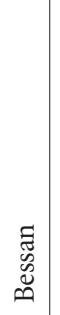 & . & 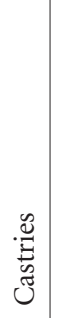 & 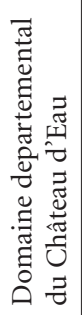 & 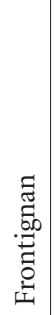 & 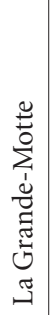 & 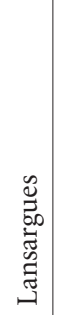 \\
\hline DÉPARTEMENT & & & & تีّ & & & & & & & & 节 & & & \\
\hline RÉGION & & & & & & & & 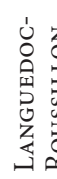 & & & & & & & \\
\hline
\end{tabular}




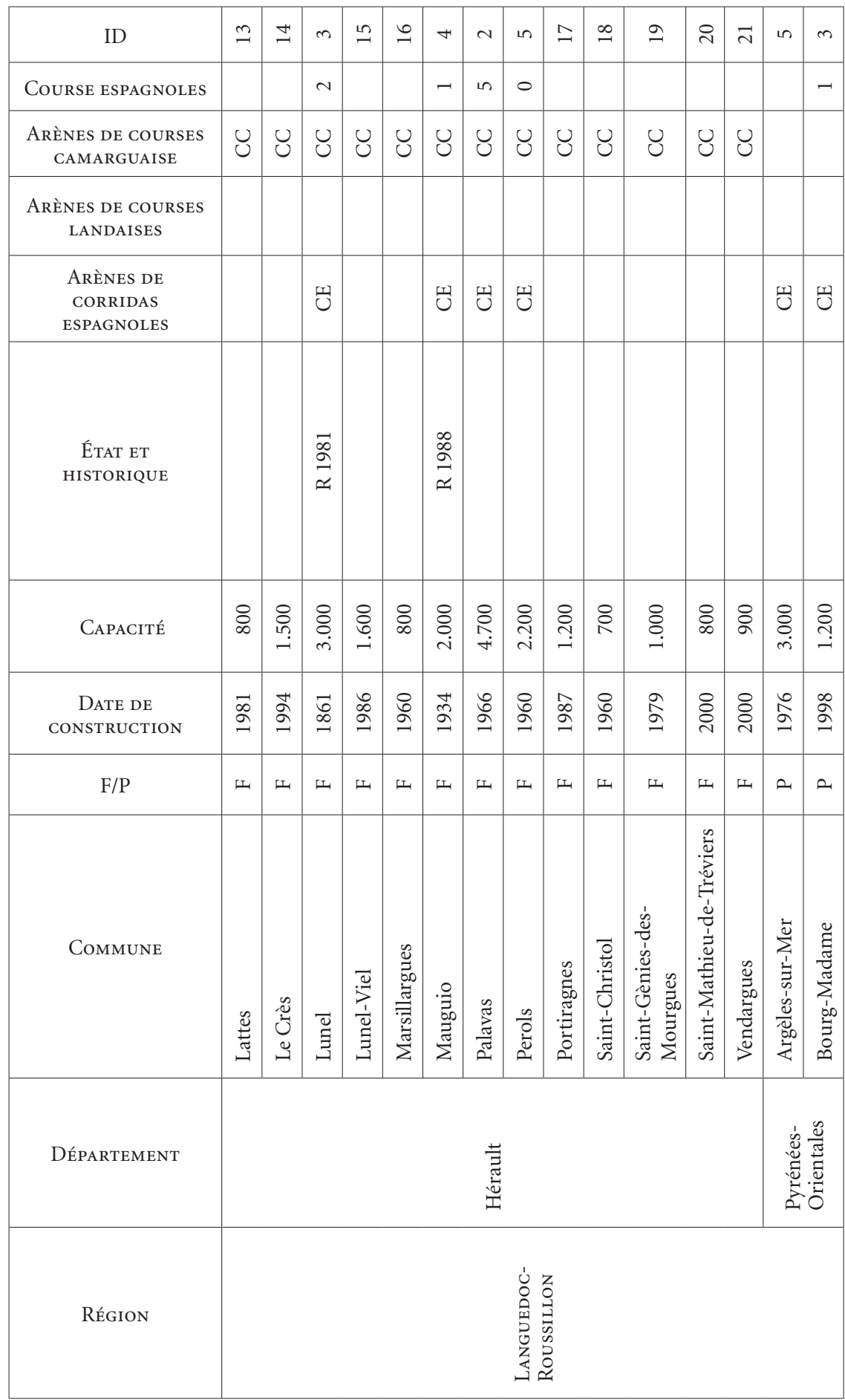




\begin{tabular}{|c|c|c|c|c|c|c|c|c|c|c|c|c|c|c|c|c|}
\hline ID & - & N & $H$ & $m$ & $a$ & 으 & $=$ & 6 & $\sim$ & 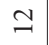 & $\stackrel{m}{2}$ & $H$ & $\Xi$ & $\Lambda$ & $\stackrel{10}{2}$ & $\stackrel{0}{-}$ \\
\hline COURSE ESPAGNOLES & in & $m$ & - & $\mathrm{N}$ & & & & - & $H$ & & & - & & - & & \\
\hline \multicolumn{17}{|l|}{$\begin{array}{l}\text { ARÈNES DE COURSES } \\
\text { CAMARGUAISE }\end{array}$} \\
\hline $\begin{array}{c}\text { ARÈNES DE COURSES } \\
\text { LANDAISES }\end{array}$ & & & & ت્ & $\vec{U}$ & 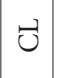 & 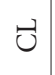 & U. & U & $\theta$ & $\vec{U}$ & $\theta$ & $\vec{U}$ & $\theta$ & 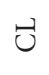 & U \\
\hline $\begin{array}{l}\text { ARÈNES DE } \\
\text { CORRIDAS } \\
\text { ESPAGNOLES }\end{array}$ & ஸ્ & 떤 & பึ & પ્યુ & & & & 더 & પ્ & & & પ્પ & & & & \\
\hline $\begin{array}{l}\text { ÉTAT ET } \\
\text { HISTORIQUE }\end{array}$ & & 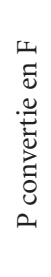 & & & & & & & & 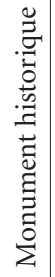 & & & & & & \\
\hline CAPACITÉ & $\underset{+}{8}$ & $\begin{array}{l}8 \\
i n \\
n \\
m\end{array}$ & @ & 辛 & & $\begin{array}{l}8 \\
i n \\
i\end{array}$ & 辛 & $\stackrel{\circ}{\stackrel{n}{n}}$ & $\begin{array}{l}8 \\
\dot{0} \\
\dot{m}\end{array}$ & @ & 옷 & $\begin{array}{l}8 \\
\substack{10 \\
m} \\
m\end{array}$ & & ì & & \\
\hline $\begin{array}{c}\text { DATE DE } \\
\text { CONSTRUCTION }\end{array}$ & స్ & & ळे & & & & & & $\begin{array}{l}\infty \\
\stackrel{2}{\Omega}\end{array}$ & Бे & $\stackrel{\widehat{\Omega}}{ }$ & & & & & \\
\hline $\mathrm{F} / \mathrm{P}$ & 工 & a & $a$ & $\Phi$ & 工 & I & 山 & 山 & 山 & 山 & 山 & $a$ & 山 & 山 & 山 & 山 \\
\hline Commune & Uัँ & 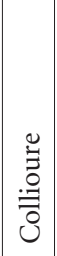 & 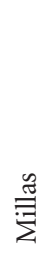 & 芯 & 节 & 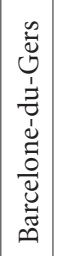 & 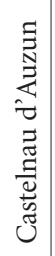 & 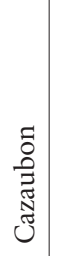 & 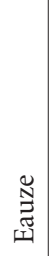 & 焉 & 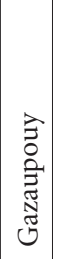 & 泀 & 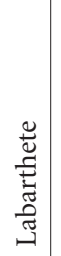 & 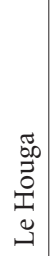 & 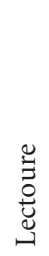 & : \\
\hline DÉPARTEMENT & & 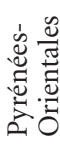 & & & & & & & & ڤ્⿹ & & & & & & \\
\hline RÉGION & & 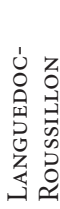 & & & & & & & & 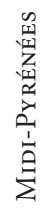 & & & & & & \\
\hline
\end{tabular}




\begin{tabular}{|c|c|c|c|c|c|c|c|c|c|c|c|c|c|c|c|}
\hline ID & $\beth$ & $\stackrel{\infty}{=}$ & 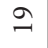 & 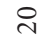 & $\vec{N}$ & $\widetilde{N}$ & $\tilde{\sim}$ & $\stackrel{\Xi}{\sim}$ & $\infty$ & in & $\stackrel{\stackrel{2}{\sim}}{ }$ & $\stackrel{\sim}{\sim}$ & $\widehat{\curvearrowright}$ & $\stackrel{\infty}{\sim}$ & - \\
\hline COURSE ESPAGNOLES & & & & & & & & & - & - & & & & & $\infty$ \\
\hline $\begin{array}{l}\text { ARÈNES DE COURSES } \\
\text { CAMARGUAISE }\end{array}$ & & & & & & & & & & & & & & & \\
\hline $\begin{array}{c}\text { ARÈNES DE COURSES } \\
\text { LANDAISES }\end{array}$ & $\overrightarrow{0}$ & $\vec{U}$ & U & $\theta$ & $\vec{U}$ & $\overrightarrow{0}$ & $\theta$ & $\theta$ & $\theta$ & $\vec{U}$ & $\vec{U}$ & $\overrightarrow{0}$ & U & త & \\
\hline $\begin{array}{c}\text { ARÈNES DE } \\
\text { CORRIDAS } \\
\text { ESPAGNOLES }\end{array}$ & & & & & & & & & & પ્યુ & & & & & 덩 \\
\hline $\begin{array}{l}\text { ÉTAT ET } \\
\text { HISTORIQUE }\end{array}$ & & & & & & & & & & 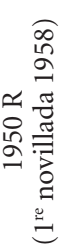 & & & \begin{tabular}{l}
\multicolumn{1}{c}{} \\
$\infty$ \\
$\stackrel{2}{-}$
\end{tabular} & & \\
\hline CAPACITÉ & 官 & : & & & & 竞 & ஷे & 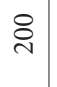 & 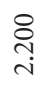 & 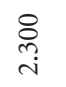 & & $\begin{array}{l}\stackrel{8}{\infty} \\
\infty \\
- \\
-\end{array}$ & $\stackrel{8}{\circ}$ & 융 & 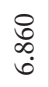 \\
\hline $\begin{array}{c}\text { DATE DE } \\
\text { CONSTRUCTION }\end{array}$ & & & & & & & ลે & §̊ & & ஓ & & & $\underset{\substack{1 \\
\rightarrow \infty}}{-1}$ & & 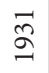 \\
\hline $\mathrm{F} / \mathrm{P}$ & 山 & 工 & 山 & 山 & 工 & $\omega$ & $\omega$ & 山 & L & 工 & 工 & 山 & 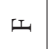 & 工 & 山 \\
\hline Commune & 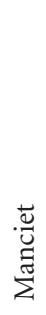 & 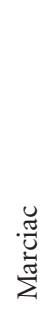 & 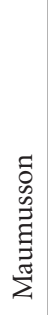 & 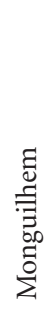 & 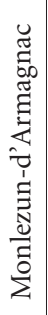 & 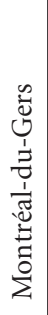 & 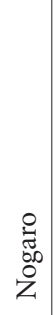 & . & 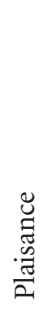 & $\begin{array}{l}\frac{u}{u} \\
\check{2}\end{array}$ & 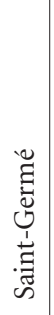 & 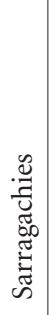 & 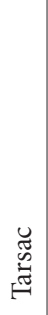 & 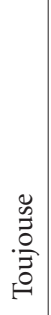 & 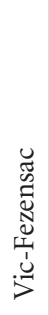 \\
\hline DÉPARTEMENT & \multicolumn{15}{|c|}{$\infty$} \\
\hline RÉGION & & & & & & & & 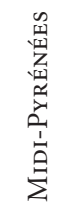 & & & & & & & \\
\hline
\end{tabular}




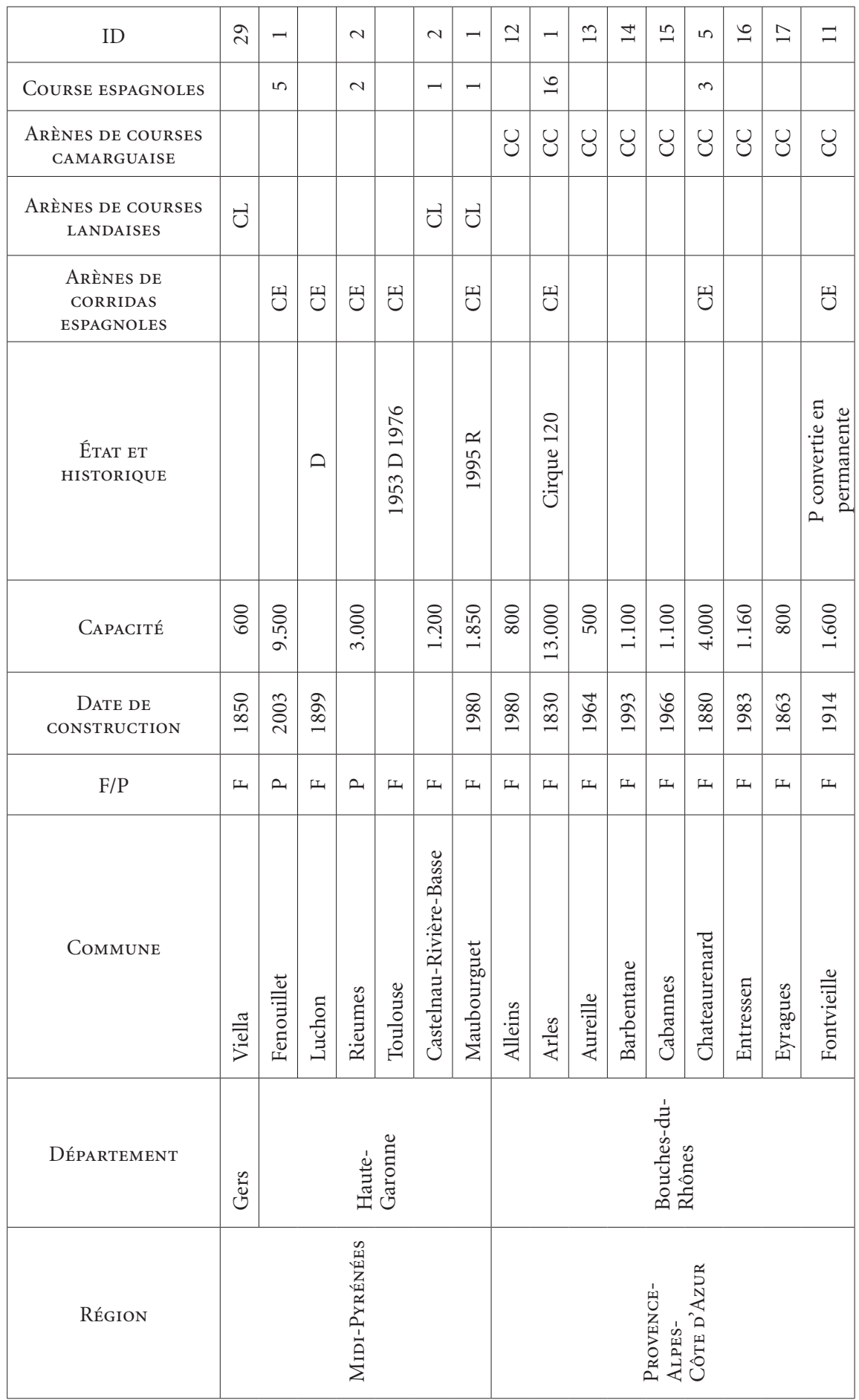




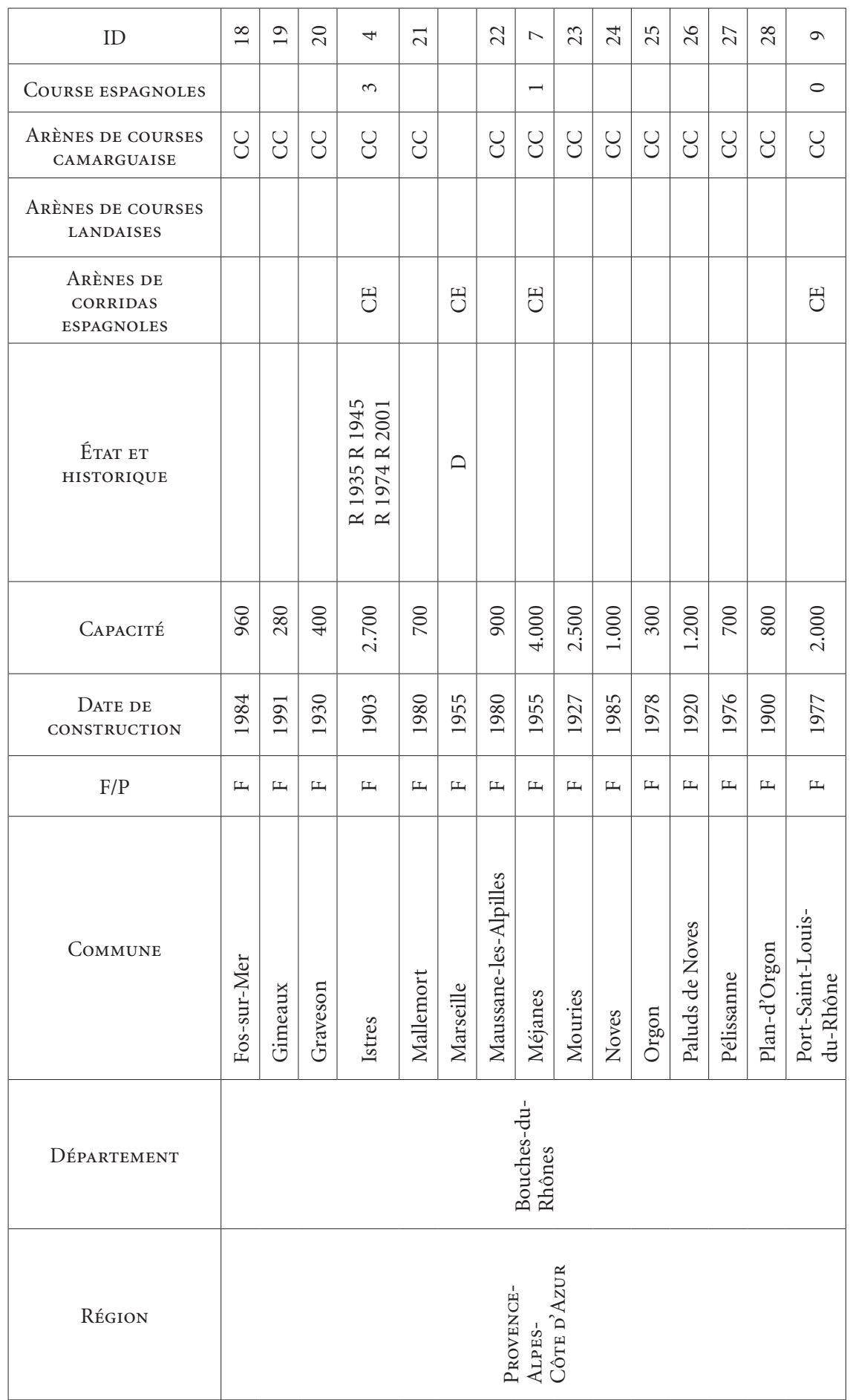


ANNEXES

\begin{tabular}{|c|c|c|c|c|c|c|c|c|c|c|c|c|c|}
\hline ID & શે & ळे & $\vec{m}$ & n & 6 & $\approx$ & $\sim$ & 으 & $\infty$ & - & & - & $\sim$ \\
\hline COURSE ESPAGNOLES & & & & n & - & & H & 0 & - & H & & & \\
\hline $\begin{array}{l}\text { ARÈNES DE COURSES } \\
\text { CAMARGUAISE }\end{array}$ & ن & U & ن & U & U & $\dot{U}$ & U & U & & & U & $\cup$ & ن \\
\hline \multicolumn{14}{|l|}{$\begin{array}{l}\text { ARÈNES DE COURSES } \\
\text { LANDAISES }\end{array}$} \\
\hline $\begin{array}{l}\text { ARÈNES DE } \\
\text { CORRIDAS } \\
\text { ESPAGNOLES }\end{array}$ & & & & 번 & 더 & & 떤 & 떠 & 떠 & ્ુ & & & \\
\hline $\begin{array}{l}\text { ÉtAT ET } \\
\text { HISTORIQUE }\end{array}$ & & & & & & & & & & & D & & \\
\hline CAPACITÉ & 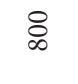 & ஓ & 8 & 总 & $\underset{\substack{0 \\
i}}{\stackrel{8}{1}}$ & 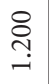 & 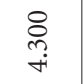 & ¿ & $\underset{7}{\stackrel{8}{\leftrightarrows}}$ & $\begin{array}{l}8 \\
\vdots \\
\dot{m}\end{array}$ & 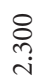 & 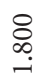 & 8 \\
\hline $\begin{array}{c}\text { DATE DE } \\
\text { CONSTRUCTION }\end{array}$ & $\stackrel{\infty}{\stackrel{2}{\beth}}$ & $\stackrel{\curvearrowright}{\curvearrowright}$ & $\stackrel{2}{2}$ & ঃ & $\underset{\infty}{\infty}$ & $\stackrel{m}{\infty}$ & $\bar{\Omega}$ & $\stackrel{\infty}{\varrho}$ & $\stackrel{\Re}{2}$ & $\stackrel{2}{2}$ & $\stackrel{\llcorner}{2}$ & $\stackrel{\curvearrowright}{\stackrel{\partial}{\sigma}}$ & $\stackrel{\circ}{\circ}$ \\
\hline$F / P$ & I & 山 & 工 & 工 & $\omega$ & $\omega$ & w & 山 & 山 & 山 & 山 & 山 & 山 \\
\hline Commune & 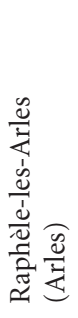 & 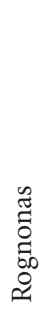 & 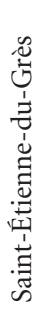 & 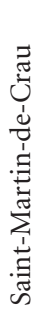 & 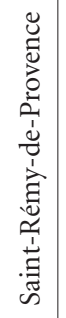 & 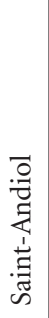 & 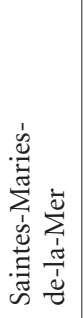 & 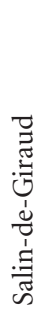 & 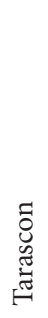 & : & 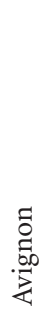 & 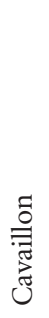 & 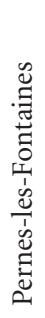 \\
\hline DÉPARTEMENT & & & & & 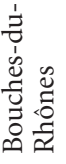 & & & & & के & & 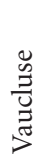 & \\
\hline RÉGION & & & & & & & 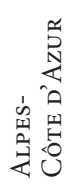 & & & & & & \\
\hline
\end{tabular}


ANNEXES

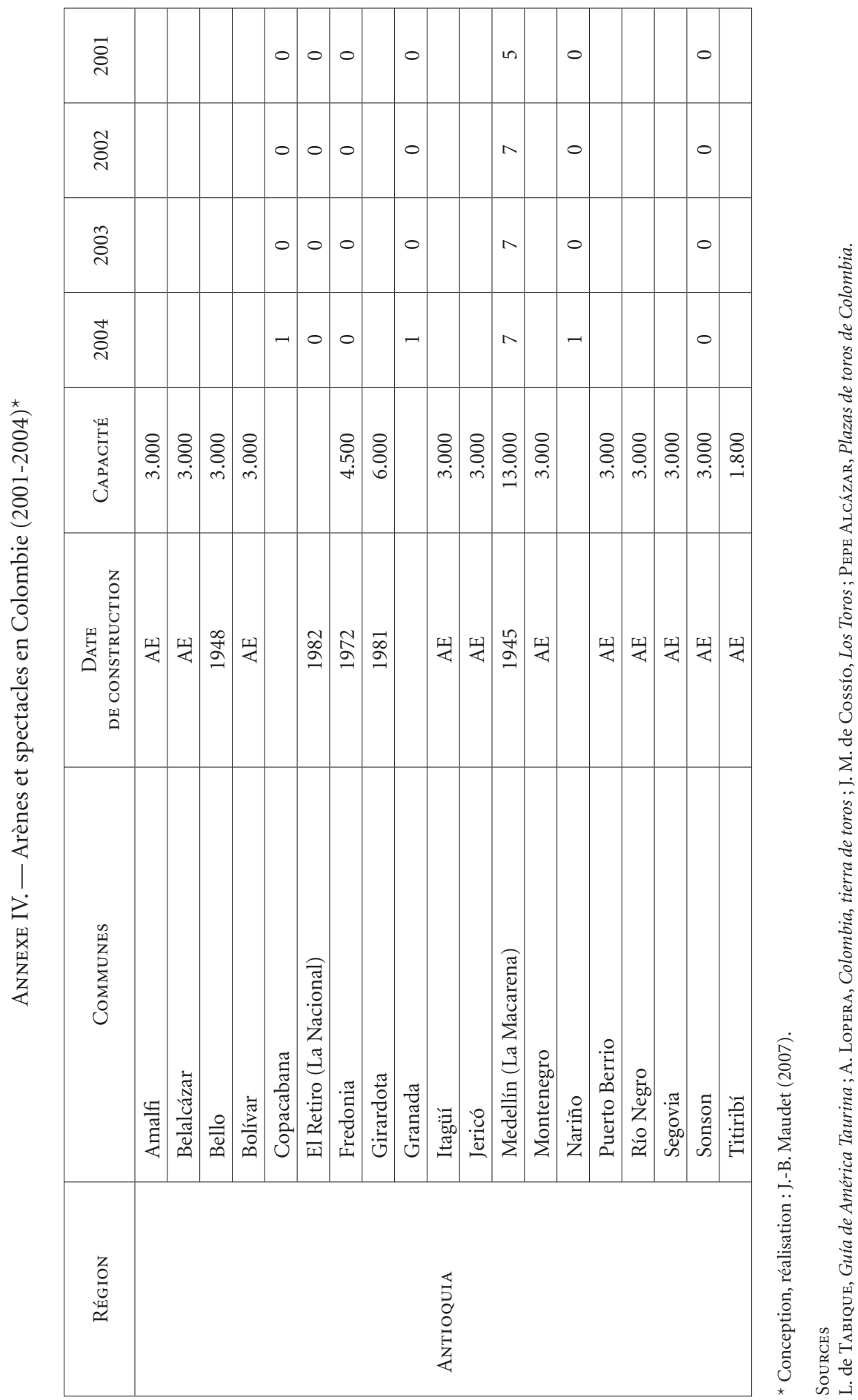




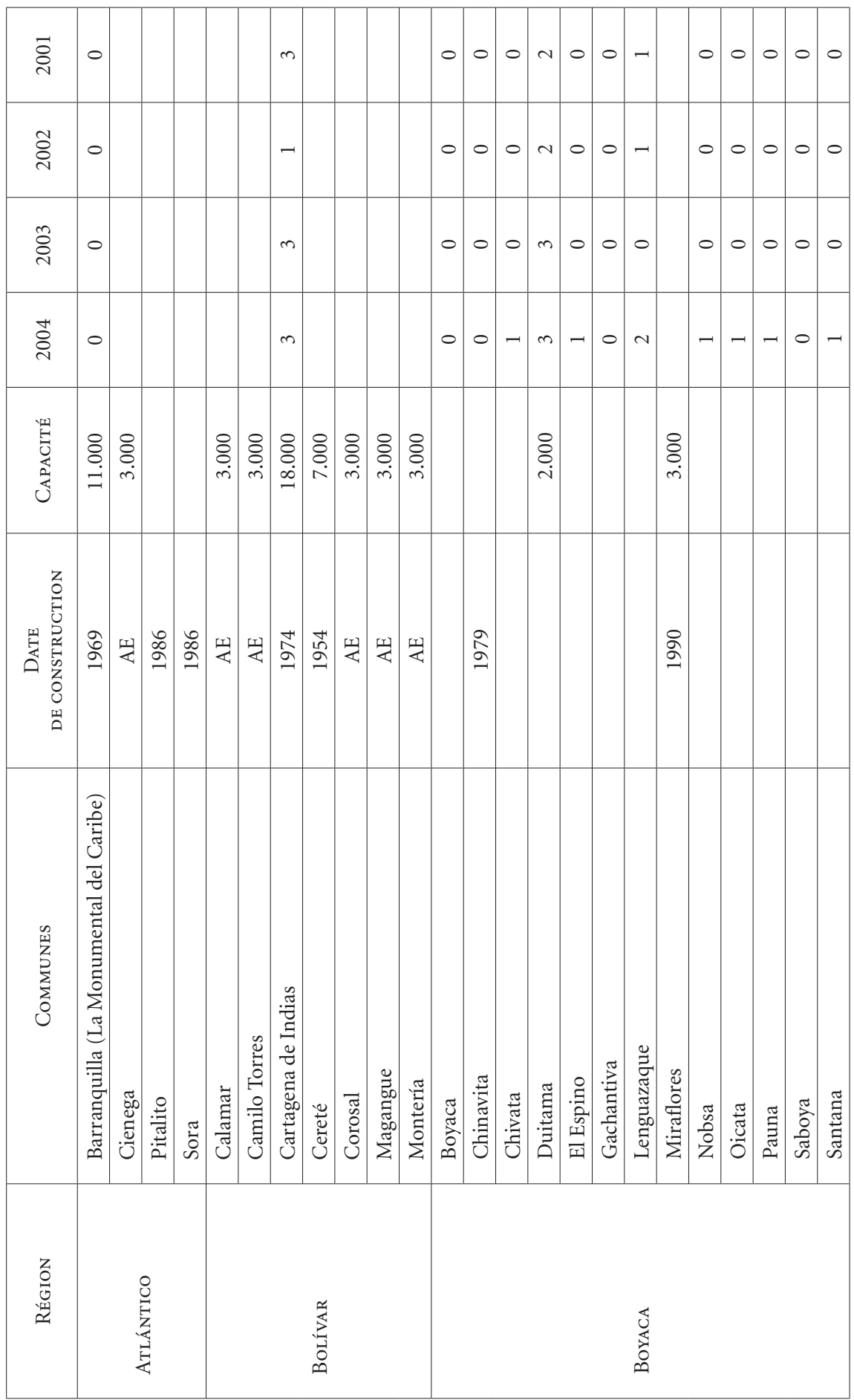




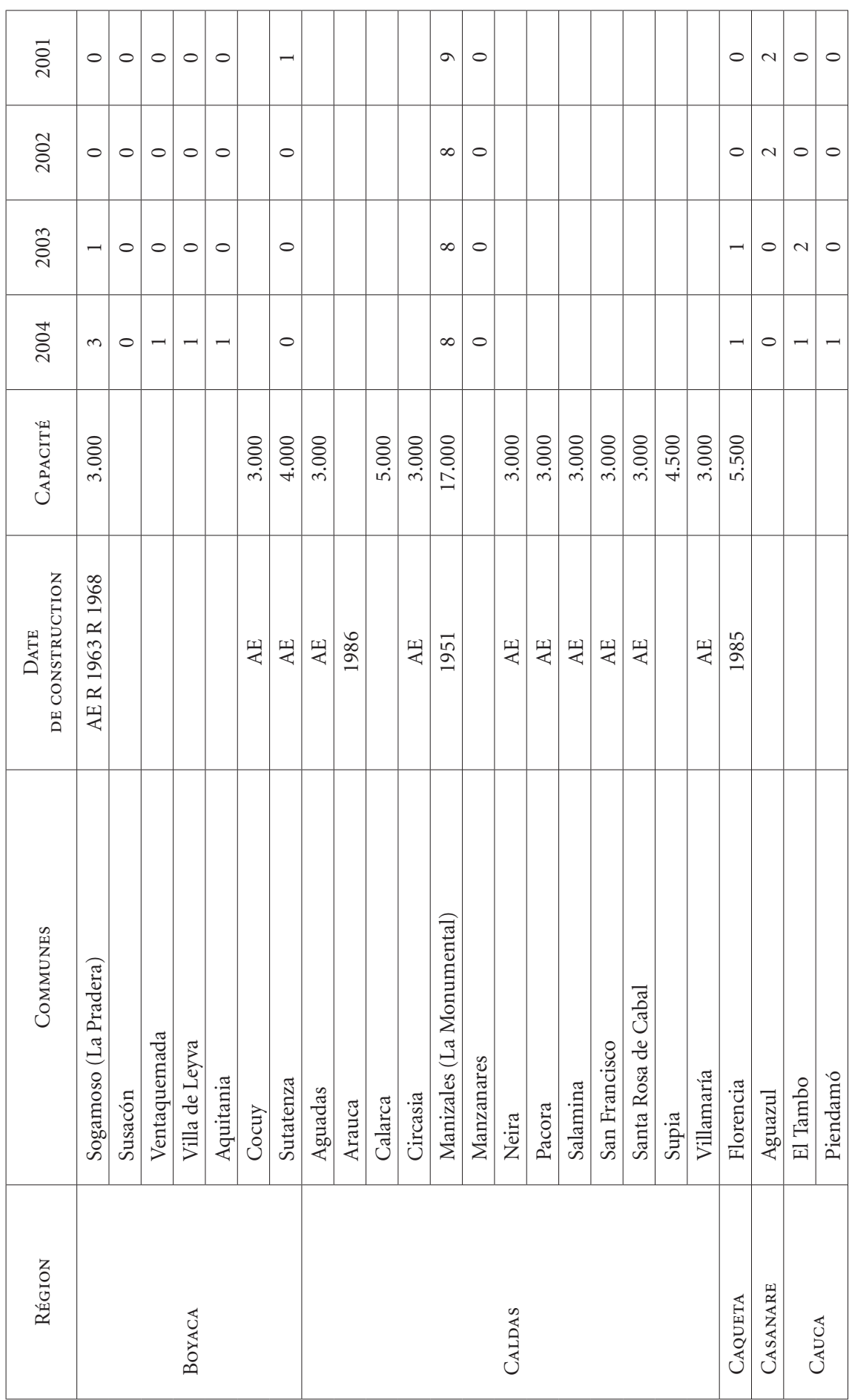




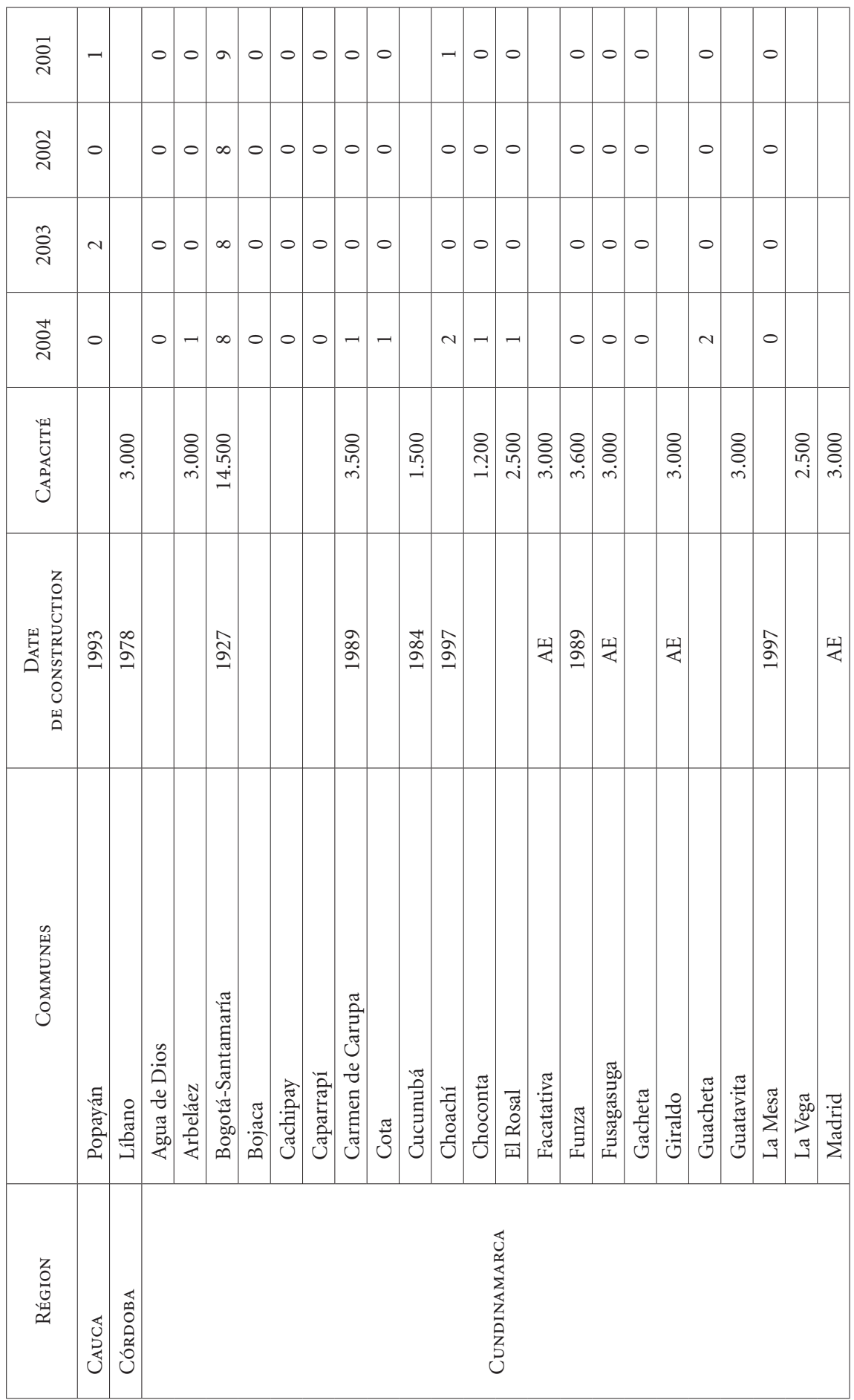




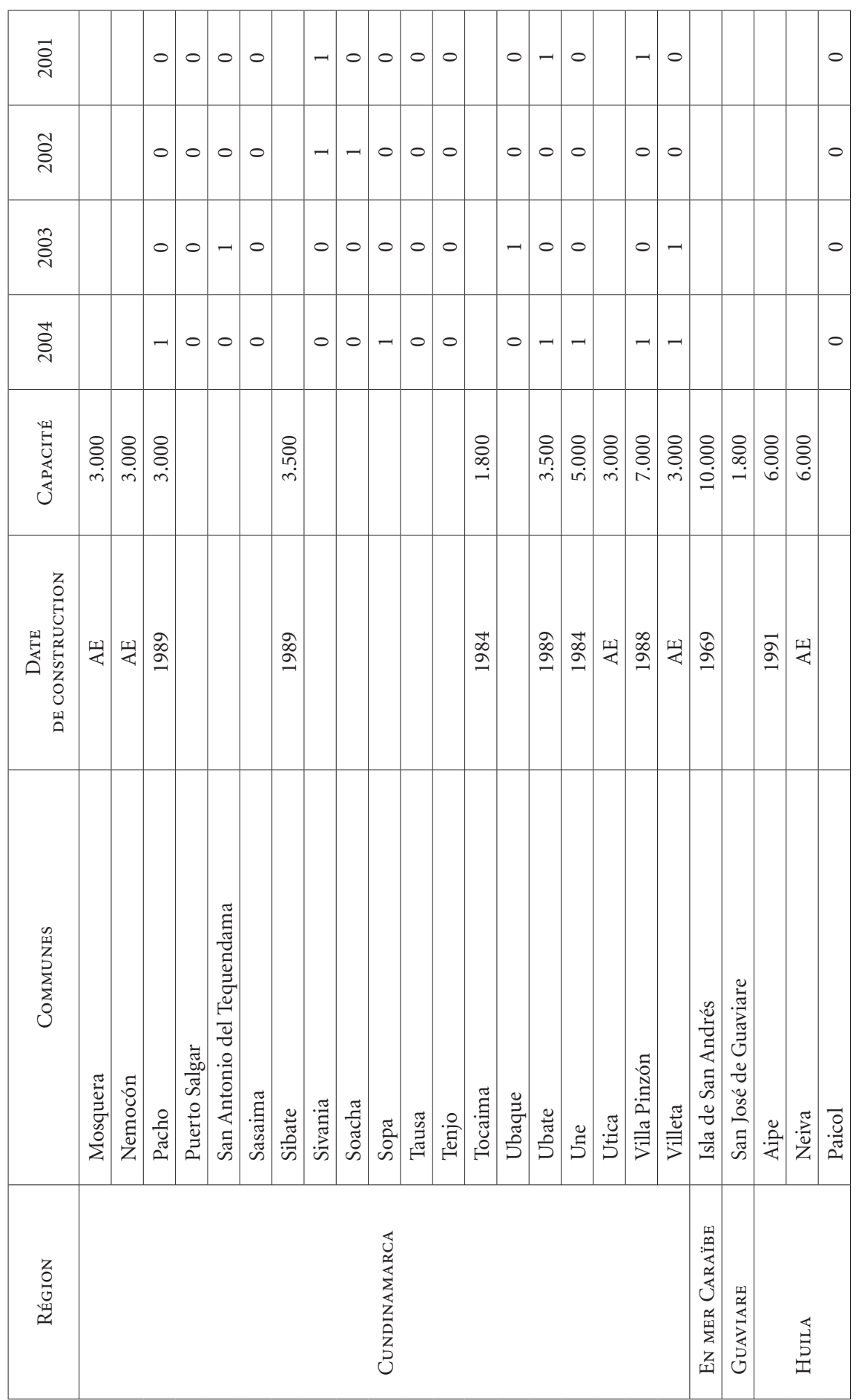




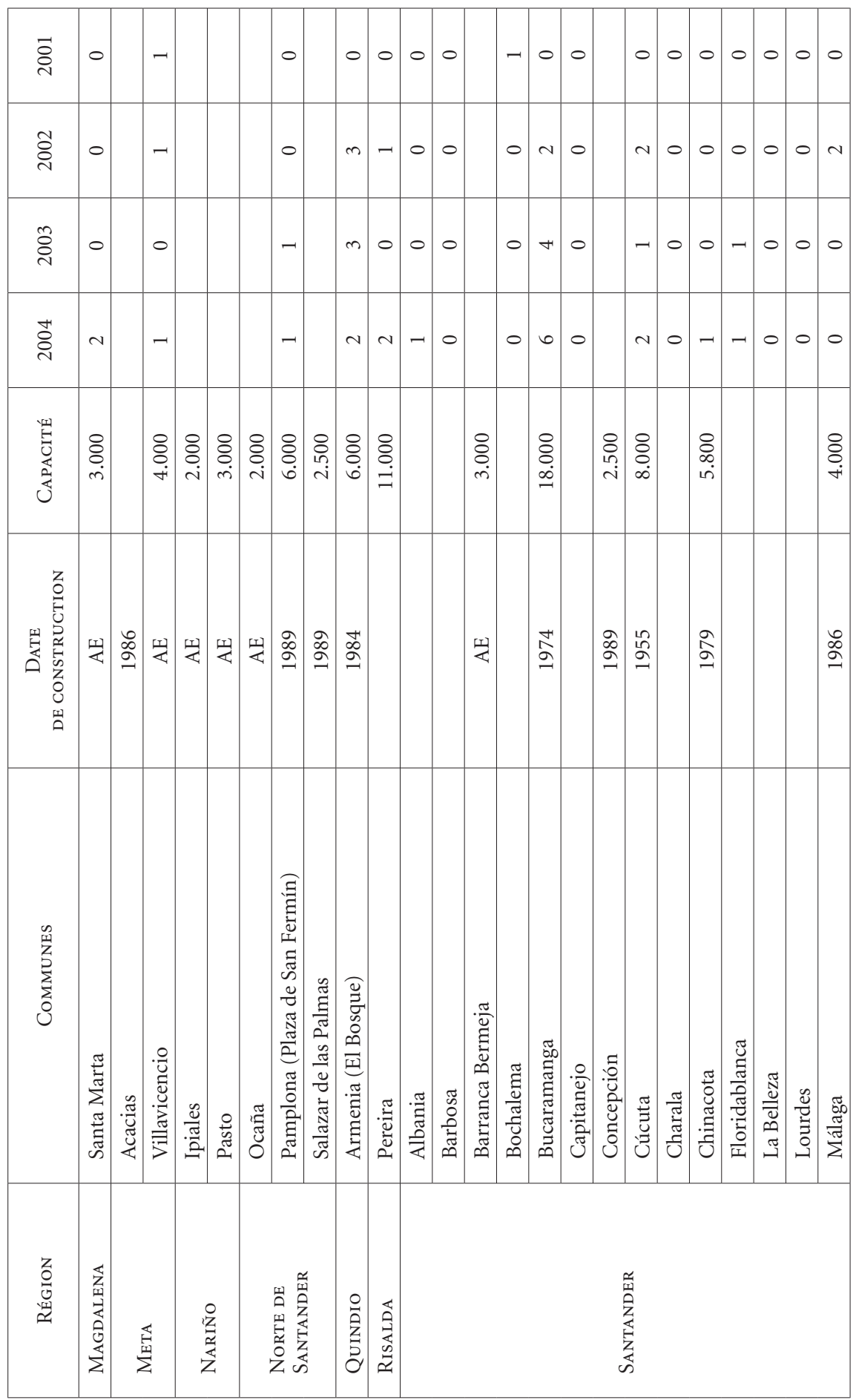




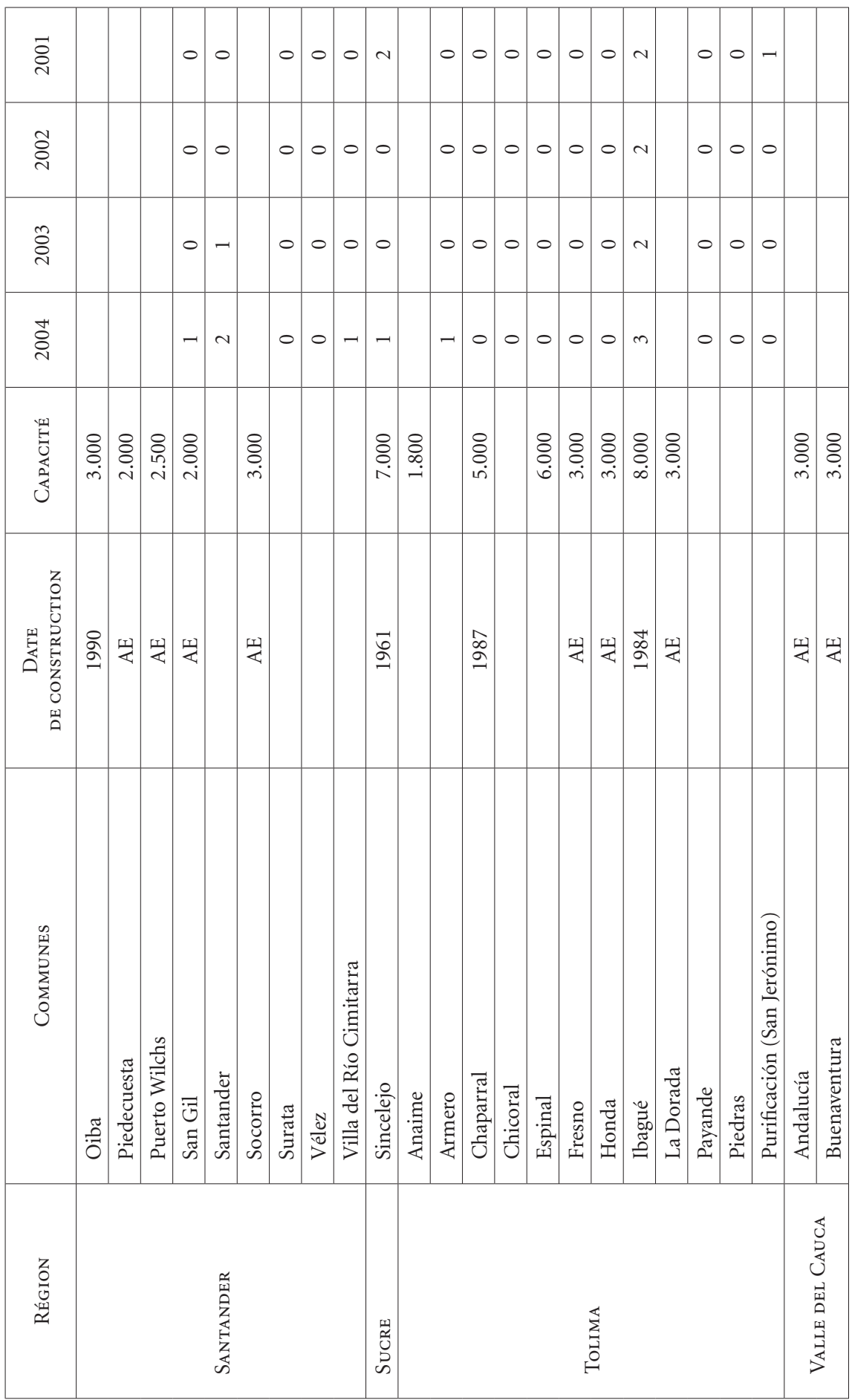




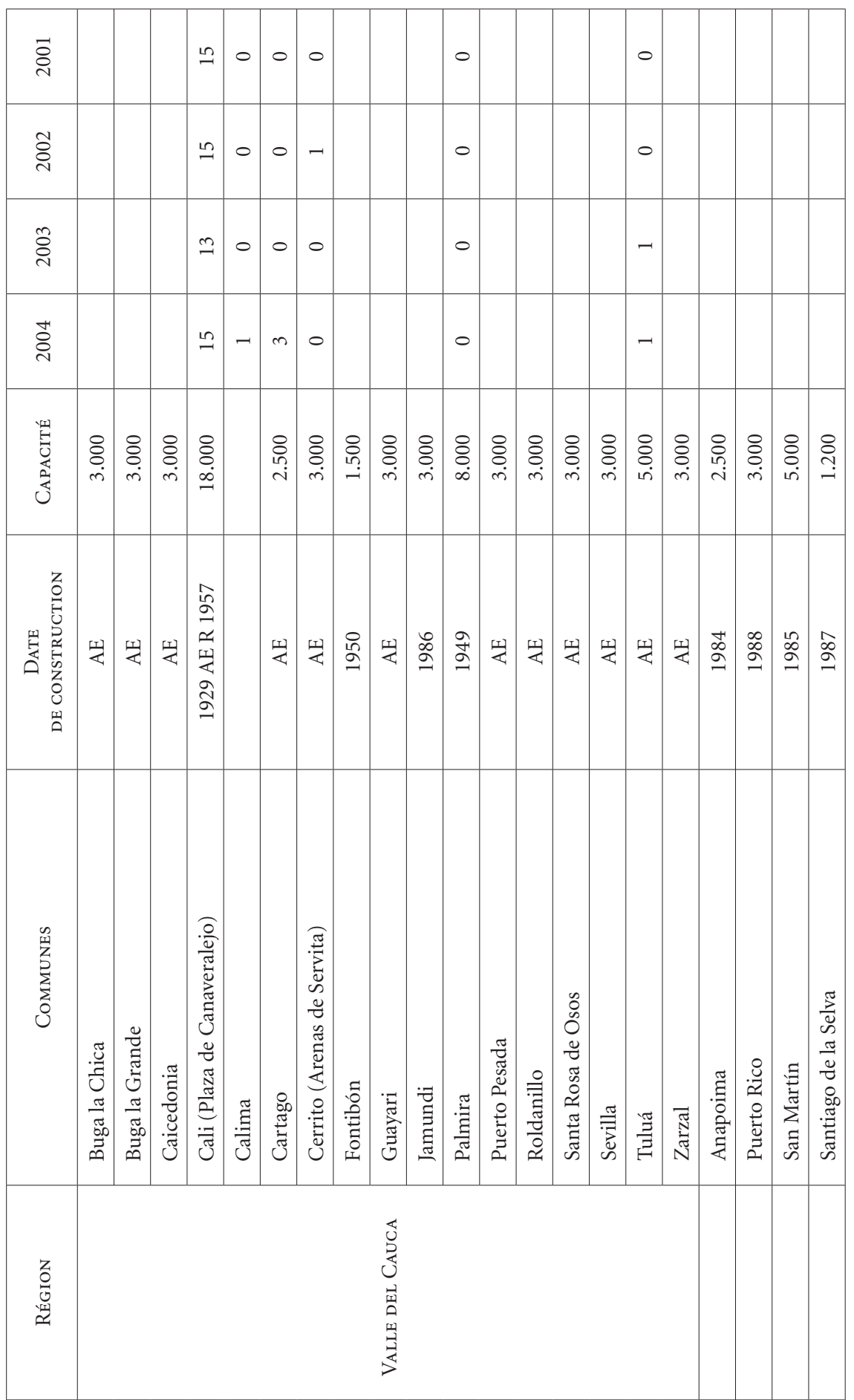




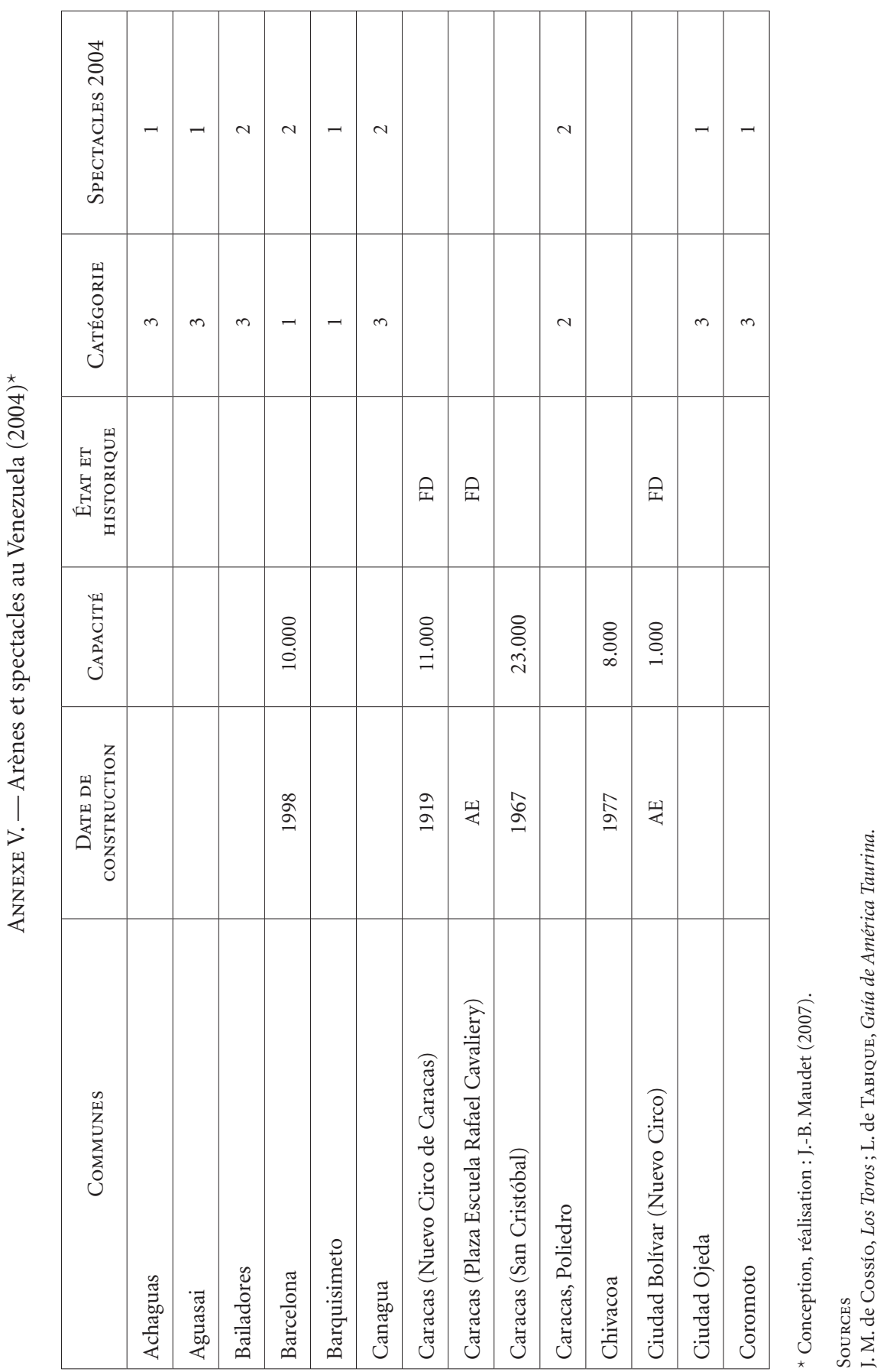




\begin{tabular}{|c|c|c|c|c|c|c|c|c|c|c|c|c|c|c|c|c|c|c|}
\hline 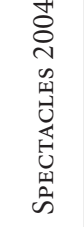 & - & - & - & $m$ & & - & - & $\neg$ & & $\sim$ & $\sim$ & . & & - & & . & & . \\
\hline 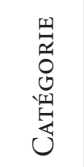 & $n$ & n & n & $m$ & & $n$ & 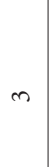 & $m$ & & $m$ & 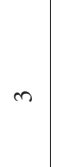 & $\sim$ & & $n$ & & $n$ & & - \\
\hline 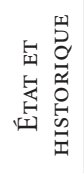 & & & & & & & & & & & & & 吕 & & $\underline{\varphi}$ & & & \\
\hline 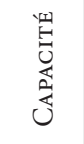 & & & & & & & & & & & & & 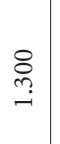 & & & & & \\
\hline 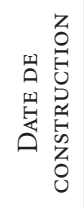 & & & & & 岁 & & & & 今ે & & & & ૂે & & 贸 & & Iે & \\
\hline 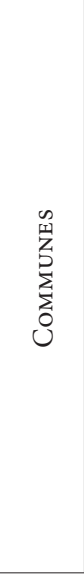 & 得 & 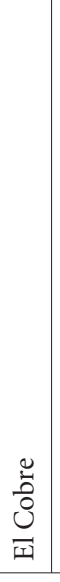 & 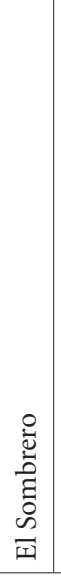 & 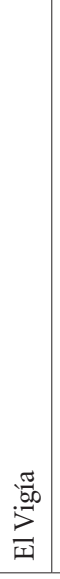 & 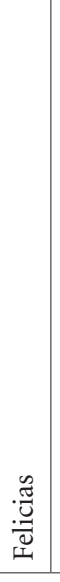 & 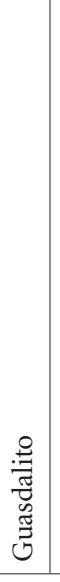 & 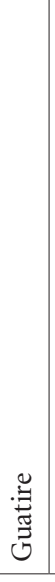 & 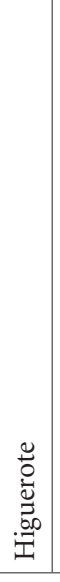 & 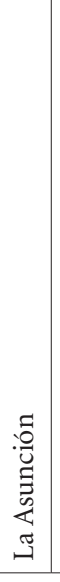 & 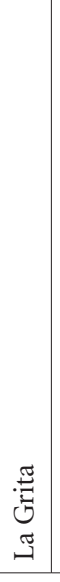 & 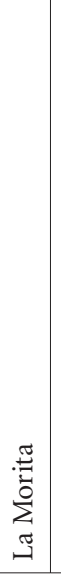 & 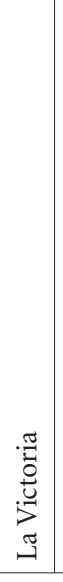 & 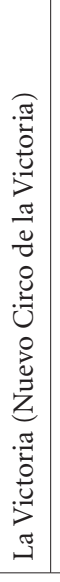 & 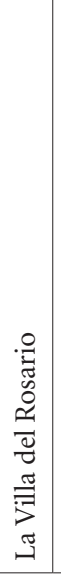 & 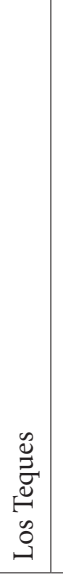 & 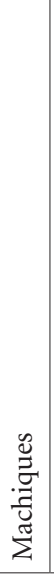 & 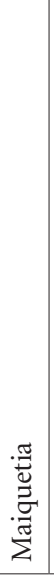 & 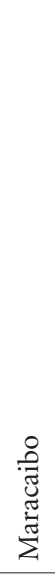 \\
\hline
\end{tabular}




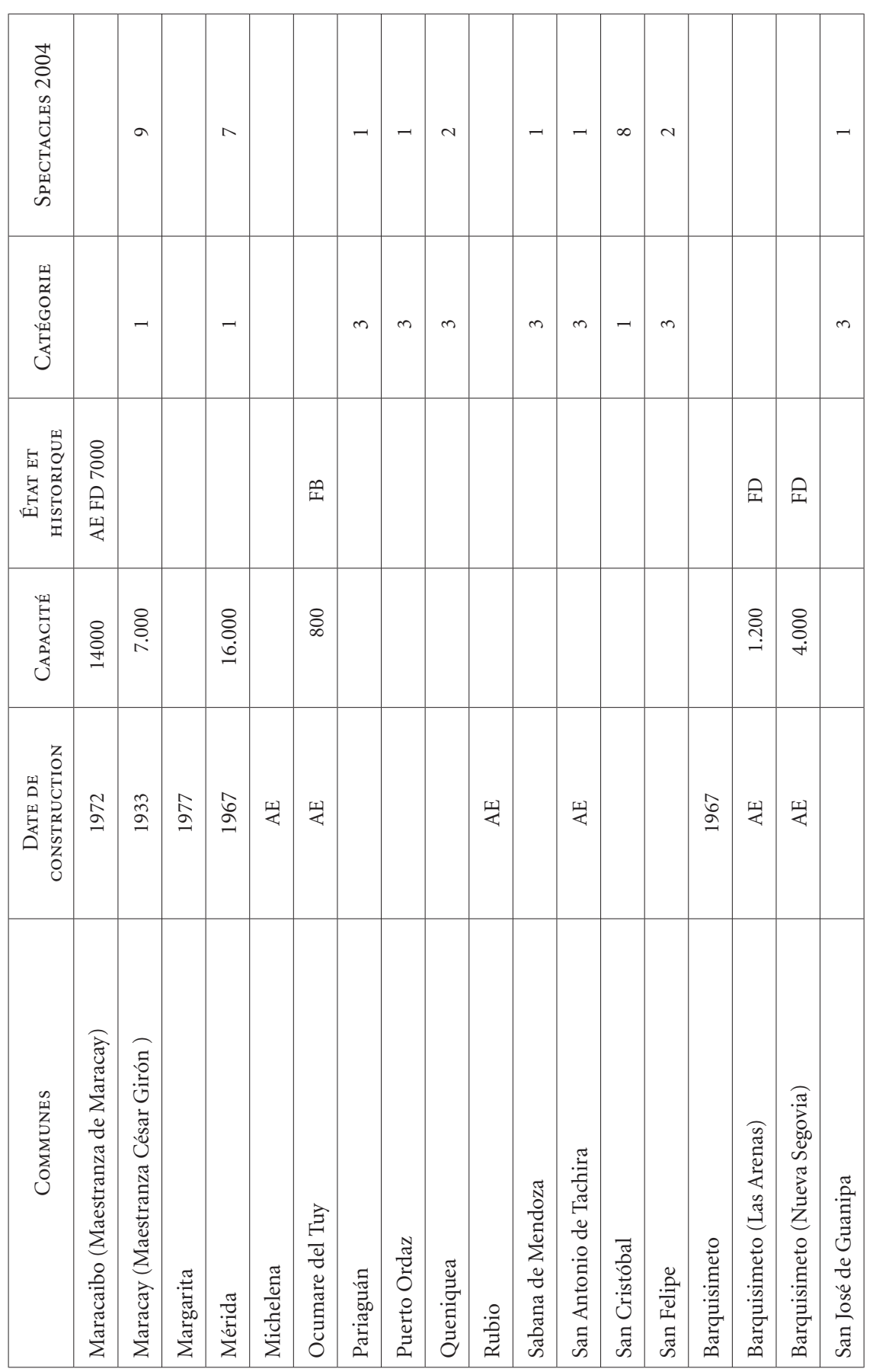




\begin{tabular}{|c|c|c|c|c|c|c|c|c|c|c|c|c|c|c|c|c|c|c|c|}
\hline 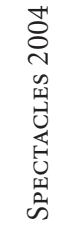 & & - & - & $\sim$ & - & - & - & - & $n$ & - & $\neg$ & & $\neg$ & & in & . & - & $n$ & \\
\hline 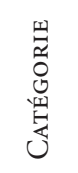 & & $n$ & $n$ & $n$ & $n$ & $\sim$ & $\sim$ & $m$ & - & $\sim$ & $\sim$ & & $m$ & & $\neg$ & $\sim$ & $m$ & $\sim$ & \\
\hline 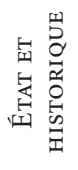 & & & & & & प्रि & & & & & 罗 & 分 & & 只 & & & $\mathscr{\varphi}$ & & 呈 \\
\hline 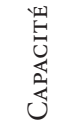 & & & & & & & & & & & $\begin{array}{l}\stackrel{8}{0} \\
\text { in }\end{array}$ & $\underset{-}{\stackrel{8}{\circ}}$ & & $\begin{array}{l}0 \\
\stackrel{0}{0} \\
\stackrel{0}{0}\end{array}$ & $\begin{array}{l}8 \\
0 \\
\text { ¿्d } \\
\text { in }\end{array}$ & & 号 & 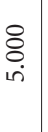 & \& \\
\hline 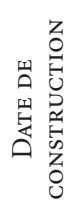 & 焉 & & & & & ळ̆ & & & & & & 琴 & & 正 & $\begin{array}{l}\infty \\
\stackrel{0}{\circ}\end{array}$ & & 贸 & $\stackrel{\text { }}{\stackrel{2}{2}}$ & 琴 \\
\hline 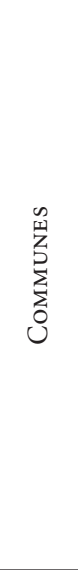 & 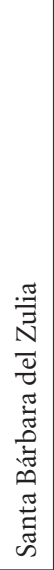 & 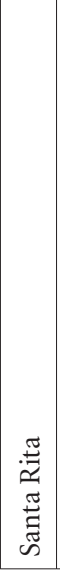 & 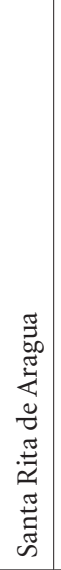 & $\begin{array}{l}8 \\
\ddot{0} \\
0 \\
0 \\
\infty\end{array}$ & 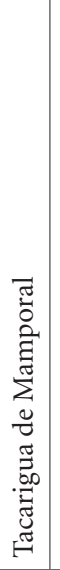 & 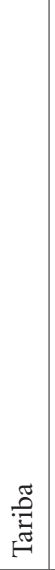 & $\begin{array}{l}\stackrel{\widetilde{Z}}{\overparen{Z}} \\
\text { ज }\end{array}$ & $\begin{array}{l}\stackrel{0}{\Xi} \\
\stackrel{\Xi}{\Xi} \\
\stackrel{\Xi}{\Xi}\end{array}$ & $\stackrel{\overrightarrow{\tilde{z}}}{\stackrel{\overrightarrow{0}}{\rho}}$ & 营 & 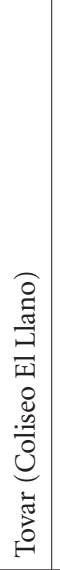 & 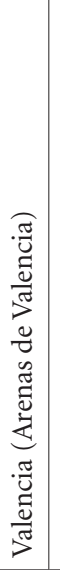 & 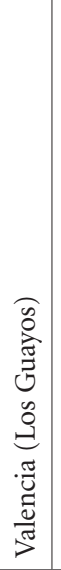 & 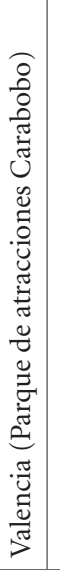 & 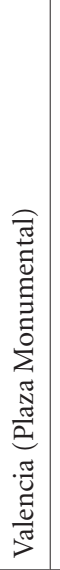 & 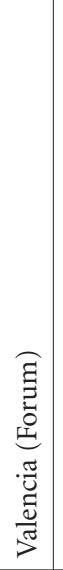 & $\frac{\frac{\pi}{2}}{\frac{\pi}{\pi}}$ & 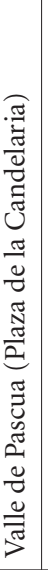 & 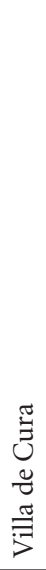 \\
\hline
\end{tabular}


ANNEXES

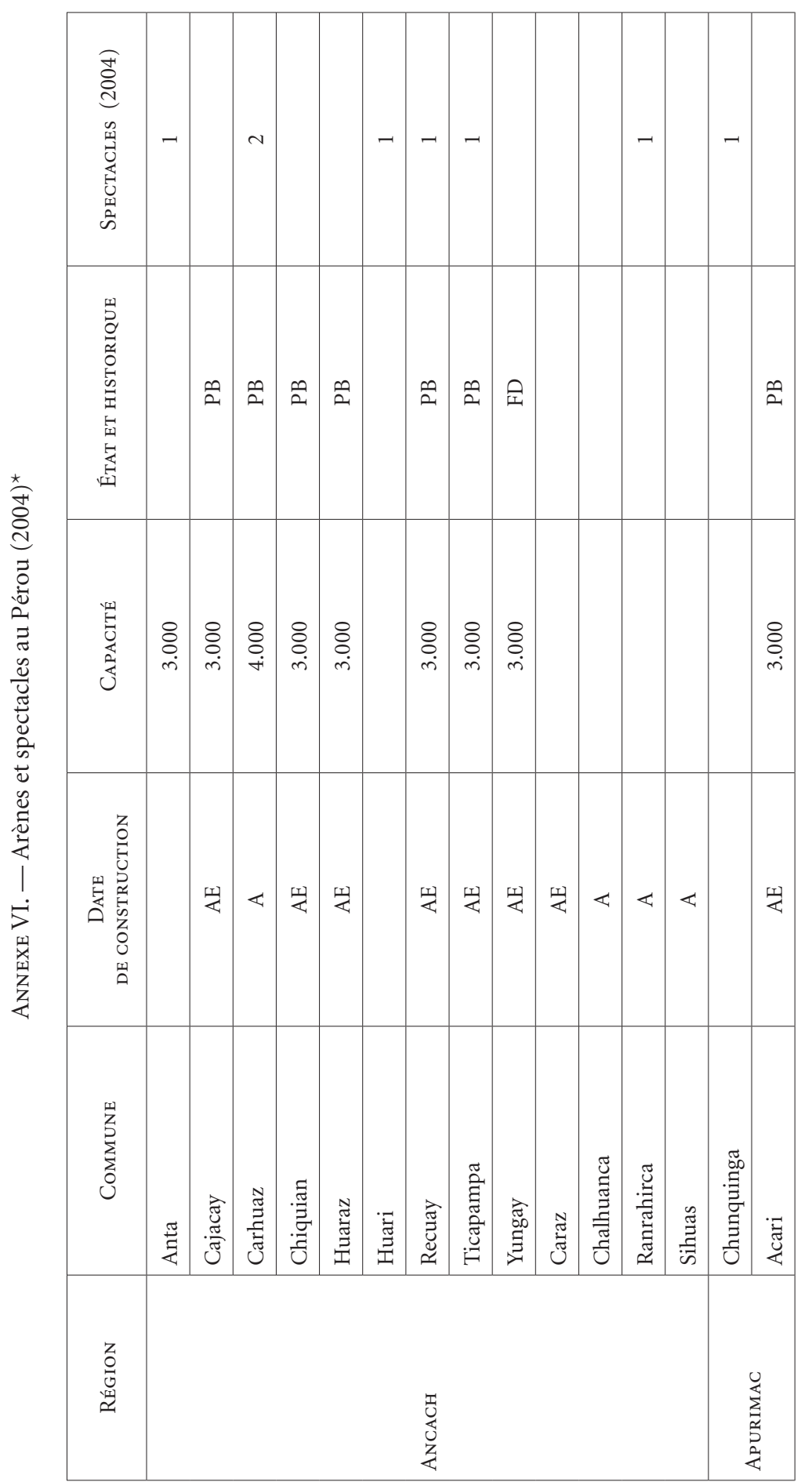

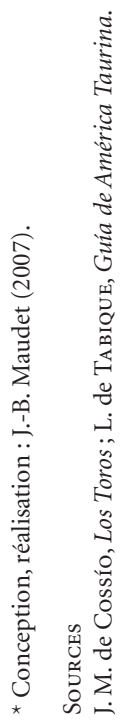




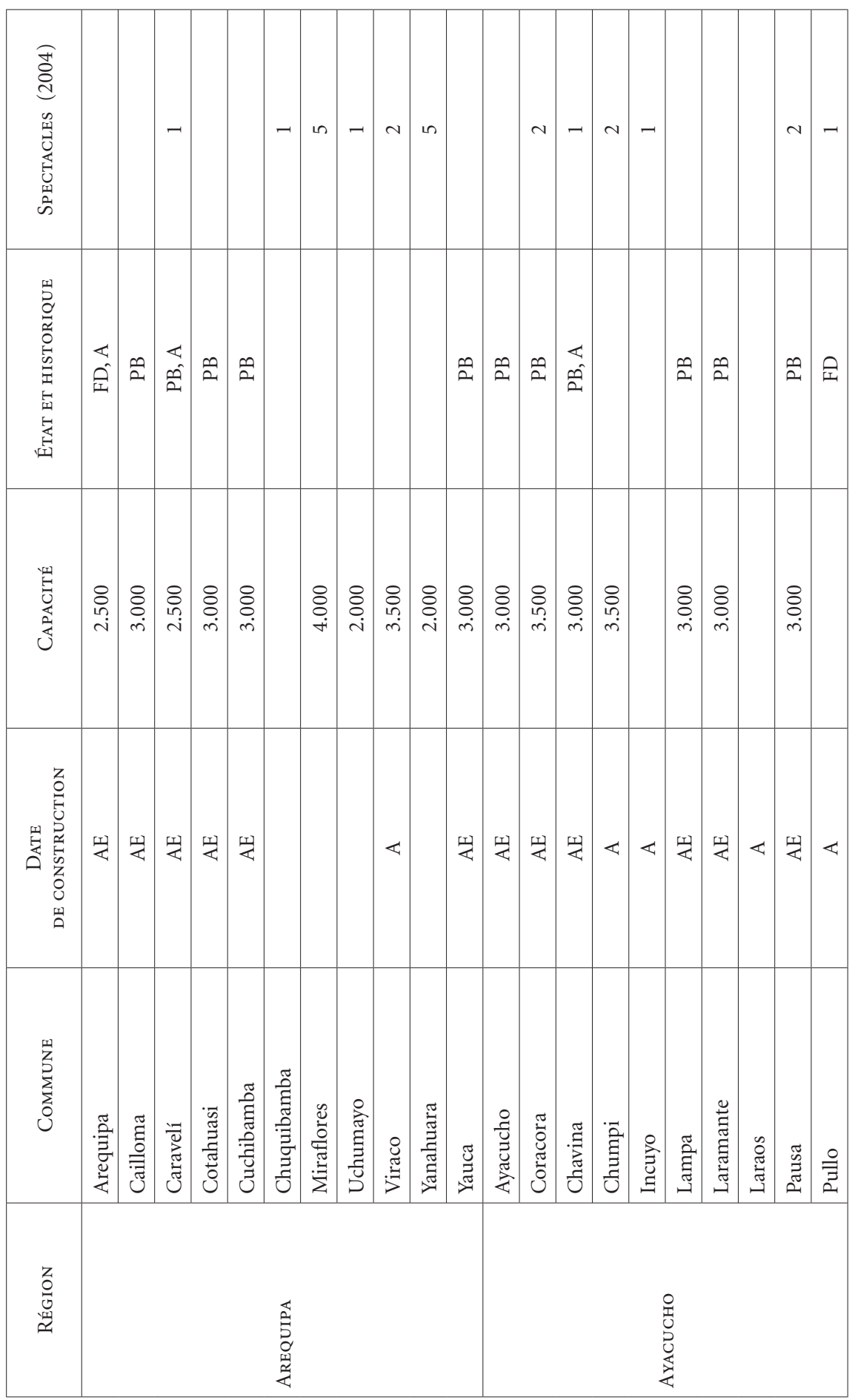


ANNEXES

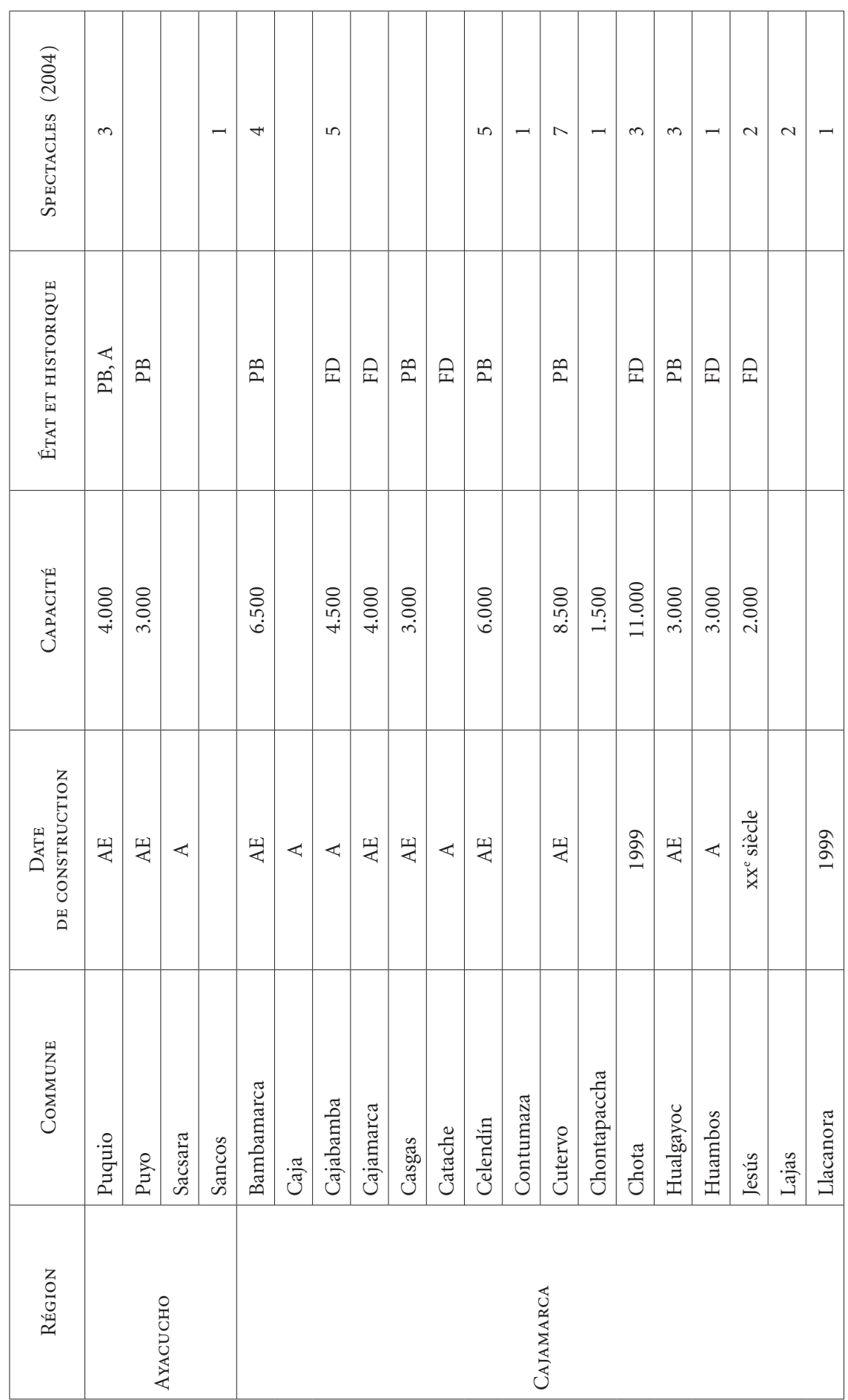




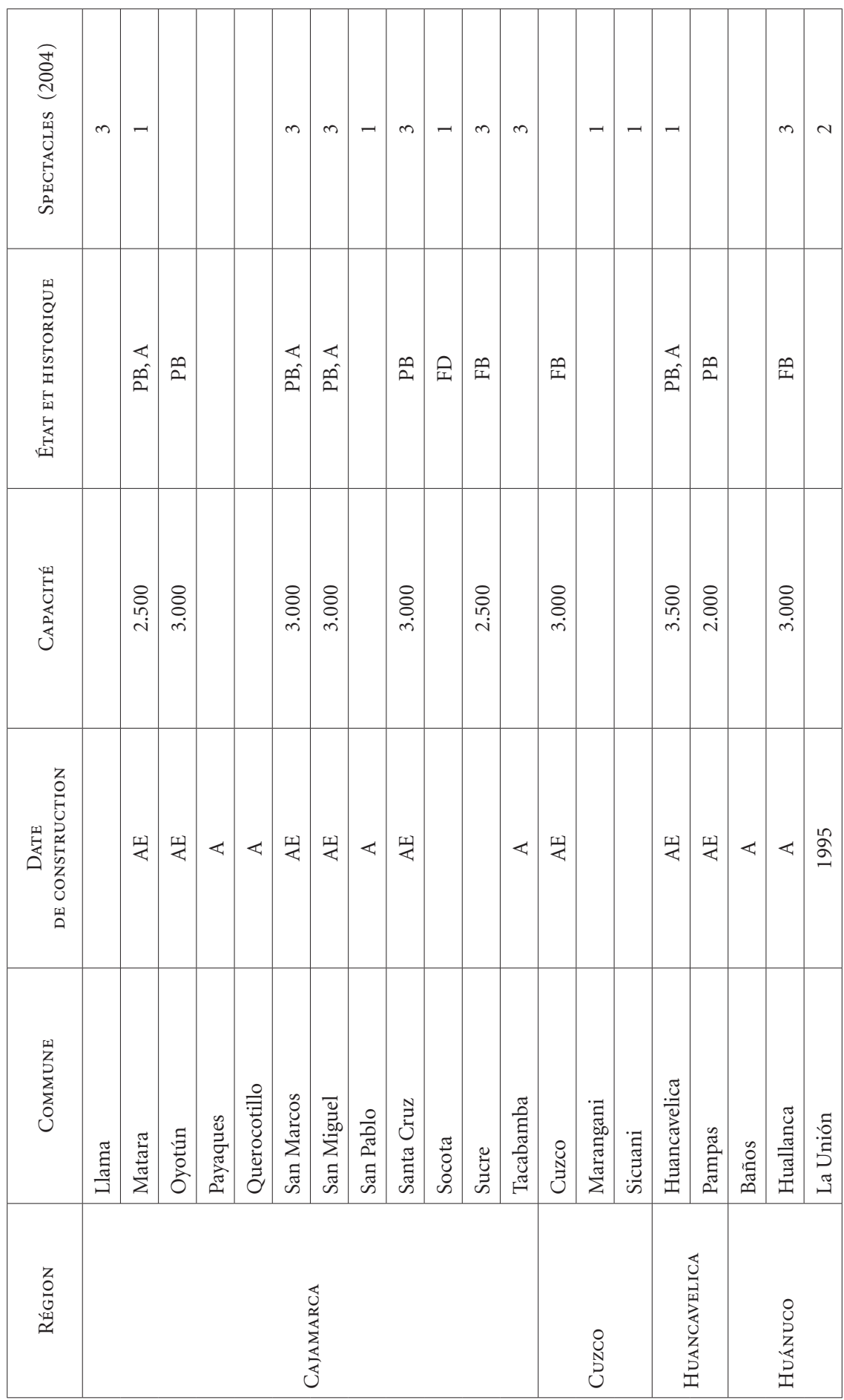


ANNEXES

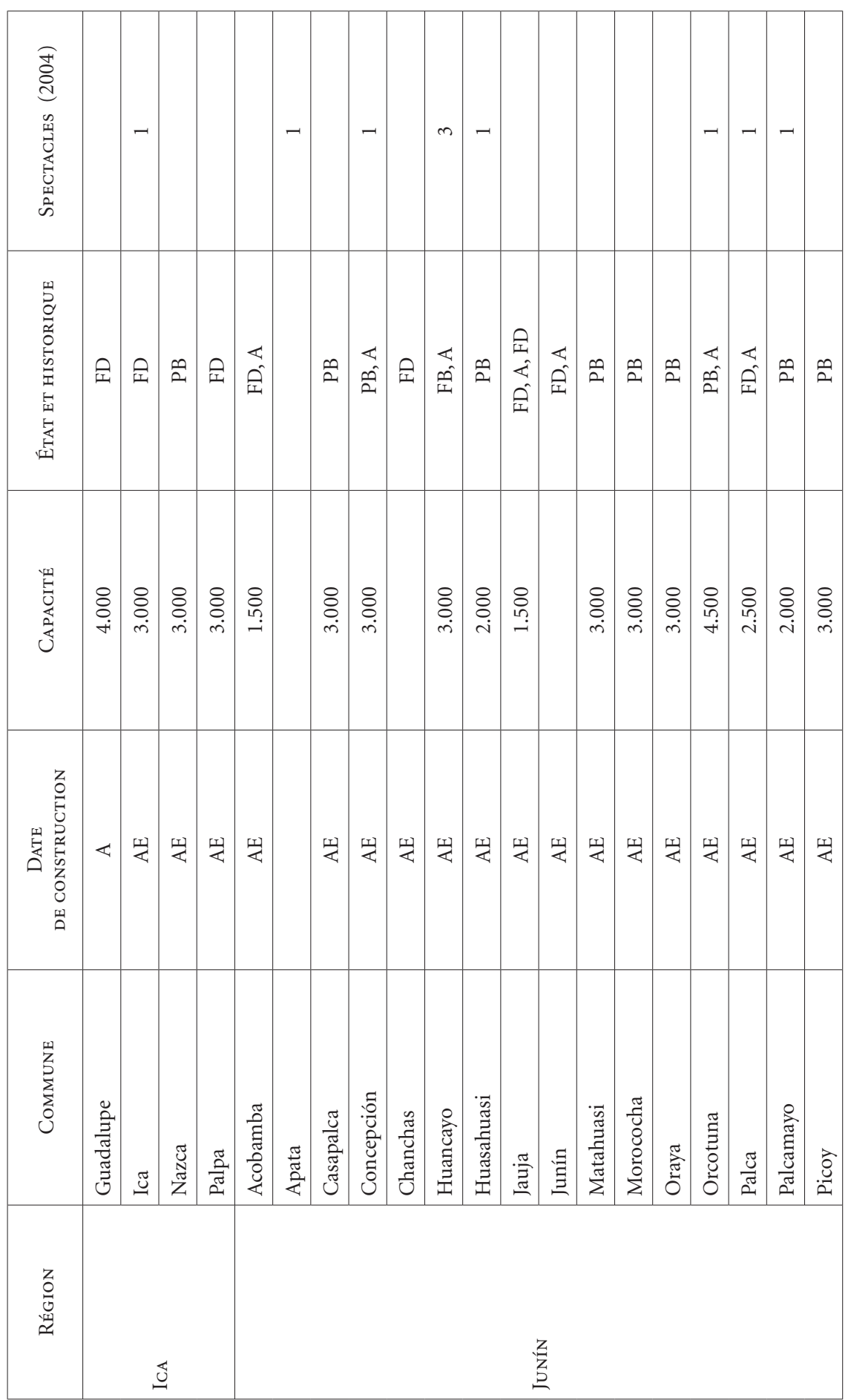




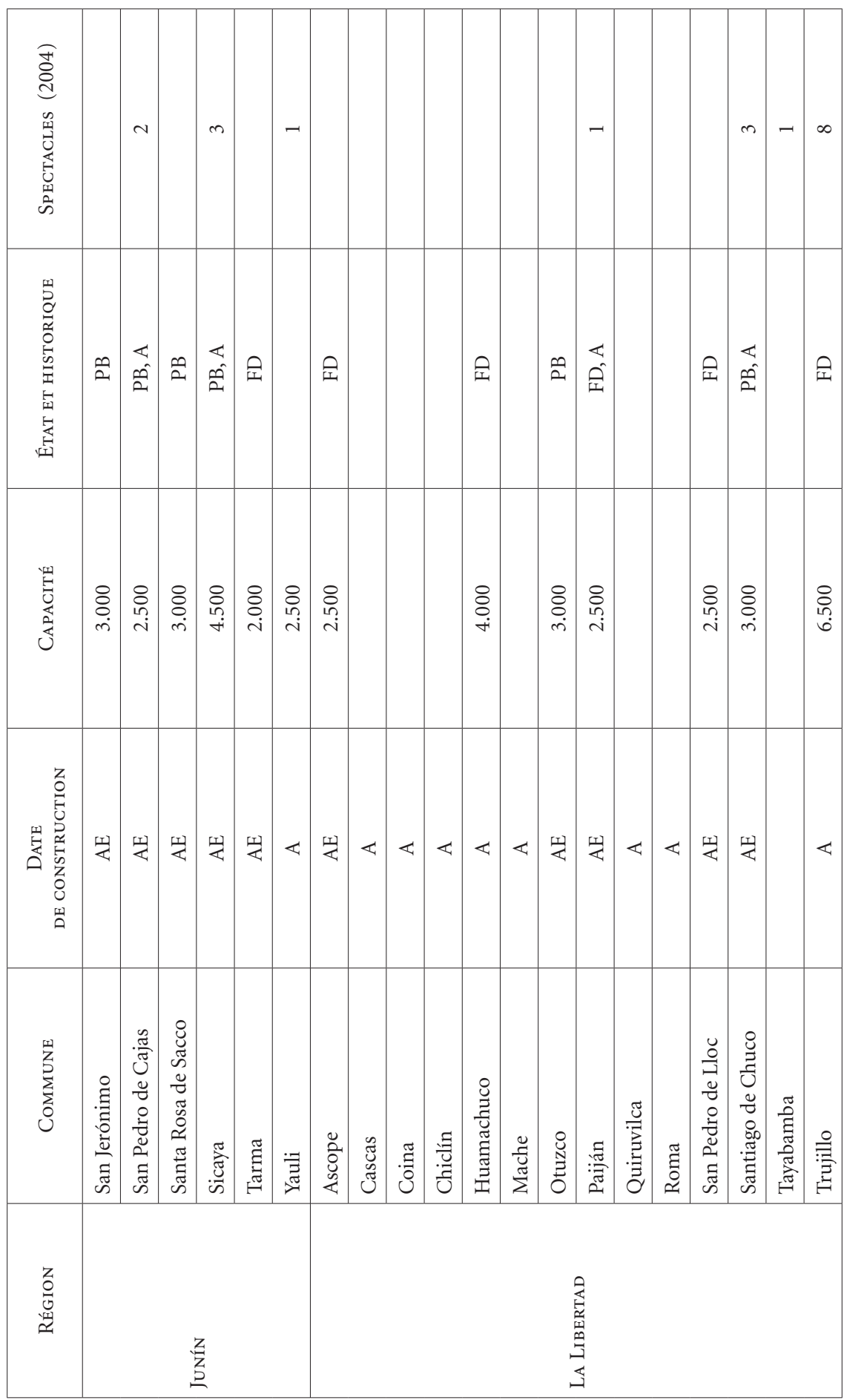


ANNEXES

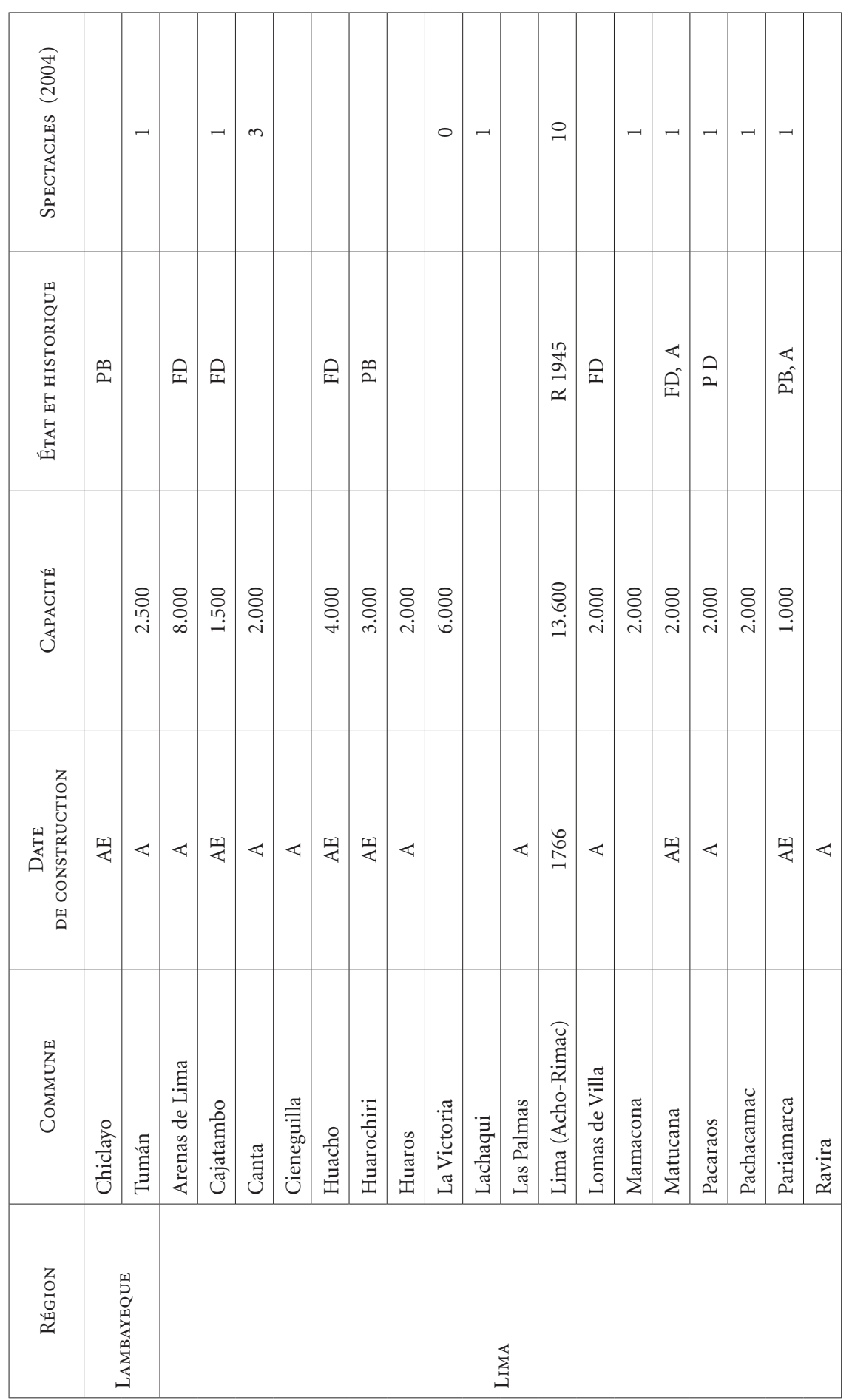




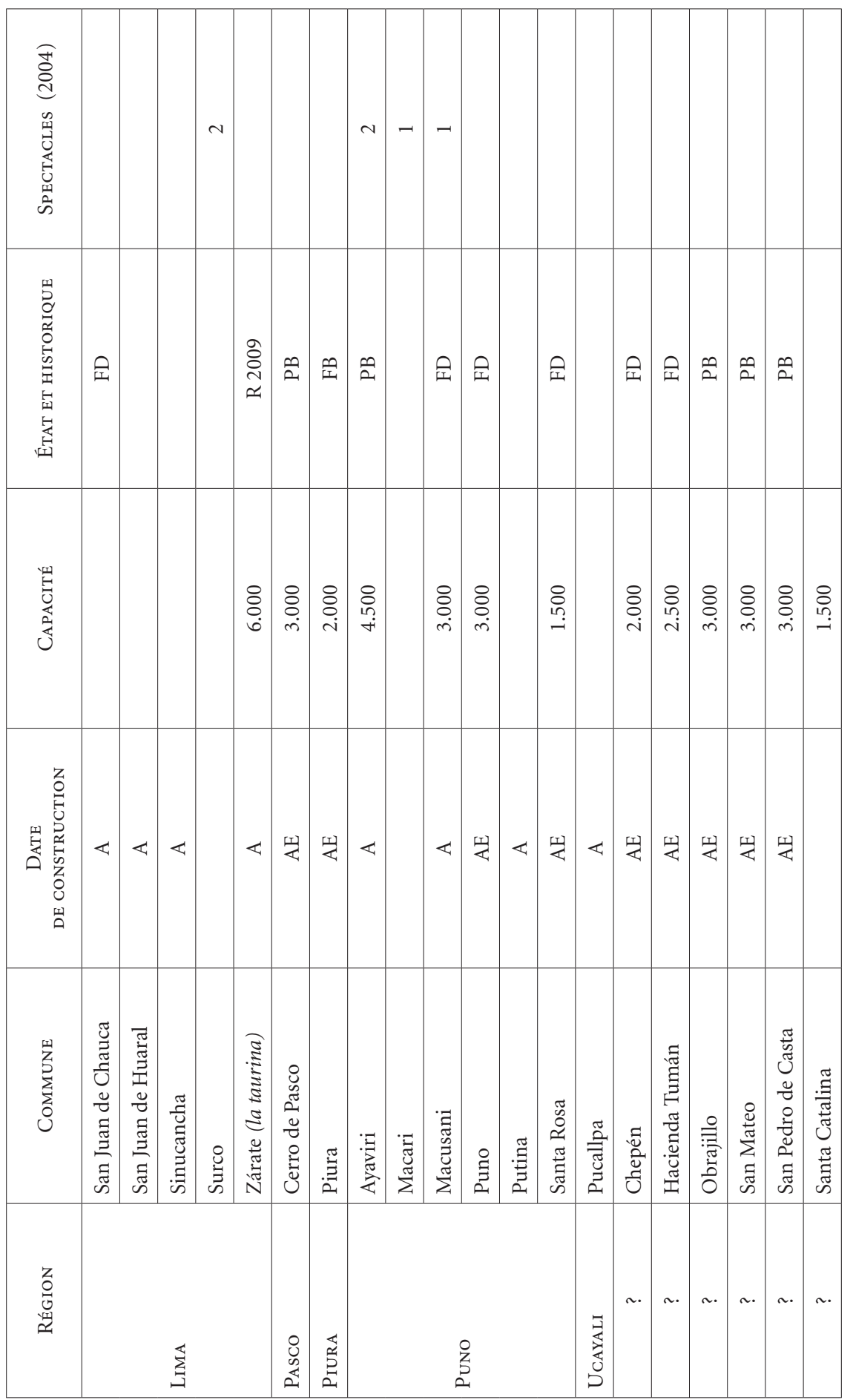




\section{Ce quarante-sixième volume de la Bibliothèque de la Casa de Velázquez a été imprimé en février 2010}

par Closas Orcoyen à Paracuellos de Jarama et broché par Ramos à Madrid.

Dépôt légal : M. 8.685-2010.

Imprimé en Espagne - Printed in Spain Impreso en España 
